$$
\text { Ag8मAh }
$$

Ag8 1 Ah

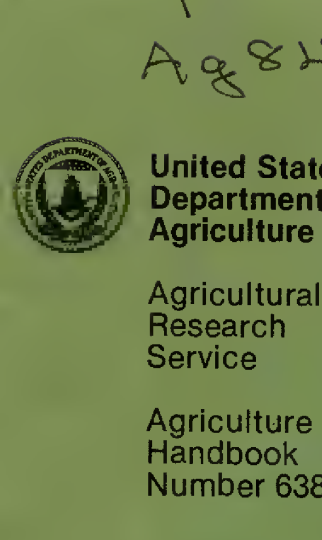

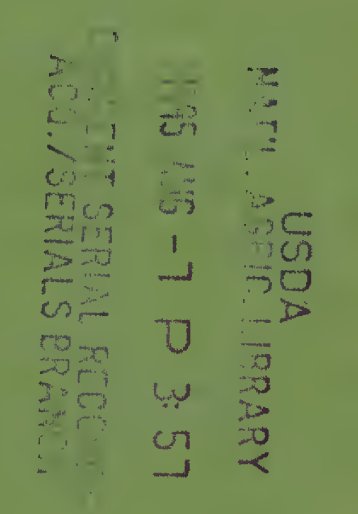

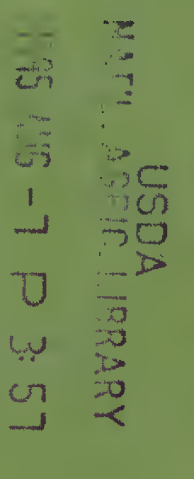

$$
\text { r }
$$ (18) United States
Department of
Agriculture
Agricultural
Research
Service
$\begin{aligned} & \text { Agriculture } \\ & \text { Handbook } \\ & \text { Number } 638\end{aligned}$ United States
Department of
Agriculture
$\begin{aligned} & \text { Agricultural } \\ & \text { Research } \\ & \text { Service }\end{aligned}$
$\begin{aligned} & \text { Agriculture } \\ & \text { Handbook } \\ & \text { Number 638 }\end{aligned}$ United States
Department of
Agriculture
$\begin{aligned} & \text { Agricultural } \\ & \text { Research } \\ & \text { Service }\end{aligned}$
$\begin{aligned} & \text { Agriculture } \\ & \text { Handbook } \\ & \text { Number 638 }\end{aligned}$ United States
Department of
Agriculture
$\begin{aligned} & \text { Agricultural } \\ & \text { Research } \\ & \text { Service }\end{aligned}$
$\begin{aligned} & \text { Agriculture } \\ & \text { Handbook } \\ & \text { Number 638 }\end{aligned}$ United States
Department of
Agriculture
$\begin{aligned} & \text { Agricultural } \\ & \text { Research } \\ & \text { Service }\end{aligned}$
$\begin{aligned} & \text { Agriculture } \\ & \text { Handbook } \\ & \text { Number 638 }\end{aligned}$ United States
Department of
Agriculture
$\begin{aligned} & \text { Agricultural } \\ & \text { Research } \\ & \text { Service }\end{aligned}$
$\begin{aligned} & \text { Agriculture } \\ & \text { Handbook } \\ & \text { Number 638 }\end{aligned}$

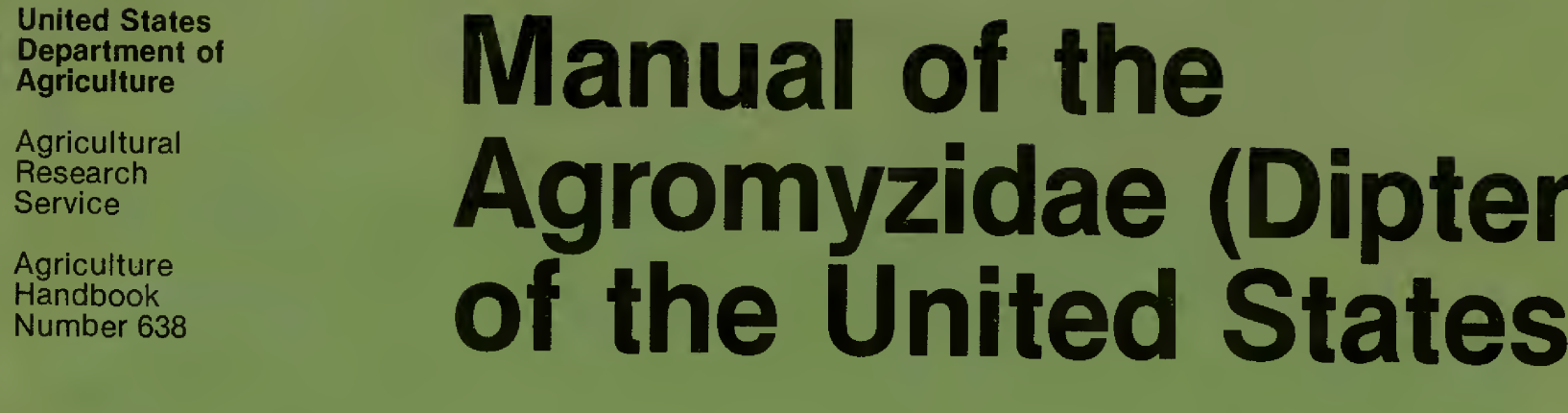
Manual of the
Agromyzidae (Diptera)
of the United States Manual of the
Agromyzidae (Diptera)
of the United States Manual of the
Agromyzidae (Diptera)
of the United States

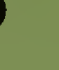

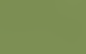

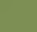

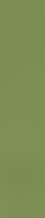

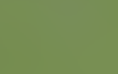

s .

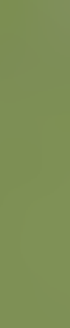

西 
Spencer, Kenneth A., and George C. Steyskal. 1986. Manual of the Agromyzidae (Diptera) of the United States. U.S. Department of Agricu1ture, Agriculture Handbook No. 638, $478 \mathrm{pp}$.

In part 1 , keys to genera and species are provided for the 531 species of
Agromyzidae, with a list of their host plants. In part 2, 85 new species are described, and 22 new synonymies and 16 new combinations are established.

KEYWORDS: Agromyzidae, biology, distribution, entomology, identification, insects, keys, North America, taxonomy. 
United States

Department of

Agriculture

Manual of the

Agricultural

Research

Service

Agriculture

Handbook

Number 638

\section{Agromyzidae (Diptera) of the United States}

By

Kenneth A. Spencer and

George C. Steyskal 

Preparation of this manual has been possible as a result of a USDA Cooperative Agreement made through the active support and interest of R. H. Hodges, formerly Chief, Systematic Entomology Laboratory, U.S. Department of Agriculture. R. J. Gagné, lead scientist of the Diptera Unit of the Systematic Entomology Laboratory, has given advice and assistance throughout the preparation of the publication. Sincere thanks are due both Dr. Hodges and Dr. Gagné.

We are grateful to the authorities of the Smithsonian Institution for facilities to study types. Types and other material have unhesitatingly been lent by the following institutions: Biosystematics Research Institute, Agriculture Canada, Ottawa; British Museum (Natural History), London; California Academy of Sciences, San Francisco; Cornell University, Ithaca, N.Y.; Illinois Natural History Survey, Urbana; Iowa State University, Ames; Museum of Comparative Zoology, Harvard University, Cambridge, Mass.; Naturhistorisches Museum, Vienna, Austria; Naturhistoriska Riksmuseet, Stockholm, Sweden; Oregon State University, Corvallis; Pennsylvania State University, University Park; Rijksmuseum van Natuurlijke Historie, Leiden, Netherlands; Utah State University, Logan; and Zoological Institute, Lund, Sweden. To the curators of the collections of these institutions we express our gratitude for their helpful cooperation.

We are also grateful to the following individuals for substantial assistance in many ways, without which this publication could not have been completed: The senior author's wife Ann, who made most of the drawings; Daphne Allen, Plymouth, England; K. E. Frick, Southern Weed Science Laboratory, U.S. Department of Agriculture, Stoneville, Miss.; G. C. D. Griffiths, University of Alberta, Edmonton; S. Tavormina, University of Texas, Austin; and K. R. Valley, Pennsylvania Department of Agriculture, Harrisburg. The direct experience of these persons with live Agromyzidae has enabled them to contribute in a very real sense to the scientific value of this publication.

It is inevitable that two systematic entomologists have some differences of opinion. This manual has been left substantially as the senior author wrote it, mainly for consistency with his numerous other works.

The outstanding point of difference pertains to the rank of supraspecific taxa. The junior author believes that recent workers in Agromyzidae have recognized too many taxa as of full generic rank. This is especially true of Chromatomyia, inasmuch as there is nothing unscientific in requiring that genera be recognizable by characters that apply to both sexes.

However, a work of this nature is not one in which to propose any major change in supraspecific classification. 

Part 1 - Introduction and keys---Review of 1 iterature------------ 1

Diagnostic characters of the Agromyzidae---------- 3

External morphology---------- 3

Male terminalia-- 5

Immature stages--_-- 7

Biology------ 8

Life history----

Leaf mines------------------- 8

Host selection-- 9

Host plants----------- 9

Economic importance------------ 9

Distribution-------- 10

Rearing Agromyzidae------------ 11

Leaf mines---------------- 12

Stems and flower heads------- 12

Preserving adults-------------- 13

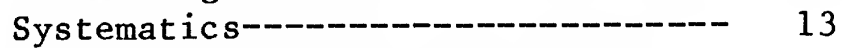

Key to Agromyzidae subfamilies and genera----- 15

Subfamily Agromyzinae------------ 18

Genus Melanagromyza Hende1------ 18

Key to Melanagromyza species---- 19

Genus Hexomyza Enderlein------ 34

Key to Hexomyza species-------- 35

Genus Ophiomyia Braschnikov----- 37

Key to Ophiomyia species------- 39

Genus Japanagromyza Sasakawa---- 53

Key to Japanagromyza species--- 54

Genus Agromyza Fallén-------- 57

Key to Agromyza species------- 58

Subfamily Phytomyzinae-------- 71

Genus Phytobia Lioy--_--- 71

Key to Phytobia species------- 73

Genus Amauromyza Hende1-------- 78

Key to Amauromyza subgenera----- 79

Key to Amauromyza subgenera species--

Genus Nemorimyza Frey---------- 87

Genus Cerodontha Rondani------- 87

Key to Cerodontha subgenera----- 88

Cerodontha, subgenus Icteromyza
Key to Cerodontha, subgenus Icteromyza species----------- 89

Cerodontha, subgenus Cerodontha Rondani-- 91

Cerodontha, subgenus Xenophytomyza Frey------ 91

Cerodontha, subgenus Poemyza Hendel-- 92

Key to Cerodontha, subgenus Poemyza species-------------

Cerodontha, subgenus Phytagromyza Hende 1----------------

Cerodontha, subgenus Butomomyza Nowakowski-- 97

Key to Cerodontha, subgenus Butomomyza species-Cerodontha, subgenus Dizygomyza Hende1-- 100

Key to Cerodontha, subgenus Dizygomyza species----------Genus Liriomyza Mik--------Key to Liriomyza species------Genus Galiomyza Spencer--------Key to Galiomyza species------Genus Haplopeodes Steyskal----Key to Haplopeodes species-----Genus Calycomyza Hendel-----Key to Calycomyza species------Genus Phytoliriomyza Hende1----Key to Phytoliriomyza species--Genus Metopomyza Enderlein-----Key to Metopomyza species------Genus Paraphytomyza Enderlein--Key to Paraphytomyza species---Genus Pseudonapomyza Hende 1----Key to Pseudonapomyza species--Genus Napomyza Westwood------Key to Napomyza species-----Genus Phytomyza Fallén-------Genus Chromatomyia Hardy------Key to Phytomyza and Chromatomyia species----

101

107

108

136

137

138

139

140 141 151 152 161 161 162 163 166 167 167 168 172 173 


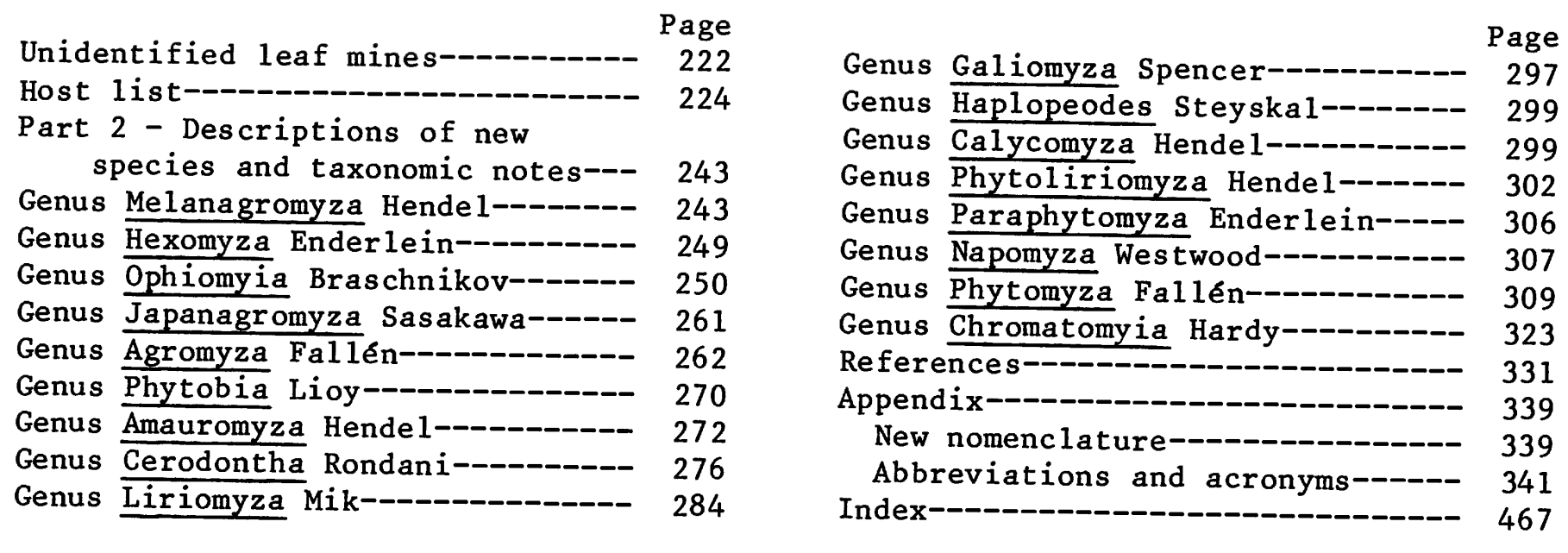

Copies of this publication may be purchased from the National Technical Information Service, 5285 Port Royal Road, Springfield, Va. 22161.

ARS has no additional copies for free distribution. 
MANUAL OF THE AGROMYZIDAE (DIPTERA) OF THE UNITED STATES

by Kenneth A. Spencer and George C. Steyskall/

\section{Part 1 - Introduction and Keys}

Several major papers have been published on the Agromyzidae of the United States, but our knowledge of the family remains inadequate. The family is widely distributed throughout this country, larval leaf-mining damage occurs on many cultivated crops and ornamentals, and numerous alternate hosts facilitate reservoirs for polyphagous pest species. No single key has hitherto been available, and identification of United States species has thus been difficult. This manual attempts to fill this gap, and new keys are provided for all genera. Much additional material has been studied, particularly from high elevations in Colorado and from the Southern States, including the results of our collecting.

In the keys are synoptic descriptions, information on host and larval feeding, distribution, reference to illustrations of male genitalia, and other essential references. We discuss 531 species, of which 85 are described as new in part 2. A generic breakdown of species is given in table 1 . Forty-three of these species are recorded as new to the United States (see appendix), mostly known previously in Canada. The types of all species in the United States have been examined, and 22 new synonymies and 16 new combinations are established. Two species (each in the genera Ophiomyia, Agromyza, Cerodontha

1/Respectively, Department of Biological Sciences, University of Exeter, Exeter, England, and Systematic Entomology Laboratory, Agricultural Research Service, U.S. Department of Agriculture, c/o National Museum of Natural History, Washington, D.C. 20560.
(Dizygomyza), and Phytomyza) are now deleted from the United States list. Liriomyza flaveola, recorded by Frick (1959)2 from California, was found by Spencer (1981) to represent a misidentification of L. septentrionalis, which was original $\overline{l y \text { described }}$ from Alberta, Canada.

\section{REVIEW OF LITERATURE}

Following publication of short papers describing the first species of Agromyzidae in the United States by Loew $(1863,1869,1872)$ and Coquillett (1902, 1910), two important revisionary papers on the family were published by Melander (1913) and Malloch (1913a), the latter including descriptions of 31 new species.

The first comprehensive study of leaf-mining Diptera in the United States was made by Frost (1924). This represented an important review of species, but it is misleading, as many identifications are inaccurate, having been based on European literature, particularly the work of the Austrian dipterist Hendel (1920). A useful, more general discussion of leaf-mining insects was given by Needham, Frost, and Tothill (1928), and a more detailed discussion of their biology was reported by Hering (1951b). Many short papers describing new species were published by Aldrich, Frost, and Malloch between 1914 and 1962 .

A major advance came with Frick's (1952a) generic revision of North American Agromyzidae, followed by a synopsis of species in the United States (1959). These two papers provided a solid basis for future work on the family, but important generic changes have been made in recent years. Frick's papers nevertheless are invaluable for the completeness of their references. Frick also published

$2 /$ The year in parentheses after the author's name refers to References, p. 331 . 
Table 1.--Number and status of Agromyzidae species in the United States

\begin{tabular}{|c|c|c|c|c|c|c|}
\hline $\begin{array}{c}\text { Subfamily, } \\
\text { genus, and } \\
\text { subgenus }\end{array}$ & $\begin{array}{c}\text { New } \\
\text { species }\end{array}$ & $\begin{array}{l}\text { New to } \\
\text { United } \\
\text { States }\end{array}$ & $\begin{array}{l}\text { Number } \\
\text { of } \\
\text { species } \\
\end{array}$ & $\begin{array}{c}\text { New } \\
\text { synonyms }\end{array}$ & $\begin{array}{c}\text { New } \\
\text { combinations }\end{array}$ & $\begin{array}{c}\text { Species not } \\
\text { formally } \\
\text { described } \\
\end{array}$ \\
\hline \multicolumn{7}{|l|}{ Agromyzinae: } \\
\hline Melanagromyza-- & 8 & 3 & 59 & 2 & --- & 1 \\
\hline Hexomyza------- & --- & -- & 6 & 1 & 1 & -- \\
\hline Ophiomyia------ & 14 & 5 & 55 & 7 & 5 & -- \\
\hline Japanagromyza-- & --- & -- & 6 & 1 & 1 & -- \\
\hline Agromyza-- & 6 & 6 & 37 & 4 & -- & 1 \\
\hline Subtotal---- & 28 & 14 & 163 & 15 & 7 & 2 \\
\hline \multicolumn{7}{|l|}{ Phytomyzinae: } \\
\hline Phytobia----- & 1 & 1 & 15 & 1 & --- & 1 \\
\hline Annimyzella-- & --- & -- & 2 & -- & --- & --- \\
\hline Catalpomyza-- & -- & --- & 1 & -- & --- & -- \\
\hline Cephalomyza-- & 2 & -- & 17 & --- & 2 & 1 \\
\hline Nemorimyza--- & -- & --- & 1 & --- & -- & -- \\
\hline \multicolumn{7}{|l|}{ Cerodontha: } \\
\hline$\frac{\text { Icteromyza--- }}{\text { Cerodontha--- }}$ & -- & -- & 5 & -- & -- & -- \\
\hline Xenophytomyza & -- & -- & 1 & -- & -- & -- \\
\hline Phytagromyza- & -- & -- & $\begin{array}{l}1 \\
1\end{array}$ & -- & -- & -- \\
\hline Poemyza----- & 3 & 1 & 10 & --- & --- & -- \\
\hline Butomomyza--- & 4 & --- & 8 & --- & --- & -- \\
\hline Dizygomyza--- & 3 & 1 & 16 & -- & -- & -- \\
\hline Liriomyza---- & 11 & 5 & 80 & 3 & --- & 1 \\
\hline Galiomyza------ & 2 & 1 & 4 & --- & --- & -- \\
\hline Haplopeodes---- & --- & -- & 4 & 1 & --- & -- \\
\hline Calycomyza---- & 2 & --- & 30 & --- & --- & -- \\
\hline Phytoliriomyza- & 2 & 3 & 22 & --- & 5 & -- \\
\hline Metopomyza---- & -- & --- & 3 & --- & -- & -- \\
\hline Paraphytomyza-- & 1 & 1 & 8 & --- & -- & 2 \\
\hline Pseudonapomyza- & -- & -- & 2 & -- & -- & -- \\
\hline Napomyza-- & 2 & 1 & 12 & --- & 1 & -- \\
\hline Phytomyza------ & 16 & 11 & 97 & 1 & --- & 2 \\
\hline Chromatomyia--- & 8 & 4 & 28 & -- & 1 & 1 \\
\hline Subtotal- & 57 & 29 & 368 & 7 & 9 & 8 \\
\hline Total------- & 85 & 43 & 531 & 22 & 16 & 10 \\
\hline
\end{tabular}


many shorter papers between 1951 and 1958, including a valuable revision of the difficult genus Calycomyza (1956).

Little further work was done on North American Agromyzidae until a comprehensive revision of species in Canada and Alaska was published by Spencer (1969a). Here many United States species were further clarified, particularly by the illustrations of the male genitalia, which had assumed cardinal significance for the identification of many species.

The Agromyzidae of Florida were reviewed by Spencer and Stegmaier (1973) after they had collected throughout the State, particularly in the Miami area by Stegmaier. A detailed revision of California species was given by Spencer (1981) and 132 new species were described. Sehgal (1968, 1971) described several new species in Alberta, and Griffiths $(1973 a-80)$ published a series of 14 papers on boreal Agromyzidae. Several species described in these papers have been identified in the United States. Steyskal (1972-81) published several short papers describing some new species, including a useful revision of the new genus Haplopeodes.

\section{DIAGNOSTIC CHARACTERS OF THE AGROMYZ IDAE}

\section{Externa1 Morphology}

The terms used in the descriptions and diagnoses have been adopted by most recent workers on the Agromyzidae and are familiar to dipterists. For the convenience of other entomologists, the terms used are indicated in figures 1 and 2 and are briefly defined here.

Acrostichals (acr)--Minute hairs lying between the two lines of dorsocentral bristles (dc).

Arista--Slender, bristlelike appendage to dorsal surface of third antennal segment.
Coxa--First or basal section of leg; femur is third section of leg (color frequently is important).

Frons--Rectangular area between rear of head (vertex) and lunule and laterally between orbits.

Gena (jow1, cheek)--Membranous or lightly sclerotized area below eye (width and shape are frequently important). (See also parafacial.) In descriptions, entire surface from lower edge of eye to lower edge of head is termed "gena." Its height is given as a decimal, because the simplest accurate way to determine this is to measure the gena and the eye with a microscope, using a measuring device, and to divide the former measurement by the latter.

Ha1ter--Knoblike, modified hindwing functioning as a balancing organ; only found in Diptera (color can be significant).

Humerus--Shoulder or anterior angle of mesonotum anterior to notopleuron. Recent morphological usage terms this "postpronotum."

Lower orbital bristles (ori)--One to four partially incurved or reclinate bristles on lower half of orbits.

Lunule--Semicircular sclerotized surface above antennae and adjoining lower margin of frons.

Mesonotum--Dorsal area of thorax bearing important dorsocentral bristles and acrostichals (color is important in many species).

Mesopleuron--Large rectangular area below notopleuron (color is important in most genera of Phytomyzinae.)

Notopleuron--Triangular area on side of thorax immediately below or lateral to transverse suture of mesonotum.

Ocellar triangle--Triangular plate on 


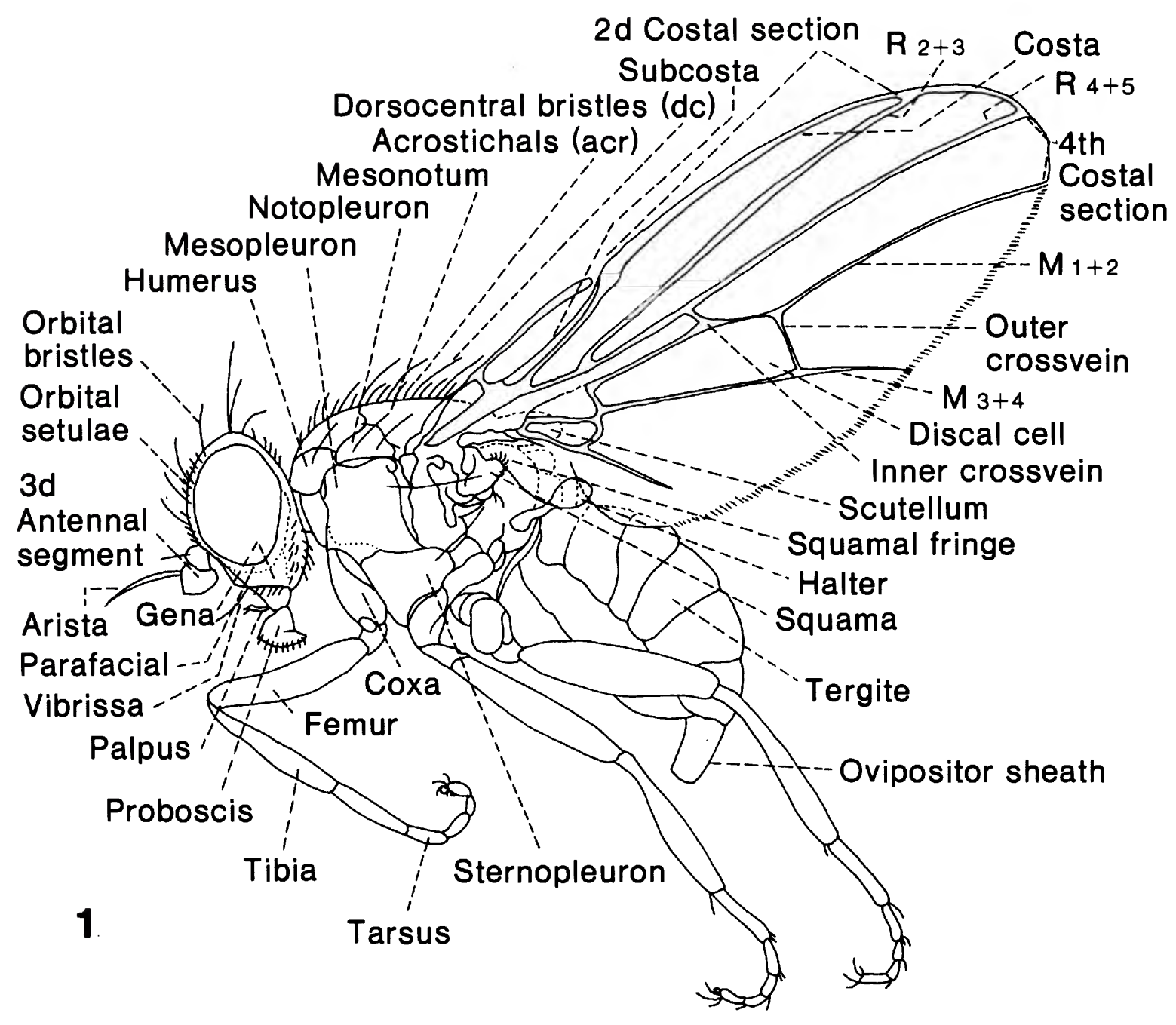

Figure 1.--Female agromyzid, side view. 
upper half of frons bearing three ocelli above and with apex sometimes greatly extended toward margin of lunule.

Orbit(s)--Narrow plates between eye margin and frons bearing orbital bristles and setulae, varying in width and sometimes raised or broadened.

Orbital bristles--(See lower and upper orbital bristles.)

Orbital setulae--Rows of minute hairs on orbits normally between eye margin and orbital bristles (direction of inclination of these setulae can be of generic significance).

Ovipositor sheath--Cylindrical structure into which ovipositor is withdrawn.

Palpus--Paired processes adjoining mouthparts.

Parafacial (cheek)--Membranous area immediately below eye, not always clearly separable from gena, which extends below to margin of head.

Prescutellar bristles (prsc)--Pair of bristles lying at center rear of mesonotum.

Proboscis--Feeding organ, greatly elongated in some species.

Scutellar bristles--Normally two pairs of strong bristles on scutellum.

Scutellum--Rear, dorsal section of thorax.

Squamae--Two lobes of wing membrane behind wing base, usually folded to lie one above the other (color is frequently significant).

Squamal fringe--Fringe of hairs on squamae (color is important specific character).

Tarsus--Fifth section of leg (itself divided into five sections).
Tergite(s)--Dorsal sclerites of abdomen.

Tibia--Fourth section of leg.

Trochanter--Second section of leg, between coxa and femur.

Upper orbital bristles (ors)--One to three strong, normally reclinate bristles on upper half of orbits.

Vibrissa--Usually strong bristle at lower, inner corner of gena.

Vibrissal fasciculus--Fused group of vibrissal bristles found in males of most Ophiomyia species.

Wing (fig. 1)--Traditional taxonomic terminology is maintained. Costa (C): First longitudinal vein forming upper margin of wing and normally conspicuously thickened, either to termination of vein $R 4+5$ or to $M 1+2$; second costal section lying between apex of veins $R 1$ and $R 2+3$; and fourth costal section lying between apex of veins $R 4+5$ and $M 1+2$.

Male Terminalia

No uniform terminology has been current for discussing male genitalia. The terms used here are mostly those proposed by Nowakowski (1962) or Griffiths (1972b). Detailed descriptions have not been included except for a few critical species. However, the aedeagus in the Agromyzidae is in most species so highly evolved and characteristic that illustrations in side, ventral, or both views suffice to permit immediate identification. The general arrangement of the male genitalia is shown in figure 3 , and the terms are briefly defined here.

Aedeagus--Actual intromittent organ, consisting basically of four sections--basiphallus, mesophallus, hypophallus, and distipha1lus; aedeagal apodeme is long, 


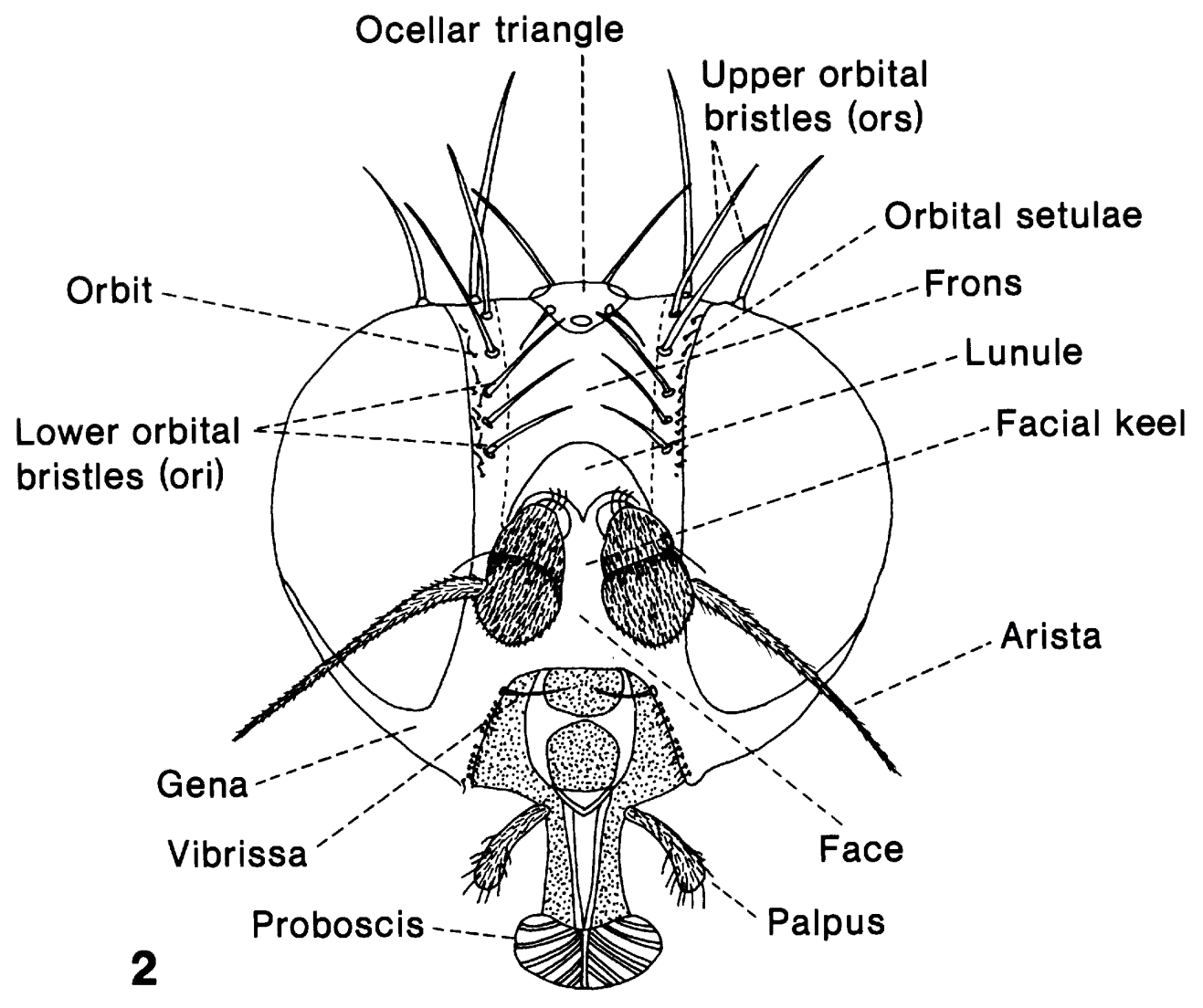

Figure 2.--Head of Agromyza sp., frontal view.

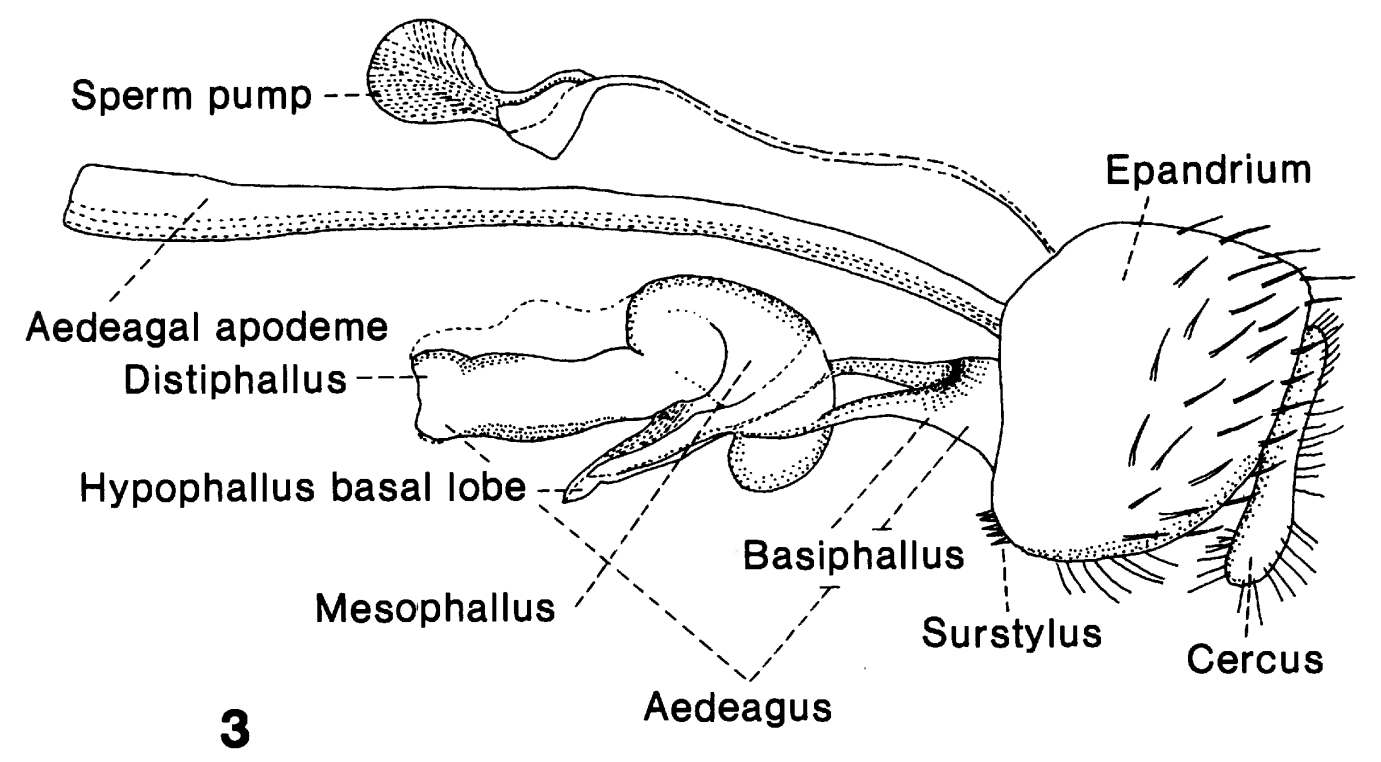

Figure 3.--Male genitalia of typical Agromyza sp. 
rodlike process extending far into abdomen and to which flexible aedeagus is articulated; basal sclerites of aedeagus are basal section attached to aedeagal apodeme, entire or divided into two asymmetrical arms.

Cerci--Paired, external appendages of epandrium, sometimes bearing stout bristles or one or more long hairs.

Distiphallus--Distal section of aedeagus, normally consisting of paired tubules of varying size and shape that sometimes may be partially fused or, particularly in Ophiomyia, highly asymmetrical. (See also mesophallus.)

Epandrium--Ninth tergite, frequently of distinctive shape.

Hypandrial apodeme--Elongate extension of hypandrium sometimes found at apex of ninth sternite.

Hypandrium--(See ninth sternite.)

Hypophallus--(See ventral lobe.)

Mesophallus--Median section of aedeagus, of relatively secondary importance diagnostically and frequently not differentiated from distiphallus.

Ninth sternite or hypandrium--Triangular or rounded, highly modified, terminal ventral sclerite of abdomen, lying internally between aedeagal apodeme and aedeagus, generally U-, V-, or Y-shaped.

Paramesophallus (paraphalli)--Paired sclerites at base of distiphallus complex.

Phallophore--Muscular attachment of base of aedeagus to aedeagal apodeme.

Postgonite--Variously formed paired lobes near base of aedeagus, sometimes greatly enlarged and with teeth or hook at end.
Sperm pump--Membranous structure lying within abdomen, with variable sclerotic blade and base and connected by long seminal duct to distiphallus.

Surstylus--Process at lower inner corner of epandrium, sometimes discrete, and with spines or strong hairs.

Ventral lobe--Ventrally directed sclerites near junction of distiphallus complex and end of basal sclerites.

Immature Stages

Few detailed descriptions have been given of larvae or puparia of United States Agromyzidae species. Important characters have been referred to under individual species here. Descriptions (in German) are available of most holarctic species in the series of 11 papers by de Meijere (1925, 1950, with index, 1955), and additional species are described, with good illustrations, in 3 short papers by Hering (1954, 1956, 1957). Descriptions of the larvae of Japanese species are given by Sasakawa (1961). Keys to genera are given by Frick (1952a) and, for Indian species, Singh and Ipe (1973), including many figures of larvae.

The larvae of Agromyzidae may be distinguished from those of other mining and boring insects by the following characters: Legs, prolegs, and sclerotized head capsule wholly lacking; mouthparts consisting of a pair of vertically functioning mouth hooks at the anterior end of the characteristic internal cephalopharyngeal skeleton; breathing organs consisting of a pair of spiracles situated dorsally on the prothoracic segment and a pair on the ultimate body segment. 


\section{B IOLOGY}

\section{Life History}

Al1 Agromyzidae are feeders on plant tissue. The white or yellowish oval egg is laid beneath the epidermis at the appropriate oviposition site, which may be at the leaf margin, at the leaf tip, indiscriminately anywhere in the leaf blade, or occasionally in the stalk, as in Phytomyza ilicis. Normally the larva emerges within a few days and feeds to form a channel or mine within the plant, which often will be characteristic of the particular species. Stem-boring species may oviposit in the leaf. The larva quickly feeds toward a vein and then works downward through the petiole to the stem. Some species may oviposit directly in the stem. The larval feeding can continue from about 1 week to several months, depending partly on the temperature, and is essentially a fundamental aspect of the life cycle. Species with several generations in quick succession, as in Liriomyza sativae, will feed rapidly, whereas those with only a single generation per year normally feed more slowly and sometimes intermittently throughout the cold winter months.

Pupation normally occurs on the ground. The larva either drops from the plant and transforms after some hours or transforms immediately on leaving the leaf and falls to the ground as a puparium. A few leaf miners pupate within the leaf at the end of the mine, particularly in the genus Chromatomyia. Most seed feeders and stem borers pupate within the plant at the feeding site. The pupal period varies greatly with the species, but it is most frequently about 2 weeks, although it may be as long as 6-10 months.

The Agromyzidae are well known as leaf miners. However, all parts of the plant are attacked, including stems, seeds, and more rarely roots, and in the case of trees, young twigs or the outer layer of the trunk.

\section{Leaf Mines}

The characteristic form of these mines or feeding tracks is a substantial aid in identifying Agromyzidae. Although individual mines may vary considerably, the essential pattern remains constant for each species. The main types of mines are linear or serpentine (figs. $649,776)$, blotchlike, approximately circular or elongate (figs. 903, 908), or more irregular, with short offshoots (fig. 935). In many instances, the larva forms a short linear mine in the first instar, and after the first molt the feeding instinct changes and the larva then forms a blotch mine (fig. 359). This may completely envelop the earlier linear section, which will nevertheless remain detectable from the frass arrangement .

The part of the leaf that is mined is frequently characteristic. Most mines are formed on the upper leaf surface, in the area of the palisade parenchyma. More rarely, very shallow mines are made immediately beneath the epidermis of the leaf, as in Liriomyza schmidti, or in a few species the larva feeds exclusively on the lower leaf surface, in the spongy parenchyma, as in Chromatomyia lactuca. Some mines are invariably associated with the margin of the leaf, others occur exclusively along the midrib or possibly one of the stronger lateral veins; this is the normal form in the pest species Liriomyza huidobrensis (fig. 713).

The arrangement of frass in the mine is frequently characteristic. It may be deposited in single, widely spaced pellets, or it may be in rather continuous strips, or all frass may be excreted immediately prior to pupation at the end of the mine, to which the puparium is sometimes firmly glued, as in Calycomyza humeralis or $\underline{\mathrm{C}}$. promissa (fig. 883). 


\section{Host Selection}

Most Agromyzidae are monophagous, that is, limited in their choice of host to a single plant or more often several species within the same genus, or they are oligophagous, feeding on several genera within a single family or on several related families of the same order. Very few species are truly polyphagous, feeding on a wide range of unrelated hosts. Common polyphagous species in the United States are Liriomyza huidobrensis, L. sativae, and $\underline{L}$. trifolii.

\section{HOST PLANTS}

Agromyzidae feed on a wide range of hosts, including ferns and both main groups of angiosperms, the Dicotyledoneae and Monocotyledoneae. Until recently there has been no evidence to suggest that any species might feed on conifers. However, Süss (1979) has identified feeding tracks in Tertiary fossil wood of Juniperoxylon silesiacum (Pril1) Krausel from the Netherlands as belonging to an agromyzid ancestral to the modern genus Phytobia, which he has named Protophytobia cupressorum. He also considered feeding tracks in modern Callitris drummondii Benth. \& Hook. (Cupressaceae) from Western Australia as belonging to a Phytobia species. If these findings can be substantiated, they will represent a remarkable discovery and the possibility that Phytobia species are present in Coniferae in the United States and cannot be entirely excluded, although it is improbable.

Of the 531 species of Agromyzidae recognized in the United States, the host is known for 250, or slightly less than 50 percent. The plant species in the United States exceed 15,000 , many of which will serve as hosts for the Agromyzidae, either of species already known or of those to be discovered. The scope for further collecting is thus enormous. Table 2 shows the total number of plant families and species (Shetler and Skog, 1978) and the number of families and genera known as agromyzid hosts. A complete host 1 ist is given on page 224 .

\section{ECONOMIC IMPORTANCE}

The economic importance of the Agromyzidae was discussed in detail by Spencer (1973a). Over 150 species of cultivated plants throughout the world are attacked by a similar number of agromyzid species. Such pest species occur on a wide variety of crops, but the most serious damage is caused by leaf miners in the genus Liriomyza in warm temperate areas in North and South America and by the stem feeder Ophiomyia phaseoli (Tryon) on beans in the old World tropics. This species has recently reached Hawaii. In temperate areas of Europe and North America, many species whose regular hosts include several crop plants occur only periodically in sufficiently large numbers to cause significant damage.

In the United States, Liriomyza sativae, L. trifolii, and L. huidobrensis are the most serious pests. L. sativae is highly polyphagous, attacking many truck crops, and occurs widely throughout the Southern States from Florida to California. L. trifolii is a regular pest in $\bar{F} 1$ orida, particularly on celery and chrysanthemum, and appears to have been introduced to California from Florida, where it is a constant problem in chrysanthemum nurseries. L. huidobrensis is not known in the East, but it is common in southern California and also in South America, attacking many cultivated plants, including ornamentals.

Chromatomyia syngenesiae regularly builds up large populations in California (Frick, 1972) and can be a serious problem on lettuce. Cucurbits and leguminous crops, including peas and alfalfa, can be seriously damaged by the three Liriomyza species. Also, L. trifoliearum and Agromyza frontella can occur in pest populations on alfalfa. All cereals may be attacked by $\underline{A}$. 
Table 2.--Plant families and genera known as hosts of Agromyzidae in the United States

\begin{tabular}{|c|c|c|}
\hline \multirow{2}{*}{$\begin{aligned} \text { Total families } \\
\text { (species in parentheses) }\end{aligned}$} & \multicolumn{2}{|c|}{ Hosts of Agromyzidae } \\
\hline & Families & Genera \\
\hline Pteridophyta--1- & --- & $\ldots$ \\
\hline Gymnospermae--n-_(115) & --- & --_ \\
\hline Angiospermae: & & \\
\hline Dicotyledoneae----- $174(11,551)$ & 51 & 223 \\
\hline Monocotyledoneae-- $37(3,068)$ & 8 & 46 \\
\hline
\end{tabular}

parvicornis and Cerodontha dorsalis, but most plants are believed to recover from the initial leaf-mining damage, and yield is probably little affected.

In Europe, damage to poplar, birch, and willow timber by the larval feeding of Phytobia species can be considerable. The hosts are known of only 5 of the 15 Phytobia species recorded in the United States ( $p .72$ ), and there appear to be no records of damage to commercial timber by agromyzid larvae. However, the nature of the damage to wood elsewhere is not always immediately apparent, and foresters should be aware of the potential damage that may be caused by agromyzid larvae.

\section{DISTRIBUTION}

Affiliations of the Agromyzidae in the United States are primarily with species in the Palaearctic Region and reflect the uniform fauna that existed between eastern Asia and North America across the Bering land bridge through much of the Tertiary period. Despite the water gap, which first opened up between eastern Asia and Alaska about 13 million years ago, there has repeatedly been a land connection during the interglacials of the Pleistocene during the past 3 million years. This has facilitated a dispersal in both directions of animals, plants, and of course Agromyzidae (see
Hopkins, 1967). Many United States species are present also in Europe and even more show close relationships. Species with a holarctic distribution in Canada were analyzed by Spencer (1969a), and boreal species in Europe and North America are currently being reported by Griffiths (1972-80) in his continuing series of papers.

Species with neotropical affinities are particulaly evident in southern Florida, and 31 species, representing 36 percent of the total known in Florida, are considered to be of southern origin (Spencer and Stegmaier, 1973). On1y a few species in southern California might fall into the same category (Spencer, 1981: 6). The fauna of other southern States is not well enough known to assess affiliations in detail, but one new Phytobia species described from Arizona (part 2, p. 271) appears to be related to species in Central America.

Over 2,000 described species are known in the Agromyzidae, but the total must exceed double that number. The Agromyzidae are distributed throughout the world from the north of Greenland to Patagonia and the subantarctic is lands south of New Zealand. The largest number of species are found in temperate areas of the Northern Hemisphere, where the dominant genera are Liriomyza and Phytomyza. However, many species are also in the Tropics, distributed from high mountainous areas to tropical forests at sea 
level, although the rain forest has been sparsely colonized.

In the United States, the Agromyzidae are present in all States but unevenly distributed. Detailed surveys have been made in Florida (Spencer and Stegmaier, 1973), where 86 species have been recorded. In California (Spencer, 1981), the number is substantially larger, with 252 species, reflecting its diverse geographical and climatic conditions. Little collecting has been undertaken in the Northern States, such as Maine and the Dakotas, but from the few records available from Michigan, Minnesota, Montana, and Wisconsin, the family is well represented in all northern areas. Our collecting in Mississippi and Arkansas has suggested that relatively few species are present there, comparable to the limited fauna in Florida, but leaf mines have recently been found on many hosts in Texas by $S$. Tavormina, although no undescribed species were discovered.

During our research, approximately 700 specimens were examined from high elevations in Colorado, particularly from the Mt. Evans area up to the summit at 4,260 m (14,200 ft). Many species were found there, but unfortunately not a single species was reared and the hosts remain unknown. From the small amount of material seen from the Western States, including Idaho, Utah, New Mexico, and Arizona, there is clearly a rich, largely unexplored fauna, and many undescribed species can be expected in these States.

A notable fact that has emerged during our study is the wide distribution within the United States of most genera and many individual species, both from east to west and north to south. Details are given under the genera concerned, but some striking examples may be cited here.

Melanagromyza buccalis occurs from New York to California and M. 1ippivora, described from Florida, is synonymous with M. riparella from Illinois. Ophiomyia texana occurs from Ontario to Texas, Liriomyza lathyri was discovered in Arkansas, previous1y known only in Alberta, and in an area of relict forest in Mississippi a large population of Phytomyza penstemonis was found, previously known in Alberta and California. Conversely, many species have a more restricted distribution. Many of neotropical origin appear to be limited to southern F1orida. The important pest species L. huidobrensis, which is common in California, is absent from Florida and other southeastern States.

Several high-elevation species previously known in the Sierra Nevada and farther north in Canada or even Alaska have been confirmed in Colorado. There does, however, appear to have been some speciation on either side of the Sierra Nevada, but the true distribution of such species requires further investigation with additional collecting.

About 200 species are known in Canada and Alaska, but not yet in the United States. A considerable proportion of these may be found in the United States when the family is better known.

Despite the clearer concept of the distribution of Agromyzidae in the United States, our knowledge is still fragmentary. Specialized collecting in all areas will produce more State records of known species, and certain1y many undescribed species await discovery.

\section{REARING AGROMYZ IDAE}

Many adults can be caught by general sweeping, but the most satisfactory method is to rear them from larvae or puparia that can be found on their host plants. Frick (1951c) outlined a technique he employed in rearing Agromyzidae in California. The notes given here somewhat modify and elaborate on his procedure. 


\section{Leaf Mines}

Even empty leaf mines will frequently permit identification of the particular species, provided the host plant can be identified reliably. With a hand lens it is easy to establish whether or not a leaf mine contains a larva or puparium. If it is empty, a representative sample of mines should be pressed and dried between sheets of newspaper, preferably with the flower, so that the identification of the plant can be confirmed.

If mines still contain living larvae, a part of the stem containing mined leaves should be kept in a sealed plastic bag or vial until the larvae have pupated. It is better not to pick individual leaves, as they desiccate more rapidly than if left attached to the stem, and thus they prevent larvae from normally completing their feeding and pupating.

Puparia obtained in this way should be transferred with a slightly moistened brush to the actual rearing container. Vials about $3 \mathrm{~cm}$ in diameter and 6 to $10 \mathrm{~cm}$ long with a small layer of dampened sand have been satisfactory. They require attention every few days, adding a few drops of water when necessary as the sand dries out, or removing any excess moisture on the sides of the vial, which can damage emerging flies.

Healthy puparia are not normally attacked by mold, but several invariably die from other causes and mold then rapidly develops. Dead puparia should be removed before the mold spreads and affects healthy puparia.

Species that pupate in the leaves present a problem. If the entire leaf or even a small section containing the puparium is kept for more than a few days in an enclosed moist atmosphere, mold will develop rapidly on the dead plant tissue, ultimately killing the transforming larva. Remove such puparia from the mines before rearing the adults as described here. This is not always easy, but with experience the puparia normally can be removed without damage.

Mined leaves from which the larvae have pupated or from which puparia have been removed, should, of course, be pressed in the normal way. Empty pupal cases should be retained together with the adults that have emerged from them or should be appropriately labeled so that later the two can be associated correctly.

Careful, accurate 1 abeling throughout the rearing process cannot be too strongly emphasized. Whatever system of serial numbering is used, the same reference number or 1 abel can be used at six separate stages: (1) Host plant, (2) plastic bag or vial containing mines in the field or laboratory, (3) actual rearing container, (4) pressed mines, (5) emerging adults and empty puparia, and (6) any parasites obtained. Mistakes can be made, however carefully one works, particularly with large amounts of material, but the labeling outlined here, if done carefully, largely eliminates the possibility of incorrectly associating adults and host plants, which can be so misleading for future workers.

\section{Stems and F1ower Heads}

When the stems are checked, the larval feeding can be readily detected if the stem is either cut across or split lengthwise. By following the feeding track of the larva either down or up the stem, the puparium normally can be found without great difficulty and should then be removed and reared as described here. Once a given host in the field is known to contain agromyzid puparia, time can be saved by randomly selecting stems that can be examined in more detail later in the laboratory.

With flower heads and seed pods, first determine that Agromyzidae are present, then collect a large quantity for later detailed examination. If 
time permits, remove the individual puparia from the flower heads to prevent their destruction by any developing mold or desiccation if kept dry. Also this procedure insures correct association of adults with their puparia, as two species may often be present in a single flower head.

\section{PRESERVING ADULTS}

Emerging flies should not be killed too soon. They should be allowed to live for at least 24 hours to insure complete hardening and full color development. Ideally they should be mounted using minuten pins, although gluing either to a stronger pin or to a card point can be satisfactory. If time or facilities do not permit, the specimens should be kept dry in layers of cellulose acetate, in either vials or small boxes containing all relevant data, including locality, collection date, host plant, and emergence date. Handle dry specimens with greatest care to prevent damage to such parts as antennae, bristles, and legs, which may be vital for accurate identification.

Preserving specimens in alcohol is not recommended. Al though satisfactory techniques exist for drying such specimens, they are time consuming and can be avoided if necessary attention is given to preserving them dry.

\section{SYSTEMATICS}

The division of the Agromyzidae into the two subfamilies Agromyzinae and Phytomyzinae, originally proposed by Fallén (1823a, b), has been accepted in all subsequent revisionary studies of the family. The two subfamilies are characterized by the termination of the subcosta in vein $R 1$ in the Agromyzidae (figs. 4, 204) and by the subcosta running parallel to $\mathrm{R} 1$ and reaching the costa independently in the Phytomyzinae (figs. 5, 957). In the larvae, the cephalopharyngeal skeleton has two upper arms in the
Agromyzinae (figs. 6, 435) but only one in the Phytomyzinae (figs. 7, 667). These differences are not entirely clear cut, particularly in the genus Phytobia, but they are of considerable practical value as a first step in identification.

Since 1930, workers on the Agromyzidae have based their systematics on the generic framework established by Hendel (1931-36) for palaearctic species. This was partially modified by Frick (1952a) to include nearctic and neotropical species. In Frick's concept, Hendel's genus Dizygomyza was treated as Phytobia with 10 subgenera. Phytobia is now restricted to treefeeding species. Of the other subgenera, Amauromyza, Nemorimyza, and Calycomyza are accepted as full genera, whereas Icteromyza, Poemyza, and Dizygomyza are treated as subgenera of the genus Cerodontha, as proposed by Nowakowski(1962). Phytagromyza is also now included in Cerodontha, and for its well-understood original concept, the available name Paraphytomyza is adopted.

Other generic changes that should be noted are the erection of Galiomyza Spencer (1981) for a small segregate of Liriomyza, the new name Haplopeodes Steyskal (1980) for Haplomyza Hendel, and the acceptance of Chromatomyia Hardy, proposed by Griffiths (1974a) as a segregate of Phytomyza, based essentially on characters of the male genitalia and in part on the method of pupation.

During our research, generic limits have been slightly modified in three genera following the study of fresh material. The essential character of the small genus Hexomyza was previously considered to be the production of twig galls by the larvae. The male genitalia of the species forming conspicuous galls on Tilia americana (fig. 192), "Agromyza" tiliae, associate it with species accepted in Ophiomyia, and Hexomyza is now restricted to gall causers on Salicaceae. "Agromyza" rutiliceps is 
shown by the male genitalia to belong correctly in Japanagromyza. This species has $3+1$ dorsocentral bristles (dc) and is the first in the genus to be known with a strong presutural dc. The color and head shape also differ from the form previously known in the genus.

In Melanagromyza, the normal arrangement of dorsocentral bristles is two in the postsutural area (rarely three); two species, $\underline{\text { M. }}$ quadrisetosa and $\underline{M}$. trispinella, were recorded in California with a strong presutural dc bristle. They are the only species in the world in this genus known to possess this bristle.

Many groups of closely related species in the genera Melanagromyza, Ophiomyia, Cerodontha (Dizygomyza), Liriomyza, Calycomyza, and Phytomyza are known that cannot be reliably separated on external characters, and identification is only possible from examination of the male genitalia. It is apparent that in several instances even the genus cannot be accurately established on external characters alone.

There can be difficulty in deciding between Agromyza and Phytobia owing to the course of the subcosta in the latter genus approaching that normal in the Agromyzinae. However, the stridulating mechanism in both sexes of Agromyza (figs. 346-348) provides a certain means of identifying the genus.

Hende1 (1920: 114; 1931: 16) differentiated Ophiomyia and Melanagromyza by the presence in the former of a raised facial keel dividing the antennae and, in the male, the presence of a vibrissal fasciculus (figs. 221, 304) replacing the simple vibrissa in Melanagromyza. In the 1960 's, these external characters were found to be inadequate for differentiating these two genera. The male genitalia and larval morphology and biology clearly showed that several species, although appearing like typical Melanagromyza, correctly belonged in Ophiomyia (see Ophiomyia key, p. 39).

Some species in Liriomyza and Phytoliriomyza can appear virtually identical in external characters. The only certain way of assigning them to the correct genus may be the presence or absence of the stridulating mechanism in males of Liriomyza (figs. 621, 622). As an example, L. marginalis and $\underline{P}$. melampyga may be cited. Each is largely yellow, with a black or gray banded mesonotum, and the central area adjoining the scutellum is yellow (fig. 673). The male genitalia, however, will frequently indicate the correct genus.

With adults of some species of Phytomyza and Chromatomyia that are indistinguishable in external characters, examination of the male genitalia is necessary to identify the genus. However, in a few instances, the genus is not immediately apparent even from the genitalia. One such species is $\mathrm{P}$. clematoides, in which the divided and dorsally directed distiphallus (fig. 1217) might be mistaken for the "supporting sclerites" present in all species of Chromatomyia (figs. 1275, 1290). In this species, the correct generic position was finally confirmed by examining the puparia.

However, the type of aedeagus of the feeders on Caprifoliaceae (figs. 1285, 1295) and on Poaceae (figs. 1258, 1261 ) is distinctive and readily recognizable. Unfortunately with our present knowledge it will not be possible in some instances to identify females in these two genera even to genus. Therefore, Phytomyza and Chromatomyia species, for convenience, have been included in a single key (p. 175). 
KEY TO AGROMYZIDAE SUBFAMILIES AND GENERA

1. Subcosta developed throughout its length (fig. 4), coalescing with $R 1$ before reaching costa (subfamily Agromyzinae)

Subcosta becoming a fold distally (fig. 5), ending ing in costa separately, and based on R 1 (subfamily Phytomyzinae)

Prsc present

Mesonotum or abdomen frequently with some metallic coloration, greenish, bluish, or coppery; costa extending strongly to vein $M 1+2$ (except in $M$. tamia and $M$. trispinosa); antennae not normally separated by raised keel, male never with vibrissal fasciculus; aedeagus symmetrical, with basal sclerites U-shaped (fig. 23); larval posterior spiracles on flat plate, with numerous bulbs, from 6 to 20, normally surrounding a strong black horn, the latter rarely atrophied to a mere scar (fig. 32)----------Melanagromyza Hendel (p. 19) Uniformly black species (mesonotum and abdomen faintly greenish only in 0 . jacintensis group); costa ending at $R 4+5$ or continuing to $M 1+2$; aedeagus frequently asymmetrical, with basal sclerites frequently diverging (fig. 226); posterior spiracles of larva normally on distinct stalks, each process with either 3 or more (normally with numerous) bulbs, never with central horn (fig. 199)

4(3). Antennae normally separated by conspicuous facial kee 1; male frequently with distinct vibrissal fasciculus (fig. 221); larval posterior spiracular process normally with between 6 and 12 bulbs-------_--_-----Ophiomyia Braschnikov (p. 37) Antennae adjoining; vibrissal fasciculus never present; larval posterior spiracles always with 3 bulbs on each process (fig. 158) ------_---_-

5(2). At least 3 pairs of dorsocentrals (dc); prsc always present; presutural dc frequently present; stridulating mechanism present in both sexes (figs. 346-348)-----------Agromyza Fallén (p. 57)

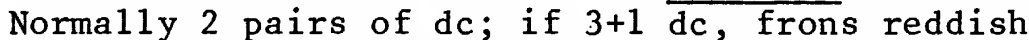
( $\mathrm{J}$. rutiliceps); prsc present or lacking; stridulating mechanism lacking

6 (1). Orbital setulae erect, reclinate, or absent (fig. 394)------------- 7

Orbital setulae distinctly proclinate (fig. 1163)- 18

7 (6). Vein $R 4+5$ ending nearest wing tip-----

Vein $M 1+2$ ending nearest wing tip-- 8

8 (7). Wing with costa extending to apex of vein $M .1+2$; if only to $R \quad 4+5$, then lunule substantially higher than semicircle (Cerodontha frankensis)--- 9 Costa extending only to $\mathrm{R}$ 4+5- 17 
9 (8). Male genitalia: Sperm pump with enlarged, bow1shaped base (figs. 509, 514); distiphallus (fig. 516) frequently with numerous spinules (subgenus Cephalomyza); halter often black (see p. 79 for other characters)--------Amauromyza Hendel (p. 78) Male genitalia: Sperm pump without such enlarged base; distiphallus never with spinules---_-

10 (9). 3d antennal segment with spine (fig. 549), scute1lum with only 1 pair of bristles; or lunule conspicuously higher than a semicircle, either narrow (fig. 540, A) or triangular ( $\mathrm{fig} .540, \mathrm{~B}$ ); or lunule conspicuously large and broad (fig. 540 , C), antennal bases widely separated, and $3 \mathrm{~d}$ antennal segment in male normally greatly enlarged (fig. 593)-Not so-

11 (10). Foretibia with lateral bristle; abdomen in male conspicuously yellow---------Nemorimyza Frey (p. 87)

Only midtibia sometimes with lateral bristle, foretibia never

12 (11). No presutural dc; epandrium with conspicuous patch of spines at hindcorner (fig. 898)

Presutural dc present; epandrium without such spines Calycomyza Hende1 (p. 140)

Stridulating mechanism present in male (figs. 621,

622); frons and scutellum characteristically bright yellow (black only in L. nigriscutellata and $\underline{L}$. nigrissima); humerus usually yellow with more or less black spot--------Liriomyza Mik (p. 107)

Stridulating mechanism lacking; frons and scute1lum variable from black to yellow--_-_---_--- 14

14 (13). Scutellum largely black, at most faintly yellow between basa1 scutellars----Galiomyza Spencer (p. 136)

Scutellum yellow or at most gray-- 15

15 (14). 1 upper orbital bristle (ors); male genitalia with aedeagus weakly developed ( $\mathrm{fig}$. 868)

----- Haplopeodes Steyska1 (p. 138)

2 ors; male genitalia with aedeagus strongly developed (figs. 1025, 1042)

16 (15). Orbit broad, raised above frons; orbital setulae reclinate------------Metopomyza Enderlein (p. 161)

Orbit largely in plane of frons; orbital setulae upright, slightly proclinate, or absent-----------

17 (8). 2d costal section at least twice length of 4 th (fig. 1039); outer crossvein either absent, or if present, wel1 beyond 1st section-------_---------

--

Second costal section conspicuously short, less than 1.5 times length of 4th (fig. 1052); outer crossvein basal to 1 st section-

-

18 (6). Costa extending to vein $\mathrm{R} 4+5$

-- Costa extending to vein M 1+2; orbital setulae con- 
spicuously proclinate (fig. 975)

-- - - - Phytoliriomyza Hende1 (p. 151) (in part)

19 (18). Male genitalia with distal section of aedeagus simple (not bifid), lying below a lobe with supporting sclerites on dorsal side of aedeagus ( $f$ igs. 1258,1262 ); outer crossvein lacking (present in a few species in Europe)---Chromatomyia Hardy (p. 173)

-- Male genitalia wth distal section of aedeagus bifid, without such lobe above-_-_- 20

20 (19). Outer crossvein present (fig. 1059); frons always strong1y projecting; 2d costal section short, always less than twice length of 4 th-

Napomyza Westwood (p. 168)

Outer crossvein normally absent; if present, frons not projecting; 2d costal section longer, 2.5 times length of 4 th ( $\underline{P}$. davisii, $\underline{P}$. duplex)-----Phytomyza Fa11en (p. 172) 


\section{SUBFAMILY AGROMYZ INAF}

\section{Genus Melanagromyza Hendel}

Melanagromyza Hende1, 1920: 120. Type of genus: Agromyza aeneoventris Fallén, 1823a (Europe).

This genus was erected by Hendel for black-haltered species previously included in Agromyza. Normally there are only two pairs of postsutural dorsocentrals (dc), but a few species are known with three or even four, and in the United States, quite exceptionally, two species, M. quadrisetosa and $M$. trispine $\bar{l} 1 \mathrm{a}$, were discovered in California having a strong presutural dc. The costa invariably extends to vein $M 1+2$, but two exceptions are known, $\underline{M}$. tamia and M. trispinosa, where the costa ends at vein $\mathrm{R} \mathrm{4+5.} \mathrm{Although} \mathrm{most} \mathrm{species} \mathrm{are}$ almost entirely black, in many the mesonotum or abdomen may be distinctly greenish or even bluish.

Genitalia studies have shown that a number of species, which on external characters would be placed in Melanagromyza, correctly belong in Ophiomyia. This has been confirmed by biological and larval characters. A detailed revision of European species was given by Spencer (1966a).

The larvae of all known Melanagromyza species are internal feeders, mainly in the stem but also in the flower head or root, and pupation occurs within the plant. The posterior spiracles of the larva and puparium consist of two sclerotized plates, each with an ellipse of pores or "bulbs," normally surrounding a conspicuous "horn," which is sometimes strongly sclerotized (fig. 127).

Hosts are known of 32 United States species, 15 in the Asteraceae and the others in 13 other families, even in the Orchidaceae. Two species were described from California (Spencer, 1981), M. scrophulariae and $M$. urticelīa, which can be accepted as feeding on Scrophularia and Urtica, respectively, but no reared specimens are known. Four other probably undescribed species were also seen after this study was completed. They were from the genera Ambrosia, Eupatorium, Lactuca, and Solidago (Asteraceae). With further collecting of puparia from stems, many additional species remain to be discovered.

Melanagromyza is one of the largest genera in the family, with over 300 described species. This is certainly a small proportion of those existing. Fifty-nine species are discussed here, of which 8 are new. Other random caught species have been seen, but as they have no distinctive characters, it has not seemed desirable to describe them at this stage. Two new synonyms are established and two species, $\underline{M}$. miranda and $\underline{M}$. tetrica, have been identified, known previously only in Canada (part 2, pp. 245, 247). Ten other species are known in Canada (Spencer, 1969a; Sehgal, 1971).

The genus is particularly well represented in the Tropics and extends south to Chile, Australia (Spencer, 1977d), and New Zealand (Spencer, 1976b). More species are known in Venezuela (Spencer, 1973b) than in Canada (Spencer, 1969a). The fewer species in temperate areas will also apply in the United States, and more will be found in the Southern States than in the north. 
Key to Melanagromyza Species
1.

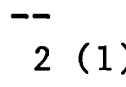

$3(2)$.

$-$
4 (3). Mesonotum greenish------------ tamia (Melander)

Costa ending at or at least attenuated beyond vein $\mathrm{R} 4+5$, not reaching $M 1+2-$

Costa extending strongly to vein $\mathrm{M} 1+2--$

$2+1 \mathrm{dc}--------1$. trispinosa Spencer Synopsis. Large species, wing length 2.5-2.75 mm; mesonotum greenish; squamal fringe white; male genitalia as in figures 8,9 .

Host/Early Stages. Unknown.

Distribution. California, widespread from Yolo Co. to Los Angeles Co.

Reference. Spencer, 1981: 61 .

2 or 3 postsutural dc, no presutural--_-_------ 3

- Squamal fringe white--_-_- 4

Squamal fringe black------Ophiomyia similata (Ma1loch) (Costa sometimes appearing to continue to vein M 1+2, couplet 61) Synopsis. Large species, wing length $\overline{2.75} \mathrm{~mm} ; 3$ postsutural dc; mesonotum greenish bluish; male genitalia as in figure 10 .

Host/Early Stages. Unknown.

Distribution. Washington.

References. Melander, 1913: 258; Frick, 1959: 366.

-- Mesonotum black; small species, wing length $2 \mathrm{~mm}---$

5 (1). 3 or 4 dc-Hexomyza winnemanae (Malloch)

$-$

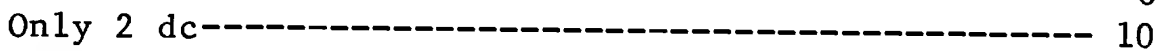

6 (5). Strong presutural dc present---------------------- 7

-- No presutural but up to 4 postsutural dc----------- 8

7 (6). 3 postsutural dc; antennae divided by facial keel;

large species, wing length $3.5-4.25 \mathrm{~mm}-$

--

Synopsis. Frons broad, twice width of eye, conspicuously projecting above eye; mesonotum and abdomen greenish; squama and fringe white; male genitalia as in figures 11,12 .

Host/Early Stages. Unknown.

Distribution. California.

Reference. Spencer, 1981: 54.

2 postsutural dc; no facial keel; smaller species, wing length 2.5-2.75 mm------M. trispinella Spencer Synopsis. Frons broad, twice width of eye, projecting above eye; mesonotum and abdomen greenish; squama and fringe whitish; male genitalia as in figures 13, 14 .

Host/Early Stages. Unknown.

Distribution. California.

Reference. Spencer, 1981: 59 .

8 (7). Distinct, raised keel dividing base of antennae---- 9

Antennae not divided by raised keel-_-_-_-_--_--M. setifrons (Melander)

Synopsis. Stout; orbit distincty projecting above

$3 /$ Includes certain Hexomyza and Ophiomyia species; see also Melanagromyza sp. (p. 249). 
eye; 2 ors, 4 strong ori, orbital setulae long, reclinate; gena broad, 0.25 height of eye, parafacial forming broad ring below eye; $3 d$ dc strong, nearer 2d than suture; squama gray, margin and fringe black; male genitalia as in figures $15,16$.

Host/Early Stages. Unknown.

Distribution. Idaho; Canada.

References. Melander, 1913: 260; Frick, 1957b: 200; 1959: 366; Spencer, 1969a: 75 .

9 (8). Large species, wing length $2.7-3.1 \mathrm{~mm}$

Synopsis. Frons broad, greatly projecting above eye; 2 ors, 4 ori; parafacial broad, up to 0.5 height of eye, facial keel raised, dividing bases of antennae; mesonotum and abdomen greenish; squama gray, margin blackish; male genitalia with aedeagus as in figures 17,18 .

Host/Early Stages. Scrophularia. Larva internal stem borer but characters unknown.

Distribution. California.

Reference. Spencer, 1981: 57.

Smaller species, wing length 2.1-2.3 mm-----------

Synopsis. Closely resembling $\underline{M}$. $\frac{\text { scrophulariae, }}{\text { sagehenensis }}$ also with raised facial keel, but distinctly smaller; male genitalia with aedeagus as in figures 19,20 .

Host/Early Stages. Potentilla glandulosa. Larva internal stem borer; puparium yellowish, posterior spiracles widely separated, each process with ellipse of some 20 bulbs and round, long, outwardly curving horn.

Distribution. California, Montana (new record).

References. Spencer, 1981: 56; part 2, p. 247.

10 (5). Squama and fringe pale, whitish----- 11

-- Squama and fringe darker, gray, or blackish-------- 46

11 (10). Proboscis conspicuously long and slender (fig. 21)11

--------- proboscidata Spencer, n. sp.

synopsis. Wing length $2.3 \mathrm{~mm}$; mesonotum shining black, abdomen blackish green or coppery; male genitalia with aedeagus as in figures 22,23 .

Host/Early Stages. Unknown.

Distribution. Texas.

Reference. Part 2, p. 246.

-- Proboscis short, normal------------------------ 12

12 (10). Ocellar triangle brilliantly shining------------- 13

-- Ocellar triangle at most moderately shining-------- 15

13 (12). Gena deep, 0.25 height of eye; orbit distinctly shining---

Synopsis. Small to medium-sized species, wing length $2-2.5 \mathrm{~mm} ; 2$ ors, 2 ori, orbital setulae in single row, reclinate; lunule higher than a semicircle; ocellar triangle long, narrow, extending to level of upper ori; male genitalia with aedeagus as in figures 24,25 . 
Host/Early Stages. Asteraceae, recorded from Calendula, Encelia, Helianthus. Tithonia, Zinnia. Larva feeding in seed heads; puparium pale, straw colored, posterior spiracular processes widely separated, with ellipse of about 10 well-defined bulbs around strong central horn.

Distribution. California, Florida; new records : Colorado, Larimer Co., Estes Park, 7,500 ft, $1 \mathrm{~m}$. , 21.VII.61 (C. H. Mann, CNC); North Carolina, Macon Co., Highlands, Little Bear Pen, $1 \mathrm{~m}$, 5.VIII.57 (W. H. Richards, CNC); Chile, Cuba, Mexico, Trinidad.

References. Frost, 1931b: 277; Spencer, 1981: 63. Gena narrow, 0.14-0.08 height of eye---_-_---- 14

14 (13). Ocellar triangle black, mesonotum brilliantly shining blackish green---------M. heliotropii Spencer Synopsis. Small species, wing length 2.2-2.3 mm; frons not projecting above eye; 2 ors, 2 or $i$; gena narrow, about 0.14 height of eye, bare in both sexes; male genitalia with aedeagus as in figures $26,27$.

Host/Early Stages. Heliotropium leavenworthii. Larva internal stem borer; puparium whitish, posterior spiracles each with ellipse of 4 or 5 large bulbs around weak black horn, separated by almost 3 times their own diameter.

Distribution. Florida.

Reference. Spencer and Stegmaier, 1973: 39.

Ocellar triangle greenish blue, mesonotum strongly shining green, blue, or greenish blue-c-

Synopsis. Wing length $1.9-2.4 \mathrm{~mm}$; frons not projecting above eye; gena narrow, 0.08 height of eye; ocellar triangle broad, large; male genitalia with aedeagus as in figures 28, 29; hypandrium with long apodeme (fig. 30); epandrium with spines along inner margin (fig. 31).

Host/Early Stages. Blechnum, Ruellia, Strobilanthes (Acanthaceae). Larva feeding in seed heads; puparium bright orange, posterior spiracles each with ellipse of 7 bulbs (fig. 32).

Distribution. Florida; Bahamas; new record: Texas, Brewster Co., Big Bend National Park, Panther Junction, 3,500 ft, $1 \mathrm{~m}$., 14.V.59 (J. F. McAlpine, CNC).

References. Spencer, 1966d: 14; Spencer and Stegmaier, 1973: 47.

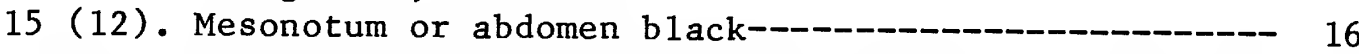

-- Mesonotum or abdomen greenish, bluish, or coppery-- 17

16 (15). Mesonotum and abdomen entirely black; frons strongly projecting above eye; orbital setulae all proclinate (fig. 33)--- inornata Spencer

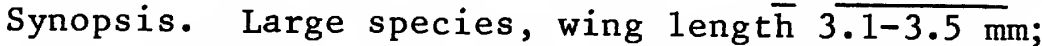
male genitalia with aedeagus as in figure 34 .

Host/Early Stages. Unknown.

Distribution. Michigan; Canada. 
References. Spencer, 1969a: 88; Steyskal, 1972b: 1 . Mesonotum black, abdomen greenish; frons not significantly projecting above eye; orbital setulae in 2 rows, outer row reclinate------M. panacis Steyskal

Synopsis. Medium-sized species, wing length 2.4-2.8 $\mathrm{mm}$; male genitalia as in figures 35,36 .

Host/Early Stages. Panax quinquefolius. Larva internal stem borer.

Distribution. Indiana, Ohio.

Reference. Steyska1, 1981: 39.

17 (15). Foretibial bristle strongly developed-------------

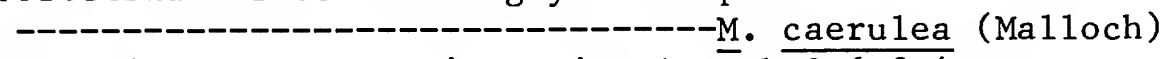

Synopsis. Large species, wing lenḡth $\overline{2.6-3.4} \mathrm{~mm}$; frons equal to width of eye, not projecting in profile; eye in male conspicuously pilose; mesonotum greenish blue, abdomen green; male genitalia with aedeagus as in figures 37,38 .

Host/Early Stages. Ipomoea spp. (Convolvulaceae). Larva feeding in seeds; posterior spiracular processes widely separated, each with ellipse of 9 bulbs, without central horn (fig. 39).

Distribution. Florida, Texas; widespread in Caribbean area, Costa Rica, Mexico, Venezuela.

References. Malloch, 1913a: 322; Spencer and Stegmaier, 1973: 33; Spencer, 1973a: 104.

Foretibial bristle normally absent, at most weak--- 18

18 (17). Large species, wing length 2.5-3.75 mm--------- 19

Smaller species, wing length $1.75-2.5 \mathrm{~mm}----------29$

19 (18). Exceptiona1ly large species, wing length in male 3 $\mathrm{mm}$, up to $3.75 \mathrm{~mm}$ in female----_--------- 20

Smaller species, wing length from $2.5 \mathrm{~mm}$ in male to $3.25 \mathrm{~mm}$ in female------------------------

20 (19). Orbit and parafacial brilliantly shining--------------------------------------M. miranda Spencer

Synopsis. Large species, wing length $3-\overline{3.5 \mathrm{~mm}}$ frons broad, strong1y projecting above eye ( $\mathrm{fig}$. 40); 2 ors, 5 or 6 ori; gena deep, 0.33 height of eye; eye in male pilose; mesonotum blackish green, abdomen greenish or coppery; male genitalia with aedeagus as in figure 41 .

Host/Early Stages. Lithospermum arvense, also unknown species of Boraginaceae. Puparium straw colored, posterior spiracles widely separated, each with ellipse of some 20 bulbs around short central horn.

Distribution. Montana (new record); Canada. References. Spencer, 1969a: 72; part 2, p. 245. Orbit and parafacial not shining-------------- - - diantherae (Ma1loch)

Synopsis. Exceptionally large species, wing length 3-3.75 mm; frons broad; 2 ors, 3 ori; gena narrow, 0.14 height of eye; mesonotum and abdomen greenish, only moderately shining; male genitalia with aedeagus as in figures 42, 43 (not previously illustrated).

Host/Early Stages. Justicia (=Dianthera) ameri- 
cana. Larva internal stem borer (details not

recorded) in plants growing beside streams.

Distribution. Illinois, Indiana, Maryland (new

record).

References. Malloch, 1920: 147; Frick, 1959: 364

(as M. diantherae).

21 (19). Mesonotum bluish black--------------------- 22

$--$

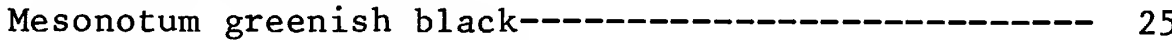

(21). Frons almost twice width of eye--------------

Frons 1.5 times width of eye---------_----------

- - walleyi Spencer, n. sp.

Synopsis. Wing length in male $2.7 \mathrm{~mm}$ last section of vein $M 3+4$ little more than 0.5 penultimate;

frons less than 1.5 times width of eye; 2 ors, 3 ori; eye in male with distinct pilosity at level of ors; gena narrow, 0.20-0.17 height of eye; male genitalia with aedeagus as in figures 44,45 . Host/Early Stages. Unknown.

Distribution. Tennessee.

Reference. Part 2, p. 249.

$23(22)$.

Synopsis. Large species, wing length $2.7-3.5 \mathrm{~mm}$; gena 0.25-0.20 eye height; eye in male with slight pilosity above; arista bare; male genitalia with aedeagus as in figure 46.

Host/Early Stages. Althaea rosea, possibly other Malvaceae. No record of larva or puparium.

Distribution. New York; Canada.

References. Spencer, 1969a: 78 (as sp. (Steyska1)); Steyskal, 1981: 36 .

Male genitalia with aedeagus as in figures $47,48--$

------ vernoniae Steyskal

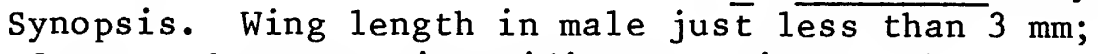
2 ors and upper ori equidistant, distance between upper and lower ori double that between upper 3 bristles; orbital setulae in 2 rows, row nearest eye margin sparse, reclinate, inner row more numerous, proclinate; gena relatively narrow, 0.17 height of eye, this in male with patch of short pilosity opposite ors; arista long, 0.83 height of eye, appearing bare; male genitalia with aedeagus having virtually no gap between basal sclerites and distiphallus complex (fig. 47). Host/Early Stages. Vernonia noveboracensis. Larva internal stem borer.

Distribution. District of Columbia, Maryland. Reference. Steyska1, 1981: 41.

Male genitalia with aedeagus as in figure 49-------

------- vernoniana Steyskal

Synopsis. Essentially as in M. vernoniae, possibly smaller, wing length about $2.5 \mathrm{~mm}$.

Host/Early Stages. Vernonia noveboracensis. Larva internal stem borer.

Distribution. District of Columbia.

Reference. Steyska1, 1981: 41.

25 (21). Frons distinctly projecting above eye------------- 
26 (24). Ocellar triangle not developed beyond ocellar plate--- urticella Spencer

Synopsis. Mesonotum and abdomen green, wing length

from $2.5 \mathrm{~mm}$ in male to $3.1 \mathrm{~mm}$ in female; gena

deepest in center below eye, 0.25-0.20 eye

height; male genitalia with aedeagus as in

figures 50,51 .

Host/Early Stages. Urtica dioica. Larva

feeding internally in stem but unknown.

Distribution. California.

Reference. Spencer, 1981: 62 .

-- Ocellar triangle well defined, apex extending to

leve 1 of lower ors or upper ori-_-

27 (26). Orbital setulae sparse, reclinate; 3 ori----------

Synopsis Wing length 2.5-3. cirsiophila Spencer

twice twice width of eye; gena deepest in center, $0.25-0.20$ height of eye; arista long, virtually bare; male genitalia with aedeagus as in figures $52,53$.

Host/Early Stages. Cirsium spp. Puparium straw colored in front, b $\overline{1 \text { ackish }}$ at segment boundaries and on segments 5 and 6 ; posterior spiracles closely adjoining, separated by less than own diameter, each with ellipse of some 12 bulbs around strong central horn.

Distribution. California; new records: Colorado, 5 miles S. of Boulder, 5,800 ft, $1 \mathrm{~m}$., $1 \mathrm{f}$., 16.VI.61 (C. H. Mann, CNC); Nebraska, Valley Co., $1 \mathrm{~m}$., ex Carduus nutans, 24.III.77 (W. O. Hamp, USNM).

Reference. Spencer, 1981: 41.

Orbital setulae in several rows, inner row at least partially proclinate, those nearest eye margin reclinate; 4 ori-ar. angelicae (Frost)

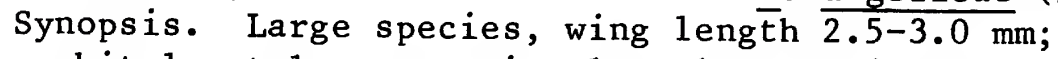
orbital setulae appearing largely proclinate; mesonotum blackish green, abdomen more shining green; male genitalia with aedeagus (not previous1y illustrated) having conspicuously wide gap between basal sclerites and distiphallus complex (fig. 54).

Host/Early Stages. Angelica atropurpurea, probably other Angelica spp. Larva feeding and pupating in stem; puparium whitish, posterior spiracles widely separated, each with ellipse of some 16-20 bulbs around strong central horn (fig. 55).

Distribution. New York, Ohio (new record), many reared specimens (D. Witwer).

References. Frost, 1934: 40; Frick, 1959: 362; part 2, p. 243.

28 (25). 3 ori; frons 1.5 times width of eye; arista appearing bare----

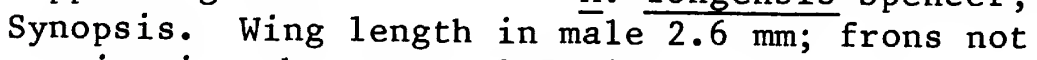
projecting above eye, 1.5 times width of eye; gena 
narrow, 0.20 height of eye; ocellar triangle and orbit distinctly but moderately shining; male genitalia with aedeagus as in figures 56, 57 . Host/Early Stages. Unknown.

Distribution. Long Island, N.Y.

Reference. Part 2, p. 245.

-- 2 ori; frons little wider than eye; arista distinct-

ly pubescent-------- virginiensis Spencer, n. sp.

Synopsis. Large species, wing length $2.75 \mathrm{~mm}$ in

male; orbital setulae in 2 rows, inner row largely proclinate, outer more reclinate; gena about 0.20 height of eye; male genitalia with aedeagus as in figures $58,59$.

Host/Early Stages. Unknown.

Distribution. Virginia.

Reference. Part 2, p. 248.

29 (18). Gena deepest in front ( $\mathrm{fig} .60$ )----M. buccalis Spencer Synopsis. Small to medium-sized species, wing length from 2 to $2.3 \mathrm{~mm}$ in male to $2.8 \mathrm{~mm}$ in $\mathrm{fe}-$ male; frons not projecting above eye; 2 ors, either 2 or 3 ori; eye in male distinctly pilose; mesonotum appearing matt, blackish from front, more greenish from rear; abdomen greenish or coppery; male genitalia with aedeagus as in figures 61,62 .

Host/Early Stages. Unknown (possibly Arnica and other Asteraceae, see Spencer, 1981).

Distribution. California; new records: Colorado, Maryland, New York, Virginia; Canada.

References. Spencer, 1969a: 67; 1981: 38; part 2, p. 243.

-- Gena deepest in center or rear--- 30

30 (29). Orbits conspicuously widening at midpoint---.-- 31

-- Orbits not so widening-- 32

31 (30). Each orbit over 0.33 width of frons; orbital setulae numerous, conspicuously proclinate above---- virens (Loew)

Synopsis. Medium-sized species, wing length 2.2-3 $\mathrm{mm} ; 2$ ors, 2 ori; gena narrow, eye pilose in male; mesonotum and abdomen greenish; male genitalia with aedeagus as in figures 63, 64; surstylus projecting inward, with 3 or 4 strong, spinelike projections at end (fig. 65).

Host/Early Stages. Eupatorium capillifolium, Heterotheca subaxillaris. Puparium straw colored, posterior spiracles widely separated, each with about 8 well-defined bulbs around strong central horn (fig. 66).

Distribution. Florida, Pennsylvania, South Carolina (confirmed), certainly more widespread; widespread in Canada.

Reference. Spencer and Stegmaier, 1973: 51. Orbit broad but less than 0.33 width of frons; orbital setulae less numerous, less proclinate above-Synopsis. Medium-sized species, wing length 2.2-2.7 mm; frons not projecting; 2 ors, 2 ori; 
eye conspicuously pilose in male; gena narrow, 0.17 height of eye; mesonotum and abdomen greenish; male genitalia with aedeagus as in figures 67,68 .

Host/Early Stages. Asteraceae, confirmed on Ambrosia, Bidens, Erechtites, Flaveria, Gaillardia, Gnaphalium, Helianthus, Parthenium, Tagetes; in Hawaii also on Lactuca and Apium (Apiaceae). Puparium straw colored, posterior spiracles with 8 bulbs but central horn rudimentary (figs. 69, 70).

Distribution. Florida, Michigan; new records: New York, South Carolina.

References. Frick, 1953b: 207; Spencer and Stegmaier, 1973: 48; Spencer, 1981: 58.

32 (30). Mesonotum without metallic sheen, predominantly

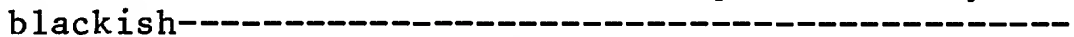

Mesonotum at least slightly greenish or coppery---- 36

33 (32). Last and penultimate sections of vein $M 3+4$ variable, last section longer, equal to or at most little shorter than penultimate----M. riparella Hendel Synopsis. Small species, wing length $1 . \overline{1.9-2.25 \mathrm{~mm}}$; frons little wider than eye, orbit prominent but not significantly projecting above eye; 2 ors, 3 ori, orbital setulae reclinate; gena deepest at rear, 0.20 height of eye; mesonotum black, abdomen green; male genitalia with aedeagus as in figures 71, 72; hypandrium broad (fig. 73).

Host/Early Stages. Phyla nodiflora, probably $\underline{P}$. lanceolata. Larva interna1 stem borer; puparium entirely white or faintly reddish; anal segments conspicuously curving ventrally (fig. 74); posterior spiracular processes separated by own diameter, each with ring of 9 well-defined bulbs, without trace of central horn or scar.

Distribution. Florida, Illinois, Texas (new record).

Reference. Part 2, p. 247.

Last section of M $3+40.5$ or at most 0.67 length of

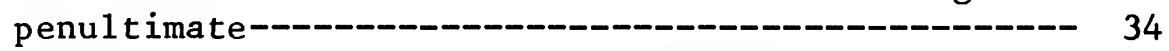

34 (33). 2 ori-C. chillcotti Spencer, n. sp. Synopsis. Wing length in male $2.1 \mathrm{~mm}$, last section of M $3+40.67$ of penultimate; frons narrow, equal to width of eye; generally closely resembling $\mathrm{M}$. subvirens; male genitalia with aedeagus as in figures 75,76 .

Host/Early Stages. Unknown.

Distribution. North Carolina.

Reference. Part 2, p. 244.

35 (34). Abdomen brilliantly shining green; 3 ori---------

Synopsis. Wing length $2.5 \mathrm{~mm}, 1$ ast section of M $3+4$ little over 0.5 penultimate; gena deepest in center, 0.20 height of eye; eye pilose in male; 
mesonotum blackish, abdomen brilliantly shining green; male genitalia with aedeagus as in figure 77; hypandrium triangular (fig. 78, paralectotype).

Host/Early Stages. Unknown.

Distribution. Illinois; reported by Frick (1959)

from Iowa, Pennsylvania, Virginia.

References. Malloch, 1915a: 105; Frick, 1959: 366.

Abdomen blackish coppery, weakly shining; normally

4 ori---

Synopsis. Closely resembling $M$. subvirens but

readily distinguishable by less shining abdomen;

male genitalia with aedeagus as in figures 79,80 .

Host/Early Stages. Unknown.

Distribution. Kansas.

Reference. Part 2, p. 244.

36 (32). Arista appearing bare (X 38)

Arista appearing distinctly pubescent- 37

$--$

$37(36)$.

Eye bare in both sexes; minute species, wing

$1.75-2 \mathrm{~mm}---$

$--$

Eye distinctly pilose in male-_-_-_..-_-_-_-_-_-

38 (37). Male genitalia with aedeagus as in figure 81-------

Synopsis. Frons equal to width of eye, not projecting above eye; 2 ors, 2 ori, orbital setulae in single row, reclinate; mesonotum and abdomen green; ocellar triangle weakly shining; male genitalia with aedeagus as in figure 81 ; hypandrium with extended apodeme (fig. 82).

Host/Early Stages. Bidens pilosa, Calendula sp. Larva feeding and pupating in seed heads; puparium straw colored, posterior spiracles widely separated, each with ellipse of about 10 bulbs around broad central horn.

Distribution. Florida; widespread in Caribbean area; Costa Rica, Mexico.

References. Spencer, 1963a: 313; Spencer and Stegmaier, 1973: 38 .

Male genitalia with aedeagus as in figures $83,84--$

-

Synopsis. Closely resembling $\underline{M}$. flōris but male genitalia entirely distinct.

Host/Early Stages. Medicago sativa. Larva internal stem borer; puparium straw colored, posterior spiracles separated by own diameter, each with 4 or 5 bulbs around central horn.

Distribution. Arizona, California; also Colorado, Texas (according to Frick, 1959).

References. Malloch, 1915a: 106; Spencer, 1981: 43.

39 (37). Frons narrow, less than 1.5 times width of eye----- chaptaliae Spencer Synopsis. Wing length $2.2 \mathrm{~mm} ; 2$ ors, 2 ori; gena narrow, 0.10 height of eye; eye distinctly pilose in male; mesonotum shining, greenish coppery, abdomen more matt; squama and fringe silvery 
white; male genitalia with aedeagus as in figures 85,86 .

Host/Early Stages. Chaptalia dentata. Larva feeding in seed heads; puparium pale orange yellow, posterior spiracles (fig. 87) on long projections, each with strongly chitinized ellipse of 9 regular bulbs around short but stout central horn.

Distribution. Florida.

References. Spencer, 1966d: 9; Spencer and Stegmaier, 1973: 35.

40 (39). 3 ori--

Synopsis. Wing length in male $2 \mathrm{~mm}$; frons 1.5

times width of eye; gena deepest in center, 0.25

height of eye; mesonotum greenish black, abdomen more shining green; male genitalia with aedeagus as in figures 88,89 .

Host/Early Stages. Unknown.

Distribution. California.

Reference. Spencer, 1981: 53.

2 ori

41 (40). Ocellar triangle slightly but not brilliantly

shining------------------M. gnaphalii spencer

Synopsis. Small species, wing length $\frac{\text { 2-2.3 mm; }}{2-2 .}$ gena narrow, 0.20-0.17 height of eye, this conspicuously pilose in male; mesonotum greenish black, abdomen more distinctly green; male genitalia with aedeagus as in figures $90,91$.

Host/Early Stages. Conyza canadensis, Gnaphalium leucocephalum. Larva internal stem borer;

further details not available.

Distribution. California.

Reference. Spencer, 1981: 45.

-- Ocellar triangle defined in outline only, at most

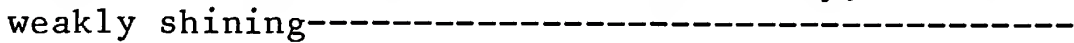

42 (41). Male genitalia with aedeagus (figs. 92, 93) with 2 spinulelike projections at each side of distiphallus dorsally-------------M. corralensis spencer Synopsis. Wing length $2.3-2.5 \mathrm{~mm}$; frons 1.5 times width of eye, this distinctly pilose in male; gena 0.25 height of eye; mesonotum and abdomen greenish. Host/Early Stages. Unknown.

Distribution. California.

Reference. Spencer, 1981: 42.

Male genitalia with aedeagus (figs. 94, 95) not having such projections----------M. muguensis Spencer

synopsis. Wing length in male $2.3 \mathrm{~mm}$, closely resembling $\underline{M}$. corralensis.

Host/Ear1y Stages. Unknown.

Distribution. California; new record: Oregon, Benton Co., Corvallis, $1 \mathrm{~m} ., 19 . \mathrm{IV} .75$

(G. Ferguson, CUI).

Reference. Spencer, 1981: 52.

43 (36). Eye in male bare---_---_---- 44

-- Eye in male pilose-------------------------------- 45

44 (43). Orbital setulae minute, similar to pubescence of 
arista------- floridensis Spencer

Synopsis. Small, shining, greenish species, wing

length $2.2 \mathrm{~mm}$; frons little wider than eye; 2 ors,

2 ori, orbital setulae reclinate; gena narrow,

0.10 height of eye; male genitalia as in figures

96,97 .

Host/Early Stages. Desmodium tortuosum. Larva

feeding and pupating in single seed within pod;

puparium pale orange, posterior spiracles separated by slightly more than own diameter, each with 10-12 irregular bulbs around small central scar (fig. 98).

Distribution. Florida; Caribbean islands, Guyana, Venezuela.

References. Spencer, 1963a: 312; Spencer and Stegmaier, 1973: 37.

Orbital setulae long, substantially longer than pubescence of arista-----_----M. miamensis Spencer

Synopsis. Closely resembling M. flöridensis but male genitalia distinctive as in figures 99, 100; conspicuous row of spines along inner margin of epandrium (fig. 101).

Host/Early Stages. Species of orchid. Holotype labeled "orchid-larva destroys bloom"; larva not known.

Distribution. Florida.

Reference. Spencer and Stegmaier, 1973: 43.

45 (43). Gena narrow, 0.10 height of eye; mesonotum shining green---- vectabilis Spencer

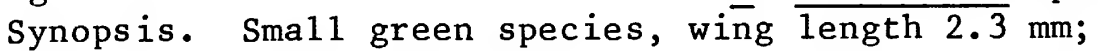
frons only slightly wider than eye, this conspicuously pilose in male; gena narrow, 0.10 height of eye; male genitalia as in figures 102, 103.

Host/Early Stages. Unknown.

Distribution. Florida; Bahamas.

Reference. Spencer and Stegmaier, 1973: 50.

Gena broader, 0.25 height of eye; mesonotum with only faint greenish reflections, largely matt blackish---Synopsis. Closely resembling $\underline{M}$. vectabilis but frons wider, 1.5 times width of eye, and gena broader; male genitalia with aedeagus as in figures 104, 105.

Host/Early Stages. Verbesina alternifolia. Larva internal stem borer; puparium straw colored, posterior spiracular plates closely adjoining, each with ellipse of 12 bulbs.

Distribution. Ohio.

Reference. Part 2, p. 248.

46 (10). Gena with conspicuous fringe of peristomal hairs (fig. 181)--

Peristomal hairs norma1- 47

47 (46). Exceptionally large species, wing length 3.6-4.25 $\mathrm{mm}--------1$ marinensis Spencer

Synopsis. Frons broad, 2.5 times width of eye, conspicuously projecting above eye; 2 ors, up to 6 
strong ori; gena about 0.33 height of eye; arista appearing bare; male genitalia with aedeagus as in figures 106, 107.

Host/Early Stages. Cirsium occidentale, probably other Cirsium spp. Larva internal stem borer but unknown.

Distribution. California, (?) New York.

Reference. Spencer, 1981: 49.

Remarks. A single female from New York, Tioga Co., Owego, 13.V.22 (L. R. West, CUI), possibly represents this species, but confirmation is desirable from a male.

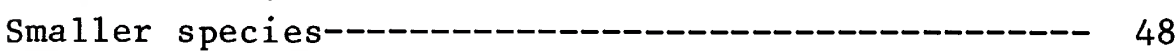

48 (47). Distinct facial keel dividing base of antennae----- 49

-- No obvious keel present----------------------- 50

49 (48). Frons not projecting above eye-----M. palaensis Spencer Synopsis. Small species, wing length in male 1.9 mm; 2 ors, 4 strong ori, orbital setulae sparse, reclinate; gena narrow, 0.17 height of eye; facial keel raised below antennal bases, lower and broader above; mesonotum and abdomen shining black; male genitalia with aedeagus as in figures 108,109 .

Host/Early Stages. Unknown.

Distribution. California.

Reference. Spencer, 1981: 54.

Frons strongly projecting above eye

--------

Synopsis. Large species, wing lengt $\overline{\mathrm{h}} \overline{2.6-3.25} \mathrm{~mm}$;

2 ors, 4 ori, orbital setulae numerous, in 2 rows, inner row conspicuously proclinate; gena broad, 0.33 height of eye; facial keel broad, moderately raised; mesonotum and abdomen greenish black; male genitalia with aedeagus having conspicuous gap between basal sclerites and distiphallus complex (fig. 110).

Host/Early Stages. Lomatium nudicaule. Larva internal stem borer.

Distribution. Oregon.

Reference. Steyska1, 1981: 38.

50 (48). Mesonotum or abdomen greenish or coppery----------- 51

-- Mesonotum and abdomen black---_--- 59

51 (50). Orbital setulae in 2 rows, inner row proclinate, outer reclinate---_- 52

Orbital setulae in single row, proclinate or reclinate--------------

52 (51). Frons broad, twice width of eye, strongly projecting above eye-------------M. maligna Spencer Synopsis. Small species, wing length in male 2.3 $\mathrm{mm} ; 2$ ors, 3 ori; gena broad, 0.33 height of eye; mesonotum matt, faintly greenish from rear, abdomen weakly shining, greenish or coppery; male genitalia with aedeagus as in figures $111,112$. Host/Early Stages. Unknown.

Distribution. California.

Reference. Spencer, 1981: 49. 


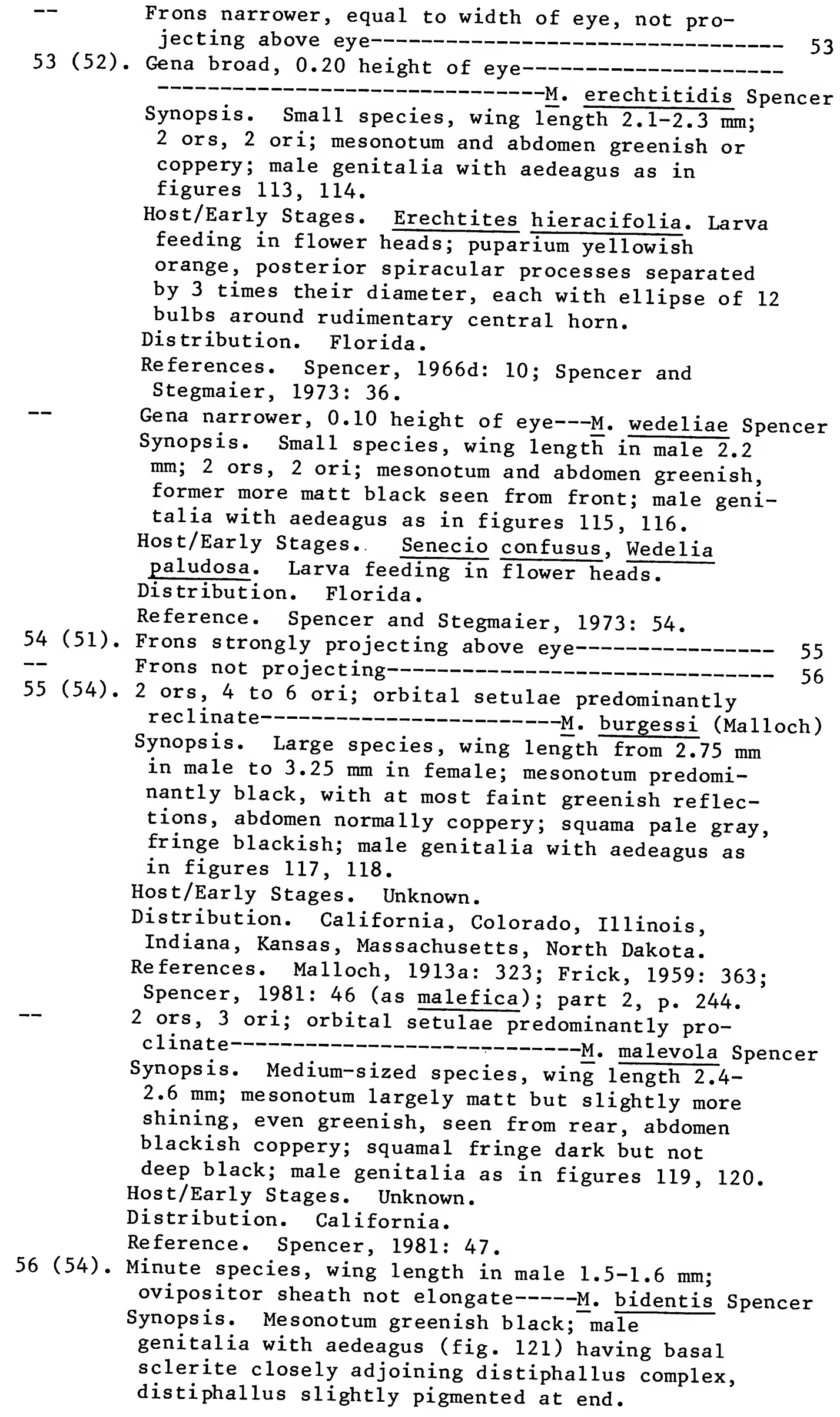


Host/Early Stages. Bidens pilosa, Verbesina sp. Larva feeding and pupating in seed heads; puparium essentially as in $M$. minimoides (fig. 127).

Distribution. Florida; Costa Rica.

References. Spencer, 1966d: 7; Spencer and Stegmaier, 1973: 32 .

Slightly larger species; ovipositor sheath distinctly elongate (figs. 122, 124)

57 (56). Male genitalia with aedeagus having conspicuous gap between basal sclerite and distiphallus complex (fig. 123)--

Synopsis. Minute species, with wing length generally less than $2 \mathrm{~mm}$; mesonotum generally bluish black, abdomen shining green; squama gray, fringe black; male genitalia with aedeagus having small area of pigmentation at extreme distal end (fig. 123).

Host/Early Stages. Wedelia paludosa, W. trilobata, probably Eupatorium odoratum, and other genera of Asteraceae. Larva feeding and pupating in seed heads; puparium essentially as in $\underline{\text { }}$. minimoides (fig. 127).

Distribution. Florida; Caribbean islands, Costa Rica.

References. Ma1loch, 1913a: 328; Spencer and Stegmaier, 1973: 44.

Male genitalia with aedeagus having only slight gap between basal sclerite and distiphallus complex (fig. 123)--_-_-_-_-_-

58 (57). Wing length in male normally $1.9-2.2 \mathrm{~mm}-$

- minimoides Spencer

Synopsis. Small greenish species, frons little wider than eye; 2 ors, 2 ori, orbital setulae in single row, reclinate; eye distinctly pilose in male, less so in female; gena 0.17 height of eye; ovipositor slightly elongate (fig. 124); male genitalia with aedeagus as in figures 125,126 , only slight gap between U-shaped basal sclerite and distiphallus complex, pigmentation at end of distiphallus variable, scarcely detectable in type series ex Verbesina (Florida), obvious in populations in Arkansas and Maryland ex Heliopsis and Rudbeckia.

Host/Early Stages. Many genera of Asteraceae, confirmed on Aster simmondsii, Borrichia frutescens, Helenium nudiflorum, Heliops is helianthoides, Melanthera deltoidea, Rudbeckia laciniata, and Verbesina virginica. Larva feeding and pupating in seed head; puparium whitish yellow, posterior spiracles (fig. 127) on widely separated conical projections, each with ring of 9 bulbs around central horn.

Distribution. Florida, Ohio; new records: Arkansas, Maryland; Venezuela.

References. Spencer, 1966d: 13; Spencer and Stegmaier, 1973: 45; part 2, p. 246 (under M. radicicola). 
Wing length in male $1.85 \mathrm{mm-----M.} \mathrm{radicicola} \mathrm{Steyska1}$

Synopsis. Essentially as in M. minimoides; male genitalia with aedeagus having distinct pigmentation distally (fig. 128).

Host/Early Stages. Unconfirmed, single available specimen obtained in rearing container with roots of Urtica but no pupal case found.

Distribution. Maryland.

References. Steyska1, 1981: 40; part 2, 246.

59 (50). Minute species, wing length in male less than $2 \mathrm{~mm}-$ - - caribbea Spencer

Synopsis. Shining black; gena narrow, 0.12 height of eye; male genitalia with aedeagus as in figures 129,130 .

Host/Early Stages. Unknown.

Distribution. Florida; Costa Rica, Dominica.

References. Spencer and Stegmaier, 1973: 34; part

2 , p. 244 .

60 (59). Gena exceptionally narrow, 0.08 height of eye-----M. ultima Spencer, n. sp.

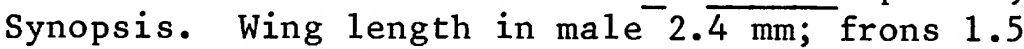
times width of eye, not projecting above eye; 2 ors, 2 ori; mesonotum and abdomen moderately shining black; squama gray, fringe black; male genitalia with aedeagus as in figures 131, 132. Host/Early Stages. 'Unknown.

Distribution. Virginia.

Reference. Part 2, p. 247.

61 (60). Frons only slightly wider than eye; (costa normal$1 y$ ending at vein $R 4+5$, couplet 3 ) ---------------- Ophiomyia similata (Malloch)

Frons broader, 1.5 times width of eye-- 62

62 (61). Mesonotum and abdomen shining black--M. tetrica Spencer Synopsis. Generally resembling M. setifrons (couplet 8 ) but with only 2 pairs of dc; male genitalia with aedeagus as in figures 133, 134 . Host/Early Stages. Unknown. Distribution. Colorado (new record); Canada. References. Spencer, 1969a: 76; part 2, p. 247. Mesonotum and abdomen matt------M. marel1ii (Brèthes) Synopsis. Frons 1.5 times width of eye, not significantly projecting above eye; 2 ors, 3 ori; ocellar triangle il1 defined; gena broad, 0.25 height of eye, this bare in both sexes; mesonotum matt black, with $2 \mathrm{dc}$, abdomen entirely black; squama pale gray, fringe dark, ochreous to black; wing length $2.3-2.7 \mathrm{~mm}$, last section of $M 3+4$ distinctly shorter than penultimate, inner crossvein at midpoint of discal ce11; male genitalia with aedeagus as in figures 135, 136. Host/Early Stages. Achyranthes (=Alternanthera) philoxeroides. Larva forming conspicuous stem galls (fig. 137); puparium yellowish orange, posterior spiracles (fig. 138) each on stout 
conical projection, with ellipse of some 18 bulbs. Distribution. Florida; Argentina, Bahamas, Brazil. References. Spencer, 1963a: 317; Spencer and

Stegmaier, 1973: 452; part 2, p. 245.

Genus Hexomyza Enderlein

Hexomyza Enderlein, 1936: 179. Type of genus: Melanagromyza sarothamni Hendel, 1923 (Europe).

Enderlein erected the genus Hexomyza for three species previously included in Melanagromyza, in which the costa ends at vein $R 4+5$ and which have three dorsocentrals. Hendel (1931-36: 570), followed by Frick (1952a: 376), synonymized Hexomyza with Melanagromyza, but the genus was revived by Spencer (1966a) for four species forming twig galls, based on their uniform biology and the differing form of the male genitalia. The formation of a gall clearly cannot be considered now as of generic significance. One such species in the United States, "Agromyza" tiliae Couden, forming twig galls on Tilia americana that appear similar to those of the other species retained in Hexomyza, has now been transferred to ophiomyia, in view of its clear association with stem-mining species (part 2 , p. 260).
Six species are retained in Hexomyza in the United States, three feeding on Salix, one on Populus, and one introduced from Japan on Wistaria. The biology of another, H. toschiae, is unconfirmed, but the aedeagus closely resembles that of the Salix feeders. Externally these species are not separable from Melanagromyza, but the differing biology and genitalia are associated with differences in larval characters, in which the posterior spiracles are on short stalks (as opposed to the flat plate in Melanagromyza) and each normally has three bulbs (fig. 158). The larval characters suggest the genus is more closely related to Ophiomyia than to Mel anagromyza.

Two of the North American species, $\underline{H}$. schineri and $\underline{H}$. simplicoides, are holarctic, and single species are known in Japan, South Africa, and New Zealand. In addition, $\mathrm{H}$. centaureae Spencer (1966c) was described from Italy, forming stem galls on Centaurea solstitialis, but the generic position of this species may require revision (holotype in USNM). 
Key to Hexomyza Species

1. Costa ending shortly after termination of vein $\mathrm{R} 4+5$, possibly attenuated toward $\mathrm{M} 1+2--------2$

Costa extending strongly to vein $\mathrm{M} 1+2-$

2 (1). Wing conspicuously white, veins pale or colorless---H. winnemanae (Malloch)

Synopsis. Frons broad, twice width of eye, projecting above eye in profile; 2 equal ors, 2 slender ori, orbital setulae reclinate above, more upright below; gena deepest in center, almost 0.33 height of eye; 3d antennal segment small, round, arista short, appearing almost bare; mesonotum with $2 \mathrm{dc}$, black; abdomen more shining; wing length 2-3 mm; male genitalia with aedeagus as in figures $143,144$. Host/Early Stages. Salix spp. Larva forming twig gall; puparium gray, posterior spiracles in form of 2 short, stout projections (fig. 145), which are adjoining and largely fused, probably each with 3 minute bulbs (although not detectable in only available specimen).

Distribution. Maryland; Canada.

References. Malloch, 1913a: 314; Spencer, 1969a: 79 (as H. albicula); part 2, p. 250 .

-- Wing hyaline, normal, veins pale or darker brown----- 3

3 (2). Small species, wing length in male 1.9-2.3 mm----- 4

-- Larger species, wing length 2.5-3.8 mm--

-

Synopsis. Frons matt black, broàd, twice width of eye, strongly projecting above eye in profile; normally 2 ors and 2 or 3 ori, orbital setulae conspicuously long, reclinate or frequently distinctly incurved; gena broad, up to 0.33 height of eye; mesonotum shining black, normally with $2 \mathrm{dc}$, $2 \mathrm{~d}$ close to 1 st and well behind level of supra-alar, frequently a 3d also present; costa normally ending just beyond vein $R 4+5$, sometimes continuing weakly toward M 1+2; outer crossvein often lacking; male genitalia with aedeagus as in figures 146, 147.

Host/Early Stages. Salix spp. Larva forming oval twig gall (see fig. 157), each posterior spiracular process having 3 minute bulbs on short, broad stalk, front segments conspicuously cut away and in puparium bright reddish brown (fig. 148).

Distribution. California, New York, Ohio.

Reference. Spencer, 1981: 31 .

4 (3). 2 ors, 3 ori; 2 dcSynopsis. Frons unusually broad, $2.5^{-}$times width of eye, conspicuously projecting above eye; orbital setulae sparse, upright or reclinate; ocellar triangle large, apex extending to middle ori; gena angular, 0.20 height of eye; mesonotum matt viewed from front, weakly shining from rear, abdomen more shining black; squama gray, margin and fringe black; wing length in male $1.9 \mathrm{~mm}$; male genitalia with aedeagus as in figures 149, 150.

Host/Early Stages. Unknown.

Distribution. California. 
Reference. Spencer, 1981: 34 .

-- 2 ors, 2 ori; 3 dc--- salicis (Malloch)

Synopsis. Frons twice width of eye, conspicuously projecting above eye in profile (fig. 151); orbit broad, orbital setulae sparse, reclinate; gena angular, deepest in center, slightly over 0.33 height of eye, parafacial prominent; ocellar triangle not shining; mesonotum weakly shining black, with dc 3 strong, immediately behind suture; abdomen entirely black; squama gray, margin and fringe black; wing with costa ending immediately after termination of vein $R 4+5$, last and penultimate sections of $M 3+4$ equal; length given by Malloch as $2 \mathrm{~mm}$; (both wings now missing in holotype but illustrated by Malloch, 1913a); male genitalia with aedeagus as in figures $152,153$.

Host/Early Stages. Salix sp. Early stages unknown. Distribution. Massachusetts; new record: British Columbia, Kootenay District, Carbonate Column, Columbia River, 2,600 ft, $1 \mathrm{~m}$., 7.VII.12.08 ( J. C. Bradley, CUI).

References. Malloch, 1913a: 314; part 2, p. 249.

5 (1). Last section of vein M $3+4$ longer than penultimate---

- websteri (Malloch)

Synopsis. Large species, wing length $3.5 \mathrm{~mm}$; frons almost twice width of eye; normally 5 orbital bristles (rarely 6); gena 0.25 height of eye; mesonotum moderately shining black, 3 strong dc, sometimes small further ones in same line that are distinctly stronger than acr; squama gray, margin black, fringe blackish; male genitalia illustrated by Sasakawa (1961: fig. 35).

Host/Early Stages. Wistaria spp. Posterior spiracles of larva each with 3 minute bulbs; galls found at base of twig buds (fig. 154).

Distribution. Washington (introduced); Japan.

References. Malloch, 1913a: 325; Sasakawa, 1961: 353.

-- Last section of M 3+4 shorter than penultimate------- 6

6 (5). Last section of vein M $3+40.67$ penultimate

-

Synopsis. Frons broad, twice.width of eye, narrowly projecting above eye in profile; 2 ors, 2 or 3 ori; gena angular, deepest in center below eye, $0.20-$ 0.25 eye height; mesonotum grayish black, weakly shining, with 2 strong dc but not infrequently a $3 d$ of varying length also present; wing length from $2 \mathrm{~mm}$ in male to $3.1 \mathrm{~mm}$ in female; squama dark gray, fringe black; male genitalia with aedeagus as in figures $155,156$.

Host/Early Stages. Populus fremontii, $\underline{\text { P. tremu- }}$

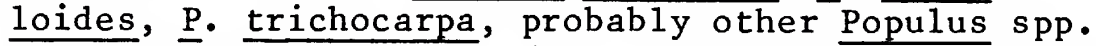
Larva forming oval gall (fig. 157) in current year's growth of young twigs, pupating in gall, emerging in spring or early summer; puparium grayish brown, 
with conspicuous scar bands at segment borders; posterior spiracles on short projections, each with 3 bulbs (fig. 158).

Distribution. California, Colorado, Massachusetts, New Mexico, Washington; Canada, widespread in $\mathrm{N}$. Europe, (?) Japan.

References. Frick, 1959: 365; Spencer, 1966a: 43; 1969a: 81; Brown and Eads (1969, biology).

Last and penultimate sections of $M 3+4$ equal

Note. Although species form twig galls (fig. 192), male genitalia clearly associate this species with Ophiomyia abutilivora (p. 250), a stem miner on Abutilon.

Genus Ophiomyia Braschnikov

Agromyza Fallén, subgenus Ophiomyia

Braschnikov, 1897: 40. Type of subgenus: Agromyza maura Meigen, 1830 (misidentified as $\underline{A}$. pulicaria Meigen). No types extant. Ophiomyia Braschnikov, Hendel, 1920. Type of genus: Agromyza maura Meigen (misidentified as A. curvipalpis Zetterstedt, 1848). Ophiomyia Braschnikov, subgenus

Tylomyza Hende1, 1931: 181. Type of subgenus: Agromyza pinguis Fallén, $1823 a$, in NRS.

Tylomyza Hendel, Enderlein, 1936

(synonymy established by Spencer, 1964a: 775).

Hende1 (1920) first treated Ophiomyia as a distinct genus, differentiating it from Melanagromyza by the raised facial keel dividing the base of the antennae and the fused vibrissal fasciculus in the male (figs. 221, 235). These characters are well defined in most species, but in others, in which the male genitalia, larval characters, or biology clearly indicate assignment to Ophiomyia rather than to Melanagromyza, one or the other of these characters or even both may be lacking.

Other important characters are the termination of the subcosta in vein $R 1$ (figs. 4, 204); the black halter (the only exceptions known are 0 . maculata from California, 0 . aeneonitens Strob 1 from Europe, and $\underline{\underline{0}}$. aberrans Spencer from South Africa and Sri Lanka); the uniform black of both mesonotum and abdomen (except in the 0 . jacintensis group discussed here, in which some greenishness occurs more frequently in Melanagromyza); and the presence of only two pairs of strong dorsocentrals. The costa normally extends to vein $M 1+2$ (to $R \quad 4+5$ in four U.S. species), and the squamal fringe is normally black (white in four U.S. species).

Characteristically in the male genitalia, the basal sclerites are long (figs. 208, 229) and the distiphallus complex is asymmetrical (figs. 209, 246). In the United States, a group of five species is now known (0. jacintensis group), in which the aedeagus is complex and asymmetrical but differing substantially from the typical form. They are $\underline{0}$. bernardinensis, $\underline{0}$. shastensis, $\underline{0}$. yolensis, $\underline{0}$. tiliae, and $\underline{0}$. abutilivora feeding on Abutilon and Sida and are described here (part 2, p. 250). The morphological characters of the larva (fig. 197) and the feeding habit of 0 . abutilivora confirm its close relationship to typical ophiomyia species.

Although the generic limits of Ophiomyia and Melanagromyza may not be obvious from external characters, the combination of morphological, larval, and biological characters will invariably indicate the correct generic position of doubtful species. 
With the distinctive differences between typical Ophiomyia and typical Melanagromyza species, including larval characters and biology, there is no justification for uniting the genera despite the blurred generic limits. The larvae and biology are known of relatively few of the species included in Ophiomyia, but as the genus becomes better known throughout the world, a number of distinctive species groups may well be given separate generic status.

Recent studies have shown that Ophiomyia is a larger genus than had previously been suspected. Over 160 species have now been described, and the genus occurs in all faunal regions. In the United States, 55 species are now known, of which 14 are described here as new (part 2, p. 250), and 5 species are recorded as new to the United States but previously known in Canada. The genus is widely distributed throughout the United States.

Seven new synonyms and five new combinations are established. A number of species have been seen from random caught males, which are almost certainly undescribed. However, with no distinctive characters, formal description seems undesirable at this time. Fifteen other species are known in Canada (Spencer, 1969a; Sehgal, 1971), some of which may be expected to occur in the United States.

The majority of Ophiomyia species form shallow external stem mines, pupating at the end of the mine, with the anterior spiracles projecting through the epidermis. Species feeding in this way are $\underline{0}$. coniceps (Sonchus), 0 . labiatarum (several genera of $\overline{\text { Lamiaceae), }}$ o. simplex (Asparagus), and a new species described here from Chondrilla. Several are leaf miners, including $\underline{0}$. camarae (Lantana), $\underline{0}$. nasuta (Taraxacum), and $\underline{0}$. quintáa

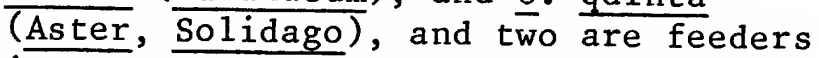
in seed heads--0. lantanae (Lantana) and 0 . lippiae (Phyla). One gall causer, $\underline{0}$ tiliae, which was previously placed in Hexomyza based on its biology, is transferred here to Ophiomyia (part 2, p. 260), as the male genitalia clearly associate it with the $\underline{0}$. jacintensis group. This new species described in part 2, page 250 , is of particular interest, as it forms a modified stem mine on Abutilon and Sida, which is somewhat raised and approaches the character of a stem gall.

The host is known of only 11 of the 55 United States species. This is the smallest proportion in any of the larger genera and is due to the inconspicuous nature of stem mines, which are difficult to detect and can only be discovered with careful searching.

All known larvae have the posterior spiracles on a slender stalk, normally with few bulbs (fig. 177), whereas in Melanagromyza the spiracles are on a flat, normally strongly chitinized plate, frequently with numerous bulbs (figs. 39, 55).

Two European species have previously been misidentified in the United States--o. major (Strob1) and $\underline{0}$. maura (Meigen) ${ }^{-} \overline{\text { rick, }}$ 1959)--and must now be deleted from the United States list: $\underline{0}$. major was confused with $\underline{0}$. vibrissata from Georgia by Hendel (1931) and o. maura with o. quinta, which occurs from Canada to Arkansas (part 2, p. 257). 
Key to Ophiomyia

Species
1. Halter variegated, white on upper half, black below---- maculata Spencer Synopsis. Wing length in male $2.5 \mathrm{~mm}$; gena broad, 0.33 height of eye; vibrissal fasciculus in male short, uniformly curving; mesonotum and abdomen moderately shining, with faint coppery tinge; squama dark gray, fringe black; male genitalia with aedeagus as in figures $159,160$. Host/Early Stages. Unknown.

Distribution. California.

Reference. Spencer, 1981: 89.

-- Halter uniformly dark, brown or black-------_---- 2

2 (1). Costa ending at or just beyond vein $R 4+5-\cdots+-$

$-$

3 (2). Squama and fringe whitish------0. banffensis spencer Synopsis. Wing length $1.9-2.2 \mathrm{~mm}$, last section of $M 3+4$ longer than penultimate, in ratio $20: 15$; frons slightly wider than eye, not projecting above eye; 2 ors, 2 ori; gena slightly projecting forward (fig. 161); facial keel narrow but high; ocellar triangle, mesonotum, and abdomen brilliantly shining black; male genitalia with aedeagus as in figures 162, 163 (holotype).

Host/Early Stages. Unknown.

Distribution. Colorado (new record); Canada.

References. Spencer, 1969a: 84; part 2, p. 252.

Squama gray, fringe black

Facial keel broad----- monticola Sehgal

Synopsis. Wing length from $2.1 \mathrm{~mm}$ in male to 2.7 $\mathrm{mm}$ in female, last section of $\mathrm{M} 3+4$ slightly longer than penultimate; frons broad, twice width of eye, not projecting above eye; 2 ors, 2 (occasionally 3 ) ori; gena distinctly projecting forward (fig. 164); male with single slender vibrissa, no fasciculus; facial keel broad but flat, with distinct furrow above; mesonotum shining black; squama pale gray, margin black, fringe whitish ochreous; male genitalia with aedeagus as in figures $165,166$.

Host/Early Stages. Unknown.

Distribution. Colorado (new record); Alaska, Canada.

References. Sehgal, 1968: 60; Spencer, 1969a: 90; part 2, p. 256.

Facial keel sharp

$5(4)$

--

Synopsis. Medium-sized, shining black species, wing (fig. 167) length 2.2-3 mm; gena broad, not projecting forward; normally 2 strong dc, small 3d and 4th rarely also present; squama gray, margin and fringe black; male genitalia with aedeagus as in figures 168, 169 .

Host/Early Stages. Asparagus officinalis. Larva external stem miner; puparium pale or darker brown, posterior spiracles each having about 16 bulbs (fig. 170). 
Distribution. Widespread, probably present wherever asparagus is regularly cultivated; confirmed in California and New York.

References. Spencer, 1973a: 108 (economic importance); 1981b: 99 .

Orbit and parafacial not shining-_-_-

- similata (Ma11 och)

Synopsis. Wing length from $2.4 \mathrm{~mm}$ in male to 2.7

$\mathrm{mm}$ in female; costa normally ending at $\mathrm{R} 4+5$,

though sometimes continuing weakly to $M 1+2 ; 2$

ors, 2 ori; frons not projecting above eye; meso-

notum and abdomen entirely black; squama dark

gray, margin and fringe black; male genitalia with aedeagus as in figures 171, 172; hypandrium broadly fused at apex (fig. 173).

Host/Early Stages. Unknown.

Distribution. Illinois, New York (new record);

Canada.

References. Ma1loch, 1918b: 178; Frick, 1959: 366;

Spencer, 1969a: 73 (as M. orientalis); part 2, p. 251.

6 (2). Squama and fringe white (see also 0 . atriplicis

Spencer, 1984, Ent. Soc. Wash. Proc. 86:664)---- 7

Squama and fringe dark-- 9

7 (6). Proboscis elongate (figs. 174, 178)-_. 8

Proboscis short, normal-- lippiae Spencer

Synopsis. Very small, shining black species, wing length $1.5-1.9 \mathrm{~mm}$; vibrissal corner forming angle of approx. $90^{\circ}$, fasciculus in male long ( $\mathrm{fig}$. 174); squama white, fringe silvery white to ochreous; male genitalia with aedeagus as in figures 175,176 .

Host/Early Stages. Phyla nodiflora. Larva reported as seed feeder but possibly a stem miner, pupating in upper part of stem; puparium pale, yellowish white, posterior spiracles on short, raised stalks, each process with 5 bulbs (fig. 177).

Distribution. Florida; Bahamas.

References. Spencer, 1966a: 6; Spencer and Stegmaier, 1973: 61.

8 (7). Last section of vein $M 3+4$ slightly shorter than penultimate--- haydeni Spencer

Synopsis. Sma11 black species, wing length in male $1.9 \mathrm{~mm}$; gena narrow, 0.125 height of eye; proboscis elongate ( $\mathrm{fig}$. 178); mesonotum and abdomen only moderately shining; squama whitish gray, margin brown, fringe white; male genitalia with aedeagus as in figure 179 .

Host/Early Stages. Unknown.

Distribution. Florida; Bahamas.

Reference. Spencer and Stegmaier, 1973: 57.

Last section of M $3+4$ times length of penultimate--

Synopsis. Minute black species, $\frac{\text { texella } \text { wing length in }}{n}$. sp. female $1.4 \mathrm{~mm}$; gena conspicuously projecting forward (fig. 180); proboscis elongate; squama and 
fringe white; male genitalia unknown.

Host/Early Stages. Unknown.

Distribution. Texas.

Reference. Part 2, p. 259.

9(6). Male without vibrissal fasciculus----_-_--_---- 10

-- Male with distinct vibrissal fasciculus (see figs.

$221,235)-----------$

10 (9). Prominent, raised facial keel present------------ 19

-- Facial keel lacking or if present, linear and

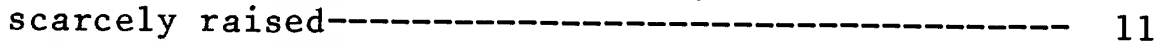

11 (10). Male with conspicuous fringe of peristomal hairs

(figs. 181, 184)---_-_-_- 12

-- Vibrissa and peristomal hairs normal--_-_-_-_-_--- 13

12 (11). Vibrissa not differentiated from peristomal hairs, these numerous, mostly equal (fig. 181)---------

--c-c- lauta (Spencer)

Synopsis. Appearing generally as a Melanagromyza but correct generic position indicated by male genitalia (figs. 182, 183); fringe of peristomal hairs distinctive; ocellar triangle scarcely shining; mesonotum and abdomen moderately shining; squama gray, margin and fringe black.

Host/Early Stages. Unknown.

Distribution. Georgia (new record), Michigan; Canada.

References. Spencer, 1969a: 70; Steyskal, 1972b: 2 ; part 2, p. 255.

Vibrissa strong, peristomal hairs strong numerous, becoming weaker toward rear (fig. 184) -

Synopsis. Wing length $2.4-2.5 \mathrm{~mm}$; $\frac{0}{\text { ocellar tri- }}$ angle, mesonotum, and abdomen shining black; arrangement of peristomal hairs distinctive; male genitalia with aedeagus as in figures 185, 186. Host/Early Stages. Unknown. Distribution. Arizona, Colorado (new record); Canada.

References. Malloch, 1913a: 328; part 2, p. 254.

13 (11). Mesonotum and abdomen entirely black-- 14

-- Mesonotum and abdomen at least slightly greenish--- 22

14 (13). Ocellar triangle weakly shining---_-_-_--_----- 15

Ocellar triangle brilliantly shining---_---_----- 17

15 (14). 2 ori (see couplet 5)-------- similata (Malloch) 3 ori------ 16

16 (15). Last section of vein $M 3+40.67$ length of penultimate----- frosti spencer, n. sp. Synopsis. Small black species, without vibrissal fasciculus in male or raised facial keel; male genitalia with aedeagus as in figures $187,188$. Host/Early Stages. Unknown, probably leaf miner on Asteraceae.

Distribution. New York.

Reference. Part 2, p. 255.

Last and penultimate sections of $M 3+4$ equal-------

-

Synopsis. Frons 1.5 times width of eye, not pro- 
jecting above eye in profile; 2 ors, 3 ori; gena not extended at rear, rounded in front, slightly projecting forward, about 0.25 height of eye; single strong vibrissa present; antennae divided by low facial keel; mesonotum shining black, with $2 \mathrm{dc}$; wing length in male $2.4 \mathrm{~mm}$, costa extending to vein $M 1+2$, inner crossvein at distal $3 d$ of discal cell, outer crossvein less than own length from inner; male genitalia with aedeagus as in figures 189, 190; epandrium and cercus as in figure 191 .

Host/Early Stages. Tilia americana. Larva forming oval twig gall, several galls adjoining on same twig (fig. 192).

Distribution. Illinois, Indiana, Missouri, Pennsylvania, Virginia.

References. Couden, 1908: 34 (description and biology); Frick, 1959: 367 (as Melanagromyza);

Spencer, 1981: 33 (as Hexomyza); part 2, p. 260.

17 (14). Gena broad, almost 0.25 height of eye--

-

Synopsis. Closely resembling 0 . abūtilivora, possibly generally larger, wing length up to $2.8 \mathrm{~mm}$; gena broader (fig. 193); male genitalia as in figures 194, 195.

Host/Early Stages. Unknown, possibly Brassica.

Distribution. Colorado (new record); Canada.

References. Spencer, 1969a: 67; part 2, p. 255.

Gena narrow, 0.17-0.14 height of eye-- 18

18 (17). Male genitalia with aedeagus as in figures 197 , 198-

Synopsis. Small black species, wing length 2-2.3 $\mathrm{mm}$; head as in figure 196; brilliantly shining ocellar triangle; mesonotum and abdomen without any greenish reflections.

Host/Early Stages. Abutilon theophrasti, Sida cordifolia. Larva forming raised, external stem mine or "welt," pupating in stem with anterior spiracles projecting through epidermis; puparium straw colored, posterior spiracles (fig. 199) each with 4-6 bulbs.

Distribution. Florida, Illinois, Minnesota, Mississippi, Wisconsin.

Reference. Part 2, p. 250.

Male genitalia with aedeagus as in figures 200, 201--

Synopsis. Small black species, not distinguishable on external characters from $\underline{0}$. abutilivora.

Host/Early Stages. Unconfirmed, possibly Lantana. Larva forming long, pale leaf mine (fig. 202), pupating in leaf.

Distribution. Florida; Bahamas.

References. Spencer and Stegmaier, 1973: 46; part 2 , p. 256.

19 (10). Orbital setulae proclinate; ors lacking in male---- 
Synopsis. Orbit strongly differentiated; in male ors lacking (fig. 203), orbital setulae particularly strong and numerous in male; male without vibrissal fasciculus but facial keel bulbous, a1most spherical; proboscis elongate; 3 strong postsutural dc; mesonotum and abdomen shining black; squama gray, fringe black; wing (fig. 204) length 2-2.3 mm; male genitalia with aedeagus as in figures 205, 206.

Host/Early Stages. Taraxacum officinale. Larva mining in stalks and midrib of leaves (Frost, 1924: 46), pupating at leaf base but unknown.

Distribution. Widespread in northern half of United States from California to New York (Frick, 1959: 372); new and most southerly records: Colorado, Mt. Evans area, up to $10,600 \mathrm{ft}, 14 \mathrm{~m}$., $8 \mathrm{f}$., VI-VII.1961 (CNC); North Carolina, Highlands, $1 \mathrm{~m}$. , 13.V.57 (J. C. Vockeroth, CNC); Virginia, Pulaski Co., Pulaski, 4 m., 1 f., $7 . V .79$ (USNM); widespread in Canada, N. Europe.

References. Melander, 1913: 260; Frick, 1959: 372; Spencer, 1969a: 91.

Orbital setulae reclinate; ors present in both sexes----------------------------------------

20 (19). Frons strongly projecting above eye, parafacial prominent---- boulderensis Spencer, n. sp.

Synopsis. Large species, wing length in male 2.5 $\mathrm{mm}$; gena and parafacial broad (fig. 207); strong vibrissa, triplicated, but not fully fused; facial keel raised, conspicuous; male genitalia with aedeagus as in figures 208, 209.

Host/Early Stages. Unknown.

Distribution. Colorado.

Reference. Part 2, p. 252.

Frons not projecting above eye, parafacial linear-- 21

Mesonotum and abdomen black----0. commendata Spencer

Synopsis. Wing length in male $2.3 \mathrm{~mm}$; frons broad, twice width of eye; gena deepest in center, 0.25 height of eye; vibrissa slender; mesonotum and abdomen shining black; squama gray, margin and fringe black; male genitalia with aedeagus as in figures 210,211 .

Host/Early Stages. Unknown.

Distribution. California.

Reference. Spencer, 1981: 73.

Mesonotum and abdomen greenish

- bernardinensis Spencer

Synopsis. Wing length in male $2 . \overline{25 \mathrm{~mm} \text {; frons } 1.5}$ times width of eye; facial keel broad, gena narrow, 0.20 height of eye; mesonotum and abdomen brilliantly shining, greenish black; male genitalia with aedeagus as in figures $212,213$.

Host/Early Stages. Unknown.

Distribution. California.

Reference. Spencer, 1981: 73.

22 (13). Frons broad, twice width of eye 
Frons narrow, 1ittle wider than eye--_-_-_-_---

- - shastensis Spencer

Synopsis. Wing length in male $2.5^{-} \mathrm{mm}$; frons narrow, little wider than eye; gena narrow, 0.17 height of eye; facial keel inconspicuous; antennal bases not widely separated; both mesonotum and abdomen distinctly greenish; male genitalia with aedeagus as in figures $214,215$.

Host/Early Stages. Unknown.

Distribution. California.

Reference. Spencer, 1981: 97.

23 (22). Gena narrow, 0.20 height of eye (fig. 216)

Synopsintensis Spencer Synopsis. Wing length in male $2 . \overline{5} \mathrm{~mm}$; frons broad, twice width of eye; male with single slender vibrissa; facial keel narrow, only slightly raised below base of antennae; male genitalia with aedeagus as in figures 217,218 .

Host/Early Stages. Unknown.

Distribution. California.

Reference. Spencer, 1981: 85.

-- Gena broader, 0.25-0.33 height of eye-----------

-

Synopsis. Wing length in male $2.3 \mathrm{~mm}$; frons broad, twice width of eye; gena almost 0.33 height of eye; facial keel narrow, only slightly raised below bases of antennae; mesonotum and abdomen shining blackish green; male genitalia with aedeagus as in figures 219,220 .

Host/Early Stages. Unknown.

Distribution. California.

Reference. Spencer, 1981: 100.

24 (9). Vibrissal angle acute, at most $60^{\circ}$

Vibrissal angle between $70^{\circ}$ and 90 $90^{\circ}$

$25(24)$.

Vibrissal angle approx. $45^{\circ}$, conspicuously elongate, extending beyond antennae--_---_--------- 26

Gena broad, 0.5 height of eye (fig. 221)

Synopsis. Relatively large species, praecisa Spencer

Synopsis. Relatively large species, wing length 2.1-2.7 mm; frons projecting above eye; gena projecting forward; vibrissal fasciculus in male short but broad at base; facial keel narrow with slight furrow above; male genitalia with aedeagus as in figures 222, 223.

Host/Early Stages. Unknown.

Distribution. California (as o. aestimabilis); Canada.

References. Spencer, 1969a: 92; 1981: 69 (as ㅁ. aestimabilis); part 2, p. 256 .

-- Gena narrower, 0.25-0.33 height of eye---------- 27

27. 2 ori-- 3 ori--_- 28

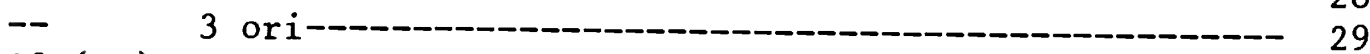

28 (27). Facial keel broad-----Synopsis. Frons narrow, equal to width of eye; gena 0.25 height of eye, distinctly projecting 
forward; vibrissal angle approx. 450; fasciculus in male short but broad (fig. 224); facial keel broad, low; wing length in male $1.9 \mathrm{~mm}$, last section of $\mathrm{M} 3+4$ little shorter than penultimate; male genitalia with aedegus as in figures $225,226$. Host/Early Stages. Unknown.

Distribution. Northern California.

Reference. Spencer, 1981: 72.

Facial keel narrow----- 0 . chondrillae Spencer, n. sp. Synopsis. Frons narrow, equal to width of eye; gena broad, projecting forward, with unfused vibrissal fasciculus (fig. 227); wing length in male $2 \mathrm{~mm}, 1$ ast and penultimate sections of vein $\mathrm{M} 3+4$ equal; male genitalia with aedeagus as in figures $228,229$.

Host/Early Stages. Chondrilla juncea. Larva forming external stem mine.

Distribution. Washington.

Reference. Part 2, p. 253.

29 (27). Frons broad, twice width of eye--_-_-_-_-- 30

-- Frons narrower, at most 1.5 times width of eye---- 31

30 (29). Gena broad, 0.33 height of eye-- subpraecisa Spencer, n. sp.

Synopsis. Frons not projecting above eye; fasciculus in male short; facial keel broad, conspicuously raised, with low furrow on upper half; wing length $2.1-2.3 \mathrm{~mm}$; ocellar triangle weakly shining; mesonotum and abdomen more distinctly shining black; male genitalia with aedeagus as in figures 230, 231.

Host/Early Stages. Unknown.

Distribution. Colorado.

Reference. Part 2, p. 258.

Gena narrow, 0.20 height of eye----0. ambrosia Spencer Synopsis. Wing length in male $2.1 \mathrm{~mm}$; ori;

fasciculus in male broad at base, with conspicuous bend beyond midpoint (fig. 232); facial keel narrow, raised below base of antennae, without furrow; mesonotum and abdomen moderately shining black, latter with faint coppery reflections; male genitalia with aedeagus as in figures 233, 234. Host/Early Stages. Unknown.

Distribution. California.

Reference. Spencer, 1981: 71.

31 (29). Facial keel high, rounded-_-_-_-_-_-_- 32

-- Facial keel only slightly raised-_-_-_-___-_-_-_ 33

32 (31). Vibrissal fasciculus in male long, slender (fig. 235)

Synopis Wing quinta Spencer ( approx. $45^{\circ}$, fasciculus regularly curving ( $\mathrm{fig}$. 235); facial keel high, without central furrow; mesonotum and abdomen shining black; male genitalia with aedeagus as in figures 236, 237. Host/Early Stages. Aster, Solidago. Larva forming long, narrow, upper surface leaf mine, with frass in conspicuous, widely spaced black pellets (fig. 
238); puparium more rarely black, posterior spiracles each with some 15 bulbs on 2 arms, each process on a short stalk.

Distribution. New records: Arkansas, New York, Pennsylvania; Canada.

References. Frost, 1924: 42 (as O. curvipalpis

var. texana); Spencer, 1969a: 96; part 2, p. 257.

Vibrissal fasciculus short, broad--

- coniceps (Malloch)

Synopsis. Wing length $2-2.2 \mathrm{~mm}$; frons little wider than eye; gena extending forward, forming angle of approx. 45 ; fasciculus in male frequently not fully fused (fig. 239); facial keel bulbous at base of antennae, furrowed above; mesonotum and abdomen shining black; male genitalia with aedeagus as in figures $240,241$.

Host/Early Stages. Sonchus asper (doubtless other related Asteraceae). Larva mining stem but unknown.

Distribution. California, Indiana, Louisiana, Utah (Frick, 1959); widespread in Canada.

References. Malloch, 1915a: 107; Frick, 1959: 369; Spencer, 1969a: 851; 1981: 75.

33 (31). Facial keel with prominent central furrow--

- virginiensis Spencer, n. sp.

Synopsis. Wing length from $2.1 \mathrm{~mm}$ in male to 2.5 $\mathrm{mm}$ in female; short but prominent vibrissal fasciculus in male, vibrissal corner forming angle of approx. $45^{\circ}$; gena narrow, 0.14 height of eye; mesonotum and abdomen only moderately shining; ocellar triangle moderately shining black; male genitalia with aedeagus as in figures 242, 243. Host/Early Stages. Unknown.

Distribution. Virginia.

Reference. Part 2, p. 260.

Facial keel without furrow-_-no obstipa Spencer Synopsis. Wing length $2 \mathrm{~mm}$; vibrissal fasciculus in male long, slender (fig. 244); gena broad, almost 0.25 height of eye; ocellar triangle brilliantly shining black; mesonotum and abdomen strongly shining; male genitalia with aedeagus as in figures $245,246$.

Host/Early Stages. Unknown.

Distribution. Florida.

Reference. Spencer and Stegmaier, 1973: 62 .

34 (25). Very small species, wing length less than $2 \mathrm{mm-----} 35$ Slightly larger species, wing length at least $2 \mathrm{~mm}-41$

35 (34). Veins conspicuously pale- - debilis Spencer Synopsis. Wing length $1.4-1.5 \mathrm{~mm}$, last section of M 3+4 normally slightly longer than penultimate; ocellar triangle large, broad, apex extending to level of lower ors; gena narrow, at most 0.20 height of eye; vibrissal fasciculus in male short, tapering (fig. 247); ocellar triangle, mesonotum, and abdomen shining black; wing and veins pale; male genitalia with aedeagus as in figures 248,249 . 
Host/Early Stages. Possibly Gilia (Asteraceae), on which 3 females were caught in Washington.

Distribution. California, Washington.

Reference. Spencer, 1981: 76.

Veins darker, normal-

(fig male conspicuously long

(fig. 250)-- camarae Spencer

Synopsis. Wing length $1.6 \mathrm{~mm}$, last section of

M $3+4$ only slightly shorter than penultimate; gena

0.17 height of eye; vibrissal fasciculus in male

broad at base but then slender, unusually long

(fig. 250); facial keel narrow but distinctly raised; ocellar triangle only moderately shining; mesonotum more so; male genitalia with aedeagus as in figures 251,252 .

Host/Early Stages. Lantana camara. Larva forming leaf mine along midrib, with offshoots into leaf blade (fig. 253); whitish puparium remaining in mine.

Distribution. Florida; Trinidad.

References. Spencer, 1963a: 326; Spencer and Stegmaier, 1973: 55.

-- Vibrissal fasciculus shorter (figs. 254, 259)------

37 (36). Facial keel low, only slightly raised-

- genitalis Spencer

Synopsis. Wing length 1.6-1.75 mm, last and penultimate sections of $\mathrm{M} 3+4$ equal or last slightly shorter; gena 0.20 eye height; vibrissal fasciculus in male slender, with distinctive bend near midpoint (fig. 254); facial keel narrow but slightly raised and widening below base of antennae; mesonotum shining black; male genitalia with aedeagus as in figure 255 .

Host/Early Stages. Unknown.

Distribution. Florida; Costa Rica, Dominica.

Reference. Spencer and Stegmaier, 1973: 126.

-- Facial keel conspicuously raised--_- 38

38 (37). Facial keel with distinct furrow----- devia spencer

Synopsis. Wing length in male $1.7 \mathrm{~mm}$, 1 ast section of $\mathrm{M} 3+4$ shorter than penultimate, in ratio $13: 16$; head (fig. 256) with gena broad, 0.33 height of eye; facial keel broad, conspicuously raised below base of antennae, with distinct furrow at highest point; ocellar triangle only weakly shining; mesonotum matt viewed from front, only slightly shining black from rear; male genitalia with aedeagus as in figures $257,258$.

Host/Early Stages. Unknown.

Distribution. California.

Reference. Spencer, 1981: 80 .

Facial keel without furrow-_-

Last section of M 3+4 longer than penultimate------

Synops of $3+4$ last section trian penultimate; ocellar triangle ill-defined, scarcely shining; vibrissal 
fasciculus in male short, broad at base, finely tapering (fig. 259); facial keel conspicuously raised, almost spherical at base of antennae; male genitalia with aedeagus as in figures 260,261 . Host/Early Stages. Unknown.

Distribution. North Carolina.

Reference. Part 2, p. 253.

-- Last section of M 3+4 shorter than penultimate-----

40 (39). Vibrissal fasciculus in male short (fig. 262)------

- vockerothi Spencer, n. sp.

Synopsis. Wing length in male $1.9 \mathrm{~mm}$, last

section of M $3+4$ little shorter than penultimate;

frons narrow, approx. equal to width of eye; gena

broad, 0.33 height of eye; facial keel broad, moderately raised, without central furrow; male genitalia with aedeagus asymmetrical (figs. 263, 264).

Host/Early Stages. Unknown.

Distribution. North Carolina.

Reference. Part 2, p. 261.

-- Vibrissal fasciculus in male longer--

- (Malloch)

(Vibrissal angle normally less acute, forming angle of $80^{\circ}$, couplet 52 )

41 (34). Facial keel with distinct central furrow--------

Synopsis. Wing length $2.4 \mathrm{~mm}$, last section of

M 3+4 little shorter than penultimate; gena 0.17

height of eye, parafacial broad, more than half

width of gena; vibrissal fasciculus in male short, regularly curving (fig. 265); facial keel of medium width, conspicuously raised above base of antennae; ocellar triangle moderately, mesonotum distinctly shining black; male genitalia with aedeagus as in figures 266, 267.

Host/Early Stages. Unknown.

Distribution. Florida; Canada.

Reference. Spencer and Stegmaier, 1973: 59.

Facial keel without furrow-- 42

42 (41). Facial keel broad--- - o. subdefinita spencer, nom. nov. Synopsis. Wing length in male $2.1 \mathrm{~mm}$, last section of $\mathrm{M} 3+4$ shorter than penultimate, in ratio $17: 21$; gena narrow, 0.17 height of eye; fasciculus in male tapering, scarcely curving; ocellar triangle, mesonotum, and abdomen moderately shining black; male genitalia with aedeagus as in figures 268 , 269.

Host/Early Stages. Unknown.

Distribution. California.

Reference. Spencer, 1981: 78 (as 0. definita preocc. Spencer, 1971a: 148 - England).

$--$

Facial keel narrow-

43 (42). Male genitalia with aedeagus as in figures 294, 295, distiphallus complex with distinctive cylindrical projection at rear---- texana (Malloch) (Vibrissal angle normally less acute, forming angle of approx. $80^{\circ}$, couplet 52 ) 
44 (43). Frons broad, twice width of eye-----0. delecta Spencer Synopsis. Wing length in male $2.1 \mathrm{~mm}$, last section of $\mathrm{M} 3+4$ slightly shorter than penultimate, in ratio 9:11; ocellar triangle short, broad, not reaching level of lower ors; head (fig. 270) with gena narrow, 0.17 height of eye; fasciculus in male slender, only slightly curving; facial keel narrow but distinctly raised above base of antennae, unfurrowed; ocellar triangle, mesonotum, and abdomen moderately shining black; male genitalia with aedeagus as in figures $271,272$.

Host/Early Stages. Unknown.

Distribution. California.

Reference. Spencer, 1981: 79.

Frons at most 1.5 times width of eye--_-_-_-_-_--

- -

Synopsis. Wing length in male $2 \mathrm{~mm}$; head (fig. 273) with gena 0.125 height of eye; ocellar triangle large, apex extending to level of lower ors, dull, paler than frons; facial keel of medium width and height; male genitalia with aedeagus characteristically asymmetrical in ventral view (figs. 274, 275).

Host/Early Stages. Unknown.

Distribution. North Carolina.

Reference. Part 2, p. 252.

45 (24). Large species, wing length in male at least $2.5 \mathrm{~mm}-$ Smaller species, wing length in male less than 2.5 $\mathrm{mm}$

46 (45). Exceptionally large species, wing length in male 2.8-3.0 mm-------------

Smaller species, wing length in male $2.5 \mathrm{~mm}-$

Synopsis. Frons strongly projecting abov̄e eye, broad, 2-2.5 times width of eye; 3 or 4 ori; oce 1lar triangle ill-defined, scarcely detectable beyond apex of ocellar plate; gena broad, 0.33 height of eye; fasciculus in male short, sometimes incompletely fused (fig. 276); facial keel of medium width, distinctly furrowed, only slightly raised; male genitalia with aedeagus as in figures 277,278 .

Host/Early Stages. Unknown (possibly Urtica on which holotype was caught).

Distribution. California.

Reference. Spencer, 1981: 83.

47 (46). 3 ori-- vibrissata (Malloch) Synopsis. Wing length $2.8-3.0 \mathrm{~mm}$; frons broad, twice width of eye, at most very slightly projecting above eye; normally 5 orbital bristles, (not 6 as stated in original description); ocellar triangle scarcely shining, not extending below level of upper ors; gena broad, 0.33 height of eye; fasciculus in male broad, tapering (fig. 279); facial keel high but narrow, with distinct furrow 
above; mesonotum and abdomen shining black; squama gray, margin and fringe black; male genitalia with aedeagus as in figures 280, 281 (holotype).

Host/Early Stages. Unknown.

Distribution. Georgia, New York (new record).

References. Malloch, 1913a: 316; Frick, 1959:

370 ; part 2, p. 260.

2 ori--

Synopsis. Agreeing closely with o. vibrissata,

possibly slightly larger, wing length in male 3

$\mathrm{mm}$; gena forming angle of approx. $90^{\circ}$; fasci-

culus in male strong but tapering; facial keel

narrow, rather low, with furrow throughout; male

genitalia with aedeagus as in figures 282,283 .

Host/Early Stages. Unknown.

Distribution. North Carolina.

Reference. Part 2, p. 253.

48 (45). Frons distinctly projecting above eye----------- 49

-- Frons not projecting----- 51

49 (48). 2 ori-- eldorensis Spencer, n. sp. Synopsis. Frons twice width of eye; ocellar triangle ill-defined, matt; gena forming angle of $80^{\circ}$; vibrissal fasciculus in male short ( $\mathrm{fig}$. 284); wing length in male $2.1 \mathrm{~mm}$, inner crossvein at distal 3d of discal cell; male genitalia with aedeagus bow1 shaped (figs. 285, 286).

Host/Early Stages. Unknown.

Distribution. Colorado.

Reference. Part 2, p. 254. 3 ori

50 (49).

Gena broad, up to 0.5 height of eye

Synopsis. Frons broad, twice width of $\frac{\text { fastella }}{\text { eye; gena }}$ forming angle of $80^{\circ}-90^{\circ}$; fasciculus in male short, incompletely fused; ocellar triangle and mesonotum strongly shining; wing length in male $2.1 \mathrm{~mm}$; male genitalia with aedeagus as in figures 287-289.

Host/Early Stages. Unknown.

Distribution. California.

Reference. Spencer, 1981: 82.

Gena 0.33 height of eye----------0. sexta Spencer

Synopsis. Frons broad, twice width of eye; gena

forming angle of $90^{\circ}$; fasciculus in male short, with normal curvature (fig. 290); ocellar triangle scarcely shining; facial keel moderately raised, with distinct central furrow; wing length from 2.3 $\mathrm{mm}$ in male to $2.7 \mathrm{~mm}$ in female; male genitalia with aedeagus as in figures 291, 292.

Host/Early Stages. Unknown. Distribution. Colorado (new record); Canada. References. Spencer, 1969a: 98; part 2, p. 257.

51 (48). Gena relatively broad, 0.33-0.25 eye height-------- 52

-- Gena narrower, 0.20-0.17 eye height---_---------- 54

52 (53). Facial keel without furrow------ 0 . texana (Ma1loch) Synopsis. Frons not projecting above eye, width 
variable, from 1.5 to 2 times width of eye; gena variable, normally forming angle of about $80^{\circ}$ (fig. 293), rarely more acute, forming angle of about $60^{\circ}$ (couplets 40, 43); vibrissal fasciculus in male strong at base but tapering and very fine at end; facial keel of variable width but never with furrow; wing length from $1.75 \mathrm{~mm}$ to 2.3 $\mathrm{mm}$; male genitalia with aedeagus (figs. 294, 295) having distinctive cylindrical projection at rear of distiphallus complex.

Host/Early Stages. Brassicaceae, recorded from stem mine in Rorippa sp. and Descurainia sp.

Distribution. California, Texas; Canada; reported by Frick (1959) as widespread from Washington to Michigan, but these records require confirmation.

References. Malloch, 1913a: 319; Frick, 1959: 371; part 2, p. 259.

53 (52). Ocellar triangle and orbits brilliantly shining---

Synopsis. Closely resembling 0 . malitiosa, differing primarily in more shining ocellar triangle and orbits; wing length in male $2.1 \mathrm{~mm}$; male genitalia with aedeagus as in figures 296 , 297.

Host/Early Stages. Unknown.

Distribution. California.

Reference. Spencer, 1981: 92.

Ocellar triangle and orbits only moderately shining----

Synopsis. Frons broad, twice width of eye; gena forming angle of $90^{\circ}$ ( $\mathrm{fig} .298$ ), 0.25 height of eye; fasciculus in male short, tapering; facial keel low, narrow, deeply furrowed; wing length $2 \mathrm{~mm}$; male genitalia with aedeagus as in figures 299,300 .

Host/Early Stages. Unknown.

Distribution. California.

Reference. Spencer, 1981: 91.

54 (51). Facial keel shining black in upper half-_-_-

-

Synopsis. Frons 1.5 times width of eye; gena narrow, 0.20-0.125 height of eye, forming angle of $70^{\circ}$; vibrissal fasciculus in male long, with distinctive curvature (fig. 301); facial keel of medium width but distinctly raised and furrowed; wing length up to $2.4 \mathrm{~mm}$; male genitalia with aedeagus as in figures 302,303 .

Host/Early Stages. Lamiaceae, genera recorded include Calamintha, Galeopsis, Lamium, Nepeta, and Stachys (in U.S. only Nepeta). Posterior spiracles of larva each with 7 bulbs; pupation occurs in stem near node.

Distribution. Indiana (Spencer, 1964a: 794); widespread in Canada and Europe.

References. Spencer, 1964a: 793; 1969a: 87. 
Facial keel not shining above---_--_-------- 55

55 (54). Minute species, wing length in male $1.6 \mathrm{mm-------} 56$

-- Larger species, wing length at least $1.9 \mathrm{mm-------} 57$

56 (55). Frons 1.5 times width of eye------ 0 . 1assa Spencer Synopsis. Head (fig. 304) with gena narrow, 0.17 height of eye, forming angle of $80^{\circ}$; fasciculus short, straight; facial keel narrow but conspicuously raised below base of antennae; male genitalia with aedeagus as in figures 305,306 .

Host/Early Stages. Unknown.

Distribution. California.

Reference. Spencer, 1981: 88 .

Frons little wider than eye---0. parva Spencer, n. sp. synopsis. Agreeing closely with 0.1 lassa but fas-

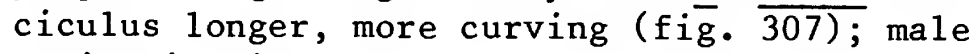
genitalia with aedeagus as in figures 308,309 . Host/Early Stages. Unknown.

Distribution. New Mexico.

Reference. Part 2, p. 256.

57 (55). Mesonotum normally matt, almost brownish

- - 1antanae (Froggatt)

Synopsis. Wing length $1.9-2.2 \mathrm{~mm}$; frons 1.5 times width of eye; gena narrow, about 0.20 height of eye, forming angle of about $80^{\circ}$; fasciculus in male long, slender, with distinctive curvature (fig. 310); male genitalia with aedeagus as in figures 311,312 .

Host/Early Stages. Lantana camara and some other

Lantana spp. Larva feeding in seed heads, either in pulp of immature berries or lower in peduncle, where pupation takes place.

Distribution. Southern California, Florida, Texas;

widespread in Brazil, Caribbean area, Costa Rica, Mexico, Panama; introduced widely in old World tropics to control Lantana.

References. Frick, 1957b: 201; 1959: 370; Spencer, 1973a: 358-363; Spencer and Stegmaier, 1973: 60.

-- Mesonotum more distinctly shining

58 (57). Facial keel low, scarcely raised----- 0 . levata Spencer Synopsis. Wing length in male $2.4 \mathrm{~mm}$; frons 1.5 times width of eye; gena narrow, 0.20 height of eye, forming angle of $80^{\circ}$; fasciculus in male long, curving ( $f i g .313$ ); male genitalia with aedeagus as in figures 314,315 .

Host/Early Stages. Unknown.

Distribution. California.

Reference. Spencer, 1981: 84.

Facial keel narrow but high-

- arizonensis Spencer, n. sp.

Synopsis. Wing length in male $1.9 \mathrm{~mm}$; frons 1.5 times width of eye; gena narrow, 0.17 height of eye, forming angle of approx. $70^{\circ}$; fasciculus in male short, slender ( $f i g .316$ ); male genitalia with aedeagus as in figures 317,318 .

Host/Early Stages. Unknown.

Distribution. Arizona.

Reference. Part 2, p. 251 . 
Genus Japanagromyza Sasakawa

Japanagromyza Sasakawa, 1958: 138. Type of genus: Japanagromyza duchesneae Sasakawa, 1954 (Japan).

Japanagromyza appears to be intermediate between Melanagromyza and Agromyza, normally with only two pairs of dorsocentrals (most common arrangement in Melanagromyza) and prescutellars frequently present (never in Melanagromyza, always in Agromyza). The halter is normally pale (dark in one species in Australia and one in Jamaica).

The discovery from the examination of males of Agromyza rutiliceps that this species correctly belongs in Japanagromyza has necessitated a substantial expansion of the generic concept. Hitherto all known species have been dark, either black or with greenish reflections; the head characteristically has the frons not projecting, with gena narrow (fig. 335). In J. rutiliceps, in which the male genitalia indicate its generic affiliation (see fig. 320 and fig. 336 of $\mathrm{J}$. inaequalis), the frons is reddish, strongly projecting and with the gena deeply extended, and there are $3+1$ strong dorsocentrals. It seems possible that the largely yellowish species in the small genus known only in the Caribbean area, Geratomyza Spencer in Spencer and Stegmaier (1973), should also be included in Japanagromyza. This would indicate an even wider extension of the generic limits, but time is not available now for the necessary study of this genus to formally propose its synonymy with Japanagromyza.

Japanagromyza was erected for a small group of species in Japan and was later found to be sparsely represented in much of the oriental Pacific area; five species were recorded in Australia (Spencer, 1977d). Twenty species are known in the Neotropical Region (Spencer and Stegmaier, 1973: 144). In the United States, the genus is present with five species in
Florida, and its range now extends to the west, with $\mathrm{J}$. rutiliceps known in California, Idah̆o, Montana, and Utah.

Hosts are known of four of the six United States species, three in the Fabaceae and one in the Fagaceae. The larva in all instances, including three known in Japan and the Oriental Region, forms a conspicuous blotch mine, pupating externally. The larval morphology has not been studied in detail, but the posterior spiracles show considerable differentiation and can be specifically diagnostic (see fig. 345, J. aequalis, and fig. 339, J. inaequalis, both on Vigna). J. viridula is a well-known leaf miner on Quercus, and recently specimens have been reared from introduced Castanea mollissima (Chinese chestnut). Unfortunately no larvae or puparia of specimens from oak are available for comparison with those from chestnut (fig. 334), but it is here accepted that only a single species is involved. 
Key to Japan-

agromyza Species
3+1 dc; frons reddish--------J. rutiliceps (Melander)

Synopsis. Head (fig. 319) with frons reddish

orange, strongly projecting above eye; 2 ors, 2

ori; gena extended at rear, 0.25 height of eye; $3 \mathrm{~d}$

antennal segment orange brown, small, round, only

slightly longer than broad; mesonotum shining

black, 3+1 dc; legs entirely black; squama whitish gray, margin pale brown, fringe pale, whitish;

male genitalia with aedeagus ( $f i g$. 320) having paired

distal tubules, large, forming single coil, fused

toward base; hypandrium triangular, with extended apodeme; epandrium with short, stout bristles at lower corner; cercus without bristles; sperm pump with small, strongly pigmented blade and welldeveloped base (fig. 321).

Host/Early Stages. Unknown.

Distribution. Montana; new records: California;

Idaho, Franklin Co., Cub River Canyon, 2 m., 1.VII.61 (W. J. Hanson, USU); Utah, Garfield Co., Clear Creek Canyon, 9,300 ft, 1 f., 4.VIII.41

(D. E. Hardy, UKaL); Panguitch Creek, $1 \mathrm{~m}$., 2-15.VI.67 (G. F. Know1ton, USU).

References. Melander, 1913: 261; part 2, p. 261.

-- Only 2 postsutural dc; frons black or brown--_-_---- 2

2 (1). Prescutellars lacking-_- 3

-- Prescutellars present--_-_-_-_-_-_-_-_-_-_- 4

3 (2). Mesonotum distinctly greenish------J. perpetua Spencer Synopsis. Wing length $1.9-2.4 \mathrm{~mm}$; frons narrow, equal to width of eye; 2 equal ors, 2 similar ori; gena narrow, 0.10 height of eye; frons matt black, lunule silvery; prsc sometimes weak; mesonotum greenish black, abdomen more shining green; squama and fringe whitish yellow; halter white; male genitalia with aedeagus (fig. 322, A) divided apically, with single coil of paired tubules; hypandrium (fig. 322, B) without hypandrial apodeme; surstylus extending downward, with few stout bristles internally (fig. 323); cercus large, with numerous bristles.

Host/Early Stages. Unknown.

Distribution. Florida; Bahamas, Costa Rica, Dominica, Puerto Rico.

Reference. Spencer and Stegmaier, 1973: 28.

Mesonotum primarily matt grayish black, only faint-

ly greenish--

Synopsis. Resembling J. perpetua but frons brownish black, mesonotum 1argely grayish black, rarely faintly greenish; male genitalia with aedeagus having single, short distal tubule (fig. 324); hypandrium (fig. 325) rounded at end, without apodeme; surstylus short and stout, with some 12 spines (fig. 326); cercus bearing unusually long hairs; sperm pump as in figure 327 .

Host/Early Stages. Desmodium tortuosum. Larva

forming irregular b lotch mine (fig. 328); puparium orange brown, posterior spiracles each with 3 slightly raised bulbs (fig. 329). 
Distribution. Florida; ? Colombia, Venezuela.

References. Spencer, 1966d: 4; Spencer and

Stegmaier, 1973: 25.

4(2). Mesonotum greenish-

Mesonotum predominantly black----J. viridula (Coquillett)

Synopsis. Frons not projecting above eye, matt black, lunule conspicuously silvery; 2 equal ors, 2 ori; gena narrow, little more than linear; 2 strong $\mathrm{dc}$, rarely a minute $3 \mathrm{~d}$, prsc strong; mesonotum moderately shining, with virtually no trace of metallic coloration; abdomen shining green; squama and fringe silvery; halter white; wing length from $2.3 \mathrm{~mm}$ in male to $3.1 \mathrm{~mm}$ in female; male genitalia with aedeagus (figs. 330, 331) moderately sclerotized basally, distiphallus long, slightly curving, unpigmented tubule, ventral sclerite well developed; hypandrium with extended apodeme (fig. 332); surstylus (fig. 333, A) extending downward, with about 10 strong bristles at end and another group at upper corner; cercus (fig. 333, B) without bristles.

Host/Early Stages. Quercus rubra, possibly some other Quercus spp.; Castanea mollissima (Chinese chestnut), possibly other Castanea spp. Larva forming primary blotch mine; puparium (ex Castanea) yellowish brown, posterior spiracles widely separated, each with 3 or 4 irregular bulbs (fig. 334).

Distribution. Georgia, Indiana, Massachusetts; new records: Kansas, Maine, North Carolina, Pennsy1vania, South Carolina, Tennessee, Virginia; Canada.

References. Frick, 1959: 359; Spencer and Stegmaier, 1973: 29.

Note. It is possible that the population on Castanea represents a sibling species of J. viridula. However, there are no apparent differences between specimens from oak and chestnut externally or in the male genitalia and other postabdominal characters. Unfortunately no larvae or pupal cases obtained from oak appear to have been preserved in any museum and no comparison with those from chestnut has been possible. The significance of differences in the female terminalia between specimens from oak and chestnut detected by G. Steyskal cannot be fully assessed at this time.

5 (4). Upper orbital bristle shorter than lower (fig. 335)--

Synopsis. Wing length 2.3-2.4 - inaequalis (Ma1loch) dis distinctly shorter than lower; frons matt black, rarely brownish; lunule whitish gray; 3d antennal segment large, distinctly longer than broad; mesonotum matt greenish gray; 2 strong dc, normally pair of strong prsc (these sometimes weak, present on one side or greatly reduced, similar to acr); abdomen shining greenish or faintly bluish; squama and fringe white, halter white; male genitalia with aedeagus (fig. 336) having distiphallus in form of paired, unpigmented tubules, forming almost complete 
coil, ventral sclerite strongly developed; hypandrium with short but distinct apodeme; surstylus (fig. 337, A) elongate, directed ventrally, with 2-5 strong spines; cercus (fig. 337, B) with 20 or more internally directed spines.

Host/Early Stages. Vigna luteola (=repens). Larva forming conspicuous white blotch mine (fig. 338); puparium oval, dark brown, posterior spiracles each with 3 bulbs (fig. 339).

Distribution. Florida; Dominica, Puerto Rico.

References. Malloch, 1914a: 89; Spencer and Stegmaier, 1973: 26.

Upper orbital bristles equal------J. aequalis Spencer Synopsis. Wing length $2-2.4 \mathrm{~mm} ; 2$ ors equal; gena narrow, 0.08 height of eye; 3d antennal segment distinctly covered with short, white pilosity; 2 strong dc, frequently small $3 d$ also present, prsc well developed; frons uniformly brown; ocellar triangle and orbit distinctly shining black; mesonotum greenish or coppery black; squama and fringe, also halter entirely white; male genitalia with aedeagus (fig. 340) membranous basally, chitinized at sides toward short distiphallus; hypandrium (fig. 341) with broad arms, upcurved at end; surstylus (fig. 342) curving inward, not downward, without bristles; cercus (fig. 343) without internal bristles but 5 or 6 short, black teeth grouped at end; epandrium (fig. 344) with distinctive narrow extension toward cercus, showing surstylus (A) and cercus (B). Host/Early Stages. Vigna luteola. Larva forming whitish blotch mine; puparium pale, straw colored, posterior spiracles each with 3 bulbs on widely separated protuberances (fig. 345).

Distribution. Florida; Bahamas, Dominica.

References. Spencer, 1966d: 3; Spencer and Stegmaier, 1973: 23 . 
Genus Agromyza Fallén

Agromyza Fallén, 1810: 21. Type of genus: Agromyza reptans Fallén, 1823b (Sweden).

Domomyza Rondani, 1856: 121 (synonymy established by Hende1, 1927). Type of genus: Domomyza cincta Rondani, 1856 (Italy).

The accepted concept of Agromyza can be defined by the following combination of characters: Subcosta complete, joining vein $R 1$ before reaching costa (fig. 4); halter white or yellow (darker in a few tropical species); orbital setulae reclinate; either $3+1$ strong dorsocentrals or 3 or more postsutural dc, greatly decreasing in size, with any presutural small and weak; costa ending at vein $R 4+5$ or continuing to $\mathrm{M} 1+2$; outer crossvein normally present; color in most species black, less frequently head or also body partially yellowish; wing length $2-4 \mathrm{~mm}$.

The course of the subcosta in some species of Phytobia ( $p$. 72) may appear similar to that in Agromyza, and in consequence the generic position of such species may be incorrectly interpreted. Tschirnhaus (1971) discovered that a structure considered as a stridulating mechanism is present in both sexes of Agromyza, but it is entirely lacking in Phytobia. This structure consists of a band of chitinized scales lying immediately below the lower margin of the fused tergites 1 and 2 (figs. 346, 347) and a scraper, a sharp chitinized ridge on the inner surface of the hindfemur (fig. 348). This structure can be accepted as a definitive generic character of Agromyza.

Agromyza is a large genus, with about 170 species throughout the world. In the United States, 37 described species are known and 1 additional species from Wyoming is diagnosed but not formally described. Ten other species are known in Canada (Spencer, 1969a; Sehga1, 1971). In Europe, 72 species are known, but the smaller number in
North America almost certainly reflects insufficient collecting. Six new species are described here and four new synonyms are established (part 2, p. 262). In addition, six species are recorded, previously known only in Canada: A. albertensis, A. bispinata, A. hockingi, A. masculina, A. tacita, and A. vockerothi. Two species-A. albitarsis Meigen (part 2, p. 262, under A. isolata) and A. nigripes Meigen (part 2, p. 26 $\overline{2}$, under A. albipennis)--must now be deleted from the United States list, as previous records have been found to be based on misidentifications.

Except for two species in Europe, one known as a gall causer and the other as a stem miner, all Agromyza larvae whose feeding habits have been discovered are leaf miners. The two main groups that are well known in Europe (Spencer, 1976a) are also the largest groups in the United States. The largest is that feeding on Poaceae. Of these, 6 species have been reared, but 18 species in a11 belong here. The presutural dorsocentral is lacking and the male genitalia are characteristic (figs. $368,388)$. There may be a distinctive arrangement of spines within the epandrium (figs. 410, 413).

Another large group is that associated with the Rosaceae, and closely related to them are feeders on Fabaceae, Salicaceae, and Ulmaceae. Seven species have been reared in this group and four others clearly belong here. Five feeders on Boraginaceae and Urticaceae form a compact group, of which only the two leaf miners on Urtica have been reared. Only a single species, Agromyza ambrosivora, is known to feed on Asteraceae, but A. virginiensis probably has its host in the same family. A. pallidiseta is known only from females and its exact affiliation is uncertain.

Agromyza is poorly represented in the Southern States, with only four species known in Florida (Spencer and 
Stegmaier, 1973). In California, 18 described species were recorded and 3 more were tentatively diagnosed from females (Spencer, 1981). Many other species await discovery.
Key to Agromyza Species
1. Mesonotum with 3 to 6 dc significantly decreasing in size, presutural ones when present little longer than acr--------- 2

Mesonotum with $3+1 \mathrm{dc}$, presutural not significantly weaker than 3d------- 33

2 (1). 3d antennal segment pale, yellow or orange brown--- 3

-- 3d antennal segment dark, black or brownish black-- 8

3 (2). Legs largely black----

-- Legs largely yellow----------------------- 5

4 (3). Mesonotum matt gray--------A. sulfuriceps Strobl Synopsis. Frons yellow below, black above together with orbit; 3d antennal segment (fig. 349) small, round, bright yellow; mesonotum matt gray, with $3+0$ dc, occasionally small presutural present; legs black, only foreknee slightly yellowish; wing length $1.6-2.3 \mathrm{~mm}$, costa extending to vein $M 1+2$, last section of $M 3+4$ long, equal to or little shorter than penultimate; male genitalia with aedeagus as in figure 350 .

Host/Early Stages. Rosaceae, known from genera Potentilla, Rubus, and Sanguisorba. Larva forming linear blotch mine (fig. 351, A), with frass in 2 rows; puparium reddish brown, posterior spiracles each with 6 bulbs (fig. 352); larval mouth hooks as in figure 353.

Distribution. California, Idaho, Washington; new records: Kansas, Louisiana, Texas.

References. Frick, 1959: 357 (as A. rubi Brischke, 1881); Spencer, 1969a: 58 .

-- Mesonotum shining black------A. varifrons Coquillett Synopsis. Frons reddish orange or yellow in front, darkened above; all antennal segments pale, orange or yellowish; mesonotum shining black; legs largely black but knees, tibiae, and tarsi paler; wing length about $2.3 \mathrm{~mm}$, costa extending strongly to vein $M 1+2$; male genitalia with aedeagus as in figure 354 ; cercus (fig. 355) with distinctive arrangement of short, stout bristles on lower, inner corner (Florida).

Host/Early Stages. Celtis laevigata. Larva forming irregular linear blotch mine (fig. 356) externally; posterior spiracles each with 3 bulbs; puparium orange.

Distribution. District of Columbia, Florida, Illinois, Iowa, Pennsylvania; new records: Arkansas, Kansas, Mississippi, Texas.

References. Frick, 1959: 358; Spencer and Stegmaier, 1973: 21; part 2, p. 269.

5 (3). All body bristles yellow---- pallidiseta Malloch 
Synopsis. Head largely yellow but upper half of frons blackish; mesonotum matt gray, scutellum yellow, legs entirely yellow; squama and fringe whitish; wing length $2-3 \mathrm{~mm}$, costa extending to M 1+2; male unknown.

Host/Early Stages. Unknown.

Distribution. District of Columbia; new record:

California, Alameda Co., Berkeley, 1 f., 16.V.15

(M. C. VanDuzee, USNM).

References. Malloch, 1924: 192; Frick, 1959: 356.

A11 body bristles black--

Mesonotum yellow or brownish--_-_-

Mesonotum gray--------

(If arrangement of dorsocentral bristles is considered to be $3+1$, see couplet 34. )

Synopsis. Frons yellow in front, blackish on upper half; antenna yellow, rather large, arista very short; legs, including coxae, bright yellow; $3+1$ or $3+2 \mathrm{dc}$, anterior ones small, sometimes little differentiated from acr; abdomen black; wing length about $2.3 \mathrm{~mm}$, costa ending short $1 \mathrm{y}$ after vein $R$ 4+5; male genitalia with aedeagus as in figures 357,358 .

Host/Early Stages. Ulmus americana (not Celtis as suggested by Frick, 1959: 354). Larva forming initially long, narrow linear mine, which in final instar develops into a blotch (fig. 359);

larval posterior spiracles each with 8-10 bulbs. Distribution. Illinois, Indiana, Iowa, New York, Ohio, Pennsylvania, Virginia; new record:

Arkansas, Hot Springs, empty leaf mines on Ulmus americana, $17 . \mathrm{V} .79$ (KAS).

References. Malloch, 1915b: 13; Frick, 1959: 354; Spencer, 1969a: 38 .

7 (6). 3d antennal segment and mesonotum yellow----------

- - - - diversa Johnson

Synopsis. Frons narrow, equal to width of eye, black, orbit distinctly paler, yellowish; scute1lum largely yellow, legs entirely yellow; abdomen basically yellow but tergites sometimes darkened, almost blackish centrally; squama and fringe yellow; wing length from $2.5 \mathrm{~mm}$ in male to $3 \mathrm{~mm}$ in female; male genitalia with aedeagus as in figure 360 (resembling A. pseudoreptans, fig. 399).

Host/Early Stages. Unknown.

Distribution. Illinois, Indiana, Iowa, Massachusetts, Vermont; new records: New York, North

Carolina, Virginia; Canada.

References. Johnson, 1922: 26; Frick, 1959: 355; Spencer, 1969a: 41 .

3d antennal segment and mesonotum darker, brownish or blackish brown-------- canadensis Malloch

Synopsis. Closely resembling A. diversabut frons, $3 \mathrm{~d}$ antennal segment, and mesoñotum matt blackish brown; somewhat larger, wing length up to $4 \mathrm{~mm}$; male genitalia with aedeagus as in figures 361 , 362 . 
Host/Early Stages. Unconfirmed but certainly Boraginaceae, possibly Cynoglossum officinale (empty blotch mines found in Ontario (KAS)).

Distribution. California; Canada.

References. Malloch, 1913a: 299; Frick, 1959: 354; Spencer, 1969a: 40; 1981: 110 .

8 (2). Costa ending at or shortly after vein $R \quad 4+5-$

Costa continuing strongly to vein $\mathrm{M} 1+2----------$

9 (8). Squamal fringe pale, white or ochreous-----------

Squamal fringe dark-_-_-_- 18

10 (9). 3d antennal segment with conspicuous point or angle at upper corner (fig. 363)--_-_- 11

3d antennal segment essentially rounded------------ 15

11 (10). Wing and at least medial veins pale----------- 12

-- Wing and veins normal

12 (11). Radial veins dark, normal medial veins virtually colorless----Synopsis. Frons projecting above eye; $3 \mathrm{~d}$ antennal segment frequently finely pointed (fig. 363); 4 orbital bristles, upper ors reclinate, lower of 2 ori normally incurved; gena extended at rear, 0.25 height of eye; mesonotum brilliantly shining black, with 3 strong dc and several smaller anterior ones; wing length $2.5-3 \mathrm{~mm}$, radial veins dark, normal, medial veins virtually colorless, costa ending just after end of vein $R \quad 4+5$; squama and fringe silvery; male genitalia with aedeagus (figs. 364, 365) having distinctive curvature of distiphallus complex, basal sclerites bending gradually (not at right angles, contrast A. ambigua, fig. 369) toward distiphallus complex; surstylus angular, with about 5 stout bristles along inner margin, none at corner ( $\mathrm{fig}$. 366). Host/Early Stages. Unconfirmed but certainly Poaceae.

Distribution. Colorado, Utah.

Reference. Part 2, p. 268 .

All veins pale, yellowish---- ambigua Fallén

Synopsis. Closely resembling $A$. utahensis but without contrast between radial and medial veins, all veins pale, yellowish; wing length 2.5$3 \mathrm{~mm}$; 3d antennal segment angular but bluntly so, not finely pointed (fig. 367); male genitalia with aedeagus having medial section bending at obtuse angle to paired tubules of distiphallus ( $f i g$. 368), basal sclerites ( $\mathrm{fig}$. 369) bending at right angle at apical $3 \mathrm{~d}$; surstylus essentially as in $\underline{A}$. utahensis (fig. 366).

Host/Early Stages. In America, reared from Agropyron repens, Hordeum jubatum, Secale cereale, and Trit icum aestivum (Frick, 1959). In Europe, also from Avena sativa. Larva forming broad blotch mine; posterior spiracles widely separated, each with 3 bulbs (see A. nigrella, fig. 384); puparium reddish orange or paler, yellowish brown. 
Distribution. California, Washington; widespread in Europe.

References. Frick, 1959: 356 (as A. niveipennis); Spencer, 1973a: 240 (economic importance); 1981: 107.

13 (11). 1 ors--- tularensis Spencer

Synopsis. Frons conspicuously projecting above eye, 1 ors, 2 ori; parafacial shining black; 3d antennal segment finely pointed (fig. 370); mesonotum brilliantly shining black, 3 strong dc; all veins dark, last and penultimate sections of $M 3+4$ equal; squama and fringe silvery; male genitalia with aedeagus (figs. 371, 372) having distinctive curvature.

Host/Early Stages. Unconfirmed but larva certainly feeding on Poaceae.

Distribution. California.

Reference. Spencer, 1981: 130 .

2 ors--------------------

14 (13). Male genitalia with aedeagus having long, slender tubules and distinctive curvature (figs. 373, 374)----- albertensis Spencer Synopsis. Frons slightly projecting above eye; 2 ors, 2 ori; gena extended at rear, 0.40 height of eye; 3d antennal segment distinctly angulate (fig. 375); mesonotum brilliantly shining black; wing with veins brown, length 2.3-2.8 mm; squama and fringe white.

Host/Early Stages. Unconfirmed but certainly Poaceae.

Distribution. Colorado (new record); Canada. References. Spencer, 1969a: 32; part 2, p. 262. Male genitalia with aedeagus having distal tubules broader, less curving (see couplet 12)

Synopsis. (?) ambigua Fallén ynopsis. Larger species, wing length $2 . \overline{5-3 \mathrm{~mm}}$ resembling $\underline{A}$. ambigua but wing and veins not pale. Host/Early Stages. Unconfirmed but certainly Poaceae.

Distribution. California.

Remarks. With no reared material available, it is uncertain whether this represents a distinct species or possibly merely a darker form of $\underline{A}$. ambigua.

15 (10). Exceptionally large species, wing length from 3.25 $\mathrm{mm}$ in male to $4.1 \mathrm{~mm}$ in female----A. kincaidi Malloch Synopsis. Frons black, slightly projecting above eye in front; normally 5 orbital bristles, more rarely 4 or 6 ; arista distinctly pubescent; gena deep at rear, 0.33 height of eye; all antennal segments black; mesonotum distinctly matt viewed from front, more shining black from rear; legs normally entirely black, foreknee sometimes paler; squama white, fringe variable, whitish, sometimes at least partially ochreous or brownish; wing veins dark; male genitalia with aedeagus as in 
figures 376, 377; distiphallus broad in side view; surstylus essentially as in A. utahensis ( $f$ ig. $366)$.

Host/Early Stages. Unconfirmed, probably Bromus. Larva forming long blotch mine; puparium reddish orange, $3.2 \mathrm{~mm}$ long, posterior spiracles on short stalks, widely separated, each with 3 bulbs.

Distribution. California; new records:(?)

Arkansas, Colorado, Tennessee, Utah.

References. Malloch, 1913a: 285; Spencer, 1969a:

45; 1981: 115; part 2, p. 264.

Smaller species, wing length up to $3.1 \mathrm{~mm}$ in $\mathrm{fe-}$

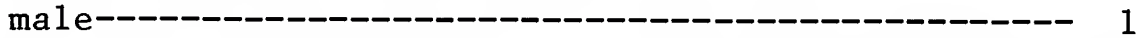

16 (15). Mesonotum entirely matt------A. schlingerella Spencer

Synopsis. Wing length in male $\overline{2} .5 \mathrm{~mm}$; frons distinctly projecting above eye; gena 0.33 height of eye; frons and all antennal segments black; 3 strong dc, 2 smaller anterior ones; squama and fringe pale, whitish; male genitalia with aedeagus as in figures 378,379 .

Host/Early Stages. Unconfirmed, certainly Poaceae. Distribution. California.

Reference. Spencer, 1981: 128.

Mesonotum shining black

17 (16). Last section of vein $M 3+4$ distinctly shorter than penultimate-------------------A. nigrella (Rondani)

Synopsis. Frons matt black or dark brown, distinctly projecting above eye in profile; gena extended at rear, 0.33 height of eye; all antennal segments black, 3d slightly longer than broad, rounded at end (fig. 380); arista moderately pubescent; mesonotum shining black, with up to 5 differentiated postsutural dc, greatly decreasing in size, sometimes 1 or 2 presutural dc; wing (fig. 381) length from $2.5 \mathrm{~mm}$ in male to $3.1 \mathrm{~mm}$ in female; legs entirely black; squama whitish, fringe white to ochreous; male genitalia with aedeagus (figs. 382, 383) having paired distal tubules elongate, distiphallus relatively short in side view (contrast A. kincaidi, fig. 376).

Host/Early Stages. Poaceae, but no hosts recorded in America; in Europe, larva feeding on Avena, Dactylis, Festuca, Glyceria, Holcus, Hordeum, Lolium, Phleum, Poa, Secale, Setaria, Trisetum, Triticum. Leaf mine starts as narrow channel running toward apex of leaf but later develops into broad blotch running downward, with frass largely diffused, giving mine greenish appearance; puparium reddish brown, spiracular processes widely separated, each with 3 bulbs (fig. 384); pupation takes place externally.

Distribution. California, New Mexico; widespread in Europe.

References. Spencer, 1981: 117; part 2, p. 265. Last and penultimate sections of vein $M-3+4$ approx. equal---- aprilina Malloch 
Synopsis. Closely resembles A. nigrella; frons distinctly brownish centrally; $\overline{2 \text { ors, } 3}$ ori; $3 \mathrm{~d}$ antennal segment longer than broad, rounded at end (Malloch, 1915c: fig. 4); mesonotum with 3 strong postsutural dc, normally 2 smaller ones in presutural area; wing length from $2.5 \mathrm{~mm}$ in male to $3.1 \mathrm{~mm}$ in female, last and penultimate sections of M $3+4$ equal; male genitalia with aedeagus (figs. $385,386)$ weakly pigmented, distiphallus relative1y short (contrast A. nigrella, figs. 382, 383). Host/Early Stages. Unknown, certainly Poaceae. Distribution. Illinois, New Hampshire; widespread in Canada.

References. Ma11och, 1915c: 359; Spencer, 1969a: 36; part 2, p. 262.

18 (9). Very large species, wing length $3.25-4.1 \mathrm{~mm}------$

(Squamal fringe normally pale, couplet 15)

Smaller species, wing length up to $2.4 \mathrm{~mm}$

(Costa normally (Costa normally clearly extending to vein $\mathrm{M} 1+2$, couplet 32).

19 (8). Squamal fringe pale, whitish or ochreous----------- 20

$--$ Squamal fringe dark, brown or black-_-_-_-_-_-_-- 31

20 (19).

Legs and all antennal segments entirely black

Synopsis. Frons dark brown or sooty black, at least slightly projecting above eye in profile; all antennal segments black, 3d enlarged, rounded below (fig. 387); mesonotum normally somewhat matt, though more shining black from rear, with up to 7 differentiated dc, 1-4 strong presutural ones sometimes little differentiated from acr; legs normally entirely black, forefemur at most with knee slightly paler; wing length from 2 to 3 $\mathrm{mm}$, last section of $\mathrm{M} 3+4$ about 0.67 penultimate; squama and fringe white; male genitalia with aedeagus as in figures 388,389 .

Host/Early Stages. Poaceae, recorded in America only from Triticum aestivum; in Europe, known on hosts in tribes $\mathrm{Ag} r$ osteae, Aveneae, Festuceae, Hordeae, Panicaeae, and Phalarideae occurring most frequently on Phalaris arundinacea, common also on Hordeum and Poa. The larva forms upper surface mine, which develops into broad blotch; pupation takes place either in leaf or externally, with puparium then loosely glued to leaf; puparium reddish, brown, or black, with posterior spiracles closely adjoining, each with 3 bulbs (fig. 390). Distribution. California, Illinois, Iowa, Massachusetts, Michigan, New York, Ohio (as A. nigripes sensu Frick, 1959); Colorado (new record), doubtlessly present in many other States; Canada, Europe. References. Spencer, 1969a: 32; 1981: 105; part 2, p. 262 . 
Tibia, tarsi, or antennal segments paler, at least slightly yellowish brown--

21 (10). Frons brownish orange-- 21

-- Frons darker, black or dark brown------------------ 23

22 (21). 1 ors-Synopsis. Frons distinctly projecting above eye; 1 reclinate ors, 4 or 5 inclined ori, all slender; gena deeply extended at rear, 0.25-0.33 height of eye; mesonotum shining black, with 2 strong dc and 2 or 3 beyond, differentiated from acr but short; acr numerous, in at least 8 rows; legs with femur black but knee yellowish, tibia and tarsus paler, brownish; squama and fringe silvery white; wing length 2.7-3 mm; male genitalia with aedeagus having long, paired tubules, uniformly curving, fused at base, separating apically (fig. 391). Host/Early Stages. Ambrosia artemisiifolia, probably Artemisia douglasiana (Calif.). Several larvae frequently feeding together to form blackish leaf mine that extends from apex of pinnately divided leaves toward midrib; pupation externally, puparium brown, posterior spiracles each with 3 bulbs; larval mouth parts with 3 teeth (fig. 392). Distribution. California; Canada. References. Spencer, 1969a: 35; 1981: 109.

-- 2 ors-- $\quad$ - virginiensis Spencer Synopsis. Frons not projecting above eye; 2 ors, 3 ori; gena 0.33 height of eye; all antennal segments pale, orange; mesonotum shining black, with 4 postsutural dc, acr in 8 rows; femora black, knees paler, tibiae and tarsi brownish orange; wing with all veins pale; squama whitish yellow, fringe silvery; wing length in male $3 \mathrm{~mm}$, costa extending only weakly to vein $M 1+2$, last section of M $3+4$ short, 0.5 length of penultimate; male genitalia with aedeagus (fig. 393) having distiphallus exceptionally long, paired tubules sinuate, fused to slightly beyond midpoint. Host/Early Stages. Unknown.

Distribution. Virginia.

Reference. Spencer, 1977c: 237.

Remarks. This species generally resembles and is obviously closely related to A. ambrosivora but different arrangement of orbital bristles and longer aedeagus justify treating it as distinct.

23 (21). Mesonotum predominantly matt, blackish gray or

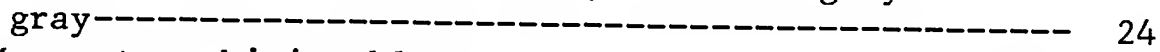

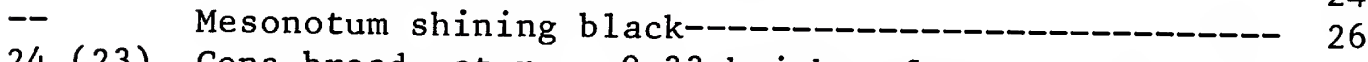

24 (23). Gena broad, at rear 0.33 height of eye--_26

Synopsis. Frons distinctly projecting above eye (fig. 394), about 1.5 times width of eye, with 2 ors and 3 (sometimes 2) ori; gena extended at rear, 0.33 height of eye; $3 \mathrm{~d}$ antennal segment brownish black, sometimes paler, distinctly brown; 
mesonotum blackish gray, with only weak subshine; legs with femora black, foreknee yellowish, tibiae and tarsi yellowish brown; wing veins pale; squama and fringe whitish; male genitalia with aedeagus ending in long tubule, divided only at apex (figs. 395 , 396), with 2 tubules then diverging at right angles.

Host/Early Stages. Urtica spp. 1st instar larva forming narrow leaf mine beside margin and this later develops into broad blotch (fig. 397); larval posterior spiracles each with 3 bulbs (without groups of hairs as in A. pseudoreptans); puparium reddish brown.

Distribution. California, Washington, almost certainly more widespread; Canada, Europe.

References. Spencer, 1969a: 55; 1981: 126, part 2 , p. 267 .

25 (24). Frons 1.5 times width of eye; gena narrow, at most 0.25 height of eye------A. pseudoreptans Nowakowski Synopsis. Head (fig. 398) with frons matt black or more brownish, not projecting above eye; gena exceptionally narrow, 0.08 height of eye in center, up to 0.11 at rear; $3 \mathrm{~d}$ antennal segment normally black, occasionally more brownish; mesonotum with 3 strong dc, grayish black; legs with femora black and yellowish foreknees, tibiae and tarsi paler, brownish yellow; wing length from 2.8 to $3.9 \mathrm{~mm}$ in both sexes, veins pale; squama and fringe silvery yellow; male genitalia with aedeagus (fig. 399) distinctive, asymmetrical (illustrated by Frick, 1952a: 348, as A. reptans).

Host/Early Stages. Urtica spp. Larval mine normally commences along leaf margin, widening into irregular blotch, turning black when old; larval posterior spiracles each with 3 bulbs and 4 patches of hairs (fig. 400); puparium reddish brown.

Distribution. California, Idaho, Oregon, Washington; Canada, Europe.

References. Frick, 1952a (as A. reptans); Spencer, 1969a: 54; 1981: 124.

Remarks. This species was confused by earlier authors with the true $A$. reptans Fallen. They occur together in America and in Europe and both feed on Urtica.

Frons broader, twice width of eye--

-

Synopsis. Frons dark brown, not projecting above eye; 2 ors, 3 ori; gena narrow, 0.17 height of eye; 3d antennal segment small, round, black; mesonotum matt grayish black, with 3 strong postsutural dc; legs brownish, with all knees yellow, tibiae and tarsi conspicuously yellow; squama and fringe silvery white; male genitalia with aedeagus as in figures 401,402 . 
Host/Early Stages. Unknown, possibly in

Boraginaceae.

Distribution. Utah.

Reference. Part 2, p. 263.

26 (23). 3d antennal segment distinctly pubescent

27 (26)

. 3d antennal segment normally black-_-

3d antennal segment paler, brownish---A. tacita Spencer

Synopsis. Frons narrow, less than $1.5^{-}$times width

of eye, not projecting above eye; gena narrow, slightly extended at rear, 0.125 height of eye;

$3 d$ antennal segment large, round, with conspicuous pubescence (fig. 403); mesonotum shining black, with $3 \mathrm{dc}$; wing length $2.5 \mathrm{~mm}$, veins pale; male genitalia with aedeagus as in figures 404, 405; epandrium with 3 short, stout bristles in center, none at either corner (fig. 406).

Host/Early Stages. Poaceae, but species unrecorded. Puparium reddish brown, posterior spiracles adjoining, each with 3 bulbs (fig. 407).

Distribution. Montana (new record); Canada.

References. Spencer, 1969a: 59; part 2, p. 268.

28 (27). Small species, wing length 2.3-2.6 mm-

Synopsis. bispinata Spencer

Synopsis. Frons black, 1.5 times width of eye, not projecting above eye in profile; 2 ors, 2 ori; gena extended at rear, up to 0.33 height of eye; $3 \mathrm{~d}$ antennal segment round, rather large, black, with distinct fringe of pubescence; mesonotum shining black, with $3 \mathrm{dc}, 3 \mathrm{~d}$ small; legs with femora black, tibiae and tarsi paler, brownish; squama and fringe white; male genitalia with aedeagus as in figures 408, 409; epandrium with 2 strong bristles (fig. 410).

Host/Early Stages. Certainly Poaceae but unconfirmed.

Distribution. New records: Georgia, Maryland, New

York, North Carolina, Utah, Virginia; Canada.

References. Spencer, 1969a: 39; part 2, p. 263.

Larger species, wing length $2.7-3.2 \mathrm{~mm}-$

Synopsis. Agreeing closely with A. Bispinata but slightly larger; squama pale or (in Canada) somewhat darker; male genitalia with aedeagus as in figures 411, 412; epandrium with row of bristles, normally 4, internal ones shorter (fig. 413).

Host/Early Stages. Certainly Poaceae but unconfirmed.

Distribution. Colorado; new records: Texas, Utah; widespread in Canada.

References. Spencer, 1969a: 44; part 2, p. 264.

29 (28). 3d antennal segment rounded, slightly longer than broad-----

$3 \mathrm{~d}$ antennal segment rounded below, almost angu-

late at upper corner (fig. 414)-_-

- A. pagana Spencer, n. sp. 
Synopsis. Frons black, not projecting above eye; gena 0.25 height of eye at rear; 3d antennal segment (fig. 414) largely black but paler, brownish on inside; mesonotum brilliantly shining black; wing length in male $2.4 \mathrm{~mm}$; male genitalia with aedeagus having distal tubules narrow with distinctive constriction near apex (figs. 415, 416). Host/Early Stages. Unconfirmed but certainly Poaceae.

Distribution. Kansas .

Reference. Part 2, p. 265.

30 (29). 3d antennal segment largely brown---A. parvicornis Loew Synopsis. Frons brown or brownish black, broad, almost twice width of eye, distinctly projecting above eye anteriorly; 2 strong reclinate ors, 2 weaker incurved ori; gena extended at rear, 0.33 height of eye; $3 d$ antennal segment round, virtually bare, pale brown; mesonotum shining black, with 4 or 5 dc greatly decreasing in size; legs black, foreknee paler, all tarsi distinctly brownish; wing base, squama, and fringe yellowish white; wing length $2.5-3 \mathrm{~mm}$; male genitalia with aedeagus short, broad, distiphallus uniformly rounded (figs. 417, 418); surstylus (fig. 419) with group of 12-15 short, stout bristles.

Host/Early Stages. Echinochloa crusgalli, Panicum miliaceum, Zea mays, probably other cultivated cereals and wild grasses. Larva forms linear blotch mine running down leaf; as many as 4 generations have been confirmed; puparium reddish brown, with posterior spiracles on small subsidiary projection arising from large protuberance, 2 processes slightly separated, each with 3 bulbs (fig. 420).

Distribution. Widespread, present in most States (Frick, 1959); Canada.

References. Phillips, 1914 (biology); Frick, 1959: 357; Spencer, 1969a: 51; 1973a: 252 (economic importance).

$3 \mathrm{~d}$ antennal segment largely black--- A. proxima Spencer Synopsis. Closely resembling A. parvicornis but 3d antennal segment and frons generally darker; wing length $2.4-2.7 \mathrm{~mm}$; male genitalia with aedeagus (figs. 421, 422) having distal tubules narrow and relatively long (contrast $\underline{A}$. parvicornis, figs. 417, 418).

Host/Early Stages. Echinochloa walteri, Panicum dichotomiflorum, almost certainly other grasses. Puparium reddish brown, posterior spiracles closely adjoining on conspicuous protuberance, each with 3 bulbs (fig. 423).

Distribution. Florida, New York; Canada.

References. Spencer, 1969a: 53; Spencer and Stegmaier, 1973: 19.

31 (19). Gena broad, 0.25 height of eye-- 
Gena narrower, 0.17 height of eye--_-_-_--_---_-

A. parca Spencer, n. sp.

Synopsis. Closely resembling A. parilis but 3d antennal segment entirely black, smaller, round; arista distinctly pubescent; 3 differentiated dc; squama pale gray, margin and fringe dark; wing length $2.5 \mathrm{~mm}$, costa extending strongly to vein M 1+2; male genitalia with aedeagus (figs. 424, 425) having distal tubules pale.

Host/Early Stages. Unconfirmed but certainly Poaceae.

Distribution. North Carolina, Tennessee. Reference. Part 2, p. 265.

32 (31). Antennal segments partially pale, brownish---------

Synopsis. parilis Spencer, n. sp. slightly projecting above eye; 2 ors and 2 ori; 3d antennal segment slightly longer than broad, largely black but paler below; arista bare; mesonotum shining black, with 2 strong dc; squama pale gray, margin and fringe brownish black; wing length in male $2.4 \mathrm{~mm}$, costa extending strongly to vein $M 1+2$; male genitalia with aedeagus (figs. 426,427 ) having distal tubules strongly pigmented. Host/Early Stages. Unconfirmed but certainly Poaceae.

Distribution. Tennessee.

Reference. Part 2, p. 266.

Antennae entirely black------A. pudica spencer, n. sp. (If costa appearing to end at vein $R 4+5$, see couplet 18 .)

Synopsis: Frons narrow, equal to width of eye, not projecting above eye; 2 ors, 3 ori; mesonotum shining black, with 3 strong postsutural dc; wing length $2.2-2.4 \mathrm{~mm}$, costa extending weakly to vein M $1+2$, last section of M $3+4$ shorter than penultimate; male genitalia with aedeagus as in figures 428,429 ; sperm pump with long narrow stalk and small blade.

Host/Early Stages. Unknown.

Distribution. Arkansas, Georgia, Minnesota, North Carolina.

Reference. Part 2, p. 267.

33 (1). Costa ending at or shortly after vein $R \quad 4+5-$

Synopsis. Head (fig. 430) with frontella (Rondani) frons reddish, sometimes more black at rear, broad, twice width of eye, conspicuously projecting above eye in profile; gena deeply extended at rear, up to 0.5 height of eye; 3d antennal segment rounded, slightly longer than broad, with conspicuous fringe of pubescence in male, pubescent only at upper corner in female; mesonotum with $3+1$ strong dc, blackish, distinctly shining; legs with femora black and yellow knees, tibiae and tarsi slightly paler, brownish; squama pale gray, fringe ochreous 
to brownish; wing length 1.9-2.2 mm (fig. 431) with last section of $M 3+4$ equal to or slightly longer than penultimate; male genitalia with aedeagus as in figures 432, 433.

Host/Early Stages. Medicago spp., particularly M. sativa, less frequently Melilotus and Trifolium. Larva forms characteristic linear blotch mine (fig. 434), cephalopharyngeal skeleton (fig. 435) with mouth hooks having 2 strong teeth and rudimentary $3 \mathrm{~d}$ one below; puparium reddish brown, posterior spiracles each with 3 bulbs (fig. 436). Distribution. Massachusetts, New Jersey, New York; widespread in Europe.

References. Steyska1, 1972a; Spencer, 1973a: 85; Guppy, 1981: 593.

34 (33). 3d antennal segment yellow--------A. aristata Malloch (Arrangement of dorsocentral bristles normally $3+2$, see couplet 6. ) 3d antennal segment black or dark brown---------- 35

35 (34). Squamal fringe pale, whitish---_--_--_-_-_-_---- 36

-- Squamal fringe dark, brown or black------------- 39

36 (35). Frons dark, brownish black------- A. isolata Malloch Synopsis. Frons dark, brownish black; gena narrow, at rear 0.125 height of eye; 3d antennal segment sma11, round, black, 1 st and $2 \mathrm{~d}$ paler, more brownish; mesonotum blackish gray, presutural dc only slightly weaker than $3 \mathrm{~d}$; legs with femora largely black, tibiae and tarsi paler yellowish; squama and fringe yellowish; wing length 2.4-3.1 mm; male genitalia with aedeagus as in figures 437, 438. Host/Early Stages. Populus spp., Salix spp. Larva forming large, irregular blackish blotch, pupating externally; puparium reddish brown, posterior spiracles each with 3 bulbs.

Distribution. California, Pennsylvania (as A. albitarsus, Frick, 1959: 353); new records: Colorado, Clear Creek Co., Mt. Evans, Doolittle Ranch, 9,800 ft, 1 m., 1 f., 3.VIII.61 (J. G. Chillcott and C. H. Mann, CNC); Minnesota, Hennepin Co., Minneapolis, Minnehaha Creek, empty leaf mines on Populus deltoides, 10.IX.78 (KAS); Washington; Canada.

References. Spencer, 1969a: 52 (as A. populoides);

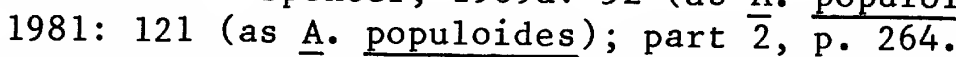

37 (36). Mesonotum shining black

Mesonotum matt gray-Japanagromyza rutiliceps (Melander)

38 (37). Small species, wing length about $2.3 \mathrm{~mm}$ in female--

Synopsis Frons reddish, ori; 3d antennal mesonotum gray, 3+1 strong dc; legs black, only foreknee narrowly paler; squama and fringe white; 
wing with costa extending to vein $M 1+2$, last and penultimate sections of $M 3+4$ equal.

Host/Early Stages. Unknown.

Distribution. Wyoming.

Reference. Part 2, p. 270.

Larger species, wing length in male $3 \mathrm{~mm}$

A. nevadensis Spencer

ecting above eye, upper orbit black; 2 ors, 2 or 3 ori; $3 d$ antennal segment pale, brownish, darker on outside; gena extended at rear, 0.40 height of eye; presutural dc strong, equal to $3 \mathrm{~d}$; legs black but all knees distinctly yellow; squama whitish or pale gray, fringe ochreous; male genitalia with aedeagus as in figures 439,440 .

Host/Early Stages. Unknown.

Distribution. California.

Reference. Spencer, 1981: 116.

Remarks. The male genitalia show this species to be closely related to A. nearctica Sehgal (1971: fig. 10), but in A. nearctica the costa extends only to vein $R 4+\overline{5}$.

39 (35). Frons predominantly black---_--- 40

-- Frons brown------_---_--- 42

40 (39). Midtibia with strong lateral bristles------------

Synopsis. Small dark species, wing length 2.3-2.5 $\mathrm{mm}$, essentially as in A. potentillae (couplet 41); male genitalia with aedeagus (figs. 441, 442) ending in 2 discrete sclerotized processes; sperm pump (fig. 443) with narrow blade.

Host/Early Stages. Unknown.

Distribution. North Carolina (new record); Canada. References. Spencer, 1969a: 60; part 2, p. 269.

-- Midtibia without differentiated bristle-

41 (40). Male genitalia with aedeagus ( $\mathrm{fig}$. 445) ending in

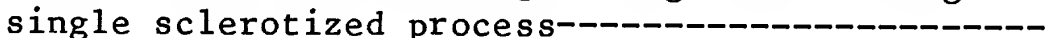

A. potentillae (Ka1tenbach)

Synopsis. 3d antennal segment sma11, round, with distinct pubescence at upper corner (fig. 444); gena extended at rear, 0.20 height of eye; mesonotum blackish gray, with $3+1 \mathrm{dc}$; legs largely black, foreknee occasionally paler, tibiae and tarsi generally dark; squama gray, margin and fringe black; wing length 2.2-3 mm; male genitalia with aedeagus (fig. 445) having long membranous gap between pigmented distiphallus and basal sclerites; sperm pump (fig. 446) minute with narrow blade.

Host/Early Stages. Only recorded host in America Potentilla gracilis, known on many genera of Rosoideae in Europe. Larva with 2 equal teeth on mouth parts (fig. 447), forming linear blotch mine (fig. 448); puparium reddish brown, posterior spiracles each with 3 bulbs. 
Distribution. California; new records: Colorado, Utah; widespread in Canada, Europe; Japan.

References. Frick (1959: 358, as A. spiraeae); Spencer, 1969a: 55 (as A. spiraeae); 1981: 122; part 2, p. 266.

Male genitalia with aedeagus (figs. 449, 450) ending in 2 discrete sclerotized processes-

Synopinalina Sehgal

Synopsis. Sma11 dark species, wing $\bar{l}$ ength up to $2.5 \mathrm{~mm}$; male genitalia with aedeagus (figs. 449, 450) having distal process enlarged, only short gap between distiphallus and well-defined U-shaped process behind; sperm pump (fig. 451) with large blade.

Host/Early Stages. Unknown.

Distribution. Utah (new.record); Canada.

References. Sehgal, 1968: 59; Spencer, 1969a: 48; part 2, p. 265.

42 (39). Frons pale brown or almost reddish----A. leechi spencer Synopsis. Agreeing closely with A. potentillae but frons distinctly paler; wing length up to $2.5 \mathrm{~mm}$; male genitalia with aedeagus (figs. 452, 453) having distiphallus at right angles to basal sclerites; sperm pump minute.

Host/Early Stages. Unknown.

Distribution. N. California; Alaska, Canada. References. Spencer, 1969a: 47; 1981: 115.

Frons dark brown-- fragariae Malloch

Synopsis. Small black species, wing length about $2.4 \mathrm{~mm}$; agreeing closely with A. isolata, but tibiae and tarsi darker, brown $\bar{i}$ sh black; male genitalia with aedeagus (figs. 454, 455) having single area of pigmentation distally; sperm pump (fig. 456) greatly enlarged.

Host/Early Stages. Fragaria spp. Larva forming linear blotch mine (fig. 457); (details of puparium not recorded).

Distribution. California, certainly more widespread; Canada.

References. Malloch, 1913a: 307; Frick, 1959: 358 (as A. spiraeae); Spencer, 1969a: 42; 1981: 42.

\section{SUBFAMILY PHYTOMYZ INAE}

Genus Phytobia Lioy

Phytobia Lioy, 1864: 1313. Type of genus: Agromyza errans Meigen, 1830 (Europe).

Dizygomyza (Dendromyza) Hende1, 1931: 22.
Shizukoa Sasakawa, 1963a (synonymy established by Spencer, 1965d).

Essential characters of this genus are the erect or reclinate orbital setulae, strongly developed, presutural, dorsocentral prescutellars, and vein $R \quad 4+5$ ending nearer the wingtip than $M 1+2$. The frons is normally dark but sometimes reddish and the 
lunule often silvery. The venation is variable, with the costa ending at $R 4+5$ or extending to $M 1+2$, and the outer crossvein is present or lacking. The subcosta normally runs parallel to vein $R 1$ directly to the costa (fig. 5), but in some species appears to join $\mathrm{R} 1$, as in Agromyza (fig. 4). Such specimens can be misidentified as Agromyza species, and Sasakawa (1963a) even erected the genus Shizukoa owing to the difficulty of detecting the correct generic position in such instances.

When Tschirnhaus (1971) discovered the stridulating mechanism in both sexes in Agromyza ( $p .57$ ), he also established that this is lacking in Phytobia, and this provides a reliable means of separating the genera when in doubt.

Phytobia in its present concept is restricted to the species whose larvae bore in the cambium of twigs or trunks of trees. Of the 15 species known in the United States, the hosts of only 5 have been established: Acer, Amelanchier, Betula, Crataegus, and Prunus. Greene (1914, 1917) discussed the biology in some detail of three species and also recorded finding larvae in Ilex monticola and Quercus rubra. It is virtually certain that Populus and Salix are additional hosts of American species. $\underline{P}$. flavohumeralis was described from an area in Alberta, where the dominant tree was Populus balsamifera, which was probably the host. A single specimen caught on Salix in New York is included in the key here, but it is not formally described pending discovery of sufficient material for accurate diagnosis.

A detailed account of 1 arval feeding in Prunus was given by Grossenbacher (1915), and he also discussed his rearing technique. Other hosts are known in Europe, with species recorded from Alnus, Malus, Populus, Salix, and Sorbus, and in Canada a specimen has also been reared from Fraxinus.

Larval feeding damages wood subsequent- ly used commercially, causing so-called "pith flecks." These were illustrated by Spencer (1973a: pl. 9) when discussing the economic importance of the genus.

Phytobia is widely distributed in all faunal regions, with a total of 50 species. Striking species with pictured wings are known in Brazil (Spencer, 1966e), and a key to 11 species in the Neotropical Region was given by Spencer and Stegmaier (1973: 174). Eight species have been recorded in Europe (Spencer, 1976a: 147), eight in the Papuan subregion (Sasakawa, 1963b, as Shizukoa), and nine in Australia (Spencer, 1977d: 122). Remarkably, four species are present on Lord Howe Island, but the genus appears not to have reached New Zealand.

Phytobia is generally accepted as one of the most primitive genera in the family. Süss and Müller-Stoll (1975) described Palaeophytobia platani from larval feeding tracks in wood identified as Platanoxylon sp. in Hungary from Upper Miocene, but no modern species is known in platanus. Sïss (1979) also described a new species, Protophytobia cupressorum, from the Netherlands from feeding tracks in wood of Tertiary age considered to belong to the Cupressaceae, identified as Juniperoxylon silesiacum (Pril1) Krausel. Similar feeding tracks are recorded from modern Callitris (Cupressaceae) in Australia, and references are given to the possible feeding of Phytobia spp. in Abies, Cunninghamia, Cupressus, Juniperus, Picea, and Pinus. It must, however, be emphasized that there may be difficulty in establishing even the order of insect producing feeding tracks in wood, particularly when a fossil. An insect identified as a Phytobia sp. has been found feeding in Nothofagus in Chile, but it was subsequently established that the species was a microlepidopteron in the family Opostegidae. It is therefore to be hoped that every effort will be 
made to confirm the presence of Phytobia on Coniferae by rearing adults or at least discovering larvae or larval remains.

One new species, described here from Arizona, is of interest as the only species in the United States with a yellow scutellum, which associates it with a number of others with this character in Central America.

One species previously known in Canada has been identified from Utah, and one new synonym is established (part 2, p. 270). No additional species are known in Canada.
Key to Phytobia Species
1 .

$--$

$3(2)$.

$-$

4(3).

2 (1). Costa extending only to vein $\mathrm{R} 4+5---$

Scutellum dark, black or gray--_-_-_-_-_-_--- 2

Scutellum yellow------P. pallida Spencer, n. sp.

Synopsis. Frons black, lunule silvery; mesonotum broadly yellow centrally, scutellum entirely ye1low; side of thorax largely yellow; legs black; abdomen predominantly yellow but hindtergites blackish centrally; squama yellow, margin and fringe black; male genitalia with aedeagus as in figures $458,459$. Host/Early Stages. Unknown. Distribution. Arizona. Reference. Part 2, p. 271. Costa extending to M 1+2--_- 8

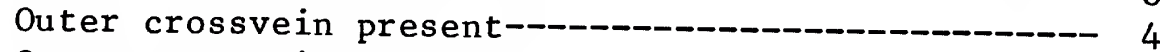
Outer crossvein lacking-- 7

Last section of M $3+41.5$ times length of penultimate------

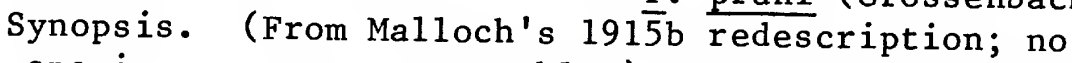
specimens are now traceable.) Frons somewhat wider than eye, not projecting above eye; 2 ors, 3 ori; gena about 0.17 height of eye; $3 \mathrm{~d}$ and $4 \mathrm{th} \mathrm{dc}$ small, anterior pair well in front of suture, prsc half length of lst dc; frons blackish, becoming brown in front, there similar to $3 \mathrm{~d}$ antennal segment and palpus; wing length $3.5-4 \mathrm{~mm}$, costa ending shortly after vein $R 4+5$, last section of M $1+21.5$ times penultimate; inner crossvein well beyond center of discal cell, little more than own length from outer; male genitalia unknown.

Host/Early Stages. Crataegus sp., Prunus avium, $P$. domestica. Larva feeding in cambium of previous year's growth of stem, pupating externally near ground level; eggs laid in mid-June, larvae feed until autumn, overwintering in stem, completing feeding the following spring.

Distribution. New York.

References. Grossenbacher, 1915: 227-238; Malloch, 1915c: 349; Frick, 1959: 376.

Last section of $M 3+42.5$ times length of penultimate----------------------------------- 5

5 (4). Frons not projecting above eye--P. indecora (Malloch)

Synopsis. Frons matt black, 1.5 times width of eye; orbit only weakly shining, with 2 ors, 3 ori; lunule gray; gena narrow, $0.125-0.10$ height of 
eye; $3+1$ strong $d c$, prsc equal to dc 3 ; mesonotum deep black, moderately shining; pleura and legs black; wing length $2.6-3.25 \mathrm{~mm}$, last section of M $3+4$ about twice length of penultimate, discal cell thus small; male genitalia with aedeagus as in figure 460 .

Host/Early Stages. Unknown.

Distribution. Illinois.

References. Ma1loch, 1918a: 132; Frick, 1959: 375.

Frons strongly projecting above eye---------------

Orbit and parafacial only moderately shining

----- vanduzeei Spencer

Synopsis. Frons broad, almost twice width of eye; 2 ors, 4 ori; gena angular, 0.25 height of eye at rear; 3d antennal segment rounded, only slight1y longer than broad; mouth margin normal, without epistoma; $3+1$ strong dc, prsc strong; wing length in male $3 \mathrm{~mm}$, last section of $M 3+4$ twice length of penultimate; frons dark brown, orbit more black, moderately shining; antennal segments dark brown, almost black; legs entirely black; squama gray, margin and fringe black; male genitalia with aedeagus having distiphallus narrow (fig. 461); bladder at base of distiphallus only partially divided; basal sclerites entirely fused ( $f i g$. 462).

Host/Ear1y Stages. Unknown.

Distribution. California; new record: Colorado, Boulder, 1 f., 8.VII.49 (L. D. Beamer, UKaL). Reference. Spencer, 1981: 140.

Orbit and parafacial brilliantly shining----------

------- confessa Spencer

Synopsis. Closely resembling $P$. vanduzeei but on $1 \mathrm{y}$ 3 ori; gena somewhat narrower, 0.33 to 0.20 height of eye; frons and all antennal segments black; wing length from $3.3 \mathrm{~mm}$ in male to $3.5 \mathrm{~mm}$ in female; male genitalia with aedeagus having distiphallus broad in ventral view; bladder at base of distiphallus clearly divided (contrast $\underline{P}$. indecora, fig. 460); 2 basal sclerites discrete (figs. 463, 464).

Host/Early Stages. Unknown.

Distribution. Utah (new record); Canada.

References. Spencer, 1969a: 105; part 2, p. 271.

7 (3). 2 ors, 2 ori-- powelli Spencer Synopsis. Costa ending at vein $R 4+5$, outer crossvein lacking; small species, wing length 2.2-2.3 $\mathrm{mm}$; frons twice width of eye, not projecting above eye in profile, black, with brownish undertone; lunule slightly grayish, orbit and cheeks distinctly shining; gena 0.20 height of eye; $3+1$ strong dc; mesonotum, scutellum, and pleura shining black; squama dark gray, margin and fringe black; male genitalia with aedeagus as in figures $465,466$.

Host/Early Stages. Unknown. 
Distribution. California.

Reference. Spencer, 1981: 137.

2 ors, 4 ori--

Synopsis. Costa extending to just beyond apex of

vein $R 4+5$, outer crossvein lacking; wing length

from $2.5 \mathrm{~mm}$ in male to $2.75 \mathrm{~mm}$ in female; frons

1.5 times width of eye; orbit distinctly project-

ing above eye, increasingly so toward antennae;

gena 0.20 height of eye; frons matt black, orbit

and parafacial distinctly shining; mesonotum bri1-

liantly shining black; squama gray, margin and

fringe black; male genitalia with aedeagus as in

figures 467,468 .

Host/Early Stages. Prunus 1yonii, almost certainly

other Prunus spp. Early stages unknown.

Distribution. California.

Reference. Spencer, 1981: 138.

8 (2). Humerus yellow--

Synopsis. Frons grayish black, equal to width of

eye, at least slightly projecting above eye; 2

ors, 2 ori; gena 0.125 height of eye, slightly

yellowish; antennae entirely black; mesonotum matt, grayish black; humerus and upper margin of mesopleuron du11 yellow; legs black, femora slightly yellow distally; wing length in male 2.8-3.1 mm (female unknown), costa extending strongly to vein $M 1+2,1$ ast and penultimate sections of M 3+4 equal; male genitalia with aedeagus as in figures 469,470 , with 2 distinctive patches of black pigmentation at end of distipha1lus.

Host/Early Stages. Unknown.

Distribution. California; widespread in Canada.

References. Sehga1, 1968: 62; Spencer, 1969a: 106; 1981: 136 .

$\begin{array}{rlr}-- & \text { Humerus dark- } & 9 \\ -- & \text { Frons and antennae reddish orange-- } & 10 \\ - & \text { Frons and antennae predominantly dark- }\end{array}$

10 (9). Palpus yellow; small species, wing length in male $2.5 \mathrm{~mm}----$ pruinosa (Coquillett)

Synopsis. Frons reddish brown, twice width of eye, strongly projecting above eye in profile; 5 orbital bristles, only upper ors reclinate, others incurved; face and gena reddish yellow; epistoma 0.5 height of that in $P$. betulivora (see fig. 473); mesonotum grayish $\bar{b} 1 \mathrm{ack}$, with $3+1$ strong dc, only about 3 rows of acr, prsc distinct; legs reddish yellow but base of femora, apex of tibiae, and entire tarsi brown; last and penultimate sections of vein $M 3+4$ equal, inner crossvein at midpoint of discal cell; male genitalia with aedeagus having distal tubules parallel, not diverging (fig. 471). Host/Early Stages. Unknown (misidentified as

Betula nigra by Greene, 1914).

Distribution. Colorado.

References. Ma1loch, 1913a: 291; Frick, 1959: 376. 
Palpus reddish orange; larger species, wing length in male over $4 \mathrm{~mm}-\cdots$

Synopsis. Frons broad, twice width of eye, conspicuously projecting above eye in profile (fig. 472); 5 or 6 long, slender orbital bristles, only upper ors reclinate, others incurved; gena broad, 0.25 height of eye; oral margin with conspicuous epistoma (fig. 473); 3d antennal segment quadrate; arista with small but distinct swelling at base; frons predominantly reddish orange, including orbit; upper orbit and area adjoining ocellar triangle blackish gray; lunule, gena, parafacial, and all antennal segments reddish orange; legs blackish brown, all knees paler, more orange; squama pale gray, margin and fringe black; last section of vein $M 3+4$ at least 1.5 times length of penultimate, inner crossvein beyond center of discal cell; male genitalia with aedeagus having long, paired distal tubules (fig. 474).

Host/Early Stages. Betula nigra. Larva mining in cambium of twig and trunk down to root, mine can be as long as $40 \mathrm{ft}$; pupation takes place externally, larva leaving tree at or below ground leve 1; puparium reddish brown, $4-5 \mathrm{~mm}$ in length, posterior spiracles each with 3 bulbs (fig. 475). Distribution. District of Columbia, Illinois, New York; new record: Kansas, Douglas Co., Natural History Reserve, Lawrence, $1 \mathrm{~m} ., 28 . \mathrm{V} .56$ (J. G. Chillcott, CNC); Canada.

References. Greene, 1914: 471 (biology, as Agromyza pruinosa); Spencer, 1969a: 103.

11 (9). 2 crossveins approximate, outer crossvein less than own length from inner (fig. 476)--_-

-

Synopsis. Large species, wing length $\overline{4 \mathrm{~mm}, 1}$ ast section of $M 3+4$ slightly longer than penultimate; frons broad, twice width of eye, only slightly projecting above eye in area of ori; 2 ors, 3 ori; gena 0.17 height of eye; epistoma lacking; frons and all antennal segments entirely black, lunule slightly paler; mesonotum blackish gray, side of thorax entirely black; squama brownish gray, margin and fringe black; male genitalia with aedeagus membranous, as in figures 477,478 .

Host/Early Stages. Unknown.

Distribution. Iowa, New York; new records: North Carolina, South Carolina; Canada.

References. Malloch, 1913a: 303; Frick, 1959: 377;

Spencer, 1969a: 109; part 2, p. 272.

12 (11). 3d antennal segment and frons black-_-_- 13

3d antennal segment brownish, frons normally at

least faintly reddish adjoining lunule-_-_--- 15

13 (12). Mesonotum matt, grayish black-14

-- Mesonotum deeper black------P. californica Spencer Synopsis. Frons 1.5 times width of eye, not pro- 
jecting above eye in profile; 2 ors, 2 ori (sometimes 3); gena narrow, 0.125 height of eye; epistoma lacking; $3+1$ strong dc; frons matt, sooty black; ocellar triangle and orbit weakly shining, lunule silvery; antennae black; mesonotum and scutellum blackish, weakly shining; squama gray, margin and fringe deep black; last and penultimate sections of vein $M 3+4$ variable, but last slightly longer or at most 1.5 times as long; male genitalia with aedeagus as in figures 479,480 , distiphallus strongly pigmented; sperm pump with large blade and strongly developed base. Host/Early Stages. Unknown, possibly Salix. Distribution. California. Reference. Spencer, 1981: 134.

14 (13). Small species, wing length 2.5-2.6 mm-----------

Synopsis. Agreeing closely with $\frac{\bar{P}}{\mathrm{P}}$. calyptrata (Hen mesonotum more grayish; male genitalia with aedeagus (figs. 481, 482) having distiphallus weakly pigmented.

Host/Early Stages. Unknown, possibly Salix. Distribution. Illinois, Maryland; Canada. Reference. Part 2, p. 270.

-- Large species, wing length in female $3.75 \mathrm{~mm}-$

---

Synopsis. Agreeing closely with $\underline{P}$. setosā but al $\bar{l}$ antennal segments deep black, frons also uniformly black; male genitalia unconfirmed, possibly as in figure 483.

Host/Early Stages. Almost certainly Salix spp. Distribution. New York, certainly more widespread. Reference. Part 2, p. 272.

15 (12). Normally 4 orbital bristles, 2 ors reclinate-------

Synopsis. Frons matt or at most slightly projecting above eye in profile, sooty black but normally at least faintly reddish adjoining lunule, this large, silvery or frequently suffused with reddish; 3d antennal segment reddish brown, never entirely black; orbital bristles strong, normally 2 ors and 2 ori, occasionally a $3 \mathrm{~d}$ ori on one side, more rarely on both; mouth margin with only narrow epistoma; mesonotum ash gray, with $3+1$ strong dc, prsc strong; legs largely black, knees only slightly paler; male genitalia with aedeagus having long paired distal tubules, only slightly diverging, virtually straight in side view (figs. 484, 485, holotype).

Host/Early Stages. Acer rubrum, probably other Acer spp. Larva mining in trunk and root, leaving tree to pupate at ground level; larva full grown in July and August (Virginia), overwintering as puparium, adults emerging in April or May; length of larva 15-17 mm; puparium pale yellow, cylindrical, posterior spiracles each with 3 bulbs (fig. 486, A, B ). 
Distribution. Widespread in north from Wisconsin to New York, most southerly record Virginia;

Canada.

References. Greene, 1917: 313 (Agromyza as aceris); Frick, 1957b: 202 (synonymy of aceris Greene with P. setosa); Spencer, 1969a: 107 .

5 orbital bristles, only upper reclinate, others incurved---

Synopsis. Closely resembling $\overline{\mathrm{p}}$. Setosa but smaller, wing length $3-4.3 \mathrm{~mm}$; orbital bristles more slender; lunule smaller; epistoma more pronounced, approaching form present in $\underline{P}$. betulivora (see fig. 473); male genitalia with aedeagus having long paired tubules (fig. 487).

Host/Early Stages. Amelanchier canadensis. Larva boring in cambium of trunk down to roots, leaving "threadlike reddish line in cambium"; larvae full grown in West Virginia in April, in Great Smoky Mts., Tennessee, North Carolina in July; puparium pale yellow, posterior spiracles slightly more slender than in $P$. setosa, each with 3 bulbs ( $f i g$. 488, $A, \underline{B}$ ).

Distribution. Massachusetts, Michigan, North Carolina, Tennessee, Washington, West Virginia (Frick, 1959); widespread in Canada.

References. Greene, 1917: 316 (biology); Frick, 1959: 375; Spencer, 1969a: 102.

Genus Amauromyza Hendel

Dizygomyza (Amauromyza) Hende1, 1931: 39. Type of subgenus (now genus): Agromyza lamii Kaltenbach, 1858 (Europe) (see part 2, p. 272). Amauromyza Nowakowski, 1962: 97.

Subgenera:

Cephalomyza Hendel, 1931: 32. Type of subgenus: Dizygomyza luteiceps Hendel, 1920 (Europe).

Catalpomyza Spencer, 1977c: 242. Type of subgenus: Agromyza pleuralis Malloch, 1914b (USA).

Annimyzella Spencer, 1981: 144. Type of subgenus: Agromyza maculosa Ma1loch, 1913a (USA).

Hendel's concept of this genus (originally as a subgenus) was based primarily on the halter being black. However, in many species with the halter white or yellow, such as
Amauromyza auriceps (fig. 518), the male genitalia are of the same distinctive form as in black species, such as A. abnormalis (fig. 489), and in all species the sperm pump has a characteristically enlarged, bowlshaped base (figs. 502, 509). With this expanded concept, over 40 species are known throughout the world; 16 species have been recorded in Europe and 2 have reached Australia (Spencer, 1977d). Twenty species are recorded in the United States. Two others are known in Alberta, Canada, one having been reared from Shepherdia (E1 aeagnaceae) (Sehgal, 1971).

Essential characters of the genus are costa extending to vein $\mathrm{M} 1+2$, which ends nearest the wingtip; orbital setulae reclinate or inclined (never proclinate). Normally there is a strongly developed presutural dorsocentral, but this may sometimes be weak or even lacking, as in Amauromyza pleuralis. 
Three subgenera are recognized here, based largely on genitalia characters (see key). The largest is Cephalomyza, with 17 named species, of which 9 were recently described in California and 2 other new species are described here (part 2, p. 273). A new subgenus, Catalpomyza, was erected by Spencer $(1977 c)$ for the isolated species feeding on Catalpa. Another subgenus, Annimyzella, was erected by Spencer (1981) to embrace the nearctic and neotropical species A. maculosa, which is common on many genera of Asteraceae, and a distinctive new species known only in California, A. lathyroides, feeding on Lathyrus. The latter possibly deserves separate subgeneric status but is included provisionally in Annimyze1la.

The larvae in this genus either form large blotch mines or feed internally in stems. The host is known only of 5 of the 20 recorded species, in the families Amaranthaceae, Asteraceae, Bignoniaceae, Caryophyllaceae, and Fabaceae. The small proportion with known hosts suggests that most of those of unknown biology are internal feeders rather than leaf miners.

Two new combinations are established here. Three other species are known in Canada (Spencer, 1969a; Sehgal, 1971).
Key to Amauromyza Subgenera
1. Male genitalia with ejaculatory duct enlarged, in form of paired tubules (figs. 493, 494, 512, 513)---

- Annimyze1la Spencer Male genitalia with ejaculatory duct reduced, without such large tubules-----

2 (1). Distiphallus with associated spiculate membrane of varying form (figs. 489-491, 498-500, 504, 516-527, $529,532,533,535,536)----------C e$ phalomyza Hendel Distiphallus largely membranous, without any spiculate membrane (figs. 506, 507)------Catalpomyza Spencer Note. The species now known in the United States are included in the single key here based on artificial external characters, which may be without subgeneric significance. 
Key to Amauromyza Subgenera Species
1 .

$-$

$2(1)$

Halter entirely black-----

Halter at least partially pale, white or yellow---- 3

Frons strongly projecting above eye in profile-----

- - - - (Cephalomyza) abnormalis (Malloch)

Synopsis. Frons and orbit strongly projecting above eye, matt black; 2 ors, 3 ori; gena angular, deeply extended at rear, up to 0.5 height of eye; mesonotum dull black with only faint subshine, $3+1$ $\mathrm{dc}$, acr irregularly in 4 rows; pleura entirely black, moderately shining; legs black; squama gray, margin and fringe black; halter black; wing length from $2.2 \mathrm{~mm}$ in male to $3.2 \mathrm{~mm}$ in female, costa extending strongly to vein $M 1+2$, last section of M $3+4$ twice penultimate; male genitalia with aedeagus having paired distal sclerites enlarged, enclosed in strongly spiculate membrane (fig. 489, California; fig. 490, Quebec).

Host/Early Stages. Amaranthus. Larva feeding in stem and "roots."

Distribution. California, District of Columbia, Iowa, Kansas; Canada.

References. Malloch, 1913a: 320 (fig. of wing); Spencer, 1969a: 158; 1981: 148; part 2, p. 273.

Frons only narrowly projecting toward base of antennae------A. (Cephalomyza) insularis Spencer

Synopsis. Frons dark brown, orbit more black; 5 orbital bristles on one side, 7 on other; gena extended at rear, almost 0.33 height of eye; mesonotum with $3+2 \mathrm{dc}$, black, weak1y shining; legs and halter black; squama gray, margin and fringe black; wing length in male $2 \mathrm{~mm}$, last section of M $3+4$ just over 1.5 times penultimate; male genitalia with aedeagus as in figures 491, 492.

Host/Early Stages. Unknown.

Distribution. California.

Reference. Spencer, 1981: 154.

3 (1). Knob of halter partially dark-- 4

-- Knob of halter entirely white or yellow---_-_--- 7

4 (3). Knob of halter darkened on lower half---------- 5

Knob of halter white with black spot above--------

- Annimyzella) maculosa (Malloch)

Synopsis. Head entirely black, frons narrow, little more than width of eye; orbital bristles strong, 2 ors, 2 or 3 ori; mesonotum shining black, with $3+1$ strong dc, acr irregularly in 4 rows, prsc weak; legs black; wing length from 2.1 $\mathrm{mm}$ in male to $2.5 \mathrm{~mm}$ in female; squama and fringe silvery white, halter white, with conspicuous oval black spot above; male genitalia with aedeagus (figs. 493, 494) having diverging paired tubules; sperm pump with reduced base (fig. 495).

Host/Early Stages. Asteraceae, recorded from following genera: Aster, Baccharis, Bidens, Chrysanthemum, Conyza, Emilia, Erechtites, Erigeron, Eupatorium, Gaillardia, Gnaphalium, 
Helianthus, Lactuca, Melanthera, Senecio, Solidago, Sonchus, and Tagetes. Larva forming large blotch mine (fig. 496) and frequently feeding together; puparium brown, posterior spiracles each with 3 bulbs.

Distribution. Widespread from Florida to New York and Wisconsin, present in all southern States, common in California; common in Caribbean area, extending to Brazil, Chile, and Argentina;

Canada, Hawaii.

References. Stegmaier, 1967a: 99; Spencer and Stegmaier, 1973: 94; Spencer, 1981: 144.

Note. The blotch mines of this species may be confused with those of Nemorimyza posticata (fig. 539), but the adults can hardly be confused.

5 (4). Mesonotum entirely matt, grayish-Mesonotum shining black-

Synopsis. Frons brownish black, distinct ly above eye (fig. 497); orbital bristles slender, 2 ors, 2 ori; gena extended at rear, 0.33 height of eye; $3 \mathrm{~d}$ antennal segment small, round mesonotum and scutellum deep black, moderately shining; 3 strong postsutural dc, additional bristles beyond in presutural area minute, scarcely differentiated from acr, these in 4 rows; wing length in female $1.5-1.6 \mathrm{~mm}$, last section of M $3+4$ from twice to less than 1.5 times penultimate; male genitalia unknown.

Host/Early Stages. Unknown.

Distribution. California.

Reference. Spencer, 1981: 154.

6 (5). Gena extended at rear, 0.33 height of eye---------

- (Cephalomyza) nevadensis spencer

Synopsis. Frons brownish black, about 1.5 times width of eye, orbit blacker; normally 2 ors, 3 or $i$; gena 0.33 height of eye at rear; $3 \mathrm{~d}$ antennal segment small, round; mesonotum gray black, dc irregular, $2+3$ or $3+4$, additional presutural ones distinctly stronger than acr, these irregularly in 4 rows; pleura and legs black; squama gray, margin and fringe black; halter and stalk black, knob blackish below, white above; wing length $2-2.1 \mathrm{~mm}$, last section of M $3+4$ from 1.5 to 2 times penultimate; male genitalia with aedeagus as in figures $498,499$.

Host/Early Stages. Unknown.

Distribution. California.

Reference. Spencer, 1981: 155.

Gena narrower, 0.14 height of eye----_-_-_-_--_-

- (Cephalomyza) subinfumata (Malloch) Synopsis. Small $\bar{b}$ lack species, closely resembling
A. ( . . ) nevadensis but gena distinctly narrower, only $3+1 \mathrm{dc}$; male genitalia with aedeagus as in figures 500, 501; sperm pump (fig. 502) with very large blade. 
Host/Early Stages. Unknown.

Distribution. I11inois; Canada.

References. Frick, 1953a: 71; 1959: 395; Spencer, 1969a: 159 .

7 (3). Frons brown---_- 8

-- Frons orange or yellow-__- 10

8 (7). Upper ors lacking; ocellar bristles long, diverging extending beyond eye margin ( $\mathrm{fig}$. 503)

-

Synopsis. Frons brownish black, strongly projecting above eye; upper orbital lacking, 4 inclined ori, orbit brilliantly shining black; postverticals directed largely forward (fig. 503); gena deeply extended at rear, up to 0.5 height of eye; mesonotum deep black, with only faint subshine, $3+1 \mathrm{dc}$, acr with only isolated hairs in front; pleura and legs black; squama gray, margin and fringe black; halter white; wing length in female $1.5 \mathrm{~mm}$, last section of $\mathrm{M} 3+4$ twice penultimate; male genitalia unknown.

Host/Early Stages. Unknown.

Distribution. California.

References. Spencer, 1981: 150; part 2, p. 274 (under A. confondata).

Upper ors present; ocellar bristles shorter, normal

9 (8). Mesonotum brilliantly shining black-_-_-_-

- - - (Cephalomyza) elsinorensis Spencer

Synops is. Frons orange brown or darker brown, slightly projecting above eye in profile; 5 orbital bristles, upper directed upward and slightly outward, lower 4 inward; orbit conspicuously black; gena rounded, almost 0.33 height of eye; broad epistoma present, equal to depth of gena; mesonotum and scutellum shining black, with 3 postsutural dc, several smaller ones in presutural area; pleura and legs black; squama gray, margin and fringe black; halter white; wing length $2 \mathrm{~mm}$, last section of M $3+4$ from just less than 2 to 1.5 times length of penultimate; male genitalia with aedeagus as in figures 504, 505 .

Host/Early Stages. Unknown.

Distribution. California.

Reference. Spencer, 1981: 153.

Mesonotum matt grayish black-_-_-_-_-_-_-_-_-

- (Cephalomyza) sp. (Colo.)

Synopsis. Frons distinctly projecting above eye, dark brown; 1 ors, 3 ori; gena deeply extended at rear, 0.4 height of eye; 3d antennal segment enlarged, b1ack; mesonotum black, weakly shining; legs and pleura black; wing slightly infuscated basally, length $2.1 \mathrm{~mm}$ in female, last section of M $3+4$ just less than twice length of penultimate; male genitalia unknown.

Host/Early Stages. Unknown.

Distribution. Colorado. 
Reference. Part 2, p. 276.

10 (7). Notopleuron yellow- 11

Notopleuron dark, black or gray-_-_-_-_-_-_-_-_ 13

11 (10). Entire side of thorax yellow13

- (Catal pomyza) pleuralis (Malloch)

Synopsis. Frons, gena, and face yellow; 3d antennal segment large, round, normally yellow, sometimes slightly brownish; mesonotum matt gray, only 2 fully developed $\mathrm{dc}$, anterior ones not differentiated from acr; pleura and halter yellow; wing length from $2.2 \mathrm{~mm}$ in male to $2.5 \mathrm{~mm}$ in $\mathrm{fe}-$ male; genitalia with aedeagus as in figures 506, 507; hypandrium broad, rounded, with minute apodeme (fig. 508); sperm pump with greatly enlarged base (fig. 509).

Host/Early Stages. Catalpa bignonioides, C. bungei, and other catalpa spp. Larva forming small linear blotch (fig. 510), as many as 5 mines have been seen in a single leaf; puparium brown, posterior spiracles each with some 30 minute bulbs. Distribution. Illinois, Ohio; Canada. References. Frick, 1953a: 70; Spencer, 1969a: 161; 1977c: 242 .

12 (10). All antennal segments black-_- Annimyzella) 1athyroides Spencer Synopsis. Head (fig. 5i1) with frons slightly projecting above eye, normally orange yellow, more rarely clearer yellow or more darkened, blackish on upper half; orbit black, 2 ors, 2 ori; gena 0.33 height of eye, similar in color to frons; face and all antennal segments black; mesonotum and scutellum deep black, $3+1$ strong $d c$, acr in 4 rows; pleura black apart from yellow notopleuron; legs black, only forefemur narrowly yellow; squama grayish black, margin and fringe deep black; halter yellow; wing length $2-2.3 \mathrm{~mm}$, last section of M $3+4$ almost twice length of penultimate; male genitalia with aedeagus (figs. 512, 513) having distiphallus divided into 2 long, sinuate tubules; sperm pump (fig. 514) large.

Host/Early Stages. Lathyrus vestitus. Larva forming large greenish blotch mine; puparium reddish brown, posterior spiracles each with about 10 minute bulbs on heavily sclerotized plate.

Distribution. Central and northern California. Reference. Spencer, 1981: 145.

All antennal segments yellow

-

Synopsis. Frons, orb̄it, gena, antenna, and palpus yellow; 4 strong orbital bristles; frons distinctly projecting above eye (fig. 515); gena deeply extended at rear, almost 4.5 height of eye; mesonotum and scutellum matt gray, $3+1 \mathrm{dc}$, acr irregularly in 2-3 rows; rear of humerus, entire notopleuron, upper margin of mesopleuron yellow, 
last largely blackish gray; legs with coxae yellow, femora streaked yellow and gray, tibiae and tarsi gray; abdomen largely black but all tergites narrowly yellow bordered; squama and fringe yellow; halter yellow; wing length $2 \mathrm{~mm}$ in female, discal cell small, last section of vein $M 3+4$ twice length of penultimate; male genitalia unknown.

Host/Early Stages. Unknown.

Distribution. New Mexico.

References. Malloch, 1913a: 292; Frick, 1959: 379 (as Phytobia (Cephalomyza)).

13 (10). Mesonotum matt gray--_- 14

-- Mesonotum black---_--_-_-_-_-_-_-_-_-_-_-_--- 17

14 (13). 3d antennal segment black-_-_-_-_-_-_-_------ 15

-- 3d antennal segment yellow-_-_-_-_-_-_-_-_-_- 16

15 (14). Discal cell small, last section of vein $M 3+4$ almost twice length of penultimate--.--_-_-_-_-_--

A. (Cephalomyza) scleritica Spencer

Synopsis. Frons yellow, broad, twice width of eye, conspicuously projecting above eye; 1 reclinate ors, 3 inclined ori; gena deeply extended at rear, 0.5 height of eye; 3d antennal segment black, 1st and 2d yellowish; palpus and legs black; scutellum and pleura grayish, similar to mesonotum; squama yellowish gray, margin and fringe black; wing length $1.75-2.1 \mathrm{~mm}$; male genitalia with aedeagus as in figures 516,517 , having strong, paired ventral sclerite.

Host/Early Stages. Unknown.

Distribution. Central and northern California. Reference. Spencer, 1981: 160.

-- Discal cell large, last section of vein M 3+4 lit-

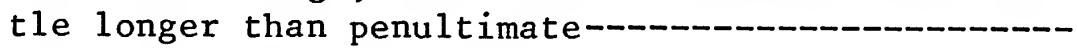

- - A. (Cephalomyza) auriceps (Melander)

Synopsis. Indistinguishable on external characters from A. (‥ ) scleritica, apart from differing wing venation; male genitalia with aedeagus (figs. 518, 519) without ventral sclerite.

Host/Early Stages. Unknown.

Distribution. Colorado, Idaho.

References. Melander, 1913: 262; Frick, 1957b:

202; 1959: 379 (as Phytobia (Cephalomyza)); part 2, p. 274 .

16 (14). Legs entirely black---

- - (Cephalomyza) schusteri Spencer

Synopsis. Frons conspicuously projecting above eye, bright yellow; gena, antennae, and palpus yellow; 1 ors, 4 inclined ori; gena deeply extended at rear, about 0.5 height of slanting, small eye; mesonotum and scutellum matt, grayish black; pleura black; squama whitish gray, margin and fringe dark; halter yellow; male genitalia with aedeagus as in figures 520,521; sperm pump with very large base.

Host/Early Stages. Unknown. 
Distribution. California (San Bernardino Mts.).

Reference. Spencer, 1981: 159.

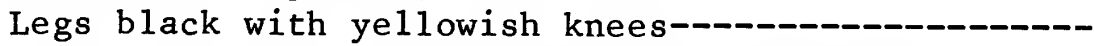

--- - (Cephalomyza) knowltoni spencer, n. sp.

Synopsis. Frons, gena, face, palpus, and all an-

tennal segments yellowish orange; 4 slender orbi-

tal bristles; mesonotum and scutellum matt gray,

3 strong postsutural dc, presutural weak; legs

black but all femora with faintly yellowish

knees; squama and fringe yellowish; wing length

in male $2.1 \mathrm{~mm}$, discal cell large, last section of

M $3+4$ about 1.5 times length of penultimate; male

genitalia with aedeagus as in figures $522,523$.

Host/Early Stages. Unknown.

Distribution. Utah.

References. Spencer, 1981: 160 (as sp., Utah); part

2, p. 275 .

17 (13). 3d antennal segment largely black------------- 18

-- 3d antennal segment pale, yellow or orange--------- 20

18 (17). Mesonotum shining black--

-

Synopsis. Frons, orbit, gena, and fringe yellow;

frons greatly projecting above eye; 1 ors, 4 in-

clined ori; gena extended at rear, 0.5 height of

eye; 3d antennal segment small, entirely black;

palpus black; 3 postsutural dc, presutural scarce-

ly differentiated from acr, these in 4 rows; scu-

tellum, pleura, and legs entirely black; squama

white, margin and fringe scarcely darker; halter

whitish yellow; wing length from $1.7 \mathrm{~mm}$ in male

to $2.3 \mathrm{~mm}$ in female, last section of $M 3+41.5$

times length of penultimate; male genitalia with

aedeagus as in figures 524, 525 .

Host/Early Stages. Unknown.

Distribution. California (San Bernardino Mts.).

Reference. Spencer, 1981: 157.

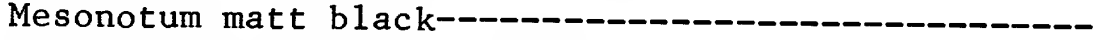

19 (18). Squamal fringe pale, whitish---_-_-_-_-

p.

synopsis. Closely resembling $\frac{\text { A. (C.) remus but }}{\text { (C.) }}$

smaller, with wing length in male $1.4 \mathrm{~mm}$, discal

cell smaller, last section of $M 3+4$ twice length

of penultimate; frons darker, pale brownish to

orange; mesonotum less shining, largely matt; male

genitalia with aedeagus as in figures 526, 527.

Host/Early Stages. Unknown.

Distribution. California (San Bernardino Mts.).

Reference. Part 2, p. 274.

Squamal fringe deep black-

----- (Cephalomyza) flavifrons (Meigen)

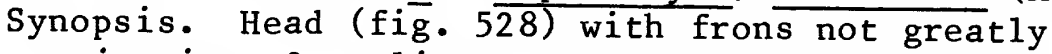
projecting; 2 reclinate ors, 3 or 4 inclined ori; frons and gena yellow; 1 st and $2 \mathrm{~d}$ antennal segments yellowish, 3d largely black, slightly paler on inside; mesonotum deep black, moderately shining, $3+1 \mathrm{dc}$, presutural strong; pleura largely 
black but upper margin of mesopleuron and wing base bright yellow; legs black, only foreknee at most slightly yellowish; squama yellowish gray, margin and fringe black; wing length from $1.9 \mathrm{~mm}$ in male to $2.7 \mathrm{~mm}$ in female, discal cell small, last section of vein $M 3+4$ up to 3.5 times length of penultimate; male genitalia with aedeagus as in figure 529; sperm pump (fig. 530) with small bow1shaped base.

Host/Early Stages. Many genera in the Caryophy1laceae; in America, reared only from Lychnis alba and Saponaria officinalis. Larva forming white linear blotch mine (fig. 531); puparium reddish brown, posterior spiracles each with 3 bulbs.

Distribution. New records: Delaware, Ohio, Wisconsin; Canada.

References. Spencer, 1969a: 160; part 2, p. 275.

20 (17). Tibiae and tarsi yellowish- - - - A. (Cephalomyza) albidohalterata (Ma1loch)

Synopsis. Frons yellowish orange, strongly projecting above eye; 1 reclinate ors, 4 incurved or $i$; gena extended at rear, 0.25 height of large eye; 3d antennal segment smal1, round, orange; palpus black; distinct epistoma present; mesonotum black, moderately shining, with 3 strong postsutural dc, no presutural, acr in 4 rows; legs with femora black, tibiae and tarsi yellowish; squama pale gray, margin slightly darker, fringe whitish, halter white; wing length in male $2.2 \mathrm{~mm}$, 1 ast section of M $3+41.5$ times penultimate; male genitalia with aedeagus as in figures 532, 533 .

Host/Early Stages. Unknown.

Distribution. Illinois, Iowa.

References. Malloch, 1916: 52; Frick, 1953a: 69; 1959: 379; part 2, p. 274.

Tibiae and tarsi black

A. (Cephalomyza) romulus Spencer

Synopsis. Closely resembling A. (C.) remus but a11 antennal segments and palpus orange yellow; head as in figure 534, differing from A. (C.) albidohalterata by darker tibiae and tarsi and brilliantly shining black mesonotum; wing length in male and female about $1.7 \mathrm{~mm}$; male genitalia with aedeagus as in figures 535,536 .

Host/Early Stages. Unknown.

Distribution. California (San Bernardino Mts.). Reference. Spencer, 1981: 158. 
Genus Nemorimyza Frey

Dizygomyza, subgenus Nemorimyza Frey, 1946: 42. Type of genus: Agromyza posticata Meigen (Europe). Nemorimyza Nowakowski, 1962: 97.

Hende1 (1931: 30) included the obviously isolated leaf-mining species posticata, together with the cambiumboring tree feeders (now Phytobia), in the subgenus Dendromyza of his genus Dizygomyza. Frey proposed the subgenus Nemorimyza and Nowakowski (1962) raised this to full generic rank on the basis of the distinctive genitalia of N. posticata.

The single species in our area, $N$. posticata (Meigen), has foretibia with lateral bristle, lunule silvery, and abdomen in male with anterior tergites yellow.

Synopsis. Frons matt black, narrow, equal to width of eye, not projecting above eye in profile; 2 strong reclinate ors, 2 incurved ori; lunule small, semicircular; gena narrow, 0.10 height of eye; all antennal segments black, 3d ovoid; mesonotum with $3+0 \mathrm{dc}$, shining black; wing length from $2.7 \mathrm{~mm}$ in male to $3.3 \mathrm{~mm}$ in female, costa extending strongly to vein $M 1+2$; legs largely black, foreknee yellowish; squama and fringe silvery white; halter yellow; male genitalia with aedeagus as in figures 537, 538 .

Host/Early Stages. Aster, Baccharis, Erechtites, Solidago, possibly other Asteraceae. Larva forming large blotch mine with conspicuous feeding lines (fig. 539); puparium reddish brown, posterior spiracles on conical projections, each with 3 bulbs.

Distribution. Widespread, probably present in all States; Canada, Costa Rica, Europe, Japan.

References. Frick, 1959: 377; Spencer, 1969a: 161; 1981: 162.
Genus Cerodontha Rondani

Cerodontha Rondani, 1861: 10. Type of genus: Chlorops denticornis Panzer, 1806 (Europe).

Traditionally this genus has been restricted to species with the third antennal segment having a spine (fig. 549) or at least conspicuously angulate (Spencer, 1976a: fig. 314) and only one pair of scutellar bristles. However, Nowakowski (1962: 102) noted the similarity in the male genitalia of many species included in different subgenera of Dizygomyza by Hendel (1931) and in Phytobia by Frick (1952a, 1959) and in particular the presence within the epandrium of a characteristic hooked or L-shaped paired structure in all species (fig. 578). Furthermore, all species he studied had hosts exclusively in the Monocotyledoneae in the four families Cyperaceae, Iridaceae, Juncaceae, and Poaceae. Nowakowski therefore proposed including all such species in a single "natural genus," and then after reporting of further revisionary studies in 1967 and 1972, he produced a comprehensive monograph of European species of Cerodontha in 1973. In this, seven subgenera were recognized, treated in phylogenetic order: Icteromyza, Cerodontha, Xenophytomyza, Poemyza, Phytagromyza, Butomomyza, and Dizygomyza. All these subgenera are represented in the United States and no subgenera have been added elsewhere subsequently. Nowakowski's concept has been followed by Spencer (1969a, 1976a, 1977d) and is accepted here, but the possibility of future splitting of the genus is briefly discussed in part 2, p. 272.

Cerodontha is a cosmopolitan genus, with species known in Alaska (Spencer, 1969a), Chile, Australia (Spencer, 1977d), and New Zealand (Spencer, 1976b). Nowakowski (1973) recorded 76 species in Europe. Forty-two species have now been identified in the United States, of which 21 were recorded in California (Spencer, 1981). Ten new species are described here; 1 new 
synonym has been discovered in

Xenophytomyza (part 2, p. 276), and 2 species are recorded as new to the United States, $\underline{\mathrm{C}}$. ( $\underline{\mathrm{P}}_{\text {. ) }}$ pygmaea and $\underline{\mathrm{C}}$. (D.) fasciata. Two species must be deleted from the United States list: C. (Dizygomyza) iraeos (R.-D.) and C. (D. ) iridis (Hende1).

A number of random caught males have been examined that probably represent other undescribed species, but differences in the genitalia and external characters are slight and not distinctive and it seems preferable not to describe them formally without confirmatory biological evidence.

The seven subgenera are keyed and briefly discussed here.
Key to Cerodontha 1 . Subgenera
1. Scutellum with 1 pair of scutellar bristles; 3d antennal segment with spine or at least angulate---- 2

Scutellum with 2 pairs of scutellar bristles------- 3

2 (1). 3d antennal segment with spine (fig. 549); partial1y yellow species--------------Cerodontha Rondani 3d antennal segment angulate; black species-------

3 (1) Conophytomyza Frey Costa ending at vein $R$ 4+5; lunule distinctly higher than semicircle-------- Phytagromyza Hende1 Costa extending strongly to vein $M$ 1+2- 4

4 (3). Lunule broad, in form of semicircle (fig. 540, C) or slightly higher but still broad (fig. 540, $\bar{B}$ )-- 5 Lunule high and narrow (fig. 540, A); if lower (fig. $540, \underline{B}$ ), 3d antennal segment angulate (fig. 555) and notopleuron yellow (C. (P.) chillcottiella)------------

5 (4). Lunule higher than semicircle (fig. 540, B); 3d antenal segment never enlarged in male-----------

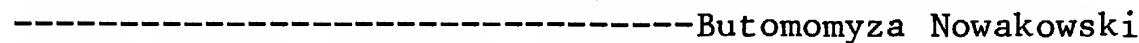
Lunule nearly semicircular (fig. 540, C)--- 6

6 (5). Frons dark, black or brown, lunule gray; ocellar triangle not extended; $3 d$ antennal segment in male enlarged in United States species (except C. (D.) bicolorata)---------------------Dizygomyza Hende1 Frons normal1y yellow (black only in C. [I.] montanoides), lunule always yellow; ocellar triangle extended, with apex near margin of lunule; $3 d$ antennal segment not enlarged in male----Icteromyza Hendel
Cerodontha, Subgenus Icteromyza Hende 1

Dizygomyza (Icteromyza) Hende1, 1931:

51. Type of subgenus: Agromyza geniculata Fallén, 1823a (Europe).

Distinguishing characters of this subgenus are the widely separated antennae, large semicircular lunule, generally yellowish frons, extended ocellar triangle that normally reaches the margin of the lunule, and large discal cell. Although lacking the two important characters of Cerodontha s.s., the spine on the third antennal segment and only a single pair of scutellar bristles, the male genitalia confirm the close relationship between Icteromyza and Cerodontha. 
Fifteen species are now known in Icteromyza, of which 5 are in the United States. This subgenus is widely distributed, occurring in Australia, Europe, New Guinea, New Zealand, South Africa, and Sri Lanka. A new species was recently described from the high mountains of Mexico (Spencer, 1977c) and two others from
California. One species, C. (I.) fuscifrons Spencer, 1969a, was described from northern Quebec and may possibly also be present at high elevations in the United States. C. (I.) pollinosa Melander, 1913, was described from Alaska and has also been recorded from northern Canada (Spencer, 1969a).
Key to Cerodontha, Subgenus Icteromyza Species
1. Palpus black------

-- Palpus yellow------

2 (1). Large species, wing length $2.7-3.5 \mathrm{~mm} ; 5$ or more orbital bristles--Synopsis. Frons yellowish orange, sometimes with slight grayish patches, orbit darkened in upper half, normally with 2 ors and 3 ori but in largest females up to 11 bristles can occur; lunule large, higher than a semicircle, yellow; $3 \mathrm{~d}$ antennal segment black, 1st and 2d slightly yellowish; mesonotum matt, grayish black; legs black but all knees contrastingly yellow for femoral width; squama yellow, margin and fringe brownish black; wing length up to $3.5 \mathrm{~mm}$; male genitalia with aedeagus (fig. 541) having end of distiphallus divided by membranous connection from curved section below.

Host/Early Stages. Almost certainly Juncus spp. But no larvae have been found, despite intensive searching in Europe.

Distribution. Widespread, occurring in many states (Frick, 1959); common in California; new records: Colorado, Mississippi, North Carolina, Wyoming.

References. Frick, 1959; Spencer, 1969a: 138; 1981: 167.

Smaller species, wing length $2.5-2.6 \mathrm{~mm} ; 2$ ors, 2 ori (rarely 3)-

Synopsis. Frons and orbit uniformly matt black, lunule, gena, and face yellow; 1 st and $2 d$ antennal segments yellow, 3d black; palpus black; legs largely black, only foreknees distinctly yellow, on midleg and hindleg only indistinctly paler; squama gray, margin and fringe black; male genitalia with aedeagus (figs. 542, 543) having distal tubules curving dorsally, diverging.

Host/Early Stages. Unknown.

Distribution. California.

Reference. Spencer, 1981: 171.

3 (1). Forefemur yellow on distal 3d-

(I.) longipennis (Loew)

Synopsis. Frons largely yellow, upper orbit blackish, eye largely bare, at most with scattered hairs; 3d antennal segment round, slightly enlarged, varying from yellowish brown to black; palpus yellow; meso- 
notum matt gray; femora blackish gray but yellow on apical 3d on $p 1$, yellow for slightly more than femoral width on $\mathrm{p} 2$ and $\mathrm{p} 3$; squama and fringe ye1low; wing length from $2.5 \mathrm{~mm}$ in male to $3 \mathrm{~mm}$ in $\mathrm{fe}-$ male; male genitalia with aedeagus (fig. 544) having long, slender, paired distal tubules, recurved to midpoint of mesophallus.

Host/Early Stages. Juncus spp., once recorded from J. xiphioides. Larva forming leaf and stem mines, pupating internally; details of larva (puparium) unknown.

Distribution. Widespread, recorded by Frick (1959) from "many States"; new records: Arkansas, Louisiana, Texas, Virginia.

References. Spencer, 1969a: 140; 1981: 170.

-- Forefemur yellow only for width of femora-- 4

4 (3). Frons entirely yellow-- - C. (I.) temeculensis spencer Synopsis. Frons 1.5 times width of eye, 2 ors, 2 or $i$; gena broad, 0.33 height of eye; frons, lunule, gena, face, and palpus yellow, 3d antennal segment black, 1st and 2d yellowish black; mesonotum grayish black, weakly shining; legs black but all femora with knees bright yellow for slightly more than femoral diameter; squama yellowish gray, margin and fringe dark; wing length from $2.5 \mathrm{~mm}$ in male to 2.9 $\mathrm{mm}$ in female, last and penultimate sections of $\mathrm{M} 3+4$ equal; aedeagus (figs. 545, 546) with distal tubules diverging at angle of $90^{\circ}$.

Host/Early Stages. Unknown.

Distribution. California.

Reference. Spencer, 1981: 173.

Frons blackish on upper half-

- (I.) churchillensis spencer

Synopsis. Head (fig. 547) with frons 1.5 times width of eye, 2 ors, 2 ori; gena narrow, about 0.17 height of eye; frons yellow below adjoining lunule but above and entire orbit black; gena, face, and palpus yellow; $3 \mathrm{~d}$ antennal segment black, 1 st and $2 \mathrm{~d}$ paler, yellowish; mesonotum grayish black; legs black but all knees yellow, less conspicuously so on midleg and hindleg; squama yellowish gray, margin and fringe black; wing length 2.3-2.7 mm, last section of M $3+41.5$ times penultimate; male genitalia with aedeagus ( $\mathrm{fig}$. 548) having distal tubules Sshaped in side view, diverging apically, forming angle of $90^{\circ}$.

Host/Early Stages. Unknown.

Distribution. California; Alaska, Canada, northern Sweden, Leningrad area of U.S.S.R. (Spencer, 1976a: 172).

References. Spencer, 1969a: 138; 1976a: 172; 1981: 169 . 
Cerodontha, Subgenus Cerodontha Rondani

Cerodontha Rondani, 1861: 10. Type of subgenus: Chlorops denticornis Panzer (Europe).

Distinctive characters of this subgenus are the spine on the third antennal segment (fig. 549) and the presence of only one pair of scutellar bristles. There is considerable color variation, from largely black through gray to yellow.

Cerodontha is virtually cosmopolitan, with species known from Australia, southern Chile, and New Zealand and is widespread across the northern hemisphere. In Africa, species have been described from several high mountains and two undescribed species have recently been seen from Kenya (Tschirnhaus collection).

Only the single species, C. dorsalis (Loew), is present in the United States. The species is remarkable in having a pale eastern form and a more black western form, both of which meet in Colorado. This color difference has not been satisfactorily explained, although it was investigated in some detail by Aldrich (1918). Two other

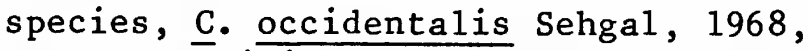
and C. gracilis Spencer, 1969a, are known in Alberta and the Yukon, respectively. Three new species were recently described from high mountains in Durango Province, Mexico (Spencer, 1977c).

Cerodontha (Cerodontha) dorsalis (Loew) (Figs. 549-552)

Synopsis. 3d antennal segment elongate, with short spine at upper corner (fig. 549); frons, face, and gena orange yellow; 3d antennal segment black; gena broad, 0.33 height of eye; mesonotum either uniformly matt grayish black (western form) or in typical (eastern) form yellowish centrally adjoining scutellum; $3+1 \mathrm{dc}$, acr lacking; scutellum either yellow centrally or entirely black; notopleural area yellow, pleura otherwise dark; legs with femora bright yellow; wing length variable, 2.4-3.2 mm; male genitalia with aedeagus (figs. 550, 551) having distal tubules greatly enlarged at end.

Hosts/Early Stages. Poaceae, with records from following cultivated and wild genera: Avena, Bromus, Dactylis, Eleusine, Elymus, Hordeum, Panicum, Phalaris, Phleum, Poa, Secale, Triticum, and Zea. Larva is a leaf miner, pupating internally in leaf sheath; puparium shining, yellowish brown, somewhat flattened, with posterior spiracles irregularly branching, each with about 15 minute bulbs (fig. 552).

Distribution. Probably present in all States, locally abundant in early summer; Brazil, Canada, Central America, Mongolia, Puerto Rico.

References. Luginbill and Urbahns, 1916 (biology); Aldrich, 1918; Spencer, 1969a: 142; 1981: 174.

Note. As many as eight generations a year have been confirmed in southern California, and with complete overlapping, all stages can be found simultaneously. This species may be of some economic importance, with all cereal crops serving as hosts. However, despite initial damage to young plants, they tend to recover and it is not certain whether there is any reduction in yield (Spencer, 1973a: 293-296).

Cerodontha, Subgenus Xenophytomyza Frey

Cerodontha, subgenus Xenophytomyza

Frey, 1946: 51. Type of subgenus:

Haplomyza atronitens Hendel, 1920

(Europe).

This subgenus has in common with Cerodontha only one pair of scutellar bristles; it was differentiated from Cerodontha by Frey (1946) by the lack of a spine on the third antennal segment and by being entirely black. Three species are known in Europe (Spencer, 1976a: 180) with the third 
antennal segment at most slightly angulate.

Cerodontha (Xenophytomyza) illinoensis (Malloch)

This is the only species known from the United States.

Synopsis. Frons not noticeably projecting above eye (in holotype and neoallotype examined); orbit pronounced, with 2 ors and 2 ori, not greatly widening anteriorly; lunule small, narrow, upper margin near level of lower ors; 3d antennal segment (fig. 553) distinctly angulate at upper corner; mesonotum with normally $3+1$ slender dc but $3 \mathrm{~d}$ and $4 \mathrm{th}$ not infrequently weak; prsc lacking; wing length from 2 to $2.5 \mathrm{~mm}$, costa extending strongly to vein $M 1+2$, last section of M $3+4$ from 1.5 to just less than twice as long as penultimate; male genitalia with aedeagus (fig. 554) having long, fused, distal tubules, enlarged and bending ventrally at end.

Host/Early Stages. Unconfirmed, almost certainly Poaceae; larva mining in leaf sheath.

Distribution. Illinois; new record: Tennessee, Hamilton Co., East Ridge, 2 m., 5.V.52 (G. Peck, CNC); Canada.

References. Frick, 1952c: 150; Spencer, 1969a: 135 (as C. (Poemyza) simcoensis); part 2, p. $\overline{2} 76$.

Cerodontha, Subgenus Poemyza Hendel

Dizygomyza (Poemyza) Hende1, 1931: 35.
Type of subgenus: Agromyza pygmaea Meigen, 1830 (Europe).

Essential characters of this subgenus are the high, narrow lunule (fig. 540, A) and the prominent orbit. A number of species previously included in Poemyza are now placed in the subgenus Butomomyza, following Nowakowski's (1973) detailed revision of the genus. Two such North American species are Cerodontha (B..) angulata and $\underline{\mathrm{C}}$. ( B. $_{\text {) }}$ subangulata.

The majority of species in Poemyza are leaf miners on Poaceae, but one new species described here was found on Carex. The host is known of 6 of the 10 recorded United States species, and of these, only 5-- $\underline{C}$. ( $\left.\underline{P}_{.}\right)$incisa, inconspicua, macminni, muscina, and superciliosa--have been actually reared in the United States. Pupation normally occurs within the mine, and several larvae frequently feed together. No less than 16 puparia of $\underline{C}$. ( $\underline{P}_{.}$) macminni were found in a single leaf of Carex (part 2, p. 278).

The subgenus is widely distributed, with 234 species known in Europe. It is present in Sri Lanka (Spencer, 1975) and New Guinea (Spencer, 1977b) but has not reached Australia. It is also absent from the Neotropical Region. Three new species are described here and one, $\underline{C} .\left(\underline{P}_{.}\right)$ pygmaea, is recorded as new to the United States. 
Key to Cerodontha, Subgenus Poemyza Species
1. Notopleuron yellow-

-- Notopleuron black--

2 (1). Orbit black; outer crossvein lacking-

-

Synopsis. 3d antennal segment conspicuously angulate (fig. 555); frons ochreous above, darkened below; lunule dark, slightly higher than a semicircle; mesonotum grayish black, acr in 2-3 rows; legs black, with all knees yellow; wing length in female $1.9 \mathrm{~mm}$ (fig. 556); male genitalia unknown.

Host/Early Stages. Unknown.

Distribution. Colorado.

Reference. Part 2, p. 277.

Orbit yellow; outer crossvein present-_

-

Synopsis. 3d antennal segment weakly angulate;

frons blackish below, yellow above adjoining ocellar triangle, orbit yellow; 2 reclinate ors, 2 or 3 ori, at least lower slightly proclinate; lunule bright yellow, face and 3d antennal segment black; mesonotum matt black; notopleuron and upper margin of mesopleuron bright yellow; legs black, with all femora distally yellow; squama, fringe, and wing base rectangular plate (fig. 557); male genitalia with aedeagus (fig. 558) having paired distal tubules long, S-shaped.

Host/Early Stages. Poaceae, including Avena sativa, Elymus canadensis, Hordeum jubatum, Triticum aestivum, and Zea mays; in Europe many other species recorded by Nowakowski (1973: 113). Larval leaf mine frequently in upper half of leaf, pupation internally; puparium metallic black, posterior spiracles each with 3 bulbs, 2 lower ones curving round base of hooklike upper one.

Distribution. Widespread in northern half of United States (Frick, 1959: 381, as $\underline{P}$. lateralis); California; new records: Colorado, incl. Mt. Evans, Timberline, $11,700 \mathrm{ft}, 2 \mathrm{~m}$, 2 f., 22.VII.61 (C. H. Mann, CNC); northern Europe.

Reference. Spencer, 1981: 183.

Note. This species was referred to in the literature as $\underline{P}$. lateralis Macquart until its correct identity was established in Europe by Nowakowski (1973).

3 (1). Femora yellow on distal half or $3 d-$

-- Femora at most yellow at knees for approx. femoral
width, or largely black with only foreknee yellowish

-- Femora at most yellow at knees for approx. femoral
width, or largely black with only foreknee yellowish

4 (3). Orbit yellow on upper half-
Synopsis. Orbit conspicuously differentiated, yellow above, more blackish below; lunule high, narrow, brown; mesonotum and side of thorax black; legs with femora black on lower half, bright yellow for distal half or 3d; squama yellow, margin and fringe dark; wing length $1.9-2.3 \mathrm{~mm}$; male genitalia with aedeagus (fig. 559) having distal tubules short and conspicuously rotated to right. 
Host/Early Stages. Many genera of Poaceae; Agropyron repens, Dactylis glomerata, Echinochloa crusgalli, Ehrharta erecta, and Hordeum murinum recorded in North America by Frick (1959: $\overline{382,438) ; ~ i n ~ E u r o p e, ~}$ also known on Bromus, Dactylis, Festuca, Hierochloe, Holcus, Lolium, Milium, and Poa. Larva forms long mine on lower surface of leaf, pupating in leaf; puparium black, with posterior spiracles conspicuously projecting dorsally (fig. 560).

Distribution. California, District of Columbia, Idaho, Illinois, Indiana, Maryland, Massachusetts, Michigan, New York, Pennsylvania (Frick, 1959); new records: Georgia, North Carolina, Tennessee (CNC), and Maryland, Montgomery Co., Bethesda, leaf mines with puparia on Dactylis glomerata, 25.VI.80

(KAS, USNM); occurring throughout Canada (Spencer, 1969a); Europe.

References. Frick, 1959: 382; Spencer, 1969a: 132; 1981: 181 .

Orbit entirely yellow------C. (P.) malaisei Spencer

Synopsis. Closely resembling $\mathrm{C}$. Muscina but orbit entirely yellow; femora black basally, merging into yellow on distal $3 \mathrm{~d}$ or $4 \mathrm{th}$; wing length in male 2 $\mathrm{mm}$; male genitalia with aedeagus (fig. 561) having distal tubules long, sinuous, not rotated (as in $\underline{C}$. muscina).

Host/Early Stages. Unconfirmed, almost certainly Poaceae.

Distribution. California (E1 Dorado Co.), (?) Texas (see Note below).

Reference. Spencer, 1981: 180.

Note. Two females from Texas, Kerr Co., Kerrville, 21. IV.59 (J. F. McAlpine, CNC) agree with C. malaisei in the bright yellow orbit, but the femora are almost entirely yellow, only narrowly black basally. It is possible that these represent another species in this complex, but their status can only be estab1 ished when a male that can be associated with them becomes available.

5 (3). Legs largely black, only forefemora distally yellow

All femora distally yellow-- 6

-- All femora distally yellow- 7

6 (5). Squamal fringe black-Synopsis. Dark species; frons $1 . \overline{5}$ times width of eye, orbit widening below lower ors; gena narrow; $3 \mathrm{~d}$ antennal segment small, round; $3+1 \mathrm{dc}$, mesonotum shining black; legs largely black, only forefemora distally yellowish; squama dark gray, margin and fringe brownish black; wing length about $2 \mathrm{~mm}$, last section of vein M 3+4 long, 2-2.5 times length of penultimate, discal cell thus small; male genitalia with aedeagus (fig. 562) sinuous, having almost circular curvature distally.

Host/Early Stages. Poaceae, favorite hosts (in Europe) are Brachypodium sylvaticum, Dactylis glomerata, Deschampsia cespitosa, al so recorded 
on Agropyron, Agrostis, Festuca, Holcus, Phleum, and Poa. Severa1 larvae normally feed together, pupating in mine; puparium shining black, resembling that of C. incisa (see fig. 564).

Distribution. New York (new record); Canada, Europe. References. Spencer, 1969a: 133; part 2, p. 279.

Squamal fringe pale, yellowish--

-

Synopsis. Agreeing closely with c- pygmaea, including black femora with only forefemora distally yellow, but differing in pale squamal fringe; wing length 2.1-2.8 mm, last section of M 3+4 about twice length of penultimate; male genitalia with aedeagus (fig. 563) having curvature of distiphallus genera1ly somewhat flat, distal tubules sometimes slightly rotated to right (but not conspicuously so, as in $\underline{C}$. (P.) muscina, fig. 559).

Host/Early Stages. Many genera of Poaceae, including in United States Agropyron repens, Bromus tectorum, Elymus glaucus, Hordeum vulgare, Phalaris arundinacea, Phleum pratense, Setaria 1utescens, and Triticum aestivum (Frick, 1952a, 1959). Larva mines normal 1 year apex of leaf, pupating interna11y, frequently several larvae feeding together; puparium blackish, posterior spiracles (fig. 564) on conspicuous protuberance, each with normally 3 bulbs.

Distribution. Widespread in northern United States (Frick, 1959: 381); new records: North Carolina, Wyoming ( $\mathrm{CNC}$ ); widespread in Canada, Europe;

Pakistan.

Reference. Spencer, 1969a: 128.

7 (5). Tarsi yellowish-Synopsis. Frons not projecting above eye, 2 ors, 2 ori; gena narrow, $3 \mathrm{~d}$ antennal segment sma11, round, arista long; frons, a11 antennal segments, mesonotum, and pleura black; legs with femora black but knees bright yellow for slightly more than femoral width; squama and fringe yellowish; wing length $2.3-2.5 \mathrm{~mm}$, 1ast section of vein M $3+41.5$ times length of penultimate; male genitalia with aedeagus as in figure 565 .

Host/Early Stages. Carex crus-corvi. Larvae forming large communal mine, pupating internally; puparium black, elongate at anal end, with posterior spiracles each having 3 bulbs, lower hooklike (fig. 566).

Distribution. Mississippi.

Reference. Part 2, p. 278 .

Tarsi dark, blackish brown--

8 (7). Discal cell small, last section of vein $M 3+4$ almost twice length of penultimate--C. ( $P_{.}$) pygminoides Spencer Synopsis. Small black species, with 3 d antennal segment slightly angulate; all knees bright yellow, tarsi dark; wing length in male $1.75 \mathrm{~mm}$, discal cell small, last section of vein $M 3+4$ almost twice length of penultimate; male genitalia with aedeagus as in figure 567. 
Host/Early Stages. Unknown, almost certainly Poaceae. Distribution. California (Inyo Co.); new record:

Colorado, Boulder Co., Boulder, Flagstaff Canyon, $5,000 \mathrm{ft}$, on side of stream, I m., 10.VI.61 (C. H. Mann, CNC).

References. Spencer, 1981: 183; part 2, p. 279.

-- Discal cell larger, last section of vein $M 3+4$ almost 1.5 times penultimate

9 (8). Last and penultimate sections of vein $M 3+4$ equal; medial veins dark; $2 d$ antennal segment yellowish

Synopsis. Generally resembling commoner $c$. inconspicua, with all knees yellow but clearly differentiated by key characters; male genitalia with aedeagus as in figures 568,569 .

Host/Early Stages. Unknown, almost certainly Poaceae.

Distribution. South Carolina, Tennessee. Reference. Part 2, p. 276.

Last section of $M 3+4$ longer than penultimate; medial veins pale; all antennal segments black

Synopsis. Smal1 dark $\mathrm{mm}$; frons not projecting above eye, 2 ors, 2 ori; orbit only slightly widening toward base of antennae; lunule high, narrow; gena narrow, slightly extended at rear; frons and all antennal segments black, orbit narrowly yellow along inner margin; mesonotum black, only slightly shining, pleura black, apart from narrow upper margin of mesopleuron; legs black but all femora bright yellow; medial veins pale, last section of M $3+4$ approx. 1.5 times length of penultimate; male genitalia with aedeagus (fig. 570) having paired distal tubules parallel, not diverging, with low curvature, slightly widening distally.

Host/Early Stages. Agropyron repens (details of leaf mine not recorded), probably other grasses. Puparium black, posterior spiracles on widely separated projections, each with 3 bulbs (see Frick, 1959: figs. 71-73).

Distribution. California, Colorado; new records: North Carolina, Utah.

References. Malloch, 1913a: 310; Frick, 1959: 381; Spencer, 1981: 178; part 2, p. 277. 
Cerodontha, Subgenus Phytagromyza Hende 1

Phytagromyza Hende1, 1920: 145. Type of subgenus: Agromyza (Domomyza) flavocingulata Strobl, 1909 (Europe).

Al1 but the type-species of Phytagromyza are now included in the genus Paraphytomyza Enderlein (p. 162). However, the type-species, flavocingulata, correctly belongs in the genus Cerodontha. Only two species are known in the subgenus, flavocingulata, which occurs in Europe and Canada, and frankensis Spencer, described from Canada and now known from California and Wyoming. These two species are distinguishable by the costa ending at vein $R \quad 4+5$ and are readily separable because the squamal fringe is dark in c. frankensis and yellow in C. flavocingulata.

Cerodontha (Phytagromyza) frankensis Spencer

(Fig. 571)

Synopsis. Frons not projecting above eye, 2 ors, 3 ori; lunule narrow, slightly higher than a semicircle; $3 d$ antennal segment small, round; mesonotum with $3+1 \mathrm{dc}$, prsc weak; wing length 2.1-2.6 mm, costa ending at vein $R 4+5$, last section of $M 3+4$ less than 3 times penultimate, inner crossvein slightly before midpoint of discal cell; frons ochreous yellow behind, blackish in front, orbit and all antennal segments black; mesonotum black, faintly tinged with gray, only weakly shining; pleura black, only upper margin of mesopleuron narrowly bright yellow; femora black, all knees yellow; squama yellow, margin and fringe brown, wing base bright yellow; male genitalia with aedeagus (fig. 571) having paired tubules of distiphallus parallel, not diverging but slightly dilated at apex.

Host/Early Stages. Unknown, almost certainly Poaceae.

Distribution. Widespread in California; new record: Wyoming, Carbon
Co., Battle Lake Road, Sierra Madre Range, 8,000 ft, $1 \mathrm{~m} ., 18$. VII.61 "on side of stream" (J. G. Chillcott, CNC); Canada.

References. Spencer, 1969a: 119; 1981: 191.

Cerodontha, Subgenus Butomomyza Nowakowski

Cerodontha (Butomomyza) Nowakowski, 1967: 633. Type of subgenus: Agromyza angulata Loew, 1869 (Europe).

The most significant character separating this subgenus from Poemyza is the broader, higher lunule (fig. 540, B). At the hindcorner of the epandrium there is normally a conspicuous patch of bristles (figs. 578, 584), never present in Poemyza. The third antennal segment is never enlarged in the male as is frequently found in Dizygomyza ( $\mathrm{fig}$. 593).

The larvae normally feed on Cyperaceae, particularly Carex, but a few species in Europe have been found on Poaceae.

of the eight species now recorded in the United States, the host is known of two (in Europe), but none have been recorded here. With further collecting, the characteristic yellowish green leaf mines will certainly be found on many species of Carex. In most species the puparium remains in the mine.

Butomomyza is a small subgenus, occurring mainly in Europe and North America, but a single species has been described in Australia (Spencer, 1977d). Eleven species have been recorded in Europe (Nowakowski, 1973). Four new species are described here. 
Key to Cerodontha, Species
1. Frons strongly projecting above eye (fig. 572)------- 2

-- Frons not projecting above eye-----

2 (1). Mesonotum black--- (B.) gibbardi Spencer Synopsis. Head (fig. 572) with frons and orbit

black; lunule distinctly higher than a semicircle;

3d antennal segment small, round in both sexes; mesonotum black, with $3+1$ strong dc; acr in 4 rows, prsc present but irregular; pleura black but upper margin of mesopleuron narrowly bright yellow; legs black, even foreknee not noticeably pale; squama, fringe, and wing base bright yellow; wing length 2.4-2.8 $\mathrm{mm}$, last and penultimate sections of vein M 3+4 equal; male genitalia with aedeagus (fig. 573) having characteristic curvature, distal tubules diverging at angle of $90^{\circ}$.

Host/Early Stages. Unknown.

Distribution. California, Idaho; Canada.

References. Spencer, 1969a: 119; 1981: 188.

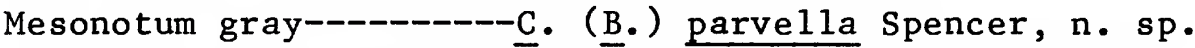

Synopsis. Agreeing closely with C. gibbardi but substantially smaller, wing length in male $2 \mathrm{~mm}$; entirely black legs are important character; male genitalia with aedeagus ( $f i g .574$ ) having distal tubules short.

Host/Early Stages. Unknown.

Distribution. Colorado.

Reference. Part 2, p. 281.

3 (1). Squamal margin and fringe dark, brownish black------ 4

Squamal margin and fringe pale, yellowish----------- 5

4 (3). Male genitalia with aedeagus short, distal tubules greatly enlarged at end (fig. 575)

C. (B. ) scirpi $(\operatorname{Kar} 1)$

Synopsis. Orbit with 2 ors, lower directed upward and slightly inward, 2-4 strong ori; lunule higher than a semicircle, almost U-shaped; 3d antennal segment small in both sexes, arista long, equal to height of eye; mesonotum black, prsc normally well developed but variable; legs black, only foreknee yellow; squama gray, fringe brownish to black; wing length 2.2-2.8 mm; male genitalia with aedeagus having distinctive curvature, distal tubules greatly enlarged at end (figs. 575, 576).

Host/Early Stages. Scirpus spp. (in Europe mainly S. sylvaticus). Larva forming very long narrow mine running down leaf and into leaf sheath, where pupation takes place; puparium yellowish to reddish brown, posterior spiracles each with 3 fingerlike bulbs arising from conical projection.

Distribution. California; Canada, widespread in Europe.

References. Spencer, 1969a: 123; 1981: 189. Male genitalia with aedeagus having long distal tubules, almost vertical to mesophallus with only slight curvature at end (fig. 577)----C. (…) fusculata spencer, n. sp. Synopsis. Closely resembling both $\underline{\text { C. scirpi }}$ and $\underline{\mathrm{C}}$. 
angulata, separable from former by male genitalia and from latter by black squamal fringe; male genitalia with hindcorner of epandrium having patch of strong bristles and row of long hairs along lower margin (fig. 578).

Host/Early Stages. Unknown.

Distribution. North Carolina.

Reference. Part 2, p. 279.

5 (3). Gena broad, almost 0.25 height of eye------------

-

Synopsis. Frons broad, twice width of eye; 2 ors, 2 ori; 3d antennal segment slightly longer than broad, arista appearing bare; legs largely black, only foreknee narrowly yellow; wing length in male $2.1 \mathrm{~mm}$, last and penultimate sections of $\mathrm{M} 3+4$ equal, inner crossvein at midpoint of discal cell; male genitalia with aedeagus ( $\mathrm{fig}$. 579) having distal tubules short with distinctive curvature, diverging; sperm pump with broad stalk and narrow blade (fig. 580).

Host/Early Stages. Unknown, almost certainly Carex. Distribution. Colorado, North Carolina.

Reference. Part 2, p. 281.

-- Gena narrower, 0.17-0.10 height of eye--------------

6 (5). Frons broad, twice width of eye----

Synopsis. 3d antennal - . B. $_{\text {. }}$ impercepta Spencer, n. sp. segment black, small, round, without noticeable pubescence, 2d slightly yellowish; arista distinctly pubescent; legs largely black, only foreknee narrowly yellow; wing length in male $2.3 \mathrm{~mm}$, last section of $\mathrm{M} 3+4$ slightly longer than penultimate, inner crossvein slightly beyond center of discal cell; male genitalia with aedeagus ( $f$ igs. 581 , 582) having distal tubules paler toward end, distinctly diverging; sperm pump pale, fanlike. Host/Early Stages. Unknown, almost certainly Carex. Distribution. North Carolina.

Reference. Part 2, p. 280.

-- Frons narrower, at most 1.5 times width of eye-------

7 (6). Outer crossvein more than own length from inner------

Synopsis. Frons 1.5 times width off eye, brownish black, orbit black, not widening toward base of antennae; 2 ors, 2 ori; $3 d$ antennal segment small, round in both sexes, with small tuft of hairs at upper corner; $3+1$ strong dc, prsc variable, frequently well developed; legs with femora black, knees variable, yellowish on foreleg, sometimes also distally paler on midleg and hindleg; squama and fringe invariably yellow, rarely somewhat darker; male genitalia with aedeagus (figs. 583, 585, 586) having distal tubules rising almost vertically from mesophallus and conspicuously widening distally; sperm pump pale, fanlike; inner corner of epandrium with patch of short bristles (fig. 584).

Host/Early Stages. Carex spp. Larva forming long, narrow, yellowish mine, pupating externally, with 
puparium frequently lightly attached to leaf near end of mine; puparium brownish, oval (fig. 587), posterior spiracles (fig. 588) with 4 bulbs, 1 long, hooklike, other 3 wrapped around it.

Distribution. Widespread, probably present in most States; new records: North Carolina, Tennessee, Virginia; Canada, Europe.

References. Frick, 1957b: 202; 1959: 380; Spencer, 1969a: 113, as Dizygomyza; part 2, p. 279.

Outer crossvein own length from inner

(B..) subangulata (Ma1loch)

Synopsis. Agreeing in all essential external characters with $\mathrm{C}$. angulata; male genitalia with aedeagus (figs. $589,590,592$ ) having distal tubules forming long low curve, scarcely enlarged at end, largely fused; sperm pump pale, fanlike; bristles on hindcorner of epandrium at end of inwardly curving process (fig. 591).

Host/Early Stages. Unknown, almost certainly Carex. Distribution. Illinois, Maryland (new record). References. Malloch, 1916: 51; Frick, 1959: 382 (as Phytobia subg. Poemyza); Spencer, 1969a: 297 (as Dizygomyza); part 2, p. 282 .

Cerodontha, Subgenus Dizygomyza Hendel

Dizygomyza Hende1, 1920: 130. Type of genus: Agromyza morosa Meigen, 1838 (Europe).

Dizygomyza (Dizygomyza) Hende1, 1931: 83.

Phytobia (Dizygomyza) Frick, 1952a: 396; 1959: 383.

Cerodontha (Dizygomyza) Nowakowski, 1962: 102 .

Three main characters distinguish this subgenus: Lunule large, broad, semicircular (fig. 540, C); 3d antennal segment enlarged in male ( $f i g$. 600); and antennal bases widely separated. Dizygomyza is essentially as defined by Hende1 (1931: 83). Hendel's original concept (1920) represented an assemblage of unrelated species that were later included in seven subgenera, and this artificial grouping was treated as the genus Phytobia by Frick (1952a, 1959). Differences between species are frequently slight, even in the male genitalia, but in Europe, Nowakowski (1973) segregated 40 distinct species, with the host plants and biology discussed in detail.

of the 16 species now known in the United States, only 2 have been reared, both from Iris. Three others, with a holarctic distribution, are known to feed on Carex in Europe. A11 known species are leaf miners in the families Cyperaceae, Iridaceae, Juncaceae, and Poaceae. Pupation takes place in the mine. Characters of the larva (and puparium), particularly the posterior spiracles, are of considerable diagnostic importance.

Dizygomyza is primarily of nearctic and palaearctic distribution. On1y two species are known from the Neotropical Region (Spencer, 1963a: 334 ), and the subgenus has not reached Australia. Three new species are described here, and one, C. (D.) fasciata, is recorded as new to the United States. Two European species, misidentified by Frick (1959)--C. iraeos (R.-D.) and $\underline{C}$. iridis Heñdel-must be deleted from the United States 1 ist. 
Key to Cerodontha, 1 Subgenus Dizygomyza Species
1. Notopleuron yellow-- Notopleuron dark-

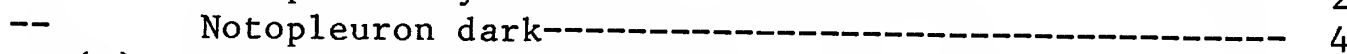

2 (1). All tergites broadly yellow laterally--_--_-_-

-C. (D.) pecki Spencer

Synopsis. Frons broad, twice width of eye, 2 ors,

2 ori; lunule semicircular; gena narrow, 0.17

height of eye; 3d antennal segment (fig. 593)

enlarged in both sexes, conspicuously pubescent;

mesonotum ash gray, humerus and notopleuron

bright yellow; legs black but foreknee bright

yellow; abdomen with all tergites broadly yellow

laterally and narrowly so on hindmargin; squama

and fringe yellow; wing length $1.8-1.9 \mathrm{~mm}$, last

and penultimate sections of $M 3+4$ approx. equal;

male genitalia with aedeagus having distal

tubules diverging and enlarged at end (fig. 594).

Host/Early Stages. Unknown.

Distribution. Florida.

Reference. Spencer and Stegmaier, 1973: 161.

Tergites largely black-_-_-_-_-

3 (2).

Large species, wing length 3.2-3.4 mm-

(Notopleuron normally darker, couplet 8)

Smaller species, wing length less than $2.5 \mathrm{~mm}----$

(D.) magnicornis (Loew)

synopsis. Frons broad, twice e wid̄th of eye, 2 ors,

2 ori; lunule semicircular, gena narrow, about

0.125 height of eye; mesonotum grayish black;

rear of humerus and notopleuron yellow; abdomen largely black, hindmargin of tergites only narrow1y yellow; legs black but all knees yellow; squama and fringe yellow; wing length $2.2-2.4 \mathrm{~mm}$, last section of M $3+4$ distinctly longer than penultimate; male genitalia with aedeagus (fig. 595)

having paired tubules of distiphallus only slightly curving at base and apex.

Host/Early Stages. Unknown.

Distribution. Illinois, Indiana, Michigan, New Hampshire, Pennsylvania; new records: Colorado, Boulder Co., Vrain Creek, 3 miles S.W. of Raymond, 8,200 ft, 1 f., 11.VIII.61 (J. G. Chillcott, CNC); Tennessee, Knox Co., Knoxville, University Farm, $1 \mathrm{~m} ., 20 . \mathrm{V} .57$ (J. R. Vockeroth, CNC).

References. Frick, 1959: 384; Spencer, 1969a: 121

(as C. morosa); Nowakowski, 1973: 222; part 2, p. $2 \overline{8} 3$.

4 (1). Al1 knees conspicuously yellow--

-- Only foreknee conspicuously yellow-- $\quad$ -

5 (4). Frons broad, 3 times width of eye---------_--9

Synopsis. Frons distinct orbit yellow, strongly differentiated from dark brown frons; 2 ors, 3 ori; gena 0.20 height of eye; $3 d$ antennal segment enlarged in male, thickly pubescent; mesonotum matt gray, abdomen slightly yellow laterally and tergites narrowly yellow 
bordered; legs black, all knees yellow; squama and fringe yellow; wing length in male $3 \mathrm{~mm}$; male genitalia with aedeagus (fig. 596) having distal tubules short, diverging.

Host/Early Stages. Unknown.

Distribution. North Carolina.

Reference. Part 2, p. 283.

Frons twice width of eye-- 6

6 (5). Frons pale brownSynopsis. Frons 1.5 times width of eye, orbit conspicuously differentiated, black; lunule semicircular, brownish gray; gena 0.20 height of eye; 3d antennal segment ( $\mathrm{fig}$. 597) enlarged in both sexes, all segments black; mesonotum matt black, upper margin and hindcorner of mesopleuron orange yellow; legs black but all knees yellow; abdomen entirely shining black; squama and fringe yellowish; wing length in female $2.5 \mathrm{~mm}$, last section of M 3+4 slightly longer than penultimate, in ratio $5: 4$; male genitalia unknown.

Host/Early Stages. Unknown.

Distribution. Florida.

Reference. Spencer and Stegmaier, 1973: 65.

Frons darker, brown or black-_- 7

2 ors, 3 ori- 8

2 ors, 2 ori-nor. (D.) morosa (Meigen)

(If knees darker, see couplet $1 \overline{0}$. )

Synopsis. Frons brownish black, at most twice width of eye, not significantly projecting above eye; orbit black; gena narrow, about 0.125 height of eye; lunule large, semicircular; 3d antennal segment greatly enlarged in male, covered with short pubescence; mesonotum grayish black, pleura largely dark, notopleuron at most faintly yellowish, upper margin of mesopleuron narrowly yellow; legs black but all femora normally yellow at knees for femoral diameter, occasionally darker; abdomen largely black; squama and fringe yellow; wing length $2.2-2.4 \mathrm{~mm}$, last and penultimate sections of M 3+4 approx. equal; male genitalia with aedeagus (fig. 598) having paired distal tubules not diverging, forming large semicircular curve, bending slightly downward at apex.

Host/Early Stages. Carex spp. Larva feeding singly, forming long, narrow greenish mine, pupating internally; posterior spiracles each with 3 bulbs, lower 2 curving round base of longer, puparium variable, yellowish, reddish to blackish brown.

Distribution. California, Montana; certainly more widespread, recorded by Frick (1959) from Illinois, Indiana, Maryland, and South Dakota, but these records require confirmation; Europe.

Reference.Spencer, 1981: 197.

Note. Spencer (1969a: 121) confused C. morosa with C. magnicornis (see above). In Europe, Nowakowski 
(1973) treats C. morosa as a superspecies with 4 distinct species. Further splitting in the United States may be justified but only when reared material is available.

8 (7). Orbit conspicuously widening toward lunule at

level of upper ori-_-_-_-C. (D.) thompsoni (Frick) Synopsis. Frons twice width of eye, narrowly projecting above eye in profile; orbit broad with 2 ors, 3 ori; gena angular, at rear 0.20 height of eye; 3d antennal segment enlarged in male, without conspicuous pubescence; frons dull brown to blackish, orbit slightly paler, sometimes yellowish but frequently little differentiated from dark pleura; legs black, all knees bright yellow; abdomen shining black; squama and fringe yellow; wing length $3.2-3.4 \mathrm{~mm}$; male genitalia with aedeagus (fig. 599) having distal tubules rising vertically from mesophallus, curving ventrally at apex.

Host/Early Stages. Iris versicolor and Typha sp. (Typhaceae). Larva feeding exclusively as leaf miner, mine continuing down to leaf base, where pupation takes place; gall develops around puparium when growth is resumed in spring; puparium pale brown, slender, somewhat flattened, posterior spiracles each with 3 minute bulbs (contrast $\underline{\mathrm{C}}$. iridophora, fig. 602).

Distribution. Illinois, Massachusetts, Michigan, New York, Pennsylvania; Canada.

References. Claassen, 1918 (detailed discussion of biology, as Agromyza laterella); Frick, 1959: 385; Spencer, 1969a: 124 .

Orbit scarcely widening----C. (D.) iridophora spencer Synopsis. Head (fig. 600) with frons scarcely projecting above eye; orbit with 2 ors and 3 ori; gena 0.125 height of eye; $3 \mathrm{~d}$ antennal segment greatly enlarged in male with short pubescence, arista short, only slightly pubescent; frons dark, more brown behind, black in front; mesonotum matt black; tibiae and tarsi slightly paler, more brownish than black femora; abdomen black; squama and fringe yellow; wing length 2.2-2.5 mm; male genitalia with aedeagus (fig. 601) having distal tubules rising vertically, with circular curvature at apex.

Host/Early Stages. Iris sp. Larva forming irregular whitish leaf mine, pupating toward base of leaf; puparium dark brown, scarcely tapering, posterior spiracles arising from projecting base, each with 3 distinct bulbs, lower 2 curving laterally (fig. 602).

Distribution. Florida; new record: Louisiana, Terrebonne Co., Houma, $1 \mathrm{~m}$, in Iris (no date or collector, AC).

Reference. Spencer and Stegmaier, 1973: 66.

9 (4). Frons 4 times width of eye- 
- (D. ) poolei spencer, n. sp.

Synopsis. Frons strongly projecting above eye in profile (fig. 603); 2 ors, 3 ori; 3d antennal segment enlarged in male, only finely pubescent, distinctly elongate in female; gena broad, 0.25 height of eye; mesonotum predominantly matt black; legs largely black, only foreknee yellowish; wing length $3.2-3.4 \mathrm{~mm}$; male genitalia with aedeagus (fig. 604) having conspicuous curvature, distal tubules not diverging.

Host/Early Stages. Unknown.

Distribution. Colorado.

Reference. Part 2, p. 284.

Frons twice width of eye-

10 (9). Arista conspicuously thickened basally (fig. 605)--

Synopsis. Frons twice width of eye, scarcely projecting above eye; gena broad, almost 0.25 height of eye; 3d antennal segment enlarged in male, round, with fine pubescence, arista distinctly enlarged in lower 3d; legs largely black, only foreknee narrowly yellow; mesonotum matt grayish black, pleura entirely black; wing length $2.5 \mathrm{~mm}$ in male, last section of vein $M 3+4$ about 0.67 length of penultimate; male genitalia with aedeagus ( $f i g$. 606) having distal tubules with large upper curve, ventrally directed at end; epandrium with patch of bristles at hindcorner, anal projection prominent (fig. 607).

Host/Early Stages. Unknown.

Distribution. North Carolina.

Reference. Part 2, p. 282.

-- $\quad$ Arista slightly widening uniformly toward base (fig. 608)--

11 (10). Last section of vein M 3+4 1.5 times penultimate--- 12

-- Last and penultimate sections of $M \quad 3+4$ approx. equal

12 (11). and penultimate sections of $M 3+4$ approx.

Orbit grayish black------Synopsis. Head (fig. 608) with frons twice width of eye, only narrowly projecting above eye at base of antennae; 2 ors, 2 ori, orbit broad, grayish black; gena 0.17 height of eye; $3 \mathrm{~d}$ antennal segment enlarged in male, only slightly pubescent; legs black, with only foreknee yellowish; abdomen entirely black; squama and fringe yellow; wing length 2.5-3 mm; male genitalia with aedeagus (fig. 609) having distal tubulars with large upper curve, turning ventrally at end.

Host/Early Stages. Unknown.

Distribution. California.

Reference. Spencer, 1981: 199.

Orbit yellowish-- (D.) fasciata (Strob1)

Synopsis. Frons brownish yellow, orbit and lunule yellowish; 3d antennal segment enlarged in male, with distinct pubescence; mesonotum matt gray, $3+1$ $\mathrm{dc}$, prsc weak; pleura dark, upper margin of meso- 
pleuron bright yellow; legs black, but foreknee bright yellow; squama and fringe yellow; wing length $1.9-2.5 \mathrm{~mm}$, discal cell small, with last section of M $3+41.5$ times penultimate; male genitalia with aedeagus (fig. 610) having distal tubules distinctly enlarged at end.

Host/Early Stages. Poa spp. (in Europe $\underline{P}$. chaixii Vil1., no specimens reared in North America). $\overline{\mathrm{Pu}}-$ parium brownish, elongate, posterior spiracles with 3 bulbs, 2 upper bulbs wrapped around base of longer, lower one.

Distribution. New record: North Carolina, Mitchell Co., Roan Mts., 6,300 ft, $1 \mathrm{~m}$., 13.VIII.57 (J. G. Chillcott, CNC); Canada (as C.. (D.) chaixiana Groschke), Europe.

References. Spencer, 1969a: 115 (as C. chaixiana); 1978: 1; part 2, p. 282.

13 (11). Tergites $1-3$ or 1-4 entirely yellow-Tergites at most yellow laterally or narrowly yellow bordered------------------------

14 (13). Tergites $1-3$ conspicuously yellow; 3d antennal segment in male not enlarged--C. . (D.) bicolorata Spencer Synopsis. Frons black, twice width of eye, not projecting; orbit blackish gray, with 2 ors, 2 ori; gena narrow, 0.10 height of eye; $3 \mathrm{~d}$ antennal segment round, normal in both sexes; mesonotum blackish; legs black, only foreknee yellowish; abdomen conspicuously yellow on tergites $1-3$, though sometimes darkened centrally; squama and fringe yellow; wing length $2.7-3 \mathrm{~mm}$, last penultimate sections of M $3+4$ approx. equal; male genitalia with aedeagus as in figure 611 .

Host/Early Stages. Unknown.

Distribution. North Carolina, Tennessee; Canada. Reference. Spencer, 1969a: 114.

Tergites 1-4 conspicuously yellow; 3d antennal segment in male enlarged------ C. (D.) maclayi Spencer

Synopsis. Head agreeing closely with that of $\underline{\mathrm{C}}$. bicolorata but $3 \mathrm{~d}$ antennal segment enlarged in male, frons slightly projecting and gena deeper, 0.17 height of eye; mesonotum grayish black; legs black, with only foreknee yellow; squama and fringe yellow; wing length $2.5-3 \mathrm{~mm}$, last section of M $3+4$ slightly longer than penultimate; male genitalia with aedeagus (fig. 612) having distal tubules with only low curvature, distinctly enlarged at end.

Host/Early Stages. Unknown.

Distribution. California.

Reference. Spencer, 1981: 196.

15 (13). Inner margin of epandrium with prominent projection behind surstylus (fig. 614)----C. (D.) questa Spencer Synopsis. Frons brownish black, twice width of eye, only narrowly projecting; orbit deeper black, narrowly yellow along inner margin, 2 ors, 2 ori; gena angular, 0.17 height of eye; $3 \mathrm{~d}$ antennal segment 
enlarged in male; mesonotum grayish black, pleura largely black but notopleural area with faint brownish undertone, upper margin of mesopleuron narrowly bright yellow; legs black, only foreknee inconspicuously yellow; squama, fringe, and wing base yellow; wing length $2.25-2.4 \mathrm{~mm}$, last section of M 3+4 slightly longer than penultimate; male genitalia with aedeagus (fig. 613) having paired distal tubules not diverging, inner margin of epandrium with conspicuous projection at midpoint (fig. 614).

Host/Early Stages. Unknown.

Distribution. California.

Reference. Spencer, 1981: 201.

Inner margin of epandrium curving uniformly to hindmargin

16 (15). Male genitalia with aedegus having large upper curve (fig. 598)-- (D.) morosa (Meigen) (All knees more frequently yellow; see couplet 7.) Male genitalia with aedeagus having short upper curve (fig. 616)

17 (16). Male genitalia with distal tubules of aedeagus diverging at angle of $45^{\circ}$ (fig. 617); host Juncus------- (D.) luctuosa (Meigen) Synopsis. Frons twice width of eye; dark brown, orbit gray or more black; 3d antennal segment (fig. 615) enlarged in male; mesonotum matt grayish; legs black, with only foreknee narrowly yellowish; abdomen normally with anterior tergites yellow laterally and narrowly yellow bordered, rarely entirely black; squama and fringe yellow; wing length $2-3 \mathrm{~mm}$, last and penultimate sections of M $3+4$ equal or last slightly longer; male genitalia with aedeagus (figs. 616,617) having distal tubules dividing at upper curve. Host/Early Stages. Juncus spp. Larva forming yellowish leaf mine, pupating in leaf blade, frass deposited in single large lump, posterior spiracles of larva (puparium) each with 3 elongate bulbs, lower one shorter (fig. 618); puparium slender (fig. 619).

Distribution. California, Colorado (new record), New York, Oregon, Washington; Canada, Europe.

References. Frick, 1959: 384; Spencer, 1969a: 120; 1981: 195; Nowakowski, 1973: 195.

Male genitalia with distal tubules of aedeagus parallel or at most slightly divergent (fig. 620); host Carex hirta (in Europe)-------------

-

Synopsis. Adult not satisfactōily distinguishable on external characters from $C$. luctuosa, well separable only by male genitalia (fig. 620). Host/Early Stages. Carex hirta (in Europe), other Carex spp. in United States.

Distribution. California, widespread in mountains. References. Nowakowski, 1973: 198; Spencer, 1981: 194. 
Genus Liriomyza Mik

Liriomyza Mik, 1894: 284. Type of genus: Liriomyza urophorina Mik, 1894 (Europe).

In earlier literature (Hende1, 1931; Frick, 1952a; 1959) the concept of this genus appeared relatively simple, with the frons and scutellum yellow, the orbital setulae reclinate, and the costa extending to vein $M 1+2$. Study of much additional material in recent years, both in the Northern Hemisphere and also in Australia and New Zealand, has revealed a number of species correctly belonging in Liriomyza, in which either the frons or the scutellum is entirely black and the outer crossvein is lacking.

In most species, Liriomyza is readily recognizable, but a clear-cut segregation from Calycomyza, Galiomyza, Metopomyza, and Phytoliriomyza has become virtually impossible on external charcters alone. The characteristic form of the aedeagus (figs. 625, 697), with the surstylus discrete and bearing one or two spines and sometimes a few weak hairs, is also distinctive. However, in a few species the distiphallus is greatly elongated (fig. 633), and there may be doubt about the generic position as between Liriomyza and Phytoliriomyza.

The only consistent character for identifying Liriomyza is the presence of the stridulating mechanism in males, a structure that may sometimes be detectable only at a magnification of $400 \mathrm{X}$. This is similar to but not identical with that present in Agromyza (p. 57), with a "file" or line of scales along the center of the membrane connecting the abdominal tergites and sternites (fig. 621) and a "scraper" or ridge on the hindfemur (fig. 622).

Liriomyza is a large genus, with over 300 described species. It is the second largest genus in the Northern Hemisphere, but it is also well represented in the Neotropical Region
(Spencer, 1963a; 1973b), in Australia (Spencer, 1977d), and in New Zealand (Spencer, 1976b), where there has been a remarkable proliferation with 22 of the total 41 agromyzid species known there. In the United States, 80 (including 1 unnamed sp.) described species are now recorded here, of which 11 are new (part 2, p. 288). In addition, five species are recorded as new to the United States: $\underline{\text { L }}$ arctii, $\underline{L}$. endiviae, L. 1athyri, L. philadelphivora, and $\underline{\text { L. taraxaci }}$, of which $\underline{L}$. endiviae is new to North America. Twenty-three other species are known in Canada (Spencer, 1969a; Sehga1, 1971). Three new synonyms have been uncovered (part 2, p. 288).

The majority of species are leaf miners, but one, $\underline{L}$. angulicornis, forms stem mines. The type of the genus, L. urophorina, and some others known only in Europe, feed in seeds. Thirty-five species have been recorded as leaf miners in the United States, and the host of several others is known from Canada or can be deduced from the male genitalia, for example L. graminaceae, which certainly feeds on Poaceae. The favorite host family is Asteraceae, from which 16 species have been recorded, followed by Fabaceae, with 7 species. Other families in which hosts are known include Acanthaceae (1), Asclepiadaceae (2), Brassicaceae (1), Lamiaceae (1), Saxifragaceae (1), and Solanaceae (1).

Some species are strictly host specific, such as L. asclepiadis on Asclepias and L. s. stachyos on Stachys. The majority are oligophagous, feeding on one or more genera in the same family, but three are polyphagous, feeding on a number of unrelated families. These three, L. huidobrensis, L. sativae, and $\underline{\text { L. trifolii, }}$ are the most serious pests in the genus, attacking a wide range of cultivated crops (see here and also Spencer, 1973a).

Although most species are restricted to the Dicotyledones, four--L. angulicornis, L. commelinae, L. marginalis, 
and L. septentrionalis--feed exclusively on Monocotyledones. The first is host specific on Triglochin, the second on Commelina and Tradescantia, whereas $\mathrm{L}$. marginalis and $\mathrm{L}$. septentrionalis have been found on several genera of grasses.

Within the United States the majority of species are known in the north or at high elevations. Ten species are known in Florida, but 48 were recorded in California (Spencer, 1981), of which 31 were described as new. The true distribution of most species remains to be established with further collecting, and it seems clear that the distribution of many species will prove to be more widespread than is apparent from present records, both from north to south and from east to west. For example, L. assimilis, previously known from $\mathrm{I} 11$ inois and Pennsylvania, is now recorded from Texas; L. 1athyri, known only from Alberta, was discovered in Arkansas; and two species, L. ptarmicae and L. septentrionalis, known from California and Canada, have now been recorded from Colorado.
Key to Liriomyza Species $\begin{array}{ll}\text { 1. Scutellum black1/- } & 2 \\ -- & \text { Scutellum at least partially yellow------------- } 3\end{array}$

2 (1). Frons yellow---- nigriscutellata Spencer Synopsis. Frons twice width of eye, conspicuous1y projecting above eye toward base of antennae; 1 ors, 3 ori, orbital setulae sparse or lacking; gena extended at rear, 0.33 height of eye, pale yellow, similar to face and palpus; 3d antennal segment yellowish brown, sma11, quadrate, arista fine, conspicuously swollen at base; mesonotum matt black but with weak subshine, acr in 2-3 rows; side of thorax black, apart from narrow yellow upper margin of mesopleuron; legs with femora black and all knees yellow, tibiae and tarsi largely black, slightly paler on $\mathrm{p} 1$; squama yellowish gray, margin and fringe dark; wing length $1.5 \mathrm{~mm}$; male genitalia with aedeagus as in figures 623, 624 . Host/Early Stages. Unknown. Distribution. California. Reference. Spencer, 1981: 253. Frons dark brown--------------L. nigrissima Spencer Synopsis. Frons twice width of eye, narrowly paler along inner margin of black orbit; 4 orbital bristles (either 1 ors, 3 ori or 2 ors, 2 ori); a11 antennal segments black; mesonotum deep black, moderately shining, acr in 4 rows; humerus and pleura black; scutellum essentially black but with faint brownish undertone; legs and abdomen black; squama dark gray, margin and fringe black; wing length in male $1.8 \mathrm{~mm}$; male genitalia with aedeagus as in figures 625,626 ; surstylus (fig. 627) elongate, with 2 short hairs at end; sperm pump with strongly developed base (fig. 628). 
Host/Early Stages. Unknown.

Distribution. California.

Reference. Spencer, 1981: 254.

3 (1). Mesonotum at least partially yellow adjoining scutellum (figs.632, 650)-_-_-_-_-_ 4

-- Mesonotum dark to margin of scutellum--_--_---_- 17

4(3). 3d antennal segment angulate (fig. 629)

ynopsisulicornis (Malloch)

nal segment yellow; mesonotum black, largely

matt, with V-shaped area yellow adjoining scute1lum; male genitalia with aedeagus as in figures 630,631 .

Host/Early Stages. Triglochin maritimum and $\mathrm{T}$. palustre. Larva forming narrow linear mine that runs round filiform leaves and can penetrate deeper into parenchyma; puparium reddish, remaining in leaf, posterior spiracles each with 3 rather large bulbs.

Distribution. Illinois; Canada, widespread in

Europe (as L. triglochinae Hende1).

References. Frick, 1959: 401 and fig. 96 (3d antennal segment); Spencer, 1969a: 166.

$3 \mathrm{~d}$ antennal segment rounded-----------

$3 \mathrm{~d}$ antennal segment infuscated distally--.---.---

L. variata (Malloch)

Synopsis. Frons lemon yellow, 2 or $\bar{s}, \overline{3 \text { ori, orbit }}$ darkened on upper half; gena pale yellow, at broadest point less than 0.25 height of eye; 3d antennal segment yellow below, distinctly darkened above (see Frick, 1959: fig. 126); mesonotum matt blackish gray (fig. 632), broadly ye1low adjoining scutellum; legs with femora bright yellow, tibiae and tarsi constrastingly black; wing length $2.3 \mathrm{~mm}$ (not 1.5 as stated by Malloch, 1913a: 277); male genitalia unknown.

Host/Early Stages. Unknown.

Distribution. Maine.

References. Malloch, 1913a: 277; Frick, 1959: 411. Note. The unique holotype is now in poor condition and lacks its head.

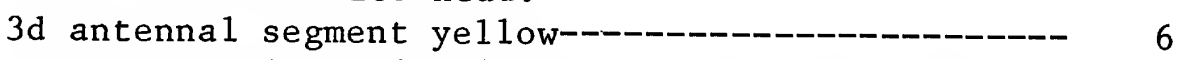

6 (5). Frons strongly projecting above eye (fig. 635)---- 7 Frons not or at most slightly projecting above eye 8

7 (6). Squamal fringe yellow-----L. flavonigra (Coquillett) Synopsis. Head largely yellow, apart from short area along hindmargin of eye; frons distinctly projecting above eye, 2 ors, 3 ori; gena broad, almost half height of eye, parafacial prominent; mesonotum grayish black broadly yellow adjoining scutellum, dark area divided into bands in type specimens from New Mexico (Malloch, 1913a), more solid in specimens from Utah; legs with femora largely yellow but with variable brown markings, tibiae and tarsi brown; squama and fringe yellow; wing length $2.3 \mathrm{~mm}$ in males from Utah, 2.5- 
$2.75 \mathrm{~mm}$ (Frick, 1959) in types from New Mexico; male genitalia with aedeagus having paired elongate tubules (figs. 633, 634, Ut ah).

Host/Early Stages. Unknown.

Distribution. New Mexico, Utah (new record).

References. Malloch, 1913a: 281; Frick, 1959: 405; part 2, p. 289.

Squamal fringe dark-------L. pechumani Spencer, n. sp. Synopsis. Frons conspicuously projecting above eye (fig. 635), yellow, together with all other parts of head; 3d antennal segment small, round, arista pubescent; mesonotum with dark areas blackish gray or matt black, divided into bands (see $\underline{\text { L. margin- }}$ alis, fig. 673), with $3+1$ or $2+1$ dc; pleura ye1low, mesopleuron and sternopleuron with numerous hairs; legs with femora yellow, tibiae and tarsi darker; wing length $3-3.25 \mathrm{~mm}$; male genitalia with aedeagus as in figures 636, 637; epandrium and surstylus as in figure 639; sperm pump large, as in figure 638.

Host/Early Stages. Unknown.

Distribution. Florida.

Reference. Part 2, p. 291.

8 (7). Mesonotum entirely yellow-------L. flavicola Spencer Synopsis. Agreeing closely with L. bella, with following essential characters: Frons, gena, face, palpus, all antennal segments, and face bright yellow; 2 ors, 2 ori, orbital setulae lacking; mesonotum with same pattern as L. bella (fig. 657) but paler, dark area at most rusty brown; scutellum and legs entirely yellow; wing length 2-2.25 $\mathrm{mm}$; male genitalia with aedeagus as in figures 640,641 ; surstylus with short stout bristle at end ( $\mathrm{fig}$. 642).

Host/Early Stages. Unknown.

Distribution. California (Mono and Nevada Cos.). Reference. Spencer, 1981: 231 .

Mesonotum partially dark, either gray, black, or with gray or black and yellow bands-ark to

9 (8). Mesonotum with central dark area extending to
scutellum (fig. 643)-

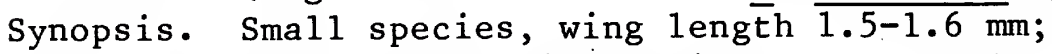
head, including entire hindmargin of eye, yellow; mesonotum (fig. 643) with 3 grayish black bands; scutellum yellow centrally; legs with femora largely yellow but with faint, variable dark striations, tibiae and tarsi contrasting brownish black; squama, including margin and fringe, yellow; male genitalia with aedeagus as in figures 644,645 .

Host/Early Stages. Unknown.

Distribution. California (Riverside Co.). Reference. Spencer, 1981: 211. Mesonotum yellow centrally adjoining scutellum (although central dark band may nearly reach scutellum (fig. 646))--- 
10 (9). Mesonotum predominantly dark, either narrowly yellow or with $\mathrm{V}$-shaped or rectangular patch adjoining scutellum-----------------------------

Mesonotum with distinct bands, dark areas either shining black or gray, or more broadly yellow adjoining scutellum

11 (10). Mesonotum narrowly yellow adjoining scutellum (fig. 646)-Synopsis. Head entirely ye llow, including entire hindmargin of eye; mesopleuron with dark bar along lower margin; tibiae and tarsi darker, brownish yellow; wing length $1.75 \mathrm{~mm}$, discal cell small, last section of vein M $3+43$ times length of penultimate; male genitalia with aedeagus as in figures 647,648 .

Host/Early Stages. Ageratina altissima (=Eupatorium rugosum). Larva forming long, narrow linear mine (fig. 649); puparium yellowish, posterior spiracles each with about 8 bulbs.

Distribution. Minnesota, Virginia, Wisconsin.

Reference. Part 2, p. 288.

Note. This species superficially resembles Phytoliriomyza felti (fig. 989), but male terminalia clearly indicate the correct generic position of P. felti (part 2, p. 303).

Mesonotum with $\mathrm{V}$-shaped or rectangular yellow patch adjoining scutellum

12 (11). Mesonotum with $\mathrm{V}$-shaped or yellow patch adjoining scutellum (fig. 650)--------L. assimilis (Malloch)

Synopsis. Small species, wing length in male 1.6 $\mathrm{mm}$; head, side of thorax, and scutellum lemon ye1low; legs largely yellow but tibiae and tarsi more brownish on p 2 and p 3; male genitalia with aedeagus as in figures 651, 652; sperm pump with large dark blade; surstylus elongate, with single short spine. Host/Early Stages. Helianthus sp. Larva forming linear mine (further details unrecorded).

Distribution. Illinois, Pennsylvania; new record: Texas, Travis Co., Austin, $1 \mathrm{~m}$, $1 \mathrm{f}$., ex leaf mines on Lindheimera texana, 20.IV.80 ( $\mathrm{S}$. Tavormina).

References. Malloch, 1918a: 80; Frick, 1959: 401. Mesonotum with rectangular yellow patch adjoining scutellum-----------------L. schmidti (Aldrich)

Synopsis. Very small species, wing length 1.25$1.35 \mathrm{~mm}$; frons, orbit, all antennal segments yellow; mesonotum matt gray, yellow behind adjoining scutellum (fig. 653); legs with coxa and femora entirely yellow, tibiae and tarsi slightly darker, brownish; squama and fringe dark; male genitalia with aedeagus as in figures 654, 655; surstylus (fig. 656) with 2 stout bristles and additional 1 at base.

Host/Early Stages. Polyphagous, confirmed hosts in Florida include Albizia lebbeck, Bauhinia purpurea, 
Stizolobium deeringianum (Fabaceae); Smilax sp. (Liliaceae); Bougainvillea spectabilis (Nyctaginaceae); and Passiflora caerulea (Passifloraceae). There are other possible hosts in the Apocynaceae and Euphorbiaceae (Stegmaier, 1966c), but confirmation was not obtained from reared adults. Larva forms irregular, silvery, epidermal mine, pupating externally; puparium is brown, posterior spiracles each with 3 minute equal bulbs.

Distribution. Florida; Bahamas, Costa Rica (type locality), Jamaica.

References. Frick, 1959: 409; Spencer and Stegmaier, 1973: 106.

13 (10). Dark areas of mesonotum gray (fig. 657)--_-_---

Synopsion bella Spencer

Synopsis. Small species, wing length $1.8-2 \mathrm{~mm}$, discal cell large, last section of vein $M 3+4$ only twice length of penultimate; head entirely bright yellow; mesonotum banded, broadly yellow adjoining scutellum; side of thorax bright yellow, apart from lower 0.67 of sternopleuron; legs with femora and tibiae yellow, tarsi slightly darker, brownish yellow; abdomen with all tergites broadly yellow; halter yellow; male genitalia with aedeagus ending in long paired tubules (fig. 658); sperm pump with large dark blade; surstylus with single stout spine.

Host/Early Stages. Unknown.

Distribution. California (predominantly in mountains); new record: Colorado, Gunnison Co., Gothic, 9,500 ft, $1 \mathrm{~m}$., 30.VII.61 (J. G.

Chill cott, CNC).

Reference. Spencer, 1981: 219.

Dark areas of mesonotum shining black, sometimes paler toward rear----

14 (13). Central dark band approaching margin of scutellum (fig. 660)-

Synopsis. Head (fig. 659) yellow, frons not projecting above eye, 2 ors, 2 ori; gena 0.25 height of eye; 3d antennal segment in male (fig. 659) greatly enlarged, with conspicuous pubescence, normally yellow, sometimes more brownish, in female small, round, similarly pubescent; mesonotum (fig. 660) with $3+2 \mathrm{dc}$, prsc lacking, shining black area approaching scutellum ventrally but broadly yellow each side, appearing banded; pleura, scutellum, and legs entirely yellow; abdomen blackish centrally but variably yellow laterally; squama and fringe yellow; wing length $1.6-2 \mathrm{~mm}$, discal cell large, last section of $M 3+4$ twice length of penultimate; male genitalia with aedeagus (fig. 661) very small, ending in paired, straight, unpigmented tubules; ventral sclerites long, narrow, in 2 loops; sperm pump large, equal to length of aedeagus, blade and also hypandrium and aedeagal apodeme almost entirely unpigmented; 
hindcorner of epandrium with 2 short teeth; surstylus with single stout spine.

Host/Early Stages. Commelina elegans (almost certainly other Commelina species), Tradescantia fluminensis. Larva forming long, narrow, whitish mine, with frass in conspicuous black lumps; puparium black, remaining in mine, posterior spiracles arising from stout protuberance, each with 3 bulbs, 1 hooklike, smaller 2 reduced to minute pores near midpoint (fig. 662).

Distribution. Florida; Argentina, Brazil, Cuba, Jamaica, St. Vincent, Venezuela.

References. Frost, 1931: 72; Frick, 1959: 403; Spencer and Stegmaier, 1973: 101; part 2, p. 286. Mesonotum centrally broadly yellow adjoining scutellum (fig. 673)-

15 (14). Epandrium in male with sclerotized bar at hindcorner (fig. 676)

Epandrium in male with short, stout bristle at hindcorner (as normal in genus)

---

Synopsis. Frons not projectin̄g above eye, bright yellow, as also entire hindmargin of eye, all antennal segments, and palpus; mesonotum shining black, divided into bands, broadly yellow adjoining scutellum (see fig. 653), either $2+1 \mathrm{dc}$ or less frequently $3+1$; legs entirely yellow, femora without lateral bristles; wing length $1.9-2.2 \mathrm{~mm}$, last section of M 3+4 1.5-2 times length of penultimate; male genitalia with aedeagus as in figures 663,664 ; surstylus with long curving spine (fig. 665); sperm in figures 663, 664; surstylus with long curving spine (fig. 665); sperm pump with narrow stalk and moderately widening blade.

Host/Early Stages. Philadelphus grandiflorus and other $\underline{P}$. species. Larva forming large white

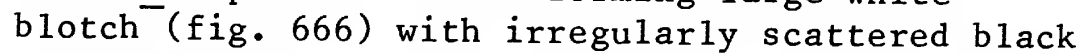
frass; puparium orange, posterior spiracles each with irregular ellipse of about 10 bulbs, 1 extended; cephalopharyngeal skeleton as in figure 667.

Distribution. New records: District of Columbia, New York; Canada.

References. Frost, 1924: 47 (as Agromyza melampyga); Spencer, 1969a: 182; part 2, p. 291.

16 (15). Cercus in male with long hair at end (fig. 668)----

Synopsis. Essentially as in P. philadelphivora; male genitalia with aedeagus as in figures 669 , 670 , distiphallus somewhat elongate; epandrium and surstylus as in L. marginalis ( $\mathrm{fig}$. 676); sperm pump with narrow blade and broad base. Host/Early Stages. Blechum brownei, B. pyramidatum; other possible hosts are Heliotropium (Boraginaceae), Lippia (Verbenaceae), Plantago 
(Plantaginaceae), and Spigelia (Loganiaceae) (see part 2, p. 285). Larva forming irregular blotch mine, frequently associated with midrib and lateral veins (fig. 671), invariably several mines in leaf; pupation in leaf, with anterior spiracles projecting through epidermis; anterior spiracles pale brown (fig. 672), with base short and 2 arms of divided section relatively long; posterior spiracles each with 3 bulbs, outer 2 conspicuously elongate, 2 processes widely separated.

Distribution. Florida, Texas (new record), possibly also District of Columbia, Mississippi; Canada. References. Spencer and Stegmaier, 1973: 98; part 2, p. 285 .

Cercus in male without such hair--_-

-

Synopsis. Essentially as in L. philadephivora; mesonotum with dark area shining black, rarely paler (fig. 673), normally with only 2 strong postsutural dc but 3d sometimes present; male genitalia with aedeagus as in figures 674,675 , distiphallus short, broad; epandrium (fig. 676) with chitinized bar at hindcorner; surstylus with strong blunt chitinized area at inner corner; sperm pump with linear blade, base exceptionally broad.

Host/Early Stages. Poaceae, including Panicum miliaceum, Digitaria, Eleusine, Euchlaena, Paspalum, and probably other genera. Larva forming broad, linear mine, pupating in leaf, with black anterior spiracles (fig. 677) projecting through epidermis, posterior spiracles each with 3 bulbs. Distribution. Florida, South Carolina; Bahamas, Brazil, Dominica, Jamaica.

References. Stegmaier, 1967b: 133 (as L. sorosis); Spencer and Stegmaier, 1973: 102.

17 (3). 3d antennal segment black, brown or at least partially darkened, dark orange--_-_-- 18

$3 d$ antennal segment pale, yellow or orange-------- 42

18 (17). 1 ors--- quadrisetosa (Malloch) Synopsis. Frons orange, strongly projecting above eye, 1 reclinate ors, 4 or 5 inclined ori; gena extended at rear, almost 0.5 height of eye; all antennal segments black; $3+1$ strong dc, sometimes additional smaller ones in presutural area; mesonotum deep black, only moderately shining, scutellum bright yellow; rear of humerus, notopleuron, and upper margin of mesopleuron bright yellow, pleura otherwise black; legs entirely black; squamal margin deep black; wing length from $2.1 \mathrm{~mm}$ in male to $2.5 \mathrm{~mm}$ in female; male genitalia with aedeagus as in figures 678,679 ; hypandrium elongate (fig. 680).

Host/Early Stages. Unknown, possibly Clematis.

Larva feeding internally, either in stem or inflorescence.

Distribution. California, New York, Texas; Canada. 
References. Spencer, 1969a: 183; 1981: 260 .

2 ors------ 19

19 (18). Al1 antennal segments black--_---_--_--_-_-_-- 20

-- At least 1 st and $2 \mathrm{~d}$ antennal segments partially paler-------------- 23

20 (19). Legs entirely black-_- 21

-- Foreknee yellowish-------------L. stachyos Spencer

Synopsis. Frons yellow, broad, almost twice width of eye; gena extended at rear, at least 0.33 height of eye; $3 \mathrm{~d}$ antennal segment small, round; mesonotum black matt, though with some subshine; mesopleuron black apart from narrow yellow upper margin; abdomen entirely black; wing length 1.6$2.4 \mathrm{~mm}$; male genitalia with aedeagus as in figures 681,682 ; sperm pump with conspicuously large black blade.

Host/Early Stages. Stachys, with records on $\underline{s}$. albens, $\underline{s}$. bullata, and $\underline{s}$. rigida. Larva forming an irregular linear mine that may widen, appearing blotchlike at end (fig. 683); pupation internally, puparium orange, posterior spiracles each with 3 bu1bs.

Distribution. California.

Reference. Spencer, 1981: 276.

21 (20). Frons strongly projecting above eye------------

- - bellissima (Spencer)

Synopsis. Large species, closely resembling $\underline{L}$. quadrisetosa with following points of difference: Orbital bristles irregular, varying from 5 to 7 ; orbit pronounced, widening toward lunule; $4 \mathrm{dc}$, decreasing in size regularly, acr in $4-6$ rows; frons brownish; wing length 2.4-2.7 mm; male genitalia with aedeagus as in figures 684,685 ; hypandrium with slender sidearms, U-shaped; sperm pump with large blade, almost triangular (fig. 686).

Host/Early Stages. Unknown.

Distribution. California; Canada. References. Spencer, 1969a: 196 (as Metopomyza);
1981: 221.

-- Frons not significantly projecting above eye------- 22

22 (21). Mesonotum distinctly matt black-----L. n. sp. (Colo.)

Synopsis. Al1 antennal segments and légs black; frons brownish, orbit black; pleura black, humerus and notopleuron bright yellow; wing length $2.4 \mathrm{~mm}$, discal cel1 large, last section of $M 3+4$ on $1 \mathrm{y} 1.5$ times penultimate; male genitalia unknown. Host/Early Stages. Unknown.

Distribution. Colorado.

Reference. Part 2, p. 297.

Mesonotum shining black-- baptisiae (Frost)

Synopsis. Color of frons and orbit variable, ranging from entirely yellow to brownish black, orbit normally distinctly black but legs frequent1y entirely yellow; 2 ors, 2 ori; mesonotum deep black; humerus and notopleuron yellow, pleura 
otherwise black; legs almost entirely black; abdomen black; squama dark gray, margin and fringe black; wing length $1.8-2 \mathrm{~mm}$; male genitalia with aedeagus as in figures 687, 688; s perm pump (fig. 689) with enlarged blade.

Host/Early Stages. Baptisia tinctoria, Lupinus spp. Larva forming linear mine in 1st instar, which later develops into regular blotch (fig. 690), conspicuously greenish when fresh, with frass deposited in irregular grains and strips; puparium yellowish white, posterior spiracles each with ellipse of about 10 bulbs on low protuberance. Distribution. Central California, Montana, Pennsylvania; new records: Colorado, Jefferson Co., Mt. Vernon Canyon, near Golden, 7,200 ft, 1 f., 31.VII.61 (C. H. Mann, CNC); Raymond, Boulder Co., 5,400 ft, $1 \mathrm{~m}$., 10.VI.61 (B. H. Poole, CNC). References. Spencer, 1969a: 169; 1981: 218 .

23 (19). Femora predominantly black but at least partially

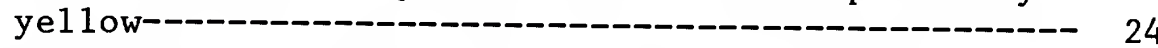

Femora predominantly yellow, at most variably marked with blackish striations----_- 30

24 (23). Femora black, apart from yellow knees-----_------ 25

-- Femora yellowish below or at least faintly on foreleg------------------------------------ 26

25 (24). Outer crossvein lacking; small species, wing length $1.5 \mathrm{~mm}$ in male-------- chlamydata (Melander) Synopsis. Frons yellow, orbit $\bar{l}$ argely black; 3d antennal segment dark orange; mesonotum shining black; mesopleuron almost completely black; legs largely black, but femora with distinctly yellowish knees; wing length $1.5 \mathrm{~mm}$ in male; male genitalia with aedeagus as in figures 691,692 .

Host/Early Stages. Unknown.

Distribution. Washington.

Reference. Frick, 1959: 403.

-- Outer crossvein present; larger species, wing length 1.9-2.5 mm-- - septentrionalis Sehgal (3d antennal segment normally yellow, see couplet 44.)

26 (24). Outer crossvein lacking-----------L. frigida spencer Synopsis. Frons, gena, and face yellow, hindmargin of eye black, both vt on black ground; orbit distinctly darkened from vertex to base of antenna, 2 ors, 2 ori; mesonotum black, only moderately shining; mesopleuron largely black; legs with femora almost completely black, only knees faintly yellowish, tibia and tarsi black; abdomen black; wing length $1.3-1.4 \mathrm{~mm}$; male genitalia with aedeagus as in figures 693, 694; sperm pump with long, narrow stalk and large, rounded blade.

Host/Early Stages. Unknown.

Distribution. Central California.

Reference. Spencer, 1981: 234. 
Synopsis. - L. elevata Spencer, n. sp. ittle wider than eye, orange to brownish, orbit frequently paler, more yellow; 3d antennal segment varying from orange to brown, 1 st and $2 \mathrm{~d}$ predominantly pale; pleura largely dark, humerus and notopleuron at least faintly yellowish; legs largely black but knees at least slight1y yellowish; abdomen matt black, epandrium and ovipositor sheath contrasting, shining black; wing length 1.6-2 mm; male genitalia with aedeagus as in figures $695,696$.

Host/Early Stages. Unknown.

Distribution. Colorado.

Reference. Part 2, p. 286.

Mesonotum shining black

28 (17). Minute species, wing length from $1.25 \mathrm{~mm}$ in male to $1.4 \mathrm{~mm}$ in female------ L. 1upiniphaga Spencer

Synopsis. Closely resembling L. ârtemisiae; orbital bristles may be reduced in smallest specimens, with 1 ors, 2 ori; mesonotum also with dc sometimes reduced, $2+1$; male genitalia with aedeagus as in figures 697,698 .

Host/Early Stages. Lupinus albicaulis and probably other high elevation Lupinus spp. Larva forming narrow linear mine, normally running along midrib, on either upper or lower surface, sometimes extending irregularly into leaf blade.

Distribution. California; new record: Colorado, Boulder Co., Nederland, Science Lodge, 9,500, 1 m., 2.VII.61 (W. R. M. Mason, CNC).

Reference. Spencer, 1981: 248.

Larger species, wing length from $1.4 \mathrm{~mm}$ in male to $1.8 \mathrm{~mm}$ in female

29 (28). Male genitalia with aedeagus as in figures 699, 700 , distiphallus large-------L. artemisiae Spencer Synopsis. Frons yellow, not projecting above eye; 2 ors, 2 ori; gena slightly more than 0.33 height of eye; $3 d$ antennal segment always darkened, normally brownish above, yellowish below, never entirely black; mesonotum shining black, without yellow patches at hindcorners; rear of humerus and notopleural area yellow, other pleura black; legs largely black but at least foreknee yellowish and in palest specimens femora distinctly yellow below; abdomen black; wing length $1.4-1.6 \mathrm{~mm}$, last section of $M 3+43$ times penultimate; male genitalia with aedeagus (figs. 699, 700) having distipha1lus distinctly divided.

Host/Early Stages. Artemisia douglasiana. Larva forming short, upper surface, linear mine, pupating internally.

Distribution. California, widespread.

Reference. Spencer, 1981: 213.

Male genitalia with aedeagus as in figures 701 , 702, distiphallus smaller-------L. paumensis Spencer Synopsis. Agreeing in external characters with $\underline{\text { L. }}$ 
artemisiae, possibly slightly larger; wing length

from $1.5 \mathrm{~mm}$ in male to $1.8 \mathrm{~mm}$ in female.

Host/Early Stages. Unknown.

Distribution. Southern California.

Reference. Spencer, 1981: 255.

30 (23). Femora variably marked with blackish striations----

Femora entirely yellow or at most narrowly black basally

31 (30). Both vt on black ground--

$--$ vti on yellow-- 36

Synopsis. Frons orange yellow; $\overline{3} \mathrm{~d}$ antennal segment variable, normally brownish yellow, rarely darker, more blackish or paler, more yellowish; mesopleuron (fig. 703) largely black, only narrowly yellow along upper margin; legs with coxae, tibiae, and tarsi black, femora basically yellow, particularly below, but variably darkened, often appearing largely black; wing length $1.4-1.9 \mathrm{~mm}$ in male, up to $2.2 \mathrm{~mm}$ in female, discal cell small, last section of M $3+4$ about 2.5 times length of penultimate; male genitalia with aedeagus as in figures 704, 705, left basal sclerite characteristically triangular (viewed ventrally in rest position); sperm pump with narrow stalk and small blade.

Host/Early Stages. Most commonly Medicago sativa, also Coronilla varia, Pisum, Trifolium incarnatum, and $\mathrm{T}$. repens; recorded once on Solanum sarrachoides. Larva forms whitish linear mine (fig. 706), normally pupating externally, occasionally in leaf.

Distribution. California, Florida, Massachusetts, New York, Pennsylvania, Washington, Wisconsin; Canada.

References. Spencer and Stegmaier, 1973: 107; Spencer, 1981: 278; part 2, p. 296.

34 (33). Frons not projecting above eye; gena 0.20 height of eye----

Synopsis. Frons and gena yellow, hindmargin of eye black, both vt on black ground; orbit black or variably darkened, at least to level of lower ors; 3d antennal segment varying from dark brown but more yellow on lower half to mainly dark orange yellow; face black, palpus yellowish; mesonotum appearing somewhat matt, only moderately shining; humerus and notopleuron yellow, pleura otherwise shining black; legs appearing blackish above but femora distinctly yellow below and with all knees yellow; squama gray, margin and fringe black; wing length $1.6-1.75 \mathrm{~mm}$ in male to $2.25 \mathrm{~mm}$ in female, last section of $M 3+4$ twice penultimate; male 
genitalia with aedeagus as in figures 707,708 . Host/Early Stages. Lupinus pratensis, L. varicolor, probably L. arboreus, L. latifolius, and other species. Larva forming irregular linear mine on both upper and lower surface.

Distribution. California.

Reference. Spencer, 1981: 244.

Frons slightly projecting above eye; gena 0.33

height of eye-----------L. huidobrensis (B1 anchard)

Synopsis. Frons orange yellow, upper orbit slight$1 y$ darkened at least to upper ors; 3d antennal segment normally brownish yellow, in palest specimens only slightly infuscated; mesonotum black, appearing somewhat matt viewed from front; mesopleuron variable but generally black on lower 0.75 (fig. 709), in darkest specimens only narrow yellow band along upper margin; legs with coxae ye1lowish black, femora basically yellow but variably darkened with black striations, sometimes appearing almost completely black but yellow always detectable below, tibiae and tarsi brownish to black; squama yellow, margin and fringe black; wing length $1.7-2.25 \mathrm{~mm}$, discal cell relatively large (fig. 710), last section of M 3+4 only 2-2.5 times length of penultimate; male genitalia with aedeagus as in figures 711,712 .

Host/Early Stages. A highly polyphagous species, with confirmed records on 10 families - Apiaceae, Asteraceae, Chenopodiaceae, Cucurbitaceae, Fabaceae, Liliaceae, Linaceae, Solanaceae, Tropaeolaceae, and Violaceae; crop plants attacked include Allium cepa, Apium graveolens, Beta vu1garis, Capsicum annuum, Cucumis melo, Lactuca sativa, Linum usitatissimum, Lycopersicon esculentum, Pisum sativum, Solanum tuberosum, Spinacia oleracea, and Vicia faba. Leaf mines also occur

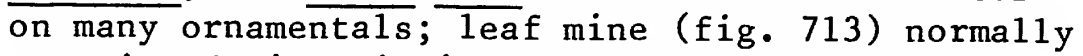
associated with midrib but can be obscured when many larvae feed together in same leaf; puparium yellowish or reddish brown, posterior spiracles each with ellipse of 6-9 bulbs (fig. 714).

Distribution. California, Utah, Washington; widespread in South America.

References. Frick, 1951a: 81 (as L. 1angei); 1958: 1 (as L. dianthi); Spencer, 1973a: 215; 1981: 241.

35 (32). Outer crossvein lacking-------L. frickella Spencer Synopsis. Frons, gena, and face yeîllow; orbit yellow though base of bristles may be darkened; 3d antennal segment brownish but more yellow basally; mesonotum deep black, distinctly shining; mesopleuron largely black, only narrowly yellow along upper margin; legs with femora basically yellow, heavily striated with black, tibiae and tarsi brownish yellow; abdomen black in male, tergites narrowly yellow bordered in female; squama yellowish gray, margin and fringe dark; wing length 1.4- 
$1.3 \mathrm{~mm}$; male genitalia with aedeagus as in figures

715, 716; sperm pump with short, narrow stalk,

blade large, rounded.

Host/Early Stages. Unknown.

Distribution. California.

Reference. Spencer, 1981: 231.

Outer crossvein present------L. californiensis Spencer

Synopsis. Closely resembling $\underline{\underline{L}}$. frickella; wing

length 1.4-1.5 mm; male genitalia with aedeagus as

in figures 717,718 .

Host/Early Stages. Unknown.

Distribution. California.

Reference. Spencer, 1981: 225.

Acr present

Acr lacking---- denudata Spencer

Synopsis. Frons yellow, almost twice width of eye, pronounced orbit at most faintly darkened, brownish or dark orange; mesonotum matt gray; mesopleuron gray on lower half, yellow above; legs with femora basically yellow but variably darkened with blackish striations; wing length in male 1.6 $\mathrm{mm}$, last section of M $3+42.5$ times length of penultimate; male genitalia with aedeagus distinctive (figs. 719, 720).

Host/Early Stages. Unknown.

Distribution. California.

Reference. Spencer, 1981: 226.

37 (36). 1 ors, 3 ori-- minor spencer Synopsis. Frons, orbit, and gena yellow, frons about twice width of eye; $3 d$ antennal segment brownish, 1 st and $2 \mathrm{~d}$ entirely yellow; mesonotum black, matt but more shining from rear; mesopleuron largely black, only narrowly yellow along upper margin, scutellum bright yellow; legs with femora basically yellow but darkened with blackish striations; abdomen in male entirely black, tergites narrowly yellow bordered in female; wing length 1.4-1.5 mm, last section of M 3+4 2.5-3 times length of penultimate; male genitalia with aedeagus having mesophallus long (figs. 721, 722).

Host/Early Stages. Unknown, probably Asteraceae Liguliflorae.

Distribution. California (mountains).

Reference. Spencer, 1981: 250 .

38 (37). 3d antennal segment with short, normal pubescence

-

Synopsis. Closely resembling $L$. minor, differentiated by presence of 2 ori; wing $\overline{\text { length } 1.6-1.75}$ $\mathrm{mm}$, discal cell small, last section of $M 3+43$ times length of penultimate; male genitalia with aedeagus as in figures 723,724 .

Host/Early Stages. Unknown.

Distribution. California (mountains).

Reference. Spencer, 1981: 272 . 
$3 d$ antennal segment with pubescence longer than basal width of arista (fig. 725)

(a) ptarmicae de Meijere

Synopsis. Frons and orbit yellow; hindmargin of eye black, with both vt on black ground; 3d antennal segment normally slightly darkened, brownish (more rarely yellow), with distinct fringe of pubescence (fig. 725); mesonotum shining black; mesopleuron normally largely yellow, only black in lower front corner, sometimes darker; legs with femora either yellow or with variable brownish striations; wing length $1.5-2.0 \mathrm{~mm}$, last section of M 3+4 3 times length of penultimate; male genitalia with aedeagus (figs. 726, 727) at conspicuous angle to basal sclerites, these fully developed on both sides; epandrium angular at hindcorner ( $\mathrm{fig} .728, \underline{B}$ ), surstylus narrow ( $\mathrm{fig}$. 728, A).

Host/Eärly Stages. Achillea spp., Chrysanthemum spp. Larva forming narrow linear mine, puparium yellow.

Distribution. California, Washington; new records: Colorado, Clear Creek Co., Mt. Evans, Echo Lake, $10,600 \mathrm{ft}, 1 \mathrm{f} ., 25 . V I I .61$ (C. H. Mann); Mt. Evans, rocky slope, 13,600 ft, $1 \mathrm{f} ., 4 . V I I I .61$ (J. G. Chillcott); Grand Co., Fraser, 9,500 ft, $1 \mathrm{m.,} 7 . V I I .61$ (J.G. Chillcott); Mont. Co., Lane Co., 20 miles $\mathrm{S}$. of Swan Lake, $1 \mathrm{~m}$, , emerged 11.XI.68, host not recorded, "Biol. Note 675" (S. Whitney).

References. Spencer, 1969a: 178 (as L. millefolii Hering); 1981: 258.

39 (30). Discal cell unusually small, last section of vein M 3+4 times length of penultimate

Synopsis. Frons, orbit, face, and palpus bright yellow; hindmargin of eye black to base of vti; 3d antennal segment brownish along upper margin, ye1low below, 1st and 2d segments yellow; mesonotum brilliantly shining black; mesopleuron grayish black on lower half, yellow above; legs with coxae and femora yellow, tibiae and tarsi brownish; wing length in male $1.75 \mathrm{~mm}$; male genitalia with aedeagus as in figures 729,730 .

Host/Early Stages. Unknown.

Distribution. Southern California.

Reference. Spencer, 1981: 212 .

-- Discal cell larger, last section of $M 3+4$ at most 3

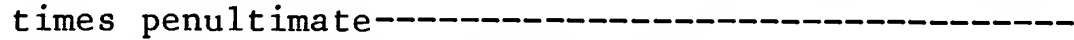

40 (39). 3d antennal segment blackish distally

L. lupini Spencer

Synopsis. Frons, gena, and face orange yellow; hindmargin of eye black to base of vti, orbit not darkened; 3d antennal segment blackish on outside and apically; mesonotum black but somewhat matt; mesopleuron broadly yellow above, black on lower 
half and extending along front margin and hindmargin; legs with femora bright yellow, narrowly darkened basally, tibiae and tarsi darker, particularly on $\mathrm{p} 3$; wing length $1.9-2 \mathrm{~mm}$, discal cell large, last section of M $3+4$ only twice length of penultimate; male genitalia with aedeagus as in figures 731,732 .

Host/Early Stages. Lupinus, including L. caulis, L. 1atifolius, and $\underline{\text { L. pratensis. }}$ Larva forming upper surface linear mine (fig. 733), frequently associated with midrib; puparium brown, straw colored when empty.

Distribution. California (mountains).

Reference. Spencer, 1981: 247.

-- $\quad 3 d$ antennal segment at most brownish or dark orange-

$41(40)$. 3d antennal segment brownish(Norma1ly yellow, see couplet 72. )

$3 \mathrm{~d}$ antennal segment dark orange----- $\underline{\text {. }}$ helenii Spencer Synopsis. Frons, orbit, gena, face, and palpus yellow; hindmargin of eye black to base of vti; mesonotum matt black, with only weak subshine; mesopleuron black below bristle, bright yellow above; legs with femora bright yellow, narrowly black basally, tibiae and tarsi dark brown; wing length $1.9 \mathrm{~mm}$, last section of M 3+4 2-2.5 times length of penultimate; male genitalia with aedeagus as in figures 734,735 .

Host/Early Stages. Helenium hoopesii. Larva forming linear mine, frequently, though not always, following midrib; (details of puparium not known). Distribution. California (mountains).

Reference. Spencer, 1981: 239.

42 (17). Femora predominantly black-_- 43

-- Femora predominantly yellow, at most darkened with blackish striations-- 46

43 (42). Mesonotum shining black--_-_-_---_--- 44

-- Mesonotum matt black-_- 45

44 (43). Femora entirely black---_-----Synopsis. Frons, orbit, and antenna yellow; hindmargin of eye black to base of vte; mesonotum black, broadly yellow at hindcorners; mesopleuron at least 0.75 black, yellow above; legs largely black, femora at most faintly yellowish at knees; wing length $2 \mathrm{~mm}$, discal cell large, last section of M $3+4$ only twice length of penultimate; male genitalia with aedeagus as in figures $736,737$. Host/Early Stages. Arctium minus. Larva forming narrow white linear mine (fig. 738); puparium dark brown.

Distribution. New records: Minnesota, Wisconsin; Canada.

References. Spencer, 1969a: 167; part 2, p. 285. Femora with a11 knees bright yellow-

(If $3 \mathrm{~d}$ antennal segment darkened, see couplet 25.) 
Synopsis. Frons bright yellow, antenna normally similar but $3 d$ segment sometimes darkened, brownish, on upper half; mesonotum shining black; mesopleuron black on lower 0.75; femora black but yellow at knees for distance equal to femoral width; wing length variable, from 1.9 to $2.5 \mathrm{~mm}$ in both sexes; male genitalia with aedeagus as in figures 739,740 ; surstylus not separated from epandrium, slender, with single hair at end ( $f i g$. 741).

Host/Early Stages. Poaceae, recorded in California on Bromus, Hordeum, and Lolium. Larva forming whitish linear mine; puparium reddish brown.

Distribution. California; new record: Colorado, Clear Creek Co., widespread at high elevations, up to $11,300 \mathrm{ft}$ on Mt. Evans (CNC); Canada.

References. Spencer, 1969a: 184; 1981: 271.

45 (43). Discal cel1 large, last section of vein $M 3+4$ twice length of penultimate----L. pulloides Spencer, n. sp.

Synopsis. Frons yellowish orange, orbit more ye1low, all antennal segments yellow; mesonotum matt black; mesopleuron largely black; femora black in female, with slightly yellow knees, more yellow below in male; abdomen largely matt black; wing length $1.6 \mathrm{~mm}$, last section of vein $M 3+4$ twice length of penultimate; male genitalia with aedeagus distinctive as in figures $742,743$.

Host/Early Stages. Unknown.

Distribution. Colorado.

Reference. Part 2, p. 292.

Discal cell small, last section of M $3+4$ slightly more than 3 times length of penultimate--

-

Synopsis. Closely resembling L. pulloides, dif-

fering, apart from wing venation, by darker

legs; wing length in male $1.5 \mathrm{~mm}$; male genitalia

with aedeagus as in figures 744,745 .

Host/Early Stages. Unknown.

Distribution. Colorado.

Reference. Part 2, p. 290.

46 (42). 3d antennal segment with distinct pubescence------- 47

-- 3d antennal segment with norma1, short pubescence-- 49

47 (46). Both vt on yellow ground-(Pubescence of 3d antennal segment not exceptionally long, see couplet 81. )

-- At least vte on black ground

48 (47). Discal cell sma11, last section of vein M $3+4$ times length of penultimate; both vt on dark ground----

(3d antennal (3d antennal segment normally därkened, see couplet 38.)

Discal cell larger, last section of vein $M 3+4$ only twice length of penultimate; vte on dark ground, vti on yellow-- L. archboldi Frost Synopsis. Frons and orbit yellow; mesonotumbrilliantly shining black, with large yellow patches 
at hindcorners, acr in 4 rows; side of thorax largely yellow but mesopleuron black along lower and front margin (see Frost, 1962: fig. 1); legs with coxae and femora yellow, tibiae and tarsi brownish; wing length $1.8-2 \mathrm{~mm}$; male genitalia with aedeagus as in figures 746,747 ; surstylus with 2 stout bristles (fig. 748).

Host/Early Stages. Bidens pilosa. Larva forming

blotch mine (further details not recorded).

Distribution. Florida.

References. Frost, 1962; Spencer and Stegmaier, 1973: 97.

49 (46). Mesonotum black, shining or more matt-------------- 50

-- Mesonotum distinctly gray-------------------------- 82

50 (49). Mesopleuron at least 0.75 black------------------- 51

-- Mesopleuron at most 0.5 black---------_----------- 58

51 (50). Mesopleuron solidly black------------------------- 52

-- Mesopleuron at most 0.75 black--------_------- 55

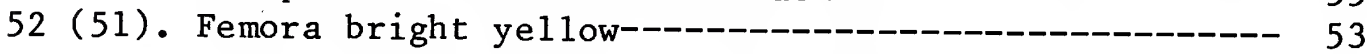

Femora darker, with some blackish striations-------

----

Synopsis. Frons and antenna yellow, hindmargin of eye and upper orbit black, both vt on dark ground; mesonotum shining black, acr in 4 rows; mesopleuron almost completely black, only narrowly yellow along suture; legs with coxae black, femora basically yellow but variably darkened above, tibiae and tarsi brownish black; wing length $1.9-2 \mathrm{~mm}$, last section of vein M $3+4$ twice length of penultimate; male genitalia with aedeagus as in figures 749,750 .

Host/Early Stages. Asteraceae, recorded from Artemisia douglasiana, Baccharis douglasii, B. pilularis, and Conyza canadensis. Larva forming dark upper linear mine that not infrequently starts at apex of leaf and there appears blotchlike (fig. 751); puparium orange.

Distribution. California, widespread; new record: Washington, Yakima Co., Wapato, $2 \mathrm{~m} ., 26 . V I .51$ (K. E. Frick, Lot 229 - 2); Colombia, Venezuela. Reference. Spencer, 1981: 216.

53 (52). Orbit predominantly black-------L. deceptiva (Malloch) Synopsis. Head (fig. 752) with frons conspicuously projecting above eye, dull yellow, together with gena, face, and palpus; orbit considerably widening toward base of antenna, 2 ors, 3 ori, all long, slender; mesonotum entirely shining black; presutural dc little differentiated from acr, these irregularly in 4 rows; scutellum, notopleuron, and femora conspicuously bright yellow; mesopleuron entirely black; wing length in female 2.25 $\mathrm{mm}$, discal cell large, last section of $\mathrm{M} 3+4$ about 1.5 times length of penultimate, in ratio $25: 17$; male genitalia unknown.

Host/Early Stages. Unknown. Distribution. Illinois, Virginia. 
References. Frick, 1959: 403; part 2, p. 286.

54 (53). Acr present in 4 rows; squamal margin black-

Synopsis. Frons, gena, face, palpus, monoensis Spencer antennal segments yellow; hindmargin of eye black to base of vti; orbit narrowly darkened to upper ors; mesonotum moderately shining black, with distinct yellow patches at hindcorners; notopleural area bright yellow, mesopleuron entirely black; legs with femora bright yellow, narrowly black at base, tibiae and tarsi blackish brown; wing length $1.7 \mathrm{~mm}$, 1ast section of $\mathrm{M} 3+4$ slightly less than 2.5 times length of penultimate; male genitalia with aedeagus as in figures 753, 754; surstylus with long, curving spine at end (fig. 755).

Host/Early Stages. Unknown.

Distribution. California (mountains).

Reference. Spencer, 1981: 251.

Acr lacking; squamal margin yellow

Synopsis. Frons, face, and antenna yellow; mesonotum deep black matt; scutellum yellow centrally but broadly black at sides; notopleuron bright yellow, mesopleuron entirely black; legs with femora bright yellow, tibiae and tarsi slightly darker, pale brownish; abdomen shining black, tergites narrowly yellow bordered but 3,4 , and 5 with small yellow patch centrally, increasing in size toward tergite 5; ovipositor sheath brilliantly shining black; wing length in female $1.6 \mathrm{~mm}$. Host/Early Stages. Unknown.

Distribution. Arizona.

References. Malloch, 1913a: 277; Frick, 1959: 404. Note. The unique holotype in the USNM now lacks its head.

55 (51). Minute species, wing length $1.25-1.75 \mathrm{~mm}----------56$

$--$ Larger species, wing length $1.75 \mathrm{~mm}-2 \mathrm{mm---------} 57$

56 (55). Orbit grayish black----_-_---- pictella (Thomson) Synopsis. Frons, gena, and antenna bright yellow; hindmargin of eye black to base of vti; mesopleuron 1argely black (fig. 756); mesonotum shining black, with distinct yellow patches at hindcorners; legs with femora predominantly black but yellowish below, tibiae and tarsi brownish ye1low; wing length $1.6 \mathrm{~mm}$, last section of vein M 3+4 3 times penultimate; male genitalia with aedeagus as in figures 757,758 ; sperm pump ( $\mathrm{fig}$. 759) with exceptiona11y broad blade.

Host/Early Stages. Unknown. Distribution. California ("San Francisco"). Reference. Spencer, 1981: 257.

Note. Only known from unique holotype; other

literature references represent misidentifications. Orbit predominantly yellow-- - - L sativae Blanchard 
(Mesopleuron normally paler, see couplet 63.)

57 (55). Femora entirely yellow or at most narrowly black basally; mesonotum shining--------L. sabaziae Spencer Synopsis. Frons, orbit, and antenna yellow; hindmargin of eye black, with both vt on black ground; 3d antennal segment with slight but not conspicuously long pubescence; mesonotum shining black, yellow patches at hindcorners small, acr in 4 rows; mesopleuron solidly black on lower 0.75 (fig. 760); legs with femora yellow, at most narrowly black basally, tibiae and tarsi brownish black; wing length $1.75-2 \mathrm{~mm}$; male genitalia with aedeagus as in figures 761, 762; surstylus with 2 strong bristles.

Host/Early Stages. Asteraceae, recorded on Baccharis, Carduus, Cirsium, Dahlia, Gnaphalium, and Silybum (in California). Larva forming irregular linear mine (fig. 763), not infrequently associated with midrib and stronger lateral veins; puparium orange.

Distribution. California, Washington; Colombia, Venezuela.

Reference. Spencer, 1981: 262.

Femora yellow, with dark striations; mesonotum matt----- montis Spencer, n. sp. Synopsis. Closely resembling $\bar{L}$. Sabaziae but femora darker and mesonotum more matt; wing length in male $2.3 \mathrm{~mm}$; male genitalia with aedeagus as in figures 764,765 .

Host/Early Stages. Unknown.

Distribution. Colorado.

Reference. Part 2, p. 290.

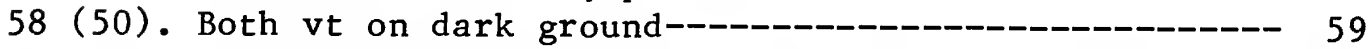

-- At least vti on yellow------------------------ 67

59 (58). Mesonotum distinctly matt black----L. helianthi Spencer Synopsis. Frons, gena, and antenna bright yellow; mesonotum deep black, appearing matt viewed from front, though more shining from rear; scutellum bright yellow with only minute lateral black patches; mesopleuron largely yellow, black along lower margin with variable dark markings up front margin and hindmargin; legs with femora yellow, tibiae and tarsi more brownish; squama yellowish gray, margin and fringe dark; wing length 1.4-1.5 $\mathrm{mm}$, discal cell small, last section of $M 3+43$ times length of penultimate; male genitalia with aedeagus as in figures 766,767 ; sperm pump with elongate stalk and rounded blade.

Host/Early Stages. Helianthus annuus, Xanthium canadense. Larva forming narrow linear mine, with frass in conspicuous black strips.

Distribution. Central California, Washington (new record).

References. Spencer, 1981: 240; part 2, p. 289.

Mesonotum solidly black, without yellow patches at 
hindcorners----- fromeri Spencer

Synopsis. Closely resembles L. sativae, with

following essential characters: Frons, orbit, and

antenna bright yellow; hindmargin of eye black to

base of vti, this just on black ground; mesopleur-

on yellow, with black patches along front and

lower margins; legs with femora bright yellow, tibiae and tarsi brownish; wing length 1.25-1.4 $\mathrm{mm}$, discal cell small, last section of vein $M 3+4$ 3.5 times length of penultimate; male genitalia with aedeagus as in figures 768,769 ; sperm pump with short stalk, blade fanlike.

Host/Early Stages. Unconfirmed (specimens caught on Chilopsis linearis and Melilotus indica).

Distribution. Southern California.

Reference. Spencer, 1981: 235.

$--$

Mesonotum with yellow patches at hindcorners------- 61

$61(60)$. 5 orbital bristles----------- L. specifica Spencer Synopsis. Frons 1.5 times width of eye, orbital bristles somewhat irregular, probably 2 ors, 3 ori; frons, gena, antenna, and palpus yellow; hindmargin of eye black to base of vti; upper orbit faintly darkened to lower ors; mesonotum brilliantly shining black, with prominent yellow patches at hindcorners; mesopleuron black on lower half, yellow above; legs with femora yellow, slightly black basally, tibiae and tarsi brownish yellow on $\mathrm{p} 1$, brownish black on $\mathrm{p} \mathrm{2,} \mathrm{p} 3$; wing length in male $1.6 \mathrm{~mm}$, last section of $\mathrm{M} 3+42.5$ times penultimate; male genitalia with aedeagus as in figures 770, 771; sperm pump with short stalk and rounded blade.

Host/Early Stages. Unconfirmed, possibly Sambucus mexicana (on which type series was caught).

Distribution. Southern California.

Reference. Spencer, 1981: 275.

4 orbital bristles---- 62

62 (61). Smal1 species, wing length 1.25-1.8 mm- 63

-- Larger species, wing length $1.6-2.1 \mathrm{~mm}------------64$

63 (62). Male genitalia with aedeagus as in figures 772 , 773; sperm pump with blade broad (fig. 774)-----Synopsis. Head (fig. 775) with 2 equal ors, 2 ori, lower weaker; frons and antenna bright yellow; hindmargin of eye black, both vt normally on black ground, upper orbit frequently darkened; mesopleuron normally dark only on lower $3 \mathrm{~d}$, though dark area may extend up hindmargin; mesonotum brilliantly shining black, with small yellow patches at hindcorners; legs with femora bright yellow, tibiae and tarsi brownish; squama yellow, margin and fringe dark; wing length from $1.25 \mathrm{~mm}$ in male to $1.8 \mathrm{~mm}$ in female, discal cell small, last section of $M 3+43$ times length of penultimate.

Host/Early Stages. Primarily Brassicaceae, many 
genera being attacked, cultivated genera include Brassica, Cheiranthus, Nasturtium, Raphanus, and Rorippa; a favorite host is Tropaeolum

(Tropaeolaceae), and Capparis, Cleome, and Gynandropsis (Capparidaceae) also commonly serve as hosts. Larvae have been found on Pisum in several countries in the old World tropics; leaf mine linear, upper or lower surface (fig. 776); puparium pale yellowish orange.

Distribution. Widespread, probably present in most

States; new record: Wisconsin (S. Tavormina);

Canada; semicosmopolitan.

References. Stegmaier, 1967c: 257; Spencer, 1973a:

153 (economic importance); 1981: 222.

Male genitalia with aedeagus as in figures 777 , 778; sperm pump with blade narrow (fig. 779) -......

-... sativae Blanchard

Synopsis. Closely resembling $L$. brassicae, with following essential characters: Head (fig. 780) with frons bright yellow, about 1.5 times width of eye; gena almost 0.33 height of eye; $3 d$ antennal segment small, round, yellow; orbit normally faintly darkened, at least to lower ors; darkening of hindmargin of eye variable, normally dark to base of vti, this may be on brown ground rather than black; mesonotum shining black, acr in 4 rows; mesopleuron predominantly yellow, variably dark, blackish gray on lower half, sometimes with extension up front margin and hindmargin, in holotype of L. munda as in figure 781, A (from Frick, $195 \overline{7}$ a), in holotype of L. propepusilla as in figure $781, \underline{B}$; legs with coxae and femora bright ye1low; wing (fig. 782) length $1.25-1.7 \mathrm{~mm}$, discal cell very small, last section of $M 3+43-4$ times length of penultimate; male genitalia with aedeagus (figs. 777, 778), distiphallus with characteristic indentation at sides; sperm pump with short narrow stalk, blade somewhat asymmetrical, not greatly widening (fig. 779) (contrast L. brassicae, fig. 774).

Host/Early Stages. A highly polyphagous species, found most frequently on genera of the 3 families, Cucurbitaceae, Fabaceae, and Solanaceae, but also commonly on Asteraceae, Brassicaceae, Euphorbiaceae, and Malvaceae. The main plants attacked are Asteraceae: Aster, Baccharis, Galinsoga; Brassicaceae: Brassica, Raphanus sativus; Cucurbitaceae: Cucurbita pepo, possibly Marah; Euphorbiaceae: Ricinus communis; Malvaceae: Gossypium (cotton); Fabaceae: Indigofera, Medicago sativa, Phaseolus lunatus; Solanaceae: Capsicum annuum (pepper), Datura, Lycopersicon esculentum (tomato). A complete list of confirmed hosts in Florida is given by Stegmaier (1966b). Leaf mine short, irregular, linear (fig. 783); posterior spiracles of puparium (fig. 784) each with 3 bulbs. 
Distribution. Alabama, California, Florida, Ohio (?), South Carolina, Texas; Argentina, Barbados, Brazil, Central America, Chile (previously unrecorded), Hawaii, Jamaica, Peru, Venezuela.

References. Spencer, 1973a: 219-225 (economic importance); 1981: 264; part 2, p. 292.

64 (62). Discal cell sma11, last section of vein $M 3+43$ times length of penultimate; femora conspicuously pale, lemon yellow----------L. venegasiae Spencer

Synopsis. Frons broad, twice width of eye, yellow, together with orbit, face, and antenna; hindmargin of eye black to base of vti; mesonotum deep black, strongly shining, yellow patches at hindcorners smal1; mesopleuron (fig. 785) black on lower half; legs with femora entirely bright yellow, tibiae and tarsi brownish yellow on $p 1$, darker, brownish black on $\mathrm{p} 2$ and $\mathrm{p} \mathrm{3}$; wing length from $1.6 \mathrm{~mm}$ in male to $2.1 \mathrm{~mm}$ in female, last section of $M 3+4$ 3-4 times penultimate; male genitalia with aedeagus as in figures 786, 787; surstylus with 2 stout spines.

Host/Early Stages. Bidens pilosa, Venegasia carpesioides. Larva forming long, irregular linear mine, with several mines in same leaf.

Distribution. Southern California.

Reference. Spencer, 1981: 284.

Discal cell larger, last section of $\mathrm{M} 3+4$ only 22.5 times length of penultimate; femora yellow, sometimes with darker striations--

65 (64). Upper orbit sometimes slightly darkened--_-_-

Synopsis. Frons and antennal. eupatorii (Kaltenbach) darkened, hind faintly darkened, hindmargin of eye black to base of vti; mesonotum deep black, matt viewed from front, distinctly shining seen from rear, yellow patches at hindcorners large; mesopleuron black on lower half, yellow above; legs with femora yellow, normally with some brownish-black striations; wing length $1.6-2 \mathrm{~mm}$, last section of M 3+4 2-2.5 times length of penultimate; male genitalia with aedeagus as in figures 788,789 ; sperm pump with short, narrow stalk and considerably widening blade; surstylus with single spine.

Host/Early Stages. Aster, Eupatorium, Solidago (in Europe also Galeops $\overline{\text { is }) . ~ L a r v a ~ f o r m i n g ~ l o n g ~ l i n e a r ~}$ mine, at beginning sometimes a small spiral (figs. 790,791 ) (in Europe this is more pronounced); puparium yellow.

Distribution. California, Washington; new records: Georgia, Mississippi, North Carolina, South

Carolina, Tennessee; Canada, Europe.

References. Frick, 1959: 404; Spencer, 1969a: 174; 1981: 230; part 2, p. 288 .

tinctly widening at distal end-C.- 
. asclepiadis Spencer

Closely resembling L. eupatorii, differing in paler upper orbit and consistently yellow femora; wing length $1.6-2 \mathrm{~mm}$.

Host/Early Stages. Asclepias spp. Larva forming small, predominantly regular blotch mine (fig. 794), with greenish diffused frass, pupating externally; puparium pale, yellowish, posterior spiracles each with ellipse of about 10 bulbs; larval mouth parts as in figure 795.

Distribution. Indiana, New Hampshire; new records: Minnesota, Minneapolis, Minnehaha Creek, empty leaf mines on Asclepias ovalifolia, 29.VI.80 (KAS); New York, Albany Co., airport, 2 m., 1 f., reared ex Asclepias incarnata, July 1969 (G. and K. Eickwort).

Reference. Spencer, 1969a: 169.

Note. The record for Washington given by Spencer, 1969a, is erroneous and represents $\underline{L}$. subasclepiadis, n. sp. (see below).

Male genitalia with aedeagus (figs. 796, 797) of uniform width at distal end-C.

- - -

Synopsis. Not distinguishable externally from $\mathrm{L}$. asclepiadis, with following essential characters: Frons, orbit, and $3 \mathrm{~d}$ antennal segment yellow, 2 ors, hindmargin of eye black, with both vt on black ground; mesonotum shining black, acr in 4 rows; femora entirely yellow, tibiae and tarsi brownish.

Host/Early Stages. Asclepias speciosa. Larva forming greenish, irregular interparenchymal mine, partially linear (fig. 798).

Distribution. Washington.

Reference. Part 2, p. 293.

67 (58). Vti on yellow ground, vte on black---_- 68

-- Both vt on yellow-- $\quad 79$

68 (67). Minute species, wing length 1.4-1.6 mm----------- 69

-- Larger species, wing length $1.8-2.75 \mathrm{~mm}--------0$

69 (68). 1 ors, 3 ori--- schlingeri spencer Synopsis. Agreeing closely with $\underline{L}$. virginica with following essential characters: Frons, orbit, and antenna yellow, 1 ors, 3 ori; hindmargin at eye black to base of vte; mesonotum shining black, with large yellow patches at hindcorners; mesopleuron largely yellow, with only small dark patch at lower front corner; legs with femora yellow, tibiae and tarsi yellowish brown; wing length $1.4-1.6 \mathrm{~mm}$, discal cell small, last section of M $3+43$ times length of penultimate; male genitalia with aedeagus as in figures 799,800 .

Host/Early Stages. Unknown. Distribution. Southern California. Reference. Spencer, 1981: 269. Synopsis. Differing from $\underline{L}$ - schlingeri externally 
only by different arrangement of orbital bristles and yellow patches at hindcorners of mesonotum being smaller; wing length in male $1.4 \mathrm{~mm}$; male genitalia with aedeagus as in figures $801,802$. Host/Early Stages. Unknown.

Distribution. Virginia.

Reference. Part 2, p. 297.

70 (68). Mesonotum shining black--

$--$

$71(70)$ Mesonotum distinctly matt---- 7

Small species, wing length from $1.8 \mathrm{~mm}$ in male to $2.3 \mathrm{~mm}$ in female--

Larger species, wing length from $2.25 \mathrm{~mm}$ in male to $2.75 \mathrm{~mm}$ in female

72 (71). Mesonotum solidly black----------L. zinniae Spencer Synopsis. Frons 1.5 times width of eye, bright yellow; 3d antennal segment normally yellow (occasionally darker, couplet 41); orbit entirely yellow, hindmargin of eye black to base of vti, this just on yellow; mesonotum moderately shining black, acr in 4 rows; mesopleuron black on lower half, yellow above; legs with femora entirely yellow, tibiae and tarsi brownish yellow; abdomen with tergites yellow laterally, conspicuously yellow bordered in female; wing length from $1.8 \mathrm{~mm}$ in male to $2.1 \mathrm{~mm}$ in female, last section of $M 3+4$ from just over 2 to 2.5 times length of penultimate; male genitalia with aedeagus (figs. 803, 804) having conspicuously strong ventral sclerite; sperm pump with short stalk, blade almost triangular.

Host/Early Stages. Calendula, Heterotheca grandiflora, Zinnia elegans. Larva forming upper surface linear mine.

Distribution. Central California. Reference. Spencer, 1981: 287.

Mesonotum with conspicuous yellow patches at hindcorners--Synopsis. Frons twice width of eye, head yellow, apart from small black area up to vte; mesonotum shining black; femora yellow; mesopleuron black on lower half; wing length from $2.3 \mathrm{~mm}$ in male to 2.5 $\mathrm{mm}$ in female, last sections of $\mathrm{M} 3+4$ about twice penultimate; male genitalia with aedeagus as in figures 805, 806 .

Host/Early Stages. Unknown.

Distribution. North Carolina.

Reference. Part 2, p. 294.

73 (71). Femora entirely yellow------- L. abnormis spencer Synopsis. Frons broad, twice width of eye, yellow, together with gena, face, antenna, and palpus; hindmargin of eye only narrowly black, vte on black, vti on yellow; mesonotum shining black, acr in 4 rows; mesopleuron black on lower $3 \mathrm{~d}$; femora bright yellow, tibiae and tarsi brownish yellow on foreleg, virtually black on midleg and hindleg; wing length in male $2.5 \mathrm{~mm}$, discal cell large, 
last section of $M 3+4$ only slightly more than twice length of penultimate; male genitalia with aedeagus as in figures 807,808 ; surstylus not differentiated, only a few weak hairs at inner margin of epandrium.

Host/Early Stages. Unknown.

Distribution. Central California.

Reference. Spencer, 1981: 209.

Femora black basally---------L. graminacea Spencer

Synopsis. Frons about 1.5 times width of eye, yellow, together with orbit and antenna; hindmargin of eye black to near base of vti but this invariably on yellow; mesonotum deep black, moderately shining from rear, with large yellow patches at hindcorners; side of thorax largely yellow, mesopleuron black along lower margin; legs with femora black basally but up to 0.33 yellow, tibiae and tarsi brownish black; wing length from $2.25-2.4 \mathrm{~mm}$ in male to $2.75 \mathrm{~mm}$ in female; male genitalia with aedeagus as in figures 809,810 ; surstylus not differentiated, only isolated hairs on inner corner of epandrium.

Host/Early Stages. Poaceae (inferred from male genitalia).

Distribution. California; new record: Colorado, Boulder Co., Corona Pass, 10,600 ft, $1 \mathrm{~m}$., 11.VIII.61 (J. G. Chillcott, CNC).

Reference. Spencer, 1981: 237.

74 (70). Femora largely yellow

Femora basically yellow but heavily striated with black

75 (74). Small species, wing length in male up to $1.8 \mathrm{~mm}$;

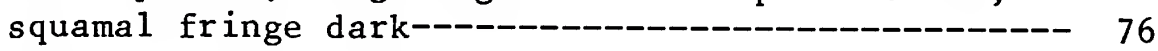

Larger species, wing length in male $2.6 \mathrm{~mm}$; squamal fringe yellow---------L. splendens spencer, n. sp. Synopsis. Frons twice width of eye, 2 ors, 3 ori; head yellowish orange, vte on black ground; mesonotum deep black; femora conspicuously bright yellow; male genitalia with aedeagus as in figures 811,812 .

Host/Early Stages. Unknown.

Distribution. New York.

Reference. Part 2, p. 293.

76 (74). Mesonotum largely matt

Mesonotum shining black

(Both vt normally on black ground, see couplet 63.)

77 (76). Mesonotum predominantly matt but with distinctive subshine------------------L. venturensis Spencer

Synopsis. Head largely yellow, hindmargin of eye black to base of vte; mesonotum black, only weakly shining; mesopleuron black on lower 3d; femora entirely yellow, tibiae and tarsi brownish; wing length in male $1.8 \mathrm{~mm}$, discal cell small, last section of M $3+43$ times length of penultimate; male genitalia with aedeagus as in figures 813, 
814 ; sperm pump with blade not greatly widening. Host/Early Stages. Unknown.

Distribution. Southern California.

Reference. Spencer, 1981: 286.

Mesonotum entirely matt-----L. texella Spencer, n. sp.

Synopsis. Closely resembling $\underline{L}$. venturensis but mesonotum more uniformly matt; wing length $1.5 \mathrm{~mm}$ in male, discal cell very small, last section of M 3+4 3.5 times length of penultimate; male genitalia with aedeagus as in figures 815,816 ; sperm pump with blade conspicuously widening.

Host/Early Stages. Unknown.

Distribution. Texas.

Reference. Part 2, p. 295.

78 (74). 5 orbital bristles----- chemsaki Spencer Synopsis. Head largely yellow, hindmargin of eye black to near base of vti; frons broad, twice width of eye; broad epistoma along mouth margin; gena deep at rear, 0.5 height of eye; mesonotum matt black, with $3+1 \mathrm{dc}$, acr in 4 rows; mesopleuron black on lower half; legs with femora yellow below but black above except at knees, these all bright yellow, tibiae and tarsi brownish black; wing length in male $2.25 \mathrm{~mm}$, last section of $\mathrm{M} 3+4$ just over twice length of penultimate; male genitalia with aedeagus as in figures 817,818 .

Host/Early Stages. Unknown.

Distribution. Northern California.

Reference. Spencer, 1981: 226.

4 orbital bristles----- L. tubula Spencer

Synopsis. Generally resembling L. chemsaki but epistoma narrow; acr only in 2 rows; legs appearing generally dark but femora basically yellow, with extensive black striations above; wing length from $1.9 \mathrm{~mm}$ in male to $2.25 \mathrm{~mm}$ in female; male genitalia with aedeagus (figs. 819, 820) having long paired tubules; sperm pump strongly pigmented, with very broad blade.

Host/Early Stages. Unknown.

Distribution. California.

Reference. Spencer, 1981: 282.

79 (67). Mesonotum shining black---------L. sativae Blanchard (Both vt normally on black ground, see couplet 63.)

Mesonotum distinctly matt------------------- 80

80 (79). Mesonotum uniformly black----------L. lathyri Sehgal

Synopsis. Head yellow, both vt on yellow ground, hindmargin of eye narrowly black beyond vte; mesonotum black, only moderately shining, acr sparse, irregularly in 3 rows; scutellum yellow centrally, broadly black at sides; mesopleuron predominantly yellow, black along lower and front margins; legs with femora yellow, tibiae and tarsi either similar or more brownish; wing length in male 1.6-1.7 mm, last section of M 3+4 2.5 times length of penultimate; male genitalia with aedeagus as in figures 821,822 ; sperm pump large, with broad, dark blade. 
Host/Early Stages. Lathyrus spp. Larva forming large greenish blotch mine (fig. 823); puparium orange, posterior spiracles each with ellipse of about 10 bulbs and minute hornlike projection on anal side.

Distribution. Arkansas (new record); Canada. References. Sehga1, 1971: 334; part 2, p. 289. Mesonotum with conspicuous yellow patches at hind corners

81 (80). Male genitalia with aedeagus having mesophallus long, slender (figs. 824, 825)-----L. endiviae Hering Synopsis. Closely resembling $\underline{L}$. lathyri but clearly distinguished by yellow patches at hindcorners of mesonotum; wing length less than $2 \mathrm{~mm}$, discal cell small, last section of vein $M 3+43$ times length of penultimate; male genitalia with sperm pump (fig. 826) unusually large.

Host/Early Stages. Lactuca, probably Cichorium intybus, possibly other genera of Liguliflorae. Larva forming small, irregular blotch (fig. 827); posterior spiracles each with ellipse of 9 bulbs. Distribution. New records: Maryland, Washington. References. Hering, 1955: 205; part 2, p. 287. Male genitalia with aedeagus having mesophallus shorter, broader (figs. 828, 829)---L. taraxaci Hering Synopsis. Not distinguishable externālly from $\underline{L}$. endiviae but in view of differences in male genitalia certainly distinct.

Host/Early Stages. Taraxacum spp. Larva forming upper surface blotch mine that may be more or less circular (fig. 830) or slightly more elongate (see Spencer, 1976a: fig. 491, Europe); puparium yellowish, posterior spiracles each with ellipse of 8 or 9 bulbs.

Distribution. (?) Washington, Wisconsin (new record); Canada, Europe.

References. Spencer, 1969a: 188; 1976a: 274; part 2 , p. 294.

82 (49). Outer crossvein 1acking-----------L. togata (Melander)

Synopsis. Head largely yellow, 1 ors and 2 or 3 ori; hindmargin of eye only narrowly grayish beyond base of vte, both vt clearly on yellow ground; mesonotum ash gray, acr sparse, in 2 or 3 rows; scutellum bright yellow centrally, narrowly black at sides; legs with femora either entirely yellow or with slight brownish striations; squama yellow, margin and fringe only slightly darker, ochreous; wing length 1.6-1.75 mm; male genitalia with aedeagus as in figures 831,832 .

Host/Early Stages. Baccharis douglasii. Details of leaf mine not known.

Distribution. Central California, Washington.

References. Steyska1, 1980: 141; part 2, p. 295.

83 (82). Squamal fringe pale, yellowish white------------ 
Synopsis. Head yellow, including entire hindmargin of eye; mesonotum matt blackish gray, with large yellow patches at hindcorners, acr in 2 rows; mesopleuron largely yellow, with small black bar along lower margin; legs with femora bright ye1low, tibiae and tarsi brownish; wing length 1.6 $\mathrm{mm}$, last section of vein M $3+43$ times length of penultimate; male genitalia with aedeagus as in figures 833,834 .

Host/Early Stages. Unknown.

Distribution. Idaho, South Dakota; Canada; new record: Montana, Petroleum Co., 1.5 miles S., 5 miles W. of Winnett, 1 m., 30.VII.69 (N. E. Rees, USNM).

References. Frick, 1959: 406; Spencer, 1969a: 176.

84 (83). Frons broad, twice width of eye---L. smilacinae Spencer Synopsis. Head almost entirely yellow; gena broad, almost half vertical height of eye; mesonotum mat but variable, normally pale gray, sometimes more blackish gray; acr also variable, normally in 2 rows or lacking, rarely more numerous; mesopleuron largely yellow, with small dark bar on lower margin; femora bright yellow, tibiae and tarsi brownish black; wing length $1.75-2 \mathrm{~mm}$, discal cell large, last section of $M 3+4$ ittle more than twice length of penultimate; male genitalia with aedeagus as in figures 835, 836; sperm pump with greatly enlarged blade.

Host/Early Stages. Smilacina. Larva forming long, whitish, linear mine (fig. 837); posterior spiracles of larva (puparium) each with ellipse of 8-10 bulbs.

Distribution. Northern California; Canada.

References. Spencer, 1969a: 186; 1981: 273.

-- Frons at most 1.5 times width of eye, not project-
ing above eye-

85 (84). Acr in 4 rows--- 85

Synopsis. Closely resembling $L$. fricki but acr invariably more numerous, in 4 rows in front; wing length $1.25-1.9 \mathrm{~mm}$; male genitalia with aedeagus as in figures 838,839 .

Host/Early Stages. A highly polyphagous species, recorded in Florida on Amaryllidaceae (as Liliaceae), Apiaceae, Asteraceae (particularly Chrysanthemum), Caryophyllaceae, Chenopodiaceae, Cucurbitaceae, Fabaceae, Malvaceae, and Solanaceae; once on Poaceae (Avena). Larva forms upper surface linear mine (fig. 840); puparium yellowish, posterior spiracles each with 3 bulbs (contrast $\underline{L}$. fricki with 4 or 5 ).

Distribution. California, Delaware, District of Columbia, Florida, Indiana, New Jersey, Ohio, Pennsylvania; new records: Arkansas, Garland Co., Hot Springs, 1 m., 17.V.79 (KAS); New York, Long Island, Babylon, 30 spms., 26.XI.35 (S.W. Frost, 
CUI); Wisconsin, Dane Co., Madison, ex Medicago sativa, May 1975 (S. Tavormina); Bahamas, Barbados, Canada, Guyana, Venezuela; as introduction from Florida: Canary Islands, Kenya, Malta, many countries in $W$. Europe (all on cultivated chrysanthemums).

References. Spencer, 1973a: 226; 1981: 281; part 2, p. 297.

86 (85). Wing length 1.4-1.6 mm---------L. fricki Spencer

Synopsis. Head largely yellow, hindmargin of eye only narrowly black, both vt clearly on yellow; mesonotum matt gray, acr in 2 rows, occasionally more numerous; mesopleuron largely yellow, with only small black bar on lower margin; femora entirely yellow; wing with last section of vein M 3+4 3 times length of penultimate; male genitalia with aedeagus as in figures 841,842 ; sperm pump with large blade.

Host/Early Stages. Fabaceae, recorded on Lathyrus, Medicago, Trifolium, and Vicia. Larva forming short linear mine, with frass in greenish-black strips; puparium yellowish orange, posterior spiracles with 4 or 5 bulbs.

Distribution. California, Washington; new record: Wisconsin, Dane Co., Madison, $1 \mathrm{~m} ., 2 \mathrm{f} .$, ex leaf mines on Trifolium repens, $27 . V I I-8 . V I I I .76$ (s. Tavormina); Canada.

References. Spencer, 1969a: 175; 1981: 233. Wing length $1.2 \mathrm{~mm}--$ Synopsis. Closely resembling L. fricki; indistinguishable on external characters, apart from possibly smaller size; male genitalia with aedeagus as in figures 843, 844; sperm pump small, with narrow blade.

Host/Early Stages. Unknown.

Distribution. Kansas.

References. Frost, 1943: 257; Frick, 1955: 88; part 2, p. 284.

Genus Galiomyza Spencer

Galiomyza Spencer, 1981: 288. Type of genus: Agromyza morio (Brischke) (Europe).

The leaf miners on Galium known in Europe and North America are close to but clearly distinct from Liriomyza, lacking the stridulating mechanism and with the scutellum largely black. Their generic position has been problematic, but the new genus
Galiomyza was erected to accommodate them by Spencer, 1981 .

A new species feeding as a leaf miner on Viola is apparently widespread in North America and is described here as Galiomyza violivora. It also lacks the stridulating mechanism, and with no discrete surstylus it cannot be included in Liriomyza. The male genitalia and epandrium differ slightly in form from those of the Galium feeders, but it seems 
appropriate to include the two groups in a single genus. One species is known in Europe as a leaf miner on Viola and was described as Liriomyza violiphaga Hende1, 1932; this has the scutellum yellow. However, it also lacks the stridulating mechanism, and the aedeagus and epandrium (Spencer, 1976a: figs. 515-517) closely resemble those of G. violivora. This species is therefore transferred here to Galiomyza. Another new species in this complex has been discovered in the Austrian Alps and will be described later by Tschirnhaus.
Key to Galiomyza Species
1. 3d antennal segment black--

-- 3d antennal segment yellow--

2 (1). Frons and notopleuron black-------G. turneri spencer Synopsis. Frons dark brown, 1.5 times width of eye, not projecting above eye in profile, orbit black; all antennal segments black; mesonotum and scutellum shining black; humerus and pleura black, only notopleuron slightly paler, brownish; legs black; wing base whitish, squama dark gray, margin and fringe black; halter white; male genitalia with aedeagus as in figures 845, 846; epandrium ( $\mathrm{fig}$. 847) with black projection and several hairs at inner corner and further blunt projection at hindcorner.

Host/Early Stages. Unknown.

Distribution. Central California.

Reference. Spencer, 1981: 290.

Frons brown, notopleuron yellow

Synopiolivora Spencer, n. sp. brom (fig. 848) with frons brownish, broad, twice width of eye; gena 0.33 height of eye, angular at rear; $3 d$ antennal segment black; mesonotum deep black; scutellum sometimes faintly brownish centrally viewed from rear; rear of humerus and notopleuron bright yellow or at least ochreous; legs black but foreknee yellow; squama yellowish gray, margin and fringe black; male genitalia with aedeagus as in figures 849,850 ; sperm pump as in figure 851; epandrium ( $\mathrm{fig}$. 852) with curving process attached at midpoint of inner margin.

Host/Early Stages. Viola sororia. Larva forming white blotch mine ( $\overline{\text { fig. }} 8 \overline{53}$ ) with finely scattered black frass; puparium brownish orange, posterior spiracles each with 3 bulbs.

Distribution. Mississippi, Pennsylvania.

Reference. Part 2, p. 298.

3 (1). Male genitalia with distiphallus enlarged (fig. 854), extended centrally (fig. 855)----G. galiivora (Spencer)

Synopsis. Frons sooty black, orbit black, weakly shining, pronounced, slightly raised above frons; 2 ors, 2 ori, all approx. equal; orbital setulae sparse, reclinate; gena brown, angular at rear, about 0.20 height of eye; $3 \mathrm{~d}$ antennal segment bright yellow, 2d darker, yellowish gray, 1st black; face and palpus black; mesonotum shining 
black, with $3+1 \mathrm{dc}$, acr in 4 rows; notopleuron predominantly dark, at most faintly paler, pleura otherwise black; legs black; wing base yellowish, margin and fringe black; halter bright yellow; male genitalia with aedeagus as in figures 854, 855, with single pair of ventral lobes, fully developed bladder enclosed by basal sclerites; sperm pump with narrow blade and stalk, base well developed; epandrium (fig. 856) with single short spine at inner corner, similar one at midpoint, and longer one below.

Host/Early Stages. Galium boreale, G. triflorum, and probably other Galium spp. (in Europe G. rotundifolium and G. rubioides). Larva forms upper surface linear mine; puparium brownish, posterior spiracles each with 3 bulbs.

Distribution. Minnesota (new record); Canada, Corsica.

References. Spencer, 1969a: 199; part 2, p. 298. Male genitalia with distiphallus smaller ( $\mathrm{fig}$. 857) not extended centrally (fig. 858)---

-- - - vockerothi spencer, n. sp.

Synopsis. Externally as in $\bar{G}$. galiivora; male genitalia with aedeagus as in figures 857, 858; epandrium (fig. 859) with single strong projection at inner corner and slightly stronger one at midpoint.

Host/Early Stages. Unknown, probably Galium.

Distribution. North Carolina.

Reference. Part 2, p. 299.

\section{Genus Haplopeodes Steyskal}

Haplopeodes Steyskal, 1980. Type of genus: Phytomyza minuta Frost, 1924.

This genus was erected to provide a new name for Haplomyza Hendel, 1914 , after it was discovered that the type of Haplomyza, Antineura togata Melander, 1913, correctly belongs in Liriomyza.

Frick (1959) differentiated Haplomyza from Liriomyza by the combination of four characters: Mesonotum matt gray, acrostichals in two rows, outer crossvein lacking, and only a single upper orbital present. This concept was amended by Spencer (1963a) when two species were discovered in Brazil with the mesonotum black.

The essential differences between
Haplopeodes and Liriomyza are that the stridulating mechanism of the males of Liriomyza is lacking and the male genitalia are simplified and reduced (see fig. 860); the sperm pump is narrow and elongate (fig. 870); and the surstylus bears three or four minute bristles (fig. 866).

Haplopeodes is known only in North and South America, and 12 species have now been described. The larva forms a short, irregular, linear mine, and hosts are in the Amaranthaceae, Chenopodiaceae, Portulacaceae, and Solanaceae. Of the four species known in the United States, two feed on Amaranthaceae (one of these also on Chenopodiaceae), one on Portulacaceae, and one on Solanaceae. 
Key to Haplopeodes Species
1. Mesonotum yellow adjoining scutellum, at least at hindcorners, inner postalar on yellowMesonotum largely gray adjoining scutellum, with at most small yellow patches at hindcorners, inner postalar on dark area

2 (1). Mesonotum entirely yellow adjoining scutellum, with central yellow area extending forward slightly beyond level of dc 1--

Synopsis. Frons, orbit, and all antenna $\bar{l}$ segments entirely yellow; 1 ors, 3 ori; acr in 2 rows; wing length $1.5 \mathrm{~mm}$; male genitalia with aedeagus having extended distal tubule (fig. 860); hypandrium with broad lobe at base (fig. 861); surstylus with 4 closely adjoining teeth (fig. 862).

Host/Early Stages. Physalis viscosa (Texas), Solanum triquetrum (Texas), s. umbelliferum (California). Larva forming short linear mine.

Distribution. California; new records: Texas, Travis Co., Austin, $3 \mathrm{~m} ., 1 \mathrm{f}$., ex leaf mines on Solanum triquetum, 9.IV.80;2 m., ex leaf mines on Physalis viscosa, 21.IV.80; (all S. Tavormina). References. Steyskal, 1980: 146; part 2, p. 299. Note. In all specimens seen from Texas and in some of type series from California, mesonotum is distinctly yellow adjoining scutellum (particularly when viewed from rear). Only in darkest specimens does mesonotum appear largely gray (see couplet 4) and such specimens may be difficult to differentiate from $\underline{H}$. minutus.

Mesonotum dark centrally adjoining scutellum but inner postalar arising from yellow area

Synopsis. Small species, wing $\bar{l}$ - palliatus (Coqu $1.5 \mathrm{~mm}$; all antennal segments and entire hindmargin of eye yellow; mesonotum with $3+1$ well-developed dc, largely grayish, with large yellow area at hindcorners; acr irregularly in 2 rows; scutellum largely yellow; male genitalia unknown.

Host/Early Stages. Portulaca sp. No information on leaf mine or early stages.

Distribution. New Mexico.

Reference. Steyskal, 1980: 149 (including statement that it is not certain that this species correctly belongs in Haplopeodes).

3 (1). Surstylus with 3 teeth and 1 seta widely spaced (fig. 865)-C. philoxeri (Spencer)

Synopsis. Head (fig. 863) with eye slanting, gena extended at rear, 0.33 height of eye; frons orange yellow, orbit similar or more grayish; 3d antennal segment orange yellow or slightly brownish; mesonotum uniformly grayish black, only faintly paler at hindcorner; humerus yellow behind; notopleuron yellowish gray, mesopleuron grayish below, more yellow above; scutellum yellow centrally, variably grayish at sides; wing length from $1.25 \mathrm{~mm}$ in male to $1.5 \mathrm{~mm}$ in female; squama silvery white, fringe pale but 
margin slightly darker; meaningful illustration of male genitalia aedeagus not possible with available material; sperm pump with long, narrow blade;

hypandrium (fig. 864) broadened and with membranous lateral extension at midpoint.

Host/Early Stages. Philoxerus vermicularis. Larva forming mine deep within leaf, preparing exit hole and then pupating some distance back in mine; puparium white, thin walled, posterior spiracles each with ellipse of 10-12 minute bulbs.

Distribution. Florida.

References. Spencer and Stegmaier, 1973: 112; Steyskal, 1980: 150.

-- Surstylus with 2-4 teeth very close together (figs. $862,866)---14$

4 (3). Surstylus with 2 or 3 teeth (fig. 866); hypandrium with narrow lobe at base (fig. 867)-- - - minutus (Frost) Synopsis. Agreeing closely on external characters with $\underline{H}$. philoxeri, not reliably distinguishable; male genitalia with aedeagus having distiphallus extended as narrow tubule (figs. 868, 869); sperm pump long, narrow (fig. 870).

Host/Early Stages. Amaranthus species, Chenopodium species. Larva forming initial linear mine, which normally develops later into irregular blotch; puparium pale yellowish brown, posterior spiracles each with ellipse of 9-12 minute bulbs.

Distribution. California, Florida, North Dakota, Texas; Canada.

References. Spencer, 1969a: 201 (as Haplomyza togata); Spencer and Stegmaier, 1973: 112 (as Haplomyza minuta); Steyskal, 1980: 148 .

Surstylus with 4 closely adjoining teeth (fig. 862); hypandrium with broad lobe at base (fig. 861)-- - - (Mesonotum normally yellow adjoining scutelellum, see couplet 1.)

Genus Calycomyza Hendel

Dizygomyza (Calycomyza) Hende1, 1931: 65. Type of subgenus: Agromyza artemisiae Kaltenbach, 1856 (Europe). Phytobia (Calycomyza) Frick, 1952a: 394.

Calycomyza Nowakowski, 1962: 97.

This genus is characterized by having the frons and notopleural area yellow and the scutellum entirely black. The only known exception is Calycomyza obscura, in which both frons and notopleuron are dark, but its correct generic position is indicated by the male terminalia. The aedeagus itself in this genus shows some variation, with the distiphallus normally short and compact (figs. 875, 881), more rarely with extended tubules (figs. $900,933)$. However, a consistent generic character in the terminalia is the presence of a patch of strong bristles on the inner hindcorner of the epandrium (fig. 924).

Identification of many species is virtually impossible on external characters, but they can normally be 
separated by the male genitalia. In his revision of North American Calycomyza species, Frick (1956) attempted to differentiate all species on minor color differences, particularly the degree of darkening of the orbit, but in practice his key has not proved reliable. With additional species now known, 10 species in what may loosely be termed the C. malvae group are included in couplets $21-30$, separated only by differences in the aedeagus.

Steyskal (1973a) examined for the first time the male genitalia of $\underline{C}$. jucunda, and it then became clear that this species had been misinterpreted. The species feeding commonly on several genera of Asteraceae and common in Florida and California is

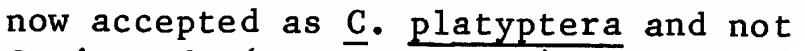
C. jucunda (Spencer, 1981). The synonymy of $\mathrm{C}$. coronata with $\mathrm{C}$. jucunda established by Frick (1956: 288) is tentatively accepted. Another species, closely related to $\underline{\mathrm{C}}$. jucunda, is $\underline{\text { C. menthae. }}$

Illustrations are given of the genitalia of the holotypes of $\mathrm{C}$. jucunda (figs. 884, 885) and C.. coronata (figs. 886, 887) and of a topotypical paratype of $\mathrm{C}$. menthae (figs. 888, 889). As will be seen, the distiphallus differs only in minor detail. A final decision as to whether the differences noted are specific or within the range of variation of a single species feeding on Lamiaceae can only be made when additional reared material becomes available; therefore, no nomenclatural changes are proposed now.

All known species of Calycomyza are leaf miners in the six families-Asteraceae, Boraginaceae, Convolvulaceae, Lamiaceae, Malvaceae, and Verbenaceae. Of the 30 species in the United States, the host is known of 20; of these, 10 feed on the As teraceae.

Calycomyza is a relatively small genus of 54 known species occurring predominantly in the Nearctic and Neotropical Regions. Only single endemic species are known in Africa and in Europe. Of the Nearctic species, only $\mathrm{C}$. humeralis (semicosmopolitan) and C. solidaginis are present also in Europe. Only two

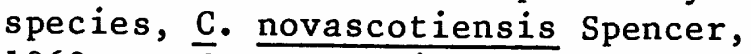
1969a, and c. : sonchi Spencer, 1969a, are endemic in Canada. Two new species are described here (part 2, p. 299).
Key to Calycomyza 1 . Species
1. Squamal fringe at least partially pale, white or yellow------------ 2

-- Squamal fringe dark, brown or black---_--_------ 10

2 (1). Discal cell unusually small, last section of vein M 3+4 3-4 times length of penultimate; minute species, wing $1.1-1.25 \mathrm{~mm}$ long------C. minor Spencer Synopsis. Frons and gena orange yellow, upper orbit conspicuously black; 1 ors, 2 ori; gena 0.33 height of eye; all antennal segments black, face grayish, more yellow below; mesonotum moderately shining black; humerus, notopleuron, and hindcorner of mesopleuron grayish yellow; legs black; squama and fringe whitish yellow; wing length from $1.1 \mathrm{~mm}$ in male to $1.25 \mathrm{~mm}$ in female, outer crossvein sometimes only slightly distad of inner; male genitalia with aedeagus as in figures 871,872 .

Host/Early Stages. Conyza canadensis. Larva 
forming upper surface blotch that may completely fill smaller leaves, pupating in mine; puparium blackish brown, posterior spiracles on conical projections, each with 5 bulbs.

Distribution. Florida; new records: South Carolina: Aiken Co., Aiken, 1 m., 12.VI.57; Jasper Co., Coosawhatchie, 1 f., 1.I.72; (both J. R. Vockeroth, $\mathrm{CNC}$ ).

Reference. Spencer and Stegmaier, 1973: 85.

-- Discal cell larger, last section of vein $M 3+4$ at most 3 times length of penultimate; larger species, wing $1.25 \mathrm{~mm}$ or longer-----_----- 3

3 (2). 3d antennal segment round--_-_---_-_-_-_-_-_-- 4

-- 3d antennal segment angulate at upper corner------- 9

4(3). Foreknee yellow---_- 5

5 (4). All antennal segments black-------- C. irwini Spencer Synopsis. Frons, gena, and face pale yellow, frons 1.25 times width of eye; eye large, upright, gena 0.25 height of eye, not extended at rear; $3 \mathrm{~d}$ antennal segment with distinct fringe of short pubescence; mesonotum shining black, with 2 strong dc, 3d, if present, scarcely differentiated from acr; squama and fringe whitish yellow; wing length $1.7-2.1 \mathrm{~mm}$, last section of $M 3+4$ twice length of penultimate; male genitalia with aedeagus (figs. $873,874)$ having mesophallus conspicuously curving and swollen basally; anterior pair of ventral

lobes long, slender, forming complete loop, posterior pair short.

Host/Early Stages. Unknown.

Distribution. Southern California (Riverside Co.). Reference. Spencer, 1981: 305.

1 st and $2 \mathrm{~d}$ antennal segments yellow

-

Synopsis. Agreeing in all essential characters

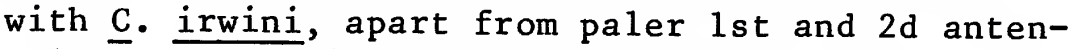
nal segments; wing length from $1.25 \mathrm{~mm}$ in male to $2 \mathrm{~mm}$ in female; male genitalia with aedeagus (figs. 875, 876) having mesophallus short, conspicuously enlarged basally.

Host/Early Stages. Lantana camara, Lippia helleri, Verbena 1itoralis. Larva forming irregular upper surface blotch (fig. 877); puparium reddish brown to more yellowish, posterior spiracles each with 3 bulbs.

Distribution. Florida, Texas, doubtless present in other Gulf States; Bahamas, Trinidad, Venezuela. References. Frick, 1956: 287; Spencer and Stegmaier, 1973: 78.

6 (4). Mesonotum strongly shining black---------------

---

Synopsis. Frons yellow, narrow, little over width of eye; all antennal segments black; orbit largely yellow, normally darkened to level of upper ors; face black in upper half, yellow below; notopleur- 
on, rear of humerus, upper margin of mesopleuron yellow; squama and fringe whitish yellow; wing length $1.5-2 \mathrm{~mm}$, discal cell small, last section of M 3+4 3 times penultimate; male genitalia with aedeagus (figs. 878, 879) having distal process short, mesophallus not greatly widening toward rear; short anterior ventral lobes distinctly broadened, posterior lobes narrow, not fused (contrast C. jucunda, figs. 884, 885).

Host/Early Stages. Asteraceae, recorded in Florida (as C. jucunda) on Ambrosia, Aster, Bidens, Gnaphalium, Helianthus, Solidago, Xanthium, Zinnia (Stegmaier, 1967a), caught in California in association with Artemisia; additional hosts recorded by Frick (1956): Arctium, Artemisia douglasiana, A. vulgaris, Baccharis viminea, Conyza canadens is, Grindelia squarrosa, Heterotheca grandiflora (as C. jucunda). Larva forming whitish blotch, frequently toward base of leaf, with characteristic irregular offshoots (fig. 880); puparium brownish, attached to pile of frass at center of mine, posterior spiracles each with 3 bulbs; young leaves become characteristically deformed as result of larval mining activity.

Distribution. Confirmed from California, Florida; new record: Mississippi, Washington Co., Stoneville, $8 \mathrm{~m}$., $6 \mathrm{f}$,, ex Xanthium strumarium, 3.IX. 71 (K. E. Frick); probably widespread in other southern States; numerous States listed by Frick (1956: 288) require confirmation; Cuba, Grand Caman Is., Jamaica.

References. Frick, 1956: 288 (as Phytobia (Cal.) jucunda); Spencer and Stegmaier, 1973: 77 (as C. jucunda); Spencer, 1981: 307; part 2, p. 300 (under C. jucunda).

Mesonotum somewhat matt blackish gray, particularly when viewed from front

7 (6). Male genitalia with aedeagus (figs. 881, 882) having mesophallus short, distinctly widening at rear, posterior paired ventral lobes long, slender, not fused; basal sclerites fully fused-- - - - promissa (Frick)

Synopsis. Closely resembling c. platyptera but mesonotum less shining; wing length 1.9-2.3 mm. Host/Early Stages. Aster chilensis, A. 1ateriflorus, and certainly other Aster spp. Larva forming white blotch, circular but generally irregular at edges; puparium attached to raised pile of frass in center of mine ( $\mathrm{fig} .883$ ); larval posterior spiracles each with 5-8 bulbs.

Distribution. California, Florida; new records: Louisiana, Rapides Co., 11 miles S.W. of Alexandria, 3 m., 21. III.60 (J. G. Chillcott, CNC); New York, Ithaca, 1 m., 1 f., 5.VIII.69 (A. G. Wheeler, CUI); Canada. 
References. Frick, 1956: 287; Spencer, 1969a: 153;

1981: 310; Spencer and Stegmaier, 1973: 87.

Male genitalia with aedeagus having mesophallus longer, more slender (figs. 884, 885)-----------

8 (7). Male genitalia with aedeagus (figs. 884, 885)

having distiphallus only slightly extended at rear; posterior ventral sclerites fully fused, forming large, flaplike process (see Steyska1, 1973a: fig. 1 , rear of aedeagus in ventral view); basal sclerites membranous between lateral margins---------C. jucunda (Wulp)

Synopsis. Closely resembling c. platyptera on external characters but mesonotum less shining; wing length in unique holotype $2.2 \mathrm{~mm}$, last section of M 3+4 2-2.5 times length of penultimate.

Host/Early Stages. Unknown.

Distribution. Pennsylvania, Wisconsin.

References. Steyska1, 1973a: 191; part 2, p. 300. Male genitalia with aedeagus having distiphallus relatively long, distinctly extended at rear (figs. 888, 889); posterior ventral sclerites fully fused; basal sclerites more strongly sclerotized at lateral margins----(Squamal fringe sometimes appearing partially dark, see couplet 30. )

Synopsis. Closely resembling C. platyptera apart from less shining mesonotum; wing length 1.9-2.2 $\mathrm{mm}$, last section of vein $M 3+42.5-3$ times length of penultimate.

Host/Early Stages. Hyptis mutabilis, Monarda fistulosa, Monardella odoratissima, Ocimum sp. Larva forming brownish blotch mine adjoining margin of leaf (fig. 890), with frass in scattered black grains, pupation externally; puparium brown, posterior spiracles each with 3 bulbs.

Distribution. Florida, California (new record); Canada.

References. Spencer, 1969a: 152; Spencer and Stegmaier, 1973: 83; part 2, p. 301.

9 (3). Face largely black-- humeralis (Roser) Synopsis. Frons bright yellow, upper orbit broadly black to upper ors, not infrequently darkened to area of upper ori; $3 \mathrm{~d}$ antennal segment ovoid, angular at upper corner (fig. 891); face black or gray, at most slightly yellow below; mesonotum shining black; legs entirely black; squama and fringe white; wing length $2-3.5 \mathrm{~mm}$; male genitalia with aedeagus (figs. 892, 893) having mesophallus narrowing distally and only slight ventral curvature.

Host/Early Stages. Aster, Conyza, Heterotheca, Madia, Solidago; in Europe, Bellis and Erigeron; in Argentina, Tithonia, Zinnia. Larva forming sma11, elongate blotch, frequent $1 y$ associated with midrib, pupating internally on mass of black frass; posterior spiracles at end of conspicuous 
dorsal projection, each with 8 bulbs in 2 groups, upper ones more elongate (see Frick, 1956: fig. 4, B).

Distribution. Widely distributed through most of United States; Argentina, Canada, Chile, Europe.

References. Frick, 1956: 290; Spencer, 1969a: 149, 302 .

Face largely yellow--------C. solidaginis (Kaltenbach)

Synopsis. As in $\underline{C}$. humeralis a part from largely yellow face; male genitalia with aedeagus as in figures $894,895$.

Host/Early Stages. Solidago spp. Leaf mine and larval characters essentially as in C. humeralis. Distribution. According to Frick (1959), "found throughout United States"; Canada, Europe.

References. Frick, 1956: 292; 1959: 392; Spencer, 1969a: 155; 1981: 312; Spencer and Stegmaier, 1973: 89 .

10 (1). Frons and notopleuron largely dark---c. obscura spencer Synopsis. Frons brownish black or in palest specimens brownish yellow, orbit more shining black; gena narrow, 0.125 height of eye; all antennal segments black; mesonotum shining black; pleura normally entirely black but in paler specimens, particularly females, humerus and notopleuron dull yellowish; legs black; squama gray, fringe jet black; halter yellow; wing length from $1.6 \mathrm{~mm}$ in male to $2 \mathrm{~mm}$ in female, last section of vein $M 3+4$ twice length of penultimate; male genitalia with aedeagus as in figures 896,897 ; surstylus and epandrium with conspicuous bristles (fig. 898). Host/Early Stages. Unknown. Distribution. Florida; Costa Rica, Jamaica. Reference. Spencer and Stegmaier, 1973: 86. Frons and notopleuron bright yellow---------- 11

11 (10). Mesonotum distinctly matt grayish----------------- 12

-- Mesonotum black, at least moderately shining------- 13

12 (il). Large species, wing length $2.5-3.25 \mathrm{~mm}-$ C. gigantea (Frick)

Synopsis. Upper orbit darkened; mesonotum matt grayish black; legs either entirely black or foreknee slightly paler, yellowish; squama gray, margin and fringe black; male genitalia with aedeagus (figs. 899, 900) having distiphallus conspicuously divided.

Host/Early Stages. Unknown.

Distribution. California, Illinois, Maryland, Virginia; new records: New York, Tompkins Co., Danby, $1 \mathrm{~m}$., 3.VI.64 (L. L. Pechuman, CUI); Virginia, Montgomery Co., Blacksburg, 1 f., 28.V.62 (J. G. Chill cott); Canada.

References. Frick, 1956: 296; Spencer, 1969a: 149; 1981: 301 .

Smaller species, wing length $1.6 \mathrm{mm--------------}$

Synopsis. Orbit darkened at least to upper, nor- 
mally to lower ors; 3d antennal segment small, round; face grayish but yellow below; acr sparse, in only 4 rows; legs largely black, foreknee faintly yellowish; discal cell small, last section of $M 3+4$ slightly more than twice length of penultimate; male genitalia with aedeagus as in figures 901, 902 .

Host/Early Stages. Ipomoea alba. Larva forming circular blotch, with strips of black frass deposited in almost regular spiral (fig. 903); puparium pale, yellowish brown, posterior spiracles each with 3 bulbs, outer long, hooklike.

Distribution. Florida.

Reference. Spencer and Stegmaier, 1973: 90.

13 (11). Hindcorners of mesonotum broadly yellow, black only narrowly reaching scutellum centrally

Synopsis. Large species, wing length in male C. flavinotum (Frick) 2.3-2.6 mm, in female $2.75-3 \mathrm{~mm}$; orbit entirely yellow, even vti just on yellow ground; $3 d$ antennal segment black, 1 st and $2 \mathrm{~d}$ partially yellowish; legs black but knee on forefemur bright yellow; mesonotum shining black but hindcorners yellow, with inner postalar on yellow and dc 1 at margin of black and yellow; male genitalia with aedeagus as in figures $904,905$.

Host/Early Stages. Ageratina, Arctium, Eupatorium (not Viburnum pubescens as recorded by Frick, 1959: 390). Larva forming whitish blotch mine, with several larvae in same leaf, mines frequently merging and giving appearance of single very large mine; puparium reddish brown, posterior spiracles each with 3 bulbs, prominent anal lobes below (see Frick, 1956: fig. 3, c).

Distribution. Massachūsetts, New York, Pennsy1vania; new records: Maine; Minnesota, Minneapolis, Minnehaha Creek, leaf mines on Arctium minus, 10.IX.78 and 29.VI.80; $1 \mathrm{m.,} 2$ f., ex Arctium and Eupatorium rugosum, July 1980 (KAS); Wisconsin, Dane Co., Madison, 1 f., reared from Arctium minus, 13.IX.76 (S. Tavormina); Canada; (not Brazil, Jamaica, and Venezuela as stated by Spencer, 1963a: 343, see Spencer and Stegmaier, 1973: 184).

References. Frick, 1956: 297; Spencer, 1969a: 147; Spencer and Stegmaier, 1973: 184 (under C. eupatorivora Spencer).

-- Hindcorners of mesonotum at most narrowly and faintly yellow, mesonotum broadly black adjoining

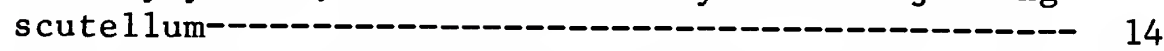

14 (13). Orbit entirely yellow---- c. frickiana Spencer, n. sp. Synopsis. Frons narrow, litte wider than eye, normally 2 ors, 2 ori; mesonotum shining black, with only inconspicuous yellow patches at hindcorners adjoining scutellum; legs largely black, foreknee only inconspicuously yellowish; male genitalia with aedeagus as in figures 906, 907. 
Host/Early Stages. Bidens, Helianthus, Rudbeckia. Larva forming greenish blotch mine.

Distribution. Illinois, Indiana, Maryland, Minnesota, New York, Pennsylvania, Virginia, (?) Wisconsin.

References. Frick, 1956: 298 (as C. allecta); part 2 , p. 299.

Orbit partially dark, at least to upper ors--------

15 (14). Discal cell large, last section of vein $M 3+4$ thus relatively short, less than twice length of penultimate---------------------------

Discal cell smaller, last section of $M 3+4$ at least twice length of penultimate-C-

16 (15). Last section of M $3+41.5$ times length of penultimate----

Synopsis. Orbit conspicuously black, normally to midway between upper and lower ori; face grayish above, yellow below; 3d antennal segment small, round; mesonotum moderately shining black; legs with foreknee slightly yellowish; wing length 1.9$2.2 \mathrm{~mm}$, last section of M $3+41.5$ times length of penultimate; squama yellowish gray, margin and fringe black; male genitalia with aedeagus as in figure 909.

Host/Early Stages. Mikania batatifolia. Larva forming whitish elongate blotch (fig. 910); posterior spiracles of larva (puparium) on distinctly elongated extension, each with 3 bulbs, terminal one greatly enlarged.

Distribution. Florida; Jamaica. Reference. Spencer and Stegmaier, 1973: 84. Last section of $M 3+41.8$ times length of penultimate----Synopsis. Medium-sized species, wing length 1.75$2.5 \mathrm{~mm}$, discal cel1 large, last section of $\mathrm{M} 3+4$ thus short, less than twice length of penultimate; orbit conspicuously black, at least to lower ori, sometimes to base of antennae; legs entirely black; mesonotum shining black; male genitalia with aedeagus (figs. 911, 912) having rear ventral sclerites separate and basal sclerites fully fused. Host/Early Stages. Senecio triangularis (California); in Alaska Petasites and $\underline{S}$. lugens. Larva forming pale green blotch, up to $\overline{4}$ larvae in single leaf; posterior spiracles each with 3 bulbs. Distribution. California, Florida, Idaho, Washington; Canada.

References. Frick, 1956: 295; Spencer and Stegmaier, 1973: 80; Spencer, 1981: 306.

17 (15). Orbit darkened to lower ori-----C. cynoglossi (Frick)

Synopsis. Small species, wing length from $1.75 \mathrm{~mm}$ in male to $2.25 \mathrm{~mm}$ in female, last section of M 3+4 from 2 to 3 times length of penultimate; orbit conspicuously darkened, at least slightly to lower ori; mesonotum moderately shining black; legs almost completely black; squama with margin 
and fringe black; male genitalia with aedeagus (figs. 913, 914) having rear ventral lobe only partially fused, basal sclerites discrete.

Host/Early Stages. Cynoglossum. Details of leaf mine and larva not recorded.

Distribution. Indiana; (not Quebec as recorded by Frick, 1956; see Spencer, 1969a: 145).

Reference. Frick, 1956: 295.

-

Synopsis. Frons yellow, orbit black to upper ors; all antennal segments black, face yellow; mesonotum shining black; legs largely black, foreknee at most faintly yellowish; discal cell small, last section of vein $M 3+42.5$ times length of penultimate; male genitalia with aedeagus as in figures 915,916 .

Host/Early Stages. Sida spp. Larva forming short, irregular elongate blotch mine (fig. 917); puparium pale, yellowish brown, posterior spiracles each with 3 bulbs.

Distribution. Florida; Bahamas, Dominica, Dominican Republic.

Reference. Spencer and Stegmaier, 1973: 88.

19 (18). Relatively large species, wing length from 2.3-2.5 $\mathrm{mm}$ in male to $2.7 \mathrm{~mm}$ in female---- 20

Orbit darkened to lower ors

C. orientalis Spencer, n. sp.

Synopsis. Large species, wing length $2.4-2.6 \mathrm{~mm}$; orbit consistently darkened to lower ors; mesonotum deep black, shining; legs entirely black; male genitalia with aedeagus (figs. 918, 919) having rear ventral lobe entire, flaplike, basal sclerites discrete.

Host/Early Stages. Unknown.

Distribution. Maine, New York, Tennessee, Virginia. Reference. Part 2, p. 301. Orbit darkened only to upper ors------------

Synopsis. Large species, wing length $2.5-2.7 \mathrm{~mm}$, last section of $M 3+4$ slightly longer than penultimate; $2 \mathrm{~d}$ and $3 \mathrm{~d}$ antennal segments black, 1 st slightly yellowish; mesonotum black but distinctly matt; legs entirely black; male genitalia with aedeagus (figs. 920, 921) conspicuous1y asymmetrical in both side and ventral views, ssp. artemisiae, symmetrical in ventral view, ssp. marcida (figs. 922, 923); hindcorner of epandrium with conspicuous patch of bristles, typical of genus (fig. 924).

Host/Early Stages. Artemisia, Eupatorium. Larva forming large, whitish blotch mine (fig. 925), 
pupating externally; puparium brown, posterior spiracles each with 3 bulbs.

Distribution. California, Pennsylvania; new records: Minnesota, Minneapolis, Minnehaha Creek, 1 f., 24.VII.80, ex leaf mine on Ageratina altissima, coll. 29.VI.80 (KAS); Wisconsin, Dane Co., Madison, 2 m., 2 f., ex Eupatorium rugosum, Sept. 76 (S. Tavormina); Canada; records given by Frick (1956: 294) require confirmation.

Reference. Spencer, 1981: 294.

21 (19). Male genitalia with aedeagus having paramesophalli strongly developed (figs. 926, 927)-_-_-_-_---

- enceliae Spencer

Synopsis. Wing length $2-2.3 \mathrm{~mm}$, last section of vein $M 3+4$ twice length of penultimate; mesonotum only moderately shining; legs black, foreknee at most narrowly yellow.

Host/Early Stages. Encelia californica. Adults common on this host in March 1977 in southern California but no mines found (KAS).

Distribution. California.

Reference. Spencer, 1981: 300 .

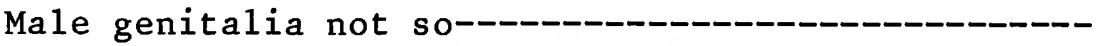

22 (21). Frons narrow, equal to width of eye--

C. michiganensis Steyskal

Synopsis. Generally resembling C. malvae; wing length in unique holotype $2 \mathrm{~mm}$; male genitalia with aedeagus (figs. 928, 929) having distiphallus relatively large, with central membranous area conspicuous in side view.

Host/Early Stages. Unknown.

Distribution. Michigan.

Reference. Steyskal, 1972b: 3 .

-- Frons broader, approx. 1.5 times width of eye-----

23 (22). Male genitalia with mesophallus short, distiphallus with lateral extension at each side (figs. 930, 931)------

Synopsis. Wing length $1.6-2 \mathrm{~mm}$, last section of vein $M 3+42.5$ times length of penultimate; orbit black to upper ors or slightly beyond; mesonotum moderately shining black; legs entirely black; squama gray, margin and fringe deep black; male genitalia with aedeagus as in figures 930, 931. Host/Early Stages. Abutilon, Althaea, Malva, Malvastrum, Sida, and probably other genera of Malvaceae. Larva forming long narrow leaf mine (fig. 932); puparium yellowish brown, posterior spiracles each with 3 bulbs.

Distribution. Florida, Indiana, New York, Pennsylvania; new records: Mississippi, Bolivar Co., Shaw, $2 \mathrm{~m} ., 1 \mathrm{f}$,, ex Abutilon theophrasti, 24.X.79 (H. Walker); Wisconsin, Dane Co., Madison, $1 \mathrm{~m}$. , ex Abutilon, 26.VII.76 (S. Tavormina); Bahamas, Brazil, Canada, Jamaica.

References. Frick, 1956: 298; Spencer, 1969a: 146 (as C. althaeae); Spencer and Stegmaier, 1973: 81. 
24 (23). Male genitalia with distiphallus having long, paired narrow tubules (figs. 933, 934)

C. hyptidis Snencer

Synopsis. Wing length $2 \mathrm{~mm}$; mesonotum shining black; legs black, foreknee bright yellow.

Host/Early Stages. Hyptis pectinata. Larva forming upper surface star-shaped blotch (fig. 935) with frass all deposited in center.

Distribution. Florida; Brazil, Dominica, Venezuela.

References. Spencer and Stegmaier, 1973: 74; Spencer, 1973b: 42.

Male genitalia not so

25 (24). Male genitalia with mesophallus short, rounded, distiphallus with extended, short tubules (figs. 936, 937)-- i pomaeae (Frost)

Synopsis. Wing length 1.8-2.2; mesonotum only moderately shining appearing more matt viewed from front; legs black, foreknee slightly yellowish; squama yellowish gray, margin and fringe black. Host/Early Stages. Ipomoea spp., recorded on $I$. alba, I. batatas, I. cathartica, and I. tiliacea. Larva forming irregular elongate blotch (fig. 938); puparium dark brown.

Distribution. Florida; Brazil, Dominica, Jamaica, Puerto Rico, Venezuela.

References. Spencer, 1969a: 150; 1973b: 44; Spencer and Stegmaier, 1973: 76.

Male genitalia not so-

26 (25). Male genitalia with aedeagus having mesophallus short, broad, conspicuously bending ventrally at rear (figs. 939, 940)---_----C. ambrosiae (Frick)

Synopsis. As in C. malvae; wing leñgth normally slightly less than $2 \mathrm{~mm}$.

Host/Early Stages. Ambrosia artemisiifolia, A. trifida, probably other Ambrosia spp. Larva forming small greenish blotch mine (fig. 941).

Distribution. California, Florida, Indiana, Tennessee, doubtless widespread in south.

References. Frick, 1956: 299; Spencer and Stegmaier, 1973: 72; Steyskal, 1973: 329.

27 (26). Male genitalia with distiphallus having short central extensions (figs. 942, 943)

--

Synopsis. Wing length $1.6-1.9 \mathrm{~mm}$; frons orange yellow, orbit conspicuously darkened at least to upper ors; mesonotum shining black; legs black, foreknee narrowly yellowish.

Host/Early Stages. Duranta repens. Larva forming upper surface whitish blotch (fig. 944); puparium reddish brown with band of short stout spinules around center of each segment ( $\mathrm{fig}$. 945). Distribution. Florida. Reference. Spencer and Stegmaier, 1973: 73. 
28 (27). Male genitalia with aedeagus conspicuously asymmetrical, mesophallus bending at right angles (figs. 946, 947)--- melantherae Spencer Synopsis. Wing length about $1.7 \mathrm{~mm}$; male genitalia with epandrium and surstylus as in figure 948. Host/Early Stages. Melanthera deltoidea. Larva forming regular primary blotch.

Distribution. Florida; Bahamas.

Reference. Spencer and Stegmaier, 1973: 82.

29 (28). Male genitalia wot soconspicuously black centrally (figs. 949, 950)----

Synopsis. Wing length $1.75-2 \mathrm{~mm}$; me ately shining; legs black, foreknee narrowly ye1lowish; male genitalia with sperm pump as in figure 951 .

Host/Early Stages. Verbena sp. Details of leaf mine not recorded.

Distribution. New Mexico; records given by Frick (1956: 300), particularly of northern States, require confirmation.

References. Frick, 1956: 300; Spencer, 1963a: 349.

30 (29). Male genitalia with distiphallus large, 1ittle 30 shorter than mesophallus (figs. 952 ,

-

Synopsis. Wing length in male $2 \overline{\mathrm{mm}}$; legs entirely black; discal cell small, last section of vein M $3+4$ slightly more than 2.5 times penultimate. Host/Early Stages. Unknown.

Distribution. California.

Reference. Spencer, 1981: 298.

-- Male genitalia with distiphallus at most half length of mesophallus (figs. 888, 889)---

(Squamal fringe normally pale, see couplet

Genus Phytoliriomyza Hende 1

Liriomyza (Phytoliriomyza) Hendel, 1931: 203. Type of subgenus: Agromyza perpusilla Meigen, 1830 (Europe); as genus, Frey, 1941: 19. Pteridomyza Nowakowski, 1962: 97. Type of genus: Agromyza hilarella

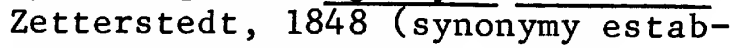
1ished by Tschirnhaus, 1971). Lemurimyza Spencer, 1965b: 26. Type of genus: Liriomyza enormis Spencer, 1963b (Madagascar) (synonymy established by Tschirnhaus, 1971).
Hendel (1931) first noted the forward inclination of the orbital setulae in Phytoliriomyza arctica in Europe and included this species in a monotypic subgenus of Liriomyza, in which the orbital setulae are predominantly reclinate. Subsequent authors have accepted Phytoliriomyza as a full genus, and in its present concept it embraces species with a considerable range of morphological characters.

The color is generally pale, yellow and gray, but entirely black species 
are known in Australia. The orbital setulae may be distinctly proclinate, upright, or lacking (but not

reclinate); the arista may be long and drooping; the eye is frequently

slanting; the mesonotum may be uniformly gray or gray with a yellow patch centrally adjoining the scutellum; the halter is frequently partially or entirely dark; and the costa always extends to vein $M 1+2$, but the outer crossvein may be present or lacking. In the male genitalia, the aedeagus is frequently greatly extended (figs. 997, 1000), and in most species there is a characteristic arrangement of spines either on the surstylus or within the epandrium (figs. 974, 996).

Normally, species can be assigned either to Liriomyza or Phytoliriomyza from assessing all these characters, but occasionally there may still be an element of doubt. In such instances, the stridulating mechanism, present in a11 males of Liriomyza (figs. 621, 622) is lacking, as far as is known, in all species of Phytoliriomyza, and will indicate the correct generic position.

Phytoliriomyza is a relatively large genus, with about 60 species, and it is widely distributed from Greenland to Chile and extending from the Palaearctic Region to Nepal (Spencer, 1965b, 1977a), Sri Lanka (Spencer, 1975), and New Zealand (Spencer, 1976b). Surprisingly, a considerable proliferation of species has occurred in Australia, where 18 species have been recorded. In the United States, 22 species are now known, of which 2 new species are described here (part 2 , p. 302), 5 species are transferred to Phytoliriomyza, and 3 have been identified as new to the United States--P. conspicua, $\underline{P}$. pallida, and P. volatilis from Canada.

The host is known of only five species. Four are leaf miners--p. clara on Pteridium aquilinum (bracken), $\underline{P}$. felt $i$ on the ferns Asplenium and Camptosorus, $\underline{P}$. jacarandae on Jacaranda, and $\underline{P}$. melampyga on Impatiens--whereas $\underline{P}$. arctica has been reared from stems of Sonchus in Europe and has been caught in association with Solidago in Ontario. The small number of species of which the host is known suggests that many or most of the others form inconspicuous stem mines rather than more obvious leaf mines.

It is significant that known leaf miners on ferns are all in Phytoliriomyza, with reared species also recorded in Europe, Australia, and New Zealand. No Liriomyza has been found to feed on any species of fern.
Key to Phyto- 1 . liriomyza species
1. Bristles on head yellow------A11 bristles dark

2 (1). A11 bristles ye11ow-P. jacarandae Steyska1 and Spencer Synopsis. Head (fig. 956) entirely yellow with frons broad, twice width of eye; gena up to 0.5 height of eye; mesonotum pale, with 3 rusty reddish bands, central one extending just beyond level of dc 2, prescutellar area yellow; scute1lum and pleura yellow; legs largely yellow; squama and fringe whitish yellow; wing length (fig. 957) 1.4-1.6 mm, discal cell sma11, last section of $M 3+43$ times length of penultimate; male genitalia with aedeagus (figs. 958, 959) having distiphallus curving dorsally; epandrium (fig. 
960) with row of stout spines along inner margin.

Host/Early Stages. Jacaranda mimosifolia and certainly other Jacaranda species. Larva forming short linear mine, which then develops into irregular blotch; this completely fills a single leaflet (fig. 961); puparium pale brown, posterior spiracles each with 3 bulbs.

Distribution. Southern California; Argentina, Cordoba.

References. Steyskal and Spencer, 1978: 585; Spencer, 1981: 327.

Only bristles of head yellow------ - . flavens spencer Synopsis. Head (fig. 962) yellow, with frons and parafacial strongly projecting; orbital bristles reduced, 1 reclinate ors, 3 or 4 inclined or $i$; gena about 0.5 height of eye; mesonotum orange or orange gray in front, broadly yellow adjoining bright yellow scutellum; pleura largely yellow; legs, squama, and fringe yellow; wing length in female $1.4 \mathrm{~mm}$, costa extending to vein $\mathrm{M} 1+2$, last section of M $3+41.5$ times length of penultimate; male genitalia unknown.

Host/Early Stages. Unknown (possibly Chilopsis, Bignoniaceae).

Distribution. Southern California.

Reference. Spencer, 1981: 323.

3 (1). Outer crossvein lacking---- conjunctimontis (Frick)

Synopsis. Orbit with 2 ors, $\overline{1}$ ori, orbital setulae proclinate; 3d antennal segment small, round, distinctly pubescent; mesonotum with $3+1$ or $2+1 \mathrm{dc}$, acr lacking; frons, gena, and face grayish yellowish; 1st and 2d antennal segments yellow, 3d blackish but paler on inside; mesonotum and scutellum matt ash gray, latter with faint trace of yellow centrally; pleura yellowish merging into gray; legs with coxae and femora yellow, tibiae and tarsi brownish; halter blackish; wing length $1.25-1.6 \mathrm{~mm}$; male genitalia with aedeagus ( $\mathrm{fig}$. 963) reduced; sperm pump minute; surstylus not divided from epandrium, with single weak bristle at end ( $\mathrm{fig}$. 964).

Host/Early Stages. Unknown.

Distribution. California; Costa Rica, Dominica.

References. Frick, 1952a: 413; Spencer and

Stegmaier, 1973: 181; Spencer, 1981: 321.

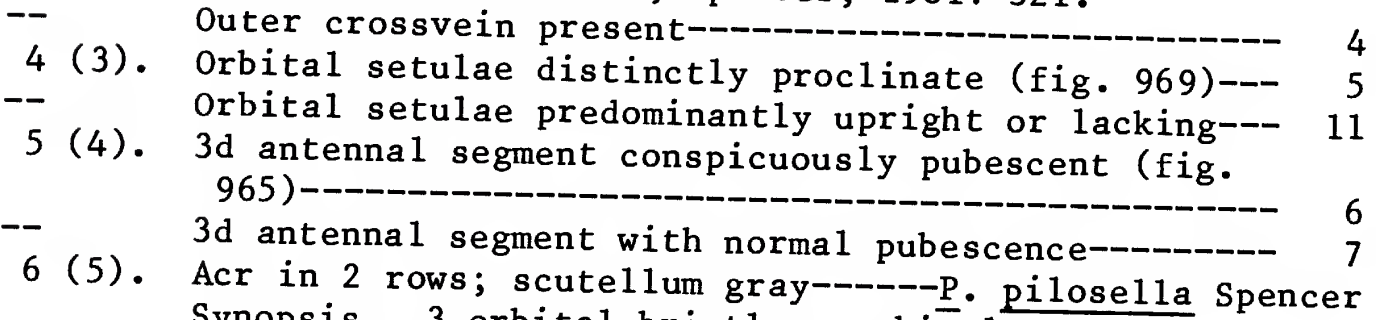
Synopsis. 3 orbital bristles, orbital setulae proclinate; $3 d$ antennal segment small, round, yellowish, conspicuously pubescent (fig. 965); mesonotum uniformly matt gray, with $3+1$ strong dc, 
scutellum also gray; legs yellow; halter uniformly yellow; wing length $1.4 \mathrm{~mm}$, last section of $M 3+4$ twice length of penultimate; male genitalia with aedeagus ending in long, unpigmented, coiled tubule (figs. 966, 967); epandrium with single stout spine at lower corner (fig. 968). Host/Early Stages. Unknown.

Distribution. Florida; Costa Rica, Puerto Rico. Reference. Spencer and Stegmaier, 1973: 116. Acr lacking; scutellum yellow centrally---------

. minutissima Spencer

Synopsis. Head (fig. 969) entirē̄y yellow; 2 ors, 1 ori, orbital setulae proclinate; eye conspicuously slanting; mesonotum largely matt gray but narrowly yellow before scutellum, this broadly yellow centrally; legs with femora yellow and brownish striate; halter yellow; wing length 1.25 $\mathrm{mm}$, last section of $\mathrm{M} 3+4$ twice length of penultimate; male genitalia with aedeagus ( $f i g .970$ ) greatly reduced, ending in asymmetrical tubules; epandrium with single bristle, surstylus oval, with 1 or 2 hairs (fig. 971).

Host/Early States. Unknown.

Distribution. California.

Reference. Spencer, 1981: 330.

7 (6). Mesonotum uniformly gray to margin of scutellum---- 8

-- $\quad$ Mesonotum partially yellow, banded ( $f i g .983$ )------ 10

8 (7). Minute species, wing length $1.3 \mathrm{~mm}$; 3d antennal segment largely black---------p. floridana Spencer Synopsis. Frons yellowish brown, 2 ors, 1 ori, orbital setulae proclinate; 1 st and $2 \mathrm{~d}$ antennal segments yellow, 3d large (fig. 972), largely black but yellow toward base; mesonotum and scutellum uniformly matt gray, acr sparse, in 2 rows; legs largely yellow but femora blackish basally; squama yellowish gray, fringe pale; halter yellowish brown, knob distinctly darkened; male genitalia with aedeagus ending in asymmetrical tubules (fig. 973); epandrium (fig. 974) with row of stout spines along inner margin, surstylus with 2 short spines at base, 3 longer ones and several hairs on inner corner.

Host/Early Stages. Unknown.

Distribution. Florida.

Reference. Spencer and Stegmaier, 1973: 114.

-- Larger species, wing length $1.6-2.5 \mathrm{~mm}$; $3 \mathrm{~d}$ antennal segment brownish yellow

9 (8). Scutellum normally gray; small species, wing length 1.6-2.2 mm----

Synopsis. Orbital setulae distinctly proclinate (fig. 975); color of head variable, from largely black (Greenland; Mt. Evans, Colorado) to largely yellow; frons and antennae normally yellowish brown; mesonotum matt gray, acr sparse, in 2 rows in front; scutellum normally gray, rarely faintly 
yellowish centrally; legs typically yellowish; wing length about $1.9 \mathrm{~mm}$, discal cell relatively large, last section of M $3+4$ rarely more than 1.5 times length of penultimate (fig. 976); male genitalia with aedeagus ( $\mathrm{fig} .977$, A) ending in pair of long, coiled membranous tubules; hypandrium characteristically narrowing distally (fig. 977, B); sperm pump minute (fig. 978); surstylus with only few weak hairs on inner corner.

Host/Early Stages. Asteraceae, once reared from Sonchus in Europe, definitely associated with Solidago in Canada. Larva stem miner, probably feeding on other genera, early stages unknown. Distribution. Widespread from New Mexico to Michigan and California to Maryland (Frick, 1959, as $\underline{\mathrm{P}}$. immaculata), probably present in most States; new records: Colorado (incl. Mt. Evans, $14,000 \mathrm{ft})$, North Carolina, Tennessee; Brazil, Canada, Chile, Europe, Greenland, Sri Lanka, Taiwan.

References. Spencer, 1969a: 202; 1981: 315.

Scutellum always yellow centrally; larger species, wing length 2-2.5 mm--------P. imperfecta (Malloch)

Synopsis. Frons yellowish or somewhat darker; 2 ors, 1 ori, orbital setulae distinctly proclinate; mesonotum largely matt gray, sometimes with faintly paler central band; scutellum yellow centrally; acr sparse, in 2 rows; pleura yellowish; legs ye1low with variable darker striations; halter predominantly yellow but knob darkened, at least faintly brown; wing length from $2 \mathrm{~mm}$ in male to $2.5 \mathrm{~mm}$ in female, last section of M 3+4 less than twice penultimate; male genitalia with aedeagus (figs. 979, 980) ending in long, paired black tubules; sperm pump (fig. 981) with blade large, fanlike; surstylus (fig. 982, A) with comb of normally 6 black bristles, inner margin of epandrium (fig. 982, B) with numerous irregular bristles. Host/Early Stages. Unknown.

Distribution. California, Florida, Idaho, Texas (new record), Washington; Chile.

Reference. Spencer, 1981: 324.

10 (7). Relatively large species, wing length up to $2.3 \mathrm{~mm}-$

Synopsis. Frons, gena, face, palpüs, and hindmargin of eye yellow; frons broad, twice width of eye; 2 ors, 1 ori, orbital setulae proclinate; $3 \mathrm{~d}$ antennal segment predominantly pale but variably brownish on outside, slightly longer than broad; eye slanting; mesonotum (fig. 983) with rectangular yellow patch centrally adjoining scutellum, narrow yellow bands extending forward along line of dc; scutellum dull yellowish centrally; humerus and notopleural area yellow, mesopleuron largely yellow, variably darkened along lower margin; legs with femora basically yellow, with variable brown- 
ish striations; abdomen largely yellow, anterior tergites slightly darkened centrally; halter yellow, knob normally distinctly darkened; wing with last section of vein $M \quad 3+41.5-2$ times length of penultimate; male genitalia with aedeagus (figs. 984, 985) ending in 2 asymmetrical tubules; epandrium (fig. 986, A) with row of stout bristles on inner margin and $\overline{2}$ longer ones basally, surstylus (fig. 986, B) with several short bristles at end and 1 below; sperm pump minute.

Host/Early Stages. Unknown.

Distribution. California.

Reference. Spencer, 1981: 317.

Small species, wing length up to $1.6 \mathrm{~mm}-----------$

- -

Synopsis. Agreeing closely with $\underline{P}$. beckerella but generally darker and distinctly smaller; banding of mesonotum as in $P$. beckerella ( $\mathrm{fig}$. 983) but pale areas less distinct; male genitalia with aedeagus ( $\mathrm{fig}$. 987) greatly reduced, ending in asymmetric tubules; epandrium (fig. 988) with 1 strong spine, some hairs and 2 shorter spines at lower corner.

Host/Early Stages. Unknown.

Distribution. Georgia, North Carolina.

Reference. Part 2, p. 302.

11 (4). Mesonotum banded or yellow centrally--------------- 12

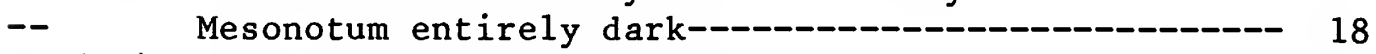

12 (11). 3d antennal segment yellow-------------------- 13

-- $\quad 3 d$ antennal segment black or at least darkened,

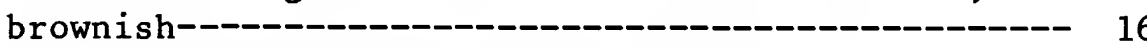

13 (12). Dark area of mesonotum shining black--

Synopsis. Head entirely yellow, inclüding entire hindmargin of eye; mesonotum shining black, apart from yellow area adjoining scutellum (fig. 989); side of thorax and legs yellow; abdomen with tergites yellow bordered; squama yellow, margin and fringe dark; wing length in male $2 \mathrm{~mm}$; male genitalia with aedeagus ( $f i g$. 990) almost entirely unpigmented, divided distally into 2 short, broad tubules; sperm pump with long stalk and broad blade, largely unpigmented; epandrium with large group of bristles at hindcorner ( $\mathrm{fig}$. 991, A), surstylus discrete, with row of 3 or more shor $\bar{t}$, stout bristles (fig. 991, B).

Host/Early Stages. Ferns Asplenium pinnatifidum and Camptosorus rhizophyllus. Larva forming blotch mine, pupating in leaf (details of larva not known).

Distribution. Illinois, New York.

References. Frick, 1959: 404; Spencer, 1969a: 297; part 2, p. 303.

Dark area of mesonotum gray or blackish gray------- 14

14 (13). Large species, wing length from $2.8 \mathrm{~mm}$ in male to $3.5 \mathrm{~mm}$ in female-- 
Synopsis. Head largely yellow but eye margin may be narrowly black; mesonotum entirely yellow adjoining scutellum, central yellow area quadrate, dark area blackish gray, on $1 y$ weak1y shining; acr in 4 rows in front, at most isolated hairs on ye1low area; mesopleuron black on lower 0.75 ; legs with femora bright yellow, tibiae and tarsi black; abdomen with all tergites broadly yellow bordered; wing with discal cell large, last section of vein M 3+4 1.5 times length of penultimate; male genitalia with aedeagus as in figures 992, 993; epandrium with numerous bristles along inner margin (fig. 994); sperm pump with blade dark, broader than long.

Host/Early Stages. Unknown.

Distribution. Michigan (new record); Canada. References. Spencer, 1969a: 171; part 2, p. 302. Smaller species, wing length at most $2.2 \mathrm{~mm}$

15 (14). Mesonotum predominantly matt gray, with central area adjoining scutellum yellow----P. pallida (Sehga1) Synopsis. Frons, orbit, entire hindmargin of eye, and antenna yellow; 2 ors, 1 or 2 ori, orbital setulae sparse, proclinate; mesonotum largely matt gray, with $\mathrm{V}$-shaped or rectangular area adjoining scutellum yellow; scutellum and pleura largely yellow; legs with femora yellow, tibiae and tarsi darker, brownish; squama yellowish, margin and fringe dark; wing length $2.4-2.6 \mathrm{~mm}$, last section of M $3+4$ twice length of penultimate; male genitalia with aedeagus as in figure 995; epandrium and surstylus with distinctive arrangement of bristles (fig. 996).

Host/Early Stages. Unknown.

Distribution. Montana (new record); Canada. References. Sehga1, 1968: 72; Spencer, 1969a: 195; part 2, 304.

Mesonotum banded, yellow and gray---p. melampyga (Loew) Synopsis. Head entirely yellow, including entire hindmargin of eye; 2 ors, 2 ori, orbital setulae reclinate at rear, more incurved below; mesonotum with $3+1$ strong dc, acr irregularly in 3 or 4 rows, matt gray, banded, with broad central area adjoining scutellum yellow; legs and scutellum largely yellow; squama yellow, margin and fringe black; halter yellow; wing length $2.2 \mathrm{~mm}$, discal cell large, last and penultimate sections of $M 3+4$ approx. equal; male genitalia with aedeagus (fig. 997) ending in long, coiled tubules which are entirely unpigmented, curvature of coil changing at midpoint; epandrium with 4 bristles on inner margin, surstylus with comb of about 12 black bristles (fig. 998).

Host/Early Stages. Impatiens capensis, I. nolitangere. Larva forming whitish linear blotch mine (fig. 999) with frass in irregular black lumps. Distribution. District of Columbia, Maryland, 
Massachusetts, Michigan, New Jersey, New York; new records: Minnesota, Minneapolis, Minnehaha Creek, leaf mines on Impatiens capensis, 28.VIII.76 (KAS); Wisconsin, Dane Co., Madison, leaf mines on Impatiens capensis, 2.IX.76 (S. Tavormina); Europe (as Liriomyza impatientis Bri.).

References. Spencer, 1969a: 178 (as Liriomyza);

Tschirnhaus, 1971: 562 (transfer to Phytoliriomyza).

16 (12). Costal area between costa and vein $R \quad 2+3$ at least faintly infuscated (see Frick, 1959: fig. 108)----

--- fumicosta (Ma11och)

Synopsis. Frons and palpus yellow; 2 ors, 1 ori, orbital setulae virtually lacking; mesonotum variable, either distinctly banded gray and ye1low, or largely gray but always broadly yellow adjoining scutellum, $3+1 \mathrm{dc}$, acr in 2 rows; scutellum yellowish gray; pleura and legs largely yellow; squama yellowish, margin dark, fringe pale; wing length $1.5-1.9 \mathrm{~mm}$; male genitalia with aedeagus as in figures 1000, 1001; epandrium ( $\mathrm{fig}$. 1002) with conspicuously strong bristles on inner margin.

Host/Early Stages. Unknown.

Distribution. Illinois, Kansas.

References. Ma1loch, 1914b: 310; Frick, 1959: 405; part 2, p. 304.

No darkening of costal area-

17 (16). Mesonotum banded, broadly yellow adjoining scute1lum------ dorsata (Siebke)

Synopsis. Head yellow, including entire hindmargin of eye; 1 st and $2 \mathrm{~d}$ antennal segments yellow, $3 \mathrm{~d}$ completely black; orbital setulae minute, sparse, mostly upright or incurved; mesonotum (fig. 1003) with pale gray bands, yellow adjoining scutellum, $3+1 \mathrm{dc}$, acr in 2 rows; pleura largely yellow; wing length $1.8-2 \mathrm{~mm}$, last section of $M 3+4$ slightly less than twice length of penultimate; male genitalia with aedeagus (fig. 1004) ending in distinctive, symmetrical tubules; epandrium (fig. 1005) with comb of strong bristles and shorter ones scattered along inner margin, surstylus with 2 strong spines.

Host/Early Stages. Unknown.

Distribution. (?) Illinois, (?) Indiana, Maryland; new records: Louisiana, Michigan, Pennsylvania. References. Spencer, 1969a: 194; 1976a: 294; part 2 , p. 303.

Mesonotum with yellow patch before scutellum------------ pacifica (Melander)

Synopsis. Closely resembling $\underline{P}$. dorsata but mesonotum uniform1y gray apart from yellow patch before scutellum; frons and palpus bright yellow; $3 \mathrm{~d}$ antennal segment deep black, 1 st and $2 \mathrm{~d}$ bright yellow; halter yellow; wing length $2 \mathrm{~mm}$; male genitalia with aedeagus as in figure 1006; 
epandrium (fig. 1007) with conspicuous comb of spines, surstylus with single spine at base and several hairs along margin.

Host/Early Stages. Unknown.

Distribution. Washington; new record: Idaho, Blaine Co., Galena, 7,600 ft, 15.VII.61 (J.G. Chillcott, CNC).

References. Melander, 1913: 264; Spencer, 1969a: 195.

18 (11). Scutellum grayish black----_--_---_---_-_--- 19

-- Scutellum at least faintly yellow centrally-------- 20

19 (18). Discal cell small, last section of vein $M 3+4$ twice length of penultimate------------P. clara (Melander)

Synopsis. Frons bright yellow in front, somewhat darker at rear; upper orbit blackish, 2 ors, 1 ori, orbital setulae sparse, reclinate; 3d antennal segment normally bright yellow, sometimes more brownish; mesonotum uniformly matt grayish black, $3+1 \mathrm{dc}$, acr sparse, in 2-4 rows; legs and pleura yellow; wing length $2 \mathrm{~mm}$, last section of $\mathrm{M} 3+4$ twice length of penultimate; male genitalia with aedeagus having 2 long, curving tubules, slightly dilated at end (fig. 1008); epandrium (fig. 1009) with row of strong bristles along inner margin, surstylus with comb of about 8 strong spines.

Host/Early Stages. Pteridium aquilinum. Larva forming narrow upper surface mine, pupating externally; puparium brown, posterior spiracles each with 3 bulbs.

Distribution. Widespread, known from California, Maryland, Michigan, Tennessee, Washington; new records: Arizona, Cochise to Chiricahua Mts., $6,500 \mathrm{ft}$, near Portal, leaf mines on bracken; Coconino Co., Mount Humphrey, near Flagstaff, 8,800 ft; New Mexico, Catron Co., Gila Wilderness, 7,500 ft; all Aug.-Sept. 1979 (J. H. Lawton).

References. Spencer, 1969a: 200 (as Pteridomyza hilarella); 1981: 319 .

-- Discal cell larger, last section of M $3+4$ only 1.5 times length of penultimate--_-

- - pulchella spencer, n. sp.

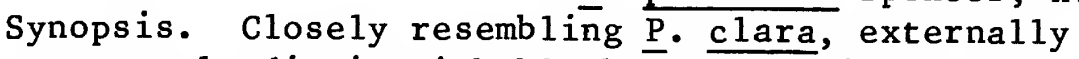
apparently distinguishable by larger discal cell; head as in figure 1010; male genitalia with aedeagus as in figure 1011, with distal tubules relatively short, recurved and dilated at end; epandrium and surstylus as in figure 1012 .

Host/Early Stages. Unconfirmed, probably Pteridium aquilinum.

Distribution. New York.

Reference. Part 2, p. 304.

20 (18). Palpus yellow--

Synopsis. Frons, hindmargin of eye, gena, face, and palpus yellow; $3 d$ antennal segment large, black, 1 st and 2d yellow; 2 ors, 1 ori, orbital setulae sparse, upright, or even lacking; mesono- 
tum matt gray, almost silvery, $3+1$ strong $d c$, acr in 2 rows; scutellum grayish yellow, paler centrally; pleura largely yellow, apart from black band on lower margin of mesopleuron and sternopleuron; legs with femora yellow, tibiae and tarsi slightly darker, brownish yellow; knob of halter yellow below, black above; wing length from $2 \mathrm{~mm}$ in male to $2.25 \mathrm{~mm}$ in female; male genitalia with aedeagus (figs. 1013, 1014) curving dorsally at end; epandrium with line of short bristles along inner margin, surstylus with comb of 8 strong bristles (fig. 1015).

Host/Early Stages. Unknown.

Distribution. Northern California.

Reference. Spencer, 1981: 328.

Palpus black---------------------------- 21

21 (20). Femora yellow--

Synopsis. Frons brownish behind, more yellow below; face and gena yellow; 2 ors, 1 ori, orbital setulae sparse, upright; $3 d$ antennal segment black, 2d yellow; mesonotum matt, brownish gray, $3+1$ strong dc, acr sparse, in 2 rows in front; scutellum predominantly dark but faintly yellow centrally, particularly when viewed from rear; legs yellowish brown (holotype); knob of halter black above; wing length $2.5 \mathrm{~mm}$ in female; male genitalia with sperm pump as in figure 1022, with blade broad.

Host/Early Stages. Unknown.

Distribution. Idaho.

References. Melander, 1913: 264; part 2, p. 305. Femora black------------_----- . volatilis Spencer Synopsis. Frons yellowish, 3d antennal segment black, 2d at most faintly paler, grayish; orbital setulae upright, a few in front more proclinate; mesonotum matt brownish black; notopleural triangle yellowish, mesopleuron largely black, narrowly yellow along upper margin; legs black; knob of halter black; male genitalia with aedeagus (figs. 1016, 1017) bending dorsally at end; epandrium with comb of 5 or 6 bristles at midpoint, lined with slender hairs, surstylus long, slender, not greatly widening at end (fig. 1018); sperm pump small, with narrow blade (figs. 1019-1021). Host/Early Stages. Unknown.

Distribution. Colorado (new record); Canada, northern Sweden.

References. Spencer, 1969a: 202; part 2, p. 305. 
Genus Metopomyza Enderlein

Metopomyza Enderlein, 1936: 180. Type of genus: Agromyza flavonotata Haliday, 1831 (Europe).

This small genus of only 12 known species resembles Liriomyza in having the scutellum yellow, but all species are otherwise darker, with the head black, brown, or at palest ochreous, and the legs are generally black, with at most the knees yellow. The stridulating mechanism in Liriomyza is lacking here.
When revising European species, Spencer (1976a) noted that the male terminalia, particularly the arrangement of characteristic bristles within the epandrium (figs. 1027, 1031), indicate closer relationship with Phytoliriomyza than with Liriomyza.

Three species are known in the United States, of which one is holarctic and one is known only in California and Washington. Metopomyza scutellata has been reared from Carex in Europe and the two other American species probably have hosts in the same genus or in the Poaceae.
Key to Metopomyza Species
1. Legs entirely black Synopsis. Frons dark, brownish black, orbit weakly shining, 1 reclinate ors, 3 more slender incurved ori; gena dark brown, 0.33 height of eye ( $\mathrm{fig}$. 1023); antenna entirely black; mesonotum with $2+1$ or $3+1 \mathrm{dc}$, brilliantly shining black; pleura largely black, only notopleuron slightly paler, brownish; scutellum lemon yellow centrally, black at sides; squama gray, margin and fringe black; halter white; wing length in female $1.6-1.75 \mathrm{~mm}$, last section of M $3+4$ twice length of penultimate; male genitalia unknown.

Host/Early Stages. Unknown.

Distribution. California, Washington.

Reference. Spencer, 1981: 335.

$2(1)$.

Femora with yellow knees-.--

Discal cell large, last section of vein $M 3+4$ only twice length of penultimate (fig. 1024)

Synopsis. interfrontalis (Melander) (1.7-2.3 $\mathrm{mm}$; frons centrally ochreous yellow, orbit prominent, darker; antenna black, with 3 or 4 postsutural dc; scutellum lemon yellow; side of thorax black; legs black apart from yellow knees; squama yellow, margin and fringe black; wing base and halter yellow; male genitalia with aedeagus as in figures 1025, 1026; surstylus angular, fringed with strong hairs or weak bristles ( $\mathrm{fig}$. 1027, A), hindcorner of epandrium with group of short, stout bristles and further row of bristles along lower margin ( $f i g$. 1027, B).

Host/Early Stages. Unknown but presumably either Cyperaceae or Poaceae.

Distribution. Northern California, Illinois, Kansas, Michigan, Texas, Virginia, Washington; widespread in Canada; Europe.

References. Frick, 1959: 412; Spencer, 1969a: 198; 


\begin{tabular}{|c|}
\hline $\begin{array}{l}\text { Discal cell small, last section of } \mathrm{M} 3+43 \text { times } \\
\text { length of penultimate ( } \mathrm{fig} \text {. 1028) }\end{array}$ \\
\hline $\begin{array}{l}\text { Synopsis. Minute black species, agreeing closely } \\
\text { with M. interfrontalis but generally smaller, wing } \\
\text { length } 1.2-1.6 \mathrm{~mm} \text {; male genitalia with aedeagus } \\
\text { (figs. 1029, 1030) having slender tubules distally, } \\
\text { curving dorsa11y; surstylus long, narrow, with } \\
\text { strong projection at midpoint ( } \mathrm{fig} \text {. 1031, A), inner } \\
\text { margin of epandrium with double band of strong } \\
\text { sclerotization, each with short, stout bristles } \\
\text { (fig. 1031, B). } \\
\text { Host/Early Stages. Probably Carex spp. Details of } \\
\text { leaf mine not confirmed. } \\
\text { Distribution. Northern California, Oregon; Canada. } \\
\text { References. Sehgal, 1971: } 342 ; \text { Spencer, 1981: } 336 \\
\text { (as scutellata); Tschirnhaus, 1981: } 332 \text {. }\end{array}$ \\
\hline
\end{tabular}

\section{Genus Paraphytomyza Enderlein}

Paraphytomyza Enderlein, 1936: 180 .

Type of genus: Phytagromyza luteoscutellata de Meijere, 1924 (as Phytagromyza xylostei RobineauDesvoidy, 1851).

This genus has in common with Phytomyza the costa extending only to vein $R 4+5$, but the orbital setulae are erect, reclinate, or lacking (never proclinate); the outer crossvein is occasionally lacking but is normally present, with the discal cel1 sma11 (fig. 1034).

The original generic name, Phytagromyza, proposed by Hendel and followed by Frick (1952a, 1959) for North American species, is sunk in Cerodontha, where the type-species, Agromyza flavocingulata Strobl, was found to belong, following study of the male genitalia (Nowakowski, 1962: 100).

The larvae are leaf or stem miners in the families Caprifoliaceae, Dipsacaceae, Rubiaceae, and Salicaceae. Six species are known in the Salicaceae in Europe and, with the male genitalia differing in structure from those of the other species in the genus, further revisionary work will doubtless lead to their being segregated in a separate genus. Paraphytomyza populicola (Walker) has been recorded in Ontario (Spencer, 1969a: 208) and is believed to have been introduced early in the 1960's.

of the eight described species known in the United States, four have been reared from leaf mines, either on Lonicera or Symphoricarpos. An undescribed species from Montana has also been reared from Lonicera.

The genus is exclusively holarctic, with 30 species known in Europe and 10 in North America. Species placed in Phytagromyza in the Afrotropical and Oriental Regions are not congeneric with Paraphytomyza, but all belong in Pseudonapomyza.

One new species is described here from Colorado, and Paraphytomyza luteoscutellata, previously known on $1 y$ in Canada and Europe, apppears to be widespread in the Northern States. In the key, two undescribed species are included that cannot be adequately diagnosed with the limited material available. Another species is known in Alberta feeding on Lonicera, $\underline{P}$. spenceri Sehgal, 1971 . 
Key to Paraphytomyza Species

1. Outer crossvein present-----

-- Outer crossvein lacking--------------------------- 8

2 (1). Mesonotum shining black--------- - nitida (Malloch) Synopsis. Largely black, frons and gena paler, brownish yellow; 3d antennal segment small, arista conspicuously short; mouth parts elongate (see Frick, 1959: fig. 131); only 2 dc; humerus yellowish; legs black but at least foreknee distinctly yellow; wing length up to $2.6 \mathrm{~mm}$ in female; male genitalia with aedeagus as in figure 1032 .

Host/Early Stages. Unknown.

Distribution. Illinois, Iowa, Maryland, North Carolina, Virginia; Canada.

References. Frick, 1959: 417; Spencer, 1969a: 207. Mesonotum matt grayish black-_- 3

3 (2). Scutellum yellow- praecox Spencer Synopsis. Frons broad, conspicuously projecting above eye in profile (fig. 1033); gena angular, deeply extended at rear, 0.5 height of eye; frons, gena, and face orange yellow; 3d antennal segment largely black, 1st and 2d yellowish; palpus black; $3+2 \mathrm{dc}, 4 \mathrm{th}$ little weaker than $3 \mathrm{~d}, 5$ th substantially smaller; mesonotum uniformly matt gray, scutellum largely yellow; humerus and notopleuron grayish yellow; mesopleuron and sternopleuron gray, only narrowly yellow on upper margin; legs black, with all knees bright yellow; squama yellow, margin and fringe dark; wing length in female $3.1 \mathrm{~mm}, 2 \mathrm{~d}$ costal section long, 4 times length of $4 \mathrm{th}$, outer crossvein very close to inner, discal cell thus small and last section of $M 3+44.5$ times length of penultimate (fig. 1034); male genitalia unknown.

Host/Early Stages. Unknown, possibly Galium.

Distribution. California.

Reference. Spencer, 1981: 346.

Scutellum gray or black

Humerus and notopleuron yellowish

Humerus and notopleuron dark, brownish black 3

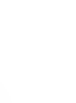


References. Spencer, 1969a: 205 (as $\underline{P} \cdot$ lonicerae); 1981: 341 .

Note. This species was treated by earlier authors as

P. lonicerae R. - D. until Griffiths (1973b) dis-

covered that this represented a misidentification.

5 (4). Legs with only foreknee yellowish--

-

Synopsis. Frons bright yellow above , black or gray in lower half; antenna black; gena extended at rear; mesonotum with 5 or $6 \mathrm{dc}$ but only posterior 3 strongly developed; humerus, notopleuron, upper margin of mesopleuron dull yellow; scutellum entirely dark, black; squama yellowish, fringe black; wing (fig. 1039) length $2.8 \mathrm{~mm}, 2 \mathrm{~d}$ costal section long, 4.5 times length of 4 th discal cell very small, crossveins closely approximated, last section of M $3+4$ long, 4.5 times length of penultimate; male genitalia unknown.

Host/Early Stages. Unknown.

Distribution. Idaho, Montana; Canada.

References. Melander, 1913: 273; Frick, 1959: 417

(as Phytagromyza); Spencer, 1969a: 208.

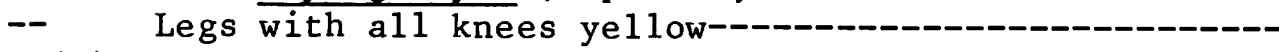

6 (5). 3d antennal segment and frons brownish orange-------

-

Synopsis. Frons distinctly projecting above eye; gena angular, 0.25 height of eye; mesonotum grayish; humerus bright yellow, notopleuron faintly yellowish; wing length in female $2.5 \mathrm{~mm}$; male genitalia unknown.

Host/Early Stages. Unknown.

Distribution. Kansas.

Reference. Part 2, p. 307.

-- 3d antennal segment black-

7 (6). Male genitalia with aedeagus having distiphallus asymmetrical (fig. 1041)---------P. lonicerina Spencer

Synopsis. Frons and orbit ochreous yellow, rarely somewhat darkened below; 3d antennal segment with fringe of white pubescence; gena extended at rear, almost 0.33 height of eye; mesonotum matt blackish gray, with up to $5 \mathrm{dc}$; humerus and notopleuron ochreous yellow; mesopleuron dark, with narrow yellow upper margin; wing length from $2.6 \mathrm{~mm}$ in male to $3 \mathrm{~mm}$ in female, discal cell small, last section of M 3+4 4 times length of penultimate; male genitalia with aedeagus as in figures 1040, 1041; sperm pump with broad blade.

Host/Early Stages. Unconfirmed but almost certainly Lonicera involucrata, on which specimens were caught in California (K. E. Frick). Larva believed to be responsible for greenish mines that are abundant on this host in Alameda and Contra Costa Counties, Calif., pupation externally; puparium brown, posterior spiracles with ellipse of some 20 minute bulbs arranged in 2 groups, with conspicuous anal projection below. 
Distribution. California.

Reference. Spencer, 1981: 342 .

Male genitalia with aedeagus having distiphallus symmetrical (fig. 1043)----- - coloradensis Spencer, n. sp.

Synopsis. Frons and orbit bright yellow, former slightly darkened toward base of antennae; generally closely resembling $\underline{P}$. lonicerina; wing length in male $2.75 \mathrm{~mm}$; male genitalia with aedeagus as in figures $1042,1043$.

Host/Early Stages. Unknown, almost certainly Lonicera.

Distribution. Colorado.

Reference. Part 2, p. 306.

8 (1). 3d antennal segment partially yellowish-------------

-

Synopsis. Frons yellow above, normally darkened below; scutellum either entirely yellow or more gray but small area between basal scutellar bristles (viewed from rear) normally yellow, rarely entirely black; outer edge of humerus and notopleuron yellowish; mesonotum gray, with conspicuous yellow patches at hindcorners adjoining scutellum; femora dark with yellow knees, tibiae and tarsi paler, yellowish; small species, wing length $1.75-2.4 \mathrm{~mm}$; male genitalia with aedeagus (figs. 1044, 1045) ending in single long tubule.

Host/Early Stages. Lonicera spp., Symphoricarpos spp. Larva forming short, funne1-shaped mine (fig. 1046) filled centrally with dark green frass; puparium pale yellowish, posterior spiracles each with ellipse of about 12 bulbs, conspicuously raised anal projections below.

Distribution. New records: Minnesota, New York, Wisconsin; Canada, Europe.

References. Spencer, 1969a: 206; part 2, p. 306.

$9(8)$. Yellow spots at hindcorners of mesonotum small--

Synopsis. Frons normally yellow above, darkened below, if largely darkened, orbit entirely yellow; normally 3 strong orbital bristles, sometimes weak lower ori present; $3 \mathrm{~d}$ antennal segment black; mesonotum and scutellum matt grayish black; humerus and notopleuron yellow, pleura otherwise dark; femora black with yellow knees, tibiae and tarsi yellowish brown; wing length from $2 \mathrm{~mm}$ in male to $2.6 \mathrm{~mm}$ in female, 2d costal section long, 3.5-5 times length of 4th; male genitalia with aedeagus as in figures 1047,1048 .

Host/Early Stages. Lonicera spp., Symphoricarpos spp. Larva forming linear mine in 1st instar, which later widens, funnellike, normally at margin of leaf (fig. 1049) or becomes more blotchlike; puparium pale yellowish, posterior spiracles each with e1lipse of about 10 bulbs, low anal projections below. Distribution. California, Idaho, Washington, 


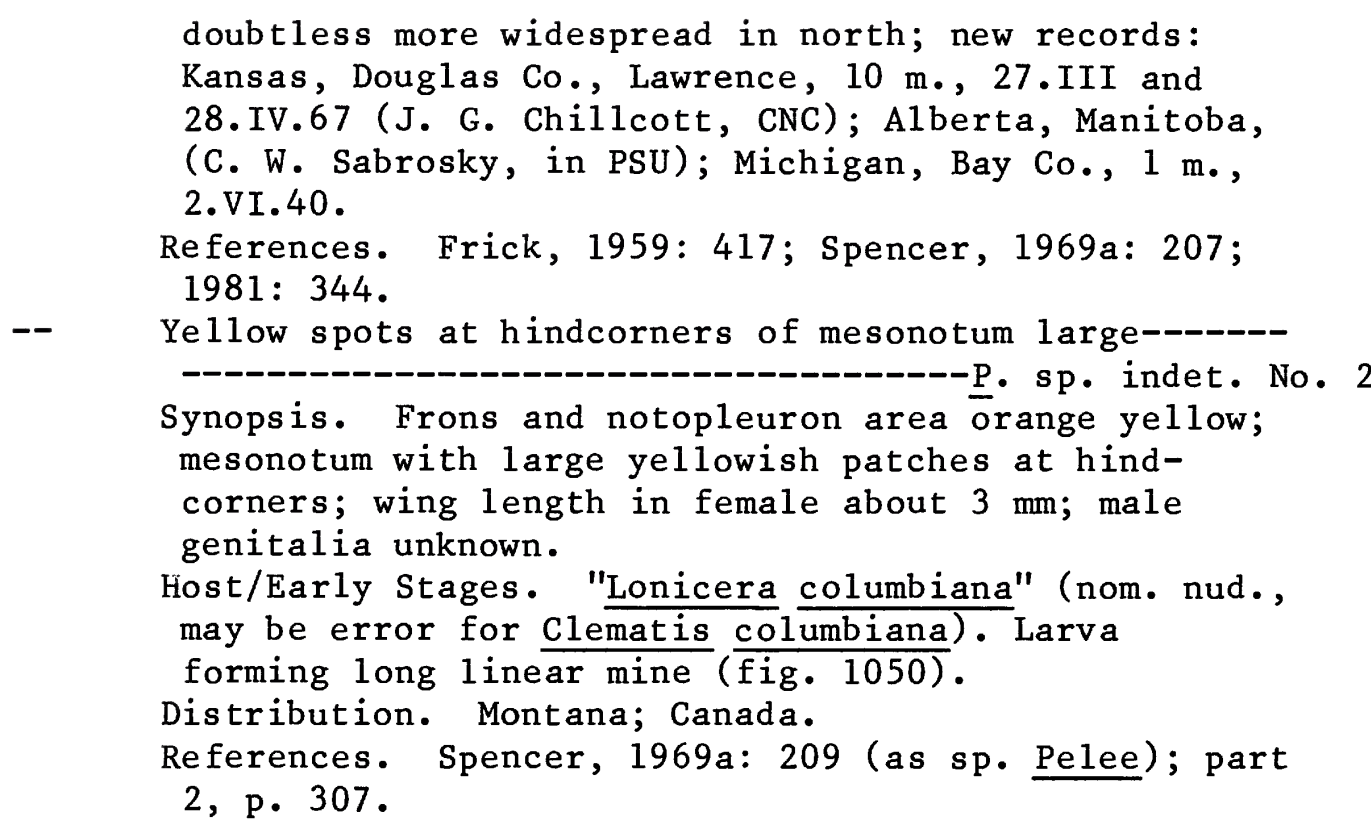

Genus Pseudonapomyza Hendel

Pseudonapomyza Hende1, 1920: 115. Type of genus: Phytomyza atra Meigen, 1830 (Europe).

This genus falls into two distinct groups. The first has the third antennal segment angulate (fig. 1051) and host plants are exclusively in the Poaceae; in the second, the third antennal segment is rounded and known hosts are all in the Acanthaceae. Only the first group is known in North America and the Palaearctic Region; the second is well represented in Africa and Australasia.

An important generic character in the larva and puparium (fig. 1055) is the spicules around each segment.

Nine new species were described by Spencer (1973a), of which six occur in Europe and six others were recently discovered in Australia (Spencer, 1977d). Twenty-seven species are known throughout the world, but only 2 are in North America. 
Key to Pseudonapo- 1 . myza Species
1. Tarsi dark brown or black; mesonotum black

-

Synopsis. Small black species; frons little wider than eye, conspicuously projecting above eye, increasingly so toward antennae; up to 5 orbital bristles, orbital setulae reclinate; 3d antennal segment angulate (fig. 1051); mesonotum deep black, largely matt but more shining from rear, with 3 strong postsutural dc; wing length $1.3-1.7 \mathrm{~mm}, 2 \mathrm{~d}$ costal section short (fig. 1052), generally about 1.5 times length of 4 th; legs entirely black; squama, fringe, and halter white; male genitalia with aedeagus as in figures 1053, 1054. Host/Early Stages. Poaceae; larva feeding on many genera, recorded in America on cultivated cereals Avena sativa, Hordeum vulgare, Secale cereale, and Triticum aestivum, also on Agropyron repens; in Europe also known on Apera, Lolium, and Poa. Larva forms narrow linear mine, pupating externally; both it and brownish puparium have rows of distinctive papilli around segmental boundaries (fig. 1055), posterior spiracles each with 7 bulbs (fig. 1056). Distribution. California, New Mexico, North Dakota, Washington; doubtless more widespread.

References. Frick, 1959: 418; Spencer, 1969a: 209; 1981: 348.

Tarsi conspicuously yellowish; mesonotum gray--------------------_----------P. lacteipennis (Malloch)

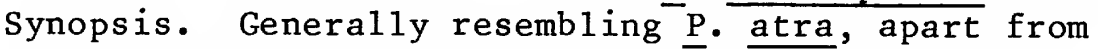
paler tarsi and grayish mesonotum; wing shining white; male genitalia unknown.

Host/Early Stages. Unknown, presumably Poaceae. Distribution. Kansas, Michigan, New Mexico, North Dakota, Washington (Frick, 1959); widespread in Canada; England.

References. Malloch, 1913b: 152; Frick, 1959: 419; Spencer, 1969a: 210 .

\section{Genus Napomyza Westwood}

Napomyza Westwood (Haliday ms.), 1840 : 192. Type of genus: Phytomyza elegans Meigen, 1830 (as P. festiva Meigen, 1830).

This genus was actually proposed earlier by Curtis with a different species as type, but this has been universally overlooked or disregarded and application has therefore been made to the International Commission on Zoological Nomenclature for suspension of the rules to maintain stability.
In the widely accepted concept of this genus, the essential characters--orbital setulae proclinate and costa extending only to vein $R$ 4+5--are as in Phytomyza, and Napomyza was differentiated by the presence of the outer crossvein, with the discal cell small (fig. 1059). Examination of male genitalia in recent years has shown that a few leaf-mining species with the crossvein present correctly belong in Chromatomyia or Phytomyza, and two such species in the United States are $\underline{P}$. davisii and $\underline{P}$. duplex. 
Conversely, another small group of species previously placed in Phytomyza, with the crossvein lacking, are referable to Napomyza on the basis of the male terminalia, in which a narrow vestige of sternum 8 is present, partially fused to tergites 6-8 (see Spencer, 1976a: fig. 623, N. nigritula). Three such species are recorded here.

Three general characteristics of Napomyza--but which are not of strict generic significance--are the projecting frons (fig. 1062), the short second costal section in the wing (fig. 1059), and the normally ash-gray mesonotum. The scutellum is generally gray but is yellow in $N$. pallens (and also in a sister species N. elegans in the Palaearctic Region).

Typical Napomyza species feed and pupate internally in stems or flower heads. This explains why no species have been reared in the United States, with little or no specialized collecting involving the examination of stems having been undertaken. How- ever, both $N$. evanescens and N. marginalis have been reared from Ranunculus stems in Canada, the former also in Europe.

Less than 50 species are known throughout the world, mainly in the Nearctic and Palaearctic Regions. The genus is largely absent from the Southern Hemisphere, but four species have been recorded in the Afrotropical Region. Twelve species have now been identified in the United States, of which 2 are new. One new combination is established (part 2, p. 307). Six other species are known in Canada (Spencer, 1969a).

Differences in external characters and in the male genitalia between species, which are certainly distinct and reared from unrelated host plants, are frequently slight. Other such probably undescribed species have been seen, but their formal description is undesirable until additional material, preferably reared, is available for accurate diagnosis and recognition of significant differentiating characters.
Key to Napomyza Species
1. Outer crossvein present-- 2

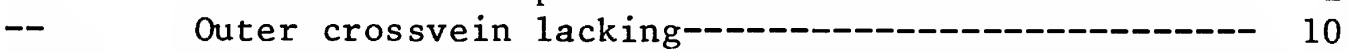

2(1). Scutellum partially or largely yellow----------

Synopsis. Frons broad, 3 times width of eye, distinctly projecting above eye in profile; gena broad, up to 0.5 height of eye at rear; wing length from $2.75 \mathrm{~mm}$ (Colorado) to $3.8 \mathrm{~mm}$, discal cell minute, outer crossvein in continuation of or even basad of inner; frons, orbit, gena, and face entirely yellow; 1st antennal segment yellowish, $2 \mathrm{~d}$ and $3 \mathrm{~d}$ black; mesonotum matt grayish black; scutellum broadly yellow centrally, with small black patches at sides; notopleuron, humerus, and upper 3d of mesopleuron yellow; legs black, all knees bright yellow; abdomen largely yellow, tergites variably grayish centrally; squama yellow, fringe blackish; male genitalia with aedeagus as in figures 1057, 1058; sperm pump with narrow blade.

Host/Early Stages. Unknown.

Distribution. California; new record: Colorado, Clear Creek Co., Mt. Evans, 9,800 ft, Doolittle Ranch, 1 m., 12.VII.61 (C. H. Mann, CNC); 5 m., 
3 f., 23.VII.61; 1 m., 3.VIII.61; (a11 B. H. Poole, CNC); Alaska, widespread in Canada.

References. Spencer, 1969a: 215; 1981: 359.

Scutellum uniformly dark-_-

3 (2). Notopleuron and upper half of mesopleuron yellow--

Synopsis. Exceptionally large species, wing length 4-5 mm; scutellum entirely black; squamal margin and fringe yellow; male genitalia not examined.

Host/Early Stages. Unknown.

Distribution. New records: Michigan, South Dakota; Canada.

References. Spencer, 1969a: 217; part 2, p. 309. Notopleural triangle black or gray, only upper mar$g$ in of mesopleuron narrowly yellow--_--_---_--

4 (3). $2 d$ costal section exceptionally short, less than length of 4 th (fig. 1059); 1 ori----N. minuta Spencer

Synopsis. Frons, gena, and face bright yellow; 2 ors, 1 ori; mesonotum deep black, with weak subshine, 3+1 dc, acr lacking; pleura black, only upper margin of mesopleuron broadly yellow; legs black, all knees yellow; squama and margin yellow; wing length in male $1.9 \mathrm{~mm}$; male genitalia with aedeagus as in figures 1060,1061 .

Host/Early Stages. Unknown.

Distribution. California.

Reference. Spencer, 1981: 355.

$2 \mathrm{~d}$ costal section longer, from equal to 1.5 times length of $4 \mathrm{th} ; 2$ ori----

5 (4). 3d antennal segment with fringe of short pubescence (fig. 1062)----- 6

6 (5). Exceptionally large species, wing length $3.5-4 \mathrm{~mm}-$

Synopsis. Frons broad, $3.5^{-}$times width of eye, strongly projecting above eye; gena extended at rear, about 0.5 height of eye; 3d antennal segment with distinct pubescence (fig. 1062); wing with $2 d$ costal section only slightly longer than 4th; male genitalia with aedeagus (fig. 1063) having end of distiphallus large, black; sperm pump (fig. 1064) small.

Host/Early Stages. Unknown.

Distribution. Arizona, Wyoming.

Reference. Part 2, p. 307.

Smaller species, wing length at most $3.1 \mathrm{~mm}$ in female--------- plumea Spencer Synopsis. Frons broad, twice width of eye, conspicuously projecting above eye in profile; frons, orbit, gena, and face yellow; 2 ors, 2 (sometimes 3) ori, orbital setulae short, sparse, proclinate; gena deep at rear, 0.33 height of eye; 3d antennal segment black, pubescence short but distinct; mesonotum ash gray, with $3+1$ strong dc, acr in $2-4$ rows; pleura grayish black, apart from narrow yel- 
low upper margin of mesopleuron; legs black, knees yellow; squama yellow, fringe black; wing length from $2.5 \mathrm{~mm}$ in male to $3.1 \mathrm{~mm}$ in female; $2 \mathrm{~d}$ costa 1 section equal to or slightly longer than $4 \mathrm{th}$, discal cell minute; male genitalia with aedeagus (figs. 1065, 1066) having distiphallus elongate, wel1-developed paired triangular sclerite below its base.

Host/Early Stages. Achillea, possibly other genera of Asteraceae. Larva feeding and pupating in stem.

Distribution. California; new record: Colorado, Teller Co., Cameron Pass, 2 m., 11.VIII.55

(G. F. Knowlton, CNC); widespread in Alaska, northern Canada, Finland, Norway, Sweden north of Arctic Circle.

References. Spencer, 1969a: 217; 1981: 359.

7 (5). Male genitalia with distiphallus elongate (fig. 1067)-C. manni Spencer, n. sp.

Synopsis. Frons 1.5 times width of eye, orbital setulae numerous in area of lower ori; gena extended at rear, almost 0.5 height of eye; wing length in male $2.75 \mathrm{~mm}$; male genitalia with distiphallus elongate (fig. 1067) having narrow process below (contrast N. plumea, fig. 1065); sperm pump rounded (fig. 1068), strongly pigmented.

Host/Early Stages. Unknown.

Distribution. Colorado.

Reference. Part 2, p. 308.

-- Male genitalia with distiphallus not elongate (see figs. 1069, 1071)

8 (7). Male genitalia with distiphallus conspicuously swollen basally in side view (fig. 1069)

Synopsis. schusteri Spencer Synopsis. On external characters agreeing closely with $N$. plumea, apart from less pubescent 3d antennal segment; male genitalia with aedeagus having connecting tubule between base and apex of distiphallus short, with characteristic curvature (fig. 1069), in ventral view distiphallus angular (fig. 1070).

Host/Early Stages. Unknown.

Distribution. California (San Bernardino Mts.). Reference. Spencer, 1981: 361.

Male genitalia with distiphallus only slightly swollen basally in side view (see figs. 1071, 1074)

9 (8). 2d costal section about 1.6 times length of 4 th----

Synopsis. On external characters agree $\overline{i n g}$ closely with $\underline{N}$. plumea but $3 \mathrm{~d}$ antennal segment appearing bare; wing length $2.3-3.3 \mathrm{~mm}$; male genitalia with aedeagus as in figures 1071, 1072, right basal sclerite with distinctive angular bend at midpoint; sperm pump smal1 (fig. 1073). 
Host/Early Stages. Unknown.

Distribution. California (see part 2); new

records: Colorado, North Carolina; widespread in

Canada.

References. Spencer, 1969a: 215; 1981: 357; part 2 , p. 309 .

$2 \mathrm{~d}$ costal section shorter, equal to $4 \mathrm{th}-$

- - - -

Synopsis. Agreeing closely with $\bar{N}$. $\overline{\text { nugax but } 2}$ d

costal section shorter, wing length $3 \mathrm{~mm}$; male genitalia with aedeagus (figs. 1074, 1075) having distiphallus not widening basally.

Host/Early Stages. Unknown, possibly Helenium hoopesii (from which specimens were aspirated). Distribution. California.

Reference. Spencer, 1981: 356.

10 (1). Squamal fringe at least partially yellow--------

-

Synopsis.: Frons ochreous yellow or more normally pale brown, distinctly projecting above eye, increasingly so toward base of antenna; orbit more grayish; 2 equal ors; gena 0.33 height of eye, parafacial prominent; all antennal segments black, $3 \mathrm{~d}$ elongate; mesonotum ash gray, acr in 2 rows; legs black, knees inconspicuously yellowish; wing length 2.2-2.7 mm, 2d costal section twice length of $4 \mathrm{th}$; squama gray, margin dark, fringe yellowish; male genitalia with aedeagus as in figures $1076,1077$.

Host/Early Stages. Ranunculus spp. Larva internal stem feeder; puparium brownish, elongate (reared in Ontario, no puparia preserved).

Distribution. New York, Pennsylvania (new record);

Canada.

References. Frick, 1959: 431 (as Phytomyza); part 2, p. 308 .

$\overline{11}(10)$. Squamal fringe uniformly dark-

Frons yellow-- - - blairmorensis (Sehgal)

Synopsis. Frons yellow, broad, about 2.5 times width of eye, greatly projecting above eye; 2 ors, 2 ori; gena angular at rear, about 0.5 height of eye; 3d antennal segment elongate, black, 2d brownish, 1st yellow; mesonotum ash gray, acr in 2 rows; legs with forecoxa yellowish, femora black with all knees bright yellow; squama yellowish gray, margin and fringe dark; wing length from 2.3 $\mathrm{mm}$ in male to $2.9 \mathrm{~mm}$ in female, $2 \mathrm{~d}$ costal section less than 1.5 times length of $4 \mathrm{th}$; male genitalia with aedeagus having distiphallus long, narrow, only slightly dilated at apex (fig. 1078).

Host/Early Stages. Ranunculus (assumed from male genitalia).

Distribution. California; Canada.

References. Sehgal, 1971: 358 (as Phytomyza); Spencer, 1981: 351 .

Frons brownish black N. evanescens 
Synopsis. Frons broad, twice width of eye, distinctly projecting above eye in profile, sometimes more yellow above beside ocellar triangle; orbit grayish, slightly yellowish on inner margin, lunule gray; 2 ors, 2 or 3 ori; gena deep at rear, $0.33-0.25$ height of eye; $3 d$ antennal segment sma11, round, black; me sonotum matt gray, with faint brownish tinge, side of thorax black; legs black, only foreknee sometimes yellowish; squama gray, margin and fringe black; wing length 2.3-2.5 mm, $2 \mathrm{~d}$ costal section slightly more than twice length of $4 \mathrm{th}$; male genitalia with aedeagus as in figure 1079; lower corner of epandrium with long, flaplike appendage (fig. 1080).

Host/Early Stages. Ranunculus californicus, almost certain1y other Ranunculus spp. Larva feeding internally in stem; puparium yellowish, slender, posterior spiracles (fig. 1081) each with ellipse of about 20 bulbs.

Distribution. California; widespread in Europe from Sicily to Scandinavia and Faroe Is.

Reference. Spencer, 1981: 354.

Genus Phytomyza Fallén

Phytomyza Fallén, 1810: 10. Type of genus: Musca ranunculi Schrank, 1803 (as $\underline{\text { P. }}$ flaveola $=$ flava Fallén, 1823b) (Europe).

The generally accepted concept of this genus is based on three characters: Orbital setulae proclinate (figs. $1112,1163)$, costa ending at vein $R \quad 4+5$, and outer crossvein lacking (fig. 1109).

Several modifications have been made in this concept after examining the genitalia of most species in this genus in recent years. A few species with the outer crossvein are now accepted as belonging in Phytomyza. Two such species in the United States are $\underline{P}$. davisii (Walton) and $\underline{P}$. duplex, n. $s \bar{p}$. (fig. 1085). Conversēely, several species previously considered in Phytomyza, lacking the outer crossvein, in fact belong in Napomyza. They are $N$. blairmorensis, N. evanescens, and N. marginaliss. For practical reasons, these three species have also been included in the Phyto- myza key given here, as based on external characters there is nothing to indicate that they correctly belong in Napomyza.

A larger group of species, with 28 known in the United States, is now included in the genus Chromatomyia (pp. 173, 373) on the basis of the male genitalia and the form of puparium and pupation site. Adults are externally inseparable from typical Phytomyza species and have therefore been included in a single key with Phytomyza.

Phytomyza is the largest world genus, and over 450 species have now been described. It is essentially of temperate regions and occurs predominantly in the Northern Hemisphere, although it is present throughout the world. In the Tropics it is largely restricted to a few species at high elevations. Only six species are known in New Zealand (Spencer, 1976b), 9 in Australia (Spencer, 1977d), 14 in the Afrotropical Region (Spencer, 1964b), and a single species from the highest 
area in Sri Lanka (Spencer, 1975). Species are certainly present in all States in America, but, as might be expected, the genus is better represented in the north than in the south. Of 99 described species recorded here ( 2 unnamed), only 7 were discovered in a detailed survey of Florida (Spencer and Stegmaier, 1973). However, 17 species are now known in Colorado, several from elevations up to $14,000 \mathrm{ft}$ on Mt. Evans.

Sixteen new species are described here and in addition 11 species are recorded as new to the United States, all previously known in Canada (part 2, p. 309); 1 species, $\underline{\text { P. wahl greni, }}$ is new to North America. One new synonym is established (part 2, p. 321).

of 78 other species in Canada and Alaska (Spencer, 1969a; Sehga1, 1971; Griffiths, 1972 a et seq.), many will be found in the Northern States or at high elevations farther south.

Three species must be deleted from the United States list, as records given by Frick (1959) are based on misidentifications. $\underline{p}$. albiceps is now known as an exclusively palaearctic species, and the record given by Frick (1959: 424) refers to $\underline{P}$. alaskana; $\underline{\text { P }}$ rufipes recorded from Oregon by Frick (1959: 435) has been found to represent $P$. flavicornis; and $P$. nigripennis, recorded by Frick (1959: 433) from Wisconsin, represents $\underline{P}$. saskatoonensis.

There is great diversity in color, head shape, size, and male genitalia in Phytomyza. The color and shape of the puparium and also the arrangement of the larval spiracles vary considerably. Nevertheless, a number of species groups cannot be satisfactorily identified on external characters alone, and for positive identification, examination of the male genitalia is necessary. Examples are the $\underline{P}$. albiceps group (comprising spécies with the frons black or yellow, with the hosts in the Apiaceae and Asteraceae), all of which have conspic- uous bristles adjoining the basal sclerites of the aedeagus (see figs. $1196,1247)$; the $\underline{P}$. robustella group, represented in America by $\underline{P}$. alamedensis, $\underline{P}$. crepidis, $\bar{P}$. flavens, $\underline{\text { P. integerrimi }} \bar{i}$, and $\underline{P}$. wahlgreni, in which the larva feeds predominantly on the midrib of the leaf, frequently forming a gall-like swelling and pupating at the leaf base; and the $\underline{P}$. ilicicola group of holly leaf miners, with six species discussed here, in which identification of adults is difficult even from the male genitalia but which are largely host specific and with characteristic leaf mines (see figs. 1305, 1316).

The host is known of 63 species in the United States, although in many instances in which relationships are apparent from the male genitalia, the host can be assumed with some confidence (at least the plant family). Examples are $\underline{P}$. nigrinervis and $P$. saskatoonensis, which are certainly feeders on Ranunculaceae.

The larvae of most Phytomyza species are leaf miners, but some feed internally in seeds or stems. Usually pupation takes place externally, the larva transforming as it leaves the mine, and the puparium either falls to the ground immediately or may for a time be loosely attached to the leaf until dislodged by wind or rain. In the $\underline{\mathrm{P}}$. ilicicola and $\underline{\mathrm{P}}$. robustella groups the anterior spiracles slightly project through the leaf epidermis.

\section{Genus Chromatomyia Hardy}

Chromatomyia Hardy, 1849: 385. Type of genus: Phytomyza periclymeni Meijere, $1 \overline{924 \text { (as } \underline{P} \text {. obscurella }}$ Fallén, 1823b) (Europe).

This genus was erected for seven species in Europe having "slippershaped pupae," which remain in the leaf at the end of the mine, as opposed to the barrel-shaped pupae of Phytomyza, which transform "on the ground." 
Hendel (1932) did not accept Hardy's genus, and it was also treated as a synonym of Phytomyza by Frick (1952a) in his generic revision of North American species. Chromatomyia was revived by Griffiths (1974a), who referred to it as "all those species of Phytomyza s.1. in whose males the distal section of the ejaculatory duct is simple (not bifid) and lies below a lobe on the "dorsal" (in anteriorly directed rest position) side of the aedeagus." (See figs. 1089, 1258.)

Characters of the puparium and method of pupation noted by Hardy remain valid for most of the Chromatomyia species, but two European species pupate externally, which Griffiths (1974a: 36) considers to be a secondary development. Other Phytomyza groups pupate as in Chromatomyia, with the anterior spiracles projecting ventrally through the leaf epidermis, and include in the United States the $P$. ilicicola group (holly leaf miners) and the P. robustella group feeding on Asteraceae (p. 173).

Chromatomyia is a predominantly north-temperate genus, with 100 species now known throughout the world. Isolated species are in the Neotropical Region as far south as Argentina and Chile and at high elevations in the Afrotropical Region. C. horticola is semicosmopolitan and is common in much of Africa and Asia, and $\underline{C}$. syngenesiae is widespread in Australia and New Zealand as an introduction. Twentyeight species are now recorded in the United States, of which 8 are described here (part 2, p. 323), 4 are recorded as new to America (C. erigerontophaga, $\underline{c}$. involucratae, $\underline{\text {. }}$ norwegica, and $\underline{\mathrm{C}}$. poae), and $1, \underline{C}$. regalensis, treated as a nomen dubium by Griffiths (1980: 8 ), is revived. C. clemativora is transferred here from Phytomyza (part 2, p. 323).

This genus is best represented at high elevations. Of the new species, five are described from Colorado, three from North Carolina, and the four species added to the United States list are all from Colorado. Thirteen species were recorded in California (Spencer, 1981), but only 2 species,

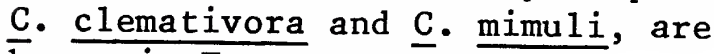
known in Texas.

of the 28 United States species, 16 have been reared in the families Asteraceae, Caprifoliaceae, Hydropyllaceae, Poaceae, Saxifragaceae, and Scrophulariaceae. In addition, random caught species can be associated with others reared from Luzula (Juncaceae) in Canada and Europe. Griffiths (1974c) has also reared species from Valeriana (Valerianaceae) in Alberta and (1976a) from Elaeagnus and Shepherdia (Elaeagnaceae) in Alberta and the Yukon. An important revision of the 22 species known in the Nearctic and Palaearctic Regions feeding on Monocotyledoneae was given by Griffiths (1980). In all, Griffiths (1972a-80) has described 39 additional species in Canada or Alaska.

There has seemed 1 ittle practical value in providing a separate key to the United States Chromatomyia species, as they are externally not distinguishable from Phytomyza, and they are therefore included in one combined key with Phytomyza. 
Key to Phytomyza and Chromatomyia Species年
A. Outer crossvein present (fig. 1085)

1. Male genitalia with aedeagus in ventral view as in figure 1083------- davisii (Walton)

Synopsis. Frons, gena, and face yellow, upper orbit darkened; 2 ors, lower weaker; all antennal segments black; mesonotum matt gray, acr in 4 rows; legs black, all knees yellow; squama and fringe yellow; wing length in male about $2.7 \mathrm{~mm}$, outer crossvein slightly beyond inner (see fig. 1085), 2d costal section long, 3.5 times length of 4 th; male genitalia with aedeagus as in figures 1082, 1083; sperm pump large.

Host/Early Stages. Ranunculus abortivus. Larva forming blotch mine (fig. 1084), adjoining margin of leaf, pupating externally through upper surface exit slit; puparium dark brown, all segments covered with broad band of spicules, posterior spiracles on widely diverging conical projections, each with circle of about 20 minute bulbs.

Distribution. Indiana, Michigan, Montana, Wisconsin; new records: Minnesota, Hennepin Co., Minneapolis, $1 \mathrm{~m} ., 20 . \mathrm{V} .77$, ex leaf mine 3.V.77 (KAS); New York, Ithaca, 1 f., 4.VIII. 16 (CUI); Canada.

Reference. Spencer, 1969a: 238.

Male genitalia with aedeagus in ventral view as in figure 1087------- . duplex Spencer, n. sp. Synopsis. Externally as in p. davisii, gena possibly broader, 0.33 height of eye; wing as in figure 1085; male genitalia with aedeagus as in figures 1086, 1087.

Host/Early Stages. Unknown, certainly Ranunculaceae.

Distribution. Tennessee.

Reference. Part 2, p. 315.

B. Outer crossvein lacking (fig. 1109)

1. Frons predominantly pale, yellow or orange, including adjoining lunule------------ 2

Frons darker, black or brown, at least in front--------------------------------- 82

2 (1). Scutellum at least partially yellow---------- 3

Scutellum entirely dark----------------------- 12

3 (2). Very large species, wing length in female $4 \mathrm{~mm}$

Synopsis. Head yellow, frons exceptionally broad, 4 times width of eye, strongly projecting above eye in profile; 1 st antennal segment yellow, $2 \mathrm{~d}$ and probably $3 \mathrm{~d}$ (this missing in only available specimen) black; mesonotum (fig. 1088) with 5 matt black bands, entirely yellow adjoining scutellum; side of 
thorax largely yellow, mesopleuron blackish along front and lower margin; legs black but all knees yellow; male genitalia unknown.

Host/Early Stages. Unknown.

Distribution. California (mountains).

Reference. Spencer, 1981: 422.

-
$-\quad(3)$
-
-

Smaller species, wing length at most $3.25 \mathrm{~mm}$ in

female-_-_-_ 4

2 ors equal- 5

Upper ors shorter than lower or absent--_----- 7

Mesonotum banded, yellow and gray (fig. 1092)--- 6

Mesonotum uniformly grayish black-

- -

Synopsis. Head yellow, apart from black 3d

antennal segment, this with distinct fringe of

whitish pubescence; mesonotum matt gray, scutel-

lum largely yellow; side of thorax and femora

bright yellow, abdomen largely yellow; wing

length 2.6-3.25 mm; male genitalia with aedea-

gus (fig. 1089) having conspicuously strong

sclerites of dorsal lobe, ejaculatory duct

extended, sinuous.

Host/Early Stages. Unknown.

Distribution. North Carolina, Virginia, Tennessee.

Reference. Part 2, p. 324.

6 (5). Frons projecting above eye (see Frick, 1959:

fig. 168); mesonotum without acr; wing with $2 d$ costal section short, equal to or at most 1.5

times length of 4 th- -

Synopsis. Head yellow, $3 d$ antenna $\overline{1}$ segment

black, 1st and 2d bright yellow; mesonotum divided into bands (see Frick, 1959: fig. 170), dark area gray, yellow adjoining scutellum; pleura largely yellow, mesopleuron faintly grayish along margins; legs yellow, tibiae and tarsi only slightly darker; wing length from about $2 \mathrm{~mm}$ in male to $2.9 \mathrm{~mm}$ in female; male genitalia with aedeagus as in figures 1090, 1091 .

Host/Early Stages. Cordylanthus nevinii (Scrophulariaceae). Larva probably feeding in flower heads, early stages unknown.

Distribution. California, Nevada; new record: Colorado, Larimer Co., Estes Park, 7,500 ft, 1 f., 20.VII.65 (C. H. Mann, CNC).

References. Frick, 1959: 436; Spencer, 1981: 430 .

Frons not projecting above eye; acr in 2-3 rows; $2 \mathrm{~d}$ costal section 2.5 times length of 4 th------

Synopsis. Head yellow, 3d antennal segment black, 1st and 2d bright yellow; dark area of mesonotum ash gray, broadly yellow adjoining scutellum (fig. 1092); side of thorax largely yellow, mesopleuron gray along front and lower 
margins; legs entirely yellow; wing length 2.3$2.8 \mathrm{~mm}$; male genitalia with aedeagus having distiphallus divided, widening at end (figs. 1093, 1094).

Host/Early Stages. Clematis columbiana, in Can-

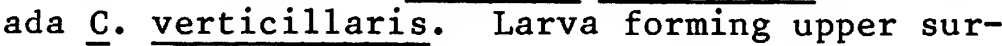
face linear mine, pupating in leaf, with anterior spiracles of puparium projecting through epidermis; puparium yellowish brown.

Distribution. Montana; Canada.

Reference. Spencer, 1969a: 236.

7 (4). 3d antennal segment brown----P. californica Griffiths

Synopsis. Frons clear yellow; mesonotum grayish black, acr in 4 rows; scutellum whitish yellow; side of thorax broadly yellow; legs with femora black, yellow at knees; wing length $2.4 \mathrm{~mm}, 2 \mathrm{~d}$ costal section about 3 times length of $4 \mathrm{th}$; male genitalia with aedeagus as in figures $1095,1096$.

Host/Early Stages. Senecio triangularis. Larva forming irregular lower surface mine (fig. 1097); puparium black, posterior spiracles each with about 40 minute bulbs.

Distribution. California (E1 Dorado Co.). References. Griffiths, 1974b: 113; Spencer, 1981: 377 .

3d antennal segment black-_-_-

8 (7). Minute species, wing length 1.4-1.75 mm-Chromatomyia clemativora

Synopsis. Head yellow, apart from black 3 d antennal segment; mesonotum banded, with dark areas moderately shining black, broadly yellow adjoining scutellum (see Frick, 1959: fig. 146); side of thorax broadly yellow; legs with femora yellow; wing length from $1.45 \mathrm{~mm}$ in male to $1.75 \mathrm{~mm}$ in female, $2 \mathrm{~d}$ costal section short, 1.5 times length of 4 th (see Frick, 1959: fig. 145); male genitalia with aedeagus as in figures 1098,1099 .

Host/Early Stages. Clematis sp. Details of leaf mine not recorded.

Distribution. Texas.

References. Frick, 1959: 426; part 2, p. 323. Larger species, wing length $2-3.3 \mathrm{~mm}-$

9 (8). $2 d$ costal section short, less than 2.5 times length of 4 thSynopsis. Frons yellow, 1 ors, 2 equal ori; 3d antennal segment black, slightly longer than broad; mesonotum grayish black, acr in 4 rows; scutellum largely black but faintly yellow between basal scutellars; legs largely black, all knees yellow; wing length in male $2 \mathrm{~mm}, 2 \mathrm{~d}$ costal section slightly over twice length of 4th; male genitalia with aedeagus as in figures 1100,1101 ; sperm pump with large blade.

Host/Early Stages. Unknown. 
Distribution. Idaho.

Reference. Part 2, p. 309.

$2 \mathrm{~d}$ costal section longer, more than 2.5 times

length of 4 th-----------_-

10 (9). Male genitalia with aedeagus having several coils (fig. 1102)----------P. ranunculi (Schrank)

Synopsis. Frons yellow, 1 strong reclinate ors, upper, if present, great $1 \mathrm{y}$ reduced, 1 inclined ori; 3d antennal segment black, 1st and 2d yellow; acr sparse, in 2 rows; color showing striking seasonal variation, with specimens from overwintering puparia darker, mesonotum solidly black, side of thorax largely so, and scutellum only narrowly yellow centrally; in pale summer form mesonotum with dark areas rusty orange or pale gray but invariably banded and broadly yellow adjoining scutellum, this and side of thorax almost entirely yellow; wide range of intermediate color forms can occur; average wing length in male $2.6 \mathrm{~mm}$, in female $2.8 \mathrm{~mm}, 2 \mathrm{~d}$ costal section long, 2.6-4 times length of 4 th; male genitalia with aedeagus (fig. 1102) divided basally but then fused and in rest position forming coil of from 3 to 8 spirals which can change direction at midpoint. Host/Early Stages. Ranunculus spp. Larva forming white linear mine (fig. 1103), with frass in closely adjoining grains; puparium grayish or brown, posterior spiracles each with 18-20 bulbs; in Europe only 2 generations.

Distribution. California (records given by Frick (1959) from Washington and Oregon almost certainly represent $\underline{P}$. humilis, which was found to be common in Cāilifornia; that from Idaho represents P. aldrichi, n. sp., couplet 9); new record: Colorado, Larimer Co., Cameron Pass, $11,500 \mathrm{ft}, 1 \mathrm{~m} ., 19-22$.VIII.43 (Sabrosky, PSU); Canada, Europe, Japan.

References. Spencer, 1969a: 271; 1981: 416. Male genitalia with aedeagus not coiled---------

11 (10). Male genitalia with aedeagus short, distiphallus straight, upright (figs. 1104, 1105)--.---.-

Synopsis. Not distinguishable externaly from $\underline{P}$. ranunculi, some variation in color comparable to that in $\mathrm{P}$. ranunculi, with overwintering generation darker.

Host/Early Stages. Ranunculus spp. Leaf mine and early stages not known (many specimens caught on Ranunculus in San Diego Co., Calif., April 1977).

Distribution. California, Oregon; Canada.

References. Spencer, 1969a: 245; 1981: 390.

Male genitalia with aedeagus in form of 2 strong tubules (figs. 1106, 1107)-----P. modocensis Spencer

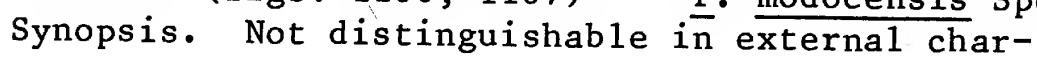


acters from pale form of $P$. ranunculi, but apparently slightly longer, wing length from 3.1 $\mathrm{mm}$ in male to $3.4 \mathrm{~mm}$ in female.

Host/Early Stages. Unknown, probably Ranuncu1aceae.

Distribution. California (mountains); new record: Wyoming, Albany Co., Centennial, $1 \mathrm{~m}$., no date (E. Fichter, D5-7, USNM).

Reference. Spencer, 1981: 403.

Note. In specimen from Wyoming, distiphallus curves ventrally, not dorsally; it is not proposed to treat this as of specific significance but it may nevertheless indicate incipient speciation.

12 (2). 1 notopleural bristle--------P. minutissima Spencer Synopsis. Head (fig. 1108) with frons distinctly projecting above eye, orange yellow; orbit broad, with 1 ors, 1 ori, orbital setulae lacking; gena extended at rear, 0.33 height of eye; 1 st and $2 \mathrm{~d}$ antennal segments yellow, 3d yellowish gray in male, black in female; mesonotum and scutellum ash gray; $2+1$ strong dc, acr lacking; side of thorax largely gray; legs with coxae yellow, femora variable, yellow to gray; wing (fig. 1109) length $1.5-1.9 \mathrm{~mm}, 2 \mathrm{~d}$ costal section short, up to 1.33 times length of 4 th; male genitalia with aedeagus (fig. 1110) short, membranous, distiphallus funnel shaped; sperm pump minute.

Host/Early Stages. Unconfirmed, possibly Artemisia californica, on which many specimens were caught in California in April 1977 (KAS).

Distribution. California.

Reference. Spencer, 1981: 402.

Note. It is possible that this species correctly belongs in the small palaearctic genus Ptochomyza Hering (see Spencer, 1966c: 306; 1969b: 24), but it seems preferable to retain it in Phytomyza at this time.

2 notopleural bristles-

13 (12). Side of thorax yellow (most of humerus, notopleuron, at least faintly, and at least upper $3 d$ of mesopleuron)

Side of thorax dark (except upper margin of mesopleuron)-

14 (13). 3d antennal segment yellowish-- $3 d$ antennal segment b-

15 (14). Hindmargin of eye black, both vt on black ground

Synopsis. Head (fig. 1111) with frons exceptionally broad, 3 times width of eye, conspicuously projecting above eye, yellowish brown in front, yellow behind; 1 ors, 3 inclined ori, orbital setulae long, numerous in front; gena deeply extended at rear, almost equal to vertical height of small eye; broad epistoma pres- 
ent, 0.5 height of eye; mesonotum matt gray, acr in 2-3 rows; side of thorax and leg largely yellow; abdomen black, with all tergites narrowly yellow bordered; squama and margin ye1low, fringe blackish; wing length $3.25 \mathrm{~mm}$ in female, $2 d$ costal section short, just over twice length of 4th; male genitalia unknown. Host/Early Stages. Unknown.

Distribution. Central California.

Reference. Spencer, 1981: 389.

Hindmargin of eye largely yellow, at most narrowly black near vertical bristles--------------

Synopsis. Head (fig. 1112) largely yellow, 3d antennal segment yellowish brown; eye small, slanting, gena broad, only slightly narrower than height of eye; acr sparse, in 2 rows; side of thorax largely bright yellow, legs yellow; wing length 2.25-2.7 mm; male genitalia with aedeagus (figs. 1113, 1114) having basal sclerites widely diverging, distiphallus largely unpigmented.

Host/Early Stages. Urtica spp. Larva feeding in pith of stem and pupating internally; puparium slender, yellowish.

Distribution. Indiana, Michigan; new records: Oregon, Columbia Co., Vernonia, 5 f., 23.IV.38, sweeping herbs under brush ( $\mathrm{K}$. Gray and $\mathrm{J}$. Schuh); Crook Co., Ochocho Ranger Station, 1 m., 1 f., 3.V.39 (Schuh and Gray); Canada, Europe.

References. Spencer, 1969a: 240; 1976c: 417. Note. The specimens from Oregon were misidentified by Frick (1959: 435) as $\underline{\text {. }}$ rufipes Mg. Mesonotum matt gray------

17 (16). Gena broad, almost equal to height of eye; abdomen matt gray-- genalis Melander Synopsis. Head yellow, frons strongly projecting above eye; 2 reclinate ors, 2 longer inclined ori; 3d antennal segment distinctly longer than broad; gena exceptionally broad, almost equal to height of eye, this unusually sma11; mesonotum and scutellum uniformly matt gray, acr sparse, in 2 rows; side of thorax largely yellow, mesopleuron with sma11, faint grayish markings on lower and front margins; legs with coxae and femora entirely yellow, tibiae and tarsi yellowish brown; squama yellow, margin and fringe slightly darker; wing length in male $3 \mathrm{~mm}$, 2d costal section 2.5 times length of 4 th; male genitalia with aedeagus as in figures 1115,1116 .

Host/Early Stages. Unknown.

Distribution. Illinois.

References. Frick, 1959: 428; part 2, p. 317. 
Gena little more than 0.5 height of eye; abdomen largely yellow-------P. coquilletti Spencer, n. sp. Synopsis. Closely resembling $\underline{P} \cdot \underline{\text { genal }} 1$ is; wing length from $3.2 \mathrm{~mm}$ in male to $3.5 \mathrm{~mm}$ in female, $2 \mathrm{~d}$ costal section shorter, twice length of $4 \mathrm{th}$; abdomen paler, predominantly yellow; male genitalia with aedeagus as in figures 1117, 1118; sperm pump minute.

Host/Early Stages. Unknown.

Distribution. Illinois.

Reference. Part 2, p. 314.

18 (14).

2 equal ors

Upper ors shorter than lower or lacking-------- 23

19 (18). Femora predominantly black, at most foreknee yellowish

Frons entirely yellow- 20 Synopsis. Frons, face, gena, and palpüs $\frac{\text { nervosa }}{\text { yellow; }}$ $3 \mathrm{~d}$ antennal segment black, 1 st and $2 \mathrm{~d}$ bright yellow; hindmargin of eye black but vti on yellow; mesonotum and scutellum ash gray, acr lacking; upper half of thorax broadly yellow; legs with femora bright yellow, tibiae and tarsi brown; squama yellow, fringe ochreous; wing length $2.4 \mathrm{~mm}$ in male, $2 \mathrm{~d}$ costal section short, 1.5 times length of $4 \mathrm{th}$; male genitalia with aedeagus as in figures 1119, 1120; sperm pump minute.

Host/Early Stages. Unknown.

Distribution. District of Columbia, Iowa, Kansas; new records: Indiana, Tippecanoe Co., Lafayette, 1 m., 5.V.16 (J. M. Aldrich); Michigan, Gladwin and Jackson Cos. (C. W. Sabrosky); Pennsylvania, Lucerne Co., Hazelton, $1 \mathrm{~m}$., 10.V.10 (Dr. Dietz Coll., PSU).

Reference. Frick, 1959: 432.

20 (19). Femora entirely black

Frons conspicuously projecting above eye; large species, wing length in male $3.1 \mathrm{~mm}$ -P. felix Spencer

Synopsis. Frons uniformly yellow, orbit slight1y darkened below, 2 ors, 3 ori; hindmargin of eye, antenna, palpus black; gena broad, 0.5 height of eye; mesonotum and scutellum matt gray, acr in 4 rows; humerus, upper $3 d$ of mesonotum, and notopleuron, at least faintly, yellowish; squama and fringe yellow; wing with 2d costal section twice length of $4 \mathrm{th}$; male genitalia with aedeagus as in figures 1121, 1122; sperm pump minute.

Host/Early Stages. Unknown.

Distribution. Northern California.

Reference. Spencer, 1981: 387.

Frons not projecting above eye; smaller species, wing length 2.1-2.5 mm------Chromatomyia castillejae ssp. nordica Spencer 
Synopsis. Upper half of frons and orbit bright yellow, frons below and particularly adjoining lunule variably darkened, blackish, lunule black; gena and face yellow, antenna and palpus black; mesonotum and scutellum ash gray; side of thorax bright yellow above, black below; squama yellowish gray, margin and fringe dark; wing with $2 \mathrm{~d}$ costal section about 2.5 times length of 4 th; male genitalia with aedeagus as in figures 1123, 1124; sperm pump large but blade narrow.

Host/Early Stages. Castilleja latifolia and certainly other $\underline{\mathrm{C}}$. species. Larva forming small linear mine, pupating at end of mine; puparium whitish, posterior spiracles each with circle of about 12 minute bulbs, anterior spiracles projecting through epidermis.

Distribution. Central California.

References. Spencer, 1973b: 34 (Phytomyza castillejae); 1981: 436.

22 (20). Wing with 2d costal section long, 3.5-4 times length of 4 th-

Synopsis. Frons and orbit yellow, hindmargin of eye and antenna black; mesonotum blackish gray; upper half of thorax yellow; wing length about $2.7 \mathrm{~mm}$; male genitalia with aedeagus as in figures 1125,1126 .

Host/Early Stages. Thalictrum polygamum. Larva forming large white blotch, normally in center of leaf, with frass in conspicuous black patch near center of mine, pupation either in mine or externally; puparium dark brown, deeply segmented.

Distribution. Pennsylvania; Canada; new record: Minnesota, Minneapolis, Minnehaha Creek, $1 \mathrm{f}$., emerged 21.VII.80 from leaf mine on Thalictrum dasycarpum, coll. 28.VI.80 (KAS).

References. Frick, 1959: 434; Spencer, 1969a: 268.

2d costal section shorter, at most 3 times length of 4 th-

Synopsis. Generally resembling $P$. plumiseta; male genitalia with aedeagus as in figures $1127,1128$.

Host/Early Stages. Aconitum spp., Delphinium spp. Up to 10 or more larvae feed together, forming large greenish-brown blotch ( $\mathrm{fig}$. 1129), pupating externally; puparium dark brown.

Distribution. New York, Ohio, Pennsylvania; Canada, Europe.

References. Frick, 1959: 427 (as $\underline{P}$. delphiniae Frost); Spencer, 1969a: 223.

23 (18). Mesonotum ash gray-- cicutella Spencer Synopsis. Frons, orbit, and face bright yellow, both vt on yellow; upper half of thorax bright yellow; legs grayish black with all knees yel- 
low; squama yellow, margin and fringe black;

wing length from $2.5-2.75 \mathrm{~mm}$ in male to $3 \mathrm{~mm}$ in female, 2d costal section 3 times length of 4th; male genitalia with aedeagus as in figures 1130,1131 , with minute spinules beside distal tubules toward base.

Host/Early Stages. Cicuta douglasii. Larva forming irregular greenish blotch mine ( $\mathrm{fig}$. 1132), which soon turns yellowish brown; details of larva (puparium) not established.

Distribution. California.

Reference. Spencer, 1981: 380.

Large species, wing length $2.5-3.4 \mathrm{~mm}-$

Synopsis. Frons, face, and orb it yellow, last at most slightly darkened; 2 ors, upper shorter than lower, normally 2 ori; $3 d$ antennal segment black, 1 st and $2 d$ frequently slightly paler; acr irregularly in 2 rows; side of thorax with upper band broadly yellow; legs black, with all knees yellow; wing with $2 \mathrm{~d}$ costal section long, 4-5 times length of 4 th; male genitalia with aedeagus (figs. 1133, 1134) having distiphallus long but largely membranous, no spinules beside basal sclerites.

Host/Early Stages. Heracleum lanatum. Larva

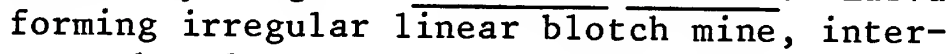
parenchymal, thus appearing yellowish green and easily overlooked, several larvae frequently feed together; puparium black, posterior spiracles each with 17-27 bulbs.

Distribution. California (Alameda Co.); Alaska.

References. Griffiths, 1973a: 227; Spencer, 1981: 428.

Smaller species, wing length 2-2.55 mm----------

-- alaskana Griffiths

Synopsis. Frons and orbit yellowish, hindmargin of eye black to base of vti; 1 strong reclinate ors, 2 inclined ori; 1 st antennal segment yellowish, 2d and 3d black; upper band of side of thorax bright yellow; legs black, all knees yellow; wing with $2 \mathrm{~d}$ costal section long, from just less than 3 to just less than 4 times length of $4 \mathrm{th}$; male genitalia with aedeagus as in figures 1135, 1136, with small group of spinules above left basal sclerite just beyond midpoint, distiphallus divided apically; sperm pump minute (fig. 1137).

Host/Early Stages. Artemisia douglasiana.

Larva forming long, narrow, white, linear mine (fig. 1138); puparium black, posterior spiracles each with 18-22 bulbs.

Distribution. California; Alaska.

References. Griffiths, 1974d: 300; Spencer, 1981: 371 . 
Upper ors weaker than lower or lacking--------- 65

3d antennal segment yellow or brown---------- 27

3d antennal segment black---_---_-_-_----- 29

Wing pale, veins yellowish; squamal fringe ye1-

low---

Synopsis. Frons and antenna yellowish orange, orbit paler yellow; 3d antennal segment virtually bare; mesonotum and scutellum matt gray, acr irregularly in 4 rows; legs black, apart from broadly yellow knees on all femora; abdomen grayish black, tergites all narrowly yellow bordered; wing length from $2.5 \mathrm{~mm}$ in male to $3.25 \mathrm{~mm}$ in female; male genitalia with aedeagus as in figures 1139, 1140 (end of distiphallus missing).

Host/Early Stages. Unknown.

Distribution. Texas.

References. Frost, 1924: 85; Frick, 1959: 428; part 2, p. 317.

Wing hyaline, normal, veins brown; squamal fringe dark-

28 (27). Large species, wing length $4.35 \mathrm{~mm}$ in female; frons strongly projecting above eye-----------

Synopsis. Frons yellow, together with orbit, face, and palpus; 3d antennal segment orange yellow, narrowly brownish distally, with slightly longer, brownish pubescence at upper corner; 2 ors, 2 ori, orbital setulae irregu1 ar, in 2 rows; gena broad, slightly over 0.5 height of eye, this bare; mesonotum and scute1lum ash gray, acr irregularly in 4 rows; pleura gray; legs black, apart from broadly yellow knees on al1 femora; abdomen blackish gray, all tergites broadly yellow bordered; squama ye1low, margin and fringe blackish; male genitalia unknown.

Host/Early Stages. Unknown.

Distribution. Colorado.

Reference. Part 2, p. 323.

Smaller species, wing length about $2.5 \mathrm{~mm}$; frons not projecting above eye-------P. auricornis Frost

Synopsis. Frons yellowish or brownish; antennal segments variable, pale yellow or yellowish brown, 3d with conspicuous fringe of white pubescence; palpus black on outside, yellow below; gena yellow, slightly less than 0.5 height of eye; mesonotum and scutellum grayish black, acr sparse but irregularly in 4 rows in front; pleura grayish black; legs black, femora yellow at knees; abdomen entirely black in female (holotype), tergites yellow bordered in male; squama yellow, margin and fringe dark; male genitalia with aedeagus as in figures 1141, 1142 . 
Host/Early Stages. Unknown.

Distribution. New York; new record: New York, Tompkins Co., Ithaca, 1 m., 1 f., 10.V.40 (E. D. McDonald, CUI).

References. Frost, 1927: 217; Frick, 1959: 426; part 2, p. 311.

29 (26). Broad epistoma present--_Epistoma lacking 30

30 (29). 2d antennal segment normally black, occasionally

slight1y yellowish--------------P. 1upini Sehga1

(Most frequently only 1 strong ors, see couplet 65.)

2d antennal segment 1argely ye11ow-------------

(

(This species lacks outer crossvein and may be mistaken for Phytomyza; see Napomyza key, couplet 11.)

31 (29) Arista conspicuously thickened (fig. 1143)------

(If frons dark, see couplet 89 crassiseta Zetterstedt

Synopsis. Frons, gena, and face normally bright yellow (frons dark brown in 1 specimen from California), orbit generally darkened to lower ors; 2 ors, 1 or 2 ori; 3d antennal segment large, longer than broad, arista distinctive (fig. 1143); mesonotum matt ash gray, acr at most in 2 rows in front or virtually lacking; legs with forecoxae yellow, femora black, and yellowish knees; squama and fringe yellow, margin possibly darker, brownish; wing length up to $2 \mathrm{~mm}$, $2 \mathrm{~d}$ costal section short, 1.5 times length of $4 \mathrm{th}$; male genitalia with aedeagus as in figure 1144.

Host/Early Stages. Hebe spp. Larva forming narrow linear mine at leaf margin, which may later widen and become blotchlike (fig. 1145), pupation in leaf on lower surface; puparium slender, white or yellowish, with conspicuous dark band running centrally along ventral surface.

Distribution. California, Idaho, Washington; new record: Virginia, Page Co., Shenandoah N.P., 3,600 ft, $1 \mathrm{f}$., 7.VI.62 (J. R. Vockeroth, $\mathrm{CNC}$ ); Europe.

References. Frick, 1959: 427; Spencer, 1981: 385 .

Note. This species is not exclusively parthenogenetic, as stated by Frick (1959).

32 (31). Squamal fringe yellow or ochreous, margin at most brownish-

Squamal margin and fringe dark---_-- 49

33 (32). 3d antennal segment with conspicuously long pubescence------ ceanothi Spencer, n. sp. Synopsis. Head largely ye $\overline{1}$ low, 3d antennal segment black, 1 st and 2 d paler; 2 ors, 1 ori; 
mesonotum and scutellum matt gray, acr in 3-4 rows; legs black, knees yellow; squama and fringe yellow, margin dark brown; wing length 1.3-1.75 mm; male genitalia with aedeagus as in figures 1146, 1147 .

Host/Early Stages. Ceanothus sp. Larva forming linear mine which develops into a secondary blotch in smal1 leaves, pupating in leaf; puparium yellow, posterior spiracles each with about 8 bulbs.

Distribution. Kansas.

Reference. Part 2, p. 311 .

3d antennal segment with short, normal pubes-

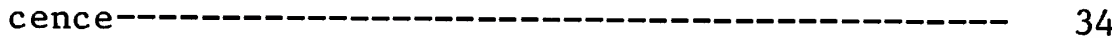

34 (33). Acr present, in 2-4 rows--- 35

-- Acr lacking------ 48

35 (34). Acr in 2 rows---_- 36

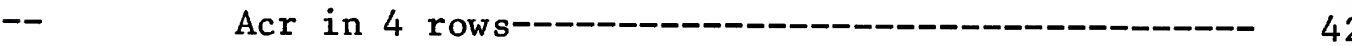

36 (35). Frons distinctly projecting above eye---------- 37

-- $\quad$ Frons at most narrowly projecting above eye----- 40

37 (36). Forecoxa at least partially yellow------------ 38

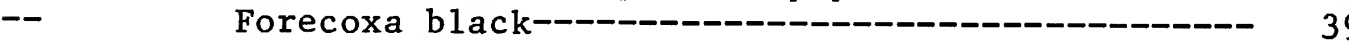

38 (37). Male genitalia with aedeagus (fig. 1148) having short, broad distiphallus--------P. subtenella Frost Synopsis. Frons, face, 1 st and $2 \mathrm{~d}$ antennal segments yellow, 3d black, slightly enlarged; gena broad, up to 0.4 height of eye; mouth margin normal, without epistoma; palpus black; mesonotum matt gray, acr in 2 rows; legs with femora black and all knees bright yellow; abdomen and all tergites yellow bordered, and in front broadly yellow latera11y; basal cone of ovipositor elongate, entirely shining with 4-6 long hairs at end; squama and fringe yellow; wing length $2.5-2.8 \mathrm{~mm}, 2 \mathrm{~d}$ costal section short, 1.5 times length of $4 \mathrm{th}$; male genitalia with aedeagus as in figures 1148,1149 . Host/Early Stages. Unknown, almost certainly internal feeder.

Distribution. California, Washington, Wisconsin; new record: Wyoming, Uinta Co., 29 miles S. of Evanston, 1 f., 20.VII.75 (G. C. Eickwort, CUI); Canada.

References. Frost, 1924: 89; Spencer, 1981: 425 .

Note. This species is clearly related to $P$. eumorpha Frey, 1946 (see Spencer, 1976a: $\bar{f}$ igs. 722,723 ), which is known from north of Arctic Circle in Sweden and Finland; however, P. eumorpha differs in having $3 \mathrm{~d}$ antennal segment yellow.

Male genitalia with aedeagus (fig. 1150) having distiphallus slender, more elongate---_------

-

Synopsis. Closely resembling $\underline{p}$. subtenella but frons less projecting, lst and $2 \bar{d}$ antennal seg- 
ments less distinctly yellow; male genitalia with aedeagus as in figures 1150,1151 .

Host/Early Stages. Unknown.

Distribution. Central California; new records:

Colorado, Clear Creek Co., Loveland Pass, $12,000 \mathrm{ft}$, summit, on tundra, $1 \mathrm{~m}$., 7.VIII.61

(C. H. Mann, CNC); Boulder Co., Nederland, Science Lodge, 9,500 ft, $1 \mathrm{~m}$., 27.VI.61 (B. H. Poole, CNC); Lake Co., Independence Pass, $12,100 \mathrm{ft}$, tundra, $1 \mathrm{~m} ., 31 . V I I .61$ ( J. G. (hillcott), 1 m., 7.VIII.61 (C. H. Mann). Reference. Spencer, 1981: 407.

39 (37). 1st and $2 \mathrm{~d}$ antennal segments yellowish----------

-----

Synopsis. Frons orange yellow, strongly projecting above eye; gena broad, 0.5 height of eye; 3d antennal segment black, longer than broad, 1st and 2d segments yellowish; mesonotum matt gray, acr in 2 rows; legs black, all knees bright yellow; squama and fringe yellow; wing length 2.5-2.7 mm, 2d costal section twice length of $4 \mathrm{th}$; male genitalia with aedeagus as in figures 1152, 1153.

Host/Early Stages. Unknown.

Distribution. Colorado.

Reference. Part 2, p. 318.

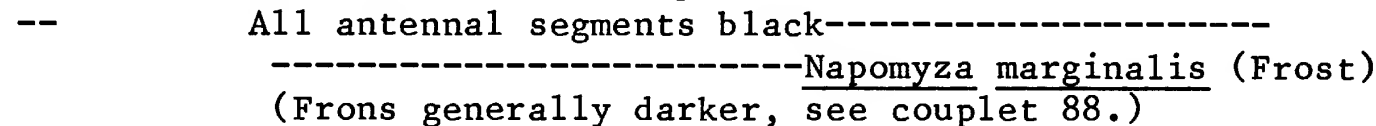

40 (36). A11 antennal segments black---

Synopsis. Frons, gena, and face $\frac{\text { carbonensis }}{\text { clear yellow }}$ orbit slightly paler; palpus black, gena extended at rear, 0.4 height of eye; mesonotum ash gray, acr in 2 rows; legs black, femora only narrowly yellow at knees; wing length $2-2.3 \mathrm{~mm}$, 2d costal section twice length of 4th; male genitalia with aedeagus as in figures 1154,1155 .

Host/Early Stages. Unknown.

Distribution. Southern California.

Reference. Spencer, 1981: 379.

1 st and 2d segments paler, partially yellowish

------------------- 41

41 (40). Palpus and forecoxa yellowish-----P. tenella Meigen Synopsis. Closely resembling $P$. carbonensis but 3d antennal segment slightly larger, distinctly longer than broad, forecoxa at least slightly yellowish, abdomen partially yellowish, at least 1aterally; wing length 2.3-2.75 mm; male genitalia with aedeagus as in figure 1156; sperm pump (fig. 1157) with base conspicuously elongated on 1 side.

Host/Early Stages. Pedicularis. Larva feeding in seed heads but pupating externally; puparium brownish, posterior spiracles enlarged, each having about 45 bulbs. 
Distribution. Colorado (new record); Europe, inc1. Iceland (Griffiths, 1964).

References. Spencer, 1976a: 512; part 2, p. 322.

Palpus and forecoxa black---- - . subalpina Sehgal

Synopsis. Frons yellow, not projecting above eye; 2 equal ors, 2 or $i$; $3 d$ antennal segment sma11, round, black, 1 st and $2 \mathrm{~d}$ segments ye1lowish; mesonotum and scutellum matt gray, acr in 2 rows; legs black, al1 knees narrowly ye1low; squama and fringe yellow; wing length 2.5 $\mathrm{mm}$ in both sexes, $2 \mathrm{~d}$ costal section slightly less than twice length of 4th; male genitalia with aedeagus as in figures 1158, 1159; in female ovipositor sheath greatly extended.

Host/Early Stages. Unknown.

Distribution. Colorado (new record); Canada.

Reference. Part 2, p. 321.

42 (35). 3d antennal segment large or distinctly elongate---

3d antennal segment small, round

43 (42). Entire orbit blackish, orbital setulae 46 numerous in area of ori ( $\mathrm{fig} .1160$ )-

Synopsis. Frons orange yellow, conspicuously projecting above eye; 2 ors, 3 or 4 ori; gena broad, 0.5 height of eye; 3d antennal segment black, elongate; mesonotum matt gray, acr in 2 rows; legs black, all knees bright yellow; squama and fringe bright yellow; wing length in male $3.1 \mathrm{~mm}$; male genitalia with aedeagus as in figures 1161, 1162 .

Host/Early Stages. Unknown.

Distribution. Michigan; new record: Michigan, Ingham Co., East Lansing, $1 \mathrm{~m}$., 30.IV.52 (C.W. Sabrosky, PSU).

Reference. Steyska1, 1972b: 5 .

At most upper orbit blackish, orbital setulae sparse

$2 d$ costal section short, twice length of 4 th$2 d$ costal section 3 times length of 4 th-

Synopsis. Head (fig. 1163) - Iigusticifoliae Spencer orange, both vt on black ground frons yellowish darkened to lower ors, palpus black; 1st and 2d antennal segments yellow, 3d black; mesonotum ash gray, acr in 4 rows; legs with coxae and femora black but all knees conspicuously yellow; abdomen black but anterior tergites laterally yellow; squama, including margin and fringe, bright yellow; wing with veins pale, yellowish, length in female $2.5 \mathrm{~mm}, 2 \mathrm{~d}$ costal section 3 times length of 4th; male genitalia unknown. Host/Early Stages. Clematis 1igusticifolia. Larva forming brownish blotch mine (fig. 1164), pupating externally; puparium yellowish brown, all segments with numerous minute papilli on 
lateral margins, posterior spiracles each with ellipse of about 15 bulbs.

Distribution. Southern California.

Reference. Spencer, 1981: 393.

45 (44). Frons distinctly projecting above eye------------

-- - - - clematisana Spencer

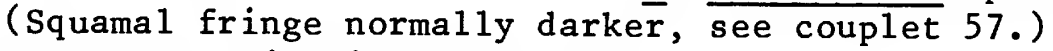

Frons not projecting above eye--

-- -

Synopsis. Frons yellow, orbit $\bar{s} 1 i$ ghtly paler, hindmargin of eye black; 1 st and $2 \mathrm{~d}$ antennal segments yellowish, 3d black, distinctly longer than broad; palpus black; mesonotum grayish black, acr irregular, in 4-5 rows; legs black, a11 knees yellow; wing length in male $2.2 \mathrm{~mm}$; male genitalia with aedeagus as in figures $1165,1166$.

Host/Early Stages. Unknown.

Distribution. Florida.

Reference. Spencer and Stegmaier, 1973: 120.

46 (42). Frons distinctly projecting above eye; all knees yellow------------ chelonei spencer

Synopsis. Frons, gena, and face bright yellow; upper orbit and hindmargin of eye black, 2 ors, 2 ori; 3d antennal segment smal1, round, black, 1st and 2d segments slightly yellowish; eye large, gena $0.5-0.33$ height of eye; mesonotum and scutellum matt gray, acr in 4-5 rows; side of thorax largely gray, notopleuron possibly slightly paler; legs largely black, femora with all knees yellow (forecoxa yellow in holotype, dark in U.S. specimens); wing length from $3 \mathrm{~mm}$ in male to $3.25 \mathrm{~mm}$ in female, $2 \mathrm{~d}$ costal section slightly over twice length of $4 \mathrm{th}$; male genitalia with aedeagus as in figures $1167,1168$. Host/Early Stages. Chelone glabra, possibly other Chelone spp. Larva feeding in seeds; puparium blackish brown, posterior spiracles each with about 15 bulbs.

Distribution. New records: Indiana, North Carolina, Tennessee; Canada.

References. Spencer, 1969a: 234; part 2, p. 312. Frons not projecting above eye; only foreknee

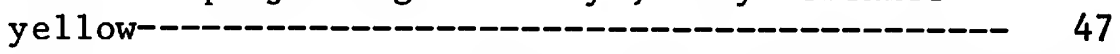

47 (46). 2 ori-- Chromatomyia eriodictyi Spencer

Synopsis. Frons orange yellow, sometimes darkened around lunule, which is black; upper orbit yellow, blackish below upper ori; gena yellow, face, palpus, and all antennal segments black, 3d segment smal1, round; mesonotum ash gray, acr in 4 rows; legs with coxae and femora black, even foreknee scarcely paler; abdomen black centrally, all tergites broadly yellow laterally; wing length $2.3-2.5 \mathrm{~mm}, 2 \mathrm{~d}$ costal section short, 1.5 times length of $4 \mathrm{th}$; male genitalia with aedeagus as in figures 1169 , 1170 . 
Host/Early Stages. Eriodictyon californicum. Larva forming irregular upper surface linear mine; puparium at end of mine on upper surface (details of puparium not available).

Distribution. Central California.

Reference. Spencer, 1981: 438.

1 ori-- Chromatomyia mimuli spencer

Synopsis. Closely resembling C. eriodictyi but frons paler, lemon yellow; wing length 2-2.5 mm, $2 \mathrm{~d}$ costal section $1.5-2$ times length of $4 \mathrm{th}$; male genitalia with aedeagus as in figures $1171,1172$.

Host/Early Stages. Mimulus, Nemophila phacelioides, Phacelia. Larva forming irregular upper or lower surface mine (fig. 1173), pupating at end of mine; puparium whitish gray, posterior spiracles each with ellipse of about 8 bulbs (on Phacelia viscida).

Distribution. Central and southern California; new record: Texas, Travis Co., Austin, $1 \mathrm{f}$., ex leaf mine on Nemophila phacelioides, 16.IV.80 (S. Tavormina).

Reference. Spencer, 1981: 443.

48 (34). 3d antennal segment unusually large (fig. 1174)-

Synopsis. Frons pale yellow above, slightly darkened, grayish, below; orbit and lunule yellow; 2 ors, 1 ori; $3 d$ antenna 1 segment black, 1 st and 2d paler, yellowish; mesonotum ash gray; legs with femora black, all knees bright yellow; small species, wing length $1.6-2 \mathrm{~mm}$, $2 \mathrm{~d}$ costal section short, about 1.5 times length of 4th; male genitalia with aedeagus as in figures 1175,1176 .

Host/Early Stages. Penstemon laxiflorus and certainly other Penstemon spp. Larva forming short, white, upper surface mine (fig. 1177), pupating in leaf; puparium whitish, anterior spiracles projecting through epidermis, posterior spiracles each with ellipse of 8 bulbs.

Distribution. California; new record: Mississippi, Washington Co., Leland Experimental Forest, $10 \mathrm{~m}$., $10 \mathrm{f}$, , emerged $20 . \mathrm{V} .79$ from leaf mine, col1. 17.V.79 (G. McMinn, KAS, USNM); Canada.

References. Spencer, 1969a: 265; 1981: 411. 3d antennal segment elongate but smaller-_-

Synopsis. Generally plantaginis Robineau-Desvoidy Synopsis. Generally as in $\underline{P}$. penstemonis, apart from smaller 3d antennal segment and slight1y darker, brownish gray mesonotum; wing length about $2 \mathrm{~mm}$; male genitalia with aedeagus as in figures 1178, 1179 (Europe; apparently entirely parthenogenetic in Australia, North America, and New Zealand, see Frick, 1951b).

Host/Early Stages. Plantago, particularly 
1anceolata and major. Larva forming long, narrow, white linear mine, pupating in leaf; puparium whitish gray, anterior spiracles projecting through epidermis, posterior spiracles with ellipse of about 12 bulbs.

Distribution. Widespread through most of United States; new record: Arkansas, Garland Co., Hot Springs, 2 f., 17.V.79 (KAS); Canada, Europe, Japan.

References. Frick, 1959: 434; Spencer, 1969a: 268; 1981: 413 .

49 (32). 3d antennal segment with conspicuously long pubescence (figs. 1180, 1181)-_-_-_-_-_-_-_- - Chromatomyia 1actuca (Frost)

Synopsis. Frons, gena, and face yellow, orbit paler, whitish yellow, 2 equal ors, 1 ori; $3 d$ antennal segment black, enlarged in female ( $\mathrm{fig}$. $1181)$, 1st and $2 d$ segments yellow or yellowish brown; mesonotum matt gray, acr in 2 rows; legs black, with all knees bright yellow; squama yellow, margin and fringe dark; wing length 2.1-2.8 mm, 2d costal section 2.5 times length of 4th; male genitalia with aedeaguas as in figures 1182,1183 .

Host/Early Stages. Crepis, Lactuca, Sonchus, and Taraxacum, possibly other genera in the subtribe Crepidinae of the tribe Cichorieae. Larva forming long linear mine on lower surface, pupating in leaf; puparium yellowish to reddish brown, posterior spiracles each with ellipse of $14-20$ bulbs on large conical protuberance (leaf mine illustrated by Needham, Frost, and Tothill, 1928: fig. 84, and Griffiths, 1977: fig. 14).

Distribution. California, Michigan, New York, Pennsylvania; Canada; new record: Maryland, Montgomery Co., Bethesda, $4 \mathrm{~m}$., $4 \mathrm{f}$., emerged 26.VI.80 from leaf mines, col1. 24.VI.80 on Lactuca biennis (Moench) Fern. (USNM).

References. Griffiths, 1974a: 37; 1977: 336; Spencer, 1981: 442.

3d antennal segment with shorter, normal pu-

50 (49). Frons yellow above, brown below-- 50

-- Frons uniformly yellow-_- 52

51 (50). Acr numerous, in 6-8 rows-- - ilicis Curtis (If frons considered as brown, see couplet 91.) Synopsis. Frons variable in color, generally somewhat brownish in front, more yellow behind; gena 0.33 height of eye; all antennal segments black; mesonotum matt black, with acr numerous; legs black, even foreknee scarcely paler; squama gray, margin and fringe black; wing length 2.8-3.3 mm, 2d costal section long, 3.5-4 times length of 4 th; male genitalia with aedeagus as in figures $1184,1185$. 
Host/Ear1y Stages. Ilex aquifolium. Larva forming upper surface linear blotch, oviposition takes place at base of midrib on lower surface and larva feeds slowly during summer, by autumn forming small greenish mine, overwintering in leaf and completing feeding and transformation following spring, pupation in mine; puparium yellowish brown.

Distribution. Oregon, Washington; Canada (introduced), Europe.

References. Frick, 1959: 429; Kulp, 1968: 19; Spencer, 1969a: 246.

Acr in 4 rows---- Chromatomyia montana Spencer

(Frons predominantly dark, see couplet 92.)

52 (50). Acr present, in 2-6 rows----------------

Acr lacking (at most 1 or 2 isolated hairs pres-

ent)-----------Chromatomyia syngenesiae Hardy

Synopsis. Frons orange yellow, orbit with 2 short, equal ors and 1 ori; 3d antennal segment sma11, round, black, 1 st and $2 d$ yellowish; mesonotum conspicuous 1 y ash gray; legs black but femora with all knees bright yellow; squama yellow, margin and fringe black; wing length 2.2-2.6 mm, 2d costal section short, 1.33-2 times length of $4 \mathrm{th}$; male genitalia with aedeagus (figs. 1186, 1187) having supporting sclerites slender, diverging apically.

Host/Early Stages. Primarily Asteraceae (apparently exclusively so in California), rarely recorded on other hosts but such records frequently referring erroneously to related species, common on Sonchus. Larva forming conspicuous white 1 inear mine, pupating in leaf.

Distribution. California, Massachusetts, Oregon, Washington; Australia, Canada, Europe, Japan, New Zealand (introduced and widespread in temperate areas).

References. Griffiths, 1967: 7; Spencer, 1969a: 278; 1981: 453; Frick, 1972: 1310.

Note. This is 1 of 2 species referred to in earlier literature as Phytomyza atricornis Mg.; 2d species, Chromatomyia horticola Goureau, does not occur in North America.

53 (52). Acr numerous, in 5-6 rows-------P. aquilegiana Frost Synopsis. Frons and orbit yellow, 2 equa1 ors, 1 or 2 ori; mesonotum and scutellum blackish; side of thorax dark, mesopleuron only narrowly yellow along upper margin; legs black, only foreknee distinct1y yellow; squama pale, margin dark brown, fringe at least partially dark; wing length $2.4-2.7 \mathrm{~mm}$, 2d costal section long, 4 times length of 4 th; male genitalia with aedeagus as in figures $1188,1189$.

Host/Early Stages. Aquilegia spp. Larva forming large greenish blotch mine, with frass 
scattered throughout mine in fine black grains; puparium reddish brown, deeply segmented, posterior spiracles widely separated, each on 1 ow protuberance, with ellipse of about 20 bulbs. Distribution. New York, Pennsylvania; Canada. References. Frick, 1959: 424; Spencer, 1969a: 227.

Note. Frick mistakenly thought that this species might also have upper ors shorter than lower; such specimens which he recorded from California, Idaho, and Washington represent $\underline{P}$. aquilegioides Sehgal (couplet 76).

$2 d$ costal section exceptionally short, little longer than 4th----- . 1atifrons Spencer, n. sp. Synopsis. Frons orange yêllow, 3 times width of eye, orbit slightly darkened to lower ors; 3d antennal segment slightly longer than broad, black, 2d black, 1st yellowish; gena broad, slightly more than 0.5 height of eye; mesonotum matt gray, acr in 2 rows; legs black, al1 knees narrowly yellow; wing length in male $2.5 \mathrm{~mm}$; male genitalia with aedeagus as in figures $1190,1191$.

Host/Early Stages. Unknown.

Distribution. Colorado.

Reference. Part 2, p. 317.

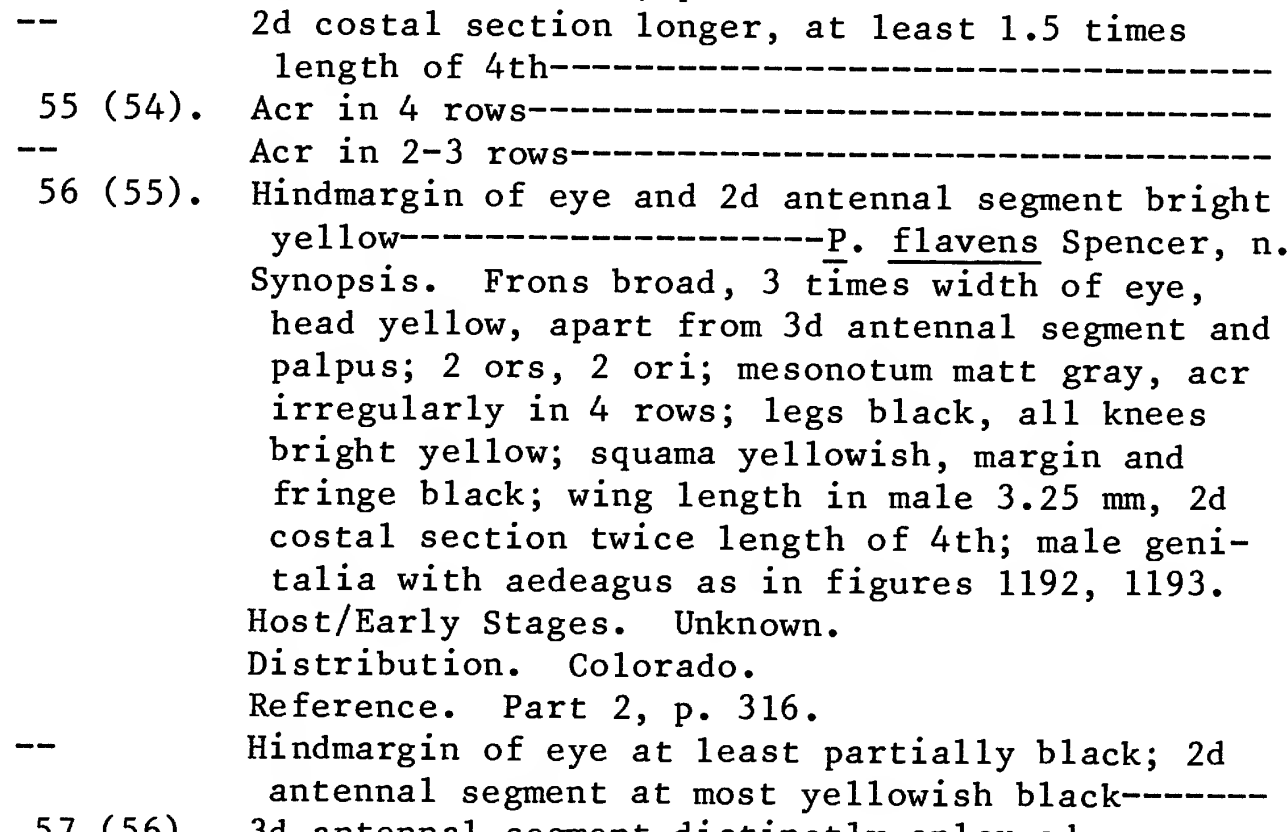

57 (56). 3d antennal segment distinctly enlarged---------

(If squamal fringe considered as $\frac{\text { clematisana }}{\text { pale, see }}$ Spencer couplet 45.)

Synopsis. Frons orange yellow, orbit slightly paler; hindmargin of eye black to base of vti; palpus, 2d and 3d antennal segments black, 1st yellowish, 3d segment enlarged, longer than broad and slightly widening distally; mesonotum 
matt gray, acr in 4 rows; legs black, a11 knees bright yellow; squama yellow, margin variable, normally brownish to black, occasionally ochreous yellow; wing length 2.1-2.5 mm, 2d costal section twice length of 4 th; male genitalia with aedeagus (fig. 1194) having distiphallus with paired, largely fused, S-shaped tubules, weakly pigmented at end.

Host/Early Stages. Clematis ligusticifolia. Larva almost certain $1 \mathrm{y}$ feeding either in stem or seeds ( 9 specimens in type series caught on foliage).

Distribution. Southern California.

Reference. Spencer, 1981: 382.

3d antennal segment small, round-

58 (57). Squamal margin brown, fringe ochreous----------

Synopsis. Frons yellowish orange or brown, orbit darkened; all antennal segments, face, and palpus black; mesonotum and scutellum matt, blackish gray, with only weak subshine; legs almost completely black, only foreknee indistinctly paler; squama gray, margin brown, fringe ochreous to black; wing length 2-2.3 mm (California), 2.6-2.8 mm (Alaska), 2d costal section 2.5 times length of 4 th; male genitalia with aedeagus as in figures $1195,1196$.

Host/Early Stages. Lathyrus ochroleucus, $\underline{\text { L. }}$ vestitus, Vicia americana. Larva forming whitish blotch, pupating externally; puparium reddish brown, posterior spiracles each with ellipse of about 20 minute bulbs.

Distribution. California; Alaska, Canada. References. Spencer, 1969a: 276; 1981: 426. Squamal margin and fringe black-

Synopsis. Frons orange yellow, twice width of eye, not projecting above eye in profile; orbit paler, with 2 ors, 2 ori; gena yellow, face grayish black; 2d and $3 \mathrm{~d}$ antennal segments black, 1st paler; mesonotum and scutellum matt black, with brownish undertone; legs black, only foreknee narrowly yellow; wing length $2.3 \mathrm{~mm}, 2 \mathrm{~d}$ costal section about twice length of 4 th; male genitalia with aedeagus as in figures 1197,1198 , basal sclerites with distinctive spicules, distiphallus divided, pigmented at end.

Host/Early Stages. Erigeron caespitosus (in Yukon Territory). Larva forming primary blotch mine on upper surface (Griffiths, 1976b: fig. 46); puparium dark brown, posterior spiracles each with 13-14 bulbs.

Distribution. California (Inyo Co.); Canada. References. Griffiths, 1976b: 255; Spencer, 1981: 409 . 
Synopsis. Frons yellow, sometimes $\bar{s}$ lightly orange centrally, orbit scarcely darkened; normally 2 strong equal ors, 2 ori, orbital setulae sparse; hindmargin of eye narrowly yellow or grayish; 3d antennal segment round, relative1y large, black, 1st and 2d generally paler, somewhat yellowish; mesonotum matt gray, acr irregularly in 2-3 rows; side of thorax dark, legs black, all knees bright yellow; squama yellowish gray, margin and fringe black; wing length 2.5-3.3 $\mathrm{mm}$ in male to $3.1-3.5 \mathrm{~mm}$ in female, $2 \mathrm{~d}$ costal section about 2.5 times length of $4 \mathrm{th}$; male genitalia with aedeagus as in figures 1199, 1200. Host/Early Stages. Taraxacum. Larva feeding in midrib, producing gall-like swelling; puparium whitish, remaining at base of midrib.

Distribution. Colorado (new record).

References. Spencer, 1976a: 526; part 2, p. 322. 1 ori, at most minute setula below-------

60 (59). Forecoxa yellowish; orbit darkened to lower ors Synopsis. Frons twice width of eye, yellowish orange; gena extended at rear, almost 0.33 height of eye, parafacial prominent; $3 d$ antennal segment black, slightly enlarged, 1 st and $2 \mathrm{~d}$ somewhat paler; mesonotum matt gray, acr in 2 rows; legs black, apart from yellowish forecoxa and narrowly yellowish knees on all femora; squama gray, margin and fringe black; wing length in male $2.5 \mathrm{~mm}, 2 \mathrm{~d}$ costal section twice length of $4 \mathrm{th}$; male genitalia with aedeagus as in figures $1201,1202$.

Host/Early Stages. Unknown.

Distribution. Colorado.

Reference. Part 2, p. 314.

Forecoxa dark; orbit entirely yellow

$61(60)$. Knees narrowly indistinctly yellowish-------

--- alamedens is Spencer

Synopsis. Frons broad, twice width of eye, bright yellow; gena yellow, antenna, face, and palpus black; mesonotum matt gray, acr in 2 rows; legs largely black; apart from narrowly yellow knees; squama gray, margin and fringe black; wing length in male $2.3 \mathrm{~mm}, 2 \mathrm{~d}$ costal section twice length of $4 \mathrm{th}$; male genitalia with aedeagus as in figures 1203, 1204.

Host/Early Stages. Unknown.

Distribution. Central California.

Reference. Spencer, 1981: 371.

Knees more conspicuously yellow

62 (61). 2d costal section long, 3 times length of 4 th---

------- Chromatomyia montella Spencer, n. sp. Synopsis. Closely resembling $P$. alamedens is but

frons broader, almost 3 times width of eye, $3 d$ antennal segment slightly longer than broad, wing with $2 \mathrm{~d}$ costal section longer, length in 
male $2.6 \mathrm{~mm}$; male genitalia with aedeagus (figs. 1205, 1206) having distinctive, curving dorsal sclerites.

Host/Early Stages. Unknown.

Distribution. Colorado.

Reference. Part 2, p. 328.

$2 d$ costal section shorter, at most twice length of 4 th------------

$63(62)$.

$2 \mathrm{~d}$ costal section twice length of $4 \mathrm{th}$

$2 \mathrm{~d}$ costal section shorter, 1.5 times length of 4 th-- -

Synopsis. Frons broad, twice width of eye, yellow but more centrally; gena and face brown; $3 d$ antennal segment small, round, black, 1st and $2 \mathrm{~d}$ slightly paler; mesonotum matt gray, acr in 2 rows; legs black, femora with all knees yellow; squama yellowish gray, margin and fringe dark; wing length 2.5-2.8 mm; male genitalia with aedeagus as in figures 1207,1208 (holotype).

Host/Early Stages. Senecio integerrimus. Details of larva and leaf mine not available.

Distribution. Oregon; new record: Colorado, Boulder Co., Niuwat Ridge, $11,300 \mathrm{ft}, 28$.VI.61 (C. H. Mann, CNC).

Reference. Griffiths, 1974b: 120.

64 (63). Male genitalia with aedeagus as in figures 1209 , $1210-0$. crepidis Spencer

Synopsis. Essentially as in $\underline{P}$. alamedensis, femora with knees slightly more yellow, and generally slightly larger, wing length 2.5-2.6 $\mathrm{mm}$.

Host/Early Stages. Crepis sp. Larva forming leaf mine, pupating in leaf (further details not recorded).

Distribution. California (mountains). Reference. Spencer, 1981: 386.

Male genitalia with aedeagus as in figures 1211 , 1212-Synopsis. Externally as in $\mathrm{P}^{-}$crepidis, differences in aedeagus distinctive. Host/Early Stages. Unknown.

Distribution. Central California.

Reference. Spencer, 1981: 405.

65 (25). Broad epistoma present-------------P. lupini Sehgal

Synopsis. Head (fig. 1213) with frons greatly projecting above eye, varying from yellow to pale brown; normally 1 strong or, less frequently 2 weaker, equal ones (couplet 30 ); gena broad, up to 0.5 height of eye; $3 \mathrm{~d}$ antennal segment distinctly elongate, black; mesonotum ash gray, acr in 2-4 rows; legs black, with all knees yellow; squama and fringe yellowish; wing length $2.7-3 \mathrm{~mm}, 2 \mathrm{~d}$ costal section short, twice length of $4 \mathrm{th}$; male genitalia with aedeagus as in figures 1214,1215 . 
Host/Early Stages. Lupinus arboreus, L. sericeus, probably other Lupinus spp. Larva feeding in pith of stem or in flower heads, pupating internally; puparium ochreous, posterior spiracles adjoining on circular, heavily sclerotized plate, each with irregular ellipse of about 12 bulbs and strong horn situated at ventral end and curving dorsally.

Distribution. California; Canada.

References. Spencer, 1969a: 252; 1981: 396. No epistoma present--_-_-_-_-_-

2 ors, upper present, 3d sometimes weak--- 72

67 (66). Squamal fringe pale------- . erigerophila Hering Synopsis. Frons orange yellow, twice width of eye, not projecting above eye; 1 ors, 2 ori; all antennal segments black, 3d rather large; palpus black; mesonotum matt black, acr irregularly in 2-4 rows; pleura largely dark but upper margin of mesopleuron and lower half of humerus yellow; legs black, all knees yellow; squama yellow, margin and fringe only slightly darker, yellowish to ochreous; wing length 1.4-1.8 mm, 2d costal section twice length of 4th; male genitalia with aedeagus (fig. 1216) having distinctive row of spicules along right basal sclerite.

Host/Early Stages. Erigeron spp. (in Europe also Aster amellus) . Larva forming narrow, upper surface linear mine; puparium black, posterior spiracles each with ellipse of 8-16 bulbs.

Distribution. Tennessee (new record); Canada, widespread in Europe.

References. Griffiths, 1976b: 256; Spencer, 1976a: 412; part 2, p. 315.

Squamal fringe dark-

$2 \mathrm{~d}$ costal section short, about 1.5 times length of $4 \mathrm{th}----$. clematoides Spencer, n. sp.

Synopsis. Frons yellow, twice width of eye; orbit dark above, with 1 ors, 2 ori; antenna black; mesonotum shining black; side of thorax and abdomen entirely black; legs black, with all knees yellow; squama yellowish, margin and fringe dark; male genitalia with aedeagus as in figures 1217, 1218, distiphallus finely divided; sperm pump minute, largely unpigmented.

Host/Early Stages. Clematis ligusticifolia. Larva forming short linear mine (fig. 1219), with frass in line of numerous fine black grains, pupating externally.

Distribution. Washington.

Reference. Part 2, p. 313.

$2 d$ costal section longer, at least twice length of 4 th----- 69

69 (68). Frons orange-- penstemonella Spencer 
Synopsis. Head (fig. 1220) with frons twice width of eye; orbit narrowly darkened beside eye margin, entire hindmargin of eye black; face, palpus, and antenna black, 3d small, round; gena 0.33 height of eye; mesonotum matt blackish gray, acr irregularly in 4 rows; legs black with all knees yellow; squama yellow, margin and fringe ochreous brown; wing length 2-2.1 mm, 2d costal section only slightly more than twice length of 4th; male genitalia with aedeagus not seen; hypandrium as in figure 1221.

Host/Early Stages. Penstemon procerus. Larva forming long, upper surface linear mine, with frass in conspicuous black line, only partial$1 y$ discontinuous, pupation externally (contrast P. penstemonis, couplet 48); details of puparium not available.

Distribution. California (mountains).

Reference. Spencer, 1981: 410.

Frons yellow, at least on upper half-_-_--- 70

70 (69). Orbit black-- aquilegivora Spencer

(Frons distinctly darkened on lower half, see couplet 126.)

Synopsis. Minute black species, closely resembling $\underline{P}$. loewii, with following essential characters: Frons ochreous yellow on upper half, black below adjoining lunule; orbit black; gena and face grayish yellow; mesonotum black, only moderately shining; legs black but al1 knees paler, brownish yellow; squama ye1lowish gray, fringe black; 2d costal section twice length of $4 \mathrm{th}$, wing length $1.5-1.75 \mathrm{~mm}$; male genitalia with aedeagus (figs. 1222, 1223) having distiphallus in form of single long tubule, with slight curvature in basal $3 \mathrm{~d}$; ventral sclerite long, narrow.

Host/Early Stages. Aquilegia spp., including A. canadensis and A. formosa var. pauciflora. Larva forming irregular whitish linear mine, with frass in scattered black grains or strips, pupating externally; puparium ovoid, dark brown, posterior spiracles each with ellipse of 10-14 bu1bs.

Distribution. California, Connecticut, District of Columbia, Illinois, New York, certainly more widespread; Canada.

References. Spencer, 1969a: 229; 1981: 376.

Note. This is probably the species misidentified by Frost (1924: 67) as $\underline{P}$. aquilegiae Hardy, which is exclusively palaearctic. Leaf mines of $\underline{P}$. aquilegivora can be confused with those of $\bar{p}$. minuscula and detailed examination of adūt $\overline{1}$ will be necessary to determine the species concerned.

Orbit yellow-

71 (70). 3d antennal segment longer than broad, virtual- 
ly bare----- saximontana Griffiths Synopsis. Frons distinctly projecting above eye (types from Alberta, scarcely so in specimens from California); orbit with 1 ors and normally 3 ori; $3 d$ antennal segment black, $2 d$ similar, lst at most faintly paler; mesonotum matt gray, acr in 3-5 rows; side of thorax dark, only upper margin of mesopleuron narrowly yellow; legs black, all knees conspicuously yellow; squama pale, margin and fringe black; wing length $2.2-2.6 \mathrm{~mm}$ in male to $2.25-2.9 \mathrm{~mm}$ in female, $2 \mathrm{~d}$ costal section 2-2.5 times length of 4th; male genitalia with aedeagus as in figure 1224 .

Host/Early Stages. Arnica, including A. mollis. Larva forming irregular linear mine (fig. 1225) which can considerably widen toward end and become almost blotchlike; puparium brown, posterior spiracles each with 17-23 bulbs.

Distribution. California (mountains); Canada. References. Griffiths, 1974b: 112; Spencer, 1981: 420 .

3d antennal segment small, round, with distinct pubescence at upper corner--------------

Synopsis. Frons yellow, not projecting above eye, with 1 strong ors at center of frons, 2 ori, lower weak; orbit entirely yellow; mesonotum blackish gray, acr irregularly in 4 rows; legs black but all knees yellow, tibiae and tarsi paler, brownish; squama yellow, margin and fringe dark; wing length $2.3-2.8 \mathrm{~mm}, 2 \mathrm{~d}$ costal section 2.5-3.5 times length of 4 th; male genitalia with aedeagus as in figures 1226, 1227, divided distiphallus pale, apart from small area of pigmentation at base.

Host/Early Stages. Aster conspicuus. Larva forming narrow, linear upper surface mine (fig. 1228), with frass in small grains or disconnected strips; puparium dull black, posterior spiracles each with ellipse of 12-15 bulbs.

Distribution. North Carolina (new record); Canada.

References. Spencer, 1969a: 230; Griffiths, 1976b: 244; part 2, p. 310 .

72 (66). Squamal fringe pale, yellow--------------- 73

-- Squamal fringe dark, brown or black----------- 74

73 (72). 2d costal section short, 1.5 times length of 4 th; orbit uniformly gray; acr in 2 rows------ - - - - - - boulderella Spencer, n. sp. Synopsis. Frons orange yellow, normal arrangement of orbital bristles probably 2 ors, with upper weaker, 1 or 2 ori; $3 d$ antennal segment black, large; mesonotum ash gray; legs black, only knees yellow; squama and fringe yellow; wing length in male $2.1 \mathrm{~mm}$; male genitalia with aedeagus as in figures 1229, 1230 . 
Host/Early Stages. Unknown.

Distribution. Colorado.

Reference. Part 2, p. 311.

$2 \mathrm{~d}$ costal section long, 3 times length of 4 th;

orbit yellow below upper ors; acr in 4 rows----

-

Synopsis. Frons clear yellow, 2 ors, upper

strong but weaker than lower, 2 ori; $3 \mathrm{~d}$ anten-

nal segment black; legs with knees broadly ye1-

low, tibiae and tarsi paler, yellowish brown;

squama and margin yellow; wing length in male

$2.5 \mathrm{~mm}$; male genitalia with aedeagus as in $\mathrm{fig}-$ ures 1231,1232 .

Host/Early Stages. Unconfirmed, probably in

Ranunculaceae.

Distribution. Washington.

Reference. Part 2, p. 317.

74 (72). Orbit black-- subtilis Spencer

(Orbit normally merely darkened, see couplet 58.)

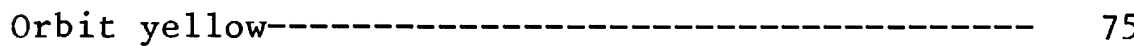

75 (74). Frons distinctly darkened in front--p. lanati spencer

(Frons normally more uniformly brown, see couplet 130.)

Frons yellow---- 76

76 (75). Acr numerous, in 5-6 rows----P. aquilegioides Sehgal Synopsis. Gena yellow, relatively narrow, 0.2-

0.17 height of eye; palpus, $3 \mathrm{~d}$ antennal segment black, lst and 2d segments slightly paler; mesonotum blackish gray, with only slight subshine; legs black, all knees yellowish; squama yellowish, margin and fringe dark; wing length 2.1-2.5 $\mathrm{mm}, 2 \mathrm{~d}$ costal section long, 3-4 times length of 4 th; male genitalia with aedeagus as in figures $1233,1234$.

Host/Early Stages. Aquilegia spp., Thalictrum polycarpum, and other Thalictrum spp. Larva forming circular blotch which can appear large and irregular, as mines of several larvae merge; puparium dark brown, all segments with bands of minute black spicules, posterior spiracles each with ellipse of about 15 minute bulbs.

Distribution. California; Alaska, Canada.

Reference. Spencer, 1981: 375.

Note. This species was misidentified in California by Frick (1959: 424) as $\underline{P}$. aquilegiana Frost. Although closely resembling P. ange1icae, $\underline{P}$. pastinacae, and $\underline{P}$. spondyli $\bar{i}$, $\overline{i t}$ is not directly related to these feeders on Apiaceae.

Acr sparse, in 2-4 rows------

Acr in 4 rows---_- 78

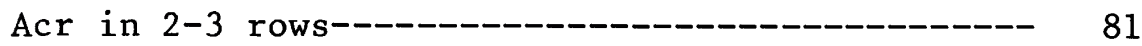

78 (77). Male genitalia with aedeagus having distiphallus

fully or at least partially pigmented (figs. 1235,1238 ) 
- Male genitalia with aedeagus having distiphallus unpigmented (figs. 1241, 1244)---_------------

79 (78). Mesopleuron only narrowly yellow along upper margin-------------- angelicae Kaltenbach

Synopsis. Frons 2-2.5 times width of eye, bright yellow, hindmargin of eye black; upper ors always more slender than lower, normally 2 ori; gena about 0.33 height of eye; $3 d$ antennal segment small, round, black, 1 st and $2 \mathrm{~d}$ segments only slightly paler; mesopleuron dark, apart from narrow yellow upper margin; mesonotum matt grayish black, acr in 4 rows; squama yellowish, margin and fringe dark; wing length from $2 \mathrm{~mm}$ in male to $3 \mathrm{~mm}$ in female, $2 \mathrm{~d}$ costal section normally about 3 times length of $4 \mathrm{th}$; male genitalia with aedeagus (fig. 1235) having distiphallus with fully pigmented, slender paired tubules in same plane as basal sclerites, these without spicules (fig. 1236).

Host/Early Stages. Most commonly Angelica spp., also Heracleum lanatum; in Europe Laserpitium, more rarely Aegopodium. Larva forming primary upper surface blotch mine (fig. 1237), several mines in same leaf may give appearance of single large mine; puparium black, posterior spiracles each with ellipse of about 20 minute bulbs.

Distribution. California; Alaska, Europe.

References. Griffiths, 1973a: 233; Spencer, 1981: 373.

Mesopleuron yellow on upper quarter-----------

-------------------- astotinensis Griffiths

Synopsis. Closely resembling p. angelicae but mesopleuron more broadly yellow, somewhat smaller, wing length up to $2.5 \mathrm{~mm}$ in female, 2d costal section shorter, about 2.5 times length of $4 \mathrm{th}$; male genitalia with aedeagus having distiphallus only partially pigmented, lying approx. at right angles to basal sclerites, these with conspicuous spicules (figs. 1238, 1239).

Host/Early Stages. Solidago flexicaulis (in Alberta, $\underline{S}$. canadensis, $\underline{S}$. gigantea). Larva forming irregular upper surface linear mine (fig. 1240); puparium black, posterior spiracles each with 15-21 bulbs.

Distribution. Wisconsin (new record); Canada. References. Griffiths, 1976b: 248; part 2, p. 310.

80 (78). Male genitalia with aedeagus having small, chitinized notch at lower 3d of distiphallus; small spiniform sclerite at end of right basal sclerite (fig. 1241)------P. spondylii Robineau-Desvoidy Synopsis. Frons and gena orange yellow, with upper orbit slightly darkened, upper ors greatly reduced (rarely lacking); 3d antennal seg- 
ment black, 1st and 2d somewhat paler; mesonotum blackish gray, acr irregularly in 4 rows; mesopleuron 1argely black but yellow along upper margin; humerus and notopleuron generally dark but sometimes faintly yellowish; legs black, with femora yellowish at knees, more distinctly so on foreleg; wing length 2.6-2.8 $\mathrm{mm}, 2 \mathrm{~d}$ costal section long, about 4 times length of 4 th; male genitalia with aedeagus as in figures 1241,1242 .

Host/Early Stages. Heracleum lanatum (in Europe, also Pastinaca sativa). Larva forming white linear upper surface mine (fig. 1243); puparium black, posterior spiracles each having 18-21 minute bulbs.

Distribution. California; Alaska, Europe.

References. Spencer, 1969a: 275 and 297 (as $\underline{P}$. heracleiphaga); Griffiths, 1973a: 225 (P. hếracleiphaga treated as new subspecies of $\underline{\underline{P}}$. spondylii); Spencer, 1981: 422.

Male genitalia with aedeagus without such notch; right basal sclerite without additional spiniform sclerite at distal end (fig. 1244)------------ pastinacae Hende1

Synopsis. Adult externally as in $\underline{P}$. spondyli $i$ male genitalia with aedeagus as in figure 1244. Host/Early Stages. Angelica atropurpurea, Heracleum lanatum (in Europe, Pastinaca sativa). Leaf mine and puparium as in $\underline{p}$. spondyli. Distribution. New York; Europe.

Reference, Griffiths, 1973a: 226 (synonymy of P. angelice11a Frost, 1927).

81 (77). Gena 0.5 height of eye--

- Chromatomyia flavida Spencer, n. sp.

Synopsis. Frons orange yellow, faint 1 y infuscated on upper half, 2 ors, upper shorter, 1 ori; $3 d$ antennal segment smal1, round, black; mesonotum grayish black, acr in 2-3 rows; legs black, with all knees yellow; squama gray, margin and fringe black; wing length in male 2.4 $\mathrm{mm}, 2 \mathrm{~d}$ costal section 1.67 times length of $4 \mathrm{th}$; male genitalia with aedeagus as in figures 1245 , 1246 .

Host/Early Stages. Unknown.

Distribution. Colorado.

Reference. Part 2, p. 325.

Gena 0.33 height of eye------ - arnicicola Lundquist Synopsis. Frons orange yellow, twice length of eye, posterior ors at most 0.67 length of anterior ors, normally 3 ori, orbit yellow or at most slightly infuscated; hindmargin of eye black; 2d and 3d antennal segments black, 1st yellowish brown; mesonotum blackish gray, acr irregularly in 2-3 rows; legs black, femora with knees contrastingly yellow; squama yellowish gray, margin and fringe dark; wing length 
2.3-2.6 mm, 2d costal section 1.5-2.5 times length of $4 \mathrm{th}$; male genitalia with aedeagus (fig. 1247) having strong patch of spinules above left basal sclerite and small patch at front end of right sclerite; distiphallus regularly curving, unpigmented except at base, divided distally.

Host/Early Stages. Arnica spp. Larva probably forming linear blotch mine (no wel1-preserved mines available, but see fig. 1365); puparium reddish brown, posterior spiracles each with 15-17 bulbs.

Distribution. Possibly Alaska, California, Colorado (new record); northern Sweden (type locality).

References. Griffiths, 1974b: 111; Spencer, 1976a: 385; part 2, p. 310 .

82 (1). 2 equal ors present----------

$83(82)$. Humerus and notopleuron bright yellow

Synopsis. Frons black, not projecting above eye, orbit similar but narrowly yellow along inner margin; 3d antennal segment small, round, black, 2d yellowish; mesonotum matt blackish gray, acr in 4 rows; legs with femora black and all knees yellow, tibiae and tarsi pale, ye1lowish brown; squama yellow, fringe black; wing length in male $2 \mathrm{~mm}$, $2 \mathrm{~d}$ costal section just less than 3 times length of 4 th; male genitalia with aedeagus as in figure 1248 .

Host/Early Stages. Unknown.

Distribution. Michigan (new record); Canada.

References. Spencer, 1969a: 259; Griffiths, 1980: 6; part 2, p. 319.

Humerus and notopleuron largely dark--------- 84

84 (83). Orbit bright yellow---Chromatomyia orbitella Spencer Synopsis. Frons and lunule black, 2 ors, 1 ori; gena 0.4 height of eye, yellow; face, palpus, and antenna black, $3 \mathrm{~d}$ segment slight1y enlarged, with fringe of short pubescence; mesonotum matt gray, with faint brownish tinge, acr in 2 rows; side of thorax blackish gray; legs black but all knees bright yellow; squama gray, margin and fringe blackish; wing length in male $2.3 \mathrm{~mm}, 2 \mathrm{~d}$ costal section slightly less than twice length of $4 \mathrm{th}$; male genitalia with aedeagus as in figures $1249,1250$.

Host/Early Stages. Unknown.

Distribution. California.

Reference. Spencer, 1981: 448.

Orbit at most narrowly yellowish on inner margin--------------------------------

85 (84). Abdomen with at least tergites $1-3$ conspicuous $1 y$ yellow-Synopsis. Frons variable, black or yellowish 
brown, orbit grayish; lunule blackish gray; gena, face, 1 st and $2 \mathrm{~d}$ antennal segments black or ochreous, 3d antennal segment black; proboscis elongate (fig. 1251); mesonotum and scutellum matt blackish gray, side of thorax black; legs entirely black; squama yellowish gray, margin and fringe black; wing length from $3.1 \mathrm{~mm}$ in male to $3.25 \mathrm{~mm}$ in female (holotype), 2d costal section 3 times length of 4 th; male genitalia with aedeagus as in figure 1252 .

Host/Early Stages. Unknown.

Distribution. New York; new records: Indiana, Tippecanoe Co., Lafayette, $1 \mathrm{~m}$., $1 \mathrm{f} ., 24 . \mathrm{V} .17$ (J. M. Aldrich, USNM); Virginia, Smyth Co., Mt. Rogers, $1 \mathrm{f.,} \mathrm{1.VI.62} \mathrm{(J.} \mathrm{R.} \mathrm{Vockeroth,} \mathrm{CNC).}$ Reference. Frick, 1959: 426.

Abdomen predominantly black or gray----_-------

Tarsi darker-

Mesonotum black, moderately shining-------------

Synopsis. Frons largely brown but somewhat ye1lowish above ocellar plate; 3d antennal segment round, black; mesonotum with acr in 5-6 rows; side of thorax dark; legs with femora black, knees yellow, tibiae and tarsi conspicuously yellow; squama whitish gray, margin and fringe black; wing length $2.1-2.5 \mathrm{~mm}, 2 \mathrm{~d}$ costal section long, 3-3.5 times length of $4 \mathrm{th}$; male genitalia with aedeagus as in figures 1253, 1254.

Host/Early Stages. Heuchera micrantha and Tellima grandiflora (Calif.), Tiarella trifoliata and Tolmiea menziesii (Alaska). Larva forming whitish linear mine up to $25 \mathrm{~cm}$ long (fig. 1255); puparium brown or white, within leaf at end of mine on lower surface, posterior spiracles each with about 20 bulbs.

Distribution. California; Alaska, Canada.

References. Griffiths, 1972a: 74; Spencer, 1981: 454 .

Mesonotum more grayish black-----P. agromyzina Meigen

Synopsis. Frons dark or ochreous brown, not projecting above eye, 2 equal ors, 1 or 2 ori; gena brown, narrow, 0.2 height of eye; $3 \mathrm{~d}$ antennal segment small, round, black or slightly paler, brownish; acr in 4 rows; side of thorax dark; femora black, with all knees yellow, tibiae and tarsi distinctly yellowish, particularly on foreleg; squama yellowish gray, margin and fringe black; wing length $2-2.25 \mathrm{~mm}, 2 \mathrm{~d}$ costal section 3-4 times length of $4 \mathrm{th}$; male genitalia with aedeagus as in figure 1256 . Host/Early Stages. Cornus californica and other Cornus spp. Larva forming long, narrow mine 
(fig. 1257), pupation normally externally but puparium sometimes remains in leaf at end of mine; puparium yellowish brown, posterior spiracles each with about 17 bulbs.

Distribution. California, Washington; Canada, Europe.

References. Spencer, 1969a: 226; 1981: 369.

88 (86). Frons uniformly pale brown-

-

(Frons considered as pale, see couplet 39.)

Frons darker, uniformly black or at least black on lower half, sometimes more yellowish above--

89 (88). Arista conspicuously thickened (fig. 1143)------

---P. crassiseta Zetterstedt (Melanic form, frons normalìy pale yellow, see couplet 31.)

$--$ Arista norma 1

90 (89). 2d costal section long, at least 3-4 times length of $4 \mathrm{th}-$

$2 d$ costal section shorter, 2-3 times length of 4 th--------------------------------

91 (90). Acr numerous, in 6-8 rows; large species, wing length 2.8-3.3 mm---- ilicis Curtis (Frons generally paler, see couplet $\overline{5} 1$. )

$92(91)$. Acr sparser, at most in $4-5$ rows

2 ors, 2 ori
2 ors, 3 ori (If frons treated as pale, see couplet 51.) Synopsis. Lower half of frons, lunule, and orbit dark, brown to blackish, upper half of frons and orbit paler, more yellowish; gena ochreous, face gray; $3 d$ antennal segment black, 1 st and $2 \mathrm{~d}$ faintly paler; mesonotum matt grayish black, acr in 4 rows; side of thorax with front of humerus and upper margin of mesopleuron yellowish, otherwise black; legs black, with only foreknee narrowly yellow; squama yellowish gray, fringe black; wing length $2.5-2.8 \mathrm{~mm}$ in male (female unknown), 2d costal section from 3 to about 4 times length of 4 th; male genitalia with aedeagus (fig. 1258) having 2 pairs of supporting sclerites, lower pair diverging by $90^{\circ}$.

Host/Early Stages. Unknown, probably a grass feeder.

Distribution. Northern California.

Reference. Spencer, 1981: 445.

93 (92). Antenna black; large species, wing length 2.7-3.3 mm----------Chromatomyia involucratae (Spencer) Synopsis. Orbit and lower frons blackish, frons on upper half frequently paler, ochreous; gena pale brown, 0.33 height of eye; mesonotum blackish gray, acr in 4 or 5 rows; side of thorax dark; legs black, only foreknee distinctly yellowish; squama whitish yellow, margin and fringe dark; wing with $2 \mathrm{~d}$ costal section long, 
3 times length of 4th; male genitalia with aedeagus (fig. 1259) having supporting sclerites large, with distinct curvature at end, divided at base in side view, largely fused and not divergent distally (fig. 1260); sperm pump minute, unpigmented.

Host/Early Stages. Almost certainly Lonicera involucrata. Leaf mine and early stages unknown.

Distribution. Colorado (new record); Canada.

References. Spencer, 1969a: 249; Griffiths, 1974a: 51; part 2, p. 326.

1st and $2 \mathrm{~d}$ antennal segments yellowish to brown; smaller species, wing length from $2.5 \mathrm{~mm}$ in male to $3 \mathrm{~mm}$ in female-_-_-_-_-_-

94 (93). Frons at most ochreous above---_--_--

Synopsis. Frons dark, brownish black in front, more ochreous above, orbit more blackish; 2 ors, upper variable, scarcely weaker than lower or more distinctly so, 2 ori; $3 d$ antennal segment black, 1st and 2d slightly paler; mesonotum grayish black with weak subshine, acr in 4-6 rows; legs largely black but femora with knees indistinctly yellowish; squama gray, margin and fringe black; wing length from $2.5 \mathrm{~mm}$ in male to $3 \mathrm{~mm}$ in female, $2 \mathrm{~d}$ costal section 3-4 times length of 4 th; male genitalia with aedeagus (fig. 1261) having supporting sclerites conspicuously expanded distally, club shaped, largely fused, pair of "wedge-shaped" sclerites below.

Host/Early Stages. Hordeum murinum, Lolium perenne in California; Agrostis and $\overline{\text { Carex }}$ in Alaska; Deschampsia in Germany. Larva forming linear mine; puparium pale white to yellow, with anterior spiracles on long, slender projections, posterior spiracles on large conical projections, each with about 25 bulbs.

Distribution. Central and northern California; new records: North Carolina, Swain Co., Clingman's Dome, 6,300-6,600 ft, Gt. Smoky Mts., N.P., 2 m., 20.V.57; Tennessee, Sevier Co., Indian Gap, 5,200 ft, Gt. Smoky Mts., N.P., $1 \mathrm{~m}$, 2.VII.57; (a11 J. R. Vockeroth); Alaska, Germany. References. Griffiths, 1980: 23; Spencer, 1981: 449.

Frons bright yellow on upper half--_-_--_-_---- - - Chromatomyia norwegica (Rydén)

Synopsis. Frons blackish below adjoining lunule, yellow above, at least adjoining orbit; orbit yellowish brown or darker, grayish black (Colorado), 2 ors, upper at most marginally shorter, 2 ori; gena and face predominantly pale; 3d antennal segment small, round, black, 
1st and 2d paler; mesonotum blackish gray, acr in 4-6 rows; legs with femora black, all knees distinctly yellow; squama yellowish white, margin and fringe black; wing length $2.5-2.9 \mathrm{~mm}$, $2 \mathrm{~d}$ costal section about 3 times length of $4 \mathrm{th}$; male genitalia with aedeagus (figs. 1262, 1263) having supporting sclerites slender, diverging slightly at distal end, "wedge-shaped" sclerites prominent, sclerites of ventral lobe tapering, with ejaculatory duct distinctly pigmented; sperm pump minute.

Host/Early Stages. Arctagrostis 1atifolia, Calamagrost is (Germany), Cinna latifolia

(British Columbia), Vahlodea atropurpurea (Alberta). Leaf mine linear, broader; puparium remaining adjacent to upper or lower leaf surface, with anterior spiracles projecting ventrally through epidermis.

Distribution. Colorado (new record); Canada, Europe - Lapland and mountains of central Europe (Germany).

References. Griffiths, 1980: 18; part 2, p. 329.

95 (90). Mesonotum shining black----- p. phaceliae Spencer Synopsis. Frons and all antennal segments deep black, orbit shining black; 2 equal ors, 1 ori, orbital setulae strong, in single row; side of thorax, legs, and abdomen black; squama yellowish gray, margin black; wing base conspicuously yellowish; wing length in female $2.3 \mathrm{~mm}, 2 \mathrm{~d}$ costal section 2.67 length of $4 \mathrm{th}$; male genitalia unknown.

Host/Early Stages. Phacelia californica. Larva forming linear blotch mine (fig. 1264), pupation externally; puparium brown, posterior spiracles each on low conical projection, with ellipse of about 12-15 bulbs.

Distribution. Central California.

Reference. Spencer, 1981: 413.

Mesonotum more matt black or grayish black------ 96

Eye conspicuously pilose--Chromatomyia nigra (Meigen)

Synopsis. Frons variable, from ochreous yellow

to dark brown, orbit frequently paler or more grayish; 2 equal ors, 1 or $i$; $3 d$ antennal segment small, round, black, at most lst segment slightly paler; mesonotum matt grayish black, acr in 2 rows; legs black but all knees distinctly yellow; squama yellowish gray, margin black; wing length in male 1.9-2.6 mm, in female $1.8-2.8 \mathrm{~mm}$, 2d costal section normally from 2 to 2.5 times length of $4 \mathrm{th}$; male genitalia with aedeagus (fig. 1265) having 2 pairs of dorsal (supporting) sclerites and additional pair of dorsally directed sclerites connected to near apex of basal sclerites; ejaculatory duct strongly pigmented, with characteristic sinuate curvature; sperm pump small, base asymmetrical. 
Host/Early Stages. Festuca, Lolium (U.S.A.), 49 other genera of Poaceae, mainly in Europe, recorded by Griffiths (1980). Larva forming narrow linear mine, with long anterior spiracles of puparium (fig. 1266) projecting through epidermis.

Distribution. California, Oregon; Canada, Europe.

References. Spencer, 1969a: 259 (as Phytomyza); 1981: 447; Griffiths, 1980: 49 (here C. nigra is treated as superspecies with 6 forms, 1 of which is North American (Pacific coast) form. Differences between these forms are slight, but Griffiths considers that they may in due course be upgraded to distinct species).

Frons conspicuously projecting above eye; 3d antennal segment slightly elongate; proboscis elongate----------Napomyza evanescens (Hendel)

98

Frons not significantly projecting above eye--- 98

98 (97). Legs entirely black-_- 99

99 (98). 2d costal section more than 2 times length of $4 \mathrm{th}$; frons paler, brownish, above-- - Chromatomyia griffithsi Spencer, n. sp. Synopsis. Frons blackish below, 2 ors, 2 ori, 3d antennal segment small, round; mesonotum matt grayish black, acr in 4 rows; wing length 2.5-3 mm, 2d costal section normally 2.3 times length of 4 th; male genitalia with aedeagus as in figure 1267, supporting sclerites as in figure 1268.

Host/Early Stages. Unknown.

Distribution. Colorado.

Reference. Part 2, p. 326.

$2 d$ costal section less than 2 times length of 4th; frons deep black---------P. nigrinervis Frost Synopsis. Frons broad, twice width of eye, deep black, orbit pronounced, generally paler, slightly gray; 2 equal ors, normally 1 ori, sometimes additional one in front; all antennal segments black, 3d slightly enlarged; mesonotum matt blackish but with faint brownish tinge, acr sparse, in 2 rows; legs entirely black; squama gray, margin and fringe deep black; wing with veins $R 2$ and $R 3$ conspicuously black, length $2.5-2.6 \mathrm{~mm}, 2 \mathrm{~d}$ costal section normally 1.7 , at most 2 times length of $4 \mathrm{th}$; male genitalia with aedeagus as in figures 1269,1270 .

Host/Early Stages. Unconfirmed but almost certainly Ranunculaceae (deduced from male genitalia).

Distribution. Colorado (type series of 10 specimens); new records: Colorado: Clear Creek Co., Mt. Evans, Summit Lake Flats, 12,800 ft, 
7 m., 6 f. (C. H. Mann, CNC); Loveland Pass, $12,000 \mathrm{ft}$, summit, $1 \mathrm{~m} ., 7 . V I I I .61$ (B. H. Poole); Lake Co., Independence Pass, 12,100 ft, tundra, $1 \mathrm{~m}$., 31.VII.671 (J. G. Chillcott). References. Frost, 1924: 87; Frick, 1959: 432. 100 (98). 3d antennal segment enlarged, somewhat angular at upper corner (fig. 1271)

Synopsis. Frons twice width of eye, blackish below, more brownish or even ochreous above; 2 equal ors, generally only 1 ori but sometimes smaller one below; gena slightly more than 0.33 height of eye, brownish; face, palpus, antenna black; mesonotum matt gray, acr variable, in 2-4 rows; legs black but all knees conspicuously yellow; squama pale gray, margin and fringe black; wing length $2.1-2.4 \mathrm{~mm}, 2 \mathrm{~d}$ costal section short, little longer than 4th; male genitalia with aedeagus (figs. 1272, 1273) having long basal sclerites, supporting (dorsal) sclerites weakly pigmented except at base, long straight sclerites lying ventrally and extending forward from end of basal sclerites, left longer than right; distiphallus with paired convergent sclerites and short semicircular sclerotized area near end of ejaculatory duct; sperm pump as in figure 1274 .

Host/Early Stages. Erigeron (high elevation species). Larva feeding mainly in petiole or base of leaf, moving from one leaf to another via short stem; puparium yellowish white, at base of leaf, with anterior spiracles projecting ventrally through epidermis.

Distribution. Colorado (new record); Alaska, Canada, northern Greenland (Peary Land).

References. Spencer, 1969a: 239; Griffiths, 1976b: 261; part 2, p. 325 .

3d antennal segment smal1, round--

101 (100). Mesonotum distinctly black-a nigrella Spencer, n. sp.

Synopsis. Small species, wing length in male 2 $\mathrm{mm}, 2 \mathrm{~d}$ costal section 2.5-2.9 times length of 4 th; frons black or slightly brownish above; 2 equal ors (normally), 1 strong ori, sometimes weak additional one below; acr variable, in 2 rows in 1 specimen, 4 rows in other; legs black, knees distinctly yellowish; male genitalia with aedeagus having slender supporting sclerites (figs. 1275, 1276); sperm pump minute (fig. 1277).

Host/Early Stages. Unknown.

Distribution. North Carolina.

Reference. Part 2, p. 328.

Mesonotum at most blackish gray-_-_-_-_-_-- 102

102 (101). Acr in 2 rows-_-_- 103

Acr in 4 rows-- 
103 (102). Frons blackish on lower half, more ochreous yellow above; $2 d$ costal section short, little longer than 4th---------------P. melane1la Frost Synopsis. Frons generally dark, paler above, 2 ors, 2 ori; 3d antennal segment small, round, black; gena yellowish, parafacial darker; mesonotum matt gray; side of thorax dark but rear or humerus, notopleuron, and upper margin of mesopleuron faintly yellowish brown; legs black, knees faintly yellowish; wing length $2 \mathrm{~mm}$; male genitalia with aedeagus (fig. 1278) having distinctive arrangement of spicules beside basal sclerites; sperm pump minute.

Host/Early Stages. Unknown.

Distribution. California.

References. Frick, 1959: 431; Spencer, 1981: 400.

Note. Frick restricted this species to type specimens from Los Angeles, considering that male from Kentucky included as paratype represented a different species. This is so, as frons in this male is yellow, but positive identification has not been possible.

Frons variable, generally orange brown, orbit paler, more yellow or gray

-- Chromatomyia poae Griffiths

Synopsis. Frons broad, about twice width of eye, not projecting above eye, 2 equal ors, 1 ori; gena about 0.33 height of eye, brownish; 3d antennal segment small, round, black, only 1st at most slightly paler; mesonotum grayish black matt, acr sparse, in 2 rows; side of thorax dark; legs black but all knees narrowly yellowish; squama pale grayish, margin and fringe black; wing length $2.1-2.6 \mathrm{~mm}$ in male to 2.2-2.7 $\mathrm{mm}$ in female, $2 \mathrm{~d}$ costal section normally about twice length of $4 \mathrm{th}$; male genitalia with aedeagus (fig. 1279) having supporting sclerites slender, ejaculatory duct at end parallel to supporting sclerites, curving ventral$1 y$; sperm pump with large, asymmetrical blade (fig. 1280).

Host/Early Stages. Poa alpina in Alberta. Larva forming narrow linear mine; puparium white to reddish brown, anterior spiracles long, projecting through epidermis, posterior spiracles each with 5-6 bulbs.

Distribution. Colorado (new record); Canada. References. Spencer, 1969a: 242; 1976a: 421;

Griffiths, 1980: 40; part 2, p. 329.

104 (102). Mesonotum distinctly brownish gray---_-_-_-_-- 105

-- Mesonotum more distinctly grayish---_---_----- 113

105 (104). Tibiae and tarsi pale, brownish-- 106

-- Tibiae and tarsi darker, concolorous with femora--------- 107

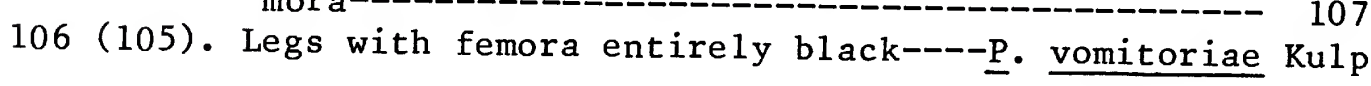


Synopsis. Frons brownish black, orbit darker, entirely black; gena brownish; antenna black, 3d segment small, round; mesonotum matt blackish gray, acr in 4-5 rows; legs with femora entirely black, tibiae and tarsi distinctly paler, brownish; wing length from $1.65 \mathrm{~mm}$ in male to $1.9 \mathrm{~mm}$ in female, 2d costal section 2.1-2.5 times length of 4th; male genitalia with aedeagus (figs. 1281, 1282) having distal, diverging section of distiphallus short, ventral sclerite small; sperm pump large.

Host/Early Stages. Ilex vomitoria. Larva forming upper surface linear blotch mine, which can entirely fill small leaves; puparium brownish black, remaining in mine with anterior spiracles projecting through leaf epidermis.

Distribution. California, Florida, Georgia; new records: Louisiana, Evangeline Co., Mamou, $1 \mathrm{~m}$, 28.III.60 (J. G. Chillcott); Mississippi, Washington Co., Leland, many specimens (K. E. Frick).

References. Ku1p, 1968: 25; Spencer and Stegmaier, 1973: 124; Steyskal, 1976: 767; Spencer, $1981: 432$.

Legs with femora having foreknee yellowish--_---

Synopsis. Frons black in front, paler $\frac{\text { persicae }}{\text { above; } 2}$ Frick equal ors, 2 ori, lower sometimes lacking; mesonotum blackish gray, acr in 4 rows; wing length about $2 \mathrm{~mm}, 2 \mathrm{~d}$ costal section twice length of 4 th; male genitalia with aedeagus (fig. 1283) having paired division of distiphallus characteristically recurved.

Host/Early Stages. Prunus persica (peach). Larva forming long, whitish linear mine (Frost, 1924: plate II, fig. 3), with frass in conspicuous black pellets (fig. 1284), pupation in leaf, with anterior spiracles of puparium projecting through epidermis.

Distribution. Connecticut, Ohio, Virginia; Canada.

References. Frost, 1924: 81 (as P. obscurella var. nigritella Zett.); Frick, 1954: 369; Spencer, 1969a: 266.

107 (105). Smal1 species, wing length $1.3-1.7 \mathrm{~mm}$ in male, $1.7-1.85 \mathrm{~mm}$ in female--

Synopsis. Frons brownish black, twice width of eye, 2 equal ors, 2 ori, lower weak; antenna black, 3d segment small, round; mesonotum blackish gray, acr irregularly in 4 rows; legs black, foreknee slightly brownish; squama gray, fringe black; male genitalia with aedeagus (figs. 1285, 1286) having supporting sclerites straight sided, uniformly tapering; sperm pump minute, unpigmented. 
Host/Early Stages. Symphoricarpos rivularis. Larva forming irregular blotch mine in leaf blade, stellate initially (fig. 1287), pupating in leaf; puparium yellowish to brown, posterior spiracles each with 12-14 bulbs.

Distribution. California, Washington.

References. Griffiths, 1974a: 53; Spencer, 1981: 439.

Larger species, wing at least $1.9 \mathrm{~mm} 1$ ong, or, if shorter with male genitalia otherwise------

108 (107). Gena broad, approx. 0.5 height of eye---------

---- Chromatomyia luzulivora Spencer, n. sp.

Synopsis. Frons brownish black, orbit grayish; 3d antennal segment small, round, black; mesonotum blackish gray, acr in 4 rows; wing length $2 \mathrm{~mm}, 2 \mathrm{~d}$ costal section twice length of $4 \mathrm{th}$; male genitalia with aedeagus as in figures 1288, 1289; sperm pump minute.

Host/Early Stages. Luzula sp. (assumed from male genitalia).

Distribution. Colorado.

Reference. Part 2, p. 327.

-- Gena narrower, about 0.33 height of eye--------

109 (108). Frons exceptionally broad, approx. 3 times width

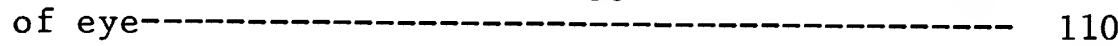

Frons at most twice width of eye-- 111

110 (109). 2 ori-----Chromatomyia mitchelli spencer, n. sp. Synopsis. Agreeing closely with $\underline{\text { C. griffithsi, }}$ slightly smaller, with wing length in male $2.3 \mathrm{~mm}$; male genitalia with aedeagus having supporting sclerites slender, diverging, not developed laterally (figs. 1290, 1291); sperm pump minute.

Host/Early Stages. Unknown, probably Luzula. Distribution. North Carolina.

Reference. Part 2, p. 327.

-- 1 ori-- $\quad$-Chromatomyia doolittlei Spencer, n. sp. Synopsis. Frons brownish black, 2 equal ors, 1 ori; gena 0.33 height of eye; $3 d$ antennal segment small, round, finely pubescent; mesonotum blackish gray, acr in 4 rows; legs with only foreknee yellowish; wing length in male $2.6 \mathrm{~mm}$, $2 \mathrm{~d}$ costal section about 2.2 times length of 4th; male genitalia with aedeagus (fig. 1292), having supporting sclerites slender but strongly developed laterally (fig. 1293); sperm pump minute (fig. 1294).

Host/Early Stages. Unknown.

Distribution. Colorado.

Reference. Part 2, p. 324.

111 (109). Male genitalia with aedeagus (fig. 1295) having supporting sclerite complex with middorsal hump in side view-------Chromatomyia gregaria (Frick)

Synopsis. Generally resembling C. fricki but slightly larger, wing length (mean) $2 \mathrm{~mm}$ in male, $2.2 \mathrm{~mm}$ in female; $2 \mathrm{~d}$ costal section nor- 
mally about 2.5 times length of $4 \mathrm{th}$; male genitalia with aedeagus (figs. 1295, 1296) distinguished from related species by middorsal hump; sperm pump minute.

Host/Early Stages. Lonicera involucrata. Larvae feeding together to form irregular mine based on midrib (fig. 1297); puparium within mine, puparium dull yellow to almost black, posterior spiracles each with 16-23 bulbs.

Distribution. Central California; Canada.

References. Frick, 1954: 371; Griffiths, 1974a: 50; Spencer, 1981: 440 .

-- Male genitalia with aedeagus (figs. 1298, 1302) having supporting sclerite complex gradually tapered in side view without such hump--.---

112 (111). Male genitalia with aedeagus (fig. 1298) having supporting sclerites slender, slightly curving toward apex----Chromatomyia symphoricarpi Griffiths Synopsis. Minute black species, agreeing close$1 \mathrm{y}$ with $\underline{\mathrm{C}}$. fricki and $\underline{\mathrm{C}}$. gregaria, marginally larger than C. fricki, not satisfactorily distinguishab $1 \mathrm{e}$ on external characters from $\underline{C}$. gregaria; male genitalia with aedeagus (figs. 1298, 1299) having basal sclerites ending before base of supporting sclerites, these broad at base and slightly curving at apex; sperm pump (fig. 1300) minute, unpigmented.

Host/Early Stages. Symphoricarpos mollis (California). Larva forming irregular blotch on midrib at base of leaf (fig. 1301); puparium yellowish or more brownish, posterior spiracles each with ellipse of 10-15 bulbs.

Distribution. California; Canada.

References. Griffiths, 1974a: 52; Spencer, 1981: 450.

Male genitalia with aedeagus (fig. 1302) having supporting sclerites broad, short--

- - - - - Chromatomyia regalensis (Steyska1)

Synopsis. Agreeing with C. symphoricarpi on external characters, wing length $2.1 \mathrm{~mm}, 2 \mathrm{~d}$ costal section 2.5 times length of 4 th; male genitalia with aedeagus (fig. 1302) having sclerites of ventral lobe largely rectangular, supporting sclerites broad at base, terminal section of ejaculatory duct strongly pigmented. Host/Early Stages. Unknown.

Distribution. Georgia, Michigan.

References. Steyskal, 1972b: 8; Griffiths, 1980: 8; part 2, p. 329 .

113 (104). 2d costal section relatively long, 2.5-3 times length of 4 th-- 114

$2 d$ costal section shorter, 2-2.5 times length of

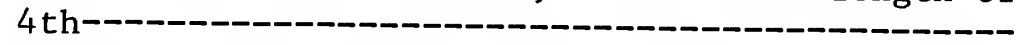

114 (113). Mesonotum pale, almost silvery gray; mesophallus

straight sided (fig. 1304)---- - - . ilicicola Loew

Synopsis. Frons black below, somewhat paler, 
brownish above; orbit yellowish along inner margin, blackish gray adjoining eye, sometimes but not always raised above eye; antenna black; acr in 4 rows; legs with femora black, tibiae and tarsi paler, yellowish brown, particularly on foreleg; squama gray, margin and fringe black; wing length from $1.6 \mathrm{~mm}$ in male to $2.3 \mathrm{~mm}$ in female; male genitalia with aedeagus (figs. 1303,1304 ) having mesophallus relatively short, tapering toward rear; sclerites of ventral lobe strongly pigmented, large, rectangular.

Host/Early Stages. Ilex aquifolium, I. opaca. Larva forming irregular linear blotch mine (fig. 1305); puparium reddish brown to black; univoltine in north, probably at least 2 generations in south (Florida).

Distribution. Widespread in northeast, extending south to Florida, Kentucky, South Carolina. References. Kulp, 1968: 16; Spencer and Stegmaier, 1973: 117; Steyskal, 1976: 767. Note. The record given by Spencer (1969a: 246 and fig. 441) for Ontario possibly refers to a distinct species.

Mesonotum slightly darker, grayish black; mesophallus with conspicuous stricture at midpoint (fig. 1307)--

Synopsis. Generally as in $\underline{P}$. ilicicola, possibly larger, wing length over $2 \mathrm{~mm}$, up to $2.5 \mathrm{~mm}$; $2 \mathrm{~d}$ costal section more than 2.5 times length of 4 th (rarely slightly over 3 times); male genitalia with aedeagus as in figures 1306, 1307. Host/Early Stages. Ilex glabra. Larva forming linear blotch mine, pupating in leaf; puparium reddish brown to black, with anterior spiracles projecting through lower epidermis of leaf.

Distribution. Connecticut, District of Columbia, Massachusetts, New Jersey, New York, Ohio. References. Ku1p, 1968: 14; Steyskal, 1976: 767.

115 (113). Male genitalia (fig. 1309) with mesophallus long, slender-- verticillatae Kulp

Synopsis. As in P. ditmani; male genitalia with aedeagus (figs. $\overline{1} 30 \overline{8,1309}$ ) having ventral lobe large, more strongly pigmented toward front; leaf mines occur from April (Florida) until November (Maryland) and are linear, only slightly widening at end (fig. 1310) or become. more distinctly blotchlike (Kulp, 1968: fig. 8, D).

Host/Early Stages. Ilex verticillata and sp. indet. (Florida). Larva forming relatively short linear blotch mine.

Distribution. District of Columbia, Florida, Maryland.

References. Kulp, 1968: 25; Spencer and Stegmaier, 1973: 123; Steyskal, 1976: 767. 
Male genitalia with aedeagus (figs. 1312, 1315)

having mesophallus shorter, broader--

116 (115). Host. Ilex decidua, I. serrata. Larva forming irregular blotch miné (fig. 1313)----p. ditmani Kulp

Synopsis. Generally as in P. ilicicola but smaller, wing length at most $1.9 \mathrm{~mm}$, mesonotum distinctly darker gray; $2 \mathrm{~d}$ costal section $2-2.5$ times length of 4th; male genitalia with aedeagus (figs. 1311, 1312) having distal tubules widely diverging, mesophallus relatively short and broad, not tapering.

Biology. Larva forms small blotch mine on lower surface of leaf in lst instar (fig. 1313), in $2 \mathrm{~d}$ and $3 \mathrm{~d}$ instar forming irregular linear blotch mine on upper surface, feeding slowly throughout summer from July until October, univoltine.

Distribution. District of Columbia.

References. Kulp, 1968: 11; Steyskal, 1976: 767 (fig. of aedeagus).

Host. Ilex aquifolium, I. cumulicola, I. opaca. Larva forming long, serpentine mine ( $f$ ig. 1316)--- -

Synopsis. As in P. ditmani; male genitalia with aedeagus (figs. $\overline{1} 31 \overline{4,1315}$ ) having distal tubules slender, widely diverging in apical 3d; mesophallus longer, more slender, ventral lobe short, broad, heavily pigmented.

Biology. Leaf mine linear in all 3 instars, 1st appearing toward end of May (Maryland).

Distribution. Delaware, District of Columbia, Florida, Maryland.

References. Kulp, 1968: 21; Spencer and Stegmaier, 1973: 119; Steyskal, 1976: 767.

117 (82). 1 ors (at most minute hairs dorsally)----_---- 118

-- 2 ors (upper at least 0.5 length of lower)------ 127

118 (117). Squamal fringe pale, yellow or white--_-_---- 119

-- Squamal fringe dark, black or brown---_-_-_-_--- 122

119 (118). 3d antennal segment round--_-_--_-_-_-_-_--- 120

-- 3d antennal segment elongated---_-_-_-_-_----- 121

120 (119). Frons matt black-------- - masoni Spencer, n. sp. Synopsis. Frons matt black, mesonotum shining black; wing length in male $2.25 \mathrm{~mm}, 2 \mathrm{~d}$ costal section 2.56 times length of $4 \mathrm{th}$; male genitalia with aedeagus as in figures 1317,1318 , tubules of distiphallus short, broad.

Host/Early Stages. Unknown, probably Ranunculaceae.

Distribution. Georgia.

Reference. Part 2, p. 318.

Frons yellowish brown

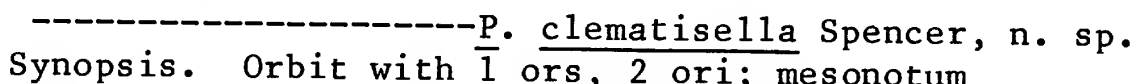
yellowish; wing length $1.75-1.9 \mathrm{~mm}$ in male, $2 \mathrm{~d}$ costal section twice length of $4 \mathrm{th}$; male geni- 
talia with aedeagus as in figures 1319, 1320, tubules of distiphallus long, slender.

Host/Early Stages. Clematis. Larva feeding in

stem (details not recorded).

Distribution. Arkansas, Connecticut.

Reference. Part 2, p. 312.

121 (119). Wing and veins distinctly yellow; 2d costal section long, 4 times length of 4 th---_------

- - pulchella Spencer, n. sp.

Synopsis. Closely resembling $\underline{P}$. saskatoonensis

but distinguished by yellow veins and wing, longer $2 \mathrm{~d}$ costal section and larger; male genitalia with aedeagus as in figures 1321,1322 .

Host/Early Stages. Unknown, probably Ranunculaceae.

Distribution. Virginia.

Reference. Part 2, p. 320.

Wing hyaline, veins brown; $2 \mathrm{~d}$ costal section shorter, at most 2.5 times length of 4 th-------

--

Synopsis. Frons dark brown to black, projecting above eye; 1 strong reclinate ors, 3 inclined or $i$; all antennal segments black, $3 \mathrm{~d}$ elongate; mesonotum deep black, only moderately shining, acr irregularly in 3 or 4 rows; legs black, femora with knee distinctly yellow on foreleg, less so on midleg and hindleg; abdomen black; squama and fringe yellow; male genitalia with aedeagus as in figures 1323, 1324 (also

Steyskal, 1972b: fig. 5); sperm pump large, blade wider than long.

Host/Early Stages. Unknown, almost certainly Ranunculaceae.

Distribution. Michigan, Wisconsin; new record:

New York, Lewis Co., Tug Hill, $1 \mathrm{~m} ., 1 . V I .66$

(L. Knutson, CUI) ; Canada.

References. Frick, 1959: 433 (as P. nigripennis); Spencer, 1969a: 272; Steyskal, 1972b: 4 (as P. cudu); part 2, p. 321 .

122 (118). 2d costal section long, 3-4 times length of 4 th-

$2 \mathrm{~d}$ costal section shorter, at most 2.5 times

length of 4 th---

123 (122). Acr in 2 rows; tarsi dark, little paler than femora ----- minuscula Goureau

Synopsis. Frons broad, twice width of eye, normally brownish black, sometimes paler, ochreous on upper half; 1 strong, outcurving ors, 1 strong inclined ori; orbit black; antenna black, 3d large; mesonotum deep black, shining from rear, appearing more matt from front; legs largely black, only foreknee sometimes yellowish; squama yellowish gray, margin and fringe black; male genitalia with aedeagus (fig. 1325) having distiphallus a single tubule, slightly curving ventrally at apex; ventral 
sclerite large, almost as long as distipha1lus; sperm pump with strongly pigmented round blade and distinct stalk.

Host/Early Stages. Aquilegia spp., Thalictrum spp. Larva forming irregular upper surface linear mine, with frass in conspicuous black strips; puparium oval, shining orange, normally at first lightly adhering to leaf near end of mine.

Distribution. Widespread in California, Washington; records given by Frick (1959: 431) from Connecticut, District of Columbia, Illinois, and Indiana probably refer to $\underline{P}$. aquilegivora (couplets 70 and 126).

References. Frick, 1959: 431; Spencer, 1981: 401. Acr in 4 rows; tarsi yellow-

--Synopsis. Frons black, orbit moderately shining; 1 ors, 2 ori; $3 \mathrm{~d}$ antennal segment small, round; mesonotum shining black; femora black, tibiae brownish; wing length $1.9-2.25 \mathrm{~mm}, 2 \mathrm{~d}$ costal section slightly more than 3 times length of 4th; male genitalia with aedeagus as in figures 1326, 1327, and distiphallus divided, recurved; sperm pump minute (fig. 1328). Host/Early Stages. Ranunculus abortivus. Larva forming white, upper surface, 1inear mine, beginning with minute spiral (fig. 1329); puparium yellowish, posterior spiracles each 2-armed, with total of 12-20 bulbs.

Distribution. Minnesota.

Reference. Part 2, p. 320.

124 (122). 2d costal section exceptionally short, less than twice length of 4th----- . thalictrella Spencer

Synopsis. Frons broad, 3 times width of eye, entirely black, orbit moderately shining; 1 reclinate ors, 1 inclined ori, sometimes minute hair below; gena broad, about 0.5 height of eye; mesonotum deep black, weakly shining, acr in 2 rows; legs black; squama grayish black, margin and fringe black; wing length from $1.25 \mathrm{~mm}$ in male to $1.75 \mathrm{~mm}$ in female, $2 \mathrm{~d}$ costal section from 1.25 to 1.67 times length of 4th; male genitalia with aedeagus (figs. 1330,1331 ) having distal tubules upright or more recurved; sperm pump small, weakly pigmented.

Host/Early Stages. Thalictrum fendleri. Details of leaf mine not available.

Distribution. California (Alpine Co.).

Reference. Spencer, 1981: 427.

125 (124). Acr in 4 rows or lacking--- loewii Hende1 Synopsis. Head black, orbit shining, 1 ors, 1 ori, at most minute hair below; mesonotum and scutellum shining black; legs black, foreknee 
inconspicuously paler; squama gray, fringe black; wing length $1.5-1.9 \mathrm{~mm}$; male genitalia with aedeagus (fig. 1332) having distiphallus directed dorsally, paired tubules strongly pigmented at base, membranous apically, strongly divergent (fig. 1333); ejaculatory duct rising dorsally from near distal end of basal sclerites to base of distiphallus; sperm pump minute.

Host/Early Stages. Clematis spp., including $\underline{C}$.

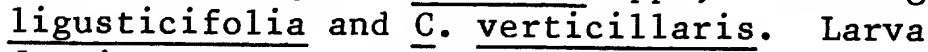
forming upper surface linear mine which greatly widens ( $\mathrm{fig}$. 1334) and can appear as blotch in small leaves; puparium brownish, posterior spiracles each with heavily sclerotized branch having about 15-20 irregular bulbs.

Distribution. California, District of Columbia, Idaho, Indiana, Louisiana, Washington; new record: Georgia, Rabun Co., Pine Mountain, $1,400 \mathrm{ft}, 1 \mathrm{~m} ., 4 . \mathrm{V} .57$ (J.R. Vockeroth, CNC); Canada.

References. Frick, 1959: 430; Spencer, 1969a: $251 ; 1981: 395$.

Acr in 2 rows or lacking

126 (125). Frons yellowish dorsally; acr lacking--_-_---

(If frons considered as predominantly pale, see couplet 70.)

Frons darker, brownish gray; acr in 2 rows------

-

Synopsis. Closely resembling $\underline{P}$. aquilegivora, differing in darker frons, more shining mesonotum, presence of acr, and longer $2 \mathrm{~d}$ costal section, which is 2.5 times length of $4 \mathrm{th}$; male genitalia with aedeagus (fig. 1335) having distiphallus long, slender, sinuate.

Host/Early Stages. Thalictrum spp. (in Alberta, T. venulosum). Larva forming upper surface linear mine, appearing blotchlike in small leaves; puparium orange brown. Distribution. (?) Northern Florida; Canada. References. Spencer, 1969a: 279; Spencer and Stegmaier, 1973: 122 (tentative identification from single caught female).

127 (117). 2d costal section long, 3-4 times length of 4 th -c- 128

$2 d$ costal section shorter, less than 3 times length of 4 th-

128 (127). Small species, wing length $1.75-2 \mathrm{~mm}-$

- saniculae Spencer

Synopsis. Frons twice width of eye, blackish, paler only beside ocellar triangle; orbit paler, sometimes yellowish; 2 ors, upper substantially shorter than lower, 2 ori; antenna black, $3 \mathrm{~d}$ segment small, round; legs with femora black, having only foreknee distinctly yellow, tibiae 
and tarsi largely dark; mesonotum blackish gray, only weakly shining, acr in 2 rows; squama gray, margin and fringe black; wing with 2d costal section about 3 times length of $4 \mathrm{th}$; male genitalia with aedeagus (fig. 1336) having 2 rows of spinules toward distal end of basal sclerites; distiphallus directed dorsally, broadly fused at base (fig. 1337); sperm pump with large blade.

Host/Early Stages. Sanicula crassicaulis (syn., S. menziesii) and $\underline{\text { s. }}$ laciniata in California, $\underline{\text { s. }}$ marilandica in Alberta. Larva forming broad, irregular upper surface mine (fig. 1338), greenish when fresh, brown when older; puparium black, posterior spiracles each with ellipse of about 15 bulbs.

Distribution. California; Canada.

References. Spencer, 1969a: fig. 536; 1981: 417.

Note. Available material suggests that P. saniculae is smaller than $\underline{P}$. lanati and $\underline{P}$. oenanthoides, but positive identification of caught specimens may only be possible from the male genitalia.

Larger species, wing length $2.1-2.8 \mathrm{~mm}$

129 (128). 1 ori-- oenanthoides Spencer Synopsis. Closely resembling $\overline{\mathrm{P}}$. $\overline{\text { saniculae but }}$ somewhat larger, wing length $\overline{2} .1 \overline{-2.4 \mathrm{~mm}, 2 \mathrm{~d}}$ costal section longer, 3-4 times length of 4 th; acr more numerous, irregularly in 4 rows; male genitalia with aedeagus (fig. 1339) having spinules above both left and right basal sclerites, those on left fewer but stronger, distiphallus largely unpigmented.

Host/Early Stages. Oenanthe sarmentosa. Larva forming irregular upper surface linear mine (fig. 1340); puparium black, posterior spiracles greatly enlarged (for aquatic environment), 2-armed, with 15 bulbs on one arm, 10 on other (see $\underline{P}$. cicutae Hendel in Europe, Spencer, 1976a: fig. 704).

Distribution. California (Alameda Co.).

Reference. Spencer, 1981: 406.

130 (129). Male genitalia with aedeagus (figs. 1341, 1342) having strip of spinulate sclerotization beside ventral lobe----- lanati spencer

Synopsis. Generally resembling both ${ }^{-}$. oenanthoides and $\underline{P}$. saniculae, possibly larger, wing length 2.1-2.8 $\mathrm{mm}$; frons variable, either almost completely yellow dorsally (see couplet 75) or more uniformly brown.

Host/Early Stages. Primarily Osmorhiza, including $\underline{0}$. chilensis (syn., o. nuda), 0 . longisty $\bar{l} i s$, and $\underline{0}$. occidentālis, possib̄ly Heracleum lanatum. Larva forming greenish 
linear mine, largely following serrations along leaf margin; puparium black.

Distribution. California; Canada.

References. Spencer, 1966b: 108; 1969a: 250;

1981: 391 .

Note. This species was described when the host was believed to be Heracleum lanatum; the normal host was found to be Osmorhiza during further collecting in California in 1977.

Male genitalia with aedeagus (fig. 1343) without secondary band of sclerotization beside ventral lobe, this with both sides conspicuously expanded at ventral end--------- - . osmorhizae Spencer Synopsis. Agreeing with $\underline{\mathrm{P}}$. $\underline{\text { lanati }} \overline{\text { in external }}$ characters but frons apparently more consistently dark brown.

Host/Early Stages. Osmorhiza claytonii and $\underline{0}$. longistylis. Larva forming upper surface linear mine (fig. 1344); puparium frequently lightly adhering to underside of leaf until dislodged by wind or rain.

Distribution. Virginia; new record: Wisconsin, Dane Co., Madison, $1 \mathrm{~m}$. ex Osmorhiza claytonii, 27.V.75 (S. Tavormina).

Reference. Spencer, 1969a: 261.

131 (127). Frons brownish black or upper half paler, ochre-

$\begin{array}{lll} & \text { ous-- } & 132 \\ -- & \text { Frons uniformly black- }\end{array}$

132 (131). Legs with femora entirely black--------------- 133

-- At least foreknee yellow----------------------- 134

133 (132). Frons dark, uniformly brownish black----------

Synopsis. Frons dark brown, orbit black; upper ors weak, slender, 2 ori; all antennal segments black, 3d slightly longer than broad; mesonotum black, predominantly matt with weak subshine; wing length in male $2.1 \mathrm{~mm}, 2 \mathrm{~d}$ costal section just over twice length of $4 \mathrm{th}$; male genitalia with aedeagus as in figures 1345 , 1346; sperm pump large.

Host/Early Stages. Unknown.

Distribution. California (mountains).

Reference. Spencer, 1981: 421.

Frons paler above, ochreous------p. columbinae Sehgal Synopsis. Frons black in lower half, orbit and lunule black; 2 ori, lower weak; antenna black, 3d segment small, round; mesonotum black, only weakly shining; wing length $1.5-1.8 \mathrm{~mm}, 2 \mathrm{~d}$ costal section twice length of $4 \mathrm{th}$; male genitalia with aedeagus as in figures 1347, 1348; sperm pump with narrow stalk and rounded blade. Host/Early Stages. Aquilegia spp., Thalictrum polycarpum, and other Thalictrum spp. Larva forming blotch mine; puparium dark brown, covered with small spicules.

Distribution. California; Canada. 
References. Sehga1, 1971: 362; Spencer, 1981: 384.

134 (132). Acr in 4 rows; $2 d$ costal section at least 2.5

times length of 4 th--------- - . aralivora Spencer

Synopsis. Frons brownish black, orbit black; 2 ori; antenna black, $3 d$ segment rounded, only slightly longer than broad; mesonotum black, heavily dusted with gray; legs with femora black, knees bright yellow, tibiae and tarsi yellow to yellowish brown; wing length about $2.2 \mathrm{~mm}$; male genitalia with aedeagus as in figures 1349,1350 .

Host/Early Stages. Aralia nudicaulis and A. racemosa. Larva forming long, whitish linear mine which becomes broad toward end; puparium black, posterior spiracles widely separated, each with irregular ellipse of about 10 bulbs, below a pair of closely adjoining low black projections above anus (Alberta, BMNH).

Distribution. Montana; Canada.

Reference. Spencer, 1969a: 230.

Note. In 3 paratypes reexamined from Alberta, $2 \mathrm{~d}$ costal section is 2.5-2.9 times length of $4 \mathrm{th}$, not 3-3.5 times as stated in original description.

Acr in 2 rows; $2 d$ costal section short, little over 1.5 times length of 4 th--_-

- evansi Spencer, n. sp.

Synopsis. Small black species, wing length $2-2.3 \mathrm{~mm}$; frons black below, paler dorsally; 2 ors, upper distinctly shorter, 2 ori; gena slightly more than 0.33 height of eye; antenna black, 3d segment slightly longer than broad; mesonotum blackish gray; squama gray, margin and fringe black; male genitalia with aedeagus (fig. 1351) having spinules along left basal sclerite, distiphallus divided, recurved (fig. 1352); sperm pump large.

Host/Early Stages. Unknown.

Distribution. Colorado.

Reference. Part 2, p. 316.

135 (131). 3d antennal segment distinctly enlarged---------

Synopsis. Frons and orbit uniformly black, latter moderately shining; 2 ors, upper distinctly weaker, 2 ori; antenna black; mesonotum black, largely matt, acr in 4 rows; legs with femora black, only foreknee slightly yellowish, tibiae and tarsi only slightly paler, brownish; squama yellowish gray, margin and fringe black; male genitalia with aedeagus as in figures $1353,1354$.

Host/Early Stages. Nepeta cataria. Larva forming upper surface linear mine which later develops into secondary blotch; puparium yellowish brown, either in leaf at end of mine or externally. 


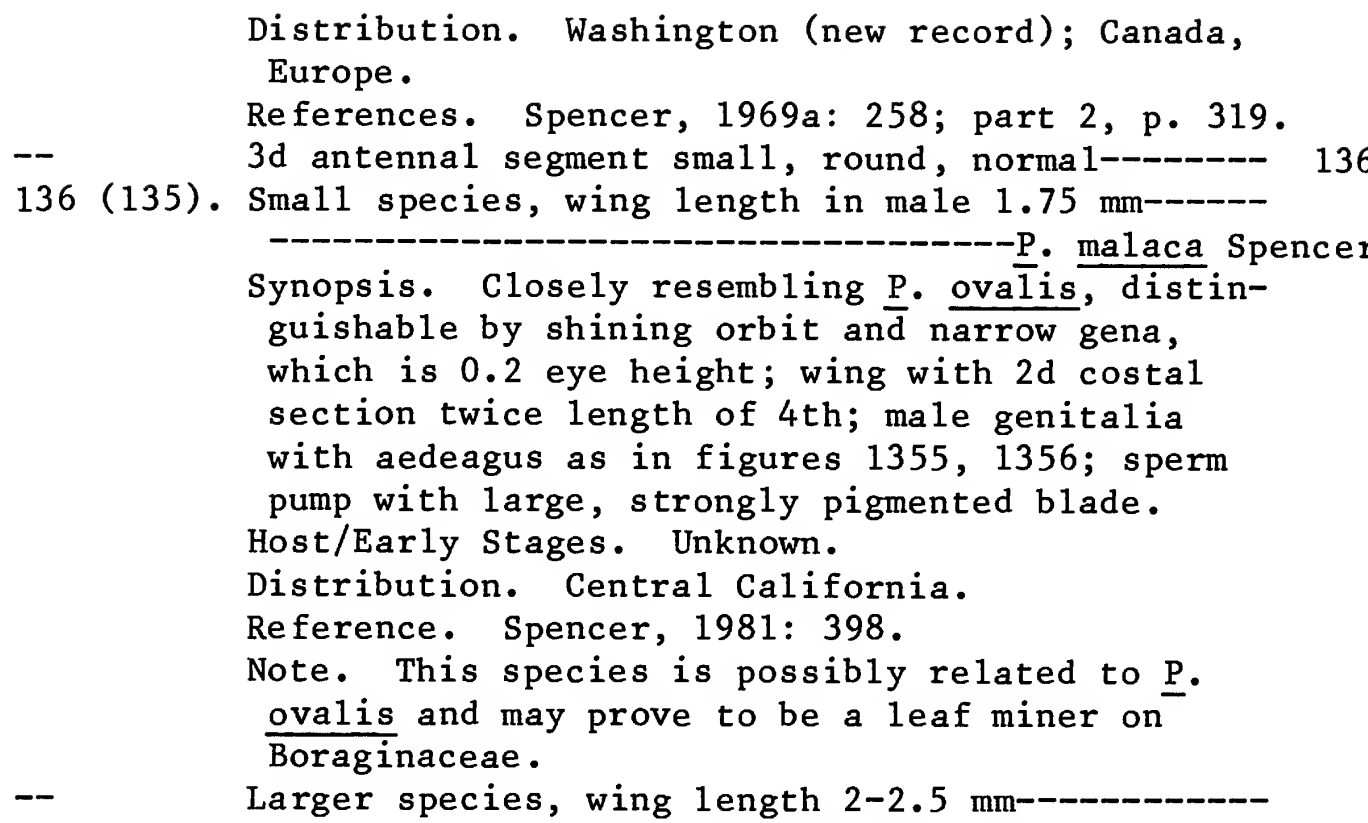

- ovalis Griffiths

Synopsis. Frons deep black, twice width of eye, not projecting above eye; orbit paler, grayish, with 2 ors, upper only slightly weaker than lower (on one side), greatly reduced on other; gena 0.33 height of eye; antenna black, 3d segment smal1, round; mesonotum matt blackish gray, acr in 4 rows; legs with femora black having only foreknee distinctly yellow, on midleg and hindleg indistinctly brownish, tibiae and tarsi brownish black; squama yellowish gray, margin and fringe black; wing with $2 d$ costal section 2.2 times length of 4th; male genitalia with aedeagus as in figures 1357, 1358; sperm pump large, with long stalk and heavily pigmented blade.

Host/Early Stages. Hackelia (in Canada and Alaska), Mertensia, Myosotis. Larva forming irregular linear blotch mine (Griffiths, 1975: fig. 15); puparium yellow to reddish brown, posterior spiracles each with 13-20 bulbs. Distribution. Colorado (new record); Alberta (Hackelia), British Columbia (Mertensia), Yukon Territory (Mertensia, Myosotis).

References. Griffiths, 1975: 133; part 2, p. 319.

\section{UNIDENTIF IED LEAF MINES}

\section{Apiaceae}

1. Angelica breweri

California, Tuolumne Co., below Sonora

Pass near stream beside road, 8,500

$\mathrm{ft}, 7 . \mathrm{IX} .78$ (KAS). The mine is an upper surface blotch at the apex of the leaf segments (fig. 1359). The only species known to feed on Angelica in eastern North America are Phytomyza angelicae, which forms circular blotch mines in the center of the leaf blade (fig. 1237), and $P$. archangelicae Hering (see Griffiths, 1973a: 230), a 
linear miner known from Alaska and $\mathrm{N}$. Europe.

2. Ligusticum grayi

Mines with larvae were found in California, Nevada Co., Sagehen Creek, 6.IX.78 (KAS). The larva forms a narrow linear mine following the serrations of the leaf (fig. 1360); the puparium is black. Similar mines have been found in Alaska by Griffiths, and these probably represent the same species, tentatively identified as Phytomyza, n. sp. ( $p \cdot 323)$.

3. Sphenosciadium capitellatum Empty mines, California, E1 Dorado Co., Luther Pass, (?) date, Lot $106-1$ (KEF). These mines (fig. 1361) closely resemble those in No. 1 on Angelica, and it is possible that both are caused by the same species.

\section{Asteraceae}

4. Ageratina altissima

Empty leaf mines on this host were common in Minnesota, Minnehaha Creek, 29.VI.80 (KAS); District of Columbia, Theodore Roosevelt Island, 2.VII.80 (KAS); and Wisconsin, Madison, June 1980 ( $\mathrm{S}$. Tavormina). The mine (fig. 1362) is long, narrow, with scarcely perceptible frass, frequently with several mines in the same leaf. It is probably caused by a Phytomyza sp.

5. Agoseris, possibly A. glauca (det. J. L. Struther, UC, Berkeley)

Empty mines were found on this host in California, Nevada Co., Sagehen Creek, $6,200 \mathrm{ft}, 6 . \mathrm{IX} .78$ (KAS). The larva

forms a blotch mine at the apex of the leaf (fig. 1363), feeding downward and pupating externally. Frass is scattered in fine particles; this arrangement is more typical of Phytomyza than Liriomyza, but the genus of this species remains to be confirmed.

6. Antennaria plantaginifolia Leaf mines with larvae were found in Arkansas, Garland Co., Hot Springs, 15.V.79 (KAS). The larva feeds primarily in the midrib forming offshoots into the leaf blade (fig. 1364), pupating at the leaf mine. The species probably belongs to the Phytomyza robustella group, but unfortunately no adults were reared.

7. Arnica chamissonis ssp. foliosa California, Nevada Co., Sagehen Creek, 6.IX.78 (KAS). This mine (fig. 1365) represents either Phytomyza arnicae Hering or $P$. arnicicola Lundquist. Both form an upper surface blotch mine, pupating externally, and positive identification of the species is not possible from the mines. $\underline{P}$. arnicae has been found in the Canadian Rockies, Alberta, and $\underline{P}$. arnicicola has been recorded from Alaska, occurs in gardens in Edmonton, Alberta, and has now been recorded from Colorado (p. 310). Positive identification of the mines found at Sagehen Creek is not possible.

8. Arnica chamissonis ssp. foliosa California, Nevada Co., Sagehen Creek, 6.IX.78 (KAS). This species represents either Phytomyza arnicivora Sehgal, 1971, or $\underline{P}$. oreas Griffiths, 1974b: 118. The mines of these two species are entirely linear, scarcely widening (fig. 1366), with the puparium remaining in the leaf on the lower surface and the anterior spiracles projecting through the epidermis. Separation of the two species by the mines alone is at the moment not possible. $\underline{P}$. oreas has been recorded from the Canadian Rockies, British Columbia, and the Yukon Territory; $\underline{P}$. arnicivora is known only from the holotype from the Canadian Rockies. Both species belong to the $\underline{P}$. robustella group (see p. 322). The aedeagus of $\underline{P}$. arnicivora was illustrated in side view by Sehgal (1971: fig. 854); the distiphallus of the holotype in ventral view is shown in figure 1367.

9. Smallanthus uvedalia

Empty blotch mines (fig. 1368) were found in the District of Columbia, Theodore Rooseve1t Island, 1.VII.80 (KAS). 
They possibly represent an undescribed Calycomyza sp. but may be caused by $\underline{C}$. artemisiae or $\mathrm{C}$. flavinotum, as the mines of one or the other of these species were at the same locality on Ageratina altissima.

Hydrophyllaceae

10. Hydrophyllum virginianum A single empty mine from Wisconsin, Madison, 27.V.75 (S. Tavormina) appears to represent an undescribed species. The mine (fig. 1369) is linear in the first instar, with scattered black frass, later developing into an irregular blotch, with distinct feeding lines. The species concerned is probably a Phytomyza.

\section{Malvaceae}

11. Sidalcea spicata

Empty leaf mines were found on this host in California, Nevada Co., Sagehen Creek, 6,200 ft, 5.IX.88 (KAS). The mine (fig. 1370) is irregularly linear, with frass in conspicuous black strips. Nine mines were in a single leaf. The generic reference of this species is uncertain.

\section{Rosaceae}

12. Rubus strigosus

White upper surface linear mines (fig. 1371) were found in Minnesota, Minneapolis, Minnehaha Creek, 20.VI. 80 (KAS). Similar mines were common in the Ottawa area, Ontario, in July 1967 (Spencer, 1969a: 62). No adults were obtained from either locality, and the identity of the species remains unknown, but it will certainly prove to be an Agromyza sp. close to A. potentillae (p. 70).

\section{Salicaceae}

13. Salix lasiandra caudata Empty mines were found in California, Sagehen Creek, 6.IX.78 (KAS). The mine (fig. 1372) is a blotch filling the apex of the leaf, with frass in scattered black grains. The puparium is brown, with the posterior spiracles on short projections, each with three bulbs. Pupation takes place externally. Examination of a dead larva showed the species to be an Agromyza.

\section{HOST LIST}

The larval feeding habit is indicated as follows: $C B$, cambium borer; F, seed feeder (flower heads); G, gall causer; $\mathrm{L}$, leaf miner; SB, stem borer; SM, stem miner.

Brackets [ ] around the host name indicate that the host is not recorded in the United States but is known elsewhere.

Plant names have been revised, $\underline{5} /$ except those followed by "ex" in author citations have been omitted according to the practice authorized by the Botanical Code.

\footnotetext{
ST/Based on Terre11, E. E., A Checklist of Names for 3,000 Vascular Plants of Economic Importance, U.S. Dept. Agr., Agr. Handbk. 505, 1977, and U.S. Dept. Agr., Soil Conserv. Serv., National List of Scientific Plant Names, 2 v., SCS-TP-159, 1982.
} 
Division Polypodiophyta (Pteridophyta)

Polypodiaceae

Asplenium pinnatifidum Nutt.---------------- Phytoliriomyza felti------ L

Camptosorus rhizophy11us (L.) Link--

Pteridium aquilinum (L.) Kuhn--_-

Division Magnoliophyta (Angiospermae)

Class Magnoliopsida (Dicotyledoneae)

Acanthaceae

B1echum brownei Juss. (pyramidatum)

Melanagromyza ruel1iae----- F

Dianthera - see Justicia. Liriomyza blechi---_---- L

Justicia americana (L.) Vah1

Melanagromyza diantherae-- SB

Ruellia brittoniana E. Leonard

M. ruel1iae--

R. caroliniensis (Wa1t.) Steud.

$\bar{M}$. rue11iae

$\underline{\text { Strobilanthes }}$

M. rue11iae

Aceraceae

Acer rubrum $\mathrm{L}$

$\underline{\text { Phytobia }}$ setosa

Amaranthaceae

Achyranthes - see Alternanthera.

A1ternanthera philoxeroides (Mart.) Griseb.------ Melanagromyza mare11ii----- G

Amaranthus spinosus L.----- Haplopeodes minutus------ I

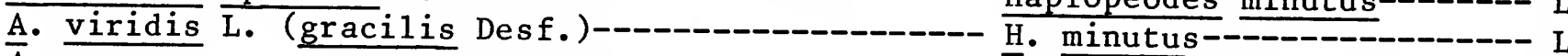

$\overline{\mathrm{A}} . \overline{\mathrm{Ap}} \cdot \mathbf{\mathrm { A }}$

Philoxerus vermicularis (L.) R. Br.-.-.- Haplopeodes philoxeri--.-. L

Apiaceae

Ange1ica atropurpurea L.

A. breweri Gray

Melanagromyza angelicae--- SB Phytomyza past inacae------ L

A. spp.

Apium graveolens L.

P. sp.---

Unidentified mine No. 1---- L

Phytomyza angelicae-------- L

Liriomyza huidobrensis----- L

L. trifolii-c--

Cicuta douglasii (DC.) J. M. Melanagromyza splendida--- SB

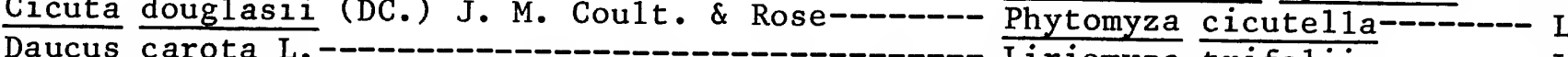

Heracl eum lanatum Michx. Liriomyza trifolii--.----- I

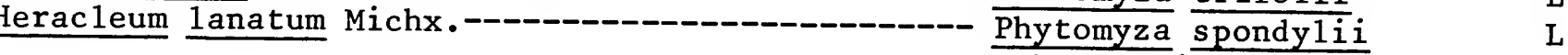
heracleiphaga.

P. tlingitica---------- L

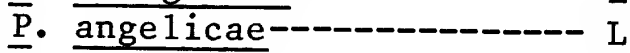

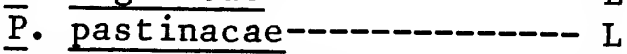

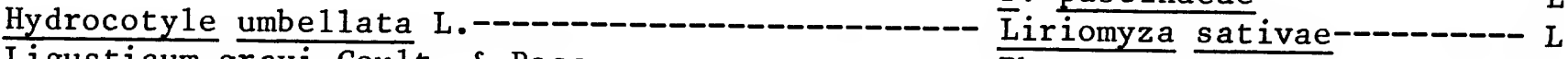

Ligusticum grayi Coult. \& Rose---Unidentified mine No. 2---- L

Lomatium nudicaule (Pursh) Cou1t.\& Rose--------- Melanagromyza lomatii----- SB

Oenanthe sarmentosa DC.------- Phytomyza oenanthoides---- L

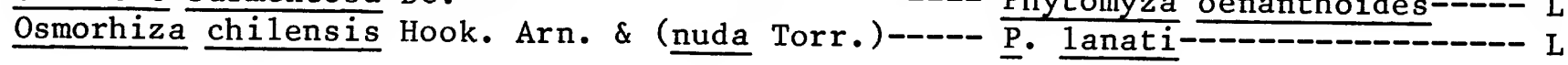


o. claytonii (Michx.) C. B. Clarke-----------

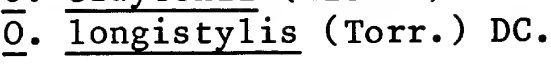

0. occidentalis (Nutt.) Torr

[Pastinaca sativa L.]

Sanicula crassicaulis Poepp. (menziesii)

S. Laciniata Hook. \& Arn.

S. marilandica L.

Sphenosciadium capitellatum Gray
P. osmorhizae------------ L

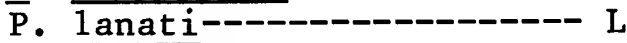

$\overline{\mathrm{P}}$. osmorhizae---_---------- L

$\bar{P}$. lanati------------ L

$\bar{P}$. pastinacae---------- L

$\overline{\mathrm{P}}$. spondy1ii--_--------- L

$\bar{P}$. saniculae------------- L

$\bar{P}$. saniculae----------- L

$\overline{\mathrm{P}}$. saniculae-------- L

$\overline{\mathrm{P}}$. sp.-c-c-- $\mathrm{L}$ Unidentified mine No. 3---- L

Apocynaceae

Vinca minor $\mathrm{L}$.

(?) Liriomyza schmidti----- L

Aquifoliaceae

Ilex aquifolium L.

I. cumulicola Sma11

Phytomyza ilicicola------- L

P. ilicis------------- L

$\overline{\mathrm{P}}$. opacae

$\overline{\mathrm{P}} \cdot$ opacae

I. decidua Walter--

$\bar{P}$. ditman $i$

I. $\overline{\text { glabra }}$ (L.) Gray

P.

I. montana Torr. \& Gray (monticola Gray)

$\bar{P}$ hytobia sp.---

I. opaca Soland. in Ait.

Phytomyza ilicicola------- L

P. opacae-

I. serrata Thunb.-------

P. ditmani----------------- L

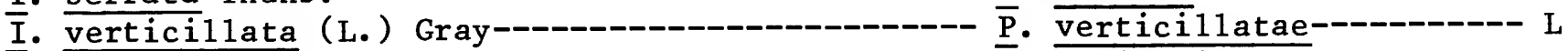

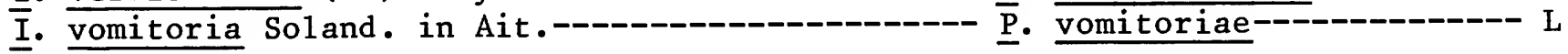

Araliaceae

Aralia nudicaulis $L$.

P. araliae-------------- L

$\bar{P}$. aralivora----------- L

$\overline{\mathrm{P}}$. araliae-------------- L

A. racemosa L.

Melanagromyza panacis---- SB

Panax quinquefolius L.

(?) Liriomyza schmidti----- L

Aristolochiaceae

Aristolochia sp.

Asclepiadaceae

Asclepias ovalifolia Decne.

L. asclepiadis--------- L

A. speciosa Tor $r$

L. $\overline{\text { subasclepiadis }}$

A. . spp.

L. asclepiadis--

Asteraceae (Compositae)

Achillea millefolium L. (1 1 anulosa Nutt.)-------- L. ptarmicae----------- L

N̄apomyza plumea---------- L

Ageratina altissima (L.) R. M. King \& H. Rob.---- Liriomyza eupatoriella---- L Calycomyza artemisiae----- L C. flavinotum-------- L Unidentified mine No. 4---- L

Ageratum conyzoides L.---------------------- Calycomyza sp.---------- L Agoseris glauca (Pursh) Raf. Liriomyza or Phytomyza sp.- L Unidentified mine No. 5---- L 
Ambrosia artemisiifolia

Melanagromyza splendida----SB

Calycomyza ambrosiae------ I

C. platyptera---------- L

[A. artemisiifolia L.]

Agromyza ambrosivora------- L

A. trifida L.

Antennaria plantaginifolia (L.) Richards

Calycomyza ambrosiae------- L

Phytomyza sp.--

Unidentified mine No. 6---- L

Arctium lappa L

Calycomyza flavinotum------ L

A. minus Bernh.

C. flavinotum---O--

[Arctium]

Arnica chamissonis Less., ssp. foliosa (Nutt.)

Maguire.

Liriomyza arctii---------- L

Calycomyza platyptera----- L

Phytomyza arnicae or $\mathrm{P}$. $\quad \mathrm{L}$ arnicicola.

$\underline{P}$. arnicivora or $P$. oreas - L

Unidentified mine Nos. I $7,8$.

A. mollis Hook.

Phytomya saximontana------ L

(?) Arnica

Melanagromyza buccalis---- SB

Artemisia californica Less

(?) Phytomyza minutis- (?) L sima.

A. douglasiana Besser (‥ vulgaris var.

(?) Agromyza ambrosivora--- L

heterophy11a Jeps.).

Calycomyza artemisiae $\quad \mathrm{L}$

marcida.

C. platyptera---_------ L

Liriomyza artemisiae------- L

L. baccharidis----_--- L L

Phytomyza alaskana--------- L

Aster chilensis Nees---

Liriomyza eupatorii------- L

A. conspicuus DC.

Phytomyza asterophaga------ L

A. 1ateriflorus (L.) Britton

Calycomyza promissa------ L

A. simmondsii Small

Melanagromyza minimoides--- F

Calycomyza platyptera----- L

A. sp., cult.

Liriomyza trifolii---_---- L

Áster

Ophiomyia quinta-------ー-ー L

Liriomyza huidobrensis----- L

L. sativae

Nemorimyza posticata-------

Amauromyza maculosa------- L

Calycomyza humeralis------- L

C. platyptera----

Baccharis douglasii DC.-----

L. togata--

B. halimifolia L.---

Nemorimyza posticata------- L

Liriomyza trifolii--_----- L

B. pilularis DC.----

Liriomyza baccharidis------ L

L. sabaziae-c-c-c- $\mathrm{L}$

B. viminea DC.

Calycomyza platyptera------ L

Baccharis

Liriomyza sativae-------- L

L. schlingeri-c--_-_-_-- L

Nemorimyza posticata------ I 
[Bellis]

Bidens frondosa L.

B. pilosa L

Borrichia frutescens (L.) DC.

Calendula officinalis

Callistephus chinensis (L.) Nees

Carduus pycnocephalus L.

Chaptalia dentata (L.) Cass.

Chondrilla juncea L.

Chromolaena odorata (L.) R. M. King \&

H. Rob. (Eupatorium).

Chrysanthemum

[Cineraria]

Cirsium arvense (L.) Scop.-

C. brevistylum Cronq. (edule

of California refs., not Nutt.).

C. occidentale (Nutt.) Jeps.--

Cirsium

Conoclinium coelestinum (L.) DC.

Conoclinium

Conyza canadensis (L.) Cronq. (Erigeron)

Crepis

Cynara scolymus L.

Dahlia imperialis Roez 1.

"Daisies"

Dugaldia hoopesii (Gray) Rydb.

Elephantopus elatus Bertol.

Emilia coccinea (Sims) G. Don (sagittata DC.)-Encelia californica Nutt.
Calycomyza humeralis------- L

C. frickiana------- L

$\overline{M e l \text { anagromyza }}$ bidentis----- L

M. floris---

$\bar{M}$. splendida-----------SB

Amauromyza maculosa-------- L

Calycomyza platyptera------ L

Liriomyza archboldi------- L

L. venegasiae--_-_-_-_ L

Melanagromyza minimoides--- $F$

M. floris-

$\bar{M}$. viridis-----_--------- F

Amauromyza maculosa------ L

Liriomyza zinniae--------- L

L. trifolii-c---_-_-_-- L

L. sabaziae----------- L

Melanagromyza chaptaliae-- SB

Ophiomyia chondrillae----- SM

Melanagromyza minima---- $\mathrm{F}$

Calycomyza jucunda-------- L

Amauromyza maculosa------ L

A. maculosa-- L

Calycomyza platyptera------ L

Chromatomyia syngenesiae--- L

Liriomyza ptarmicae------- L

L. trifolii-c- L

Chromatomyia syngenesiae--- L

Liriomyza sabaziae------- L

L. sabaziae-c-c--_- L

Melanagromyza marinensis-- SB

M. cirsiophila--- SB

Liriomyza trifolii------- L

L. trifolii-c-_-_-_--- L

Amauromyza maculosa-------- L

Calycomyza humeralis------- L

C. minor--

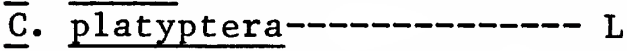

Liriomyza baccharidis------ L

Melanagromyza gnaphalii--- SB

Chromatomyia lactuca------ L

Phytomyza crepidis------- L

Chromatomyia syngenesiae--- L

Liriomyza sabaziae------- L

Chromatomyia syngenesiae--- L

Liriomyza helenii----- - L

(?) Napomyza montanoides-- SB

Calycomyza sp.-C- L

Amauromyza maculosa------- L

Calycomyza enceliae-------- L

Melanagromyza viridis----- SB 
Erechtites hieraciifolia (L.) DC

M. erechtidis------------- F

$\bar{M}$. Splendida Frick-------- SB

Nemorimyza posticata------- L

Amauromyza maculosa------- L

Liriomyza trifolii-------- L

Calycomyza platyptera------ L

Erigeron sp. (high elevation)

Chromatomyia L erigerontophaga.

Erigeron-

Amauromyza maculosa------- L

Calycomyza humeralis------- L

C. platyptera---------- L

[E. caespitosus Nutt.]

[Erigeron s pp.]

Eupatoriadelphus maculatus (L.)

Phytomyza ovimontis-------- L

Calycomyza humeralis------- L

Phytomyza erigerophila----- L

R. M. King \& H. Rob.

Calycomyza flavinotum------ L

Eupatorium capillifolium (Lam.) Smal1

E. maculatum L.

Melanagromyza virens------ SB

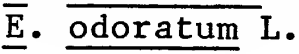

Calycomyza flavinotum----- L

Melanagromyza minima------- F

Calycomyza jucunda------ L

Amauromyza maculosa-------- L

E. rotundifolium L.

Calycomyza sp.--------- L

E. serotinum Michx

Liriomyza trifolii--------- L

E. sp.

E. spp.

Melanagromyza sp.---_---- SB

Calycomyza artemisiae------ L

C. platyptera----------- L

[Eupatorium]

Flaveria 1inearis Lag.

Liriomyza eupatorii-------- L

F. trinervia (Spreng.) C. Mohr

Gaillardia aristata Pursh-

Melanagromyza splendida--- SB

Liriomyza trifolii-------- L

Amauromyza maculosa------- L

Liriomyza trifolii------- L

G. ciliata (Raf.) Blake

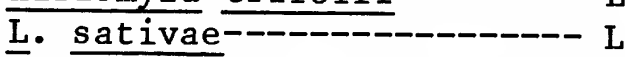

$\bar{L}$. trifolii------------ L

G. pulchella Foug. var. picta (D. Don) Gray------ Melanagromyza splendida--- SB

G. sp.---

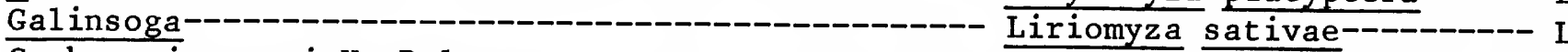

Gerbera jamesoni H. Bolus----_--

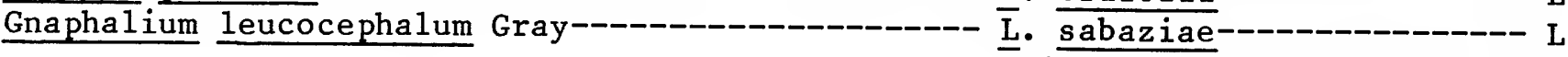

G. peregrinum Fernald (purpureum spathulatum) Melanagromyza gnaphalii--- SB

M. splendida-

Amauromyza maculosa-------- L

Liriomyza trifolii-------- L

Gnaphalium

Grindelia squarrosa (Pursh) Duna1----------------

Calycomyza platyptera------ L

Helenium flexuosum Raf. (nudiflorum Nutt.)

C. platyptera-c------ L

Helianthus annuus L. --

Melanagromyza minimoides--- F

Amauromyza maculosa------- L

Calycomyza platyptera------ L

Liriomyza assimilis------ L

L. helianthi------------- L

L. trifolii------- L

H. sp.--------

Helianthus----_-_- 
Heliopsis helianthoides (L.) Sweet Heterotheca grandiflora Nutt.

H. subaxillaris (Lam.) Britton \& Rusby Hymenopappus scabiosaeus L'HerLactuca biennis (Moench) Fernald L. canadensis $\mathrm{L}$.

L. sativa L. -

L. serriola L. (scariola L.)

$\overline{\mathrm{L}}$. sp.--c-

Lindheimera texana Gray

Madia elegans D. Don-

Matricaria sp.

Melanthera nivea (L.) Smal1 (deltoidea Michx.)----

Mikania batatifolia DC.

Parthenium argentatum Gray

[Petasites]

Rudbeckia laciniata L.

Senecio confusus Britten

S. glabellus Poir.

S. integerrimus Nutt. var. exaltatus (Nutt.)

Cronq. (lugens Richards. var.).

S. triangularis Hook

Senecio

Silybum marianum (L.) Gaertn.

Smallanthus uvedalia (L.) Mackenz.-------------

Solidago caesia L.

S. elongata Nutt.

S. flexicaulis L.

S. $\mathrm{x}$ tortifolia Elliott

$\underline{\text { S. }} \mathrm{sp}$

S. spp.

Sonchus asper (L.) J. Hi11

S. oleraceus

[S. oleraceus ]
Melanagromyza minimoides--- F

Calycomyza humeralis----- L

C. platyptera----------- L

Liriomyza zinniae---------- L

Melanagromyza virens------ SB

Liriomyza trifolii-------- L

Chromatomyia lactuca------- L

Liriomyza trifolii--------- L

L. huidobrensis----------- L

$\bar{L}$. endiviae------------ L

Amauromyza maculosa------- L

Melanagromyza sp.------- SB

Liriomyza assimilis-------- L

Calycomyza humeralis------ L

Chromatomyia syngenesiae--- L

Melanagromyza minimoides--- F

Calycomyza melantherae---- L

Amauromyza maculosa------- L

Liriomyza trifolii--------- L

Calycomyza mikaniae------- L

Melanagromyza splendida--- SB

Calycomyza majuscula------ L

C. frickiana---_-_-_-_ L

Melanagromyza minimoides--- $\mathrm{F}$

M. wedeliae---

Ámauromyza maculosa-------- L

Calycomyza majuscula------- L

Phytomyza integerrimi----- L

Calycomyza majuscula------ L

Phytomyza californica------ L

Calycomyza platyptera----- L

Liriomyza sabaziae-------- L

Chromatomyia syngenesiae--- L

Calycomyza sp.--------- L

Unidentified mine No. 9---- L

Calycomyza platyptera------ L

Liriomyza eupatorii------- L

Phytomyza astotinensis----- L

Nemorimyza posticata------ L

Calycomyza platyptera------ L

Amauromyza maculosa------- L

Calycomyza solidaginis----- L

C. platyptera----------- L

$\overline{\mathrm{C}}$. solidaginis------------ L

Ophiomyia quinta---------- L

Phytoliriomyza arctica---- SM

Liriomyza eupatorii-------- L

Ophiomyia coniceps-------- SM

Amauromyza maculosa-------- L

Liriomyza trifolii--------- L

L. trifoli i-----_-------- L

Chromatomyia syngenesiae--- L

Phytoliriomyza arctica--- SM 
S. sp.

Chromatomyia lactuca------- L

Synedrella nodiflora (L.) Gaertn.

Liriomyza trifolii--_----- L

Tagetes erecta L.---

L. trifolii--

T. patula $\mathrm{L}$.

Amauromyza maculosa------- L

Liriomyza trifolii-------- L

T. $\mathrm{sp} \cdot$ Amauromyza maculosa-------- L Melanagromyza splendida--- SB Calycomyza platyptera----- L

Taraxacum officinale G. H. Weber-

T. spp.

Ophiomyia nasuta--------- L

Chromatomyia lactuca------- L

[T. spp.]

Liriomyza taraxaci--------- L

Títhonia rotundifolia (Mi11.) S. F. Blake--------Phytomyza wahlgreni------- L

Tithonia

[Tithonia]

Venegasia carpesioides DC.

Verbesina alternifolia

Melanagromyza viridis------ F

Calycomyza platyptera----- L

C. humeralis--------- L

V. helianthoides Michx.

Liriomyza venegasiae------- L

v. virginica L. var. laciniata (Poir.)

Vernonia gigantea taeniotricha S. F. Blake (a1tissima).

V. noveboracensis (L.) Michx.

Melanagromyza verbesinae-- SB

Liriomyza sativae--------- L

Melanagromyza bidentis----- F

(?) Liriomyza trifolii----- L

Wedelia paludosa $\mathrm{L}$.

Melanagromyza vernoniae--- SB

M. vernoniana----_--- $S B$

$\bar{M}$. minima----

$\overline{\mathrm{M}}$. wedeliae--_-_-_-_-_-- F

W. trilobata (L.) A. Hitchc.

Xanthium strumarium L. var. canadense (Mi11.) Torr. \& Gray.

$\overline{\mathrm{M}}$. minima-

$\overline{\text { Liriomyza }}$ helianthi--

Calycomyza platyptera------ L

$\mathrm{X}$. sp.

Zinnia elegans Jacq.

Liriomyza trifolii-------- L

Melanagromyza viridis------ F

Liriomyza zinniae-------- L

Z. $\mathrm{sp}$.

Calycomyza platyptera------ L

Melanagromyza viridis------ F

Liriomyza trifolii---_---- L

[Zinnia ]

Calycomyza humeralis------- L

Balsaminaceae

Impatiens capensis Meerb. (biflora Walt.)

Phytoliriomyza melampyga--- L

I. noli-tangere L.

P. melampyga--

Betulaceae

Betula nigra L.

Phytobia betulivora

Bignoniaceae

Catalpa bignonioides Walter

Amauromyza pleuralis------- L

C. bunge i C. A. Meyer-

A. pleuralis-------_- L

Jacaranda mimosifolia D. Don Phytoliriomyza jacarandae-- L

Boraginaceae

Cynoglos sum sp.

Hackelia deflexa (Wahlenb.) Opiz var. americana

(Gray) Fernald \& I. Johnst.

Heliotropium curassavicum L.

Calycomyza cynoglossi------ L

Phytomyza ovalis-----_---- L

(?) Liriomyza blechi------- L 
H. polyphyllum Lehm. (1eavenworthii Torr.)

Li thospermum arvense L.

Melanagromyza heliotropii- SB

[Mertensia paniculata (Ait.) G. Don]

M. miranda-- $S B$

[Myosotis alpestris F. W. Schmidt]

Phytomyza ovalis----------- $L$

P. ovalis -

Brassicaceae (Cruciferae)

Brassica campestris L.

B. napus $\mathrm{L}$.

$\bar{B}$. oleracea L. (botrytis group)

B.

Liriomyza brassicae-------- L

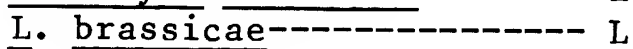

$\bar{L} \cdot \overline{\text { brassicae }}-------------\mathrm{L}$

$\bar{L}$. brassicae------------- L

B. rapa $L$.

L. sativae------------- L

Capse1la bursa-pastoris (L.) Medik.

$\bar{L}$. brassicae--------------- L

L. Sativae------------ L

Cheilanthus----c---

L. trifolii

Descurainia sophia (L.) P. B. Webb (Sophia sp.)---

L. brassicae------------ L

Erysimum inconspicuum ( $\mathrm{S}$. Wats.) Macmil.-------- Liriomyza brassicae-------- L

Lepidium virginicum $\mathrm{L}$.

L. brassicae------------- L

Nasturtium-

Raphanus sativus L.

L. brassicae-------------- L

$\bar{L}$. brassicae---_-------- L

$\bar{L}$. Sativae--------------- L

Rorippa palustris cernua (Nutt.) Stuckey

$\overline{\mathrm{L}}$. brassicae-------------- I (islandica).

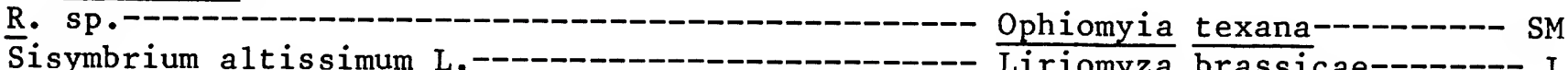

Sisymbrium altissimum L.---

\section{Capparidaceae}

Capparis

L. brassicae

Cleome-

L.

Caprifoliaceae

Lonicera canadensis Marsha11

Paraphytomyza

L. involucrata Banks

\section{luteoscutellata.}

Chromatomyia gregaria------ L

(?) C. involucratae------ L

Paraphytomyza cornigera---- L

P. involucratae--------- L

L. morrowii Gray

$\bar{P}$. 1uteoscutellata-------- L

L.

$\bar{P}$. orbitalis-------- L

$\bar{P} . \overline{\text { sp. indet. No. 2------- } \mathrm{L}}$

Sambucus mexicana $K$. Pres 1

(?) Liriomyza specifica---- L

Symphoricarpos mollis Nutt

Chromatomyia

symphoricarp.

S. parishii Rydb. (albus auctt.)

C. fricki----

Paraphytomyza orbitalis---- L

P. cornigera-

S. rivularis Suksd. (albus auctt.)

$\overline{\mathrm{P}}$. orbitalis

Chromatomyia fricki-------- L

S. spp.

Paraphytomyza

luteoscute11ata. 
Caryophyllaceae

Dianthus barbatus

Liriomyza huidobrensis----- L

D. caryophyllus L.

L. huidobrensis-----_---- L

Gypsophila sp.

L. trifolii-- -

Lychnis alba Mill.

Amauromyza flavifrons------ L

Saponaria officinalis L.

A. flavifrons---_-_-_--- L

Chenopodiaceae

Atriplex patula L.

Liriomyza huidobrensis----- L

Beta vulgaris L.

L. trifolii----

Chenopodium sp.

$\bar{L}$. huidobrensis--_-_------- L

Spinacia oleracea $\mathrm{L}$.

Haplopeodes minutus-------- L

Liriomyza trifolii--_------ L

Convolvulaceae

Ipomoea alba L. (Calonyction aculeatum)

Calycomyza ipomaeae-------- L

C. stegmaieri-c--_-_--- L

I. batatas (L.) Lam.

I. indica acuminata (Vahl) Fosb. (cathartica)

C. ipomaeae--

I. tiliacea (Willd.) Choisy

C. ipomaeae

I. triloba $\mathrm{L}$.

$\bar{C}$. ipomaeae

$\bar{M}$ lanagromyza caerulea----- F

Cornaceae

Cornus stolonifera occidentalis (Torr.\& Gray)

C. Hitchc. (californica).

Phytomyza agromyzina------- L

Cucurbitaceae

Cucumis melo L.

C. sativus L.

Cucurbita pepo L.

Liriomyza huidobrensis----- L

L. sativae----

$\bar{L}$. trifolii-_-_-_-_-_-_-_ L

L. Sativae--_-_-_-_-_- $\mathrm{L}$

L. trifolii-_-_-_-_-_-_

L. Sativae--_-_-_-_- L

L. trifolii-_-_-_-_-_-_- L

C. spp.

Marah sp.

L. sativae

L. (?) sativae---_------- L

Euphorbiaceae

Acalypha hispida Burm. f.

Breynia nivosa (W. J. Smith) Small

(?) .L. schmidti--_-_----- L

Ricinus communis L.

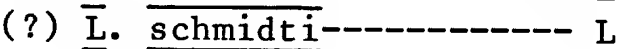

L. sătivae----

Fabaceae (Leguminosae)

Albizia lebbeck (L.) Benth.

Baptisia tinctoria (L.) R. Br.

L. schmidti--_-_-_-------- L

Bauhinia purpurea L.

. baptisiae-------------- L

Cajanus cajan (L.) Millsp.

L. schmidti-

Cassia occidentalis L

$\overline{\mathrm{L}}$. sativae-

C. tora L.

Centrosema (?) virginianum (L.) Benth.

L. Sativae

Coronilla varia L.

L. $\overline{\text { sativae- }}$

Crotalaria incana L.

(?) L. sativae-_-_-_-_-_-_-

L. trifoliaearum-_-_-_-_- L

C. lanceolata E. Meyer

L. trifolii-_-_-_-_-_-- L

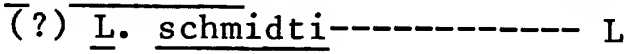


Desmodium tortuosum DC.

Galactia sp

Indigofera

[Lathyrus ochroleucus Hook.]

L. odoratus L.

L. vestitus Nutt.

L. sp.--

Lupinus albicaulis Dougl

L. arboreus Sim

L. latifolius Lindl.

L. pratensis A. Heller

L. sericeus Pursh-

L. varicolor steud. Medicago lupulina $\mathrm{L}$ M. sativa L.
Japanagromyza desmodivora-- L Melanagromyza floridensis-- $\mathrm{F}$

(?) Japanagromyza sp.---- L Liriomyza sativae--------- L L. fricki-Pिytomyza subtilis-------- L Liriomyza huidobrensis----- L Amauromyza lathyroides----- L Phytomyza subtilis------- L Liriomyza lathyri--------- L L. lupini----------- L L. Iupiniphaga------------- L L. Iupinella--_-_-_---- L Phytomyza lupini---------- L Liriomyza baptisiae-------- L L. lupinel la----_----- I $\overline{\mathrm{L}}$. Iupini--L. baptisiae--------------- L L. Iupinella-------------- L $\bar{L}$. Tupini--Phytomyza lupini----------- F Liriomyza lupinella-------- L L. trifoli i-cAgromyza frontella-------- L Liriomyza fricki---_------- L L. huidobrensis----------- L

$\overline{\mathrm{L}}$. Sativae----

$\bar{L}$. trifoliearum----------- L Melanagromyza gibsoni----- SB

Melilotus alba Medik M. indica (L.) Al 1. Mucuna deeringiana (Bort) Merrill (Stizolobium)Phaseolus lunatus L.

P. vulgaris L.-Pisum sativum L.

Liriomyza sativae-------- L L. huidobrensis---------- L Agromyza frontella--------- L Liriomyza schmidti--------- L L. sativae----------- L $\overline{\mathrm{L}} \cdot \overline{\text { sativae}}--------------\mathrm{L}$

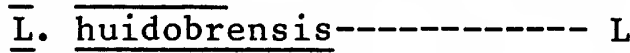

$\bar{L}$. sativae--

$\overline{\mathrm{L}}$. trifoliearum--_-------- L

L. trifolii--_-_-_-_-_- L

L. robiniae------- L

Robinia pseudoacacia L. Trifolium incarnatum L.

L. Sativae-------------- L

$\overline{\mathrm{L}}$. trifoliearum--.------- L

$\bar{L}$. trifoliearum--_------- I

Agromyza frontella--------- I Liriomyza fricki--_------- L Phytomyza subtilis--------- L Liriomyza fricki----------- L L. huidobrensis---------- L $\bar{L}$. fricki------------- L Japanagromyza aequalis----- L J. inaequalis--------- L Liriomyza sativae---------- L

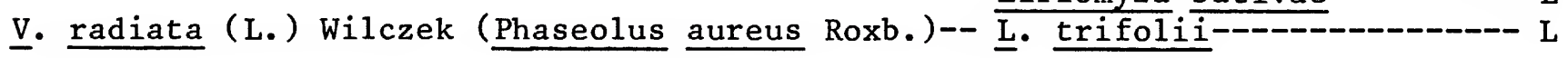


V. unguiculata (L.) Walpers (sinensis [L.] Savi)-- L. sativae---------------- L Wisteria sp.--

\section{Fagaceae}

Castanea mollissima B1.

Quercus rubra L.--

Japanagromyza viridula----- L Phytobia sp.-Japanagromyza viridula----- L

Hydrophyllaceae

Eriodictyon californicum (Hook.\& Arn.) Torr.----- Chromatomyia eriodictyi--- L

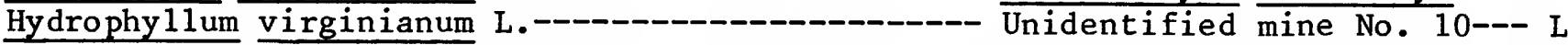

Nemophila phacelioides Nutt.-------------- Chromatomyia mimuli------- L

Phacelia californica Cham.---

P. cicutaria Greene------- Chromatomyia mimuli------- L

$\overline{\mathrm{P}}$. imbricata Greene-----

P. minor (Harv.) The11.

$\underline{\bar{p}}$. viscida (Benth.) Torr

C. $\overline{\text { mimuli }}$

C.

Juncaginaceae

Triglochin maritimum L.

Liromyza angu1icornis----- SM

T. palustre L.--

Lamiaceae (Labiatae)

Hyptis mutabilis (A. Rich.) Briq.---

H. pectinata ( L. $_{\text {. }}$ Poit.--

Monarda fistulosa L.---

M. punctata L.--

Monarde11a odoratissima Benth.-------------- $\overline{\mathrm{C}}$. $\overline{\text { menthae }}-------------$ L

Monarde11a sp.---

Nepeta cataria L.---

Phytomyza nepetae-------- L

Ocimum sp.---

Stachys albens Gray----------- Liriomyza s

S. bullata Benth. (californica Benth.)---

$\underline{\text { S. }}$ rigida Nutt.--

\section{Linaceae}

Linum usitatissimum $\mathrm{L}$

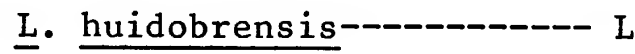

Loganiaceae

Spigelia anthelmia L.

(?) $\underline{\text { L. blechi-口- }}$

\section{Malvaceae}

Abelmoschus esculentus (L.) Moench (Hibiscus)----- L. sativae--------------- L Abutilon theophrasti Medik. (avicennae Gaertn.)--- $\overline{\text { Calycomyza }}$ malvae--------- L

Ophiomyza abutilivora----- SM

Alcea rosea L. (Althea)--

A. sp.

Anoda cristata (L.) Schlecht Calycomyza malvae-------- L Liriomyza sativae---------- $L$ Gossypium Ma1va rotundifolia L.

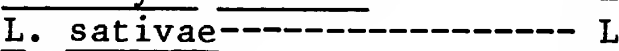

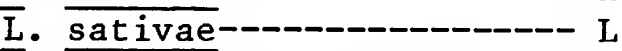
Sida acuta N. L. Burm. 
S. cordifolia L

S. rhombifolia L.

Calycomyza sidae------------ L

Ophiomyia abutilivora------ SM

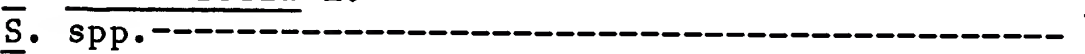

Calycomyza malvae---------- L C. sidae------------- L

Sidalcea oregana (Nutt.) Gray, ssp. spicata Melanagromyza sp.----- (?) SB (Rege1) C. Hitchc.

Nyctaginaceae

Bougainvillea spectabilis Willd.

Liriomyza schmidti---------- L

\section{Passifloraceae}

Passiflora caerulea L.

L. schmidti----------- L

Plantaginaceae

Plantago lanceolata $\mathrm{L}$.

P. major L.

Phytomyza plantaginis------- L

$\underline{\mathrm{p}} \cdot \mathrm{sp} \cdot$

P. plantaginis----------- L

(?) Liriomyza blechi-------- L

Portulacaceae

Portulaca sp.

Haplopeodes palliatus------- L

Ranunculaceae

Aconitum sp.

Aquilegia canadensis L.

A. formosa Fisch.--.

A. formosa var. pauciflora (Greene) Boothman------

$\underline{\text { A. }}$ formosa var. truncata (Fisch.\& C. A. Meyer) Baker.

A. spp.

Clematis colombiana (Nutt.) Torr.\& Gray c. ligusticifolia Nutt.

C. occidentalis (Hornem.) DC. (verticillaris)

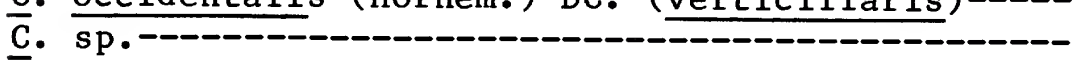

Delphinium spp.

Ranunculus abortivus L.

R. californicus Benth.

$\overline{\mathrm{R}}$. spp.

[Ranunculus ]

Thalictrum dasycarpum Fisch. \& Ave-Lall

T. fendleri Engelm.

Phytomyza aconiti----------- L

P. aquilegivora------------ L

$\underline{\mathrm{P}}$. aquilegioides----------- L

$\bar{P}$. sonorensis------------ L

$\overline{\mathrm{P}}$. aquilegivora---------- L

$\overline{\mathrm{P}}$. aquilegioides------------ L

P. aquilegiana------------- L

$\overline{\mathrm{P}}$. columbinae-------------- L

$\overline{\mathrm{P}}$. minuscula-------------- L

$\overline{\mathrm{P}}$. clematiphaga----------- L

$\bar{P}$. clematisana--- (?) SM or F

$\bar{P}$. clematoides----_-------- L

$\overline{\mathrm{P}}$. 1igusticifoliae---------- L

$\bar{P}$. loewii---------------- L

$\bar{P}$. 1oewii------------------- L

P. clematisella---------- SM

Chromatomyia clemativora--- L

Phytomyza aconiti---.------ L

P. davisii---------- L

$\bar{P}$. ranunculoides------------ L

Napomyza evanescens------- SB

$\mathrm{N}$. blairmorensis-(?) SB or $\mathrm{F}$ Phytomyza humilis----------- L

P. ranunculi-------------- L

Napomyza marginalis---- SB, F

Phytomyza plumiseta--------- L

P. minuscula---------- L

$\overline{\mathrm{P}}$. thalictrella----------- L 
T. polycarpum (Torr.) S. Wats.--

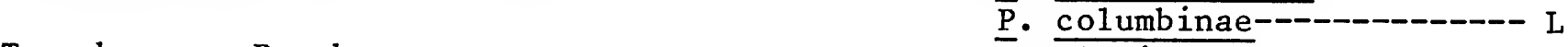

T. pubescens Pursh------_---

[T. venulosum Trelease]--

Rhamnaceae

Ceanothus sp.--

Rosaceae

Amelanchier canadensis (L.) Medik.----------- Phytobia amelanchieris--- CB

Comarum - see Potentilla.

Crataegus sp.---

[Filipendula]----_--- Ágromyza potentillae------ L

Fragaria sp.----

Potentilla glandulosa Lindl.------------ $\overline{\text { Melanagromyza }}$ SB

P. gracilis Doug1. sagehenensis.

[Potentilla $]$ gromyza potentillae------- L

[Potentilla, as Comarum]

Prunus avium (L.) I

A. su1furiceps--------- L

P. x domestica L

A. potentillae----------- L

P. lyonii (Eastw.) Sarg.

p. persica (L.) Batsch-

Rubus strigosus Michx.

Rubus sp.--

[Rubus sp.]-

[Sanguisorba]

Phytobia pruni----------- $\mathrm{CB}$

P. pruni--

$\bar{P}$. prunivora------- CB

Phytomyza persicae--------- L

Unidentified mine No. 12--- L

Agromyza sp.-------------- L

A. potentillae------------- L

$\bar{A}$. potentillae--------- L

A.

Rubiaceae

Chiococca alba (L.) A. Hitchc.-------------- Liriomyza schmidti-------- L

Galium boreale L.---

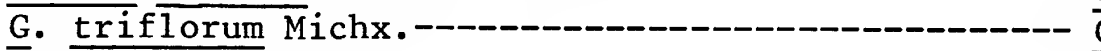

Galiomyza galiivora-------- L

$\mathrm{G}$. sp.---

(?) G. sp.

galivora----carneri--

Hamelia patens Jacq.

Paraphytomyza praecox- (?) SM

alicaceae

Populus balsamifera L., ssp. trichocarpa

(Torr. \& Gray) T. C. Brayshaw.

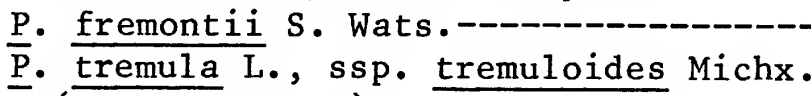
(Loeve \& Loeve).

Liriomyza schmidti-------- L

Salix breweri Bebb-

S. Iasiandra Benth

$\underline{\underline{s}}$. $\overline{\text { lasiandra }}$ caudata (Nutt.) Sudw. (bryantiana)---

s. sp.

Agromyza isolata---------- L

Hexomyza schineri---------- G

H. schineri--

H. simplicoides----------- G

Agromyza isolata----------- L

(?) A. leechi----------- L

Unidentified mine No. 13--- L

Hexomyza winnemanae-------- G

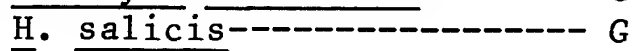

Sapindaceae

Cardiospermum microcarpum H. B.K.

Agromyzid sp. 
Saxi fragaceae

Heuchera micrantha Dougl.

Philadelphus inodorus L. var. grandiflorus (Willd.) Gray.

Tellima grandiflora (Pursh) Doug1.

[Tiarella trifoliata L.]--

[Tolmiea menziesii (Pursh) Torr.\& Gray]
Chromatomyia tiare11ae----- L Liriomyza philadelphivora-- L

Chromatomyia tiarellae----- L C. tiarellae$\overline{\mathrm{C}}$. $\frac{\text { tiarellae }}{}$

Scrophulariaceae

Castilleja latifolia Hook. \& Arn.

[Chelone glabra L.]-

Cordylanthus nevinii Gray-----------------

Hebe speciosa (R. Cunn.) J. C. Andersen

(Veronica imperialis of horticulturists).

Mimulus aurantiacus Curt

[Pedicularis sp.]-

[Penstemon confertus Dougl.]

P. laxiflorus Pennel1

(?) P. procerus Dougl.

Scrophularia californica Cham. \& Schlecht.

C. castillejae nordica----- L

Phytomyza chelonei------- F

P. trivittata------ (?) F

$\bar{P}$. crassiseta-

Chromatomyia mimuli-------- L

Phytomyza tenella-------- F

P. penstemonis----------- $\mathrm{L}$

$\bar{P}$. penstemonis ------------ L

$\bar{P}$. penstemone 11a---------- $\mathrm{L}$

Mel anagromyza scrophulariae.

Solanaceae

Capsicum annuum L.

Liriomyza huidobrensis----- L

Cestrum diurnum L.

L. sativae--------- L

C. nocturnum L.

L. trifolii

Datura innoxia Mill. (meteloides)

L. sativae-

$\overline{\mathrm{L}}$. $\overline{\text { huidobrensis }}$

Lycopersicon esculentum Mill.

L. sativae

L. $\overline{\text { huidobrensis }}$

$\bar{L}$ sativae----------

L. trifolii--_-_-_-_---- L

Petunia sp.

$\bar{L}$. huidobrensis----------- L

$\overline{\mathrm{L}}$. trifolii----------- L

Physalis viscosa $\mathrm{L}$.

Hap $\overline{l o p e o d e s}$ kefi----------- L

P. sp.--

Solanum erianthum D. Don (verbascifolium)

Liriomyza trifolii--------- L

(?) L. sativae--------- L

L. trifolii---

S. melongena $\mathrm{L}$.

$\overline{\mathrm{L}}$. sativae--------------- L

L. trifolii--

S. nigrum L.

$\overline{\mathrm{L}}$. Sativae-

L. trifolii------------- L

S. Sarrachoides Sendtn.

L. trifoliearum----------- L

$\underline{\mathrm{S}}$. triquetrum Cav.

Haplopeodes kef $i----------~ L$

$\underline{\mathrm{S}}$. $\underline{\text { tuberosum }}$ L.

Liriomyza huidobrensis----- L L. trifoli i-c--------- L

S. umbelliferum Eschsch.------

Tiliaceae

Tilia americana L.

Ophiomyia tiliae---------- G

Triumfetta semitriloba (L.) Jacq

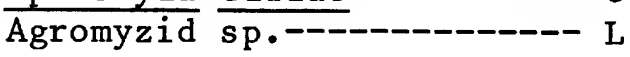


Tropaeolaceae

Tropaeolum majus L

Liriomyza brassicae-------- L

Turneraceae

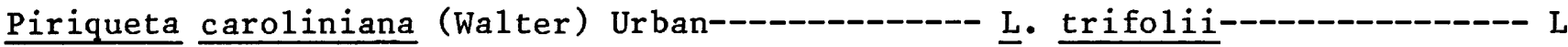

U1maceae

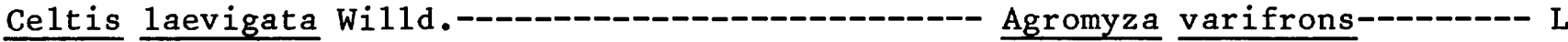

C. occidentalis L.-----

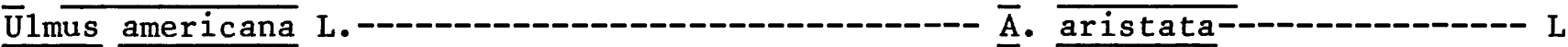

Urticaceae

Urtica californica Greene-------

$\underline{\text { U. }}$ dioica L., ssp. holosericea (Nutt.) Thorne----- $\bar{A}$. pseudoreptens--------- L

A. reptans--------- L

Melanagromyza urtice11a--- SB

(?) $\mathrm{U} . \mathrm{sp} .--$

U. $s \bar{p} .--$

Verbenaceae

Duranta repens L.

Lantana camara L.

Ca1ycomyza durantae-------- L

Ophiomyia camarae-------- L

0. 1antanae----------- F

$\overline{0} . \overline{s p \cdot---1}$

Calycomyza lantanae-------- L

L. involucrata L.

C. 1antanae----------- L

Lippia micromera helleri Britton

C. 1antanae--------- L

Phyla nodiflora (L.) Greene

Melanagromyza ripare11a--- SB

Ophiomyia lippiae-- F (?) SM

Liriomyza blechi----------- L

Verbena 1itoralis H.B.K.

Ca1ycomyza 1antanae-------- L

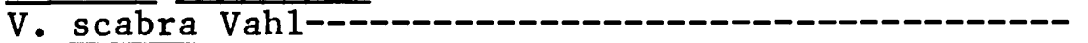

Melanagromyza sp.------ SB

$\overline{\mathrm{v}} \cdot \mathrm{sp} \cdot \mathrm{c}-\mathrm{c}$

Calycomyza verbenae------- L

Violaceae

Viola sororia Willd. (papilionacea)

Ga1iomyza violivora-------- L

Zygophy11aceae

Ka11stroemia maxima (L.) Hook. \& Arn.--------- Liriomyza trifolii------- L

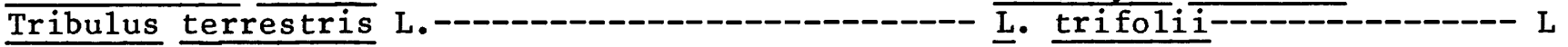

Class Liliopsida (Monocotyledoneae)

Commelinaceae

Commelina diffusa N. L. Burm.------------ L. commelinae---------- L

C. sp.--

Tradescantia fluminensis Vell.--

Cyperaceae

Carex crus-corvi Kunze

Cerodontha macminni-------- L

C. morosa--

Chromatomyia pseudomilii--- L 
Carex

C. angulata-- ---------- L

[Carex]

Cerodontha hirtae---------- L

[ Scirpus expansus Fernald (sylvaticus L. var.)]--- $\underline{\text { C. } \text { scirpi- }}$

\section{Iridaceae}

Iris versicolor L.

I. sp.

C. thompsoni

C. iridophora--

\section{Juncaceae}

Juncus xiphioides E. Meyer

(?) J. spp.

C. 1ongipennis---------- L

[J. spp.]

C.

C. 1uctuosa

Liliaceae

A1lium cepa L.

Asparagus officinalis L.

[Smilacina stellata (L.) Desf.] (1)

Smilax sp.

Liriomyza huidobrensis----- L L. trifoliiOphiomyia simplex--------- SM Liriomyza smilacinae------ L L L. (?) schmidti-C---- L

Orchidaceae

Orchid sp. Melanagromyza miamensis---- F

Poaceae (Gramineae)

Agropyron repens (L.) Beauv.

Agromyza ambigua-----ー-ー--- L

Cerodontha incisa-------- L

C. inconspicua---ー----ー-ー-- L

C. muscina-------------- L

Pseudonapomyza atra------- $\mathrm{L}$

[Agropyron]

Cerodontha pygmaea------- L

[ Agrostis] Avena sativa $\mathrm{L}$.

Chromatomyia pseudomilii--- L

C. norwegica--

Ágromyza ambigua----------- L

Cerodontha dorsalis-------- L

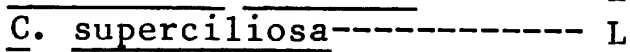

Liriomyza trifolii-------- L

Pseudonapomyza atra-----ー- L

[Avena ]

Brachypodium

Agromyza nigre 1 1a-------- L

Bromus carinatus Hook. \& Arn.

Cerodontha pygmaea-------- L

Liriomyza septentrionalis-- L

Cerodontha incisa-------- L

B. tectorum L.

C. muscina------n------- L

$\overline{\mathrm{B}}$. $\mathrm{sp}$.

[Calamagrostis]

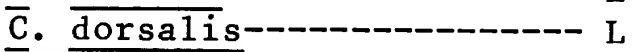

[ Cinna latifolia (Trevir.) Griseb.]

Dactylis glomerata L.--

[Dactylis]

Chromatomyia norwegica----- L

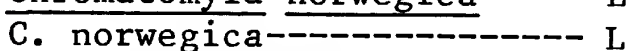

Cerodontha dorsalis------- L

C. muscina-------

Ágromyza nigre11a---------- L

Cerodontha pygmaea-------- L L

[Des champsia]

C. pygmaea-

Digitaria sanguinalis (L.) Scop. 
Echinochloa crusgalli (L.) Beauv.

E. walteri (Pursh) A. Heller

Ehrharta erecta Lam

Eleusine indica (L.) Gaertn.

E. sp.

Elymus canadensis $\mathrm{L}$

E. glaucus Buckley

E. sp.

Eragrostis sp.

Euchlaena - see Zea mexicana.

Festuca--

[Festuca]

[Glyceria]

[G.sp.]

[

Hordeum jubatum L.

[Hordeum jubatum L.]

H. leporinum Link

$\overline{\mathrm{H}}$. murinum $\mathrm{L}$.

H. vulgare L.

H. $s p$.

Lasiacis divaricata (L.) A. Hitchc

Lolium perenne L.-

L. perenne L. var. multiflorum (Lam.)

R. Parnel1.

Lolium

[Lolium]

Panicum dichotomiflorum Michx

$\underline{\text { P. miliaceum L. }}$

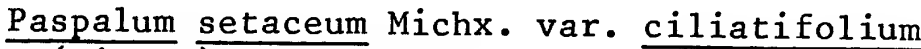
(Michx.) Vasey.

Phalaris arundinacea $\mathrm{L}$

Phalaris-
Agromyza parvicornis------- L

A. proxima--------- L

Cerodontha muscina--------- L

Agromyza proxima--------- L

A. parvicornis----------- L

Cerodontha muscina--------- L

(?) Liriomyza marginalis--- L

Cerodontha dorsalis------ L

C. superciliosa---_-_---- L

C. dorsalis---

$\overline{\mathrm{C}}$. incisa-

$\overline{\mathrm{C}}$. dorsalis-_-_-_-_-_-_---- L

C. $\overline{\text { dorsalis }}$

Chromatomyia nigra--------- L

Agromyza ambigua---_-_-_-- L

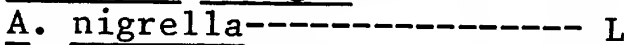

$\bar{C}$ erodontha pygmaea--------- L

Agromyza ambigua----------- $L$

A. nigrel 1 la-----_-_-_--- L

Cerodontha pygmaea--------- L

Agromyza ambigua---_------- L

A. ambigua----_-_-_-_-_- L

Cerodontha superciliosa---- L

C. dorsalis - -

Ägromyza nigrella----_-_--- L

Cerodontha muscina-------- L

C. mus cina-

Chromatomyia pseudomilii--- L

Liriomyza septentrionalis-- L

Cerodontha dorsalis----- L

C. incisa--

Pseudonapomyza atra-------- $\mathrm{L}$

Agromyza albipennis-------- L

A. ambigua-- - -

(?) A. sp.---_-_-_-_- L

Chromatomyia pseudomilii--- L

Cerdontha dorsalis-------- L

C. muscina-

Lir iomyza septentrionalis-- L

Chromatomyia nigra------- L

Agromyza ambigua----------- L

A. nigrel la--

$\bar{A}$. proxima-

$\overline{\mathrm{A}}$. parvicornis--_-_-_-_--- L

Cerodontha dorsalis-------- L

(?) Liriomyza marginalis--- L

Chromatomyia nigra-------- L

Liriomyza marginalis------- L

Agromyza albipennis-------- L

Cerodontha incisa---_----- $L$

C. dorsalis 
Phleum pratense L.
[Phleum]-
Poa alpina L. ]

[Poa $]$

Secale cereale L.

[Secale]

Setaria glauca (L.) Beauv. (1utescens)

[Setaria]

Sorghum bicolor (L.) Moench (vulgare Pers.)

[Trisetum]

Triticum $\times$ aestivum $L$.

[Triticum]

Vahlodea atropurpurea (Wahlenb.) Fr.

Zea mays

Z. mexicana (Schrad.) Reeves and Mangelsd. (Euch1aena).
C. dorsalis-----_------- L

C. muscina------------ L

Ágromyza nigre11a---------- L

Chromatomyia poae---------- L

Cerodontha dorsalis-------- L

C. incisa-

Ägromyza albipennis-------- L

A. ambigua---------- L

A. nigre11a---------- L

Cerodontha dorsalis------- L

C. pygmaea-

$\bar{C}$. fasciata----_-------- L

Ágromyza ambigua---------- L

Cerodontha dorsalis-------- L

Pseudonapomyza atra------- L

Agromyza nigrel la-------- L

Cerodontha incisa-------- $\mathrm{L}$

C. muscina----------- L

Agromyza nigre11a--------- L

Cerodontha dorsalis-------- L

Agromyza nigre11a-------- L

A. albipennis---------- L

$\bar{A}$. ambigua----D-D

Cerodontha dorsalis-------- L

C. incisa---- L

$\bar{C}$. superciliosa---------- L

Pseudonapomyza atra------- L

Agromyza nigre 1 la--

Chromatomyia norwegica----- L

Agromyza parvicornis------- L

Cerodontha dorsalis------- L

C. superciliosa------- L

(?) Liriomyza marginalis--- L

Typhaceae

Typha sp 
Part 2 - Descriptions of New Species and Taxonomic Notes

In this primarily taxonomic section, 85 new species are described, 22 new synonymies and 16 new combinations are established, and records are given, including details of original description and type location, of species new to the United States. The status of a number of species is discussed and as far as possible clarified. In addition, three lectotype designations are made, one in the genus Calycomyza and two in the Phytomyza.

\section{GENUS MELANAGROMYZA HENDEL}

Eight new species are described, two new synonyms are established, and three species are recorded as new to the United States. New State records are given for three species, and the taxonomic positions of three are briefly discussed.

\section{Melanagromyza angelicae (Frost)}

(Figs. 54, 55)

Agromyza angelicae Frost, 1934: 40. Holotype male from Ithaca, N.Y., in USNM.

Melanagromyza angelicae, Frick, 1952a: $377 ; 1959: 362$.

Melanagromyza angelicae, Steyskal, 1981: 38 .

In addition to the type series from New York, Frost included as paratypes specimens caught at Pacific Grove, Calif., by Aldrich in 1906. The latter have been examined and prove to represent M. marinensis Spencer, 1981, described from Marin County, Calif.

Specimens have been confirmed from Ohio and it seems probable that $M$. angelicae is restricted to the East.

In Europe, the name "angelicae" was originally used for the species feeding commonly in stems of Angelica sylvestris and occasionally a1so in $\underline{\text { Heracleum }}$ and Pastinaca, but it was later discovered that this represents a distinct species described as $M$. angeliciphaga by Spencer (1969b).

New record.

Ohio, Portage Co., Streetsboro, 12

m., 1 f., emerged March 1970 ex

Angelica stems (D. Witwer), in KAS.

Melanagromyza buccalis Spencer

(new to U.S.A.)

(Figs. 60-62)

Melanagromyza buccalis Spencer, 1969a:

67. Holotype male from Quebec in CNC.

M. buccalis occurs widely in Ontario and Quebec and was recently found to be widespread in California. The forwardly projecting gena and distinctive male genitalia make the species readily recognizable (figs. 60-62).

Additional material now studied shows the species to be present also in Colorado, Maryland, New York, and Virginia. In California, one host was believed to be Arnica, but it now seems clear that other genera of Asteraceae are attacked.

New records.

Colorado, Boulder Co., Valmont Butte, 5,300 ft, $1 \mathrm{f}$., 1.VII.61 (J. R. Staines, CNC); Clear Creek Co., 5 miles SW of Idaho Springs, 8,600 ft, $1 \mathrm{~m} ., 27 . V I I .61$ (J. G. Chillcott, CNC).

Maryland, Montgomery Co, Bethesda, 4 m., 1 f., 24.VIII.75 (USNM).

New York, Long Is., Babylon, 2 f., 20.VI and 5.IX.35; 1 f., 28.VI.36 (Blanton and Borders, CUI); Fair Haven Beach S.P., 1 m., 7.VI.68 (G. and K. Eickwort, CUI); Ithaca, $1 \mathrm{~m}$. , "July" (S. W. Frost, CUI).

Virginia, Montgomery Co., Blacksburg, 2,100 ft, $1 \mathrm{f} ., 28 . V .62$; Christiansburg, Montgomery Co., 1 f., 2.VII.62; (both J. G. Chillcott, CNC). 
Melanagromyza burgessi (Malloch)

(Figs. 117, 118)

Agromyza burgessi Malloch, 1913a:

322. Holotype female from

Massachusetts in USNM.

Melanagromyza burgessi, Frick, 1959: 363.

Melanagromyza malefica Spencer, 1981: 46, California. NEW SYNONYM.

Although females in this genus are frequently not satisfactorily identifiable, the large size, projecting frons, numerous orbital bristles, and dark squamal fringe satisfy us that the female holotype can be correctly associated with males seen from Illinois and California (as M. malefica). M. malefica is therefore now synonymized with $\underline{\text { M. }}$ burgessi.

Melanagromyza caribbea Spencer (Figs. 129, 130)

Melanagromyza caribbea Spencer, in Spencer and Stegmaier, 1973: 34. Holotype male from Florida in USNM.

The correct generic position of this minute species with a wing length of $1.7 \mathrm{~mm}$ is problematic. It may well be a leaf or stem miner, but we hesitate to transfer it to Ophiomyia until its biology and larval characters are known. Even the aedeagus (figs. 129, 130) does not clearly indicate the generic position.

\section{Melanagromyza chillcotti Spencer,} new species

(Figs. 75, 76)

Head. Frons narrow, equal to width of eye, not projecting above eye in profile; 2 ors, 2 more widely spaced ori, orbital setulae sparse but in 2 rows, inner row largely proclinate, row nearest eye margin reclinate; ocellar triangle well defined, apex extending only to level of lower ors; gena deepest in center, about 0.17 vertical height of eye, this distinctly pilose in male; 3d antennal segment small, round arista appearing bare.

Mesonotum. $2 \mathrm{dc}, \mathrm{acr}$ in some 8 rows.
Wing. Length in male $2.1 \mathrm{~mm}$, inner crossvein beyond midpoint of discal ceill, last section of $M 3+40.67$ of penultimate.

Color. Frons matt black, ocellar triangle and orbits distinctly but not brilliantly shining; mesonotum almost entirely black, with only faintest greenish reflections viewed from rear; abdomen distinctly green; squamae and fringe white.

Male genitalia. Aedeagus as in figures 75,76 .

Host/early stages. Unknown.

Holotype m., North Carolina, Macon Co., Wayah Gap, 4,100 ft, 29.VII.57 (J.G.C.), in CNC.

Remarks. Although somewhat resembling both M. riparella and M. subvirens, the mále genitalia are entirely distinctive. M. chillcotti also closely resembles M. chaptaliae but is distinguishable by the blacker mesonotum and the broader gena.

Melanagromyza chillcottiana Spencer, new species

(Figs. 79, 80)

Head. Frons not or only slightly projecting above eye, 1.5 times width of eye; 2 ors and normally 4 strong ori, orbital setulae sparse, irregular, mainly proclinate; ocellar triangle only weakly shining; lunule large, higher than semicircle; eye in male pilose; gena deepest in center, narrow, about 0.14 height of eye; arista long, weakly pubescent.

Mesonotum. $2 \mathrm{dc}$, acr in some 8 rows.

Wing. Length in male $2.5 \mathrm{~mm}, \mathrm{C}$ extending strongly to $M 1+2$, last section of $M 3+4$ about 0.67 of penultimate.

Color. Frons brownish black, ocellar triangle, orbits, and lunule somewhat paler; mesonotum matt black, though more shining seen from rear; abdomen blackish coppery, only weakly shining; 
squama and fringe white, margin pale brown.

Male genitalia. Aedeagus as in figures 79,80 , ventral bladder elongate; sperm pump with large blade and distinct central rod.

Host/early stages. Unknown.

Holotype m., Kansas, Douglas Co., Lawrence, 27.III.64; paratypes $9 \mathrm{~m}$., same data; (al1 J. G. Chillcott). Holotype in CNC, paratypes in KAS.

Remarks. Although this species closely resembles $\underline{M}$. chillcotti and $\underline{M}$. subvirens, it is distinguishable by the less green abdomen and the presence of normally four ori. The aedeagus resembles that of $\underline{M}$. martini Spencer, 1969a, a stem bore $\bar{r}$ in Urtica, which is widespread in Canada and might be expected to occur in the Northern United States.

Melanagromyza longensis Spencer, new species

(Figs. 56, 57)

Head. Frons not projecting above eye, 1.5 times width of eye; 2 reclinate ors, 3 inclined ori, orbital setulae predominantly reclinate, a sparse inner row more proclinate; orbits well defined but narrow, not widening anteriorly; lunule slightly higher than a semicircle; jowls narrow, 0.2 height of eye; eye distinctly pilose above; 3d antennal segment round, with short, normal pubescence, arista appearing bare.

Mesonotum. $2 \mathrm{dcr}$, acr in about 8 rows. Wing. Length in male $2.6 \mathrm{~mm}$.

Color. Ocellar triangle and orbits distinctly but moderately shining; mesonotum shining blackish green; abdomen more distinctly green; squama and fringe white.

Male genitalia. Aedeagus as in figures 56, 57; sperm pump large, with somewhat irregular blade; 9 th sternite triangular.
Host/early stages. Unknown.

Holotype m., New York, Long Is land, Babylon, 9.VIII.33 (Blanton and Borders), in CUI.

Remarks. Although this species somewhat resembles $M$. virginiensis, $n$. sp., it is readily $\bar{d}$ istinguishable by the key characters (couplet 28), and the male genitalia of the two species are entirely distinct.

Melanagromyza marellii (Brèthes) (Figs. 135-138)

Melanagromyza marellii (Brèthes), 1920: 284. Type series from Brazil, location not known.

Melanagromyza marellii, Spencer, 1963a: 317; Spencer and Stegmaier, $1973: 42$.

This is an aberrant species and its correct generic affiliation is uncertain.

The male genitalia (figs. 135, 136), the stem galls on Achyranthes (fig. 137), and the larval spiracles (fig. 138) strongly suggest that it does not belong in Melanagromyza. The genitalia are unlike those of any known Ophiomyia, but the form of the puparia does suggest affinity with Ophiomyia. 0 . tiliae, causing twig gal1s, is known to be in this genus, and a typical Ophiomyia has been described from Hong Kong forming unusual leaf galls. There appears to be some justification for erecting a monotypic genus to accommodate this species. However, splitting of this kind is undesirable until a more detailed revision of Melanagromyza and Ophiomyia on a world basis can be undertaken. For the time being, therefore, as the adults appear externally like a typical Melanagromyza, no generic change is proposed.

Melanagromyza miranda Spencer (new to U.S.A.)

Melanagromyza miranda Spencer, 1969a:

72. Holotype male from British

Columbia in CNC. 
The host of this species was established by the discovery of puparia in stems of Lithospermum arvense and another unidentified species of Boraginaceae, $1 \mathrm{~km}$ south of Polson, Mont., Lake County, 8.IV.69 (D. Witwer).

\section{Melanagromyza proboscidata Spencer,} new species

(Figs. 21-23)

Head (fig. 21). Frons 1.5 times width of eye, not projecting above eye in profile; 2 ors and 2 ori, latter widely spaced, distance between them double that between lower ors and upper ori, orbital setulae minute, in 2 rows, those nearest eye margin largely upright, inner row more proclinate; ocellar triangle narrow, apex extending to level of upper ori; gena unusually narrow, 0.08 height of eye in center, broader in front; 3d antennal segment sma11, rounded, arista appearing bare; proboscis conspicuously 1 ong and slender, labellum projecting upward to level of antennae in rest position (fig. 21).

Mesonotum. $2 \mathrm{dc}$, acr numerous, in some 10 rows.

Wing. Length $2.3 \mathrm{~mm}, \mathrm{C}$ extending strongly to $M 1+2$, inner crossvein just beyond midpoint of discal cell, last section of $M 3+4$ about 0.67 of penultimate.

Color. Frons matt black, ocellar triangle and orbits distinctly shining; mesonotum shining black; abdomen blackish green or coppery; squama and fringe white.

Male genitalia. Aedeagus as in figures 22, 23; 9th sternite broadly fused at apex; sperm pump with narrow blade.

Host/early stages. Unknown.

Holotype m., Texas, Kerr Co., Kerrville, 5.IV.59; paratypes $2 \mathrm{~m}$., 3.IV, and $4 \mathrm{~m} ., 5 \mathrm{f} ., 1-11 . I V .59$; Davis Ranch, NW. Blanco Co., $1 \mathrm{f}$.,
22.IV.59; (a11 J. F. McAlpine); San Patricio Co., Welder Wildlife Reserve, nr. Sinton, 1 f., 19-23.III.65 (J. G. C.). Holotype and paratypes in $\mathrm{CNC}, 1$ m., 2 f. in KAS.

Remarks. The long proboscis (fig. 21) makes this a distinctive species in the group with the abdomen green or coppery. The male genitalia are characteristic of species in this group and show no particular differentiation associated with that of the proboscis.

Melanagromyza radicicola Steyskal (Fig. 128)

Melanagromyza radicicola Steyskal, 1981: 40. Holotype male from Maryland in USNM.

The single male on which the description of this species was based was found dead in a rearing container, in which roots and stems, believed to be of Urtica sp., had been kept hoping to rear M. martini Spencer, 1969a, which is common in Canada.

The aedeagus is clearly of the type occurring in three species feeding in seed heads of various genera of Asteraceae--M. bidentis (fig. 121), M. minima (fig. $12 \overline{3}$ ), and $\mathrm{M}$. minimoides

(fig. 125). It appears to be indistinguishable from that of $M$. minimoides. The slight pigmentation at the end of the ejaculatory duct is also clearly present in specimens examined from Bethesda, Md., reared from Heliopsis helianthoides (fig. 126), and also from Ouachita State Park, Ark., reared from Rudbeckia sp. However, this pigmentation is not obviously apparent in the type series from the Miami, Fla., area ex Verbesina sp. (fig. 125).

The inner margin of the epandrium of M. radicicola has an acute angle and two long bristles below the surstylus (Steyska1, 1981: fig. 7, A). These additional bristles are normally lacking in $\mathrm{M}$. minimoides, but one paratype from Florida ex Verbesina 
(genitalia slide No. 52229, BMNH) has been seen with four such bristles.

There is thus no significant difference between $\underline{M}$. minimoides and M. radicicola. The suspected host, Urtica, has nevertheless led the junior author to feel justified in describing $\underline{M}$. radicicola as distinct and this is tentatively accepted here.

Melanagromyza ripare11a (Hende1)

(Figs. 71-74)

Agromyza riparia Malloch, 1915a: 105. Lectotype male from Illinois (designated by Frison, 1927: 194) in INHS. Preoccupied.

Agromyza riparella, Hendel, 1923: 145, nom. nov. for A. riparia Wulp, 1871.

Melanagromyza riparella, Frick, 1952a: 379; 1959: 365 .

Melanagromyza 1 ippivora Spencer, 1966d: 11. NEW SYNONYM. Holotype male from Florida in USNM.

Examination of the male genitalia (figs. 71, 72) of the lectotype satisfies us that this is identical with the species subsequently

described from Florida, reared from seed heads of Phyla nodiflora. This host does not occur in Illinois, where the host is no doubt $\underline{P}$. lanceolata.

This species can now also be recorded from Texas, San Patricio County, Welder Wildlife Reserve, near Sinton, 2 miles, 19-25.III.65 (J. G. Chillcott), in CNC.

Melanagromyza sagehenensis Spencer (Figs. 19, 20)

Melanagromyza sagehenensis Spencer, 1981: 56. Holotype male from California in UCD.

This species was described from four specimens caught at Sagehen Creek, Nevada County, Calif.

Five males and six females from stems of Potentilla glandulosa, 3 miles south of Ronan, Lake County, Mont., 27.VII.69 (D. Witwer), have recently been examined, which agree in all respects

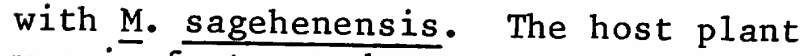
was in fact noted at Sagehen Creek during a visit there by KAS in September 1978.

Melanagromyza tetrica Spencer (new to U.S.A.)

(Figs. 133, 134)

Melanagromyza tetrica Spencer, 1969a: 76. Holotype male from Saskatchewan in CNC.

This black species has hitherto been known only from the two type specimens. A single male has now been identified from Colorado, Clear Creek County, Loveland Pass, west slope, 9,850 feet, 18.VII.61 (B. R. Poole), in CNC.

Although only two dc are present in all these specimens, the male genitalia (figs. 133, 134) closely resemble those of $M$. setifrons, and it is possible that there is variation in the arrangement of the dorsocentral bristles and that $M$. tetrica is in fact synonymous with $\bar{M}$. setifrons. This should be borne in mind as more material becomes available.

Melanagromyza ultima Spencer, new species

(Figs. 131, 132)

Head. Frons 1.5 times width of eye, not projecting above eye in profile; 2 ors, 2 ori, orbital setulae sparse, reclinate; ocellar triangle short, apex not reaching level of lower ors; gena narrow, 0.1 height of eye, this bare; antennae narrowly separated by facial keel, but this not raised.

Mesonotum. $2 \mathrm{dc}$, acr in 8 rows.

Wing. Length in male $2.4 \mathrm{~mm}, \mathrm{C}$ extending strongly to $M 1+2$, last section of $M 3+4$ distinctly shorter than penultimate, inner crossvein just beyond center of discal cell.

Color. Ocellar triangle only weakly shining; mesonotum and abdomen moderately shining black; squama ochreous gray, fringe black. 
Male genitalia. Aedeagus (figs. 131, 132) with basal sclerite reduced to narrow sclerotized strip, widely separated from distiphallus complex; sperm pump exceptionally large, slightly longer than aedeagus.

Host/early stages. Unknown.

Holotype m., Virginia, Giles Co., Stony Cross, 2,000 ft, 26.V.62 (J. R. Vockeroth), in CNC.

Remarks. Although this species appears to be a typical Melanagromyza, the male genitalia are aberrant, particularly in the form of the basal sclerite, indicating that it occupies an isolated position within the genus.

\section{Melanagromyza verbesinae Spencer,} new species

(Figs, 104, 105)

Head. Frons 1.5 times width of eye, not projecting above eye in profile; 2 ors, 2 ori, latter twice as wide apart as ors, orbital setulae numerous, in several rows, those nearest eye margin reclinate, inner rows more proclinate; orbits broad, inner margin parallel to eye margin; ocellar triangle with apex not reaching level of upper ori; gena 0.25 height of eye, this distinctly pilose in male; 3d antennal segment small, round, arista finely pubescent.

Mesonotum. $2 \mathrm{dc}$, acr numerous, in 8-10 rows.

Wing. Length in male $2.1 \mathrm{~mm}$, inner crossvein at center of discal cell, last section of M $3+4$ about 0.4 penultimate.

Color. Ocellar triangle and orbits weakly shining; mesonotum distinctly matt, only weakly shining viewed from rear; abdomen more greenish; squama and fringe white.

Male genitalia. Aedeagus as in figures 104, 105; 9th sternite with narrow sidearms and slightly extended hypandrial apodeme, somewhat curving ventrally.
Host/early stages. Verbesina alternifolia, almost certainly other species in the genus and possibly related genera. Larva internal stem borer; puparium straw colored, posterior spiracular plates heavily chitinized, closely adjoining, only narrowly divided, each with ellipse of about 12 bulbs around strong central horn.

Holotype m., Ohio, Portage Co., Jennings Woods, 6 miles NE of Rayenna, Coll. 5.V.69, emerged 21.III.70 (D. Witwer); paratypes $2 \mathrm{~m}$., Tennessee, Hamilton Co., East Ridge, 6 and 9.V.52 (G. S. Walley); Texas, Kerr Co., Kerrville, $1 \mathrm{~m}$., 10.IV.59 (J. F. McAlpine); 1 m., same locality, emerged 10.XI.69. Holotype in USNM, paratypes in $\mathrm{CNC}$ and $\mathrm{KAS}$.

Remarks. This species most closely resembles $\underline{M}$. vectabilis known from Florida and the Bahamas. Because it belongs to the large group of small greenish species, positive identification may only be possible from the male genitalia.

Melanagromyza virginiensis Spencer, new species

(Figs. 58, 59)

Head. Frons narrow, little wider than eye, not projecting above eye in profile; 2 reclinate ors, 2 inclined ori, ors and upper or $i$ equidistant, distance between ori double that between upper 3 bristles, orbital setulae in 2 rows, inner row partially proclinate, row nearest eye margin more reclinate; ocellar triangle narrow, apex extending to level of upper ori; gena deepest in center, narrow, 0.2 height of eye; eye in male pilose above; 3d antennal segment small, round, arista distinctly pubescent, long, only slightly shorter than height of eye.

Mesonotum. $2 \mathrm{dc}, \mathrm{acr}$ in about 8 rows.

Wing. Length $2.75 \mathrm{~mm}$ in male, inner crossvein slightly beyond midpoint of discal cell, last section of $M 3+4$ 0.67 of penultimate. 
Color. Frons matt black, ocellar triangle and orbits weakly shining black; mesonotum blackish green; abdomen greenish coppery; squama and fringe white.

Male genitalia. Aedeagus as in figures 58,59 .

Host/early stages. Unknown.

Holotype m., Virginia, Montgomery Co., Blacksburg, 28.V.62 (J. G. C.), in CNC.

Remarks. The aedeagus of this species is distinctive but somewhat resembles that of $M$. occidentalis Spencer

(1969a: figs. 102, 103), which is widespread in western Canada.

Melanagromyza walleyi Spencer, new species

(Figs. 44, 45)

Head. Frons less than 1.5 times width of eye, not significantly projecting above eye in profile; 2 ors, 3 ori, orbital setulae sparse but in 2 rows, those nearest eye margin reclinate, inner row more proclinate; ocellar triangle well defined but small, apex not extending beyond level of lower ors; orbits broad, widest between lower ors and upper ori; eye in male with distinct pilosity at level of ors; gena narrow, about 0.2-0.17 height of eye; 3d antennal segment small, round, arista long, appearing bare.

Mesonotum. $2 \mathrm{dc}$, acr numerous, in some 10 rows.

Wing. Length $2.7 \mathrm{~mm}$, inner crossvein distinctly beyond midpoint of discal cell, last section of $M 3+4$ short, little more than half length of penultimate, in ratio $20: 37$.

Color. Orbit and ocellar triangle weakly shining black; mesonotum shining, bluish black; abdomen more greenish; squama and fringe white.

Male genitalia. Aedeagus as in figures 44,45 .

Host/early stages. Unknown.
Holotype m., Tennessee, Hamilton Co., East Ridge, 9.V.52 (G. S. Walley), in CNC.

Remarks. This species closely resembles $M$. hicksi Steyskal feeding in stems of A $\overline{1 \text { thaea }}$, but this does not necessarily indicate that the two are closely related.

Melanagromyza sp. (Maryland) (Figs. 139-142)

A new species has been seen from Maryland, Montgomery County, Wheaton, 27-28.V.79 (A. Freidberg), in USNM, but this is not formally described and is not included in the key, as it is believed that the chaetotaxy of both head and mesonotum is abnormal. On the head the lower orbital bristles are strong1y proclinate and the outer vertical bristles are duplicated on both sides (fig. 139). The

arrangement of the dorsocentrals on the mesonotum is irregular, and it is uncertain whether the normal arrangement is two or three pairs.

The male genitalia are distinctive and the aedeagus is shown in figures 140 , 141; the surstylus bears strong bristles and a row of bristles continues along the inner margin of the epandrium (fig. 142). Such an arrangement is unusual in Melanagromyza but has been seen in a few species elsewhere, for example in $\underline{M}$. polymniae Spencer, 1973b, from Venezuela (see Spencer, 1977c: fig. 9).

It is hoped that further collecting at the locality where this specimen was obtained will produce additional material to permit full clarification of this interesting species.

\section{GENUS HEXOMYZA ENDERLEIN}

One new synonymy is established and the status of one species is discussed.

Hexomyza salicis (Malloch) (Figs. 15 $\overline{1-153)}$

Agromyza salicis Malloch, 1913a: 314. 
Holotype male from Massachusetts in USNM.

Melanagromyza salicis, Frick, 1959: 365.

Hexomyza salicis, Spencer, 1966a: 39;

1969a: 80 (under H. albicula);

$1969 \mathrm{~b}: 13$.

There has been doubt about the status of this species. Spencer (1966a)

treated the European species, $\underline{H}$. cecidogena Hering, 1927, as a junior synonym of $\mathrm{H}$. salicis and later

(1969a) suggested that $\mathrm{H}$. salicis might be synonymous with $\mathrm{H}$.

simplicoides (Hendel) rather than with

H. cecidogena.

The holotype of $\mathrm{H}$. salicis has been examined and probably represents a distinct species. Differences in the aedeagus between this group of gall causers on Salix are slight, but larval and puparial characters are distinctive. The early stages of $\underline{H}$. salicis are not known, and further clarification of this species must wait until reared material becomes available.

The aedeagus of the holotype of $\underline{H}$. cecidogena was illustrated by spencer (1966a: figs. 60,61) and the posterior spiracles of the puparium by Spencer (1969b: fig. 14). The aedeagus of $\underline{H}$. salicis is shown in figures 152,153 .

Hexomyza winnemanae (Ma1loch), new combination

(Figs. 143-145)

Agromyza winnemanae Malloch, 1913a: 314. Holotype female from Maryland in USNM.

Melanagromyza winnemanae, Frick, 1952a: 380; 1959: 367.

Hexomyza albicula Spencer, 1969a: 79. Holotype male from Ontario in CNC. NEW SYNONYM.

H. albicula, a distinctive species with unusually pale wings, the costa ending at vein $R \quad 4+5$, and the squamal fringe pale, was described before the true identity of $\underline{\mathrm{H}}$. winnemanae was known. The female holotype has now been examined and we are satisfied that it is identical with $\underline{H}$. albicula, a gall causer on Salix.

Shewel1 (1953: 465) correctly wrote of H. winnemanae: "This is a problematic species" and suggested that it might belong in Ophiomyia. However, its correct status is now clarified and it is formally transferred to Hexomyza, with $\underline{\mathrm{H}}$. albicula as a junior synonym.

\section{GENUS OPHIOMYIA BRASCHNIKOV}

Fourteen new species are described, 7 new synonyms are established, and 5 new combinations are proposed. In addition, five species are recorded as new to the United States.

Ophiomyia abutilivora Spencer, new species

(Figs. 196-199)

Head (fig. 196). Frons little wider than eye, not projecting above eye in profile; 2 ors, 2 ori, orbital setulae sparse, reclinate; ocellar triangle broad, large, apex extending to level of lower ors; gena not projecting forward, deepest in center, 0.14 height of eye; antennal bases narrowly separated; facial keel variable, sometimes distinctly raised; 3d antennal segment small, round.

Mesonotum. $2 \mathrm{dc}$, acr sparse, in 6-8 rows.

Wing. Length from $2 \mathrm{~mm}$ in male to 2.3 $\mathrm{mm}$ in female, $\mathrm{C}$ extending strongly to $M 1+2$, last section of M $3+40.67$ penultimate, inner crossvein well beyond center of discal cell.

Color. Black, with greenish trace; ocellar triangle brilliantly shining; both mesonotum and abdomen strongly shining; squama gray, margin and fringe black.

Male genitalia. Aedeagus as in figures 197, 198; hypandrium with conspicuously extended apodeme; sperm 
pump with narrow blade, similar in length to aedeagus.

Host/early stages. Abutilon theophrasti and probably other genera of Malvaceae. Larva forming characteristic raised mine or "welt" in stem, feeding both upward and downward, pupating with anterior spiracles projecting through epidermis, length of visible welt varies from 2.5 to 8 $\mathrm{cm}$; puparium yellowish, posterior spiracles on 2 short stalks, each with row of 4-6 bulbs (fig. 199).

Holotype m., Minnesota, Dakota Co., Rosemount, Agricultural Research Station, 13.IX.78; paratypes $1 \mathrm{~m} ., 3$ f., same data; 4 m., 2 f., same locality, Aug. 79; (a11 R. N. Andersen and R. Ralston); Mississippi, Washington Co., Stoneville and Merigold, $8 \mathrm{~m} ., 2 \mathrm{f}$., ex "stem gal1s" on Abutilon theophrasti, 22.X.79 (K. E. Frick); Florida, Broward Co., Fort Lauderdale, $2 \mathrm{~m} ., 2 \mathrm{f} .$, on flowers of Sida cordifolia, 16.III.80 (H. E. Walker). Holotype and paratypes in USNM; other paratypes in Department of Entomology, University of Minnesota, St. Paul; Southern Weed Science Laboratory, Stoneville, Miss.; and KAS.

Mines on Abutilon theophrasti have also been found in Minnesota, Goodhue Co., Red Wing, and Wisconsin, Crawford Co., Barnum (R. N. Andersen and R. Ralston); also in central Illinois ( $R$. M. Wax).

Remarks. This is the first species of which the host and biology are known in the complex group comprising 0 . similata (p. 258), 0. 1auta (p. 255), and the 0 . jacintensis group in California. The latter were assigned to Ophiomyia rather than Melanagromyza on the basis of their male genitalia, and confirmation of the correct generic position of the group has been obtained from the discovery of the host and feeding habit of $\underline{0}$. abutilivora.

Although native species of Abutilon occur in California, it seems certain that 0 . abutilivora feeds on other genera of Malvaceae, from which it has transferred to the introduced A. theophrasti.

It has also been discovered that 0 . tiliae, a gall causer on twigs of Tilia americana that was previously placed in the genus Hexomyza, has very similar genitalia to those of $\underline{0}$. abutilivora and clearly belongs to the same group.

Ophiomyia arizonensis Spencer, new species

(Figs. 316-318)

Head (fig. 316). Frons about 1.5 times width of eye, not projecting above eye in profile; 2 ors, 2 ori, orbital setulae sparse, reclinate; gena narrow, 0.17 height of eye, forming angle of approx. $70^{\circ}$; vibrissal fasciculus in male short, slender; facial keel narrow, distinctly raised, without furrow but flattened above base of antennae.

Mesonotum. 2 dc (acr missing).

Wing. Length in male $1.9 \mathrm{~mm}, \mathrm{C}$ extending to $M 1+2$ (relative lengths of $M 3+4$ and position of inner crossvein not detectable, as one wing missing and the other damaged).

Color. Black, ocellar triangle and orbits moderately shining; mesonotum and abdomen more strongly so; squama gray, margin and fringe black.

Male genitalia: Aedeagus as in figures 317,318 .

Host/early stages. Unknown.

Holotype m., Arizona, Yuma Co., Wellton, 5-6.V.18 (J. C. Bradley), in CUI.

Remarks. There are minor external differences between this species and $\underline{0}$. levata, but the male genitalia of the two are entirely distinct. 
Ophiomyia asymmetrica Spencer, new species

(Figs. 273-275)

Head (fig. 273). Frons 1.5 times width of eye, not projecting above eye in profile; 2 ors, 2 ori, orbital setulae sparse, reclinate; ocellar triangle large, apex extending to leve1 of lower ori; gena forming angle of $60^{\circ}$, narrow, about 0.125 height of eye; vibrissal fasciculus in male long, curving beyond antennae; facial keel of medium width and height and without central furrow.

Mesonotum. $2 \mathrm{dcr}$, acr in 8 rows.

Wing. Length in male $2.0 \mathrm{~mm}$, last section of M 3+4 little shorter than penultimate, inner crossvein only slightly beyond midpoint of discal cell.

Color. Ocellar triangle and orbits paler than frons, entirely dull; mesonotum and abdomen only moderately shining black; squama gray, margin and fringe black.

Male genitalia. Aedeagus highly asymmetrical as in figures 274, 275 .

Host/early stages. Unknown.

Holotype m., North Carolina, Highlands, 3,888 ft, 12.VII.57 (J. G. Chillcott); paratype m., same data; both in CNC.

Remarks. Although closely resembling 0. delecta and $\underline{0}$. carolinensis, the male genitalia of this new species immediately show that it is distinct.

Ophiomyia banffensis Spencer (new to U.S.A.)

(Figs. 161-163)

Ophiomyia banffensis Spencer, 1969a:

83. Holotype male from Alberta in CNC.

This distinctive species has hitherto been known only from Alberta and Saskatchewan. A single male can now be recorded from Colorado, Boulder, 6,000 feet, 16.V.61 (B. H. Poole), in CNC.

With the costa ending at vein $R 4+5$ and the squamal fringe white, 0 . banffensis superficially resembles Hexomyza winnemanae (p. 250), but it is distinguishable by the distinct, though narrow, facial keel.

Ophiomyia boulderensis Spencer, new species

(Figs. 207-209)

Head (fig. 207). Frons broad, twice width of eye, conspicuously projecting above eye in profile; 3 ors, 2 ori, al1 slender, approx. equal, orbital setulae sparse, reclinate; ocellar triangle detectable in outline on $1 y$; gena broad, rounded, not projecting forward, parafacial unusually broad, filling about half distance between lower margin of eye and of gena; vibrissa and 2 other bristles strong but not fully fused into fasciculus, few slender peristomal hairs behind; $3 \mathrm{~d}$ antennal segment smal1, round, arista short, bare; facial keel raised but narrow.

Mesonotum. 2 dc, acr sparse.

Wing. Length in male $2.5 \mathrm{~mm}$, in female up to $3 \mathrm{~mm}$, C extending strongly to $M 1+2$, inner crossvein well beyond center of discal cell, last section of M 3+4 only slightly shorter than penultimate, in ratio $6: 7$.

Color. Black, ocellar triangle not shining; mesonotum and abdomen on1y moderately shining; squama gray, margin and fringe black.

Male genitalia. Aedeagus as in figures 208, 209.

Host/early stages. Unknown.

Holotype m., Colorado, Boulder Co., Boulder, 6,900 ft, 17.VI.61, "mountain meadow with Ponderosa Pine" (C. H. Mann); paratypes, 2 f., Boulder, 4.VI.61; $1 \mathrm{~m}$., Boulder, 5,500 ft, 
16.VI.61; Nederland, 8,500 ft, $1 \mathrm{f}$. , 15.VI.61; (a11 B. H. Poole). Holotype and paratypes in $\mathrm{CNC}, 2$ paratypes in KAS •

Remarks. This large, distinctive species cannot be confused with any others known at the present time.

Ophiomyia carolinae Spencer, new species

(Figs. 282, 283)

Head. Frons broad, twice width of eye, not projecting above eye in profile; 2 ors, 2 ori, orbital setulae reclinate; ocellar triangle short, apex extending only slightly beyond apex of upper ors; gena broad, 0.33 height of eye; vibrissal angle approx. $90^{\circ}$, fasciculus in male broad at base, finely tapering; facial keel narrow, furrowed, only slightly raised.

Mesonotum. $2 \mathrm{dc}$, acr in some 8 rows.

Wing. Length in male $3 \mathrm{~mm}$, costa extending strongly to $M 1+2$, last section of $\mathrm{M} 3+40.67$ penultimate, inner crossvein only slightly beyond midpoint of discal cell.

Color. Black, ocellar triangle and orbits only faintly shining; mesonotum and abdomen moderately shining; squama gray, margin and fringe black.

Male genitalia. Aedeagus as in figures 282, 283 .

Host/early stages. Unknown.

Holotype m., North Carolina, Macon Co., Highlands, 3,800 ft, 7.VI.57 (W. R. M. Mason), in CNC.

Remarks. This is the largest species in the genus known in the United States, and it can be compared only with 0 . vibrissata. The two agree close $\overline{1}$, but in 0 . carolinae the vibrissal fasciculus is shorter and more slender. The aedeagi of the two species are distinctively different.

A female from North Carolina, High- lands, Horse Cove, 3,000 feet (H. C. Huckett), in CNC, agrees closely with the holotype, but it is smaller, with a wing length of $2.6 \mathrm{~mm}$ and is not treated as a paratype.

Ophiomyia carolinensis Spencer, new species

(Figs. 259-261)

Head (fig. 259). Frons 1.5 times width of eye, not projecting above eye in profile; 2 ors, 2 ori, orbital sparse, reclinate; ocellar triangle ill defined beyond ocellar plate, apex just reaching level of lower ors; vibrissal corner forming angle of approx. $60^{\circ}$, vibrissal fasciculus in male short, broad at base, finely tapering; gena about 0.2 vertical height of eye (both antennae missing); facial keel narrow but highly raised at base of antennae.

Mesonotum. $2 \mathrm{dc}$, acr in about 8 rows.

Wing. Length in male $1.9 \mathrm{~mm}, 1$ ast section of $M 3+4$ longer than penultimate, in ratio $19: 16$, inner crossvein at distal $3 \mathrm{~d}$ of discal cell.

Color. Ocellar triangle scarcely, mesonotum and abdomen distinctly, shining black; squama gray, margin and fringe black.

Male genitalia. Aedeagus as in figures 260,261 .

Host/early stages. Unknown.

Holotype m., North Carolina, Macon Co., Highlands, 3,800 ft, 23.VIII.57 (J. G. Chillcott), in CNC.

Remarks. Distinctive characters of this species are the very high facial keel and the long last section of vein M 3+4. The male genitalia are diagnostic.

Ophiomyia chondrillae Spencer, new species

(Figs. 227-229)

Head. Frons narrow, equal to width of 
eye, not projecting above eye; 2 ors, 3 ori; gena broad, almost 0.25 height of eye, conspicuously extending forward, forming angle of approx. 450; vibrissal fasciculus not fully fused, consisting of bunch of some 10 bristles (fig. 227); facial keel narrow, scarcely raised; 3d antennal segment smal1, round, arista bare.

Mesonotum. $2 \mathrm{dc}$, acr in about 8 rows.

Wing. Length in male $2 \mathrm{~mm}, \mathrm{C}$ extending strongly to $M 1+2,1$ ast and penultimate sections of $M 3+4$ equal, inner crossvein at midpoint of discal cell.

Color. Black, mesonotum moderately shining; squama pale gray, margin and fringe dark.

Male genitalia. Aedeagus as in figures 228, 229; sperm pump with blade almost triangular; inner corner of epandrium narrow, with some 10-12 minute bristles.

Host/early stages. Unknown.

Holotype m., Washington, Spokane Co., 9 miles from Falls, ex stem of Chondrilla juncea, 11.VII.79 (G. L. Piper), in USNM.

Remarks. The unique holotype is in poor condition, but with the host known, its description appears justifiable and desirable.

The male genitalia are remarkably similar to those of $\underline{0}$. undecima Spencer (1969a: figs. 174, 175) from Alberta, but the two species differ substantially in external characters.

\section{Ophiomyia congregata (Malloch)}

\section{(Figs. 184-186)}

Agromyza congregata Malloch, 1913a: 328. Holotype male from Arizona in USNM.

Ophiomyia congregata, Frick, 1952a: 382; 1959: 369 .

Ophiomyia pulicarioides Sehgal, 1968: 61. Holotype male from Alberta in CNC. NEW SYNONYM.
Ophiomyia decima Spencer, 1969a: 85. Holotype male from Alberta in CNC. NEW SYNONYM.

Examination of the holotype of 0 . congregata shows it to be the senior synonym of $\underline{0}$. pulicarioides and $\underline{0}$. decima. The distinctive arrangement of strong peristomal hairs (fig. 184) makes the species readily distinguishable. The asymmetrical aedeagus (figs. 185, 186) is characteristic of the genus.

New records.

Colorado, Clear Creek Co., Mt. Evans, Doolittle Ranch, 9,800 ft, $1 \mathrm{~m} ., 2$ f., 15.VII.-10.VIII.61 (B. H. Poole), in CNC; Grand Co., Granby, 7,900 ft, $1 \mathrm{f}$., 15.VI.61 (C. H. Mann), in CNC.

Three males have also been seen from British Columbia, Kootenay District, Carbonate, Columbia River, 2,600 feet, 7-12.VII.08 ( J. C. Bradley), in CUI.

Ophiomyia eldorensis Spencer, new species

(Figs. 284-286)

Head (fig. 284). Frons broad, twice width of eye, distinctly projecting above eye in profile; 2 ors, 2 ori, orbital setulae sparse, reclinate; ocellar triangle ill defined, apex not extending to level of lower ors; gena broad, slightly more than 0.25 height of eye, forming angle of approx. $80^{\circ}$ at vibrissal corner; vibrissal fasciculus in male short, only slightly curving; facial keel broad, only moderately raised, without central furrow.

Mesonotum. $2 \mathrm{dc}$, acr in 8 rows.

Wing. Length in male $2.1 \mathrm{~mm}, \mathrm{C}$ extending to $M 1+2$, last section of M $3+4$ only slightly shorter than penultimate, in ratio $4: 5$, inner crossvein at distal $3 \mathrm{~d}$ of discal cell.

Color. Ocellar triangle matt, scarcely shining; mesonotum and abdomen only moderately shining black; 
squama gray, margin and fringe black.

Male genitalia. Aedeagus (figs. 285, 286) with distiphallus symmetrically bowl shaped; sperm pump with unusually large blade.

Host/early stages. Unknown.

Holotype m., Colorado, Boulder Co., Eldora, 3.VI.61 (J. G. Chillcott), in CNC.

Remarks. Although this species belongs to a group in which identification is difficult based on external characters, the aedeagus is of a form not known elsewhere in North America and is entirely distinctive.

Ophiomyia fastosa (Spencer), new combination (new to U.S.A.)

(Figs. 193-195)

Melanagromyza fastosa Spencer, 1969a:

67. Holotype male from Ontario in CNC.

This species lacks the two primary generic characters of Ophiomyia--the male vibrissal fasciculus and the raised facial keel. It was described in Melanagromyza before the significance of the unusual form of male genitalia was appreciated.

o. fastosa resembles $\underline{0}$. abutilivora, which has been confirmed as a true Ophiomyia feeding on Abutilon. A paratype from Alberta was caught on "wild mustard," and it is possible that the host will prove to be Brassica.

Material seen. Colorado, Eagle Co., State Bridge, 7,000 ft, near Bond, 24.VI-5.VII.61 (B. H. Poole), in CNC.

Ophiomyia frosti Spencer, new species (Figs. 187, 188)

Head. Frons 1.5 times width of eye, not projecting above eye in profile; (orbital bristles all missing but as far as can be detected from basal pits 2 ors, 3 ori), orbital setulae rec- linate; gena deepest in center below eye, 0.2 height of eye; vibrissa short, normal, peristomal hairs weak, sparse; 3d antennal segment round, without pubescence, arista appearing bare, bases of antennae narrowly separated by low facial keel.

Mesonotum. (dc missing, no evidence of more than 2), acr sparse.

Wing. Length in male $2.3 \mathrm{~mm}, \mathrm{C}$ extending strong1y to $\mathrm{M} 1+2$, last section $M 3+40.67$ penultimate, outer crossvein more than own length from inner.

Color. Black, mesonotum and abdomen distinctly shining; squama gray, margin and fringe black.

Male genitalia. Aedeagus (figs. 187, 188) asymmetrica1, characteristic of genus; sternite IX with slightly extended hypandrial apodeme; sperm pump long, only slightly shorter than aedeagus .

Host/early stages. Unknown.

Holotype m., New York, Tompkins Co., Ithaca, "16 May" (year not legible), (S. W. Frost), presented to USNM.

Remarks. This species closely resembles $\underline{0}$ - pulicaria (Meigen), which is common in Europe and is also known from Alberta and British Columbia (Spencer, 1969a: 93). The male genitalia, however, show the two to be distinct. $O$. pulicaria feeds on Taraxacum and related genera, forming a leaf mine along the midrib into the leaf blade, pupating at the base of the leaf. It seems probable that 0 . frosti will feed in the same way.

Ophiomyia lauta (Spencer), new combination

(Figs. 181-183)

Melanagromyza lauta Spencer, 1969a: 70. Holotype male from Ontario in CNC.

The fringe of numerous peristomal hairs 
(fig. 181) and the male genitalia (figs. 182, 183) associate this species correctly with Ophiomyia, to which it is now transferred.

Steyskal (1972b: 2) recorded a single male from Michigan, and another male has now been seen from Thalman, Glynn County, Ga. (CUI).

Ophiomyia monticola Sehgal (new to U.S.A.)

(Figs. 164-166)

Ophiomyia monticola Sehgal, 1968: 60; Spencer, 1969a: 90. Holotype male from Alberta in CNC.

This species is widespread in northern Canada and has also been recorded from Alaska. Two males have now been identified from Colorado, Boulder County, Nederland, 8,500 feet, and Caribou, 10,000 feet, both 18.VI.61 (C. H. Mann), in CNC.

Al though $\underline{0}$. monticola closely resembles o. banffensis, it is readily distinguishable by the darker squama and the conspicuously broad facial keel.

Ophiomyia parva Spencer, new species (Figs. 307-309)

Head (fig. 307). Frons little wider than eye, not projecting above eye in profile; 2 ors, 2 ori, orbital setulae sparse, reclinate; ocellar triangle broad but short, apex not extending to level of lower ors; gena narrow, 0.17 height of eye, forming angle of about $70^{\circ}$; vibrissal fasciculus in male (inconspicuously fused on 1 side) short, regularly curving; facial keel broad, high, flattened above but without central furrow.

Mesonotum. $2 \mathrm{dc}$, acr sparse, in some 6 rows.

Wing. Length in male $1.6 \mathrm{~mm}, \mathrm{C}$ extending to $M 1+2,1$ ast and penultimate sections of $\mathrm{M} 3+4$ equal, inner crossvein only slightly beyond midpoint of discal cell.
Color. Ocellar triangle, mesonotum, and abdomen strongly shining black; squama gray, margin and fringe black.

Male genitalia. Aedegus as in figures 308,309 , distiphallus strongly pigmented.

Host/early stages. Unknown.

Holotype m., New Mexico, Otero Co., White Sand National Monument, 5.VIII.66 (R. L. Brumley), in UCD.

Remarks. 0 . parva closely resembles O. lassa from northern California, but the heavily pigmented aedeagus is distinctive and confirms its rank as a species.

Ophiomyia parvella (Spencer), new combination

(Figs. 200-202)

Melanagromyza parvella Spencer, in Spencer and Stegmaier, 1973: 46. Holotype male from Florida in USNM.

The male genitalia show the close relationship of this species to $\underline{0}$. abutilivora, which is confirmed as an Ophiomyia. The minute size of $\underline{0}$. parvella suggests that it may be a leaf miner, possibly the species forming long narrow mines on Lantana (fig. 202). Unfortunately it has never been reared, but it is common in Florida (Spencer and Stegmaier, 1973: 63). Another comparable leaf miner now accepted in Ophiomyia is $\underline{0}$. pfaffiae (Spencer, 1963a), known in Venezuela (Spencer, 1973b: 28).

Ophiomyia praecisa Spencer

(Figs. 221-223)

Ophiomyia praecisa Spencer, 1969a: 92. Holotype male from Alberta in CNC. Ophiomyia aestimabilis Spencer, 1981: 69. Holotype male from California in CAS. NEW SYNONYM.

This species was described from the Banff area in the Canadian Rockies at 4,500 feet. 
It is now apparent that $\underline{0}$. aestimabilis, described from California, Mono County, 9,300 feet, although slightly smaller, is identical with 0 . praecisa, and this new synonymy is established here.

The population in the Mt. Evans area, Colo., is now accepted as distinct from 0 . praecisa (see $\underline{0}$. subpraecisa, p. 258), with a number of external differences supported by small but distinctive differences in the male genitalia.

Ophiomyia quinta Spencer (new to U.S.A.)

(Figs. 235-238)

Ophiomyia quinta Spencer, 1969a: 96. Holotype male from Ontario in CNC.

This species was described from a single caught male, with the vibrissal corner acute, forming an angle of approximately $45^{\circ}$. It represents the common leaf miner on Aster and Solidago that was misidentified by Frost (1924: 42) as Agromyza curvipalpis var. texana Malloch and in Canada by Spencer (1969a: 89) as the European species 0 . maura (Meigen, 1838). ‥ maura forms long, narrow mines on Solidago and the vibrissal corner forms an angle of $80^{\circ}-90^{\circ}$. It has been puzzling that in North America 0 . maura should also feed on Aster, which is never attacked in Europe.

It has now been possible to examine the genitalia of males reared from Solidago by Frost from Ithaca, N.Y., and from Aster at Hot Springs, Ark. (KAS). The aedeagus of these two populations is identical to that of 0 . quinta; it is of the same general form as in $\underline{0}$. maura but more strongly pigmented.

Four distinct populations of this complex can now be identified:

(1) True $\underline{0}$. maura occurring in Europe and feeding exclusively on Solidago. The aedeagus is pale, without pigmentation. The vibrissal angle is approximately $80^{\circ}-90^{\circ}$.
(2) In Japan, Sasakawa (1961: 358) has recorded $\underline{0}$. maura as feeding on both Aster and Solidago, and he synonymized 0. asteris Kuroda, 1954, with 0 . maura. The form of the gena and the aedeagus (Sasakawa, 1961: figs. 38, a and 38, d) agrees with that of true $\overline{0}$. maura, but this population has already colonized Aster.

(3) In Alberta, G. C. D. Griffiths has reared specimens from Solidago (the species also occurs there on Aster). The aedeagus is as in European 0 . maura, but the gena is acute.

(4) In eastern North America (Ontario, New York, Arkansas), the gena forms an angle of approximately $45^{\circ}$ (fig. 235), and the aedeagus is heavily pigmented. Aster is attacked as frequently as Solidago.

With the name 0 . quinta available for the eastern North American population, it is proposed to treat this as distinct from the European 0. maura. The status of the population in Japan and western Canada requires further clarification. No representative of this complex has so far been found in California.

Specimens examined.

Arkansas, Garland Co., Hot Springs National Park, $2 \mathrm{~m}$., emerged 20 and 21.V.79 from leaf mines on Aster sp., coll. 15.V.79 (KAS), in KAS.

New York, Tompkins Co., Ithaca, $1 \mathrm{~m}$., "linear Solidago," 7.IX.18 (S. W. F.), in PSU.

Pennsylvania, Adams Co., Arendtsville, 1 f., "Solidago," 1.VIII. 26 (S.W. F.), in PSU.

Ophiomyia sexta Spencer (new to U.S.A.) (Figs. 290-292)

Ophiomyia sexta Spencer, 1969a: 98. Holotype male from Alberta in CNC.

This distinctive species is known from Alberta, Manitoba, Northwest Territories, and Quebec in Canada. The first specimen from the United States can now be recorded from Colorado, Boulder County, Boulder, Flagstaff Canyon, 5,800 feet, male, 10.VI.61 (C. H. Mann), in CNC. 
Ophiomyia similata (Ma1loch), new combination

(Figs. 171-173)

Agromyza similata Ma1loch, 1918b: 178. Holotype male from Illinois in INHS. Melanagromyza similata, Frick, 1952a: 379; 1959: 366 . Melanagromyza orientalis spencer, 1969a: 73. Holotype male from Ontario in CNC. NEW SYNONYM.

This species was described from a single male. Frick (1959) noted that $C$ ends at vein $R \quad 4+5$, although this was not referred to in the original description. We believe the reason why Malloch failed to mention this character is that $\mathrm{C}$ could be taken as continuing weakly to $M 1+2$.

It is clear from the male genitalia that 0 . orientalis is identical with 0. similata, and this new synonymy is formally established here. The termination of $C$ at $R 4+5$ was not noticed when describing $\underline{0}$. orientalis, but reexamination of male and female paratypes by KAS confirms that there can be doubt as to the exact extent of the costa, and it appears to be more strongly defined to $M 1+2$ in the female. The species has therefore been included in both couplets 5 and 15 to allow for both interpretations.

Two new illustrations of the aedeagus given in figures 171,172 , as those included with the description of $\underline{M}$. orientalis (Spencer, 1969a: figs. 104, 105), are not entirely in side and ventral views and may appear misleading. The form of the aedeagus strongly suggests that this species correctly belongs in Ophiomyia, somewhat resembling that of $\underline{0}$. abutilivora, which is now known as a stem miner on Abutilon, and the species is now formally transferred to Ophiomyia.

The second record of this uncommon species in the United States has now been confirmed from New York, Ithaca, 1 male, 1 female (no head), in copulation, 28.V.62 (L. V. Knutson), CUI.
0. Similata closely resembles $\underline{0}$. fastosa (p. 255), and the male genitalia show the two to be closely related. However, in $\underline{0}$. fastosa, C extends very strongly to $\mathrm{M} 1+2$, there are three ori, and the species is larger. It has possibly speciated at its present high elevation in Colorado.

Ophiomyia subpraecisa Spencer, new species

(Figs. 230, 231)

Head. Frons broad, twice width of eye, not projecting above eye in profile; 2 ors, 2 ori, orbital setulae sparse, reclinate; ocellar triangle with apex extending to lower ors; gena broad, 0.33 height of eye; vibrissal corner forming angle of $45^{\circ}$, fasciculus in male short; facial keel broad, conspicuously raised, with low furrow on upper half.

Mesonotum. Normally 2 dc (rarely a smal1 3d present), acr in some 8 rows.

Wing. Length from $2.1 \mathrm{~mm}$ in male to $2.3 \mathrm{~mm}$ in female, $\mathrm{C}$ extending strongly to $M 1+2$, last section of $M 3+4$ about 0.67 penultimate, inner crossvein we11 beyond midpoint of discal cell.

Color. Black, ocellar triangle weakly shining; mesonotum and abdomen more distinctly shining; squama gray, margin and fringe black.

Male genitalia. Aedeagus as in figures 230, 231, distiphallic complex round behind, narrowing centrally, and then again widening.

Host/early stages. Unknown.

Distribution. Colorado, Mt. Evans area.

Holoptype m., Colorado, Lake Co., Independence Pass, 12,100 ft, tundra, 7.VIII.61 (C. H. Mann); paratypes 27 m., 2 f., same data; 9 m., 11 f., 31.VII. and 7.VIII.61 (J. G. Chillcott and B. H. Poole); Gunnison-Chaffee Co. dividing 1 ine, Cottonwood Pass, 2 m., $1 \mathrm{f} ., 31$.VII and 7.VIII.61 (J. G. 
Chillcott and B. H. Poole); Mt. Evans, Doolittle Ranch, 9,800 ft, $2 \mathrm{f}$., 29.VII and 3.VIII.61 (C. H. Mann and B. H. Poole); Mt. Evans, Timberline, $11,700 \mathrm{ft}, 1 \mathrm{~m} ., 1 \mathrm{f},, 22$ and 29.VII.61 (S. M. Clark and W. R. M. Mason); Mt. Evans, Rocky Slope, 13,600 ft, $1 \mathrm{f}$., 4.VIII.61 (J. G. Chillcott); Mt. Evans, Echo Lake, 10,600 ft, $2 \mathrm{~m} ., 1$ f., 20.VII.61 (B. H. Poole); Loveland Pass, summit, $12,000 \mathrm{ft}, 2 \mathrm{f}$., 12.VII.61 (C. H. Mann and B. H. Poole). Holotype and paratypes in $\mathrm{CNC}$, other paratypes in KAS.

Remarks. This species closely resembles $\underline{0}$. praecisa and the male genitalia confirm their direct relationship. $\underline{0}$. subpraecisa is generally smaller, the frons is not projecting, the gena is narrower, and the vibrissal fasciculus is shorter; in the aedeagus the cylindrical projection at the rear of the distiphallic complex present in 0 . praecisa is lacking (see figs. $2 \overline{2} 2$, 223).

0. subpraecisa has probably speciated relatively recently in isolation in the Rockies in the Mt. Evans area.

\section{Ophiomyia texana (Malloch)}

(Figs. 293-295)

Agromyza texana Ma1loch, 1913a: 319. Holotype male from Texas in USNM. Ophiomyia texana, Shewel1, 1953: 465; Frick, 1957b: 201; 1959: 371. Ophiomyia shiloensis Spencer, 1969a: 98. Holotype male from Manitoba in CNC. NEW SYNONYM.

Ophiomyia arguta Spencer, in Spencer and Stegmaier, 1973: 169. Holotype male from Eleuthera Is., Bahamas, in AMNH. NEW SYNONYM.

Ophiomyia modesta Spencer, 1981: 94. Holotype male from California in CAS. NEW SYNONYM.

This is a small species, with a wing length ranging from $1.75 \mathrm{~mm}$ in the male to $2.3 \mathrm{~mm}$ in the female.

It has only now been possible to examine the male holotype (the head is detached and in poor condition and difficult to orientate for accurate illustration, as stated by Frick (1959: 452)). In consequence, Frick's figure (1959: fig. 53) is somewhat inaccurate in showing the vibrissal fasciculus to be broad and blunt at the end, whereas a distinctive feature is that it tapers very finely. A new illustration of the head is shown in figure 293.

Both 0. shiloensis from northern Canada and $\underline{0}$. modesta from southern California were described before 0 . texana had been examined, but the male genitalia, with the distinctive cylindrical projection at the rear of the distiphallic complex, show conclusively these species are synonyms of 0. texana. 0 . arguta from the Bahamas (see Spencer and Stegmaier, 1973: figs. 417-419) is also now recognized as a synonym of $\underline{0}$. texana.

This is a striking example of the extensive north-south range of a number of species, which had not previously been suspected.

Ophiomyia texella Spencer, new species

(Fig. 180)

Head (fig. 180). Frons narrow, equal to width of eye, not projecting above eye; 2 ors, 2 ori, all slender and equal, orbital setulae minute, sparse, reclinate; eye unusually elongate; gena narrow, extending forward below antennae, with single slender hair at end in female; facial keel narrow but high.

Mesonotum. $2 \mathrm{dc}$, acr sparse, in 6 rows.

Wing. Length in female $1.4 \mathrm{~mm}, \mathrm{C}$ extending to $M 1+2$, last section of M 3+4 1.5 times length of penultimate.

Color. Black, ocellar triangle and orbits weakly shining; mesonotum and abdomen strongly shining; squama, including margin and fringe, white.

Male genitalia. Unknown. 
Host/early stages. Unknown.

Holotype f., Texas, Kerr Co., Kerrville, 21.IV.59 (J. F. McAlpine), in $\mathrm{CNC}$.

Remarks. This unusual species is distinctive in its projecting gena, elongate proboscis, and long last section of vein $M 3+4$.

Ophiomyia tiliae (Couden), new combination

(Figs. 189-192)

Agromyza tiliae Couden, 1908: 33. Lectotype female, designated by Frick, 1957b: 200, in USNM.

Melanagromyza tiliae, Frick, 1952a: $380 ; 1959: 367$.

Hexomyza tiliae, Spencer, 1973a: 299; 1981: 33 .

In view of the characteristic galls formed by this species on twigs of Tilia americana, it was placed in the ga11-forming genus Hexomyza by Spencer (1973a).

Examination of the male genitalia of one of the type series shows that the aedeagus (figs. 189, 190) is of the characteristic form of Ophiomyia abutilivora, a stem miner on Abutilon (p. 250). In view of this clear association, 0 . tiliae must be transferred to Ophiomyia. The epandrium ( $\mathrm{fig} .191$ ) is unusually square on the lower corner, with a row of stout bristles and some longer hairs, and the cercus is particularly broad (fig. 191).

It is now apparent that formation of galls is not a valid character for generic delimitation, as had been believed. The galls, of course, merely represent a reaction by the plant to the larval feeding, and different plants react differently to feeding by larvae even in the same genus. However, even in $\underline{0}$. abutilivora, the stem mine is not shallow and flat immediately below the epidermis, as in most Ophiomyia species, but it is in the form of a raised "we1t." This almost certainly represents a reaction of the plant to the presence of the larva and can be accepted as a minor type of gall. Similar galls are formed by Ophiomyia fici Spencer and Hill, 1976, in the leaves of Ficus microcarpa in Hong Kong (Spencer and Hil1, 1976: fig. 3).

Ophiomyia vibrissata Malloch (Figs. 279-281)

Agromyza vibrissata Malloch, 1913a: 316. Holotype male from Georgia in USNM.

Hende1 (1920: 130) synonymized $\underline{0}$. vibrissata with 0 . major Strobl from Europe.Frick (195 $\overline{2 \mathrm{a}: 382} ; 1959$ : 370) followed this synonymy. However, after comparison of a male paratype of $\underline{0}$. vibrissata (now NMW) with the male lectotype of 0 . major, this synonymy was rejected by Spencer (1964a: 794).

Examination of the holotype of 0 . vibrissata (aedeagus: figs. 280, 281) confirms that this is a distinct species despite the superficial similarity of external characters with those of $\underline{0}$. major.

The only record of this species has been from Georgia. A male and female have now been identified from New York, Long Island, Farmingdale, 5.VI.35 and 6.VI.36 (Blanton and Borders), in CUI. Although the species is obviously uncommon, it probably will be present at suitable localities between Georgia and New York.

Ophiomyia virginiensis Spencer, new species (Figs. 242, 243)

Head. Frons little wider than eye, not projecting above eye in profile; 2 ors, 2 equal and slightly weaker ori, orbital setulae sparse, reclinate; ocellar triangle with apex extending to level of lower ors; gena extending forward, forming angle of approx. $45^{\circ}$, in center below eye narrow, 0.14 height of eye; vibrissal 
fasciculus broad at base, short, regularly curving; facial keel moderately raised, with conspicuous furrow throughout its length.

Mesonotum. $2 \mathrm{dc}$, acr in some 8 rows.

Wing. Length from $2.1 \mathrm{~mm}$ in male to $2.5 \mathrm{~mm}$ in female, $\mathrm{C}$ extending to $\mathrm{M} 1+2$, last and penultimate sections of $M 3+4$ equal, inner crossvein at distal $3 \mathrm{~d}$ of discal cell.

Color. Black; frons matt, ocellar triangle, and orbits moderately shining; mesonotum and abdomen only moderately shining; squama gray, margin and fringe black.

Male genitalia. Aedeagus as in figures 242, 243; sperm pump with large, asymmetrical blade.

Host/early stages. Unknown.

Holotype m., Virginia, Hawksbi11, Shenandoah Nat1. Park, 3,600-4,050 ft, 7.VI.62 (J. R. Vockeroth); paratypes 1 m., 1 f., same data. Holotype and f. paratype in CNC, m. paratype in KAS.

Remarks. Although this species generally resembles both $\underline{0}$. quinta and o. obstipa, there are sma 11 but significant external differences and the aedeagus is entirely distinctive.

Ophiomyia vockerothi Spencer, new species

(Figs. 262-264)

Head (fig. 262). Frons narrow, 1ittle wider than eye width, not projecting above eye in profile; 2 ors, 2 ori, orbital setulae sparse, reclinate; ocellar triangle short, apex extending only midway between upper and lower ors; gena broad, 0.33 height of eye; vibrissal fasciculus in male short; facial keel broad, moderately raised, without furrow.

Mesonotum. $2 \mathrm{dc}$, acr sparse, in about 6 rows.

Wing. Length in male $1.9 \mathrm{~mm}$, last section of $M 3+4$ 1ittle shorter than penultimate, inner crossvein only slightly beyond midpoint of discal ce11.

Color. Ocellar triangle moderately shining; mesonotum and abdomen shining black; squama gray, margin and fringe black.

Male genitalia. Aedeagus as in figures 263, 264 .

Host/early stages. Unknown.

Holotype m., North Carolina, Macon Co., Highlands, 3,800 ft, 22.V.57 ( J. R. Vockeroth), in CNC.

Remarks. This species somewhat resembles 0 . texana, but the male genitalia of the two are entirely distinct.

\section{GENUS JAPANAGROMYZA SASAKAWA}

One new synonym and one new combination are established here.

Japanagromyza rutiliceps (Melander), new combination

(Figs. 319-321)

Agromyza rutiliceps Melander, 1913:

261. Holotype male from Montana in USNM.

Agromyza irwini Spencer, 1981: 113. Holotype female from California in CAS. NEW SYNONYM.

This species has the appearance of a typical Agromyza, with reddish, strongly projecting frons, deep gena, and $3+1$ strong dorsocentral bristles. In Japanagromyza, normally the frons is black or brown and does not project, the gena is narrow, and there are invariably only two postsutural dc. However, with males now available, the aedeagus has been found to be atypical of Agromyza and, with the coiled distiphallus, closely resembles that of a number of Japanagromyza species, such as $\mathrm{J}$. inaequalis (Ma1loch) in Florida (fig. 336). The 
stridulating mechanism present in all Agromyza species is also lacking. Despite the uncharacteristic external morphology, J. rutiliceps is therefore transferred to Japanagromyza here.

Agromyza irwini was described from three females in California and before the holotype of A. rutiliceps had been examined. There was no reason to suspect that it was not an Agromyza, generally resembling $A$. nevadensis (p. 70). Although the distinctive distiphallus of the holotype of $\mathrm{J}$. rutiliceps was lost during previous preparation, this species can now be associated both with males now seen from Idaho and Utah and also with the females of A. irwini, which is therefore synonymized with J. rutiliceps here.

J. rutiliceps appears to be a primitive species, retaining several morphological characters shared with Agromyza at an early stage in the evolution of the two genera but now lost in other members of the genus.

We are grateful to Michael von Tschirnhaus for valuable discussions on this interesting species and in particular for his perspicacious assessment of its correct generic position.

\section{GENUS AGROMYZA FALLÉN}

Six new species are described, four new synonyms are established, and six species are recorded as new to the United States. One undescribed species from Wyoming is briefly diagnosed. The taxonomic status of one species is clarified and new State records are given for four species.

Agromyza albertensis Spencer (new to U.S.A.)

(Figs. 373-375)

Agromyza albertensis Spencer, 1969a: 32. Holotype male from Alberta in CNC.

A single male referable to this species has been identified from Colorado, Eagle County, State Bridge, 7,000 feet, near Bond, 24-25.VI.61 (C. H. Mann), in CNC.

A. albertensis belongs to the closely related complex of grass-feeding species, including also in America $\underline{A}$. ambigua, A. tularensis, and $\underline{A}$. utahensis, in which the third antennal segment is angulate and the $C$ ends at vein $R 4+5$. The male genitalia of $A$. albertensis are shown in figures $37 \overline{3}$, 374 , and the third antennal segment is in figure 375 .

\section{Agromyza albipennis Meigen}

(Figs. 387-390)

Agromyza albipennis Meigen, 1830: 171. Two syntypes from Germany in NMW; female in MNHNP is not an agromyzid.

Agromyza dubitata Malloch, 1913a: 311. Two female syntypes from Massachusetts in USNM. NEW SYNONYM.

Frick (1959) confused this species with A. nigripes Meigen, which he considered as having the squamal fringe white, whereas it is in fact invariably darker. A. dubitata was thus synonymized with A. nigripes (Frick, 1959: 356); however, examination of the two type specimens (which are in perfect condition) immediately shows that they represent A. albipennis, and this new synonymy is established here.

A distinctive character of A. albipennis is the shape of the third antennal segment (fig. 387), which is almost angulate at the upper corner and rounded below."

Agromyza aprilina Malloch, stat. rev. (Figs. 385, 386)

Agromyza aprilina Ma1loch, 1915c: 359. Lectotype female, designated by Frison, 1927, in INHS.

Frick (1957b: 199) synonymized A. aprilina with A. subnigripes $\bar{M}$ alloch (1913a: 334). 
Malloch (1913a: 334) considered a female from Hampton, N.H., to have the squamal fringe dark and to be identical with two specimens of A. nigripes Schiner from Europe that he examined. As the name A. nigripes is preoccupied by A. nigripes Meigen, Malloch gave the species the new name of $A$. subnigripes. Hendel synonymized $\underline{A}$. subnigripes with A. morosa Meigen, a common species in Europe, in which the squamal fringe is black.

Examination of the specimens from New Hampsire treated by Malloch as $\underline{A}$. subnigripes confirms that the squamal fringe is pale, as correctly noted by Frick (loc. cit.). Although Malloch was correct to give a new name to $\underline{A}$. nigripes of Schiner, he was incorrect to apply this name to the New Hampshire specimen, which he in fact misidentified owing to its squamal fringe being pale. This specimen is indeed identical with A. aprilina, but it has no status in nomenclature and Frick's synonymy is thus rejected.

Agromyza bispinata Spencer (new to U.S.A.)

(Figs. 408-410)

Agromyza bispinata Spencer, 1969a: 39. Holotype male from Ontario in CNC.

Additional material recently studied shows this species to be widespread in America and records from six States are given here. The two strong spines at the lower corner of the epandrium (fig. 410) are distinctive, and the aedeagus is shown in figures 408 , 409. The conspicuous1y pubescent third antennal segment is another distinguishing character.

New records.

Georgia, Rabun Co., Rabun Bald, $1 \mathrm{~m}$., 13.VII.57 (W. R. Richards), in CNC. Maryland, Montgomery Co., Bethesda, 1 m., 1 f., 30.V.80 (G. Steyskal), in USNM.

New York, Tompkins Co., Ringwood, Ithaca, $1 \mathrm{~m}$., 13.VII.20 (M. D. Leonard), in CUI.

North Carolina, Jackson Co., Cherokee,
2,000 ft, $1 \mathrm{~m} ., 1 \mathrm{f} ., 29 . V I I .57$, and

2 f., 13 and 16.VII.57 (a11 J. G.

(hillcott), in CNC.

Utah, Cache Co., Green Canyon, $1 \mathrm{~m}$., 23.VII.64 (W. J. Hanson, Malaise trap), in USU.

Virginia, Shenandoah Co., Mt. Jackson, 1 m., 3 f., 25.V.62 (J. G.

Chillott), in CNC.

Agromyza hardyi Spencer, new species (Figs. 401, 402)

Head. Frons broad, twice width of eye, not projecting above eye in profile; 2 ors, 3 ori, orbital setulae sparse, reclinate; gena narrow in center below eye, somewhat extended at rear, up to 0.17 height of eye; $3 d$ antennal segment small, round, arista short, equal to width of eye in profile.

Mesonotum. 3 strong postsutural dc, prsc similar to dc 2 .

Wing. Length in male $3.25 \mathrm{~mm}, \mathrm{C}$ extending to $M 1+2$, last section of M $3+4$ short, 0.5 penultimate, inner crossvein at midpoint of discal cell.

Color. Frons dark brown, antenna black; mesonotum matt grayish black, with only faintest subshine; legs with femora blackish, knees yellow, tibiae and tarsi conspicuously yellow; veins dark brown; squama and fringe silvery white.

Male genitalia. Aedeagus (figs. 401, 402) asymmetrical, distinctive.

Host/early stages. Unknown.

Holotype m., Utah, Cache Co., Blacksmith Fork Canyon, 12.VI.38 (D. E. Hardy and A. T. Hardy), in USNM.

Remarks. The male genitalia show this species to be related to A. pseudoreptans but even more closely to a shining black species, A. pseudorufipes Nowakowski, 1964, known in Europe as a leaf miner on Myosotis (Boraginaceae). 
Agromyza hockingi Spencer (new to U.S.A. )

(Figs. 411-413)

Agromyza hockingi Spencer, 1969a: 44. Holotype male from Alberta in CNC.

Specimens have now been identified from three States and are recorded here.

In the type series the squamal fringe was somewhat variable, ochreous to brownish. In the specimens now seen it would be considered as pale, but the male genitalia (figs. 411-413) and in particular the distinctive arrangement of bristles within the epandrium (fig. 413) are diagnostic and in the specimen from Colorado exactly as in the type series.

New records.

Colorado, Clear Creek Co., Mt. Evans, Doolittle Ranch, 9,800 ft, 3.VIII.61 (C. H. Mann), in CNC.

Texas, Kerr Co., Kerrville, $1 \mathrm{~m}$., 31. III.59 (J. F. McAlpine), in CNC.

Utah, Cache Co., Green Canyon, $1 \mathrm{~m}$. , 25-31.VII.68 ( $\mathrm{J}$. Hanson, Malaise trap), in USU.

Agromyza isolata Malloch

(Figs. 437, 438)

Agromyza isolata Malloch, 1913a: 306. Holotype female from California in USNM.

Agromyza populoides Spencer, 1969a:

52. Holotype male from Saskatchewan in CNC. NEW SYNONYM.

With only a female known, the identity of A. isolata has long been in doubt. It was considered to be a member of the A. potentillae (= spiraeae) group, but identification in the absence of males was problematic. The one distinctive character was the yellowish tibiae and tarsi.

A female recorded from Populus deltoides at Hoquiam, Grays Harbor County, Wash. (Greene, Lot 44-22553), identified by Greene as A. isolata, presumably after comparison with the holotype, has now been examined and this identification is accepted. This species, occurring as a leaf miner on both Populus and Salix, is common and widespread in Canada and also in California and Washington. In Canada it was described as A. populoides, and west coast specimens in the United States were misidentified by Frick (1959: 353) as the European species A. albitarsis Meigen. The new synonymy of A. populoides with A. isolata can thus now be formally established.

Agromyza kincaidi Malloch

(Figs. 376, 377)

Agromyza kincaidi Malloch, 1913a:

285. Holotype female from Alaska in USNM.

Frick (1959: 353) treated A. kincaidi as a synonym of A. ambigua Fallén (in the sense of earlier authors, i.e., A. nigrella Rondani), but this synonymy is not accepted. A. nigrella is indeed very close to A. kincaidi but is substantially smaller.

From the male genitalia it is clear that $\underline{A}$. kincaidi is a grass feeder. No specimens have been reared, but two exceptionally large puparia $3.2 \mathrm{~mm}$ in length were obtained from larvae feeding on Bromus purgans at Ouachita State Park, near Hot Springs, Ark., 15.V.79 (KAS), and it is believed that these can only represent $\underline{A}$. kincaidi.

New records.

Colorado, Clear Creek Co., Mt. Evans, Doolittle Ranch, 9,800 ft, $1 \mathrm{f}$., 22.VII.61 (J. G. Chill cott), in CNC; $1 \mathrm{~m} ., 1 \mathrm{f} ., 3$ and 8.VIII.61 (W. R. M. Mason and B. H. Poole), in CNC; Echo Lake, 10,600 ft, $1 \mathrm{f}$., 25.VII.61 (C. H. Mann), in CNC.

Tennessee, Sevier Co., Indian Gap to Clingman's Dome, 5,200-6,600 ft, 1 m., 6.VIII.57 (J. G. Chill cott), in CNC.

Utah, Weber Co., North of Huntington, 1 f., 16.VI.66 (G. F. Knowlton), in USU. 
Agromyza masculina Sehgal (new to U.S.A. )

(Figs. 449-451)

This species has been known from two specimens from Alberta, one from Blairmore in the Rockies at 6,000 feet and the other from Okotoks, about 80 $\mathrm{km}$ farther east and at a lower elevation.

A single male from Utah, Cache County, Green Canyon, 9.VII.69, Malaise trap, (W. J. Hanson), in USU, is referable to A. masculina. This species belongs to the A. potentillae group and appears to be most closely related to A. vockerothi (figs. 441-443).

Agromyza nigrella (Rondani)

(Figs. 380-384)

Domomyza nigrel1a Rondani, 1875: 174. Agromyza nigre11a, Spencer, 1966c: 288; 1976a: 128; 1981: 117.

Agromyza abbreviata Malloch, 1913a: 285. Holotype male from New Mexico in USNM.

Agromyza barberi Frick, 1952a: 372. New name for $A$. abbreviata Malloch (not Fallén, 1823a). NEW SYNONYM.

Ma1loch (1913a) gave the wing length of A. abbreviata as $3.5 \mathrm{~mm}$. This has led to confusion, as with this size the species could only be compared with A. kincaidi Ma1loch (1913a), which is in fact larger, although the wing length is given as $3 \mathrm{~mm}$.

Examination of the holotype of $A$. abbreviata shows the wing lengt $\bar{h}$ to be $2.6 \mathrm{~mm}$. It agrees in all characters with A. nigrella, which was recently recorded in California (Spencer, 1981: $117)$, and the male genitalia are also identical. A. abbreviata was renamed A. barberi Frick, as the name is preoccupied, and this species therefore is synonymized with A. nigrella here.

Agromyza pagana Spencer, new species (Figs. 414-416)

Head. Frons 1.5 times width of eye, not projecting above eye in profile; 2 reclinate ors, 2 inclined ori, orbital setulae short, reclinate; ocellar triangle scarcely extended beyond foremost ocellus; gena narrow in center, extended at rear, 0.25 height of eye; 3d antennal segment longer than broad, rounded below, slightly angular at upper corner (fig. 414).

Mesonotum. Probably 3 differentiated $\mathrm{dc}$ but $3 \mathrm{~d}$ missing, acr in some 8 rows.

Wing. Length in male $2.4 \mathrm{~mm}, \mathrm{C}$ extending to $M 1+2$, last section of M $3+40.67$ penultimate, inner crossvein at center of discal cell.

Color. Frons sooty black, ocellar triangle and orbits weakly shining; $3 \mathrm{~d}$ antennal segment predominantly black, narrowly brownish on inner side, 1st and $2 \mathrm{~d}$ segments also brownish; mesonotum shining black; legs with femora black, tibiae and tarsi brownish yellow; abdomen shining black; squama and fringe whitish yellow.

Male genitalia. Aedeagus with distal tubules narrow but distinctively constricted near apex and then widening before narrowing again to junction with basal sclerites (figs. $415,416)$; surstylus with some 15 short, stout bristles continuing to inner lower corner.

Host/early stages. Unconfirmed but certainly Poaceae.

Holotype m., Kansas, Douglas Co., Natura 1 History Reserve, Lawrence, 28.IV.56 ( J. G. Chillcott), in CNC.

Remarks. The shape of the third antennal segment is the most obvious distinguishing character of this species, which otherwise somewhat resembles A. parvicornis. The male genitalia are distinctive.

Agromyza parca Spencer, new species (Figs. 424, 425)

Head. Frons projecting above eye, 1.5 
times width of eye; orbital bristles strong, 2 ors, 2 ori, orbital setulae sparse, reclinate; ocellar triangle slightly extended beyond foremost ocellus; gena narrow, only slightly extended at rear, 0.17 height of eye; $3 d$ antennal segment small, round, arista distinctly pubescent.

Mesonotum. $3 \mathrm{dc}$, small, close to suture, prsc strong, equal to $2 \mathrm{~d} d c$, acr in $8-10$ rows.

Wing. Length $2.5 \mathrm{~mm}, \mathrm{C}$ extending strongly to $M 1+2$, last section of M $3+4$ about 0.67 penultimate, inner crossvein slightly before or at midpoint of discal cell.

Color. Frons deep black, antenna black; mesonotum and abdomen shining black; legs largely black, tarsi at most slightly paler; squama pale gray, margin brown, fringe blackish; veins dark.

Male genitalia. Aedeagus as in figures 424, 425; surstylus with row of short spines continuing to lower corner.

Host/early stages. Unconfirmed but certainly Poaceae.

Holotype m., North Carolina, Mitchel1 Co., Roan Mountain, 6,200 ft, 13.VIII.57; 1 f., same data; (both J. G. Chillcott); Tennessee, Sevier Co., Great Smoky Mountains National Park, 5,200 ft, 1 m., 3.VII.57 (C. D. Hines). Holotype and paratypes in CNC.

Remarks. Distinctive characters of this species are the dark squamal fringe, narrow gena, and pubescent arista. It somewhat resembles $\mathrm{A}$. parilis, but the male genitalia of the two are entirely distinct.

Agromyza parilis Spencer, new species (Figs. 426, 427)

Head. Frons narrowly projecting above eye, 1.5 times width of eye; orbital bristles slender, 2 ors, 2 ori, orbital setulae sparse, reclinate; ocellar tri- angle not differentiated beyond foremost ocellus; gena extended at rear, 0.25 height of eye; $3 \mathrm{~d}$ antennal segment rounded, slightly longer than broad, arista appearing bare.

Mesonotum. Only 2 strong differentiated $\mathrm{dc}$, prsc slightly weaker than $2 \mathrm{~d}$ $\mathrm{dc}$, acr in 8 rows.

Wing. Length in male $2.4 \mathrm{~mm}, \mathrm{C}$ extending strongly to $\mathrm{M} 1+2$, 1 ast section of $R 3+40.67$ penultimate, inner crossvein at center of discal cell.

Color. Frons sooty black; 3d antennal segment predominantly black but slightly paler, yellowish brown on lower half both on inside and outside, $2 \mathrm{~d}$ segment similarly pale, 1st black; mesonotum and abdomen shining black; legs with femora black, tibiae and tarsi only slightly paler, brownish black; squama pale gray, margin and fringe brownish black; wing veins dark.

Male genitalia. Aedeagus (figs. 426, 427) with distal tubules broad, heavily pigmented; sternite IX with narrow sidearms, broadly fused at apex; surstylus with group of short stout bristles but bare at lower corner; sperm pump with small, circular blade and unusually long stalk, base only slightly extended on one side.

Host/early stages. Unconfirmed but certainly Poaceae.

Holotype m., Tennessee, Hamilton Co., East Ridge, 9.V.52 (G. S. Walley), in CNC.

Remarks. Distinctive characters of this species are dark squamal fringe, broad gena, and bare arista. It somewhat resembles A. parca, but the male genitalia of the two are entirely distinct.

Agromyza potentillae (Kaltenbach) (Figs. 444-448)

Phytomyza potentillae Kaltenbach, 1864: 351. Types from Germany lost. 
Agromyza spiraeae Kaltenbach, 1867: 104 (synonymy established by

Spencer, 1976a: 134).

This species is common on many genera of Rosaceae in Europe, but unfortunately no reared specimens are known in North America. The characteristic linear blotch mines were found on Potentilla gracilis in California, Nevada County, Sagehen Creek, at 6,000 feet (KAS). Although no specimens were reared, a caught male from the same locality was identified as A.

potentillae by Spencer (1981: 122).

long series from high elevations in Colorado are recorded here. In these specimens the squama and fringe are darker (deep black) than in typical A. potentillae, but the genitalia are not separable from those of European specimens, and without supporting host information, it is not consdered justifiable to treat them as distinct from A. potentillae.

Frick (1959: 358) discussed this species as $\underline{A}$. spiraeae Kalt.

New records.

Colorado, Clear Creek Co., Summit

Lake, Mt. Evans, $12,800 \mathrm{ft}, 6 \mathrm{~m} ., 3$

f., 16.VII.61 (C. H. Mann); Niwat

Ridge, $11,500 \mathrm{ft}, 1 \mathrm{f.,} \mathrm{4.} \mathrm{VII.61}$

(C. H. Mann); Mt. Evans, Timberline, $11,700 \mathrm{ft}, 2 \mathrm{f} ., 29 . V I I .61$ (C. H. Mann and B. H. Poole); Independence Pass, Lake Co., $11,500 \mathrm{ft}$, tundra, 1 m., 2 f., 31.VII.61 (J. G. Chillcott); all in CNC.

Utah, Duchesne Co., Wasatch National Forest, Mirror Lake, $10,200 \mathrm{ft}, 1$ f., 30.VII.75 (G. Eickwort), in CUI.

Agromyza pudica Spencer, new species (Figs. 428, 429)

Head. Frons not projecting above eye, narrow, equal to width of eye; 2 ors, 3 ori, orbital setulae sparse, reclinate; ocellar triangle short, not extending beyond foremost ocellus; gena narrow in center, extended at rear, 0.25 height of eye; $3 d$ antennal segment small, round.
Mesonotum. 3 strong postsutural dc, 4 th possibly just differentiated from acr, no presutural, acr numerous, in 8 rows.

Wing. Length in male $2.2 \mathrm{~mm}$, in female $2.4 \mathrm{~mm}$, C extending weakly to $M 1+2$ but sometimes appearing to end shortly after $R \quad 4+5$, last section of M 1+2 distinctly shorter than penultimate, inner crossvein near midpoint of discal cell.

Color. Head, including antenna black; mesonotum brilliantly shining black; legs largely black but tarsi distinctly pale, faintly yellowish; squama yellow, fringe black.

Male genitalia. Aedeagus (figs. 428, 429) with basal sclerites greatly expanded anteriorly, forming right angle with distiphallic complex and with narrow extension on anterolateral margin; sperm pump with long basal stalk; surstylus angular, with row of short bristles extending to lower corner (contrast A. aprilina and $\underline{A}$. utahensis (fig. 36 6 ), in which lower corner is bare).

Host/early stages. Unconfirmed but certainly Poaceae.

Holotype m., Arkansas, Garland Co., Hot Springs, 15.V.79; paratypes $1 \mathrm{f}$., same data; (both KAS and USNM); Georgia, Rabun Co., Rabun Bald, $1 \mathrm{~m}$. , 2 f., 16.VII.57 (J. G. Chillcott, CNC); Minnesota, Minneapolis, Minnehaha Creek, 1 f., 28.VIII.76 (KAS); North Carolina, Transylvania Co., Pisgah Forest, Looking Glass Lake, $1 \mathrm{~m}$., 19.VII.57 (W. R. Richards). Holotype and female paratype in USNM, male paratype in $\mathrm{CNC}$.

Agromyza reptans Fallen (Figs. 394-397)

Agromyza reptans Fal1én, 1823a: 3. Holotype male from Sweden in NRS. Agromyza haplacme Steyskal, 1972b: 2 . Holotype male from Michigan in USNM. NEW SYNONYM. 
This is a widespread leaf miner on Urtica in Europe, Canada, and the United States. Frick (1952a) and some European workers confused A. reptans with A. pseudoreptans Nowak̄owski, which also feeds on Urtica, but the male genitalia of the two species are entirely distinct (figs. 395, 399). Nowakowski illustrated the male genitalia of two specimens from Europe (1962: fig. 8, Galicia, and 1964: fig. 14, Estonia), showing the long distiphallus as narrowly divided in the distal two-thirds before the two tubules diverge at right angles at the apex. The holotype of $A$. reptans has been reexamined and the distiphallus is fully fused, apart from the apical divergence. Other specimens have been examined from Italy and from northern Germany (Tschirnhaus). They agree exactly with the holotype.

Steyskal (1972b) described A. haplacme from Michigan, showing the distiphallus as fused and treating this as distinct from A. reptans on the basis of Nowakowski's illustrations. However, this species is clearly identical with A. reptans, and this new synonymy is established here. A minor inaccuracy in Steyskal's illustration must be pointed out, as only the left side of the apical division of the distiphallus is shown, that on the right presumably being obscured. Other males have been examined from California, Washington, and Ontario and all have the distiphallus as shown in figure 396. A slight variation is noted in North American specimens. The distal end of the basal sclerites is somewhat rounded in the specimens from Michigan and California but more angular in one seen from Ontario, bending almost at right angles as is normal in European specimens. However, splitting of A. reptans on this variable character alone is not considered justifiable.

It is puzzling that Nowakowski (1oc. cit.) should have shown the distiphallus as divided in both specimens illustrated. It is conceivable but highly improbable that there is a second species in Europe, but more likely these illustrations seem to reflect an inaccurate observation.

Agromyza tacita Spencer (new to U.S.A.)

(Figs. 403-407)

Agromyza tacita Spencer, 1969a: 59. Holotype male from Ontario in CNC.

This species was described from a series from Ontario, Manitoba, and Quebec. The host was unconfirmed.

A single male reared from an unidentified grass has now been seen from Montana, Lake County, 15 miles northeast of Polson, 22.VII.67 (S. Whitney), in KAS. The posterior spiracles of the puparium are closely adjoining ( $\mathrm{fig}$. 407), in contrast to those of A. parvicornis and A. proxima (figs. 420, 423), in which they are widely separated.

A. tacita belongs to a compact group, including also $\mathrm{A}$. bispinata and A. hockingi. All have the distinctly pubescent third antennal segment, but the arrangement of bristles within the epandrium consistently differs and there are also significant differences in the aedeagus. It will be of interest to discover whether the three species are restricted to different genera of the Poaceae.

Agromyza utahensis Spencer, new species (Figs. 363-366)

Head (fig. 363). Frons 1.5 times width of eye, distinctly projecting above eye toward base of antennae; 4 orbitals, arrangement somewhat variable, upper frequently slightly longer, reclinate, other 3 more incurved, sometimes both ors reclinate and 2 ori incurved, orbital setulae sparse, reclinate; ocellar triangle short, apex not reaching level of lower ors; gena broad, extended at rear, 0.25 height of distinctly slanting eye; $3 \mathrm{~d}$ antennal segment angulate but variable, sometimes finely pointed (fig. 363) but not infrequently more obtuse. 
Mesonotum. Normally 3 strong postsutural dc, not infrequently 2 or 3 smaller anterior ones, presutural, if present, little stronger than acr, these irregularly in 6 rows, prsc approx. equal to dc 3 .

Wing. Length from $2.5 \mathrm{~mm}$ in male to 3 $\mathrm{mm}$ in female, $C$ ending abruptly just beyond end of $R 4+5$, last section of M $3+40.67$ length of penultimate, inner crossvein distinctly before midpoint of discal cell.

Color. Frons matt black, ocellar triangle and bases of orbital bristles distinctly shining; antenna black; lower orbit and parafacial normally conspicuously shining, occasionally less so; mesonotum and abdomen shining black; legs entirely black; wing unusually pale, $R$ veins dark, normal, $M$ veins white, virtually colorless; squama and fringe silvery white.

Male genitalia. Aedeagus with medial section rising abruptly from base of distiphallus (fig. 364), basal

sclerites bending gradually (fig. 365) toward distiphallus complex; surstylus angular, with row of about 5 short, stout bristles along inner margin but not extending to lower corner (fig. 366); sperm pump with large blade and long narrow base.

Host/early stages. Unknown but larva certainly a grass feeder.

Holotype m., Utah, Salt Lake City, 6.V.39 (G. F. Knowlton and R. L. Janes); paratypes $3 \mathrm{f}$, same data; 3 f., Cache Junction, 20.VII.13 (H. R. Hagen); 1 f., Promontory, 25.V.52 (G. F. Knowlton); $1 \mathrm{f}$., Green Canyon, Cache Co., 25-27.VII.64; 1 f., Cache Co., Mendon Cold Springs, 3.IX.65; (both W. J. Hanson); Colorado, Boulder Co., Boulder, 5,500 ft, $1 \mathrm{f} ., 5 . V I .61$ (B. H. Poole). Holotype in USNM, paratypes in $\mathrm{CNC}, \mathrm{KAS}$, and USU.

Remarks. This species closely resembles and is closely related to $\underline{A}$. ambigua (=niveipennis Zett.). The contrasting color of the $R$ and $M$ veins is distinctive, and there are slight but distinct differences in the genitalia (see particularly figs. 364, 368).

Agromyza varifrons Coquillett (Figs. 354-356)

Agromyza varifrons Coquillett, 1902: 189. Holotype female from District of Columbia in USNM.

The food plant of this distinctive species, Celtis laevigata, was first recorded by Spencer $(1966 \mathrm{c})$. Empty leaf mines were common but local in Arkansas and Mississippi in May 1979, and two other State records are also recorded here.

The leaf mine is an irregular linear blotch, most frequently following the leaf margin (fig. 356). One empty leaf mine found at Hot Springs, Ark., 18.V.79 (KAS) can scarcely be associated with A. varifrons. It is a more regular bloteh toward the center of the leaf blade and possibly represents an undescribed species.

\section{New records.}

Arkansas, Garland Co., Hot Springs, empty leaf mines on Celtis laevigata, 15-18.V.79 (KAS and GCS), in USNM and KAS.

Kansas, Douglas Co., Natural History Reserve, Lawrence, $1 \mathrm{~m}$., 28.IV.56 (J. G. Chillcott), in CNC.

Mississippi, Washington Co., Experimental Forest, Leland, empty leaf mines, 11.V.79 (KAS), in KAS.

Texas, Kerr Co., Kerrville, $1 \mathrm{~m}$., 5.IV.56 (J.F. McAlpine), in CNC; San Patricio Co., Welder Wildlife Reserve, nr. Sinton, $1 \mathrm{f}$., 19-23.III.65 (J. G. Chillcott), in CNC.

Agromyza vockerothi Spencer (new to U.S.A.)

(Figs. 441-443)

Agromyza vockerothi Spencer, 1969a: 60. Hollotype male from Nova Scotia in $\mathrm{CNC}$. 
This species is widespread in Canada and the type series included specimens from Nova Scotia, Ontario, Alberta, and British Columbia.

Specimens recorded here from North Carolina and Tennessee agree exactly with A. vockerothi.

Material seen.

North Carolina, Macon Co., Highlands, 1 f., 18.V.57 (J. R. Vockeroth); Highlands, Little Bear Pen Mountain, 1 m., 5.VIII.57 (W. R. Richards); 1 f., 13.VI.57 (C. J. Burden); Monroe Co., Wayah Bald, 3,500 ft, $1 \mathrm{~m}$., $6 . V I I .57$ (J. R. Vockeroth); Mitchell Co., Roan Mt., 6,200 ft, $1 \mathrm{f}$., 13.VIII.57 (J. G. Chillcott); all in CNC.

Tennessee, Swain Co., Indian Gap to Clingman's Dome, 5, 200-6,000 ft, Great Smoky Mountains National Park, 1 m., 6.VIII.57 (J. G. Chillcott), in CNC.

\section{Agromyza sp. (Wyoming)}

A single female from Wyoming, Teton County, Tugwater Pass, "shrubby vegetation along stream," 17.VII.61 (J. G. Chillcott), in CNC, appears to represent an undescribed species. However, it is also close to A. johannae de Meijere, a leaf miner on Cytisus and more rarely Genista and Lupinus in Europe (see Spencer, 1976a: 116). Positive identification is not possible in the absence of a male.

Essential characters of this species are as follows: Frons reddish in front, darker behind, orbit black; 2 ors, 2 ori; gena deep, slightly less than 0.33 height of eye; $3 d$ antennal segment small, round, blackish, lst and 2d paler, more orange; mesonotum matt gray, $3+1$ strong dc, acr irregularly in 4-5 rows, prsc strong; legs largely black, only foreknees reddish; squama and fringe whitish; wing length in female $2.4 \mathrm{~mm}$, C extending strongly to $M 1+2$, last and penultimate sections of $M 3+4$ equal.

\section{GENUS PHYTOBIA LIOY}

One new species is described, one new synonym is established, and one species is recorded as new to the United States. New State records are given for one species, and one possible new species on Salix is briefly discussed.

Phytobia calyptrata (Hendel)

(Figs. 481, 482)

Agromyza nigrisquama Malloch, 1916: 53. Holotype female from Illinois in INHS .

Agromyza calyptrata Hende1, 1923: 145, new name, not nigrisquama Malloch, 1914c (Formosa).

Phytobia (Trilobomyza) calyptrata, Frick, 1953a: 70; 1959: 393.

Phytobia septentrionalis Spencer, 1969a: 106. Holotype male from Quebec in CNC. NEW SYNONYM.

The generic position of the holotype of this species has previously been in doubt. Although Frick (1959) placed it in Phytobia, he was using this name in the sense of Dizygomyza Hendel, 1931, and he included it in the subgenus Trilobomyza (now in Amauromyza), whereas in Frick's sense it would correctly belong in the subgenus Dendromyza. It is one of the smallest of the true tree-feeding Phytobia species, and its identity is revealed by the conspicuously silvery lunule.

A male has now been examined from Maryland, Montgomery County, Colesville, 15.IX.76 (A. S. Menke), in UCD, which agrees exactly with the female holotype from Illinois, and it is now apparent that $\underline{P}$. septentrionalis Spencer, known from Quebec and Ontario, represents the same species. This new synonymy is established here.

P. calyptrata agrees closely with $\underline{P}$. californica, but with available material, treating the two as distinct appears justified. 
Phytobia confessa Spencer (new to U.S.A.)

(Figs. 463, 464)

Phytobia confessa Spencer, 1969a: 105. Holotype male from

Saskatchewan in CNC.

This species has previously been known from Alberta, Manitoba, and Saskatachewan. Three specimens can now be recorded from Utah.

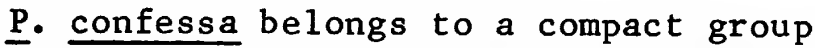
comprising $\underline{P}$. indecora from Illinois and $\underline{P}$. vanduzeei from California. The three species have $C$ ending at vein R $4+5$ and the same type of genitalia. However, distinct external differences can be associated with significant differences in the male genitalia, as indicated in the key (pp. 73, 74).

Material seen.

Utah, Box Elder Co., Willard Bay, 1

m., Malaise trap, 11.VIII.66 (K. Capelle); Cache Co., Logan Canyon, 1 f., Malaise trap, 30.VIII.65; Cache Co., Green Canyon, 1 f., Malaise trap, 26.VII-2.VIII.73; (both W. J. Hanson); all in USU.

Phytobia pallida Spencer, new species (Figs. 458, 459)

Head. Frons 1.5 times width of eye, not projecting above eye in profile; 2 ors, 1 or 2 ori, lower, when present, distinctly included, orbital setulae minute, sparse, reclinate; gena narrow, about 0.125 height of eye; 3d antennal segment small, round, arista long, appearing bare.

Mesonotum. 3+1 dc, 1st and 2d strong, 3d and 4th weak, sometimes little differentiated from acr, equidistant each side of suture, acr irregularly in 8 rows in front, sparse behind $2 \mathrm{~d}$ dc.

Wing. Length $2.1 \mathrm{~mm}$ in male, $2.5 \mathrm{~mm}$ in female, $C$ extending strongly to $M 1+2$, last section of $M \quad 3+4$ about 0.67 penultimate, inner crossvein at midpoint of discal cell.
Color. Frons sooty black, rarely slightly paler, faintly brownish; lunule silvery; antenna black; mesonotum broadly yellow centrally to level of 2d dc; outer band of black, narrowly divided at rear, with both supra-alars just on yellow; scutellum yellow; side of thorax largely bright yellow, humerus with small central dark spot, mesopleuron with triangular black patch filling lower front corner and extending along lower and front margins; sternopleuron black; legs black; abdomen largely yellow but tergites 4-6 with slightly variable blackish area centrally; squama yellow, margin and fringe black.

Male genitalia. Aedeagus (figs. 458, 459) pale, scarcely pigmented; sternite 9 rounded apically, slightly U-shaped; surstylus discrete, rounded, with fringe of hairs on inner margin; aedeagal apodeme pale; sperm pump with narrow blade and base having circular area of strong sclerotization at each end.

Host/early stages. Unknown.

Holotype m., Arizona, Yuma Co., Bill Williams Fork, "Aug."; paratypes $1 \mathrm{f}$., same data; 1 m., 1 f., same locality, "Sept." Holotype and paratypes in USNM.

Remarks. This species closely resembles P. picta (Coquillett, 1902), described from southern Mexico, but is smaller. In a key to neotropical species, Spencer (in Spencer and Stegmaier, 1973: 174) gave the wing length of $\underline{P}$. picta as $2.5-3.1 \mathrm{~mm}$. The male genitalia of the two species, although of the same general form, differ substantially in detail (loc. cit.: figs. 444,445$)$.

Malloch (1913a: 275) referred to a female of a species in this group from New Mexico in the USNM, but the specimen cannot be found and it is impossible to decide whether it represents $\underline{P}$. pallida or one of the other black and yellow species known from Central America and Mexico. 
Phytobia waltoni (Ma11och)

(Figs. 476-478)

Agromyza waltoni Ma1loch, 1913a: 303. Holotype female from New York in USNM.

Phytobia (Dendromyza) waltoni, Frick, 1959: 377 .

Phytobia waltoni, Spencer, 1969a: 109.

This species was described on the basis of its distinctive venation, with the outer crossvein less than its own length from the inner (fig. 476).

The male has not previously been critically examined, but, with the identification of two males recorded here, it has now been possible to examine the male genitalia. The distinctive aedeagus (figs. 477, 478)

fully confirms the diagnosis previously made from a single venational character. The aedeagus is almost completely membranous and entirely distinct from that of any other species known in the genus.

New records.

North Carolina, Macon Co., Highlands, $3,800 \mathrm{ft}, 1 \mathrm{f} ., 9 . \mathrm{V} .57$ (J. R. Vockeroth), in CNC; Swain Co., "Smokies," Andrews Bald, 2 m., 2 f., 9.VII.41 (A. L. Melander), in USNM.

\section{Phytobia sp. (Salix)}

(Fig. 483)

A single female has been seen from New York, Fulton County, Broadalbin, 28.IV.67 (L. L. Pechuman), in CUI, collected on Salix sp. It is reasonable to assume that Salix is the host (on which P. cambii Hende1, 1931, occurs commonly in Europe), but describing the species is not proposed until a male is available for examination of the genitalia.

This female appears to be distinguished from $P$. setosa by the deep black antennal segments and possibly by the slightly higher epistoma. The frons also is uniformly black. However, more material definitely associated with Salix will be necessary before this species can be properly diagnosed.

Males have been examined from New York (USNM) and Wisconsin (CUI), in which the distal tubules are shorter and more curving than in $\underline{P}$. setosa and the basal sclerite is shorter (fig. 483). Possibly these specimens represent the Salix feeder, but the aedeagus differs in detail between the three specimens seen, and their status requires further clarification.

\section{GENUS AMAUROMYZA HENDEL}

Subgenus Cephalomyza Hende1, 1931: 32. Type of subgenus: Dizygomyza

luteiceps Hendel, 1920, from Germany. Subgenus Trilobomyza Hende1, 1931: 71. Type of subgenus: Agromyza flavifrons Meigen, 1830, from Germany.

In the original concept of Hende1 (1931: 59), Amauromyza was a sma11, simple subgenus of Dizygomyza; the most important single character was the black halter. No complete revision of the genus has been undertaken subsequently, but several partial revisionary publications have appeared and the male genitalia of many species have been illustrated.

In its currently accepted sense, Amauromyza comprises an assemblage with diverse external characters. The color may be entirely black, or the frons may be yellow, with the third antennal segment yellow or black, the notopleuron dark or yellow, the halter black, yellow, white, or variegated (A. maculosa), and the presutural dorsocentral strongly developed or lacking. However, a constant character in the male genitalia is the large sperm pump, with a conspicuously enlarged, bow1-shaped base (figs. 509, 514).

There has been some confusion about the identity of the type of the genus Agromyza lamii Kaltenbach, 1858. This was briefly described by Kaltenbach from specimens reared in Germany from Ballota and Lamium (see Spencer and 
Stegmaier, 1973: 93). We specifica11y referred to the species as shining black and the halter as yellow.

Hendel interpreted A. $\underline{\text { lamii }}$ as a shining black species with the halter also black. Three species are known in Europe fitting this interpretation-A. morionella (Zetterstedt, 1848), which is common on Ballota; A. leonuri Spencer, 1971a, known only on Leonurus; and the third, treated as A. 1amii, feeding on Lamium and Betonica (Nowakowski, 1962: fig. 10, Pol and). These three species can be satisfactorily separated only by the male genitalia.

A second species is common in Europe feeding on Lamium and Ballota and also five other genera of the Lamiaceae, described as Dizygomyza (Trilobomyza) labiatarum Hendel, 1931. This is a paler species, with the halter yellow. On the basis of Kaltenbach's description, Spencer and Stegmaier (1973: 93) tentatively interpreted A. lamii as this species.

It seems possible or even probable that Kaltenbach had reared both this paler species and also one of the entirely black species as his description referred to characters of each. However, in the interest of nomenclatura1 stability it is now proposed that Hendel's interpretation of A. lamii should be followed and A. lamii (with the halter black) accepted as type of the genus.

Genitalia studies have revealed that two subgenera that Hendel (1931) placed in his composite genus Dizygomyza--Cephalomyza and Trilobomyza-correctly belong in Amauromyza (Spencer, 1971a, 1971b). Campanulomyza Nowakowski, 1962, was also synonymized with Amauromyza, and, with existing names available, three subgenera--Amauromyza, Cephalomyza, and Trilobomyza--were recognized by Spencer (1976a: 156). An additional European genus, described as Phytobia (Melanophytobia) Hering, 1960, is correctly synonymous with Amauromyza, but whether or not it deserves sepa- rate subgeneric status has not yet been established. Two new subgenera-Catalpomyza Spencer, 1977c, and Annimyzella Spencer, 1981--are also now accepted.

Further study of the species included in the subgenus Trilobomyza, with $\underline{T}$. flavifrons now recorded here as present in the United States, shows that there is no justification for treating this as distinct from Cephalomyza, and this new synonymy is formally established here.

Two new species are described, two new combinations established, and two other species are briefly discussed here. One species is diagnosed but not formally described.

\section{Amauromyza (Cephalomyza) abnormalis (Ma11och) \\ (Figs. 489, 490)}

Agromyza abnormalis Ma1loch, 1913a: 320. Holotype female from District of Columbia in USNM.

Phytobia (Amauromyza) abnormalis, Frick, 1959: 378 .

Amauromyza abnorma1is, Spencer, 1969a: 158.

Amauromyza (Cephalomyza) abnormalis, Spencer, 1981: 148 .

The only male that can be referred to this species in America is one from Riverside County, Calif., and the aedeagus is shown in figure 489. The only other male known in North America is from Quebec. Its genitalia are shown in figure 490. These two specimens clearly seem to represent distinct species, but it is impossible at this stage to decide which of the two represents the true $A$. abnormalis. It is hoped, therefore, that other males will become available from the type area and can be associated with the female holotype; then the status of the two known males can be clarified. 
Amauromyza (Cephalomyza) albidoha1terata (Ma11och), new combination (Figs. 532, 533)

Agromyza albidohalterata Malloch, 1916: 52 . Holotype male from Illinois in IINR. Phytobia (Cephalomyza) albidohalterata, Frick, 1953a: 69.

This is a typical Cephalomyza and therefore correctly belongs in the genus Amauromyza, to which it is now transferred.

The unique male holotype is from St. Joseph, Champaign County, Ill. There is another male in the USNM from White Heath, I11., and the aedeagus of this specimen is shown in figures 532 , 533. The genitalia indicate close relationship with A. remus (figs. 524, 525) and A. romulus (figs. 535, 536), both from California, but there are significant differences between these species.

Amauromyza (Cephalomyza) auriceps (Melander), new combination (Figs. 518, 519)

Agromyza auriceps Melander, 1913: 262. Lectotype female from Idaho, designated by Frick, 1957b: 202, in USNM.

Phytobia (Cephalomyza) auriceps, Frick, 1957b: 202 .

With the inclusion of the subgenus Cephalomyza in Amauromyza, this species is now formally transferred to Amauromyza.

A. auriceps closely resembles A. scleritica, known only from

California, but it is distinguishable by the larger discal cell and the different male genitalia (figs. 518, 519 and figs. 516, 517).

Melander included in the type series five males and six females from Idaho and one female from Colorado. Eight other specimens have now been seen from Colorado, Boulder County, 4 miles northwest of Boulder, 6,900 feet, two males, six females, 17.VI.61 (B. H. Poole), in CNC.

Amauromyza (Cephalomyza) confondata Spencer, new species (Figs. 526, 527)

Head. Frons strongly projecting above eye (head shrunk, frons/eye ratio not measurable); 1 ors, 4 ori, orbital setulae minute, sparse, virtually lacking; gena deeply extended at rear, slightly over 0.5 height of small eye; $3 d$ antennal segment small, partia1ly enclosed by projecting frons, arista distinctly swollen basally; broad epistoma present.

Mesonotum. 3 developed postsutural dc, 2 presuturals little shorter than 3d, acr lacking.

Wing. Length in male $1.4 \mathrm{~mm}$, last section of $M 3+4$ twice length of penultimate.

Color. Frons and gena pale brownish, antenna, face, and palpus black; mesonotum matt black; scutellum, pleura, and legs black, notopleuron faintly brownish; squama whitish gray, margin faintly brownish, fringe pale; halter white.

Male genitalia. Aedeagus as in figures 526,527 , illustrated as $\underline{A}$. (C.) anomala (Spencer, 1981: figs. 21 2, 213); sperm pump with large, almost triangular blade, slightly longer than aedeagus, base large.

Host/early stages. Unknown.

Holotype m., California, San Bernardino Co., San Bernardino Mts., Kramer Hills, 25.IV.57 (G. I. Stage), presented by UCB to CAS.

Remarks. This species was originally tentatively associated with $\underline{A}$. anomala Spencer (1981: 150) following hasty examination after completion of the description. It is now clear that it is distinct and is related to $A$. romulus and $\underline{A}$. remus, which occur at 
the same locality in southern

California.

\section{Amauromyza (Cephalomyza) flavifrons (Meigen) \\ (Figs. 528-531)}

Agromyza flavifrons Meigen, 1830 : 184. Holotype female from Germany in MNHNP.

Dizygomyza ( $\operatorname{Trilobomyza)~flavifrons,~}$ Hende 1, 1931: 71 .

Trilobomyza flavifrons, Spencer, 1969a: 160; Wheeler and Henry, 1981: 197.

The generic position of this distinctive species has not been entirely clear. Hendel's subgenus Trilobomyza was raised to full generic status by Spencer (1969a), but Trilobomyza was later treated as a subgenus of Amauromyza (Spencer, 1971a, 1971b).

A. flavifrons was first recorded in North America (Canada) by Spencer (1969a). Wheeler and Henry (1981) recorded the species from southeastern Pennsylvania. Three new records are given here for the United States. This species occurs commonly on cultivated plants such as Dianthus and Saponaria, and possibly it has been introduced into North America relatively recently.

Its host range is unusual, as in addition to the Caryophyllaceae it feeds commonly in Europe also on Beta vulgaris (Chenopodiaceae).

New records.

Delaware, New Castle Co., Newark, Beneficial Insects Research Laboratory grounds, 1 reared from Saponaria officinalis, 7.VII.79 (R. M. Hendrickson).

Ohio, Portage Co., Kent, $4 \mathrm{~m} ., 6 \mathrm{f}$., ex leaf mines on Lychnis alba, 10.VI.69 (s. Whitney), in USNM.

Wisconsin, Dane Co., Madison, leaf mines on Lychnis alba, 25.VI.75 (S. Tavormina).
Amauromyza (Cephalomyza) knowltoni

Spencer, new species

(Figs. 522, 523)

Head. Frons 1.5 times width of eye, conspicuously projecting above eye in profile; 4 slender orbital bristles (upper ors missing on both sides), orbital setulae lacking; ocellar triangle not developed beyond foremost ocellus; gena broad, extended at rear, 0.5 height of eye; $3 d$ antennal segment small, round, arista short, bare.

Mesonotum. 3 strong postsutural dc, up to 3 smaller anterior ones but these scarcely longer than acr, these irregular, in about 3 rows.

Wing. Length in male $2.1 \mathrm{~mm}, \mathrm{C}$ extending strongly to $M 1+2$, last section of $M 3+4$ longer than penultimate, in ratio $11: 8$, inner crossvein distinctly before center of discal cell.

Color. Frons, gena, face, palpus, and antenna yellowish orange; upper orbit blackish to near level of lower ors; mesonotum and scutellum matt gray; humerus and pleura blackish gray; legs black but femora with knees faintly yellowish; squama and fringe yellowish; halter whitish yellow.

Male genitalia. Aedeagus (figs. 522, 523) with curving area of spinulose membrane below distal end of distiphallus and separate spinose membrane below black base of distiphallus; sperm pump with blade elongate, only slightly widening distally.

Host/early stages. Unknown.

Holotype m., Utah, Utah Co., American Fork Canyon, 21.VII.60 (G. F. Knowlton), in UCD.

Remarks. The male genitalia indicate that this species is closely related to A. auriceps, but the two are immediately distinguishable by the differing color of the antennae. 
Amauromyza (Cephalomyza) sp. (Colorado)

A single caught female from Colorado, Clear Creek County, Mt. Evans, timberline, 11,700 feet, 22.VII.61 (B. H. Poole), in CNC, clearly represents an undescribed species. A brief diagnosis is given in the key at couplet 9 , but the formal description is best delayed until a male becomes available.

\section{GENUS CERODONTHA RONDANI}

Ten new species are described, 3 each in the subgenera Poemyza and Dizygomyza and 4 in Butomomyza. One new synonym is established and two species are recorded as new to the United States. The status of five species is discussed and further clarified.

Cerodontha (Xenophytomyza) illinoensis (Malloch)

(Figs. 553, 554)

Agromyza illinoensis Malloch, 1934: 483. Holotype female (neotype) and neallotype male from Illinois in INHS.

Cerodontha (Xenophytomyza) illinoensis, Frick, 1952c: 150 .

Cerodontha (Poemyza) simcoensis Spencer, 1969a: 135. Holotype male from Ontario in CNC. NEW SYNONYM.

Malloch (1934: 483) briefly referred to a North American species, Agromyza illinoensis Malloch, commenting on its black color and adding "the third antennal segment is angulate but not spinose at the upper apical angle." Frick (1952c) accepted this as a valid description, giving a detailed redescription of the species and correctly placing it in the subgenus Xenophytomyza. Spencer (1969a) overlooked the presence of only a single pair of scutellar bristles and described Cerodontha (Poemyza) simcoensis from Ontario, but comparison of this with the holotype and neallotype of $\underline{\mathrm{C}}$. illinoensis shows the two to be identical, and $\mathrm{C}$. simcoensis is synonymized with $\underline{\text { C. }}$ illinoensis here. The male genitalia show that among the three European species, $\underline{\text { C. }}$ ililinoensis is most closely rélated to $\underline{C}$. atronitens (Hende1, 1920).

\section{Cerodontha (Poemyza) attenuata}

Spencer, new species

(Figs. 568, 569)

Head. Frons about 1.5 times width of eye, not projecting above eye in profile; 2 ors, 2 ori, orbital setulae sparse, reclinate; orbit conspicuously widening toward midpoint of lunule, this high, upper margin at level of lower ors; gena narrow, at rear only 0.1 height of eye; $3 \mathrm{~d}$ antennal segment small, round; arista long, almost 1.5 times width of eye.

Mesonotum. 3 postsutural dc, presutural apparently lacking, acr in about 6 rows, no prsc.

Wing. Length in male $2.2 \mathrm{~mm}, \mathrm{C}$ extending strongly to $M 1+2$, last and penultimate sections of $M 3+4$ equal, discal cell thus large.

Color. Frons matt black, orbit faintly paler along inner margin; lunule grayish; $3 \mathrm{~d}$ and 1 st antennal segments black, 2d distinctly yellowish; mesonotum largely matt black, only weakly shining; pleura black, apart from narrow yellow upper margin of mesopleuron; legs black, apart from knees on all femora, which are narrowly yellow; squama, including margin and fringe, also wing base, yellow; halter white.

Male genitalia. Aedeagus (fig. 568) with distal tubules membranous at end; basal and also ventral sclerites fully fused (fig. 569); sperm pump with large, lightly pigmented blade, wider than high; anal projection prominent.

Host/early stages. Unknown.

Holotype m., South Carolina, Oconee Co., Mountain Rest, 1,500 ft, 26.VII.57; paratype m., Tennessee, Transylvania Co., Looking Glass Rock, 
near Pisgah Forest, 2,500 ft, 19.VII.57; (both J. G. Chillcott), in CNC.

Remarks. This species closely resembles $\mathrm{C}$. ( $\underline{P}_{.}$) inconspicua, but it is distinguishable by its paler second antennal segment, darker $M$ veins, and two sections of vein $M 3+4$ being equal. The male genitalia confirm the differentiation of the two species.

\section{Cerodontha (Poemyza) chillcottiella}

Spencer, new species

(Figs. 555, 556)

Head. Frons twice width of eye, distinctly projecting above eye in profile; orbit broad, strongly differentiated; 2 widely spaced ors, 2 ori, orbital setulae sparse, reclinate; lunule (deformed) apparently higher than a semicircle; gena angular, broad, only slightly less than 0.5 height of eye; $3 \mathrm{~d}$ antennal segment (fig. 555) angulate.

Mesonotum. $3+1$ weak dc, acr sparse, in $2-3$ rows.

Wing (fig. 556). Length in female 1.9 $\mathrm{mm}, \mathrm{C}$ extending to $\mathrm{M} 1+2$, outer crossvein lacking.

Color. Frons ochreous above, more grayish on lower half, orbit and entire hindmargin of eye black; gena ochreous, parafacial black; al1 antennal segments black; mesonotum and scutellum grayish black, largely matt, with only weak subshine; notopleuron bright yellow, side of thorax otherwise black; legs black but knees bright yellow; abdomen black, weakly shining, all tergites narrowly yellow bordered; squama yellowish gray, margin and fringe black; ovipositor sheath moderately shining but with distinct gray dusting.

Male genitalia. Unknown.

Host/early stages. Unknown.

Holotype m., Colorado, Boulder Co., Corona Pass, 10,600 ft, $1 \mathrm{f.,} \mathrm{6.VII.61}$ (J. G. Chill cott), in CNC.
Remarks. In the absence of males the generic position of this most distinctive species is not entirely clear. It is tentatively assigned to Cerodontha, subgenus Poemyza, although the lunule more closely resembles that of Butomomyza species (fig. 540, B). Characters associating the species with Poemyza are angulate third antennal segment, broad orbit, yellow notopleuron, and small size. The species is unique in Cerodontha in lacking the outer crossvein. This is possibly teratological but more probably represents a specific character.

There will be no difficulty in associating other specimens with the unique female holotype, and it is hoped that a male will be obtained to further clarify this interesting species.

\section{Cerodontha (Poemyza) inconspicua (Malloch) \\ (Fig. 570)}

Agromyza inconspicua Malloch, 1913a: 310. Holotype male from Colorado in USNM.

Phytobia (Poemyza) inconspicua, Frick, 1959: 381.

Cerodontha (Poemyza) inconspicua, Spencer, 1969a: 129; Nowakowski, 1973: 93.

This species was described from a single male, but the material now seen shows it to be widespread, with records from New York to Utah and California.

Spencer (1969a) recorded C. inconspicua as widespread in Canada, and the aedeagus of a specimen from Alberta was illustrated (Spencer, 1969a: fig. 227). This identification was accepted by Nowakowski (1973), who recorded the species in Poland and Finland. The illustration of the aedeagus given by Nowakowski (1973: fig. 127) agrees closely with that of the specimen from Canada, and it can be accepted that the same species is involved. However, the aedeagus of 
specimens now seen from California, Colorado (fig. 570), New York, and Utah is identical but differs considerably from that previously accepted as $\underline{\mathrm{C}}$. ( $\underline{\mathrm{P}}_{\text {. ) }}$ inconspicua by Nowakowski and Spencer. In his monograph of this genus, Nowakowski treats $\underline{C}$. inconspicua as one of five species under the C. (P.) atra superspecies. Differences are slight, but if this splitting is accepted, then it seems probable that the Canadian and European form represents another undescribed species in this complex.

Cerodontha (Poemyza) macminni Spencer, new species

(Figs. 565, 566)

Head. Frons almost 1.5 times width of eye, not projecting above eye in profile; 2 ors, 2 ori; orbit considerably widening below level of lower ors; lunule high, narrow, upper margin at level of lower ors; gena narrow, at rear only 0.1 height of eye; $3 \mathrm{~d}$ antennal segment small, round, arista long, only slightly pubescent, longer than wide, only slightly shorter than height of eye.

Mesonotum. $3+1 \mathrm{dc}$ or presutural lacking, acr in some 6 rows, prsc undeveloped.

Wing. Length $2.3-2.5 \mathrm{~mm}, \mathrm{C}$ extending strongly to $M 1+2$, last section of M 3+4 almost 1.5 times length of penultimate.

Color. Frons, antenna, and palpus black, lunule faintly grayish; orbit either uniformly black or narrowly yellowish along inner margin; mesonotum black, only weakly shining; upper margin and hindcorner of mesopleuron bright yellow, pleura otherwise black; legs with femora black but bright yellow distally for slightly more than femoral width, tibiae narrowly yellow adjoining femora, otherwise black, tarsi paler, yellowish brown; abdomen uniformly black, moderately shining; squama, including margin and fringe, yellow; wing with all veins moderately dark, brown.

Male genitalia. Aedeagus (fig. 565) with distal tubules not diverging, with uniform ventral curvature and distinct dilation distally; sperm pump with small blade, only lightly pigmented.

Host/early stages. Carex crus-corvi. Larvae forming long, brownish blotch, up to $35 \mathrm{~cm}$ in length, with from 4 to 16 larvae in single mine; pupation internal, with black puparia lying horizontal in leaf, completely unattached, posterior spiracles on common elongate extension, each with 3 bulbs, lower hooklike (fig. 566).

Holotype m., Mississippi, Washington Co., Experimental Forest, Leland, emerged 25.V.79 from puparia coll. V.11 from Carex crus-corvi growing in water-filled ditch beside road; paratypes $5 \mathrm{~m}$., $11 \mathrm{f}$., emerged 25-28.V.79, otherwise same data (G. McMinn). Holotype and paratypes in USNM, 4 paratypes in KAS.

Remarks. This species is unique in being the only known Poemyza to feed on Carex. Externally it generally resembles $\underline{C}$. phragmitidis Nowakowski, a leaf miner on Phragmites in Europe, particularly in the form of the aedeagus (see Nowakowski, 1973: fig. 122). The elongate extension of the puparium is also as in C. phragmitidis, but the arrangement of the posterior spiracles is different, with the two processes diverging from the common base and with the lower of the three bulbs conspicuously hooklike (fig. 566). In this character it closely resembles C. alpina Nowakowski (see Nowakowski, 1973: fig. 230), belonging to Nowakowski's muscina group. The jump in host from Poaceae to Cyperaceae, which has occurred in this species, has thus been accompanied by evolutionary changes in the larva, including the feeding habit, and it therefore cannot be accurately included in any of the special groups into which Nowakowski divided this subgenus. 
We take pleasure in naming this interesting species after Greg McMinn from the Southern Weeds Infestation Laboratory, Leland, Miss., who accompanied us to the Experimental Forest and discovered the mines.

Cerodontha (Poemyza) pygmaea (Meigen) (new to U.S.A.)

(Fig. 562)

Agromyza pygmaea Meigen, 1830: 183. Holotype from Germany 1ost. Cerodontha (Poemyza) pygmaea, Spencer, 1969a: 133; Nowakowski, 1973: 127.

This species can now be confirmed in the United States from New York, Ithaca, two males, one female, 21.V.67 (R. A. Dunn), in CUI. It is common in Ontario and has been recorded in Quebec, Labrador, and Alaska; it is widespread in Europe. $\underline{\text { C. pygmaea is a }}$ typical Poemyza with a narrow, high lunule (fig. 540, A), closely resembling $\underline{C}$. incisa and other species, but it is immediately distinguishable by the dark squamal fringe.

\section{Cerodontha (Poemyza) pygminoides}

\section{Spencer}

(Fig. 567)

Cerodontha (Poemyza) pygminoides

Spencer, 1981: 183. Holotype male

from Inyo Co., Calif., in UCD.

The unique holotype was slight1y damaged, lacking the third antennal segment and all orbital bristles. Another male has now been identified from Colorado, Boulder, Flagstaff Canyon, 5,800 feet, "on side of stream," $10 . \mathrm{VI} .61$ (C. H. Mann), in CNC. The arrangement of orbital bristles is normal, with two ors and two ori, and the third antennal segment is black, small, obtusely angulate, with the arista slightly pubescent and as long as the eye width.

Cerodontha (Butomomyza) angulata (Loew) (Figs. 583-588)

Agromyza angulata Loew, 1869: 47.
Lectotype female from Pennsylvania, designated by Frick, 1957b: 202, in MCZ.

Phytobia (Poemyza) angulata, Frick, 1957b: 202 .

Cerodontha (Dizygomyza) angulata, Spencer, 1969a: 113.

Cerodontha (Butomomyza) angulata, Nowakowski, 1973: 152.

Cerodontha (Butomomyza) neptis (Loew, 1869: 50). Lectotype female, from District of Columbia, designated by Frick, 1957b: 202, in MCZ.

Cerodontha (Butomomyza) semiposticata (Hende1, 1920: 131). Lectotype female from Germany, designated by Nowakowski, 1967: 634.

In his monograph of this genus, Nowakowski (1973) includes three closely related species from Europe in the $\underline{c}$. angulata group. The male genitalia of eight specimens have now been examined from North Carolina, and it seems clear that several species close to $C$. angulata are also present in the United States. The aedeagus of one such specimen from North Carolina, Swain County, Indian Gap, Great Smoky Mountains National Park, 5, 200 feet, 3.VII.57 (C. D. Hines), in CNC, is shown in figures 585, 586. Another specimen from North Carolina is described here as $\underline{C}$. ( n. sp., but furthe $\bar{r}$ splitting is considered undesirable in the absence of information on host plants and early stages. Most, if not a11, of these species will prove to be leaf miners on Carex, but surprisingly not a single specimen has so far been reared in this group in North America.

A male syntype, which can be associated with the female lectotype, although it was not mentioned in the original description, has now been examined, and the aedeagus and epandrium are shown in figures 583, 584.

Cerodontha (Butomomyza) fusculata

Spencer, new species

(Figs. 577, 578)

Head. Frons narrow, 1ittle wider than eye, not projecting above eye in 
profile; 2 ors, 2 ori, orbital setulae well developed, reclinate; lunule extending to level of upper ori; gena narrow but slightly extended at rear, 0.1 height of eye; 3d antennal segment small, round, slightly pubescent, arista long, little shorter than height of eye.

Mesonotum. $3+1$ strong dc, prsc well developed, acr irregularly in 5 rows.

Wing. Length in male $2.3 \mathrm{~mm}, 1$ ast section of $M 3+4$ slightly shorter than penultimate, in ratio $6: 7$, inner crossvein at midpoint of discal cel1.

Color. Head black but lunule gray; mesonotum grayish black, weakly shining; pleura black, only hindcorner of mesopleuron yellow; legs black apart from yellow knee on foreleg; squama yellowish gray, margin and fringe deep black.

Male genitalia. Aedeagus (fig. 577) with distal tubules vertical to mesophallus, only slightly widening and curving at apex; sperm pump large, fan1ike; epandrium (fig. 578) with conspicuously strong group of bristles at hindcorner and very long hairs along outer margin.

Host/early stages. Unknown.

Holotype m., North Carolina, Transy1vania Co., Looking Glass Rock, near Pisgah Forest, 2,500 ft, 30.VII.57 ( J. G. (hillott), in CNC.

Remarks. This species is distinguishable from $C$. angulata by the dark squamal fringe, and both aedeagus and epandrium (see figs. 583, 584) are also distinctively different. It may be reliably distinguishable from $\underline{C}$. scirpi only by the genitalia.

\section{Cerodontha (Butomomyza) impercepta}

Spencer, new species

(Figs. 581, 582)

Head. Frons broad, twice width of eye, not projecting above eye in profile; 2 ors, 2 ori, orbital setulae sparse, reclinate; orbit pronounced, conspicuously widening toward base of antennae; lunule narrow but high, upper margin at level of upper ori; gena extended at rear, 0.17 height of eye; 3d antennal segment small, round, without noticeable pubescence, arista distinctly pubescent.

Mesonotum. $3+1 \mathrm{dc}$, presutural shorter than $3 d$, acr in some 6 rows, prsc strong, equal to $3 \mathrm{~d} d c$.

Wing. Length in male $2.3 \mathrm{~mm}, C$ extending to $M 1+2$, last section of $M 3+4$ slightly longer than penultimate, inner crossvein slightly beyond center of discal cell.

Color. Frons and orbit black, 3d antennal segment black, 2d slightly paler, faintly yellowish; mesonotum grayish black, on $1 y$ weakly shining; pleura black, only upper margin of mesopleuron narrowly yellow; legs with femora black but knees bright yellow on foreleg and midleg, scarcely paler on hindleg, tibiae brownish, tarsi yellowish; wing with $R$ veins dark, $M$ veins pale, $M 3+4$ almost colorless; squama, fringe, and wing base bright ye11ow.

Male genitalia. Aedeagus as in figures 581, 582, distal tubules pale at end, only slightly diverging; sperm pump elongate, with narrow stalk and blade not greatly widening.

Host/ear1y stages. Unknown.

Holotype m., North Carolina, Macon Co., Highlands, 3.VI.57 (J. R. Vockeroth), in CNC.

Remarks. Although generally resembling other species with the squamal fringe pale, $\underline{C}$. ( $\left.\underline{B}_{.}\right)$ impercepta is clearly distinguishable by the flat curve of the distiphallus (fig. 581). Positive identification may only be possible from the male genitalia. 
Cerodontha (Butomomyza) impolita

Spencer, new species

(Figs. 579, 580)

Head. Frons broad, almost twice width of eye, not projecting above eye in profile but finely setulose above lunule; 2 ors, 2 ori, orbital setulae sparse, reclinate; orbit not significantly widening anteriorly; gena broad, extended at rear, there almost 0.25 height of eye; $3 \mathrm{~d}$ antennal segment rounded, longer than broad, with only short pubescence, arista appearing bare.

Mesonotum. $3+1$ strong dc, presutural only slightly shorter than $3 d$, acr sparse, in only 4 rows, prsc weak, shorter than presutural dc.

Wing. Length in male $2.1 \mathrm{~mm}, C$ extending to $M 1+2,1$ ast and penultimate sections of $M 3+4$ equal, inner crossvein at midpoint of discal cell.

Color. Frons, orbit, and antenna black; mesonotum matt gray; pleura black, with only upper margin of mesopleuron yellow; legs largely black, only foreknee narrowly yellow; squama, fringe, and wing base bright yellow.

Male genitalia. Aedeagus (fig. 579) S-shaped, distal tubules short, only slightly diverging; sperm pump with thick stalk, blade only slightly widening ( $\mathrm{fig}$. 580).

Host/early stages. Unknown.

Holotype m., Colorado, Boulder Co., Longmont, 5,000 ft, 3.VI.61 (B. H. Poole); paratype m., North Carolina, Yancey Co., Mills Creek, 2, $100 \mathrm{ft}$, 14.VIII.57 (J. G. Chillcott); both in CNC.

Remarks. The relatively broad gena, bare arista, and matt gray mesonotum are distinctive characters of this species. The S-shaped distiphallus (fig. 579) is significantly different from that of the species it most closely resembles, C. impercepta (fig. 581).
Cerodontha (Butomomyza) parvella

Spencer, new species

(Fig. 574)

Head. Frons broad, twice width of eye, distinctly projecting above eye in area of ori (see fig. 572); 2 ors, 2 ori, orbital setulae sparse, reclinate; lunule broad, higher than a semicircle, characteristic of subgenus (fig. 540, B); gena extended at rear, about 0.25 height of eye; $3 \mathrm{~d}$ antennal segment small, longer than broad, rounded below, obtusely angulate at upper corner, arista appearing bare, short, swollen at base.

Mesonotum. $3+1$ strong dc, presutural equal to $3 \mathrm{~d}$, acr irregularly in 4 rows, prsc well developed.

Wing. Length in male $2 \mathrm{~mm}, \mathrm{C}$ extending strongly to $M 1+2$, last and penultimate sections of $M 3+4$ equal, inner crossvein at midpoint of discal cell.

Color. Frons, orbit, and antenna black; mesonotum almost entirely matt, gray; pleura grayish black, mesopleuron only narrowly yellow along upper margin; legs entirely black; squama and fringe yellow.

Male genitalia. Aedeagus (fig. 574) with paired distal tubules short, not diverging; sperm pump with broad stalk and blade but greatly widening, heavily pigmented; surstylus with 2 or more short bristles on inner corner.

Host/early stages. Unknown.

Holotype m., Colorado, Clear Creek Co., Mt. Evans, Echo Lake, 10,600 ft, 20.VII.61 (B. H. Poole), in CNC.

Remarks. This species is distinguishable by the small size, projecting frons, and gray mesonotum. The short distiphallus ( $\mathrm{fig}$. 574) is also distinctive. 
Cerodontha (Butomomyza) subangulata

(Malloch)

(Figs. 589-592)

Agromyza subangulata Ma1loch, 1916: 51. Holotype male from Illinois in IINR. Phytobia (Poemyza) subangulata, Frick, 1959: 382 .

Cerodontha (Dizygomyza) subangulata, Spencer, 1969a: 297.

Malloch described this species from a single male, differentiating it from $\underline{C}$. angulata primarily by a venational character, the outer crossvein being only its own distance from the inner. Frick (1959) used this character, also considering the femora on the midleg and hindleg to be dark in $\underline{C}$. subangulata and yellow in C. angulata. However, on external characters, differentiation of $\underline{c}$. subangulata and others in the angulata group may be difficult, but the male genitalia of c. subangulata are distinctive (figs. 589,590 ), with the distal tubules largely fused and the curve shallow. The bristles on the lower corner of the epandrium are also arranged differently, being at the end of an inwardly curving process (fig. 591), not at the actual corner (contrast angulata, fig. 584).

The aedeagus (fig. 592) of a specimen from Maryland, Montgomery County, Cabin John Creek, 24.VIII.76 (KAS), somewhat resembles that of $\underline{C}$. ( B. $^{\text {) }}$ subangulata but possibly represents another undescribed species in this group.

\section{Cerodontha (Dizygomyza) aristosa}

Spencer, new species

(Figs. 605-607)

Head. Frons twice width of eye, only very slightly projecting above eye at base of antennae; orbit slightly widening toward lunule, with 2 ors, 2 ori, orbital setulae sparse, reclinate; lunule semicircular; gena broad, almost 0.25 height of eye; 3d antennal segment enlarged in male, round with fine pubescence, arista conspicuous $1 \mathrm{y}$ swollen in lower 3d (fig. 605).
Mesonotum. $3+1 \mathrm{dc}$, presutural weaker than 3d, acr short, sparse, in 6 rows in front, prsc lacking.

Wing. Length in male $2.5 \mathrm{~mm}$, last section of $M 3+4$ distinctly shorter than penultimate, in ratio $5: 8$, inner crossvein slightly before midpoint of discal cell.

Color. Frons brownish black, orbit darker, blackish, weakly shining; lunule brownish, slightly grayish seen from front; antenna black; mesonotum matt grayish black; pleura entirely black, even upper margin of mesopleuron not paler; legs almost entirely black, only foreknee narrowly yellowish; abdomen black; squama and fringe yellow.

Male genitalia. Aedeagus (fig. 606) with upper curve of distal tubules large, more than a semicircle, distal end not divided, directed ventrally; sperm pump with large blade, fanlike; surstylus with row of 5 or 6 short spines; anal projection prominent (fig. 607).

Host/early stages. Unknown.

Holotype m., North Carolina, Haywood Co., Pisgah National Forest, Chestnut Bald, 5,900 ft, 2.VIII.57 (J. G. (hillcott), in CNC.

Remarks. The male genitalia suggest close relationship with c. ultima Spencer, 1969a, described from Ontario, but the two species differ in a number of morphological characters.

Cerodontha (Dizygomyza) fasciata

(Strob1) (new to U.S.A.)

(Fig. 610)

Phyllomyza fasciata Strobl, 1880: 38. Lectotype male from Austria in coll. Strobl, Admont, Austria.

Cerodontha (Dizygomyza) fasciata, Spencer, 1971a: 153 (1ectotype designation); 1976a: 220 .

This species was misidentified in Alberta and Ontario as C. (D.) 
chaixiana (Groschke) by Spencer (1969a: 115). This misidentification resulted from Groschke having reared two separate species from the same host, Poa chaixii Villars, and at the same locality in southern Germany. The specimens of $\mathrm{C}$. chaixiana available at the time in fact represented $c$. fasciata. This confusion in Groschke's type series of $\mathrm{C}$. chaixiana was discussed and clarified by spencer (1978).

A single male of $\mathrm{C}$. fasciata has now been seen from North Carolina, Mitchell County, Roan Mountain, 6,200 feet, 13.VIII.57 (J. G. Chillcott), in CNC. The species is distinctive in having yellowish orbits, and the thickened end of the distal tubules of the aedeagus is also characteristic (fig. 610).

Nowakowski (1973: 244) considered that C. fasciata, C. grisea Rydén from Sweden, and the nearctic species here treated as $\underline{\mathrm{C}}$. fasciata might represent three sibling species. However, the differences are so slight that we consider such splitting unjustified without more substantial biological evidence and therefore treat the populations in Canada and America as identical with the European $\underline{C}$. fasciata.

\section{Cerodontha (Dizygomyza) 1atifrons}

Spencer, new species

(Fig. 596)

Head. Frons exceptionally broad, 3 times width of eye, not projecting above eye in profile; orbit broad, strongly differentiated, with 2 ors and 3 ori, all equal, orbital setulae sparse, reclinate; gena extended at rear, 0.2 height of eye; 3d antennal segment greatly enlarged in male, slightly longer than broad, thickly covered with pubescence as long as basal width of arista, this bare, only slightly widening basally.

Mesonotum. $3+1 \mathrm{dc}$, presutural strong, equal to $3 d$, acr sparse, in 6 rows in front, 4 behind, prsc strong, equal to $3 \mathrm{~d} \mathrm{dc}$.
Wing. Length in male $3 \mathrm{~mm}$, last section of $M 3+4$ only slightly longer than penultimate, inner crossvein at midpoint of discal cell.

Color. Frons dark brown, orbit contrastingly yellow; lunule brownish, more silvery below; antenna black; mesonotum matt gray; pleura dark apart from narrow yellow upper margin of mesopleuron; legs black, knees bright yellow, slightly darker on p 3; abdomen slightly yellow laterally, all tergites narrowly yellow on hindmargin; squama and fringe, also wing base, yellow.

Male genitalia. Aedeagus (fig. 596) with proximal and distal curves equal, distal tubules diverging at angle of $90^{\circ}$, with slight gap at distal end; sperm pump fanlike, unusually broad.

Host/early stages. Unknown.

Holotype m., North Carolina, Sevier Co., C1ingman's Dome, Great Smoky Mountains National Park, 6.VIII.57 (C. J. Durden), in CNC.

Remarks. This species is immediately distinguishable from $\underline{C}$. poolei, n. sp., from Colorado, with the frons even broader and by the conspicuously yellow knees on all legs. The male genitalia (figs. 596, 604) indicate that the two species are not closely related.

Cerodontha (Dizygomyza) magnicornis (Loew)

(Fig. 595)

Agromyza magnicornis Loew, 1869: 46. Holotype male from Pennsylvania in MCZ .

Phytobia (Dizygomyza) magnicornis, Frick, 1952a: 396; 1959: 384. Cerodontha (Dizygomyza) magnicornis, Nowakowski, 1973: 222.

This species was previously synonymized with C. morosa Meigen (Spencer, 1969a: 121) before the correct identity of $C$. morosa was known. Nowakowski (1973) pointed out that the two species are distinct. 
Among nearctic species, $\underline{c}$. magnicornis is recognizable by the yellow notopleuron. The holotype has been reexamined, and it is noted that the third antennal segment is missing on both sides, but the aedeagus is extruded and its identity is not in doubt. A new illustration of the aedeagus is given in figure 595. The species is not common and two new records are given here.

\section{Cerodontha (Dizygomyza) poolei Spencer, new species \\ (Figs. 603, 604)}

Head (fig. 603). Frons conspicuously projecting above eye below lower ors, exceptionally broad, 4 times width of eye; orbit broad, distinctly widening toward lunule; 2 ors, 3 ori (occasionally 4), orbital setulae sparse, somewhat irregular but predominantly reclinate; lunule semicircular; gena broad, extended at rear, 0.25 height of eye; 3d antennal segment enlarged in male, only finely pubescent, more elongate in female, arista fine, bare in upper half, conspicuously widening below.

Mesonotum. $3+1$ strong dc, presutural little weaker than $3 d$, acr sparse, in approx. 4 rows, but coarse, particularly in presutural area, prsc und eveloped.

Wing. Length from $3.2 \mathrm{~mm}$ in male to $3.4 \mathrm{~mm}$ in female, last section of M $3+4$ shorter than penultimate, in ratio $6: 7$, inner crossvein slightly oblique, just beyond center of discal cell.

Color. Frons brownish, normally dark, occasionally paler; orbit and antenna black; mesonotum matt blackish, only slightly grayish; pleura black, only mesopleuron narrowly yellow on upper margin; legs almost entirely black, only foreknee narrowly yellow; abdomen shining black, with all tergites narrowly yellow bordered; squama and fringe yellow.
Male genitalia. Aedeagus (fig. 604) with distinctive curvature.

Host/early stages. Unknown.

Holotype m., Colorado, Clear Creek Co., Mt. Evans, 12,000 ft, 3.VIII.61 (B. H. Poole); paratypes 1 f., same data; Mt. Evans, Timberline, 11,700 ft, 3 m., 2 f., 22.VII.61 (B. H. Poole); Boulder Co., Hoosier Pass, $12,000 \mathrm{ft}, 1 \mathrm{~m} ., 8$. VIII.61 (C. H. Mann). Holotype and paratypes in $\mathrm{CNC}$, 2 paratypes in KAS.

Remarks. The unusually broad frons and large size make this a distinctive species.

\section{GENUS LIRIOMYZA MIK}

Eleven new species are described, 3 new synonyms are established, and 5 species are recorded as new to the United States and 1 as new to North America. One probable new species from Colorado is briefly diagnosed but not formally described in the absence of males. Eight other species are discussed. The full synonymy is given of $\underline{\text { L }}$ sativae in view of its economic importance and the confusion caused in the past by the eight names that have been used for populations reared from different hosts at different

localities.

Liriomyza allia (Frost)

(Figs. 843, 844)

Agromyza allia Frost, 1943: 257. Holotype male from Kansas in USNM. Liriomyza allia, Frick, 1955: 90.

Following a heavy infestation of onions in Iowa in 1932, Frost described L. allia, surprisingly selecting as holotype a caught male from Kansas and including 27 as paratypes, of which 20 had been reared from onion. Frick (1955) noted that the holotype of L. allia was not identical with the onion leaf miner, which he described as a new species, L. allivora. The latter has proved to 
be identical with L. trifolii and the synonymy was estabis ished by Spencer (1973a: 226).

Examination of the holotype of L. allia has confirmed that it is distinct from L. trifolii (allivora), and the male genitalia are shown in figures 843,844 . This species--with a misleading name as the host is unknown and certainly not onion--is indistinguishable on external characters from L. fricki, a feeder on Fabaceae, but the genitalia of the two do not suggest that they are closely related.

Liriomyza arctii Spencer (new to

U.S.A.)

(Figs. 736-738)

Liriomyza arctii Spencer, 1969a: 167. Holotype male from Ontario in CNC.

This species is distinctive in having the frons and antennae bright yellow and the femora and mesopleuron predominantly black. The aedeagus is shown in figures $736,737$.

Leaf mines on Arctium minus are common in August and September in Minnesota, Minneapolis, Minnehaha Creek, and one male was reared 3.X.78 (KAS). Another male was reared $21 . I X .76$ at Madison, Wis. (s. Tavormina).

Liriomyza blechi Spencer

(Figs. 668-672)

Liriomyza blechi Spencer, in Spencer and Stegmaier, 1973: 98. Holotype male from Florida in USNM.

This species was described as a leaf miner on Blechum (Acanthaceae). The male genitalia (figs. 669, 670) and long anterior spiracles of the puparium (fig. 672) show that $\underline{L}$. blechi is closely related to L- marginalis (figs. 673-677), a leaf miner on grasses.

A leaf miner on Plantago has previously been $\overline{\text { recorded }}$ in the 1iterature (Coquillett, 1898: 77;
Ma11och, 1913a: 282; Frost, 1924: 47; al1 as $\underline{L}$. melampyga). Specimens ex Plantago in Florida were tentatively recorded as L. blechi (Spencer and

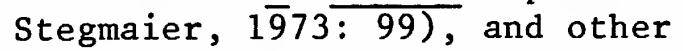
specimens from Mississippi (K. E. Frick) have now been examined. The single character that appears to differentiate $\underline{L}$. blechi is the long hair at the end of the cercus (fig. 668); this is also present in the male recorded here from Texas. It is lacking in specimens seen ex Plantago, but all other characters, including the color of the adults, details of the aedeagus and epandrium, and both anterior and posterior spiracles of the puparium, appear to agree exactly. In view of this, it is not considered desirable to treat the population on $\mathrm{Plantago}$ as distinct at this stage. Other specimens have been reared from Heliotropium (Boraginaceae), Lippia (Verbenaceae), and Spigelia (Loganiaceae), and in none of these is the long cercal hair present. A caught male from São Paulo, Brazil (in BMNH), appears to be identical to these specimens.

In all species in this group the entire terminalia are conspicuously pale, virtually without pigmentation. A further significant character is that the normal arrangement of the dorsocentral bristles is two strong postsutural dc and one equally strong presutural. However, in a few specimens an additional third postsutural is present.

The leaf mine on Blechum is an irregular blotch (fig. 671), normally but not exclusively associated with the midrib or one of the lateral veins. Insufficient material is available from Plantago and the other hosts to be certain whether this larval feeding habit applies here also.

It is barely possible that a second polyphagous species is present, distinguishable only by the cercal hair, but further detailed study is desirable before such splitting can be justified. 
Another species in this group, L. andina Malloch (see Spencer, 1963a: 353), is present in Chile, and the host was discovered to be Plantago during collecting in Chile by KAS in January 1978 .

New record.

Texas, San Patricio Co., Welder Wildlife Res., 1 m., 2 f., 19-23.III.65 (B. H. Poole), in CNC.

Liriomyza commelinae (Frost)

(Figs. 659-662)

Agromyza commelinae Frost, 1931: 72. Holotype male from St. Vincent in USNM.

Liriomyza commelinae, Frick, 1952a: 402; Silva and Oliveira, 1952: 293; Spencer, 1963a: 361; Spencer and Stegmaier, 1973: 101.

Adults of this species are unusual in having the third antennal segment greatly enlarged in the male (fig. 659). However, the general coloration is typical of Liriomyza, and the stridulating mechanism is developed normally.

The larva, puparium, and leaf mine are atypical of Liriomyza. The puparium is black, elongate, and remains in the mine after pupation. Each posterior spiracle (fig. 662) has one greatly elongated, hooklike "bulb" and two minute ones at its base, generally resembling the form found in many species of Butomomyza and Dizygomyza (see fig. 588). The frass is deposited in large widely spread lumps, whereas the normal practice in Liriomyza is deposition in slender strips at alternate sides of the mine.

It is suggested that this is a primitive annectant species between Liriomyza and Cerodontha, subgenus Dizygomyza, with the adult having developed normal characters of Liriomyza apart from the enlarged male third antennal segment, whereas primitive characters of the larva, including its feeding habit, have been retained, showing clearly the link with Dizygomyza. This is thus a unique species, with evolution in the adult and larva not having proceeded in parallel. Although the essential genetic structure of Liriomyza must be present in the larva, the larva remains dominated by primitive genes of the Dizygomyza type.

L. commelinae is essentially a neotropical species, occurring widely in suitable habitats from Argentina to Venezuela and the Caribbean and just reaches the United States in Florida.

Liriomyza deceptiva (Ma1loch) (Fig. 752)

Agromyza deceptiva Malloch, 1918a: 78. Holotype female from Illinois in INHS.

Frick (1959: 403) commented that this "is the most diverse North American species of Liriomyza known to me." This comment is justified, as, despite the characteristic color of Liriomyza, the shape of the head ( $\mathrm{fig} . \overline{752 \text { ) and }}$ the long, slender orbital bristles suggest that the species may in fact belong in Amauromyza. However, no formal generic change appears justified until the correct status of the species can be established from examining the male genitalia.

Liriomyza elevata Spencer, new species (Figs. 695, 696)

Head. Frons slightly but not significantly projecting above eye, little wider than eye in profile; 2 ors, 2 or $i$ in male, normally, 3 ori in female, orbital setulae sparse, reclinate; orbit well differentiated; gena 0.33 height of eye; 3d antennal segment small, round.

Mesonotum. Normally $3+1$ dc but occasionally additional ones in postsutural area and line of smaller ones beyond strong presutural, acr in 4-6 irregular rows, intra-alar lacking.

Wing. Length $1.6-2 \mathrm{~mm}$, discal cell 
variable, last section of $M 3+4$ from 1.67 to 2.5 times penultimate.

Color. Frons orange to brownish, orbit frequently paler, yellow; 3d antennal segment varying from orange to brown; palpus yellowish brown; mesonotum blackish gray, with only moderate subshine, scutellum dark at sides, always at least slightly yellowish centrally; humerus and notopleuron usually slightly yellowish, sometimes almost completely blackish gray, pleura otherwise dark; legs almost entirely black, apart from yellowish knee and foreleg, sometimes also on midleg; abdomen matt black; epandrium in male and ovipositor sheath in female brilliantly shining black; squama gray, margin and fringe dark; halter whitish yellow.

Male genitalia. Aedeagus (figs. 695, 696) with slightly sinuate paired tubules; sperm pump with large black blade; surstylus elongate, with single spine at end.

Host/early stages. Unknown.

Holotype m., Colorado, Clear Creek Co., Mt. Evans, Timberline, 11,700 ft, 22.VII.61 (W. R. Mason and B. H. Poole); paratypes $7 \mathrm{~m} ., 11 \mathrm{f}$., same data. Holotype and paratypes in $\mathrm{CNC}$, other paratypes in KAS.

Remarks. The form of the male genitalia associates this species with $\underline{L}$. equiseti de Meij. (as L. kenti Spencer, 1969a), L. nordica Spencer, 1969a, and L. balcanicoides Sehgal, 1971, known in Canada, and with L. freye11a Spencer, 1976a, from Finland. However, it is readily distinguishable by the darker frons and third antennal segment from all these species.

Liriomyza endiviae Hering (new to U.S.A. and North America)

(Figs. 824-827)

Liriomyza endiviae Hering, 1955: 205. Holotype male from Spain in BMNH.

The type series was reared from blotch mines on Sonchus oleraceus. In addition, Hering referred to the mines of this species on Crepis capillaris (L.) W11r. from northern France and on Lactuca virosa from northern Germany.

A series of 23 specimens from Washington, reared from Lactuca sativa, L. serriola (as scariola), and Taraxacum vulgare, are now identified as L. endiviae. The aedeagus (figs. 824 , 825) agrees exactly with that of the holotype; the distiphallus is relatively small and the mesophallus is long and slender. The sperm pump is conspicuously large and characteristically angular at the upper corners (fig. 826). Characters of the adult show some variation. In the holotype, the third antennal segment is distinctly darkened and orange brown; four of the American specimens are similar, but in the others all antennal segments are yellow. The arrangement of the orbital bristles is normally two ors and two ori, but, as pointed out by Hering in the description, the lower ori may be weak or even lacking. This variation also occurs in the American specimens. The third antennal segment is slightly pubescent but not conspicuously so. L. endiviae is readily distinguishāble from L. ptarmicae (couplet 38), in which the third antennal segment is treated as having long pubescence, as this has both vt on black ground.

L. endiviae belongs to a group of closely related species known in Europe feeding on Hieracium, Lactuca, Scorzonera, Sonchus, Taraxacum, and Tragopogon. In $\underline{L}$. sonchi Hendel the mesophallus is broader and shorter than in L. endiviae (Spencer, 1976a: fig. 481), whereas in $L$. taraxaci Hering the mesophallus is even shorter (figs. $828,829)$.

The different form of leaf mine in $\underline{L}$. scorzonerae Rydén, which starts as $\bar{a}$ lower surface mine, confirms the distinctness of this species, although the aedeagus is scarcely separable from that of $\underline{L}$. endiviae (see Spencer,

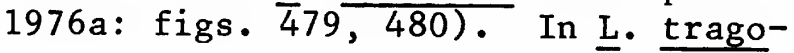


pogonis de Meij. the larva forms a long linear blotch mine along the midrib (Spencer, 1976a: fig. 493). In two other species feeding on

Hieracium, L. hieracii (Kaltenbach) and L. hieracivora Spencer (see Spencer, 1976a), the aedeagus is more clearly differentiated. Hering (1955) described L. scariolae feeding on Lactuca serriola in western Germany. This is very closely related to $\underline{L}$. endiviae, but it appears to be distinct, as the distiphallus (of the holotype) is considerably larger. $\underline{L}$. endiviae is clearly related to $\underline{L}$. minor, known from California (see figs. 721,722$)$, but this is a darker species.

New records.

Maryland, Montgomery Co., Bethesda, empty leaf mines on Lactuca, prob. serriola, 25.VI.80 (KAS), in KAS.

Washington, Benton Co., Prosser, 23 specimens ex Lactuca sativa, 26.VIII.49 (K. E. Frick, Lot 218 $1)$, in CAS.

Liriomyza eupatorie11a Spencer, new species

(Figs. 646-649)

Head. Frons 1.5-2 times width of eye, not projecting above eye in profile; 2 ors, 2 ori, distance between ors greater than that between lower ors and 2 ori, which are equidistant; orbital setulae sparse, reclinate; 3d antennal segment small, round.

Mesonotum. $3+1$ strong dc, acr in 4 rows, inner postalar strong.

Wing. Length $1.75 \mathrm{~mm}$ in both sexes, discal cell small, last section of M 3+4 3 times length of penultimate.

Color. Head bright yellow, both vt on yellow ground, hindmargin of eye normally black beyond vte; mesonotum strongly shining black but narrowly yellow centrally adjoining scutellum and broadly so at hindcorners (fig. 646); inner postalar at margin of black and yellow; side of thorax largely yellow, mesopleuron with narrow black bar along lower margin; legs with coxae and femora bright yellow, tibiae and tarsi brownish yellow, darker on hindleg; abdomen largely black, tergites narrowly yellow laterally; squama yellow, margin and fringe black.

Male genitalia. Aedeagus (figs. 647, 648) small, largely unpigmented; surstylus with 2 strong spines at inner corner.

Host/early stages. Eupatorium rugosum. Larva forming long, narrow linear mine (fig. 649); puparium yellowish, posterior spiracles each with about 8 bulbs, 1 elongated.

Holotype m., Wisconsin, Dane Co., Madison, 25.IX.76, ex leaf mine on Ageratina altissima (= Eupatorium rugosum) col1. 12.IX.76; paratypes 1 f., emerged 27.IX, otherwise same data; $1 \mathrm{~m} ., 3 \mathrm{f}$. , caught on host 13.IX.76; (a11 S. Tavormina).

Holotype in USNM, paratypes in KAS and S. Tavormina collection.

Empty leaf mines have also been seen from Minnesota, Minneapolis, Minnehaha Creek, 29.VI.80, and Virginia, Arlington County, Roosevelt Island, 1.VII.80.

Remarks. Although this distinctive species closely resembles Phytoliriomyza felti (fig. 990), a leaf miner on ferns, the male genitalia confirm that the two are not related.

We are grateful to Dr. Tavormina for allowing us to describe this interesting species.

Liriomyza eupatorii (Kaltenbach) (Figs. 788-791)

Agromyza eupatorii Kaltenbach, 1874 (see Hendel, 1931: 217, as Liriomyza). Types from Europe lost. Liriomyza eupatorii, Frick, 1959: 404; Spencer, 1969a: 174; 1981: 230.

In Europe, this species feeds most commonly on Eupatorium, more rarely on Aster and Solidago (surprisingly also 
on Galeopsis, Lamiaceae), and the leaf mine commences with a distinctive spiral (see Spencer, 1981: fig. 313).

In America, typical mines (fig. 790) have been found on Aster chilensis in California, Alameda County, Albany ( $\mathrm{K}$. E. Frick). Longer mines are also frequent on Solidago (Washington County, Miss.), with the initial spiral minute or more frequently entirely lacking (fig. 791). No differences can be found between the adults from Solidago and Aster or in their male genitalia, and the Solidago feeder is therefore treated as L. eupatorii despite the different form of the leaf mine.

The species has hitherto been known in the United States only in California and Washington. Study of additional material now shows that $L$. eupatorii is widespread also in the southern States. During a visit to Mississippi in May 1979, leaf mines were common on Solidago, but all were recently empty in mid-May.

New records.

Georgia, Rabun Co., Rabun Bald, $1 \mathrm{~m}$, 3 f., 14.VII-4.VIII.57 (J. G. (hillcott), in CNC.

Mississippi, Washingt on Co., Leland, 1 m., 2 f., 11.V.79, also numerous empty leaf mines on Solidago

(K. A. S.), KAS.

North Carolina, Macon Co., Highlands, $3,800 \mathrm{ft}, 1 \mathrm{~m} ., 12 . \mathrm{V} .57$ (J.R. Vockeroth); Wayah Bald, 3,500 ft, 1 f., 13.VII.57 (W. R. Richards); Mitchel1 Co., Roan Mts., 6,200 ft, 2 f., 13.VIII.57 (J. G. Chill cott); all in CNC.

South Carolina, Aiken Co., $1 \mathrm{~m}$., 12.VI.57 (J. R. Vockeroth), in CNC.

Tennessee, Hamilton Co., Chapin Sanctuary, East Ridge, $1 \mathrm{~m} ., 12 \mathrm{f}$., 5-9.V.52 (0. Peck and K. S. Walley), in CNC.

Virginia, Shenandoah Co., Mt. Jackson, 1 f., 25.V.62 (J. G. Chillcott); Hawksbill State Park, 3,600 ft, 1 f., 4.VI.62 (J.R. Vockeroth); Montgomery Co., Brush Mt., 2,800 ft, Blacksburg, 1 f., 27.V.62 (J.G. (hill cott), in CNC.

\section{Liriomyza flavonigra (Coquillett) (Figs. 633, 634)}

This species was described from two females from New Mexico, and an additional male was subsequently recorded by Melander (1913: 258) at an elevation of 9,000 feet (Frick, 1959: 405). The latter is now mislaid.

Coquillett (1902) gave the wing length of the female as $3 \mathrm{~mm}$, whereas Frick (1959) recorded it as $2.5-2.75 \mathrm{~mm}$. Distinctive characters of the species are the projecting frons (see Malloch, 1913a: p1. XXX, fig. 27) and the yellow squamal fringe. Two males have been seen from Utah, Cache County, Providence, 25.V.64 (G. F. Knowlton), in UCD. They agree in these and other characters, but they are somewhat smaller, with a wing length of $2.3 \mathrm{~mm}$. It seems reasonably certain that they represent $\underline{L}$.

flavonigra. The distinctive aedeagus is shown in figures 633,634 .

Liriomyza helianthi Spencer (Figs. 766, 767)

Liriomyza helianthi Spencer, 1981: 240 . Holotype male from California in CAS.

The type series was caught on Helianthus annuus and it seemed probable that this was the host. Additional specimens reared from this host and also from Xanthium strumarium in Washington have been examined and identified as $\underline{\text { L. helianthi. }}$

New records.

Washington, Yakima Co., Yakima, 4 m., 5 f., ex Helianthus annuus, Lot 203 -

$1,8 . V I .50$ (K. E. Frick), in CAS; 22

m., $18 \mathrm{f}$. , ex Xanthium strumarium, Lot $50-1 \overline{5,6-10 . I X .50(\mathrm{~K} .} \mathrm{E}$. Frick), in CAS.

\section{Liriomyza lathyri Sehgal (new to} U.S.A.)

(Figs. 821-823)

Liriomyza 1athyri Sehga1, 1971: 334. Holotype male from Alberta in CNC. 
This species has previously been known only from the type series. Leaf mines (fig. 823) were found in Arkansas, Hot Spring County, Lake Catherine State Park, on Lathyrus sp., 18.V.79 (KAS and GCS), in KAS, and a single male emerged 11.VI.79. It is distinctive in having both vertical bristles on a yellow ground and the mesonotum uniformly matt black. The aedeagus is shown in figures 821,822 .

Liriomyza montella Spencer, new species (Figs. 744, 745)

Head. Frons twice width of eye, projecting only narrowly above eye in profile; 2 reclinate ors, 2 inclined ori, orbital setulae sparse, reclinate; gena about 0.33 height of eye; $3 d$ antennal segment small, round.

Mesonotum. $3+1 \mathrm{dc}$, acr sparse, in 2 rows.

Wing. Length in male $1.5 \mathrm{~mm}$, discal cell small, last section of vein $M 3+4$ slightly more than 3 times length of penultimate.

Color. Frons, orbit, face, and antenna yellow; hindmargin of eye narrowly black, with vte on dark ground, vti on yellow; mesonotum matt black, scutellum broadly dark at sides, narrowly yellow in center; humerus and notopleuron yellow, pleura otherwise blackish gray; legs largely black but femora with all knees yellow; squama and fringe yellowish, margin only slightly darker; abdomen largely black.

Male genitalia. Aedeagus as in figures 744,745 .

Host/early stages. Unknown.

Holotype m., Colorado, Clear Creek Co., Mt. Evans, Timberline, $11,700 \mathrm{ft}$, 22.VII.61 (C. H. Mann), in CNC.

Remarks. The male genitalia indicate close relationship between this species and L. balcanoides Sehgal, 1971, but the latter has the meso- pleuron and legs largely yellow. L. montella somewhat resembles $\underline{L}$. elevata, n. sp., caught at the same locality, but it has the third antennal segment darkened.

Liriomyza montis Spencer, new species (Figs. 764, 765)

Head. Frons 1.5-2 times width of eye, narrowly projecting above eye in profile; normally 2 ors (on one side in one specimen 3 ) and 2 ori, orbital setulae sparse, reclinate; gena extended at rear, 0.33 height of eye; $3 d$ antennal segment slightly longer than broad, rounded at end.

Mesonotum. 3+1 strong dc, acr irregularly in 4 rows.

Wing. Length in male $2.3 \mathrm{~mm}$, discal cell large, last section of vein $M 3+4$ only slightly more than twice length of penultimate, in ratio $30: 13$.

Color. Frons, orbit, and antenna bright yellow, hindmargin of eye black to base of vti; mesonotum uniformly black, predominantly matt, with only weak subshine, scutellum completely yellow; mesopleuron yellow on upper quarter, black below; legs with femora largely dull yellow, with blackish striations, tibiae and tarsi black; squama yellowish gray, margin and fringe black.

Male genitalia. Aedeagus as in figures 764 , 765; sperm pump with narrow stalk and only slightly widening blade; surstylus with 2 stout bristles and several hairs at end.

Host/early stages. Unknown.

Holotype m., Colorado, Clear Creek Co., Mt. Evans, Summit Lake, 12,000 ft, 24.VII.61 (B. H. Poole); paratype m., Colorado, Boulder Co., near Ward, $11,500 \mathrm{ft}$, on tundra, 4.VII.61 (C. H. Mann); both in CNC.

Remarks. Although this species closely resembles L. sabaziae, the male genitalia indicate that the two are not closely related. 
Liriomyza pechumani Spencer, new species

(Figs. 635-639)

Head (fig. 635). Frons only slightly wider than eye, conspicuously projecting above eye in profile; orbital bristles strong, 2 ors, 2 ori, orbital setulae reclinate, irregularly in 2 rows; gena 0.33 height of eye at rear; $3 d$ antennal segment small, round, arista long, drooping, distinctly pubescent.

Mesonotum. $3+1 \mathrm{dc}$ (male), 2+1 (female) in only specimens seen, acr in some 6 rows in front, intra-alar hairs numerous, in 4 rows, intra-alar bristle well developed.

Side of thorax. Mesopleuron and sternopleuron both with numerous hairs in addition to normal bristle.

Wing. Length $3 \mathrm{~mm}$ in male, $3.25 \mathrm{~mm}$ in female, discal cell large, last section of vein $M 3+4$ less than 1.5 times length of penultimate, in ratio $31: 26$.

Abdomen. Thickly covered with exceptionally numerous hairs, particularly in male, strongest laterally and on last tergite, where they are almost bristlelike.

Color. Head entirely yellow; mesonotum blackish gray in male, matt black in female, dark area divided into 5 bands, 2 other bands divided from dark humeral area, broadly yellow adjoining scutellum to level of $2 \mathrm{~d} d c$, scutellum entirely yellow; pleura entirely yellow, apart from normal black triangle on sternopleuron; legs with coxae and femora bright yellow, tibiae and tarsi darker, yellowish brown; abdomen predominantly yellow, darker centrally on each tergite, giving appearance of dark central line; squama yellowish gray, margin and fringe black; ovipositor sheath shining black; halter yellow.

Male genitalia. Aedeagus as in figures 636, 637; sperm pump large, with blade becoming unpigmented distally (fig. 638); epandrium (fig. 639) with stout projection at lower corner; surstylus ending in long, curving projection.

Host/early stages. Unknown.

Holotype m., Florida, Collier Co., Copeland, 31.III.69; paratype f., same data; (both L. L. Pechuman). Holotype and paratype in CUI.

Remarks. This large, distinctive species is shown by the male genitalia to be closely related to $\underline{L}$. blechi and L. marginalis. Its size suggests that it is not a true leaf miner, and the larva may well feed in the midrib.

Liriomyza philadelphivora Spencer

(new to U.S.A.)

(Figs. 663-667)

Liriomyza philadelphivora Spencer, 1969a: 182. Holotype male from Ontario in CNC.

The species forming conspicuous white blotch mines on Philadelphus was recorded as Agromyza melampyga by Coquillett (1898: 77) and Frost (1924: 47). It was described as a new species from a series reared from Philadelphus at the Biosystematics Research Institute, Ottawa.

L. philadelphivora has the dark areas of the mesonotum more shining black than in L. melampyga (which is in the genus Phytoliriomyza) but closely resembles both L. marginalis feeding on various grasses and L. blechi feeding on Blechum and possibly other hosts ( $\mathrm{p}$. 285).

New records.

District of Columbia, Washington (see Malloch, 1913a: 282).

New York, Long Island, Babylon, $1 \mathrm{~m}$., 1 f., 16 and 17.VII.36 (Blanton and Borders); 1 f., 23.VI.37 (F. S. Blanton); all CUI. 
Liriomyza pulloides Spencer, new species

(Figs. 742, 743)

Head. Frons at least 1.5 times width of eye, slightly projecting above eye in profile; 2 ors, 2 ori, upper ors reclinate, lower 2 ori incurved, orbital setulae sparse, reclinate; gena extended at rear, 0.4 height of eye; 3d antennal segment small, round, arista short.

Mesonotum. $3+1 \mathrm{dc}$, with several smaller setulae in same line beyond presutural, acr irregularly in 4 rows.

Wing. Length $1.6 \mathrm{~mm}$, last section of vein $M 3+4$ twice length of penultimate.

Color. Frons yellowish orange, orbit more yellow, antenna, gena, face, and palpus yellow; hindmargin of eye dark, both vt on dark ground; mesonotum entirely matt black; humerus and notopleruon dul1 yellow, pleura otherwise black, upper margin of mesopleuron only faintly yellowish; legs largely black, though femora distinctly yellowish at knees and slightly so below in male; abdomen matt black, tergites only narrowly yellow bordered; in female ovipositor sheath shining black; squama yellowish gray, margin and fringe dark.

Male genitalia. Aedeagus as in figures 742, 743, distiphallus with distinctive dorsally directed membranous tubules; sperm pump with narrow stalk and large, dark blade; surstylus narrow, elongate, with stout spine at end.

Host/early stages. Unknown.

Holotype m., Colorado, Larimer Co., Estes Park, 7,500 ft, 14.VIII.61; paratype f., same data; (both B. H. Poole), in CNC.

Remarks. The yellow frons and antenna, combined with the dark legs and mesopleuron and the matt mesonotum, make this a distinctive species. The aedeagus is distinct from any related species.
Liriomyza sativae Blanchard

(Figs. 777-784)

Liriomyza sativae Blanchard, 1938: 354. Holotype female ex Medicago sativa from Argentina in Museo de $1 a$ Plata, Buenos Aires, Argentina. Liriomyza verbenicola Hering, 1951a: 43. NEW SYNONYMY. Holotype female ex Verbena sp. from New Mexico in ZMHU.

Liriomyza pullata Frick, 1952b: 509. Holotype female ex Datura sp. from Hawaii in CAS (synonymy established by Spencer, 1973a: 219).

Liriomyza canomarginis Frick, 1952b: 511. Holotype female ex Indigofera sp. from Hawaii in CAS (synonymy established by Spencer, 1973a: 219).

Liriomyza minutiseta Frick, 1952b: 512. Holotype female ex tomato from Hawaii in CAS (synonymy established by Spencer, 1973a).

Liriomyza propepusilla Frost, 1954: 73. Holotype male from Kansas in USNM (synonymy established by Steyska1, 1973b: 735).

Liriomyza munda Frick, 1957a: 61. Holotype male ex tomato from San Joaquin County, Calif., in CAS (synonymy established by Spencer, 1973a: 219).

Liriomyza guytona Freeman, 1958: 344. Holotype male ex "beans" from Alabama in USNM (synonymy established by Spencer, 1973a: 219; synonymy of $\underline{L}$. guytona with $L$. munda established by

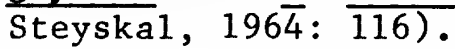

This highly polyphagous species is widely distributed in the Southern States, and its range extends south to Argentina and Chile. As is inevitable in such cases, it has been described with different names by various authors from different hosts on several occasions. In view of the economic importance of L. sativae (see Spencer, 1973a), the ful1 synonymy is given here.

L. verbenicola was described from three females reared from upper surface 1 inear mines on Verbena at Las Cruces, N. Mex. One of the female paratypes in the USNM has been 
examined. Since it is reasonably certain that $\underline{L}$. verbenicola is identical with L. sativae, this new synonymy is established here.

L. sativae shows greater color variation than has previously been appreciated. The upper orbit is normally darkened, at least to the lower ors, and both vt are on black ground; the mesopleuron is normally predominantly yellow, with small black markings along the lower, front, and hind margins; the femora are normally entirely yellow. However, darker specimens appear to be common, with the mesopleuron more extensively black and the femora having blackish striations. The species is therefore included in the key at couplets 56, 63 , and 76 .

Frick (1957a) unfortunately in part misidentified L. sativae as $\underline{\text { L }}$ pictella in California. Further, a number of hosts cited for L. "pictella" have since been found to have their own distinct species. This confusion has been discussed in greater detail by Spencer (1981).

Liriomyza splendens Spencer, new species

(Figs. 811, 812)

Head. Frons broad, twice width of eye, not projecting above eye in profile; 2 ors, 3 ori, orbital setulae sparse, reclinate; gena extended at rear, 0.4 height of eye, parafacial forming prominent ring below eye; 3d antennal segment small, round, arista long, virtually bare.

Mesonotum. $3+1$ strong dc, acr coarse, in 4 rows.

Wing. Length in male $2.6 \mathrm{~mm}$, discal cell large, last section of vein M $3+4$ just over twice length of penultimate.

Color. Head largely yellowish orange, hindmargin of eye black to just beyond base of vte, vti on yellow; mesonotum deep black, essentially matt, with only faint subshine, scutellum largely bright yellow, with only small black patches below basal scutellars; legs with femora conspicuously bright yellow, tibiae and tarsi brownish yellow; squama and fringe bright yellow.

Male genitalia. Aedeagus (figs. 811, 812) ending in long, membranous extensions; sperm pump with large, heavily pigmented blade and strong base.

Host/early stages. Unknown.

Holotype m., New York, Tompkins Co., McLean Bogs, 28.V.62 (W. W. Boyle), in CUI.

Remarks. This is a striking species, with highly differentiated male genitalia. The long aedeagus indicates that the female terminalia are also elongate and this suggests that the larva is an internal feeder rather than a leaf miner.

Liriomyza subasclepiadis Spencer, new species

(Figs. 796-798)

Head. Frons broad, almost twice width of eye, not projecting above eye in profile; 2 ors, 2 ori, orbital setulae sparse, reclinate; gena extended at rear, 0.33 height of eye; $3 d$ antennal segment small, round, with short, normal pubescence.

Mesonotum. $3+1$ strong $\mathrm{dc}$, acr in 4 rows in front.

Wing. Length $1.6-2 \mathrm{~mm}$, last section of $M \quad 3+42-2.5$ times length of penultimate.

Color. Frons, orbit, gena, and antenna bright yellow, hindmargin of eye black, with both vt on black ground; mesonotum deep black, distinctly shining, with small yellow patches at hindcorners, inner postalar on black, scutellum and side of thorax largely yellow; tibiae and tarsi darker, brownish; squama yellow, margin and fringe dark. 
Male genitalia. Aedeagus (figs. 796, 797) with distiphallus short, not widening distally; sperm pump with narrow stalk, blade small but widening at end, base well developed.

Host/early stages. Asclepias speciosa. Larva forming irregular, interparenchymal mine (fig. 798), which is frequently partially linear, with frass detectable in 2 lines of strips, but often develops into secondary blotch; mine appears greenish and may be difficult to detect as a true mine; pupation externally; several mines may be present in a single leaf.

Holotype m., Washington, Yakima Co., Donald, ex larva on Asclepias speciosa, 13.VI.49 (K. E. Frick, Lot 210 - 1); paratypes topotypical, same data, 9 m., $6 \mathrm{f}$. Holotype and paratypes in CAS, other paratypes in KAS.

Remarks. This species is indistinguishable from $\underline{\text { L. asclepiadis }}$ externally, but the male genitalia of the two species differ substantially (figs. 792, 793 and 796, 797), although of the same general form. Further confirmation that separate species are involved is provided by differences in the leaf mines. In L. asclepiadis the larva forms a primary upper surface blotch (fig. 794), whereas in L. subasclepiadis the feeding is interparenchymal, of varying depth, and at least in part linear.

This is an interesting instance of relatively recent speciation with no differences having yet developed in external characters, and the two species apparently separated by the Sierra Nevada mountains.

Liriomyza taraxaci Hering (new to U.S.A.)

(Figs. 828-830)

Liriomyza taraxaci Hering, 1927: 184. Holotype male from Germany in ZMHU.

This species belongs to the closely related group feeding on the Asteraceae Liguliflorae. It has previously been recorded in Canada (Spencer, 1969a: 188), and leaf mines on Taraxacum officinale have now been seen from Wisconsin, Dane County, Madison, 31.V.75 (S. Tavormina). With the species confirmed in the Ottawa area at approximately the same latitude as Madison, there is little reasonable doubt that the mines found at Madison represent $\underline{L}$. taraxaci.

As far as is known, L. taraxaci is host specific on Taraxacum, whereas $\underline{L}$. endiviae feeds on Lactuca, Sonchus, and possibly Crepis (p. 287). The two species are not satisfactorily distinguishable based on external characters but are distinctive in having the hindmargin of the eye largely yellow, with both vertical bristles thus on yellow ground, and the black of the occiput at most reaching the eye margin for a short distance well beyond the outer vertical. There are distinctive differences in the male genitalia of the two species (see figs. 824, 825 and 828,829 ).

Two specimens reared by K. E. Frick from Taraxacum vulgare at Washington, Benton County, Prosser, 14.VI and 10.VII.49, are possibly referable to L. taraxaci, but at this locality $\underline{L}$. endiviae was common on Lactuca and possibly this was the species concerned.

Liriomyza temperata Spencer, new species (Figs. 805, 806)

Head. Frons broad, twice width of eye, not projecting above eye in profile; 2 ors, 2 ori, orbital setulae sparse, reclinate; gena extended at rear, 0.33 height of eye; $3 d$ antennal segment small, round.

Mesonotum. $3+1$ strong dc, acr in 4 rows, inner postalar strong.

Wing. Length $2.3 \mathrm{~mm}$ in male, up to $2.5 \mathrm{~mm}$ in female, last section of vein M 3+4 just over twice length of penultimate. 
Color. Head yellow, apart from narrow black area at hindmargin of eye, vti clearly on yellow; mesonotum strongly shining black, even viewed from front, yellow patches at hindcorners large, inner postalar at margin of black and yellow, scutellum largely yellow, lateral black patches small, well below anterior scutellars; mesopleuron black on lower half; legs with femora largely bright yellow, narrowly black basally, tibiae and tarsi brownish yellow; squama yellow, margin and fringe dark; abdomen largely black but tergites normally narrowly yellow bordered; in female ovipositor sheath shining black.

Male genitalia. Aedeagus as in figures 805, 806; sperm pump with narrow stalk and conspicuously widening blade.

Host/early stages. Unknown.

Holotype m., North Carolina, Swain Co., Great Smoky Mountains National Park, Clingman's Dome, 6,600 ft, 22.VIII.57 (J. G. Chillcott); paratypes $3 \mathrm{f}$., same data; $4 \mathrm{f} ., 6,300$ ft, 28.V.57 (J. R. Vockeroth); Indian Gap, 5,200 ft, 2 f., 20.V.57 (J. R. Vockeroth); Devil's Court House, Blue Ridge Parkway, 1 f., 2.VIII.57 (W. R. Richards); Mitchel1 Co., Roan Mountain, 6,200 ft, $1 \mathrm{m.,} 2 \mathrm{f}$., 13.VIII.57 (J. G. Chillcott). Holotype and paratypes in CNC, 4 paratypes in AC.

Remarks. This species belongs to a difficult group, but the male genitalia confirm that it is distinct, although its exact relationship is uncertain.

Liriomyza texella Spencer, new species (Figs. 815, 816)

Head. Frons twice width of eye, not projecting above eye in profile; normally 2 ors ( 3 on one side), 2 ori, orbital setulae sparse, reclinate; gena approx. 0.33 height of eye; $3 \mathrm{~d}$ antennal segment small, round.
Mesonotum. $3+1$ strong $\mathrm{dc}$, acr in 4 rows.

Wing. Length in male $1.5 \mathrm{~mm}$, discal cel1 very small, last section of vein M $3+43.5$ times length of penultimate.

Color. Head yellow, hindmargin of eye black to vte, vti on yellow; mesonotum uniform1y matt, grayish black, hindcorners with very small yellow patches, scutellum largely bright yellow, on $1 y$ narrowly black at sides, a11 scutellars on yellow; mesopleuron largely yellow, with narrow black bar along lower margin which also extends along front margin; legs with femora brighter yellow, tibiae and tarsi brownish yellow on forelegs, more blackish on other legs; squama yellow gray, margin and fringe dark.

Male genitalia. Aedeagus as in figures 815,816 ; sperm pump with short stalk, conspicuously widening blade, and strongly developed base.

Host/early stages. Unknown.

Holotype m., Texas, Kerr Co., Kerrville, 3.IV.59 (J. F. McAlpine), in CNC.

Remarks. Al though $\underline{L}$. texella closely resembles $\underline{L}$. venturensis from California, the male genitalia indicate that the two species are obviously not closely related.

Liriomyza togata (Melander)

(Figs. 831, 832)

Antineura togata Melander, 1913: 250. Lectotype male from Washington in USNM.

Haplomyza togata, Hende1, 1914: 73; Frick, 1957b: 204 (1ectotype designation); 1959: 413.

Liriomyza togata, Steyskal, 1980: 141. Liriomyza doug1asii Spencer, 1981: 228. NEW SYNONYM.

Hendel transferred this species to Haplomyza in view of the absence of the outer crossvein, one of the characters subsequently used by Frick 
and other authors in defining the genus Haplomyza. However, as Steyskal (1980) rightly noted, although L. togata closely resembles true Haplomyza species (now Haplopeodes), the male genitalia indicate that togata correctly belongs in Liriomyza.

L. douglasii was described from $\bar{C}$ alifornia before the correct identity of $\underline{L}$. togata was known. The aedeagus of L. douglasii (figs. 831, 832) is obviously identical to that of the lectotype of L. togata illustrated by Steyska1 (1980): $\overline{\text { figs. } 1}$ A, 1 B ). L. douglasii, therefore, is synonymized here with $\underline{L}$. togata.

There is some variation in the arrangement of the orbital bristles in this species. In the lectotype there is only one strong reclinate ors and two inclined ori, but in three other specimens examined there are $1+3$. The wing length is not $1.2 \mathrm{~mm}$ as stated by Melander but ranges from 1.6 to 1.75 $\mathrm{mm}$.

Among the four specimens included in the type series of $\underline{L}$. douglasii, one female from California, Alameda County, Albany, was reared from Artemisia douglasiana, 13.VIII.48 ( $\mathrm{K}$. E. Frick, Lot 41-3). Details of the leaf mine are not available.

\section{Liriomyza trifoliearum Spencer}

\section{(Figs. 703-706)}

Liriomyza trifoliearum Spencer, in Spencer and Stegmaier, 1973: 107. Holotype male from Florida in USNM.

This species was originally misidentified by Frick and other American workers as $\underline{L}$. pictella. However, $\underline{L}$. pictella is now restricted to the unique holotype from "San Francisco." At the time of its description $\underline{L}$. trifoliearum was known as a lea $\bar{f}$ miner on only two hosts, Medicago and Trifolium. It was subsequently recorded on Coronilla and Pisum.

A series of six specimens has been examined from Washington, Benton
County, Prosser, reared in April 1950 from Solanum sarachoides (Frick, Lot 50 - 16). Despite the improbable host, it is clear that these specimens represent $\underline{L}$. trifoliearum, which has been recorded from the same locality on Medicago sativa (Frick, Lot 229 1). Records were given by Spencer (1981: 278) of caught specimens from 13 counties in California. It was assumed that the hosts of such specimens would be in the Fabaceae, but it now remains to be established whether the species occurs regularly on Solanum or even other hosts, or whether its occurrence on Solanum in Washington represents mere $\overline{l y}$ an unusual local transference.

\section{Liriomyza trifolii (Burgess)}

(Figs. 838-840)

Oscinis trifolii Burgess, 1880: 201. Neotype male from Indiana, designated by Spencer, 1965a: 37, in USNM. Liriomyza phaseolunata Frost, 1943: 256. NEW SYNONYM. Holotype missing, 2 paratopotypes from New Jersey in PSU.

Liriomyza trifolii, Frick, 1952a: 405; 1959: 410; Spencer, 1965a: 37; 1973a: 226; 1981: 281; Spencer and Stegmaier, 1973: 109.

Liriomyza alliovora Frick, 1955: 88. Holotype male from Iowa in USNM (synonymy established by Spencer, 1973a: 226).

This species is highly polyphagous. Stegmaier (1966a) listed 55 hosts in 10 families in Florida. L. trifolii has a wide distribution in the Eastern United States, and as often occurs in such cases, it has been described on several occasions by different authors. Distinctive characters are the yellow hindmargin of the eye and the matt grayish black mesonotum, with acrostichals irregularly in some four rows in front but sparse or even lacking behind the second dorsocentral.

An unexpected and favored host is Allium, and Frost (1943) included in his description of Agromyza allia 20 
paratypes reared from onion at Ames, Iowa. After examination of a paratype from this series, L. alliovora was synonymized with L- trifolii by Spencer (1973a).

Two paratypes of "Agromyza" phaseolunata Frost have been examined, which were recorded from lima bean at Bridgetown, N.J. This species a1so clearly represents L. trifolii. This new synonymy is formaly estab 1 ished here.

In recent years, $\underline{\text { L. trifolii has }}$ become known as a pest of truck crops in Florida, but the most significant damage is caused on cultivated chrysanthemums, particularly in Florida, California, and in greenhouses in Washington, D.C. Chrysanthemum cuttings have been regularly supplied from a nursery in Florida to a large propagating nursery in Kenya, which in turn has supplied cuttings to many European countries. In this way L. trifolii was introduced first into Kenya, where it is now widespread on many local plants, and subsequently has reached most countries in western Europe and a1so Hungary and Yugoslavia.

There are now wild populations both in southern England (where it may not have survived the 1982 winter) and in a number of districts in France. $\underline{L}$. trifolii is regarded as a serious pest in Europe because of the threat to tomato growers, as tomato is another favorite host.

Liriomyza virginica Spencer, new species

(Figs. 801, 802)

Head. Frons 1.5 times width of eye, not projecting above eye in profile; 2 ors, 2 ori; gena extended at rear, 0.33 height of eye; $3 \mathrm{~d}$ antennal segment sma1l, round, slightly pubescent.

Mesonotum. $3+1 \mathrm{dc}$, acr in 4 rows.

Wing. Length in male $1.4 \mathrm{~mm}$, discal cell small, last section of vein $M 3+4$ 3 times length of penultimate.
Color. Head yellow, only hindmargin narrowly black, but vti distinctly on yellow ground; mesonotum deep black, distinctly shining, yellow patches at hindcorners small, scutellum bright yellow, with small black patches laterally; mesopleuron largely yellow, with narrow black band along lower margin; legs with coxae and femora bright yellow, tibiae and tarsi brownish yellow; squama yellow, margin and fringe dark.

Male genitalia. Aedeagus as in figures 801,802 ; sperm pump with narrow stalk and dark, moderately widening blade.

Host/early stages. Unknown.

Holotype m., Virginia, Patrick Co., Vesta, 2,800 ft, 30.V.62 (J. R. Vockeroth), in CNC.

Remarks. L. virginica closely resembles $L$. schlingeri known from California, but the male genitalia confirm that the two species are distinct.

\section{Liriomyza sp. (Colorado)}

Colorado, Clear Creek Co., Mt. Evans, Doolittle Ranch, 9,800 ft, $1 \mathrm{f}$., 8.VII.61 (C. H. Mann), in CNC.

Essentia1 characters of this species are the entirely black antenna and legs; brownish frons with deep black orbit; matt black mesonotum, with acr in four rows; bright yellow humerus and notopleuron; other pleura entirely black; squama gray, margin and fringe black; and wing length $2.4 \mathrm{~mm}$, with discal cell large.

The matt mesonotum is the most distinctive character, readily distinguishing the species from $\underline{L}$. baptisiae, which it otherwise generally resembles.

\section{GENUS GALIOMYZA SPENCER}

Two new species are described, one 
feeding on Viola and the other probably on Galium, and one species is recorded as new to the United States.

Galiomyza galiivora (Spencer) (new

to U.S.A.)

(Figs. 854-856)

Praspedomyza galiivora Spencer, 1969a: 199. Holotype male from Alberta in CNC.

Galiomyza galiivora, Spencer, 1981: 291.

A single male has been seen from Minnesota, Hennepin County, Minneapolis, Minnehaha Creek, 28.VIII.76 (KAS), in KAS. Apart from this record, the species is only known in North America from Alberta but is doubtless widespread with its host, Galium, at least in the Northern States.

Galiomyza violivora Spencer, new species

(Figs. 848-853)

Head (fig. 848). Frons broad, twice width of eye, not projecting above eye in profile; 2 ors, 2 strong ori which are little weaker than ors, orbital setulae coarse, reclinate; orbit pronounced, little differentiated from frons, slightly widening at midpoint; gena rounded in front, angular at rear, 0.33 height of eye; $3 d$ antennal segment small, round, with only short pubescence, arista slightly longer than width of eye, appearing virtually bare.

Mesonotum. $3+1 \mathrm{dc}$, presutural small, acr coarse, irregularly in 4 rows, intra-alar present.

Wing. Length from $1.6 \mathrm{~mm}$ in male to $1.9 \mathrm{~mm}$ in female, last section of vein M 3+4 2.5 times length of penultimate.

Color. Variable; frons varying from orange brown to blackish brown, with orbit contrastingly black; gena orange brown with lower margin black; all antennal segments and palpus black; mesonotum deep black, appearing some- what matt viewed from front, more shining from rear, scutellum either entirely black but frequently paler, brownish at rear, particularly when viewed from behind; rear of humerus and notopleuron either bright yellow or at least ochreous, pleura entirely black; legs largely black but foreknee narrowly yellow; abdomen black; squama yellowish gray, margin and fringe black; halter whitish yellow.

Male genitalia. Aedeagus (figs. 849, 850 ) divided distally, with 2 pairs of ventral lobes and well-defined bladder within basal sclerites; sperm pump (fig. 851) heavily pigmented, asymmetrical, with well-developed base; epandrium (fig. 852) without discrete surstylus but rounded on inner corner bearing few short hairs, strong, curving process attached at midpoint.

Host/early stages. Viola sororia and probably other Viola species. Larva forming large white blotch mine (fig. 853) with finely scattered black frass; puparium reddish brown, posterior spiracles each with 3 bulbs.

Holotype m., Mississippi, Washington Co., Leland, emerged 4.VI.79 (K. E. Frick 79-13), col1. G. McMinn; paratypes $11 \mathrm{~m} ., 8 \mathrm{f}$., same data; Pennsylvania, Sullivan Co., Lincoln Falls, 1 m., "Blotch mine Violet," 26.VIII.53 (S. W. Frost). Holotype and paratypes in USNM, other paratypes in KAS, PSU, and Southern Weed Science Laboratory, Stoneville, Miss.

Remarks. This species is closely related to $G$. violiphaga (Hendel), a leaf miner on Viola in Europe (see Spencer, 1976a: 287 and figs. 515-517), but in the European species both the scutellum and the third antennal segment are yellow. The curving process within the epandrium in $G$. violivora ( $\mathrm{fig} .852$ ) is also present but more pronounced in $\mathrm{G}$. violiphaga.

Among the North American and European feeders on Galium, G. violivora most closely resembles $G$. turneri from 
California, both having the third antennal segment black, whereas in G. galiivora and $G$. vockerothi in America and G. morio in Europe the third antennal segment is yellow.

Galiomyza vockerothi Spencer, new species

(Figs. 857-859)

Adu1t. Externally as in G. galiivora; male genitalia with aedeagus as in figures 857,858 , with distiphallus not enlarged and not extended centrally; epandrium with small strong "tooth" and similar, strongly sclerotized projection at midpoint of inner margin of epandrium (fig. 859).

Host/early stages. Unknown.

Holotype m., North Carolina, Macon Co., Highlands, 25.V.57 (J. R. Vockeroth), in CNC.

Remarks. Differences between this species and $G$. galiivora are limited to the male genitalia and epandrium. The aedeagus is of the same general form in the two species, but the smaller size and lack of the central projection in G. vockerothi are sufficiently distinctive to justify treating this southern population as distinct. The host is almost certain1y Galium.

\section{GENUS HAPLOPEODES STEYSKAL}

One new synonym is established here.

Haplopeodes kefi Steyska1

(Figs. 860-862)

Haplopeodes kefi Steyskal, 1980: 146. Holotype male from California in CAS. Phytoliriomyza yolensis Spencer, 1981: 331. NEW SYNONYM. Holotype male from California in UCD.

P. yolensis was described from a single caught male from Yolo County, Calif., with the head badly shrunk. It was possible to see only the foremost orbital setula, which was proclinate, and this led to the conclusion that the species belonged in Phytoliriomyza. The aedeagus appeared to resemble that of $P$. conjunctimontis. However, it is now realized that the generic position in Haplopeodes is clearly indicated by the long, narrow sperm pump (Spencer, 1981: fig. 479). The partially yellow mesonotum shows that the species is in fact $\underline{H}$. kefi, which was described from Santa Cruz County, Calif., and this new synonymy is established here.

Although in the description of $\underline{\mathrm{H}}$. kefi the mesonotum was considered to be gray, the additional material now seen, including specimens from Texas, suggests that the mesonotum is norma1ly yellow adjoining the scutellum, appearing uniformly gray only in the darkest specimens.

\section{GENUS CALYCOMYZA HENDEL}

Two new species are described and the taxonomic status of two species is clarified. A male is designated as lectotype of Phytobia coronata (which is accepted as a synonym of $\underline{C}$. jucunda).

Calycomyza frickiana Spencer, new species

(Figs. 906-908)

Head. Frons narrow, little wider than eye; 2 ors, normally 2 ori (rarely a $3 d$, sometimes on one side on $1 y$ ); gena somewhat extended at rear, almost 0.25 height of eye; $3 d$ antennal segment ovoid.

Mesonotum. 2 strong dc, 3d (and also sometimes a $4 \mathrm{th}$ ) sma11, acr irregularly in 6 rows.

Wing. Length $2.25-2.5 \mathrm{~mm}$ in male, only slightly longer in female, discal cell variable, last section of $M 3+4$ varying from 1.5 to 2.5 times length of penultimate.

Color. Frons and face yellow; orbit almost entirely yellow, at most 
faintly brownish between $v t i$ and upper ors; 3d antennal segment black, 1st and 2d slightly paler, yellowish; mesonotum black, moderately shining, with only sma11, indistinctly yellowish patches at hindcorners; humerus (apart from central black area), notopleuron, upper margin of mesopleuron, and wing base bright yellow; legs black, foreknee only faintly yellowish; squama yellow, margin and fringe black.

Male genitalia. Aedeagus (figs. 906, 907) with anterior pair of ventral sclerites flaplike, posterior pair short, basal sclerites partially fused, asymmetrical; sperm pump large, with broad blade.

Host/ear1y stages. Bidens, Helianthus, Rudbeckia. Larva forming large blotch mine (fig. 908); puparium brown, posterior spiracles each with 3 bulbs.

Holotype m., New York, Seneca Co., East Varick, ex Bidens frondosa, 30.X.56 (S.W. Frost); paratypes Illinois, Champaign Co., Urbana, 1 f., 20.VI.15; Indiana, Tippecanoe Co., Lafayette, $1 \mathrm{~m} ., 27 . \mathrm{V}$. (? year); South Dakota, Kingsbury Co., Erwin, VI.08; (all J. M. Aldrich); Pennsylvania, Adams Co., Arendtsville, $1 \mathrm{f}$. , ex Bidens frondosa, 5.VIII.20; Adams Co., Colerain Park, 1 m., ex Rudbeckia 22.VII.62; (both S. W. Frost); Virginia, Montgomery Co., Blacksburg, 2,100 ft, $1 \mathrm{f}$., 28.v.62 (J. G. Chillcott). Holotype in USNM, paratypes in USNM, CNC, and PSU.

There are other specimens in poor condition in the $\mathrm{S}$. W. Frost collection from Arendtsville, $\mathrm{Pa}$., reared from Helianthus sp. Leaf mines have been seen from Maryland, Montgomery County, Cabin John Creek, 24.VIII.76 and 26.X.78, and from Minnesota, Hennepin County, Minneapolis, Minnehaha Creek, 10.IX.78, a11 on Rudbeckia laciniata.

Remarks. Frick (1956) misinterpreted this species as $\underline{\mathrm{C}}$. allecta (Melander). C. allecta was described from St. $\bar{V}$ incent, West Indies, and is a neo- tropical species, with confirmed records from Brazil, Dominica, Trinidad, and Venezuela (Spencer, 1963a: 338; 1973b: 37; Spencer and Stegmaier, 1973: 182). This misinterpretation was entirely understandable at the time, as the two species agree closely, particularly in the important character of the entirely yellow orbit, but $\underline{C}$. allecta is somewhat smaller and slightly darker. The male genitalia of the two species are entirely distinct (figs. 906, 907, and Spencer, 1963a: figs. 52, a , 52, ㅁ).

C. frickiana appears to be a more northern species, with no records south of Virginia and Pennsylvania. It can occur together with $\underline{\mathrm{C}}$. flavinotum, of which the known hosts are Arctium and Eupatorium.

Calycomyza jucunda (Wulp)

(Figs. 884-887)

Agromyza jucunda Wu1p, 1867: 161. Holotype male from Wisconsin in $\mathrm{RNH}$. Phytobia coronata (Loew, 1869: 48), synonymy established by Frick, 1956: 288. Lectotype male from Pennsy1vania in MCZ designated here. Calycomyza jucunda, Steyska1, 1973a: 191 .

This species has been confused with $\underline{C}$. platyptera, feeding on Asteraceae, until its identity was established by Steyskal (1973a). In $\underline{C}$. jucunda the mesonotum is distinct $1 \bar{y}$ less shining than in $\underline{C}$. platyptera and there are also distinctive differences in the male genitalia.

Essential characters of the aedeagus of $\underline{C}$. jucunda are the short pair of anterior ventral sclerites, the fully fused, flaplike posterior sclerites, and the almost discrete basal sclerites, which have only a light membranous connection. The distiphallus is not solidly black, as appears when using only direct 1 ight (Steyska1, 1973a: fig. 1, e) but distinctly paler distally when seen in reflected light (figs. 884, 885). The mesophallus is slender adjoining the 
distiphallus (more so than indicated by Steyskal, loc. cit.), and the anterior part of the ventral sclerites is slightly divided apically. A male and female of $\underline{P}$. coronata, of which the male is now designated as lectotype, have been examined and this species (figs. 886, 887) is tentatively accepted as identical with the true $\underline{\text { C. }}$ jucunda.

C. platyptera, which occurs commonly in California and Florida, was misidentified as C. jucunda by Frick (1956: 288) and by spencer and Stegmaier (1973). The true distribution of the two species remains to be established either from larval leaf mines or from the examination of males, which will have been identified in the past as $\underline{C}$. jucunda.

C. menthae appears to be indistinguishable from $\underline{C}$. jucunda in external characters, and differences in the aedeagus of the two species are slight. In $\underline{\mathrm{C}}$. menthae (figs. 888, 889) the base of the distiphallus is longer and the distiphallus itself is also slightly longer. In the limited material seen the discal cell is slightly smaller. However, it is not impossible that $\underline{c}$. menthae will prove to be synonymous with C. jucunda, but more reared material is necessary to decide whether the differences noted are of specific significance or within the range of variation of $\underline{\mathrm{C}}$. jucunda.

Calycomyza menthae Spencer

(Figs. 888-890)

Calycomyza menthae Spencer, 1969a:

152. Holotype male from Ontario in CNC.

This species is known in the United States only from leaf mines on Hyptis mutabilis and Monarda punctata (Florida) and on Monardella sp. (California). The type series was reared from Mentha arvensis and Monarda fistulosa. The larva forms a large brownish blotch mine (fig. 890).

The squamal fringe appears to be variable. If it is considered as pale, ‥ menthae will be separable from C. jucunda only by the male genitalia, and differences here are slight (see figs. 884-889). If it appears dark, $\underline{\mathrm{C}}$. menthae will be difficult to separate from the group of eight small species (couplets 23-30), which can only be identified by the male genitalia.

New records.

California, Alpine Co./E 1 Dorado Co., summit Luther Pass, empty leaf mines on Monardella odoratissima, 1948 ( $\mathrm{K}$. E. Frick, Lot 104-1); Stanislaus Co., Del Puerto Canyon, young leaf mines on Monardella sp., 9.IV.78 (KAS).

Florida, Dade Co., Miami, leaf mines on Ocimum sp., 14.V.80 (DPI).

Calycomyza orientalis Spencer, new species

(Figs. 918, 919)

Head. Frons narrow, only slightly wider than eye; 2 ors, 2 ori, orbital setulae sparse, reclinate; gena at rear almost 0.25 height of eye; 3d antennal segment round, arista long, almost equal to height of eye.

Mesonotum. 2 strong dc, 3d minute, acr irregularly in 6 rows.

Wing. Length from $2.4 \mathrm{~mm}$ in male to $2.6 \mathrm{~mm}$ in female, last section of vein M 3+4 slightly more than twice length of penultimate.

Color. Frons bright yellow, orbit blackish from vertex to lower ors; antenna black; mesonotum deep black, shining; legs entirely black; squama yellow, margin and fringe dark.

Male genitalia. Aedeagus (figs. 918, 919) with mesophallus long, slender; rear ventral sclerites fully fused with long flaplike lobe, basal sclerites discrete; sperm pump with broad blade.

Host/early stages. Unknown. 
Holotype m., Virginia, Giles Co., Mountain Lake, 3,800 ft, 28.V.62; paratypes $2 \mathrm{~m} ., 1 \mathrm{f} .$, same data; (a11 J. G. Chillcott); Tennessee, Hamilton Co., Chapin Sanctuary, East Ridge, 1 m., 9.V.52 (0. Peck); New York, Long Is land, Farmingdale, 1 m., 26.VI. 36 (Blanton and Borders); Tompkins Co., Beaver Creek, McLean Reserve, $1 \mathrm{~m}$., 1.25.VIII (? collector); Suffolk Co., 1 m., 30.V.64 (M. I. Blenderman); Maine, Piscataquis Co., First Roach Pond, 4 m., 4 f., 4.VIII.76 (A. S. Menke). Holotype in $\mathrm{CNC}$, paratypes in $\mathrm{CNC}$, CUI, KAS, and UCD.

Remarks. Although this species somewhat resembles $\underline{C}$. artemisiae, there are many smali differences, in particular the more extensive darkening of the orbit, the more shining mesonotum, and the entirely black forefemur. The male genitalia suggest closer relationship with $\mathrm{C}$. cynoglossi and C. majuscula. There is no indication of the host plant, but with the wide distribution of the species and its large size the leaf mine must itself be large and conspicuous, and it should be found without difficulty with further collecting.

Leaf Mines of Unidentified Calycomyza Species

(Figs. 954, 955)

(1) Ageratum conyzoides: Florida, Gads den Co., nr. Quincy, 4.X.65 (KAS). An upper surface, irregular whitish blotch (fig. 954), pupation externally.

(2) Elephantopus elatus: Florida, Putnam Co., Camp Bell Ridge, 20 miles east of Gainesville, 8.X.68 (KAS), mostly recently vacated, few larvae still feeding. Linear during first larval instar, then developing into large, greenish white blotch (fig. 955). From size of puparia this is clearly the largest species in Florida and will almost certainly prove to be undescribed.

(3) Eupatorium rotundifolium: Florida,
Alachua Co., Gainesville, grounds of Division of Plant Industry, 5.X.68 (KAS), mostly recently vacated, few larvae still feeding. An upper surface whitish blotch, which possibly represents C. eupatorivora Spencer (in Spencer and Stegmaier, 1973: 184), from Jamaica.

\section{GENUS PHYTOLIRIOMYZA HENDEL}

Two new species are described and five new combinations are established. Three species are recorded as new to the United States.

Phytoliriomyza conspicua (Sehgal), new combination (new to U.S.A.) (Figs. 992-994)

Liriomyza conspicua Sehga1, 1968: 66; Spencer, 1969a: 171. Holotype male from Alberta in CNC.

The aedeagus of this species (figs. $992,993)$ is atypical of Liriomyza, and reexamination of the terminalia has shown that it correctly belongs in Phytoliriomyza, to which it is now transferred. The inner margin of the epandrium is clothed in numerous strong bristles at the rear, and there is a small subsidiary projection distally with a row of seven to eight bristles; the surstylus is large, with a number of bristles and many strong hairs (fig. 994).

P. conspicua is widespread in Canada from Ontario to Alberta. A single female can now be recorded from Michigan, Schoolcraft County, 24.VIII.52 (R. R. Dreisbach), in USNM, determined by GCS.

Phytoliriomyza consulta Spencer, new species

(Figs. 987, 988)

Head. 2 ors, 1 ori, orbital setulae sparse, proclinate; eye conspicuously slanting; gena extended at rear, 0.25 height of eye; $3 d$ antennal segment distinctly longer than broad, without conspicuous pubescence, arista long, longer than long axis of eye. 
Mesonotum. 3+1 strong dc, acr sparse, reduced to 2 isolated hairs in front.

Wing. Length from $1.5 \mathrm{~mm}$ in male to $1.6 \mathrm{~mm}$ in female, size of discal cell variable, last section of $\mathrm{M} 3+4$ from twice to less than 1.5 times length of penultimate.

Color. Frons yellowish brown, orbit paler, clearer yellow; gena, face, and palpus entirely yellow; 3d antennal segment brownish, 1 st and $2 d$ more yellow; mesonotum distinctly but faintly banded, central band brownish in front, more yellow adjoining margin of scutellum, 2 lateral bands darker, brownish gray, narrowly divided by yellow, essentially as in figure 983 (P. beckerella), scutellum yellowish gray; pleura predominantly yellow, mesopleuron with black band along lower margin, sternopleuron black in lower half; legs with femora brownish above, yellow below, tibiae and tarsi brownish; abdomen grayish but tergites yellow bordered; halter and knob darkened, brownish.

Male genitalia. Aedeagus (fig. 987) greatly reduced, ending in asymmetrical tubules; epandrium with 1 strong spine, several hairs and 2 shorter spines at lower corner ( $f$ ig. 988).

Host/early stages. Unknown.

Holotype m., North Carolina, Macon Co., Highlands, 3,800 ft, 6.V.57 (J. R. Vockeroth); paratypes North Carolina, Swain Co., Great Smoky Mountains National Park, Clingman's Dome, 6,300 ft, 1 f., 20.V.57 (J. R. Vockeroth); Wake Co., at light, $1 \mathrm{~m}$., 20.IV.49 (no collector recorded); Georgia, Rabun Co., Pine Mountain, 1,400 ft, 1 f., 4.V.57 (J. R.

Vockeroth). Holotype and 1 paratype in CNC, 1 paratype in CUI, 1 in KAS.

Phytoliriomyza dorsata (Siebke) (Figs. 1003-1005)

Agromyza dorsata Siebke, 1864: 169. Holotype male from Norway in Zoologisk Museum, Oslo.
Agromyza reverberata Malloch, 1924:

191. Holotype lost, paratype female from Maryland in CNC (synonymy established by Spencer, 1969a: 194). Phytoliriomyza dorsata, Spencer, 1976a: 208 .

This holarctic species has previously been confirmed only in California and Maryland, although records given by Frick (1959) from Illinois and Indiana of $\underline{P}$. pacifica probably refer to $\underline{P}$. dorsata.

Records from four other States are given here.

New records.

Louisiana, Rapides Co., 11 miles SW of Alexandria, 1 f., 26.III.60 (J. G. Chillcott), in CNC.

Michigan, Cheboygan Co., Mackinac Strait, 1 f., 3.VIII.10 (M. C. Van Duzee), in CAS.

Pennsylvania, Centre Co., State College, 1 f., 20.V.34 (s. W. Frost), in PSU.

Virginia, Giles Co., Strong Creek, $2,000 \mathrm{ft}, 2 \mathrm{f} ., 26 . V .62$ ( J. G. (hillcott), in CNC.

Phytoliriomyza felti (Malloch), new combination

(Figs. 989-991)

Agromyza felti Malloch, 1914b: 310. Holotype male from New York in NYSM. Liriomyza felti, Frick, 1952a: 403; 1959: 404; Spencer, 1969a: 297.

Examination of the terminalia of a male paratype in INHS shows that this species correctly belongs in Phytoliriomyza, to which it is now transferred.

The aedeagus (fig. 990) closely resembles that of $P$. pteridii Spencer (1973c: figs. 3, 4), a leaf miner on bracken in Europe. The bristles on the surstylus and on the hindcorner of the epandrium (fig. 991) clearly indicate the generic affiliation of $\underline{P}$. felti. Known hosts are the two ferns Asplenium pinnatifidum and Camptosorus rhizophy1lus. Frost (1924: pl. V, fig. 3) in- 
cluded a photograph of the mine on the latter host.

It is interesting to note that no Liriomyza is known as a feeder on ferns, but Phytoliriomyza species are known as such in the United States ( . clara, $\underline{P}$. pulchella), in Europe ( $\underline{P}$.

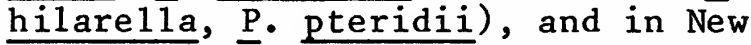
Zealand ( $P$. cyatheae, $P$. flavopleura, $\underline{P}$. tearohensis) (see Spencer, 1976b). The arrangement of the bristles in the epandrium of $P$. felti closely resembles that found in the three New Zealand species and clearly indicates relationships among this primitive group of fern feeders.

Phytoliriomyza fumicosta (Ma11och), new combination

(Figs. 1000-1002)

Agromyza fumicosta Malloch, 1914b: 310. Holotype female from Illinois in INHS.

Liriomyza fumicosta, Frick, 1952a: 402 ; 1959: 405 .

A male from Illinois, Champaign, 25.V.57 (J. F. McAlpine), in CNC, was examined in 1967, and it was then noted that the species correctly belonged in Phytoliriomyza, with the aedeagus similar to that of $\underline{p}$. dorsata and others in this group (which has been referred to as the mikii group in Europe). Five specimens in the CNC have been reexamined and the aedeagus and epandrium are shown in figures 1000-1002. A1though the darkening of the costal area may be distinct, it can also be almost imperceptible. The species may then be recognized by its small size and the lack of orbital setulae.

The data given here of four specimens "ex elm leaves" may be misleading. This does not mean that the specimens were reared from elm but merely that they were caught on the foliage. It is unlikely that $\mathrm{elm}$ is the host.

New records.

Illinois, Champaign, 2 m., 2 f., "ex elm leaves," 25.V.57 (J. F. McAlpine), in CNC.
Kansas, Douglas Co., Natural History Reserve, Lawrence, 1 f., 21.III.59 (J. G. Chill cott), in CNC.

Phytoliriomyza pallida (Sehga1), new combination (new to U.S.A.) (Figs. 995-996)

Lemurimyza pallida Sehgal, 1968: 72 . Holotype male from Alberta in CNC.

Only the male holotype has been known, from Banff, Alberta.

A single female from Montana, Madison County, 10 miles southeast of Eunis, 17.VIII.60 (D. R. Miller), in UCD, has now been seen that appears to represent P. pallida. The central yellow area adjoining the scutellum is slightly longer than indicated in the redescription (Spencer, 1969a: 195), but the specimens otherwise agree closely.

Lemurimyza was synonymized with Phytoliriomyza by Tschirnhaus (1971), and L. pallida is therefore now transferred to Phytoliriomyza.

Phytoliriomyza pulchella Spencer, new species

(Figs. 1010-1012)

Head (fig. 1010). Frons broad, twice width of eye, strongly projecting above eye in profile; normally 2 ors and 1 ori (this sometimes replaced by 2 weaker ones), orbital setulae lacking; gena extended at rear, 0.33 height of conspicuously slanting eye; $3 d$ antennal segment small, round, arista only finely pubescent.

Mesonotum. $3+1 \mathrm{dc}$, presutural sma11, acr in 4 rows.

Wing. Length $1.6-1.75 \mathrm{~mm}$, discal cell relatively large, last section of M 3+4 only 1.5 times length of penultimate.

Color. Head either entirely yellow or upper orbit darkened; mesonotum and scutellum uniformly matt gray; pleura yellow apart from large black triangle almost filling sternopleuron; legs bright yellow; squama and fringe 
yellow, margin dark; halter yellow.

Male genitalia. Aedeagus (fig. 1011) with distal tubules fused in lower 0.67 , curving dorsally and recurved at end with distal end enlarged; epandrium (fig. 1012) with comb of about 10 strong bristles at inner corner directed toward rear.

Host/early stages. Unknown, probably Pteridium aquilinum (bracken).

Holotype m., New York, Long Island, Farmingdale, 2.VI.35 (Blanton and Borders); paratypes $2 \mathrm{f}$., same data. Holotype and paratype in CUI, 1 paratype in KAS.

Remarks. This species closely resembles $\underline{P}$. clara but appears to be distinguishable by the larger discal cell and thus the shorter ratio of the last to penultimate sections of vein M $3+4$.

The aedeagus is intermediate between that of $\mathrm{P}$. clara, in which the distal tubules are substantially longer and only slightly enlarged at the end (fig. 1008), and $\underline{P}$. pteridii Spencer, $1973 c$, a leaf miner on bracken in Europe, in which the distal tubules of the aedeagus are not recurved and the terminal enlargement is more pronounced (Spencer, 1973c: figs. 3, 4).

Phytoliriomyza varia (Melander), new combination

(Fig. 1022)

Agromyza varia Melander, 1913: 264. Holotype female from Idaho in USNM. Phytobia (Trilobomyza) varia, Frick, 1957b: 203; 1959: 393.

The holotype of Agromyza varia has now been examined and it is transferred to Phytoliriomyza here.

P. varia is known from the unique female holotype. It is distinguishable from $P$. volatilis by the generally paler color, particularly the yellow femora. It is not distinguishable

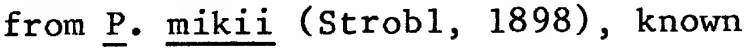

from the Austrian Alps and Denmark, and may be synonymous with it. However, formal establishment of this possible synonymy must be delayed until males from North America become available that can positively be associated with the holotype of $P$. varia. The aedeagus and epandrium of P. mikii (holotype) were illustrated by Spencer (1966a: figs. 30, 31). The sperm pump of a male from Denmark (col1. Tschirnhaus) is shown in figure 1022; the blade is characteristically broad and fanlike (contrast $P$. volatilis, figs. 1019-1021).

Phytoliriomyza volatilis Spencer (new to U.S.A.)

(Figs. 1016-1021)

Phytoliriomyza volatilis Spencer, 1969a: 202. Holotype male from British Columbia in CNC.

The type series consisted of four specimens and another male has now been seen from Colorado, Clear Creek County, Mt. Evans, Echo Lake, 10,600 feet, 23.VII.61 (C. H. Mann), in CNC.

Important characters of this species are the entirely black legs; the side of the thorax is predominantly black, but the lower half of the notopleuron and the upper half of the mesopleuron are yellowish. The male terminalia closely resemble those of $\underline{P}$. mikii, known from the Austrian Alps and also recently collected in Denmark

(Tschirnhaus). However, the surstylus is more slender and less widening distally and the distal tubules are slightly longer and more slender. The arrangement of bristles within the epandrium is similar in the two species (fig. 1018, male from Abisko, northern Sweden). The most obvious difference in the genitalia is the smaller and narrower blade of the sperm pump (figs. 1019-1021), which is broad and fanlike in $\underline{P}$. mikii ( $\mathrm{fig}$. 1022).

Specimens from Lapland in northern Sweden were previously considered to represent $\underline{\mathrm{P}}$. mikii, but the legs are 


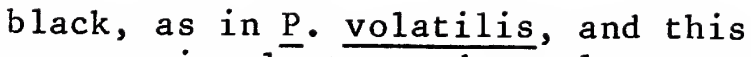
was previously treated merely as melanism (Spencer, 1976a: 299). However, it is now noted that the sperm pump had the blade narrow in these specimens, exactly as in the male from Colorado (fig. 1020). The Lapland population is therefore now considered to be identical with $P$. volatilis rather than with $\underline{\mathrm{P}}$. mikiki.

\section{GENUS PARAPHYTOMYZA ENDERLEIN}

One new species is described and one species is recorded as new to the United States. Two species are briefly discussed but not formally described in the absence of males.

\section{Paraphytomyza coloradensis Spencer,} new species

(Figs. 1042, 1043)

Head. Frons broad, twice width of eye, not projecting above eye in profile; 2 ors, 2 ori, lower ori substantially weaker (all missing, detectable only from basal pits); parafacial pronounced, gena angular, 0.33 height of eye; ( $3 d$ antennal segment missing on both sides).

Mesonotum. 4+2 dc, 4 th to 6 th small but well differentiated from acr, these in 4 rows.

Wing. Length in male $2.75 \mathrm{~mm}$, discal cell small, last section of M $3+44$ times length of penultimate.

Color. Frons largely bright yellow, somewhat darkened adjoining antennae; orbit and parafacial entirely yellow; gena and face grayish; 1st antennal segment yellowish, 2d gray, 3d (missing) almost certainly black; mesonotum and scutellum uniformly grayish black; humerus, notopleuron, and upper margin of mesopleuron yellow, pleura otherwise grayish black; legs largely black but all knees distinctively yellow; squama yellowish, margin and fringe dark.

Male genitalia. Aedeagus as in figures 1042, 1043; sperm pump with large rounded blade; surstylus large, rounded, with fringe of weak hairs.

Host/early stages. Unknown, almost certainly Lonicera.

Holotype m., Colorado, Clear Creek Co., Mt. Evans, Echo Lake, 10,600 ft, 23.VII.61 (C. H. Mann), in CNC.

Remarks. The male genitalia of this species indicate its relationship with $\underline{\mathrm{p}}$. lonicerina. The aedeagus of $\underline{P}$. coloradensis is entirely symmetrical, but in $\mathrm{P}$. lonicerina (fig. 1041) it is distinctively asymmetrical.

Paraphytomyza luteoscutellata (Meijere) (new to U.S.A.)

(Figs. 1044-1046)

Phytomyza lonicerae Brischke, 1881: 257. Types from Germany believed lost.

Phytagromyza luteoscutellata de Meijere, 1924: 143, nom. nov. for Phytomyza lonicerae Brischke, preoccupied by $P$. lonicerae Robineau-Desvoidy, 1851: 396. Phytagromyza xylostei (RobineauDesvoidy) sensu Hendel, 1932: 275. Paraphytomyza luteoscutellata, Spencer, 1969a: 206 .

The status of this species has been confused but was clarified by the synonymy given here when the species was first discovered in Canada (Spencer, 1969a).

P. luteoscutellata somewhat resembles $\bar{P}$. orbitalis but is generally paler. The scutellum is frequently, but not always, partially yellow, whereas in P. orbitalis it is consistently blackish.

Specimens have been seen from New York and Wisconsin, and leaf mines have been found in Minnesota. The species is probably widespread in the Northeastern States.

New records.

Minnesota, Minneapolis, Minnehaha 
Creek, leaf mines on Lonicera canadensis, 20.V.79 (KAS).

New York, Madison Co., Brookfield, 1 m., 2 f., 27.VIII.30 (C.U.

Expedition), in CUI.

Wisconsin, Dane Co., Madison, 4 m., 5

f., ex Lonicera morrowii, 13.IX.76

(S. Tavormina).

\section{Unnamed Species No. 1}

Kansas, Douglas Co., Lawrence, Natural History Reserve, 1 f., 28.IV.67 (J. G. (hillcott), in CNC.

Frons and all antennal segments brownish orange, humerus and notopleuron yellowish, all knees bright yellow, and outer crossvein present. This species somewhat resembles $\underline{P}$. lonicerina but is clearly distinct. Wing length in the only available specimen is $2.5 \mathrm{~mm}$.

Unnamed Species No. 2

(Fig. 1050)

Montana, Lake Co., Polson, 1 f., ex leaf mine on Lonicera columbiana (sp. dubium), 17.VII.67 (S. Whitney), in KAS .

This specimen was referred to by Spencer (1969a: 209) as Paraphytomyza sp. (Pelee). Numerous empty mines were found at Point Pelee in southern Ontario on L. dioica, 15.VII.67 (KAS), which appeared to be identical to those from Montana (fig. 1050). The single female seen somewhat resembles P. orbitalis, but it is larger, with the frons and notopleuron orange yellow, and there are conspicuous yellowish patches at the hindcorners of the mesonotum. The species is certainly undescribed, but the formal description is best delayed until a male becomes available.

\section{GENUS NAPOMYZA WESTWOOD}

Two new species are described, one new combination is established, the status of N. nugax is discussed, and one species is recorded as new to the United States.
Napomyza grande11a Spencer, new species (Figs. 1062-1064)

Head (fig. 1062). Frons exceptionally broad, almost 3.5 times width of eye, strongly projecting above eye in profile; 2 ors, 2 ori; gena broad, slightly more than 0.5 height of eye, parafacial also broad and pronounced; $3 d$ antennal segment quadrate, with fringe of fine pubescence, arista distinctly swollen at base.

Mesonotum. 3+1 strong, dc, acr irregularly in 2-3 rows.

Wing. Length $3.5 \mathrm{~mm}$ in male, $4 \mathrm{~mm}$ in female, $2 d$ costal section slightly longer than $4 \mathrm{th}$, in ratio $7: 6$, discal cell minute, outer crossvein in continuation of inner.

Color. Frons, orbit, gena, and face yellow; hindmargin of eye black to base of vti; antenna and palpus black; mesonotum and scutellum ash gray; side of thorax grayish black, upper margin of mesopleuron narrowly yellow; legs black, femora bright yellow distally for slightly more than femoral diameter; abdomen largely black, but all tergites narrowly yellow bordered; squama yellow, fringe black.

Male genitalia. Aedeagus (fig. 1063) with end of distiphallus large, heavily pigmented; sperm pump (fig. 1064) with blade small and base weakly sclerotized at each end.

Host/early stages. Unknown.

Holotype m., Wyoming, Fremont Co., Union Pass Road, 8,500 ft, 17.VII.61 (B. H. Poole); paratype f., Arizona, Coconino Co., Hart Prairie, 10 miles NNW of Flagstaff, 1.VII.64 (R. W. Poole). Holotype in CNC, paratype in CUI.

Remarks. This species is distinctive in having the third antennal segment pubescent and can be distinguished from N. plumea, which also has this character, by the exceptionally large size. 
Napomyza manni Spencer, new species (Figs. 1067, 1068)

Head. Frons 1.5 times width of eye, broadly projecting above eye in profile; 2 ors, 2 ori, orbital setulae in single row near eye margin and some additional hairs at broadest point of orbit near lower ori; gena extended at rear, just less than 0.5 height of eye; 3d antennal segment flat above, almost angular at upper corner.

Mesonotum. $3+1 \mathrm{dc}$, acr sparse, in 2 rows.

Wing. Length in male $2.75 \mathrm{~mm}, 2 \mathrm{~d}$ costal section only slightly longer than $4 \mathrm{th}$, in ratio $7: 6$.

Color. As in N. grandella.

Male genitalia. Aedeagus (fig. 1067) with distiphallus elongate, adjoined by narrow process enlarged basally; sperm pump (fig. 1068) with large blade and base strongly sclerotized at each end.

Host/early stages. Unknown.

Holotype m., Colorado, Clear Creek Co., Loveland Pass, 7.VII.61 (C. H. Mann), in CNC.

Remarks. The elongate distiphallus suggests close relationship with $\mathrm{N}$. plumea (fig. 1065), but the two species are readily distinguishable by the different form of the third antennal segment.

Napomyza marginalis (Frost), new combination

(Figs. 1076, 1077).

Phytomyza marginalis Frost, 1927:

219. Holotype female from New York in USNM.

Examination of the female holotype and a male paratype with the same data shows that this species is close to, if not identical with, Napomyza nigritula (Zetterstedt) from Sweden. A more familiar name is $\underline{N}$. cineracea
(Hendel), which was synonymized with N. nigritula by Spencer (1976a: 341). The species is known under this name from much of Europe and was also recorded in Canada (Spencer, 1969a: 235, as Phytomyza).

Although Frost described the frons as yellow, it is normally distinctly darker, at least ochreous yellow and ranging from pale to darker brown. Frick (1959: 431) referred to the frons as "dark."

It is not proposed at this stage formally to synonymize $\mathrm{N}$. marginalis with $N$. nigritula, as it is possible that $a$ complex of two or more species is involved. The only apparent difference is in the form of the distiphallus, which is virtually straight in N. marginalis (figs. 1076, 1077) but distinctly curving in the population from Alberta (see Spencer, 1969a: fig. 415). An intermediate form has been seen from England, Wiltsshire, Gastard (KAS), in BMNH. In one male of Hendel's type series from Hungary ("Aust." in Hendel's sense) (genitalia slide 5204, in NMW), the paramesophalli are shorter and more rounded, although the distiphallus itself is straight. This specimen is not designated as lectotype, pending examination of all males in Hendel's type series of nine specimens.

The species is widespread in Scandinavia, central Europe, and Britain and has been recorded from Iceland (Griffiths, 1968). Specimens have been reared from Ranunculus stems in Europe, Ontario, and Alberta. No biological differences between the populations have been detected.

Further clarification of this complex will require more detailed study than is possible at present, and an additional species that will require examination is $\underline{P}$. nigrigenis Hering, 1937, from Germany.

New records.

New York, Tompkins Co., Slaterville Wild Flower Preserve, $1 \mathrm{~m} ., 27 . \mathrm{V} .38$ (PSU). 
Pennsylvania, Luzerne Co., Hazelton, 1 m., 10.V.10 (Dr. Dietz coll., PSU).

Napomyza nugax Spencer

(Figs. 10 $\overline{71-1073 \text { ) }}$

Napomyza nugax Spencer, 1969a: 215. Holotype male from Ontario in CNC.

This species is widespread in Canada, and two specimens can now be recorded from Colorado and North Carolina. The aedeagus of the male from North Carolina agrees exactly with that of the holotype (fig. 1071), although the specimen itself is unusually large, with a wing length of $3.3 \mathrm{~mm}$.

The status of a male from California, Alpine County, Hope Valley, 8.VIII.48, Lot $91-24(\mathrm{KEF})$, in CAS, has been in doubt. At the time of the description of N. nugax it was labeled as a paratype but was not included as such in the description. This may have been inadvertent but was possibly deliberate, as the aedeagus ( $\mathrm{fig}$. 1072), though resembling that of $\mathrm{N}$. nugax, appears to be not identica $\bar{l}$ with it. It seems undesirable to treat this single specimen as representing a distinct species, and it is tentatively referred to $N$. nugax pending further clarification when additional material becomes

available.

New records.

Colorado, Boulder Co., Nederland, $8,500 \mathrm{ft}, 1 \mathrm{~m}$., 18.VI.61 (B. H. Poole), in CNC.

North Carolina, Yancey Co., Mt. Mitchel1, 6,500-6,684 ft, $1 \mathrm{~m}$., 5.VI.62 (J. R. Vockeroth), in CNC.

Napomyza suda Spencer (new to U.S.A.)

Napomyza suda Spencer, 1969a: 217. Holotype male from Ontario in CNC.

This is an exceptionally large, distinctive species, with a wing length of 4-5 $\mathrm{mm}$. The notopleuron, the upper half of the mesopleuron, and the squamal margin and fringe are yellow. It is distinguishable from $N$. pallens by the scutellum being entirely black.

New records.

Michigan, Allegan and Midland Cos.

South Dakota, Brookings Co.,

Brookings, 1 f., 17.V.12 (H.

Severing), in PSU.

\section{GENUS PHYTOMYZA FALLEN}

Sixteen new species are described, 1 new synonym is established, and 11 species are recorded as new to the United States and 1 as new to North America. A lectotype is designated of $\underline{P}$. flavinervis, and two species are diagnosed as probably new but are not formally described in the absence of males. The first male is recorded of P. auricornis, and illustrations are given of the genitalia. P. nigripennis Fallén, recorded by Frick (1959), is deleted from the United States list, representing a misidenti-

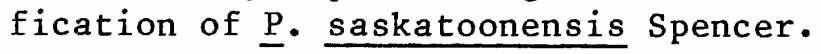

Phytomyza aldrichi Spencer, new species (Figs. 1100, 1101)

Head. Frons twice width of eye, not projecting above eye in profile; 1 strong ors, directed slightly outward, 2 equal, reclinate ori, orbital setulae sparse; gena about 0.33 height of eye; $3 \mathrm{~d}$ antennal segment slightly longer than broad.

Mesonotum. 3+1 strong $\mathrm{dc}$, acr in 4 rows.

Wing. Length in male $2 \mathrm{~mm}, 2 \mathrm{~d}$ costal section 2.2 times length of $4 \mathrm{th}$.

Color. Frons yellow, orbit slightly paler; vti on yellow, hindmargin of eye black, vte at border of black and yellow; gena and face yellow; palpus, $2 \mathrm{~d}$ and $3 \mathrm{~d}$ antennal segments black, 1 st yellow; mesonotum matt grayish black, scutellum largely similar but faintly yellow between basal scutellars; legs largely black but coxae on foreleg and midleg yellow, all knees distinctly yellow; squama yellowish, margin and fringe dark, brownish. 
Male genitalia. Aedeagus (fig. 1100) with distiphallus directed dorsally, divided (fig. 1101), supported basally by pair of broad sclerites that taper ventrally; paramesophalli directed forward, lying in same plane as basal sclerites, becoming membranous apically; sperm pump with large, rounded blade and narrow stalk.

Host/early stages. Unknown.

Holotype m., Idaho, Latah Co., Moscow, no date (J. M. Aldrich), in USNM.

Remarks. This species superficially resembles the dark form of $P$. ranunculi and was tentatively identified by Frick as $\underline{P}$. ranunculi var. praecox. However, it is distinctly smaller, the costal ratio is less, and the male genitalia show that the two species are not related.

Phytomyza arnicicola Lundquist (new to U.S.A.)

(Figs. 1247, 1365)

Phytomyza arnicicola Lundquist, 1949: 173. Holotype male from Swedish Lapland in ZIL.

This species is host specific on Arnica and has hitherto been known from northern Sweden, Alaska, and Alberta. Leaf mines on Arnica chamissonis ssp. foliosa, possibly referable to $\underline{P}$. arnicicola (fig. 1365), were found in Nevada County, Calif. (Spencer, 1981: 457).

Two specimens recorded here have been identified from Colorado. Although the aedeagus of these two specimens is virtually identical to that of the holotype (fig. 1247) and that illustrated by Griffiths (1974b: fig. 5) from Alaska, the venation of the Colorado population differs in the ratio of the second to fourth costal sections, which is appreciably less than in the Swedish and Alaskan populations (although only single specimens have been seen from each area). In these the costal ratio is approximately 2.5, whereas in the two Colorado specimens it is 2.25 and
1.5. With the limited material available, this difference can only be treated as local variation.

It seems probable that $\underline{P}$. arnicae Hering, 1925 (see Spencer, 1976a: 384), which is present in the mountains of central Europe and at Jasper, Alberta, in the Canadian Rockies (Griffiths, 1974b: 109), may also be present at high elevations in the United States.

New records.

Colorado, Lake Co., Independence Pass, $11,100 \mathrm{ft}$, tundra, $1 \mathrm{~m} ., 31$.VIII.61

(J. G. Chillcott); Larimer Co., Estes Park, 7,500 ft, $1 \mathrm{~m} ., 2$.VII.61 (C. H. Mann); both in CNC.

\section{Phytomyza asterophaga Spencer (new to U.S.A.)}

(Figs. 1226-1228)

Phytomyza asterophaga Spencer, 1969a: 230. Holotype male from Alberta in CNC.

This species has been known previously only from central Alberta, and according to Griffiths (1976b: 244) it is common around the city of Edmonton on its only known host, Aster conspicuus. A probable sister species, P. ciliolati Spencer (1969a: 234), feeds on other Aster species and may be found in the United States. Differences between the two species are slight, with the notopleuron frequently somewhat paler in $P$. ciliolati and the distiphallus with even weaker pigmentation (Griffiths, 1976b: fig. 4).

New record. North Carolina, Macon Co., Highlands, $3,000 \mathrm{ft}, 5 . \operatorname{VIII} .57$ (J. G. Chillcott), in CNC.

Phytomyza astotinensis Griffiths (new to U.S.A.)

(Figs. 1238-1240)

Phytomyza astotinensis Griffiths, 1976b: 248 . Holotype male from Lake Astotin, Alberta, in CNC. 
This species is a 1 inear miner on Solidago, recorded hosts being $\underline{S}$. canadensis and S. gigantea, and it has been known only from Alberta.

It forms part of a closely related group described from Alberta, including also $\mathrm{P}$. solidaginovora Spencer, 1969a Thost Aster modestus, rather than Solidago sp., according to Griffiths, 1976b: 246); P. phalangites Griffiths, 1976b (Aster spp.); . solidaginophaga Sehga1, 1971 (Sölidago lepida); . asterophaga Spencer, 1969a (Aster conspicuus); and $P$. ciliolati spencer, 1969a (Aster cīiolatus and other Aster spp.).

Griffiths (1976b) reviewed species mining Asteraceae and illustrated the genitalia of the species mentioned here. A male and female reared from Solidago flexicaulis, 20.IX.76, in Wisconsin, Dane County, Madison, are tentatively identified as $\underline{P}$. astotinensis. The aedeagus (figs. 1238, 1239) is not identical with that figured by Griffiths (1976b: fig. 13), with more spinules along the right basal sclerite and the angle of the divided distiphallus somewhat different. However, it seems undesirable to add yet another name to this complex without additional material, particularly from intermediate areas between Alberta and Wisconsin.

Phytomyza auricornis Frost

(Figs. 1141, 1142)

Phytomyza auricornis Frost, 1927: 217. Ho lotype female from New York in USNM.

The unique holotype has been the only specimen known of this distinctive species. A male, also from New York (CUI), has been identified and the aedeagus is illustrated in figures 1141,1142 . The form of the genitalia indicates that the species is related to feeders on Ranunculaceae ( $\underline{P}$. albomargo Hering, $\underline{P}$. calthophila

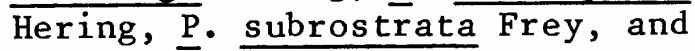
others) (see Spencer, 1976a: figs. $388,650,692$,$) , and it is reasonable$ to assume that the host is in this family.
Phytomyza bouldere11a Spencer, new species

(Figs. 1229, 1230)

Head. Frons twice width of eye, not projecting above eye in profile; orbital bristles irregular in only available specimen, 2 ors on one side, upper broken, but appearing weaker than lower, 1 only on other side, 2 or $i$ on one side, 1 only on other, orbital setulae sparse; gena about 0.33 height of eye; $3 d$ antennal segment rounded, rather large.

Mesonotum. Acr sparse, in 2 rows.

Wing. Length in male $2.1 \mathrm{~mm}, 2 \mathrm{~d}$ costal section short, about 1.5 times length of $4 \mathrm{th}$.

Color. Frons orange yellow, entire orbit pale gray, hindmargin of eye black; 3d antennal segment black, 1st and $2 \mathrm{~d}$ somewhat paler, slightly yellowish; mesonotum and scutellum matt gray, side of thorax dark, apart from narrow yellow upper margin of mesopleuron; legs black but all knees bright yellow; squama, including margin and fringe, yellow.

Male genitalia. Aedeagus as in figures 1229,1230 ; sperm pump with large, asymmetrical blade.

Host/early stages. Unknown.

Holotype m., Colorado, Boulder Co., 4 miles NW of Boulder, Ponderosa pine, $6,900 \mathrm{ft}, 8 . \mathrm{VI} .61$ (C. H. Mann), in CNC.

Remarks. This species is distinctive in having the upper ors shorter than the lower and the squamal fringe yellow. It can be compared only with p. flexuosa, n. sp., with which it is not related, but there are clear differences between the two species.

Phytomyza ceanothi Spencer, new species (Figs. 1146, 1147)

Head. Frons twice width of eye, not projecting above eye in profile; 2 equal ors, 1 strong ori, orbital 
setulae sparse, in single row; gena broad, extended at rear, 0.4 height of eye; 3d antennal segment slightly longer than broad, with long, upcurved pubescence.

Mesonotum. $3+1$ slender, $\mathrm{dc}, \mathrm{acr}$ irregularly in 3-4 rows in front.

Wing. Length from $1.3 \mathrm{~mm}$ in male to $1.75 \mathrm{~mm}$ in female, $2 \mathrm{~d}$ costal section 2.5 times length of $4 \mathrm{th}$.

Color. Frons, orbit, gena, face, and palpus yellow; hindmargin of eye black; 3d antennal segment black, 1st and 2d paler, yellowish; mesonotum, scutellum, and side of thorax gray; legs blackish gray, femora with all knees yellow; abdomen largely dark but tergites somewhat yellowish laterally; squama and fringe yellow, margin dark.

Male genitalia. Aedeagus as in figures 1146, 1147; sperm pump minute, blade round, scarcely pigmented.

Host/early stages. Ceanothus sp. ("buckbrush"). Larva forming 1 inear mine that appears as blotch virtually filling small leaves, pupating in leaf; puparium yellowish, posterior spiracles on widely separated projections, each with ellipse of about 8 bulbs.

Holotype m., Kansas, Riley Co., Manhattan, ex Ceanothus, 27.V.39; paratypes $1 \mathrm{~m} ., 1 \mathrm{f}$. (without head), 28.V. and 10.VI.39, otherwise same data; (a11 C.W. Sabrosky). Holotype presented to USNM, paratypes in PSU.

Remarks. This is the only leaf miner known on any genus of the Rhamnaceae, and the male genitalia confirm that it occupies an isolated position.

\section{Phytomyza chelonei Spencer (new to} U.S.A.)

(Figs. 1167, 1168)

Phytomyza chelonei Spencer, 1969a: 234. Holotype male from Quebec in CNC.

This species has been known only from the unique holotype. Five specimens can now be recorded from Indiana, North Carolina, and Tennessee.

The aedeagus of the United States specimens agrees exactly with that of the holotype (figs. 1167, 1168).

Essential characters of the adult are projecting yellow frons; 2 equal ors; acr in 4 rows; femora black with yellow knees and yellow squamal fringe. These are also identical in the two populations. The only detectable difference appears to be the color of the forecoxa, which is yellow in the holotype but predominantly dark in the United States specimens. Some variation in the color of this part of the leg is not considered to be of great significance. Wing length of the female, which has not previously been known, is $3.25 \mathrm{~mm}$.

New records.

Indiana, Tippecanoe Co., Lafayette, 1 m., 3.V.18 (co11. not recorded), in PSU.

North Carolina, Macon Co., Highlands, $1 \mathrm{~m} ., 21 . \operatorname{VIII.57}$ (W. R. Richards); 1 f., same data (J. G. Chil1cott); Mt. Mitche11, 6,800 ft, $1 \mathrm{~m} ., 12$.VIII. 57 ( J. G. Chillcott); in CNC.

Tennessee, Sevier Co., Great Smoky Mts. N. P., Indiana Gap to Clingman's Dome, 5, 200-6,600 ft, 1 m., 6.VIII.57 (J. G. Chi11cott), in CNC.

Phytomyza clematisella Spencer, new species

(Figs. 1319, 1320)

Head. Frons twice width of eye, not projecting above eye; 1 reclinate ors, 2 equal, inclined ori that are slightly weaker than ors, orbital setulae minute, sparse; gena 0.33 height of eye; $3 d$ antennal segment rounded, only slightly longer than broad.

Mesonotum. $3+1$ strong dc, acr numerous in front, in 4-5 rows.

Wing. Jength in male $1.75-1.9 \mathrm{~mm}, 2 \mathrm{~d}$ 
costal section twice length of $4 \mathrm{th}$.

Color. Frons brownish yellow, darker toward lunule; orbit yellowish gray, darker above ors; gena and face dull yellow, palpus black; 3d antennal segment black, 1 st and $2 \mathrm{~d}$ paler, somewhat yellowish; mesonotum, scutellum, side of thorax matt gray; legs black but knees narrowly yellowish, more definitely so on foreleg; abdomen largely black, tergites variably yellow bordered; squama and fringe yellow.

Male genitalia. Aedeagus (figs. 1319, 1320) with distiphallus divided into slender, paired, rather long tubules; mesophallus broad, short, paramesopha11 i well developed, diverging, ventral lobe strong1y sclerotized above, membranous ventrally; sperm pump weakly pigmented, with narrow blade.

Host/early stages. Unknown.

Holotype m., Arkansas, Garland Co., Hot Springs, Recreation Ground, 17.V.79; paratypes $5 \mathrm{~m}$., same data (K.A.S.); Connecticut, Litchfield Co., Sharon, "stem Clematis," 1 m., 1 f., 8.VII.50 (E. 0. Thornton). Holotype and paratypes in USNM, other paratypes in KAS and PSU.

Remarks. The male genitalia of this species resemble but are obviously distinct from those of two stem miners described from Clematis recta L. in Europe by Hering (1958), Phytomyza clematidicaulis and $\underline{P}$. novitzkyi. The aedeagus of the latter was illustrated by Spencer (1969a: fig. 548).

The two specimens from Connecticut are in poor condition; the male lacks all orbital bristles and the female lacks its head and most of both wings. However, with the data label "stem clematis" and the clear affinity with the two European enecies, the larva can be assumed with certainty to be a stem feeder on Clematis.

In view of the poor condition of the two specimens caught on Clematis (or possibly reared), with the aedeagus of the male being identical with that of the six specimens caught in Arkansas, it has seemed justified and preferable to select as holotype one of this series. These specimens were caught on vegetation beside the small creek flowing through the Recreation Ground at Hot Springs, Ark. Ranunculus was present and was originally thought to be the host. However, clematis was presumably present beside the creek--to the left of the steps leading down from the parking area--growing along the bank on the far side of the creek, which is less than a meter in width.

Phytomyza clematoides Spencer, new species

(Figs. 1217-1219)

Head. Frons twice width of eye, not significantly projecting above eye in profile; 1 strong reclinate ors, 2 inclined ori, the lower weak, orbital setulae sparse, proclinate; gena extended at rear, almost 0.5 height of eye; 3d antennal segment rounded, slightly longer than broad.

Mesonotum. $3+1$ strong $\mathrm{dc}$, acr in 4 rows in front.

Wing. Length from $1.6 \mathrm{~mm}$ in male to $1.9 \mathrm{~mm}$ in female, $2 \mathrm{~d}$ costal section 1.4 (m.) to 1.6 ( $f$. ) times length of 4 th.

Color. Frons, gena, and face yellow; hindmargin of eye black, with vti just on black ground, which extends down upper orbit to level of ors; palpus and antenna black; mesonotum shining black, scutellum black but less shining, side of thorax black; legs black, with knees yellow; squama yellowish gray, margin and fringe brownish black; abdomen black.

Male genitalia. Aedeagus (figs. 1217, 1218) with distiphallus directed dorsally to basal sclerites, slightly divided at apex; strong central sclerite attached to apical ends of basal sclerites, curving dorsally (see $\underline{\mathrm{P}}$. $\underline{\text { loewii }}$ and $\underline{\mathrm{P}}$. ranunculoides, figs. 
1332, 1326); sperm pump minute, unpigmented.

Host/early stages. Clematis ligusticifolia. Larva forming upper surface linear mine (fig. 1219) with frass in line of fine grains, pupating externally.

Holotype m., Washington, Benton Co., Prosser, emerged 19.IV.52 ex leaf mine on Clematis ligusticifolia (K. E. Frick, Lot No. 51-39); paratype f., same data; both in CAS.

Remarks. Distinctive characters of this species are the single strong ors, the shining black mesonotum, and the short second costal section. The male genitalia indicate a relationship with the two black species feeding on Clematis ( . . loewii) and Ranunculus (‥ ranunculoides, n. sp.).

Phytomyza coloradella Spencer, new species

(Figs. 1201, 1202)

Head. Frons twice width of eye, not projecting above eye in profile; 2 equal ors, 1 strong ori, orbital setulae sparse, in single row; gena extended at rear, almost 0.5 height of eye, parafacial prominent, filling half distance between lower eye margin and lower margin of gena; 3d antennal segment round, slightly enlarged.

Mesonotum. $3+1 \mathrm{dc}$, acr in 2 rows.

Wing. Length in male $2.5 \mathrm{~mm}, 2 \mathrm{~d}$ costal section twice length of $4 \mathrm{th}$.

Color. Frons yellowish orange; upper orbit black to lower ors, faintly infuscated below, entire hindmargin of eye dark; face and gena yellow, palpus black; 3d antennal segment black, 1st and 2d paler, yellowish; mesonotum and scutellum matt gray, side of thorax similar, apart from narrow yellow upper margin of mesopleuron; legs with forecoxa yellowish distally, femora black, with knees faintly and narrowly yellow; squama gray, margin and fringe black.
Male genitalia. Aedeagus (figs. 1201, 1202) with distiphallus extended into membranous tubule; ventral sclerite large but weakly developed on right side, basal sclerites fused; sperm pump with small, pale blade and extended base on right side.

Host/early stages. Unknown.

Holotype m., Colorado, Lake Co., Independence Pass, 12,100 ft, tundra, 7.VIII.61; paratype m., Clear Creek Co., Mt. Evans, Summit Lake Flats, 12,800 ft, 24.VII.61; (both C. H. Mann), both in CNC.

Remarks. Al though generally resembling $P$. alamedensis, this species is distinguishable by the yellowish forecoxa. The male genitalia indicate that the two species are not closely related.

Phytomyza coquilletti Spencer, new species

(Figs. 1117, 1118)

Head. Frons broad, twice width of eye, strongly projecting above eye in profile; 2 equal, reclinate ors, 2 longer, inclined ori, orbital setulae sparse, more numerous in area of or $i$; gena extended at rear, slightly more than 0.5 height of eye, parafacial pronounced, 0.5 width of gena; $3 d$ antennal segment distinctly longer than broad.

Mesonotum. $3+1$ strong $d c$, acr in 2 rows.

Wing. Length from $3.2 \mathrm{~mm}$ in male to $3.5 \mathrm{~mm}$ in female, $2 \mathrm{~d}$ costal section twice length of $4 \mathrm{th}$.

Color. Head entirely yellowish orange, including hindmargin of eye; mesonotum and scutellum ash gray; humerus and notopleuron yellowish orange, mesopleuron appearing pale but slightly suffused with gray; legs with coxae and femora yellowish orange, tibiae and tarsi slightly darker, more brownish; abdomen predominantly yellowish; squama and margin yellowish, fringe ochreous. 
Male genitalia. Aedeagus as in figures 1117, 1118; sperm pump minute, pale.

\section{Host/early stages. Unknown.}

Holotype m., Illinois, McHenry Co., Algonquin, 4-30-94-34; paratypes $1 \mathrm{~m}$., same data; $1 \mathrm{~m} ., 5-3-95-109 ; 1 \mathrm{~m}$. , 5-9-94-39 / 2287; $1 \mathrm{~m}$. (without head), 4-30-94-34 / 2268; 1 f., 4-30-94-34 / 2296; 1 f., 4-30-94-33; 1 f., 5-8-94; (all coll. Coquillett). Holotype and paratypes in USNM, $1 \mathrm{~m}$. paratype in KAS.

Remarks. This species closely resembles $P$. genalis but is distinguishable by the narrower gena, the larger eye, and the paler abdomen. The male genitalia do not suggest that the two species are closely related.

Phytomyza duplex Spencer, new species (Figs. 1085-1087)

Head. Frons 1.5 times width of eye, not projecting above eye in profile; 2 ors, upper only marginally weaker, 2 ori, lower substantially weaker, orbital setulae short, sparse, proclinate; gena 0.33 height of eye; $3 \mathrm{~d}$ antennal segment rounded, little longer than broad; palpus normal (not elongated).

Mesonotum. $3+1$ strong $d c$, acr in 4 rows in front, reduced to 2 rows at level of $2 \mathrm{~d} \mathrm{dc}$.

Wing (fig. 1085). Length in male 2.5 $\mathrm{mm}$, outer crossvein present, slightly beyond inner, $2 \mathrm{~d}$ costal section 3.33 times length of $4 \mathrm{th}$.

Color. Frons, lunule, gena, and face yellow; upper orbit grayish to level of lower ors, both vt on dark ground; antenna black; mesonotum and scutellum matt gray, without subshine; pleura blackish gray; legs black, knees distinctly yellow; squama and fringe yellow.

Male genitalia. Aedeagus as in figures 1086, 1087, distiphallus divided, with characteristic curvature; sperm pump with large, broad blade, base strongly sclerotized at each end.

Host/early stages. Unknown.

Holotype m., Tennessee, Hamilton Co., East Ridge, 9.V.52 (G. S. Walley), in CNC.

Remarks. Although this species appears to be identical in external characters to $\underline{P}$. davisii, differences in the aedeagus confirm that it is distinct.

Two other species in this complex with the outer crossvein present are known in Europe, $\underline{P}$. rydén $i$ Hering (Ranunculus) and P. aconitophila Hendel (Aconitum). The former is distinguishable by the elongate proboscis, the latter by the distinctly pubescent third antennal segment. In both, the aedeagus is of the same form as in $\underline{P}$. duplex and $\underline{P}$. davisii, differing primarily in the slight but distinctive curvature of the distipha1lus (Spencer, 1976a). It is reasonably certain that the host is in the Ranunculaceae.

Phytomyza erigerophila Hering (new to U.S.A.)

(Fig. 1216)

Phytomyza erigerophila Hering, 1927: 174. Two female syntypes from Germany (Berlin) in ZMHU.

This species is widespread but local in Europe and was recorded from Alberta by Griffiths (1976b: 256).

A single male has now been seen from Tennessee, Sevier County, Great Smoky Mountains National Park, Indian Gap, 5,700 feet, 20.V.57 (J. R. Vockeroth), in $\mathrm{CNC}$.

The male genitalia are distinctive (fig. 1216), with the left basal sclerite greatly reduced and a row of strong spinules along the right sclerite from the termination of the left sclerite to the distal end. The 
distiphallus is entirely membranous and unpigmented (not shown in fig. 1216). This is illustrated by Griffiths (1976b: fig. 25) though probably somewhat formalized.

Phytomyza evansi Spencer, new species (Figs. 1351, 1352)

Head. Frons twice width of eye, narrowly projecting above eye, orbit strongly differentiated; 2 ors, upper shorter (1acking in 1 of 8 specimens), 2 ors, upper equal to lower ors, lower greatly reduced; gena broad, somewhat more than 0.33 height of eye, parafacial prominent; 3d antennal segment rounded, slightly longer than broad.

Mesonotum. $3+1 \mathrm{dc}$, acr sparse, in 2 rows.

Wing. Length from $2 \mathrm{~mm}$ in male to 2.3 $\mathrm{mm}$ in female, 2d costal section short, 1.5-1.7 times length of $4 \mathrm{th}$.

Color. Frons blackish below, more brownish above and beside ocellar plate; orbit blackish gray, narrowly yellow along inner margin; gena dark brown, face, palpus, and antenna black; mesonotum and scutellum matt blackish gray, side of thorax entirely dark; legs black but forefemur narrowly yellowish, on other legs less so; squama gray, margin and fringe black.

Male genitalia. Aedeagus (figs. 1351, 1352) with row of long spinules along left basal sclerite; distiphallus membranous basally, more pigmented from midpoint, 2 tubules recurved basally but finally directed dorsally; ventral lobe swung forward, strongly sclerotized; sperm pump large, with broad, pale blade.

Host/early stages. Unknown.

Holotype m., Colorado, Clear Creek Co., Mt. Evans, 14,000 ft, 25.VII.61 (B. H. Poole); paratypes $4 \mathrm{~m}$., 2 f., same data (B. H. Poole and J. G. Chillcott); Mt. Evans, Summit Lake Flats, 12,800 ft, 1 m., 24.VII.61 (C.
H. Mann). Holotype and paratypes in $\mathrm{CNC}, 2$ paratypes in KAS.

Remarks. The male genitalia indicate that this species belongs to the $P$. albiceps group (see Griffiths, 19776b), which includes leaf miners on Apiaceae and Asteraceae having the frons either yellow or dark. A distinctive character is the arrangement of spinules beside the basal sclerites. Although closely resembling $\underline{P}$. aralivora, the two species are not closely related.

Phytomyza flavens Spencer, new species (Figs. 1192, 1193)

Head. Frons broad, almost 3 times width of eye, distinctly projecting above eye in profile; 2 equal ors, 2 strong ori, orbital setulae strong, irregularly in 2 rows; gena extended at rear, slightly more than 0.5 height of eye, parafacial forming broad ring below eye; 3d antennal segment small, round, with distinct, though short pubescence.

Mesonotum. $3+1 \mathrm{dc}$, acr irregularly in 4 rows.

Wing. Length in male $3.25 \mathrm{~mm}, 2 \mathrm{~d}$ costal section twice length of $4 \mathrm{th}$.

Color. Frons, gena, face, 1 st and $2 \mathrm{~d}$ antennal segments, and entire hindmargin of eye bright yellow; 3d antennal segment and palpus black; mesonotum and scutellum matt gray, side of thorax similar, with only upper margin of mesopleuron narrowly yellow; legs black, knees bright yellow; abdomen largely matt blackish gray but all tergites conspicuously yellow bordered; squama yellowish gray, margin and fringe black.

Male genitalia. Aedeagus (figs. 1192, 1193) with distiphallus divided, lying at right angles to basal sclerites; sperm pump large, blade triangular, weakly pigmented along upper margin.

Host/early stages. Unknown.

Holotype m., Colorado, Boulder Co., 
Hoosier Pass, 12,000 ft, willow tundra, 8.VIII.61 (J. G. Chillcott); paratype m., Clear Creek Co., Mt. Evans, $11,300 \mathrm{ft}$, wet vegetation, 10.VIII.61 (C. H. Mann); both in CNC.

Remarks. The male genitalia indicate that this distinctive species belongs to the $\underline{P}$. robustella group.

Phytomyza flavinervis Frost

(Figs. 1139, 1140)

Phytomyza flavinervis Frost, 1924: 85. Lectotype female (designated here) from Texas in USNM.

On1y the male and female syntypes are known of this species. Unfortunately the male lacks its head and the end of the distiphallus appears to be broken (the genitalia were extruded). However, the illustrations given will suffice to establish the identity of the species. The female is in perfect condition and is hereby designated as lectotype.

Phytomyza flexuosa Spencer, new species (Figs. 12 31,1232$)$

Head. Frons broad, twice width of eye, not projecting above eye in profile; 2 strong ors but upper distinctly weaker, 2 ori, lower weak, orbital setulae sparse, in single row; gena 0.33 height of eye; 3d antennal segment rounded, rather large.

Mesonotum. Acr irregularly in 4 rows.

Wing. Length in male $2.5 \mathrm{~mm}, 2 \mathrm{~d}$ costal section long, 3 times length of $4 \mathrm{th}$.

Color. Frons, gena, and face clear yellow, upper orbit yellow below upper ors, black above, confluent with hindmargin of eye; mesonotum and scutellum blackish gray; legs with femora black, knees broadly yellow, tibiae and tarsi yellowish brown; squama, including margin and fringe, yellow.

Male genitalia. Aedeagus (figs. 1231, 1232) with distiphallus divided into 2 tubules with distinctive curvature; sperm pump large, with blade broader than long, base with points of strong sclerotization at each end.

Host/early stages. Unconfirmed, probably Ranunculaceae.

Holotype m., Washington, Benton Co., Prosser, sweeping Erigeron canadensis, 27.VIII.48 (K. E. Frick, Lot $215-1$ ), in CAS.

Remarks. This species closely resembles both $\underline{P}$. davisi $i$ and $\underline{P}$. duplex (see genitalia figs. 108 3 , 1087), but in both these species the outer crossvein is present (fig. 1085). Although the unique holotype was caught on "Erigeron" (= Conyza) canadensis, it is believed that the host is in the Ranunculaceae.

Phytomyza genalis Melander (Figs. 11 15,1116 )

Phytomyza genalis Melander, 1913: 272. Lectotype male from Illinois, designated by Frick (1957b: 205), in USNM.

This species is distinctive in having the $3 \mathrm{~d}$ antennal segment yellowish, the mesonotum matt gray, and the side of the thorax largely yellow. In the male genitalia (figs. 1115, 1116) the distiphallus is a membranous, dorsally directed tubule with equal lateral extensions at the midpoint; the ventral lobe is well developed but also membranous.

Phytomyza latifrons Spencer, new species

(Figs. 1190, 1191)

Head. Frons exceptionally broad, almost 3 times width of eye, distinctly projecting above eye in profile; 2 equal ors, 3 strong ori, sometimes a smaller 4 th present, orbital setulae sparse, in single row; gena broad, slightly more than 0.5 height of eye, parafacial prominent, occupying

0.75 distance between lower margin of gena and eye margin; 3d antennal 
segment slightly longer than broad.

Mesonotum. $3+1$ strong dc, acr sparse, in 2 rows.

Wing. Length in male $2.5 \mathrm{~mm}, 2 \mathrm{~d}$ costal section very short, little longer than $4 \mathrm{th}$.

Color. Frons, gena, and face orange yellow; orbit slightly darkened to lower ors, pale below; entire hindmargin of eye black; 1st antennal segment yellowish, $2 \mathrm{~d}$ and $3 \mathrm{~d}$ black, palpus black; mesonotum and scutellum matt gray, side of thorax similar, apart from narrow yellow upper margin of mesopleuron; legs black, knees narrowly yellowish; abdomen entirely gray; squama pale gray, margin and fringe black.

Male genitalia. Aedeagus (figs. 1190, 1191) long, distiphallus divided, with characteristic curvature; main ventral sclerites joining distal end of basal sclerites strong, a weak, largely membranous lobe situated more basally, left basal sclerite short, half length of right; sperm pump small, blade slightly asymmetrical.

Host/early stages. Unknown.

Holotype m., Colorado, Lake Co., Independence Pass, 12,100 ft, 31.VII.61; paratypes $2 \mathrm{~m}$. , same data (J. G. Chillcott); Chaffee Co., Cottonwood Pass, 11,900 ft, $1 \mathrm{~m}$., 29.VII.61 (B. H. Poole). Holotype and paratypes in CNC, 1 paratype in KAS.

Remarks. This species is distinctive in the broad frons and short second costal section.

Phytomyza manni Spencer, new species (Figs. 1152, 1153)

Head. Frons twice width of eye, distinctly projecting above eye in profile; 2 ors, 3 ori (lowest may be weak), orbital setulae in single row; gena deeply extended at rear, almost 0.5 height of eye; $3 \mathrm{~d}$ antennal segment longer than broad, arista slightly swollen basally.
Mesonotum. $3+1 \mathrm{dc}$, acr in 2 rows.

Wing. Length $2.5-2.7 \mathrm{~mm}, 2 \mathrm{~d}$ costal section almost twice length of $4 \mathrm{th}$.

Color. Frons orange yellow, orbit grayish, entire hindmargin of eye dark; palpus and 3d antennal segment black, 1st and 2d yellowish; mesonotum and scutellum matt gray, side of thorax blackish gray; upper margin of mesopleuron only narrowly yellow; legs black, femora with knees bright yellow; abdomen blackish gray; squama and fringe yellow, margin scarcely darker.

Male genitalia. Aedeagus (figs. 1152, 1153) with large ventral lobes and smaller flaplike lobe attached to right ventral sclerite; distiphallus with paired black lateral sclerites, ejaculatory duct short, membranous; sperm pump with large blade and base extended on one side.

Host/early stages. Unknown.

Holotype m., Colorado, Clear Creek Co., Mt. Evans, Echo Lake, along grassy lake shore, $10,600 \mathrm{ft}$, 13.VII.61; paratype f., same data; (both C. H. Mann), in CNC.

Remarks. The affiliations of this species are unclear, but it possibly belongs to the $\underline{\mathrm{P}}$. robustella group.

Phytomyza masoni Spencer, new species (Figs. 1317, 1318)

Head. Frons broad, 2.5 times width of eye, not projecting above eye in profile; 1 strong ors, 2 ori, orbital setulae short, sparse; orbit pronounced; gena angular, 0.33 height of eye; 3d antennal segment little longer than broad, rounded, with conspicuous fringe of white pubescence.

Mesonotum. $3+1$ strong $d c, a c r$ irregularly in 4 rows.

Wing. Length in male $2.25 \mathrm{~mm}, 2 \mathrm{~d}$ costal section 2.5 times length of 4 th. 
Color. Head entirely dark, frons uniformly matt black, orbit weakly. shining, grayish black; antenna black; mesonotum deep black, moderately shining, scutellum and side of thorax black; legs black but all femora narrowly yellowish; squama and margin whitish, fringe essentially similar, at most faintly ochreous; abdomen black.

Male genitalia. Aedeagus (figs. 1317, 1318) with paired distal tubules, mesophallus conspicuously short and broad; sperm pump with large moderately pigmented blade, which is broader than high, stalk short, base shortly pigmented.

Host/early stages. Unknown.

Holotype m., Georgia, Rabun Co., Pine Mountain, 1,400 ft, 14.V.57 (W. R. M. Mann), in CNC.

Remarks. The single ors, dark color, and pale squamal fringe associate this species with $\underline{P}$. pulchella, n. sp., and it is possible that both are feeders on Ranunculaceae.

Phytomyza nepetae Hendel (new to U.S.A.)

(Figs. 1353, 1354)

Phytomyza nepetae Hendel, 1922: 70.

Syntypes from Germany in ZMHU.

The group of small, closely related black species known in Europe as leaf miners on Boraginaceae and Lamiaceae was revised in detail by Nowakowski (1959). Nine species are known in Europe. One leaf miner on Nepeta (Lamiaceae), P. nepetae, has previously been recorded in North America, with records from Ontario and Quebec (Spencer, 1969a: 258), and this species has now been identified from Washington State. The aedeagus is shown in figures 1353, 1354. Probably $\mathrm{P}$. nepetae was introduced into Cañada and the Western United States by early settlers. Another species feeding on Boraginaceae, $\underline{P}$. ovalis Griffiths, is recorded here from Colorado.
New record.

Washington, Yakima Co., Wapato, "sweeping Nepeta cataria," $13 \mathrm{~m}$., 4 f., 26.VI.52(K. E. Frick, Lot 51 - 16), in CAS.

Phytomyza notopleuralis Spencer (new to U.S.A.)

(Fig. 1248)

Phytomyza notopleuralis Spencer, 1969a: 259. Holotype male from Ontario in CNC.

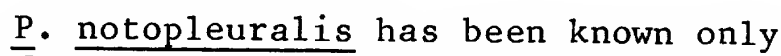
from the Ottawa area.

During his examination of Chromatomyia regalensis (Steyskal, 1972b), Griffiths (1980: 6) found that one of the two specimens treated as paratypes had been misidentified and represents this species. This specimen is from Michigan, Keweenaw County, 9.VII.57 ( $R$. W. Hodges), in USNM.

The aedeagus is shown in figure 1248, with the distiphallus slender, divided, at right angles to a large, horseshoeshaped sclerite lying beyond the apex of the basal sclerites.

Phytomyza ovalis Griffiths (new to U.S.A.)

(Figs. 1357, 1358)

Phytomyza ovalis Griffiths, 1975: 133. Holotype male from Yukon Territory in CNC.

P. ovalis belongs to a complex of four species feeding as leaf miners on Boraginaceae and known in North America from Alberta and British Columbia to Alaska. Related species on Boraginaceae are known in Europe.

A single male has been seen in this complex from Colorado, Jackson County, Rabbit Ear Pass, 7.VII.61 (J.G. (hillcott), in CNC, which is tentatively identified as $P$. ovalis. Although this specimen differs in minor detail from $\underline{P}$. ovalis, as described and illustrated by Griffiths (1975), it is clearly undesirable to describe another 
species in the complex without confirmatory biological evidence. Differences between the four described species are slight both in external morphology and in the male genitalia. More reliable differences are apparent in the leaf mines. Until reared material is available in the United States, positive identification of species in this group will remain problematic.

Phytomyza pulche11a Spencer, new species

(Figs. 1321, 1322)

Head. Frons twice width of eye, narrowly projecting above eye in profile; 1 strong reclinate ors, 3 slender inclined ori, orbital setulae short, in single row; gena extended at rear, slightly less than 0.33 height of eye; 3d antennal segment slightly elongate.

Mesonotum. $3+1$ strong dc, acr short, irregularly in 4 rows in front.

Wing. Length $3.5 \mathrm{~mm}$ in male, $3.8 \mathrm{~mm}$ in female, $2 d$ costal section long, almost 4 times length of 4 th.

Color. Head entirely black, mesonotum, scutellum, and side of thorax matt black; legs with femora black, knees contrastingly bright yellow, tibiae and tarsi more brownish; abdomen entirely black; squama and fringe golden yellow; wing and veins yellowish.

Male genitalia. Aedeagus exceptionally large (figs. 1321, 1322), distiphallus divided into 2 diverging tubules; sperm pump large, with broad blade.

Host/early stages. Unknown.

Holotype m., Virginia, Smyth Co., Mt. Rogers, 4,700-5,800 ft, 1.VI.62;

paratypes $3 \mathrm{~m} ., 2 \mathrm{f}$. , same data; (a11 J. R. Vockeroth). Holotype and 3 paratypes in CNC, 2 paratypes in KAS.

Remarks. The single ors, generally black body, and pale squama and fringe, together with the large size, make this a distinctive species. It is clearly related to $P$. saskatoonensis (see genitalia figs. $1 \overline{3} 23,1324$ ) and p. masoni, n. sp. (figs. 1317, 1318), and a11 are almost certainly feeders on Ranunculaceae.

Phytomyza ranunculoides Spencer, new species

(Figs. 1326-1329)

Head. Frons at most 1.5 times width of eye, not projecting above eye in profile; 1 strong ors, 2 ori, lower weaker, orbital setulae sparse; gena about 0.33 height of eye; $3 d$ antennal segment small, round, only finely pubescent.

Mesonotum. $3+1 \mathrm{dc}$, acr in 4 rows.

Wing. Length from $1.9 \mathrm{~mm}$ in male to $2.25 \mathrm{~mm}$ in female, $2 \mathrm{~d}$ costal section just over 3 times length of 4 th.

Color. Frons matt black, orbit weakly shining, more so around base of orbital bristles; gena, face, and antenna black; mesonotum, side of thorax, and abdomen black, mesonotum conspicuously shining; legs with coxae and femora black, tibiae brownish, more yellow at each end, tarsi conspicuously yellow; squama pale gray, margin and fringe dark.

Male genitalia. Aedeagus (figs. 1326, 1327) with distiphallus divided, slightly recurved; ventral lobe prominent, horseshoe shaped, with strong sclerite lying centrally between each side of ventral lobe, broad at base of distiphallus, finely tapering, almost hairlike at end; sperm pump largely unpigmented, minute (fig. 1328).

Host/early stages. Ranunculus abortivus. Larva forming white, upper surface linear mine commencing with minute spiral (fig. 1329), frass deposited in line of fine, closely adjoining grains, pupation externally, larva leaving leaf through upper surface exit slit; puparium yellowish brown, posterior spiracles widely separated, each on prominent projec- 
tion, 2-armed, each arm with irregular ellipse of 6-10 minute bulbs.

Holotype m., Minnesota, Minneapolis, Minnehaha Creek, emerged 20.VII.80, ex leaf mine coll. 29.VI.80; paratypes 2 f., topotypical, emerged 22.VII.80; (a11 KAS). Holotype in USNM, paratypes in KAS.

Remarks. The male genitalia indicate that $\underline{P}$. ranunculoides is the sister species of $\underline{P}$. loewii (described as $P$. clematidis Loew), a leaf miner on Clematis. Externally the two species differ in the longer costal ratio, paler tarsi, and less shining orbit in the Ranunculus feeder. The aedeagus of each species is of the same distinctive form, with the distiphallus partially recurved and the conspicuous forwardly directed sclerite at its base.

Two black species are known as leaf miners on Ranunculus in Europe, $\underline{P}$. ranunculicola Hering, 1949, and $\overline{\mathrm{P}}$. ranunculivora Hering, 1932. The latter forms a blotch mine and, with a different type of aedeagus (see Spencer, 1976a: figs. 849, 850), is not closely related to $\underline{P}$. ranunculoides. $\underline{P}$. ranunculicō̄a forms a linear mine, which is generally similar to that of $\underline{P}$. ranunculoides, but it lacks the initial spiral. The aedeagus (see Spencer, 1976a: fig. 852) has the distiphallus recurved, and this apomorphous character probably indicates a relationship with the nearctic species. One black species, $\underline{P}$. rectae Hendel, is common in the Mediterranean area on Clematis recta, and other black species in Europe are known on Anemone and Pulsatilla. They are all more closely related to $\underline{P}$. ranunculivora than to the two nearctic species.

\section{Phytomyza saskatoonensis Spencer (new} to U.S.A.)

(Figs. 1323, 1324)

Phytomyza saskatoonensis Spencer,

1969a: 272. Holotype male from Saskatoon in CNC.
Phytomyza cudu Steyskal, 1972b: 4. NEW SYNONYM. Holotype male from Michigan in USNM.

This large black species is distinctive in having a single strong ors, three strong inclined ori, the third antennal segment elongate, and the mesonotum deep black, though with some grayish dusting.

The holotype of $\mathrm{P}$. cudu has been examined and clearly represents $\underline{P}$. saskatoonensis, with which it is now formally synonymized. Steyskal considered it could not represent this species owing to the smaller size, the wing length being given as $2.25 \mathrm{~mm}$, whereas it is in fact $2.5 \mathrm{~mm}$, and the second costal section is stated to be three times the length of the fourth, although it is shown as 2.3 times in Steyskal's figure 5, a. In other distinctive characters, including the aedeagus and very large sperm pump, it agrees with $\underline{P}$. saskatoonensis.

Frick (1959: 433) identified $\underline{P}$. nigripennis Fallén, a European species, with specimens from Wisconsin and Ontario. They certainly also represent $\underline{P}$. saskatoonensis. $\underline{P}$. nigripennis appears to be a closely related species but with distinctive differences in male genitalia (Spencer, 1976a: figs. 796, 797). $\underline{\text { p }}$ nigripennis has on a number of occasions been caught on Anemone sp., and probably this is the host, the larvae feeding internally in the stem or root. It is virtually certain that

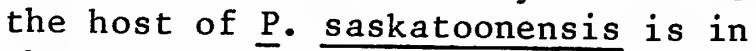
the Ranunculaceae.

Phytomyza subalpina Sehgal (new to U.S.A.)

(Figs. 1158, 1159)

Phytomyza subalpina Sehgal, 1971: 379. Holotype male from Alberta in CNC.

This species has been known only from the unique holotype from Coleman in the Canadian Rockies. Ten specimens are now recorded here from several 
localities at high elevations in Colorado.

With the frons yellow, two equal ors, and the second costal section short, P. subalpina resembles a number of other species, particularly p. manni, n. sp., but the male genitalia (figs. $1158,1159)$ are distinctive. It is interesting to note that the female has the ovipositor greatly elongated, a character found in $\underline{P}$. varipes Macquart, which feeds in seed heads of Rhinanthus spp. and which is known from Europe, including Iceland, and Nova Scotia (see Spencer, 1969a:

282). It can be assumed with certainty that $P$. subalpina feeds in the same way, but there is no indication from present data as to the host.

New records.

Colorado, Clear Creek Co., Mt. Evans,

Echo Lake, $10,600 \mathrm{ft}, 1 \mathrm{~m}$.,

13.VII.61, 2 f., 25.VII and

24.VIII.61; Mt. Evans, Timberline, $11,700 \mathrm{ft}, 1 \mathrm{~m} ., 29 . V I I .61$; Summit Flats, 12,800 ft, $1 \mathrm{~m} ., 24$.VII.61; (a11 C. H. Mann); Loveland Pass, $12,000 \mathrm{ft}$, Summit, $1 \mathrm{f} .$, 7.VIII.61 (B. H. Poole); Boulder Co., Corona Pass, $10,600 \mathrm{ft}$, marshy meadow at Timberline, $1 \mathrm{~m} ., 6 . V I I .61$ (J. G. Chillcott); Jefferson Co., Mt. Vernon, near Golden, 7,200 ft, $1 \mathrm{~m}$., 31.VII.61 (C. H. Mann); Lake Co., Independence Pass, 12,100 ft, tundra, 1 f., 31.VII.61 (J. G. Chillcott), 1 f., 7.VIII.61 (C. H. Mann); all in CNC.

Phytomyza tenella Meigen (new to U.S.A.)

(Figs. 1156, 1157)

Phytomyza tenella Meigen, 1830: 195. Holotype male from Germany in NMW.

A single male of this species can now be recorded from Colorado, Clear Creek County, Mt. Evans, Echo Lake, 10,600 feet, 24.VIII.61 (C. H. Mann), in CNC. $P$. tenella is known from northern Canada (misidentified as affinis Fallén, Spencer, 1969a: 225) and is widespread in Europe, including Ireland (Griffiths, 1964: 411).
This species belongs to a closely related group of feeders in seed heads and stems of several genera of Scrophulariaceae. Apart from P. tenella (Pedicularis, Euphrasiā), species known in Canada include $\underline{P}$. pedicularicaulis Spencer and $P$. pedicularidis Spencer (Pedicutaris) and in Europe $P$. rostrata Hering (Euphrasia, Melampyrum, Odontites, and Rhinanthus) (see Spencer, 1969a, 1976a). A distinctive character of the aedeagus (fig. 1156) is the large flaplike ventral sclerite at the end of the right basal sclerite. $\underline{P}$. tenella is obviously closely related to $\underline{P}$. orindensis and $\underline{P}$. subtenella, but in these two species this ventral lobe is lacking.

Phytomyza wahlgreni Rydén (new to North America)

(Figs. 1199, 1200)

Phytomyza wah1greni Rydén, 1944: 49. Holotype female from N. Sweden in ZIL.

Phytomyza taraxacocecis Hering, 1949:

29. Holotype male from Germany in ZMHU (synonymy established by Spencer, 1976a: 527).

This species is widespread in Europe, the larva feeding in the midrib of Taraxacum spp. Five specimens can now be recorded from Colorado. The aedeagus in side view (fig. 1199) agrees exactly with illustrations given by Griffiths (1964: fig. 18) and Spencer (1976a: fig. 921). The divided distiphallus of the Colorado specimens is as shown by Spencer (1976a: fig. 922) (male from Sweden), rather than as shown by Griffiths (1964: fig. 18b). Additional study of European material will be necessary to establish whether this difference is of specific significance.

P. wahlgreni belongs to the difficult $\overline{\mathrm{P}}$. "robustella" group, in which a number of species, virtually inseparable on external characters, are host specific on differing genera of Asteraceae and have distinctive differences in the male genitalia. It 
is thus impossible to identify

individual caught females.

New records.

Colorado, Clear Creek Co., Loveland

Pass, 12,000 ft, 1 m., $3 \mathrm{f}$,

7.VIII.61; Hoosier Pass, 12,000 ft,

1 m., 8.VIII.61; (a11 C. H. Mann), in CNC.

\section{Phytomyza new species (Colorado)}

A large, distinctive female from Colorado, Clear Creek County, Mt. Evans, 9,800 feet, Doolittle Ranch, 3.VIII.61 (J. G. Chillcott), in CNC, represents an undescribed species, but the formal description is best delayed until a male becomes available. A synoptic description is given in the key at couplet 28 .

It is believed that this species is related to $\underline{P}$. dasyops Hendel, $\underline{P}$. eumorpha Frey, and $P$. hirta Rydén (see Spencer, 1976a), which are all arctic species.

Two females can be recorded from Alaska, Matanuska Valley, 22.VII. 32 (C. 0. Berg), in CUI, one of which appears to represent $\underline{P}$. eumorpha and is the first record of this species in North America. The specimen is mounted with its puparium (but no indication is given of the host). This is brownish orange, with the distinctly enlarged posterior spiracles characteristic of species feeding on hosts in an aquatic environment (see $P$. dasyops, Spencer, 1976a: fig. 719). The second specimen can be tentatively identified as $\underline{P}$. dasyops, having yellow femora and pilose eyes, and this is also new to North America.

Phytomyza new species (Ligusticum) (Fig. 1360)

Two females were reared from leaf mines found on Ligusticum grayi, 6.IX.78, at Sagehen Creek, Nevada County, Calif., 27 and 29.IX.78

(KAS). The frons is dark, the two ors appear to be equal (although there is some irregularity and the upper may be weaker), the mesonotum is grayish with acr in four rows, and the foreknee is yellowish. The second costal section is variable, less than three times and more than 2.5 times the length of the fourth. On this diagnosis the species runs to couplet 114 and externally resembles $\underline{p}$. glabricola, a leaf miner on Ilex, with which it is not related.

The larva forms a narrow linear mine, following the serrations of the leaf (fig. 1360), pupating externally; the puparium is black. Leaf mines have been found in Alaska on Ligusticum by Griffiths, and these may represent the same species.

It is not proposed formally to describe this species until further clarification is possible from the examination of a male.

\section{GENUS CHROMATOMYIA HARDY}

Eight new species are described and one new combination is established. In addition, four species are recorded as new to the United States. The status of one species, C. regalensis, is clarified in view of its previous assessment as a nomen dubium.

Chromatomyia clemativora Coquillett, new combination

(Figs. 1098, 1099)

Phytomyza clemativora Coquillett, 1910: 131. Holotype male from Texas in USNM.

The male genitalia indicate that this species correctly belongs in

Chromatomyia to which it is now transferred.

C. clemativora was reared, presumably from leaf mines, on an unidentified Clematis sp. It somewhat resembles $\underline{P}$. clematiphaga, a leaf miner on $\underline{\mathrm{C}}$. columbiana and $\underline{\mathrm{C}}$. verticillaris , known from Montana and western Canada, but it is smaller, with a wing length of $1.4 \mathrm{~mm}$ in the male (not $1 \mathrm{~mm}$ as stated by Coquillett in his description) to 
$1.75 \mathrm{~mm}$ in the female. The male genitalia indicate that the two species are not closely related (see figs. 1098, 1099 and 1093, 1094). In C. clemativora the aedeagus lacks a ventral lobe and this is replaced by a horseshoe-shaped sclerite extending beyond the apical end of each basal sclerite. There is in addition a strong sclerite rising dorsally and slightly recurving, finely divided distally, attached to the ends of the basal sclerites, and to its rear there is another central, upright sclerite.

Only the two type specimens from Texas, Cameron County, Brownsville, have been known. A single female has now been identified from San Patricio County, Welder Wild Life Reserve near Sinton $(240 \mathrm{~km}$ north of the type locality), 19-23.III.65 (J. G. Chillcott), in CNC.

Chromatomyia compta Spencer, new species

(Fig. 1089)

Head. Frons broad, twice width of eye, not projecting above eye in profile; 2 equal, reclinate ors, 2 inclined ori; gena extended at rear, 0.33 height of eye; 3d antennal segment slightly longer than broad, rounded at end, with distinct fringe of whitish pubescence.

Mesonotum. $3+1$ strong $d c$, acr in 2 rows.

Wing. Length from $2.6 \mathrm{~mm}$ in male to $3.25 \mathrm{~mm}$ in female, $2 \mathrm{~d}$ costal section 3.5 times length of 4 th.

Color. Frons, hindmargin of eye, palpus, and lst and $2 \mathrm{~d}$ antennal segments bright yellow, 3d segment black; mesonotum uniformly matt gray; scutellum yellow, sometimes suffused with gray, but always yellow between basal scutellars viewed from rear; side of thorax largely yellow; legs with coxae and femora bright yellow, tibiae and tarsi yellowish brown; abdomen largely yellow, tergites sometimes narrowly grayish in front; squama yellow, margin and fringe dark.
Male genitalia. Aedeagus (fig. 1089) with strong, paired, diverging sclerites above front end of basal sclerites, horseshoe-shaped sclerite in same plane distally of basal sclerites; ejaculatory duct long, sinuous; sperm pump with large, weakly pigmented blade.

Host/early stages. Unknown.

Holotype m., North Carolina, Swain Co., Great Smoky Mts. National Park, Clingman's Dome, 6,300-6,642 ft, 20.V.57; paratypes $4 \mathrm{f}$., same data; 3 m., 28.V.57; (a11 J. R. Vockeroth); 1 f., 21.V.65 (J. G. Chi11cott); 1 f., 6.VIII.57 (C. J. Durden); Yancey Co., Mt. Mitche11, 6,800 ft, $1 \mathrm{~m}$., 12.VIII.57 (J. G. Chillcott); Mt. Richland-Balsam, 6,000-6,400 ft, Blue Ridge Parkway, 1 f., 30.V.65 (J. G. Chillcott); Tennessee, Sevier Co., Great Smoky Mts. National Park, Indian Gap, 5,200 ft, 1 m., 3 f., 28.V.57 (J. R. Vockeroth); Virginia, Shenandoah Co., Hawksbil1, 3,600-4,050 ft, $1 \mathrm{~m}$., 3 f., 7.VI.62; Smyth Co., Mt. Rogers, 5,300-5,700 ft, $1 \mathrm{~m}$., 1.VI.62 (J.R. Vockeroth). Holotype and paratypes in $\mathrm{CNC}$, other paratypes in KAS.

Remarks. This is the largest known Chromatomyia species and one of the few with predominantly yellow coloration. Although generally resembling a number of Phytomyza species, the strong, dorsally directed "supporting sclerites" and the distinctive ejaculatory duct below clearly indicate the correct generic position in Chromatomyia.

C. compta appears to be related to $\mathrm{C}$. clemativora and this suggests that its host is probably also Clematis sp.

Chromatomyia doolittlei Spencer, new species

(Figs. 1292-1294)

Head. Frons exceptionally broad, 3 times width of eye; 2 equal ors, 1 ori; gena 0.33 height of eye, parafacial prominent; $3 d$ antennal segment small, round, finely pubescent. 
Mesonotum. Acr irregularly in 4 rows.

Wing. Length in male $2.6 \mathrm{~mm}, 2 \mathrm{~d}$ costal section 2.25 times length of 4th.

Color. Frons brownish black, orbit darker, blackish; gena brownish black, face and antenna black; mesonotum, scutellum, and entire side of thorax blackish gray; legs black, only foreknee indistinctly yellowish; squama whitish gray, margin and fringe black.

Male genitalia. Aedeagus (fig. 1292) with left basal sclerite short, broad, with long ventral extension basally; dorsal lobe with main supporting sclerites widely divergent and further developed lateral sclerite in front (fig. 1293); sclerites of ventral lobe, broad, ejaculatory duct curving dorsally toward supporting sclerites; sperm pump (fig. 1294) with long stalk and rounded blade.

Host/early stages. Unknown.

Holotype m., Colorado, Clear Creek Co., Mt. Evans, Doolittle Ranch, 9,800 ft, 23.VIII.61 (J. G. Chill cott), in CNC.

Remarks. The most distinctive character of this species is the unusually broad frons. The male genitalia show that the species cannot be closely associated with any others in the genus, in particular the strongly developed lateral sclerites of the dorsal lobe, the characteristic shape of the left basal sclerite, and the large, long-stalked sperm pump are all significant distinguishing characters.

It is a pleasure to name this species after Mr. Doolittle, around whose ranch many interesting species were collected.

Chromatomyia erigerontophaga (Spencer) (new to U.S.A.)

(Figs. 1271-1274)

Phytomyza erigerontophaga Spencer, 1969a: 239. Holotype male from Greenland in UZMC. Chromatomyia erigerontophaga, Griffiths, 1976b: 261 .

This small black species is known as a leaf miner on Erigeron compositus, E. eriocephalus, and E. humilis, and $i t$ is widespread in the Arctic from northern Greenland to Alaska. Griffiths (1976b: 263) recorded leaf mines on E. [acris L., var.] debilis in the Rockies in Alberta but does not positively refer them to $\underline{C}$. erigerontophaga.

The species can now be recorded from Colorado, and this extension of its range suggests that there is little reasonable doubt about the identity of the population in Alberta. $\underline{\text { C. }}$ erigerontophaga is distinctive in having the third antennal segment enlarged and angulate (fig. 1271) and the second costal section unusually short, little longer than the fourth. The exceptionally long basal sclerites of the aedeagus (figs. 1272, 1273) and the heavily pigmented sperm pump (fig. 1274), with the blade little more than linear, also indicate the isolated position of the species.

New records.

Colorado, Clear Creek Co., Mt. Evans, Timber1ine, $11,700 \mathrm{ft}, 1 \mathrm{~m} ., 1 \mathrm{f}$. , 29.VII.61 (C. H. Mann); Mt. Evans, $14,000 \mathrm{ft}, 1 \mathrm{f} ., 25 . V I I .61$ (J. G. Chillcott); Boulder Co., Hoosier Pass, 12,000 ft, 1 f., 8.VIII.61 (B. H. Poole); all in CNC.

Chromatomy ia flavida Spencer, new species

(Figs. 1245, 1246)

Head. Frons broad, slightly more than twice width of eye, narrowly projecting above eye; normal arrangement of orbital bristles with upper ors shorter than lower (only single ors on one side in only available specimen), 1 ors, orbital setulae sparse, in single row; gena extended at rear, 0.5 height of eye, parafacial prominent; $3 d$ antennal segment small, round, with fringe of weak pubescence. 
Mesonotum. Acr irregular, in 2-3 rows.

Wing. Length in male $2.4 \mathrm{~mm}, 2 \mathrm{~d}$ costal section short, 1.67 length of $4 \mathrm{th}$.

Color. Frons basica1ly orange ye11ow, faintly infuscated on upper half; orbit and gena yellow, hindmargin of eye, palpus, $2 \mathrm{~d}$ and $3 \mathrm{~d}$ antennal segment black, 1st paler, slightly yellowish; mesonotum matt grayish black, side of thorax dark; legs black but a11 knees bright yellow; squama gray, margin and fringe black.

Male genitalia. Aedeagus (figs. 1245, 1246) with supporting sclerites only slightly diverging, distinctly dilated at apex but not solidly club shaped; ejaculatory duct strongly developed, with large, flaplike sclerite below extending symmetrically on either side; sperm pump minute.

Host/early stages. Unknown.

Holotype m., Colorado, Boulder Co., Nederland, Caribou, 10,000 ft, 18.VI.61 (B. H. Poole), in CNC.

Remarks. Although this species closely resembles Phytomyza arnicicola in external characters, it is distinguishable by the broader gena. Its generic position is clearly indicated by the male genitalia.

Chromatomyia griffithsi Spencer, new species

(Figs. 1267, 1268)

Head. Frons broad, twice width of eye, orbit pronounced but not projecting above eye; 2 equal ors, 2 ori, lower sometimes weak or even lacking; gena 0.33 height of eye, parafacial forming broad ring below eye; 3d antennal segment small, round.

Mesonotum. Acr short, irregularly in 4 rows.

Wing. Length from $2.5 \mathrm{~mm}$ in male to 3 $\mathrm{mm}$ in female, $2 \mathrm{~d}$ costal section slightly more than twice length of 4th, ranging from 2.2 to 2.4 times.

Color. Frons blackish brown on lower half, paler above, orbit dark brown, sometimes narrowly paler along inner margin; lunule gray; gena black to brownish, face, palpus, and antenna black; mesonotum entirely matt grayish black, side of thorax black; legs entirely black; squama gray, margin and fringe black.

Male genitalia. Aedeagus (fig. 1267) having left basal sclerite with conspicuous ventral bend in rear third; supporting sclerites with line of sclerotization at rear and broad, strongly pigmented sclerite in front in side view, in ventral (front) view as in figure 1268; at each side of curving ejaculatory duct a strongly pigmented sclerite below distal end of basal sclerites; sperm pump with lightly pigmented, rounded blade and extended base on one side.

Host/early stages. Unknown.

Holotype m., Colorado, Clear Creek Co., Mt. Evans, Summit Lake Flats, $12,600 \mathrm{ft}, 24$. VII. 61 ; paratypes $2 \mathrm{~m}$., $2 \mathrm{f}$, $1 \mathrm{~m}$., on tundra, 2.VII.61, otherwise same data; (a11 C. H. Mann). Holotype and paratypes in $\mathrm{CNC}, 2$ paratypes in KAS.

Remarks. This species superficially closely resembles Phytomyza nigrinervis, which occurs at the same locality, but the frons is paler behind, the third antennal segment is smaller, and the second costal section is consistently longer. There is no indication of the host plant.

We take pleasure in naming this species after Dr. Graham C. D. Griffiths, whose recent work has been invaluable in clarifying North American species in this genus.

Chromatomyia involucratae (Spencer)

(new to U.S.A.)

(Figs. 1259, 1260)

Phytomyza involucratae Spencer, 1969a: 
249. Holotype male from Alberta in CNC.

Chromatomyia involucratae, Griffiths, 1974a: 51 .

A single male can now be recorded from Colorado, Clear Creek County, Mt. Evans, Doolittle Ranch, 9,800 feet, 15.VII.61 (C. H. Mann), in CNC. The species has previously been known only from two series in Alberta and British Columbia, both caught on Lonicera involucrata. It was originally thought that these flies could be associated with long, greenish mines that were present at both localities, but Griffiths (1974a: 51) has

subsequently recorded a Paraphytomyza sp. from such mines, and the host of C. involucratae thus remains to be confirmed, although it will almost certainly prove to be Lonicera involucrata.

C. involucratae is a relatively large species, with the second costal section long, three times the length of the fourth. The aedeagus is distinctive, in having the supporting sclerites of the dorsal lobe large, with pronounced curvature toward the apex (figs. 1259, 1260).

Chromatomyia 1uzulivora Spencer, new species

(Figs. 1288, 1289)

Head. Frons twice width of eye, not projecting above eye; 2 equal ors or upper marginally shorter, 2 strong ori; gena extended at rear, almost 0.5 height of eye, parafacial prominent; $3 d$ antennal segment small, round.

Mesonotum. $3+1 \mathrm{dc}$, acr irregular, in 4 rows in front.

Wing. Length in male $2 \mathrm{~mm}, 2 \mathrm{~d}$ costal section twice length of $4 \mathrm{th}$.

Color. Frons uniformly brownish black, orbit paler, grayish; antenna black; gena dark brown, face black; mesonotum matt blackish gray, side of thorax similar; legs black, foreknee narrowly yellow; squama gray, margin and fringe black.
Male genitalia. Aedeagus (fig. 1288) with supporting sclerites of dorsal lobe straight in side view, relatively long, fused below, narrowly divergent apically (fig. 1289); strongly developed, slightly curving sclerite each side of base of ejaculatory duct, this curving dorsally toward apex of dorsal sclerites; sperm pump minute, blade unpigmented.

Host/early stages. Unknown.

Holotype m., Colorado, Lake Co., Independence Pass, 11,500 ft, tree line, 31.VII.61 (J. G. Chill cott), in CNC.

Remarks. This species belongs to the C. 1uzulae superspecies and to the first of the two "aedeagus types," with long supporting sclerites (Griffiths, 1980: 31), which include three species known only in Europe. Only a single species has been described from North America, C. cygnicollina Griffiths, 1980, from Alberta. $\underline{\text { C. }}$ luzulivora differs from the six known species in having the frons uniformly dark (ranging from yellowish above to entirely yellow in the other species) and the costal ratio greater than in any other species in the group. The greatest previously recorded is 2.2 in $\mathrm{C}$. tschirnhausi Griffiths, 1980, from Norway, but even in this species the second costal section is normally about 2.5 times the length of the fourth.

Chromatomyia mitche11i Spencer, new species

(Figs. 1290, 1291)

Head. Frons exceptionally broad, almost 3 times width of eye, not projecting above eye in profile; 2 ors, upper variable, apparently equal to lower on one side (lower partially broken) but distinctly shorter on other side, 2 ori; gena broad, slightly more than 0.33 height of eye, parafacial prominent; $3 d$ antennal segment sma11, round, faintly pubescent. 
Mesonotum. Acr irregularly in 4 rows.

Wing. Length in male about $2.3 \mathrm{~mm}$ (both wings partially crumpled in only available specimen, accurate measurement not possible), 2d costal section 2.3 times length of fourth.

Color. Frons dark brown, more blackish toward lunule, orbit deeper black; gena dark brown, face, palpus, and antenna black; mesonotum matt blackish gray, side of thorax entirely dark; legs black, knee of foreleg distinctly yellow, scarcely differentiated on other legs; squama dark gray, margin and fringe black.

Male genitalia. Aedeagus as in figures 1290,1291 , supporting sclerites long, diverging, paired sclerites each side of ejaculatory duct large, curving; ejaculatory duct strongly developed, recurving toward supporting sclerites; sperm pump minute, weakly pigmented.

Host/early stages. Unknown.

Holotype m., North Carolina, Yancey Co., Mt. Mitche11, 6,800 ft, 12.VIII.57 (J. G. Chillcott); paratype m., Macon Co., Highlands, Wilson's Gap, 3,100 $\mathrm{ft}, 25 . \mathrm{V} .57$ (J. R. Vockeroth); both in CNC.

Remarks. This species belongs to the C. 1uzulae superspecies and appears to be related to $\underline{\text { C. griffithsi from }}$ Colorado in having the frons exceptionally broad. Differences in the aedeagus of the two species are distinctive.

In the paratype from Highlands the frons appears narrower, but this is presumably due to the head having partially shrunk.

Chromatomyia montella Spencer, new species

(Figs. 1205, 1206)

Head. Frons broad, almost 3 times width of eye, narrowly projecting above eye in profile; 2 equal ors, 1 strong ori, orbital setulae sparse; gena extended at rear, almost 0.5 height of eye, parafacial prominent, occupying more than half distance between eye margin and lower margin of gena; 3d antennal segment rounded, slightly longer than broad, with fringe of very fine pubescence.

Mesonotum. $3+1 \mathrm{dc}$, acr sparse, in 2 rows.

Wing. Length in male $2.6 \mathrm{~mm}, 2 \mathrm{~d}$ costal section approx. 3 times length of 4th (exact measurement not possible, as both wings slightly crumpled).

Color. Frons bright yellow, orbit slightly paler; entire hindmargin of eye black; gena yellow, face and palpus black; 3d antennal segment black, 1st and 2d only faintly paler; mesonotum and scutellum matt gray, side of thorax blackish gray; legs black, all knees bright yellow; squama gray, margin and fringe black.

Male genitalia. Aedeagus (figs. 1205, 1206) with dorsal sclerites widely diverging, slender, slightly thickened at end; sperm pump sma11, with narrow, curving blade.

Host/early stages. Unknown.

Holotype m., Colorado, Clear Creek Co., Mt. Evans, $11,300 \mathrm{ft}$, wet vegetation, 10. VIII.61 (C. H. Mann), in CNC.

Remarks. Distinctive characters of this species are the broad frons and relatively long second costal section of the wing. The male genitalia are diagnostic.

Chromatomyia nigrella Spencer, new species

(Figs. 1275-1277)

Head. Frons twice width of eye, not projecting above eye; normally 2 equal ors (upper shorter on one side in 1 of 3 specimens seen), 1 strong ori, equal to ors, sometimes a weak lower also 
present; gena slightly more than 0.33 height of eye; $3 d$ antennal segment smal1, round.

Mesonotum. Acr irregular, in 2-4 rows .

Wing. Length in male $2 \mathrm{~mm}, 2 \mathrm{~d}$ costal section 2.5-2.9 times length of $4 \mathrm{th}$.

Color. Frons uniformly black or slightly paler, blackish brown, above; orbit blackish gray; gena, face, antenna black; legs black but femora with yellowish knees; mesonotum black, with weak subshine and only faintly gray dusted, side of thorax black; squama yellowish, margin and fringe ochreous brown.

Male genitalia. Aedeagus (figs. 1275, 1276) with supporting sclerites of dorsal lobe slender, relatively long, unpigmented in upper half, only narrow1y diverging; end of ejaculatory duct curving dorsally; sperm pump minute (fig. 1277).

Host/early stages. Unknown.

Holotype m., North Carolina, Macon Co., Highlands, 3,800 ft, 8.V.57; paratype m., same data; (both J. R. Vockeroth). Holotype in CNC, paratype in KAS.

Remarks. This is another species in the C. 1uzulae superspecies;

distinctive characters are the distinctly black mesonotum and in the male genitalia the form of the supporting sclerites of the dorsal lobe.

Chromatomyia norwegica (Rydén) (new to U.S.A.)

(Figs. 1262, 1263)

Phytomyza cirsiophaga Hendel var. norwegica Rydén, 1957: 1, from Lapland in Tromsoe Museum, Norway. Chromatomyia norwegica, Griffiths, $1980: 18$

The distribution of this species has been known as holarctic, with records in Europe from Norway, Sweden,
Finland, and Germany (Erzgebirge), and from North America in Alberta and British Columbia. A single male has been seen from Colorado, Clear Creek County, Mt. Evans, Doolittle Ranch, 23.VII.61 (J. G. Chillcott), in CNC.

Although Rydén associated the species with a feeder on Cirsium, it was first recognized as a grass feeder by Spencer (1976a: 447), who included it with Phytomyza milii Kaltenbach. Griffiths (1980) accepted C. norwegica as one of nine species forming part of the $\underline{C}$. milii superspecies. The only other species in this group known in the United States is $C$. pseudomilii (p. 206). A distinctive character of the genitalia of this superspecies is the presence of "wedge-shaped" sclerites between the base of the dorsal lobe (supporting sclerites) and the sclerites of the ventral lobe (fig. 1262).

A distinctive character of $\underline{\text { C. norwegica }}$ is the partially yellow frons, which is, however, black on the lower half. The specimen from Colorado is generally darker than others recorded by Griffiths, but the genitalia agree exactly with the illustrations given by Griffiths of a male from British Columbia and that are repeated here (figs. 1262, 1263).

Chromatomyia poae Griffiths (new to U.S.A.)

(Figs. 1279, 1280)

Chromatomyia poae Griffiths, 1980: 46. Holotype male from Alberta in CNC.

Griffiths (1980: 40) treated the well-known species $\underline{\text { C. fuscula as a }}$ superspecies, recognizing four distinct species, C. fuscula (holarctic), C. puccinelliae spencer (1969a: 269, Greenland), C. alopecuri Griffiths (1980: 47, Alberta), and $\underline{C}$. poae Griffiths (1980: 46, Alberta).

Differences between these species are slight even in the male genitalia. However, a population in Colorado on 
Mt. Evans can be referred with some confidence to $\underline{C}$. poae. The upcurved ejaculatory duct (fig. 1279) is shorter than in C. fuscula s.s., it is equal in length to the supporting sclerites, and the bend at the distal end is tighter.

In seven of the specimens recorded here the frons is reddish brown, but in three males (with genitalia identical to the other examined) the frons is dark brown to black. Griffiths treated as holotype a male with the frons "yellow-brown" and considered a female with the frons dark brown bred from Deschampsia cespitosa as doubtfully conspecific. It is clear from the present series that there is a considerable color range in the frons of this species, and it seems probable that the female obtained by Griffiths is correctly referable to $\underline{C}$. poae.

New records.

Colorado, Clear Creek Co., Mt. Evans, timberline, $11,700 \mathrm{ft}, 2 \mathrm{~m} ., 22$ and 29.VII.1961 (C. H. Mann); Mt. Evans, 11,000-11,800 ft, $1 \mathrm{~m} ., 22$.VII.61 (J. G. Chillcott); Mt. Evans, Rocky slope, 13,000 ft, $1 \mathrm{f.,} \mathrm{4.VIII.61}$ (J. G. Chillcott); Boulder Co., nr. Ward, Niuwat Ridge, $11,300 \mathrm{ft}, 2 \mathrm{~m}$., 28.VI.61 (C. H. Mann); Boulder Co., Corona Pass, 10,600 ft, $1 \mathrm{f}$., 6.VII.61 (J. G. Chillcott); Nederland, Caribou, 10,000 ft, 1 f., 18.VI.61 (B. H. Poole); Grand Co., Frazer, 9,500 ft, $1 \mathrm{f.,} 7 . V I I .61$ (J. G. Chillcott); Park Co., Jefferson, $9,400 \mathrm{ft}, 1 \mathrm{f.,} 21 . \mathrm{VI} .61$ (C. H. Mann); all in CNC.

\section{Chromatomyia regalensis (Steyskal),}

\section{new status}

(Fig. 1302)

Phytomyza regalensis Steyskal, 1972b: 8. Holotype male from Michigan in USNM.

Chromatomyia regalensis, Griffiths, 1980: 8 (as nomen dubium).

Three specimens were included in the type series, all from the same locality, Isle Royale, Keweenaw County, Mich. Steyskal in his description referred to variation in color, but Griffiths (1980) rightly noted that one of the paratypes, which has the notopleuron yellow, represents Phytomyza notopleuralis Spencer, 1969a. The second paratype now lacks its genitalia and cannot be positively identified, although it may well be identical with the holotype.

Griffiths (1980) pointed out that the collection date of the holotype, 10 July 1957, is not as recorded in the description, which is $19 \mathrm{July}$. However, this can reasonably be explained as a typing error. He also considered Steyskal's illustration to be "incompatible" with the aedeagus that he examined. KAS has also examined this specimen and finds that the aedeagus is damaged and the sperm pump is lost. The supporting sclerites are truncated, but nevertheless from what remains it appears that Steyskal's illustration of this important character can be accepted as accurate; the prominent ejaculatory duct was seen (but is now also lost). The one point of inaccuracy is the failure to show the prominent, large sclerite of the ventral lobe (fig. 1302), which was not readily visible in the original specimen owing to inadequate clearing.

Although an element of doubt remains about the status of this species, it seems correct to retain it as valid, but further material, if possible reared from the type locality, is obviously desirable for confirmation that it is indeed different from other related species feeding on Caprifoliaceae.

A male from Georgia, Chattooga County, Cloudland Canyon State Park, 8.V.52 (0. Peck), in CNC, is tentatively identified as $\underline{C}$. regalensis, and the aedeagus is shown in figure 1302 (see Steyskal, 1972b: fig. 8, a ). 
Aldrich, J. M. 1918. Seasonal climatic variation in Cerodontha. Ent. Soc. Amer. Ann. 11: 63-66.

Blanchard, E. E. 1938. Descripciones y anotaciones de dipteros argentinos. Agromyzidae. Soc. Cient. Argentina An. 126: 352-359.

Braschnikov, W. C. 1897. [On the biology and systematics of some species of mining Diptera.] Moskva Selsk. Khoz. Inst. Izv. 3(2): 19-43. [In Russian.]

Brèthes, J. 1920. Insectos útiles y dañinos de Rio Grande do Sul y de la Plata. Soc. Rur. Argentina An. 54: 281-290.

Brischke, C. G. A. 1881. Die Blattminierer in Danzig's Umgebung. Naturf. Gese11. Danzig Schr., n. ser., 5: 233-290.

Brown, L. R., and C. O. Eads. 1969. Unnamed and little-known insects attacking cottonwood in southern California. Jour. Econ. Ent. 62: 667-674.

Burgess, E. 1880. The clover Oscinis (Oscinis trifolii, Burgess n. sp.). U.S. Dept. Agr. Rpt. 1879: 200-201.

Claassen, P.W. 1918. Observations on the life history and biology of Agromyza latere11a Zetterstedt (Diptera). Ent. Soc. Amer. Ann. 11: 9-18.

Coquillett, D. W. 1898. On the habits of the Oscinidae and Agromyzidae, reared at the United States Department of Agriculture. U.S. Dept. Agr. Bul . 10: 70-79. 1902. New acalyptrate Diptera from North America. N.Y. Ent. Soc. Jour. 10: 177-191. 1910. New genera and species of North American Diptera. Ent. Soc. Wash. Proc. 12: 131.

Couden, E. D. 1908. A ga11-maker of the family Agromzidae (Agromyza tiliae, n. sp.). Ent. Soc.Wash. Proc. 9: 34-36.

Enderlein, G. 1936. Zweiflüler, Diptera. In Brohmer et al., Tierwelt Mitteleur. 6 (Lief. 2. Insekten, 3) : 179-183.

Fallén, C. F. 1810. Specim. entomolog. novam Diptera disponendi methodum exhibens. 26 pp. Lundae. 1823a. Agromyzides sveciae.

10 pp. Lundae. 1823b. Phytomyzides et

Ochtidiae sveciae. 10 pp. Lundae.

Freeman, C. C. 1958. Liriomyza guytona, a new species of agromyzid leaf miner. Ent. Soc. Amer. Ann. 51: 344-345.

Frey, R. 1941. Diptera. Enumeratio Insectorum Fenniae et Sueciae 6: $1-63$.

1946. Anteckningar om

Finlands agromyzider. Notulae Ent. 26: 13-55.

Frick, K. E. 1951a. Liriomyza langei, a new species of leaf-miner of economic importance in California. Pan-Pacific Ent. 21: 81.

1951b. Parthenogenetic reproduction in Phytomyza plantaginis R. $-D$., the second reported case in the family Agromyzidae (Diptera). Science 114: 576 .

1951c. A satisfactory technique for rearing agromyzid flies from the leaf mining larval stage. PanPacific Ent. 27: 187-189. 1952a. A generic revision of the family Agromyzidae (Diptera) with a catalogue of New World species. Univ. Calif. Pubs., Ent. 8 : 339-452. 1952b. Four new Hawaiian Liriomyza species and notes on other Hawaiian Agromyzidae (Diptera). Hawaii. Ent. Soc. Proc. 14: 509-518. 1952c. The status of

Agromyza illinoensis Malloch, 1934 , and its correct generic position (Agromyzidae, Diptera). Kans. Ent. Soc. Jour. 25: 150-153. 1953a. Some additions and corrections to the species 1 ist of North American Agromyzidae (Diptera). Canad. Ent. 85: 68-76. 1953b. Further studies on Hawaiian Agromyzidae (Diptera) with descriptions of four new species. Wash. State Agr. Expt. Sta. Bul. 1182: 207-215. 1954. Three North American Phytomyza species closely related to P. nigritella Zetterstedt (Agromyzidae: Diptera). Ent. Soc. Amer. Ann. 47: 367-374. 
Frick, K. E. 1955. Nearctic species in the Liriomyza pusilla complex, No. 3. L. al1iovora, new name for the Iowa onion miner. Kans. Ent. Soc. Jour. 28: 88-92.

1956. Revision of the North American Calycomyza species north of Mexico (Phytobia: Agromyzidae, Diptera). Ent. Soc. Amer. Ann. 49: 284-300.

1957a. Nearctic species in the Liriomyza pusilla complex, No. 2 . L. munda and two other species attacking crops in California. Pan-Pacific Ent. 33: 59-70.

1957b. Nomenclatural changes and type of designations of some New World Agromyzidae (Diptera). Ent. Soc. Amer. Ann. 50: 198-205. 1958. Liriomyza dianthi n. sp., a new pest of carnations in California. Ent. Soc. Wash. Proc. $60: 1-5$.

1959. Synopsis of the species of agromyzid leaf miners described from North America (Diptera). U.S. Nat1. Mus. Proc. 108: 347-465.

1972. Biological notes on

adults and eggs of Phytomyza syngenesiae in north-central coastal California. Jour. Econ. Ent. 65: 1310-1313.

Frison, T. H. 1927. A 1ist of the insect types in the collections of the Illinois State Natural History Survey and the University of I1linois. I11. State Nat. Hist. Survey Bu1. 16(IV): 137-309.

Frost, S. W. 1924. A study of the leaf-mining Diptera of North America. Cornell Univ. Agr. Expt. Sta. Mem. 78: 1-228.

1927. Three new species of Phytomyza (Agromyzidae, Diptera). Ent. Soc. Amer. Ann. 20: 217-220. 1931a. New species of West Indian Agromyzidae (Diptera). Ent. News 42: 72-75.

1931b. New North American

Agromyzidae (Dipt.) Canad. Ent. 63: 273-277.

1934. A new species re-

lated to Agromyza virens Loew (Dipt.: Agromyzidae). Ent. News 45: 40-41.

1943. Three new species of Diptera related to Agromyza pusil1a
Meig. N.Y. Ent. Soc. Jour. 51: 253-263.

1954. A new name for Phytomyza subpusilla Frost (Diptera). Ent. News 65: 73.

1962. Liriomyza archboldi, a

new species (Dipt., Agromyzidae).

Ent. News 73: 51-53.

Greene, C. G. 1914. The cambium miner in river birch. Jour. Agr. Res. 1: 471-474.

1917. Two new cambium miners

(Diptera). Jour. Agr. Res. 10:

313-317.

Griffiths, G. C. D. 1964. The agromyzid fauna of Iceland and the Faroes, with appendices on the Phytomyza milii and robuste1la groups (Diptera, Agromyzidae). Ent. Medde1. 32: 393-750.

1967. Revision of the

Phytomyza syngenesiae group (Diptera, Agromyzidae), including species hitherto known as "Phytomyza atricornis Meigen." Stuttgart. Beitr. Naturk. 177: 1-28. 1968. Further notes on

Icelandic Agromyzidae (Dipt.). Opusc. Ent. 33: 129-138.

1972a. Studies on boreal

Agromyzidae (Diptera). 1. Phytomyza miners on Saxifragaceae. Quaest. Ent. 8: 67-80. 1972b. Studies on the phylogenetic classification of Diptera Cyclorrhapha, with special reference to the male postabdomen. $340 \mathrm{pp}$. W. Junk, The Hague.

1973a. Studies on boreal Agromyzidae (Diptera). IV. Phytomyza miners on Angelica, Heracleum, Laserpitium and Pastinaca (Umbelliferae). Quaest. Ent. 9: 219-253.

1973b. Description of an unnamed Paraphytomyza (Diptera, Agromyzidae). Ent. Gaz. 24: 307-310. 1974a. Studies on boreal Agromyzidae (Diptera). V. On the genus Chromatomyia Hardy, with revision of Caprifoliaceae-mining species. Quaest. Ent. 10: 35-69. 1974b. Studies on boreal Agromyzidae (Diptera). VI. Further Phytomyza miners on Senecioneae (Compositae). Quaest. Ent. 10: 103-129. 
Griffiths, G. C. D. 1974c. Studies on boreal Agromyzidae (Diptera). VII. A new Chromatomyia miner on Valeriana. Quaest. Ent. 10: 217-222. $1974 \mathrm{~d}$. Studies on boreal Agromyzidae (Diptera). VIII. Phytomyza miners on Artemisia (Compositae). Quaest. Ent. 10: 295-314.

1975. Studies on boreal Agromyzidae (Diptera). IX. Phytomyza miners on Boraginaceae in North America. Quaest. Ent. 11: 129-142.

1976a. Studies on boreal Agromyzidae (Diptera). XI. Chromatomyia miners on E1 aeagnaceae. Quaest. Ent. 12: 211-216.

1976b. Studies on boreal Agromyzidae (Diptera). XII. Phytomyza and Chromatomyia miners on Asteraceae (Compositae). Quaest. Ent. 12: 239-278.

1977. Studies on boreal Agromyzidae (Diptera). XIII. Some Phytomyza and Chromatomyia miners on Cichoreae (Compositae). Quaest. Ent. 13: 327-345.

1980. Studies on boreal Agromyzidae (Diptera). XIV. Chromatomyia miners on Monocotyledones. Ent. Scand., Sup. 13, pp. 1-61.

Grossenbacher, J. G. 1915. Medullary spots and their cause. Torrey Bot. Club Bul. 42: 227-239.

Guppy, J. C. 1981. Bionomics of the alfalfa blotch leafminer, Agromyza frontella (Diptera, Agromyzidae), in eastern Ontario. Canad. Ent. 113: 593-600.

Haliday, A. H. 1833. Catalogue of Diptera occurring about Holywood in Downshire. Ent. Mag. (London) 1: 147-180.

Hardy, J. 1849. On the primrose-leaf miner; with notice of a proposed new genus, and characters of three species of Diptera. Ann. Mag. Nat. Hist. (2) 4: 385-392.

Hende1, F. 1914. Namensänderungen (Dipt.). Ent. Mitt. 3: 73. 1920. Die paläarktischen

Agromyziden (Prodromus einer Monographie). Arch. f. Naturgesch. A84: 109-174.

1922. Blattminierende Fliegen.

Wien. Ent. Ztg. 39: 65-72.

1923. Neue europäische Melan-
agromyza-Arten (Dipt.). Konowia 2: 142-145.

1931-36. Agromyzidae. 59. In

Lindner, E., ed., Die Fliegen der

Palaearktischen Region 6 (pt. 2;

Lief. 52, 54, 56, 58): 1-256; (Lief. 66) : 257-320.

Hering, M. 1927. Beiträge zur

Kenntnis der Ökologie und Systematik blattminierende Insekten (Minenstudien VIII). Ztschr. f. Angew.

Ent. 13: 156-198.

1932. Minenstudien XI.

Ztschr. f. Wiss. Insektenbiol. 26:

157-182.

1937. Agromyziden-Nachlese

(Dipt.) II. Deut. Ent. Gesell.

Mitt. 8: 76-77.

1949. Neue paläarktische

Agromyziden. Notulae Ent. 29: 18-32. 1951a. Neue paläarktische

und nearktische Agromyziden

(Dipt.). Notulae Ent. 31: 31-45. 1951b. Biology of the leaf

miners. 420 pp. W. Junk,

's-Gravenhage. 1954. Die Larven der

Agromyziden (Diptera) - 1.

Tijdschr. v. Ent. 97: 115-136.

1955. Die Liriomyza-Arten

von Lactuca und Sonchus (Dipt.

Agromyz.). Deut. Ent. Ztschr. 2: 204-209.

1956. Die Larven der Agro-

myziden (Diptera) - 2. Tijdschr. v.

Ent. 98: 257-281.

1957. Die Larven der

Agromyziden (Diptera) III.

Tijdschr. v. Ent. 100: 73-94.

1958. Stengelbewohnende

Larven an Clematis recta L. (Dipt. Agromyzidae). Deut. Ent. Ztschr.

(N.F.) 5: 72-78.

1960. Neue Blattminen-

Studien. Deut. Ent. Ztschr. (N.F.)

7: 119-145.

Hopkins, D. M., ed. 1967. The Bering land bridge. 495 pp. Stanford Univ. Press, Stanford, Calif.

Johnson, C. W. 1922. New North American Diptera. Boston Soc. Nat. Hist. Occas. Papers 5: 21-26.

Kaltenbach, J. H. 1858. Die Phytophagen aus der Klasse der Insekten (B). Naturhist. Ver. der Preuss. Rheinlande Verhand1. 15: 77-161. 
Kaltenbach, J. H. 1864. Die Phytophagen aus der Klasse der Insekten (B).

Naturhist. Ver. der Preuss. Rheinlande Verhand 1. 21: 228-404.

1867. Die Phytophagen aus der

Klasse der Insekten (B). Naturhist.

Ver. der Preuss. Rheinlande

Verhand1. 24: 21-117.

1874. Die Pflanzenfeinde aus

der Klasse der Insekten. 848 pp.

Stuttgart.

Kulp, L. A. 1968. The taxonomic status of holly leaf miners

(Diptera: Agromyzidae). Univ. Md. Agr. Expt. Sta. Bul. Al55: 1-42.

Lioy, P. 1864. I ditteri distribuiti secondo un nuovo metodo di classificazione naturale. I. Reale Ist. Veneto Sci., Let. Arti, Atti ser. 3, 9: 1311-1352.

Loew, H. 1863. Diptera Americae septentrionalis indigena. Centuria tertia. Berlin. Ent. Ztschr. 7: $1-55$.

1869. Diptera Americae septentrionalis indigena. Centuria octava. Berlin. Ent. Ztschr. 13: $1-52$.

1872. Diptera Americae

septentrionalis indigena. Centuria decima. Berlin. Ent. Ztschr. 16: 49-115.

Luginbill, P., and T. D. Urbahns. 1916. The spike-horned leaf miner, an enemy of grains and grasses. U.S. Dept. Agr. Bul. 432, pp. 1-18.

Lundquist, A. 1949. Blattminennotizen. Opusc. Ent. 14: 169-174.

Malloch, J. R. 1913a. A revision of the species in Agromyza Fallen and Cerodontha Rondani. Ent. Soc. Amer. Ann. 6: 269-336.

1913b. A synopsis of the genera of Agromyzidae, with descriptions of new genera and species. U.S. Natl. Mus. Proc. 46: 127-154. 1914a. Description of a new species of Agromyza from Porto Rico. Ent. Soc. Wash. Proc. 16: 89-90.

1914b. Notes on North American Agromyzidae (Diptera). Ent. News 25: 308-314.

1914c. Formosan Agromyzidae.

Mus. Nat. Hungarici Ann. 12: 306-336. 1915a. Flies of the genus
Agromyza, related to Agromyza

virens. U.S. Nat1. Mus. Proc. 49: $\overline{103-108 .}$

1915b. North American

Diptera. Canad. Ent. 47: 12-16. 1915c. Some additional records

of Chironomidae for Illinois and notes on other Illinois Diptera. I11. State Nat. Hist. Survey Bul. 11 : 305-363.

1916. Three new North American species of the genus Agromyza.

Psyche (Cambridge, Mass.) 23: 50-54. 1918a. A partial key to species of the genus Agromyza (Diptera). Canad. Ent. 50: 76-80, 130-132. 1918b. Key to the North American species of Agromyza related to simplex Loew. Canad. Ent. 50: 178-179. 1920. A new genus of Agromyzidae (Diptera). Brooklyn Ent. Soc. Bu1. 15: 147-148. 1924. Three new species of Agromyza and synonymical notes. Canad. Ent. 56: 191-192. 1934. Acslyptrata (concluded). Diptera of Patagonia and South Chile. Brit. Mus. (Nat. Hist.), pt. VI, Fasc. 2: 393-489.

Meigen, J. W. 1830. Systematische Beschreibung der bekannten europäischen zweiflügeligen. Insekten, v. 6, pp. 166-196. Hamm. 1838. Systematische Beschreibung der bekannten europäischen zweiflügeligen. Insekten, v. 7, pp. 396-407. Hamm.

Meijere, J. C. H. de. 1924. Verzeichnis der holländischen Agromyzinen. Tijdschr. v. Ent. 67: 119-155.

1925. Die Larven der Agromyzinen. Tijdschr. v. Ent. 68: 195-293.

1950. Die Larven der Agromyzinen. Nachtrag 9. Tijdschr. v. Ent. 92: 15-33. 1955. Die Larven der Agromyziden (Diptera). Genera1-Register. Tijdschr. v. Ent. 98: 1-27.

Melander, A. L. 1913. A synopsis of the dipterous groups Agromyzinae, Milichiinae, Ochthiphilinae, and Geomyzinae. N.Y. Ent. Soc. Jour. 21: 219-300. 
Mik, J. 1894. Ueber eine neue Agromyza, deren Larven in den Blïthenknospen von Lilium martagon leben. Wien. Ent. Ztg. 13: 284-290. Needham, J. G., S. W. Frost, and J. D. Tothill. 1928. Leaf-mining insects. 351 pp. Williams and Wilkins, Baltimore, Md.

Nowakowski, J. T. 1959. Studien über Minierfliegen 3. Revision der in Labiaten und Boraginaceen minierenden Arten aus der Gruppe der Phytomyza obscura Hend. Deut. Ent. Ztschr. (N.F.) 6: 185-229.

1962. Introduction to a systematic revision of the family Agromyzidae (Diptera) with some remarks on host plant selection by these flies. Polska Akad. Nauk Inst. Zool. Ann. Zoo1. 20(8): 67-183. 1964. Studien uber Minierfliegen (Dipt. Agromyzidae) 9. Revision der Artengruppe Agromyza reptans Fal1. - A. rufipes Meig. Deut. Ent. Ztschr. (N.F.) 11: 175-213.

1967. Vorläufige Mitteilung zu einer Mongraphie der europäischen Arten der Gattung Cerodontha Rond. (Diptera, Agromyzidae). Polskie Pismo Ent. 37: 633-661.

1972. Zweite vorläufige

Mitteilung zu einer Monographie der europäischen Arten der Gattung Cerodontha Rond. (Diptera, Agromyzidae). Polskie Pismo Ent. 42: 735-765.

1973. Monographie der europäischen Arten der Gattung Cerodontha Rond. (Diptera, Agromyzidae). Polska Akad. Nauk Inst. Zoo1. Ann. Zool. 31: 1-327.

Panzer, G. W. F. 1806. Faunae insectorum germanicae initia oder Deutschlands Insecten. Heft 104, 24 pp., 24 pls. Nürnberg.

Phillips, W. J. 1914. Corn-leaf blotch miner. Jour. Agr. Res. 2: 15-31.

Riley, C. V. 1884. The cabbage Oscinis. U.S. Dept. Agr. Ann. Rpt. 1884: 322.

Robineau-Desvoidy, J. B. 1851 . Descriptions d'Agromyzes et de Phytomyzés éclosés chez M. le Col. Goureau. Rev. Mag. Zool. (2) 3 : 391-405.
Rondani, C. 1856. Dipterologiae Italicae prodromus. V. I, $228 \mathrm{pp}$. Parmae. [This work and its reprints have a complex collation; see Bibliography in Stone, A., et al., 1965, p. 1393.]

1861. Dipterologiae Italicae prodromus. V. 4, 174 pp. Parmae. [See Rondani, 1856.] 1875. Dipterologiae Italicae prodromus. V. 8, Stirps 23, $26 \mathrm{pp}$. Firenze. [See Rondani, 1856.]

Rydén, N. 1944. Ti11 kännedomen om svenska bladminerare VII. Opusc. Ent. 9: 48-50.

1957. Norwegische Agromyziden 2. Astarte 15: 1-4.

Sasakawa, M. 1954. Neue Agromyzidae aus Japan. Shikoku Ent. Soc. Trans. 4: 35-49.

1958. The female terminalia of the Agromyzidae, with description of a new genus (1). Saikyo Univ. (Kyoto), Sci. Rpt., Agr. 10: 133-145. 1961. A study of the Japanese Agromyzidae (Diptera), 2. Pacific Insects 3: 307-472. 1963a. Oriental Agromyzidae (Diptera) in Bishop Museum, 1. Pacific Insects 5: 25-50. 1963b. Papuan Agromyzidae (Diptera). Pacific Insects 5: 797-835.

Schrank, F. von P. 1803. Fauna Boica. V. 3, pt. 1, 272 pp. Landhut.

Sehgal, V. K. 1968. Descriptions of new species of flies of the family Agromyzidae from Alberta, Canada (Diptera). Quaest. Ent. 4: 57-88. 1971. A taxonomic survey of the Agromyzidae (Diptera) of Alberta, Canada, with observations on host-plant relationships.

Quaest. Ent. 7: 291-405.

Shetler, S. G., and L. E. Skog. 1978. A provisional checklist of species for Flora North America. (Rev., FNA Rpt. 84.) Mo. Bot. Gard., Monog. Syst. Bot., v. 1, 199 pp.

Shewe11, G. E. 1953. Notes on the types of some American Agromyzidae (Diptera). Canad. Ent. 85: 462-470. Siebke, H. 1864. Beretning om en $i$ Sommeren 1861 foretagen entomologisk Reise. Nyt. Mag. Naturvid. 12: 169. 
Silva, G. A. da, and S. J. de Oliveira de. 1952. Sôbre um "Agromyzidae: (Diptera) cujas larvas minam folhas de Trapoeiraba (Commelinaceae). Bras. Biol. Rev. 12: 293-299.

Singh, S., and I. M. Ipe. 1973. The Agromyzidae from India. St. Johns's Col., Mem. Sch. Ent. 1: i-ii, 1-286, i-v, p1. I-CLXXVI.

Spencer, K. A. 1963a. A synopsis of the neotropical Agromyzidae. Roy.

Ent. Soc., London, Trans. 115: 291-389.

1963b. Notes on the Agromyzidae (Diptera) of Madagascar 1. Roy. Ent. Soc., London, Proc. B32: 114-116.

1963c. Notes on the

African Agromyzidae (Diptera) - 4 .

Ent. Soc. South. Afr. Jour. 26:

94-124.

1964a. A revision of the palaearctic species of the genus Ophiomyia Braschnikov (Diptera: Agromyzidae). Beitr. z. Ent. 14: 773-822.

1964b. Notes on the

African Agromyzidae (Diptera) - 5 . Deut. Ent. Ztschr. (N.F.) 11: 15-41. 1965a. A clarification of

the status of Liriomyza trifolii

(Burgess) and some related species.

Ent. Soc. Wash. Proc. 67: 32-40. 1965b. Agromyzidae. In

Diptera From Nepal. Brit. Mus.

(Nat. Hist.) Dept. Ent. Bul. 16: 25-31.

1965c. A clarification of

Fallén's types of Agromyzidae in Stockholm and Lund. Ent. Tidskr. 86 : 249-259.

1965d. Notes on the Oriental

Agromyzidae (Diptera) - 2. Agromyzidae from the Philippines. Ent.

Medde1. 34: 3-9.

1966a. A revision of

European species of the genera

Melanagromyza Hendel and Hexomyza

Enderlein, with a supplement on the genus Ophiomyia Braschnikov. Beitr. z.

Ent. 16: 3-60.

1966b. A new Phytomyza

species from California. Pan-Pacific

Ent. 42: 108-110.

1966c. Notes on European

Agromyzidae - 1. Beitr. z. Ent. 16:

285-309. 1966d. New and interesting

Agromyzidae from Florida.

Stuttgart. Beitr. Naturk. 158: 1-20. 1966e. Notes on the neo-

tropical Agromyzidae - 1. Dept.

Zool. (São Paulo) Papeis Avulsos 19: 141-150.

1969a. The Agromyzidae of

Canada and Alaska. Ent. Soc. Canad.

Mem. 64: 1-311.

1969b. Notes on the European

Agromyzidae (Diptera) - 2. Beitr.

z. Ent. 19: 5-26.

1971a. Notes on a revision of

the British Agromyzidae (Diptera),

including the description of 14 new species. Ent. Gaz. 22: 141-195.

1971b. Notes on European Agromyzidae (Dipt.) - 3. Beitr. z. Ent. 21: 249-265.

1973a. Agromyzidae (Diptera)

of economic importance. Ent. Ser.

9, 405 pp. W. Junk, The Hague.

1973b. The Agromyzidae of

Venezuela. Facult. Agron. (Maracay)

Rev. 7(2): 5-107.

1973c. A new species of

Agromyzidae mining bracken

(Pteridium aquilinum L.). Ent. Gaz. 24:315-317.

1975. Agromyzidae from Ceylon.

Ent. Scand., Sup. 4, pp. 209-220.

1976a. The Agromyzidae (Diptera)

of Fennoscandia and Denmark.

Fauna Ent. Scand. 5 (pt. 1): 1-304;

(pt. 2): 305-606.

1976b. The Agromyzidae of

New Zealand (Insecta: Diptera).

Jour. Roy. Soc. New Zeal. 6: 151-211.

1977a. A new species of

Phytoliriomyza from Nepal (Diptera,

Agromyzidae). Ent. Gaz. 28: 15-16.

1977b. Agromyzidae in New

Guinea. Pacific Insects 17: 339-369.

1977c. Notes on world

Agromyzidae (Diptera), with the

description of 16 new species.

Beitr. z. Ent. 27: 233-254.

1977d. A revision of the

Australian Agromyzidae (Diptera).

West. Austral. Mus. Spec. Pub. 8: $1-255$.

1978. Clarification of

the status of Cerodontha (Dizygomyza) chaixiana (Hering, 1956). Stuttgart. Beitr. Naturk. (Ser. A) 306: 1-3. 
Spencer, K. A. 1981. A revisionary study of the leaf-mining flies (Agromyzidae) of California. Univ. Calif., Div. Agr. Sci. Spec. Pub. 3273: 1-489. and D. S. Hill. 1976. A new species of Ophiomyia (Diptera: Agromyzidae) causing leaf falls on Ficus microcarpa in Hong Kong. Hong Kong Dept. Agr. Bu1. 1: 419-423. and C. E. Stegmaier. 1973. The Agromyzidae of Florida with a supplement on species from the Caribbean. Arthropods of Florida, v. 7, pp. 1205.

Stegmaier, C. E., Jr. 1966a. Host plants and parasites of Liriomyza trifolii in Florida (Diptera:

Agromyzidae). Fla. Ent. 49: 75-80. 1966b. Host plants and

parasites of Liriomyza munda in Florida (Diptera: Agromyzidae).

F1a. Ent. 49: 81-86. 1966c. Host plants and

parasites of Liriomyza schmidti in Florida (Diptera: Agromyzidae).

Fla. Ent. 49: 119-122. 1967a. Some new host

plant records and parasites of Phytobia (Amauromyza) maculosa in Florida (Diptera: Agromyzidae).

F1a. Ent. 50: 99-101.

1967b. Notes on new host

plant records and parasites of

Liriomyza sorosis in Florida

(Diptera: Agromyzidae). Fla. Ent.

50: 133-136.

1967c. Host plants of

Liriomyza brassicae, with records of

their parasites from south Florida

(Diptera: Agromyzidae). Fla. Ent. $50: 278-281$.

Steyska1, G. C. 1964. Descriptive and synonymical notes on Liriomyza munda (Diptera: Agromyzidae). Ent. Soc. Amer. Ann. 57: 116.

1972a. The taxonomy of the a1falfa blotch-miner, Agromyza fronte 1la (Rondani). U.S. Dept. Agr. Coop.

Econ. Insect Rpt. 22: 134-137.

1972b. New and little-

known Agromyzidae from Michigan

(Diptera: Acalyptratae). Great

Lakes Ent. 5: 1-10.

1973a. The identity of

Calycomyza jucunda (Wulp) (Diptera,
Agromyzidae). Ent. Soc. Wash. Proc. 75: 191-194.

1973b. The strange fate of the "serpentine leaf miner" (Diptera: Agromyzidae). U.S. Dept. Agr. Coop. Econ. Insect Rpt. 23: 735-736. 1976. Additional data on hol1y leafminer adults (Diptera, Agromyzidae: Phytomyza ilicis ilicicola group). U.S. Dept. Agr. Coop. P1ant Pest Rpt. 1: 767-770. 1980. Haplopeodes, a new genus for Haplomyza of authors (Diptera, Agromyzidae). Ent. Soc. Wash. Proc. 82: 140-151. 1981. Six new species of Agromyzidae. Wash. Acad. Sci. Jour. $70: 36-43$. and K. A. Spencer. 1978. A new species of Phytoliriomyza Hendel feeding on jacaranda in California (Diptera: Agromyzidae). U.S. Dept. Agr. Coop. Plant Pest Rpt. 3: 585-586.

Stone, A., C. W. Sabrosky, W. W. Wirth, and others. 1965. A catalog of the Diptera of America north of Mexico. U.S. Dept. Agr. Handb. 276, 1696 pp.

Strob1, G. 1880. Dipterologische Funde um Seitenstetten. Programme des $\mathrm{K}$. K. Obergymnasiums der Benedictiner in Seitenstetten 14: $1-65$. 1898. Die Dipteren von Steiermark. IV. Theil. Naturw. Ver. Steiermark Mitt. 34 (1897): 192-298. 1909. Neue österreichische Muscidae Acalypterae II. Wien. Ent. Ztg. 28: 283-301.

Siiss, H. 1979. Durch Protophytobia cupressorum gen. nov., sp. nov. (Agromyzidae, Diptera) verursachte Markflecke in einem Holz von Juniperoxylon aus dem Tertiär von Süd-Limburg (Nederlande) und der Nachweis von Markflecken in einer rezenten Callitris-Art. Feddes Repert., Ber1in, 90: 165-172. and W. R. Müiller-Stoll.

1975. Durch Palaeophytobia platani n.g., n. sp. (Agromyza, Diptera) verursachte Markflecken im Holz fossiler Platanen aus dem ungarischen Miozän. Humboldt Univ., 
Berlin, Wiss. Ztschr. Math.-Naturw. Reihe 24: 515-519.

Tschirnhaus, M. von. 1969. Zur Kenntnis der Variabilität, Eidonomie und Verwandtschaft bemerkenswerter Agromyzidae (Diptera). Senckenberg, Biol. 50: 143-157.

1971. Unbekannte Stridula-

tionsorgane bei Dipteren und ihre Bedeutung für Taxonomie und Phylogenetik der Agromyziden.

Beitr. z. Ent. 21: 551-579. 1981. Die Halm- und Minierfliegen im Grenzbereich Land-Meer der Nordsee. Spixiana, Sup. 6, pp. 1-405, p1s. 1-11.

Valley, K. R. 1982. A new Liriomyza mining leaflets of black locust (Diptera: Agromyzidae). Ent. Soc. Wash. Proc. 84: 781-785.

Watt, M. 1924. The leaf-mining insects of New Zealand: Pt. 5 - The genus Nepticula (Lepidoptera), and the Agromyzidae (Diptera) continued, and Gracilaria selonitis Meyr. (Lepidoptera). Beitr.z. Ent. 55: 674-687.

Westwood, J. 0. 1840. An introduction to the modern classification of insects. II. Synopsis of the genera of British insects. $158 \mathrm{pp}$. London. Wheeler, A. G., Jr., and T. J. Henry. 1981. Seasonal history and habits of the European alfalfa beetle, Subcoccinella vigintiquatuopunctata (L.) (Coleoptera: Coccinellidae). Coleopt. Bu1. 35: 197-203.

Wulp, F. M. van der. 1867. Eenige Noord-Americaansche Diptera. Tijdschr. v. Ent. 10: 125-164. 1871. Dipterologische

Aantekeningen. No. 3. VI. Muscidae Acalypterae. Tijdschr. v. Ent. 14: 186-210.

Zetterstedt, J. W. 1848. Diptera Scandinaviae 7: 2728-2844. Lundae. 


\section{APPENDIX}

New Nomenclature

1. New species

Melanagromyza:

chillcotti

chillcottiana

longensis

proboscidata

u1tima

verbesinae

virginiens is

walleyi

Ophiomyia:

abutilivora

arizonensis

asymmetrica

boulderensis

carolinae

carolinensis

chondrillae

eldorensis

frosti

parva

subpraecisa

texella

virginiensis

vockerothi

Agromyza:

hardy $i$

pagana

parca

parilis

pudica

utahens is

Phytobia pallida

Amauromyza (Cephalomyza):

confondata

knowltoni

Cerodontha (Poemyza):

attenuata

chillcottiella

macminni

C. (Butomomyza):

fusculata

impercepta

impolita

parvel1a

C. (Dizygomyza) :

aristosa

latifrons

poolei

Liriomyza:

elevata eupatoriella

montella

montis

pechumani

pulloides

splendens

subasclepiadis

temperata

texella

virginica

Galiomyza:

violivora

vockeroth $i$

Calycomyza:

frickiana

orientalis

Phytoliriomyza:

consulta

pulche1la

Paraphytomyza coloradensis

Napomyza:

grande11a

manni

Phytomyza:

aldrich $i$

bou1dere11a

ceanothi

clematisella

clematoides

colorade11a

coquilletti

duplex

evansi

flavens

flexuosa

latifrons

manni

masoni

pulchella

ranunculoides

Chromatomyia:

compta

doolittlei

flavida

griffithsi

luzulivora

mitche11i

monte11a

nigrella

2. New synonyms

Melanagromyza burgessi (Ma1loch, 1913a)

M. malefica Spencer, 1981

M. ripare11a (Hende1, 1923)

M. 1ippivora Spencer, 1966d 
Hexomyza winnemanae (Ma1loch, 1913a)

H. albicula Spencer, 1969a

Oph̄iomyia congregata (Ma1loch, 1913a)

o. pulicarioides Sehgal, 1968

o. decima Spencer, 1969a

o. praecisa spencer, 1969a

0. aestimabilis Spencer, 1981

o. similata (Malloch, 1918)

Melanagromyza orientalis Spencer, $1969 a$

O. texana (Malloch, 1913a)

o. shiloensis Spencer, 1969a

o. arguta Spencer, 1973

0. modesta Spencer, 1981

Japanagromyza rutiliceps (Melander,

1913)

Agromyza irwini Spencer, 1981

Agromyza albipennis Meigen, 1830

A. dubitata Malloch, 1913a

A. isolata Malloch, 1913a

A. populoides Spencer, 1969a
A. nigrella (Rondani, 1875)

A. barberi Frick, 1952a

A. reptans Fallén, 1823a

A. haplacme Steyskal, 1972b

Phytobia calyptrata (Hendel, 1923)

P. septentrionalis Spencer, 1969a

Cerodontha (Xenophytomyza) illinoensis

(Malloch, 1934)

C. (Poemyza) simcoensis Spencer, $1969 a$

Liriomyza sativae Blanchard, 1938

L. verbenicola Hering, 1951a

L. togata (Melander, 1913)

L. douglasii Spencer, 1981

L. trifolii (Burgess, 1880)

L. phaseolunata Frost, 1943

Hap̄lopeodes kefi Steyskal, 1980

Phytoliriomyza yolensis Spencer, 1981

Phytomyza saskatoonens is Spencer, 1969a

P. cudu Steyska1, 1972b

3. New combinations

\section{$\underline{\text { Present }}$ status}

Hexomyza winnemanae (Malloch, 1913a)

Ophiomyia fastosa (Spencer, 1969a)

Ophiomyia lauta (spencer, 1969a)

Ophiomyia parvella (Spencer, 1973)

Ophiomyia similata (Malloch, 1918)

Ophiomyia tiliae (Couden, 1908)

Japanagromyza rutiliceps (Melander, 1913)

Amauromyza (Cephalomyza) albidohalterata

(Malloch, 1916)

Aauromyza (ㅁ. ) auriceps (Melander, 1913)

Phytoliriomyza conspicua (Sehgal, 1968)

Phytoliriomyza felti (Malloch, 1914b)

Phytoliriomyza fumicosta (Malloch, 1914b)

Phytoliriomyza pallida (Sehgal, 1968)

Phytoliriomyza varia (Melander, 1913)

Napomyza marginalis (Frost, 1927)

Chromatomyia clemativora (Coquillett, 1910)

4. New name

Ophiomyia subdefinita Spencer, replacement name for $\underline{0}$. definita Spencer, 1981, preoccupied

5. Removed from synonymy

Agromyza aprilina Malloch
Former status

Melanagromyza winnemanae

Melanagromyza fastosa

Melanagromyza lauta

Melanagromyza parvella

Melanagromyza similata

Hexomyza tiliae

Agromyza rutiliceps

Phytobia (‥) albidohalterata

Phytobia (‥) auriceps

Liriomyza conspicua

Liriomyza felti

Liriomyza fumicosta

Lemurimyza pallida

Phytobia (Trilobomyza) varia

Phytomyza marginalis

Phytomyza clemativora 
Abbreviations and Acronyms

Abbreviations (acronyms) for depositories of specimens, such as museums, are as suggested by Griffiths (1980). Genus names abbreviated with an initial in binomina after their first use in a paragraph are not listed here, nor are standard abbreviations of names of periodicals and of States in the United States. A few initials and acronyms, mostly from insect labels, remain unelucidated.

acr - acrostichal (bristles)

AMNH - American Museum of Natural

History, New York

BMNH - British Museum (Natural

History), London

C - costa (costal vein of wing)

CAS - California Academy of Siences,

San Francisco

$\mathrm{CB}$ - cambium borer

CNC - Canadian National Museum, Ottawa

Co. - county

C.U. - Cornel1 University

dc - dorsocentral (bristles)

det. - determined (as, by)

DPI - Division of Plant Industry

(Florida Arthropod Collection,

Florida State Department of

Agriculture, Gainesville, Florida)

et seq. - and in the sequel

f - female

F - seed feeder, in flowers

Fig. - figure

ft - foot, feet (length or height)

$G$ - gall causer

GCS - George C. Steyskal (specimens of

his collection are in USNM)

indet. - indeterminate, undetermined

INHS - Illinois Natural History Survey, Urbana

Is. - is land

J.G.C. - J. G. Chillcott

KAS - Kenneth A. Spencer or his personal collection

KEF - Kenneth E. Frick

L- leaf miner

loc. cit. - in the place cited

m. - male

M, M $1+2, M 3+4$ - media (medial vein of

wing together with its branches)

MCZ - Museum of Comparative Zoology,

Harvard University, Cambridge, Mass.

$\mathrm{mm}$ - millimeter(s)

MNHNP - Muséum National d'Histoire

Naturelle, Paris, France ms. - manuscript

NMW - Naturhistorisches Museum, Wien, Austria

nom. nov. - new name (nomen novum)

nom. nud. - bare name, name without

nomenclature status (nomen nudum)

NRS - Naturhistoriska Riksmuseet,

Stockholm, Sweden

n. sp. - new species

NYSM - New York State Museum, Albany

ori - orbitalis inferior (lower orbital bristle)

ors - orbitalis superior (upper orbital bristle)

P 1, P 2, P 3 - foreleg, midleg, hind-

leg, respectively (Latin pes)

prsc - prescutellar (bristles)

PSU - Pennsylvania State University, University Park

$R, R 1, R 2+3, R 4+5$ - radius (radial

vein of wing and its branches)

RNH - Rijksmuseum van Natuurlijke

Historie, Leiden, Netherlands

$\mathrm{SB}$ - stem borer

Sc - subcosta (subcostal vein of wing)

s.1. - in a broad sense (sensu lato)

SM - stem miner

sp., spp. - species (singular and

plural, respectively)

spm., spms. - specimen(s)

s.s. - in a narrow sense (sensu

stricto)

ssp. - subspecies

stat. rev. - revised status

subg. - subgenus

SWF - S. W. Frost

UCB - University of California at

Berkeley

UCD - University of California at Davis

UKaL - University of Kansas, Lawrence

USNM - U.S. National Museum (of

Natural History, Washington, D.C.)

USU - Utah State University, Logan

UZMC - University Zoological Museum,

Copenhagen, Denmark

vt - vertical (bristles)

vte - outer vertical bristles (verticales exteriores)

vti - inner vertical bristles (verticales interiores)

ZIL - Zoological Institute, University of Lund, Sweden

ZMHU - Institut für Spezielle Zoologie und Zoologisches Museum der Humboldt Universität, Berlin, German Democratic Republic (East Germany) 
4
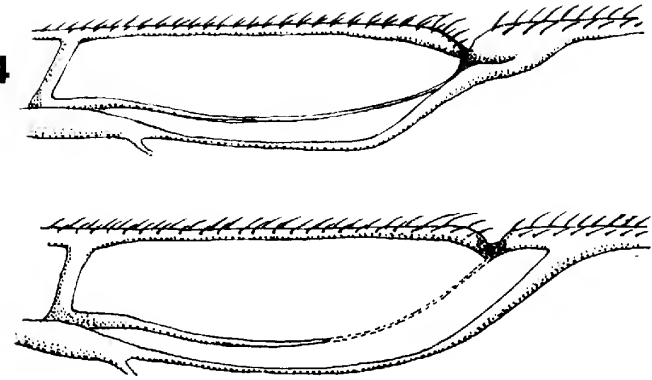

5
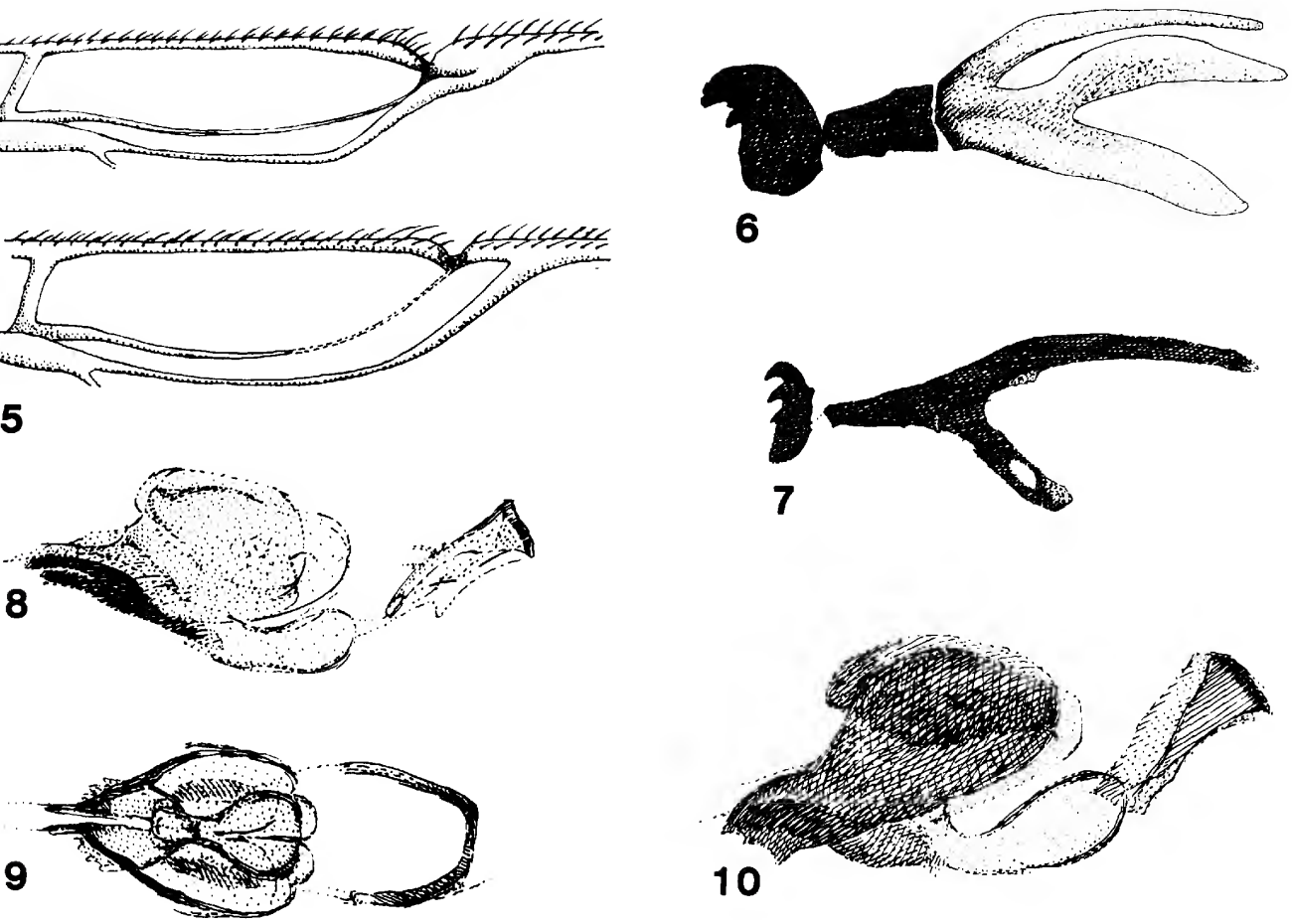

6
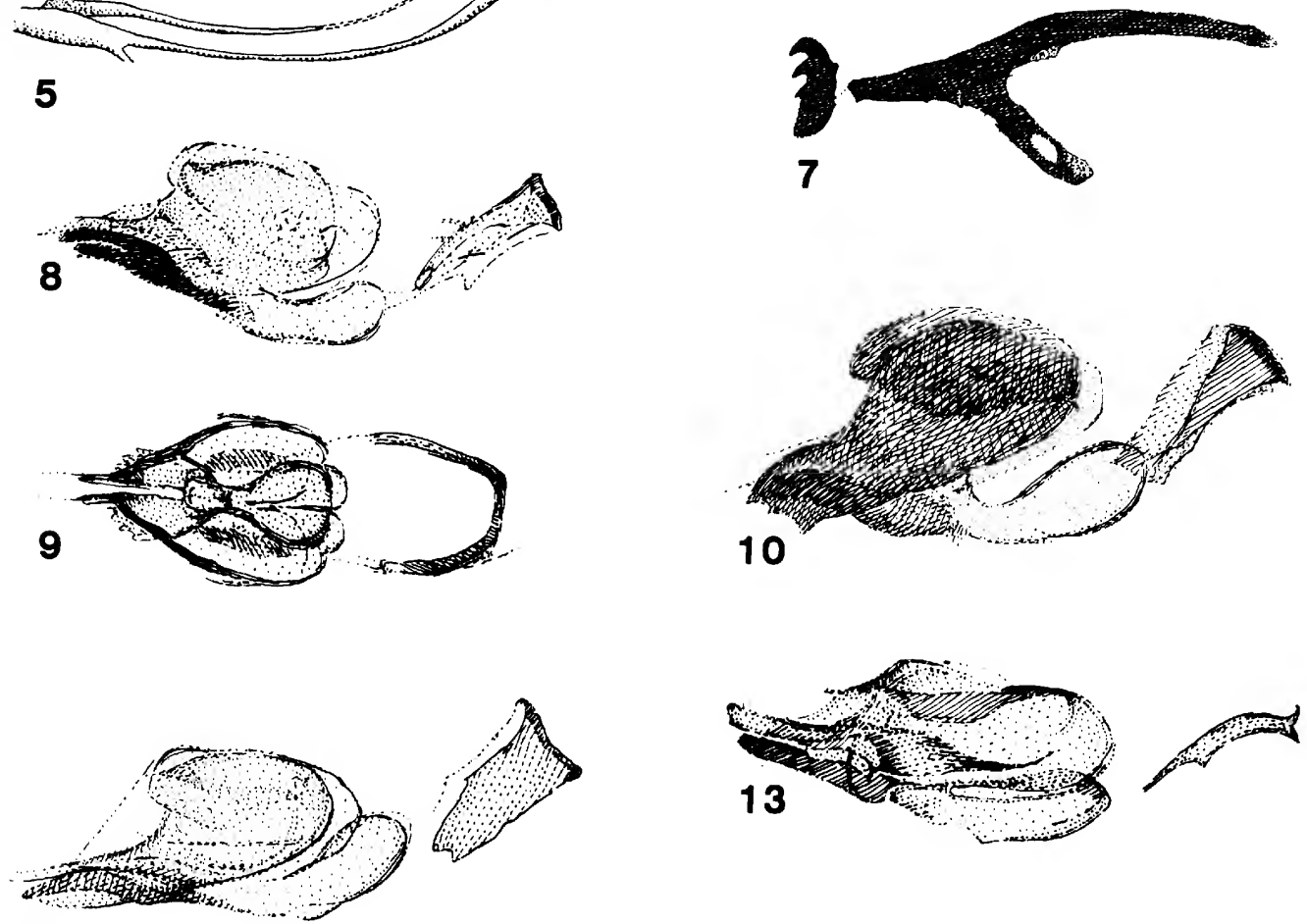

11
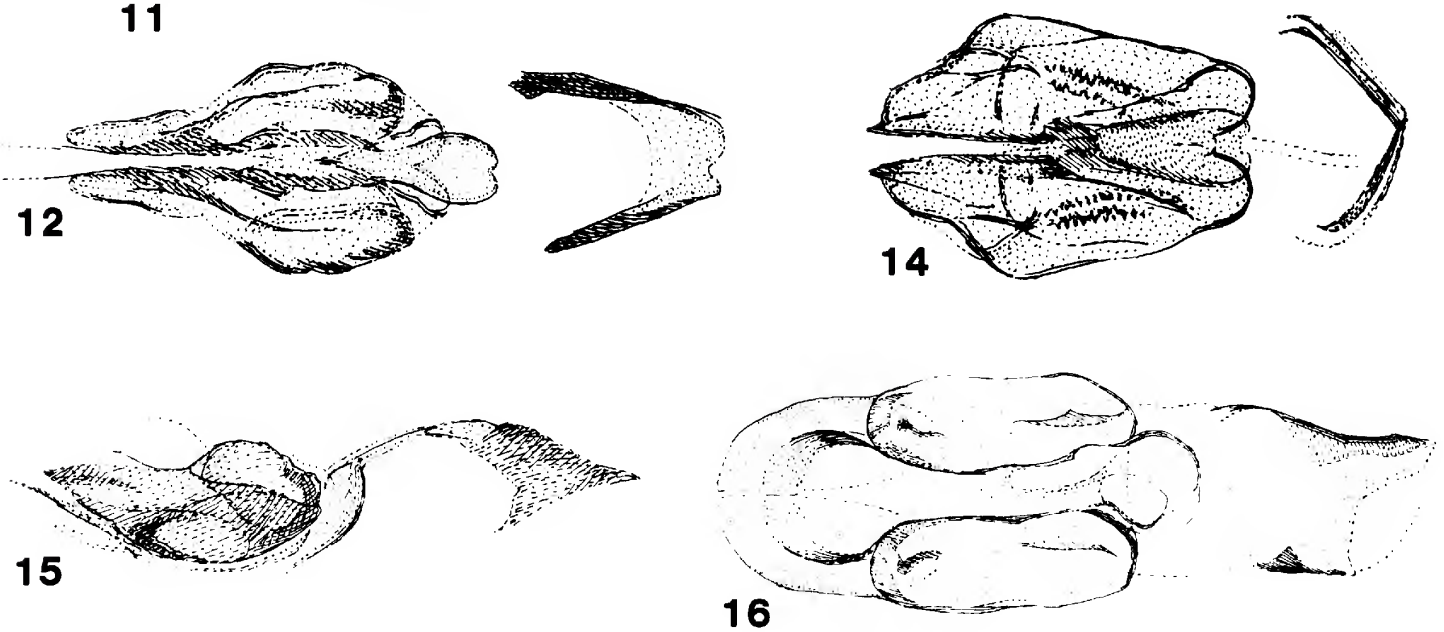

Figures 4-16.--Subcostae of subfamilies Agromyzinae

(4) and Phytomyzinae (5). Larval cephalopharyngeal

skeletons in subfamilies Agromyzinae (6) and

Phytomyzinae (7). Melanagromyza trispinosa: 8,

Aedeagus, side view; 9 , same, ventral view; $M$.

tamia: 10, Aedeagus, side view; M. quadrisetosa:

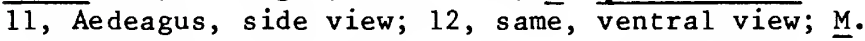

trispinella: 13, Aedeagus, side view; 14, same,

ventral view; M. setifrons: 15 , Aedeagus, side view

(holotype); 16, same, ventral view (Canada). 

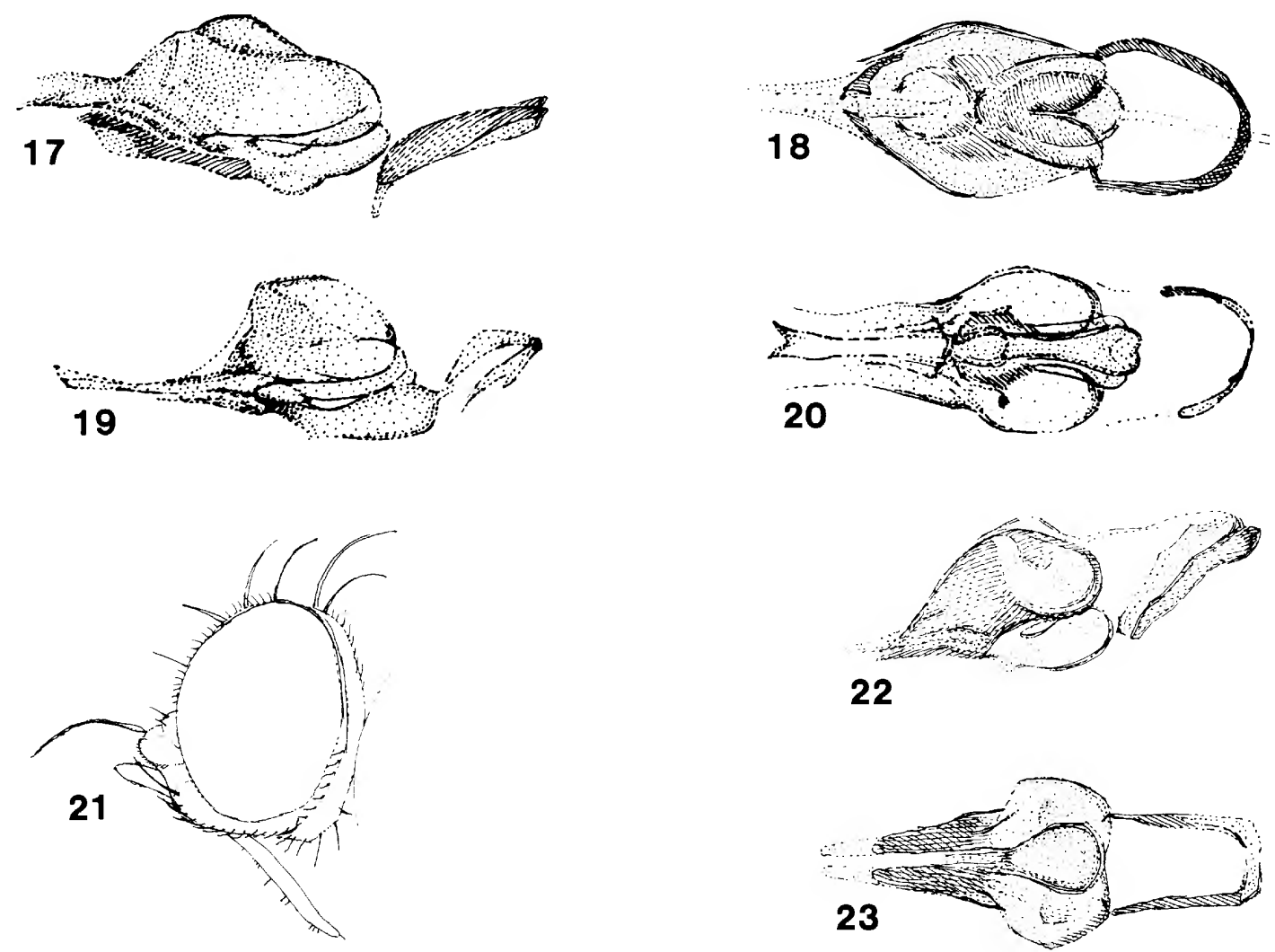

22
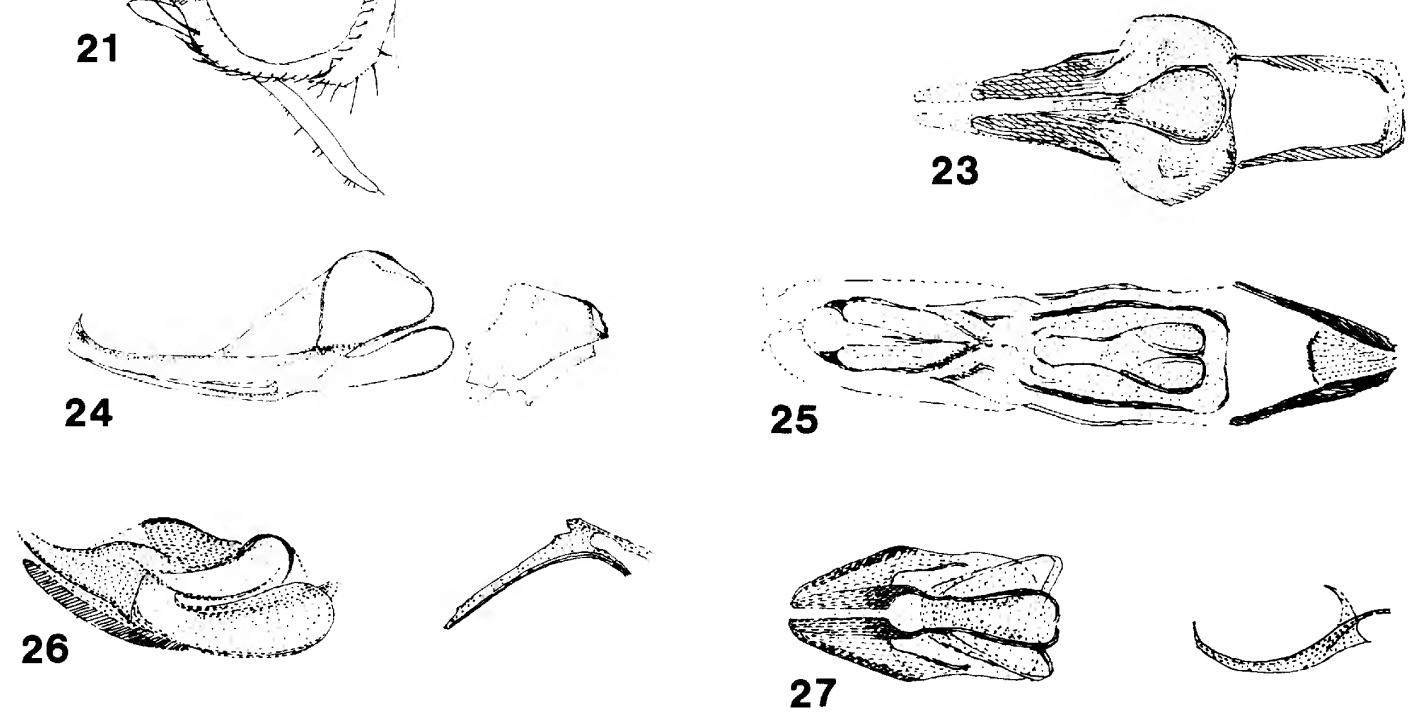

Figures 17-27.--Me lanagromyza scrophulariae: 17, Aedeagus, side view; 18 , same, ventral view (holotype); . sagehenensis: 19, Aedeagus, side view; 20 , same, ventral view (holotype); $M$. proboscidata: 21 , Head; 22, aedeagus, side view;

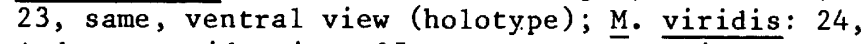
Aedeagus, side view; 25 , same, vent $\bar{r}$ al view; $M$. heliotropii: 26, Aedeagus, side view; 27 , same, ventral view (holotype). 

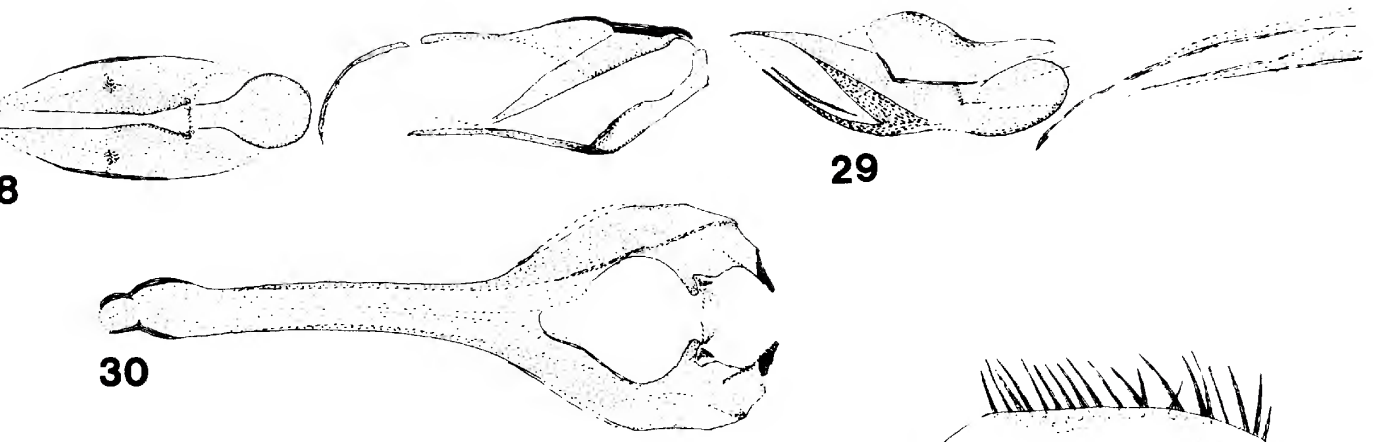

29

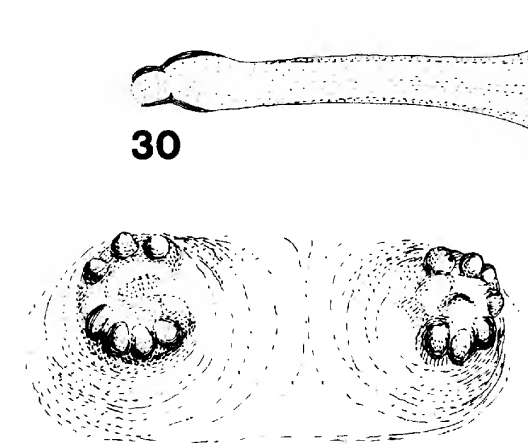

32
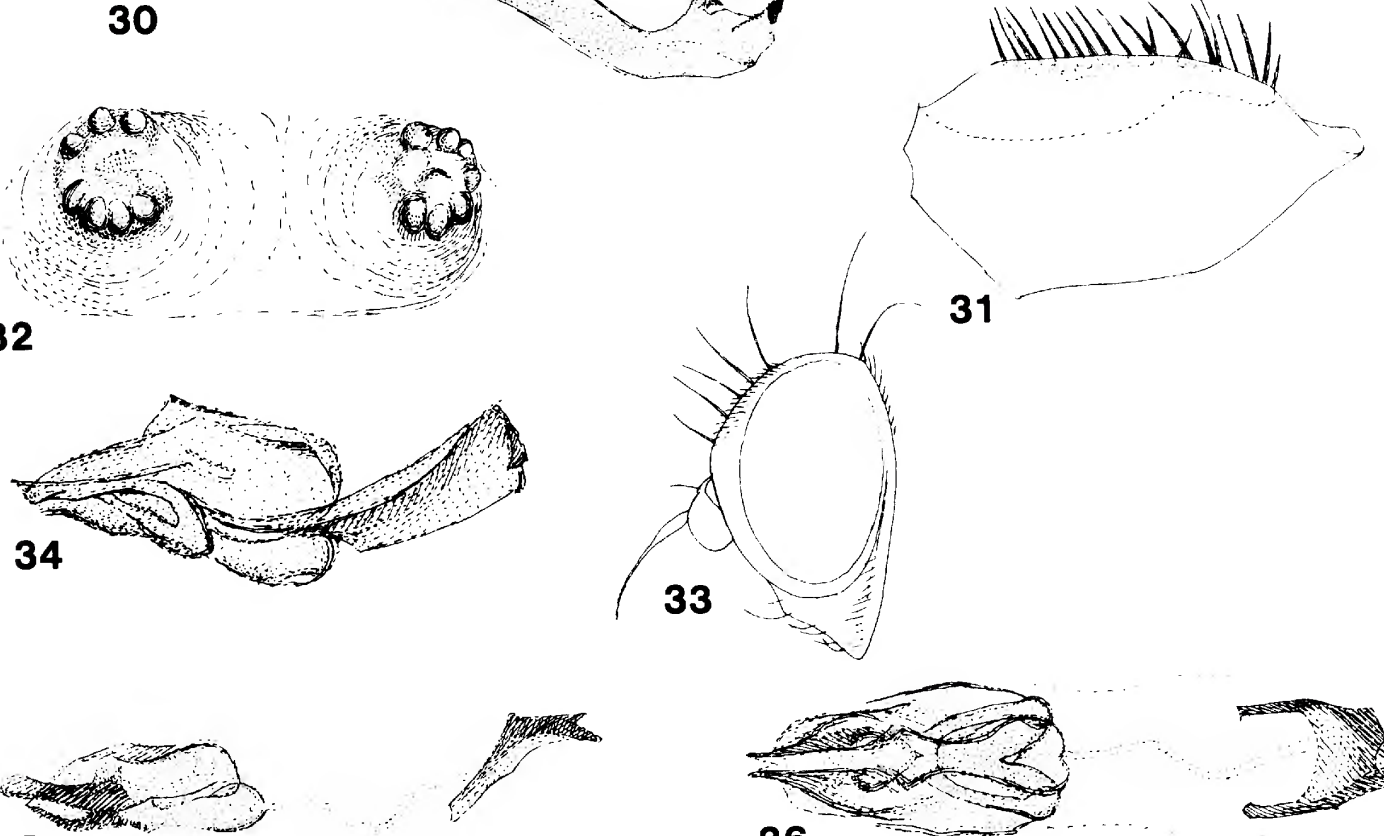

35
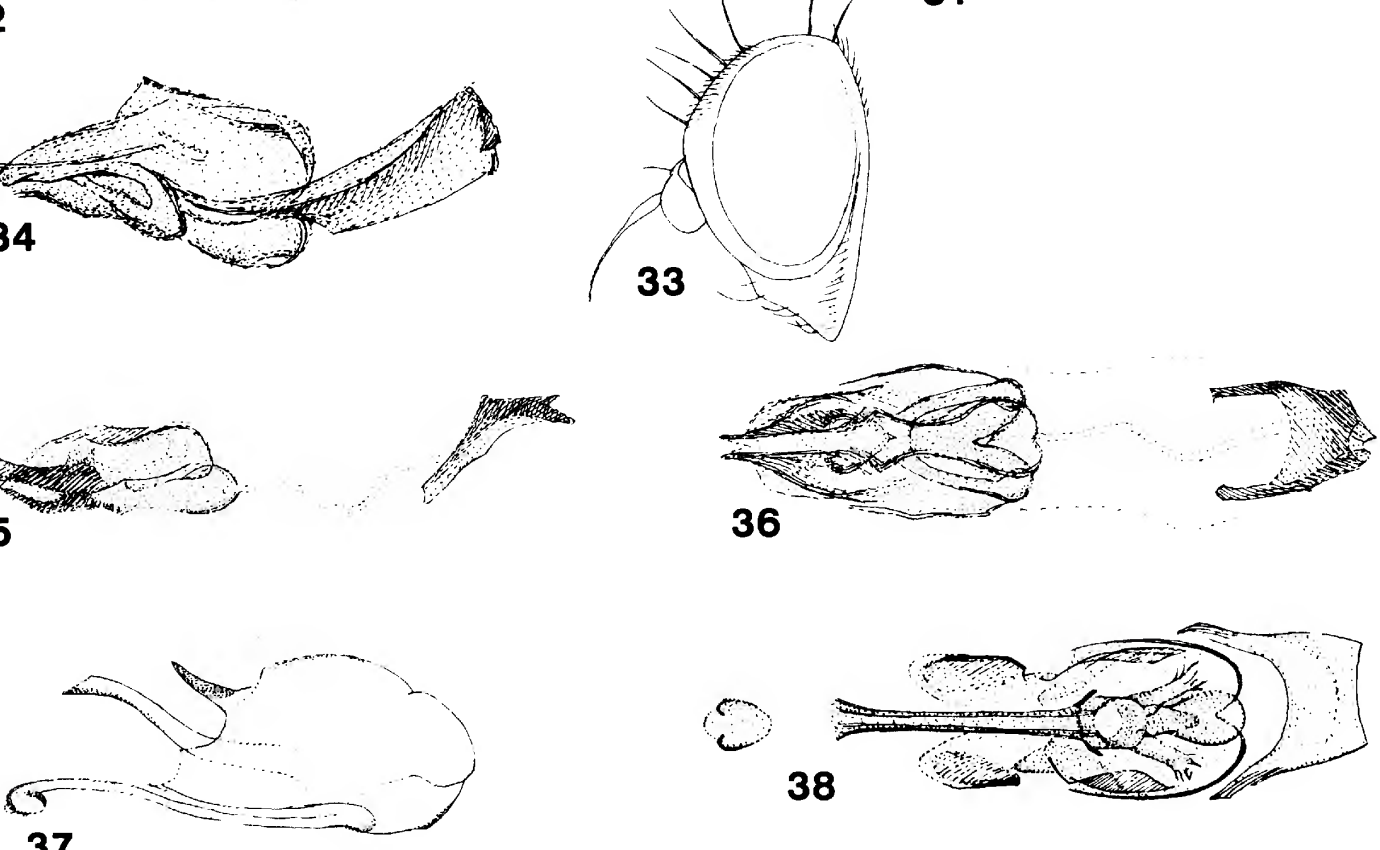

37
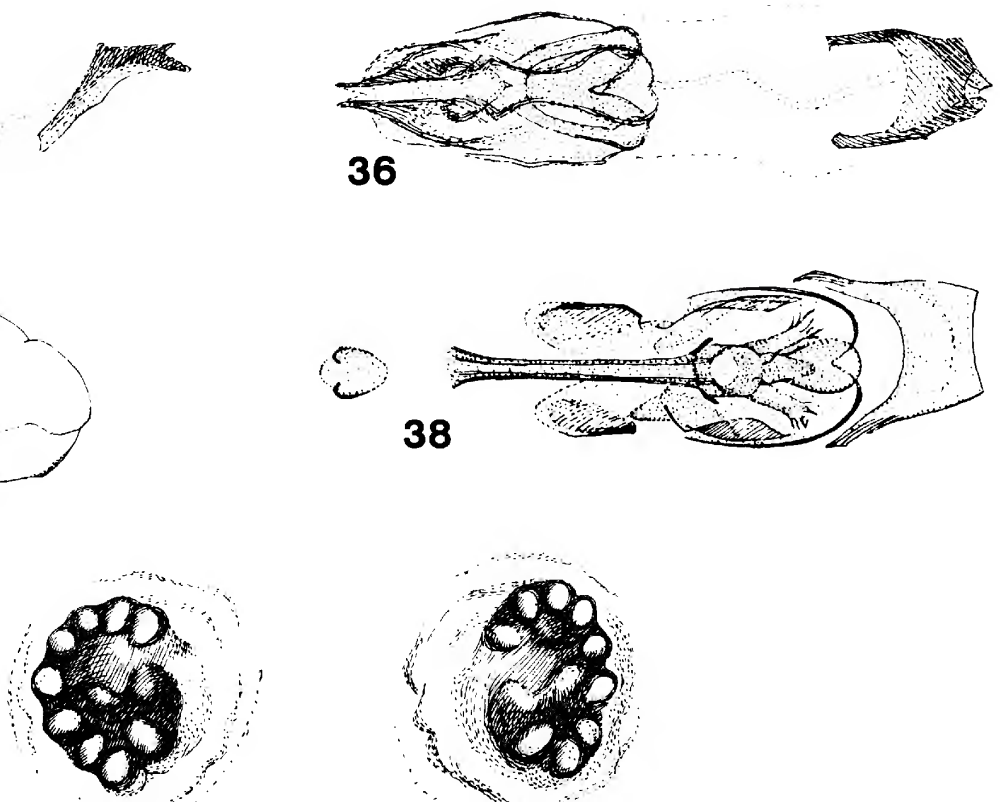

39

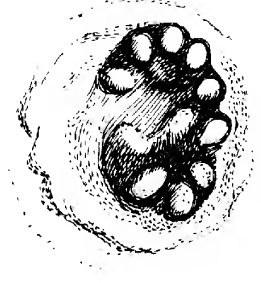

Figures 28-39.--Melanagromyza ruelliae: 28, Aedeagus, side view; 29 , same, ventral view; 30 , hypandrium; 31 , epandrium; 32 , posterior spiracles of puparium; M. inornata: 33 , Head; 34 , aedeagus, side view (Michigan); M. panacis: 35 , Aedeagus, side view; 36, same, veñtral view (holotype); M. caerulea: 37 , Aedeagus, side view; 38 , same, ventral view; 39 , posterior spiracles of puparium. 

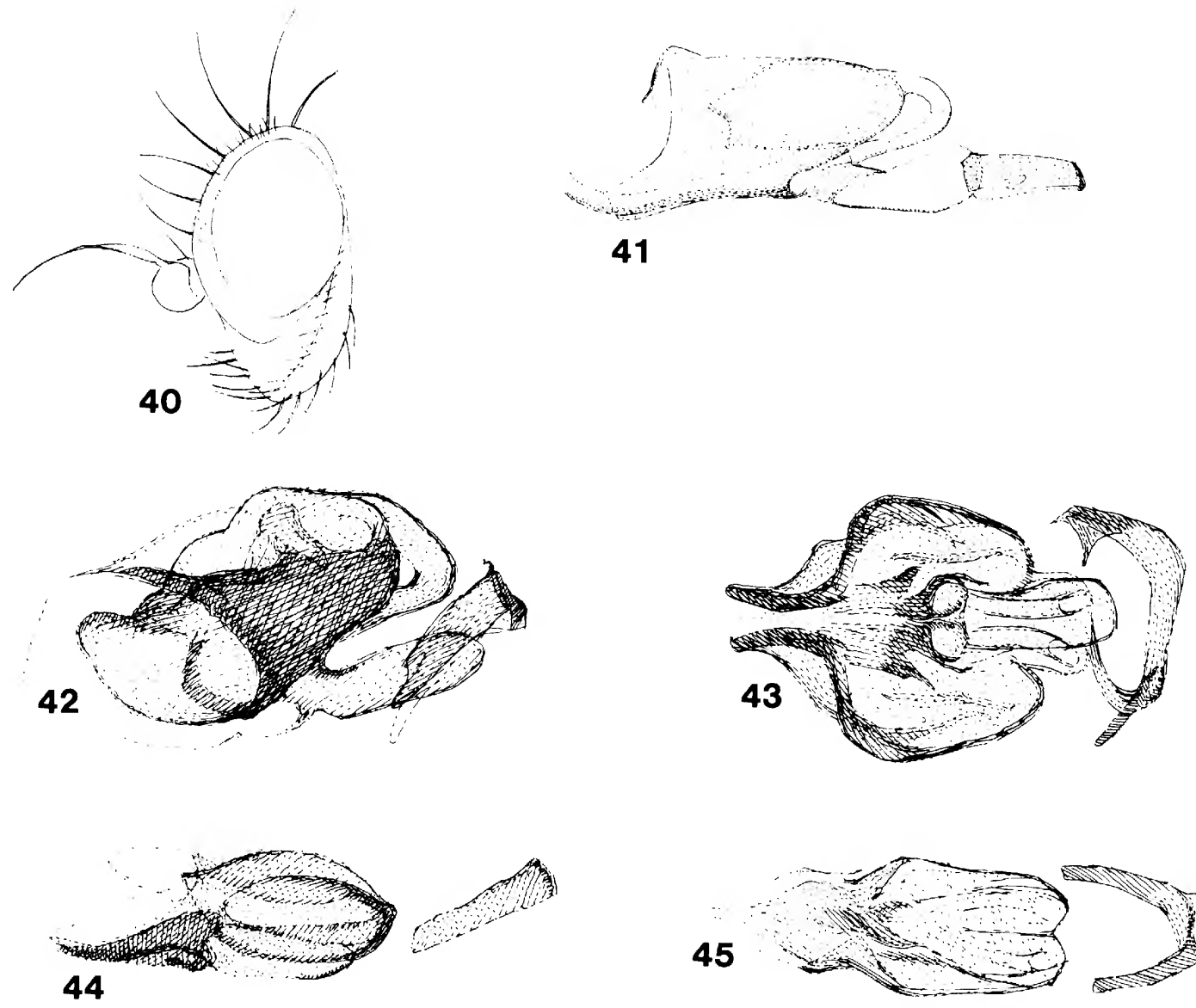

41
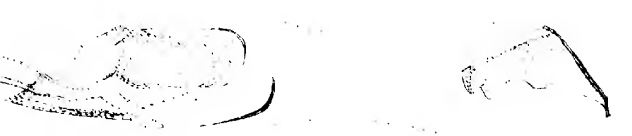

46
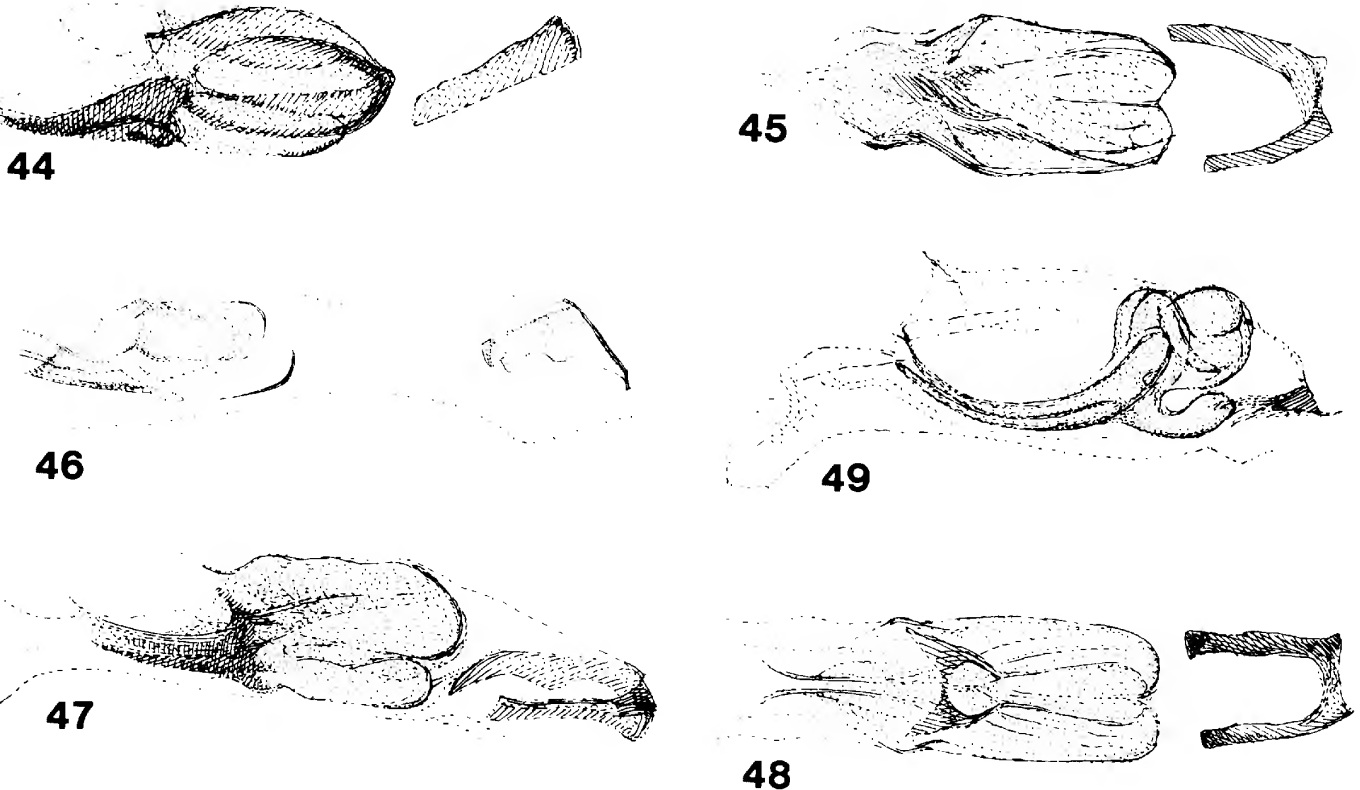

Figures 40-49.--Melanagromyza miranda: 40, Head; 41, aedeagus, side view; $\underline{\text { }}$. diantherae: 42, Aedeagus, side view; 43, same, ventral view (paratype); M. walleyi: 44, Aedeagus, side view; 45, same, veñtral view; . hicksi: 46, Aedeagus, side view (Canada); M. vernoniae: 47 , Aedeagus, side view; 48 , same, ventral view (holotype); . vernoniana: 49 , Aedeagus, side view (holotype). 


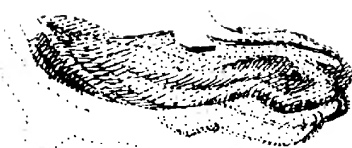

50
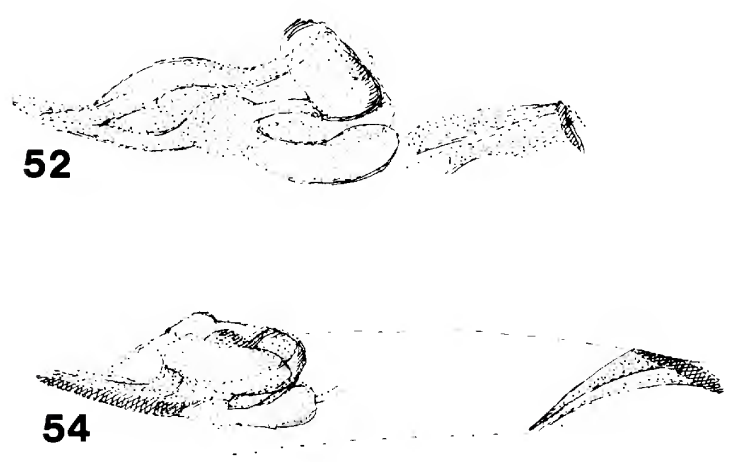

56
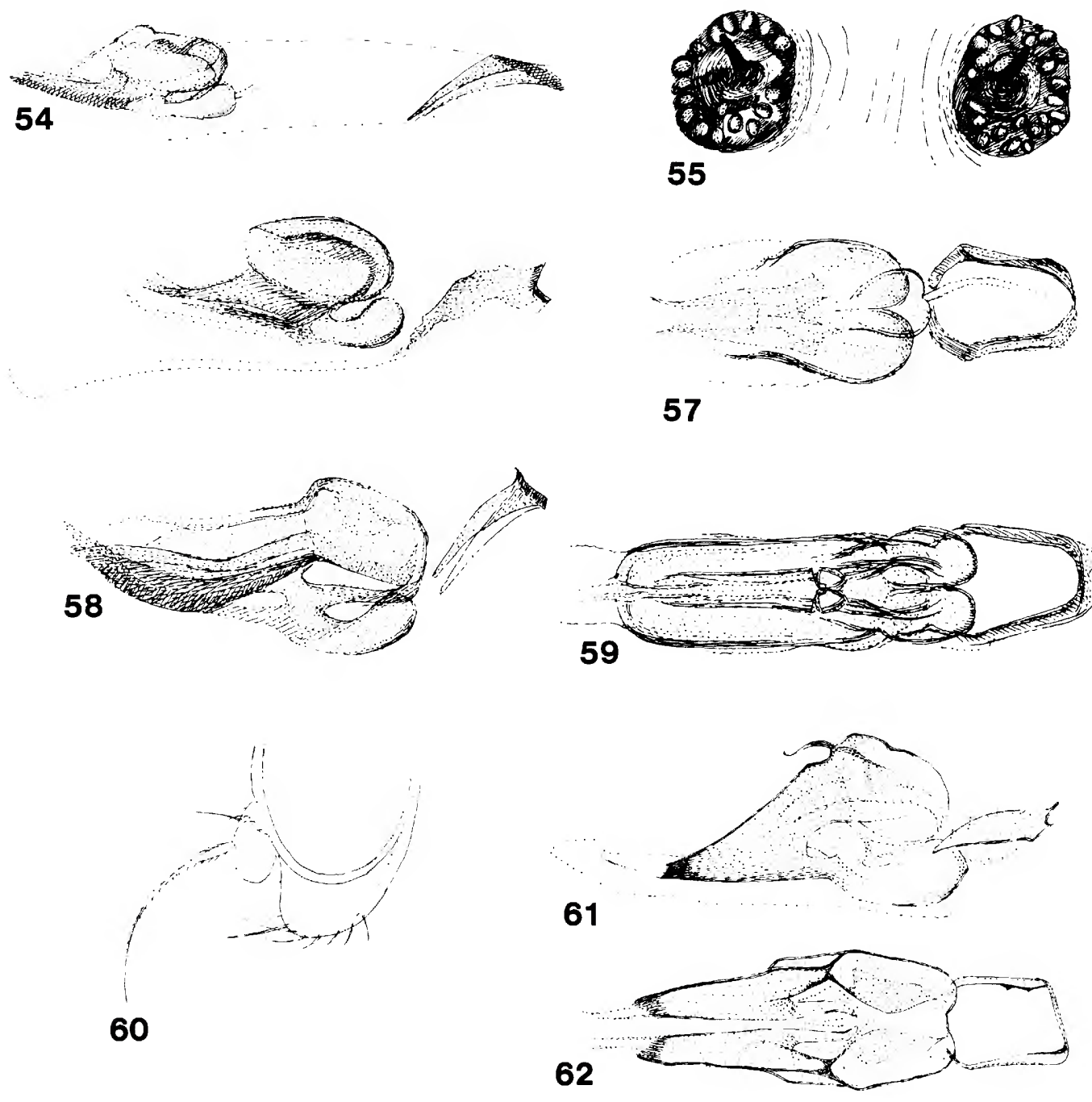

57

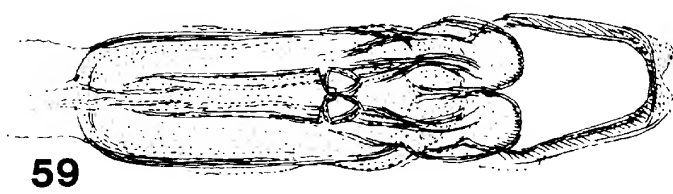

Figures 50-62.--Melanagromyza urticella: 50, Aedeagus, side view; 51 , same, ventral view (holotype); M. cirsiophila: 52, Aedeagus, side view (Colorado); 53, same, ventral view (holotype); M. angelicae: 54, Aedeagus, side view; 55, posterior spiracles of puparium; $\underline{M}$. longensis: 56 , Aedeagus, side view; 57, same, ventral view (holotype); M. virginiensis: 58, Aedeagus, side view; 59 , same, ventral view (holotype); M. buccalis: 60 , Head; 61 , aedeagus, side view; $6 \overline{2}$, same, ventral view. 

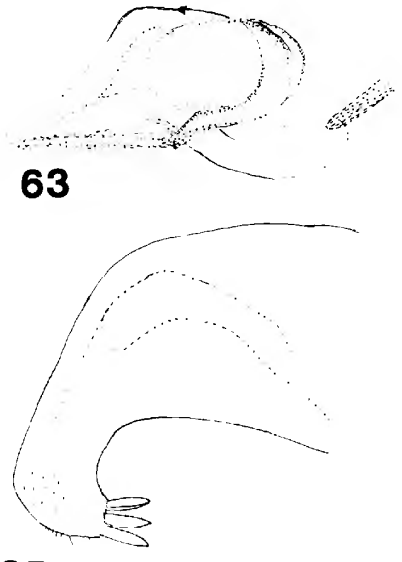

65

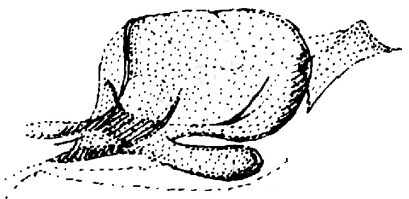

67

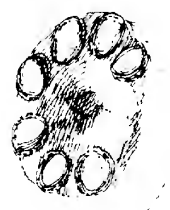

69

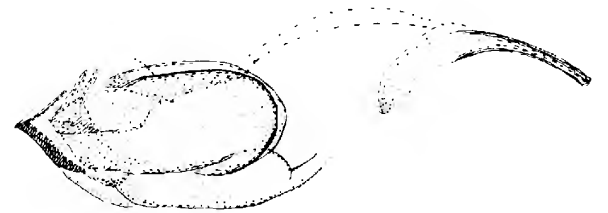

71

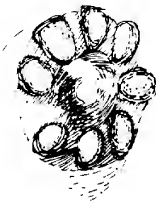

70

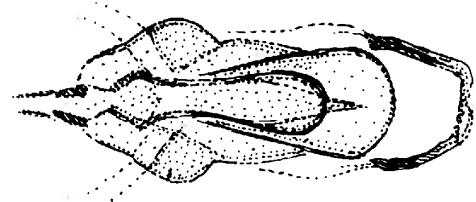

68

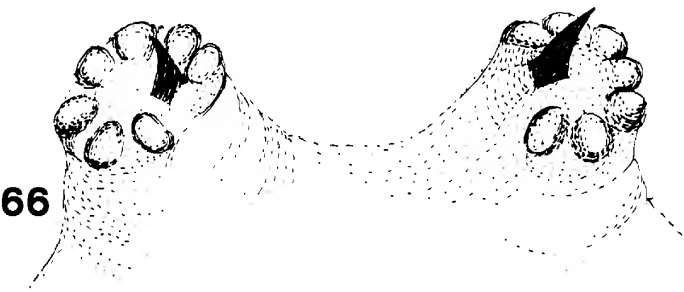

64

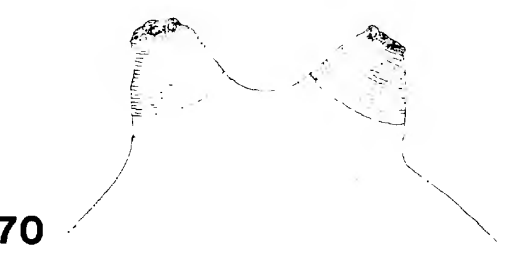

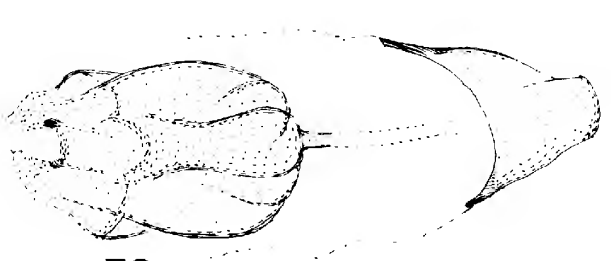

72
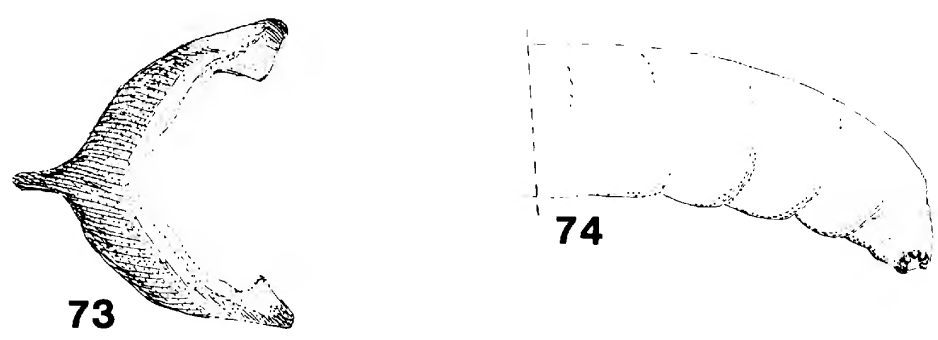

Figures 63-74.--Melanagromyza virens: 63, Aedeagus,

side view; 64, same, ventral view; 65 , surstylus;

66 , posterior spiracles of puparium; M. splendida:

67, Aedeagus, side view; 68, same, venttral view;

69,70 , posterior spiracles of puparium; $\underline{\text { M. }}$

ripare11a: 71, Aedeagus, side view; 72, same, ventral view; 73, hypandrium (holotype); 74, posterior end of puparium (Florida). 


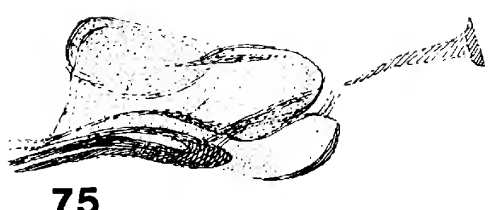

75
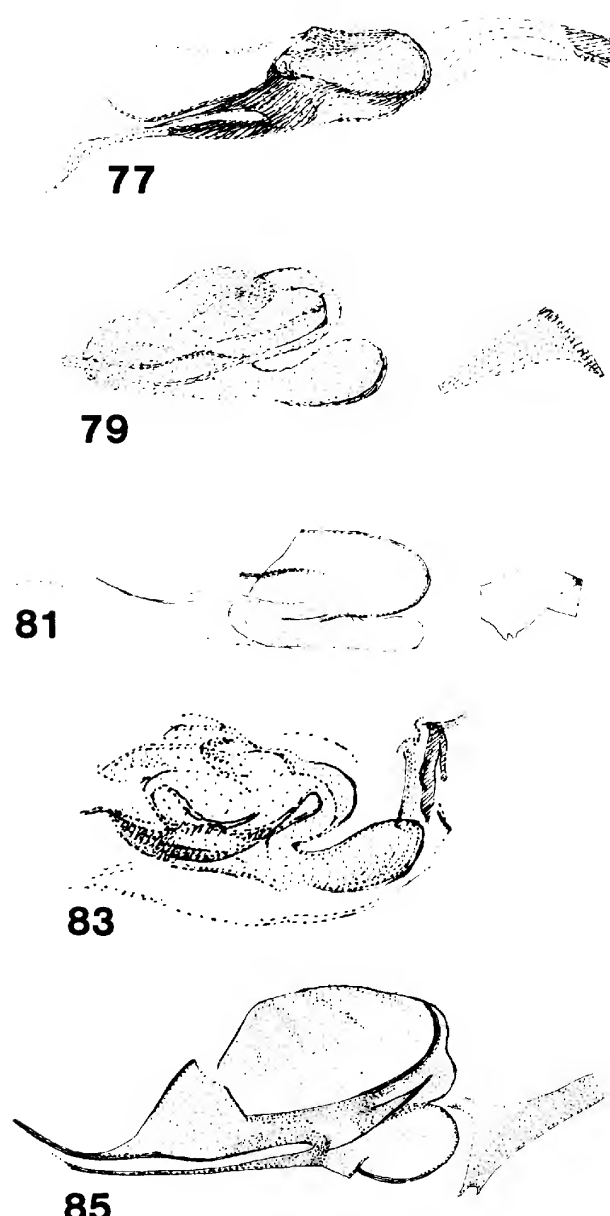
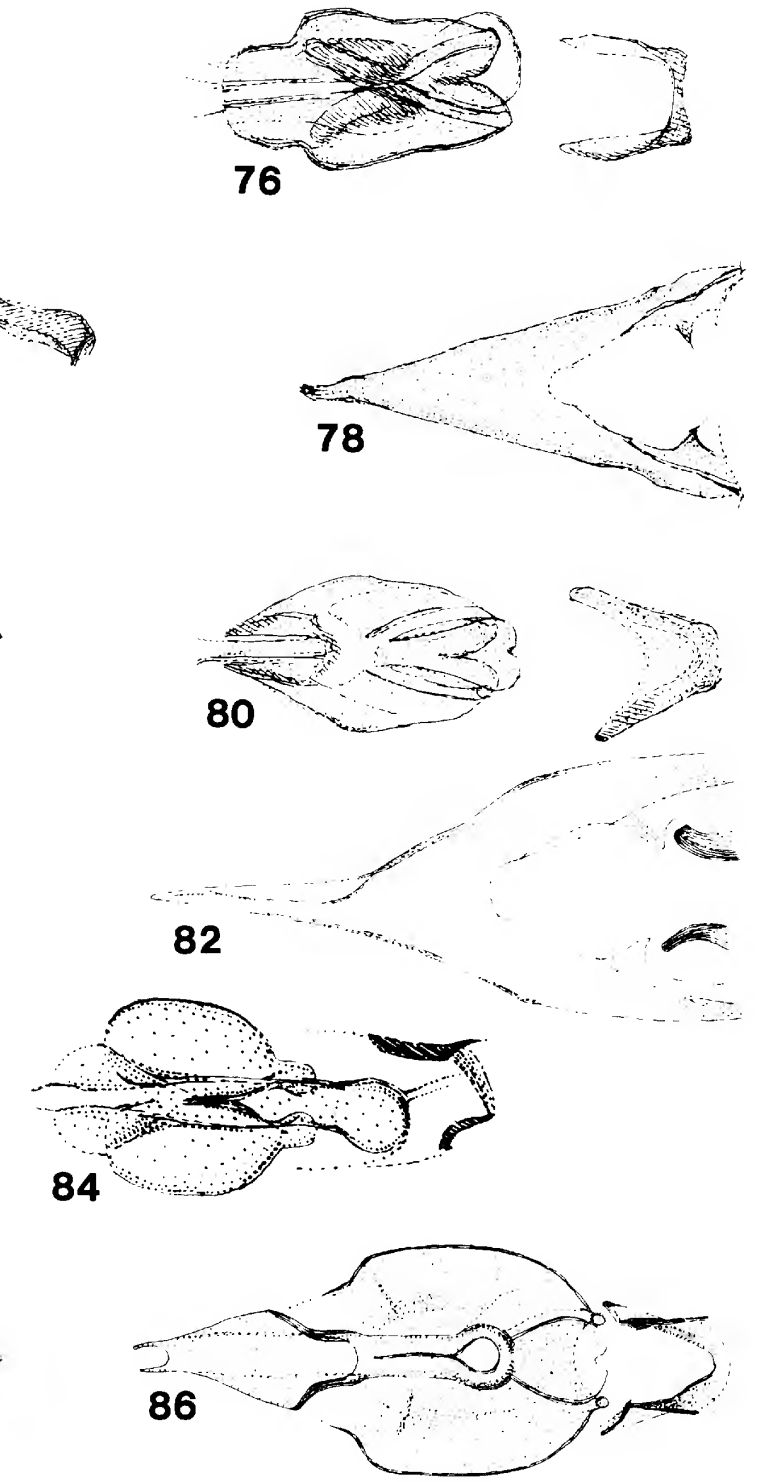

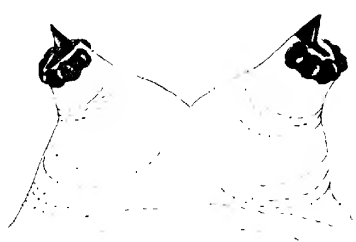

87

Figures 75-87. --Melanagromyza chillcotti: 75,

Aedeagus, side view; 76 , same, ventral view; $M$.

subvirens: 77 , Aedeagus, side view; 78, hypand̄rium

(paralectotype); . . chillcottiana: 79 , Aedeagus,

side view; 80 , same, ventral view (holotype); $M$.

floris: 81 , Aedeagus, side view; 82 , hypandrium; $\underline{M}$.

gibsoni: 83 , Aedeagus, side view; 84 , same, ventrā

view; . chaptaliae: 85 , Aedeagus, side view; 86 ,

same, ventral view (holotype); 87 , posterior

spiracles of puparium. 

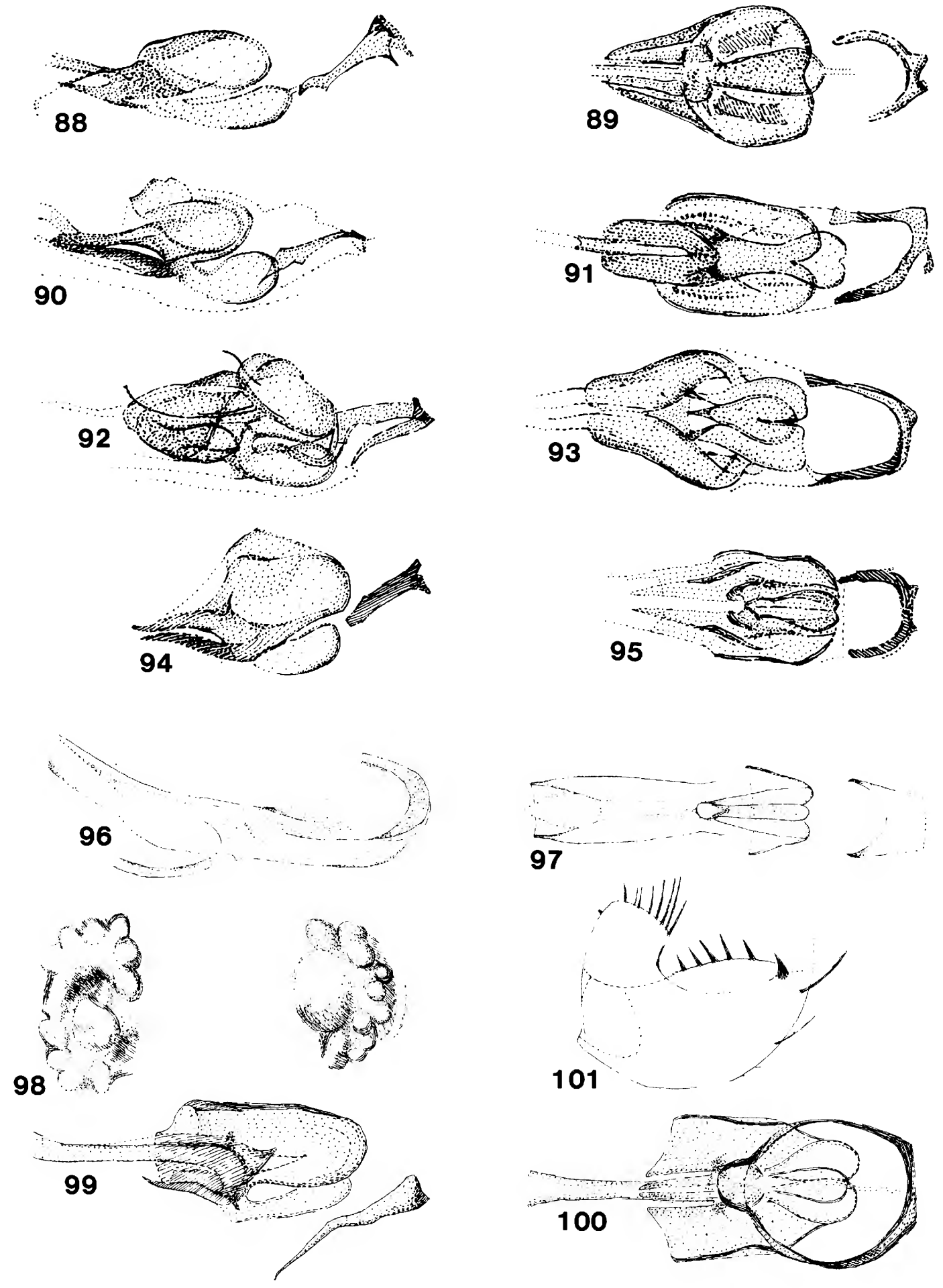

Figures 88-101.--Melanagromyza osoflacensis: 88 , Aedeagus, side view; 89, same, ventral view (holotype); M. gnaphalii: 90, Aedeagus, side view; 91, same ventral view (holotype); M. corralensis: 92, Aedeagus, side view; 93, same, ventral view (holotype); M. muguensis: 94, Aedeagus, side view; 95, same, ventral view (holotype); M. floridensis: 96, Aedeagus, side view; 97, same, ventral view (holotype); 98 , posterior spiracles of puparium; $\underline{M}$. miamensis: 99, Aedeagus, side view; 100, same, ventral view; 101, epandrium. 

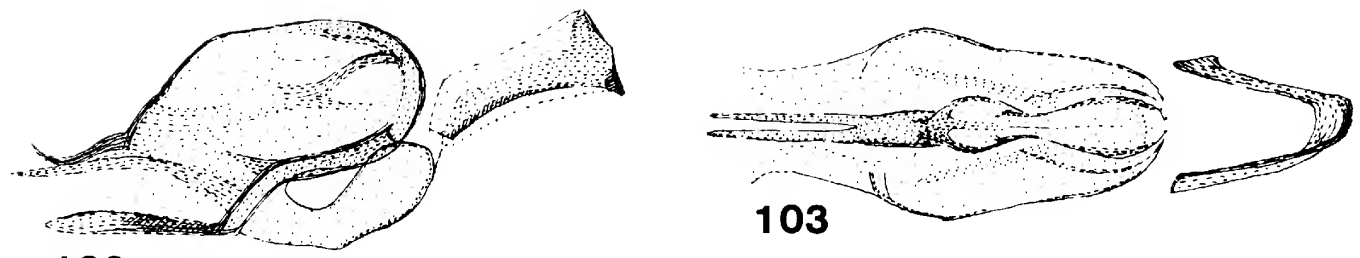

102
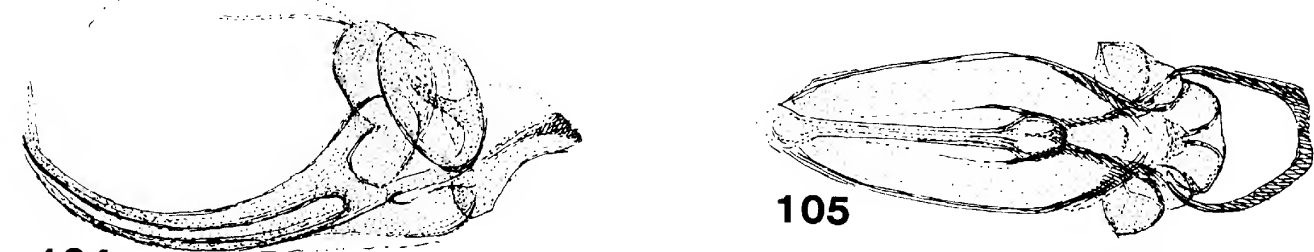

104
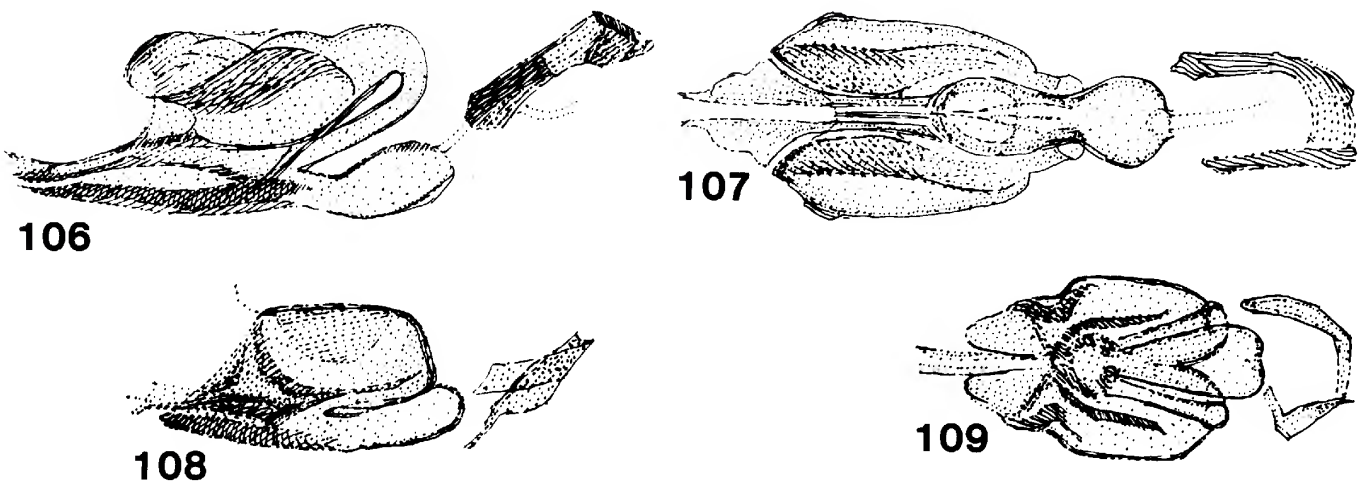

110
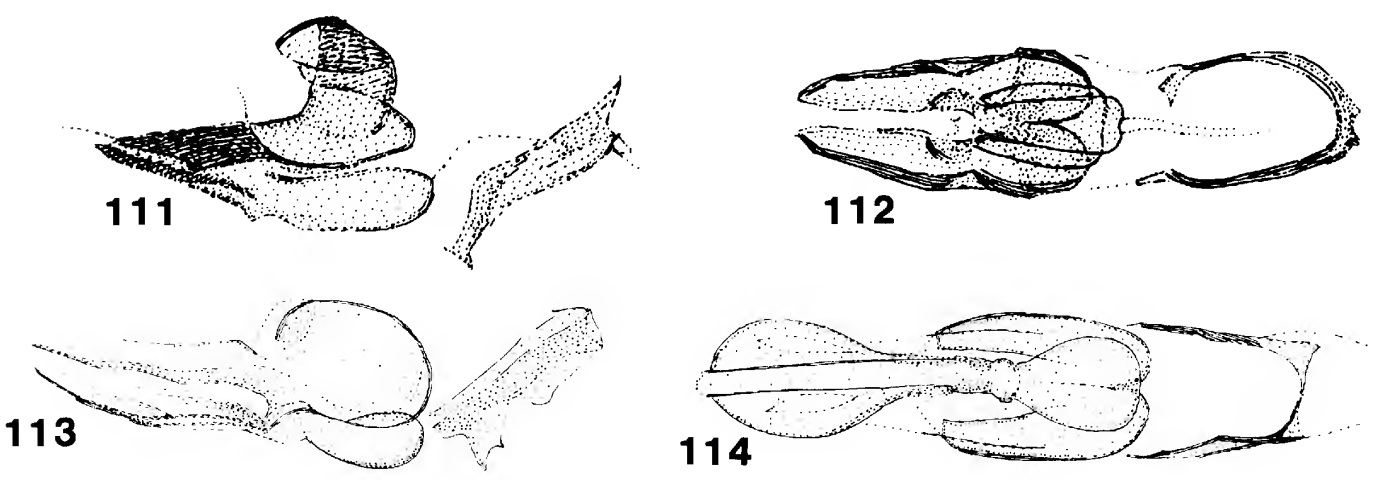

Figures 102-114.--Melanagromyza vectabilis: 102, Aedeagus, side view; 103, same, ventral view; $\underline{M}$. verbesinae: 104, Aedeagus, side view; 105, same, ventral view (holotype); - marinensis: 106, Aedeagus, side view; 107, same, ventral view (holotype); M. palaensis: 108, Aedeagus, side view; 109, same, ventral view (holotype); M. lomatii:

110, Aedeagus, side view (holotype); M. maligna:

111, Aedeagus, side view; 112, same, ventral view

(holotype); M. erechtitidis: 113, Aedeagus, side view; 114 , same, ventral view. 

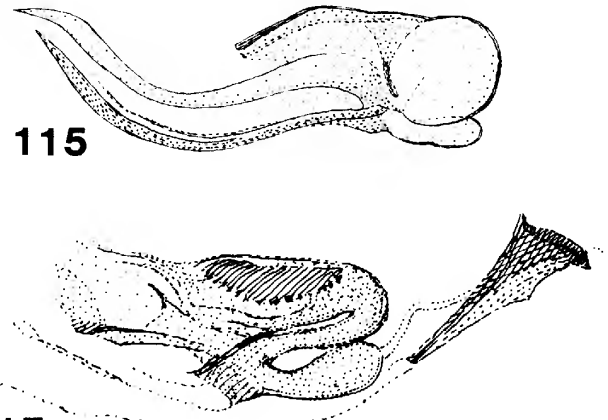
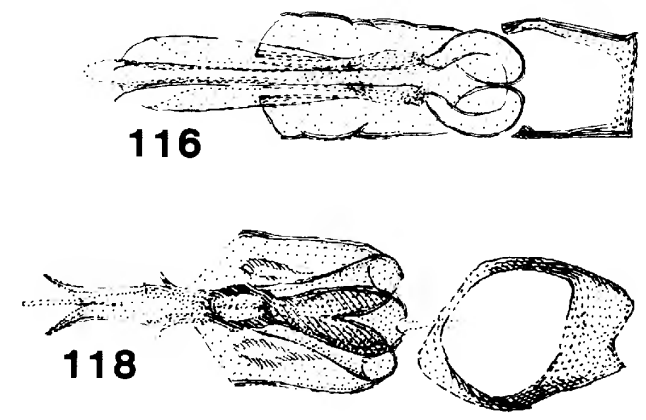

117

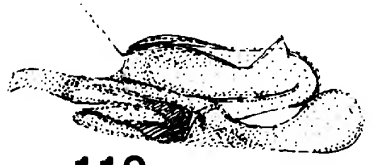

119
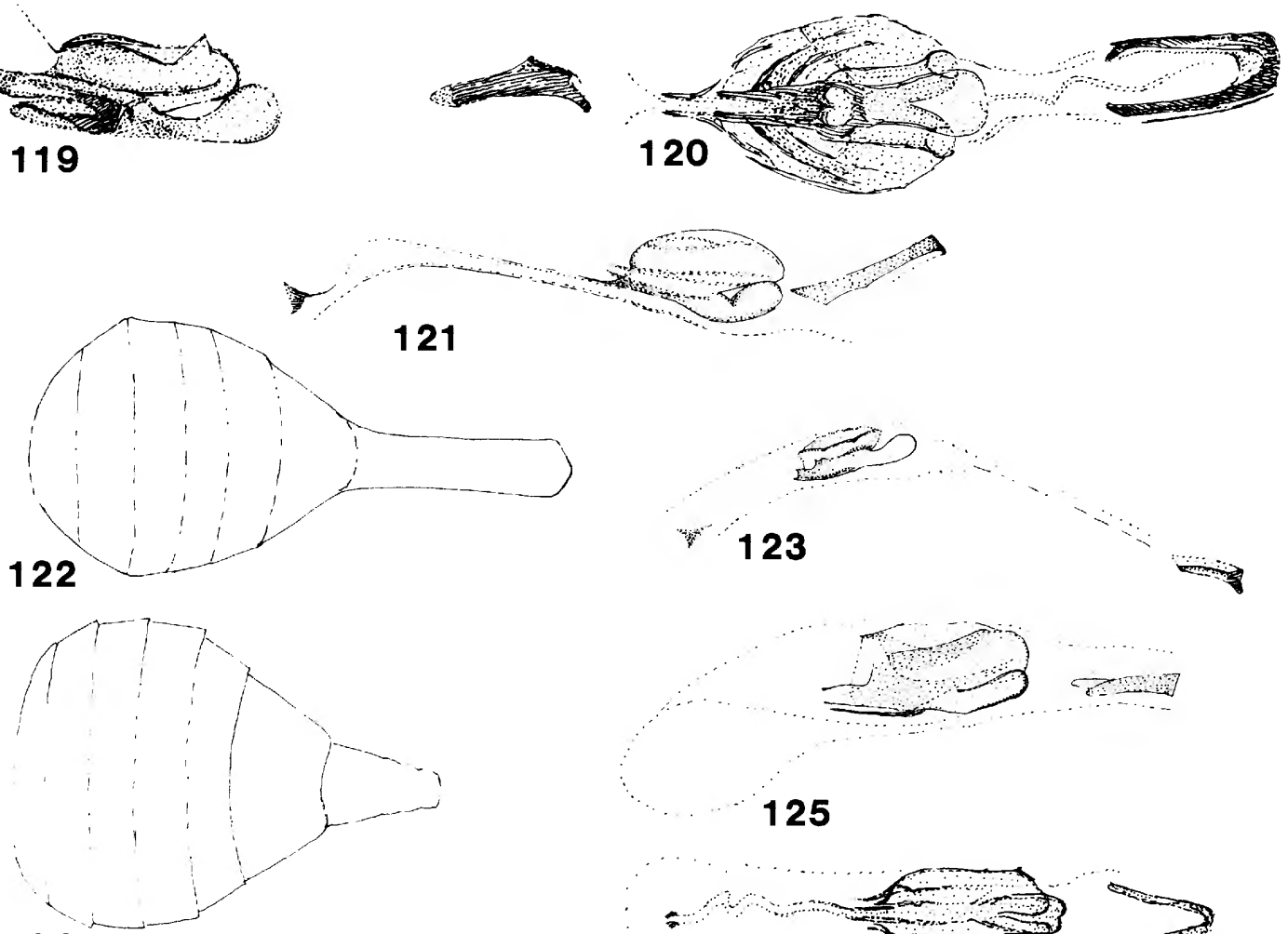

124

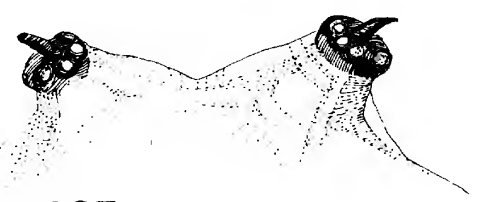

127
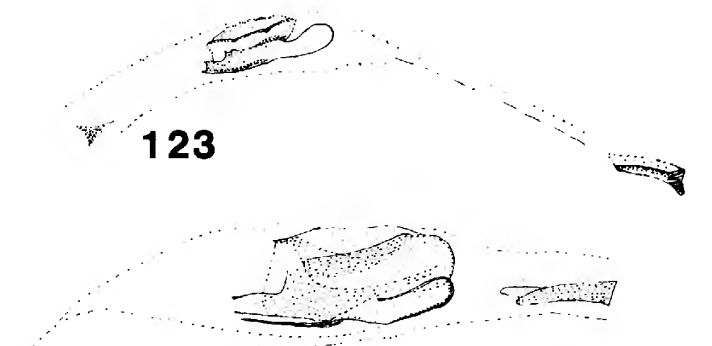

\section{5}
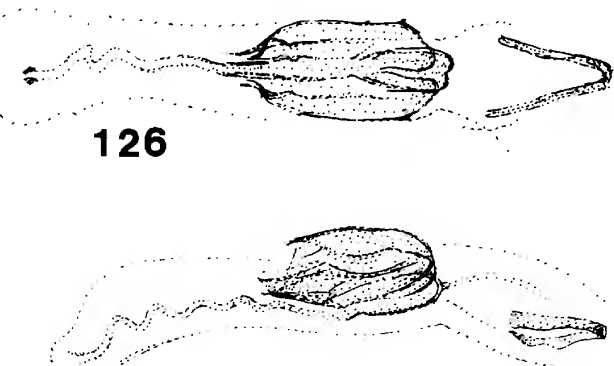

128

Figures 115-128.--Melanagromyza wedeliae: 115, Aedeagus, side view; 116 , same, ventra1 view; $M$. burgessi: 117, Aedeagus, side view; 118, same, ventral view; $M$. malevola: 119, Aedeagus, side view; 120 , same, ventral view (holotype); $M$. bidentis: 121, Aedeagus, side view; $\underline{\text {. minima: }}$ 122, Female ovipositor sheath; 123, aedeagus, side view; M. minimoides: 124, Female ovipositor sheath; 125, aedeagus, side view; 126, same, ventral view (ex He1iopsis, Maryland); 127 , posterior spiracles of puparium; M. radicicola: 128, Aedeagus, side view. 


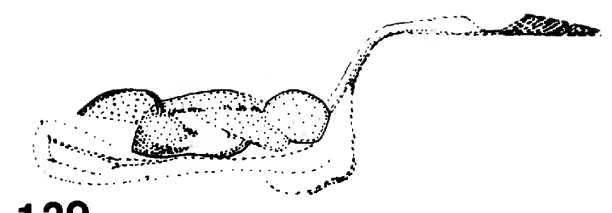

\section{9}
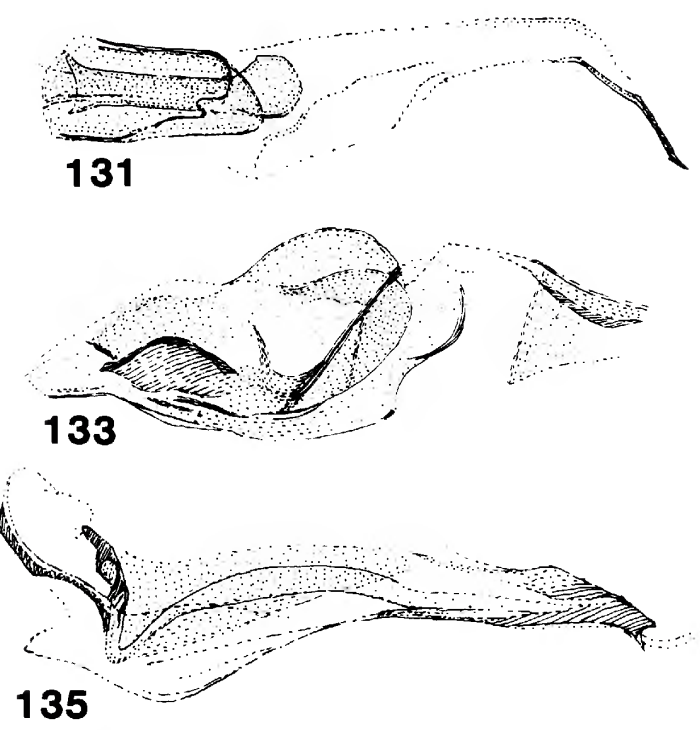

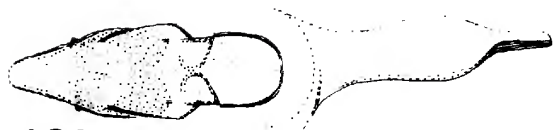

130
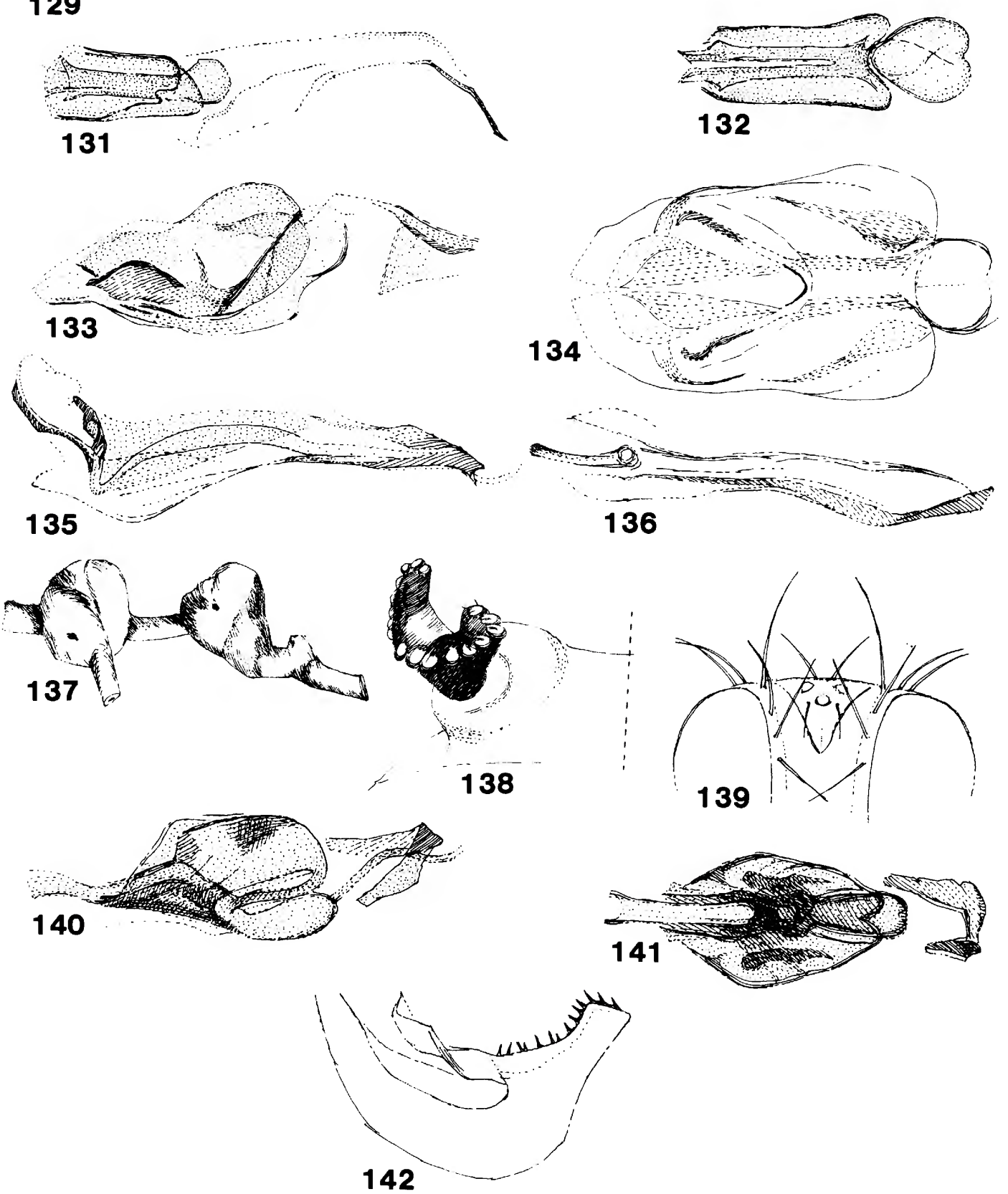

Figures 129-142.--Melanagromyza caribbea: 129,

Aedeagus, side view; 130 , same, ventral view; $\underline{M}$.

ultima: 131, Aedeagus, side view; 132, same,

ventral view; M. tetrica: 133, Aedeagus, side view;

134, same, ventral view; M. marellii: 135 ,

Aedeagus, side view; 136, same, ventral view; 137 ,

stem galls on Achyranthes (Argentina); 138,

posterior spiracles of puparium; (137, 138,

Argentina); M. sp. (Maryland): 139, Head; 140,

aedeagus, side view; 141, same, ventral view; 142,

epandrium. 


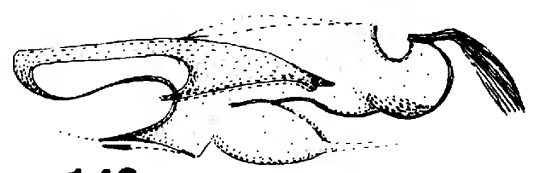

143
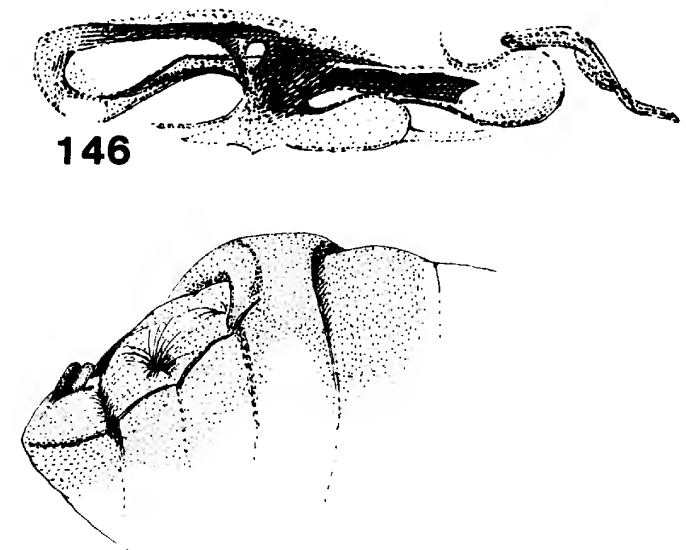

148

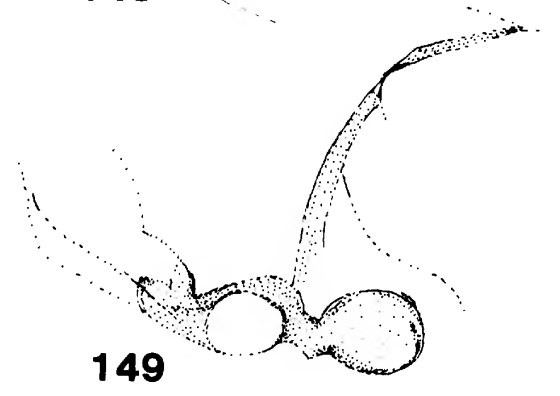

149
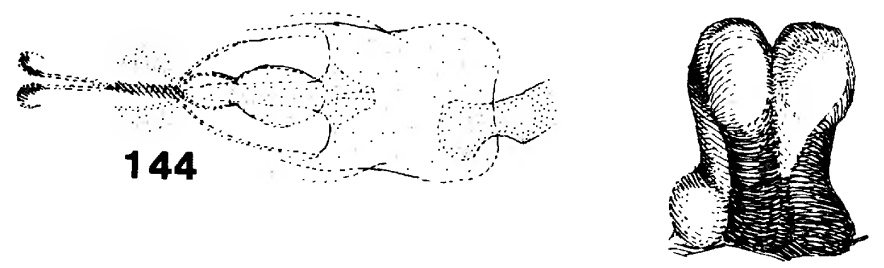

145
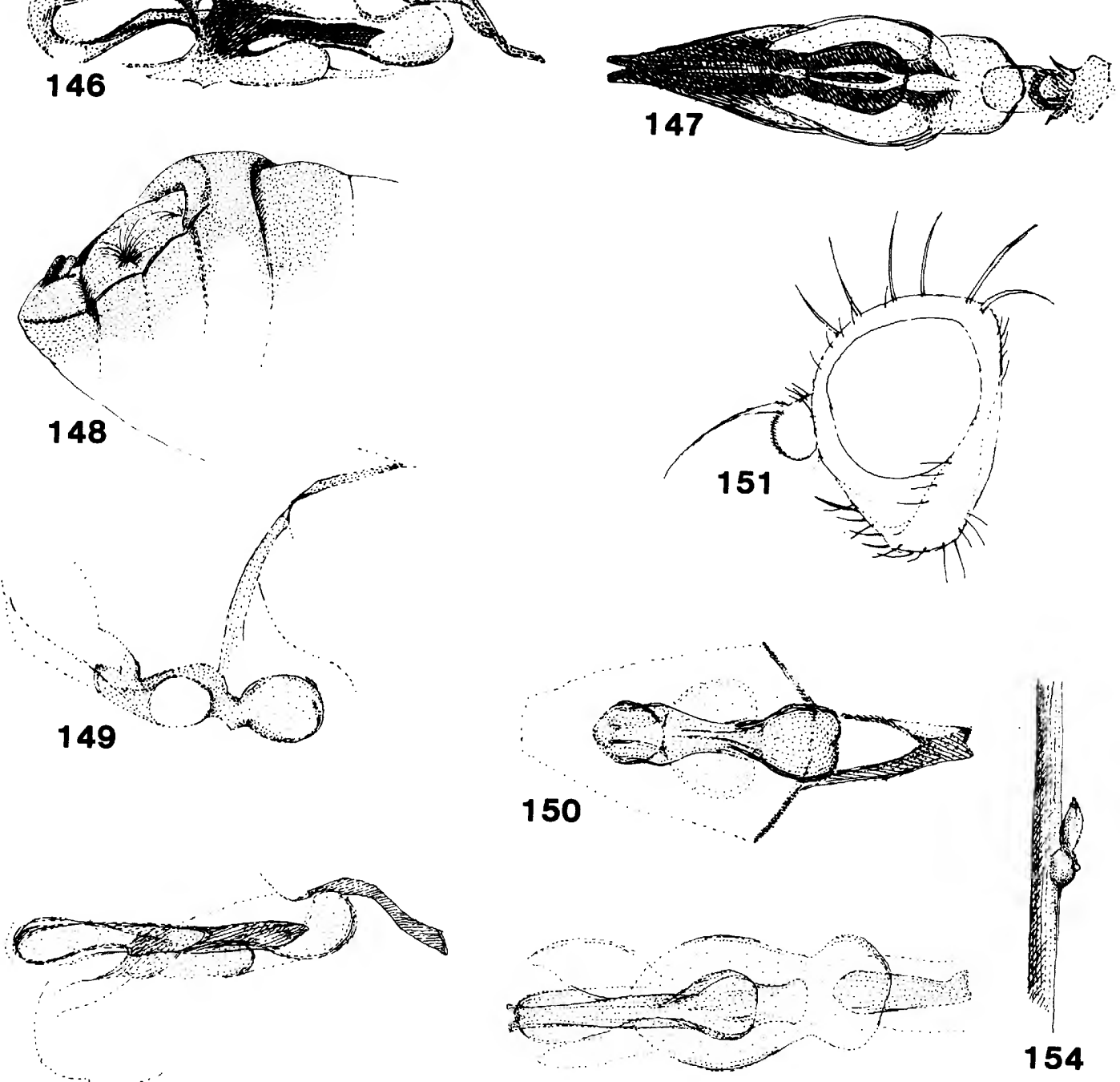

152

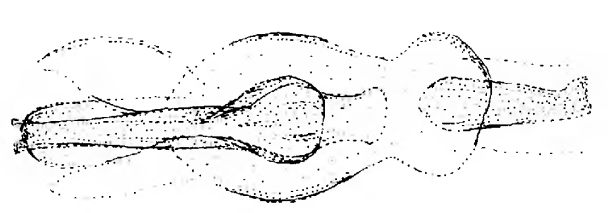

153

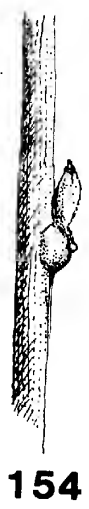

154

Figures 143-154.--Hexomyza winnemanae: 143, Aedeagus,

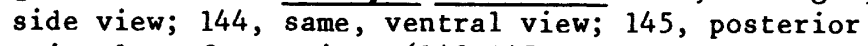
spiracles of puparium; (143-145, holotype of $\mathrm{H}$. albicula, Canada); H. simplicoides: 146, Aedeägus, side view; 147 , same, ventral view (California);

148, anterior end of puparium (Europe); $\mathrm{H}$.

toschiae: 149, Aedeagus, side view; 150, same, ventral view; $\underline{H}$. salicis: 151 , Head; 152 , aedeagus, side view; 153, same, ventral view (holotype); $\mathrm{H}$. websteri: 154 , Twig gall on Wistaria. 

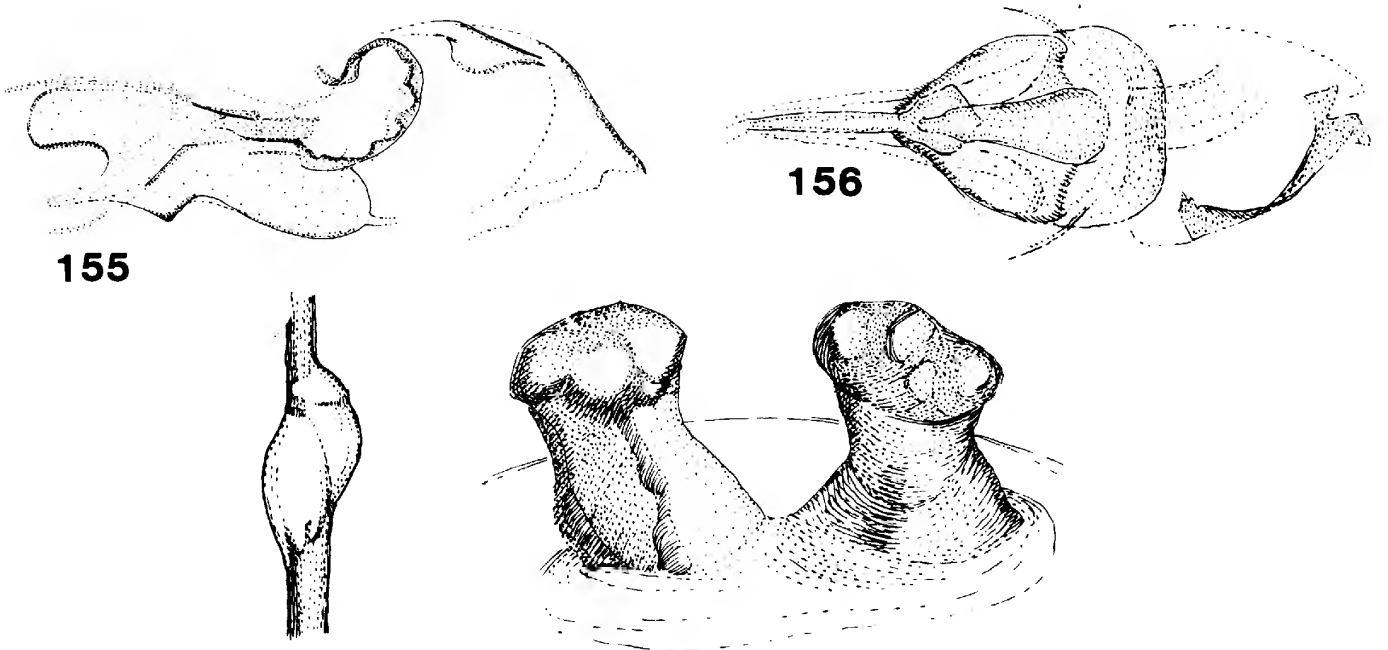

157

158

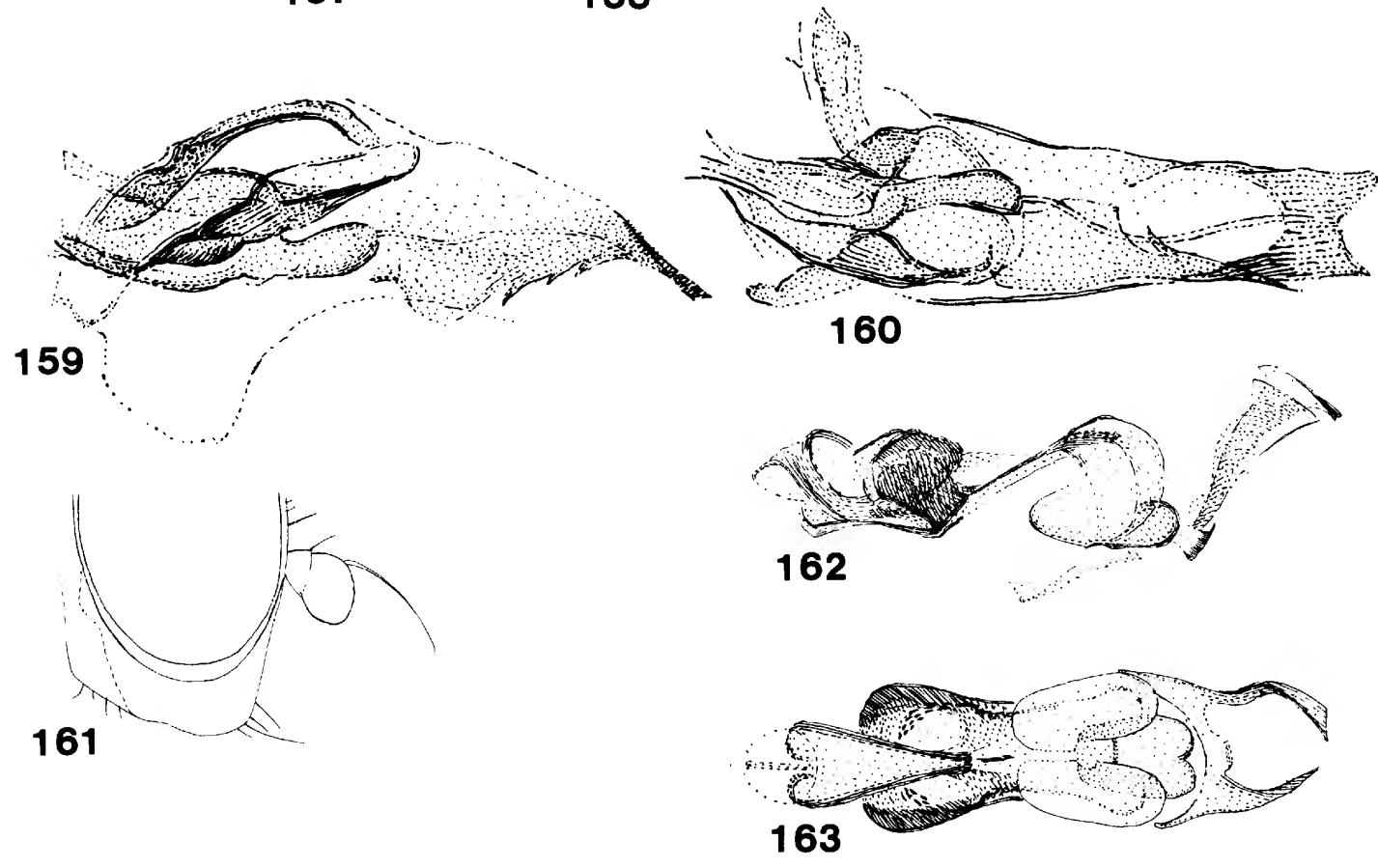

Figures 155-163.--Hexomyza schineri: 155, Aedeagus, side view; 156, same, ventral view; 157, galls on Salix sp.; 158, posterior spiracles of puparium. Ophiomyia maculata: 159, Aedeagus, side view; 160 , same, ventral view (holotype); 0 . banffensis: 161,

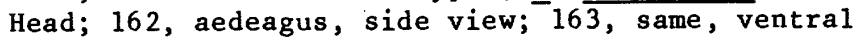
view (holotype, Canada). 

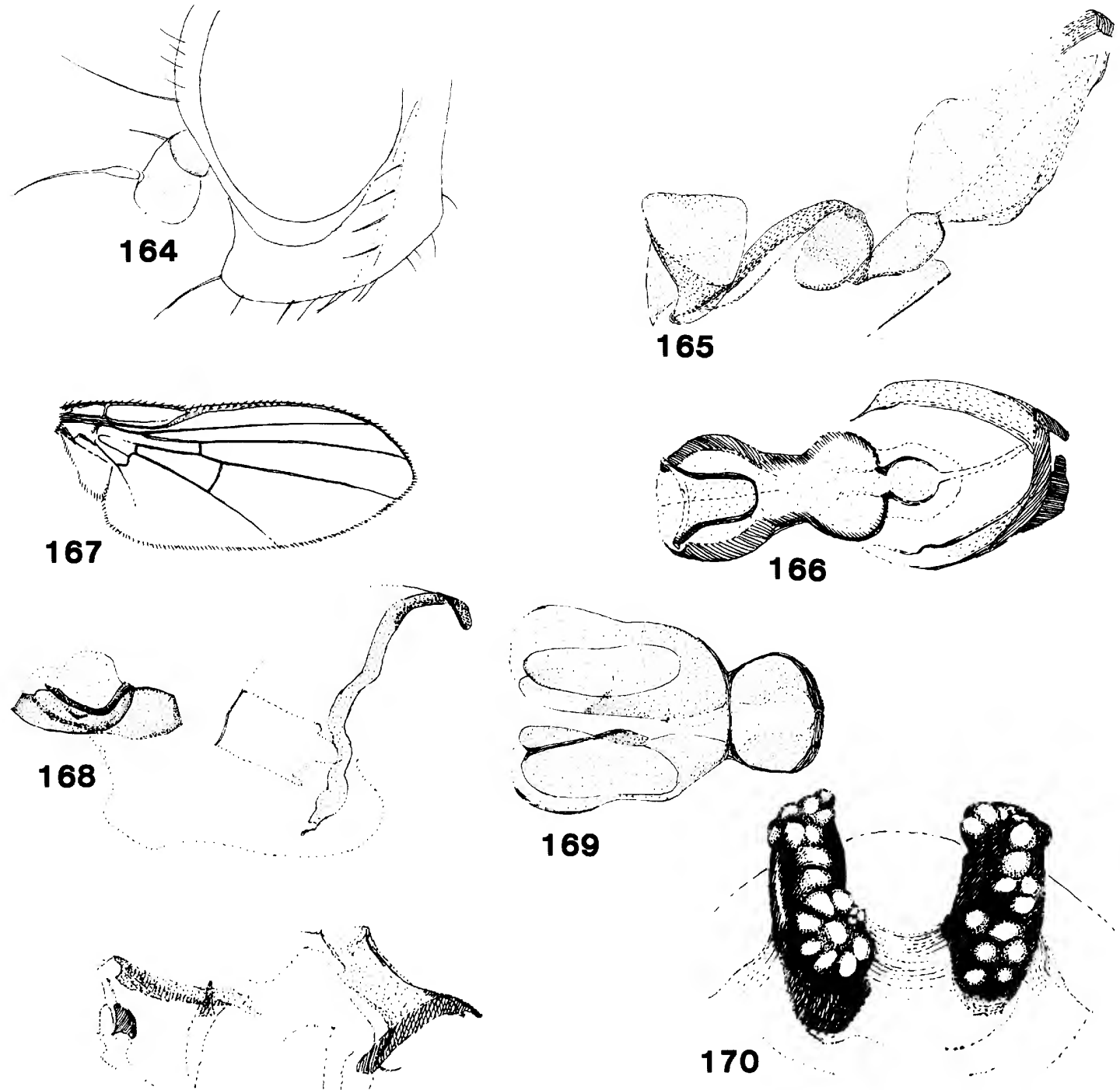

\section{1}
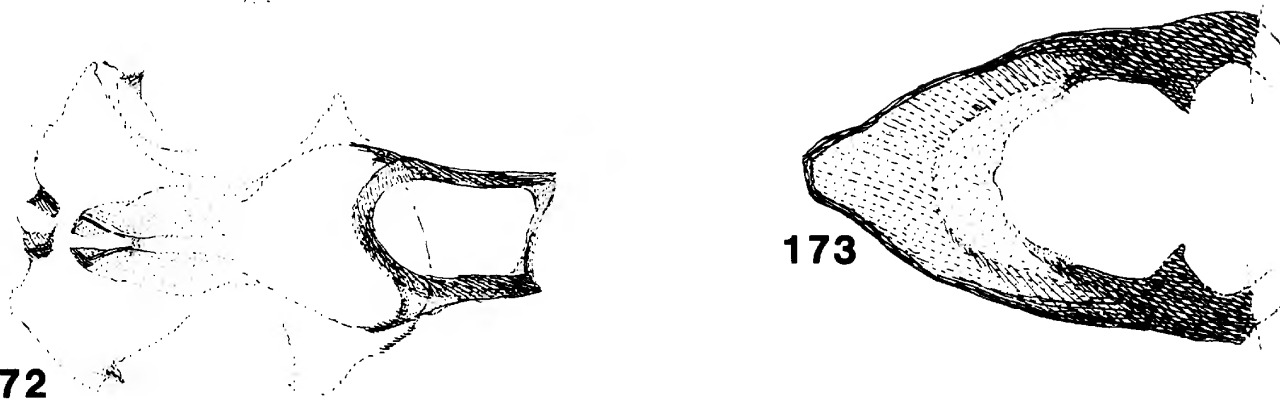

172

Figures 164-173.--0phiomyia monticola: 164, Head; 165, aedeagus, side view; 166, distiphallus, ventral view; 0 . simplex: 167 , Wing; 168 , aedeagus, side view; 169 , distiphallus, ventral view; 170 , posterior spiracles of puparium; 0. similata: 171, Aedeagus, side view; 172, same, ventral view; 173, hypandrium (New York). 

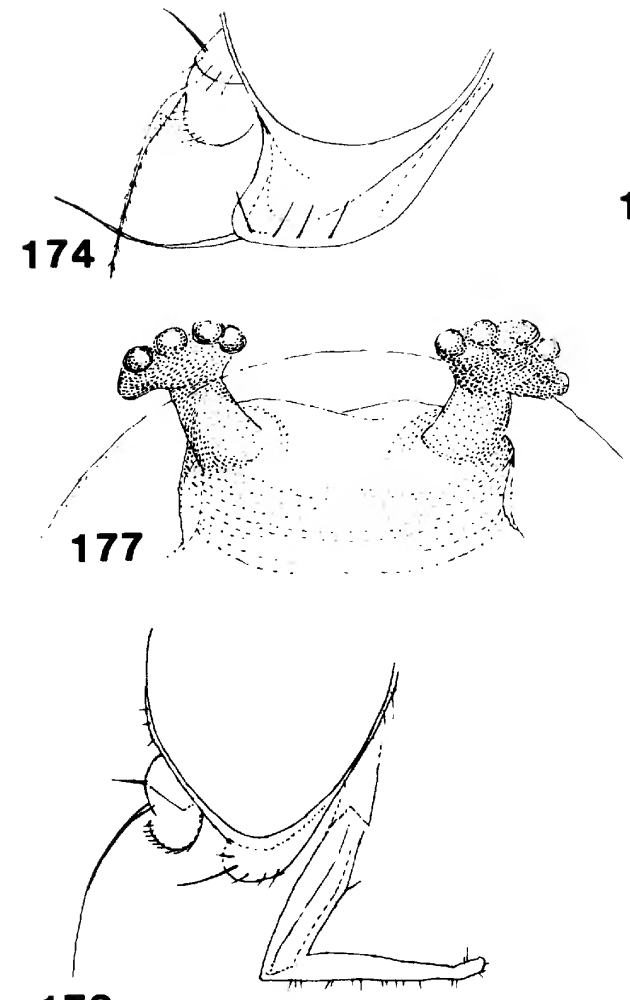

178

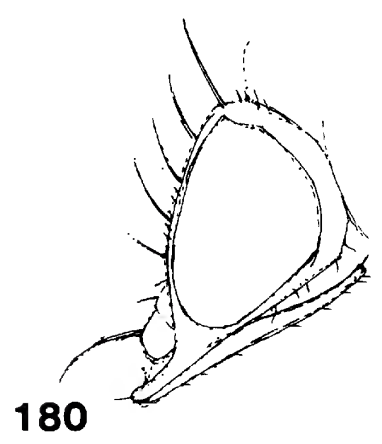

175
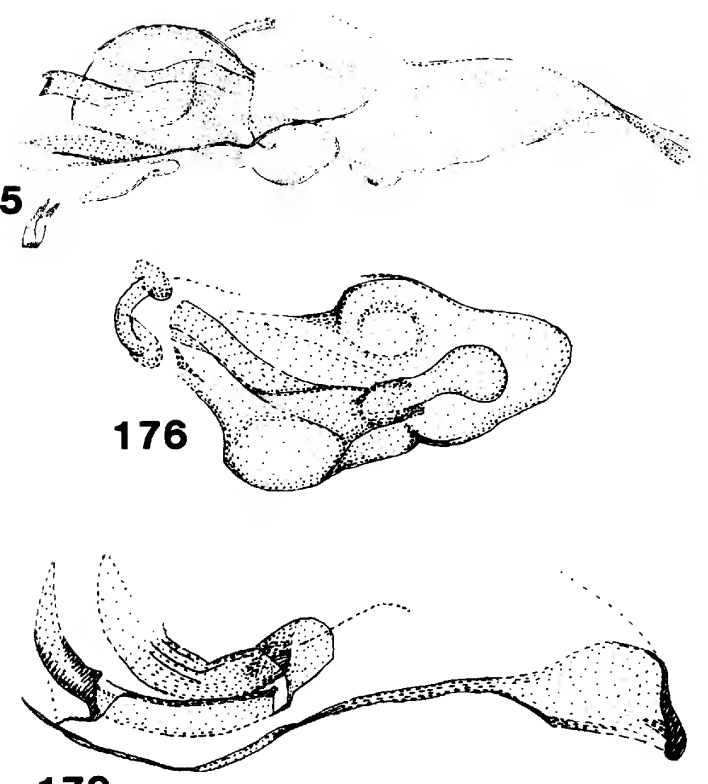

179
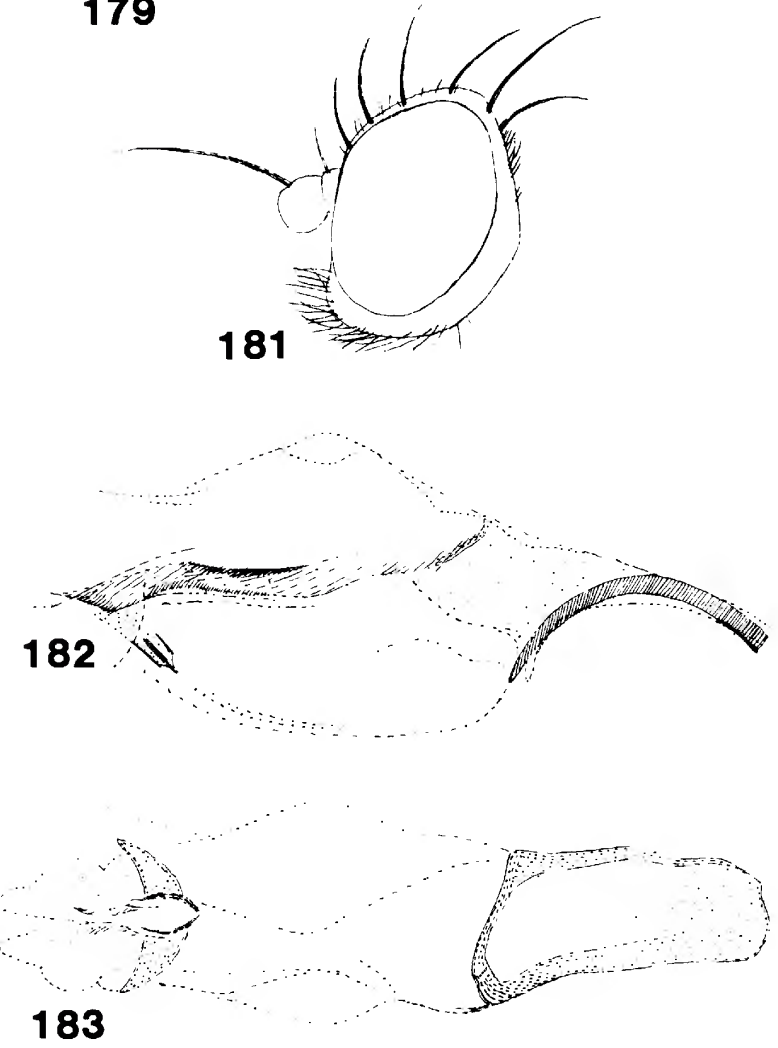

Figures 174-183.--Ophiomyia lippiae: 174, Head; 175, aedeagus, side view; 176, same, ventral view; 177 , posterior spiracles of puparium; 0 . haydeni: 178, Head; 179 , aedeagus, side view (Florida); 0 . texella: 180 , Head; 0 . lauta: 181 , Head; $1 \overline{8} 2$, aedeagus, side view; $18 \overline{3}$, same, ventral view. 


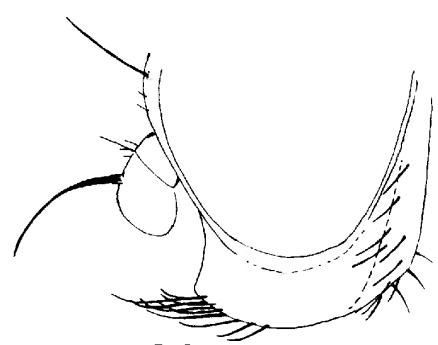

184
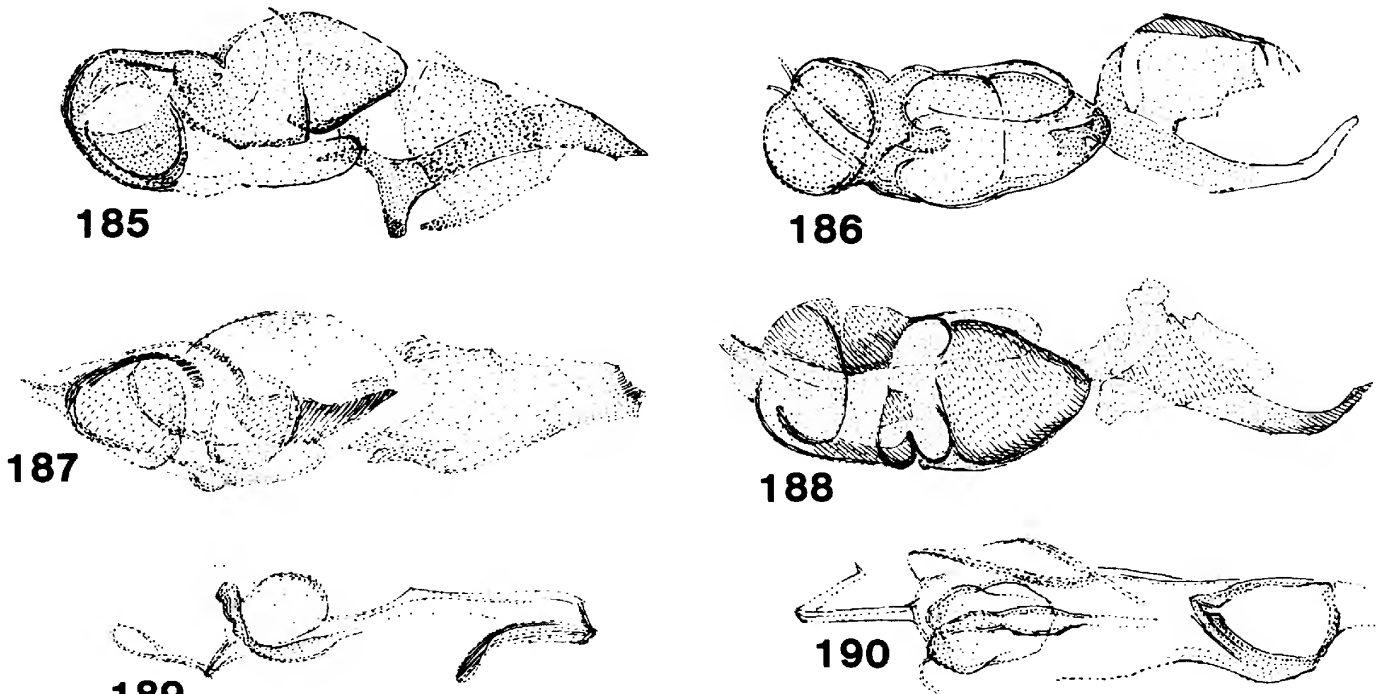

189
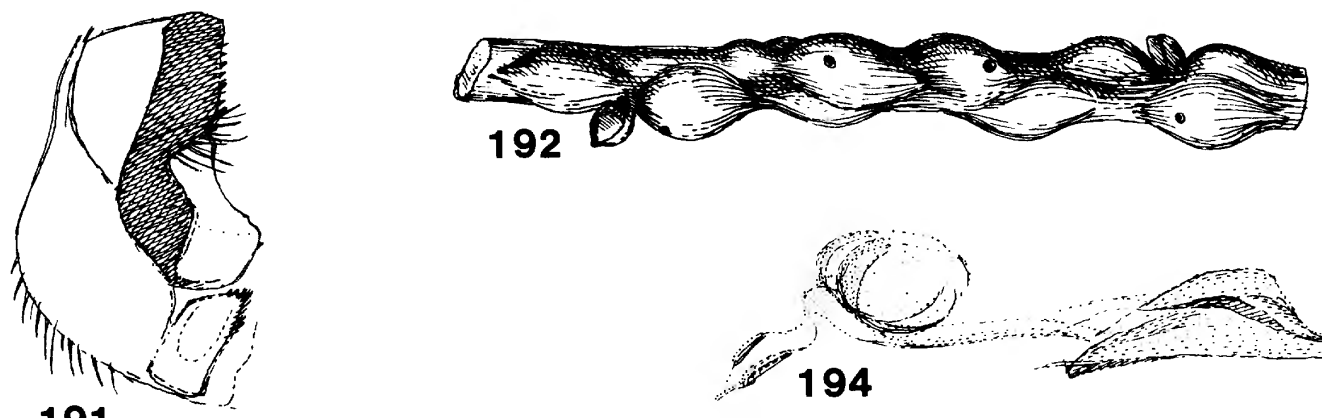

191
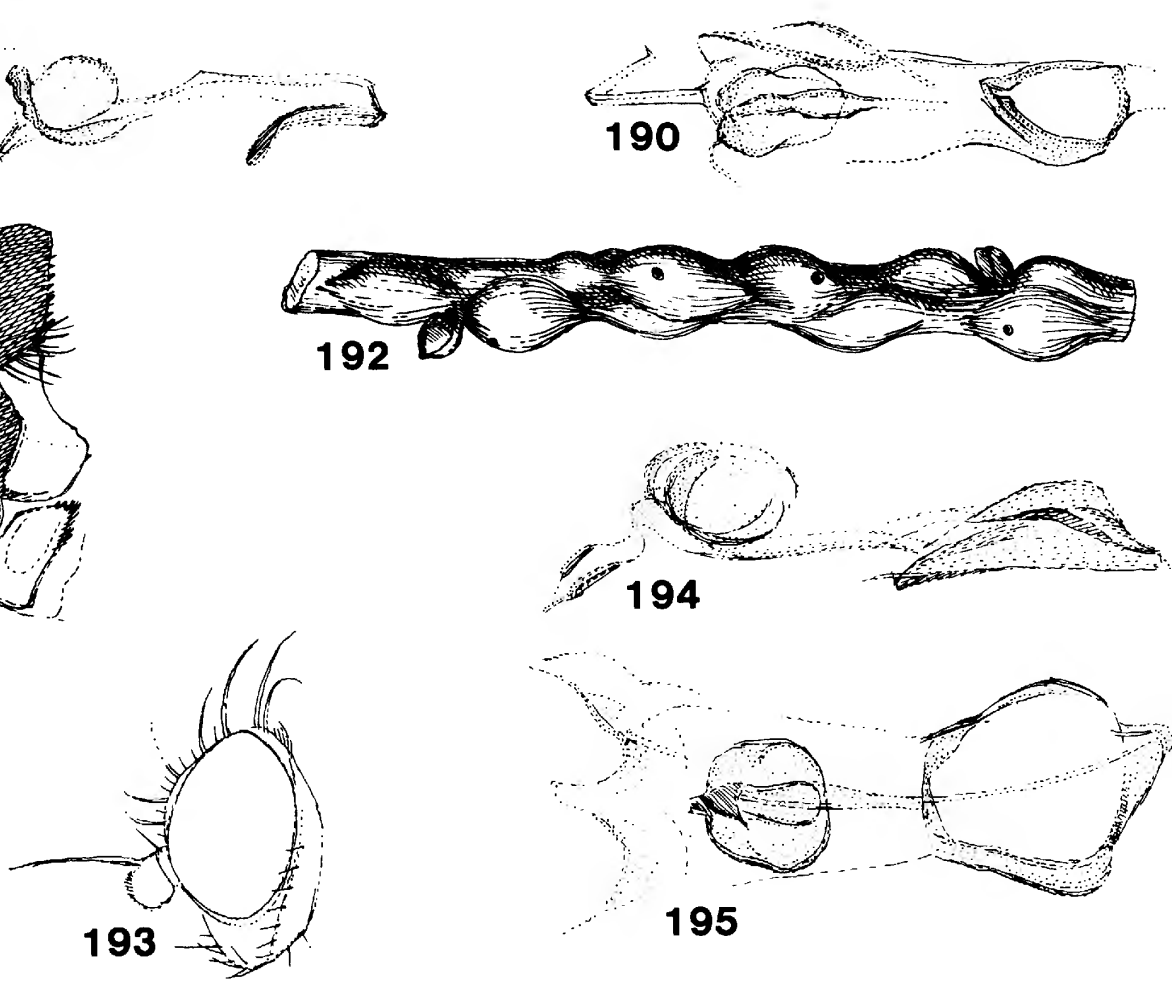

\section{4}

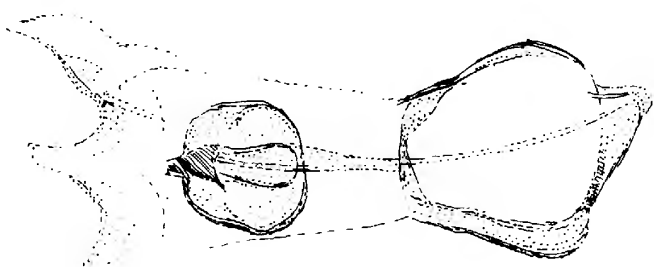

195

Figures 184-195.--Ophiomyia congregata: 184, Head;

185 , aedeagus, side view; 186 , same, ventral view;

o. frosti: 187, Aedeagus, side view; 188, same,

ventral view; 0 . tiliae: 189, Aedeagus, side view;

190, same, ventral view; 191, epandrium with

cercus; 192, twig galls on Tilia americana; 0 .

fastosa: 193, Head; 194, aedeagus, side view; 195, same, vertical view (Colorado). 

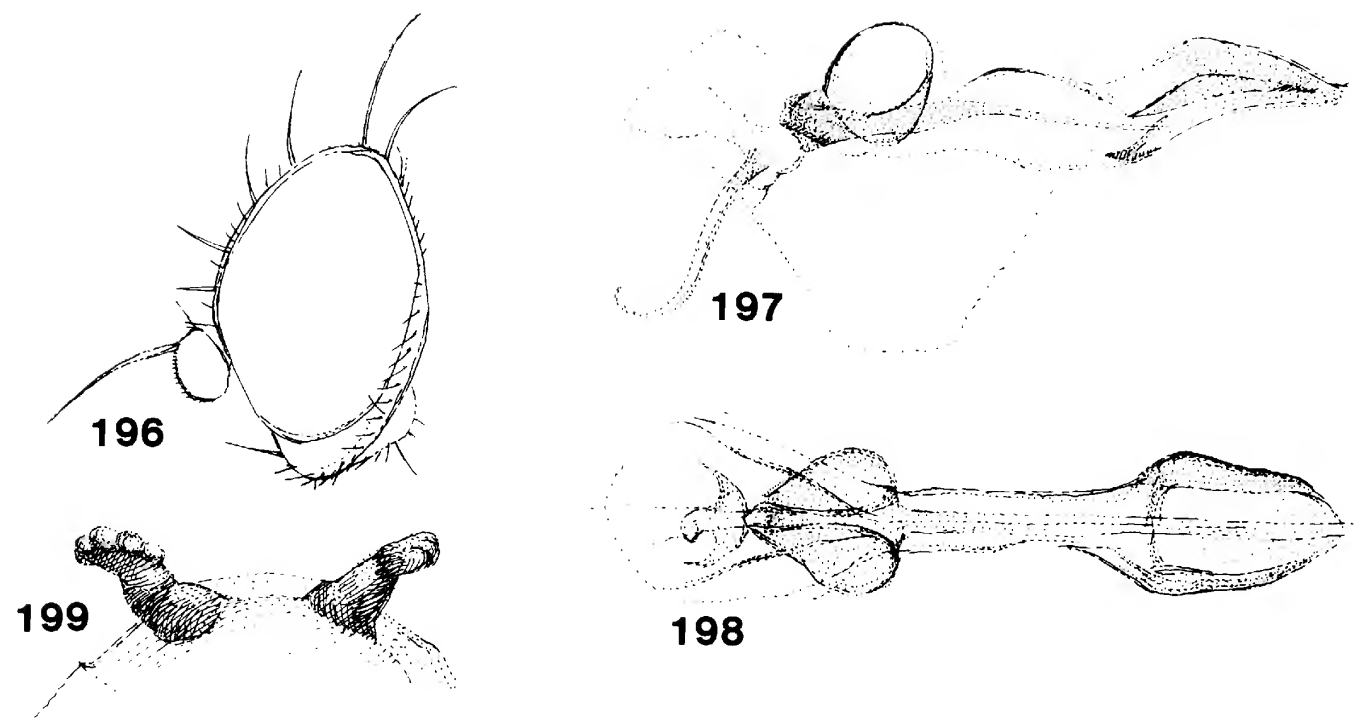

\section{8}
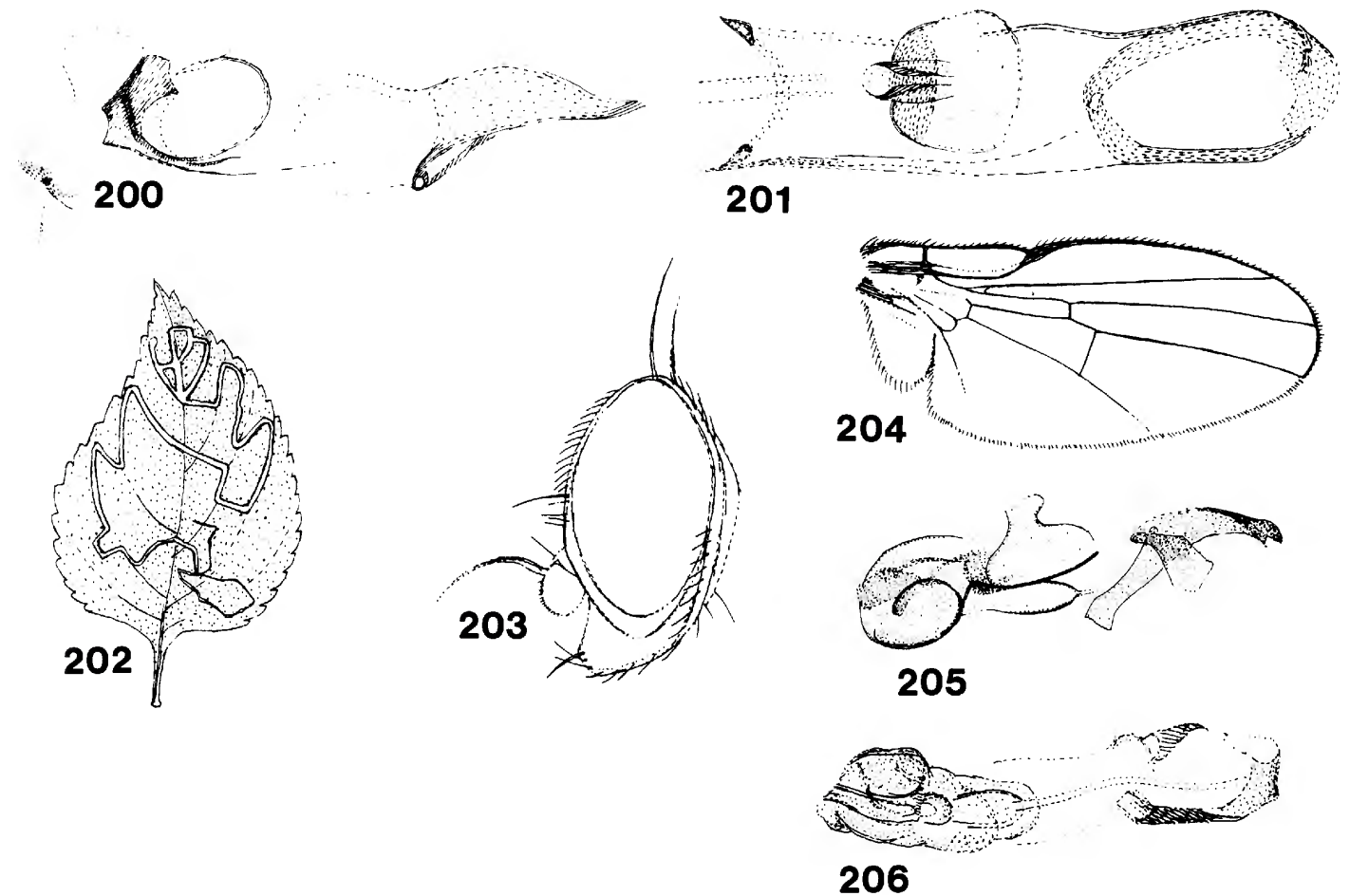

Figures 196-206.--Ophiomyia abutilivora: 196, Head;

197, aedeagus, side view; 198, same, ventral view;

199, posterior spiracles of puparium; 0. parvella:

200, Aedeagus, side view; 201, same, ventral view;

202, leaf mine on Lantana (Florida); 0. nasuta:

203, Head of male; 204, wing; 205, aedeagus, side

view; 206, same, ventral view (Colorado). 

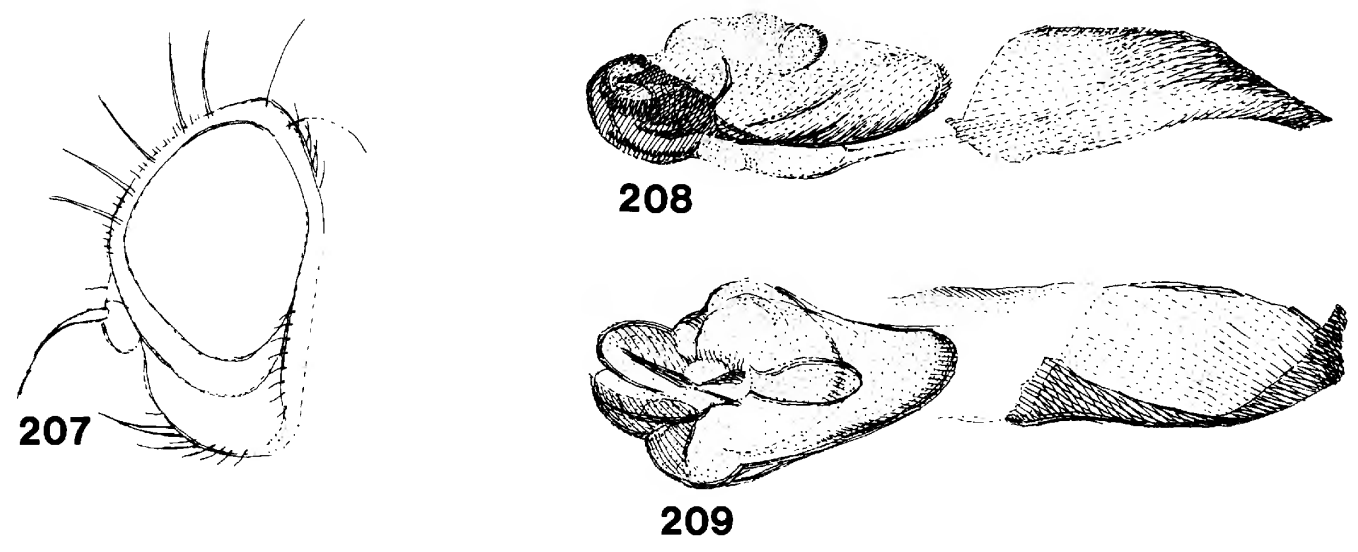

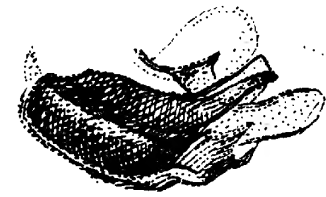

210

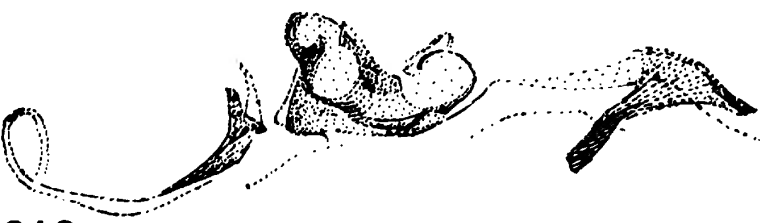

212

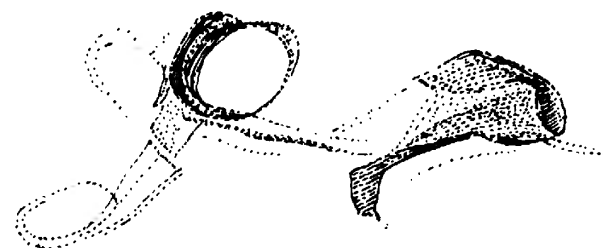

214
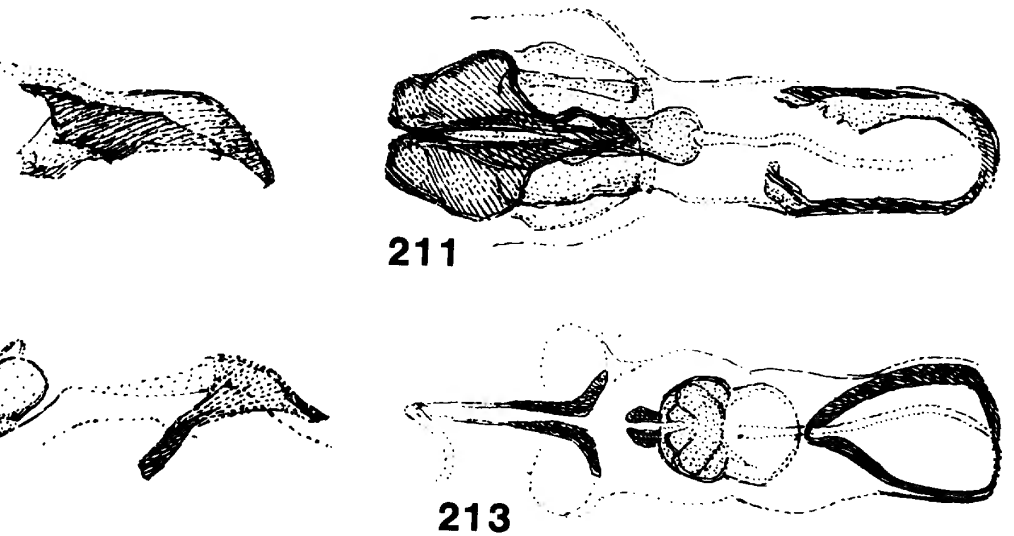

Figures 207-215.--Ophiomyia boulderensis: 207, Head; 208 , aedeagus, side view; 209 , same, ventral view; 0 . commendata: 210 , Aedeagus, side view; 211 , same, ventral view; 0 . bernardinensis: 212, Aedeagus, side view; 213 , same, ventral view; 0 . shastensis: 214, Aedeagus, side view; 215 , same, ventral view: 


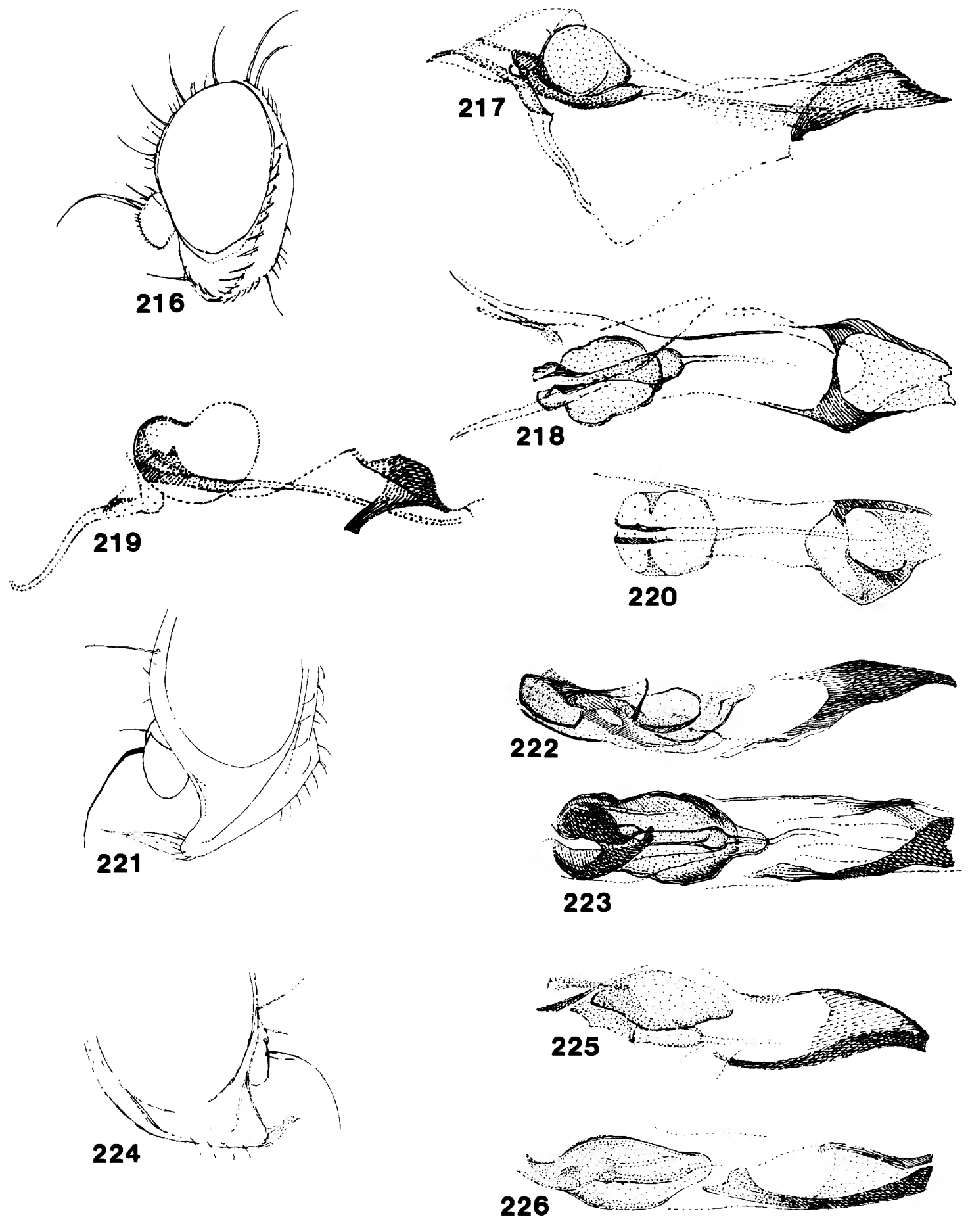

Figures 216-226.--0phiomyia jacintensis: 216, Head; 217 , aedeagus, side view; 218 , aedeagus, ventral view; 0 . yolensis: 219, Aedeagus, side view; 220, same, ventral view; 0. praecisa: 221, Head; 222, aedeagus, side view (holotype, Canada); 223, same, side view (California); 0. apta: 224, Head; 225, aedeagus, side view; 226 , same, ventral view. 

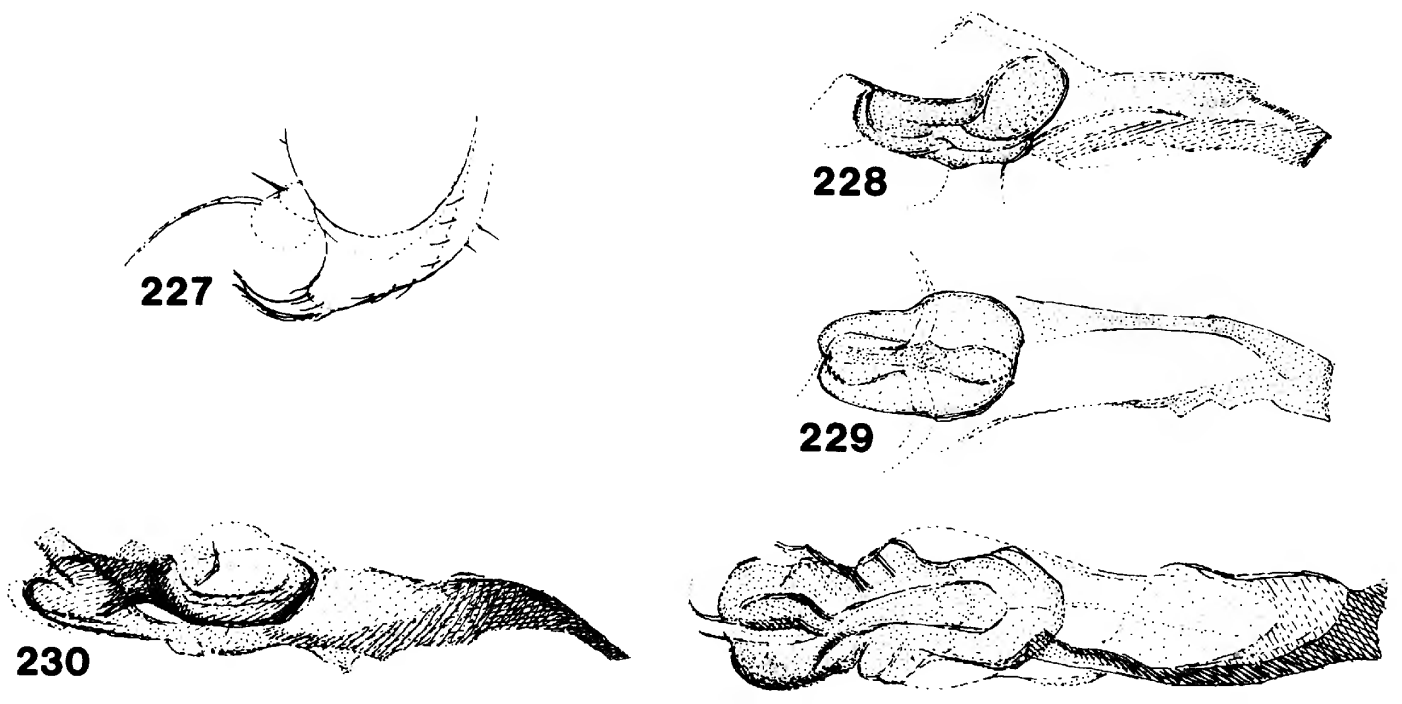

\section{1}
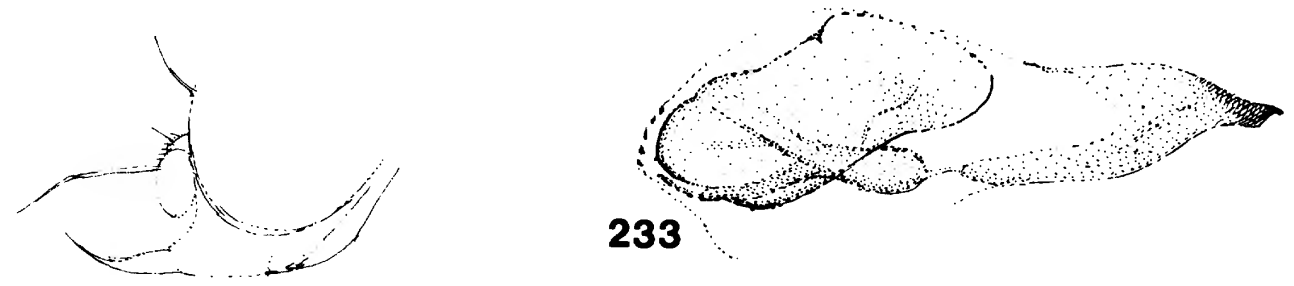

232

233
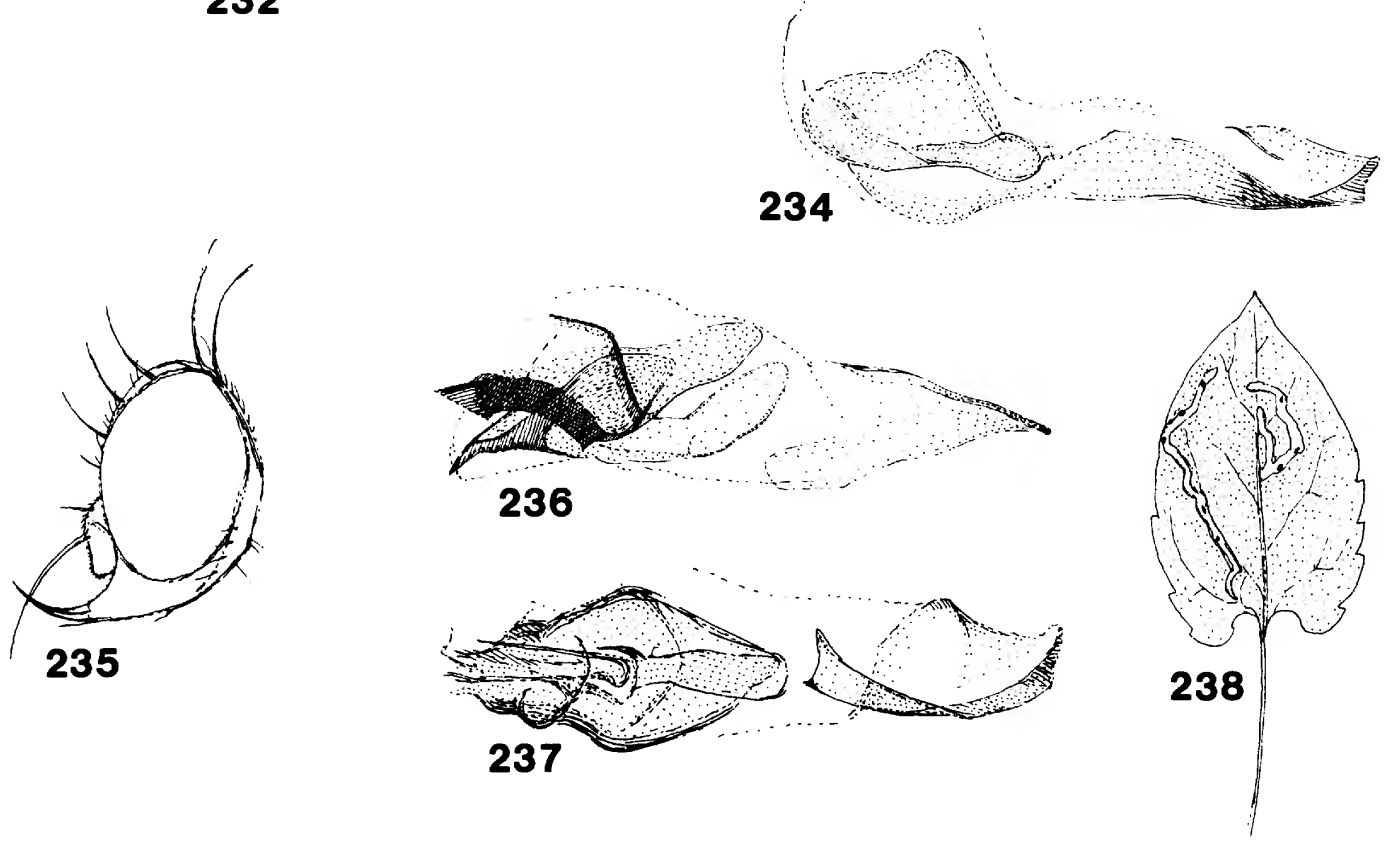

Figures 227-238.- - Ophiomyia chondrillae: 227, Head;

228 , aedeagus, side view; 229 , same, ventral view;

O. subpraecisa: 230, Aedeagus, side view; 231,

same, ventral view; $\underline{0}$. ambrosia: 232, Head; 233, aedeagus, side view; 234 , same, ventral view; $\underline{0}$.

quinta: 235, Head; 236, aedeagus, side view

(Canada); 237, same, ventral view (Arkansas); 238 ,

leaf mines on Aster; (235, 237, 238, Arkansas; 236,

Canada). 

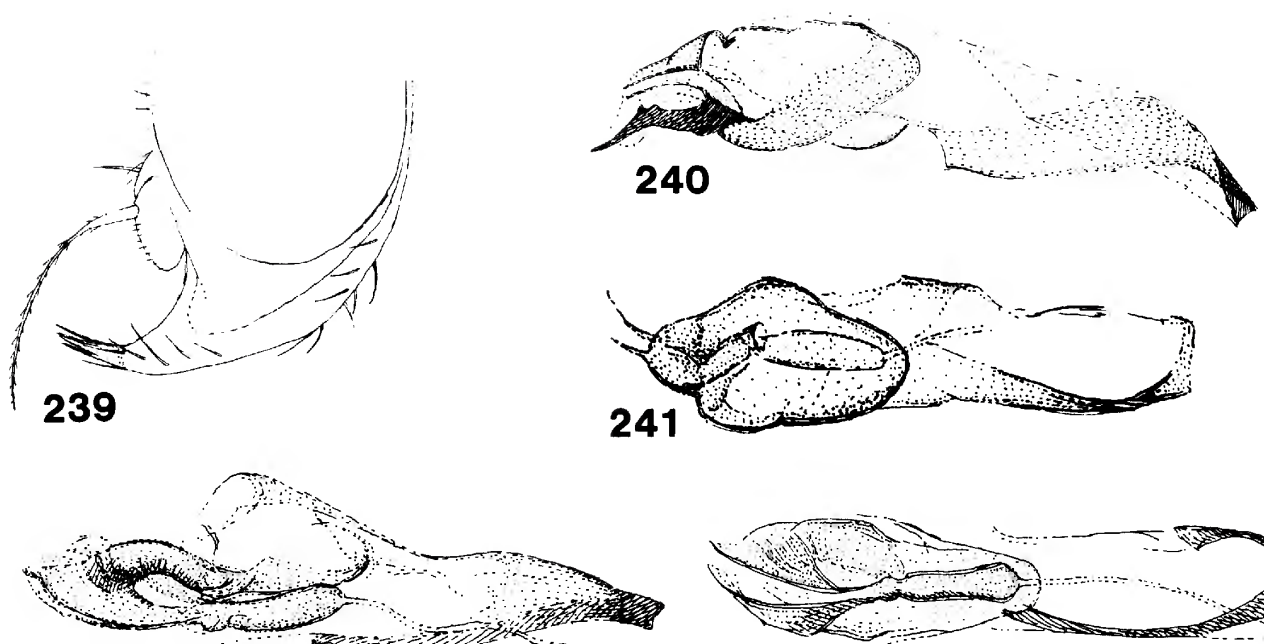

242

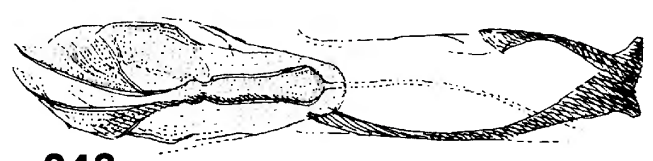

243

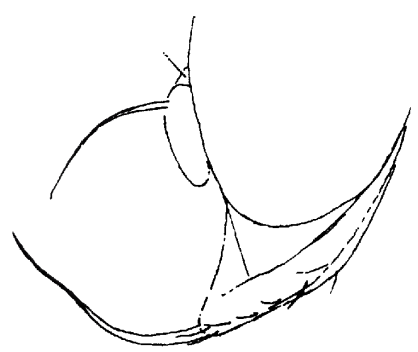

244
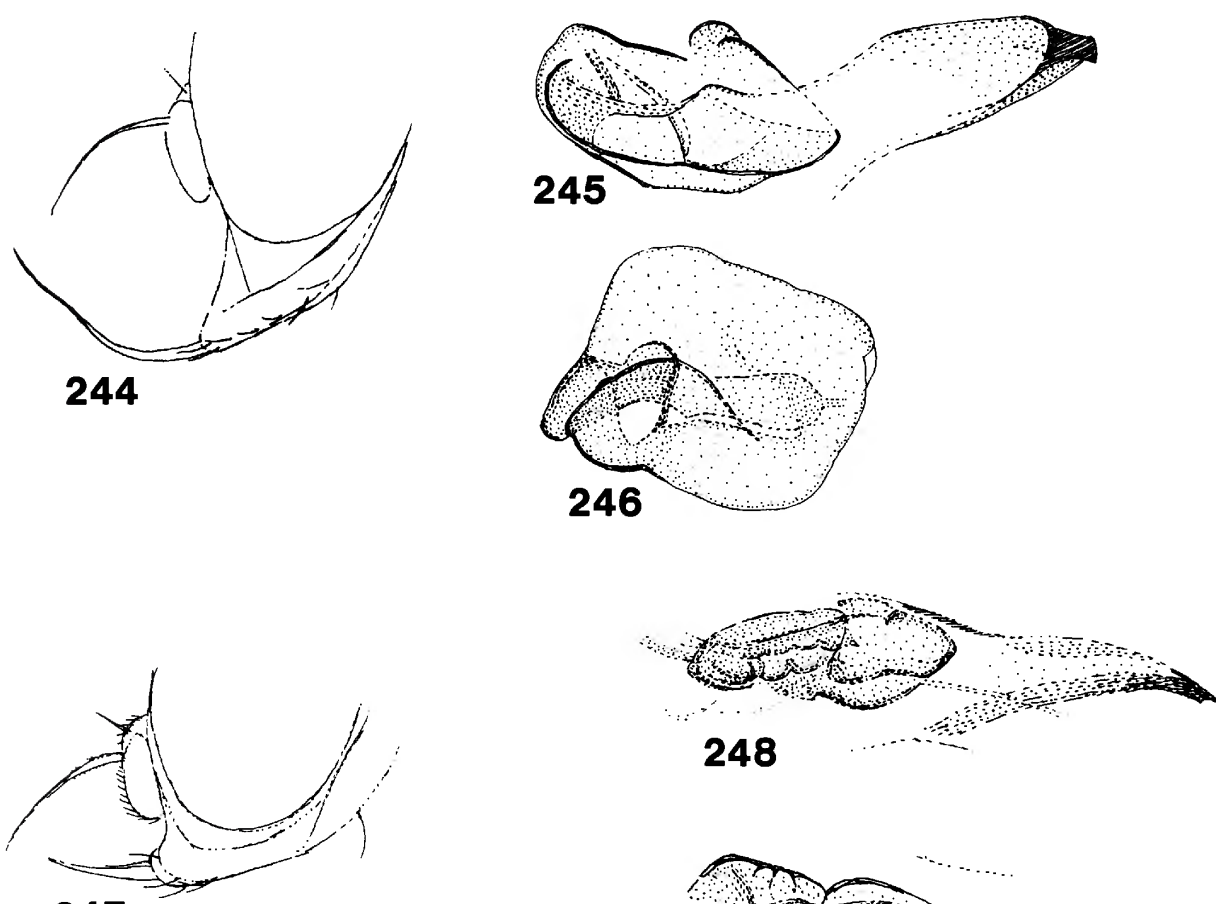

247

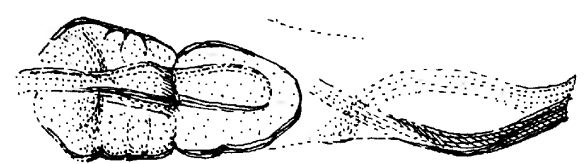

249

Figures 239-249.--Ophiomyia coniceps: 239, Head; 240, aedeagus, side view; 241 , same, ventral view; $\underline{0}$. virginiensis: 242, Aedeagus, side view; 243, same, ventral view; 0. obstipa: 244, Head; 245, aedeagus, side view; 246 , distiphallus, ventral view; 0 .

debilis: 247, Head; 248 , aedeagus, side view; 249, same, ventral view. 


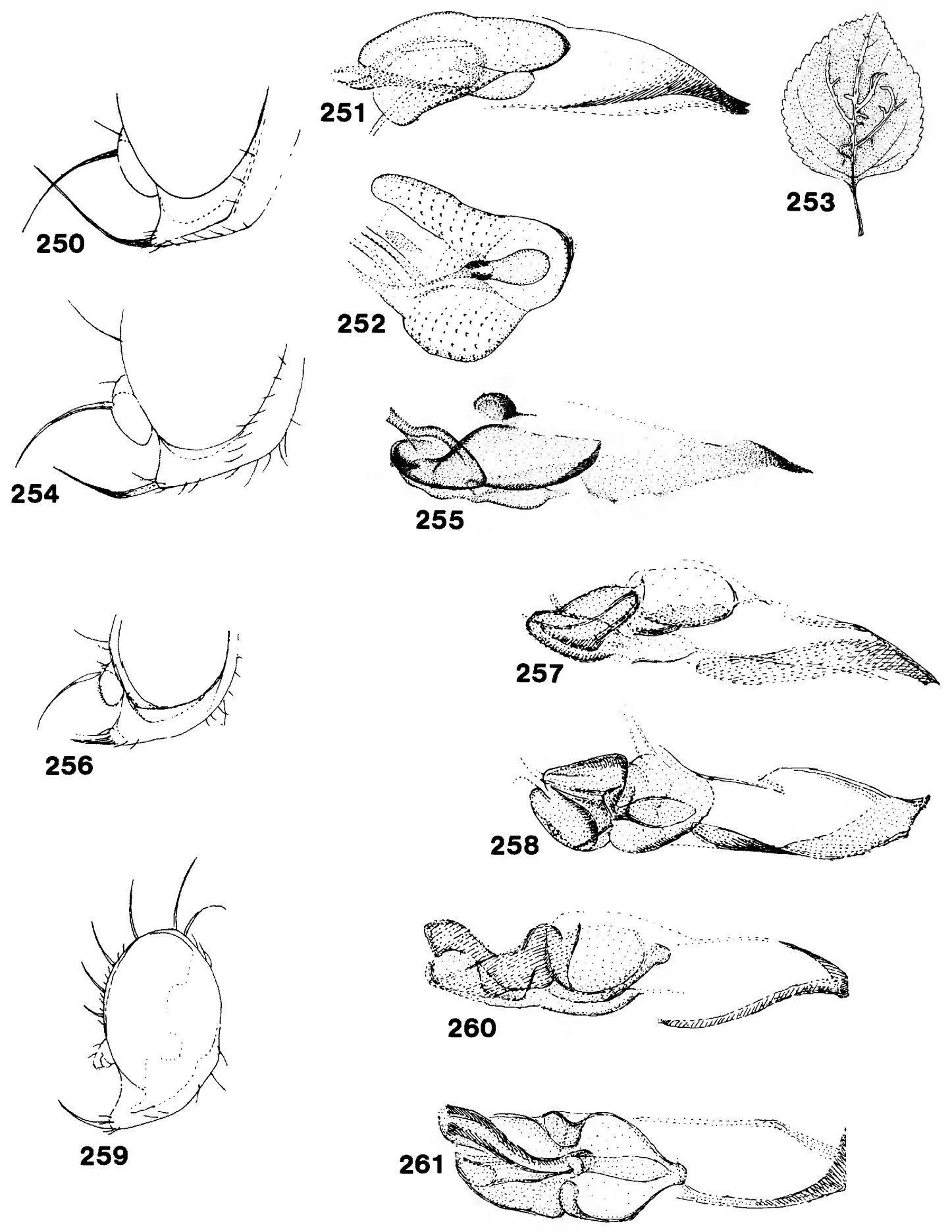

Figures 250-261.--Ophiomyia camarae: 250, Head; 251, aedeagus, side view; 252 , distiphallus, ventral view; 253, leaf mine on Lantana camara; $\underline{0}$.

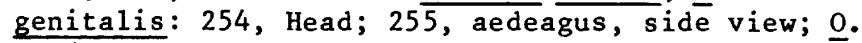
devia: 256, Head; 257, aedeagus, side view; 258, same, ventral view; $\underline{0}$. carolinensis: 259 , Head;

260, aedeagus, side view; 261, same, ventral view. 


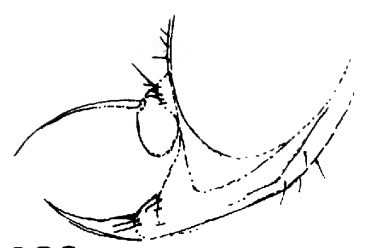

262
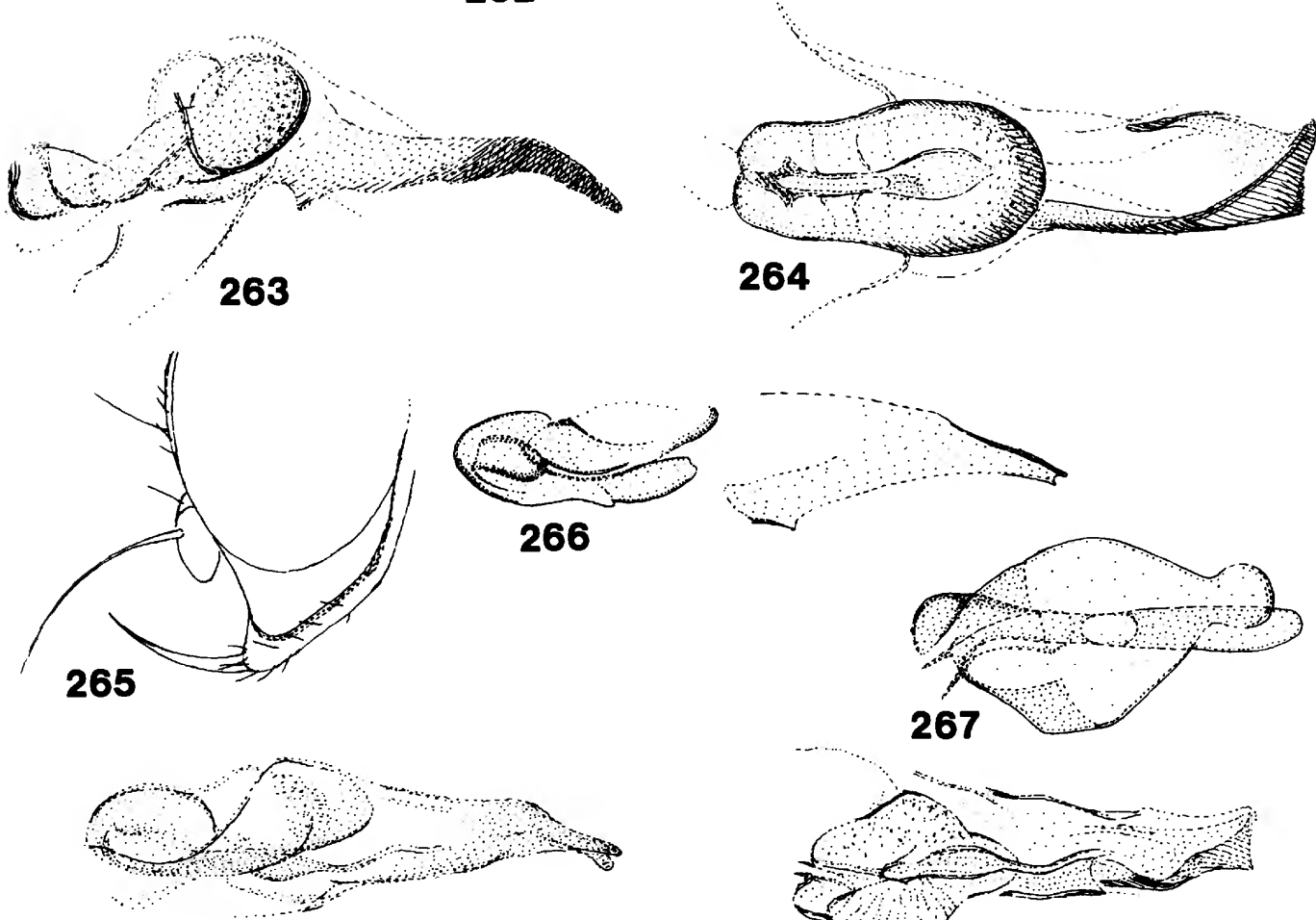

268

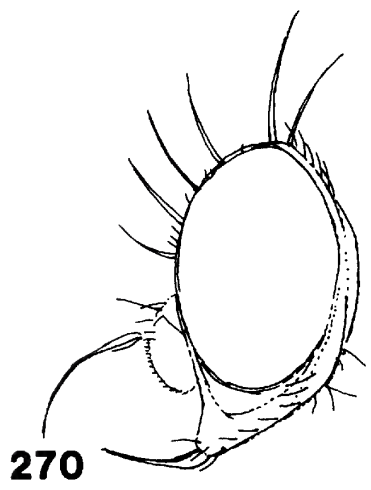

269
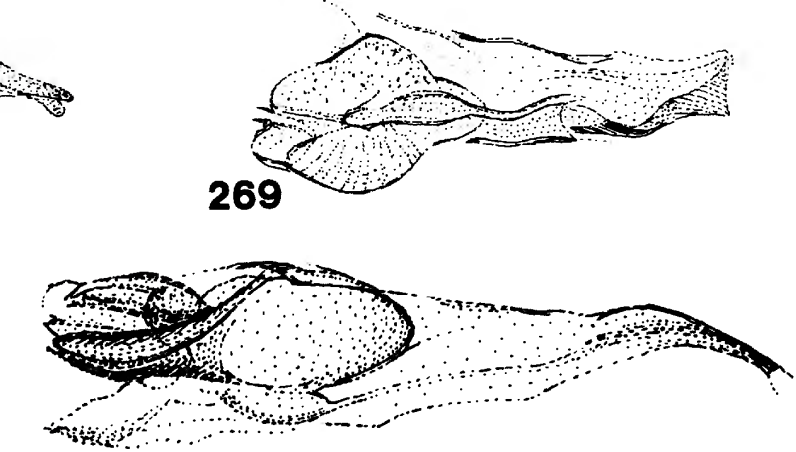

271

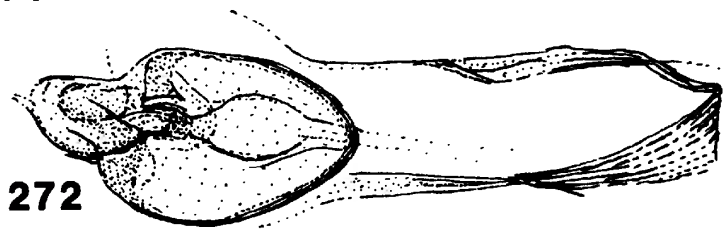

Figures 262-272.--0phiomyia vockerothi: 262, Head;

263, aedeagus, side view; 264 , same, ventral view;

0. 1acertosa: 265, Head; 266, aedeagus, side view;

267, distiphallus, ventral view; 0 . subdefinita:

268, Aedeagus, side view; 269 , same, ventral view;

0. delecta: 270, Head; 271, aedeagus, side view;

272 , same, ventral view. 


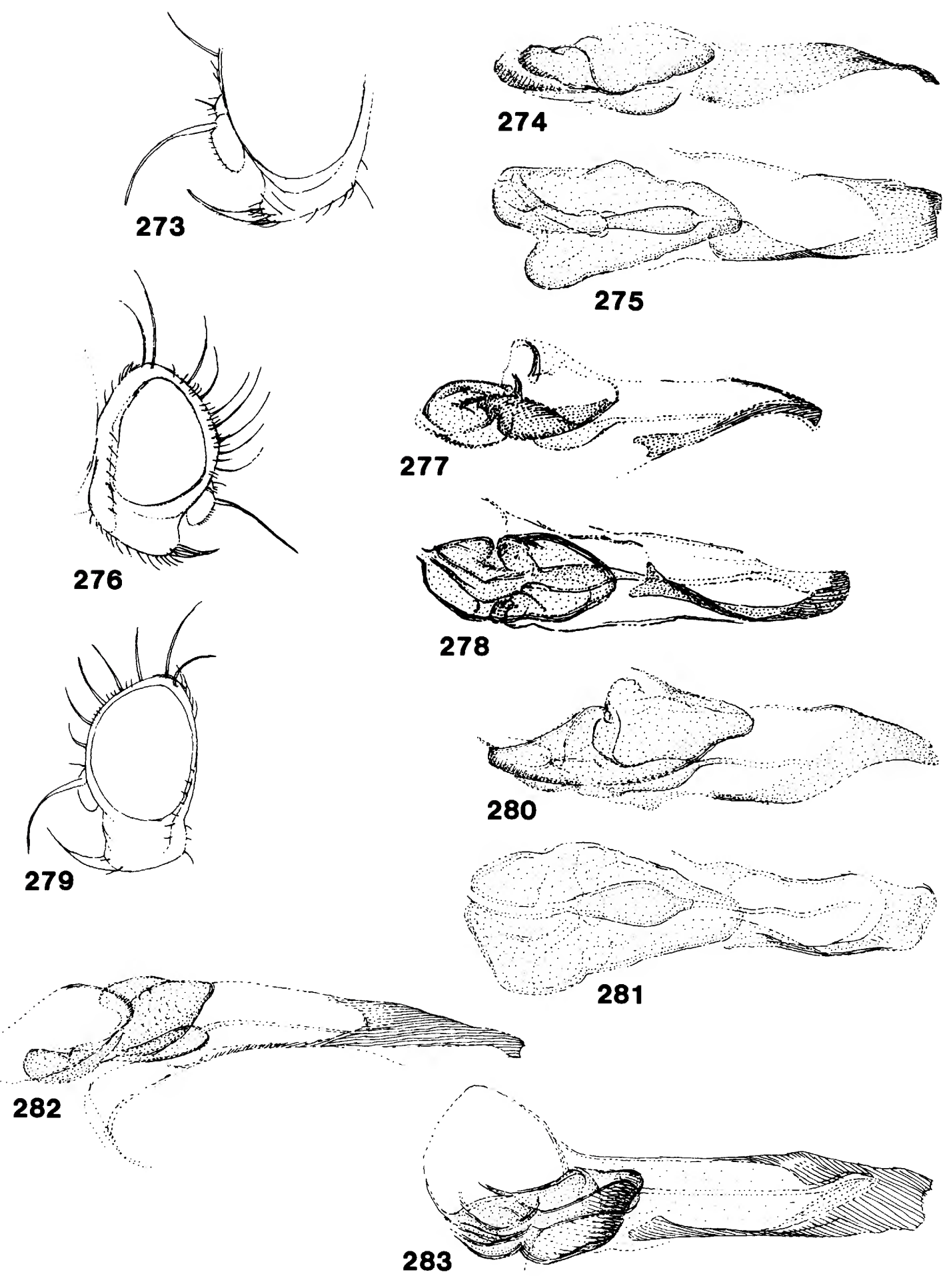

Figures 273-283.--Ophiomyia asymmetrica: 273, Head;

274 , aedeagus, side view; 275 , same, ventral view;

o. fida: 276 , Head; 277 , aedeagus, side view; 278 , same, ventral view; 0 . vibrissata: 279 , Head; 280,

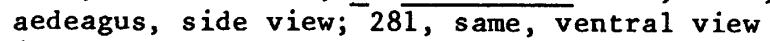

(holotype); 0 . carolinae: 282, Aedeagus, side view;

283, same, ventra1 view. 

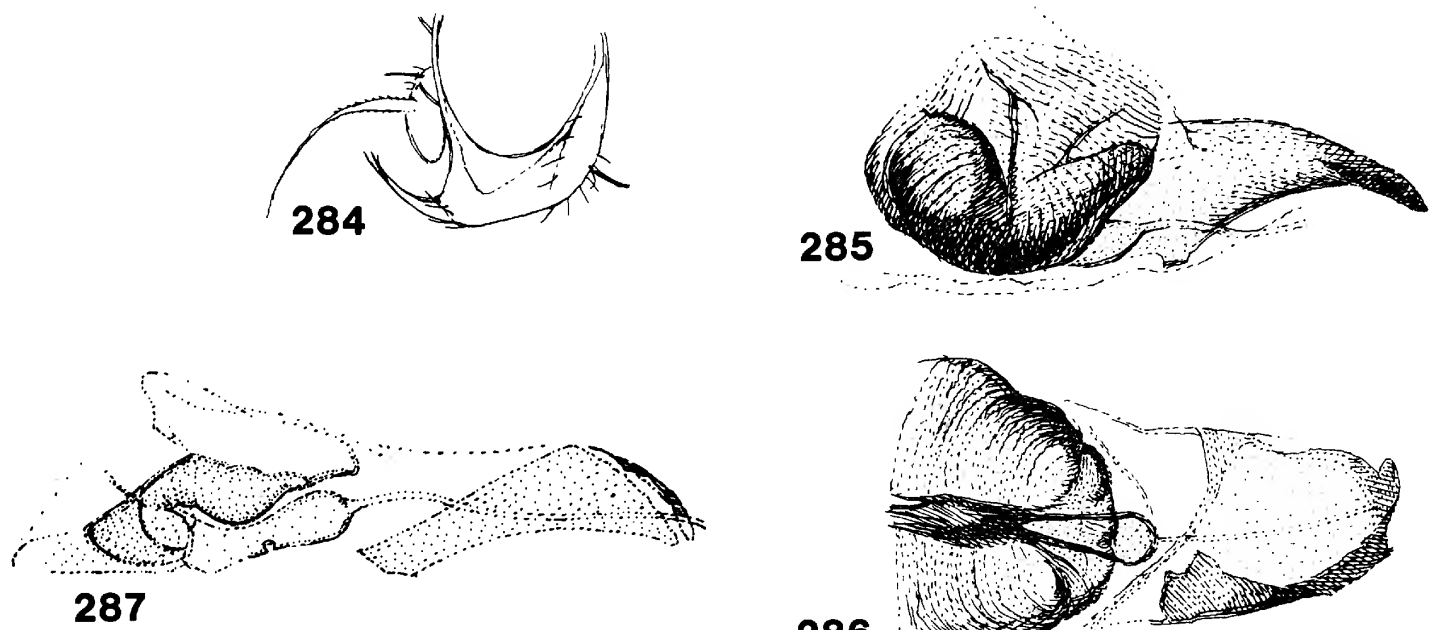

286
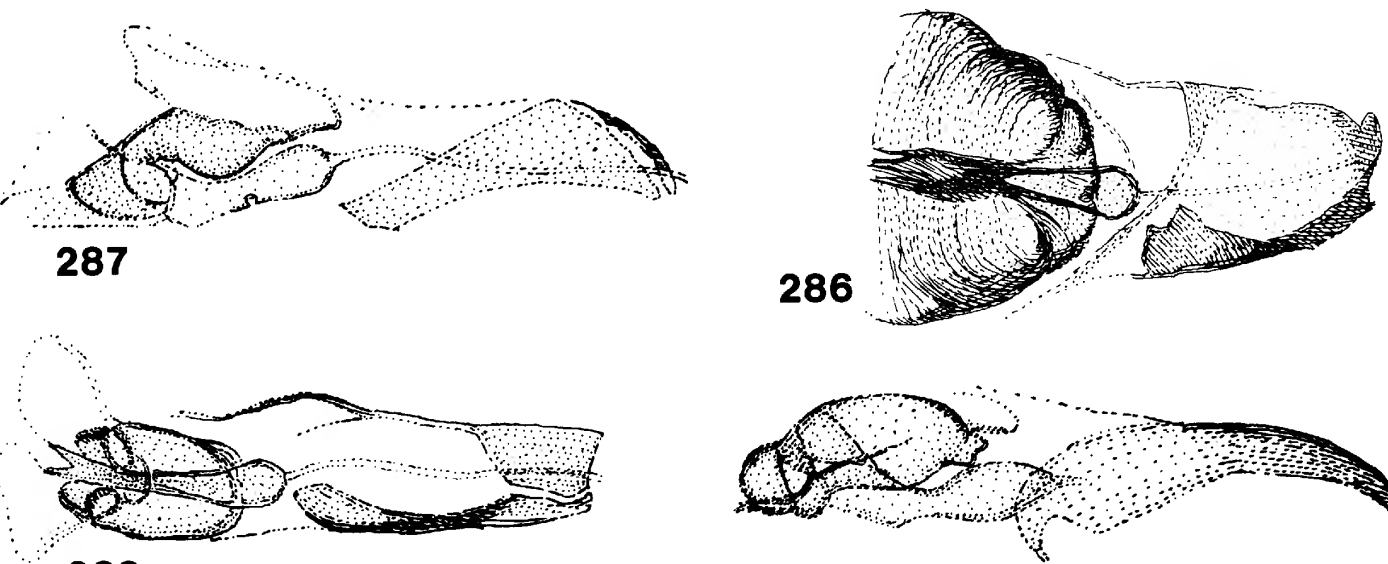

\section{8}
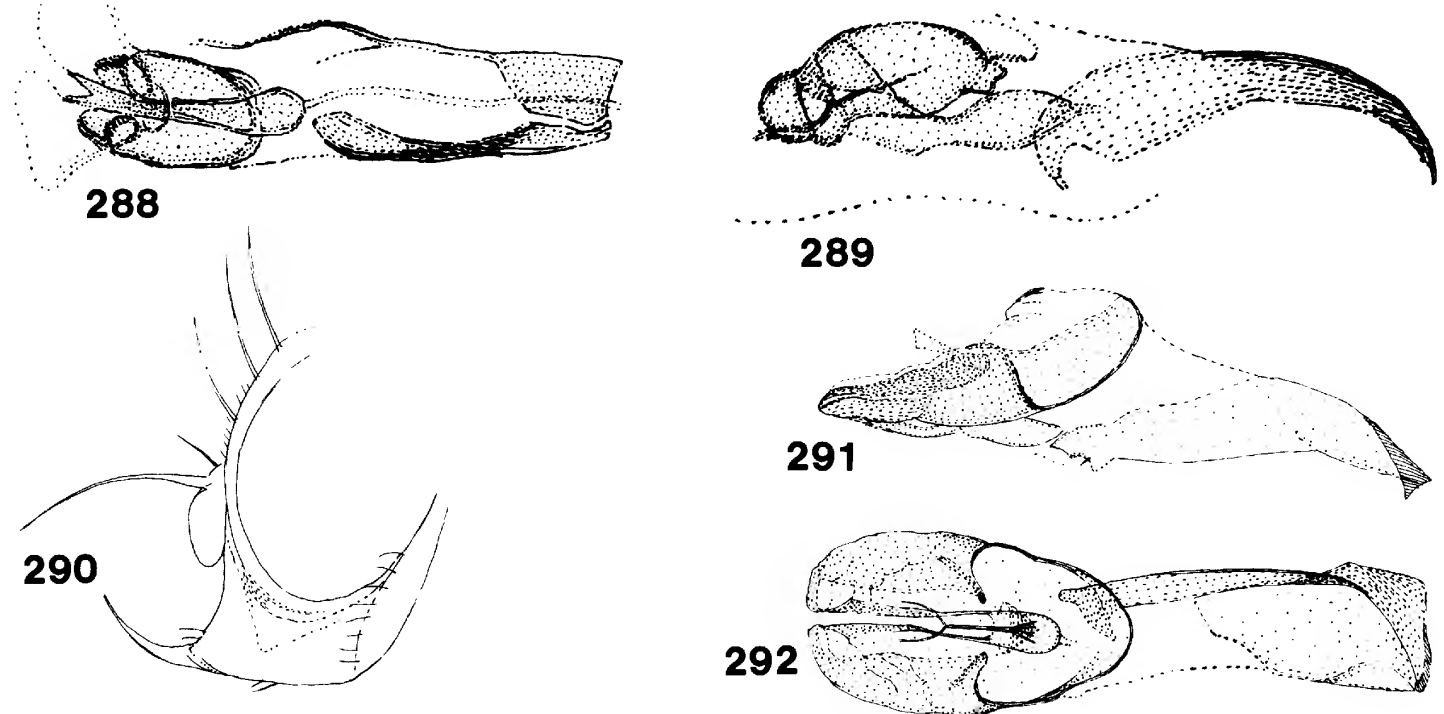

289
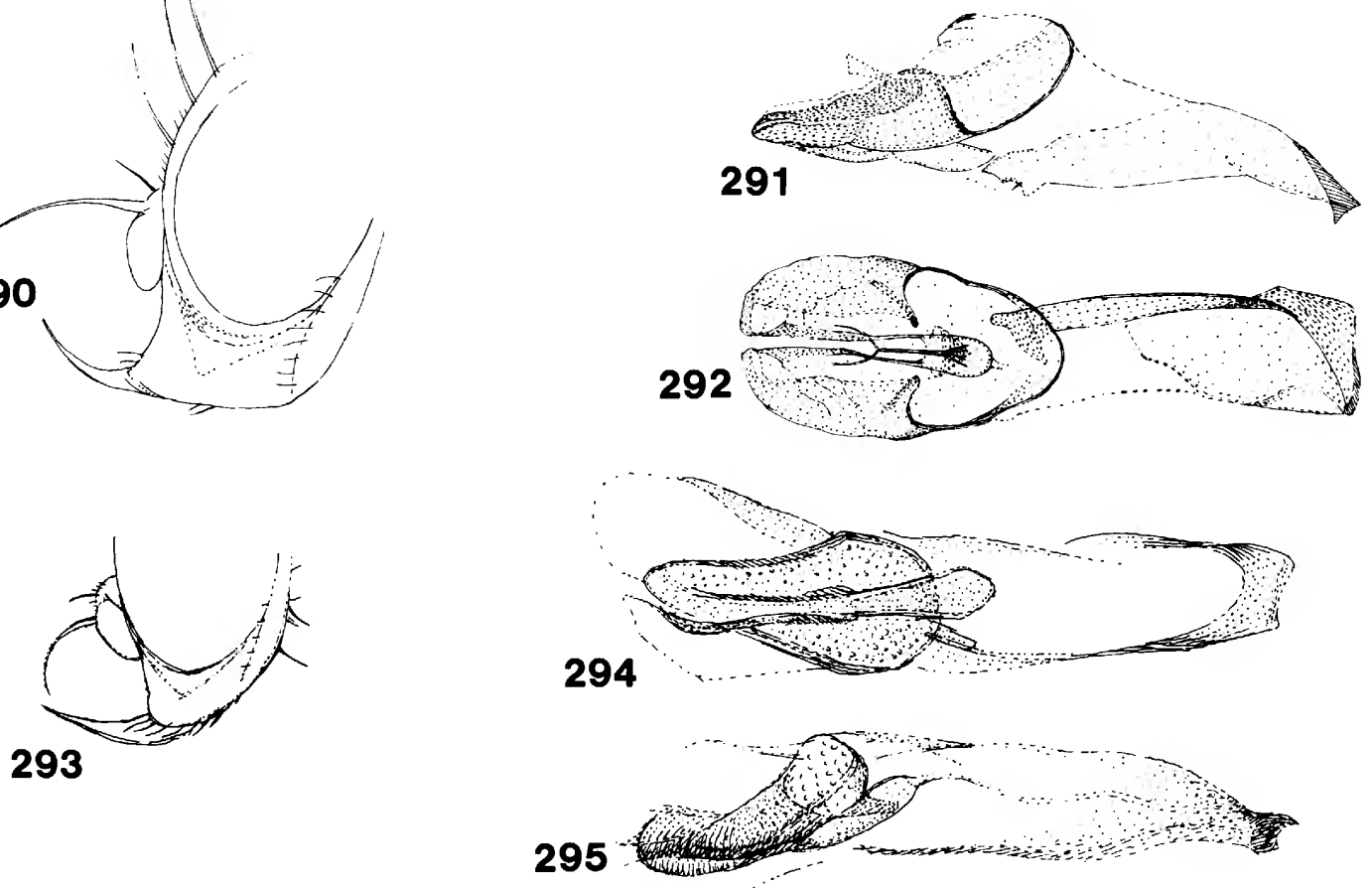

Figures 284-295.--Ophiomyia eldorensis: 284, Head; 285 , aedeagus, side view; 286 , same, ventral view; o. fastella: 287, Aedeagus, side view; 288, same, ventral view (holotype, California, Mono Co.); 289 , same, side view (California, Siskiyou Co., Mt.

Shasta); 0. sexta: 290, Head; 291 , aedeagus, side view; 292 , same, ventral view; O. texana: 293, Head

(holotype of 0 . modesta, California); 294 , aedeagus, side view; 295, same, ventral view (holotype of $\underline{0}$. texana). 

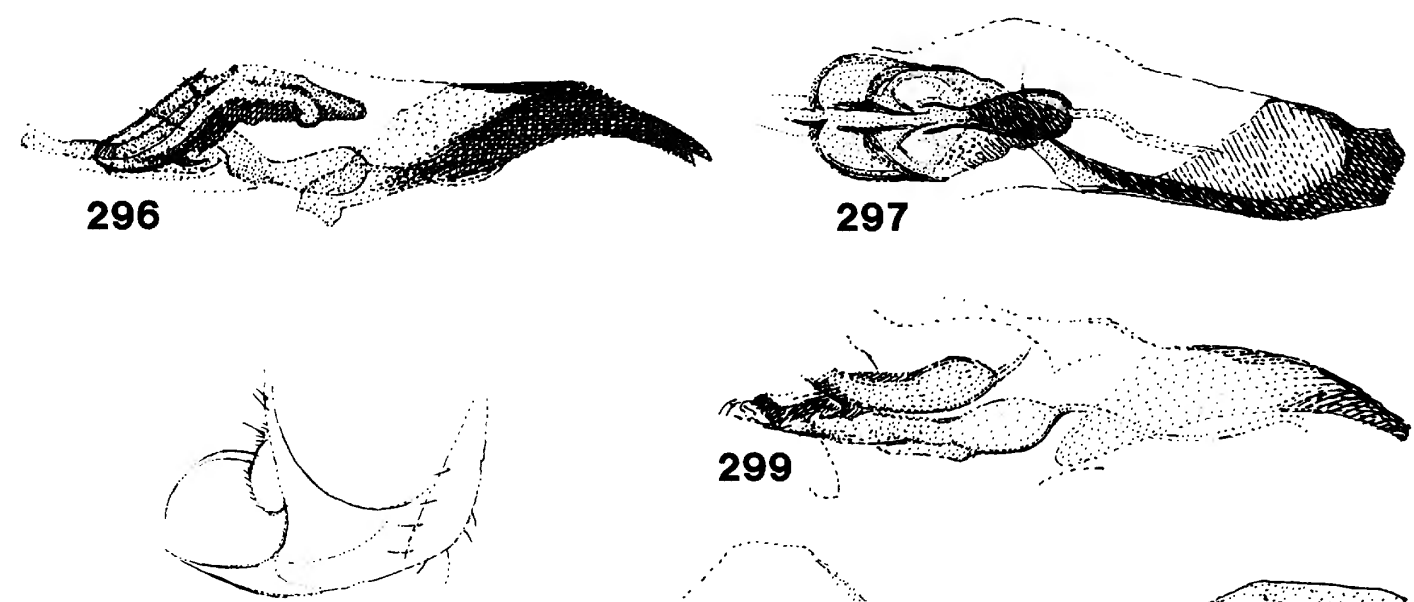

298
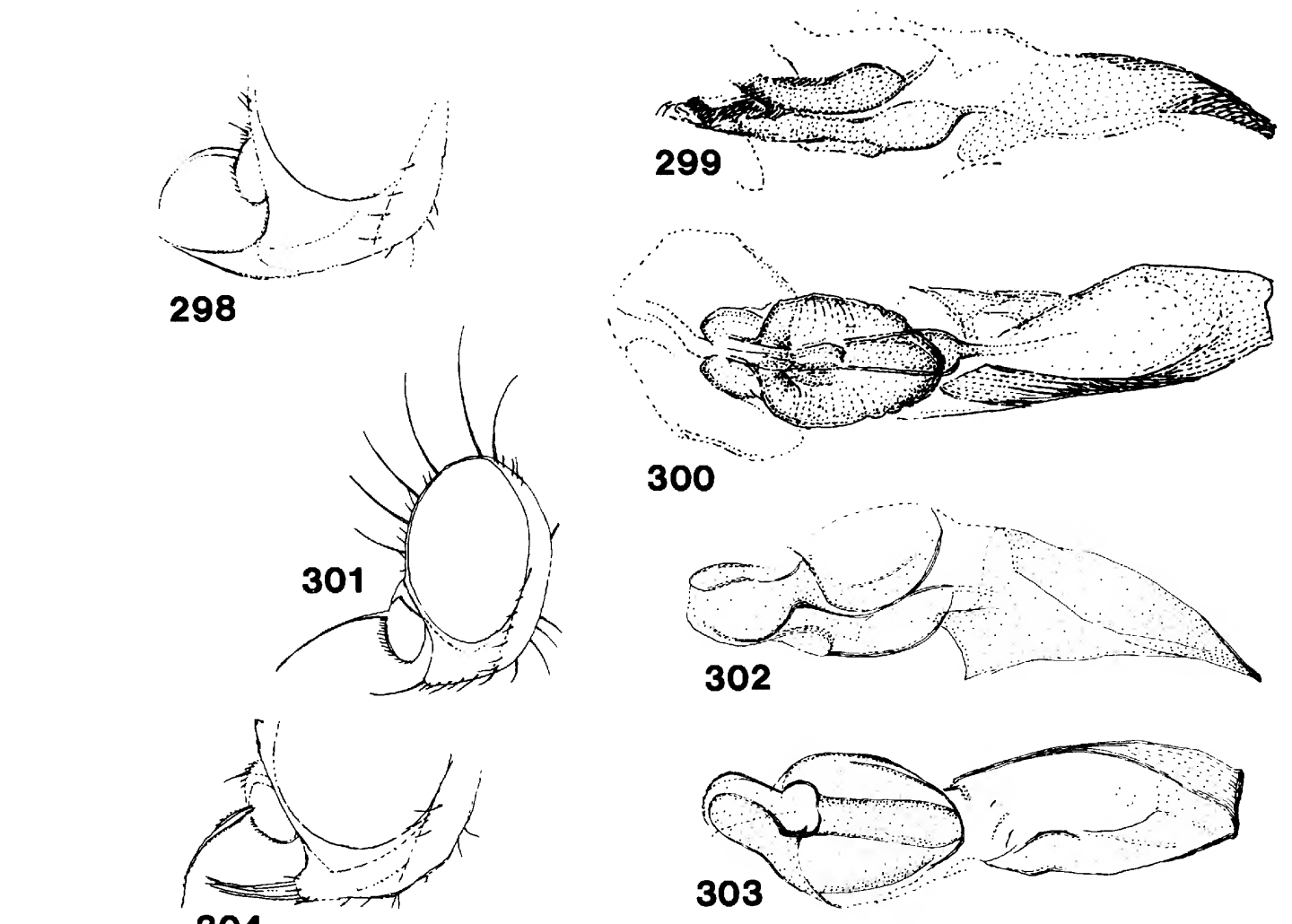

304
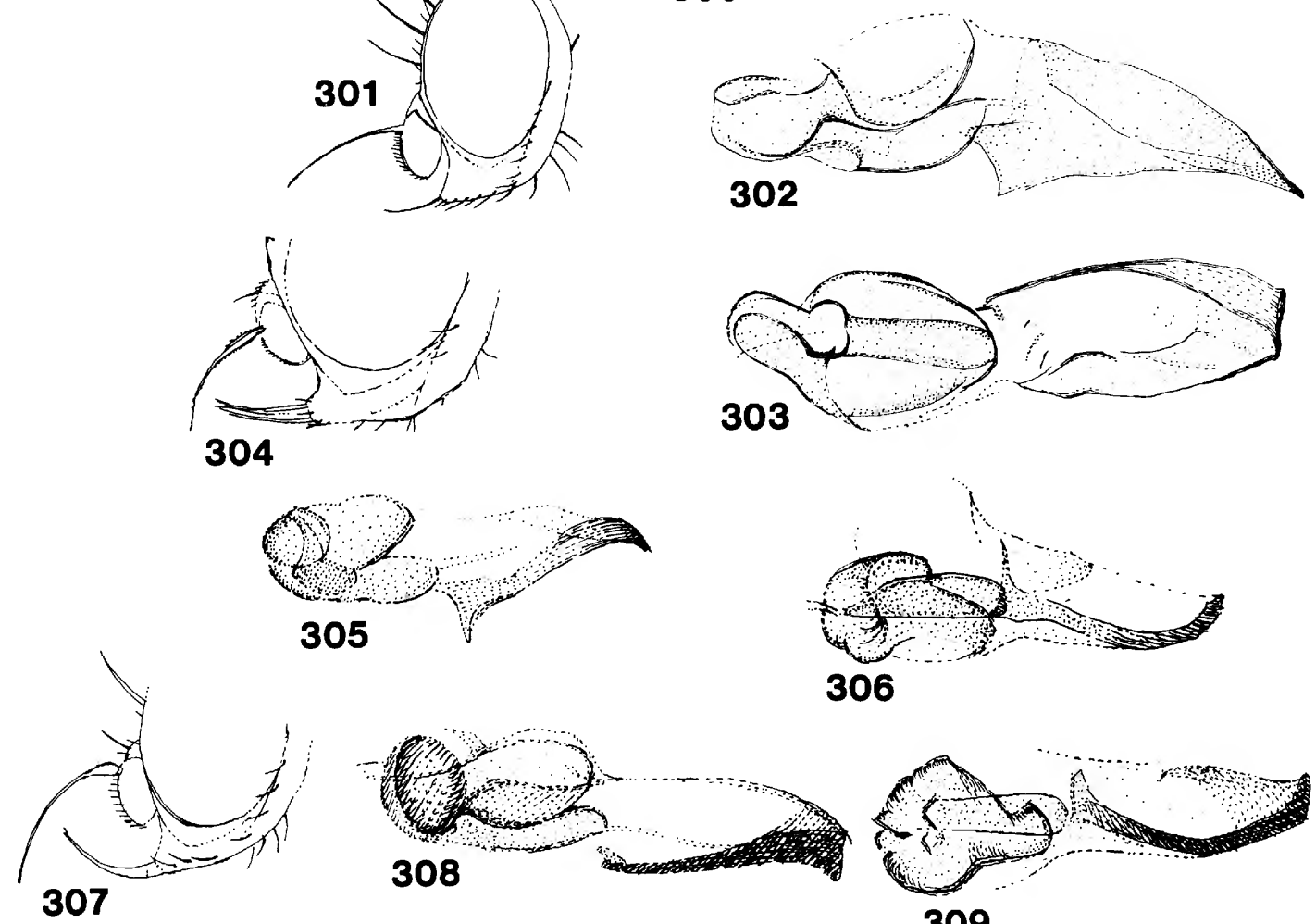

Figures 296-309.--Ophiomyia melica: 296, Aedeagus, side view; 297, same, ventral view; 0 . malitiosa: 298 , Head; 299 , aedeagus, side view; 300 , same, ventral view; $\underline{0}$. 1abiatarum: 301, Head; 302 , aedeagus, side view; 303 , same, ventral view; 0. lassa: 304 , Head; 305, aedeagus, side view; $30 \overline{6}$, same, ventral view; 0 . parva: 307 , Head; 308 , aedeagus, side view; 309 , same, ventral view. 

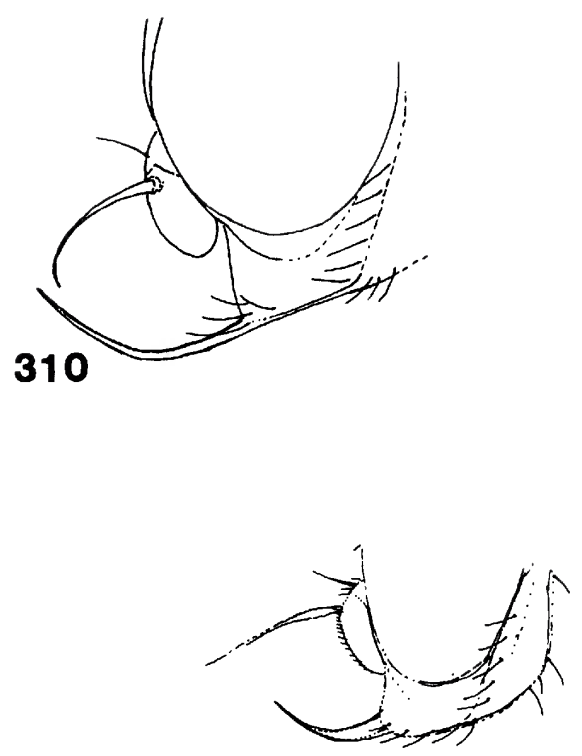

313
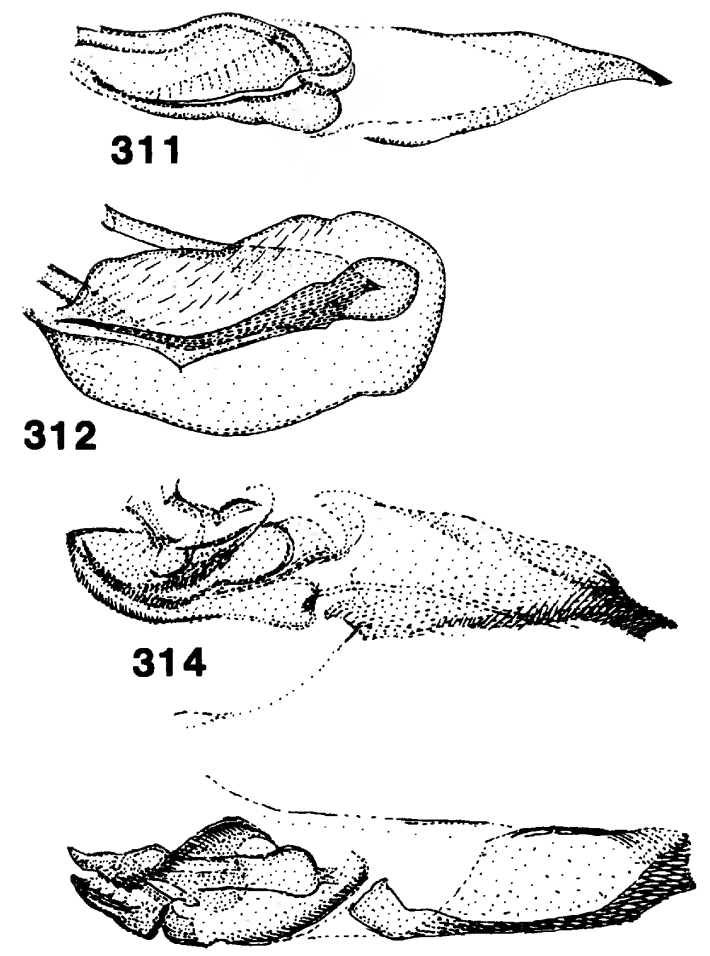

315

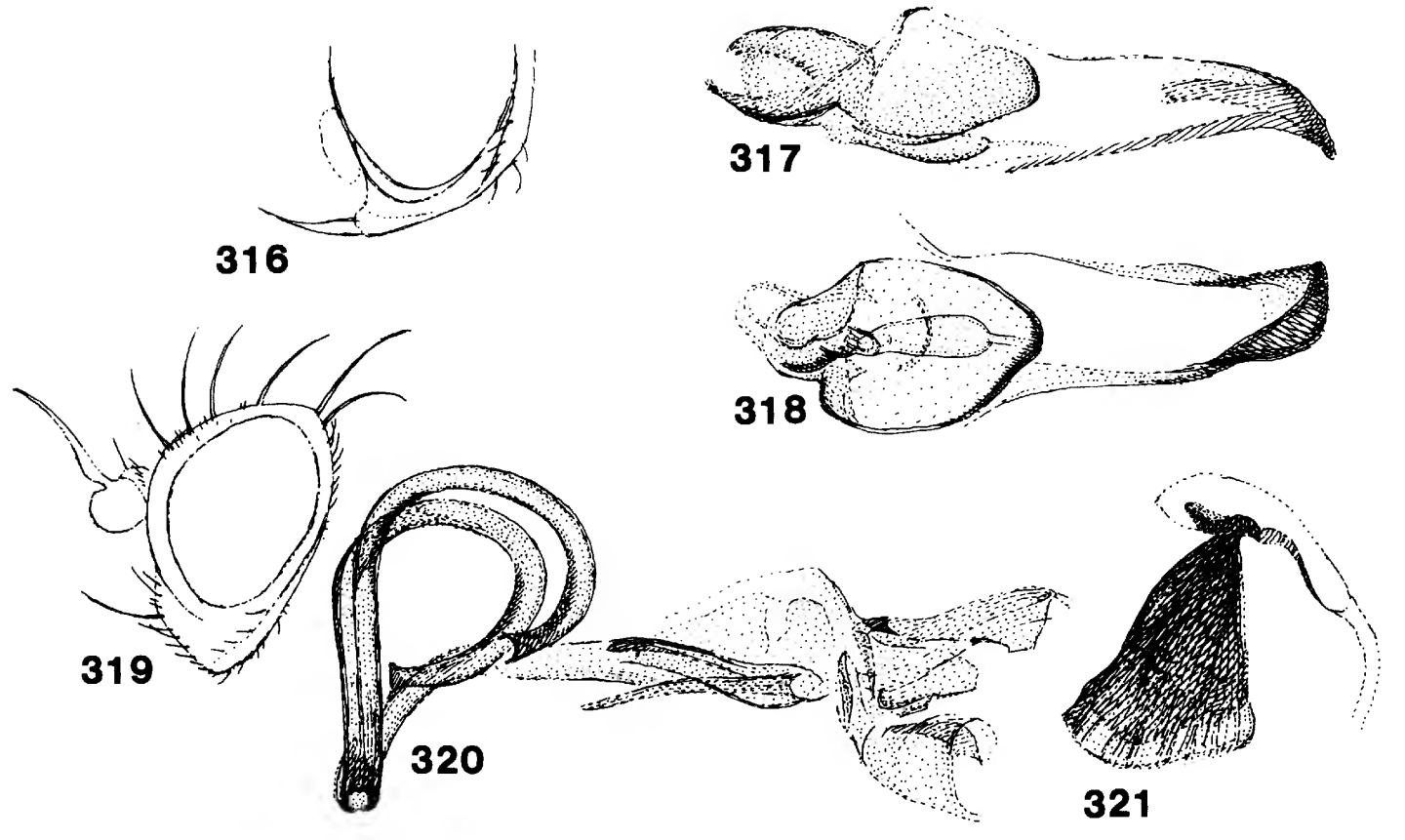

Figures 310-321.--Ophiomyia lantanae: 310 , Head; 311 , aedeagus, side view; 312 , distiphallus, ventral view; 0 . levata: 313 , Head; 314 , aedeagus, side view; $315 \overline{\text {, same }}$, ventral view; 0 . arizonensis: 316 , Head; 317 , aedeagus, side view; 318 , same, ventral view. Japanagromyza rutiliceps: 319 , Head (California); 320, aedeagus, side view (Utah); 321 , sperm pump (Idaho). 

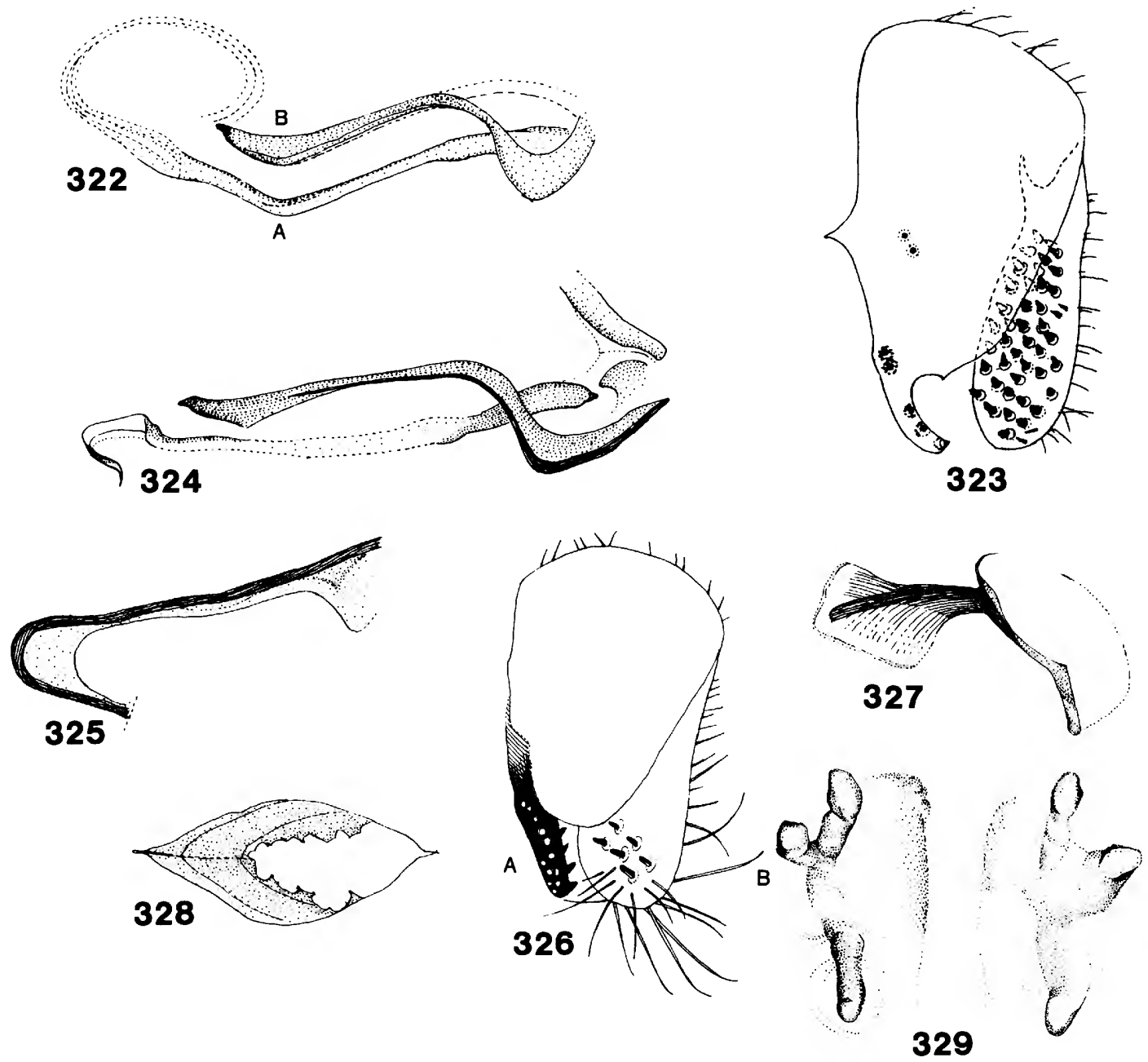

Figures 322-329.--Japanagromyza perpetua: 322 ,

Aedeagus, side view (A) with hypandrium (B) ; 323 ,

epandrium with surstylus and cercus; J. desmodivora:

324, Aedeagus, side view; 325, hypandrium; 326,

epandrium with surstylus (A) and cercus (B); 327 ,

sperm pump; 328 , leaf mine on Desmodium; 329 ,

posterior spiracles of puparium. 

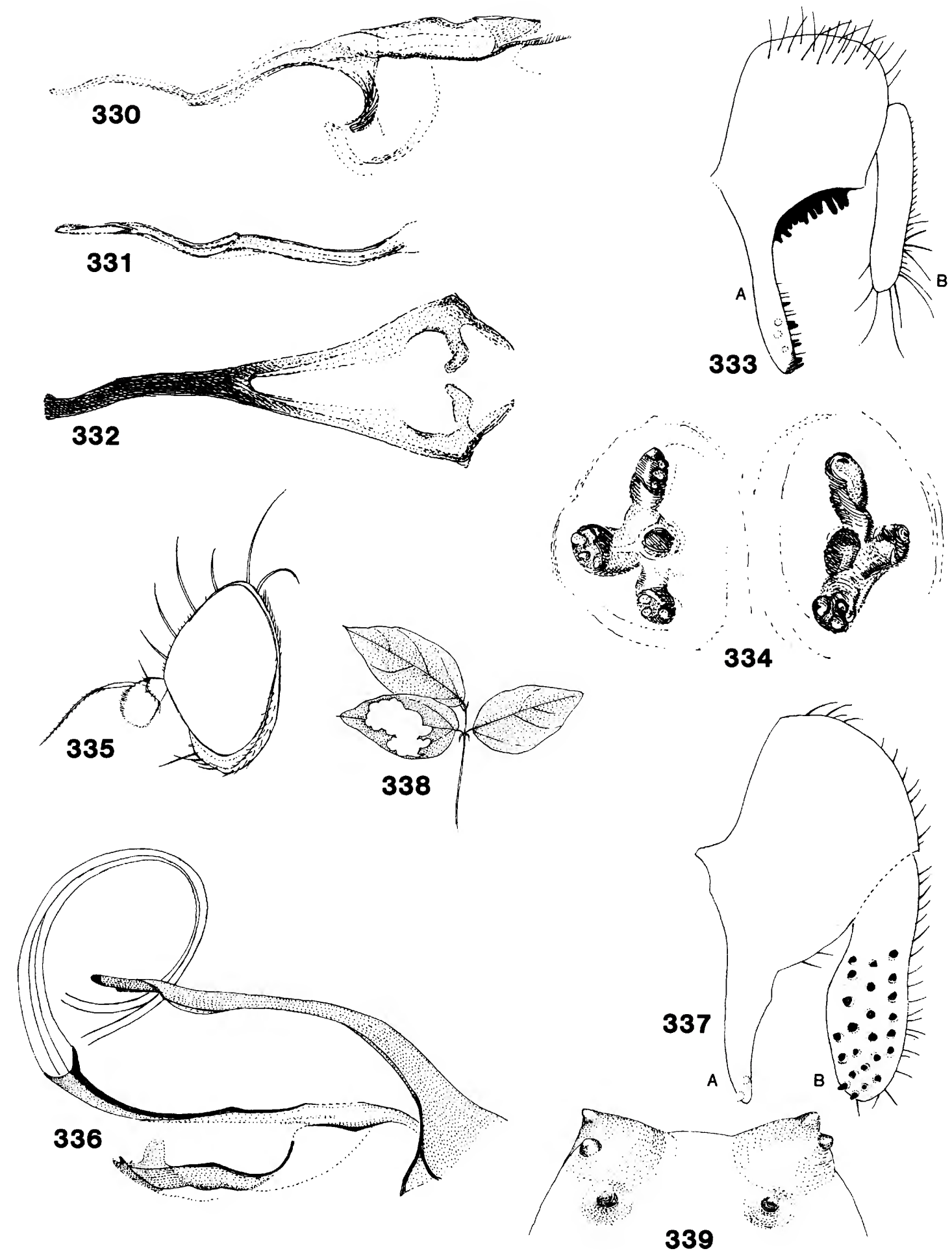

Figures 330-339.--Japanagromyza viridula: 330 ,

Aedeagus, side view; 331, distiphallus, side view

(ex Quercus rubra, Maine); 332, hypandrium; 333,

epandrium, with surstylus (A) and cercus (B); 334 ,

posterior spiracles of puparium (ex Castanea

mollissima, Pennsylvania); J. inaequalis: 335 ,

Head; 336, aedeagus, side view; 337, epandrium,

with surstylus (A) and cercus (B); 338 , leaf mine

on Vigna; 339, posterior spiractes of puparium. 


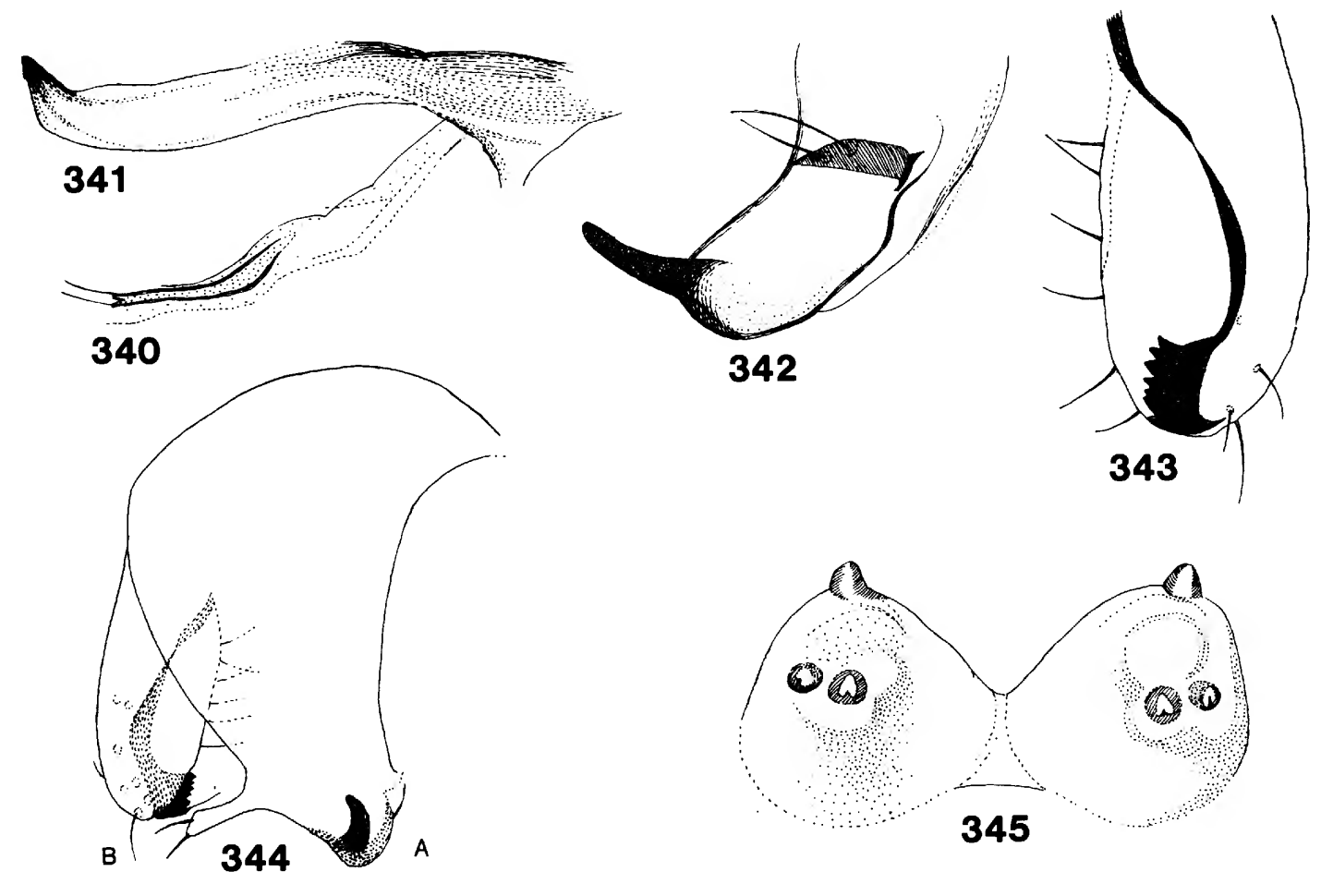

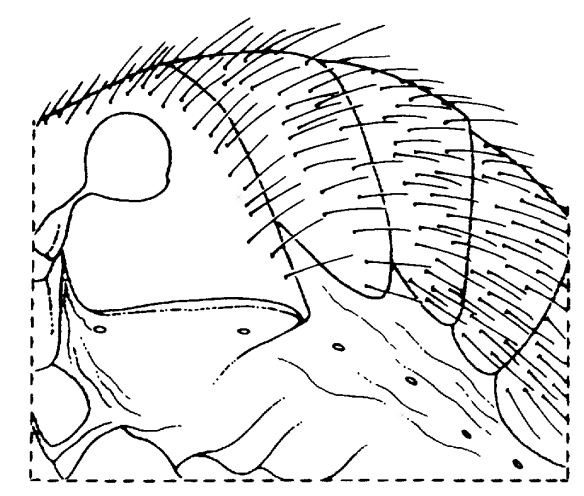

346
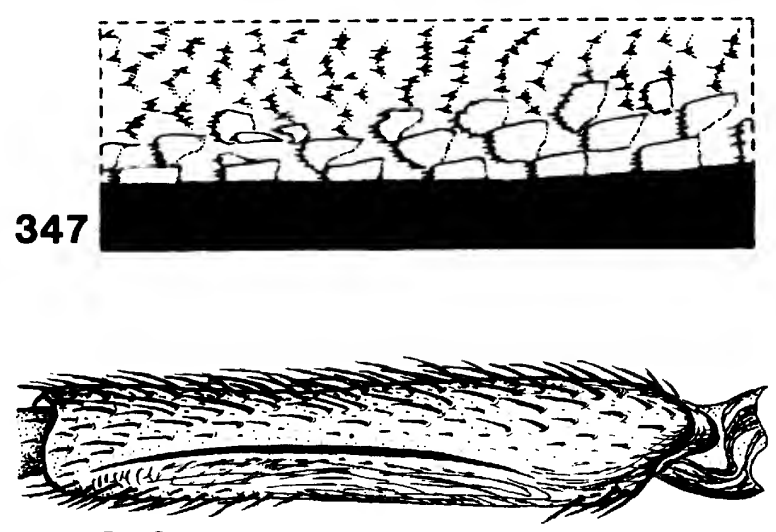

348

Figures 340-348.--Japanagromyza aequalis: 340 , Aedeagus, side view; 341, hypandrium; 342, surstylus; 343 , cercus; 344 , epandrium with surstylus $(\underline{A})$ and cercus $(\underline{B}) ; 345$, posterior spiracles of puparium. Agromyza spp., stridulating mechanism: 346 , A. nigripes female, "file" below tergites $1-3 ; 34 \overline{7}$, A. nigrescens file at higher magnification; A. nigripes: 348 , Hindfemur;

(346-348, Europe, after Tschirnhaus, 1971; 346 and 347, drawn from photographic originals). 


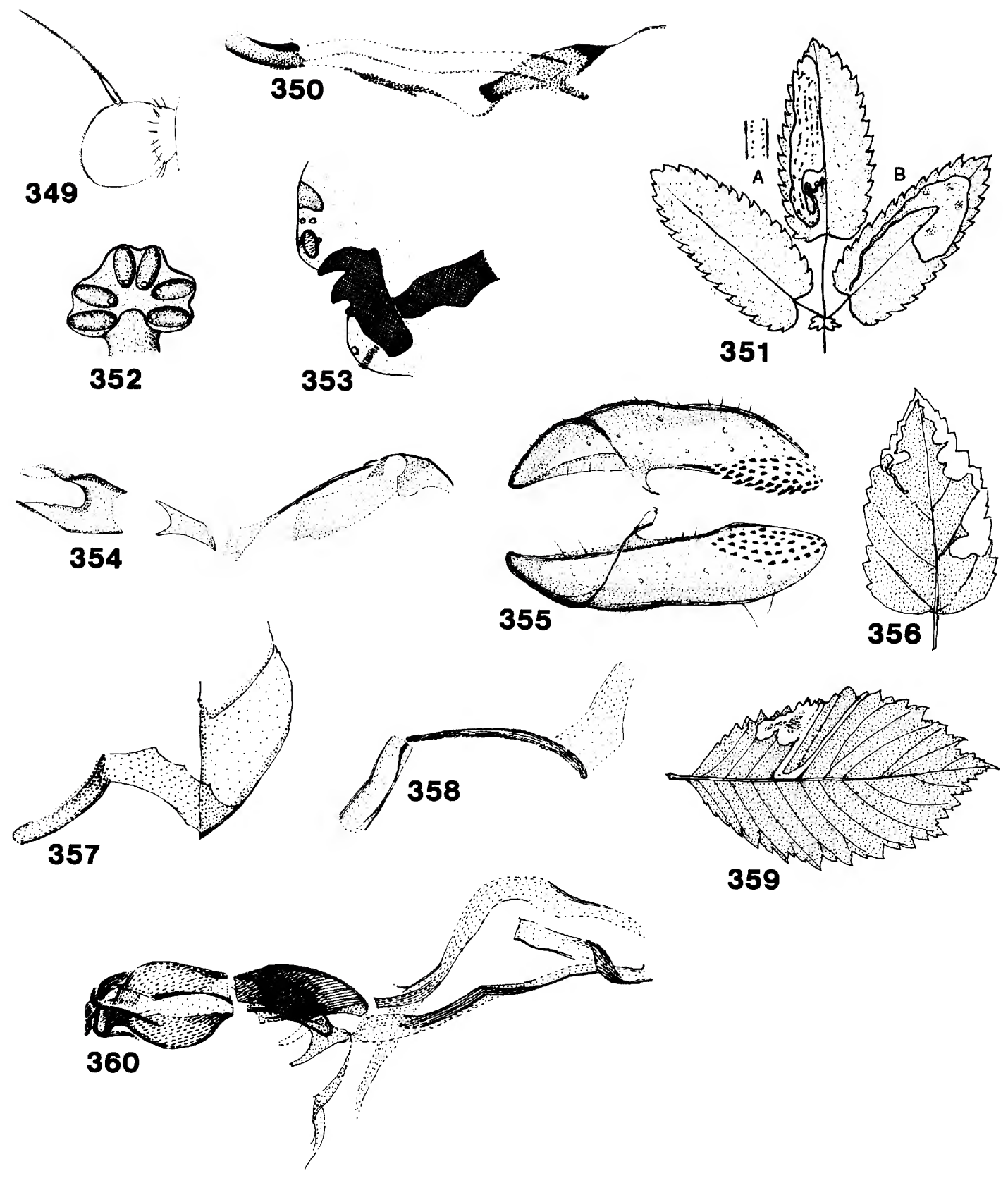

Figures 349-360.--Agromyza sulfuriceps: 349 , Third antennal segment; 350 , aedeagus, side view; 351 , leaf mines on Sanguisorba (Europe) (A) (for B, see fig. 448); 352, posterior spiracles of puparium; 353, larval mouth hooks; A. varifrons: 354, Aedeagus, side view; 355 , cerci; 356 , leaf mine on Celtis laevigata (Hialeah, Fla.); A. aristata: 357 , Aedeagus, side view (paratype); $35 \overline{8}$, same, side view (paratype of A. ulmi); 359, leaf mine on U1mus americana (Edmontoon, Alberta, KAS); $A$. diversa: 360, Aedeagus, side view. 

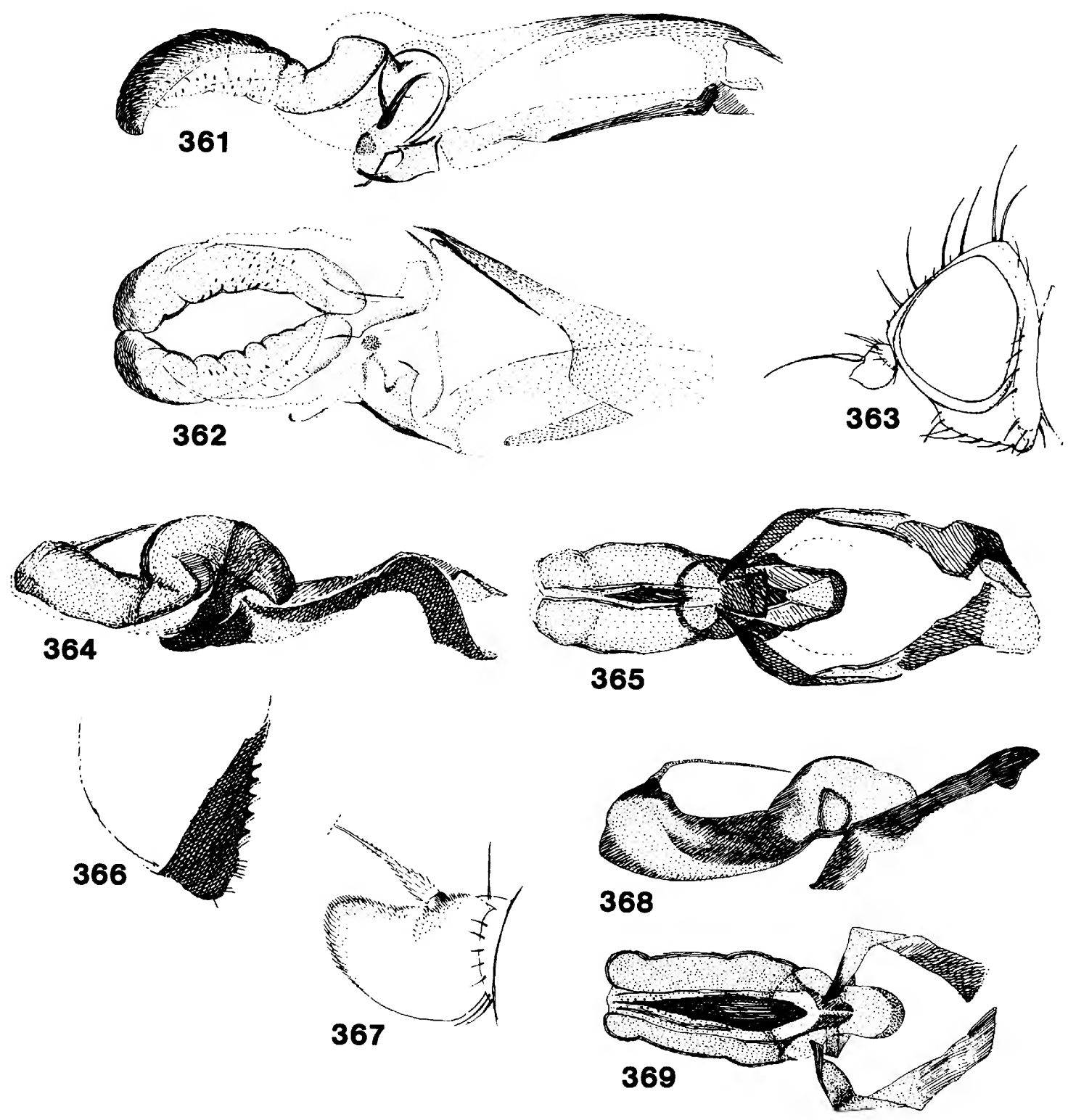

Figures 361-369.--Agromyza canadensis: 361, Aedeagus, side view; 362 , same, ventral view; A. utahensis: 363, Head; 364 , aedeagus, side view; $36 \overline{5}$, same, ventral view; 366 , surstylus; A. ambigua: 367 ,

Third antennal segment; 368 , aedeagus, side view; 369 , same, ventral view. 

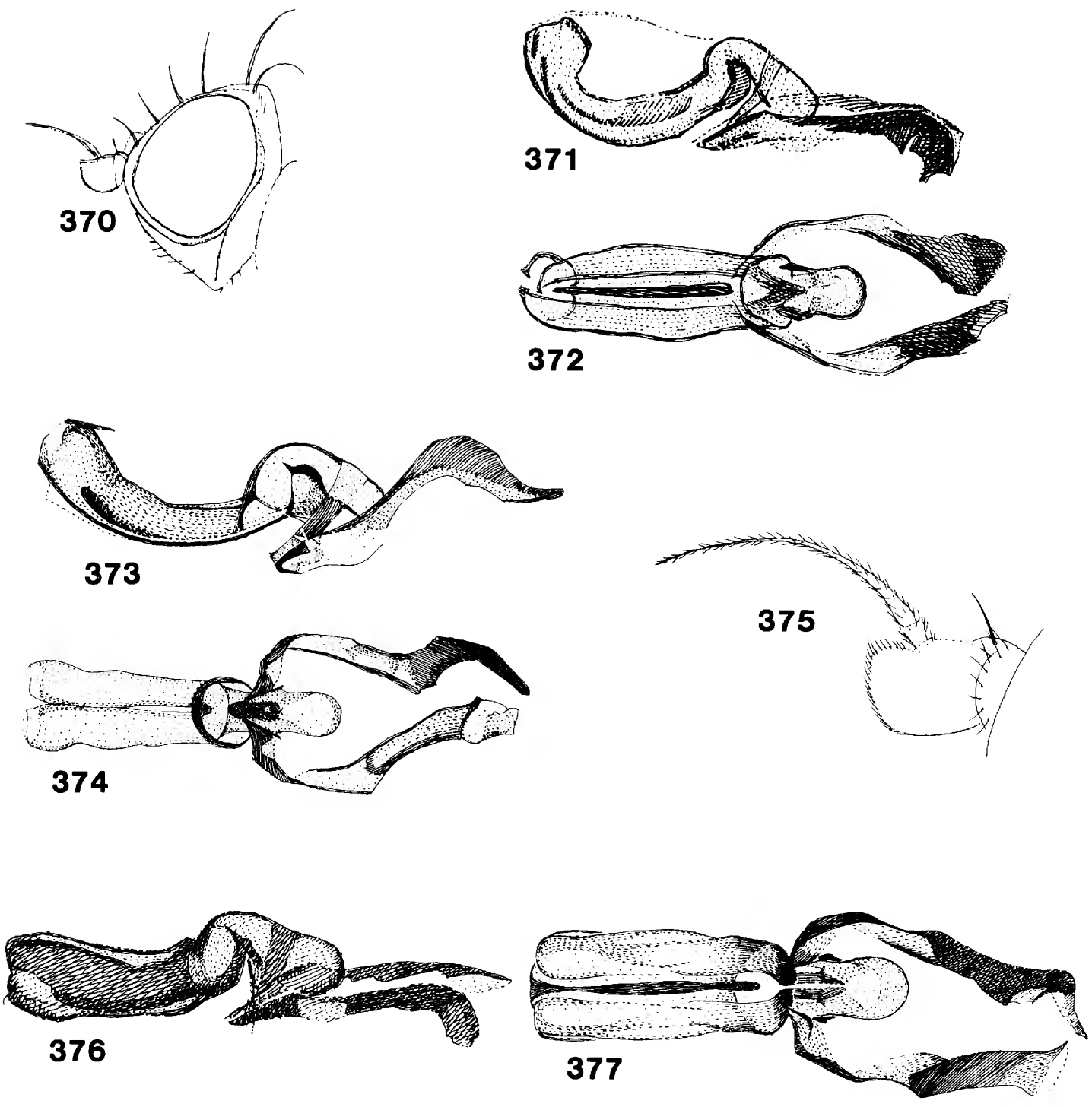

Figures 370-377.--Agromyza tularensis: 370 , Head; 371 , aedeagus, side view; 372 , same, ventral view; $A$. albertensis: 373 , Aedeagus, side view; 374 , same, ventral view; 375 , third antennal segment; $A$. kincaidi: 376 , Aedeagus, side view; 377 , same, ventral view. 

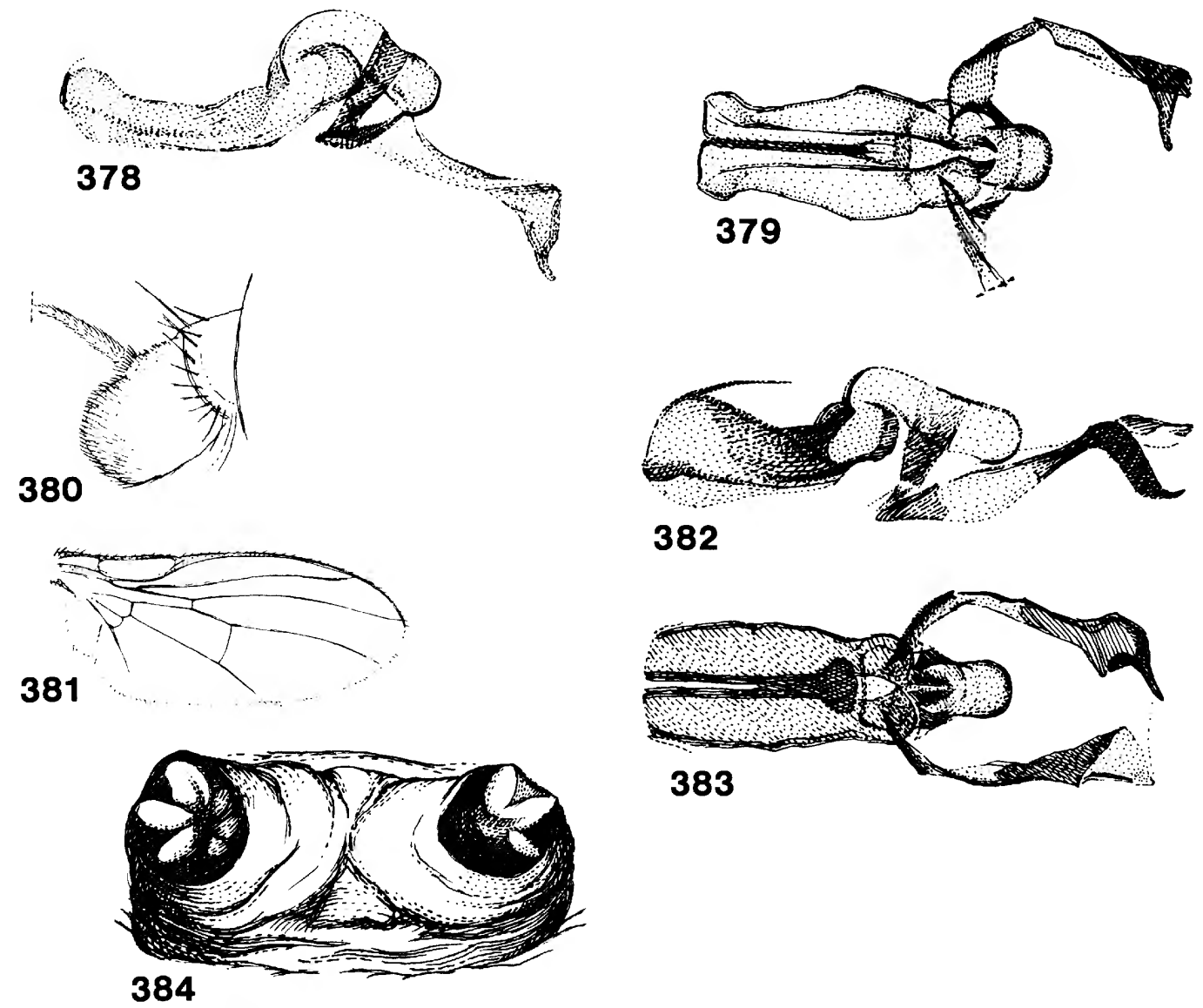

382
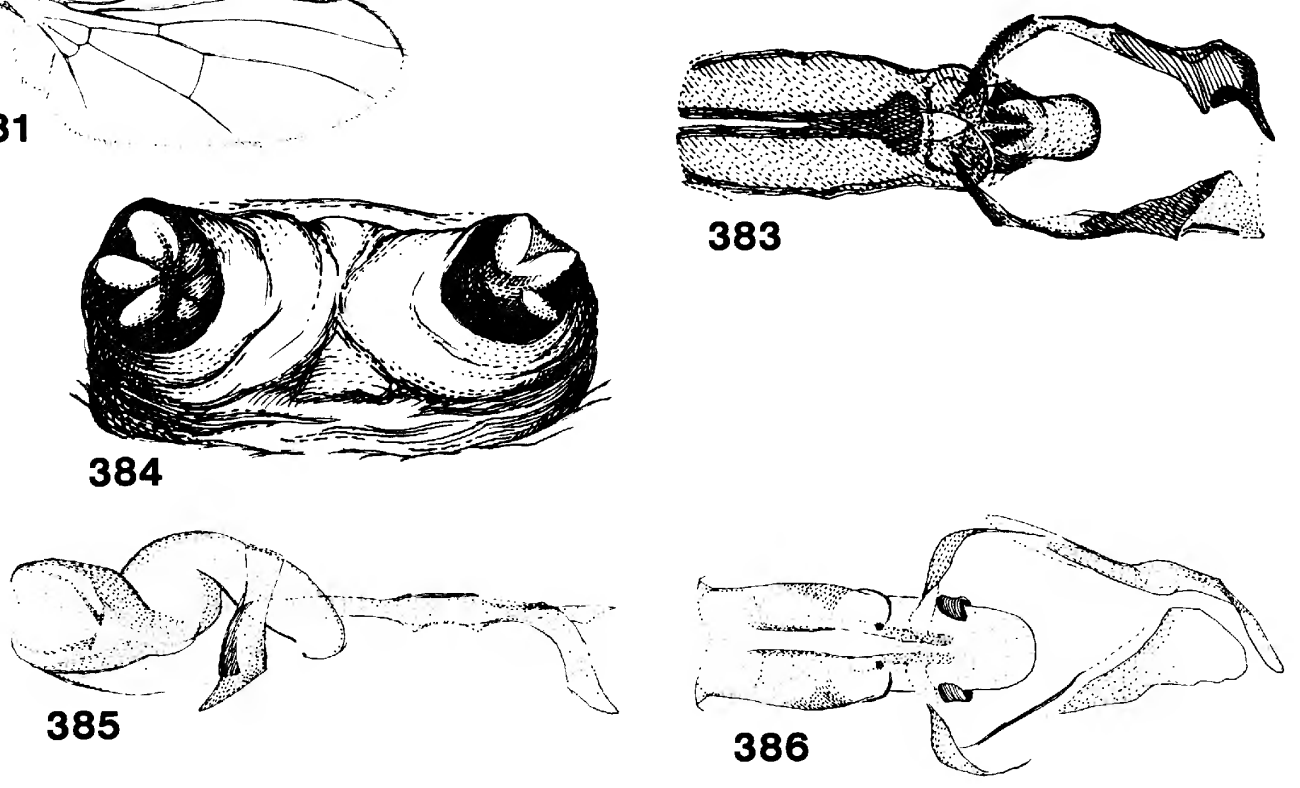

Figures 378-386.--Agromyza schlingerel1a: 378 ,

Aedeagus, side view; 379, same, ventral view; $\underline{A}$.

nigrella: 380, Third antennal segment; 381, wing;

382, aedeagus, side view; 383 , same, ventral view;

384 , posterior spiracles of puparium; (383,

California, Tuolumne Co.; 380-382, 384, Europe); A.

aprilina: 385, Aedeagus, side view; 386, same,

ventral view. 


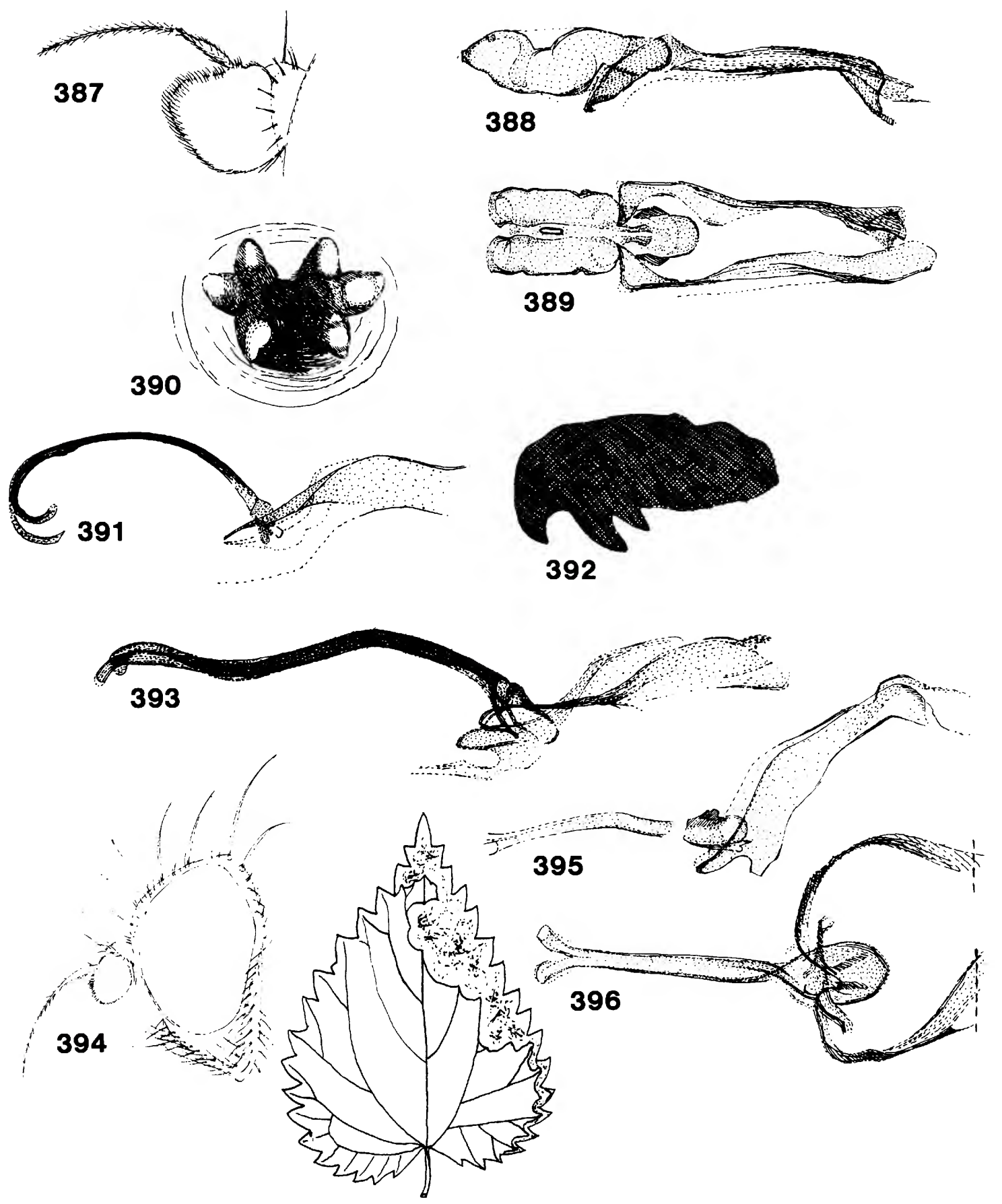

397

Figures 387-397.--Agromyza albipennis: 387, Third antennal segment; 388 , aedeagus, side view; 389 , same, ventral view (Utah); 390, posterior spiracles of puparium; A. ambrosivora: 391, Aedeagus, side view; 392, mouth hooks of larva; A. virginiensis: 393, Aedeagus, side view; A. reptans: 394, Head; 395 , aedeagus, side view; 396, same, ventral view (California); 397, leaf mine on Urtica. 

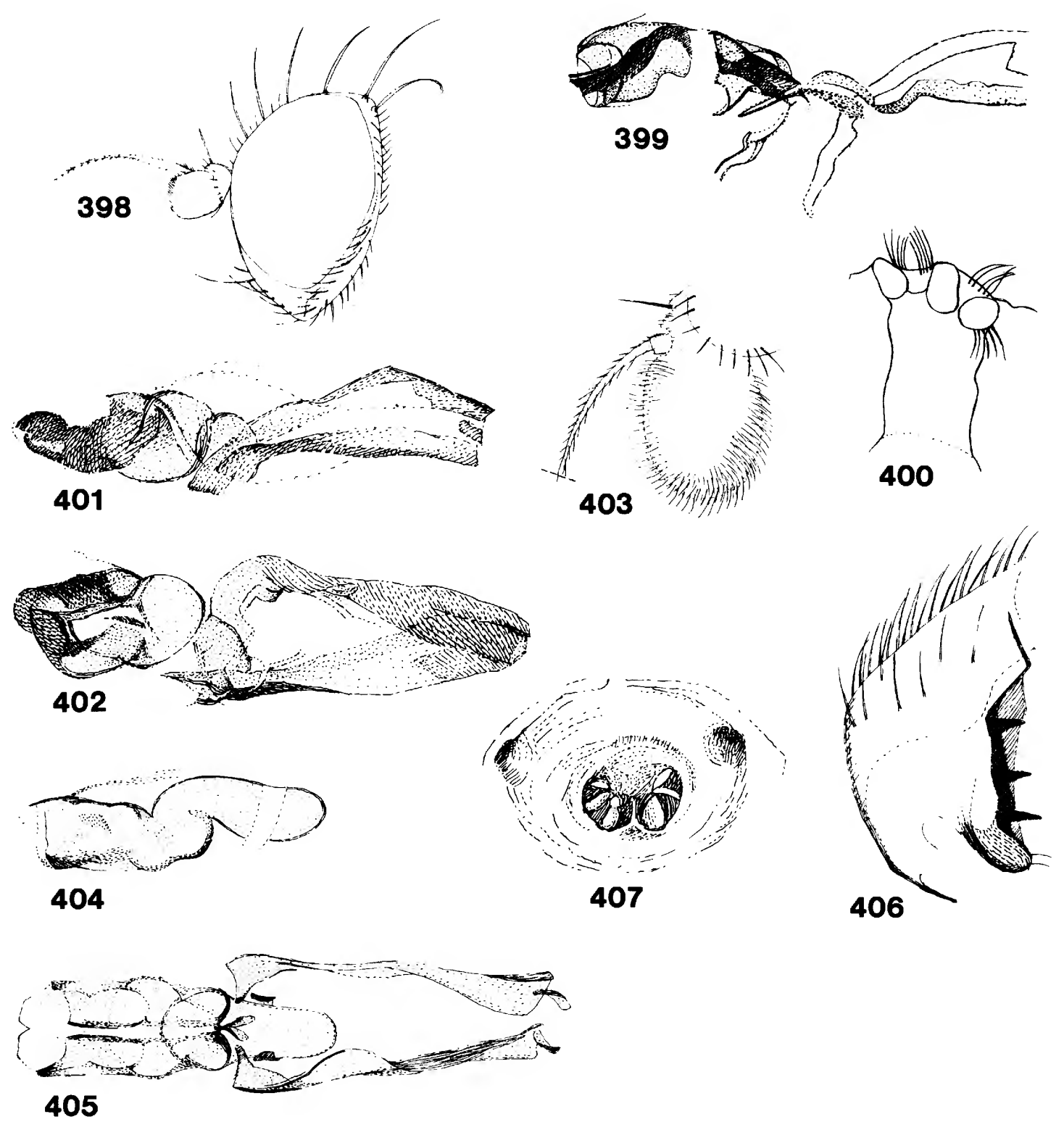

Figures 398-407.--Agromyza pseudoreptans: 398, Head; 399, aedeagus, side view; 400, posterior spiracles of larva; A. hardyi: 401, Aedeagus, side view; 402, same, ventral view; A. tacita: 403, Third antennal segment; 404, distiphal $\overline{l u s, ~ s i d e ~ v i e w ; ~} 405$, aedeagus, ventral view; 406, epandrium; 407, posterior spiracles of puparium (ex grass, Montana). 


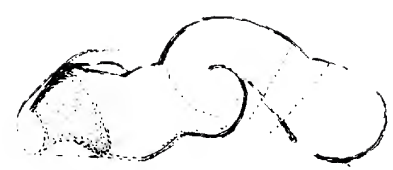

\section{8}

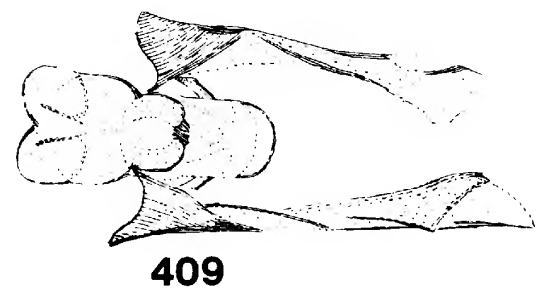

414
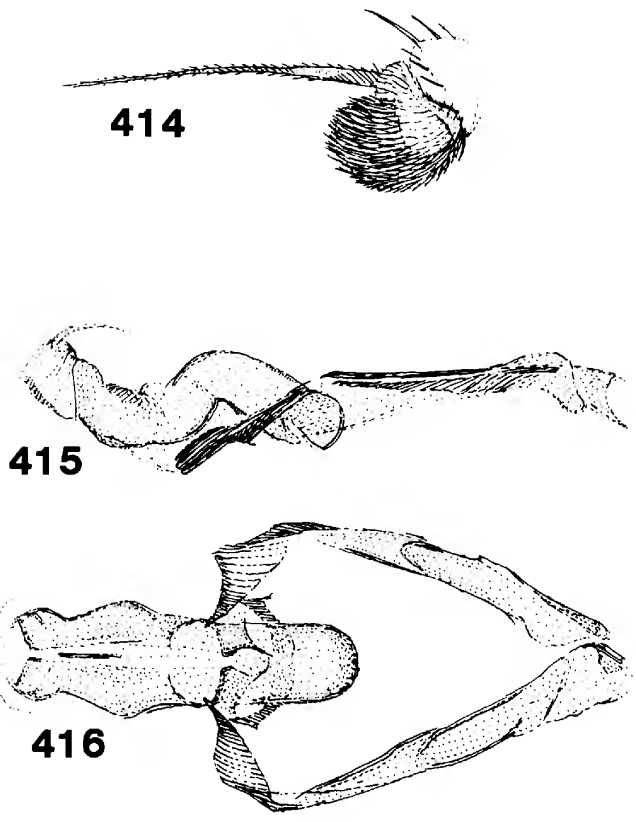
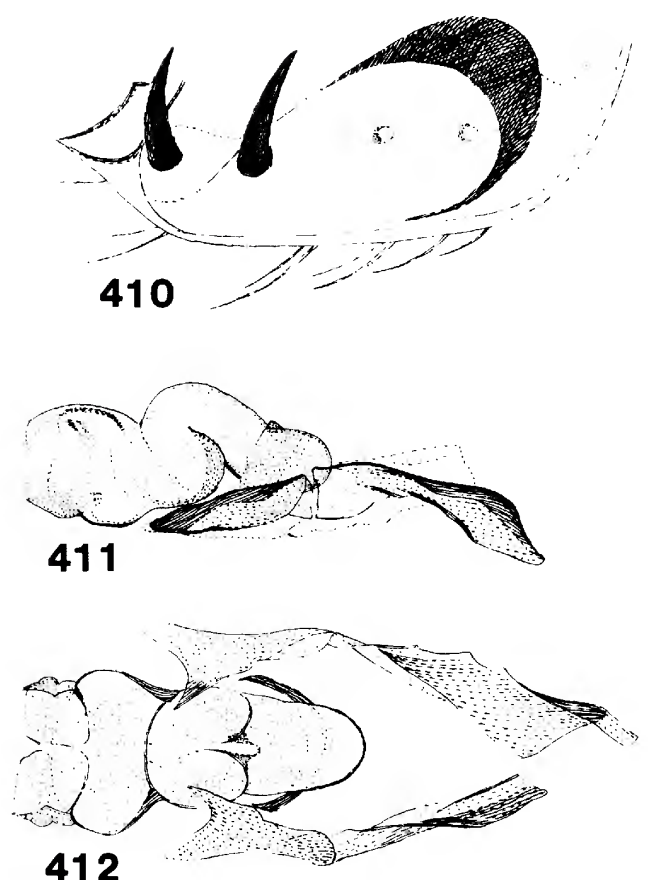

412

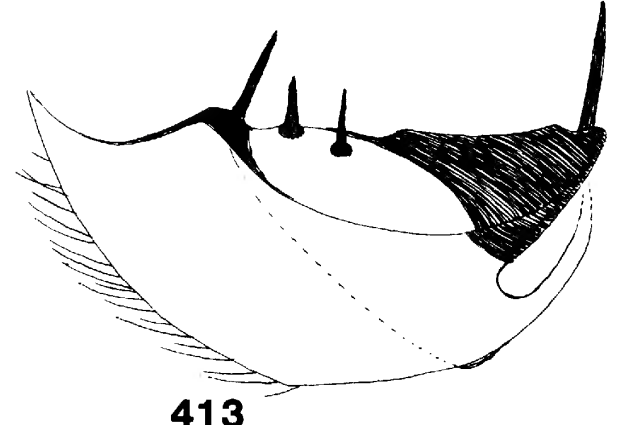

Figures 408-416.--Agromyza bispinata: 408, Distipha1lus, side view; 409 , aedeagus, ventral view; 410 , epandrium; A. hockingi: 411, Aedeagus, side view; 412, same, ventral view; 413, epandrium; A. pagana: 414, Third antennal segment; 415 , aedeagus, side view; 416, same, ventral view. 

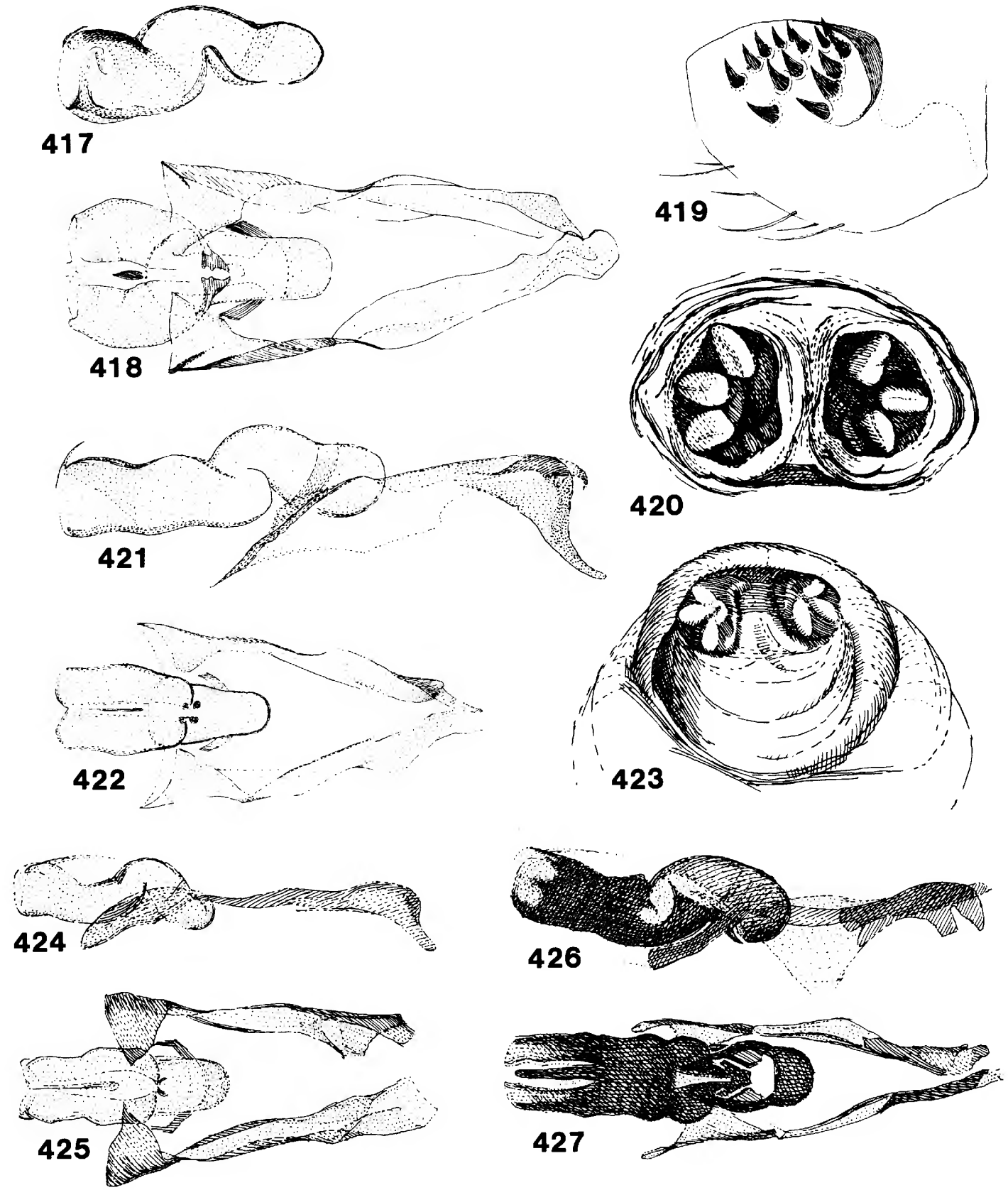

Figures 417-427.--Agromyza parvicornis: 417, Distiphallus, side view; 418, aedeagus, ventral view; 419 , surstylus; 420 , posterior spiracles of puparium; A. proxima: 421, Aedeagus, side view; 422, same, ventral view; 423, posterior spiracles of puparium; A. parca: 424, Aedeagus, side view; 425, same, ventral view; A. parilis: 426, Aedeagus, side view; 427 , same, ventral view. 


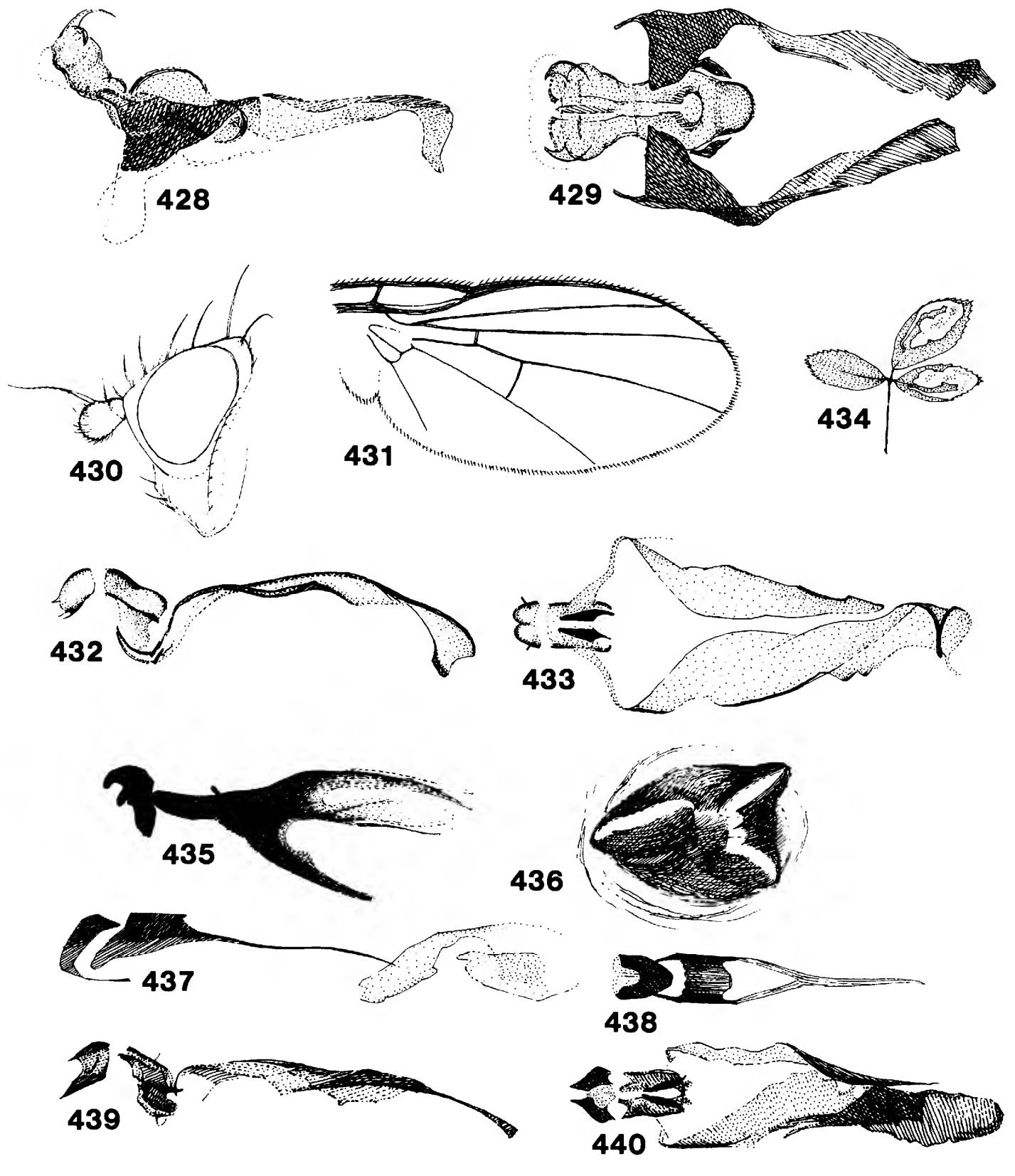

Figures 428-440.--Agromyza pudica: 428, Aedeagus, side view; 429, same, ventral view; A. frontella: 430, Head; 431, wing; 432, aedeagus, side view; 433, same, ventral view; 434, leaf mines on Trifolium; 435, cephalopharyngeal skeleton of larva; 436, posterior spiracles of puparium; (430-436, Europe); A. isolata: 437, Aedeagus, side view; 438, same, ventral view (Canada); A. nevadensis: 439,

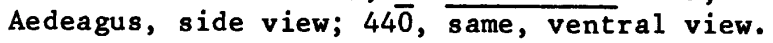



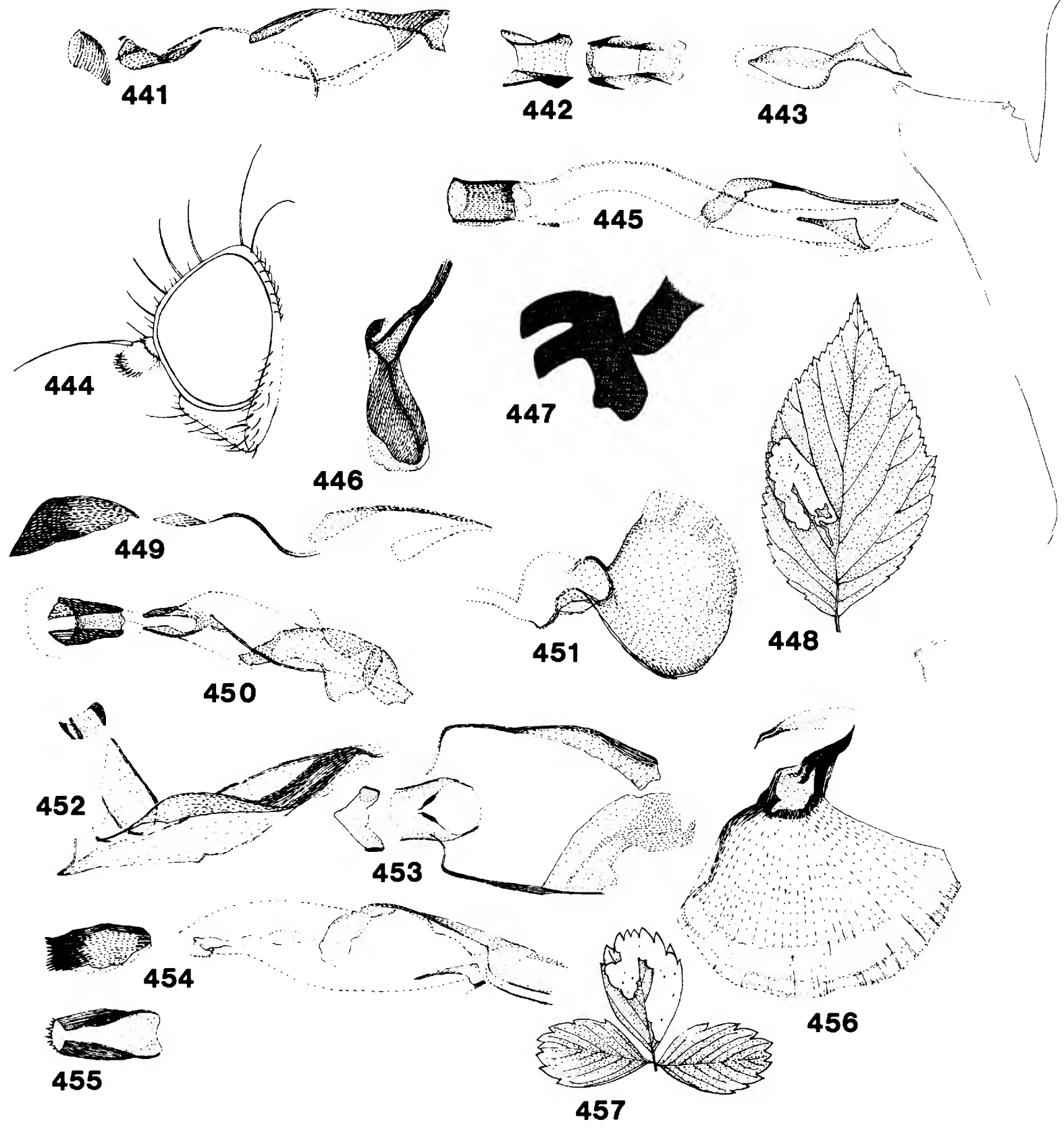

Figures 441-457.--Agromyza vockerothi: 441, Aedeagus, side view (North Carolina); 442, distiphallus, ventral view; 443, sperm pump; (442, 443, Canada); A. potentillae: 444, Head; 445, aedeagus, side view; 446, sperm pump; 447, larval mouth hooks; 448, leaf mine on Rubus (see also leaf mine on Sanguisorba (fig. $351, \underline{A})$ ); (444-448, Europe); A. masculina: 449 , Aedeagus, side view; 450 , same, ventral view; 451, sperm pump; (449, Alberta; 450 , 451, Utah); A. leechi: 452, Aedeagus, side view; 453, same, ventral view; A. fragariae: 454, Aedeagus, side view; 455 , distiphallus, ventral view; 456 , sperm pump; 457 , leaf mine on Fragaria. 

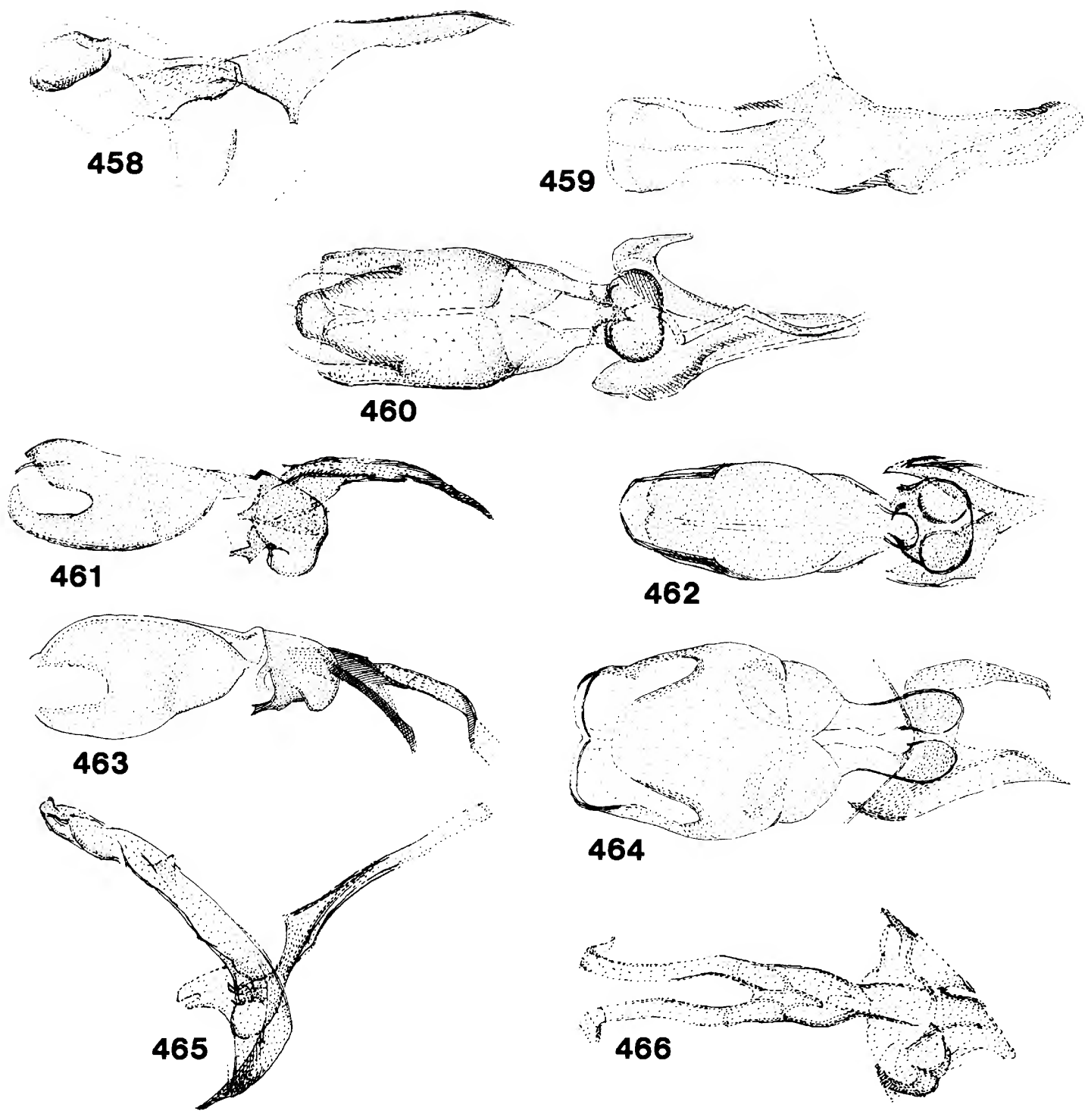

Figures 458-466.--Phytobia pallida: 458, Aedeagus,

side view; 459, same, ventral view; $P$. indecora:

460 , Aedeagus, ventral view (paratype, 0.8 scale of

P. confessa, fig. 464); P. vanduzee i: 461 ,

Aedeagus, side view; 462 , same, ventral view; $P$.

confessa: 463, Aedeagus, side view; 464, same,

ventral view; $\underline{P}$. powelli: 465, Aedeagus, side view;

466, same, ventral view. 

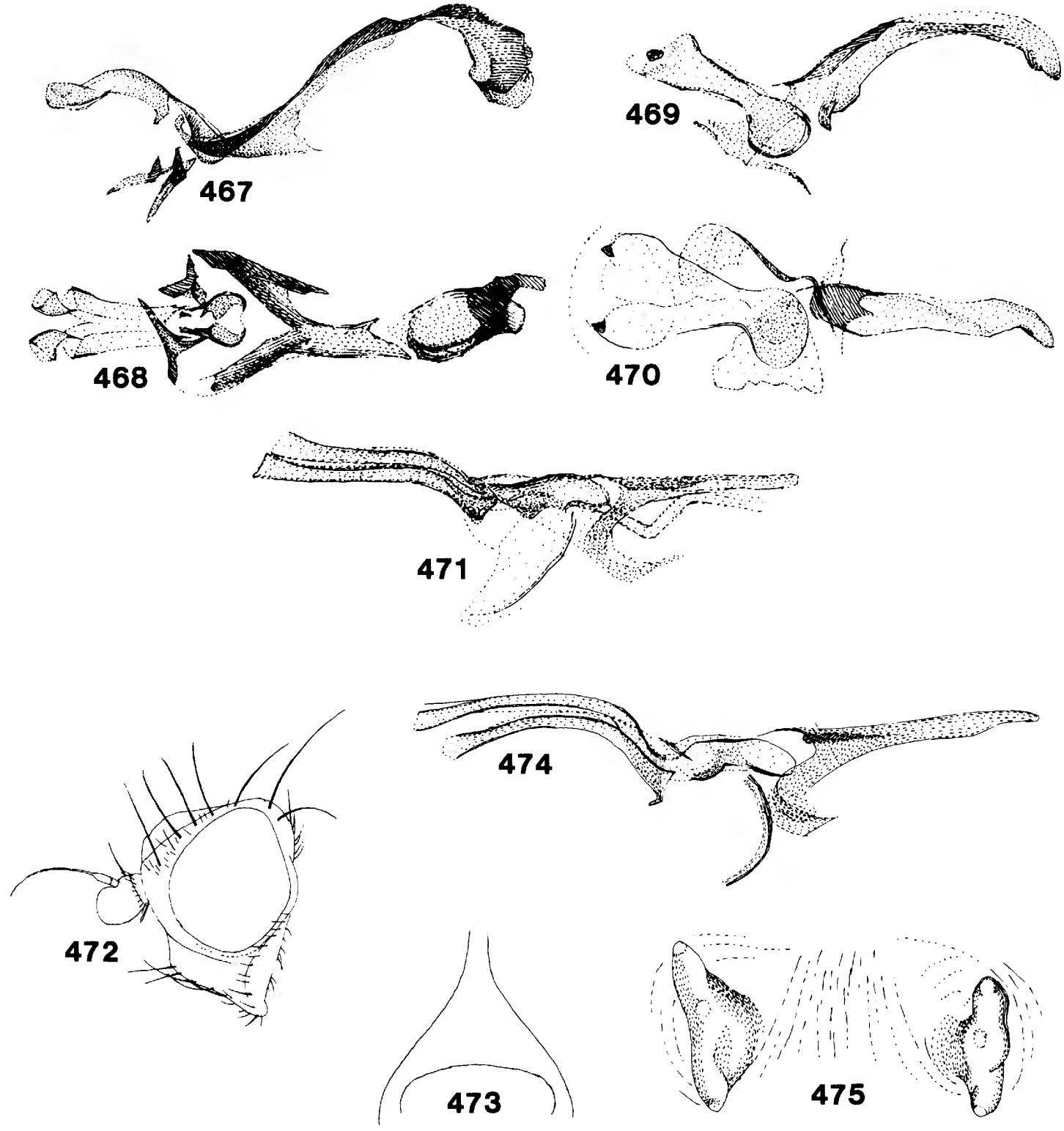

Figures 467-475.--Phytobia prunivora: 467, Aedeagus, side view; 468 , same, ventral view; $\underline{P}$.

flavohumeralis: 469, Aedeagus, side view; 470 , same, ventral view; $\underline{P}$. pruinosa: 471 , Aedeagus, side view (holotype); P. betulivora: 472, Head; 473, epistoma; 474, aedeagus, side view; 475 , posterior spiracles of puparium (ex Betula nigra, District of Columbia). 


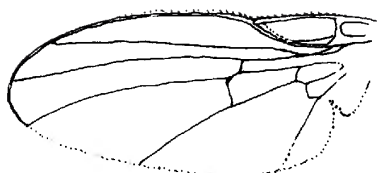

476
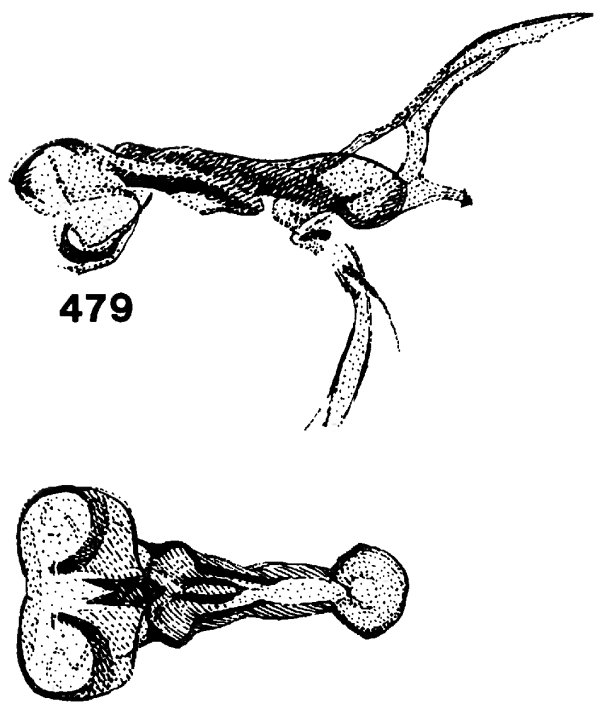

480
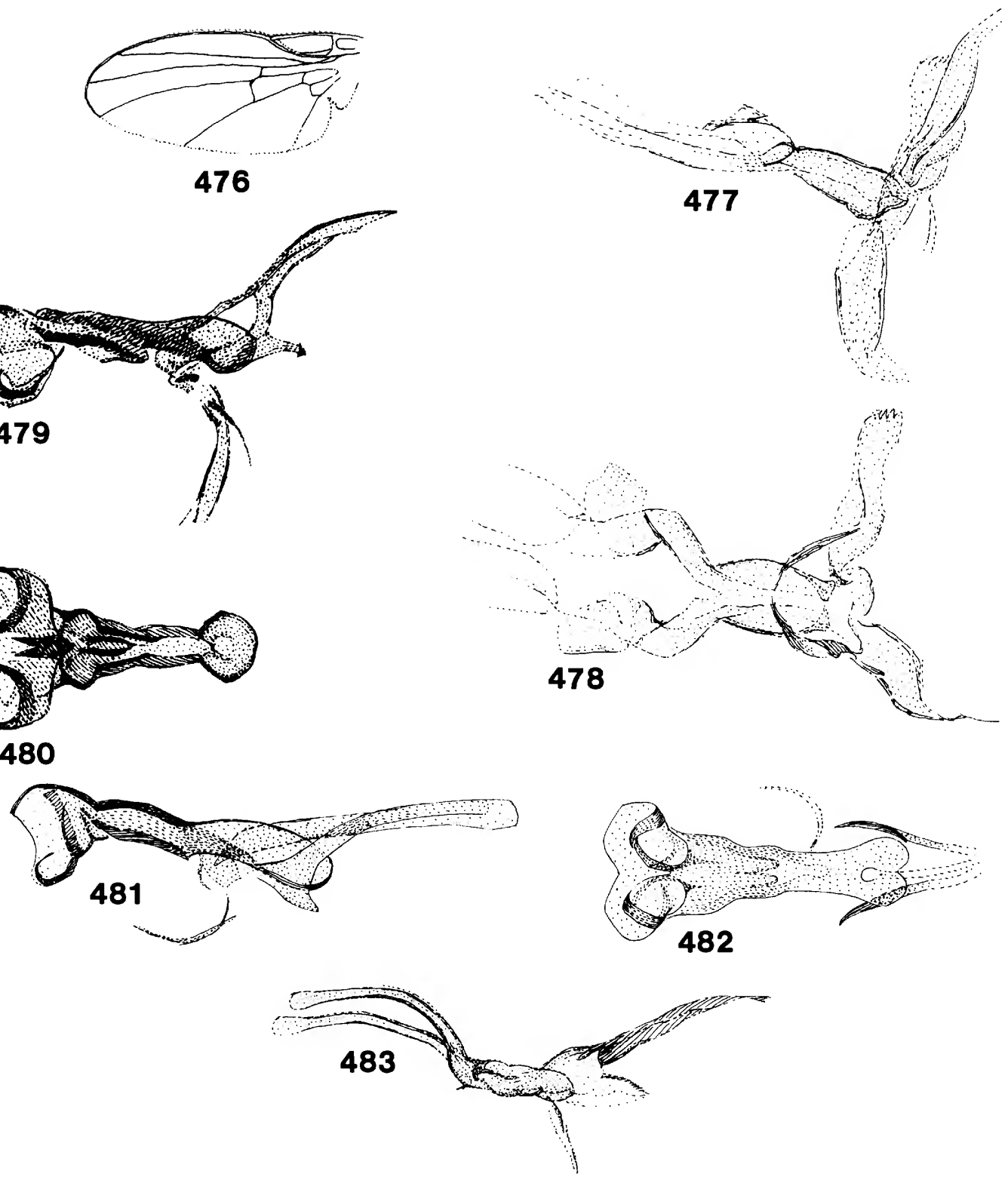

Figures 476-483.--Phytobia waltoni: 476, Wing; 477, aedeagus, side view; 478 , same, ventral view (North Carolina); P. californica: 479, Aedeagus, side

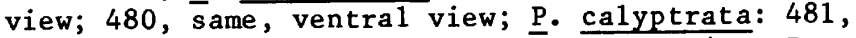
Aedeagus, side view; 482 , same, ventral view; $\underline{P}$. sp. $(\underline{\operatorname{Salix}}): 483$, Aedeagus, side view. 

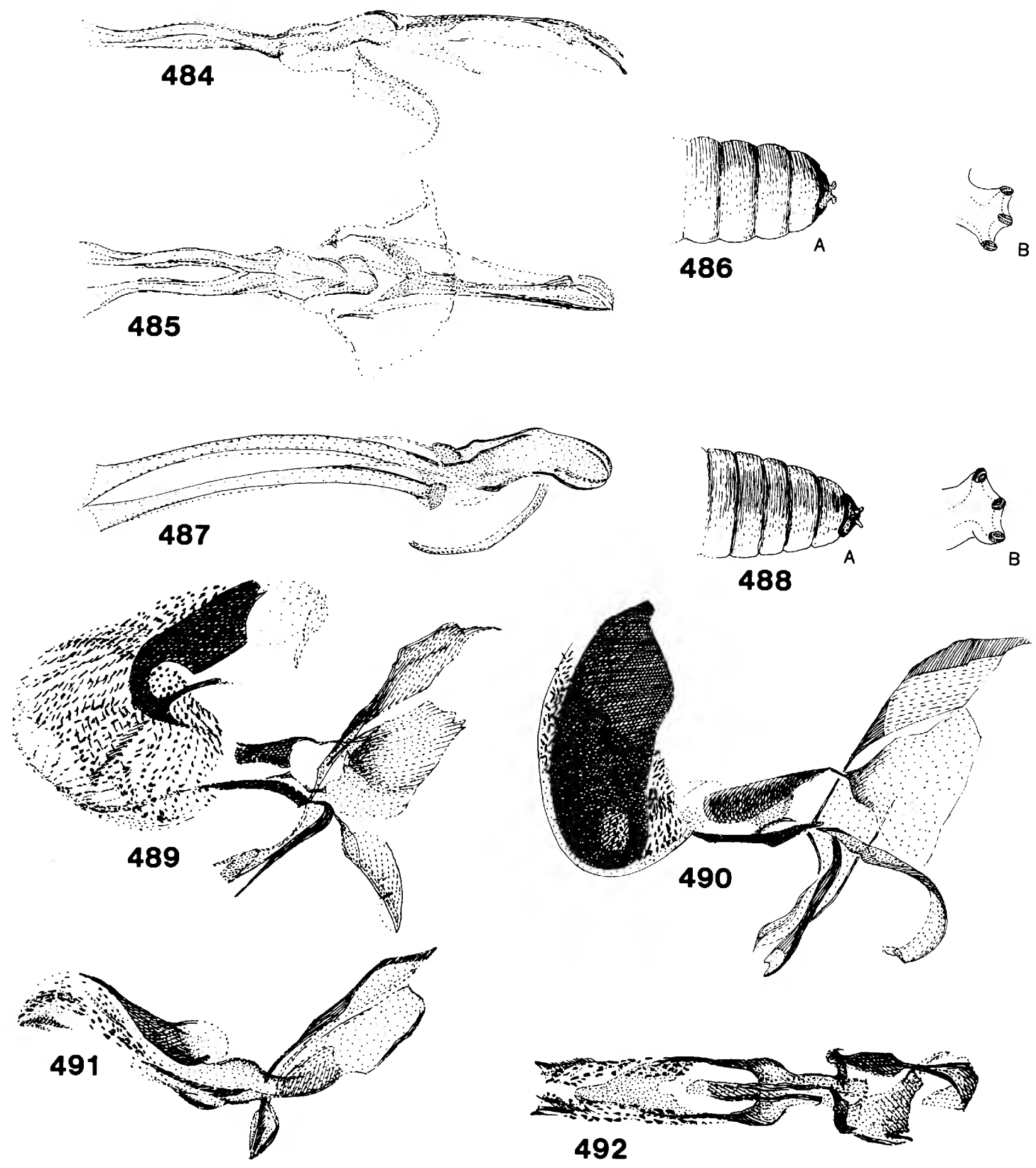

Figures 484-492.--Phytobia setosa: 484, Aedeagus, side view; 485, same, ventral view (holotype); 486, posterior spiracles (A, B) of puparium (after Greene, 1914, as Agromyza aceris); P. amelanchieris: 487, Aedeagus, side view; 488, posterior spiracles (A, B) of puparium (after Greene, 1914). Amauromyza (Cephalomyza) abnormalis: 489, Aedeagus, side view (California); 490, same, side view (Quebec); A. (c.) insularis: 491, Aedeagus, side view; 492, same, ventral view. 

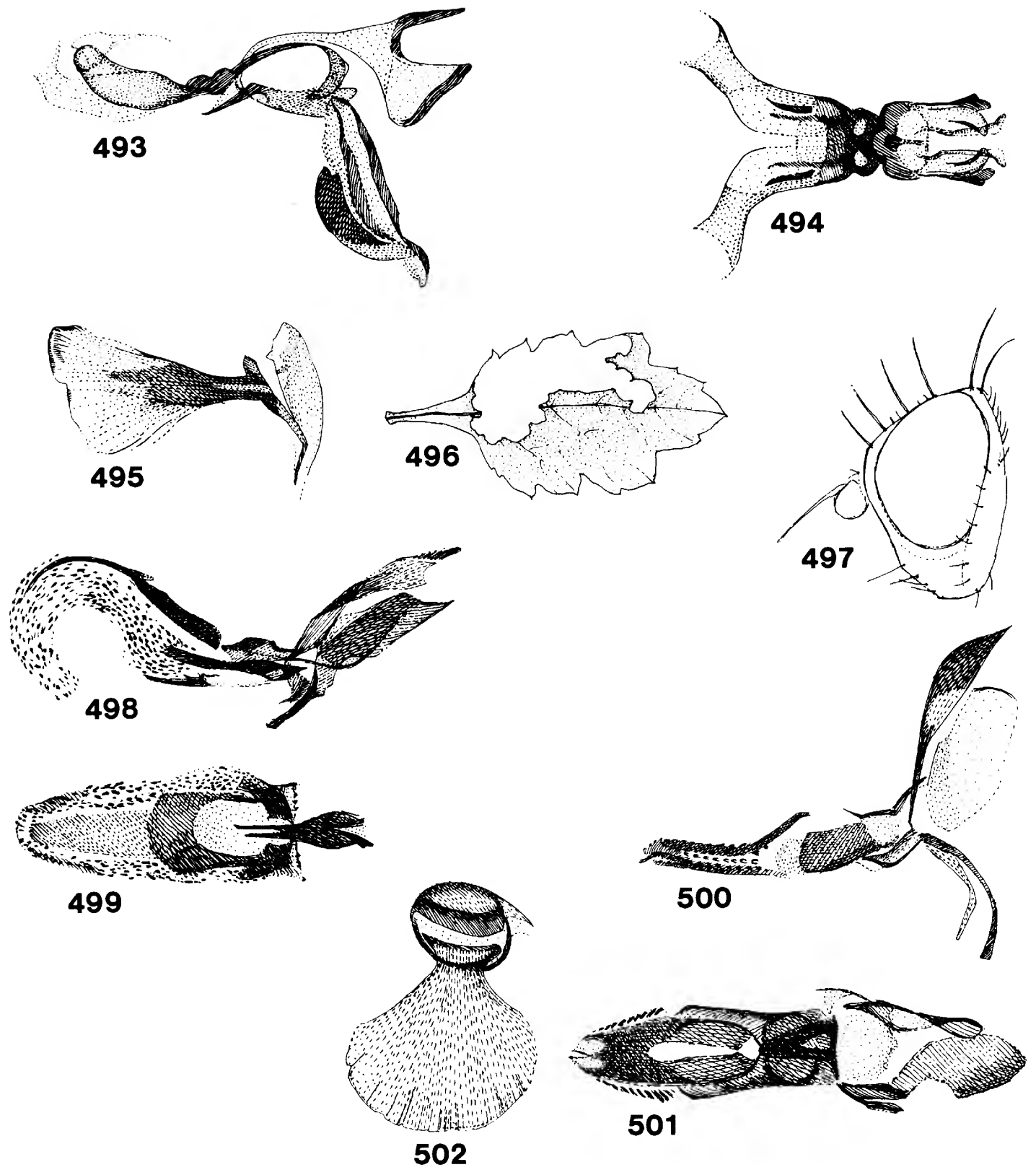

Figures 493-502.--Amauromyza (Annimyzella) maculosa:

493, Aedeagus, side view; 494, same, ventral view; 495, sperm pump; 496, leaf mine on Chrysanthemum. A. (Cephalomyza) lucens: 497 , Head; A. (C..)

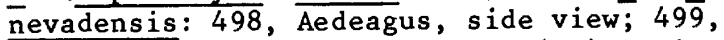
distipha11us, ventral view; $A$. ( . . ) subinfumata: 500, Aedeagus, side view; 501, same, ventral view; 502, sperm pump. 


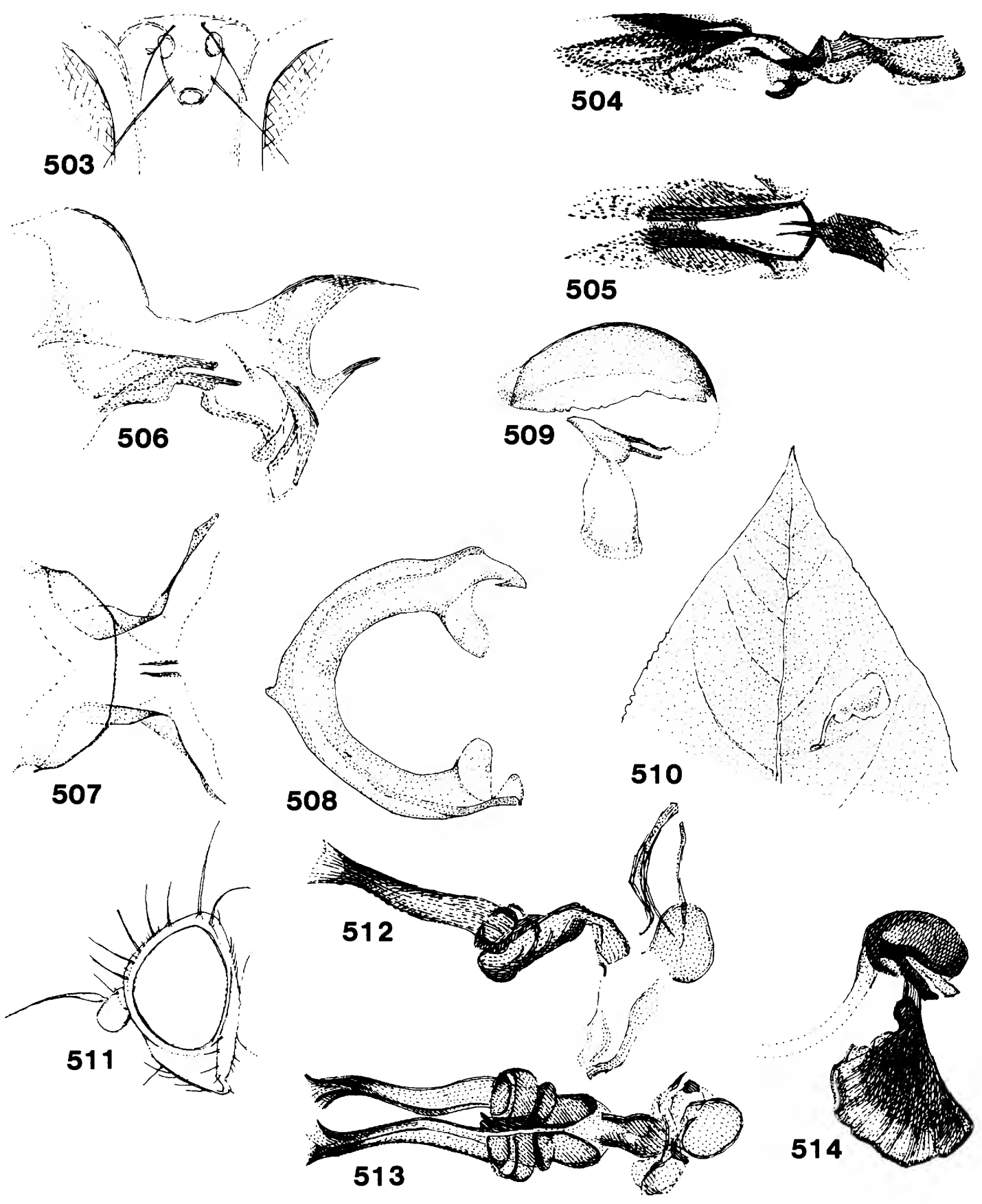

Figures 503-514.--Amauromyza (Cephalomyza) anomala:

503, Head, middorsal view; A. (‥) elsinorensis:

504, Aedeagus, side view; $5 \overline{0} 5, \bar{d}$ ist iphallus,

ventral view. A. (Catal pomyza) pleuralis: 506,

Aedeagus, side view; 507, distiphallus, ventral

view; 508, hypandrium; 509, sperm pump; 510, leaf

mine on Catalpa (Ontario). A. (Annimyzella)

lathyroides: 511 , Head; 512, aedeagus, side view;

513, same, ventral view; 514, sperm pump. 

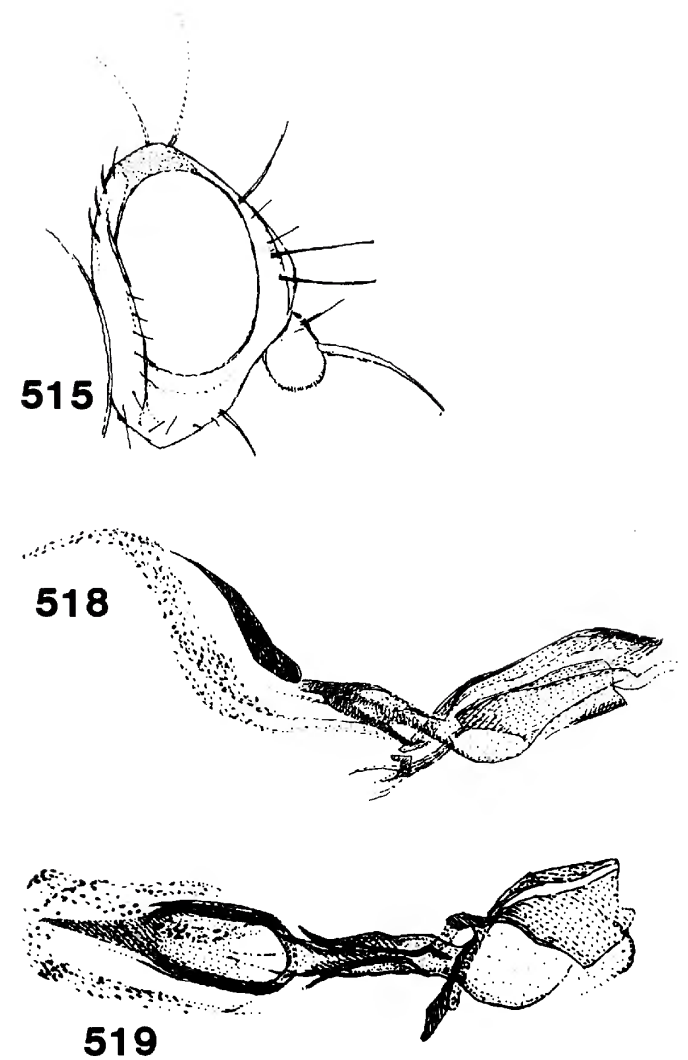

519
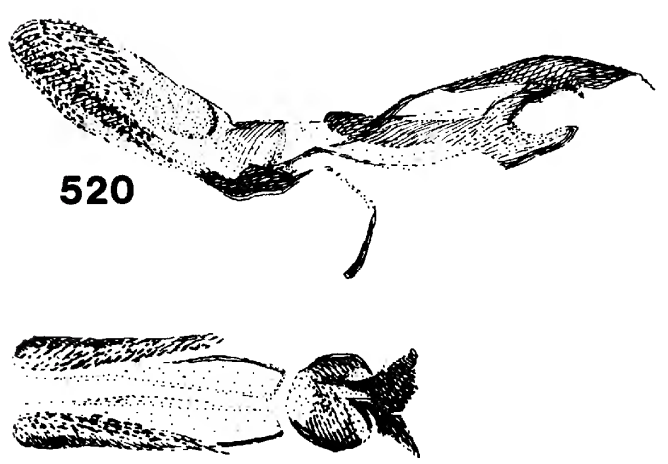

521
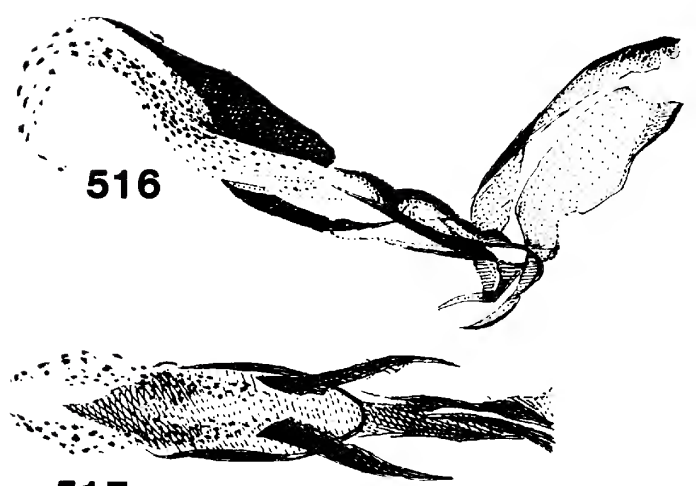

517
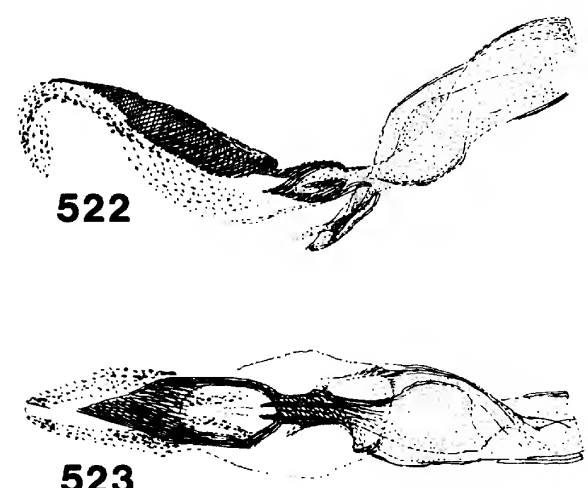

523
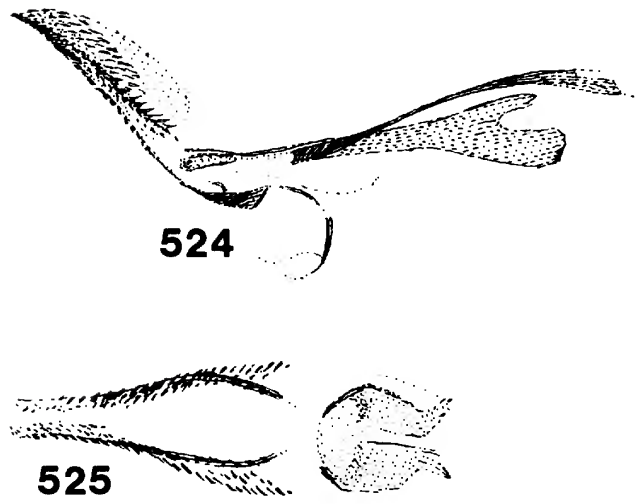

Figures 515-525.--Amauromyza (Cephalomyza) indecisa:

515, Head (holotype); A. (C.) scleritica: 516, Aedeagus, side view; $5 \overline{1} 7, \bar{d}$ istiphallus, ventral

view; A. (C..) auriceps: 518, Aedeagus, side view

(Colorado); 519, same, ventral view (paratype, Idaho); A. (C..) schusteri: 520, Aedeagus, side view; $52 \overline{1}$, distiphallus, ventral view; $\underline{A}$. (C.) knowltoni: 522, Aedeagus, side view; $52 \overline{3}$, same, ventral view; $\underline{A}$. (…) remus: 524, Aedeagus, side view; 525, same, ventral view. 

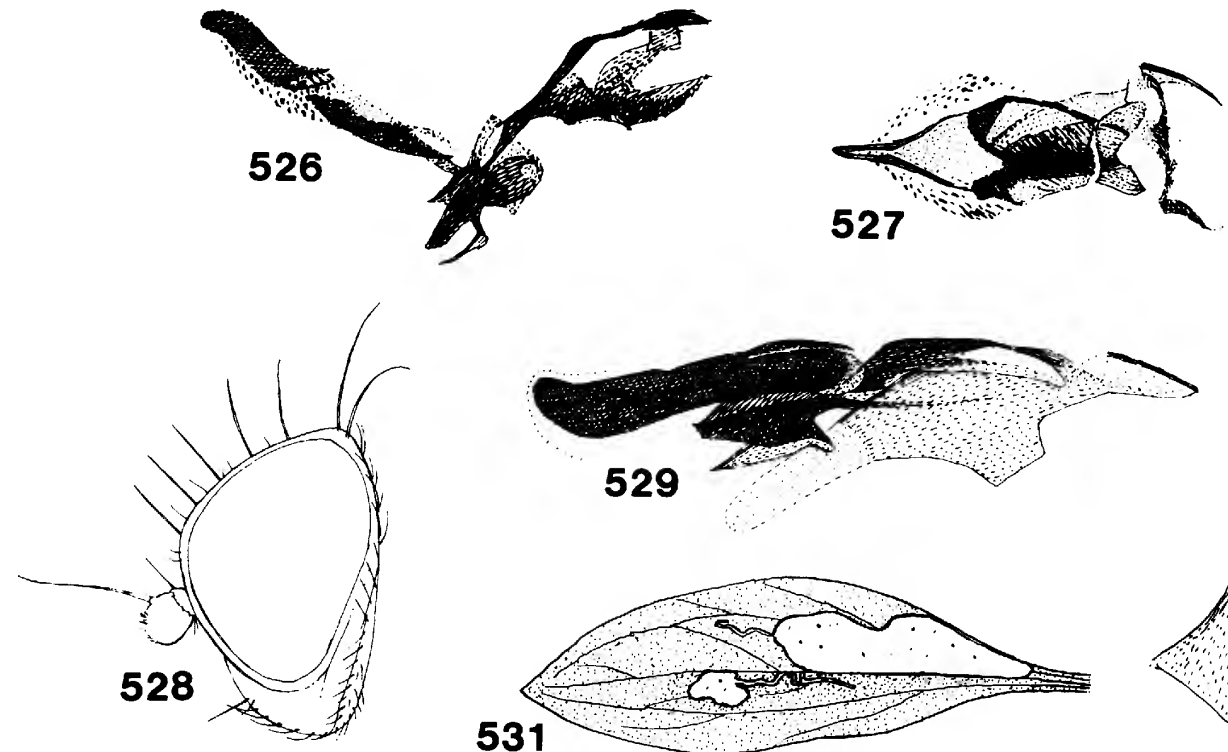

529
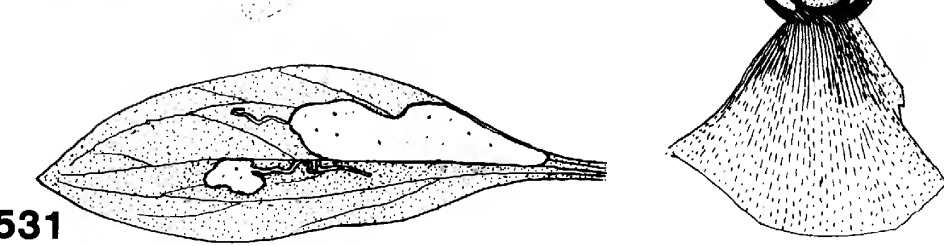

530
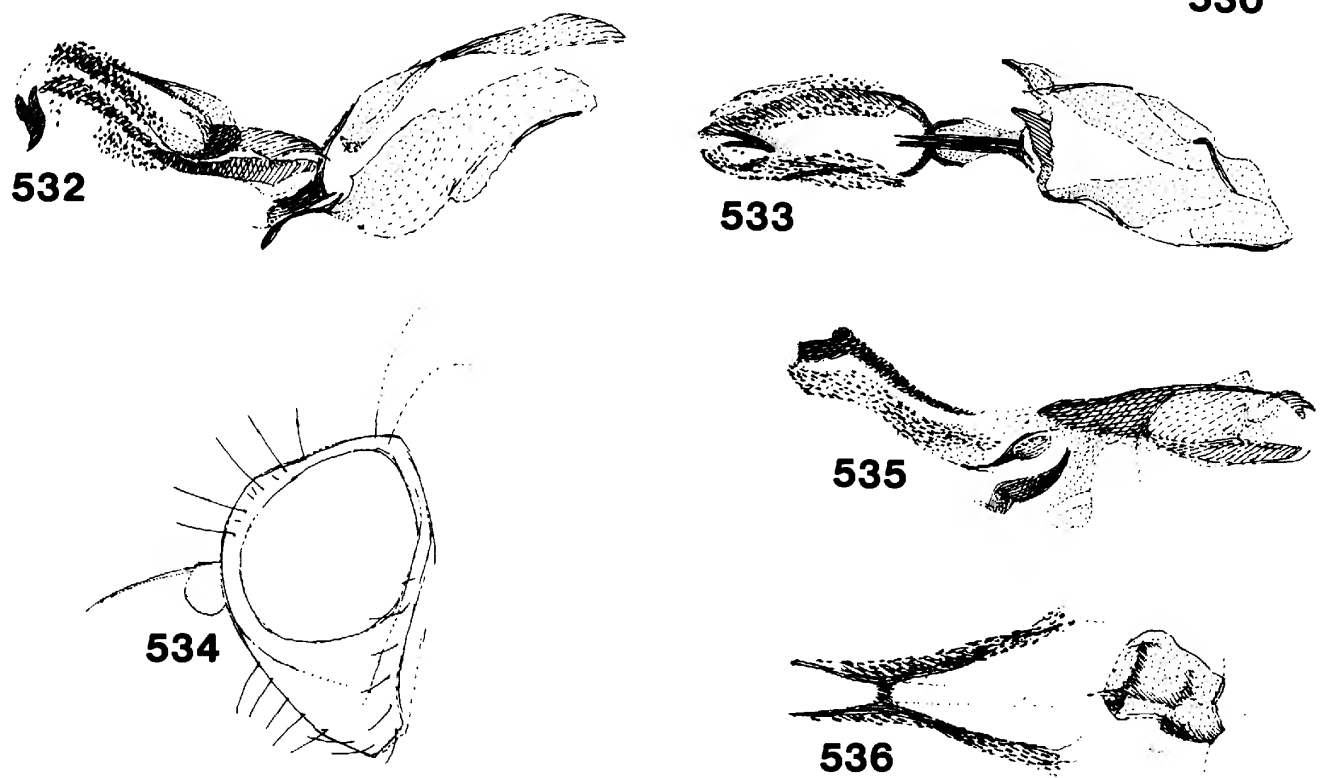

Figures 526-536.--Amauromyza (Cephalomyza) confondata:

526, Aedeagus, side view; 527 , same, ventral view

(as A. anomala, Spencer, 1981: figs. 212, 213); A.

(C.) flavifrons: 528, Head; 529, aedeagus, side

view; 530 , sperm pump; 531, leaf mines on Saponaria;

A. (C.) albidohalterata: 532, Aedeagus, side view;

533 , same, ventral view; A. (C.) romulus: 534 ,

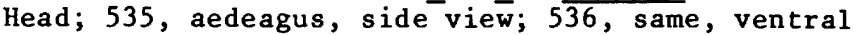

view. 


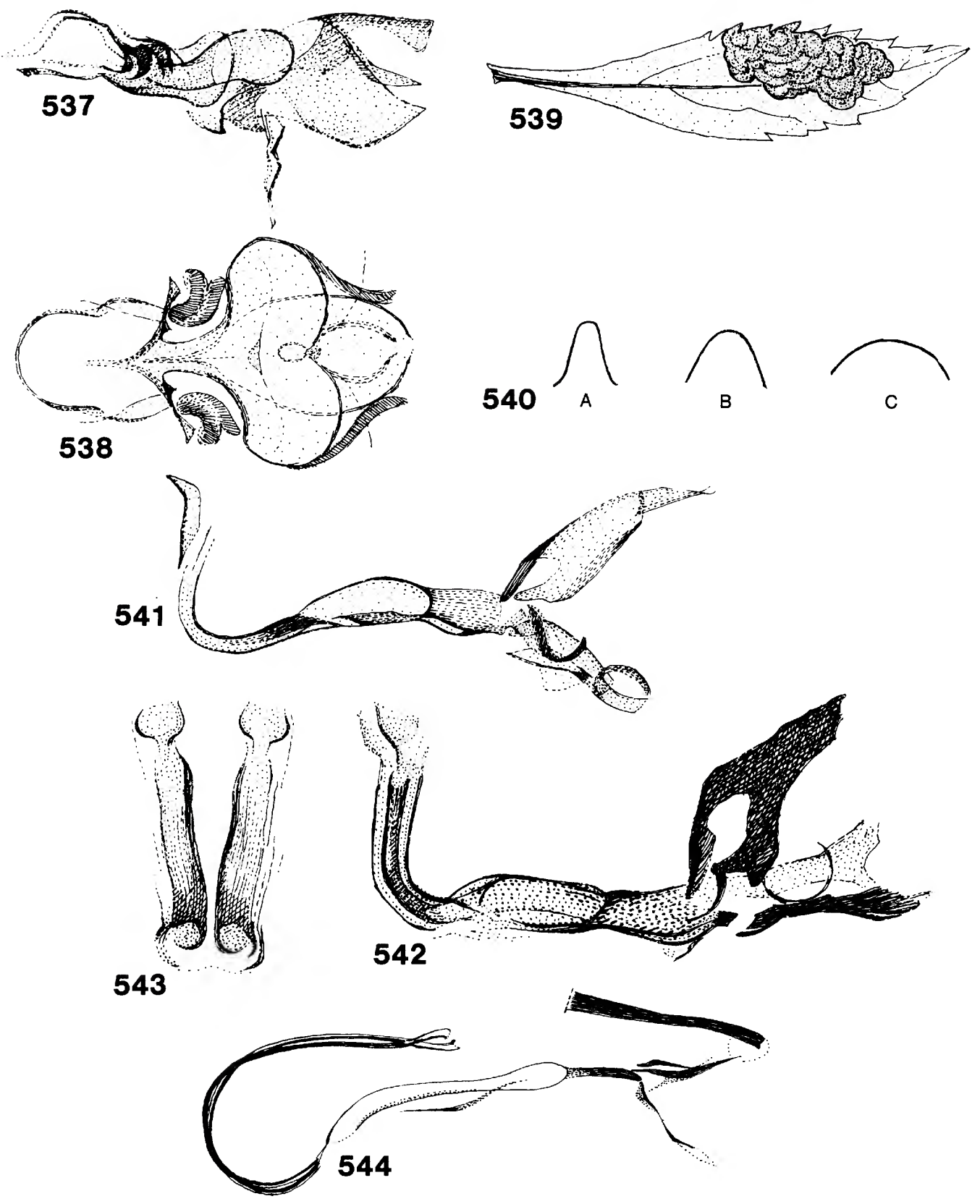

Figures 537-544.--Nemorimyza posticata: 537, Aedeagus, side view; 538 , same, ventral view; 539 , leaf mine on Solidago. 540, Lunules of Cerodontha subgenera:

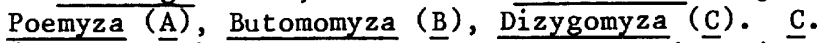
(Icteromyza) capitata: $54 \overline{1}$, Aedeagus, side view; c. (I.) montanoides: 542, Aedeagus, side view; 543, distiphallus, ventral view; $\underline{\text {. }}$ (I. ) longipennis: 544, Aedeagus, side view. 


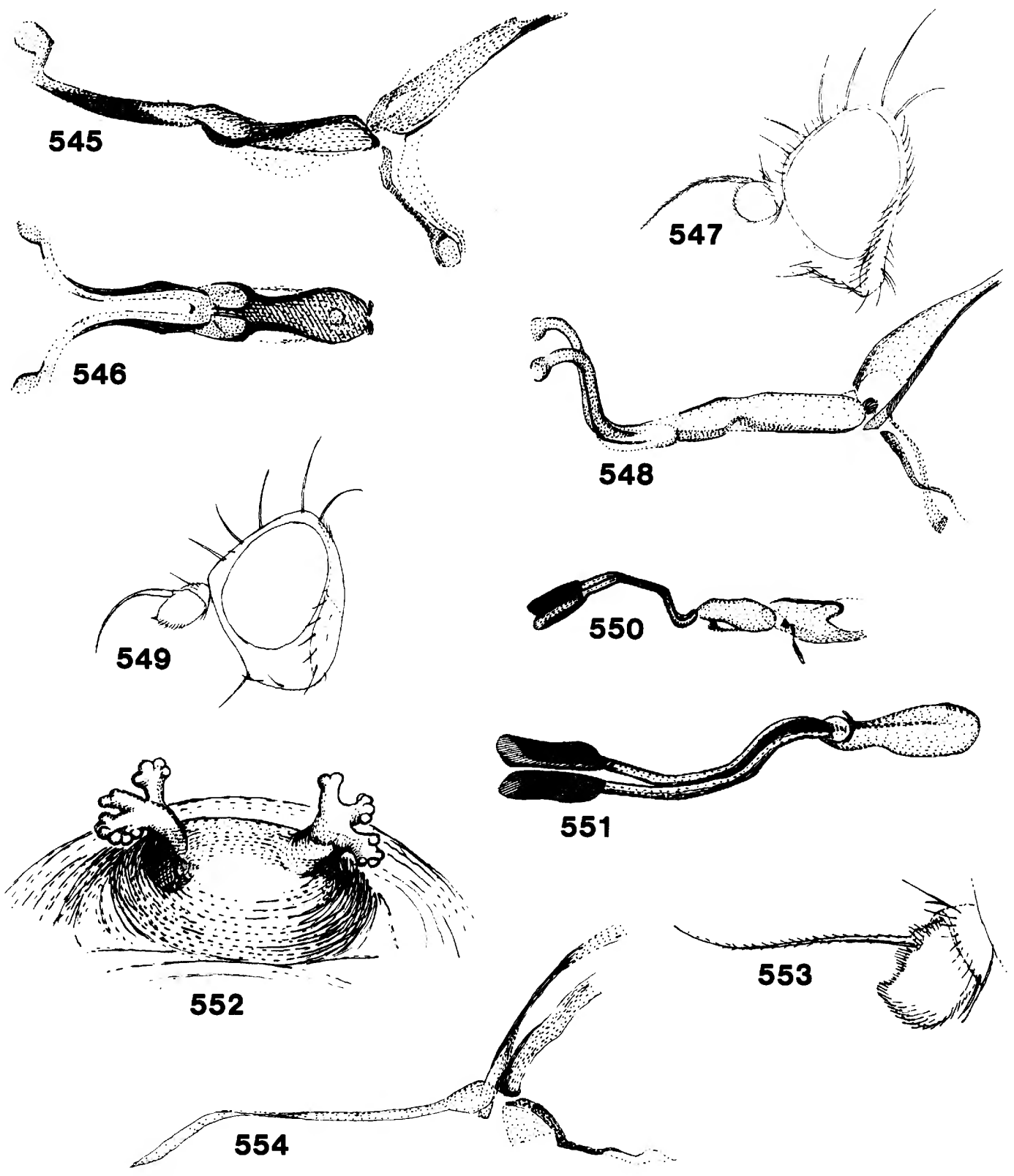

Figures 545-554.--Cerodontha (Icteromyza)

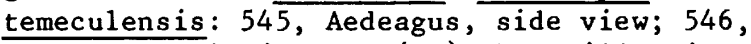
same, ventral view; C. (I.) churchillensis: 547,

Head; 548, aedeagus, side view. C. (Cerodontha)

dorsalis: 549, Head; 550, aedeagus, side view; 551,

same, ventral view; 552, posterior spiracles of

puparium. C. (Xenophytomyze) illinoensis: 553,

Third antennal segment; 554, aedeagus, side view. 


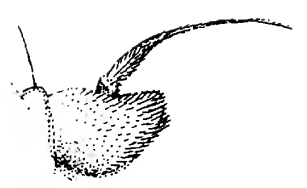

555

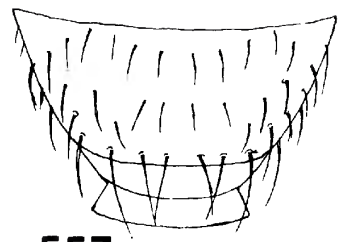

557
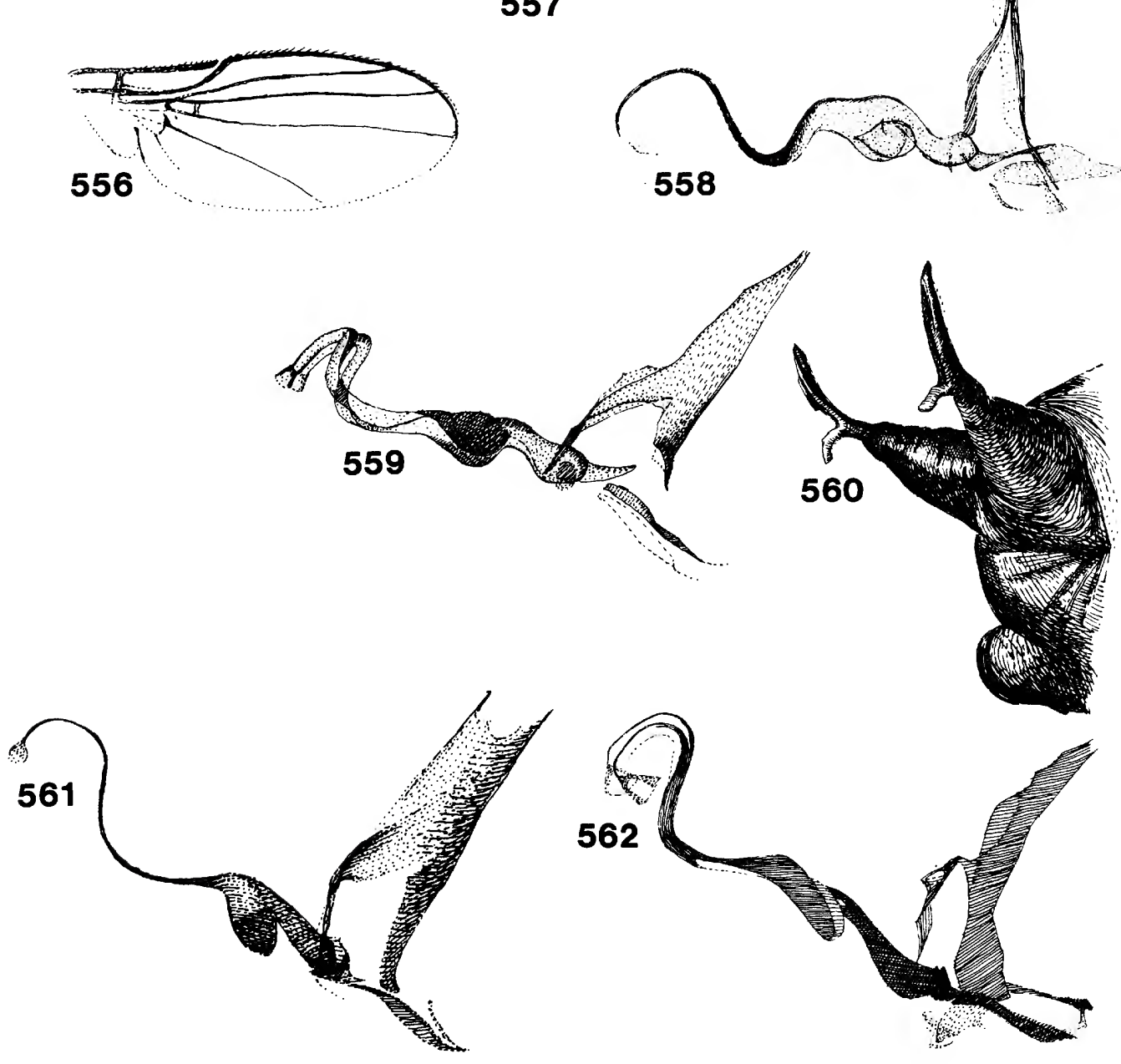

Figures 555-562.--Cerodontha (Poemyza) chillcottiella:

555, Third antennal segment; 556, wing; C. (P.)

superciliosa: 577, Epandrium; 558, aedeagus, side

view; C. (…) muscina: 559, Aedeagus, side view;

560 , posterior spiracles of puparium; C. (P.)

malaise $i$ : 561 , Aedeagus, side view; $\underline{C} .(\underline{P}$.

pygmaea: 562 , Aedeagus, side view. 

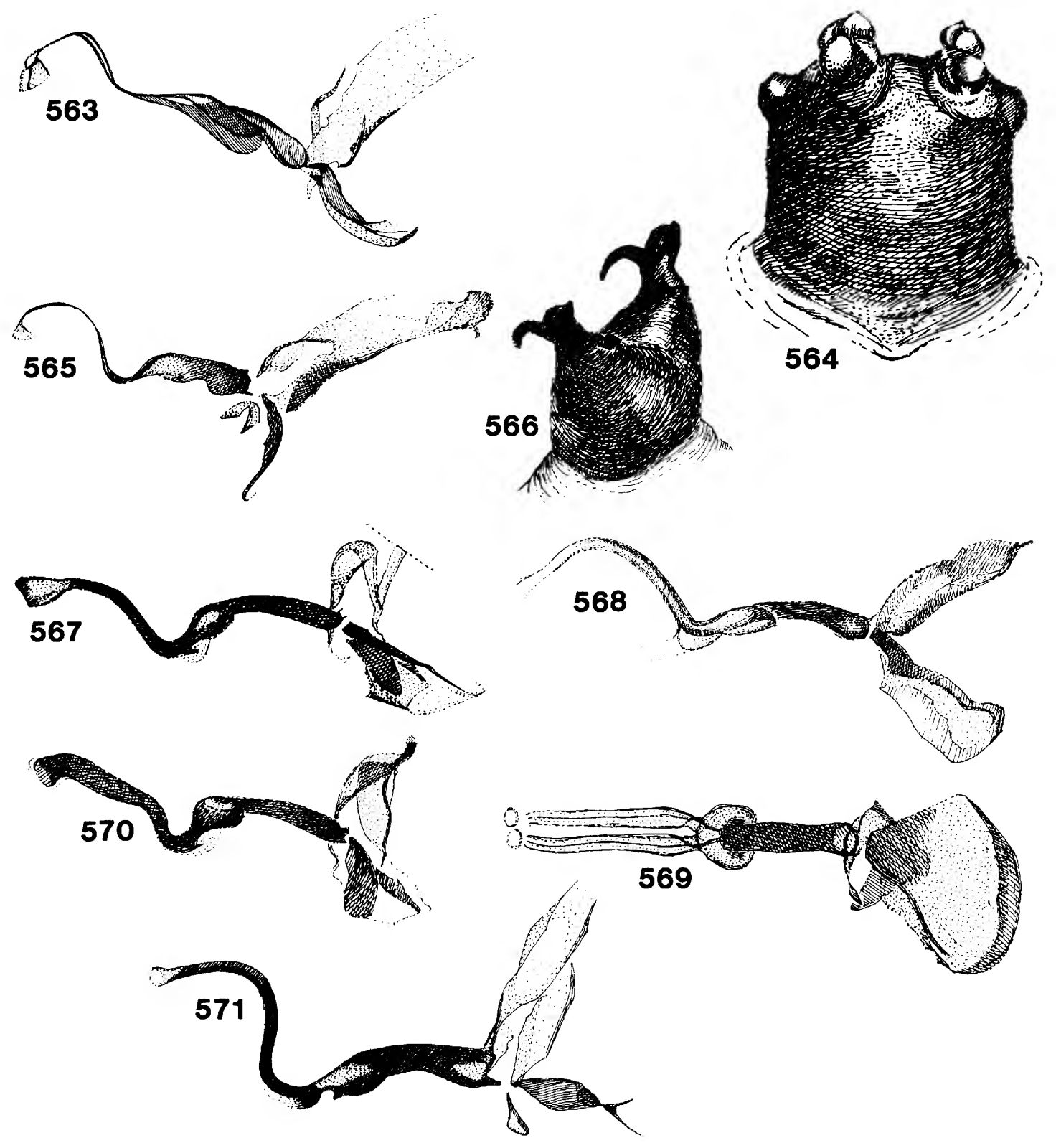

Figures 563-571.--Cerodontha (Poemyza) incisa: 563, Aedeagus, side view; 564, posterior spiracles of puparium; C. (P.) macminni: 565, Aedeagus, side view; 566 , posterior spiracles of puparium; C. (P.) pygminoides: 567 , Aedeagus, side view; C. (P..) attenuata: 568, Aedeagus, side view; $56 \overline{9}$, same, ventral view; C. (P.) inconspicua: 570, Aedeagus, side view (Colōradō). $\underline{\text { C. (Phytagromyza })}$

frankensis: 571 , Aedeagūs, side view. 


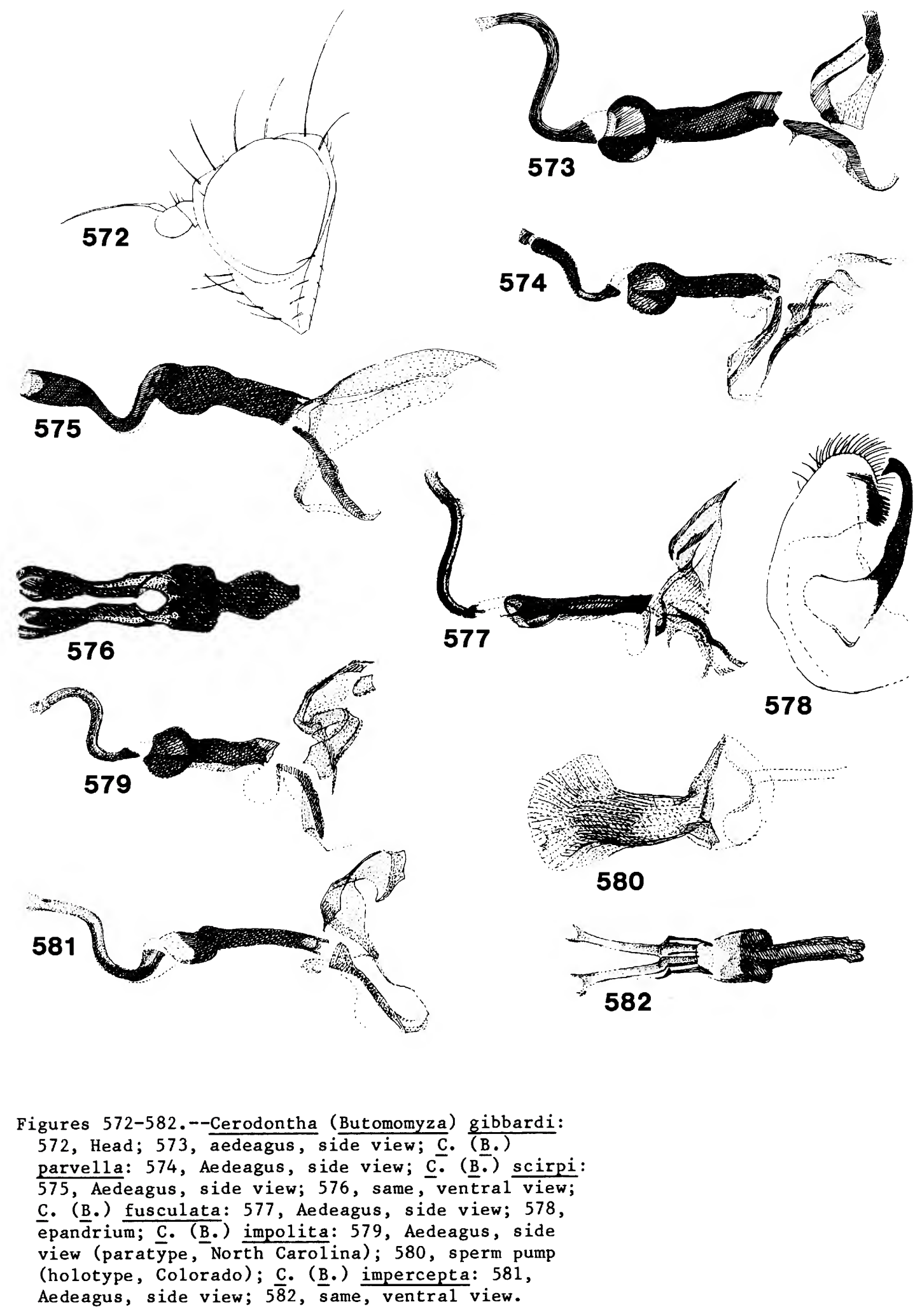



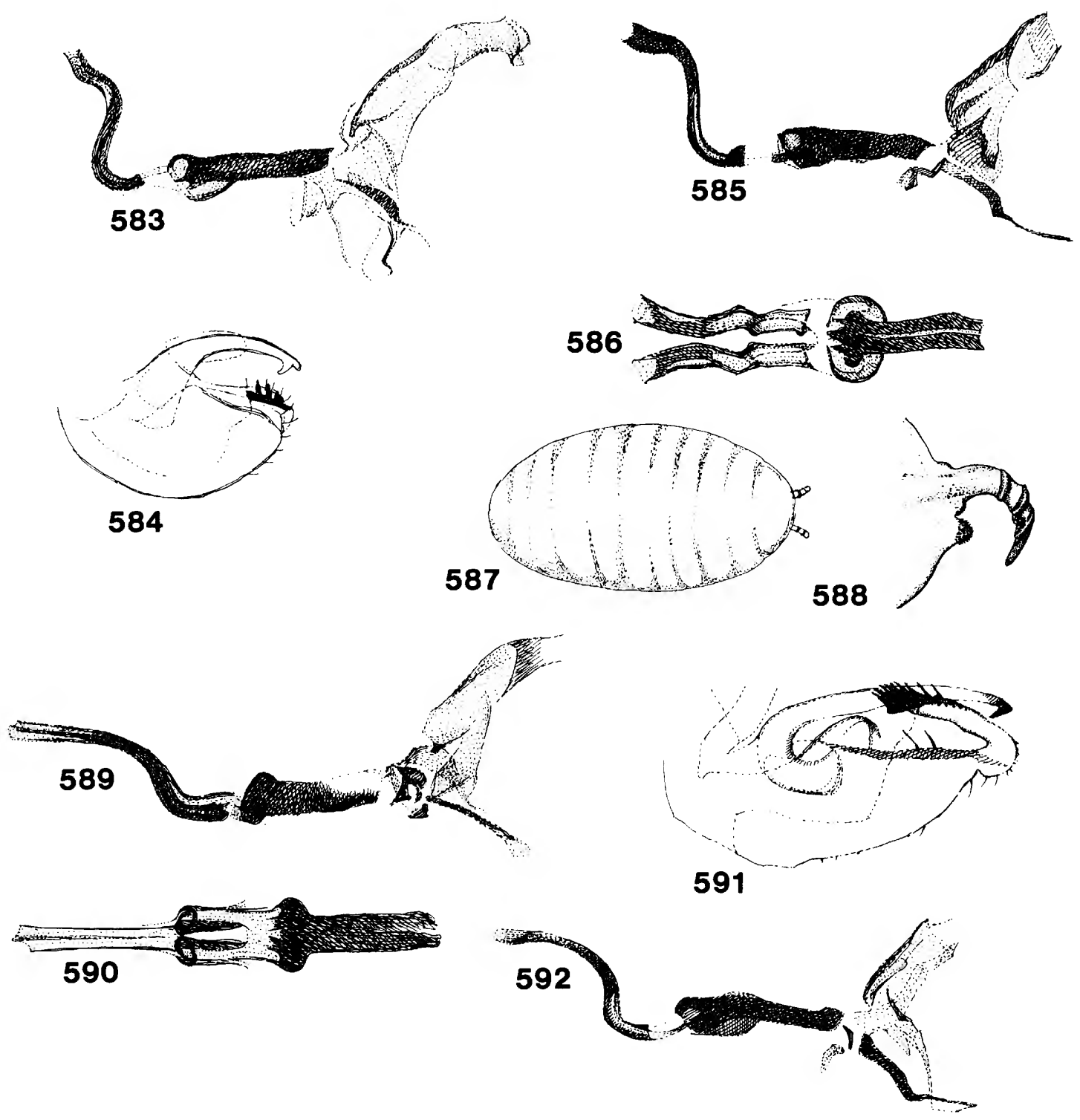

Figures 583-592.--Cerodontha (Butomomyza) angulata:

583, Aedeagus, side view; 584, epandrium; (583,

584, paralectotype, Pennsylvania); 585, aedeagus, side view; 586, same, ventral view; $(585,586$,

Indian Gap, N.C.); 587, puparium; 588, posterior

spiracles of puparium; ( 587,588 , Europe); ‥ (…)

subangulata: 589, Aedeagus, side view; 590 , same,

ventral view; 591, epandrium; (589-591, holotype);

592, aedeagus, side view (Cabin John Creek, Md.). 

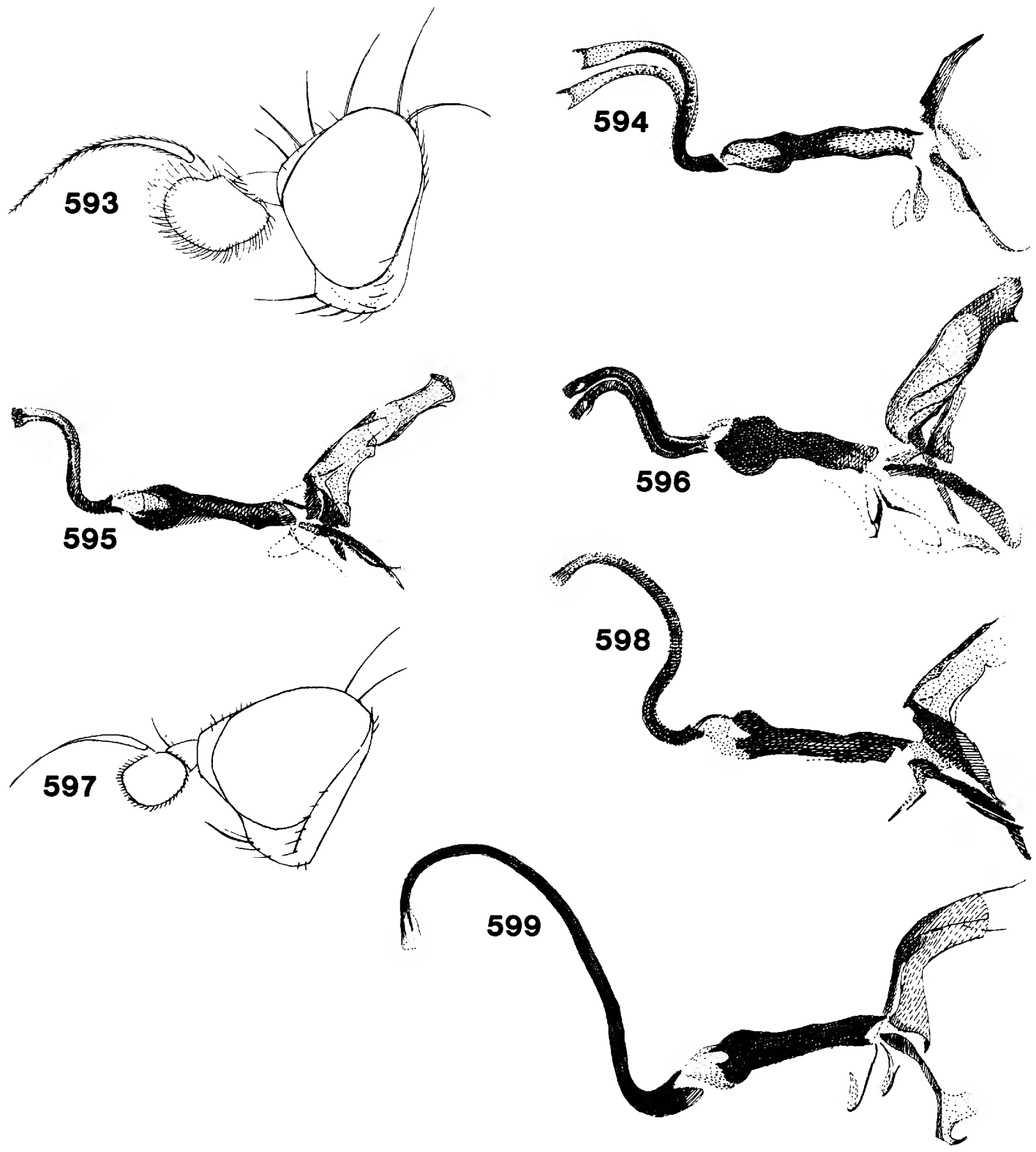

Figures 593-599.--Cerodontha (Dizygomyza) pecki: 593,

Head; 594, aedeagus, side view; C.. (D.) magnicornis:

595, Aedeagus, side view (Tennessee); C. (D. )

latifrons: 596, Aedeagus, side view; C. (D.)

frosti: 597 , Head; C. (…) morosa: $59 \overline{8}$, Aédeagus, side view (holotype); C. (D.) thompsoni: 599,

Aedeagus, side view. 

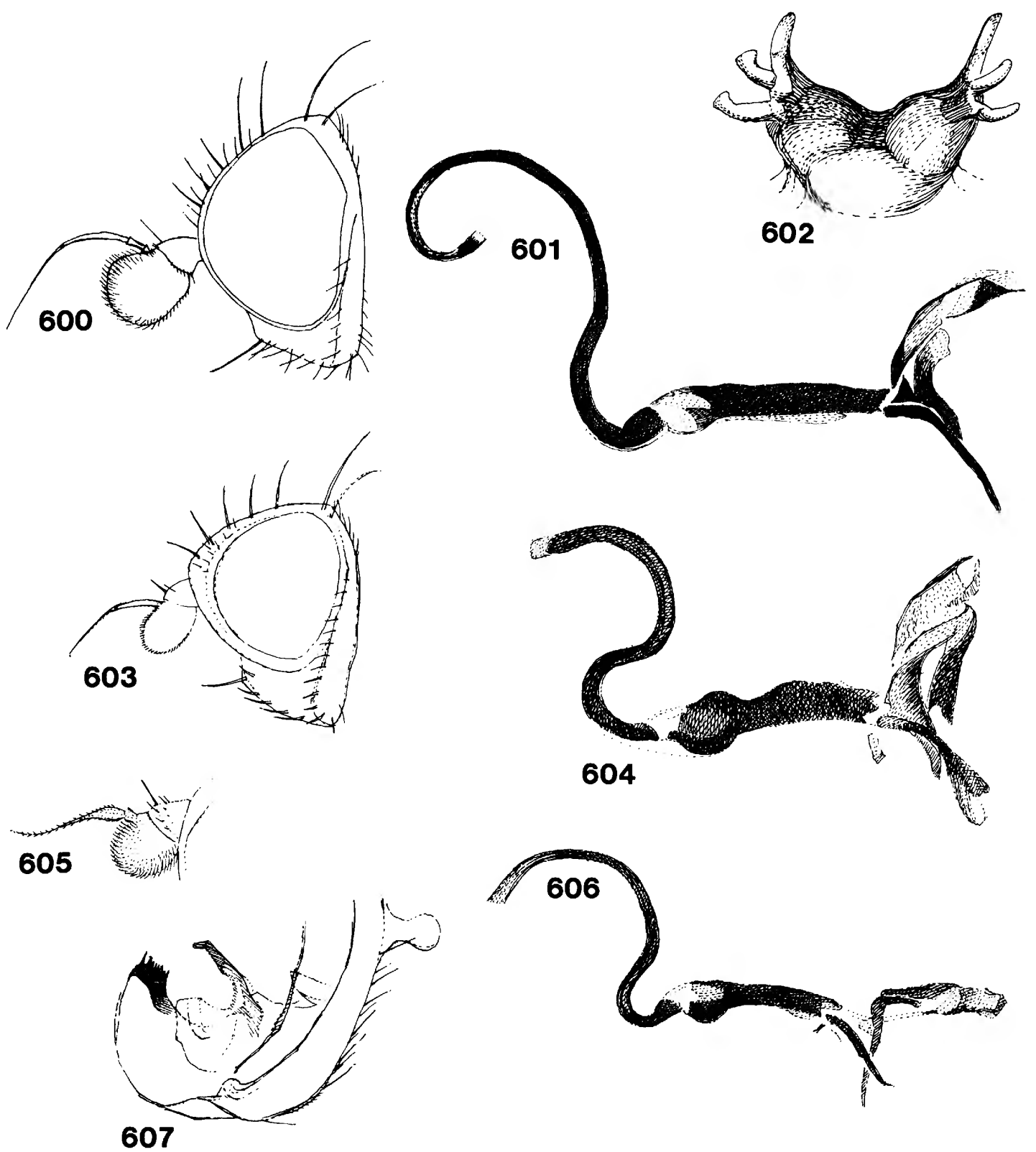

Figures 600-607.--Cerodontha (Dizygomyza) iridophora:

600, Head; 601, aedeagus, side view; 602, posterior

spiracles of puparium; C. (D.) poolei: 603, Head;

604, aedeagus, side view; c.. (D. ) aristosa: 605,

Third antennal segment; $60 \overline{6}$, aedeagus, side view;

607 , epandrium. 

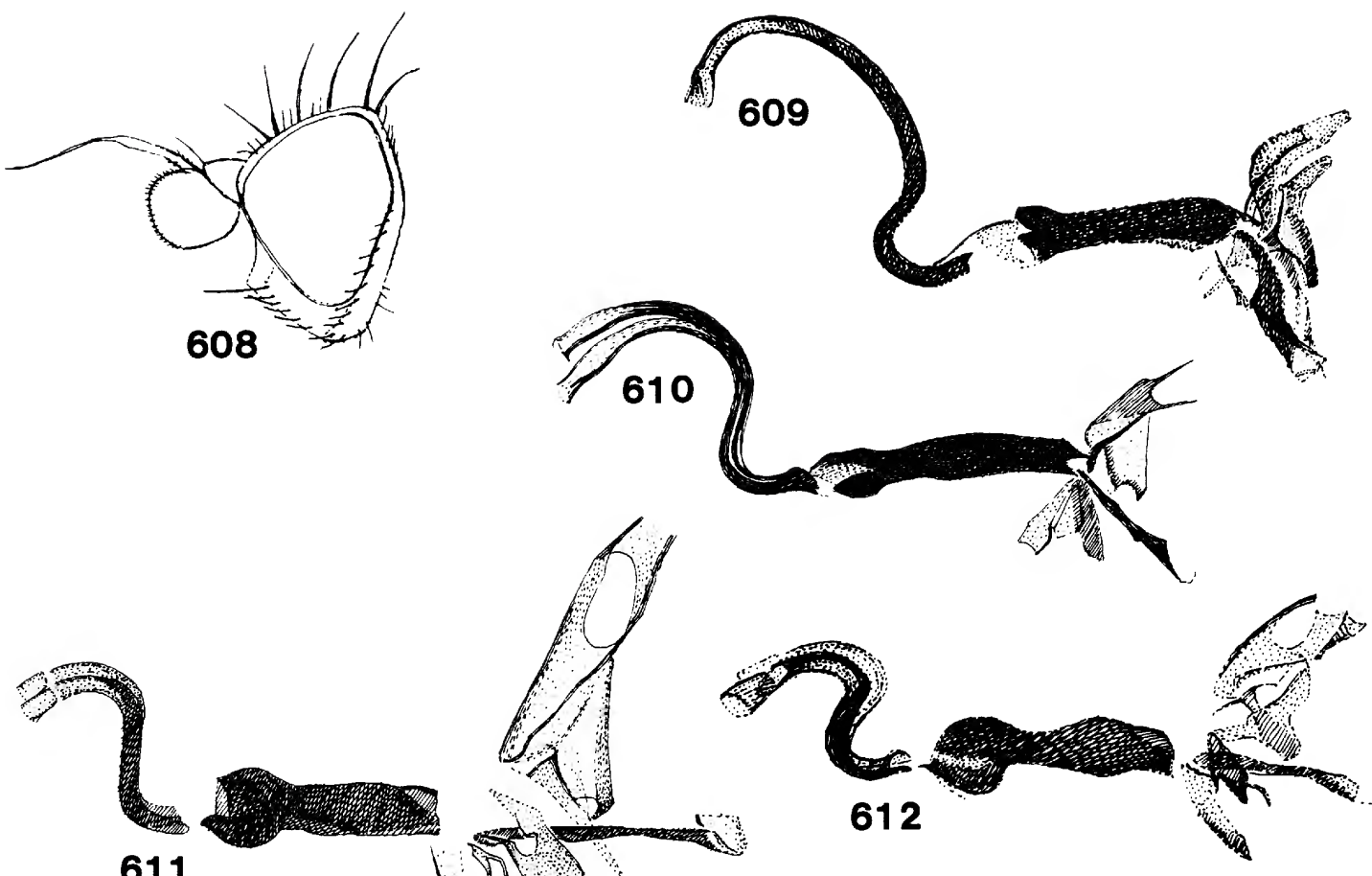

611

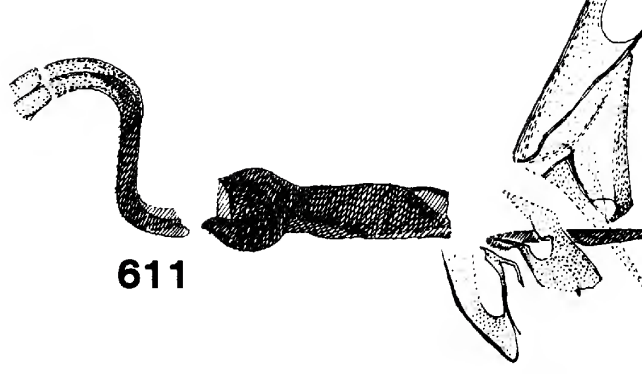

la
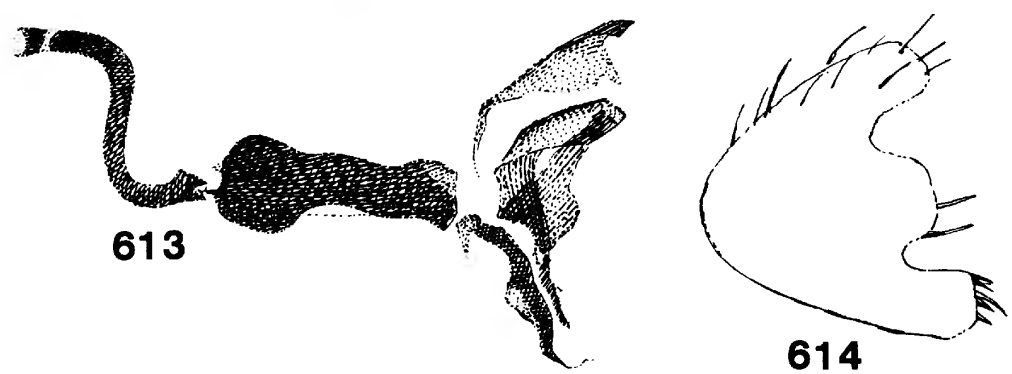

Figures 608-614.--Cerodontha (Dizygomyza) paludosa:

608 , Head; 609 , aedeagus, side view; C. (D.)

fasciata: 610 , Aedeagus, side view; C. (D.) bicolorata: 611 , Aedeagus, side view; $\underline{\mathrm{C}}$. (犃)

maclayi: 612, Aedeagus, side view; $\underline{\text { C. }}$ ( D. $^{-}$) questa:

613, Aedeagus, side view; 614, epand̄rium. 

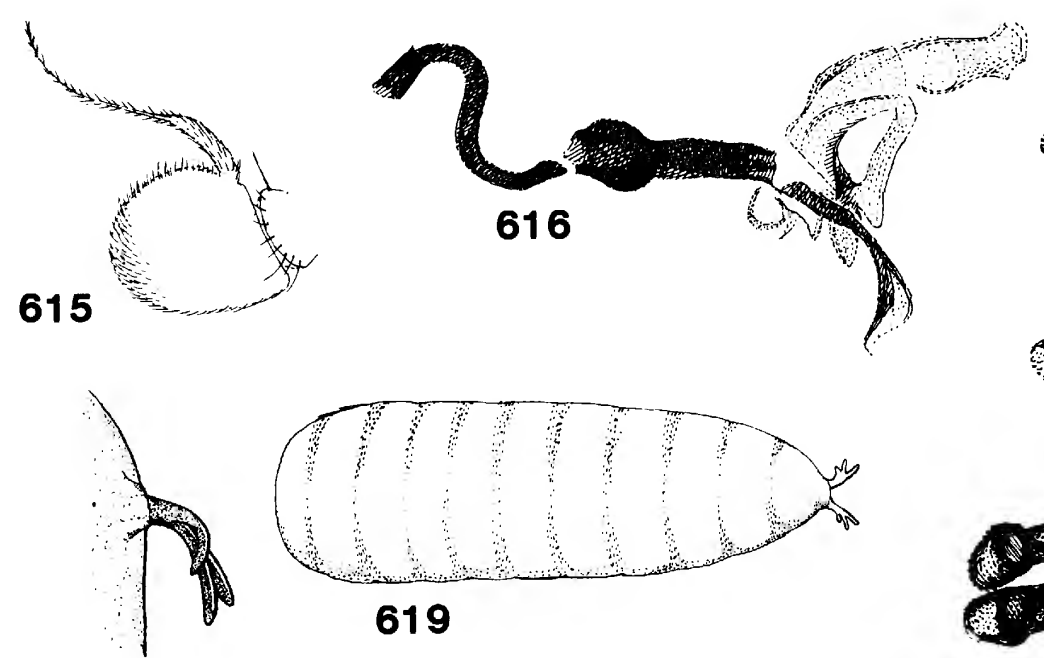

\section{8}
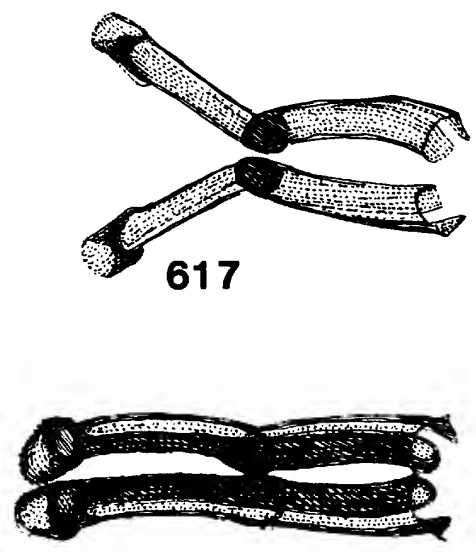

620
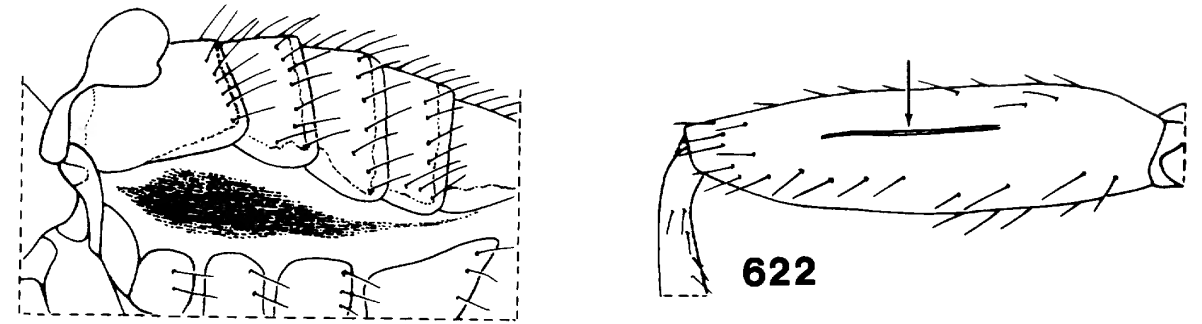

621

Figures 615-622.--Cerodontha (Dizygomyza) luctuosa: 615, Third antennal segment; 616, aedeagus, side view (Colorado); 617, distiphallus, ventral view; 618, posterior spiracles of larva; 619, puparium; C. (D.) hirtae: 620 , Distiphallus, ventral view. Liriomyza spp., stridulating mechanism in male; 621, L. virgo "file"; 622, L. orbona, arrow indicates "scraper"; $(621, \overline{6} 22$, Europe, after Tschirnhaus, 1971; drawn from photographic originals). 


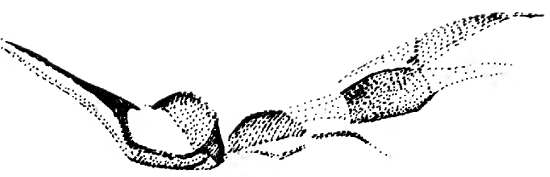

623
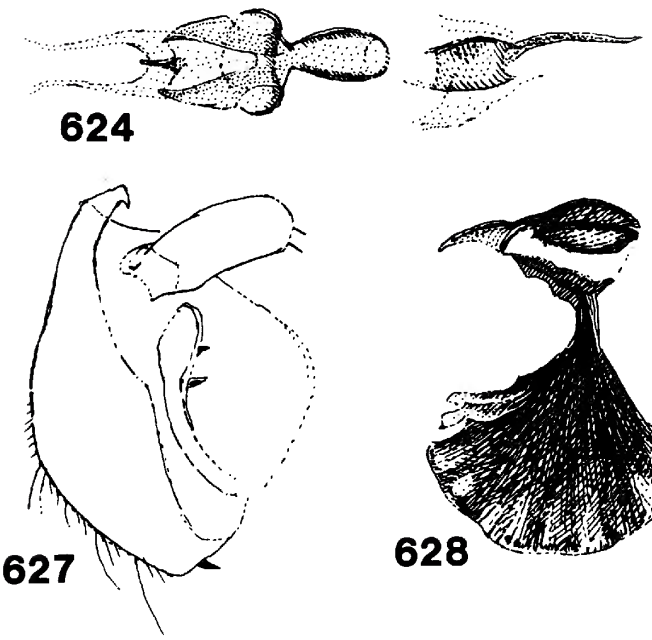

\section{8}
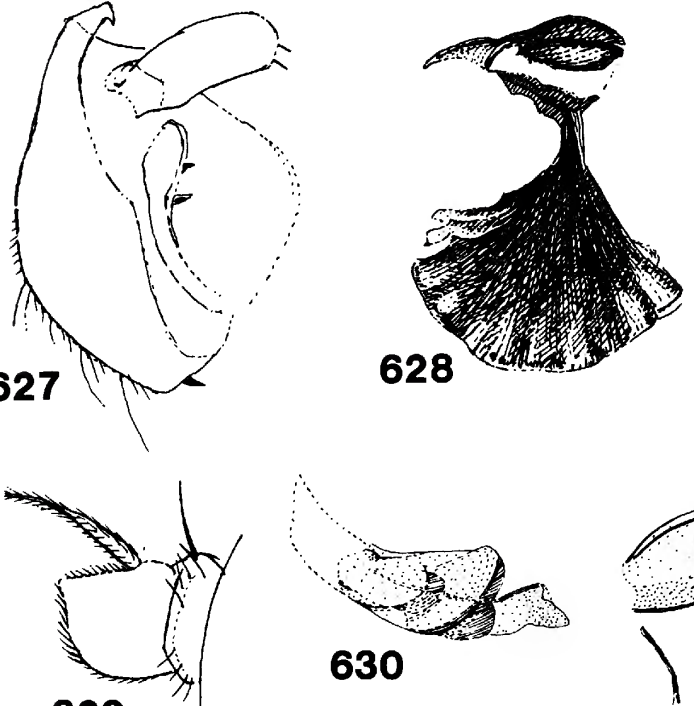

629
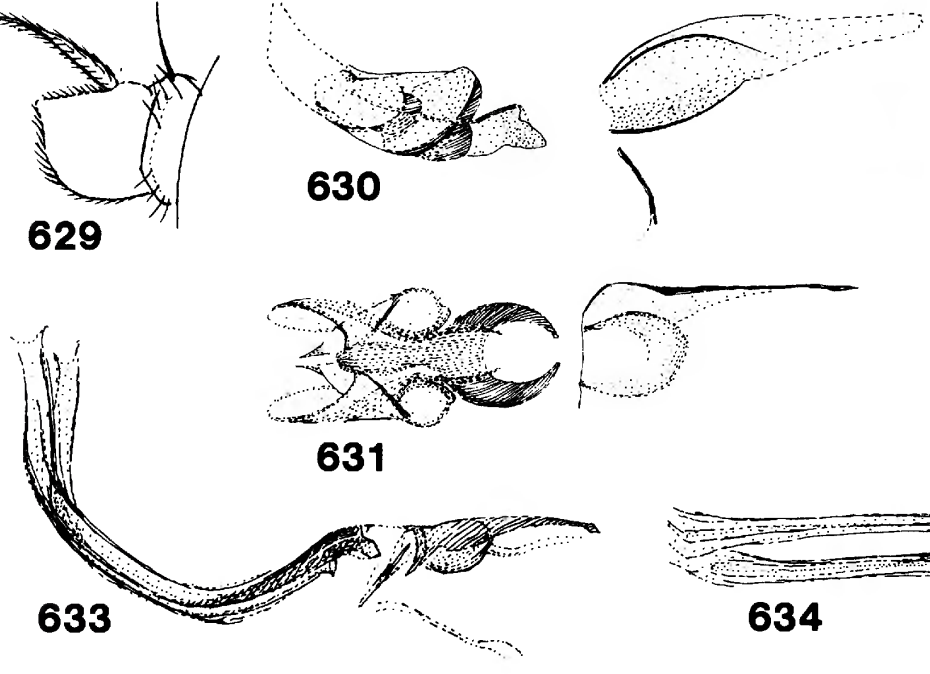

630
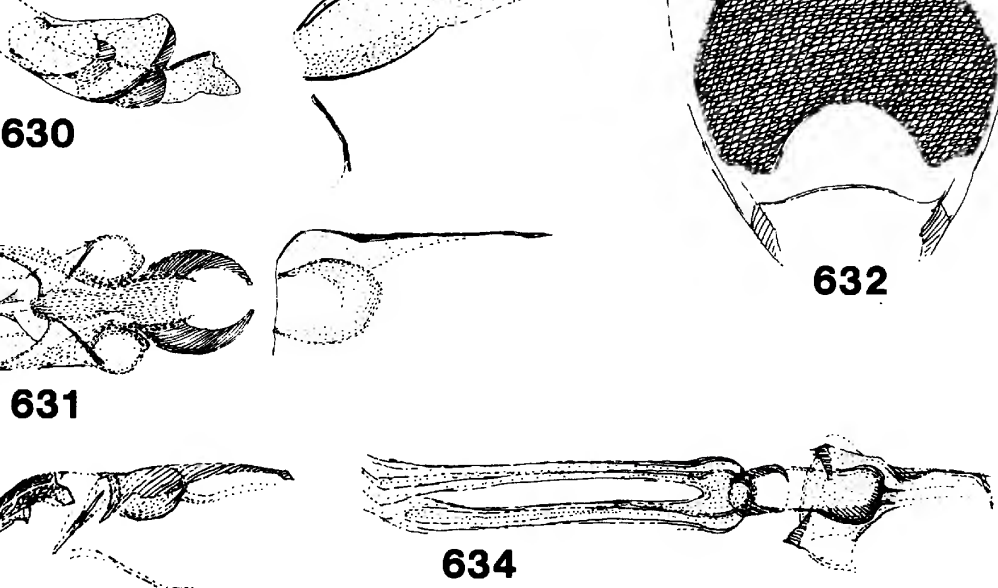

Figures 623-634.--Liriomyza nigriscutellata: 623 , Aedeagus, side view; 624, same, ventral view; $L$. nigrissima: 625, Aedeagus, side view; 626, same, ventral view; 627 , epandrium with surstylus; 628 , sperm pump; L. angulicornis: 629, Third antennal segment; 630 , aedeagus, side view; 631 , same,

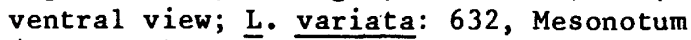
(holotype); L. flavonigra: 633, Aedeagus, side view; 634, same, ventral view (Utah). 

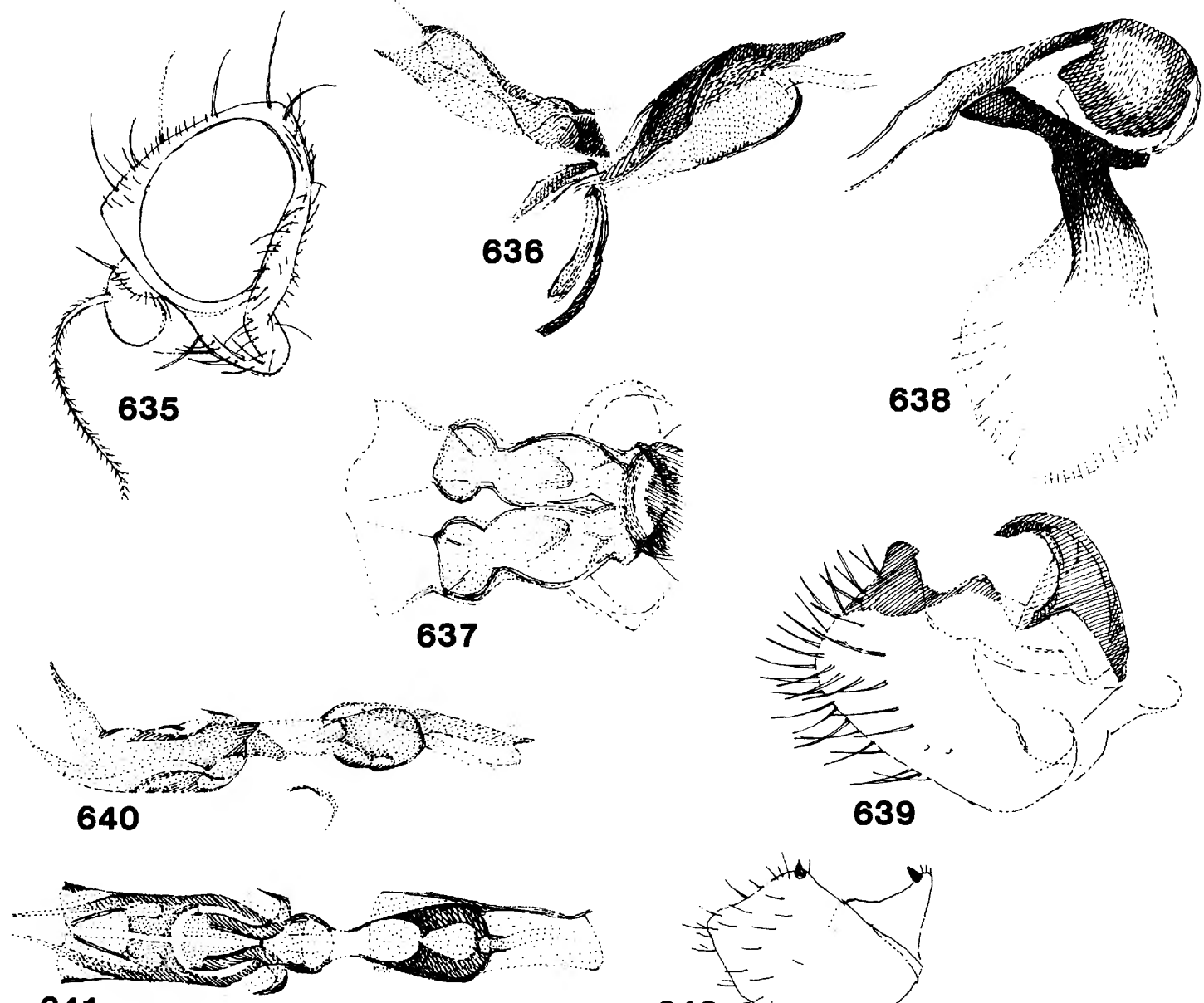

\section{1}
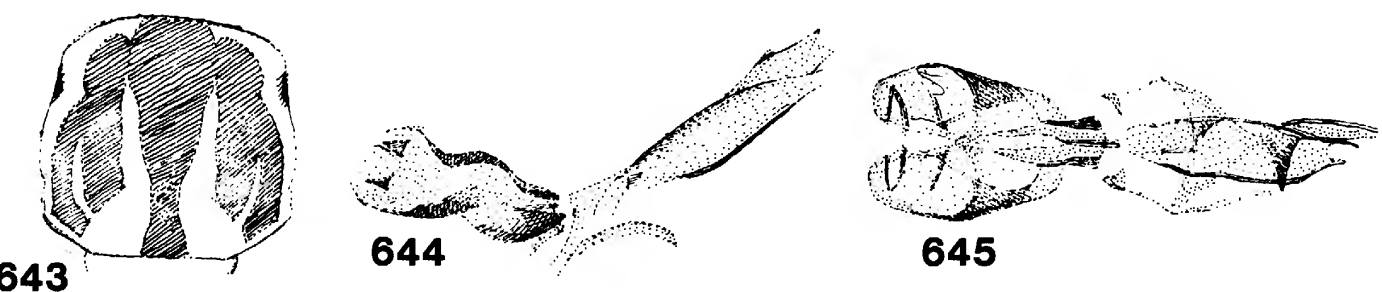

645

Figures 635-645.--Liriomyza pechumani: 635, Head;

636, aedeagus, side view; 637, distiphallus,

ventral view; 638, sperm pump; 639, epandrium with

surstylus; $\underline{L}$. flavicola: 640 , Aedeagus, side view;

641, same, ventral view; 642, surstylus; $\underline{L}$.

admiranda: 643, Mesonotum; 644, aedeagus, side

view; 645 , same, ventral view. 

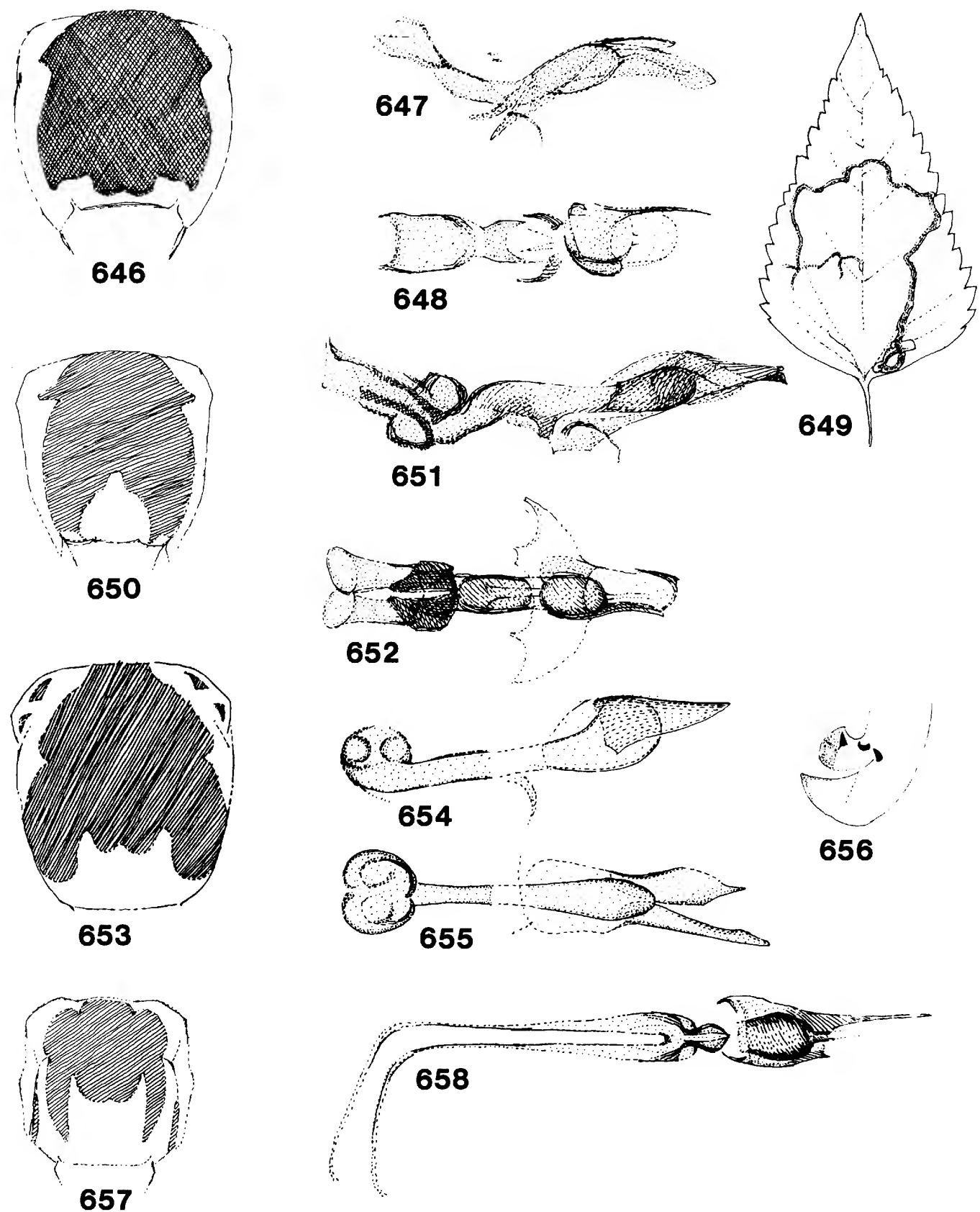

Figures 646-658.--Liriomyza eupatoriella: 646, Mesonotum; 647, aedeagus, side view; 648 , same, ventral view; 649 , leaf mines on Ageratina altissima (=Eupatorium rugosum); L. assimilis: 650, Mesonotum; 651 , aedeagus, side view; 652 , same, dorsal view (holotype); L. schmidti: 653, Mesonotum; 654, aedeagus, side view; 655, same, ventral view; 656 , epandrium with surstylus; L. bella: 657, Mesonotum; 658, aedeagus, ventral view. 


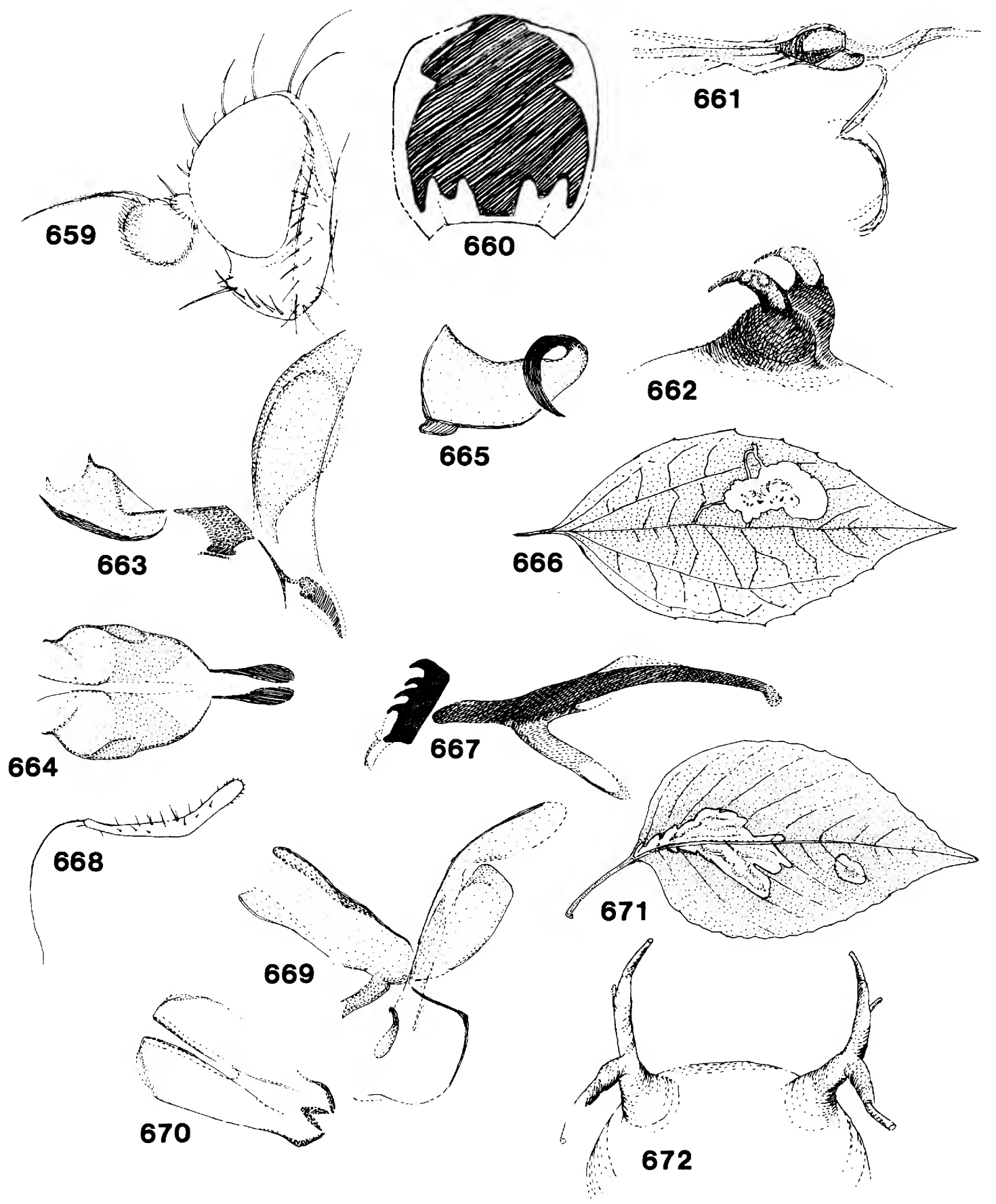

Figures 659-672.--Liriomyza commelinae: 659, Head (male); 660, mesonotum; 661 , aedeagus, side view

(Jamaica); 662, posterior spiracles of puparium; L. philadelphivora: 663, Aedeagus, side view; 664, distiphallus, ventral view; 665, surstylus; 666 , leaf mine on Philadelphus; 667, cephalopharyngea 1 skeleton of larva; L. blechi: 668, Cercus; 669,

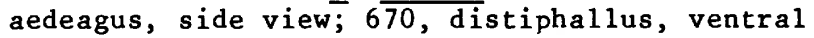
view; 671 , leaf mines on Blechum brownei; 672 , anterior spiracles of puparium. 


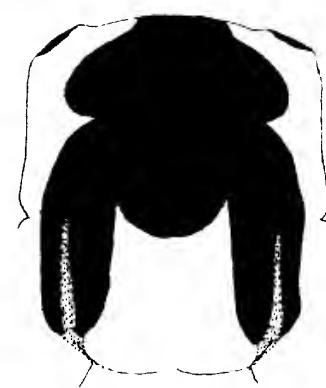

673
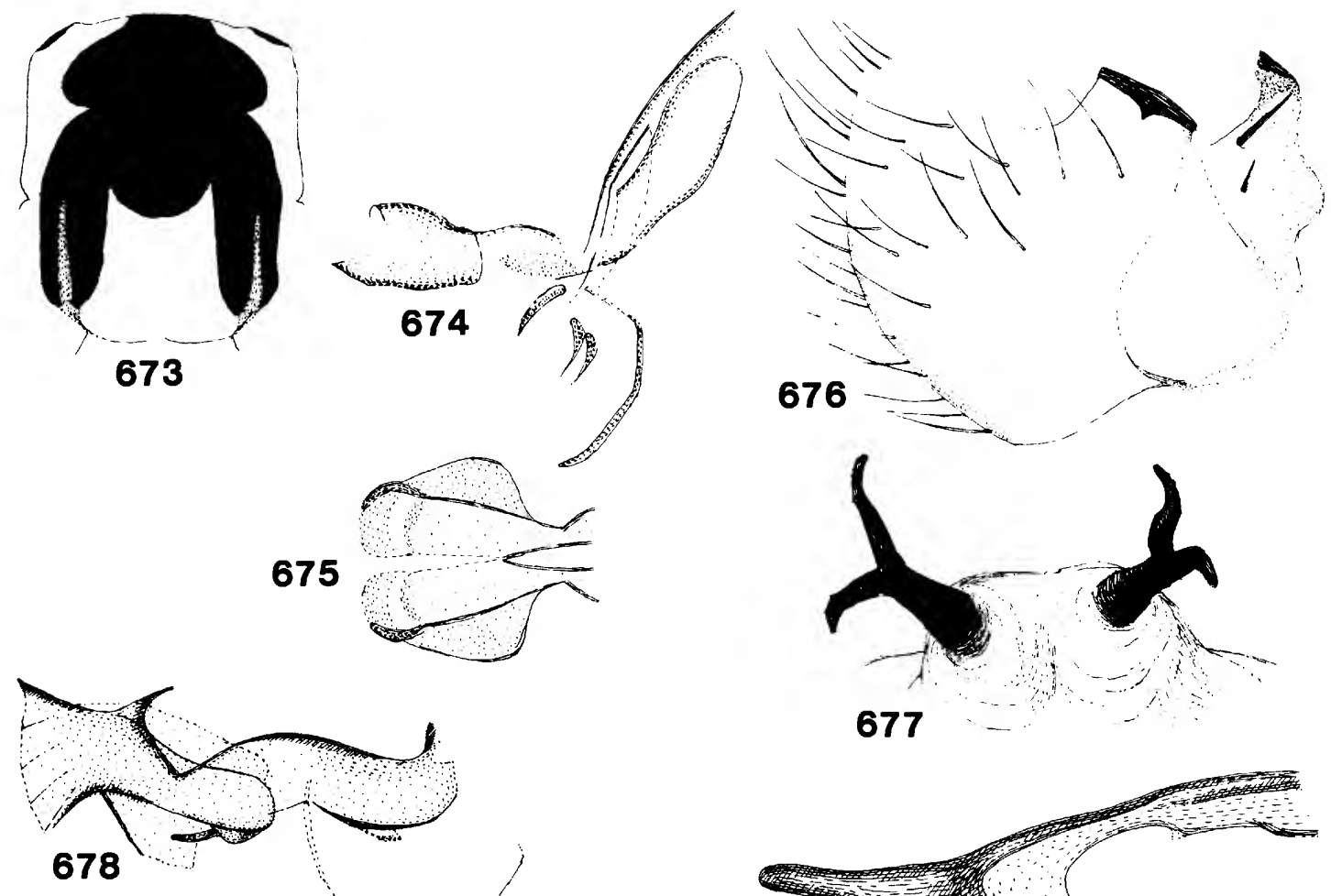

677
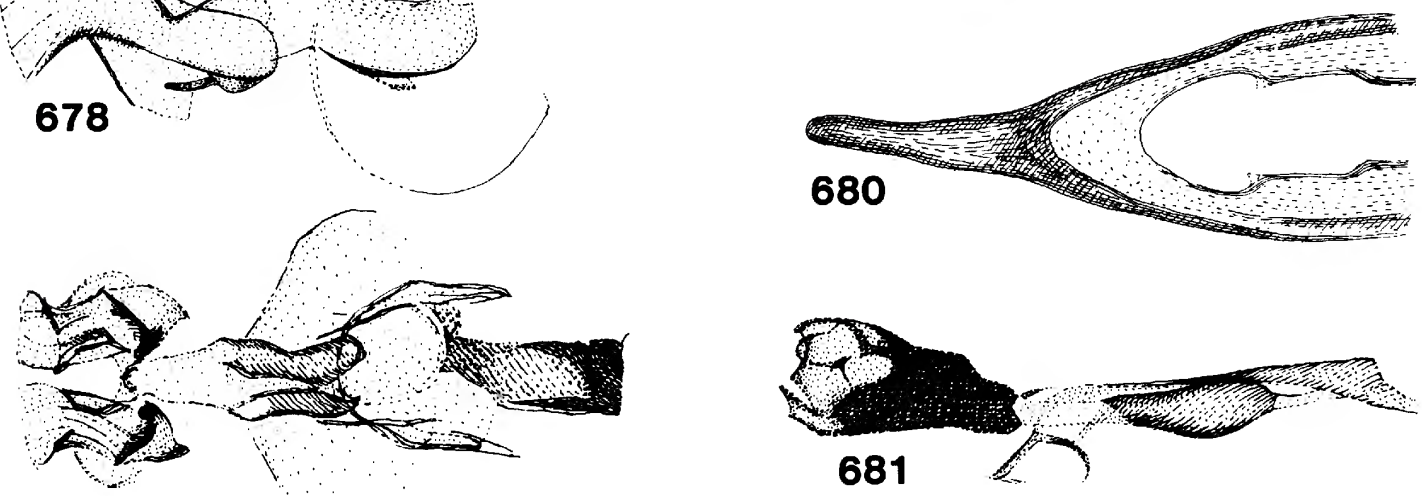

679
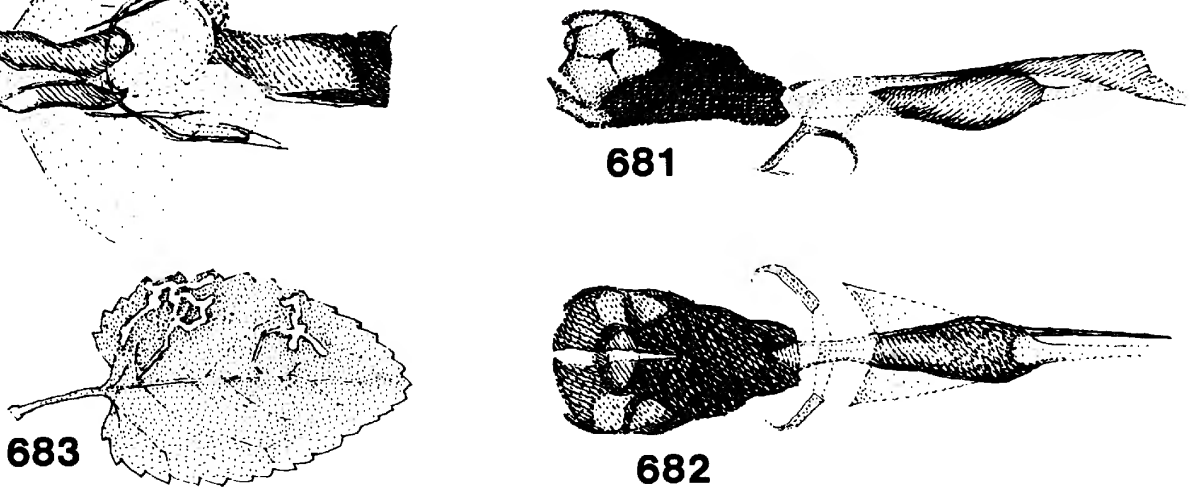

Figures 673-683.--Liriomyza marginalis: 673, Mesonotum; 674, aedeagus, side view; 675 , distiphallus, ventral view; 676 , epandrium with surstylus; 677 , anterior spiracles of puparium; L. quadrisetosa: 678, Aedeagus, side view; 679, same, ventral view; 680, hypandrium; L. stachyos: 681, Aedeagus, side view; 682, same, ventral view; 683, leaf mines on Stachys sp. (California). 


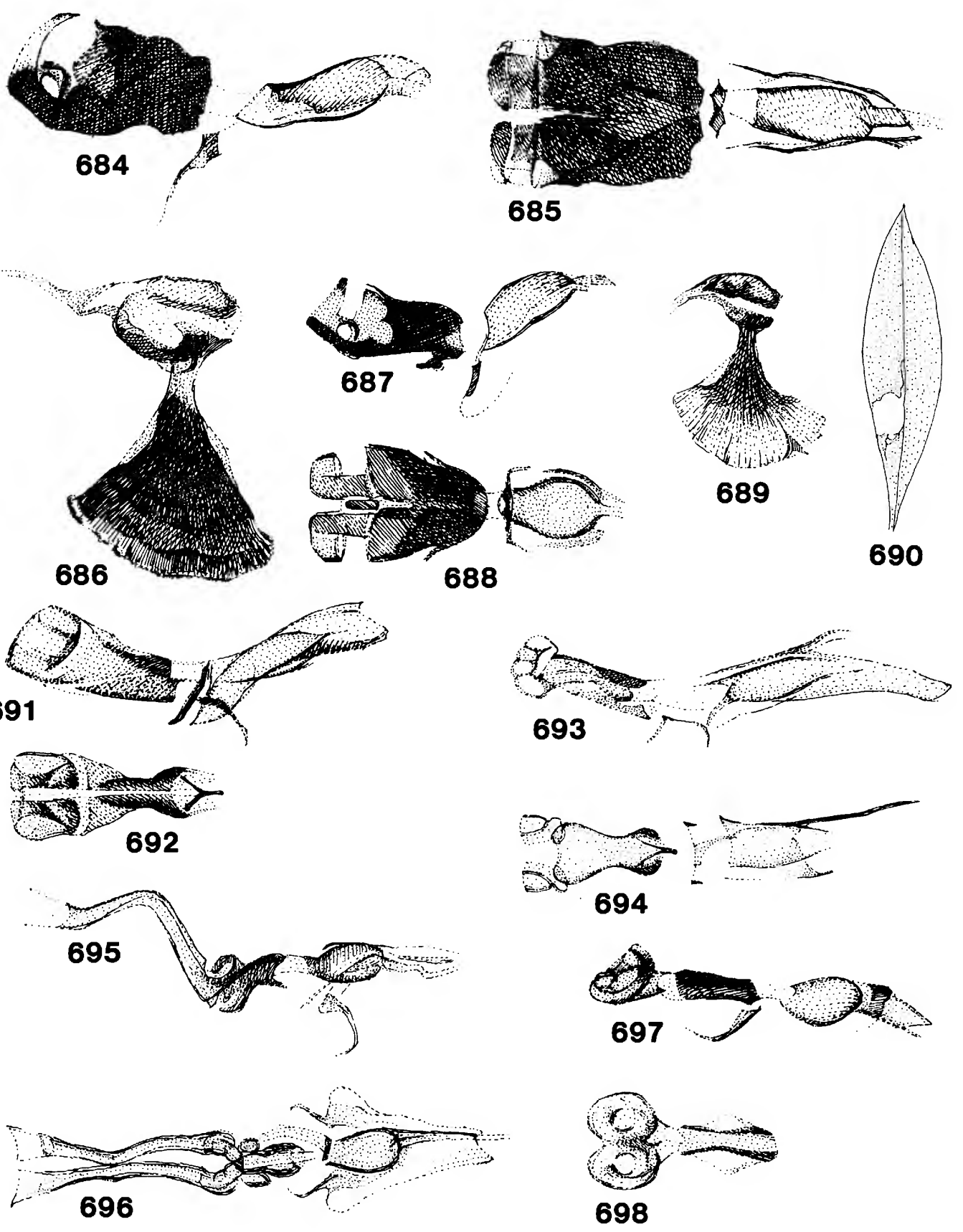

Figures 684-698.--Liriomyza bellissima: 684, Aedeagus, side view; 685 , same, ventral view; 686 , sperm pump; L. baptisiae: 687, Aedeagus, side view; 688 , same, ventral view; 689 , sperm pump; 690 , leaf mine on Lupinus sp. (California); L. chlamydata: 691, Aedeagus, side view; 692, distiphallus, ventral view (holotype); L. frigida: 693, Aedeagus, side view; 694, same, ventral view; L. elevata: 695, Aedeagus, side view; 696, same, ventral view; L. lupiniphaga: 697, Aedeagus, side view; 698, same, ventral view. 


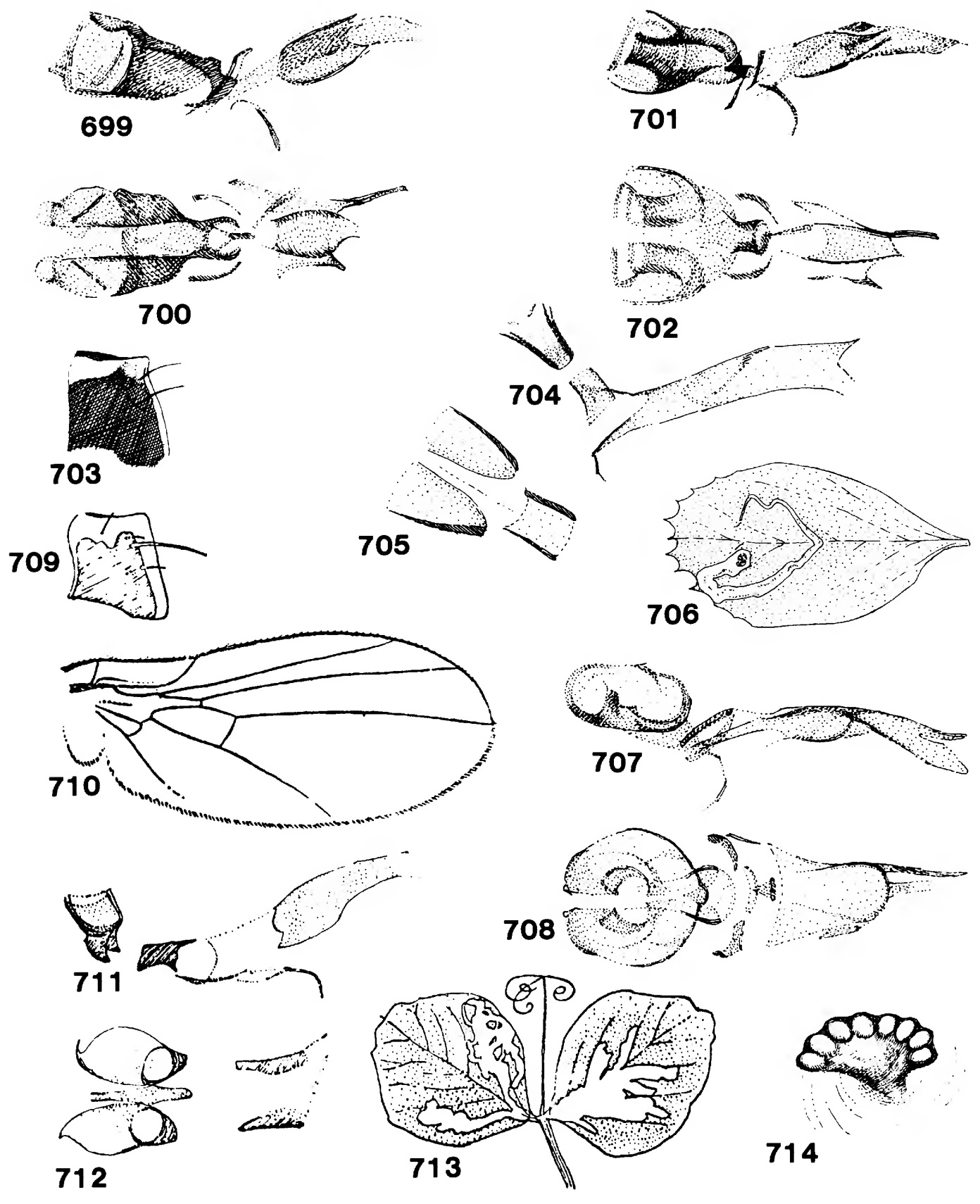

Figures 699-714.--Liriomyza artemisiae: 699, Aedeagus, side view; 700 , same, ventral view; L. paumensis:

701, Aedeagus, side view; 702, same, ventral view; L. trifoliearum: 703, Mesopleuron; 704, aedeagus, side view; 705 , distiphallus, ventral view; 706 , leaf mine on Medicago sativa; L. lupinella: 707, Aedeagus, side view; 708 , same, ventral view; L. huidobrensis: 709, Mesopleuron; 710, wing; 711, aedeagus, side view; 712 , distiphallus, ventral view; 713, leaf mines on Pisum; 714, posterior spiracles of puparium. 

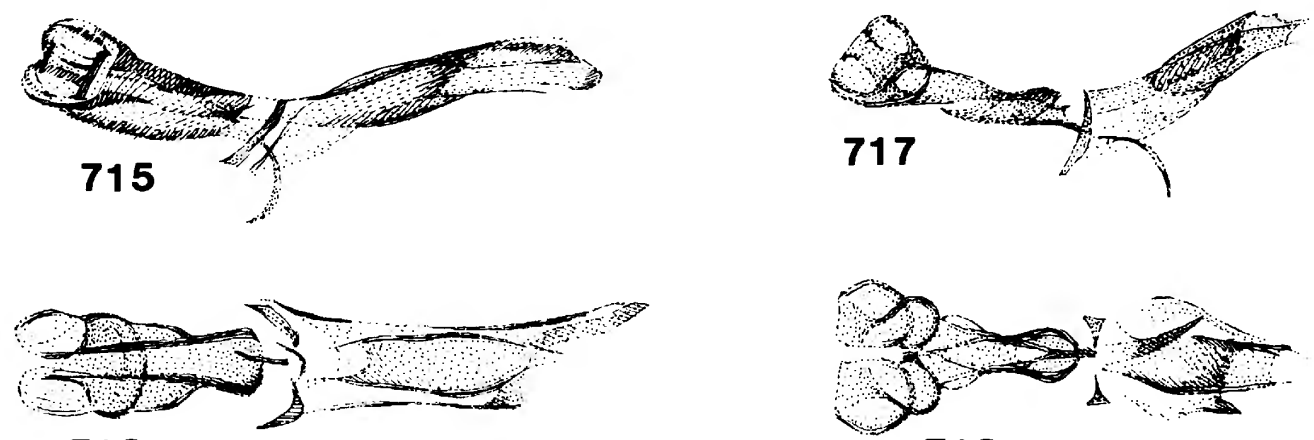

716

718
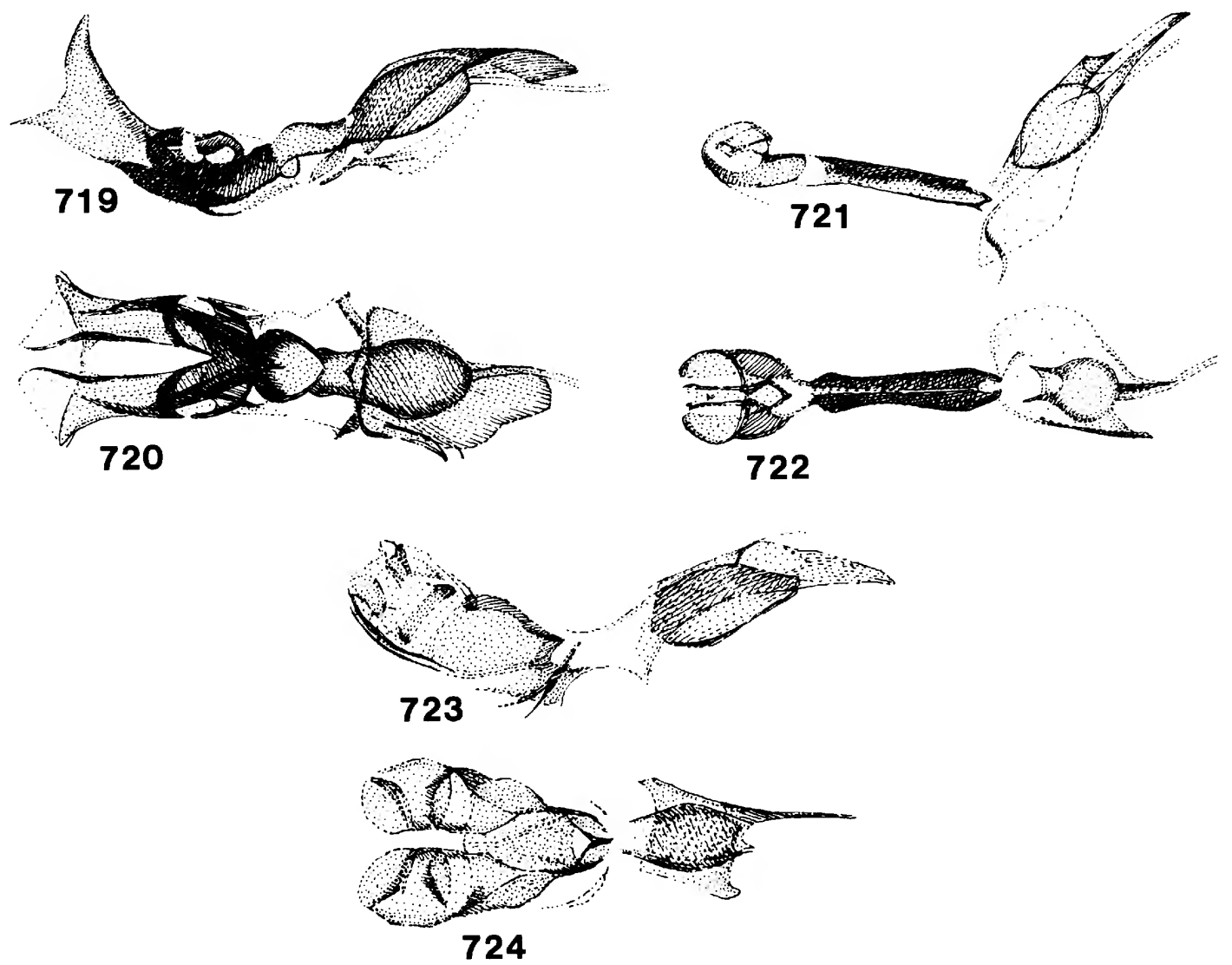

Figures 715-724.--Liriomyza fricke11a: 715, Aedeagus, side view; 716 , same, ventral view; L. californiensis: 717 , Aedeagus, side view; 718 , same, ventral view; L. denudata: 719, Aedeagus, side view; 720 , same, ventral view; L. minor: 721, Aedeagus, side view; 722 , same, ventral view; L. similis: 723 , Aedeagus, side view; 724 , same, ventral view. 

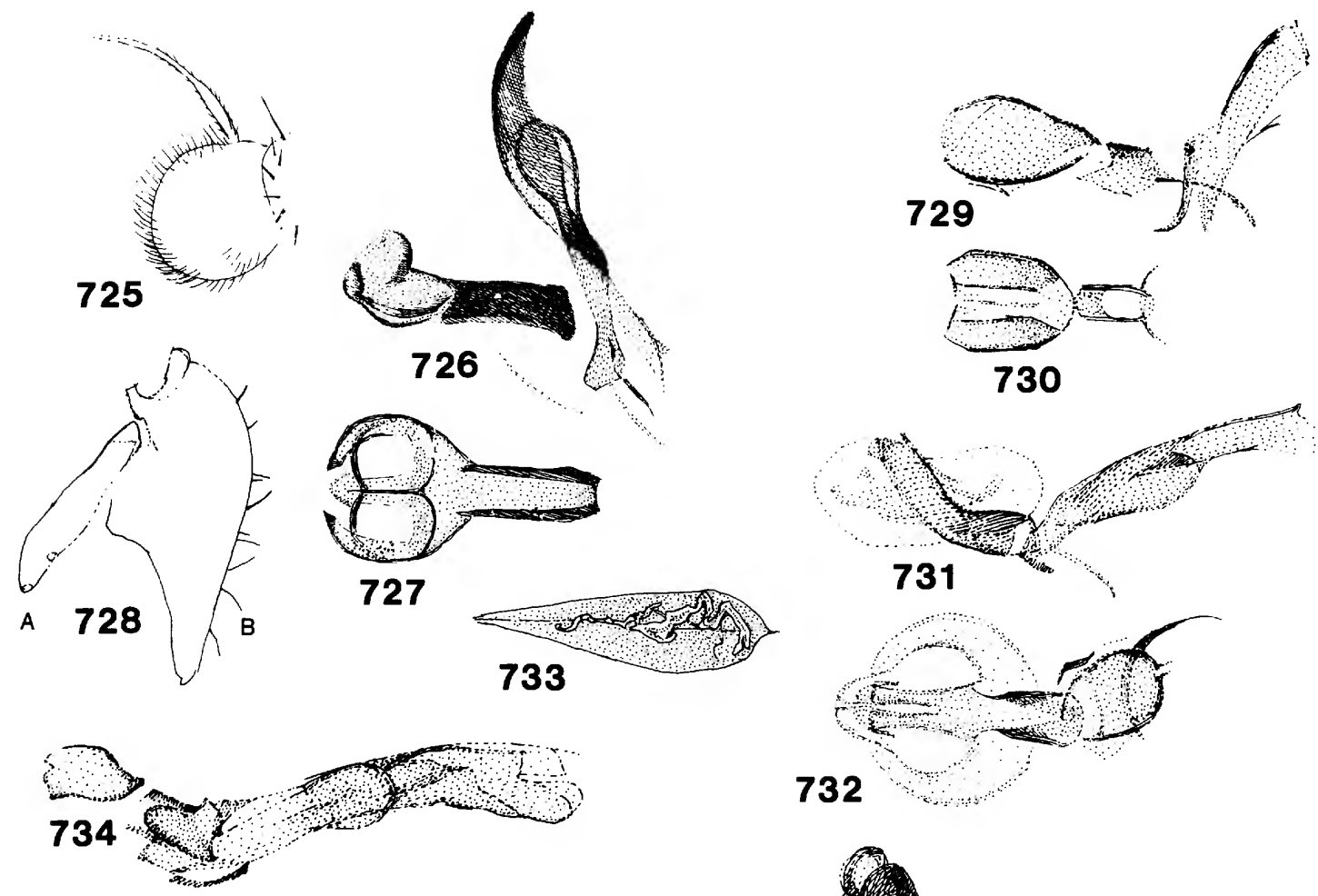

\section{2}
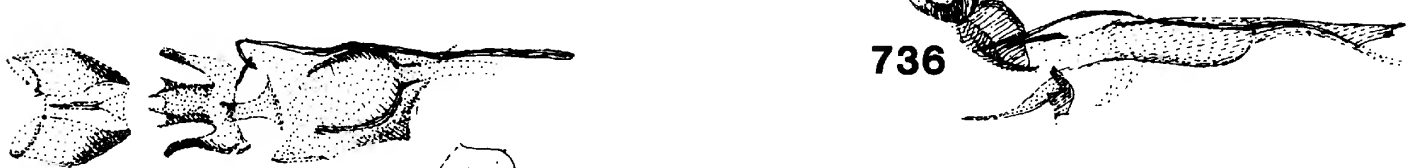

735

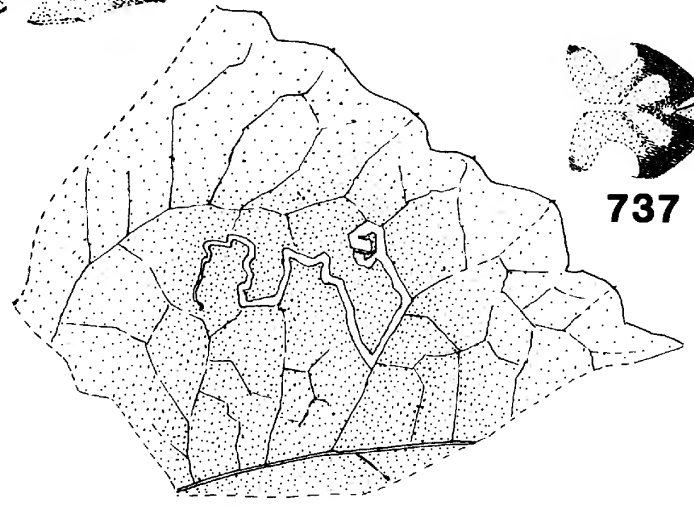

738

Figures 725-738.--Liriomyza ptarmicae: 725, Third antennal segment; 726, aedeagus, side view; 727, distiphallus, ventral view; 728 , epandrium (B) with surstylus (A); L. arnaudi: 729 , Aedeagus, side view; 730 , distiphallus, ventral view; L. lupini: 731, Aedeagus, side view; 732, distipha1lus, ventral view; 733 , leaf mine on Lupinus; $\underline{L}$. helenii: 734, Aedeagus, side view; 735, same, ventral view; $L$, arctii: 736, Aedeagus, side view

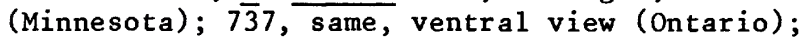
738, leaf mine on Arctium minus. 


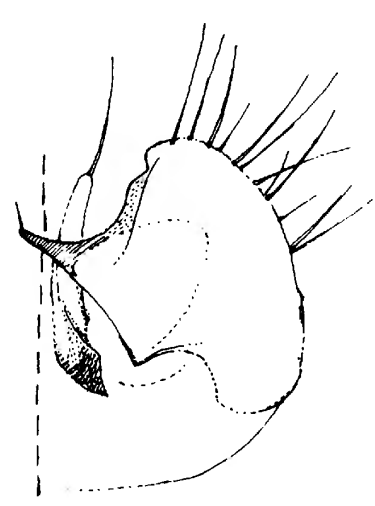

741

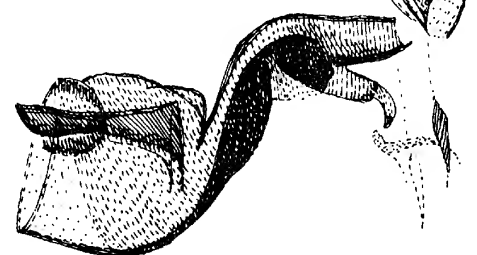

\section{9}

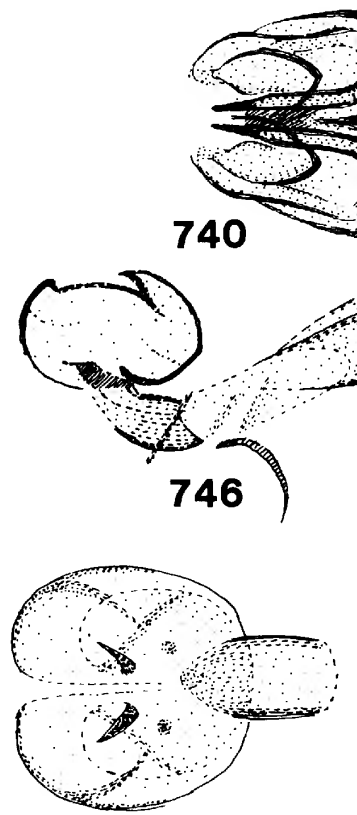

747

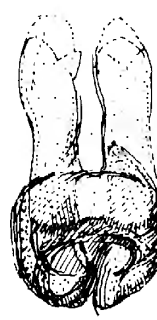

743

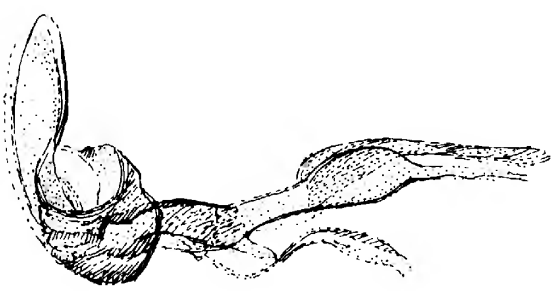

742
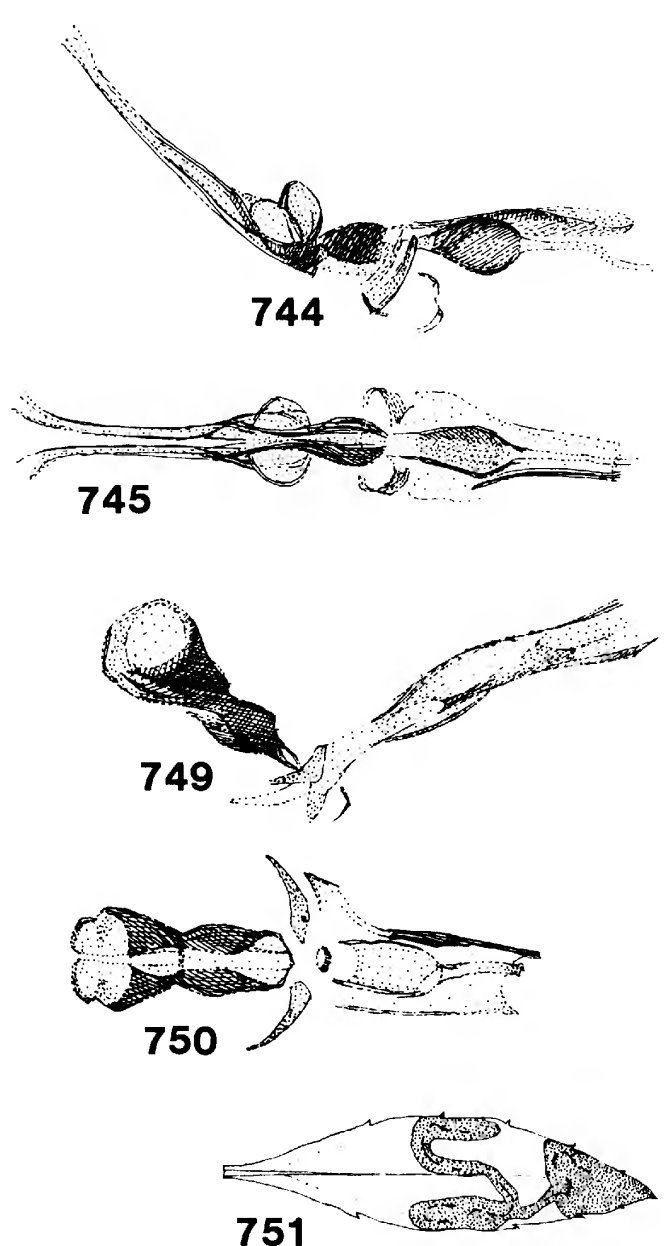

Figures 739-751.--Liriomyza septentriona1is: 739 , Aedeagus, side view; 740 , distiphallus, ventral view; 741 , epandrium with surstylus; L. pulloides: 742, Aedeagus, side view; 743, distiphallus, anteroventral view; $\underline{L}$. montel la: 744, Aedeagus, side view; 745, same, dorsal view; L. archboldi: 746, Aedeagus, side view; 747, distiphallus, ventral view; 748 , surstylus; L. baccharidis: 749 , Aedeagus, side view; 750 , same, ventral view; 751 , leaf mine on Baccharis floribunda H.B.K. (Colombia). 

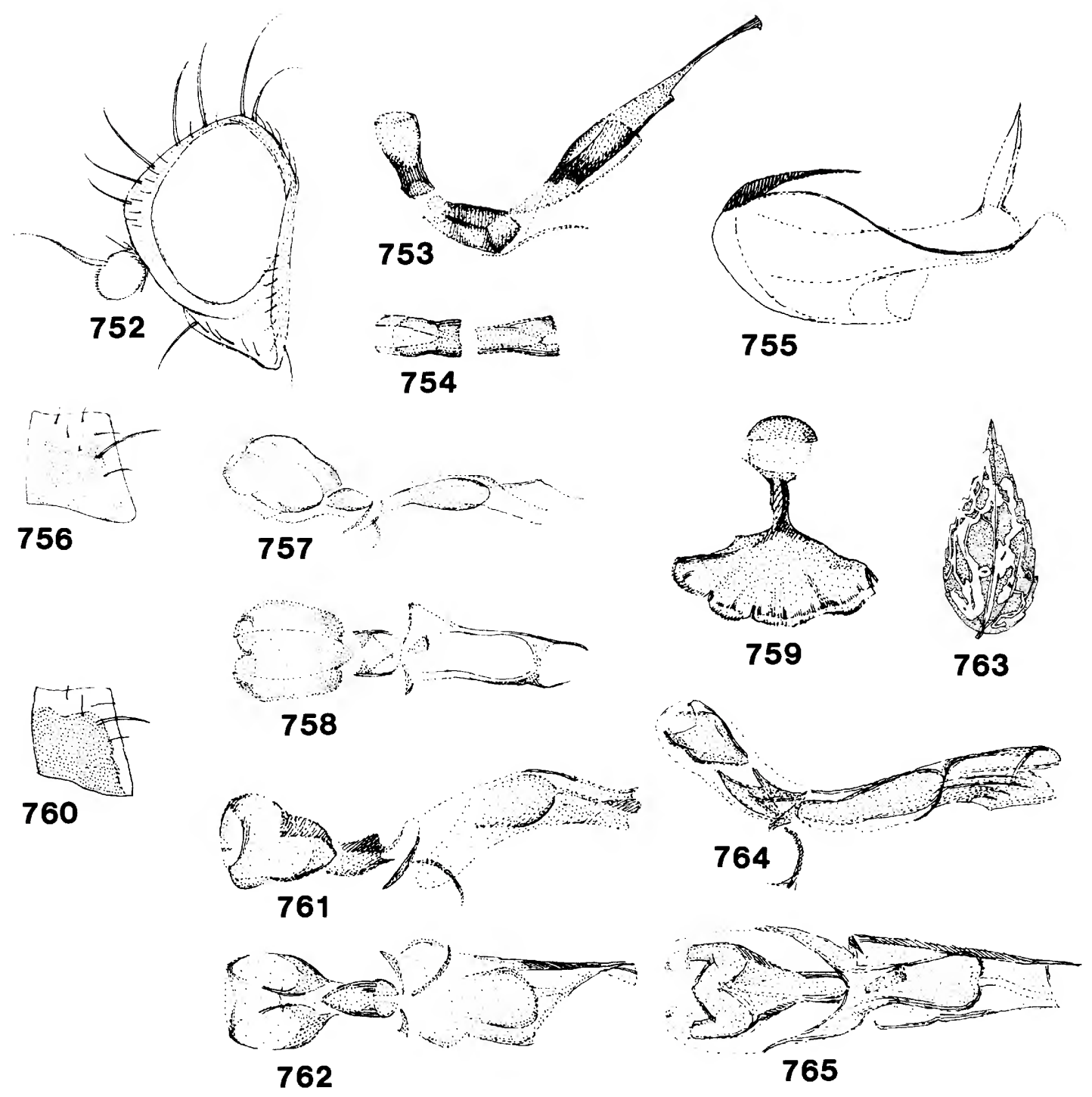

Figures 752-765.--Liriomyza deceptiva: 752, Head (holotype); L. monoensis: 753, Aedeagus, side view; 754, distiphallus, ventral view; 755 , epandrium with surstylus; L. pictella: 756, Mesopleuron; 757, aedeagus, side view; 758 , same, ventral view; 759 , sperm pump; (756-759, holotype); L. sabaziae: 760 , Mesopleuron; 761, aedeagus, side view; 762, same, ventral view; 763, leaf mines on Sabazia

(Colombia); L. montis: 764, Aedeagus, side view; 765, same, ventral view. 

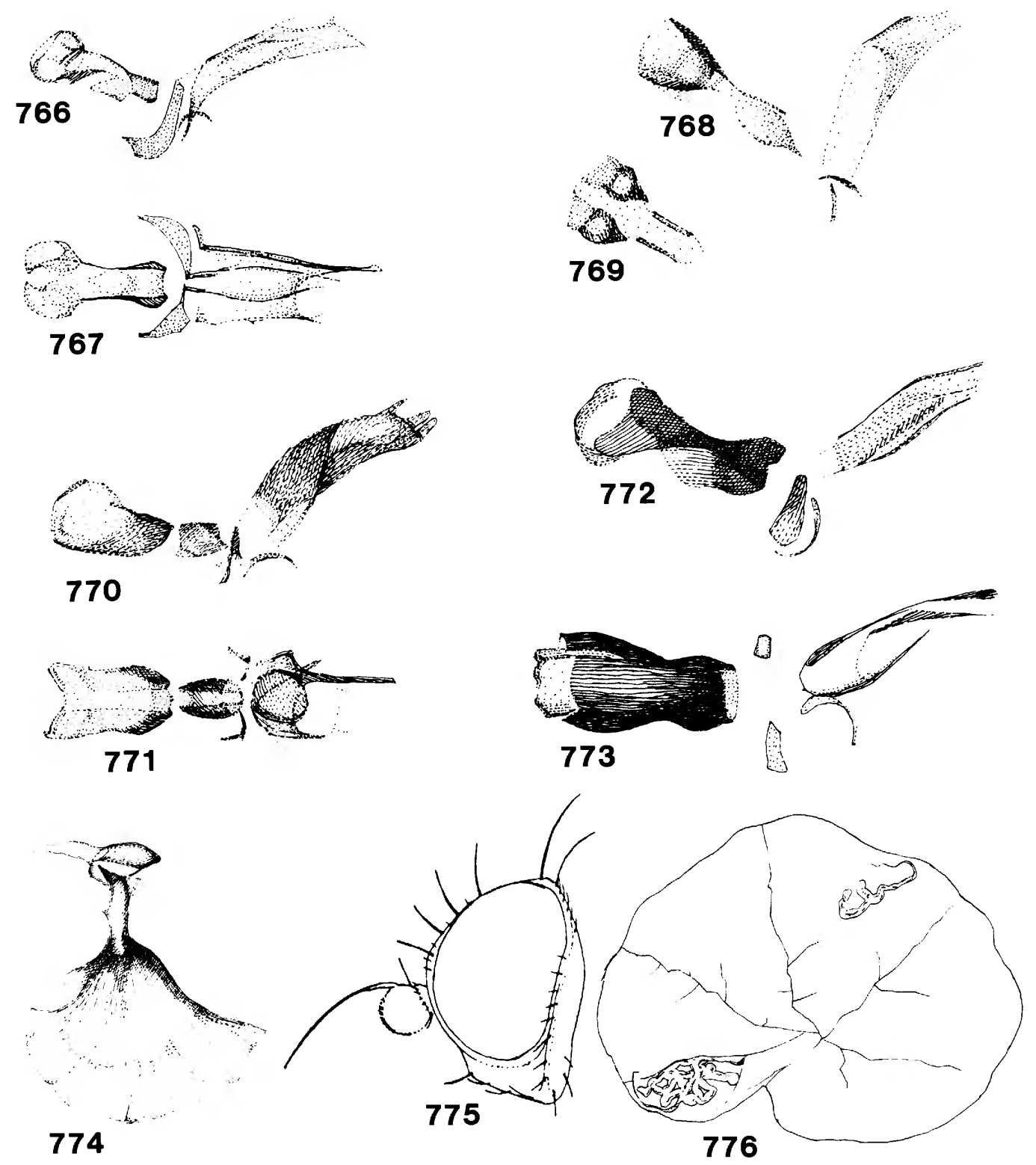

Figures 766-776.--Liriomyza helianthi: 766, Aedeagus, side view; 767 , same, ventral view; L. frommeri: 768, Aedeagus, side view; 769, distiphallus, ventral view; L. specifica: 770 , Aedeagus, side

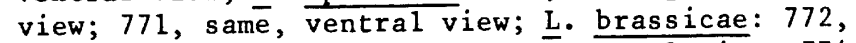
Aedeagus, side view; 773, same, ventral view; 774, sperm pump; 775, head; 776, leaf mines on Tropaeolum. 


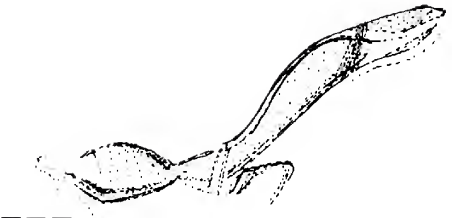

777
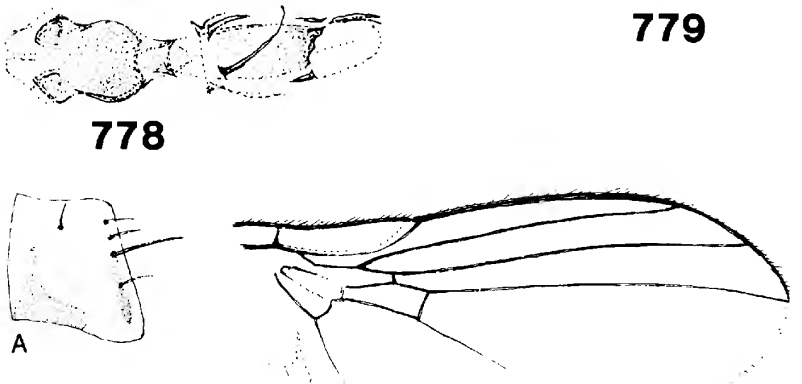

782

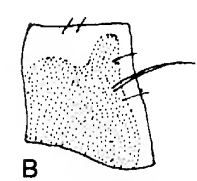

781
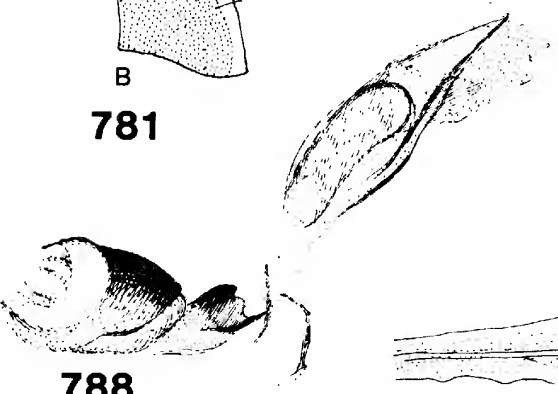

788

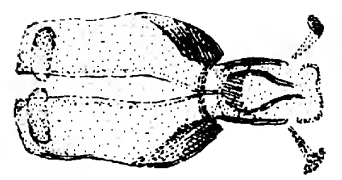

789

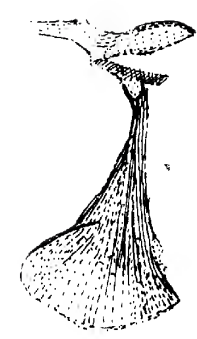

779
785

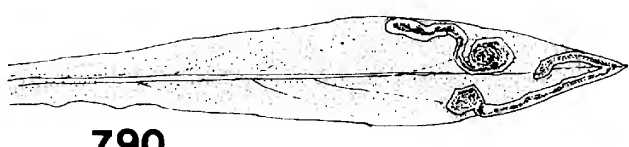

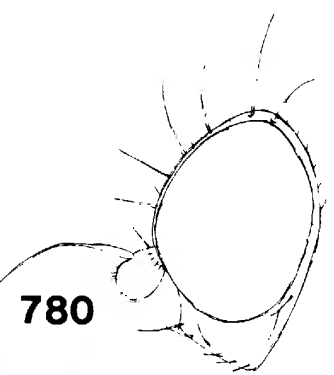

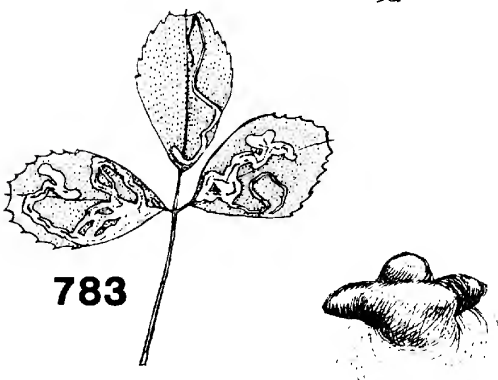

784

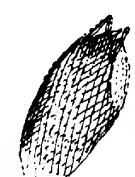

786

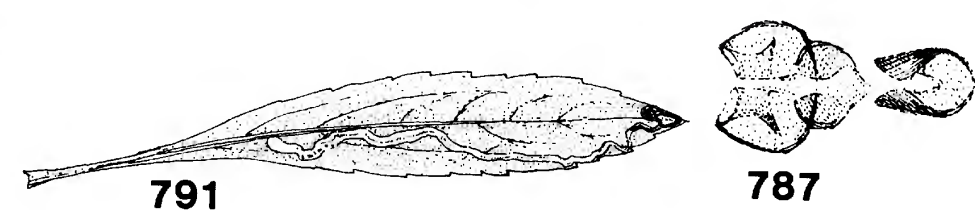

Figures 777-791.--Liriomyza sativae: 777, Aedeagus,

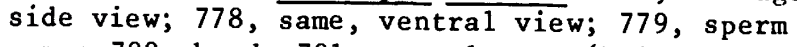
pump; 780, head; 781, mesopleuron (holotypes of $\mathrm{L}$. munda (A) and L. propepusilla (B))); 782, wing; $7 \overline{8} 3$, leaf mines on Medicago sativa; 784 , posterior spiracles of puparium; L. venegasiae: 785 , Mesopleuron; 786, aedeagus, side view; 787, distiphallus, ventral view; L. eupatorii: 788 , Aedeagus, side view; 789, distiphallus, ventral view; 790, leaf mines on Aster (California); 791, leaf mine on Solidago (Mississippi). 

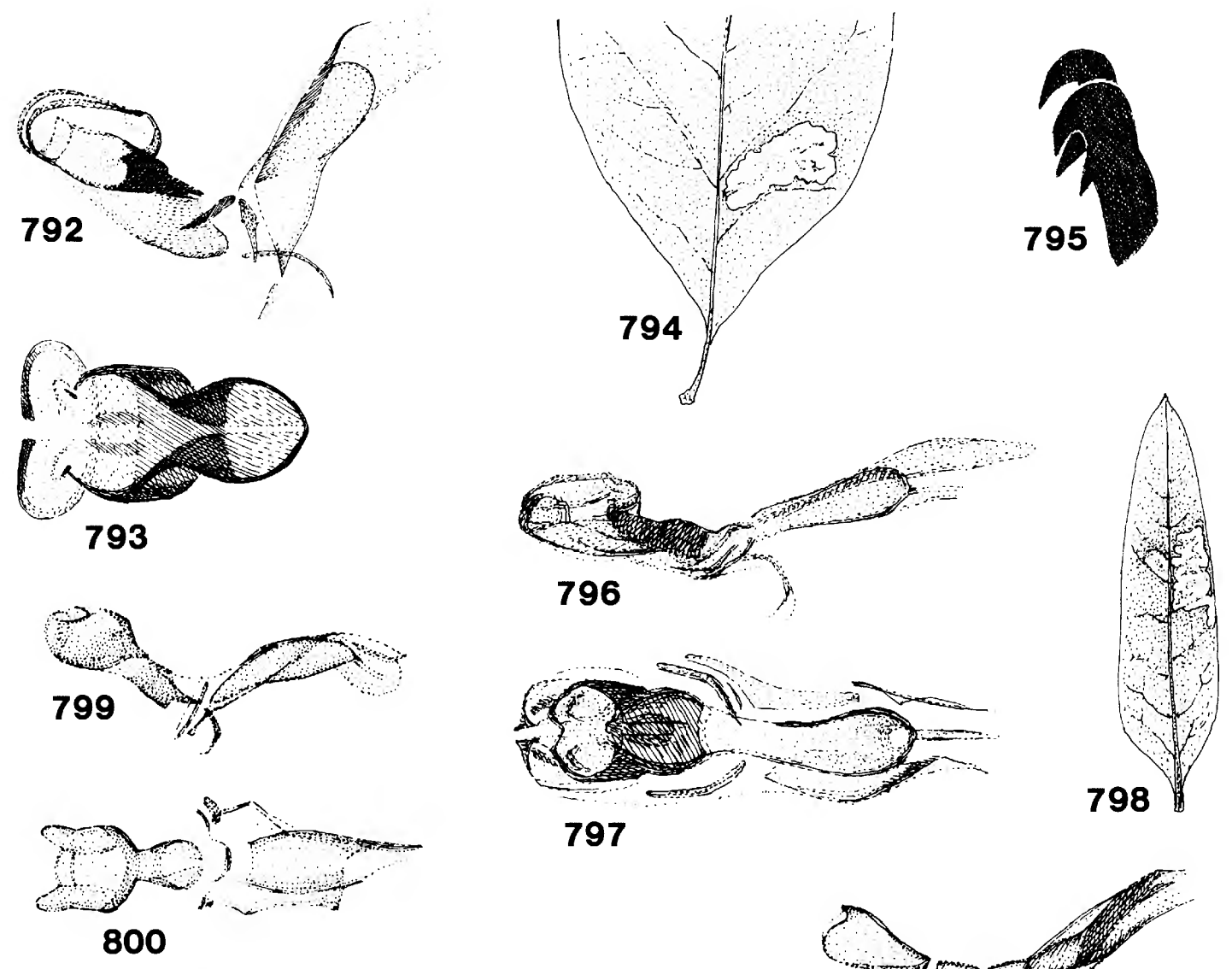

797
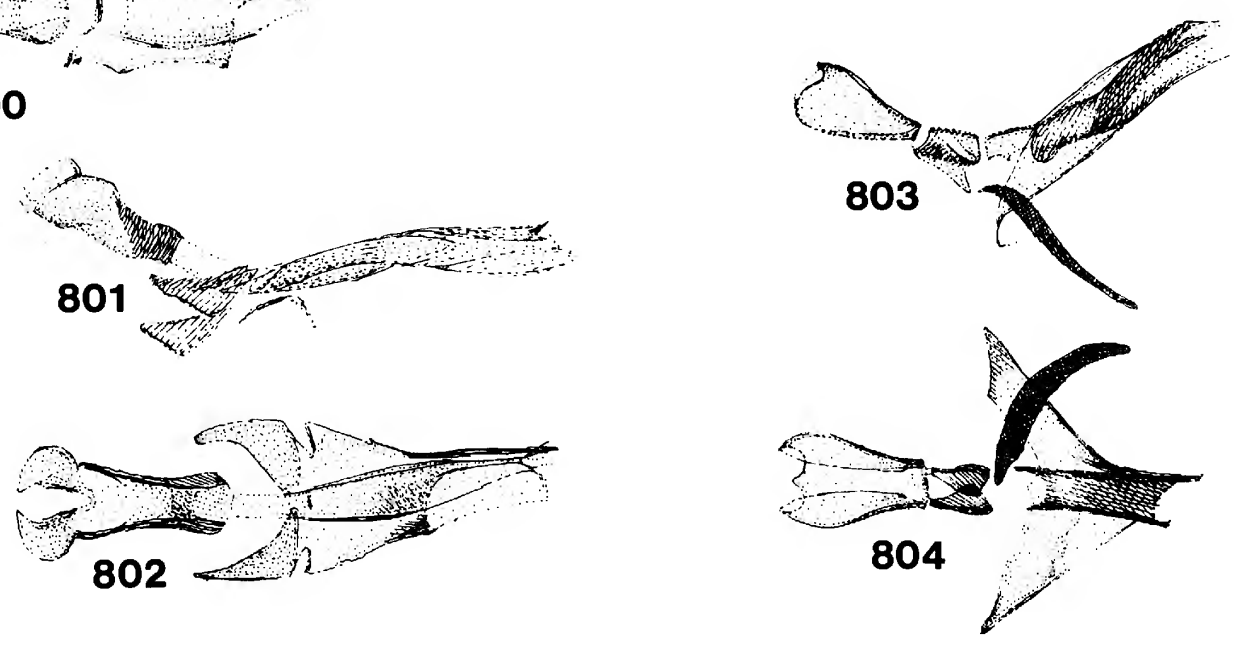

Figures 792-804.--Liriomyza asclepiadis: 792, Aedeagus, side view; 793 , distiphallus, ventral view; 794, leaf mine on Asclepias ovalifolia (Minnesota); 795, larval mouth parts; L. subasclepiadis: 796 , Aedeagus, side view; 797 , same, ventral view; 798 , leaf mine on Asclepias sp.; L. schlingeri: 799, Aedeagus, side view; 800 , same, ventral view; $\underline{L}$. virginica: 801 , Aedeagus, side view; 802, same, ventral view; L. zinniae: 803, Aedeagus, side view; 804 , same, ventral view. 

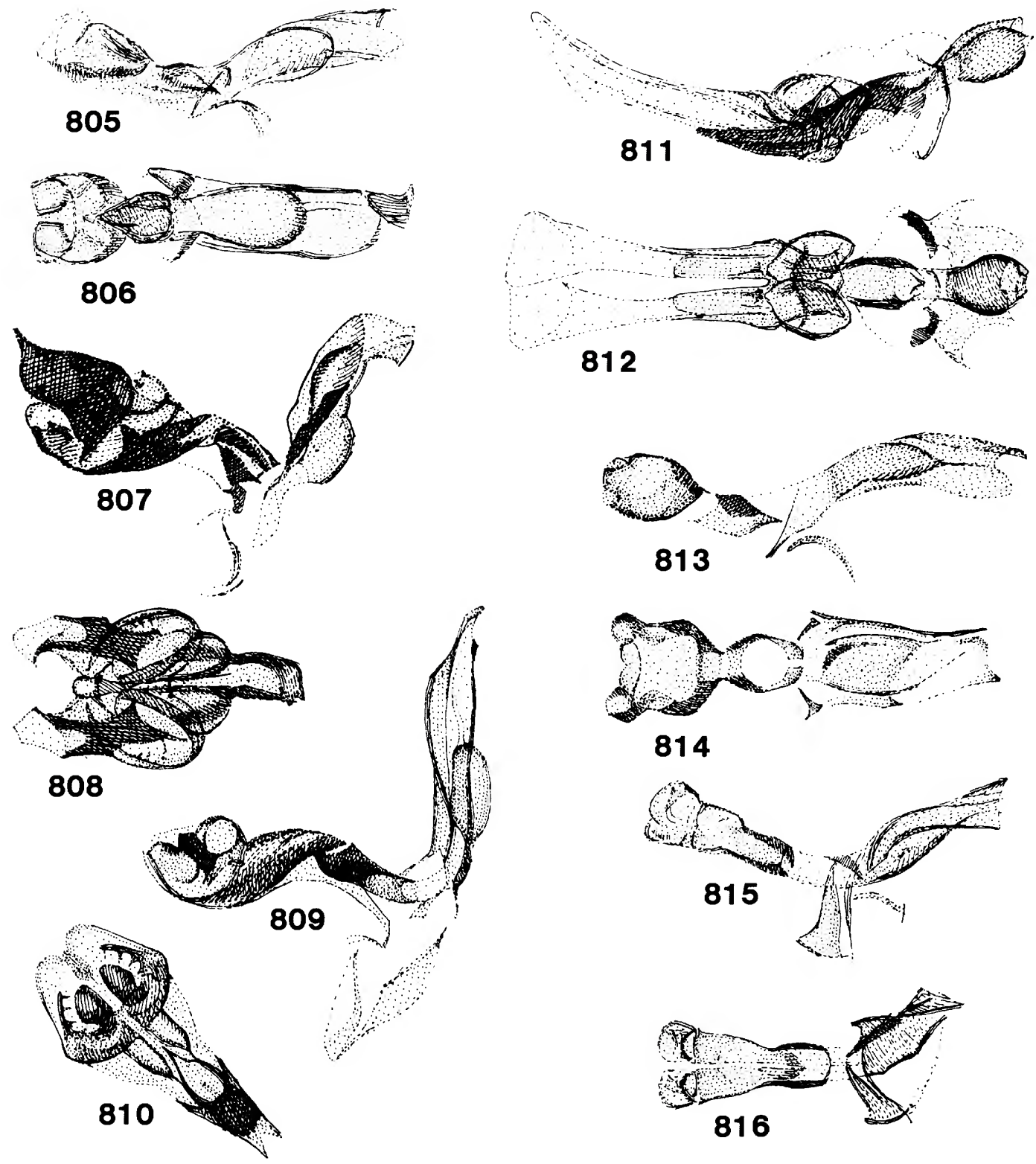

814
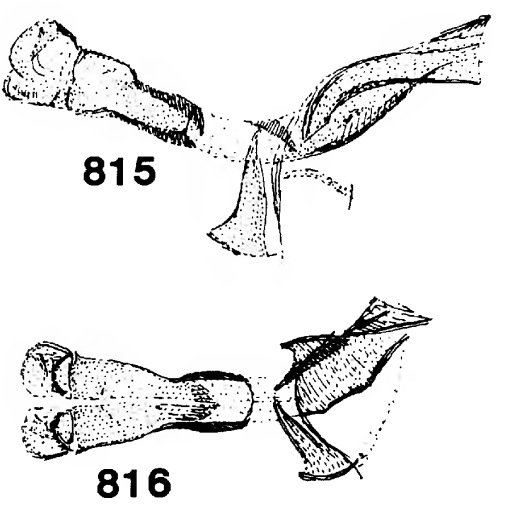

Figures 805-816.--Liriomyza temperata: 805, Aedeagus, side view (paratype, North Carolina, Roan Mts.); 806 , same, ventral view (holotype, North Carolina, Smoky Mts.); L. abnormis: 807, Aedeagus, side view; 808 , same, ventral view; L. graminacea: 809 , Aedeagus, side view; 810 , same, ventral view; $L$. splendens: 811 , Aedeagus, side view; 812, same, ventral view; $L$. venturensis: 813 , Aedeagus, side view; 814, same, ventral view; L. texella: 815, Aedeagus, side view; 816 , same, ventral view. 


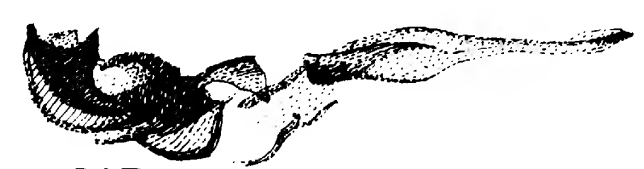

817
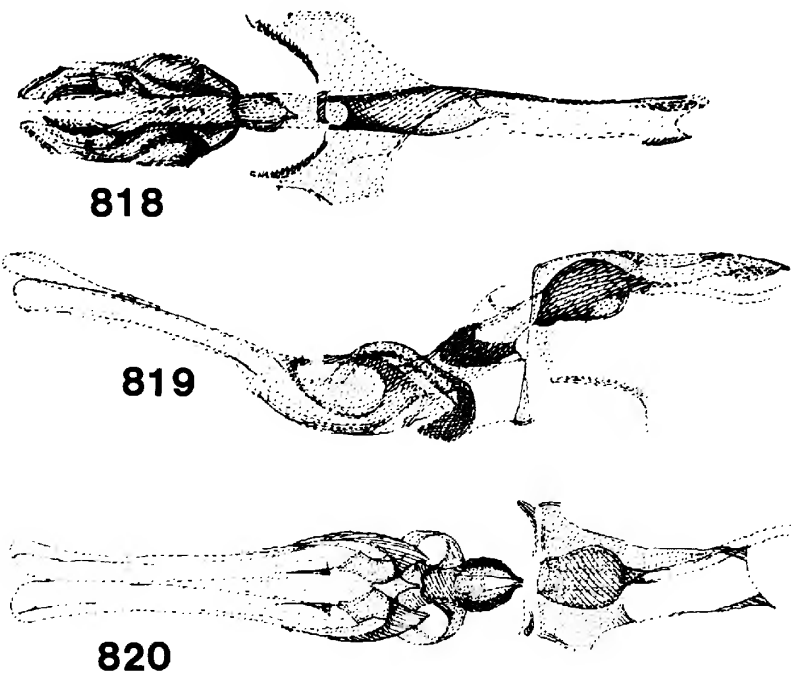

820

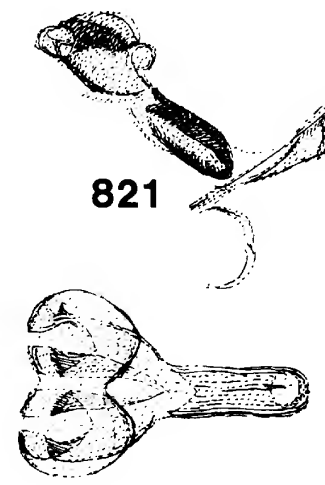

822
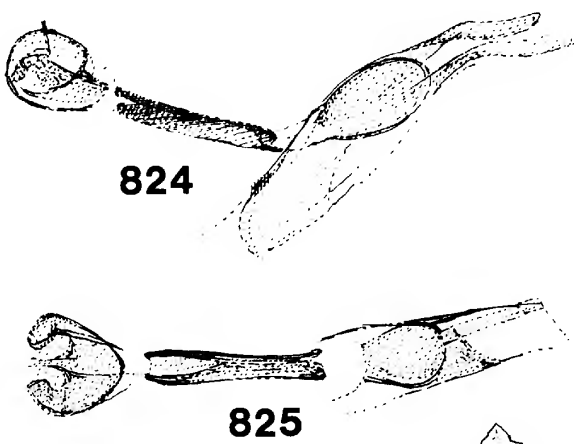

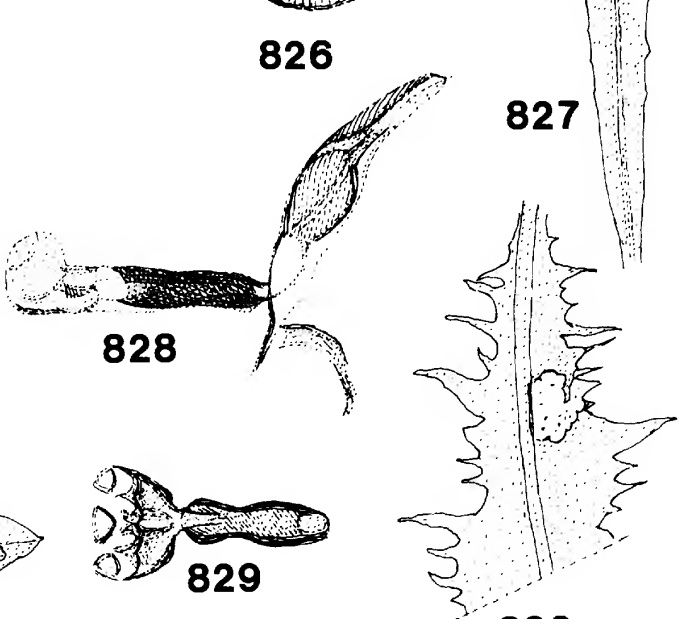

830

Figures 817-830.--Liriomyza chemsaki: 817, Aedeagus, side view; 818, same, ventral view; L. tubula: 819 , Aedeagus, side view; 820 , same, ventral view; L. lathyri: 821 , Aedeagus, side view; 822 , distiphallus, ventral view; 823, leaf mine on Lathyrus sp.

(Arkansas); L. endiviae: 824, Aedeagus, side view; 825 , same, ventral view; 826, sperm pump (same scale as aedeagus); 827 , leaf mine on Lactuca serriola; (824-827, Washington); L. taraxaci: 828 , Aedeagus, side view (Germany); $82 \overline{9}$, distiphallus, ventral view (Ontario); 830 , leaf mine on Taraxacum (Wisconsin). 

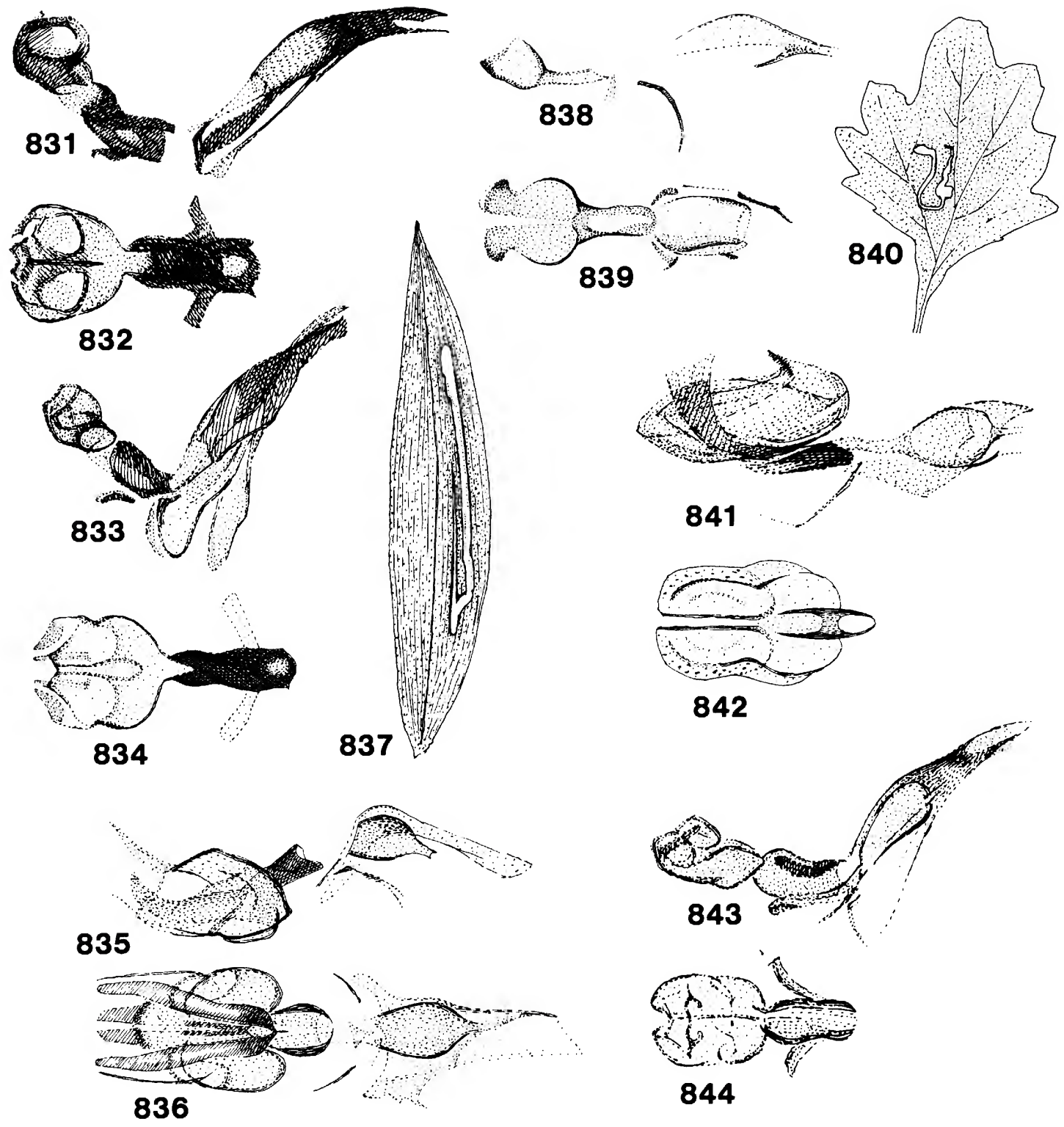

844

Figures 831-844.--Liriomyza togata: 831, Aedeagus, side view; 832 , distiphallus, ventral view (as $\underline{L}$. douglasii, California); L. 1ima: 833, Aedeagus, side view; 834, distipha1̄lus, ventral view; $\underline{L}$. smilacinae: 835, Aedeagus, side view; 836, same, ventral view; 837 , leaf mine on Smilacina

(Alberta); L. trifolii: 838, Aedeagus, side view;

839 , same, ventral view; 840 , leaf mine on

Chrysanthemum; L. fricki: 841 , Aedeagus, side view;

842, distiphallus, ventral view; L. allia: 843 ,

Aedeagus, side view; 844, distiphällus, ventral

view. 

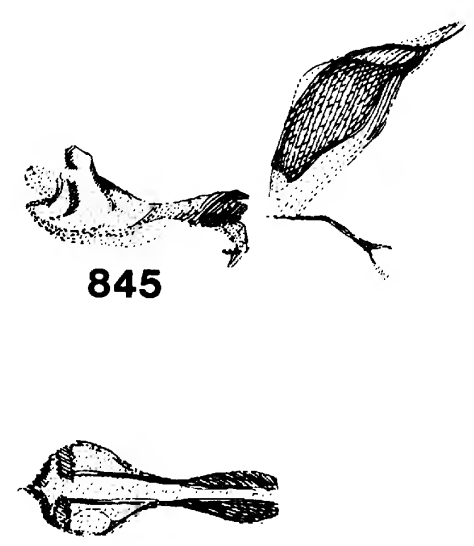

846

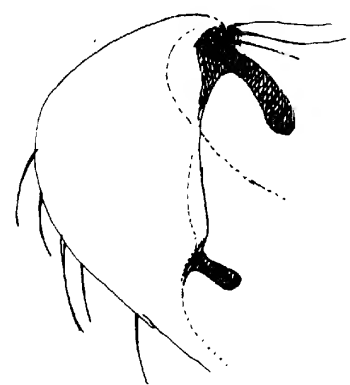

847
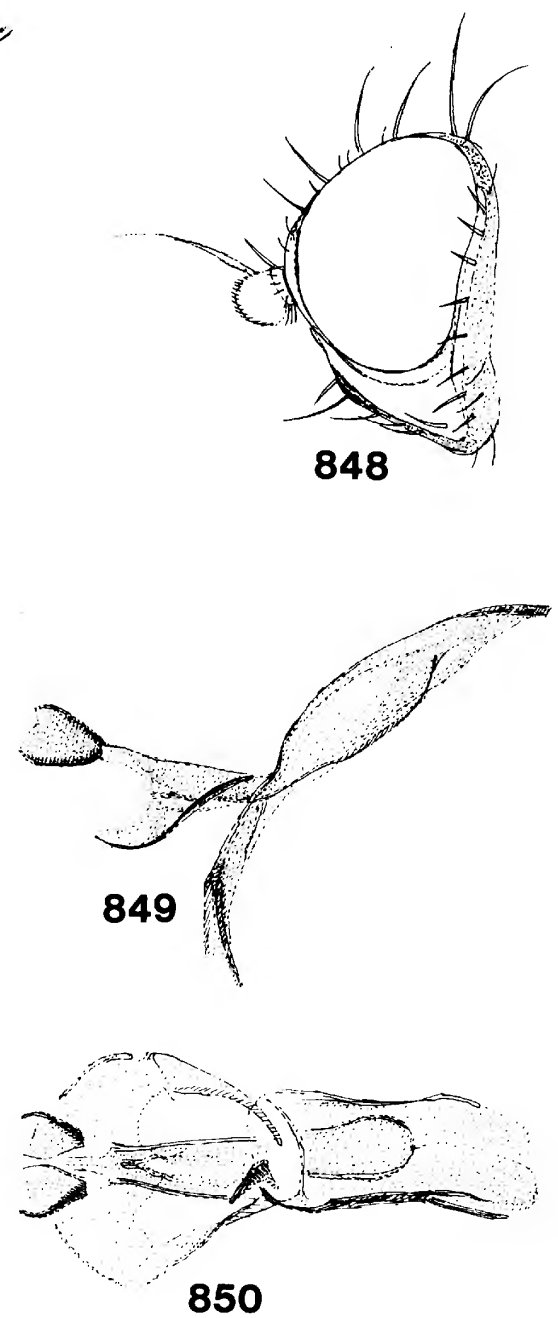

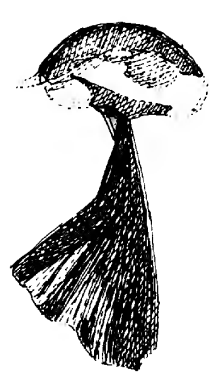

851

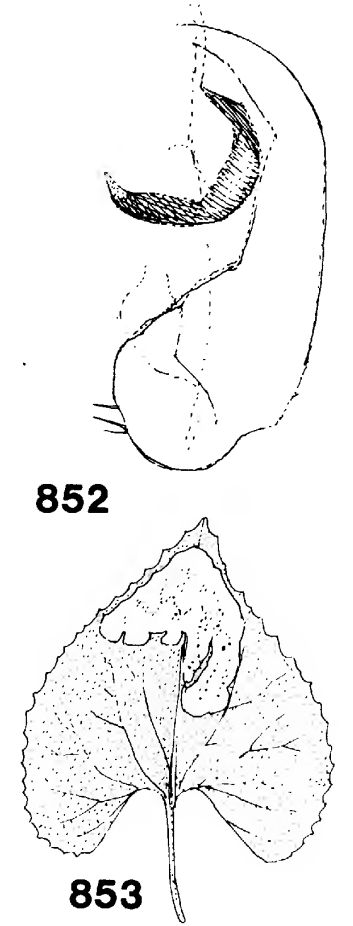

Figures 845-853.--Galiomyza turneri: 845, Aedeagus, side view; 846 , distiphallus, ventral view; 847 , epandrium; G. violivora: 848 , Head; 849 , aedeagus, side view; 850 , same, ventral view; 851 , sperm pump; 852, epandrium; 853, leaf mine on Viola sororia. 

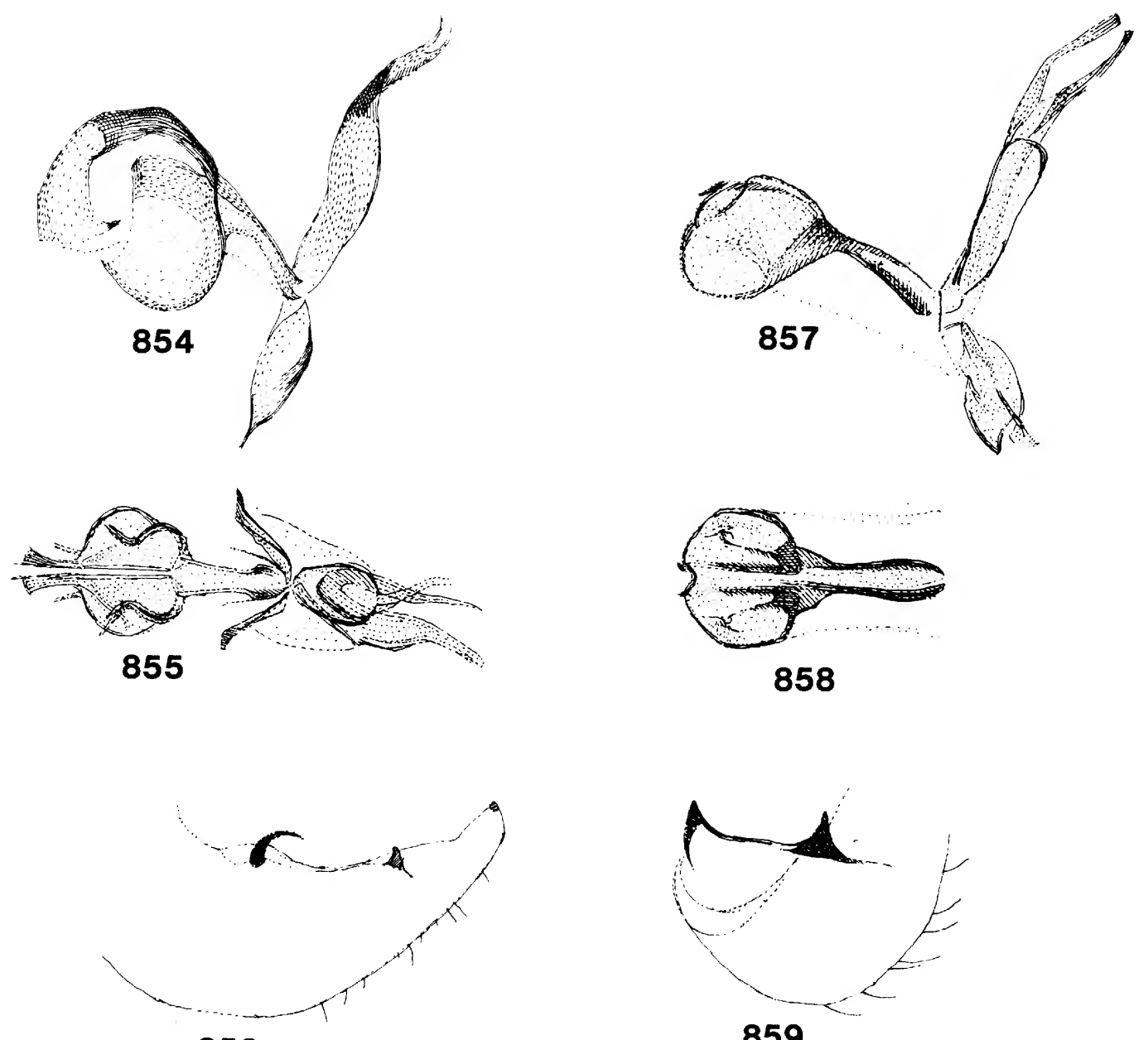

856

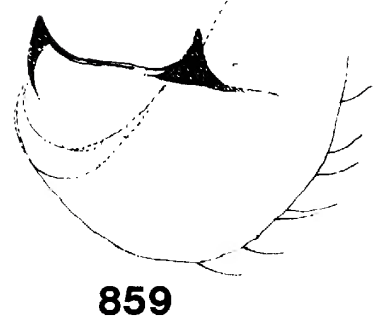

Figures 854-859.--Galiomyza galiivora: 854, Aedeagus, side view; 855 , same, ventral view; 856 , epandrium; (854-856, Alberta); G. vockerothi: 857, Aedeagus, side view; 858 , distiphallus, ventral view; 859 , epandrium. 

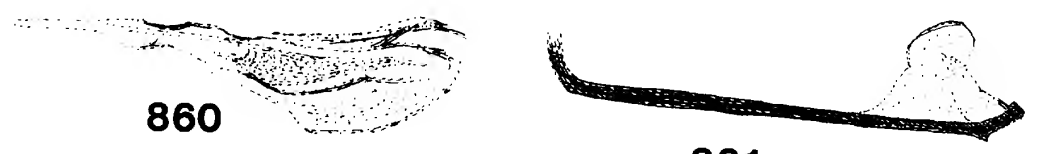

861

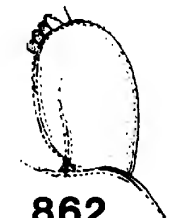

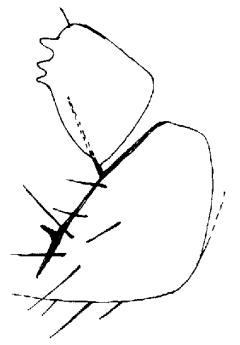

863
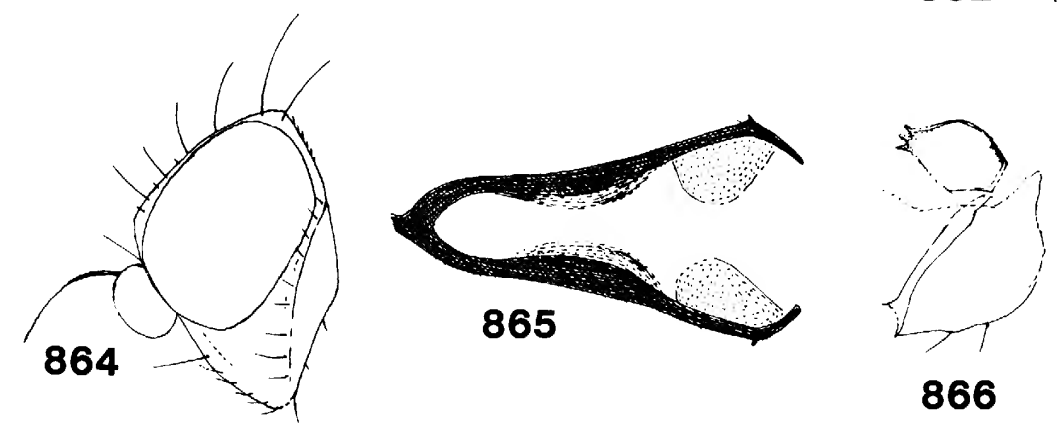

866
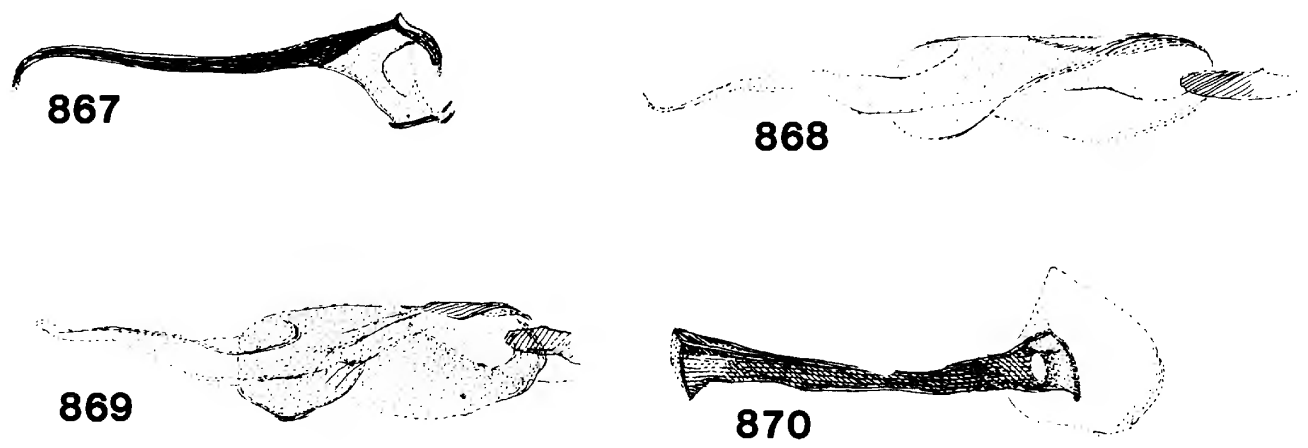

Figures 860-870.--Haplopeodes kefi: 860, Aedeagus, side view; 861 , hypandrium; 862 , surstylus;

(860-862, paratype); H. philoxeri: 863, Head; 864 , hypandrium; 865 , surstylus; $\underline{\mathrm{H}}$. minutus: 866 ,

Surstylus; 867 , hypandrium; $868 \overline{\text {, aedeagus, }}$

side view (ex Chenopodium, Florida); 869, same,

side view (on Amaranthus, California); 870, sperm pump. 

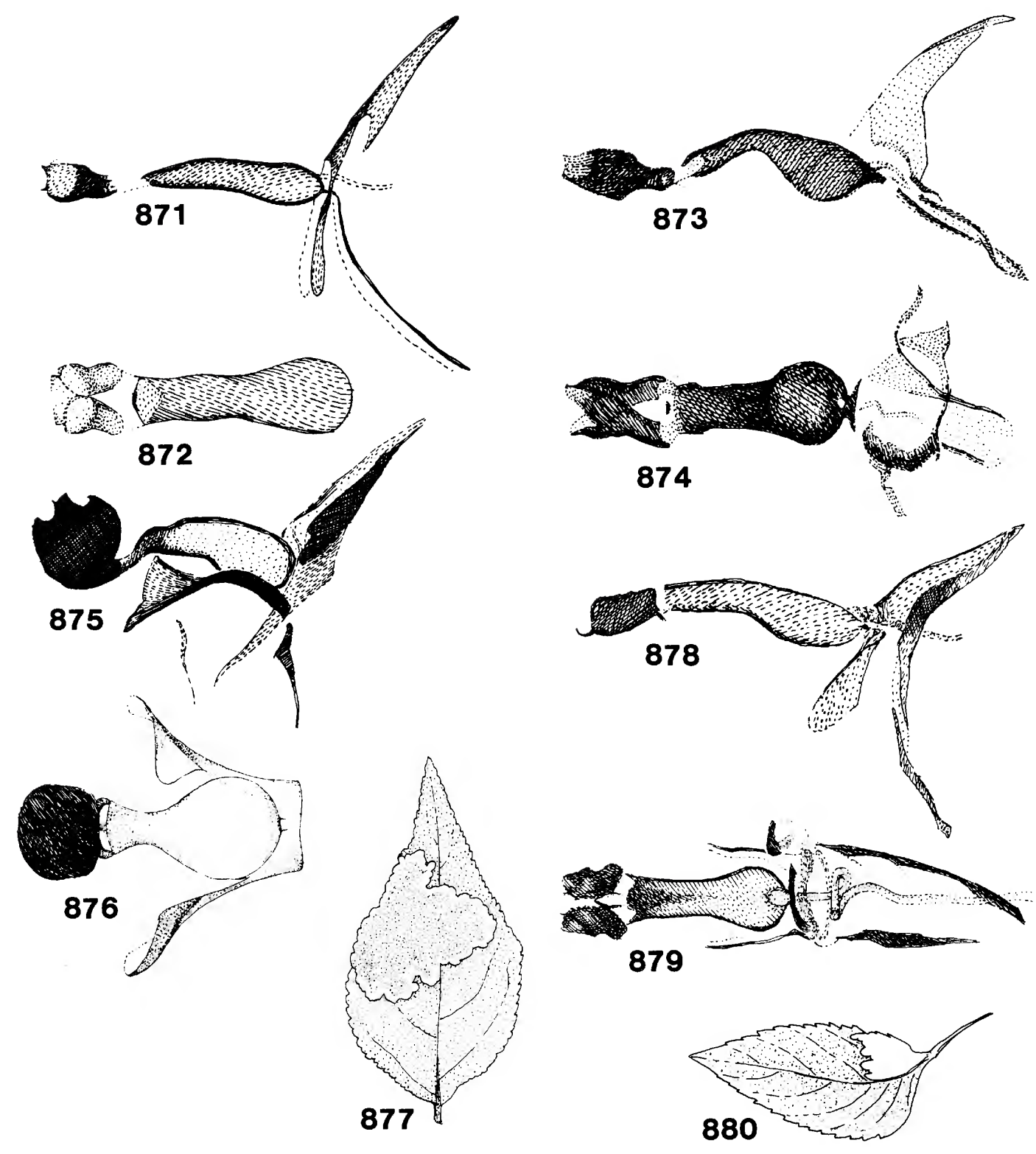

Figures 871-880.--Calycomyza minor: 871, Aedeagus, side view; 872 , distiphallus, ventral view; $\underline{\text { C. }}$ irwini: 873 , Aedeagus, side view; 874, same, ventral view; $C$. lantanae: 875 , Aedeagus, side view; 876 , dist̄iphallus, ventra1 view; 877 , leaf mine on Lantana camara; C. platyptera: 878, Aedeagus, side view (Florida); 879, same, ventral view (California); 880, leaf mine on Bidens pilosa. 


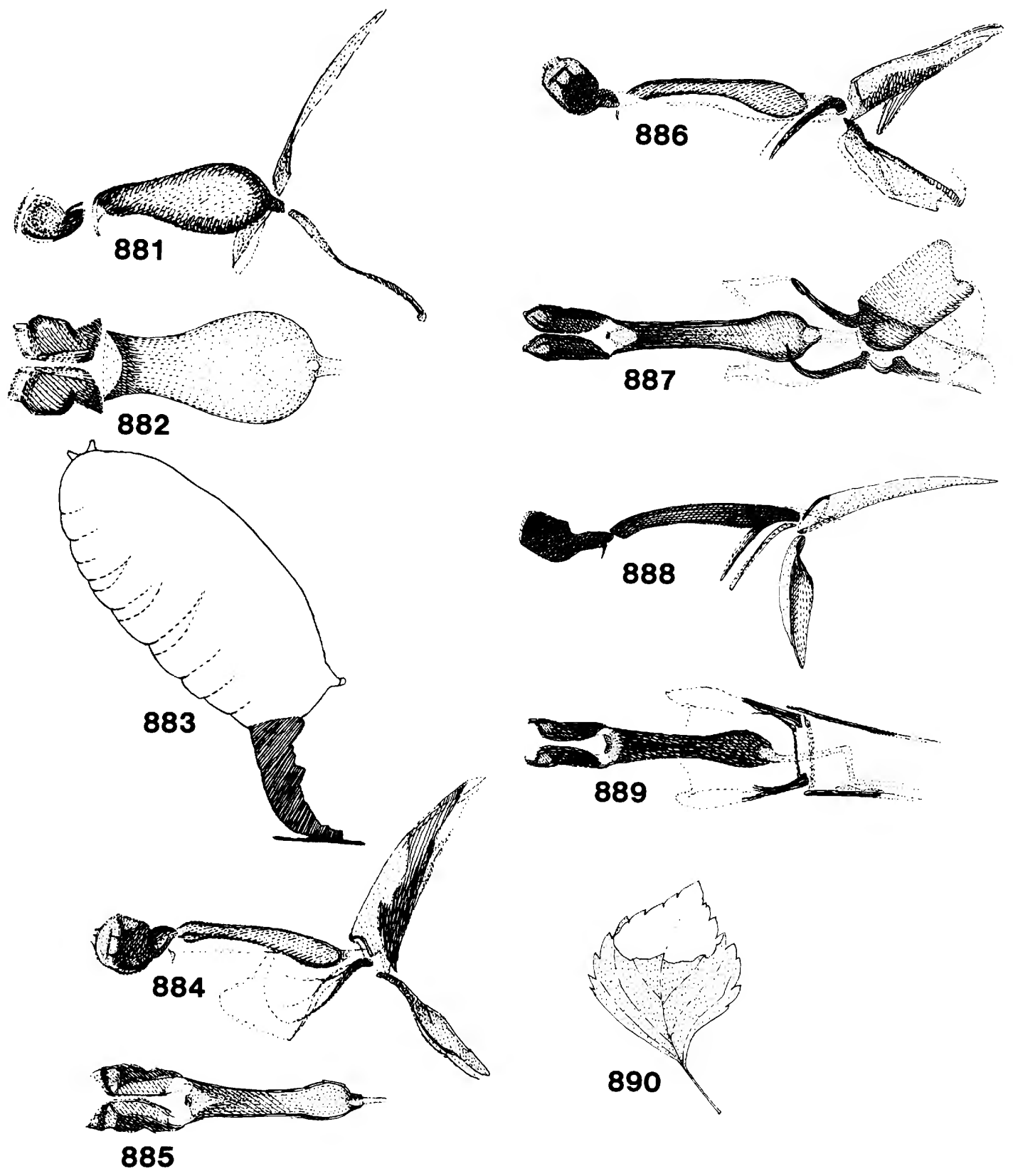

Figures 881-890.--Calycomyza promissa: 881 , Aedeagus, side view; 882 , distiphallus, ventral view; 883 , puparium; C. jucunda: 884, Aedeagus, side view; 885, distiphallus, ventral view (holotype).

Phytobia coronata: 886 , Aedeagus, side view; 887 , same, ventral view (lectotype of $\underline{P}$. coronata). C. menthae: 888, Aedeagus, side view (holotype); $88 \overline{9}$, same, ventral view (paratype, Ontario, genitalia slide No. 1786, BMNH); 890, leaf mine on Hyptis mutabilis (Florida). 

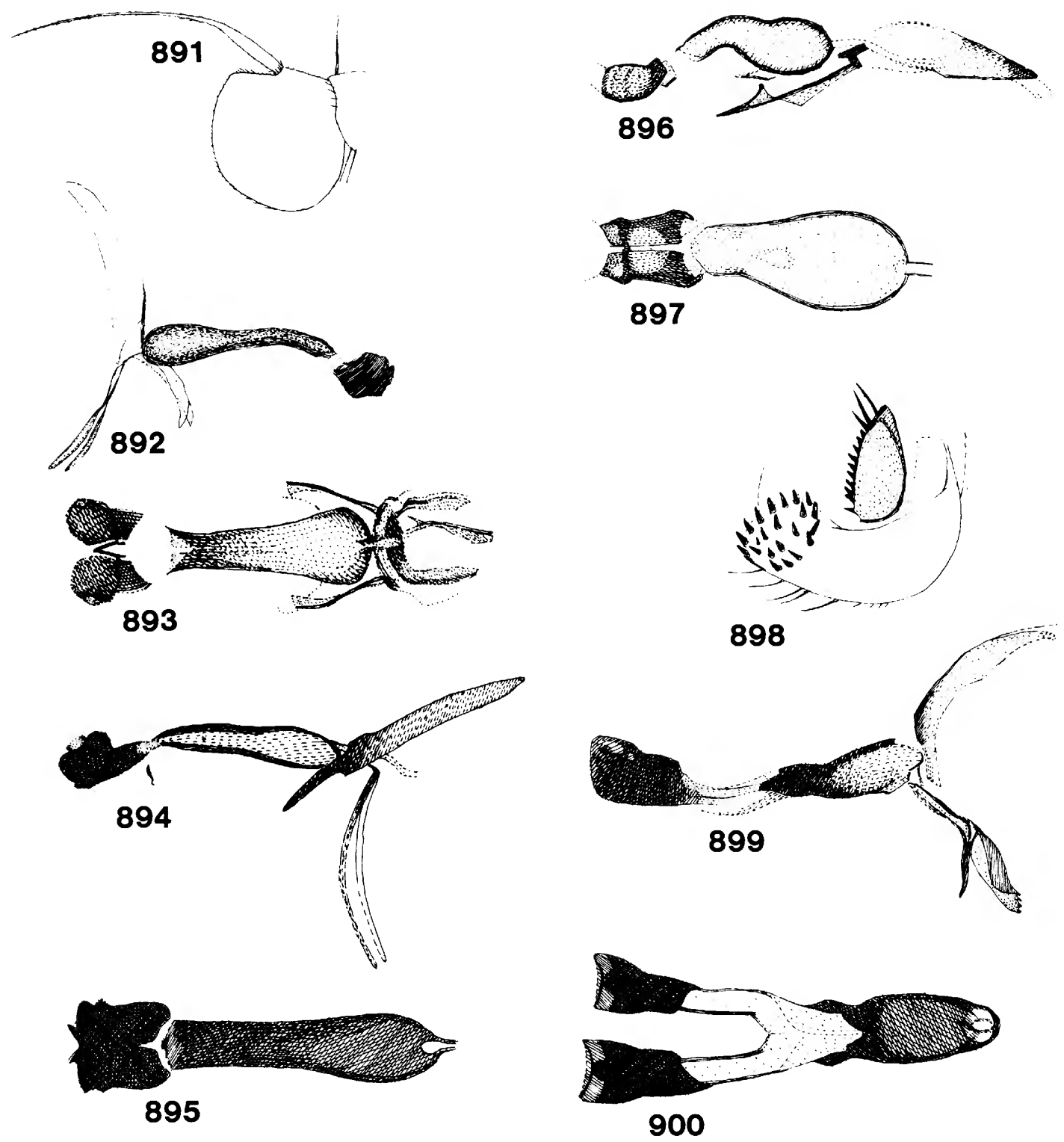

Figures 891-900.--Calycomyza humeralis: 891, Third antennal segment; 892, aedeagus, side view (Europe); 893, same, ventral view (California); . solidaginis: 894, Aedeagus, side view; 895, distiphallus, ventral view (Canada); C. obscura: 896, Aedeagus, side view; 897, distiphallus, ventral view; 898 , epandrium with surstylus; $\underline{C}$. gigantea: 899, Aedeagus, side view; 900 , distiphallus, ventral view. 


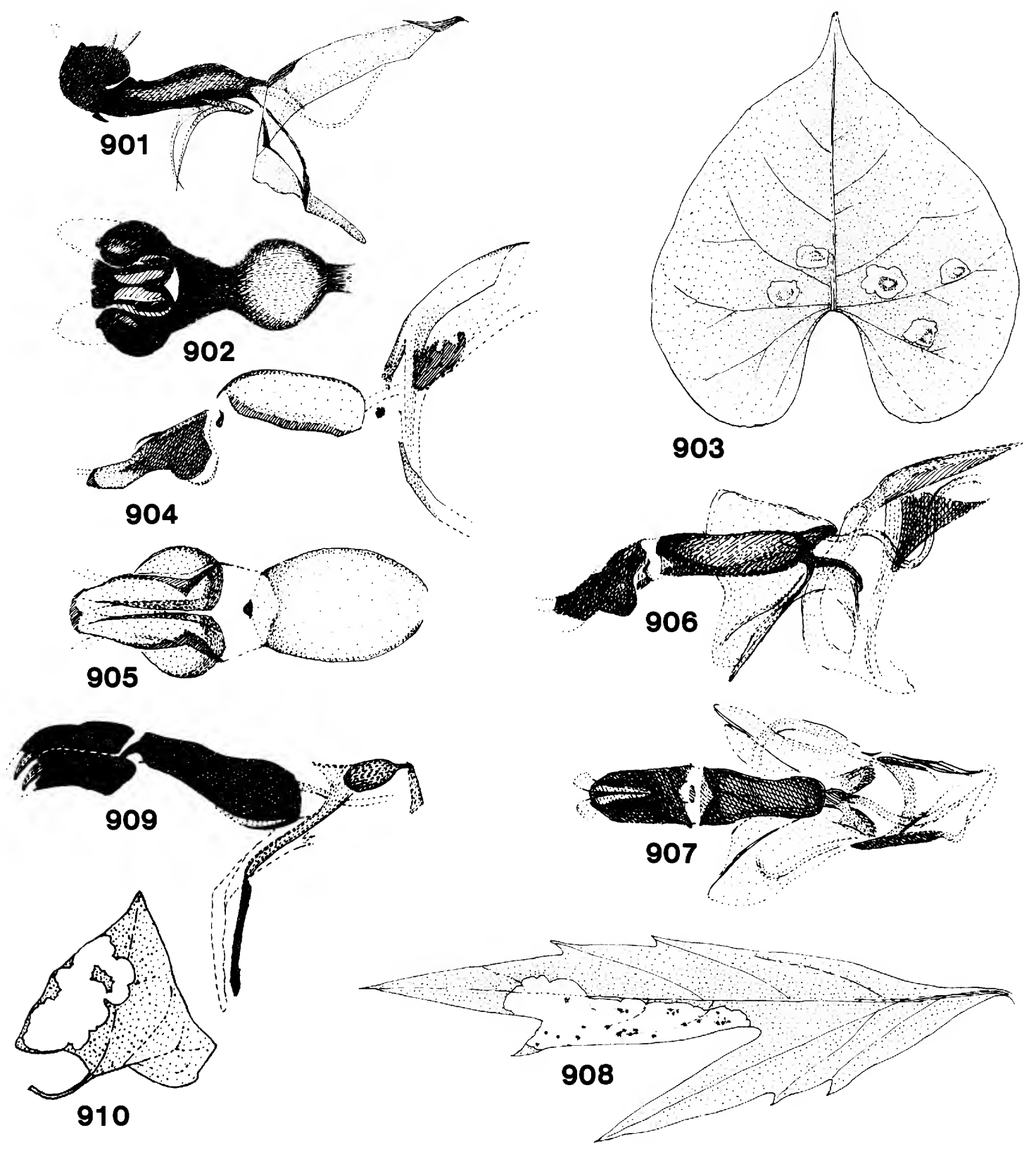

Figures 901-910.--Calycomyza stegmaieri: 901, Aedeagus, side view; 902, distiphallus, ventral view; 903, leaf mines on Calonyction aculeatum; . flavinotum: 904, Aedeagus, side view; 905, distiphallus, ventral view; C. frickiana: 906, Aedeagus, side view (paratypé, Illinois); 907, same, ventral view (paratype, South Dakota); 908 , leaf mine on Rudbeckia laciniata (Maryland); $\underline{C}$. mikaniae: 909, Aedeagus, side view; 910, leaf mine on Mikania batatifolia. 

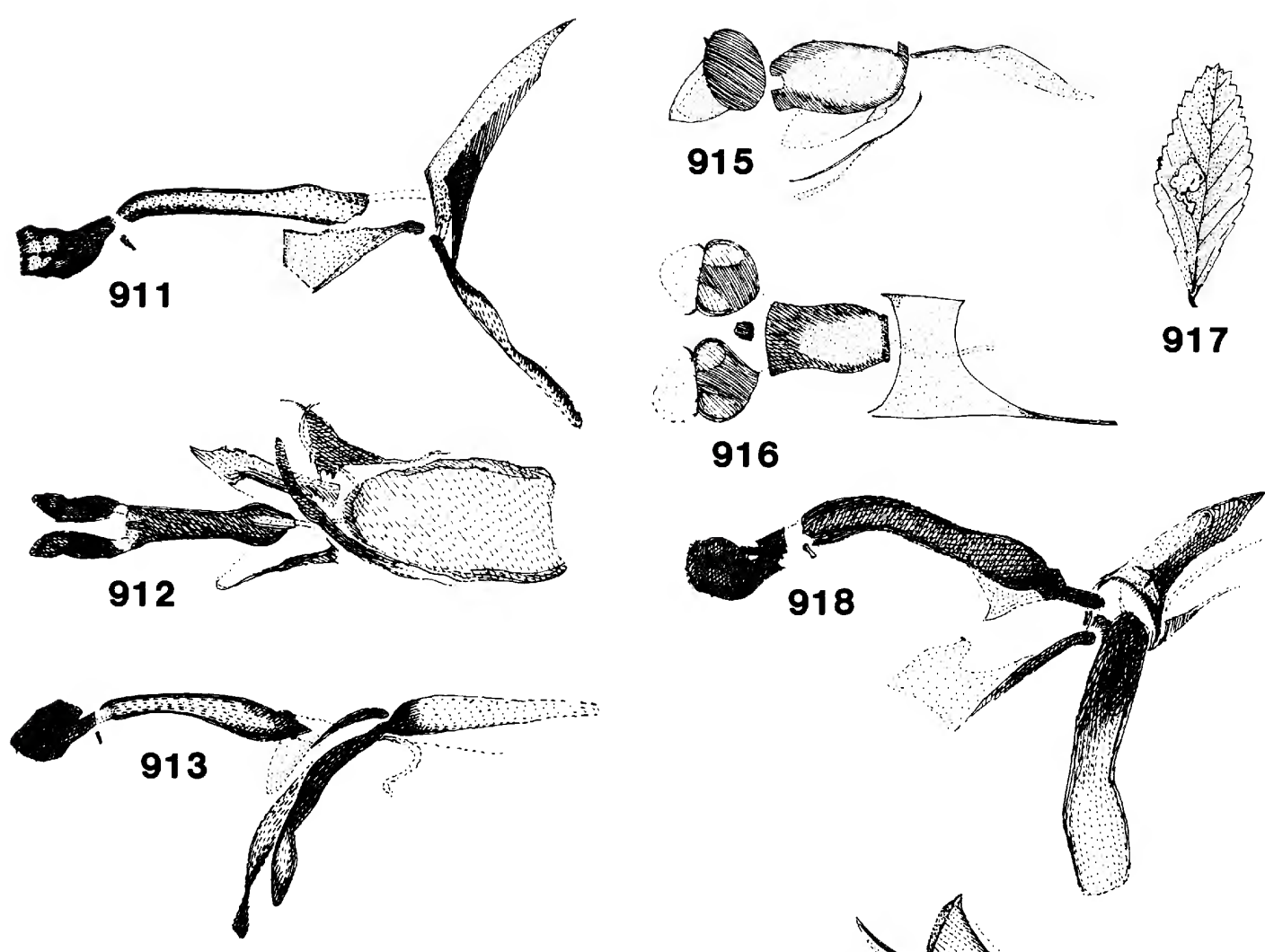

916
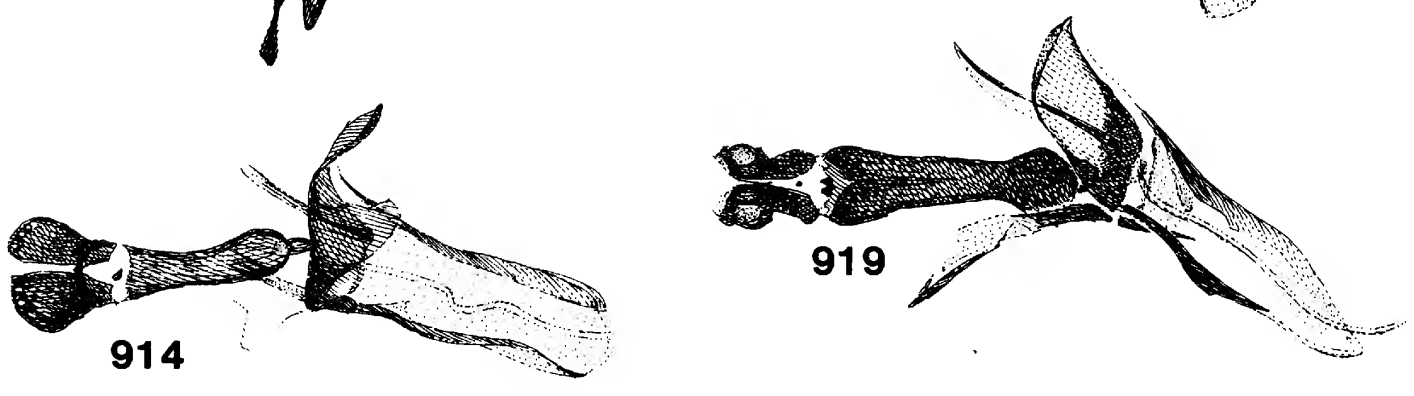

Figures 911-919.--Calycomyza majuscula: 911, Aedeagus, side view (paratype, Ontario); 912, same, ventral view (holotype, California); c. cynoglossi: 913, Aedeagus, side view; 914 , same, ventral view; $\underline{C}$. sidae: 915 , Aedeagus, side view; 916 , same, ventral view; 917, leaf mine on Sida; C. orientalis: 918, Aedeagus, side view; 919 , same, ventral view (paratype, Tennessee). 


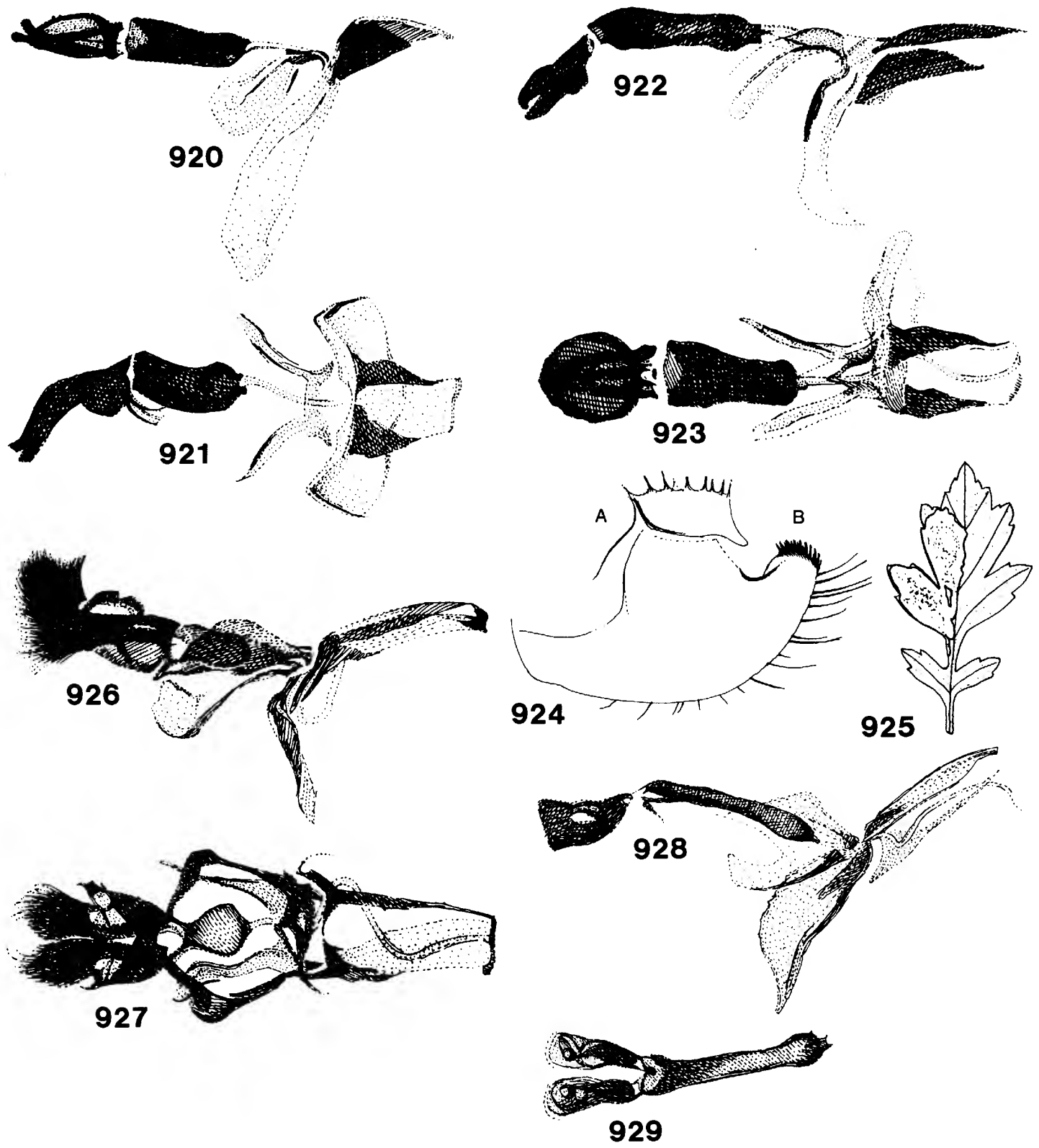

Figures 920-929.--Calycomyza artemisiae: 920, Aedeagus, side view; 921 , same, ventral view (ssp. artemisiae); 922, aedeagus, side view; 923, same, ventral view (ssp. marcida); 924, epandrium with surstylus (A) and posterior process (B) ; 925, leaf mine on Artemisia; C. enceliae: 926, Âdeagus, side view; 927 , same, ventral view; $\mathrm{C}$. michiganensis: 928, Aedeagus, side view; 929, distiphallus, ventral view (holotype). 


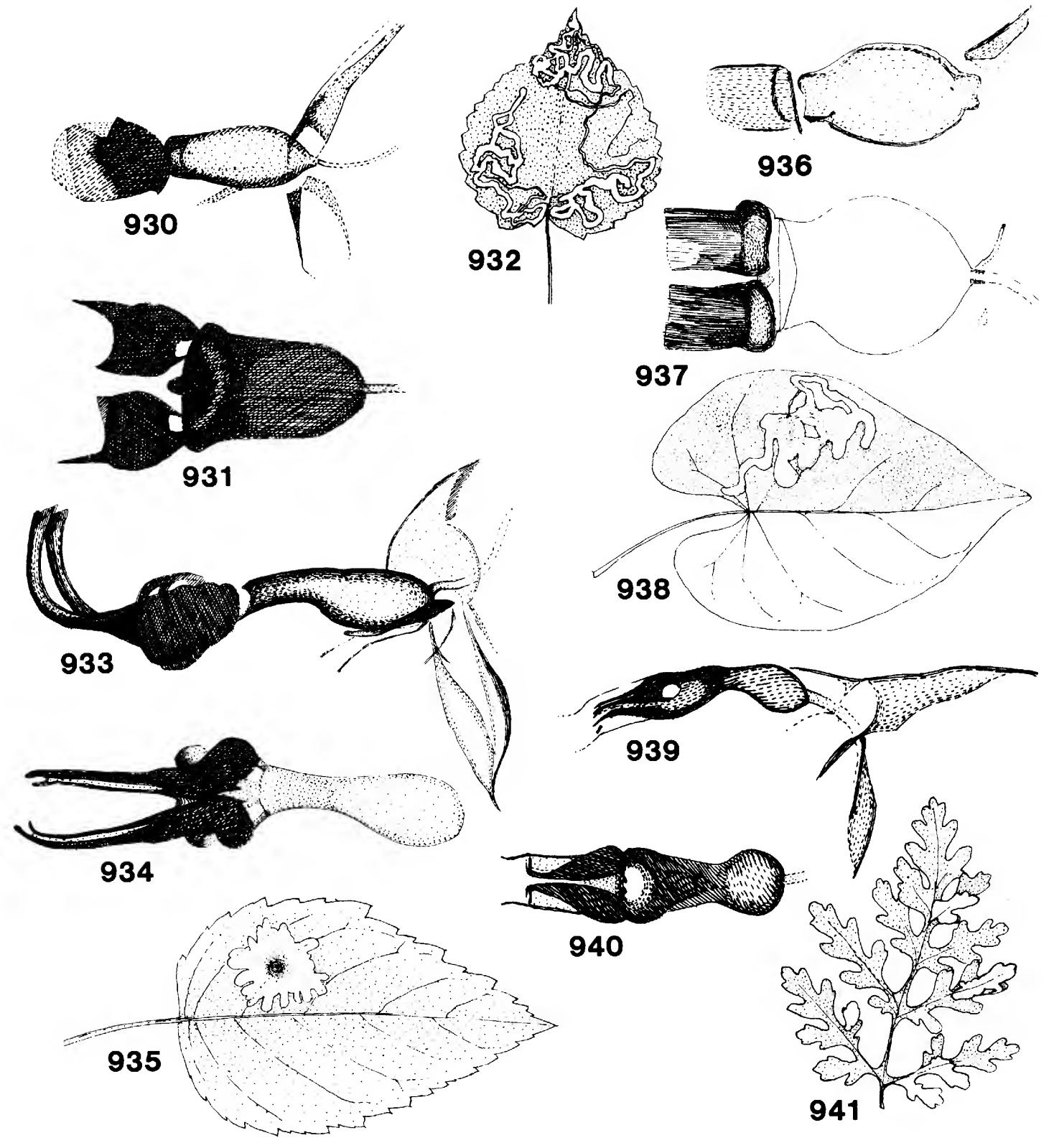

Figures 930-941.--Calycomyza malvae: 930, Aedeagus, side view; 931, distiphallus, ventral view; 932, leaf mines on Abutilon avicennae; C. hyptidis: 933, Aedeagus, side view; 934 , distiphaillus, ventral view; 935, leaf mine on Hyptis mutabilis; $\underline{C}$. ipomaeae: 936, Aedeagus, side view; 937, distiphallus, ventral view; 938, leaf mine on Ipomoea sp.; C. ambrosiae: 939, Aedeagus, side view; 940, distiphallus, ventral view; 941, leaf mines on Ambrosia artemisiifolia. 


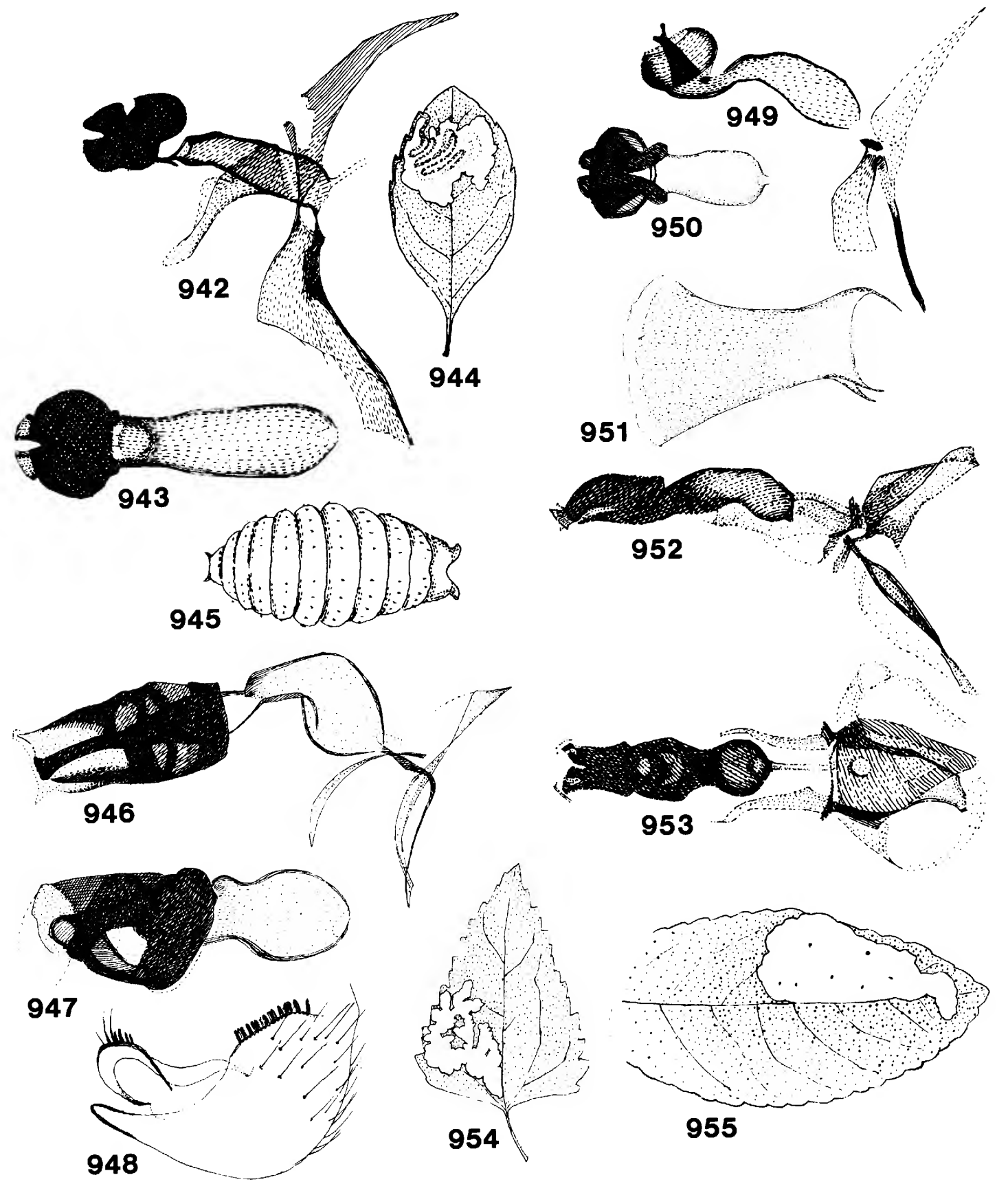

Figures 942-955.--Calycomyza durantae: 942, Aedeagus, side view; 943 , distiphallus, ventral view; 944, leaf mine on Duranta repens; 945 , puparium; $\underline{\mathrm{C}}$. melantherae: 946, Aedeagus, side view; 947, distiphallus, ventral view; 948 , epandrium with surstylus; C. verbenae: 949 , Aedeagus, side view; 950, distiphallus, ventral view; 951, sperm pump; C. barbarensis: 952, Aedeagus, side view; 953, same, ventral view; leaf mines of unidentified Calycomyza spp. on Ageratum conyzoides (954) and Elephantopus elatus (955). 

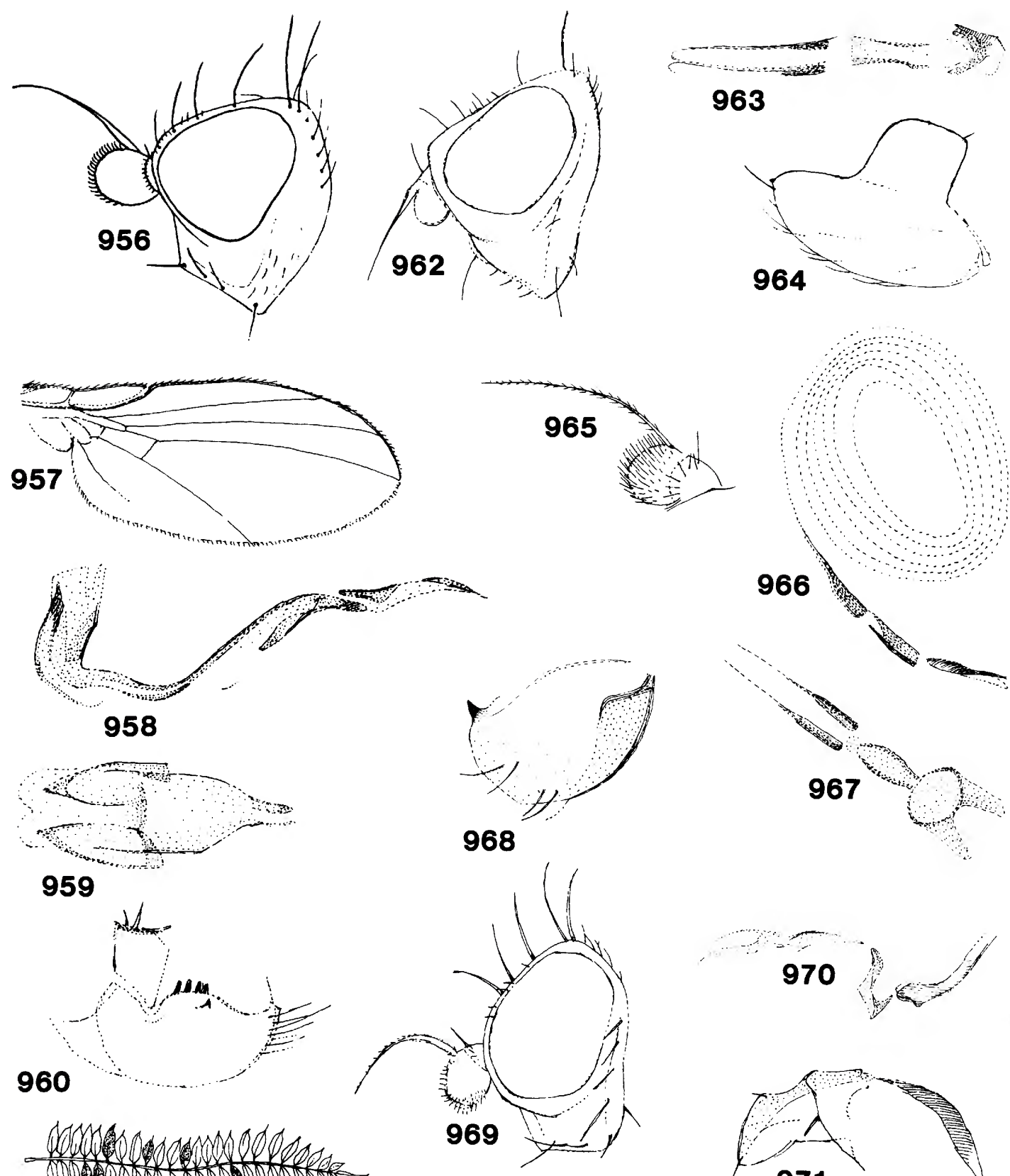

968
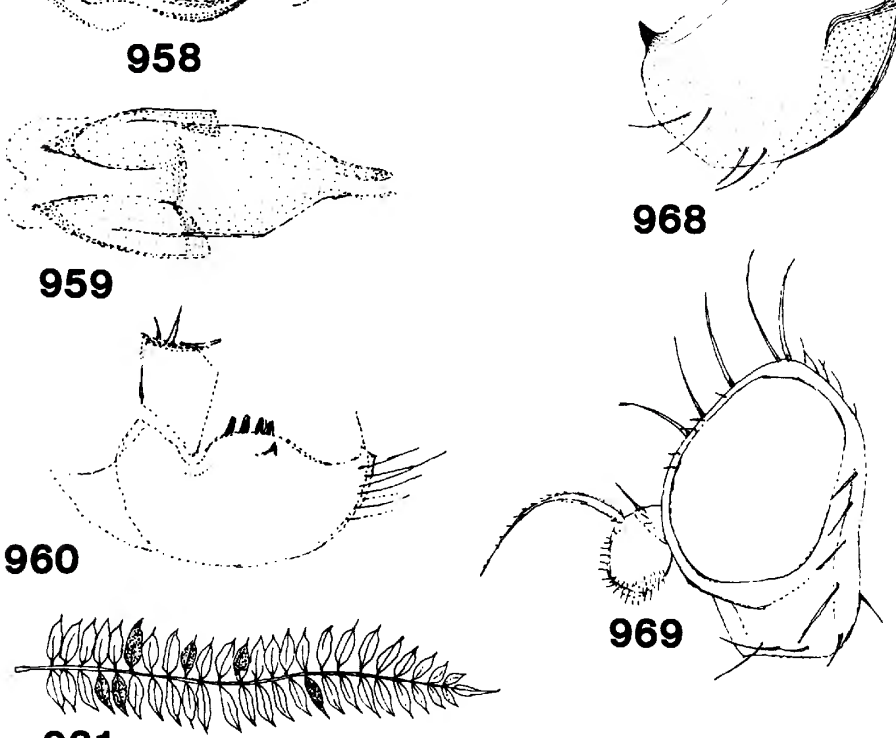

961

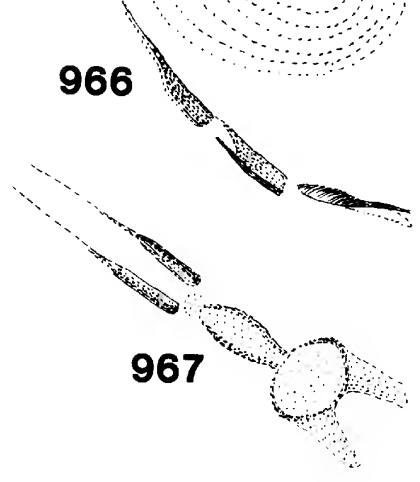

Figures 956-971.--Phytoliriomyza jacarandae: 956, Head; 957, wing; 958, aedeagus, side view; 959, distiphallus, anteroventral view; 960 , epandrium with surstylus; 961 , leaf mines on Jacaranda mimosifolia; $P$. flavens: 962 , Head; $P$. conjunctimontis: 963, Aedeagus, vent $\bar{r}$ al view; 964 , epandrium with surstylus; $\underline{P}$. pilosella: 965, Third antennal segment; 966, aedeagus, side view; 967 , same, ventral view; 968 , epandrium; $P$. minutissima: 969 , Head; 970 , aedeagus, side view; $97 \overline{1, \text { epandrium }}$ with surstylus. 


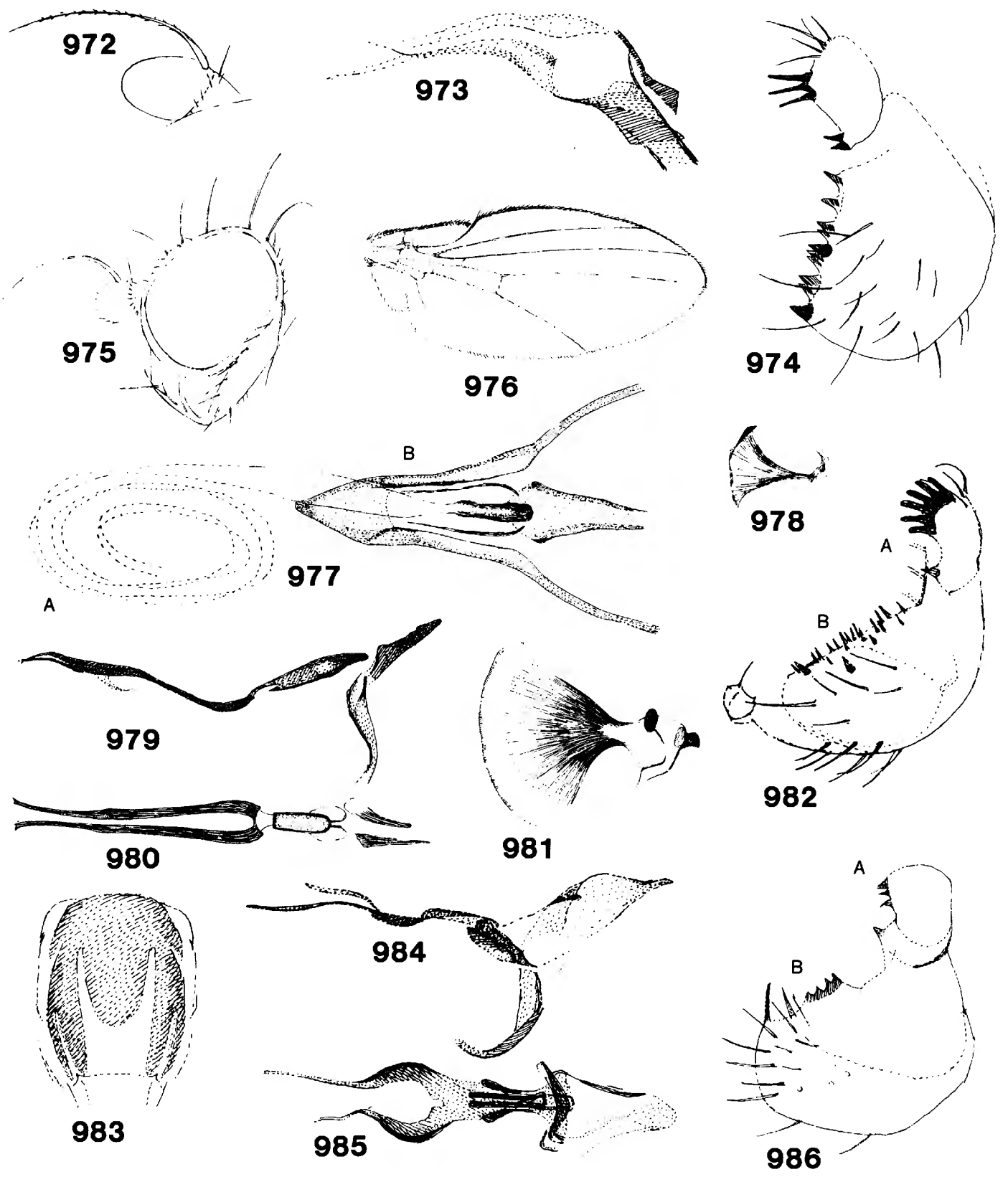

Figures 972-986.--Phytoliriomyza floridana: 972, Third antennal segment; 973, aedeagus, side view; 974, epandrium with surstylus; $\underline{P}$. arctica: 975 , Head; 976, wing; 977 , aedeagus $(\bar{A})$, ventral view, with hypandrium (B); 978, sperm pump; . imperfecta: 979, Aedeagus, side view; 980 , same, ventral view; 981 , sperm pump; 982 , surstylus (A) with epandrium (B), ventral view; $\underline{P}$. beckere1la: 983 , Mesonotum; 984, aedeagus, side view; 985 , same, ventral view; 986, epandrium (A) with surstylus ( $\underline{B})$. 


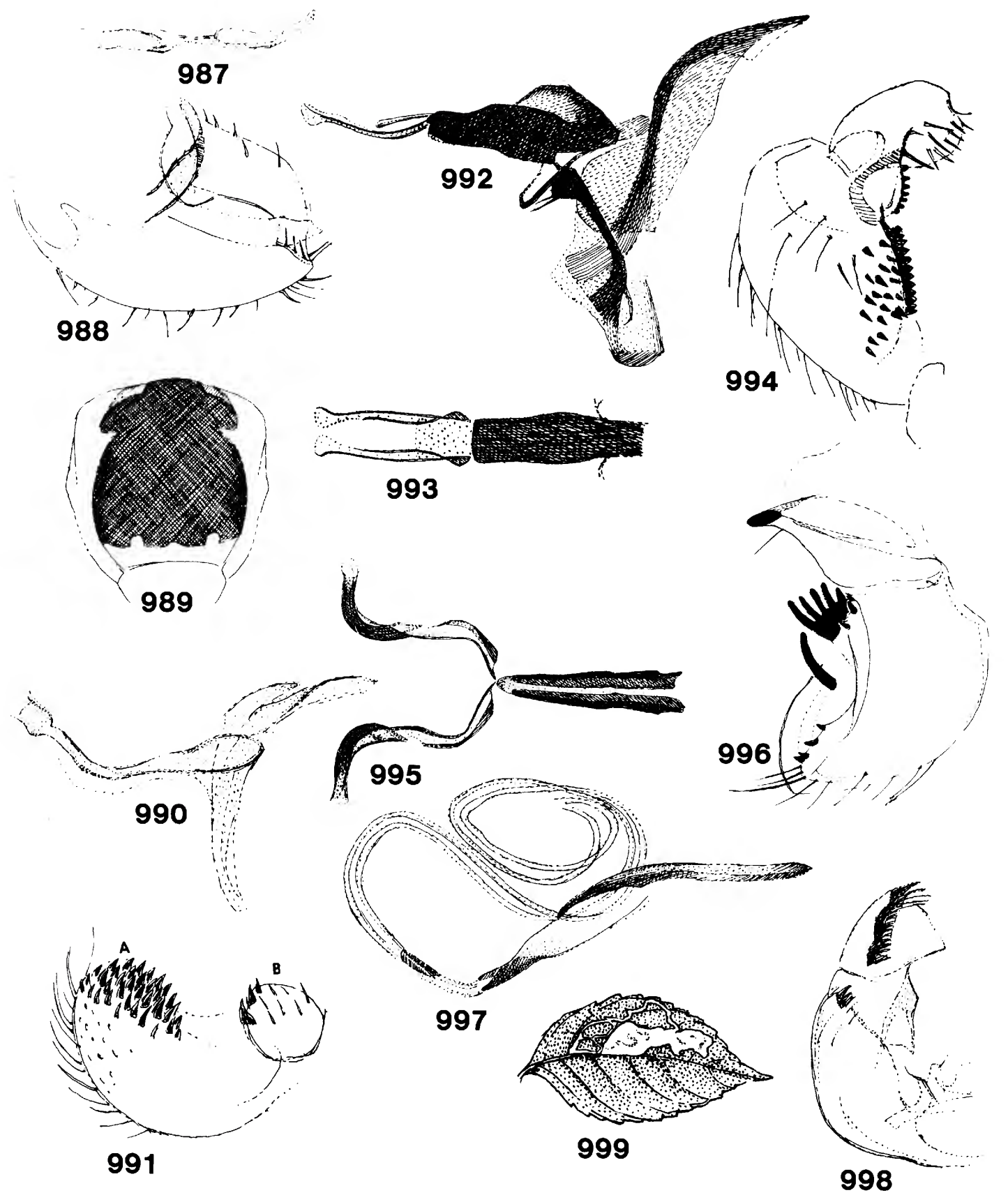

Figures 987-999.--Phytoliriomyza consulta: 987, Aedeagus, side view; 988, epandrium; $P$. felt i: 989, Mesonotum; 990 , aedeagus, side view; $\overline{991}$, epandrium (A) with surstylus ( $\underline{B})$; $\underline{P}$. conspicua: 992 , Aedeagus, side view; 993 , distiphallus, ventral view; 994, epandrium with surstylus; $P$. pallida: 995, Distiphallus, ventral view; 996, epandrium with surstylus; $\underline{P}$. melampyga: 997 , Aedeagus, side view; 998, epandrium with surstylus; 999, leaf mine on Impatiens (Europe). 

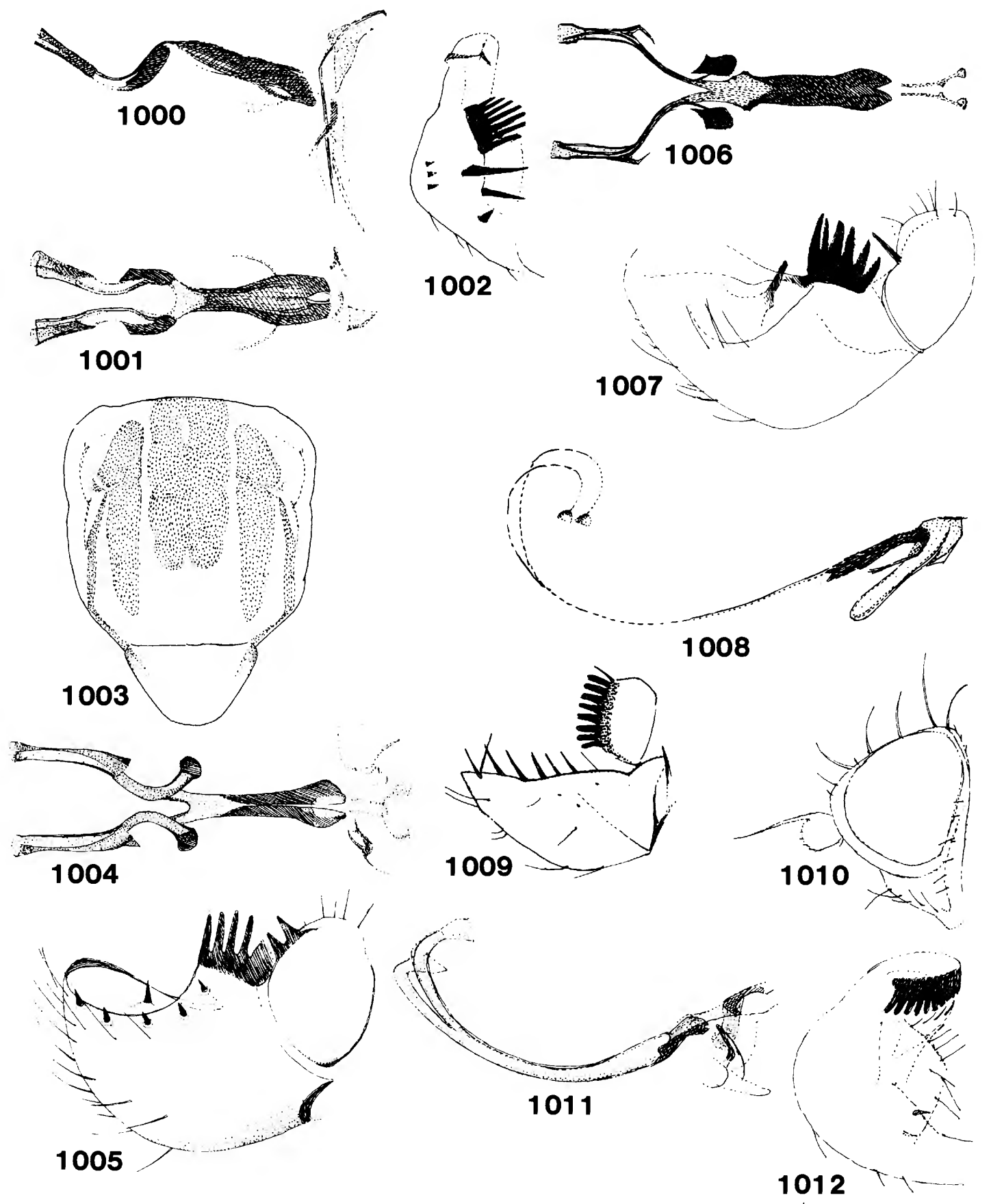

Figures 1000-1012.--Phytoliriomyza fumicosta: 1000,
Aedeagus, side view; 1001, same, ventral view;
1002, epandrium; P. dorsata: 1003, Mesonotum; 1004,
aedeagus, ventral view; 1005, epandrium with
surstylus; P. pacifica: 1006, Aedeagus, ventral
view; 1007, epandrium with surstylus; P. clara:
1008, Aedeagus, side view; 1009, epandrium with
surstylus; P. pulchella: 1010 , Head; 1011,
aedeagus, side view; 1012, epandrium with surstylus. 

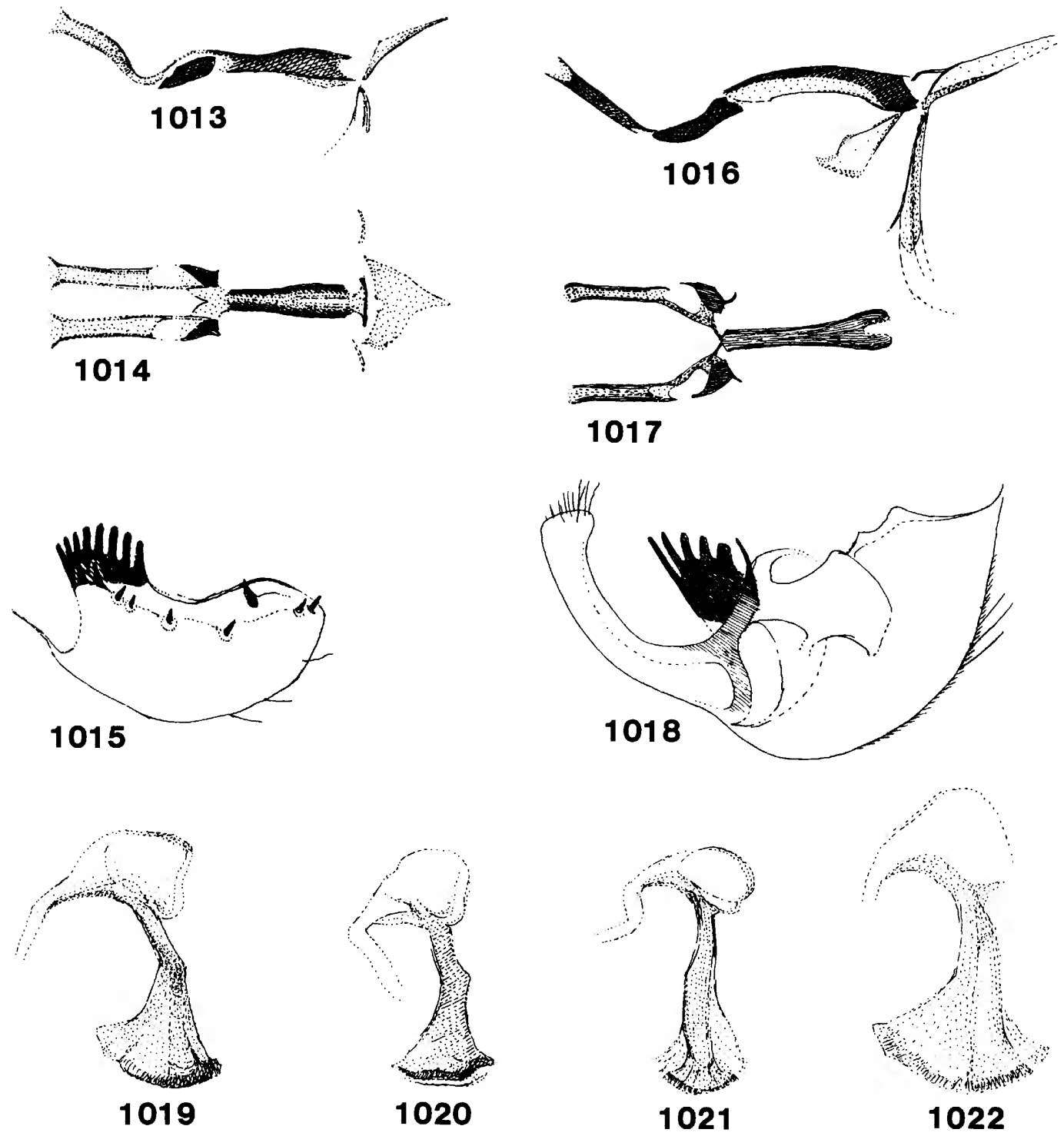

Figures 1013-1022.--Phytoliriomyza leechi: 1013, Aedeagus, side view; 1014 , same, ventral view; 1015 ,

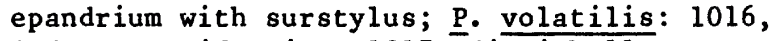
Aedeagus, side view; 1017, distiphallus, ventral view; (1016, 1017, British Columbia); 1018, epandrium with surstylus (as P. mikii, Swedish Lapland); 1019, sperm pump (paratype); 1020, same (Colorado); 1021, same (Swedish Lapland); $\underline{P}$. varia: 1022, Sperm pump (P. mikii, Denmark). 


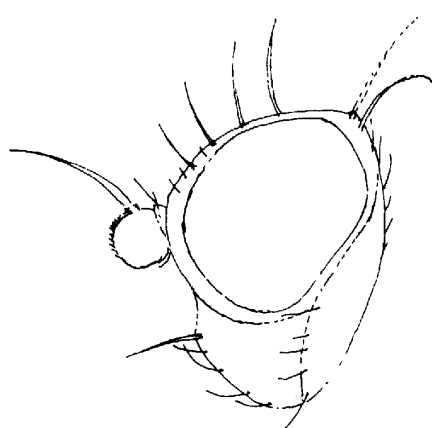

1023

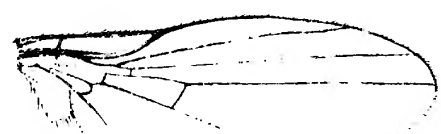

1024

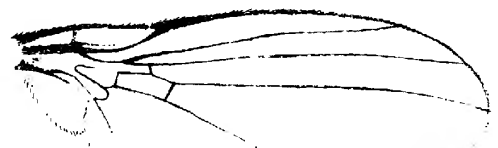

1028
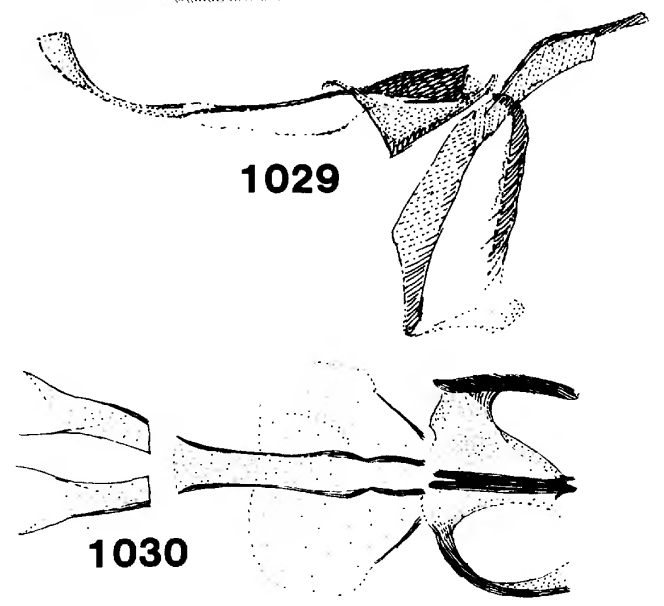
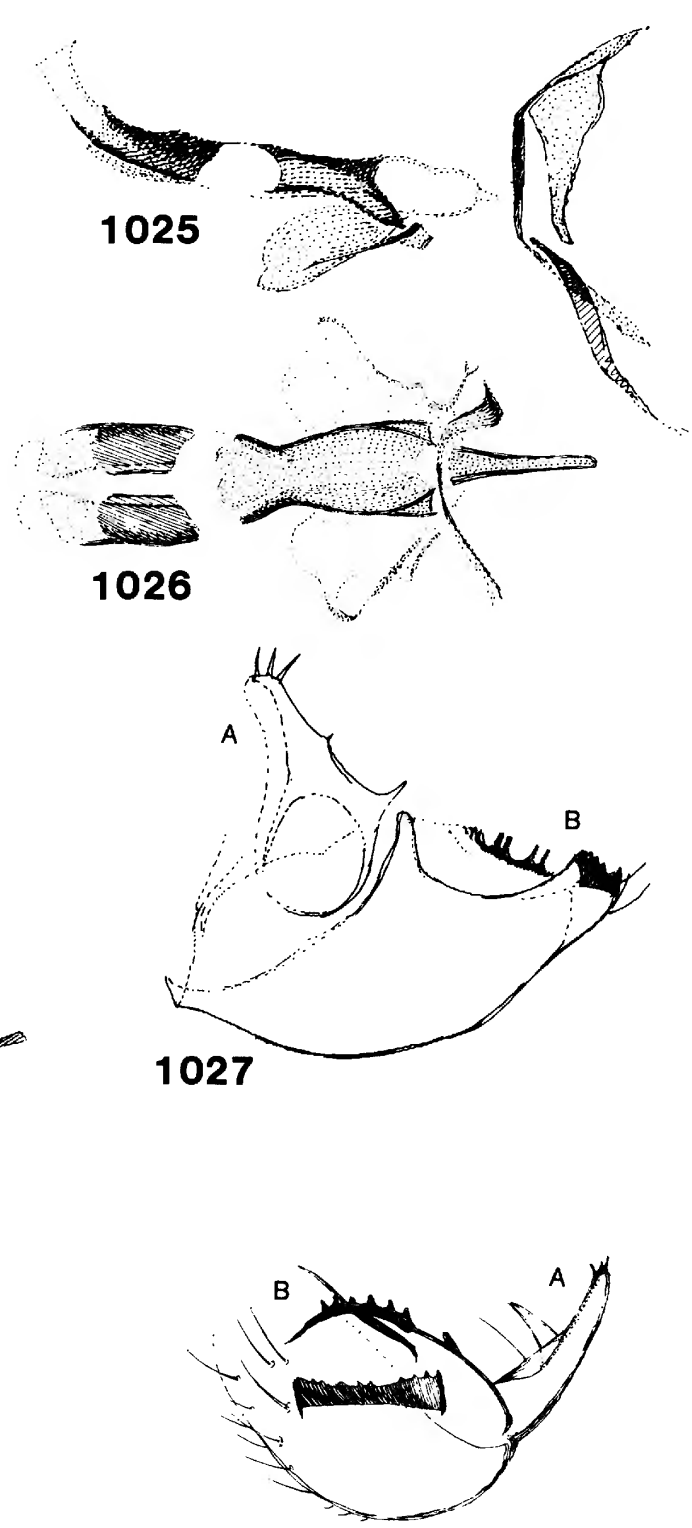

1031

Figures 1023-1031.--Metopomyza nigripes: 1023, Head; M. interfrontalis: 1024, Wing; 1025, aedeagus, side view; 1026, same, ventral view; 1027, surstylus (A) with hindcorner of epandrium (B); M. scutellata: 1028, Wing; 1029, aedeagus, side view; 1030, same, ventral view; 1031, surstylus (A) with inner margin of epandrium (B). 


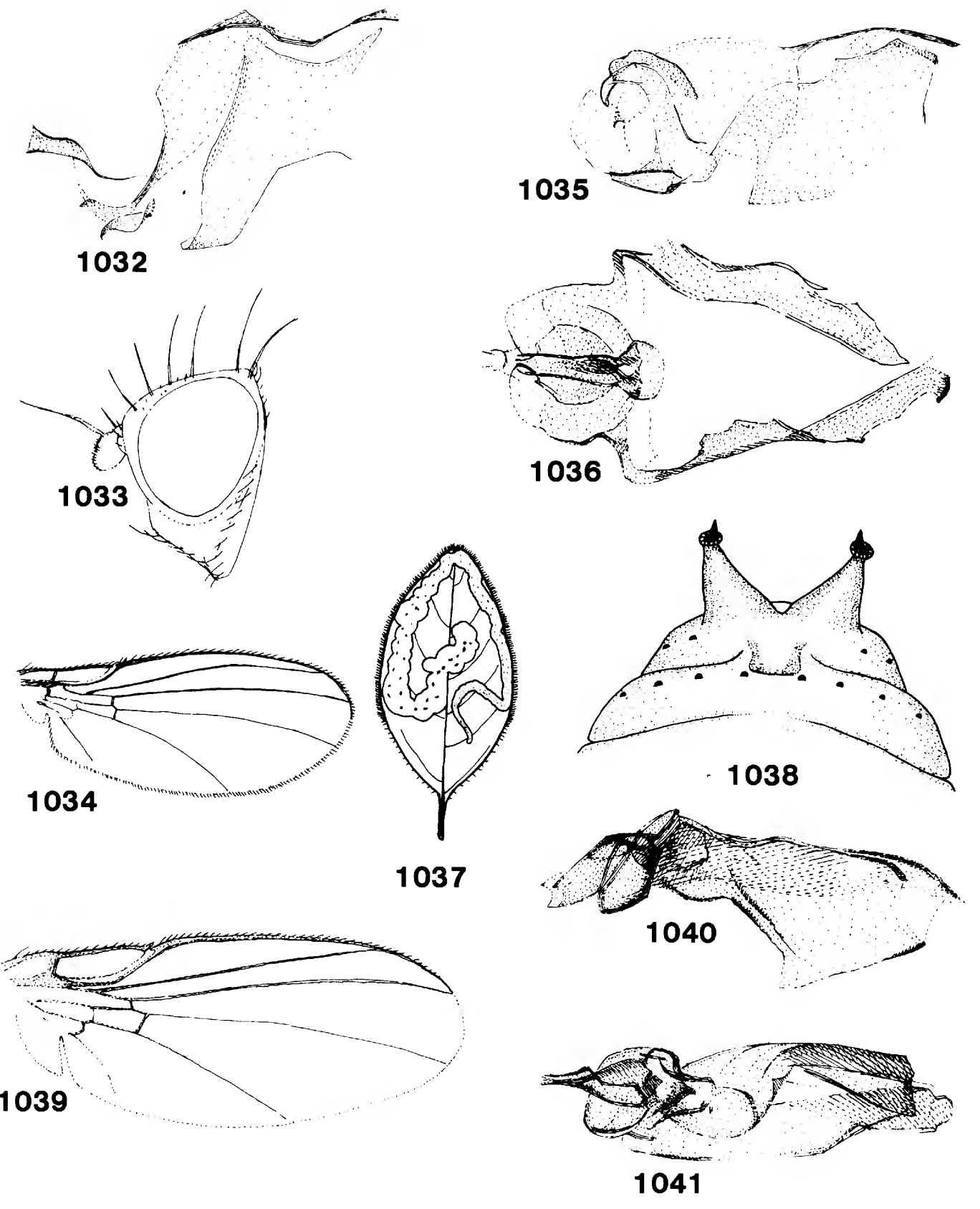

Figures 1032-1041.--Paraphytomyza nitida: 1032, Aedeagus, side view; P. praecox: 1033, Head; 1034, wing; $\underline{P}$. cornigera: $1 \overline{0} 35$, Aedeagus, side view; 1036, same, ventral view; 1037, leaf mine on Lonicera; 1038, posterior spiracles of puparium; (1037, 1038, after Griffiths, 1973b); P. plagiata: 1039, Wing (Alberta); P. lonicerina: $1 \overline{0} 40$, Aedeagus, side view; $1 \overline{0} 41$, same, ventral view. 

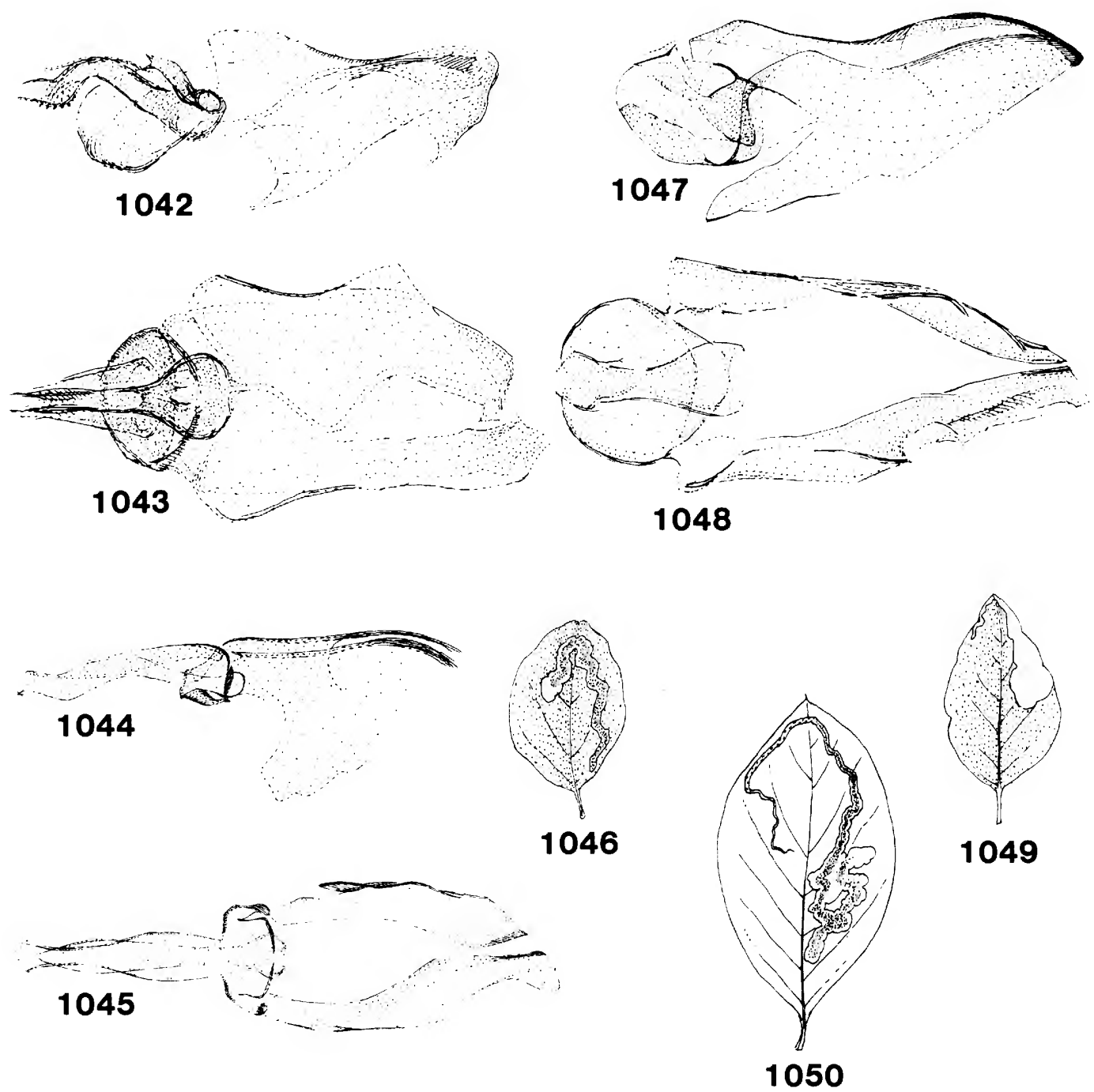

Figures 1042-1050.--Paraphytomyza coloradensis: 1042, Aedeagus, side view; 1043, same, ventral view; $\underline{P}$. luteoscutellata: 1044, Aedeagus, side view; $104 \overline{5}$, same, ventral view; 1046, leaf mine on Lonicera; P. orbitalis: 1047, Aedeagus, side view; 1048, same, ventral view; 1049, leaf mine on Lonicera; $\underline{\text { P. sp. }}$ indet. No. 2: 1050, Leaf mine on $\overline{\text { Lonicera }}$ columbiana. 

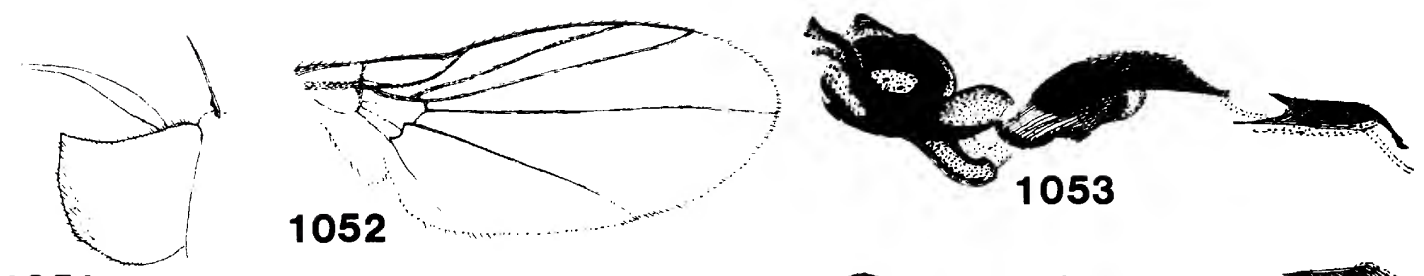

1051
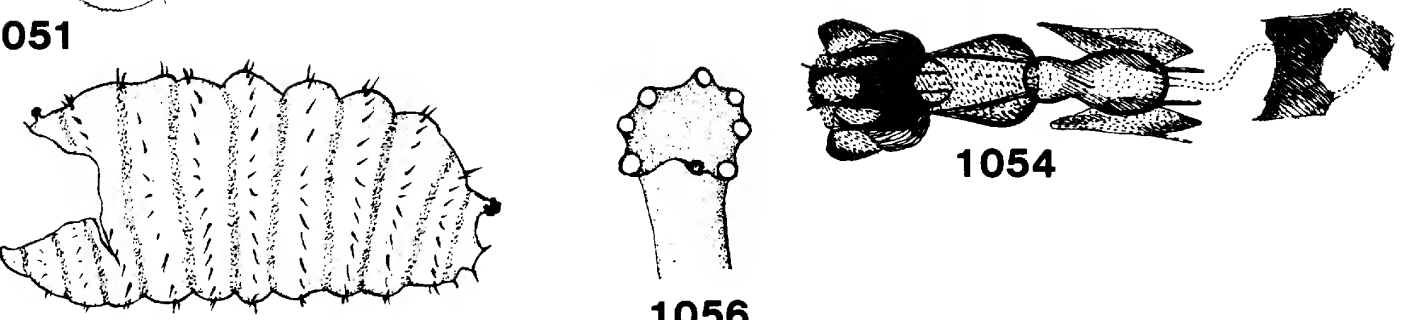

1055

1056
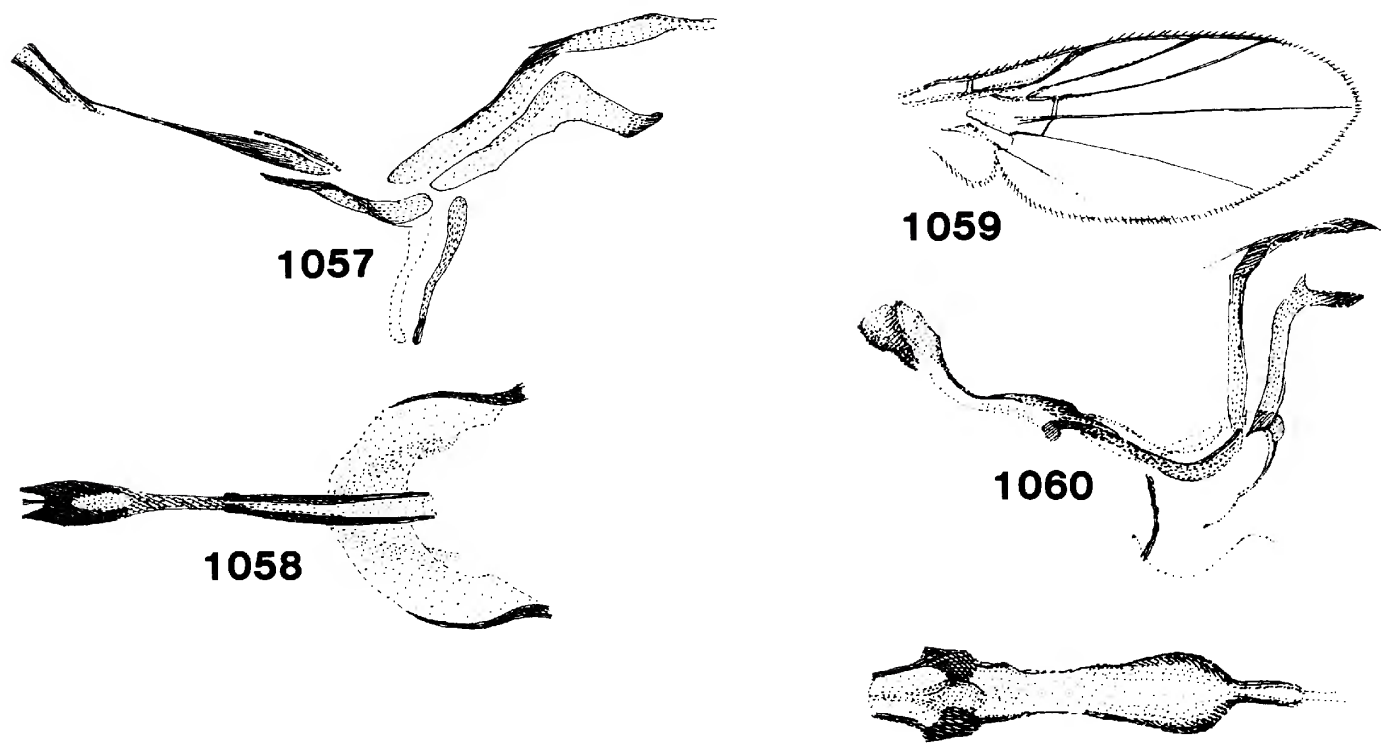

1061

Figures 1051-1061.--Pseudonapomyza atra: 1051, Third

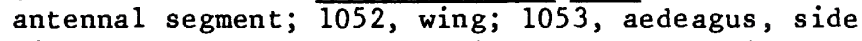
view; 1054, same, ventral view; 1055, puparium; 1056, larval posterior spiracles. Napomyza pallens: 1057, Aedeagus, side view; 1058, same, ventral view; $N$. minuta: 1059, Wing; 1060, aedeagus, side view; 1061, distiphallus, ventral view. 

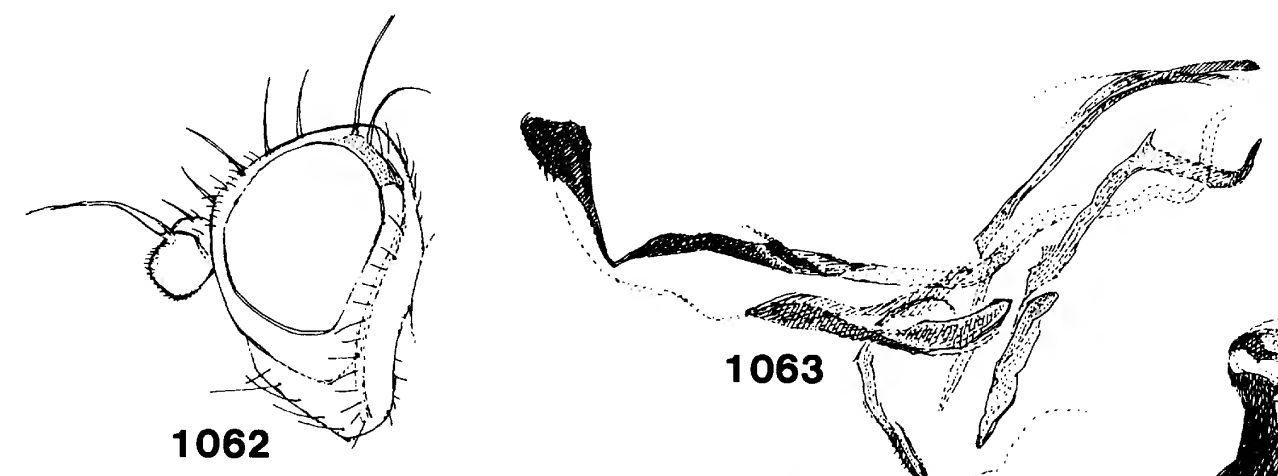

1063
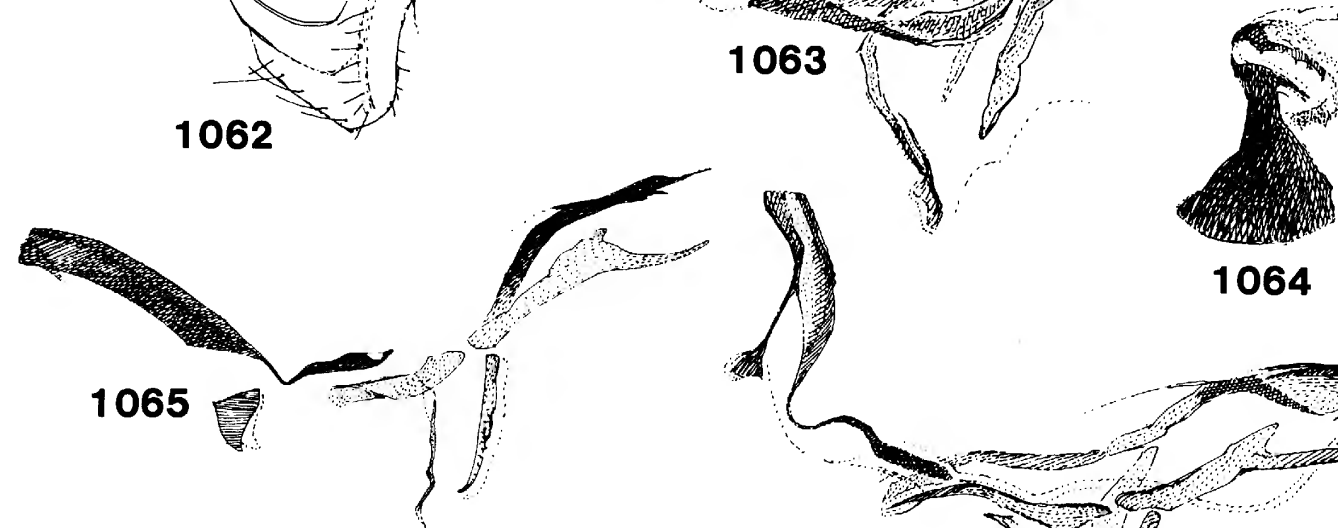

064
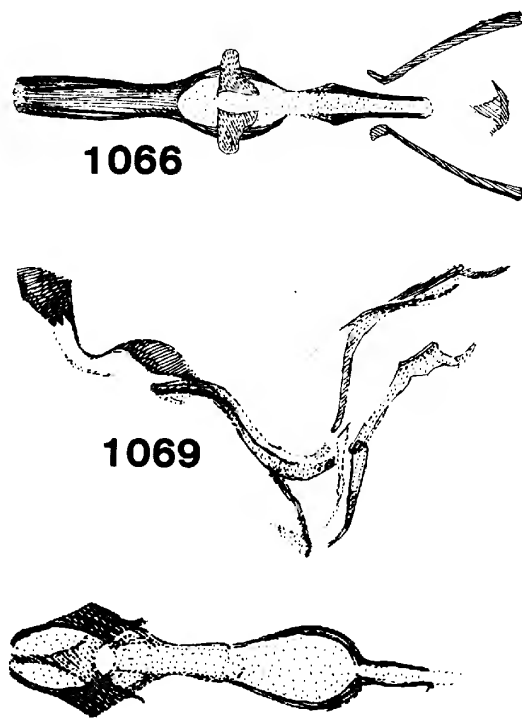

1070
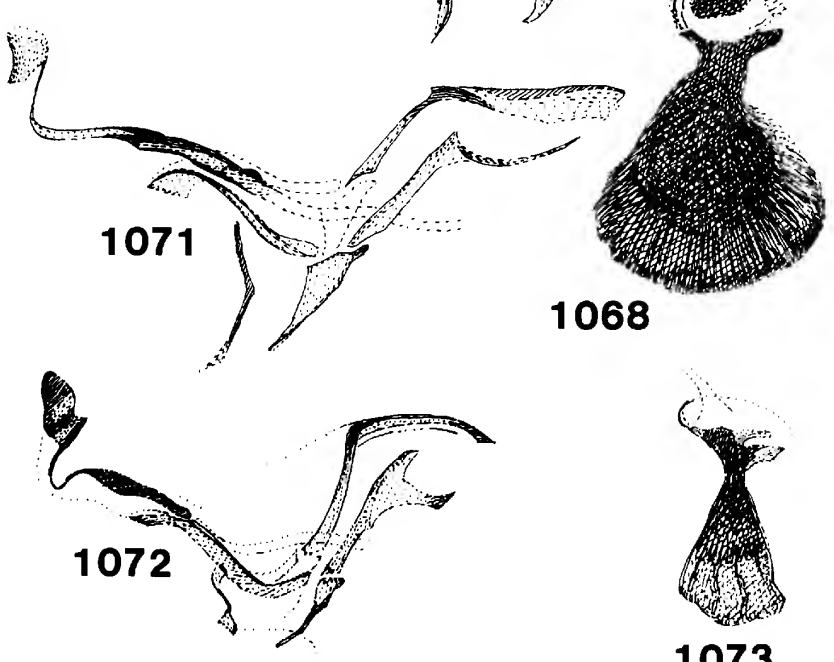

Figures 1062-1073.--Napomyza grandella: 1062, Head;

1063, aedeagus, side view; 1064 , sperm pump; $N$.

plumea: 1065, Aedeagus, side view; 1066, same,

ventral view; N. manni: 1067, Aedeagus, side view;

1068, sperm pump; N. schusteri: 1069, Aedeagus,

side view; 1070 , distiphallus, ventral view; $N$.

nugax: 1071, Aedeagus, side view (holotype,

Ontario); 1072, same, side view (California, Alpine

Co.); 1073, sperm pump (paratype, Ontario,

genitalia slide No. 5083, BMNH). 

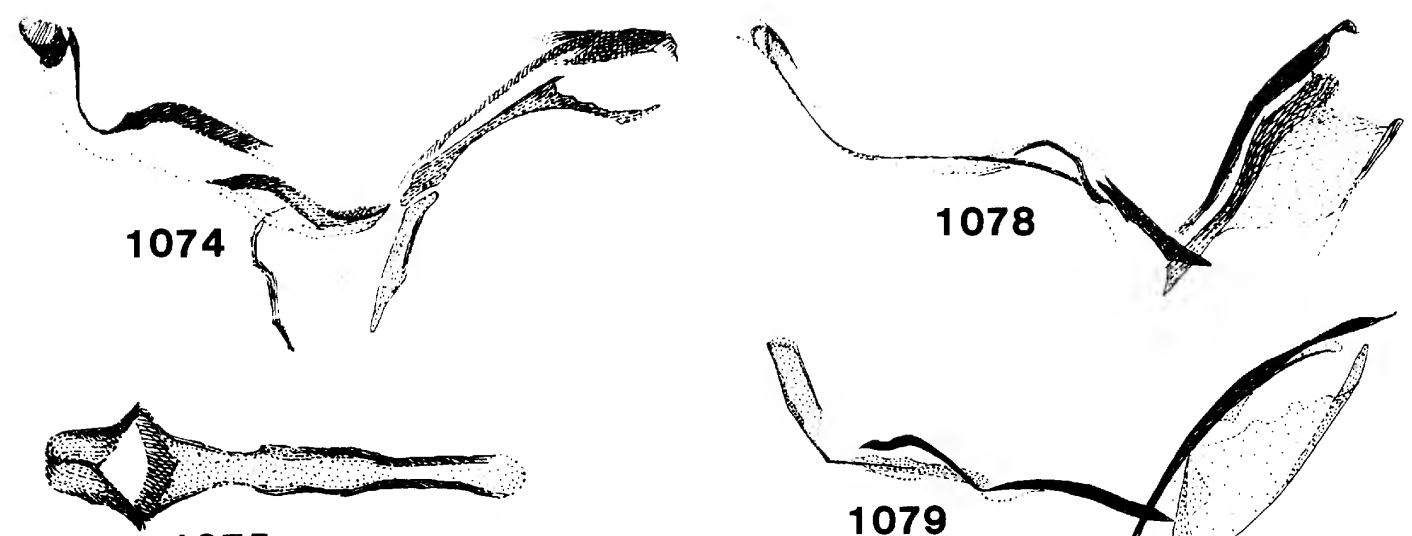

1075
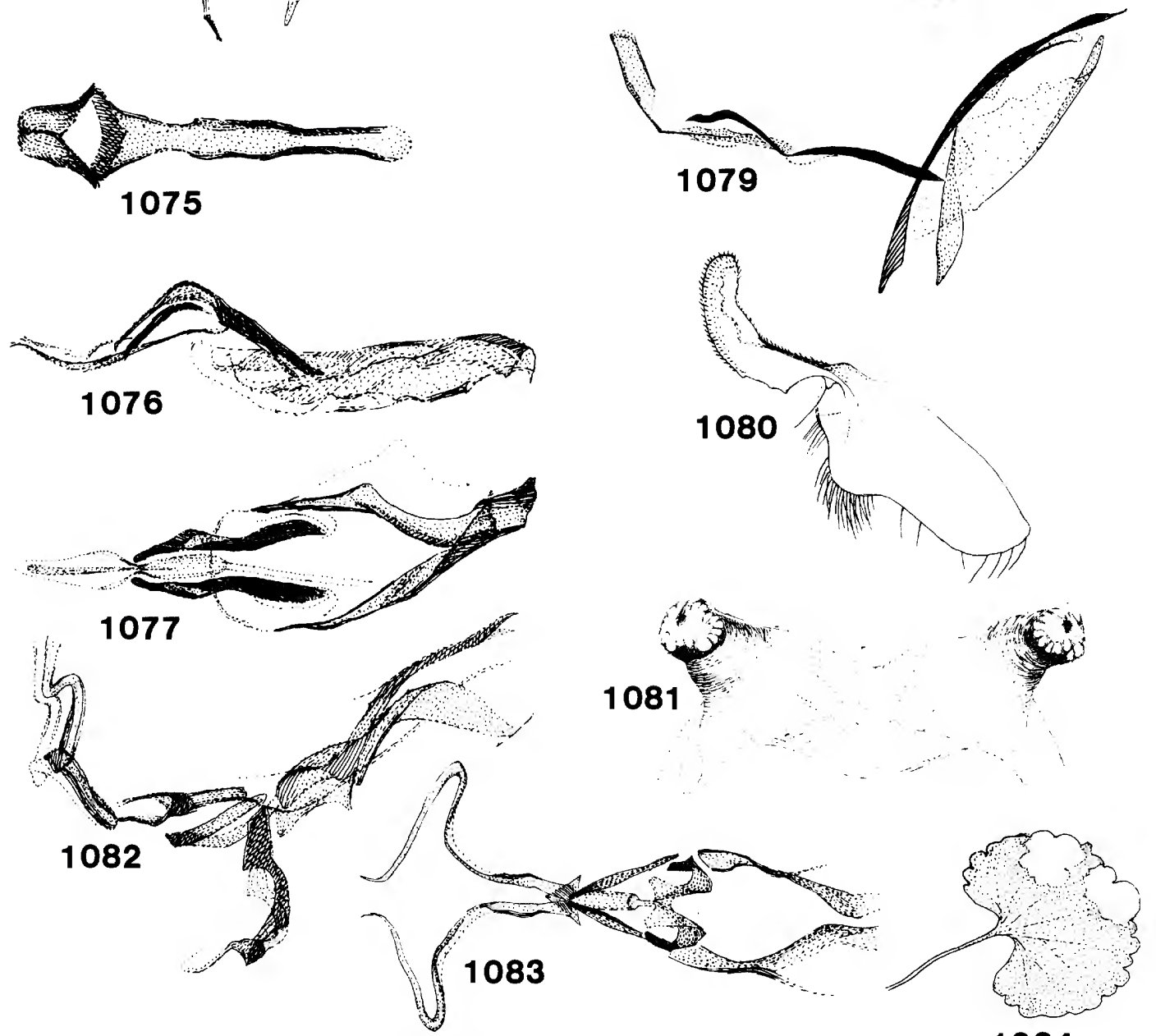

1084

Figures 1074-1084.--Napomyza montanoides: 1074, Aedeagus, side view; 1075 , distiphallus, ventral view; N. marginalis: 1076, Aedeagus, side view; 1077, same, ventral view (paratype); N. blairmorensis: 1078, Aedeagus, side view; N. evanescens: 1079, Aedeagus, side view; 1080, epandrium; 1081, posterior spiracles of puparium. Phytomyza davisii: 1082, Aedeagus, side view; 1083, same, ventral view; 1084, leaf mines on Ranunculus abortivus (Madison, Wis.). 


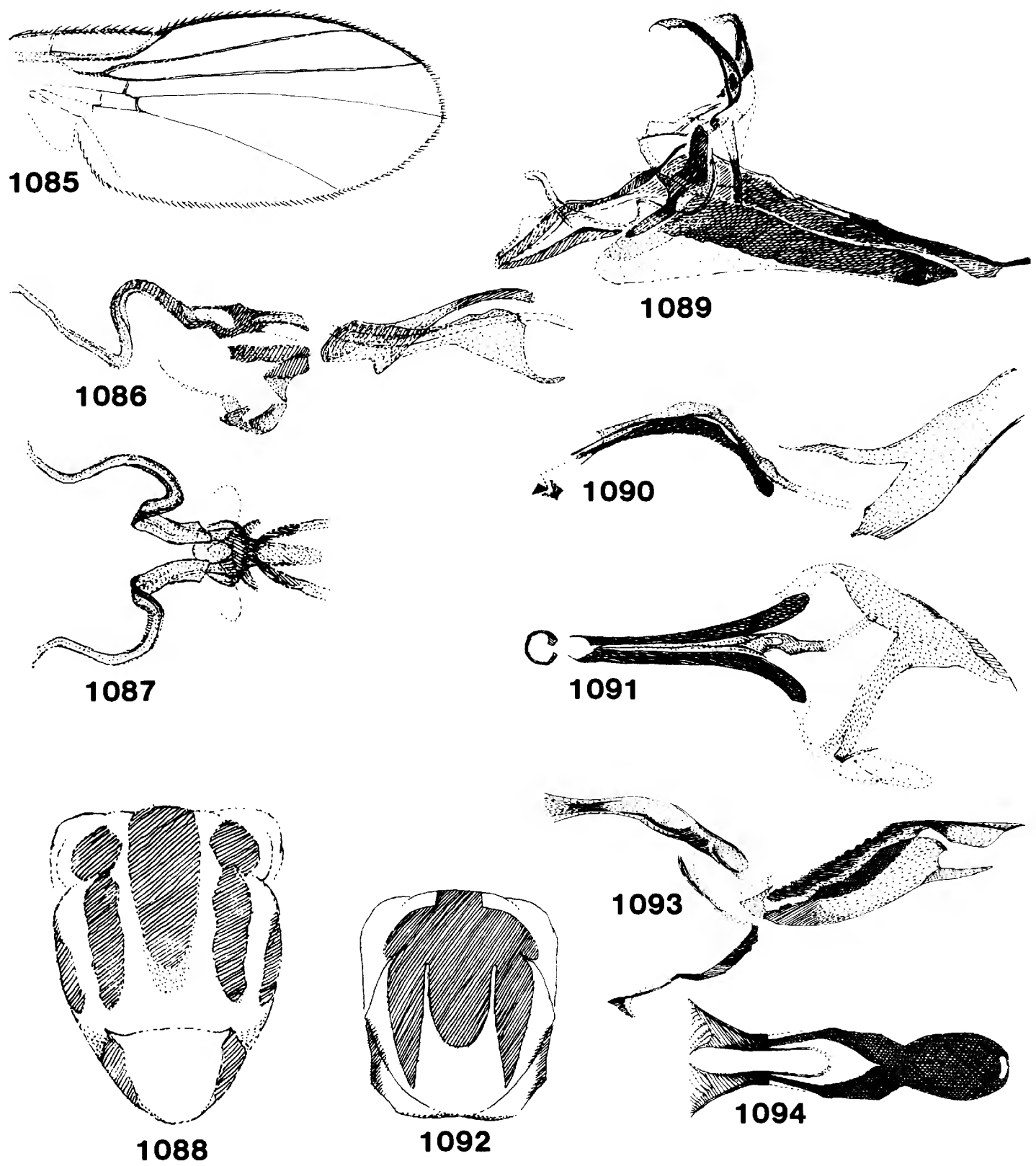

Figures 1085-1094.--Phytomyza duplex: 1085, Wing;

1086, aedeagus, side view; 1087 , same, ventral

view; P. splendida: 1088 , Mesonotum. Chromatomyia

compta: 1089, Aedeagus, side view. $\underline{p} \cdot$ trivittata:

1090, Aedeagus, side view; 1091, same, ventral

view; P. clematiphaga: 1092, Mesonotum; 1093,

aedeagus, side view; 1094 , distiphallus, ventral

view. 

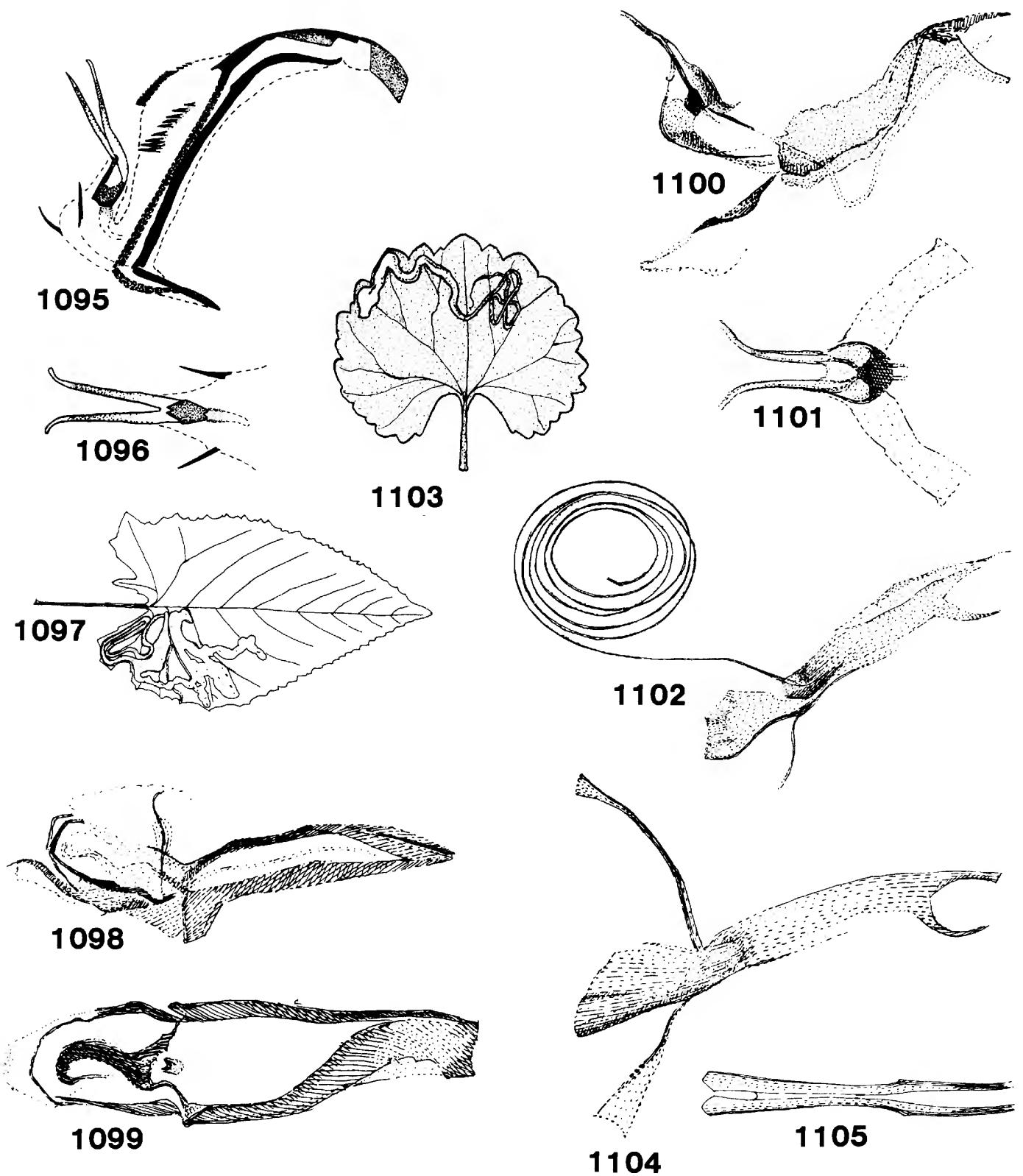

Figures 1095-1105.--Phytomyza californica: 1095, Aedeagus, side view; $10 \overline{96}$, distiphallus, anteroventral view; 1097, leaf mine on Senecio triangularis. Chromatomyia clemativora: 1098, Aedeagus, side view; 1099, same, ventral view (holotype). P. aldrichi: 1100, Aedeagus, side view; 1101 , distiphallus, anteroventral view; $\underline{P}$. ranunculi: 1102, Aedeagus, side view; 1103, lea f mine on Ranunculus; (1102, 1103, Europe); P. humilis: 1104 , Aedeagus, side view; 1105 , distiphallus, anteroventral view. 

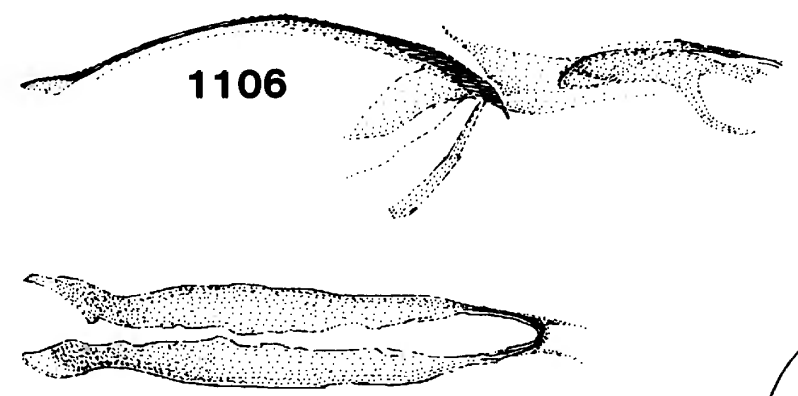

1107
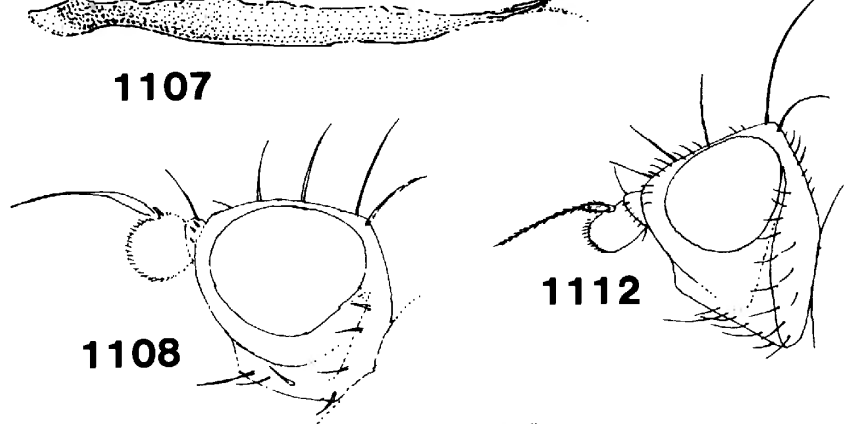

\section{3}
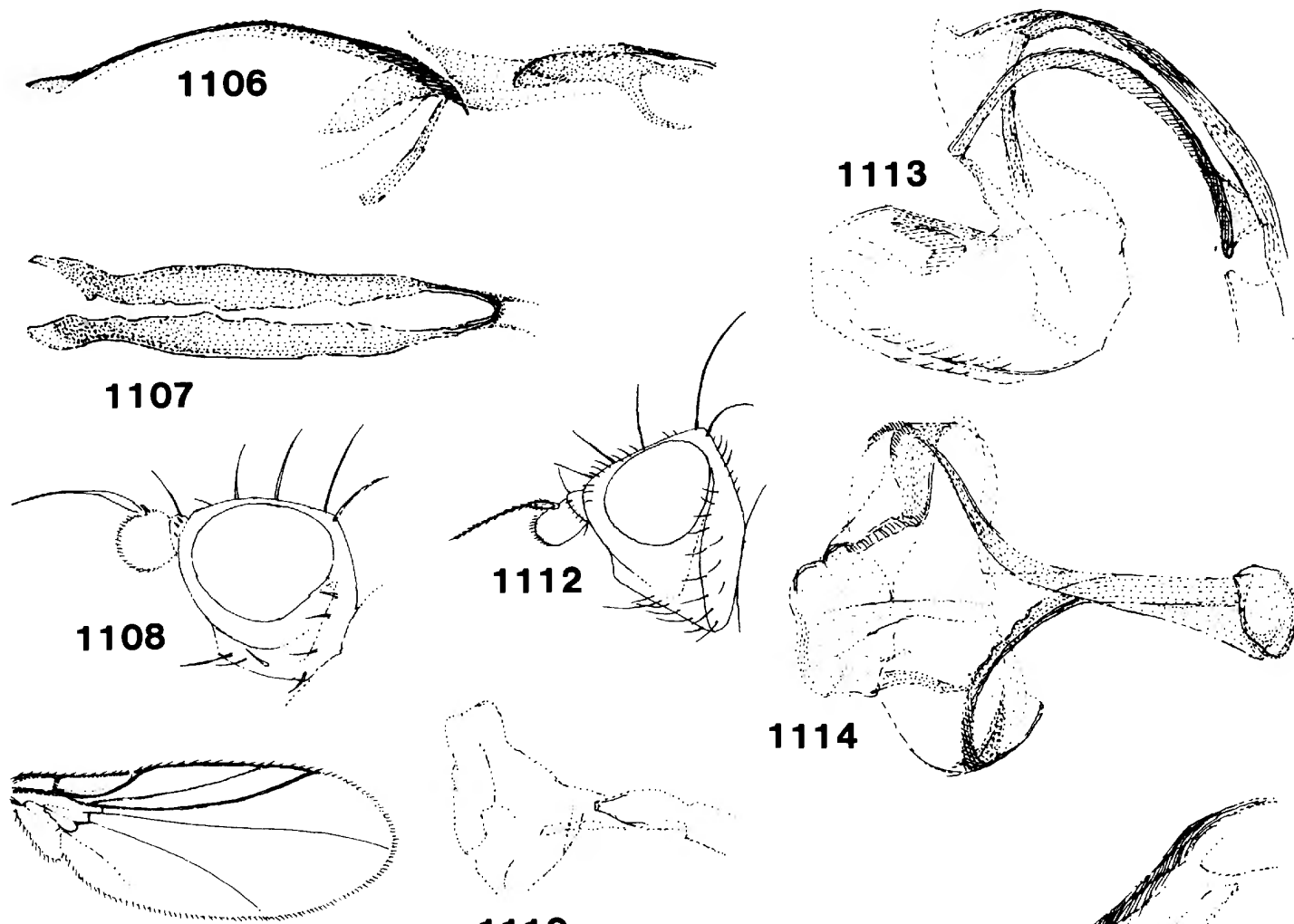

1114

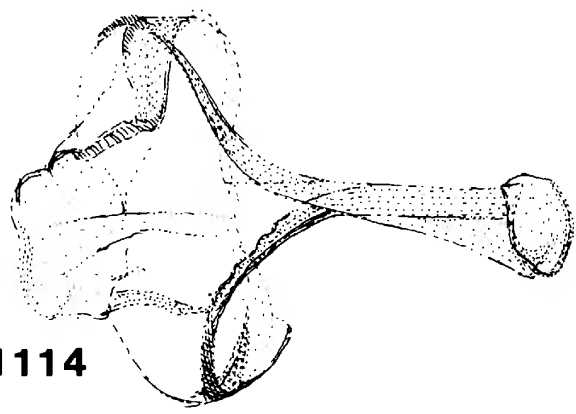

1109

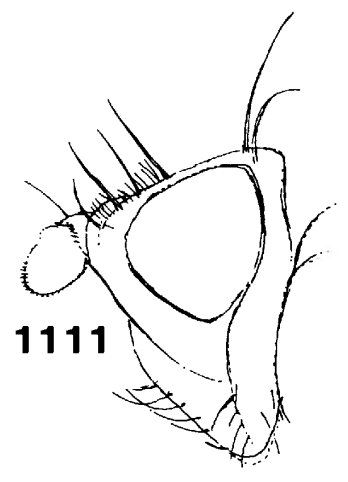

1110

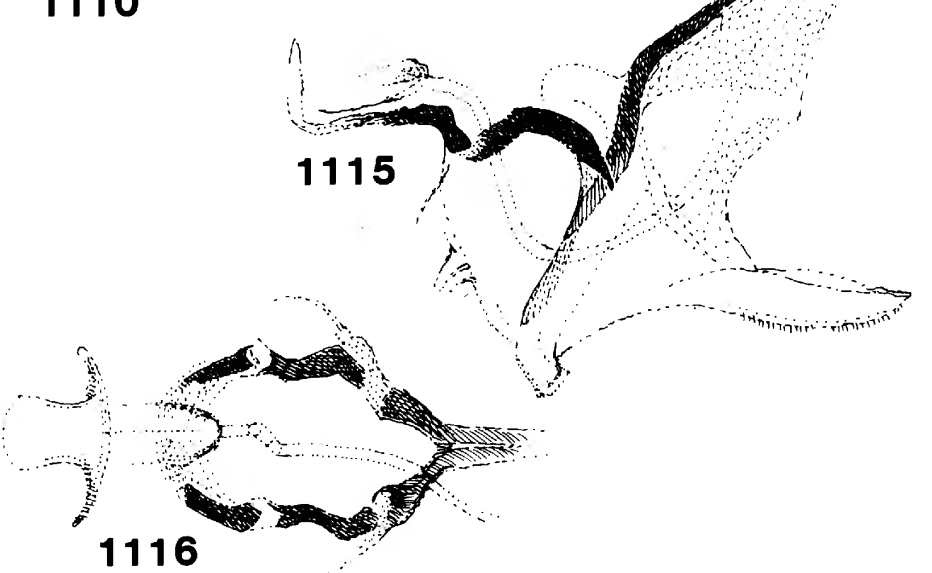

Figures 1106-1116.--Phytomyza modocensis: 1106, Aedea-

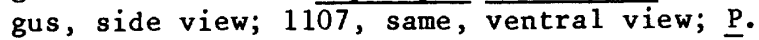
minutissima: 1108, Head; 1109, wing; 1110 , aedeagus, side view; P. flaviantennalis: 1111 ,

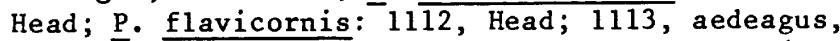
side view (Oregon); 1114 , same, ventral view (ex

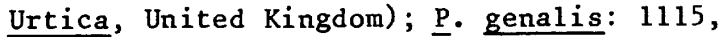
Aedeagus, side view; 1116 , same, ventral view. 


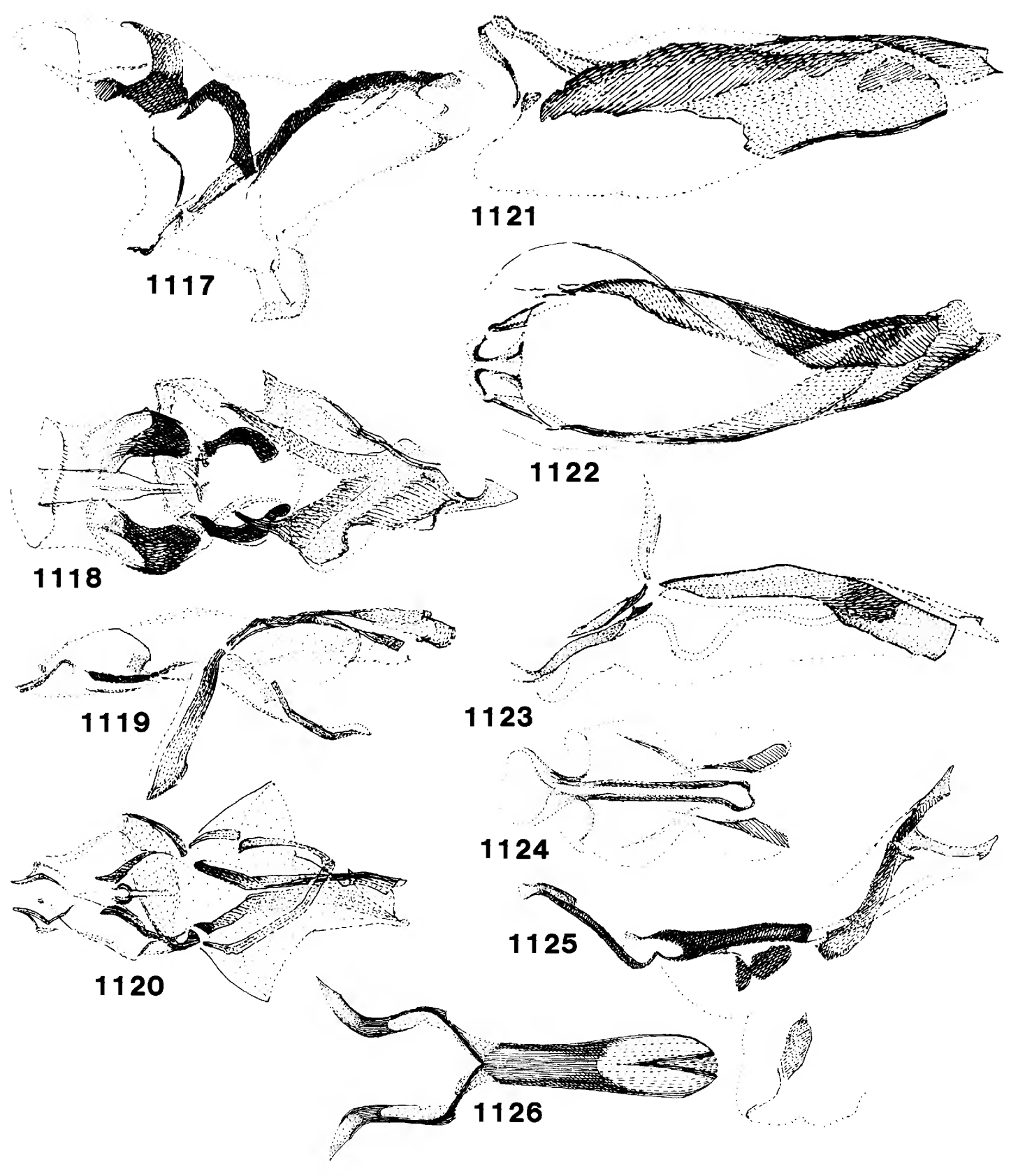

Figures 1117-1126.--Phytomyza coquilletti: 1117, Aedea-

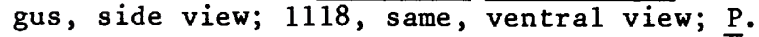
nervosa: 1119 , Aedeagus, side view; 1120, same, ventral view (Lafayette, Ind.); $\underline{P}$. felix: 1121, Aedeagus, side view; 1122, same, ventra1 view. Chromatomyia castillejae ssp. nordica: 1123 , Aedeagus, side view; 1124, same, ventral view. $P$. plumiseta: 1125, Aedeagus, side view (ex Thalict $\bar{r}$ um polygamum, Quebec); 1126, distiphallus, ventral view. 


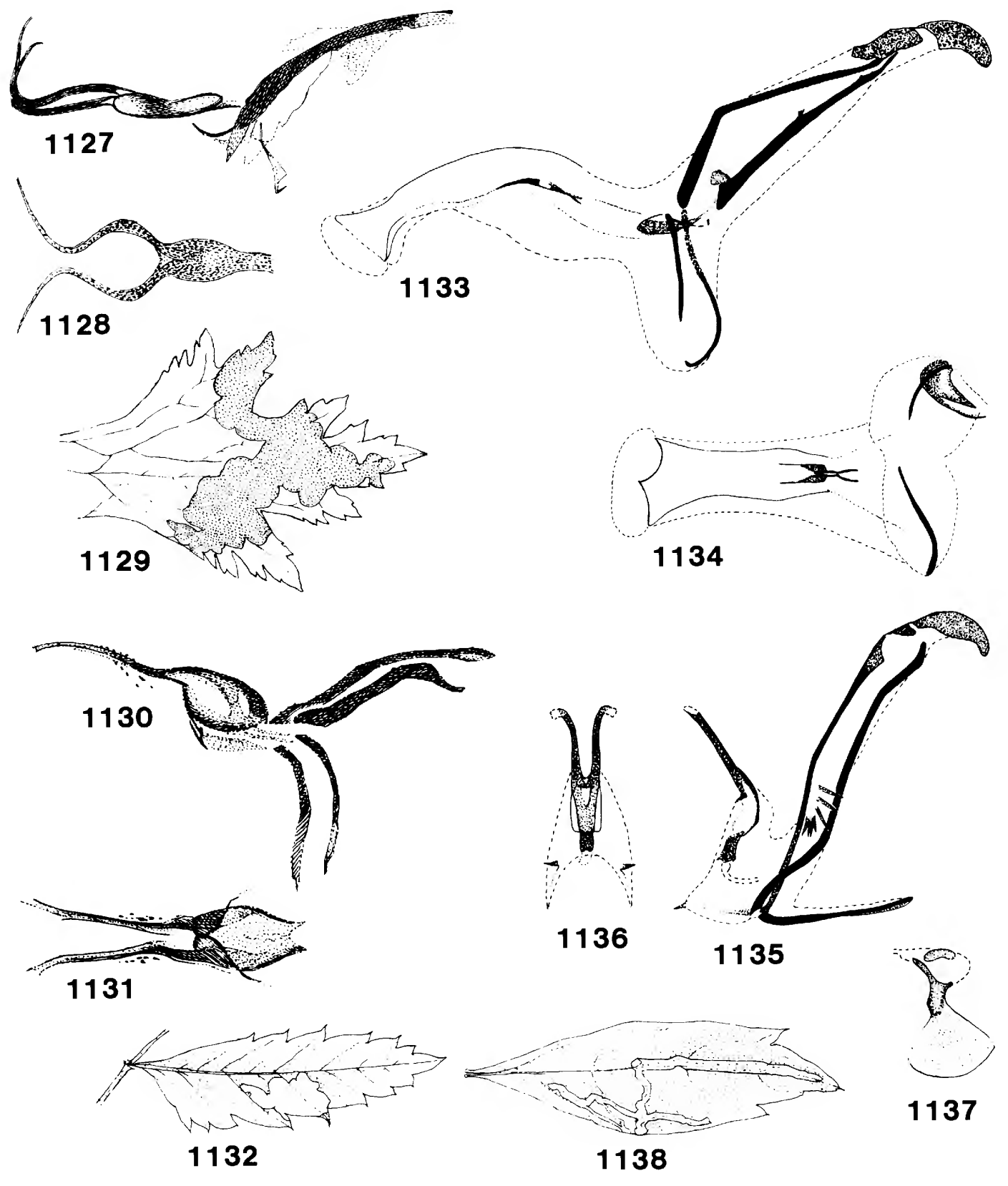

Figures 1127-1138.--Phytomyza aconiti: 1127, Aedeagus, side view; 1128 , distiphallus, ventral view; 1129 , leaf mine on Aconitum; P. cicutel1a: 1130, Aedeagus, side view; $11 \overline{3} 1$, distiphallus, ventral view; 1132, leaf mine on Cicuta douglasii; $\underline{P}$. t1ingitica: 1133, Aedeagus, side view; 1134, distiphallus, ventral view (holotype, Alaska, after Griffiths); P. alaskana: 1135, Aedeagus, side view; 1136 , distiphallus, anteroventral view; 1137 , sperm pump; (1135-1137, Alaska, after Griffiths); 1138, leaf mine on Artemisia douglasiana (California). 


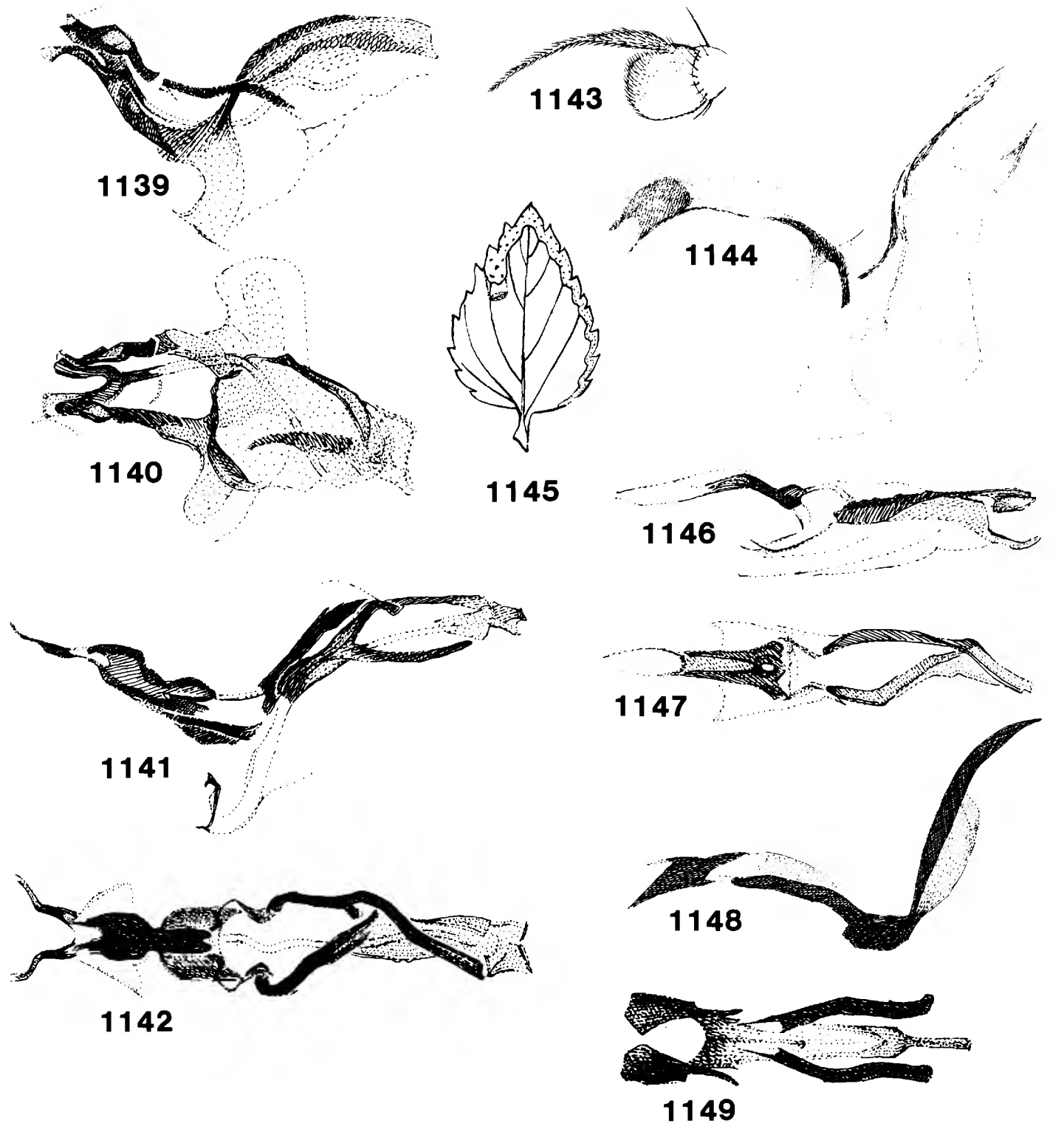

Figures 1139-1149.--Phytomyza flavinervis: 1139, Aedeagus, side view; $11 \overline{40, \text { same, ventral view }}$ (paratype); $\underline{P}$. auricornis: 1141, Aedeagus, side view; 1142, same, ventral view (new record, New York); $\underline{P}$. crassiseta: 1143, Third antennal segment; 1144 , aedeagus, side view; 1145, leaf mine on

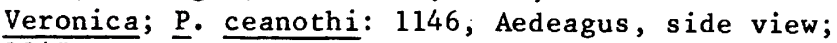
1147 , same, ventral view; $\underline{P}$. subtenella: 1148 , Aedeagus, side view (Alberta);1149, same, ventral view (N. California). 

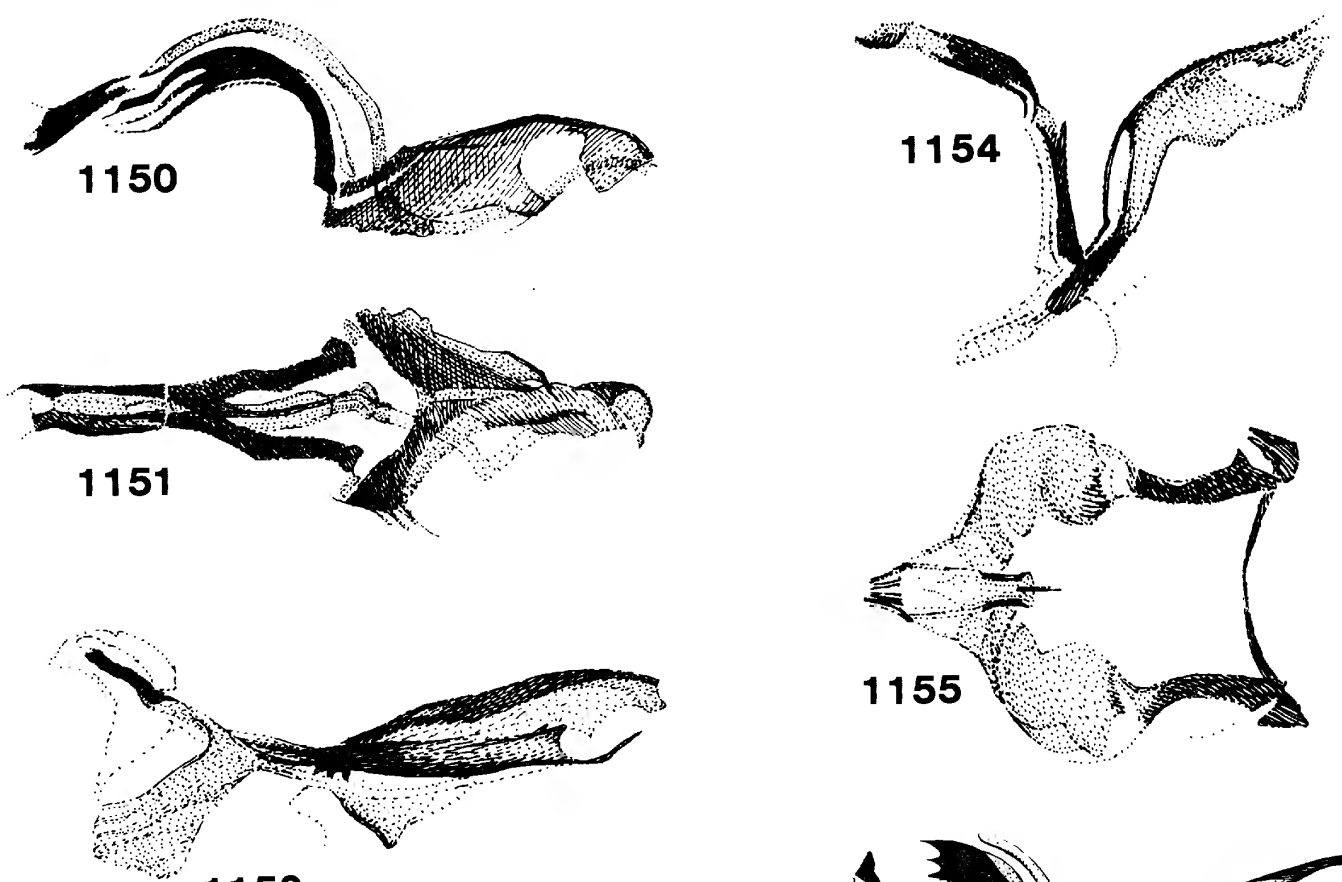

\section{2}
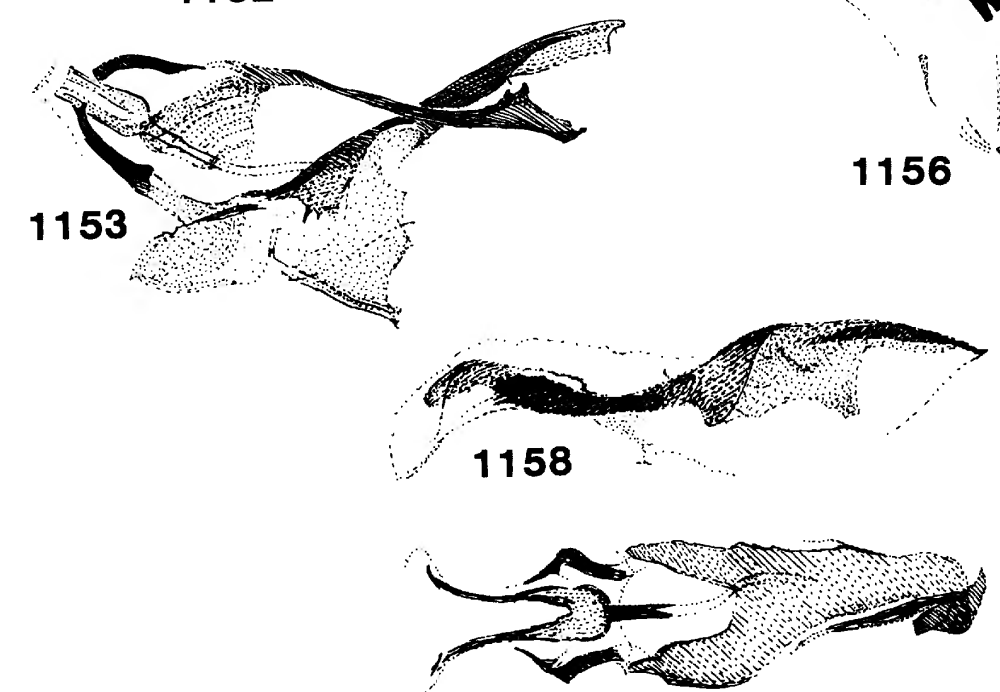

1157
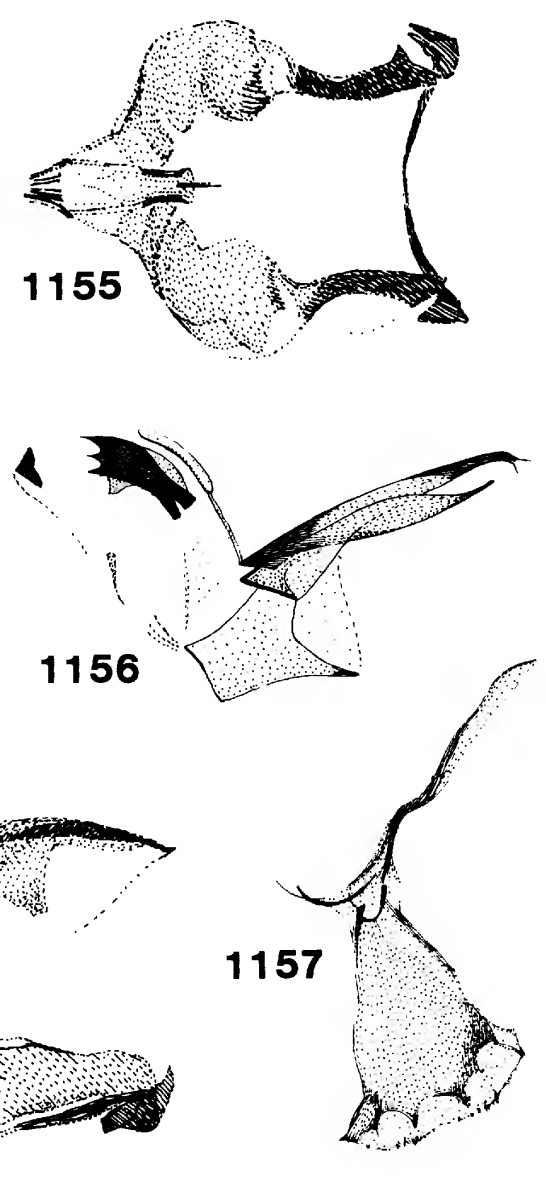

1159

Figures 1150-1159.--Phytomyza orindensis: 1150, Aedea-

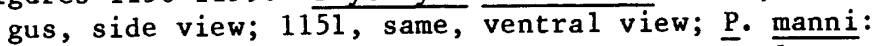

1152 , Aedeagus, side view; 1153, same, ventral

view; $P$. carbonensis: 1154 , Aedeagus, side view;

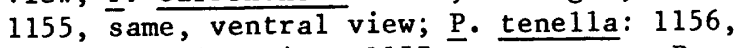

Aedeagus, side view; 1157 , sperm pump; $P$.

subalpina: 1158, Aedeagus, side view; $1 \overline{1} 59$, same, ventral view (Colorado). 

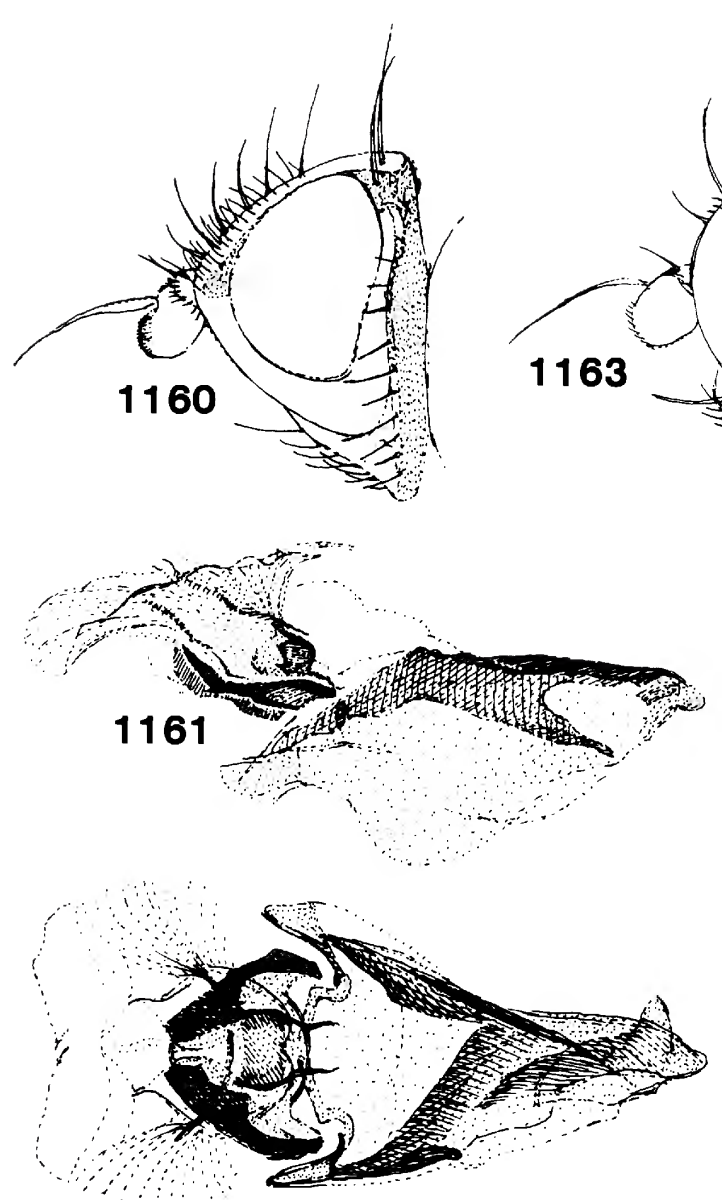

1162
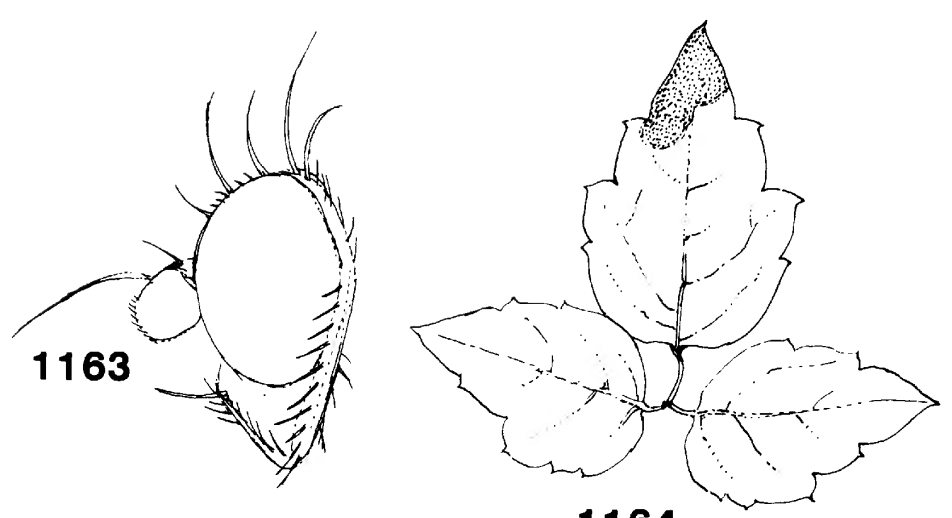

1164
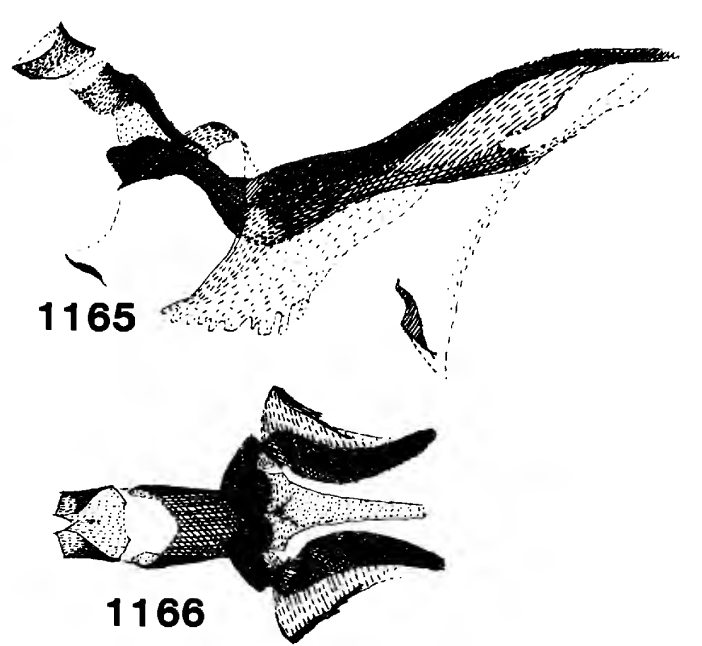

1166
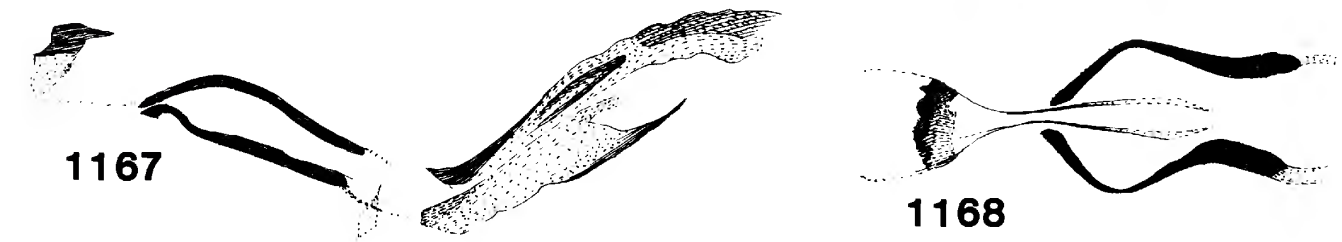

Figures 1160-1168.--Phytomyza dreisbachi: 1160, Head;

1161 , aedeagus, side view; 1162 , same, ventral

view; $\underline{P}$. 1igusticifoliae: 1163, Head; 1164, leaf

mine on clematis ligusticifolia; $\underline{P}$. orlandensis:

1165, Aedeagus, side view; 1166, same, ventra1

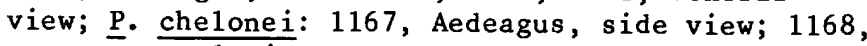
same, ventral view. 

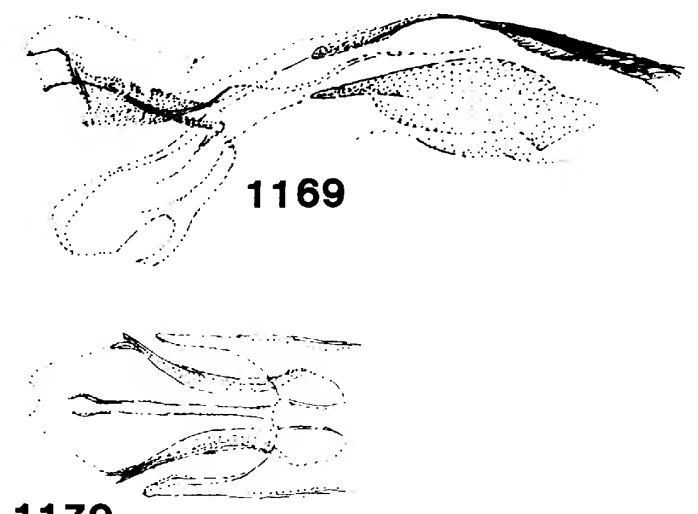

1170
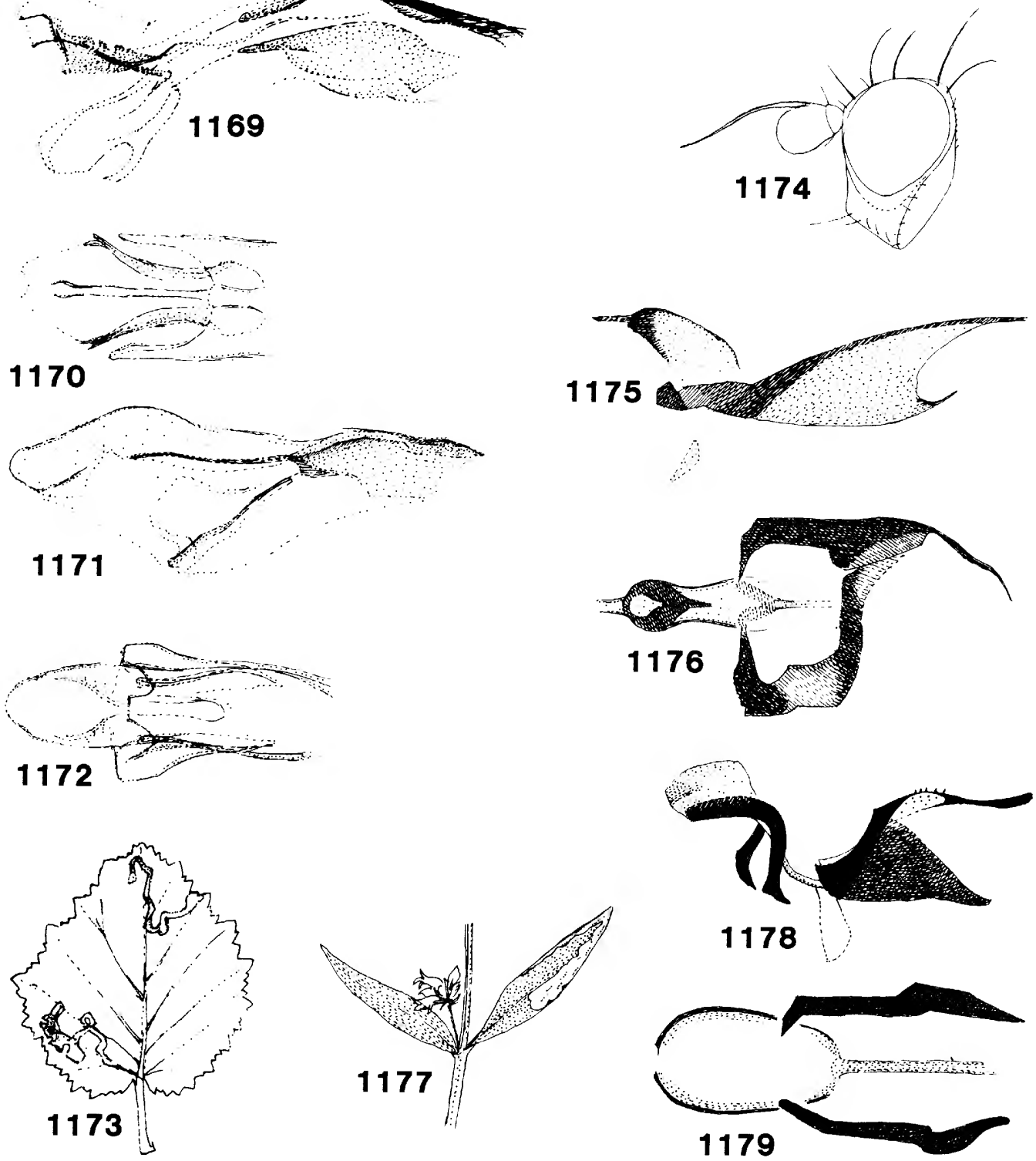

Figures 1169-1179.--Chromatomyia eriodictyi: 1169, Aedeagus, side view; 1170, distiphallus, ventral view; C. mimuli: 1171, Aedeagus, side view; 1172 , distiphallus, ventral view (holotype); 1173 , leaf mines on Phacelia viscida. Phytomyza penstemonis: 1174, Head; 1175, aedeagus, side view; 1176, same, ventral view; 1177 , leaf mine on Penstemon confertus (Alberta); $\underline{P}$. plantaginis: 1178, Aedeagus, side view; 1179 , same, ventral view (Europe). 


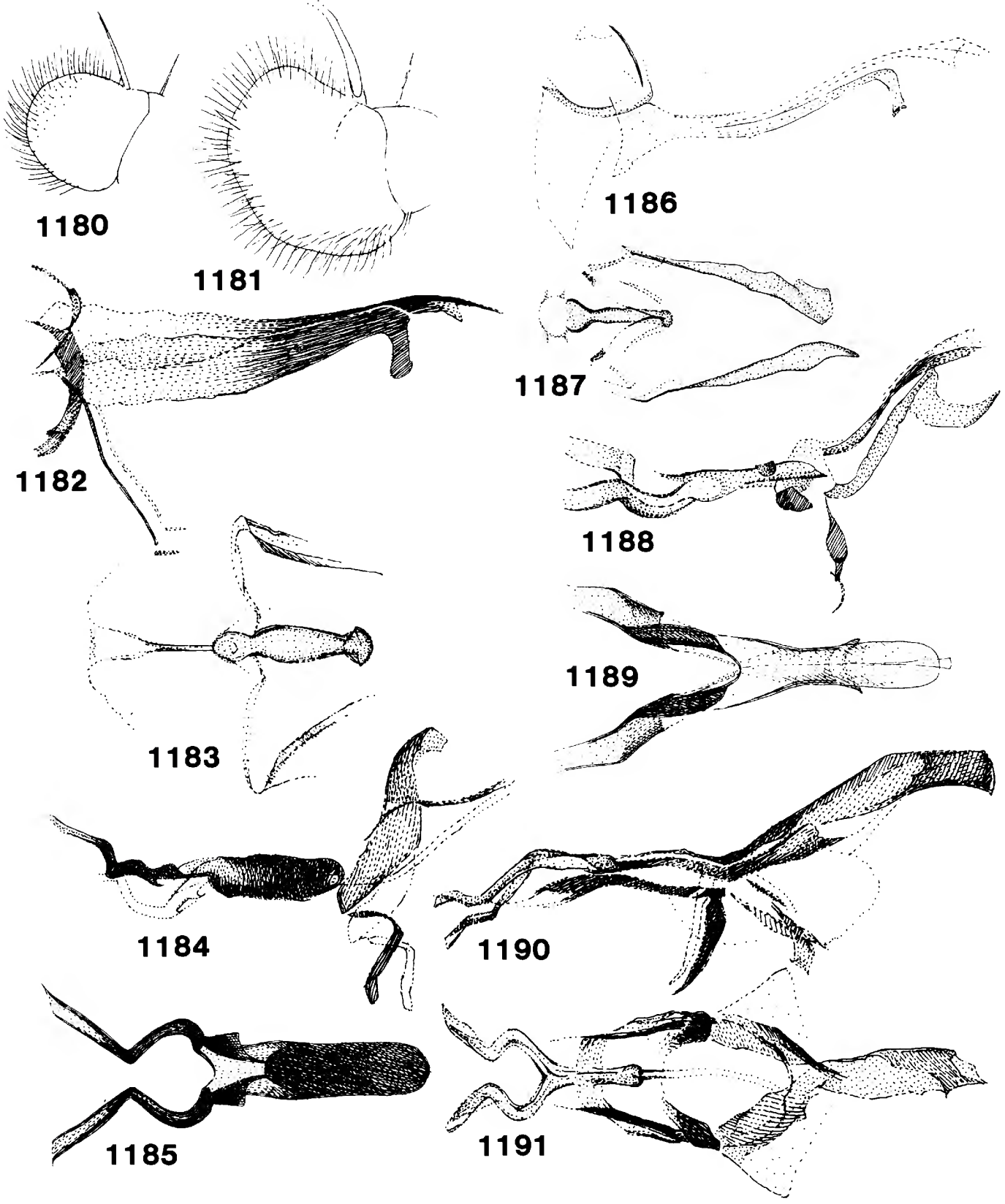

Figures 1180-1191.--Chromatomyia lactuca: 1180, Third antennal segment, male; 1181, same, female; 1182, aedeagus, side view; 1183, distiphallus, ventral view. Phytomyza ilicis: 1184, Aedeagus, side view; 1185 , distiphallus, ventral view. C. syngenesiae: 1186, Aedeagus, side view; 1187 , same, ventral view. $\mathrm{P}$. aquilegiana: 1188 , Aedeagus, side view;

1189 , distiphallus, ventral view; $\underline{P}$. latifrons:

1190, Aedeagus, side view; 1191, same, ventral view. 


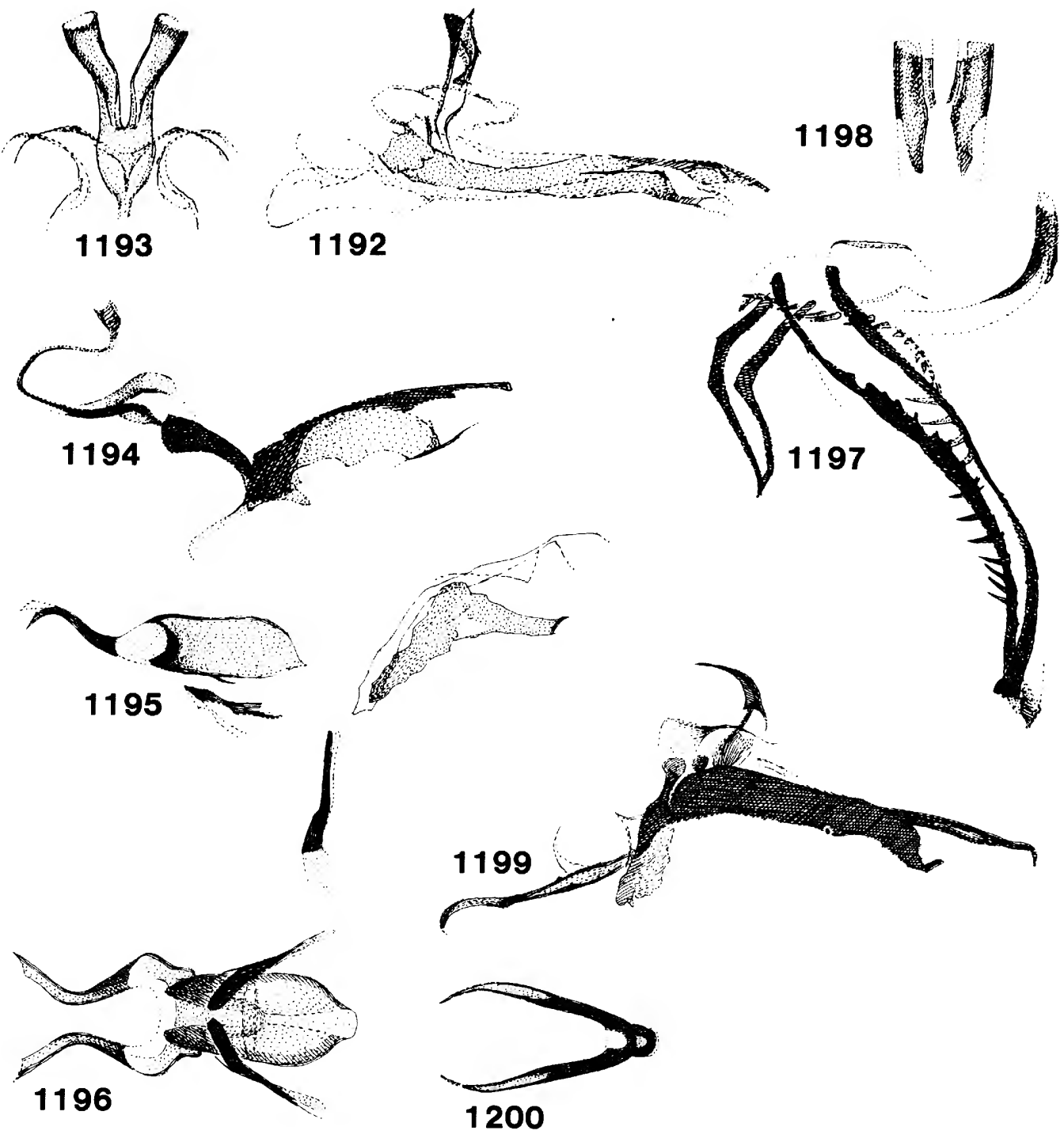

Figures 1192-1200.--Phytomyza flavens: 1192, Aedeagus, side view; 1193, distiphallus, anteroventral view; P. clematisana: 1194, Aedeagus, side view; $\underline{\text { P. }}$ subtilis: 1195, Aedeagus, side view; 1196,

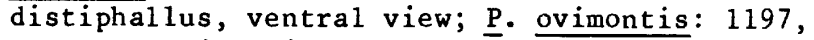
Aedeagus, side view; 1198, distiphallus, anteroventral view (California); $\underline{P}$. wahlgreni: 1199, Aedeagus, side view; 1200, distiphallus, dorsal view. 

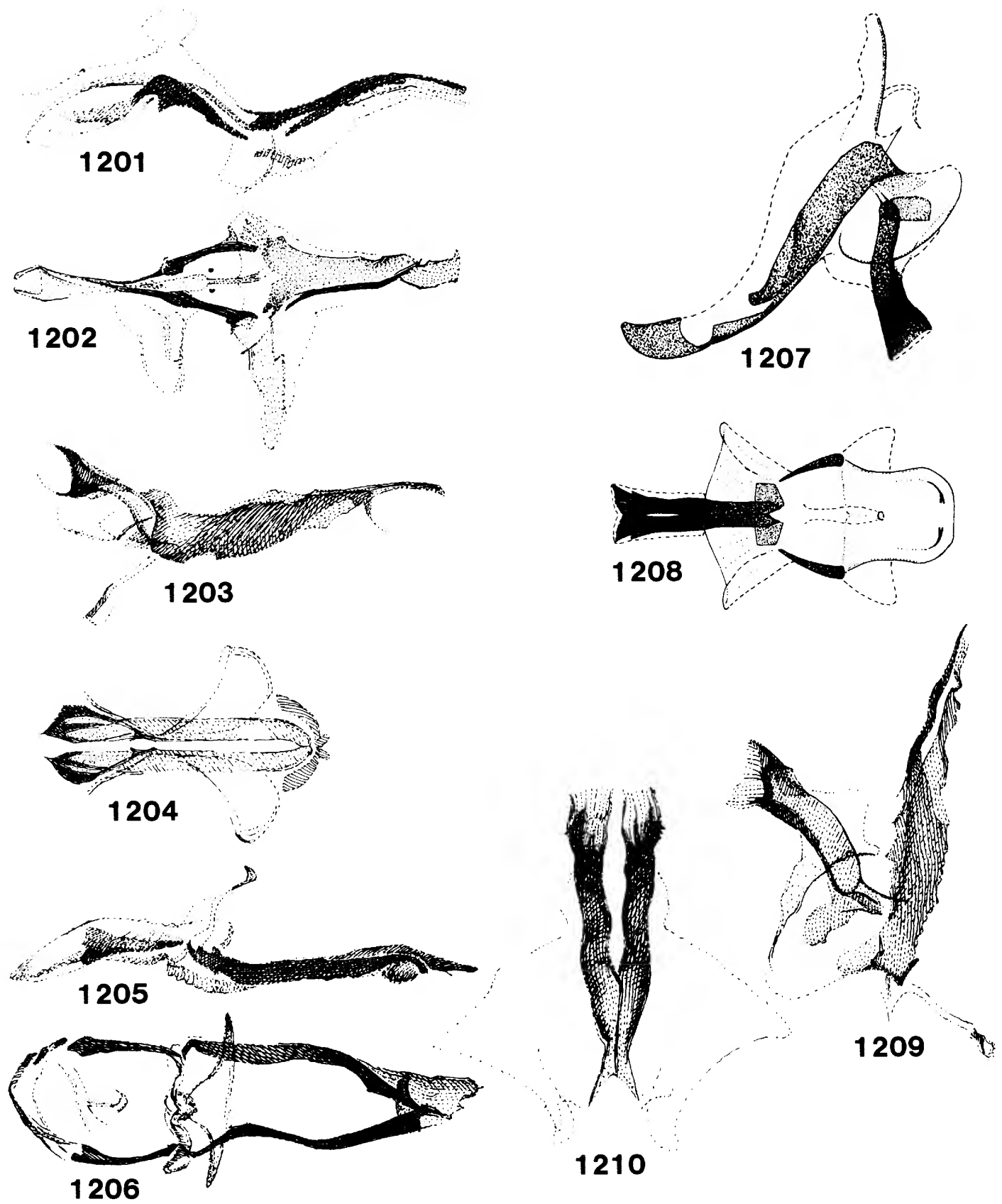

Figures 1201-1210.--Phytomyza coloradel1a: 1201, Aedeagus, side view; $12 \overline{02}$, same, ventral view; $P$. alamedensis: 1203, Aedeagus, side view; 120 4 , distiphallus, ventral view. Chromatomyia montella: 1205, Aedeagus, side view; 1206, same, dorsal view. P. integerrimi: 1207, Aedeagus, side view; 1208 , same, anteroventral view (holotype, Oregon, after Griffiths); . crepidis: 1209, Aedeagus, side view; 1210 , distiphallus, anteroventral view. 


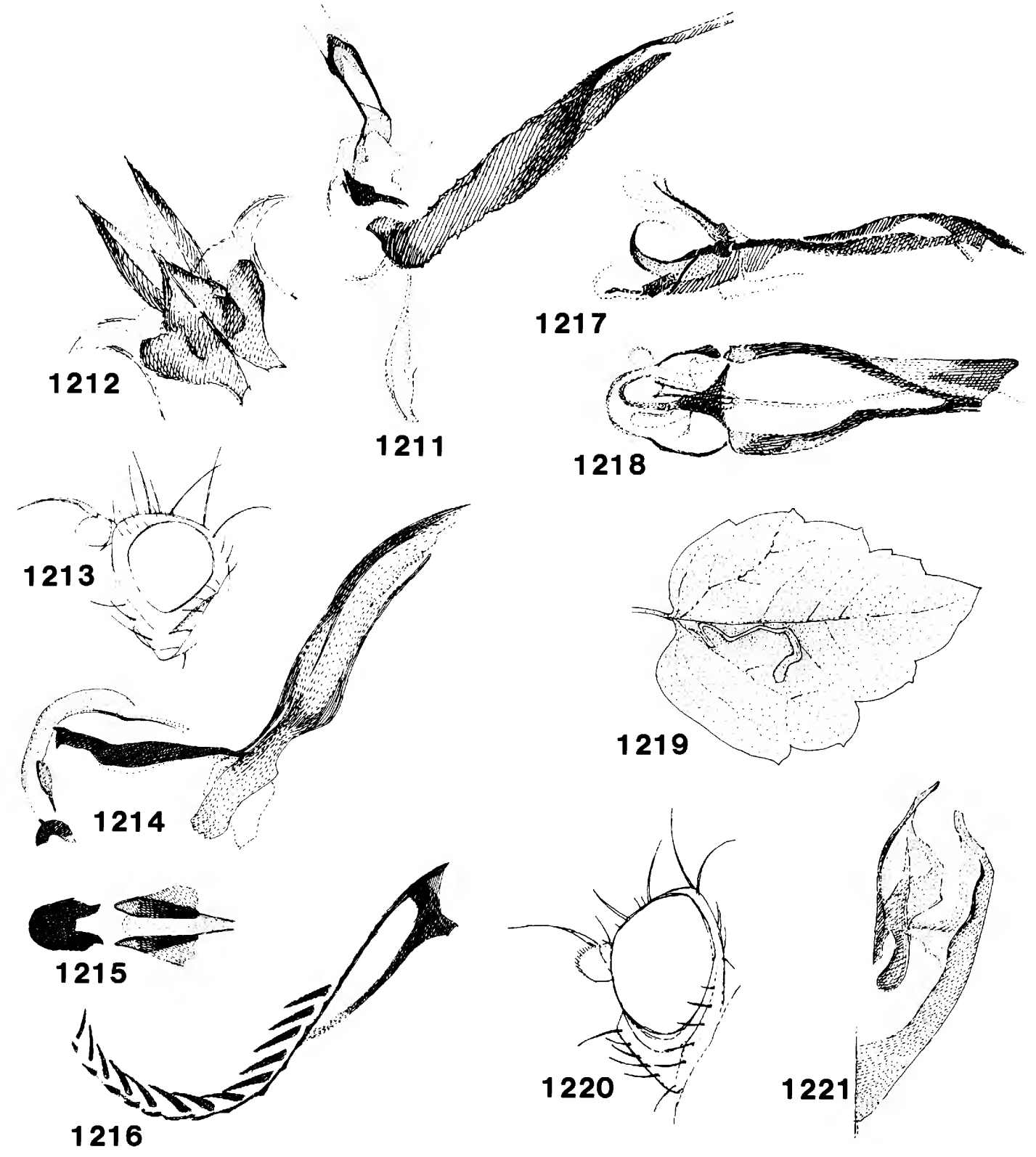

Figures 1211-1221.--Phytomyza montereyensis: 1211 , Aedeagus, side view; 1212, distiphallus, ventral

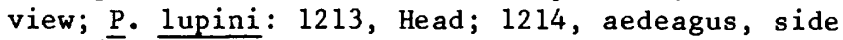
view (paratype, Alberta); 1215, distiphallus, ventral view (California); $\underline{P}$. erigerophila: 1216, Right basal sclerite of aedeagus; P. clematoides: 1217, Aedeagus, side view; 1218, same, ventral view; 1219, leaf mine on Clematis ligusticifolia; P. penstemonella: 1220 , Head; 1221 , hypandrium (holotype). 

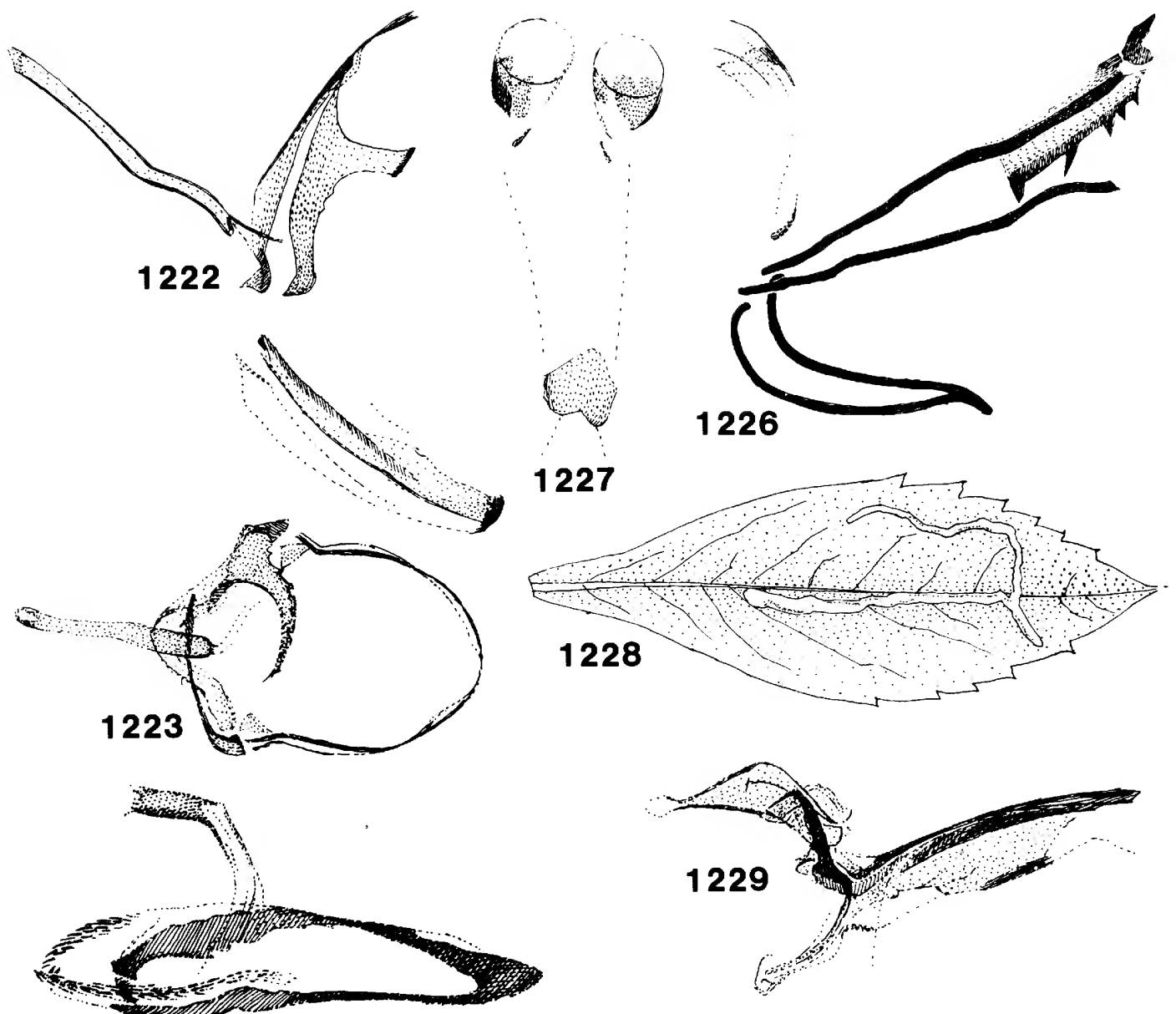

\section{4}
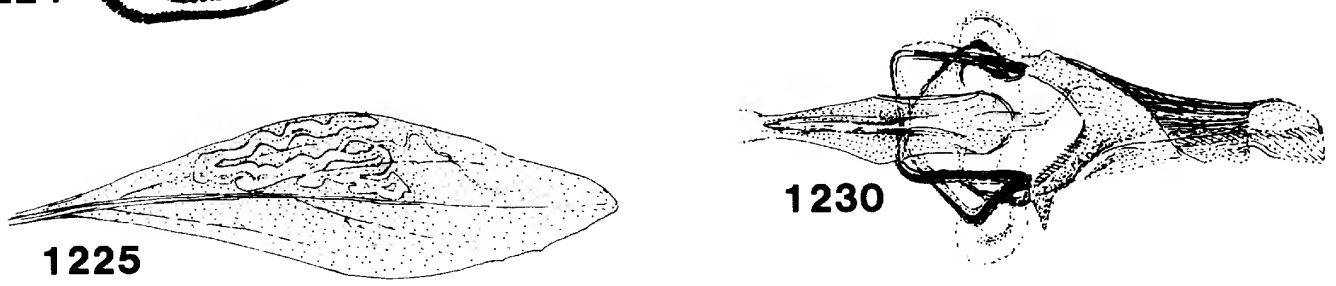

Figures 1222-1230.--Phytomyza aquilegivora: 1222, Aedeagus, side view (Ontario); 1223, same, ventral view (New York); P. Saximontana: 1224, Aedeagus, side view; 1225 , leaf mine on Arnica; $\underline{P}$.

asterophaga: 1226, Aedeagus, side view; 1227, distiphallus, anteroventral view; 1228 , leaf mine on Aster conspicuus (A1berta); $\underline{P}$. bouldere1la: $122 \overline{9}$, Aedeagus, side view; 1230 , same, ventral view. 


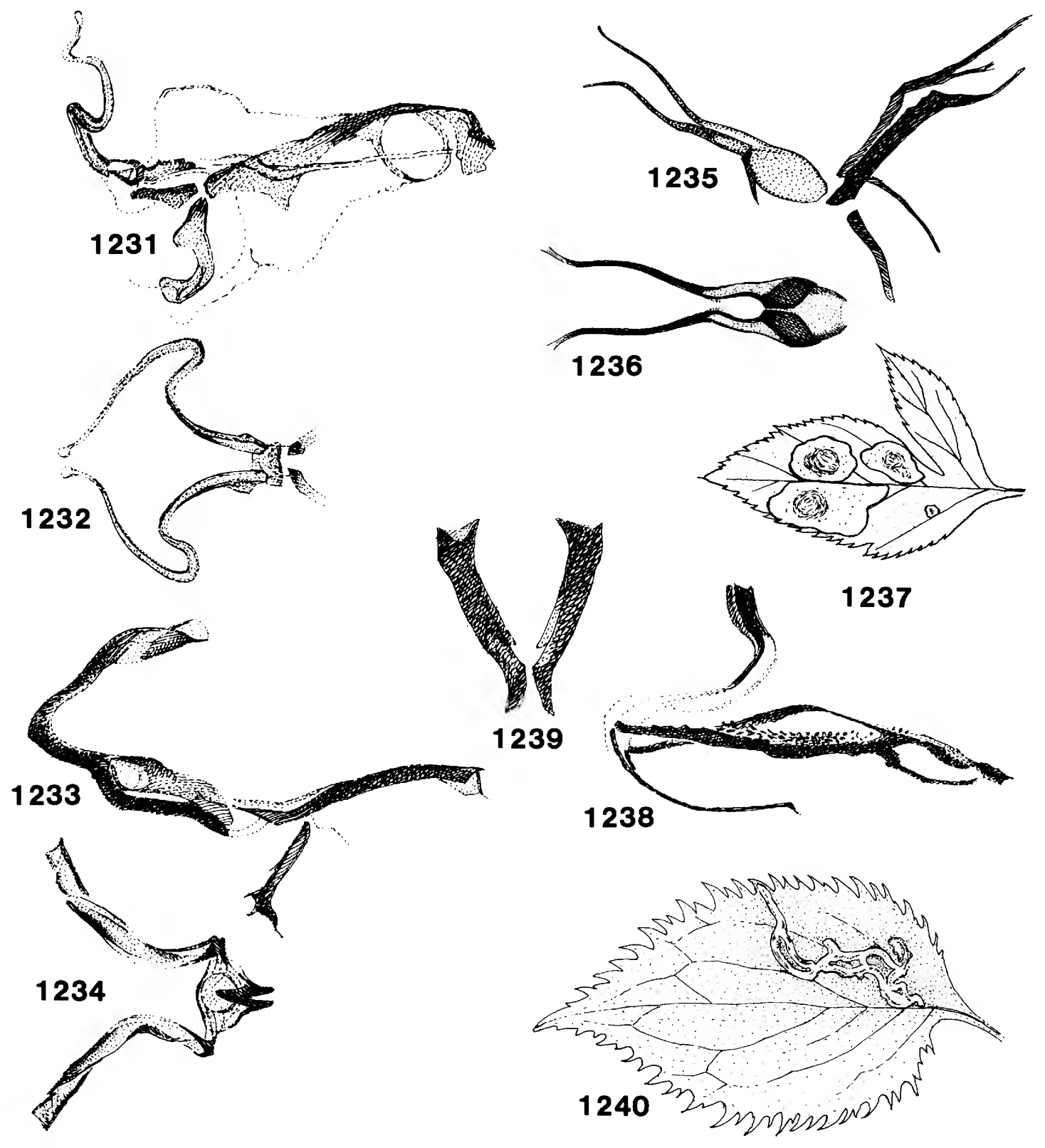

Figures 1231-1240.--Phytomyza flexuosa: 1231, Aedea-

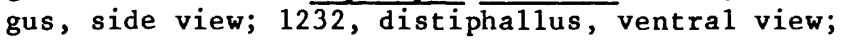
P. aquilegioides: 1233, Aedeagus, side view; 1234 , distiphallus, anterodorsal view (California); $\underline{P}$. angelicae: 1235, Aedeagus, side view; 1236, distipha1 lus, ventral view; 1237 , leaf mines on Angelica; $\underline{\mathrm{P}}$ - astotinensis: 1238 , Aedeagus, side view; 1239, distipha1lus, ventral view; 1240, leaf mine on Solidago flexicaulis (Wisconsin). 
1241
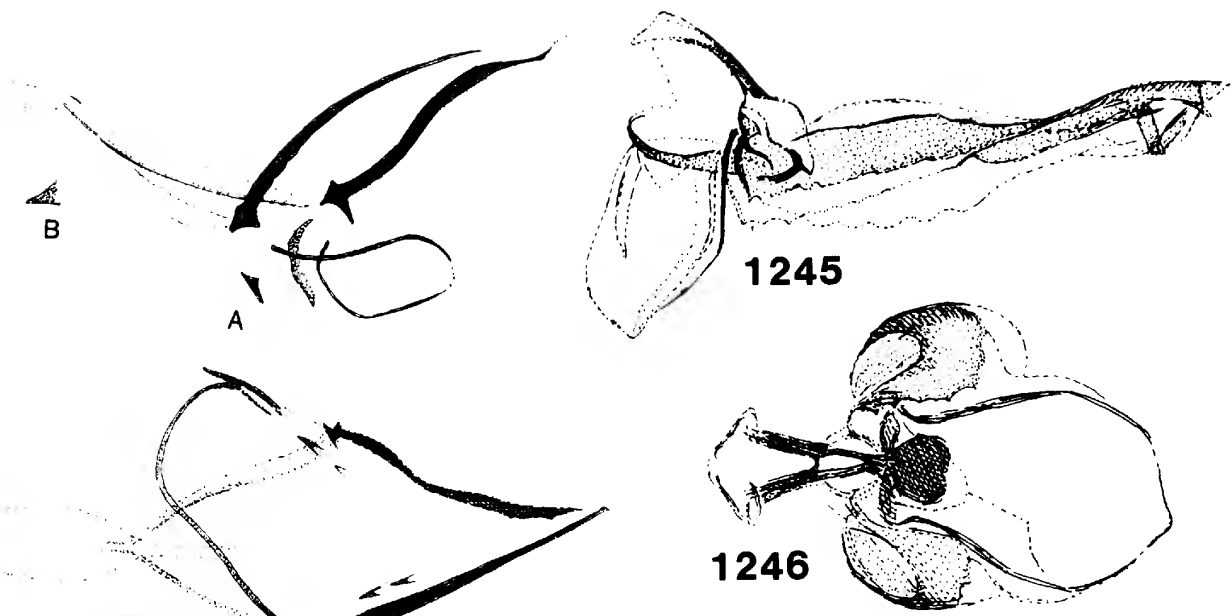

1242

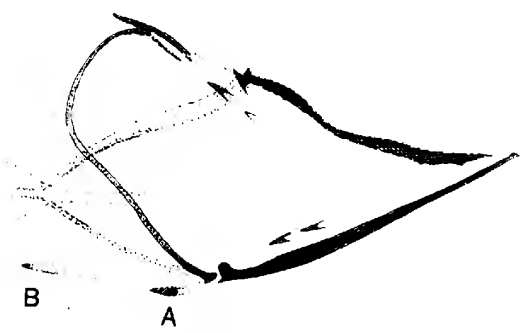

1246

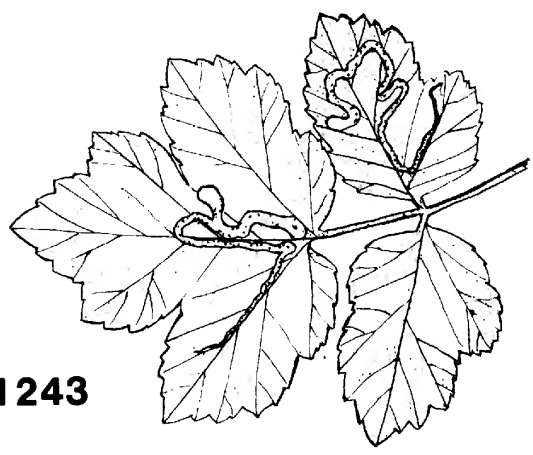

$$
\text { . }
$$
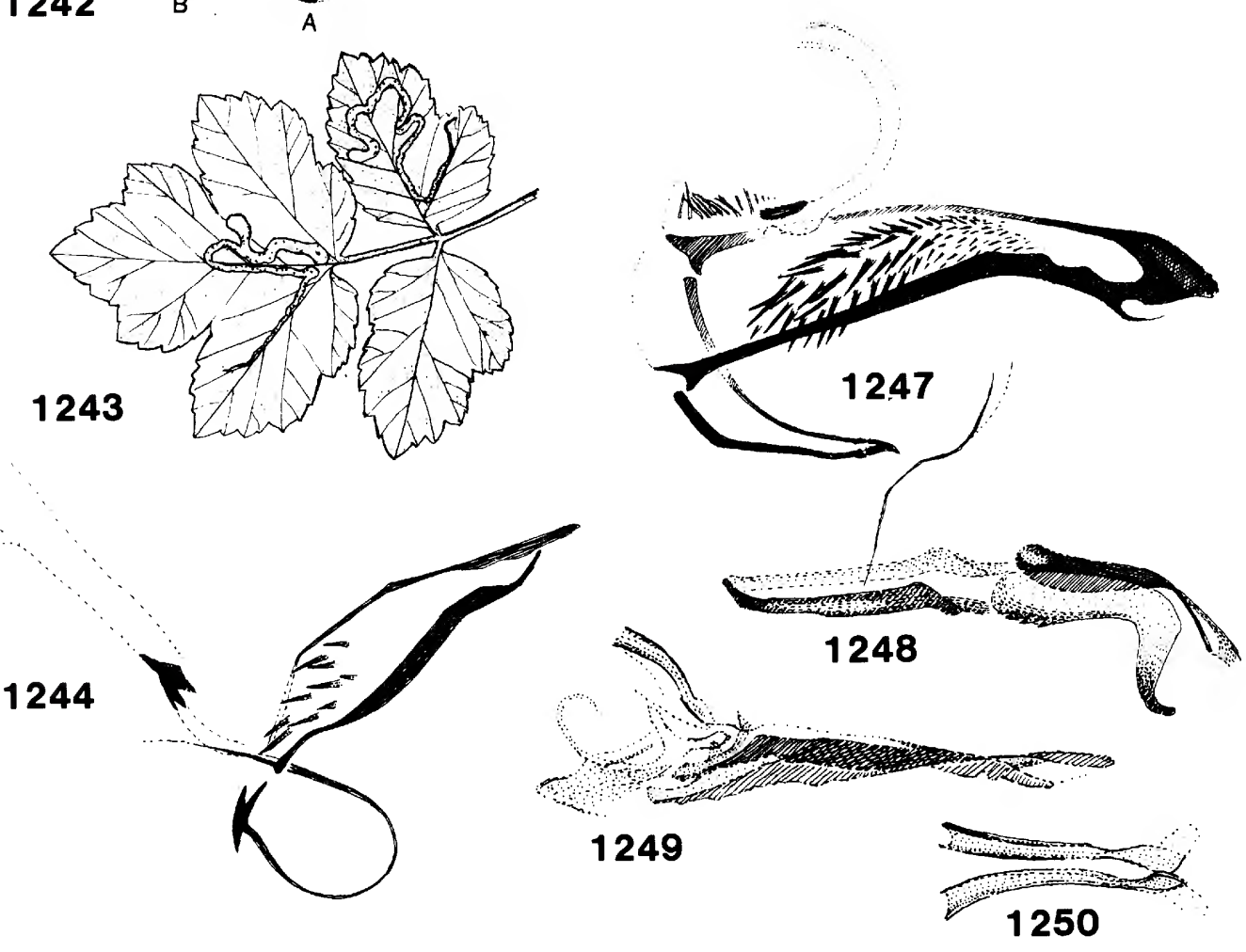

Figures 1241-1250.--Phytomyza spondylii: 1241, Aedeagus, side view, with characteristic spicules (A, B); 1242 , same, ventral view; 1243, leaf mines on Heracleum; $P$. pastinacae: 1244 , Aedeagus, side view. Chromatomyia flavida: 1245, Aedeagus, side view; 1246 , same, ventral view. $\underline{\text { p arnicicola: }}$ 1247, Aedeagus, side view (holotype, Sweden); $\underline{P}$. notopleuralis: 1248 , Aedeagus, side view. $\underline{C}$. orbitella: 1249, Aedeagus, side view; 1250, supporting sclerites, dorsal view. 

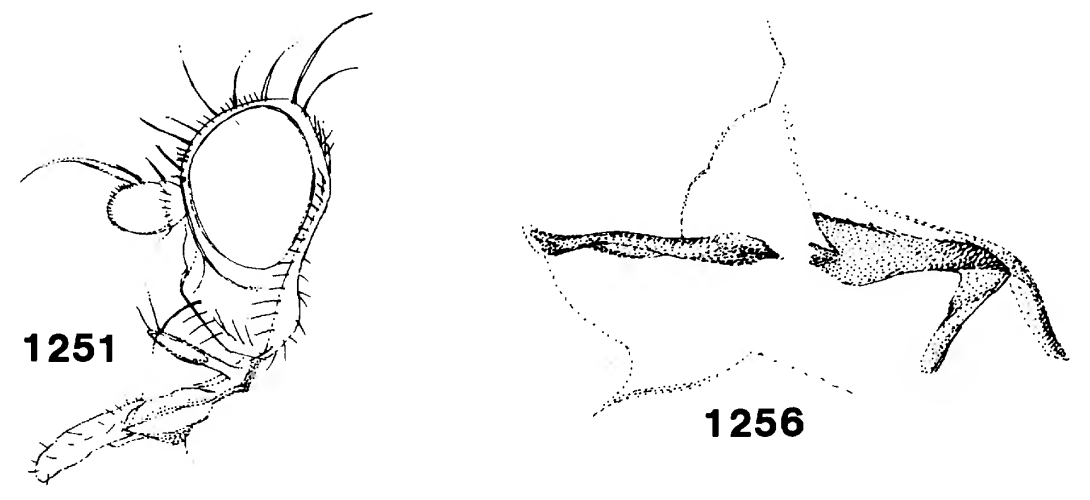

1256
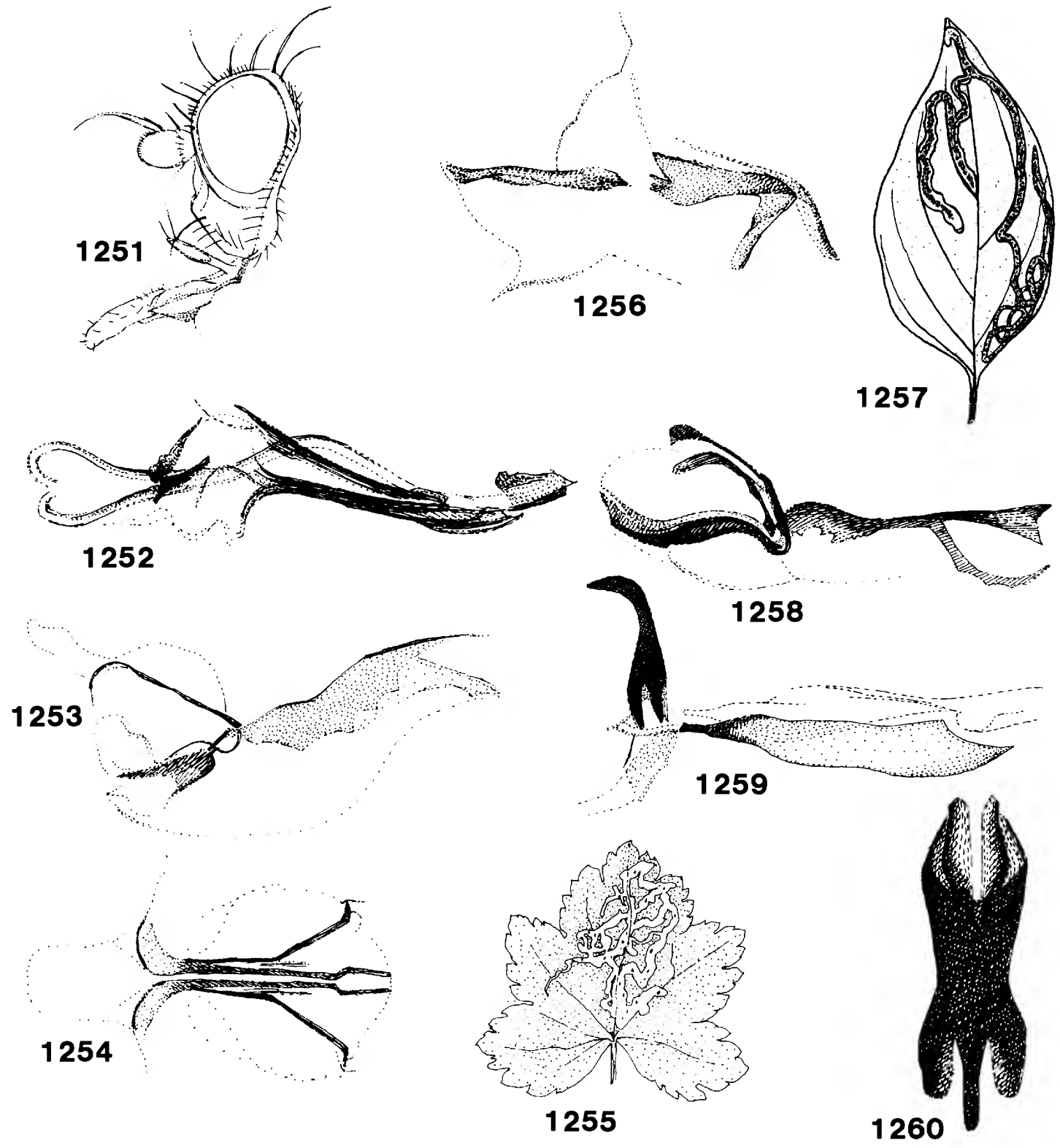

Figures 1251-1260.--Phytomyza bicolor: 1251, Head;

1252, aedeagus, ventral view. Chromatomyia

tiarellae: 1253, Aedeagus, side view; 1254,

supporting sclerites, dorsal view; 1255, leaf mines

on Tellima grandiflora (California). $\underline{\text {. }}$

agromyzina: 1256 , Aedeagus, side view; 1257 , leaf

mine on Cornus. C. montana: 1258, Aedeagus, side

view; c. involucratae: 1259, Aedeagus, side view;

1260 , distiphallus, anteroventral view. 


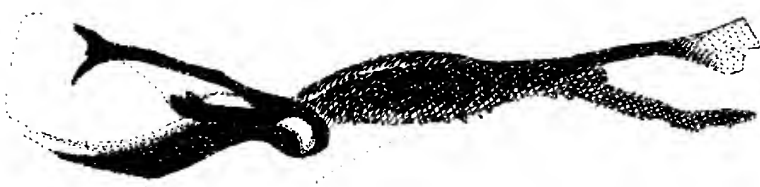

1261
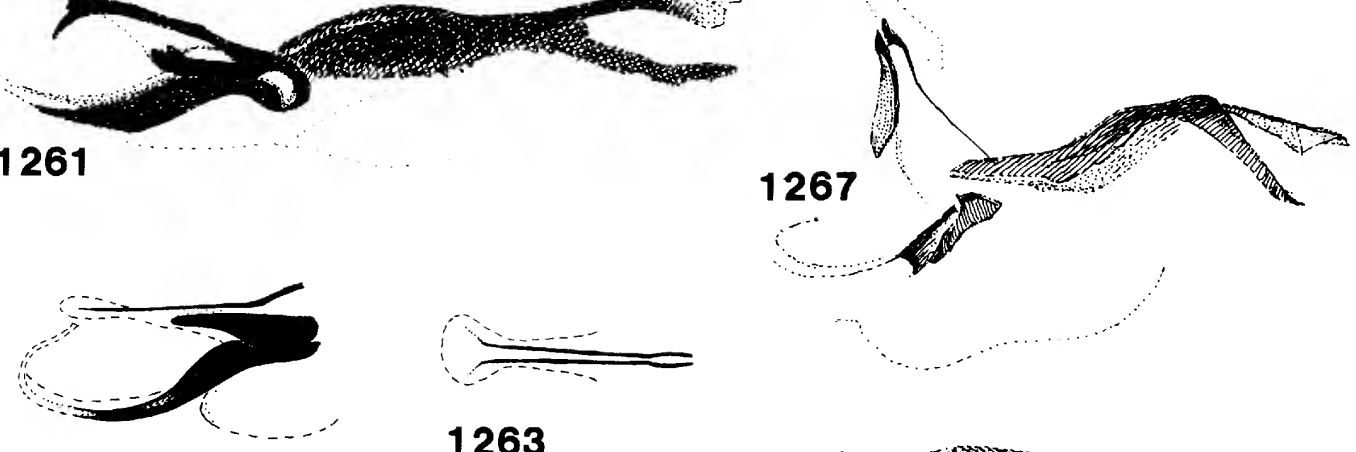

1262

\section{3}
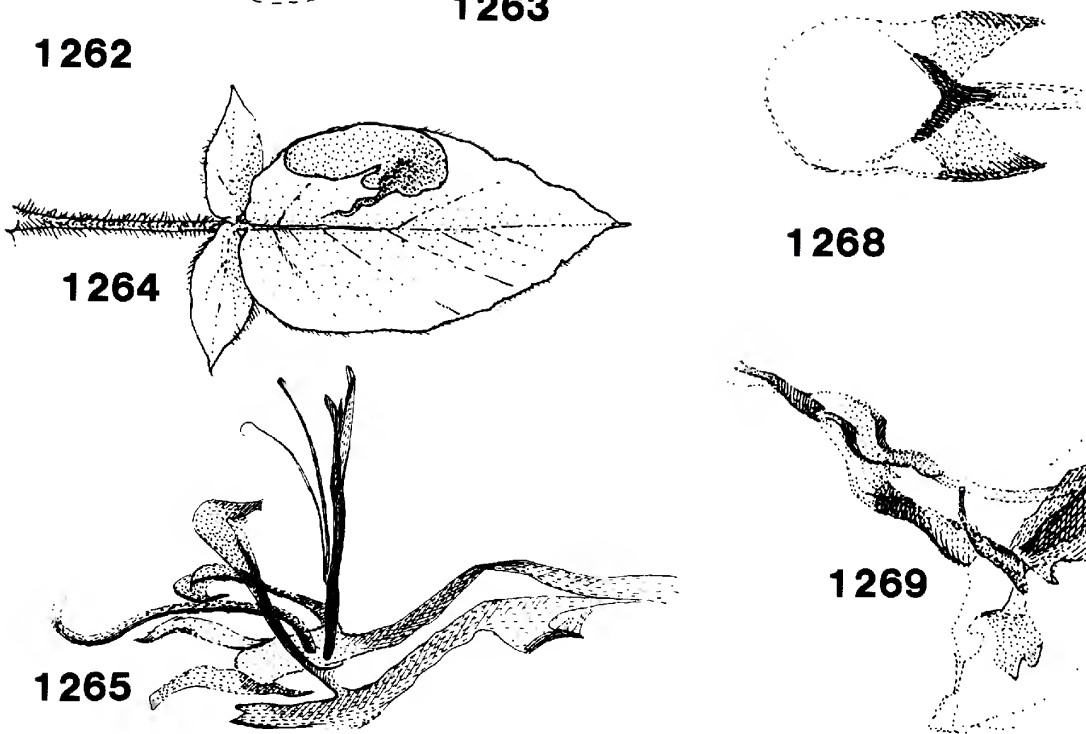

1268
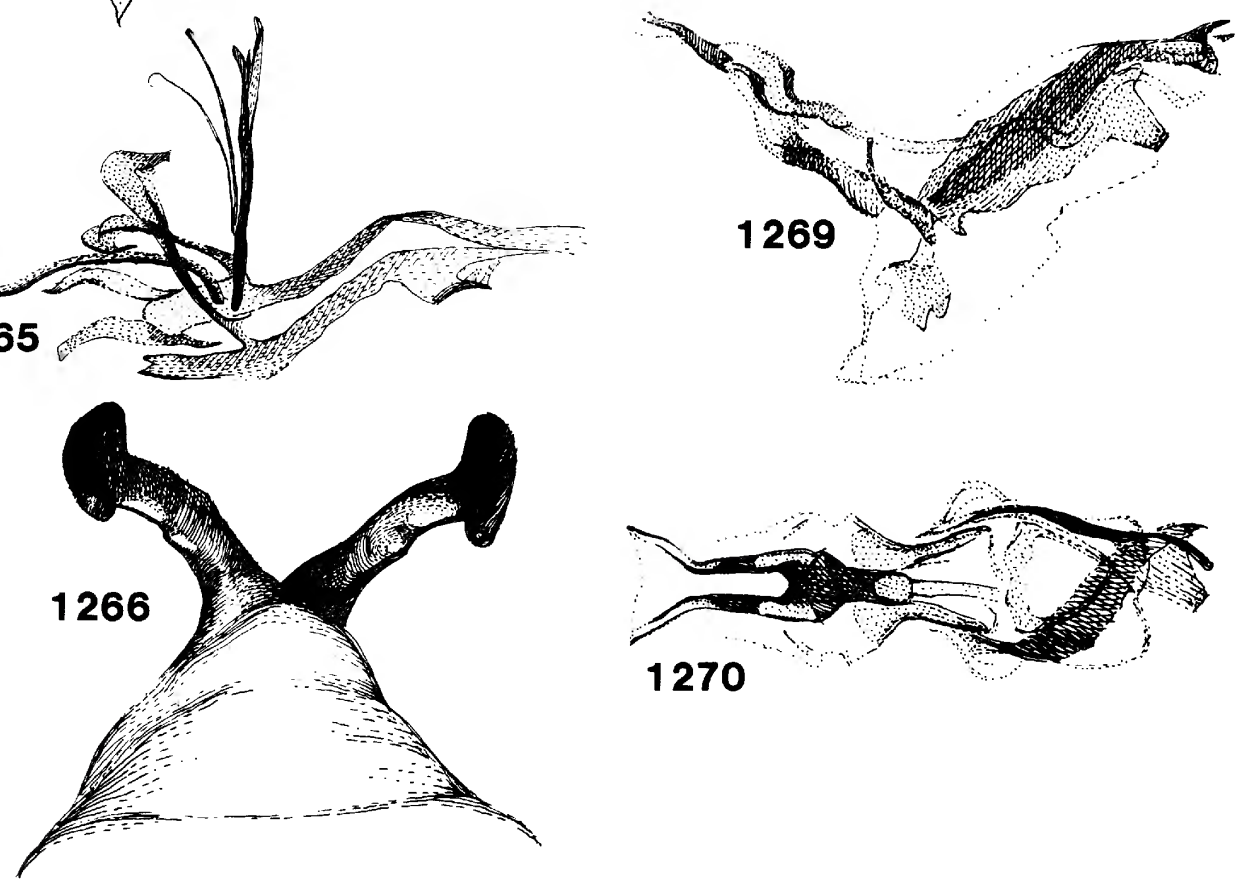

Figures 1261-1270.--Chromatomyia pseudomilii: 1261, Aedeagus, side view; $\underline{C}$. norwegica: 1262, Aedeagus, side view; 1263 , supporting sclerites, dorsal view (holotype, Norwegian Lapland, after Griffiths, 1980). Phytomyza phaceliae: 1264, Leaf mine on Phacelia californica. C. nigra: 1265, Aedeagus, side view; 1266, anterior spiracles of puparium; C. griffithsi: 1267 , Aedeagus, side view; 1268 , dorsa 1 lobe, dorsal view. P. nigrinervis: 1269, Aedeagus, side view; 1270 , same, ventral view. 

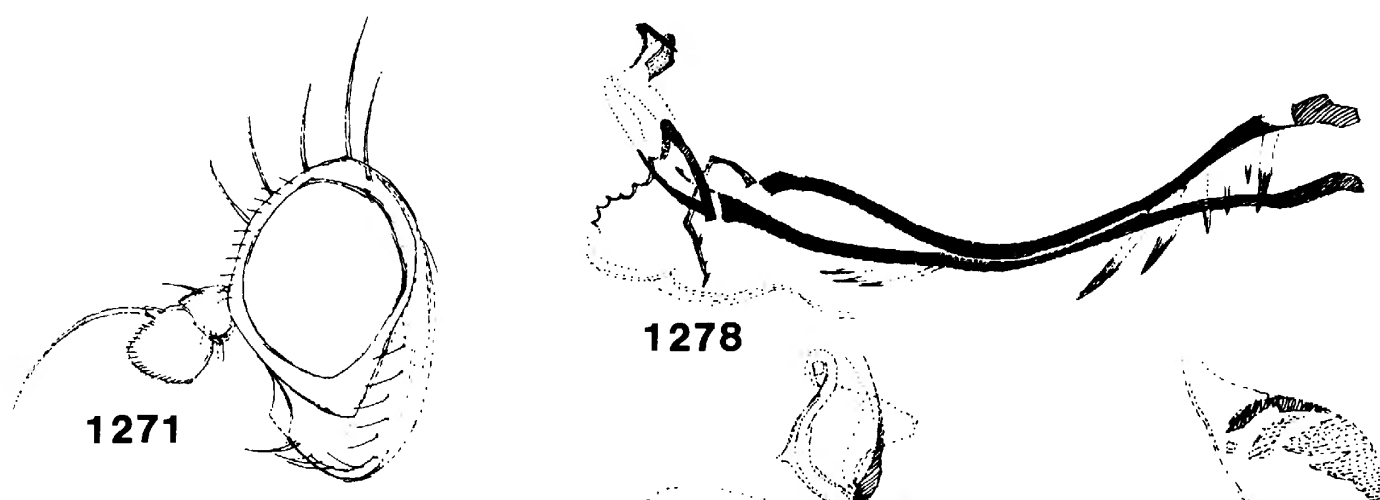

1278
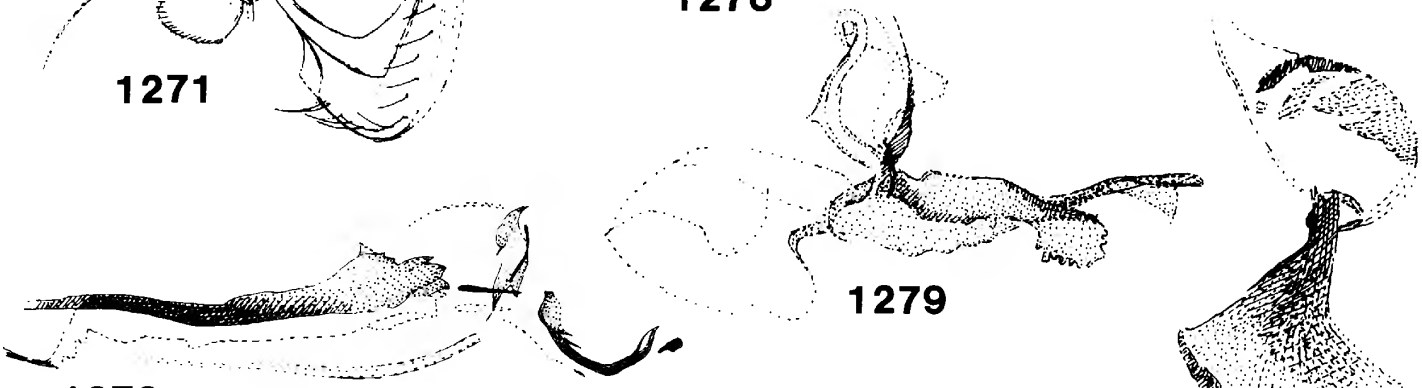

\section{2}
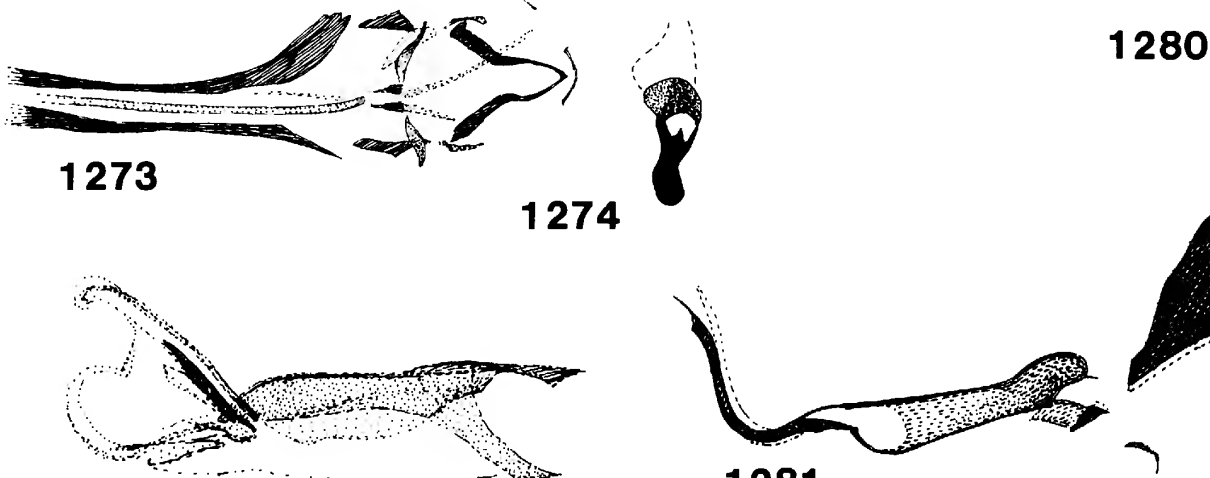

1275
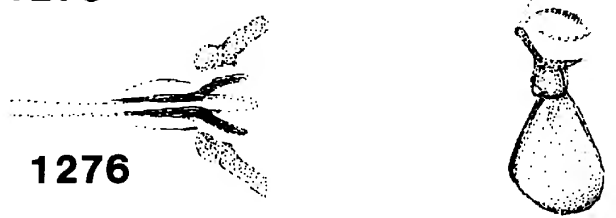

1277

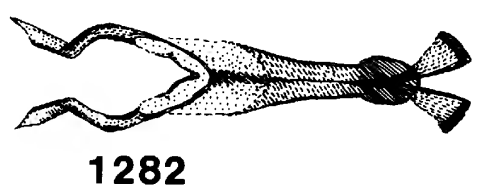

Figures 1271-1282.--Chromatomyia erigerontophaga:

1271, Head; 1272 , aedeagus, side view; 1273, same, ventral view (holotype, Greenland); 1274 , sperm pump (Alberta, after Griffiths); C. nigrella: 1275, Aedeagus, side view; 1276 , supporting sclerites, dorsal view; 1277 , sperm pump. Phytomyza melanella: 1278, Aedeagus, side view (holotype). C. poae: 1279 , Aedeagus, side view; 1280, sperm pump (Colorado). P. vomitoriae: 1281, Aedeagus, side view; 1282 , distiphallus, ventral view. 

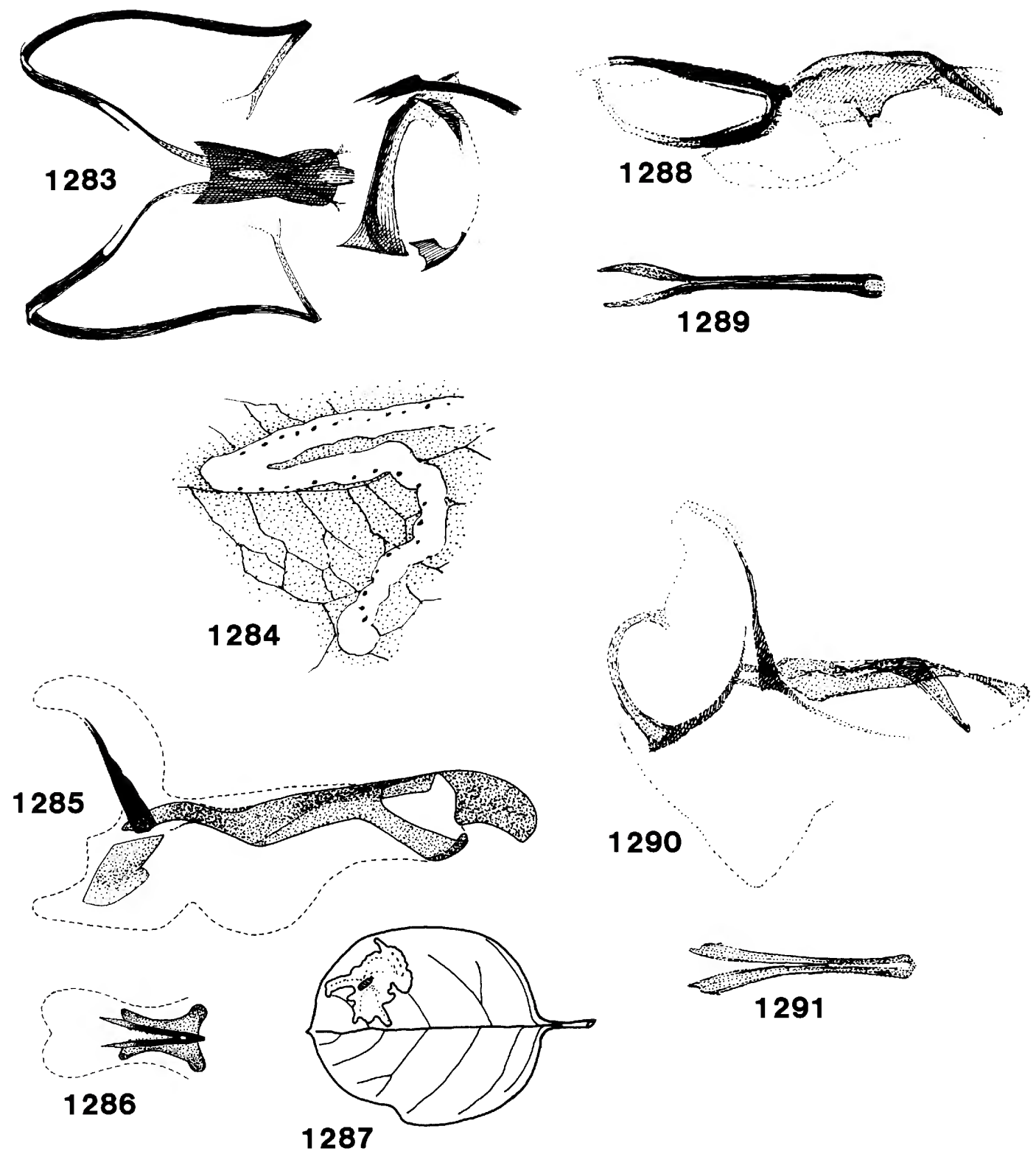

Figures 1283-1291.--Phytomyza persicae: 1283, Aedeagus, ventral view; 1284 , leaf mine on Prunus persica. Chromatomyia fricki: 1285, Aedeagus, side view; 1286, supporting sclerites, ventral view; 1287, leaf mine on Symphoricarpos rivularis (California); C. luzulivora: 1288, Aedeagus, side view; 1289 , supporting sclerites, dorsal view; $\underline{\text {. }}$ mitche11i: 1290, Aedeagus, side view; 1291, supporting sclerites, dorsal view. 


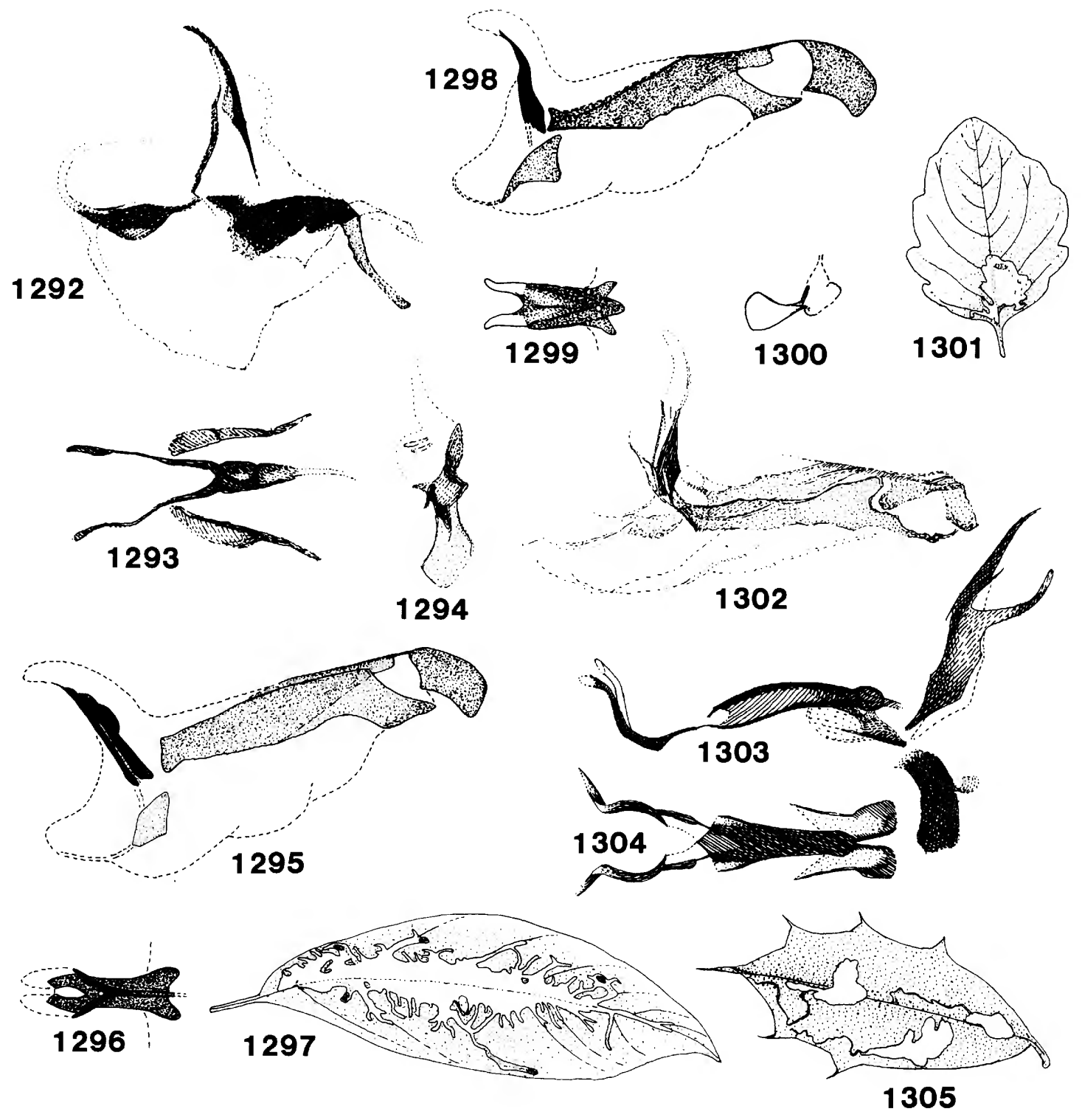

Figures 1292-1305.--Chromatomyia doolittlei: 1292, Aedeagus, side view; 1293, supporting sclerites, dorsal view; 1294, sperm pump; C. gregaria: 1295, Aedeagus, side view; 1296, supporting sclerites, ventral view; 1297, leaf mines on Lonicera involucrata (after Frick); C. symphoricarpi: 1298, Aedeagus, side view; $\overline{1} 299$, supporting sclerites, ventral view; 1300, sperm pump; 1301 , leaf mine on Symphoricarpos (after Griffiths); C. regalensis: 1302, Aedeagus, side view (Georgia). Phytomyza ilicicola: 1303, Aedeagus, side view; 1304, distiphallus, ventral view; 1305, leaf mines on Ilex opaca. 

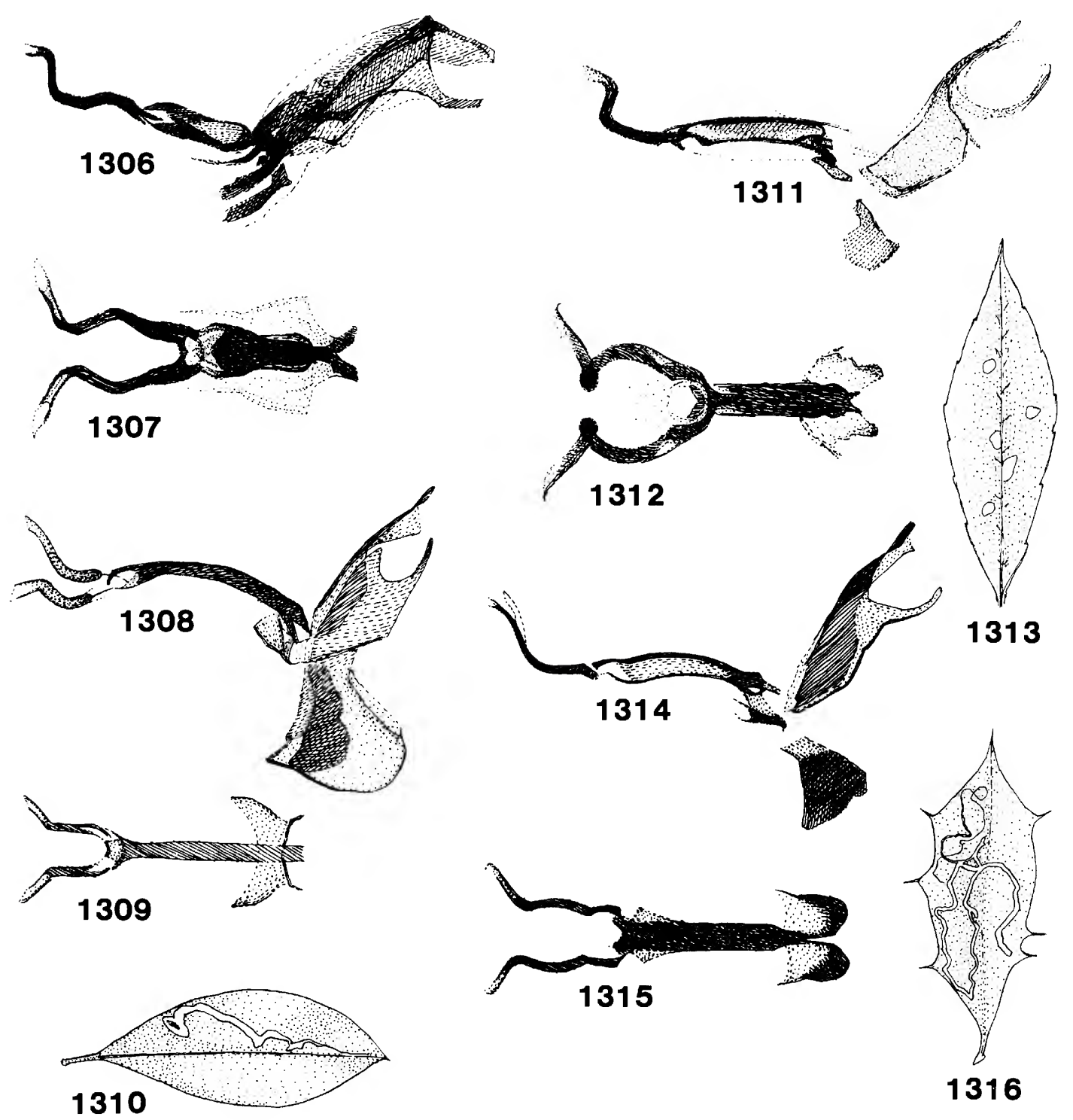

1316

Figures 1306-1316.--Phytomyza glabricola: 1306, Aedea-

gus, side view; $13 \overline{07}$, distiphallus, ventral view (paratype, Massachusetts); . verticillatae: 1308, Aedeagus, side view; 1309, distiphallus, ventral view; 1310, leaf mine on Ilex sp. (Florida); $\underline{P}$. ditmani: 1311, Aedeagus, side view; 1312, distiphallus, ventral view; 1313, young leaf mines on Ilex decidua (after Kulp, 1968); P. opacae: 1314, Ae deagus, side view; 1315, distiphallus, ventral view; 1316, leaf mine on Ilex opaca (Florida). 


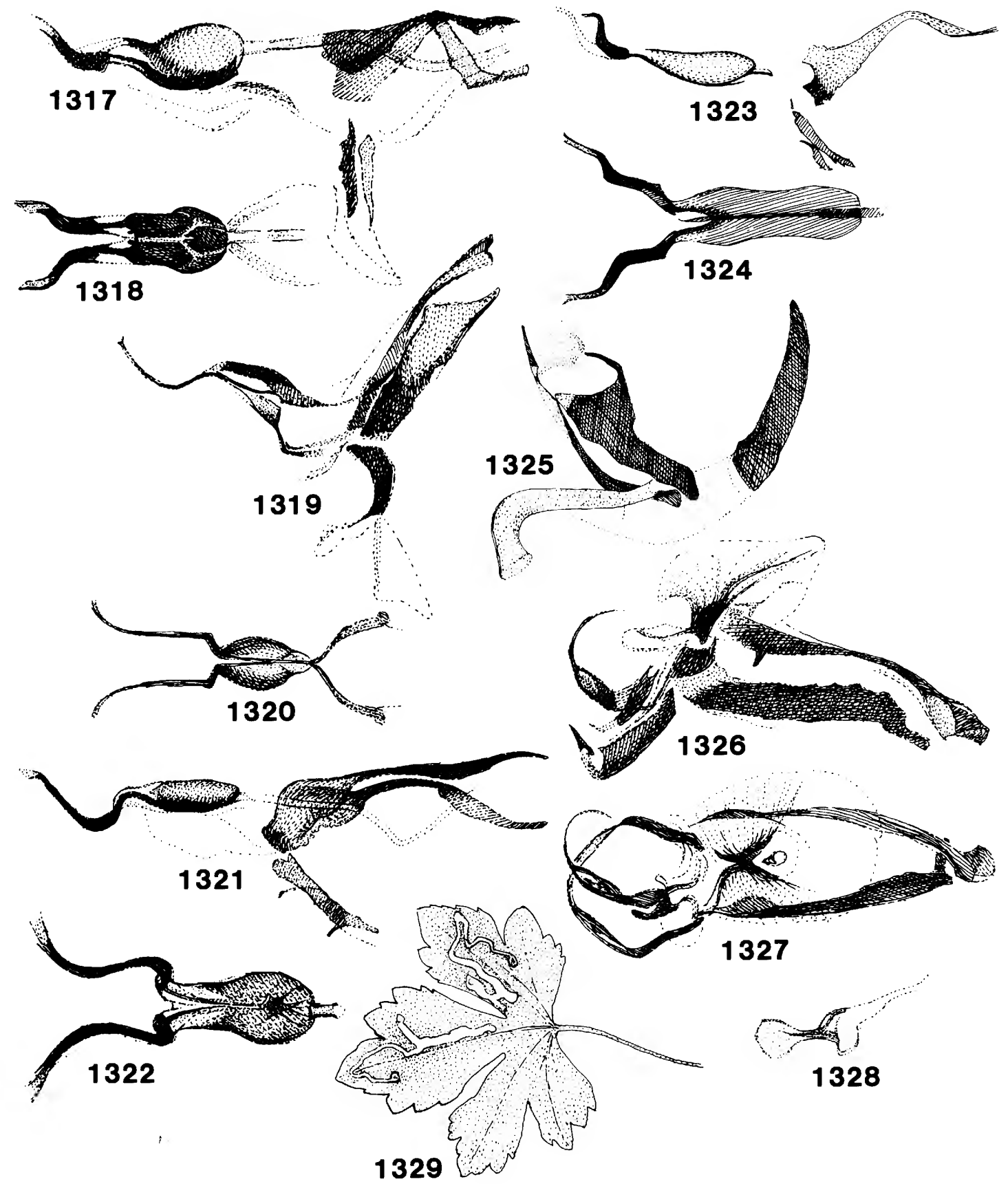

Figures 1317-1329.--Phytomyza masoni: 1317, Aedeagus, side view; 1318 , distiphallus, ventral view; $\underline{P}$. clematisella: 1319, Aedeagus, side view; 1320,

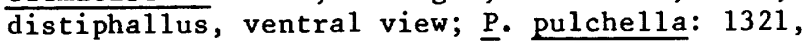
Aedeagus, side view; 1322, dîstiphallus, ventral view; $P$. saskatoonensis: 1323, Aedeagus, side view; 1324, distiphallus, ventral view; $P$. minuscula:

1325, Aedeagus, side view; $\underline{P}$. ranunculoides: 1326 , Aedeagus, side view; 1327 , same, ventral view; 1328 , sperm pump; 1329, leaf mines on Ranunculus abort ivus. 

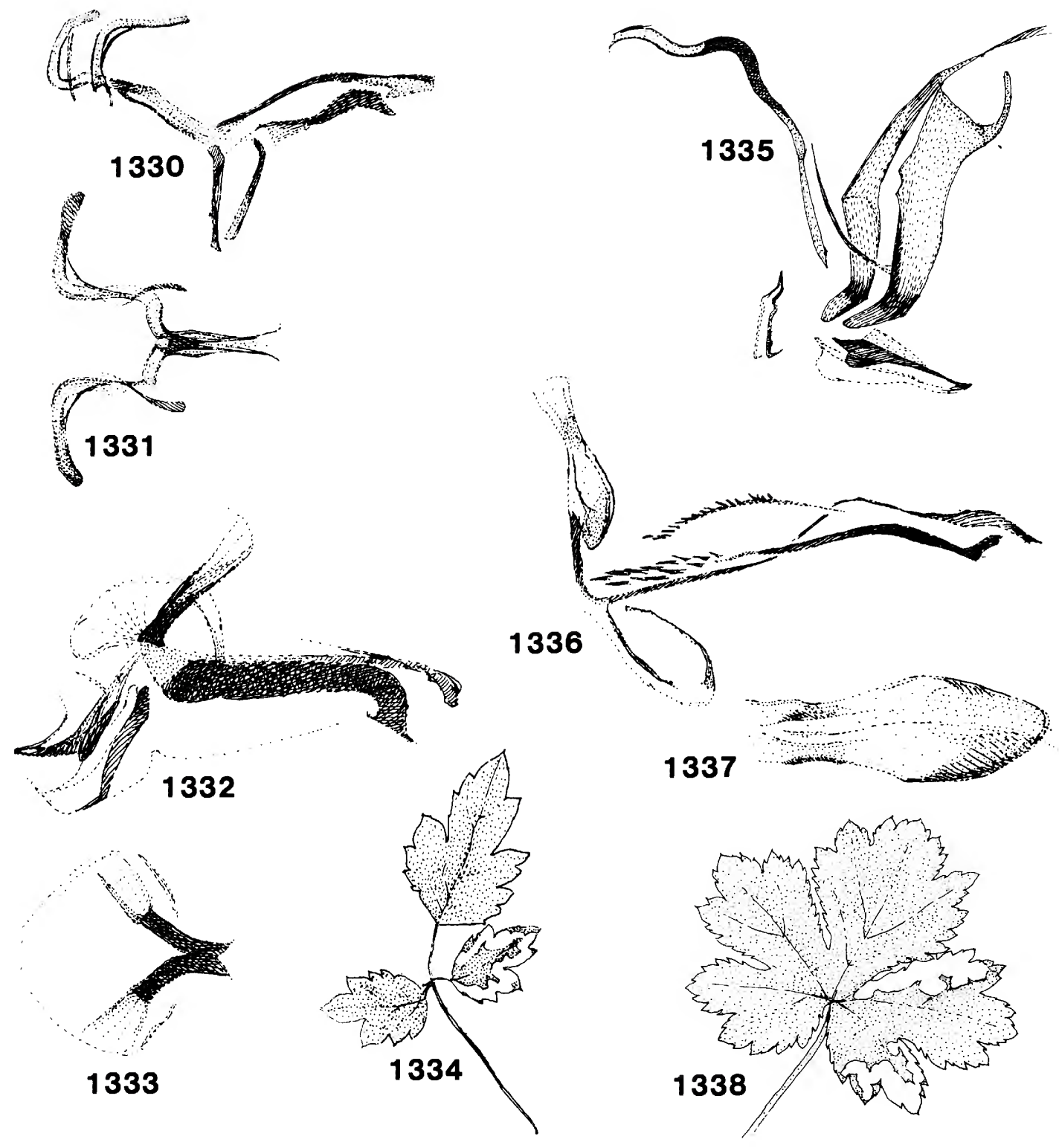

1336
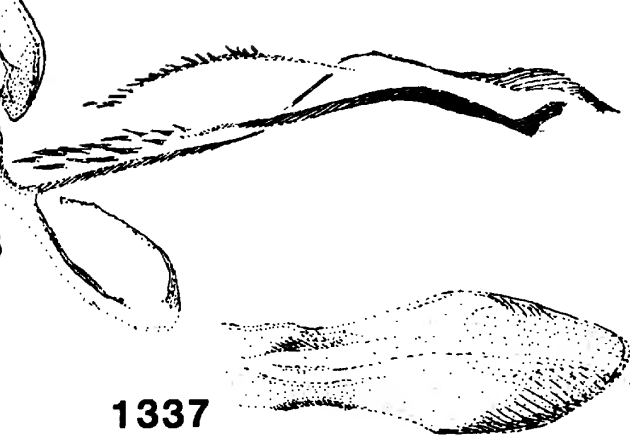

Figures 1330-1338.--Phytomyza thalictrella: 1330, Aedeagus, side view; 1331, distiphallus, ventral view; P. loewii: 1332, Aedeagus, side view; 1333, distiphallus, anteroventral view; 1334 , leaf mine on Clematis ligusticifolia (California); $P$.

thalictrivora: 1335, Aedeagus, side view (Alberta);

P. saniculae: 1336, Aedeagus, side view; 1337, distiphallus, ventral view; 1338 , leaf mines on Sanicula laciniata (California). 


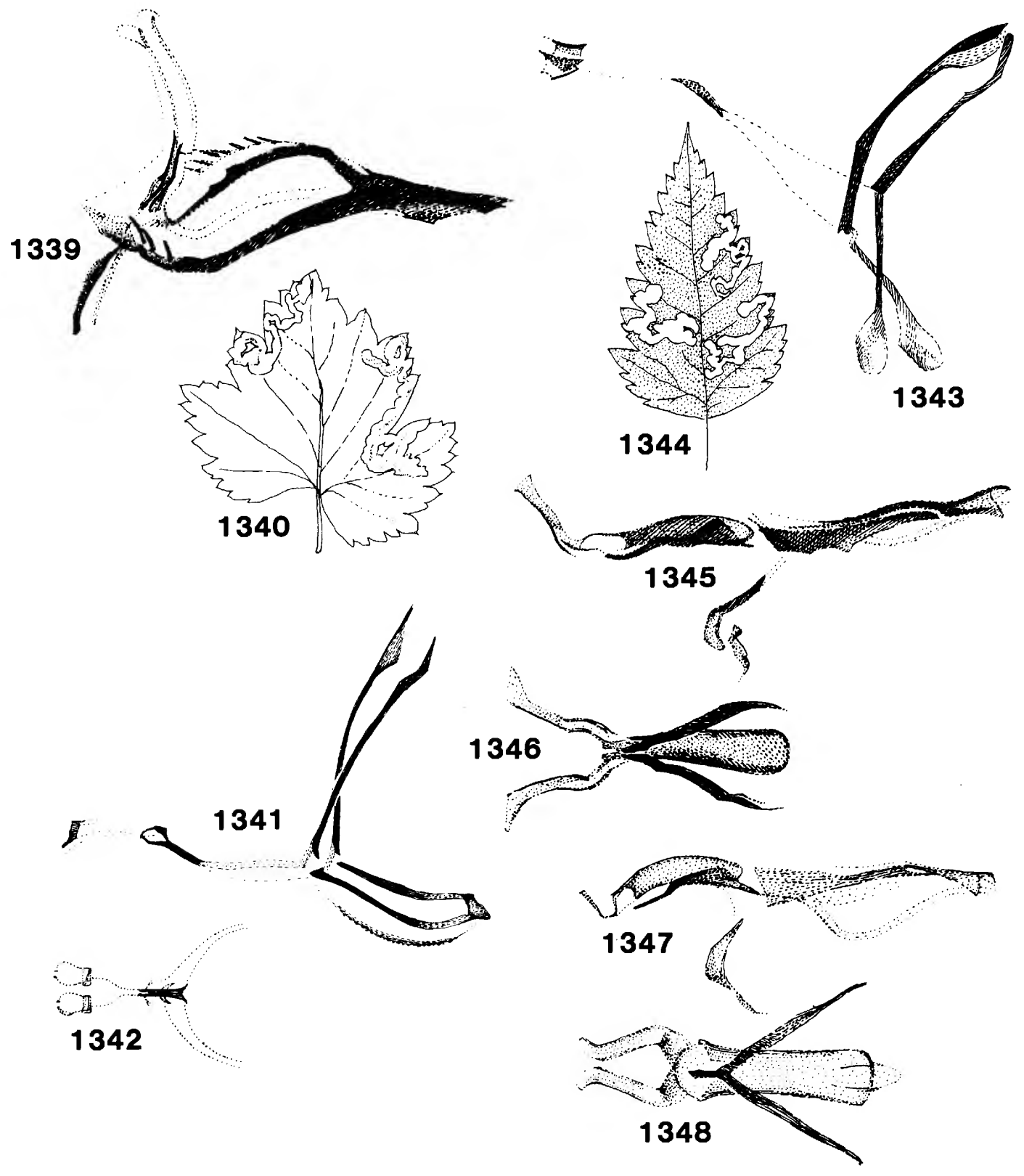

Figures 1339-1348.--Phytomyza oenanthoides: 1339 , Aedeagus, side view; 1340, leaf mines on Oenanthe sarmentosa; P. 1anati: 1341, Aedeagus, side view; 1342, distiphallus, ventral view (California); $P$. osmorhizae: 1343, Aedeagus, side view (Virginia); 1344, leaf mines on Osmorhiza longistylis; $\underline{\text { P. }}$ sonorensis: 1345, Aedeagus, side view; 1346, distiphallus, ventral view; P. columbinae: 1347 , Aedeagus, side view; 1348 , same, ventral view (California). 


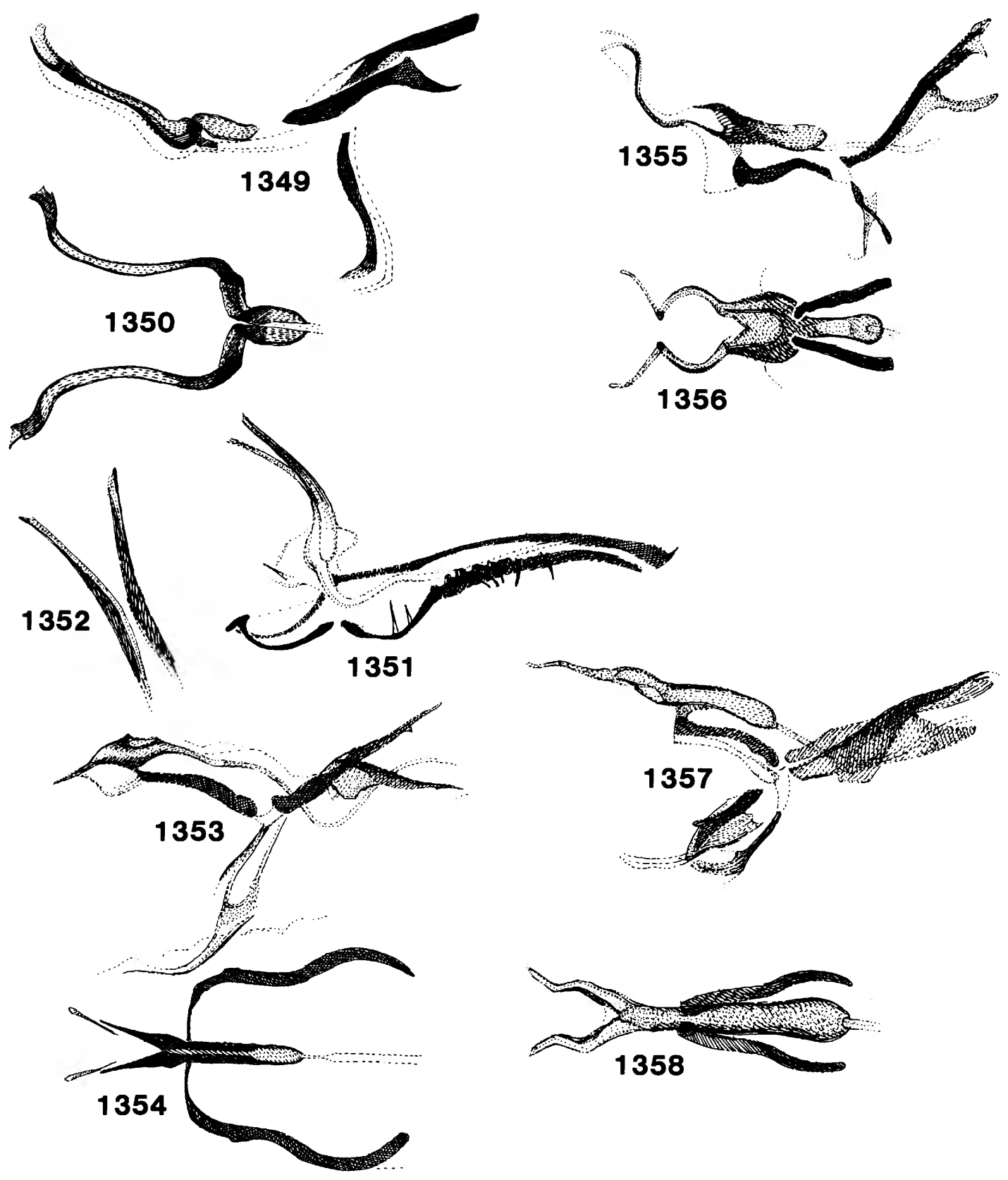

Figures 1349-1358,--Phytomyza aralivora: 1349, Aedea-

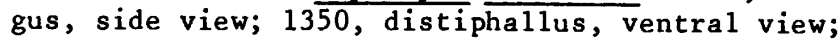
P. evansi: 1351, Aedeagus, side view; 1352,

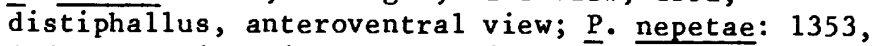
Aedeagus, side view; 1354, distiphallus, ventral view; P. malaca: 1355, Aedeagus, side view; 1356,

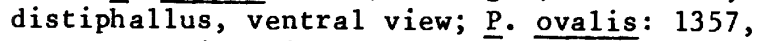
Aedeagus, side view; 1358, distiphallus, ventral view (Colorado). 


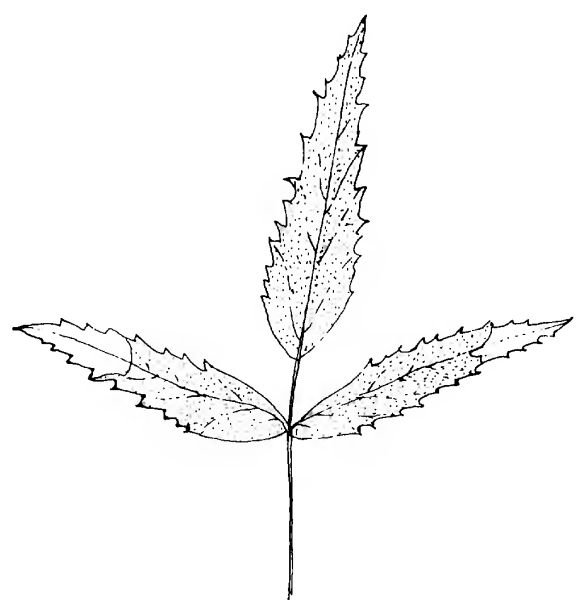

1359

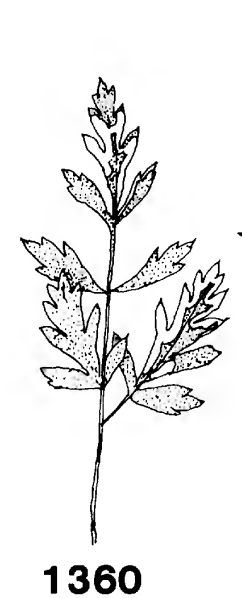

1360

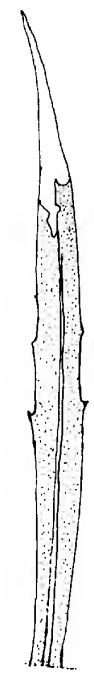

1363

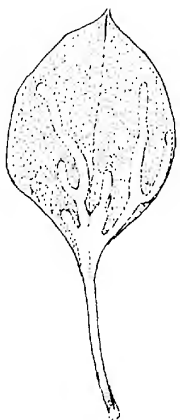

1364

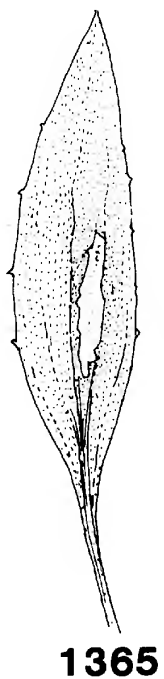

1365

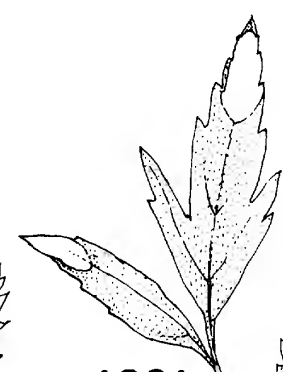

1361

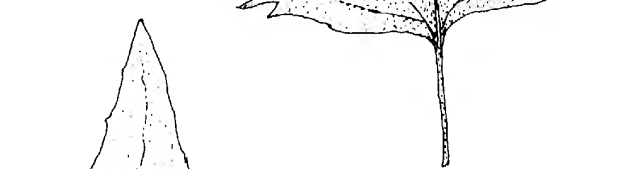

1362

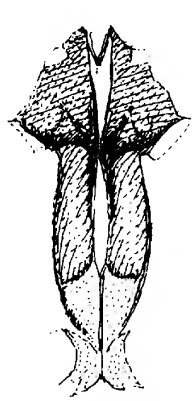

1367

Figures 1359-1367.--Unidentified leaf mines: 1359, Angelica brewer $i ; 1360$, Ligusticum gray $i$; 1361, Sphenosciadium capitellatum; 1362, Ageratina altissima; 1363, Agoseris (?) glauca; 1364, Antennaria plantaginifolia; 1365, Arnica chamissonis (Phytomyza arnicae or P. arnicicola); 1366, Arnica chamissonis (Phytomyza arnicivora or P. oreas); 1367, P. arnicivora, distiphallus, ventral view (holotype, Alberta). 


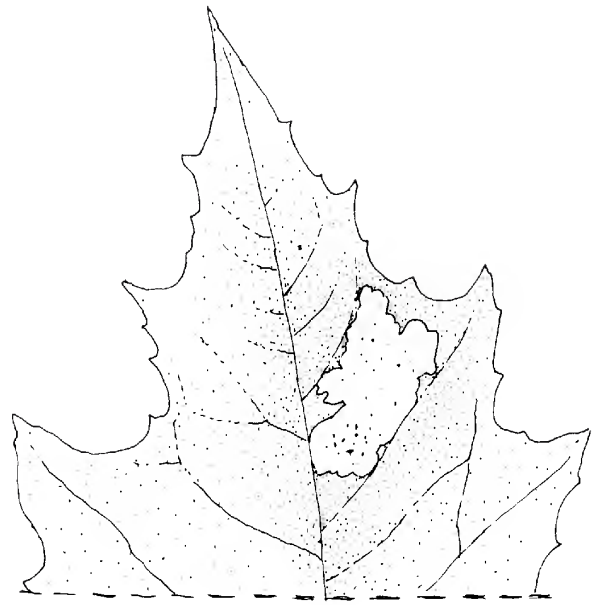

1368

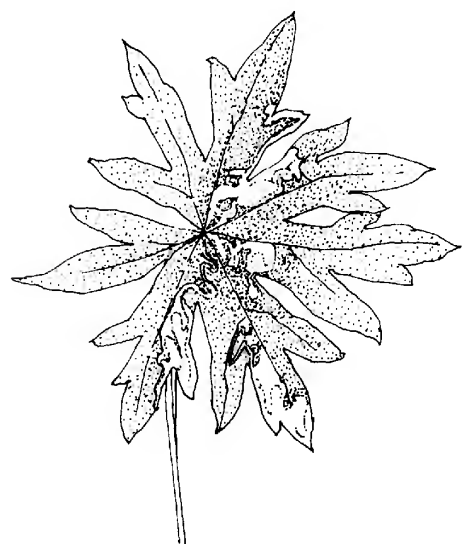

1370

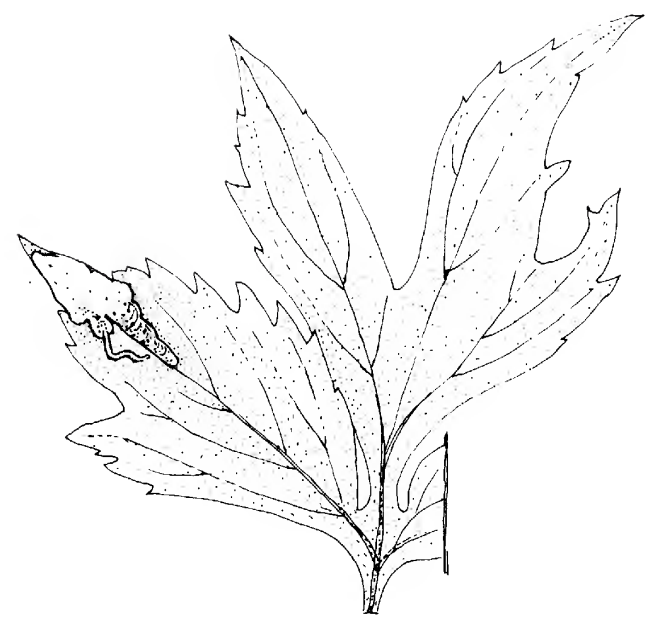

1369

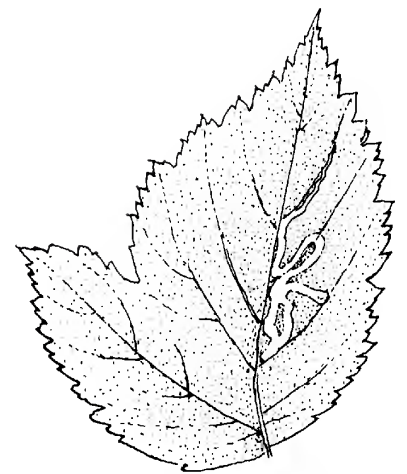

1371

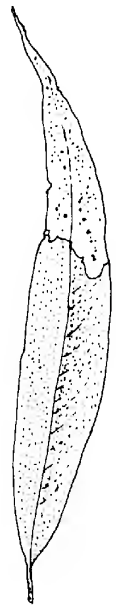

1372

Figures 1368-1372.--Unidentified leaf mines: 1368 , Smallanthus uvedalia; 1369 , Hydrophyllum virginianum; 1370, Sidalcea spicata; 1371, Rubus strigosus; 1372, Salix lasiandra caudata. 
INDEX

Host plants are indexed only by genus and family; the specific names are 1isted in their botanical classification on page 225 and under each agromyzid name elsewhere.

age

abbreviata, Agromyza--------------- 265

Abelmoschus------------------------ 235

aberrans, Ophiomyia---------------- 37

abnormalis, Amauromyza----- 78, 80, 273

abnormis, Liriomyza---------------- 131

abutilivora, Ophiomyia----- 37, 42, 250

Abutilon--------------------------- 235

Acalypha--------------------------- 233

Acanthaceae------------------------ 225

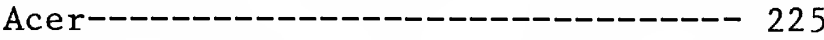

Aceraceae-------------------------- 225

aceris, Agromyza------------------- 78

Achillea--------------------------- 226

Achyranthes------------------------ 225

aconiti, Phytomyza----------------- 182

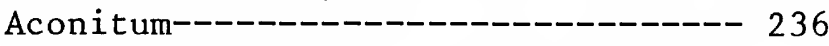

acrostichals----------------------- 3

admiranda, Liriomyza--------------- 110

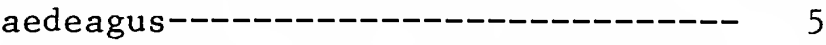

aeneonitens, Ophiomyia------------- 37

aeneoventris, Melanagromyza-------- 18

aequalis, Japanagromyza--------- 53, 56

aestimabilis, Ophiomyia-------- 44, 256

Ageratina------------------------- 226

Ageratum--------------------------- 226

Agoseris--------------------------- 226

Agromyza------------------- 14, 15, 262

agromyzina, Phytomyza-------------- 204

Agropyron-------------------------- 240

Agrostis--------------------------- 240

a lamedensis, Phytomyza-------- 173, 195

alaskana, Phytomyza----------- 173, 183

albertensis, Agromyza------ 57, 61, 262

albiceps, Phytomyza---------------- 173

albicula, Hexomyza------------- 35, 250

albidohalterata, Amauromyza---- 86, 274

albipennis, Agromyza------- 57, 63, 262

albitarsis, Agromyza------------ 57, 69

Albizia---------------------------- 235

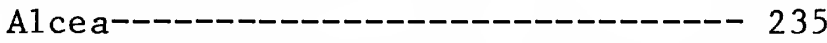

aldrichi, Phytomyza------ 177, 178, 309

alfalfa. (See Medicago (sativa).)
Page

allecta, Calycomyza----------- 147, 300

a 11 ia, Liriomyza-------------- 136, 284

al1iovora, Liriomyza-------------- 297

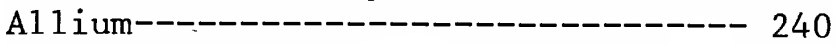

Alternanthera---------------------- 225

althaeae, Calycomyza--------------- 149

Amaranthaceae--------------------- 225

Amaranthus------------------------ 225

Amauromyza------------ 13, 16, 78, 272

ambigua, Agromyza-------------- 60, 61

Ambrosia--------------------------- 227

ambrosia, Ophiomyia---------------ー 45

ambrosiae, Calycomyza------------- 150

ambrosivora, Agromyza----------- 57, 64

Amel anchier------------------------ 237

amelanchieris, Phytobia----------- 78

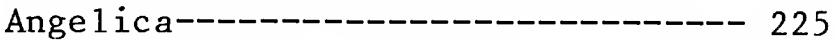

angelicae, Melanagromyza------- 24, 243

Phytomyza---------- 200, 201

angelicella, Phytomyza------------- 202

angulata, Cerodontha--- 92, 97, 99, 279

angulicornis, Li riomyza------- 107, 109

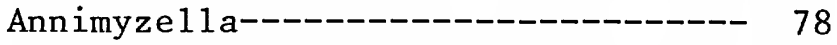

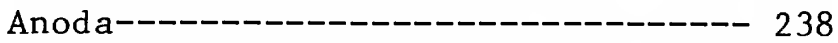

anomala, Amauromyza----------- 82, 274

Antennaria------------------------- 227

Apiaceae (Umbe11iferae)----------- 225

Apium------------------------------ 225

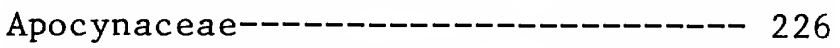

apodeme--------------------------- 5,7

aprilina, Agromyza------------- 62, 262

apta, Ophiomvia------------------- 44

Aquifoliaceae--------------------- 226

Aquilegia-------------------------- 236

aquilegiae, Phytomyza-------------- 198

aquilegiana, Phytomyza-------- 192, 200

aquilegioides, Phytomyza------ 193, 200

aquilegivora, Phytomyza-- 198, 217, 218

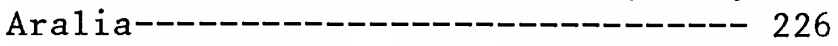

Araliaceae------------------------- 226

aralivora, Phytomyza--------------- 221

archboldi, Liriomyza-------------- 123

Arctagrostis----------------------- 240

arctica, Phytoliriomyza-- 151, 152, 154

arctii, Liriomyza-------- 107, 122, 285

Arctium---------------------------- 227

arguta, Ophiomyia------------------ 259

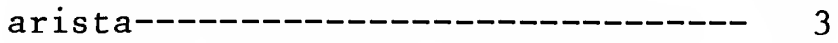

aristata, Agromyza------------- 59, 69 
Aristolochia---_-_-_- 226

Aristolochiaceae---_---_-_--D---- 226

aristosa, Cerodontha--_-_-_------ 104

arizonensis, Ophiomyia-_-_-_--- 52, 251

arnaudi, Liriomyza------------ 121

Arnica----------------------- 227

arnicicola, Phytomyza------- 202, 310

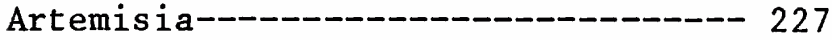

artemisiae, Agromyza--------- 140

Calycomyza------ 140,148

Liriomyza----------- 117

Asclepiadaceae------------------ 226

asclepiadis, Liriomyza------- 107, 130

Asclepias-------------------- 226

As paragus------------------- 240

Asplenium--------------------- 225

assimilis, Liriomyza---_----- 108, 111

Aster-------------------------- 227

Asteraceae (Compositae)----------- 226

asterophaga, Phytomyza------- 199, 310

astotinensis, Phytomyza------ 201, 310

asymmetrica, Ophiomyia-------- 49, 252

atra, Pseudonapomyza---_----- 166, 167

atricornis, Chromatomyia-------- 192

Atriplex--------------------- 233

atriplicis, Ophiomyia-_-_-_-_-_--- 40

atronitens, Haplomyza--_--_------ 91

attenuata, Cerodontha---------- 96, 276

auriceps, Amauromyza------- 78, 84, 274

auricornis, Phytomyza--------- 184, 311

Avena--_-_-_-_-_-_-_-_-_-_-- 240

baccharidis, Liriomyza--_-_-_-_--- 124

Baccharis--_---_-_-_-_-_-_-_----- 227

Balsaminaceae---_---_-_---_----- 231

banffensis, Ophiomyia-_-_-_-_-- 39, 252

Baptisia----------------------- 233

baptisiae, Liriomyza------------ 115

barbarensis, Calycomyza--_-_- 157

barberi, Agromyza-------------- 265

basiphallus------------------ 5

Bauhinia------ 233

beckerella, Phytoliriomyza-------- 155

bella, Liriomyza------------- 112

Bellis----_-_-_-_-_-_-_-_-_---- 228

bellissima, Liriomyza------------ 115

bernardinensis, Ophiomyia------- 37,43

Beta-------- 233

Betula--_-_-_-_-_-_-_-_-_-_-_-- 231

Betulaceae-----------_---_-------- 231

betulivora, Phytobia--_--_---_---- 76

bicolor, Phytomyza----_-- 203

bicolorata, Cerodontha-_-_-_-_--- 105

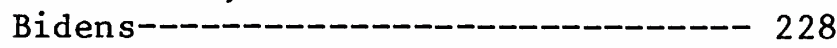

bidentis, Melanagromyza---_--- 31
Page

Bignoniaceae---_--_-_-_-_-_-_---- 231

birch. (See Betula.)

bispinata, Agromyza------- 57, 66, 263

blairmorensis, Napomyza-- 171, 172, 185

blechi, Liriomyza---------- 113, 285

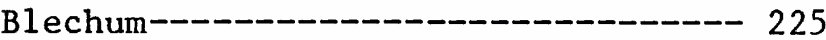

Boraginaceae----_--_---_-_---_---- 231

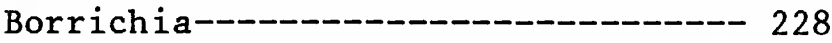

Bougainvillea----_--_-_-_-_----- 236

boulderella, Phytomyza-------- 199, 311

boulderensis, Ophiomyia----_--- 43, 252

Brachypodium---------------- 240

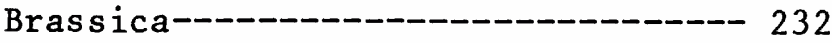

Brassicaceae (Cruciferae)-------- 232

brassicae, Liriomyza----------- 127

Breynia----

Bromus---------------------- 240

buccalis, Melanagromyza---- 11, 25, 243 burgessi, Melanagromyza------- 31, 244 Butomomyza--_-_- 87, 97, 276

caerulea, Melanagromyza---------- 22

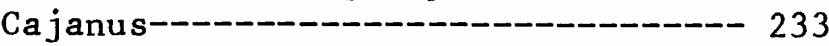

Calamagrostis---------_-------- 240

Calendula-------_--_--_-------- 228

californica, Phytobia----_----- 76

Phytomyza---------- 177

californiensis, Liriomyza--------- 120

Callistephus-------------------- 228

Callitris----_--_-_- 9, 72

Calonyction. (See Ipomoea.)

Calycomyza---_------- 13, 14, 16, 299

calyptrata, Phytobia---------- 77, 270

camarae, Ophiomyia---_-_----- 38, 47

Camptosorus--_-_-_-_-_-_-_-_-_-_- 225

canadensis, Agromyza--_-_-_-_---- 59

canomarginis, Liriomyza-_-_-_-_--- 292

capitata, Cerodontha--_-_-_-_---- 89

Capparidaceae---_---_--_-_--_------ 232

Capparis---_-_-_-_-_-_-_-_----- 252

Caprifoliaceae--_-_-_-_-_-_-_---- 232

Capsella-------_---_----_------ 232

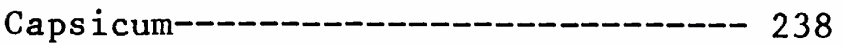

carbonensis, Phytomyza------------- 187

Cardios permum-----_---_---_------ 237

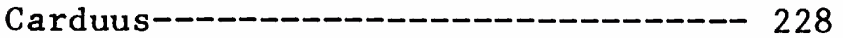

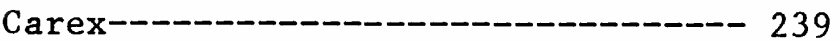

caribbea, Melanagromyza------- 33, 244

carolinae, Ophiomyia---------- 50, 252

carolinensis, Ophiomyia--_-_--- 47, 253

Caryophyllaceae------------------- 233

Cassia-------------------------- 233

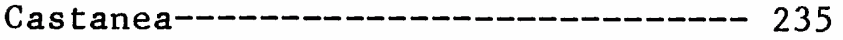

Castilleja-----_---_-- 238 
Page

castillejae, Chromatomyia---------- 181

Catalpa-------------------------- 231

Catal pomyza---------------------- 78

ceanothi, Phytomyza---------- 185, 311

Ceanothus-------------------------- 237

cecidogena, Hexomyza--------------- 250

celery. (See Apium (graveolens).)

Celtis---------------------------- 239

centaureae, Hexomyza------------- 34

Centrosema------------------------- 233

Cephalomyza--------------- 78, 272

cercus------------------------- 7

Cerodontha----- 13, 14, 15, 87, 91, 276

Cestrum--------------------------- 238

chaixiana, Cerodontha--------------- 105

chaixii, Cerodontha---------------- 105

Chaptalia-------------------------- 228

chaptaliae, Melanagromyza--------- 27

cheek------- 3,5

Cheilanthus--------------------- 232

Chelone----------------------------- 238

chelonei, Phytomyza---------- 189, 312

chemsaki, Liriomyza--------------- 133

Chenopodiaceae---------------------- 233

Chenopodium------------------------ 233

chillcotti, Mel anagromyza------ 26, 244

chillcottiana, Melanagromyza--- 27, 244

chillcottiella, Cerodontha----- 93, 277

Chiococca-------------------------- 237

ch 1amydata, Liriomyza-------------- 116

Chondrilla------------------------- 228

chondrillae, Ophiomyia-------- 45, 253

Chromatomyia-- 8, 14, 17, 173, 309, 323

Chromolaena--------------------- 228

Chrysanthemum------------------- 228

churchillensis, Cerodontha--------- 90

Cicuta----------------------------- 225

cicutae, Phytomyza----------------- 219

cicute11a, Phytomyza--------------- 182

cincta, Domomyza----------------- 57

Cineraria------------------------- 228

Cinna------------------------------ 240

cirsiophaga, Phytomyza------------- 329

cirsiophila, Melanagromyza--------- 24

Cirsium----------------------- 228

clara, Phytoliriomyza--------- 152, 159

clematiphaga, Phytomyza----------- 176

C1 ematis--------------------------- 236

clematisana, Phytomyza-------- 189, 193

clematise11a, Phytomyza------- 215, 312

clemativora, Chromatomyia-------- 174 ,

177,323

clematoides, Phytomyza---- 14, 197, 313

Cleome--------------------------- 232

coloradella, Phytomyza-------- 195, 314 coloradensis, Paraphytomyza--- 165, 306

columbinae, Phytomyza------------- 220

Comarum. (See Potentilla.)

Commelina-------------------------- 239

Comme 1 inaceae--------------------- 239

commelinae, Liriomyza---- 107, 112, 286

commendata, Ophiomyia-------------- 43

Compositae (Asteraceae)------------ 226

compta, Chromatomyia--------- 176, 324

confessa, Phytobia------------- 74, 271

confondata, Amauromyza----- 82, 85, 274

congregata, Ophiomyia---------- 14, 254

coniceps, Ophiomyia------------ 38, 46

conifers (hosts)--------------- 9

conjunctimontis, Phytoliriomyza---- 153

Conoclinium---------------------- 228

conspicua, Phytoliriomyza--------- 152 ,

156,302

consulta, Phytoliriomyza------ 156, 302

Convolvulaceae--------------------- 233

Conyza----------------------------- 228

coqui1letti, Phytomyza-------- 181, 314

Cordylanthus---------------------- 238

Cornaceae---------------------------- 233

cornigera, Paraphytomyza----------- 163

Cornus----------_---_------------- 233

coronata, Calycomyza---------- 141, 300

Coronilla------------------------- 233

corralensis, Melanagromyza--------- 28

costa------------------------- 5

costal sections------------------- 5

coxa------------------------------ 3

crassiseta, Phytomyza--------- 185, 205

Crataegus------------------------- 237

crepidis, Phytomyza----------- 173, 196

Crepis------------------------- 228

crossveins------------------------ 5

Crotalaria-------------------------- 233

Cruciferae (Brassicaceae)---------- 232

cubitus--------------------------- 5

Cucumis---------------------------- 233

Cucurbita------------------------ 233

Cucurbitaceae--------------------- 233

cucurbits-------------------------- 233

cudu, Phytomyza-------------- 216, 321

curvipalpis, Agromyza---------- 37

Cynara--------------------------- 228

cynoglossi, Calycomyza------------ 147

Cynoglossum--------------------- 231

Cyperaceae------------------------ 239

Dactylis------------------------ 240

Dah1ia-------------------_--_------ 228

daisy-------------------------------- 228

Datura----------------------------- 238 


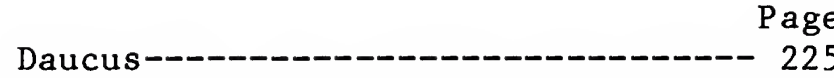

davisii, Phytomyza------- 167, 172, 175

debilis, Ophiomyia--------------- 46

deceptiva, Liriomyza---------- 124, 286

decima, Ophiomyia---------------- 254

definita, Ophiomyia------------- 48

delecta, Ophiomyia------------- 49

delphiniae, Phytomyza-------------- 182

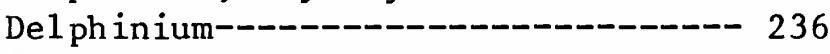

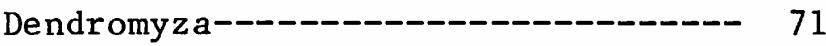

denticornis, Cerodontha-------- 87, 91

denudata, Liriomyza------------- 120

Deschampsia------------------------ 240

Descurainia--------- 232

Desmodium-_-_-_-_- 234

desmodivora, Japanagromyza-------- 54

devia, Ophiomyia------------ 47

Dianthera----------------------- 225

diantherae, Melanagromyza--------- 22

dianthi, Liriomyza------------- 119

Dianthus-------------------- 233

Digitaria--

discal cel1------------------- 5

discalis, Liriomyza---_-- 125

distiphallus-_- 5,7

distribution--------- 10

ditmani, Phytomyza------------ 215

diversa, Agromyza---- 59

Dizygomyza---- 13, 71, 78, 87, 100, 276

Domomyza---------------------- 57

doolittlei, Chromatomyia------ 212, 324

dorsalis, Cerodontha--------- 10, 100

dorsata, Phytoliriomyza------- 153, 303

douglasii, Liriomyza------------- 295

dreisbachi, Phytomyza------------- 188

dubitata, Agromyza-------------- 262

duchesneae, Japanagromyza-------- 53

Dugaldia------- 228

duplex, Phytomyza-------- 167, 175, 315

Duranta----------------------- 239

durantae, Calycomyza--------------- 150

Echinochloa------------------- 241

economic importance-------------- 9

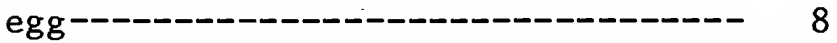

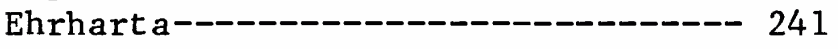

eldorensis, Ophiomyia--------- 50, 254

elegans, Napomyza--------------- 168

Phytomyza----------------- 167

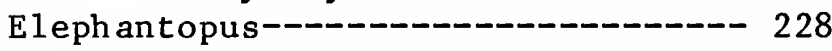

Eleus ine---_-- 241

elevata, Liriomyza-_-_-_-_-- 117,286

elsinorensis, Amauromyza---_-_-- 82

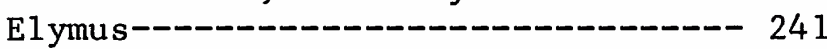

Emilia---------------------------- 228

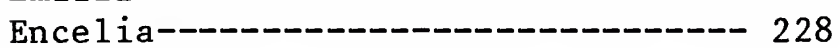

Page

enceliae, Calycomyza------------ 149

endiviae, Liriomyza------------- 107 ,

$123,134,287$

enormis, Liriomyza------------- 151

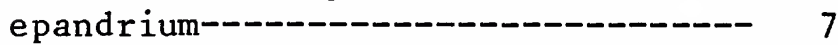

Eragrostis----------------------- 241

Erechtites---------------------- 229

erechtitidis, Melanagromyza------- 31

Erigeron--------------------------- 229

erigerontophaga, Chromatomyia----- 174 ,

209,325

erigerophila, Phytomyza------ 197, 315

eriodictyi, Chromatomyia--------- 189

Eriodictyon---------------------- 235

errans, Agromyza-------------- 71

Erysimum----------------------- 232

Euchlaena----------------------- 241

eumorpha, Phytomyza--

Eupatoriadel phus------------ 229

eupatoriella, Liriomyza------- 111, 288

eupatorii, Liriomyza--------- 129,288

Eupatorium----------------------- 229

eupatorivora, Calycomyza--------- 146

Euphorbiaceae----------------- 233

evanescéns, Napomyza-_- 168 ,

$171,172,208$

evansi, Phytomyza-----_----- 221, 316

Fabaceae (Leguminosae)-- 233

Fagaceae---------------------- 235

fasciata, Cerodontha--

$100,104,282$

fastella, Ophiomyia------------ 50

fastosa, Ophiomyia--------- 42, 255

felix, Phytomyza---------------- 181

felti, Phytoliriomyza--------- 111 ,

ferns. (See Polypodiaceae.)

$152,156,303$

festiva, Phytomyza---------------- 167

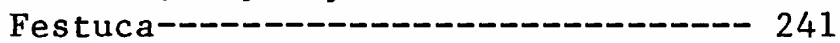

fida, Ophiomyia----------------- 49

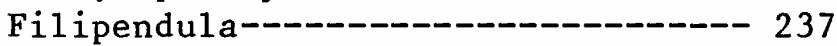

flava, Phytomyza------------- 172

flavens, Phytoliriomyza----------- 153 Phytomyza------- 173, 193, 316

flaveola, Phytomyza---------------- 172

Flaveria----------------------- 229

flaviantenna1is, Phytomyza--------- 179

flavicola, Liriomyza------------ 110

flavicornis, Phytomyza------- 173, 180

flavida, Chromatomyia-------- 202, 325

flavifrons, Amauromyza--------- 85, 275

flavinervis, Phytomyza------- 184, 317

flavinotum, Calycomyza--------- 146

flavocingulata, Agromyza---------- 162

Cerodontha-------- 97 
flower heads--------------------- 12

fossil-------_--_--_--_------- 9, 72

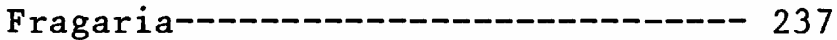

fragariae, Agromyza--------------- 71

frankensis, Cerodontha------------- 97

frickella, Liriomyza--------------- 119

fricki, Chromatomyia------------ 211

Liriomyza---------------- 136

frickiana, Calycomyza-------- 146, 299

frigida, Liriomyza-------------- 116

frommeri, Liriomyza-------------- 127

frons, front---------------- 3

frontella, Agromyza----------- 9, 68

frosti, Cerodontha------------- 102

Ophiomyia------------ 41, 255

fumicosta, Phytoliriomyza----- 158, 304

fuscifrons, Cerodontha---------- 89

fusculata, Cerodontha--------- 98, 279

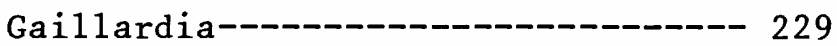

Galactia--------------------------- 234

galiivora, Galiomyza----------- 137, 298

Galinsoga--------------------- 229

Galiomyza------------------ 13, 16, 297

Galium---------------------------- 237

gena--------------------------- 3

genalis, Phytomyza----------- 180, 317

genera, key-----_-----_------ 15

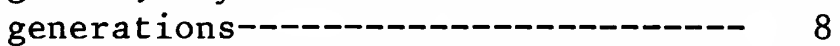

geniculata, Cerodontha----------- 83

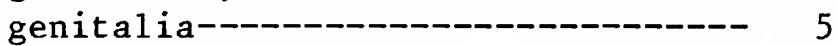

genitalis, Ophiomyia-------------- 47

Geratomyza------------------------ 53

Gerbera---------------------------- 229

gibbardi, Cerodontha------------- 98

gibsoni, Melanagromyza------------ 27

gigantea, Calycomyza--------------- 145

glabricola, Phytomyza-------------- 214

G1yceria--------------------------- 241

gnaphalii, Melanagromyza---------- 28

Gnaphalium------------------------ 229

Gossypium------------------------ 235

gracilis, Cerodontha------------ 91

graminacea, Liriomyza--------- 107, 132

Gramineae (Poaceae)-------------- 240

grandella, Napomyza--------- 169, 307

gregaria, Chromatomyia----------- 212

griffithsi, Chromatomyia------ 208, 326

Metopomyza------------ 162

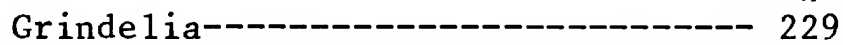

guytona, Liriomyza------------- 292

Gypsophila----------------_------- 233

Hackelia------------------------- 231

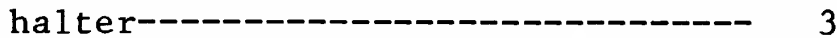

Hamelia-------------------------- 237

haplacme, Agromyza----------------- 267

Haplomyza---------------------- 13

Haplopeodes------------- 13, 16, 299

hardyi, Agromyza----------- 65, 263

haydeni, Ophiomyia--------------- 40

Hebe---------------------------- 238

helenii, Liriomyza-------------- 122

Helenium---------------------- 229

helianthi, Liriomyza---------- 126, 289

Helianthus----------------------- 229

Heliopsis-------------------- 230

heliotropii, Melanagromyza-------- 21

Heliotropium------------------- 231

heracleiphaga, Phytomyza---------- 202

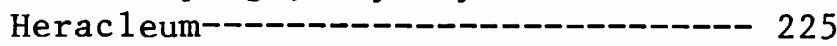

Heterotheca----------------------- 230

Heuchera--------------------------- 238

Hexomy za---------- 13, 15, 34, 249

hicksi, Melanagromyza------------ 23

hilarella, Agromyza--------- 151, 159

hirtae, Cerodontha---------------- 106

hockingi, Agromyza-------- 57, 66, 264

Holcus------------------------ 241

Hordeum------------_-------------- 241

horticola, Chromatomyia------- 174, 192

hosts---------_------- 9, 10, 224

huidobrensis, Liriomyza---_---- 8 , $9,11,107,119$

humeralis, Calycomyza------ 8, 141, 144

humerus------------------------ 3

humilis, Phytomyza-------------- 178

Hydrocotyle---_-_-_---_--------- 225

Hydrophy 11 aceae--------------------- 235

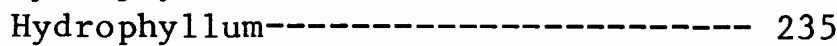

Hymenopappus-----------_----------- 230

hypandrium-------_------------- 7

hypophallus------------------ 5,7

hyptidis, Calycomyza------------- 150

Hyptis------------------------- 235

Icteromyza----- $3,87,88$

Ilex------------------------------- 226

ilicicola group-------------- 173, 174

ilicicola, Phytomyza------------ 214

ilicis, Phytomyza--------- 8, 191, 205

illinoensis, Cerodontha-------- 92, 276

immaculata, Phytoliriomyza--------- 155

immature stages--------------- 7 
ipomaeae, Calycomyza------------ 150

Ipomoea---------------------- 233

iraeos, Cerodontha------------ 88, 100

Iridaceae--------------------- 240

iridis, Cerodontha------------- 88, 100

iridophora, Cerodontha----------- 103

Iris---------------------------- 240

irwini, Agromyza------------------- 261

Calycomyza---------------- 142

isolata, Agromyza------ 57,69, 71, 264

Jacaranda-------------------------- 231

jacarandae, Phytoliriomyza-------- 152

jacintensis, Ophiomyia----- 37, 38, 44 Japanagromyza------------ 14, 15, 261 jucunda, Calycomyza----_--_----- 141 , $143,144,300$ Juncaceae----------------------- 240 Juncaginaceae---------------------- 235 Juncus--------------------------- 240

Juniperoxylon------------------- 9

Justicia---------------------- 225

Kallstroemia----------------------- 239

kefi, Haplopeodes------- 139, 140, 299 kincaidi, Agromyza--------- 61, 62, 264 knowltoni, Amauromyza--_-_---- 85, 275

Labiatae (Lamiaceae)-------------- 235

labiatarum, Ophiomyia----------- 38, 51

lacertosa, Ophiomyia--_-_-_-_---- 48

lacteipennis, Pseudonapomyza------- 167

Lactuca---

lactuca, Chromatomyia--------- 8, 191

Lamiaceae (Labiatae)--_-_-_-_---- 235

lamii, Agromyza------------------ 78

lanati, Phytomyza------------- 200, 219

langei, Liriomyza----_-_-_---- 119

Lantana-------- 239

lantanae,

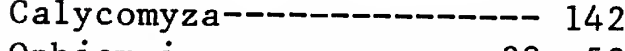

Oph iomyia----------- 38, 52

larva-------------------------- 7

Lasiacis--------------------------- 241

lassa, Ophiomyia------_--_--_--- 52

lateralis, Cerodontha--_---_----- 93

laterella, Agromyza-------------- 103

lathyri, Liriomyza-------------- 11 ,

$107,108,133,285$

lathyroides, Amauromyza------ 78, 83

Lathyrus--------------------------- 234

latifrons, Cerodontha-------- 101, 283

Phytomyza--------- 193, 317

lauta, Ophiomyia----------- 29, 41, 255

1eechi, Agromyza-------_--- 71

Phytoliriomyza------------ 159

legumes. (See Fabaceae.)

Leguminosae (Fabaceae)------------- 235

Lemurimyza------------------------- 151

Lepidium------------------------ 232

lettuce. (See Lactuca (sativa).)

levata, Ophiomyia-----_------- 52

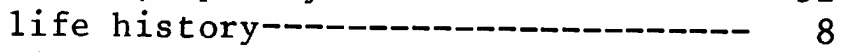

1igusticifoliae, Phytomyza-------- 188

Ligust icum----------------------- 225

Liliaceae-------------------------- 240

lima, Liriomyza------------------ 134

Linaceae------------------------- 235

Lindheimera----------------------- 230

Linum------------------------------ 235

Lippia----------------------------- 239

lippiae, Ophiomyia----------- 38, 40

lippivora, Melanagromyza------ 11, 247

Liriomyza-- 9, 10, 13, 14, 16, 107, 284

Li thos permum----------_------ 232

loewii, Phytomyza---------------- 217

Loganiaceae----------------------- 235

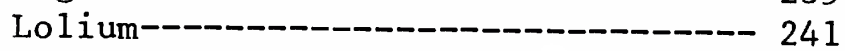

lomatii, Melanagromyza----------- 30

Lomatium------------------ 225

longensis, Melanagromyza------- 24, 245

longipennis, Cerodontha--_-_-_-- 89

Lonicera---- 232

lonicerae, Paraphytomyza----------- 164

1onicerina, Paraphytomyza---------- 164

lucens, Amauromyza----_---_----- 81

luctuosa, Cerodontha---_--_----- 106

lunule----------------------- 3

lupinella, Liriomyza------------ 118

lupini, Liriomyza------------- 121

Phytomyza--------- 185, 196

lupiniphaga, Liriomyza---------- 117

Lupinus-------------------------- 234

luteiceps, Dizygomyza---------- 78

luteoscutellata, Paraphytomyza---- 162 ,

165,306 
luzulivora, Chromatomyia------ 212, 327 Lychnis------------------------- 233 Lycopersicum----------------- 235

maclayi, Cerodontha--------- 105 macminni, Cerodontha------- 92, 95, 278 maculata, Ophiomyia--------- 37,39 maculosa, Amauromyza---------- 78, 80 Madia---------------------------- 230 magnicornis, Cerodontha-- 101, 102, 283 major, Ophiomyia---------- 38, 260 majuscula, Calycomyza---------- 147 malaca, Phytomyza-------------- 222 malaisei, Cerodontha------------ 94 malefica, Melanagromyza------------ 244 malevola, Melanagromyza----------- 31 maligna, Melanagromyza------------ 30 malitiosa, Ophiomyia----------- 51

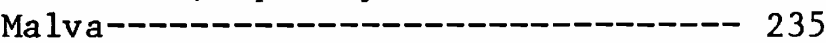

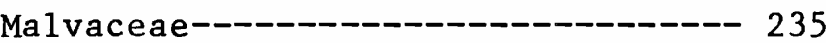

malvae, Calycomyza-----_----------- 149 manni, Napomyza---------- 170, 308 Phytomyza------------ 187,318

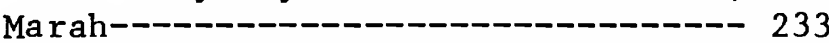
marellii, Melanagromyza-------- 33, 245 marginalis, Liriomyza----- 14, 107, 114 Napomyza---------- 168 , $171,172,187,205,308$

marinensis, Melanagromyza--------- 29 masculina, Agromyza-------- 57, 71, 265 masoni, Phytomyza------------- 215, 317 Matricaria------------------------ 230 maura, Ophiomyia---------- 37, 38, 257 Medicago----------------------- 234 medius-------------------------- 4 melampyga, Phytoliriomyza-------- 14 , $113,152,157$ Melanagromyza-------- 14, 15, 18, 243 melanella, Phytomyza-------------- 210 Melanthera---------------------- 230 melantherae, Calycomyza---------- 157 melica, Ophiomyia----------------- 51 Melilotus----------------------- 234 menthae, Calycomyza------------ 141 , $144,151,301$ Mertensia---------------------- 232 mesonotum------- 3 mesophallus---------------- 5, 7 mesopleuron-------------------- 3 Metopomyza--------------- 16, 161 miamensis, Melanagromyza----------- 29 michiganensis, Calycomyza--------- 149

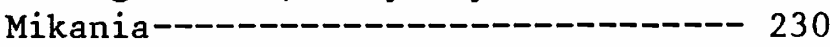
mikaniae, Calycomyza---_---_------- 147 millefolii, Liriomyza--_----_------ 121 mimuli, Chromatomyia--------- 174, 190 Mimulus--------------------------- 238

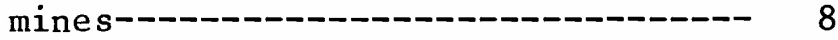
minima, Melanagromyza---------- 32 minimoides, Melanagromyza------ 32 minor, Calycomyza------------- 141 Liriomyza-------------- 120 minuscula, Phytomyza---------- 198, 216 minuta, Napomyza---------------- 169 Phytomyza--------- 138

minutiseta, Liriomyza------------- 292 minutissima, Phytoliriomyza------- 154 Phytomyza------------- 179 minutus, Haplopeodes------------- 140 miranda, Melanagromyza----- 18, 22, 245 mitchel1i, Chromatomyia------- 212, 327 modesta, Ophiomyia------------- 259 modocensis, Phytomyza------------- 178

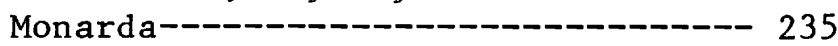

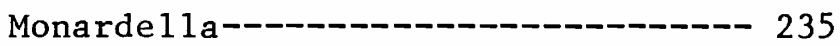
monoensis, Liriomyza-------------- 125 monophagy----------------- 9 montana, Chromatomyia--------- 192, 205 montanoides, Cerodontha----------- 89 Na pomyza-------------- 171

montella, Chromatomyia------- 195, 328 Liriomyza---------- 123, 290 montereyensis, Phytomyza--------- 196 monticola, Ophiomyia---------- 39, 256 montis, Liriomyza------------ 126, 290 morio, Galiomyza---------------- 136 morosa, Agromyza--------------- 100 , $101,102,106,283$ Mucuna------------------------- 234 muguensis, Melanagromyza--------- 28 munda, Liriomyza----------- 128, 292 muscina, Cerodontha----------- 92, 93 Myosotis---- 232

Na pomy za---------------- 17, 167, 307 Nasturtium----------------------- 232 nasuta, Ophiomyia------------ 38, 42 nearctica, Agromyza---------- 70 Nemophila------------------------- 235 Nemorimyza---------------- 13, 16, 87 Nepeta-------------------------- 235 nepetae, Phytomyza----------- 221, 319 neptis, Cerodontha------------- 279 nervosa, Phytomyza------------ 181 nevadensis, Agromyza------------- 70 Amauromyza------------ 81 nigra, Chromatomyia---------------- 207 nigrella, Agromyza----- 60, 62, 63, 265 Chromatomyia------- 209, 328 nigrinervis, Phytomyza-------- 173, 208 
nigripennis, Phytomyza-------- 173, 216 nigripes, Agromyza----------- 57, 63 Metopomyza-------------- 161 nigriscutellata, Liriomyza--------- 108 nigrisquama, Agromyza------------- 270 nigrissima, Liriomyza------------- 108 nigritella, Phytomyza-------------- 211 nigritula, Napomyza-----_---------- 168 nitida, Paraphytomyza----------- 163 niveipennis, Agromyza-------------- 61 nordica, Chromatomyia--_---_------ 181 norwegica, Chromatomyia-- 174, 206, 329 notopleuralis, Phytomyza------ 203, 319 notopleuron----- 3 novaescotiae, Calycomyza---------- 141 nugax, Napomyza------------- 170, 309 number of species-------------- 10, 11 Nyctaginaceae--------_-_-_----- 236

obscura, Calycomyza---------- 140, 145 obscurella, Phytomyza--------- 173, 211 obstipa, Ophiomyia------------- 46 occidentalis, Cerodontha---------- 91 ocellar triangle------------ 3

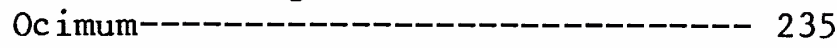

Oenanthe------------------------ 225

oenanthoides, Phytomyza------------ 219

oligophagy------------------ 9

opacae, Phytomyza----------------- 215

Ophiomyia------ $14,15,19,37,250$

orbital bristle--------- 3,5

orbitalis, Paraphytomyza--------- 165

orbitella, Chromatomyia-----_------ 203

Orchidaceae--------------------- 240

orientalis, Calycomyza-------- 148, 301 Melanagromyza------ 40, 258

orindensis, Phytomyza------_----- 186 orlandensis, Phytomyza------------ 189

Osmorhiza---------------_----- 225

osmorhizae, Phytomyza-------------- 220

osoflacensis, Melanagromyza-------- 28

ovalis, Phytomyza---------- 222, 319

ovimontis, Phytomyza--_-_-_-_---- 194

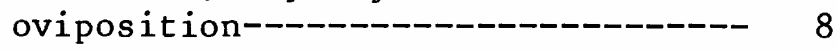

ovipositor--- 5

pacifica, Phytoliriomyza----------- 158 pagana, Agromyza-------------- 66, 265 palaensis, Melanagromyza---------- 30 pallens, Napomyza----------_----- 168 palliatus, Haplopeodes------------- 139 pallida, Phytobia----------- 73, 271 Phytoliriomyza-- 152, 157, 304 pallidiseta, Agromyza----------- 57, 58

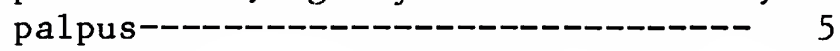

Page

paludosa, Cerodontha----_---------- 104 panacis, Melanagromyza------------ 22

Panax----------------------- 226

Panicum-------_--_-_-_-_-_------- 241

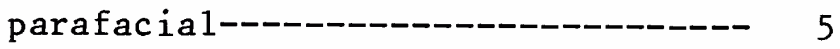

paramesophallus------------_------- 7

Paraphytomyza---_-_-_--_--- 13, 16, 306

parca, Agromyza---_--_-_-_-_-- 68, 265

parilis, Agromyza---_-_-_-_---- 68, 266

Parthenium------------------------- 230

parva, Ophiomyia--------------- 52, 256

parvella, Cerodontha----------- 98, 281

Ophiomyia--------- 42, 256

parvicornis, Agromyza----_----- 10, 67

Paspalum--------_---_-_-_-------- 241

Passiflora----_-_--_-_-_-_-_--_---- 236

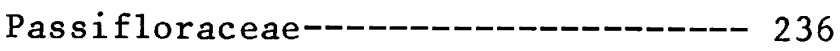

Pastinaca-----------_---_----------- 226

pastinacae, Phytomyza--------- 200, 202

paumensis, Liriomyza----_-------- 117

peas. (See Pisum.)

pechumani, Liriomyza---------- 110, 291

pecki, Cerodontha-------_--_------ 101

Pedicularis-----_---_---_-_--_----- 238

Penstemon---------------_--_------- 238

penstemone11a, Phytomyza----_----- 197

penstemonis, Phytomyza---- 11, 190, 198

periclymeni, Phytomyza------------ 173

perpetua, Japanagromyza----------- 54

perpusil1a, Agromyza------------- 151

persicae, Phytomyza-------------- 211

Petasites---------------------- 230

Petunia--------------------------- 238

Phacelia--------_--_---_---_------ 235

phaceliae, Phytomyza-------------- 207

Phalaris----------------------- 241

phallophore------------------------ 7

phaseoli, Ophiomyia--------_---- 9

phaseolunata, Liriomyza----------- 297

Phaseolus-------------------------- 234

philadelphivora, Liriomyza-------- 107,

113,291

Philadelphus-------------------- 238

philoxeri, Haplopeodes------------ 139

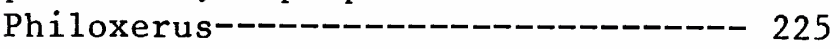

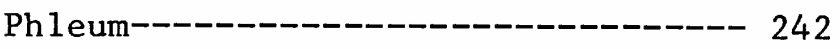

Phyla------------------------------ 239

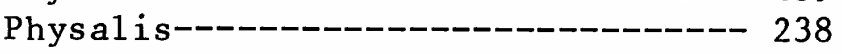

Phytagromyza----_---_-- 13, 87, 97

Phytobia-----

$10,13,14,15,71,73,270$

Phytoliriomyza---- 14, 16, 17, 151, 302

Phytomyza-------- 10, 14, 17, 172, 309

pictella, Liriomyza---_------ 125, 293

pilosella, Phytoliriomyza-------- 153 
pinguis, Agromyza----------------- 37

Piriquetta------------------------ 239

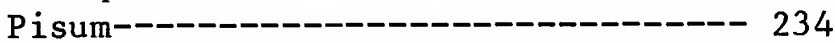

pith flecks-------------------- 10, 72

plagiata, Paraphytomyza------------ 164

Plantaginaceae---------------------- 236

plantaginis, Phytomyza------------ 190

Plantago---------------------------- 236

platyptera, Calycomyza--- 141, 142, 300

pleuralis, Amauromyza----------- 78, 83

plumea, Napomyza------------------- 169

plumiseta, Phytomyza--------------- 182

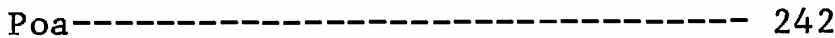

Poaceae (Gramineae)---------------- 240

poae, Chromatomyia------- 174, 210, 329

Poemyza---------------- 13, 87, 92

pollinosa, Cerodontha------------- 89

Polymnia. (See Smallanthus.)

polyphagy---------------------- 9

Polypodiaceae (ferns)-------- 225, 304

poolei, Cerodontha------------ 104, 284

poplar. (See Populus.)

populicola, Paraphytomyza---------- 162

populoides, Agromyza----------- 69, 264

Populus---------------------------- 237

Portulaca-------------------------- 236

Portulacaceae---------------------- 236

postgonite---------------------- 7

posticata, Nemorimyza---------- 81, 87

Potentilla------------------------- 237

potentillae, Agromyza------ 70, 71, 266

powelli, Phytobia---------------- 74

praecisa, Ophiomyia------------ 44, 256

praecox, Paraphytomyza------------- 163

prescutellar bristle------------ 5

preservation---------------------- 13

proboscidata, Melanagromyza---- 20, 246

proboscis--------------_----------- 5

promissa, Calycomyza----------- 8, 143

propepusilla, Liriomyza------- 128, 292

Protophytobia---_-_--_----------- 9

proxima, Agromyza----------------- 67

pruinosa, Agromyza------------ 76

Phytobia----------------- 75

pruni, Phytobia------------------- 73

prunivora, Phytobia-------------- 5

Prunus-----_--_---_---_-------- 237

pseudomilii, Chromatomyia---------- 206

Pseudonapomyza----------------- 16, 166

pseudoreptans, Agromyza-------- 65, 263

pseudorufipes, Agromyza------------ 263

ptarmicae, Liriomyza----- 108, 121, 123

Pteridium-------------------------- 225

Pteridomyza---------------------- 151

Ptochomyza------------------------ 179 pudica, Agromyza-----_--_-- 68, 267

pulchella, Phytoliriomyza----- 159, 304

Phytomyza---------- 216, 320

pulicaria, Agromyza-------------- 37

pulicarioides, Ophiomyia----------- 254

pullata, Liriomyza----------------- 292

pulloides, Liriomyza---_------ 123, 292

pupa------------------------------- 8

pupation------------------------ 8

pygmaea, Cerodontha---- 88, 92, 94, 279

pygminoides, Cerodontha-------- 95, 279

quadrisetosa, Liriomyza----------- 114

Melanagromyza-------- 14 ,

18,19

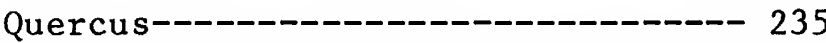

questa, Cerodontha------------ 105

quinta, Ophiomyia---------- 38, 45, 257

radicicola, Melanagromyza------ 33, 246

Radicula. (See Nasturtium.)

radius---------------------------- 5

Ranunculaceae--------------- 236

ranunculi, Phytomyza-------- 172, 179

ranunculoides, Phytomyza------ 217, 320

Ranunculus------------------------- 236

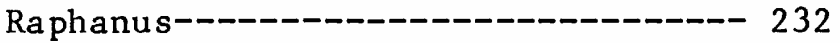

rearing------------------------- 11

regalensis, Chromatomyia------- 174 ,

213,330

remus, Amauromyza----------------- 85

reptans, Agromyza------ 57, 64, 65, 267

reverberata, Agromyza------------- 303

Rhamnaceae------------------------ 237

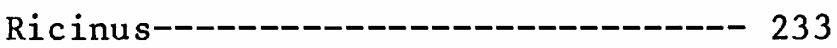

riparella, Melanagromyza------- 26, 247

riparia, Agromyza------------------ 247

Robinia----------_------_---------- 234

robiniae, Liriomyza------------- 108

robustella group, Phytomyza-------- 173

romulus, Amauromyza----_---_----- 86

Rorippa---------------------------- 232

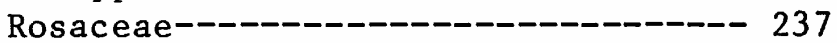

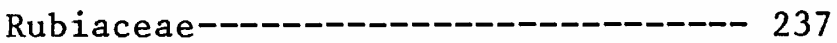

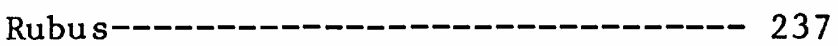

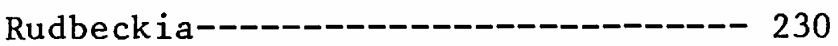

Ruellia---------------------------- 225

ruelliae, Melanagromyza----------- 21

rufipes, Phytomyza------------ 173, 180

rutiliceps, Japanagromyza-------- 13,

$53,54,69,261$

sabaziae, Liriomyza---------------- 126 sagehenensis, Melanagromyza---- 20, 247 Salicaceae----------------------- 237 salicis, Hexomyza------------ 36, 249 
Salix-_- Page

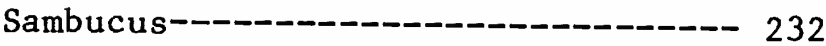

Sanguisorba-----_--_---_-_------- 237

Sanicula------------------------- 226

saniculae, Phytomyza---------- 218, 219

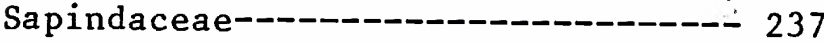

Saponaria--------------------- 233

sarothamni, Hexomyza------------- 34

saskatoonensis, Phytomyza-------- 173,

216,321

sativae, Liriomyza-------------- 8 , $9,107,125,128,132,133,292$

Saxi fragaceae-----------_------- 238

saximontana, Phytomyza------------- 199

schineri, Hexomyza----_-_------- 34, 36

schlingerella, Agromyza---------- 62

schlingeri, Liriomyza------------ 130

schmidti, Liriomyza------------- 8, 111

schusteri, Amauromyza---_--------- 84

Napomyza---------------- 170

scirpi, Cerodontha----_-_-_------ 98

Scirpus------------_---_---_----- 240

scleritica, Amauromyza------------ 84

Scrophularia--------------------- 238

Scrophulariaceae------------------- 238

scrophulariae, Melanagromyza---- 18, 20

scutellar bristle------------------ 5

scutellata, Metopomyza-------- 161, 162

scutel lum--------------------- 5

Secale---------_-------_--------- 242

semiposticata, Cerodontha---------- 279

Senecio--------------------------- 230

septentrionalis, Liriomyza------ 108 ,

116,122

Phytobia--------- 270

Setaria---------------------------- 242

setifrons, Melanagromyza---------- 19

setosa, Phytobia----_---_--_-_---- 77

sexta, Ophiomyia-----_---_--- 50, 257

shastensis, Ophiomyia---_---_--- 37, 44

sheath (of ovipositor)------------ 5

shiloensis, Ophiomyia---_--------- 259

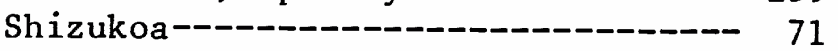

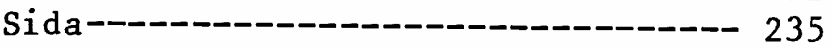

sidae, Calycomyza-----_-_-_-------- 148

Sidalcea-------------------------- 236

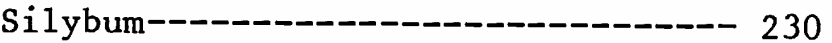

simcoensis, Cerodontha------------- 276

similata, Ophiomyia----_-

$33,40,41,258$

similis, Liriomyza---_---_------ 120

simplex, Ophiomyia------------ 38, 39

simplicoides, Hexomyza----- 34, 35, 250

Si symbrium----------------------- 232

Smallanthus------------------ 230
Pmilacina

Smilacina---n-n- 240

smilacinae, Liriomyza-------------- 135

Smilax----------------------------- 240

Solanaceae------------------------- 238

Sol anum--------------------------- 238

solidaginis, Calycomyza------- 141, 145

Solidago------------------------ 230

sonchi, Calycomyza----------------- 141

Sonchus---------------------------- 230

sonorensis, Phytomyza-------------- 220

Sophia. (See Descurainia.)

Sorghum------------------------ 242

sorosis, Liriomyza----------------- 114

specifica, Liriomyza--------------- 127

spenceri, Paraphytomyza--_---_----- 162

sperm pump--------------------- 7

Sphaenosciadium-------------------- 226

Spigelia-------------------------- 235

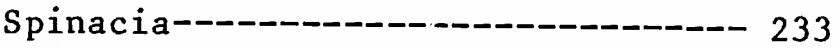

spiraeae, Agromyza------------- 71, 267

splendens, Liriomyza---------- 132, 293

splendida, Melanagromyza---------- 25

Phytomyza-----_---_- 175

spondylii, Phytomyza----- 200, 201, 202

squama------------------------- 5

squamal fringe-------------------- 5

stachyos, Liriomyza----------- 107, 115

Stachys-----------------_---------- 235

stegmaieri, Calycomyza----------- 145

stems---------- 12

sternite-------------------------- 7

stridulation----_-- 57, 72

Strobilanthes--------------------- 225

subalpina, Phytomyza--------- 188, 321

subangulata, Cerodontha--- 92, 100, 232

subasclepiadis, Liriomyza----- 130, 293

subcosta------------------------- 5

subdefinita, Ophiomyia------------ 48

subfamilies, key-------_--_------- 15

subinfumata, Amauromyza------------ 81

subpraecisa, Ophiomyia--------- 45, 258

subtenella, Phytomyza-------------- 186

subtilis, Phytomyza----------- 194, 200

subvirens, Melanagromyza--_------ 26

suda, Napomyza------------- 169, 309

sulfuriceps, Agromyza------------ 58

superciliosa, Cerodontha-------- 92, 93

surstylus------------------------ 7

symphoricarpi, Chromatomyia-------- 213

Symphoricarpos--------------------- 232

Synedrella------------------------- 231

syngenesiae, Chromatomyia-- 9, 174, 192

systemaics, general--_-_-_----- 13

tacita, Agromyza--------- 57, 66, 268 


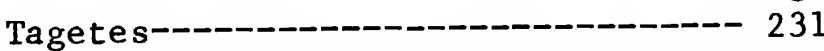

tamia, Melanagromyza------------ 18, 19

taraxaci, Liriomyza------ 107, 134, 294

taraxacocecis, Phytomyza---------- 322

Taraxacum------------------------- 231

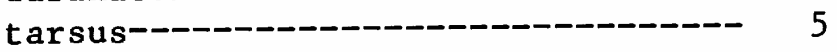

Tellima------------------------- 238

temeculensis, Cerodontha---------- 90

temperata, Liriomyza--------- 131, 294

tenel1a, Phytomyza---------- 187, 322

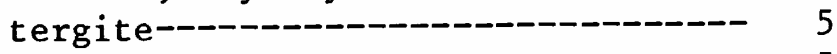

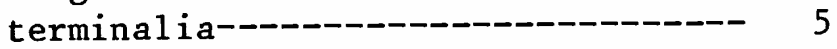

tetrica, Melanagromyza----- 18, 33, 247

texana, Ophiomya----------------- 11 , $46,48,50,257,259$

texella, Liriomyza---------- 133, 295

Ophiomyia------------- 40, 259

thalictrella, Phytomyza------------ 217

thalictrivora, Phytomyza---------- 218

Thal ictrum-------------------- 236

thompsoni, Cerodontha--------- 101, 103

Tiare 11 a-------------------------- 238

tiarellae, Chromatomyia------------ 204

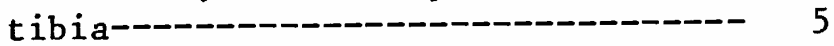

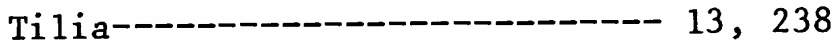

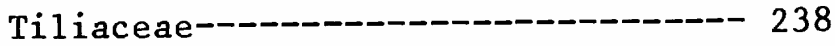

tiliae, Ophiomyia---------------- 13 ,

$$
34,37,38,41,260
$$

Tithonia------------------------- 231

tlingitica, Phytomyza------------- 183

togata, Liriomyza-------- 134, 140, 295

Tolmiea------------------------- 238

toschiae, Hexomyza------------ 34, 35

Tradescantia--------------------- 239

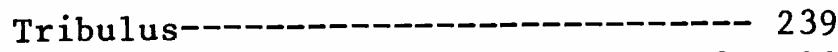

trifoliearum, Liriomyza---- 3, 118, 296

trifolii, Liriomyza--- 9, 107, 135, 297

Trifolium---------------------- 234

Triglochin------------------------- 235

triglochinae, Liriomyza----------- 109

Trilobomyza------------- 270, 272, 275

Trisetum----------------------- 242

trispinella, Melanagromyza-- 14, 18, 19

trispinosa, Melanagromyza------ 18, 19

Triticum---------------------- 242

Triumfetta--------------------- 238

trivittata, Phytomyza-------------- 176

trochanter----------------------- 5

Tropaeolaceae-------------------- 239

Tropaeolum--------------------- 239

tubula, Liriomyza----------------- 133

tularensis, Agromyza------------ 61

Turneraceae------------------- 239

turneri, Galiomyza--------------- 137

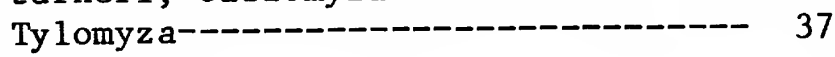

Typha---------------------------- 242

Typhaceae--------------------------- 242

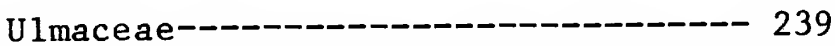

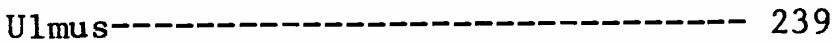

ultima, Me lanagromyza----------- 33, 247

Umbelliferae (Apiaceae)---------- 225

unidentified leaf mines------- 222, 302

urophorina, Liriomyza----------- 107

Urtica--------------------------- 239

Urticaceae----------------------- 239

urticella, Melanagromyza------- 18, 24

utahensis, Agromyza---------- 60, 268

Vahlodea------------------------ー- 242

vanduzeei, Phytobia--------------- 74

varia, Phytoliriomyza-------- 160, 305

variata, Liriomyza--------------- 109

varifrons, Agromyza------------ 58, 269

vectabilis, Melanagromyza-------- 29

venation, wing------------------ 4

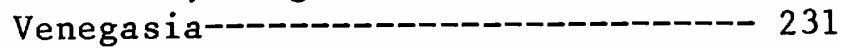

venegasiae, Liriomyza-------------- 129

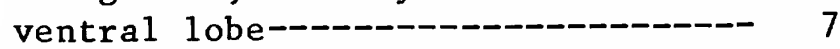

venturensis, Liriomyza------------ 132

Verbena--------------------------- 239

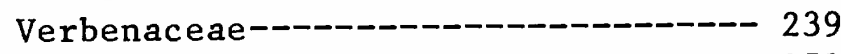

verbenae, Calycomyza-------------- 151

verbenicola, Liriomyza------------- 292

Verbesina------------- 231

verbesinae, Melanagromyza------ 29, 248

Vernonia-------------------------- 231

vernoniae, Melanagromyza---------- 23

vernoniana, Melanagromyza--------- 23

Veronica----------------------- 238

verticillatae, Phytomyza---------- 214

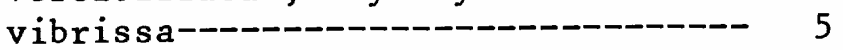

vibrissata, Ophiomyia----- $38,49,260$

Vicia---------------------------- 234

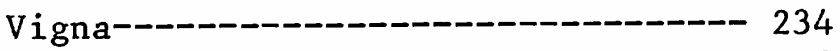

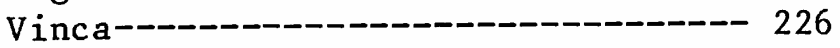

Viola--------------------------- 239

Violaceae------------------------- 239

violiphaga, Galiomyza------------ 137

violivora, Galiomyza----- 136, 137, 298

virens, Melanagromyza------------- 25

virginica, Liriomyza--------- 130, 297

virginiensis, Agromyza------ 57, 64

Mel anagromyza---- 25,248

Ophiomyia------- 46,260

viridis, Melanagromyza----------- 20

viridula, Japanagromyza-------- 53, 55

vockerothi, Agromyza------- 57, 70, 269

Galiomyza-------- 138, 299

Ophiomyia-------- 48, 261 
wood, damage to

vomitoriae, Phytomyza------------- 210

wahlgreni, Phytomyza----- 173, 195, 322 walleyi, Melanagromyza--------- 23, 249 waltoni, Phytobia------------- 76, 272 websteri, Hexomyza-------------- 36

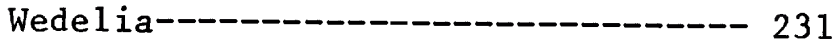
wedeliae, Melanagromyza----------- 31 willow. (See Salix.)

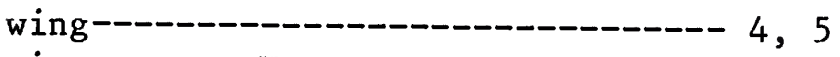
winnemanae, Hexomyza------- 19, 35, 250 Wisteria------------------------ 235

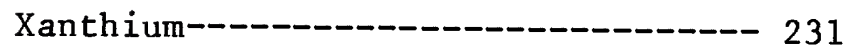

Xenophytomyza------------- 87, 91, 276 xylostei, Phytagromyza-------- 162, 306

yolensis, Ophiomyia------------ 37,44 Phytoliriomyza----------- 299

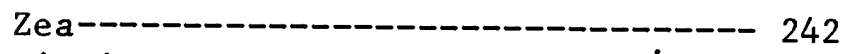

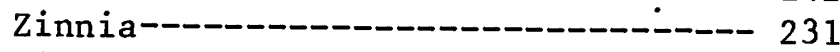

zinniae, Liriomyza------------ 122, 131

Zygophyllaceae------------------- 239 